NIST Special Publication 1239-3

NIST Conference Papers

Fiscal Year 2017

Volume 3:

Center for Nanoscale Science and Technology

Physical Measurement Laboratory

Compiled and edited by:

Andrea Medina-Smith

Kathryn Miller

Karen Wick

This publication is available free of charge from:

https://doi.org/10.6028/NIST.SP.1239-3

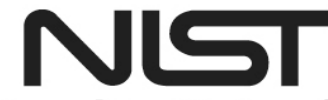

National Institute of Standards and Technology

U.S. Department of Commerce 
NIST Special Publication 1239-3

\title{
NIST Conference Papers Fiscal Year 2017
}

Volume 3:

\section{Center for Nanoscale Science and Technology Physical Measurement Laboratory}

\author{
Compiled and edited by: \\ Andrea Medina-Smith \\ Kathryn Miller \\ Karen Wick \\ Information Services Office
}

This publication is available free of charge from: https://doi.org/10.6028/NIST.SP.1239-3

August 2019

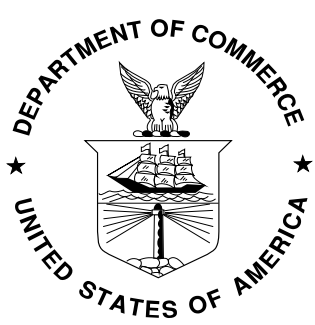

U.S. Department of Commerce Wilbur L. Ross, Jr., Secretary

National Institute of Standards and Technology Walter Copan, NIST Director and Undersecretary of Commerce for Standards and Technology 
Certain commercial entities, equipment, or materials may be identified in this document in order to describe an experimental procedure or concept adequately. Such identification is not intended to imply recommendation or endorsement by the National Institute of Standards and Technology, nor is it intended to imply that the entities, materials, or equipment are necessarily the best available for the purpose.

National Institute of Standards and Technology Special Publication 1239-3

Natl. Inst. Stand. Technol. Spec. Publ. 1239-3, 355 pages (August 2019)

CODEN: NSPUE2

This publication is available free of charge from: https://doi.org/10.6028/NIST.SP.1239-3 


\section{Foreword}

NIST is committed to the idea that results of federally funded research are a valuable national resource and a strategic asset. To the extent feasible and consistent with law, agency mission, resource constraints, and U.S. national, homeland, and economic security, NIST will promote the deposit of scientific data arising from unclassified research and programs, funded wholly or in part by NIST, except for Standard Reference Data, free of charge in publicly accessible databases. Subject to the same conditions and constraints listed above, NIST also intends to make freely available to the public, in publicly accessible repositories, all peer-reviewed scholarly publications arising from unclassified research and programs funded wholly or in part by NIST.

This Special Publication represents the work of Center for Nanoscale Science and Technology and Physical Measurement Laboratory researchers at professional conferences, as reported in Fiscal Year 2017.

More information on public access to NIST research is available at https://www.nist.gov/open. 


\section{Table of Contents}

Center for Nanoscale Science and Technology .................................. SP

Kolmakov, Andrei; Tselev, Alexander. "Probing Nanoscale Objects in Liquids

through Membranes with Near-Field Microwave Microscopy." Paper presented at

2015 European Microwave Conference (EuMC), Paris, France. September 6, 2015 -

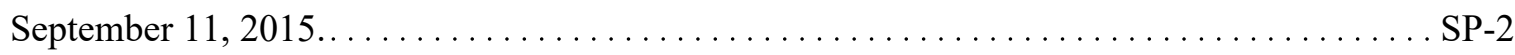

Collins, Rob; Haney, Paul; Koirala, Prakash; Yoon, Heayoung; Zhitenev, Nikolai. "

Models for Low Energy Electron Beam Induced Current Experiments in

polycrystalline thin film photovoltaics." Paper presented at 42nd IEEE Photovoltaic

Specialists Conference, New Orleans, LA. June 15, 2015 - June 19, 2015 . . . . . . . . . . . SP-9

Haney, Paul; Li, Junwen. "Molecular Alignment and Rashba Splitting in

Organometal Halide Perovskite CH3NH3PbI3 Absorbers." Paper presented at 42nd

IEEE Photovoltaic Specialists Conference, New Orleans, LA. June 14, 2015 - June

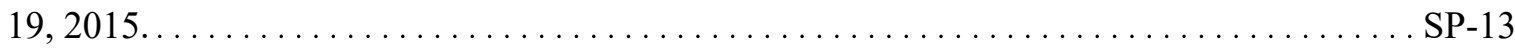

An, Sang; Basham, James; Haney, Paul; Yoon, Heayoung; Yoon, Yohan; Zhitenev, Nikolai. "Local photocarrier dynamics in CdTe solar cells under optical and electron beam excitations." Paper presented at Photovoltaic Specialist Conference (PVSC), 2015 IEEE 42nd, New Orleans, LA. June 14, 2015 - June 19, 2015. . . . . . . . . . . SP-17

An, Sang; Centrone, Andrea; Chae, Jungseok; Katzenmeyer, Aaron; Schumacher, Joshua; Yoon, Heayoung; Yoon, Yohan; Zhitenev, Nikolai. "Local measurements of photocurrent and band gap in CdTe solar cells." Paper presented at 31st European Photovoltaic Solar Energy Conference and Exhibition (EU PVSEC 2015),

Hamburg, Germany. September 14, 2015 - September 18, 2015.

Kolmakov, Andrei; Tselev, Alexander; Velmurugan, Jeyavel. "Imaging in Liquids through Ultra-thin Membranes: A Comparative Analysis of Scanning Electron and Scanning Microwave Microscopies." Paper presented at Microscopy \& Microanalysis, Columbus, OH. July 24, 2016 - July 28, 2016.

Kolmakov, Andrei; LaVan, David; Stevanovic, Ana; Velmurugan, Jeyavel; Yi, 
Feng. "Bridging the Pressure Gap in Electron Beam Induced Current Microscopy: Imaging Charge Transport in Metal Oxide Nanowires under Atmospheric Pressures." Paper presented at Microscopy \& Microanalysis, Columbus, OH. July 24, 2016 - July 28, 2016. SP-25

Alec, Talin; Kolmakov, Andrei; Marina, Leite; Yulaev, Alexander. "Li Diffusion in All-Solid-State Batteries Imaged Through Optically and Electron Transparent Electrodes." Paper presented at Microscopy \& Microanalysis, Columbus, OH. July 24, 2016 - July 28, 2016.

Kolmakov, Andrei; LaVan, David; Stevanovic, Ana; Velmurugan, Jeyavel; Yi, Feng. "Versatile fluidic cell for in-situ electrochemical measurements in SEM." Paper presented at Microscopy \& Microanalysis, Columbus, OH. July 24, 2016 July $28,2016$.

Gaury, Benoit; Haney, Paul. "Minority-carrier dynamics in semiconductors probed by two-photon microscopy." Paper presented at 43rd Photovoltaic Specialists Conference, Portland, OR. June 5, 2016 - June 10, 2016. SP-31

Aksyuk, Vladimir; Roxworthy, Brian. "Subdiffraction optical motion transduction using a scalable plasmomechanical platform." Paper presented at Frontiers in Optics 2016, Rochester, NY. October 17, 2016 - October 21, 2016.

Aksyuk, Vladimir; Michels, Thomas; Rangelow, Ivo. "Cantilever array with optomechanical read-out and integrated actuation for simultaneous high sensitivity force detection." Paper presented at International Conference on Optical MEMS and Nanophotonics, Singapore. July 31, 2016 - August 4, 2016.

Robinson, Matthew; Slaughter, Gymama; Tyson, Joel; Zhang, Chen. " Neuroelectronic Device Process Development and Challenge." Paper presented at Optical Microlithography, San Jose, CA. February 26, 2017 - March 2, 2017.

Ilic, Bojan; Krylov, Slava; Stella, Lulinski; Tsvi, Shmilovich. "Parametric amplification of acoustically-excited micromechanical oscillators using fringing electrostatic fields." Paper presented at 9th European Nonlinear Dynamics Conference, ENOC 2017, Budapest, Hungary. June 25, 2017 - June 30, 2017. 
Gilat, Rivka; Ilic, Bojan; Krylov, Slava; Medina, Lior. "Open-loop, self-excitation in a bistable micromechanical beam actuated by a DC electrostatic load." Paper presented at the 30th IEEE International Conference on Micro Electro Mechanical Systems (MEMS2017), Las Vegas, NV. January 22, 2017 - January 26, 2017.. . . . . . . . . SP-61

Hussaini, Zahra; Lin, Pin; Natarajan, Bharath; Sharma, Renu. "Measuring the Phase Transformation Kinetics Under Non-Equilibrium Conditions from Time Resolved High Resolution TEM Images." Paper presented at Microscopy \& Microanalysis 2017, St Louis, MO. July 30, 2017 - August 3, 2017. . . . . . . . . . . . . . . . . . . SP-65

Physical Measurement Laboratory . . . . . . . . . . . . . . . . . . . . . . . . . SP-67

Cular, Stefan; Lipe, Thomas. "A Simple Model for the Prediction of AC-DC

Difference of Multi-Junction Thermal Converters." Paper presented at Conference on Precision Electromagnetic Measurements (CPEM)2016, Ottawa, Canada. July 10, 2016 - July 15, 2016.

Oh, Semin; Ohno, Yoshihiro. "Vision experiment ii on white light chromaticity for lighting." Paper presented at CIE Lighting Quality and Energy Efficiency 2016, Melbourne, Australia. March 3, 2016 - March 5, 2016. SP-70

Miller, Carl; Nadal, Maria; Ohno, Yoshihiro; Tsai, Benjamin; Zong, Yuqin. "SolidState Lighting Measurement Assurance Program Summary with Analysis of Metadata." Paper presented at CIE Lighting Quality and Energy Efficiency 2016, Melbourne, Australia. March 3, 2016 - March 5, 2016.

Litorja, Maritoni; Lorenzo, Simon; Sevick Muraca, Eva; Zhu, Banghe. "Lambertian nature of tissue phantoms for use as calibrators in near infrared fluorescence imaging." Paper presented at Molecular-Guided Surgery: Molecules, Devices, and Applications II (Conference 9696), San Francisco, CA. February 12, 2016 February 18, 2016. SP-86

Brown, Thomas; Germer, Thomas; Oliver, James; Sharma, Katelynn; Smith, Christopher; Zuegel, Jonathan. "Scattered Light Analysis of Birefringent Coatings for Distributed Polarization Rotators." Paper presented at Frontiers in Optics 2016, Rochester, NY. October 17, 2016 - October 21, 2016. SP-92

Baek, Burm; Benz, Samuel; Donnelly, Christine; Dresselhaus, Paul; Hopkins, Peter; 
Pufall, Matthew; Rippard, William; Russek, Stephen; Schneider, Michael. " Stochastic Single Flux Quantum Neuromorphic Computing using Magnetically Tunable Josephson Junctions." Paper presented at 2016 IEEE International Conference on Rebooting Computing (ICRC), San Diego, CA. October 17, 2016 October 19, 2016.

Ohno, Yoshihiro. "Color Quality Metrics - Recent Progress and Future Perspective." Paper presented at the 15th International Symposium of Science and Technology of Lighting, Kyoto, Japan. May 23, 2016 - May 27, 2016.

Baumgart, Helmut; Campbell, Jason; Cheung, Kin; Kim, Jihong; Nminibapiel, David; Shrestha, Pragya. "Compliance-Free Pulse Forming of Filamentary RRAM." Paper presented at ECS PRIME 2016, Honolulu, HI. October 2, 2016 October 7, 2016. SP-105

Foldyna, Martin; Germer, Thomas; Mrazkova, Zuzana. "Mueller matrix bidirectional reflectance distribution function measurements and modeling of textured silicon surfaces." Paper presented at SPIE Optics and Photonics, San Diego, CA. August 28, 2016 - August 29, 2016. SP-117

Bhadriraju, Kiran; Nablo, Brian; Niu, Pengfei; Reyes-Hernandez, Darwin. "FineTuning Electrical Flow Rate Sensing in Deformable Channels." Paper presented at the 20th International Conference on Miniaturized Systems for Chemistry and Life Sciences, Dublin, Ireland. October 9, 2016 - October 13, 2016. SP-130

Li, Chunhui; Mickan, Bodo; Vallet, J-P; Wright, John. "Extended Data Analysis of Bilateral Comparisons with Air and Natural Gas up to $5 \mathrm{MPa}$." Paper presented at FLOMEKO 2016, Sydney, Austria. September 26, 2016 - September 29, 2016.

Gillis, Keith; Johnson, Aaron; Shinder, Ida; Wright, John. "Back Pressure Ratio and the Transonic Resonance Mechanism of Low Unchoking in Critical Flow Venturis." Paper presented at FLOMEKO 2016, Sydney, Australia. September 26, 2016 - September 29, 2016.

Ahmed, Zeeshan; Klimov, Nikolai. "Ring Resonator Thermometry." Paper presented at IEEE Photonics Conference, Waikoloa, HI. October 10, 2016 -

October 13, 2016. SP-151 
Alberding, Brian; Biacchi, Adam; Hagmann, Joseph; Heilweil, Edwin; Hight Walker, Angela; Le, Son; Richter, Curt. "Elucidating the Electronic Properties of Colloidally-Synthesized 2D Nanoelectronic Device Components." Paper presented at 2016 International Semiconductor Device Research Symposium, Bethesda, MD.

December 7, 2016 - December 9, 2016.

Gokhale, Vikrant; Gorman, Jason. "Direct Measurement of Dissipation in Phononic Crystal and Straight Tethers for MEMS Resonators." Paper presented at IEEE International Conference on Micro Electro Mechanical Systems, Las Vegas, NV. January 22, 2017 - January 26, 2017. SP-154

Dwyer, Kevin; Kim, Hyun Soo; Pomeroy, Joshua; Ramanayaka, Aruna; Simons, David; Tang, Ke. "Enriching and purifying silicon epilayers for quantum information." Paper presented at International Semiconductor Device Research Symposium, Bethesda, MD. December 7, 2016 - December 9, 2016. SP-158

Tondare, Vipin; Villarrubia, John; Vladar, Andras. "Assessing Scanning Electron Microscopy Stereophotogrammetry Algorithms with Virtual Test Samples." Paper presented at 2017 International Conference on Frontiers of Characterization and Metrology for Nanoelectronics, Monterey, CA. March 21, 2017 - March 23, 2017.

Charriere, Andree; Nadal, Maria; Zarobila, Clarence. "Comparison of specular gloss values from a spectrally resolved five-axis goniometer and a reference goniophotometer." Paper presented at Material Appearance, Burlingame, CA. December 29, 2016 - January 2, 2017. SP-163

Fonville, Blair; Hardis, Jonathan; Matsakis, Demetrios. "Time and frequency from electrical power lines." Paper presented at 48th Annual Precise Time and Time Interval Systems and Applications Meeting, Monterey, CA. January 30, 2017 -

February 2, 2017. SP-171

Allen, Richard; Hood, Colleen; Payne, Beverly. "Improvements in Accelerometer Calibration at NIST Using Digital Vibrometry." Paper presented at 87th Shock and Vibration Symposium, New Orleans, LA. October 17, 2016 - October 20, 2016 SP-186

Afridi, Muhammad; Gaitan, Michael; Geist, Jon. "Analysys and Protocol for Characterizing Intrinsic Properties of Three-Axis MEMS Accelerometers Using a 
Gimbal Rotated in the Gravitational Field." Paper presented at IMEKO 23rd TC3, 13th TC5 and 4th TC22 International Conference, Helsinki, Finland. May 30, 2017 - June 1, 2017.

Kopanski, Joseph; Obeng, Yaw; You, Lin. "Reference Materials to Enable Precise and Accurate Imaging with Electrical Scanning Probe Microscopes." Paper presented at the 2017 International Conference on Frontiers of Characterization and Metrology for Nanoelectronics, Monterey, CA. March 21, 2017 - March 23, 2017.

Egan, Patrick; Hendricks, Jay; Ricker, Jacob; Stone Jr., Jack. "Stuck in a moment: A view from the MIRE." Paper presented at ASPE 2017 Topical Meeting, Tucson, AZ. April 23, 2017 - April 26, 2017. SP-200

Barnes, Bryan; Henn, Mark Alexander; Silver, Richard; Sohn, Martin; Zhou, Hui. "Assessing Quantitative Optical Imaging for Realizing In-die Critical Dimension Metrology." Paper presented at 2017 International Conference on Frontiers of Characterization and Metrology for Nanoelectronics, Monterey, CA.

March 21, 2017 - March 23, 2017.. SP-208

Barnes, Bryan; Henn, Mark Alexander; Zhou, Hui. "Evaluating the Effects of Modeling Errors for Isolated Finite 3-D Targets." Paper presented at SPIE Advanced Lithography 2017: Metrology, Inspection, and Process Control for Microlithography XXXI, San Jose, CA. February 27, 2017 - March 2, 2017.

Barnes, Bryan; Henn, Mark Alexander; Silver, Richard; Sohn, Martin; Zhou, Hui. " Assessing the wavelength extensibility of optical patterned defect inspection." Paper presented at SPIE Advanced Lithography 2017: Metrology, Inspection, and Process Control for Microlithography XXXI, San Jose, CA. February 20, 2017 March 23, 2017. SP-221

Doiron, Theodore; Ren, Wei; Stanfield, Eric; Stoup, John. "Characterization of the Dimensions of Internal Gap in a Glass Artifact Using a Laser Triangulation Probe." Paper presented at NCSLi 2017 Workshop \& Symposium, National Harbor, MD. August 13, 2017 - August 17, 2017. SP-233

Echeverria Villagomez, Salvador; Harris, Georgia; Mercader, Flora; Veraza, Adriana. "Metrology Outreach and Training: A Fulbright Experience in Mexico." 
Paper presented at Precision and Performance with Measurement Science, Oxon Hill, MD. August 13, 2017 - August 17, 2017.

Postek, Michael; Villarrubia, John; Vladar, Andras. "Nanoelectronics Dimensional Metrology: Understanding the Differences between Secondary and Backscattered Electron Imaging." Paper presented at 2017 International Conference on Frontiers of Characterization and Metrology for Nanoelectronics, Monterey, CA. March 21, 2017 - March 30, 2017.

Cooksey, Catherine; Eppeldauer, George; Podobedov, Vyacheslav. "UV scale calibration transfer from an improved pyroelectric detector standard to field UV-A meters and $365 \mathrm{~nm}$ excitation sources." Paper presented at SPIE Defense + Security, Anaheim, CA. April 9, 2017 - April 13, 2017. SP-261

Barnes, Bryan; Henn, Mark Alexander; Silver, Richard; Sohn, Martin; Zhou, Hui. "Optimizing image-based patterned defect inspection through FDTD simulations at multiple ultraviolet wavelengths." Paper presented at Modeling Aspects in Optical Metrology, Munich, Germany. June 25, 2017 - June 29, 2017.

Cheok, Geraldine; Muralikrishnan, Balasubramanian; Rachakonda, Prem; Sawyer, Daniel; Shilling, Katharine. "An Overview of Activities at NIST Towards the Proposed ASTM E57 3D Imaging System Point-to-point Distance Standard." Paper presented at 33rd Annual Coordinate Metrology Society Conference, Snowbird, UT. July 17, 2017 - July 21, 2017.

Bao, Yiliang; Gorman, Jason; LeBrun, Thomas; Zhou, Feng. "A Photonic MEMS Accelerometer with a Low-Finesse Hemispherical Microcavity Readout." Paper presented at 2017 International Conference on Optical MEMS and Nanophotonics, Santa Fe, NM. August 13, 2017 - August 17, 2017.

Milchberg, H; Wahlstrand, Jared; Zahedpour, Sina. "Bound Electron Nonlinearity Beyond the Ionization Threshold." Paper presented at OSA Frontiers in Optics 2017, Washington, DC. September 17, 2017 - September 21, 2017. SP-300

Cooksey, Gregory; Hands, James. "Integrated Optical Waveguides for in situ Microflow Measurements." Paper presented at microTAS, Savannah, GA. October 22, 2017 - October 26, 2017. 
Bittle, Emily; Hacker, Christina; Li, Qiliang; Natoli, Sean; Pookpanratana, Sujitra; Ren, Tong; Richter, Curt; Robertson, Joseph; Zhu, Hao. "Integration of RedoxActive Diruthenium-based Molecular Layer onto Electrodes for Memory Device Applications." Paper presented at AVS 63rd International Symposium, Nashville, TN. November 6, 2016 - November 11, 2016.

Phillips, Steven; Shakarji, Craig. "Should the repeatability of the instrument under test be included in test uncertainty?." Paper presented at NCSLi 2017 Workshop \& Symposium, National Harbor, MD. August 15, 2017 - August 17, 2017.

Cooksey, Catherine; Eppeldauer, George; Hanssen, Leonard; Podobedov, Vyacheslav. "Low-NEP pyroelectric detectors for calibration of UV and IR sources and detectors." Paper presented at International Society for Optics and Photonics (SPIE) Optical Engineering and Applications Symposium, San Diego, CA. August 8, 2017 - August 9, 2017.

Shakarji, Craig; Srinivasan, Vijay. "Computational investigations for a new, constrained least-squares datum definition for circles, cylinders, and spheres." Paper presented at The ASME 2016 International Mechanical Engineering Congress \& Exposition, Phoenix, AZ. November 11, 2016 - November 17, 2016. SP-326

Shakarji, Craig; Srinivasan, Vijay. "Optimality Conditions for Constrained LeastSquares Fitting of Circles, Cylinders, and Spheres to Establish Datums." Paper presented at The ASME 2017 International Design Engineering Technical Conferences \& Computers and Information in Engineering Conference, Cleveland, OH. August 6, 2017 - August 9, 2017. 


\section{Center for Nanoscale Science and Technology}

Work of researchers at professional conferences as reported in Fiscal Year 2017 


\title{
Probing Nanoscale Objects in Liquids through Membranes with Near-Field Microwave Microscopy
}

\author{
Alexander Tselev \\ Center for Nanophase Materials Sciences \\ Oak Ridge National Laboratory \\ Oak Ridge, TN 37831, USA \\ E-mail: atselev@utk.edu \\ Andrei Kolmakov \\ Center for Nanoscale Science and Technology \\ National Institute of Standards and Technology \\ Gaithersburg, MD 20899, USA \\ E-mail: andrei.kolmakov@ nist.gov
}

\begin{abstract}
In this work, scanning near-field microwave imaging was implemented to test the feasibility of the approach for in-situ studies of nanoscale objects immersed in liquids under thin dielectric membranes. It was found that mechanical strength and stability of SiN membranes on Si frames are sufficient for contact mode imaging in a standard AFM setup. Model polystyrene particles immersed in glycerol in contact with the membrane from the cavity side could be reliably detected. The probing depth of this imaging mode can be estimated to be approximately $100 \mathrm{~nm}$.
\end{abstract}

Keywords - microwave imaging; scanning probe microscopy; impedance measurement; atomic force microscopy

\section{INTRODUCTION}

The fast development of nanotechnology enables study and control of materials and devices at the level down to single atoms. Valuable knowledge can be gained through observations of nanometer- and atomic-scale objects and events in operando such as living cells, photo- and electrochemical reactions. This need boosted the development of in-situ electron microscopy techniques with

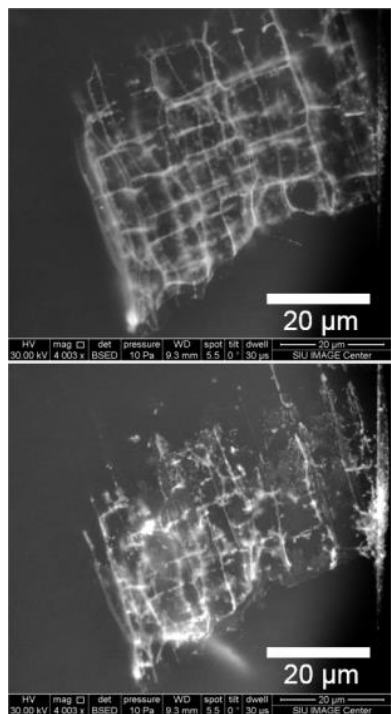

Fig. 1. Plant tissue immersed in water and imaged in an SEM through polyimide membrane. Top: Initial state; the image was acquired immediately after microscope adjustments at a neighboring spot. Bottom: the state after ca. $1 \mathrm{~min}$. of exposure to the electron beam. The beam-induced degradation of the cellular walls is presumably due to water radiolysis and formation of chemically active species such as $\mathrm{H}_{2} \mathrm{O}_{2}$. 
samples imaged in liquid cells. In such cells, objects of interest are immersed in liquids confined by capsule-like structures, where one of the capsule dimensions is small enough to be transparent to electron beams [1]. However, electron energies and intensities in these experiments need to be high enough to penetrate through such membranes with acceptable attenuation. Typically, energies are in the tens of keV range for scanning electron microscopes (SEM) and hundreds of keV for transmission electron microscopes (TEM). Such energies are far above the thresholds for breaking chemical bonds in materials, which may eventually lead to sample destruction because of radiation-induced damage and unwanted chemical reactions. For example, 79 different chemical reaction were identified as occurring concurrently under an electron beam of a TEM in water [2]. The images in Fig. 1 show, for instance, a consequence of one-minute-long exposure of a plant tissue in water to electron beam of a $30 \mathrm{keV}$ energy in an SEM. Apparent degradation of the cellular walls indicates that the radiation damage is unacceptably large for the selected imaging parameters. Similarly, electron damage and electron beam induced artifacts can be observed in many other soft matter objects such as polymers and electrolytes. Therefore, alternative imaging technique operating at smaller radiation energies are needed for radiation-sensitive specimens. Imaging using optical wavelengths would be an obvious candidate. However, the far-field optical imaging is limited in spatial resolution by diffraction effects. To achieve special resolution close to $100 \mathrm{~nm}$ and below, typical for in-situ electron microscopy of encapsulated samples, near-field imaging should be implemented. In a near-field, the special resolution limit is set by the scanning probe size rather than by the radiation wavelength, and one can use a radiation with wavelengths several orders of magnitude larger that the size of the probe or the field-concentrating element.

Microwave frequencies of about $1 \mathrm{GHz}$ are the lowest where high-resolution near-field imaging was achieved [3]. Its important distinction is a very low energy photons - in the $\mu \mathrm{eV}$ range. At this energy scale, the energy is largely adsorbed by collective excitations in matter, and only heating can potentially lead to irreversible changes in the materials. Therefore, the radiolysis as well as radiation damage associated with the electron microscopies can be completely eliminated. Simultaneously, microwave radiation can penetrate into solids and liquids including those, which are opaque for optical radiation. As an example, microwaves were proposed for medical tomography and disease diagnostics [4-6] and used for underground and through-the-wall surveillance. In light of these properties, microwaves may have a significant potential for in-situ imaging objects in their native environment including biological, reactive, toxic, and others. While the history of near-field imaging goes back a few decades [3], the recent realization of the near-field microwave imaging on atomic-force microscopy (AFM) platforms with precise probe-sample distance control not only dramatically improves the spatial resolution [7-11], but also potentially offers new imaging capabilities taking advantage of penetrating ability of microwave fields $[7,9,12,13]$

\section{METHODS}

The goal of this work was to analyze and experimentally prove the applicability of near-field microwave imaging for studies of objects immersed into liquids under a few tens nm thick dielectric membranes. The dielectric membranes serve as molecularly impermeable "walls" separating the liquids from the environment, however allowing the fields from the probe to reach the objects in the liquid. The main questions to be answered were the mechanical stability of the membrane under the AFM tip and the evaluation of the microscope sensitivity needed for informative imaging in this configuration.
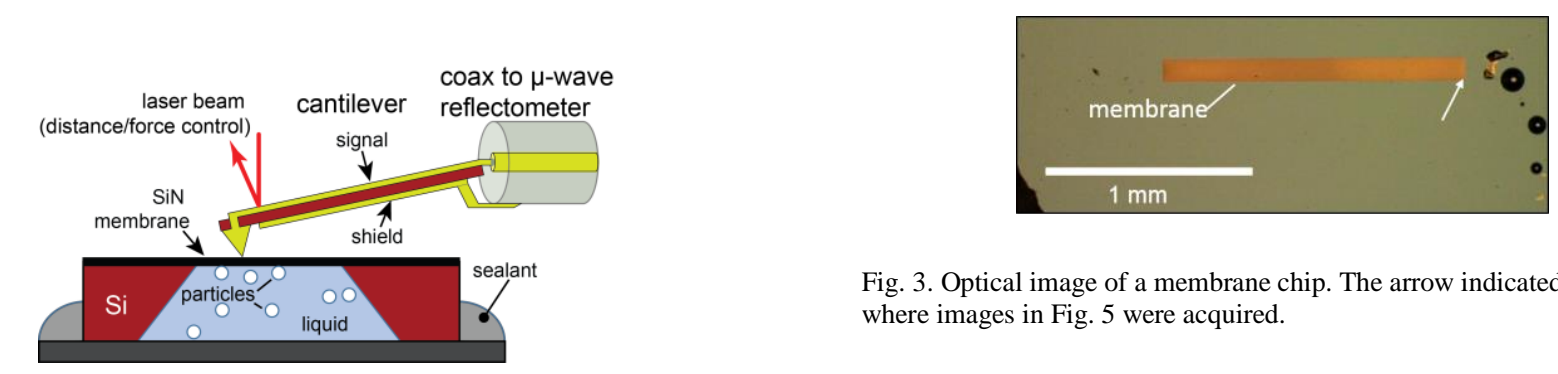

Fig. 3. Optical image of a membrane chip. The arrow indicated the corner, where images in Fig. 5 were acquired.

Fig. 2. Schematic of the experimental setup.

\section{A. Experimental Setup}

Fig. 2 displays a schematic of the near-field microwave imaging system employed in the experiments (Scan Wave, Prime Nano, Inc., Palo Alto, CA, USA). The probe is made as a standard AFM cantilever-based probe with a metallic sensing tip at the free cantilever end. The probe is completely shielded on the sample side, including the cantilever. The waveguiding structure runs to the very pyramid (sensing tip) and is geometrically close to a microstrip line. Importantly, the cantilevers are relatively soft with a spring constant of about $0.8 \mathrm{~N} / \mathrm{m}$, which can be used as advantage for imaging over thin membranes. The relatively moderate stiffness of the cantilevers allows for the formation of a mechanically and electrically stable contacts between the probe and the sample without the membrane being punched or moved by the probe while scanning, which is a necessary prerequisite for a successful AFM-based imaging. 
The probe is installed in a commercial AFM microscope with imaging performed with a probe in contact with the sample (contact mode). Generally, several images are obtained simultaneously within one scan frame corresponding to different microscope signals: height, deflection error, and microwave imaging channels.

The microwave detection system is based on the reflectometer principle. The amplitude and phase of the reflected waves are determined by the impedance of the tip-sample system, which is in turn determined by the average-over-cycle electric or magnetic energy stored in the near, evanescent, fields at the probe. For the tip-style probes, the energy is overwhelmingly stored in the electric component of the near fields, and therefore, the probes of this type are sensitive to sample dielectric permittivity and conductivity [3]. Accordingly, the output signals are provided as two channels: capacitive (C) and resistive (R), and this technique is named scanning Microwave Impedance Microscopy (sMIM). The probed sample volume is determined by the spatial extent and distribution of the fields. Since the electric field distribution has a singular character near the tip apex, the characteristic length scales in all three dimensions contributing to formation to the microscope signal and image contrast are defined by the tip apex radius. The nominal radius of the used tips is about $40 \mathrm{~nm}$ according to the manufacturer.

In the experiments, we used commercially available SiN membranes on Si frames designed as a support in TEM applications. We have chosen membranes of $50 \mathrm{~nm}$ thickness with the window shape shown in the optical image in Fig. 3. Such membranes are a good initial compromise between the membrane thickness and the mechanical strength. For the measurements, the cavities under the membranes were filled with glycerol. The high viscosity of glycerol was expected to facilitate the mechanical stability of the membrane under the localized pressure imposed by the probe. Prior to that, polystyrene particles of a diameter of $\approx 1.5 \mu \mathrm{m}$ were placed in the cavity. The cavity was covered by a $10 \mathrm{~mm}$-diameter steel disk and hermetically sealed with a glue. After that, the sMIM imaging was performed through the membrane at a microwave frequency of $\approx 3 \mathrm{GHz}$ and a generator power set to $-20 \mathrm{dBm}$. 


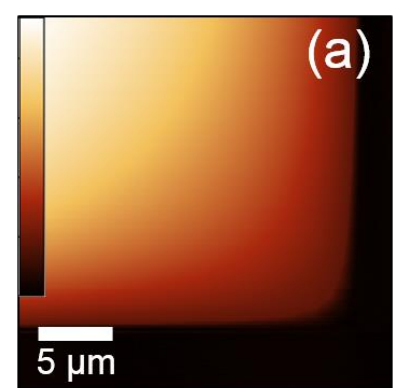

\section{B. Numerical Modeling}

To make an assessment of the applicability of the near-field microwave imaging for nanoscale objects immersed in liquids under a membrane, we have performed numerical calculations of the quasi-static electric field distribution in the tip-sample system in the presence of a particle in a glycerol. The modeling and calculations were carried out in quasi-static approximation employing a commercial finite elements analysis package. The model layout is displayed in Fig. 4. The model is axisymmetric. The tip is in contact with a membrane of a $50 \mathrm{~nm}$ thickness and a dielectric permittivity $\varepsilon=7.5\left(\mathrm{Si}_{3} \mathrm{~N}_{4}\right)$. The space under the membrane is filled with a dielectric of a permittivity $\varepsilon=40$ (glycerol). A dielectric particle $(\varepsilon=2.5)$ of a spherical shape and a diameter of $500 \mathrm{~nm}$ is in contact with the membrane from the cavity side. The tip-sample contact radius is set to $45 \mathrm{~nm}$.

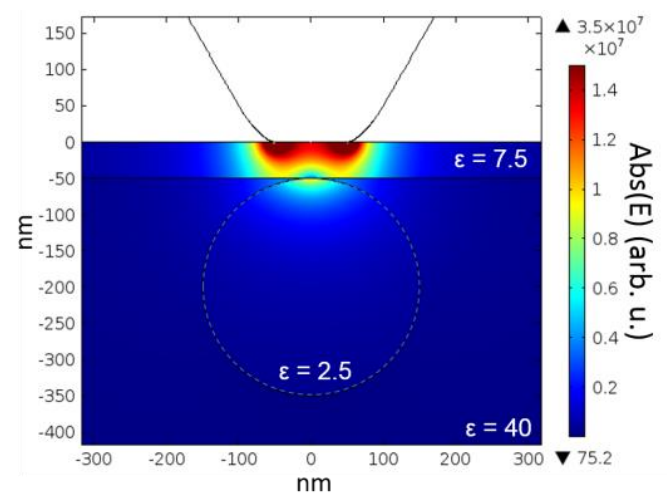

Fig. 4. Calculated distribution of the absolute value of the electric field near the probe tip apex in the presence of a dielectric particle. 


\section{RESULTS AND DISCUSSION}

Fig. 4 show a calculated distribution of the electric field in the vicinity of the tip apex. As evident, there exists an appreciable coupling between the tip and the particle, however, only the top part of the particle can be accessed by the penetrating probing field. The calculation results also reveal a significant dielectric screening by the membrane due to a relatively large difference in the dielectric permittivities of $\mathrm{SiN}$ and glycerol.

Next, we performed imaging experiments with the setup described in the previous section. Fig. 5 presents one of typical sets of simultaneously acquired $25 \times 25 \mu \mathrm{m}^{2}$ images of polystyrene particles in glycerol. The figure displays (from top to bottom) images of height, deflection error, and sMIM-C channel. In this particular case, the particles agglomerated in a corner of the membrane window. The particles are well visible against the background of glycerol. Both the particles $(\varepsilon=2.5)$ and Si frame $(\varepsilon=12)$ are darker in the image compared to glycerol $(\varepsilon=40)$, as expected due to a higher dielectric constant of glycerol for the used microscope settings. The images also reveal a high stability of the membrane under scanning in contact mode. However, a significant fraction of the particles seen in the sMIM-C image appeared in the deflection error image as well, indicating the deformation of the membrane under the probe. This also evidences that the particles seen in the microwave image are in contact with the back side of the membrane. The structure formed by the particles is ordered with the main pattern corresponding to a hexagonal close-packed structure signaling that the particles are in contact with each other. Therefore, the distance between the particles in the images is equal to particles diameter. The image analysis reveals that the distances are narrowly distributed at the expected $1.5 \mu \mathrm{m}$ corresponding to the nominal particle diameter. Since the apparent diameter of the darker spots in the sMIM-C image is about 0.25 to 0.4 of the particle diameter, the probed depth below the membrane can be preliminary estimated to be in the range from about $50 \mathrm{~nm}$ to $110 \mathrm{~nm}$ based on the spherical shape of the particles.

To further estimate the sensitivity and the spatial resolution of the microwave probing, we have imaged a smaller, $7 \mathrm{x} 7 \mu \mathrm{m}^{2}$, region farther away from the membrane widow corner. For these images, the tip-sample set force was reduced by a factor of about 2.5. The height and sMIM-C images of an area with two isolated polystyrene particles under the membrane are shown in Figs. 6a and b. As seen, any signs of the particle presence are completely absent in the topographic image Fig. 6a; however, the image is streaky indicating some degree of instability in the tip-membrane contact causing the noise in the height image at a level of a few nm. Still, good-quality microwave images could be obtained. In the microwave image, the particle contrast has a characteristic profile expected for the spherical shape. The line profile across one of the spots in Fig. $6 \mathrm{~b}$ shows a peak-like shape with base-line peak width of about $1.5 \mu \mathrm{m}$ (Fig. 6c), suggesting that the signal variation is imposed by the whole particle diameter. It could be inferred that, in the tested configuration the sensitivity of the microwave imaging is sufficient for probing up to a depth of about $1 \mu$ m and even more. However, the influence of the probe side field has to be carefully analyzed and taken into account to verify this conclusion. Further experiments are planned to test the imaging mode in different liquid-particle configurations together with the numerical modeling and varying the membrane thicknesses.

\section{CONCLUSIONS}

In conclusion, we have demonstrated the feasibility of the near-field microwave imaging for in-situ probing of nanoscale objects under thin membranes. The $50 \mathrm{~nm}$ thick SiN membrane stability and mechanical strength are adequate to enable contact mode imaging in a standard AFM setup with the membrane cavity filled with a viscous liquid. The sensitivity of the near-filed images used in the 


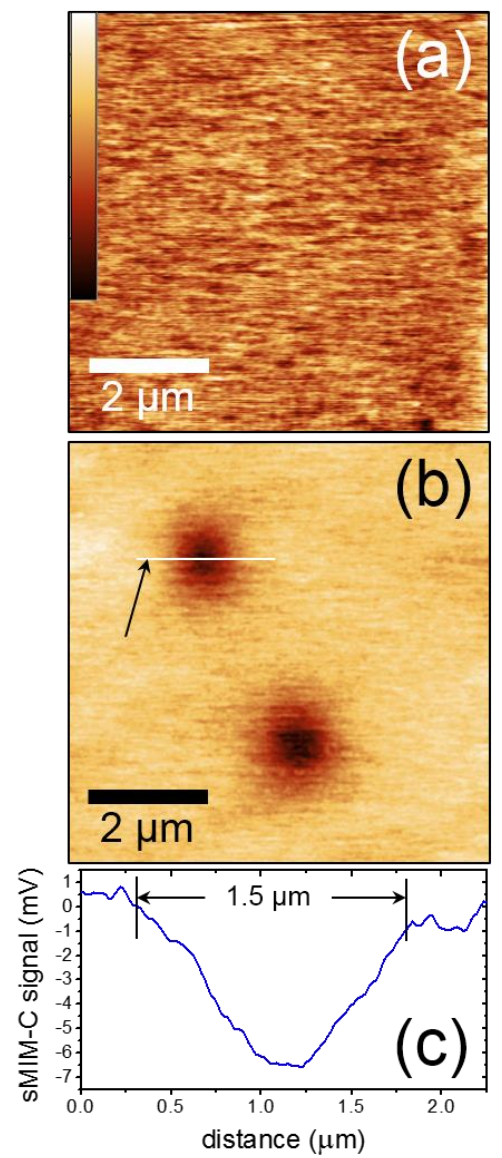

Fig. 6. (a) Height and (b) sMIM-C images of two isolated polystyrene particles under the membrane. (c) The sMIM-C signal profile along the line indicated by the arrow in (b). The full range of the color bar in (a) is $9.5 \mathrm{~nm}$.

experiments was sufficient to detect polystyrene particles in glycerol in contact with the membrane. The probing depth can be estimated to be above $100 \mathrm{~nm}$.Acknowledgment

Microwave imaging was conducted at the Center for Nanophase Materials Sciences, which also provided support (A. T.) and which is sponsored at Oak Ridge National Laboratory by the Scientific User Facilities Division, Office of Basic Energy Sciences, U. S. Department of Energy. A.T. acknowledges support by U.S. Civilian Research and Development Foundation.

Disclaimer: Certain commercial equipment, instruments, or materials are identified in this paper in order to demonstrate the experimental procedures and capabilities adequately. Such identification is not intended to imply recommendation or endorsement by the National Institute of Standards and Technology, nor is it intended to imply that the materials or equipment identified are necessarily the best available for the purpose.

\section{REFERENCES}

[1] N. de Jonge and F. M. Ross, "Electron microscopy of specimens in liquid," Nat. Nano, vol. 6, pp. 695-704, 2011.

[2] N. M. Schneider, M. M. Norton, B. J. Mendel, J. M. Grogan, F. M. Ross, and H. H. Bau, "Electron-Water Interactions and Implications for Liquid Cell Electron Microscopy," J. Phys. Chem. C, vol. 118, pp. 22373-22382, 2014.

[3] S. M. Anlage, V. V. Talanov, and A. R. Schwartz, "Principles of Near-Field Microwave Microscopy," in Scanning Probe Microscopy: Electrical and Electromechanical Phenomena at the Nanoscale, S. Kalinin and A. Gruverman, Eds., ed New York: Springer Scientific, 2007, pp. 215-253.

[4] S. Semenov, "Microwave tomography: review of the progress towards clinical applications," Philosophical transactions. Series A, Mathematical, physical, and engineering sciences, vol. 367, pp. 3021-3042, 2009.

[5] K. P. Gaikovich, "Subsurface Near-Field Scanning Tomography," Phys. Rev. Lett., vol. 98, p. 183902, 2007.

[6] A. N. Reznik and N. V. Yurasova, "Electrodynamics of microwave Near-field probing: Application to medical diagnostics," J. Appl. Phys., vol. 98, pp. 1147019, 2005.

[7] K. Lai, M. B. Ji, N. Leindecker, M. A. Kelly, and Z. X. Shen, "Atomic-force-microscope-compatible near-field scanning microwave microscope with separated excitation and sensing probes," Rev. Sci. Instrum., vol. 78, p. 063702, 2007. 
[8] F. Wang, N. Clément, D. Ducatteau, D. Troadec, H. Tanbakuchi, B. Legrand, et al., "Quantitative impedance characterization of sub-10 nm scale capacitors and tunnel junctions with an interferometric scanning microwave microscope," Nanotechnology, vol. 25, p. 405703, 2014.

[9] C. Plassard, E. Bourillot, J. Rossignol, Y. Lacroute, E. Lepleux, L. Pacheco, et al., "Detection of defects buried in metallic samples by scanning microwave microscopy," Phys. Rev. B, vol. 83, p. 121409, 2011.

[10] S. Wu and J.-J. Yu, "Attofarad capacitance measurement corresponding to single-molecular level structural variations of self-assembled monolayers using scanning microwave microscopy," Appl. Phys. Lett., vol. 97, p. 202902, 2010.

[11] A. Tselev, N. V. Lavrik, I. Vlassiouk, D. P. Briggs, M. Rutgers, R. Proksch, et al., "Near-field microwave scanning probe imaging of conductivity inhomogeneities in CVD graphene," Nanotechnology, vol. 23, p. 385706, 2012.

[12] M. Farina, A. Di Donato, T. Monti, T. Pietrangelo, T. Da Ros, A. Turco, et al., "Tomographic effects of near-field microwave microscopy in the investigation of muscle cells interacting with multi-walled carbon nanotubes," Appl. Phys. Lett., vol. 101, p. $203101,2012$.

[13] J. J. Kopanski, L. You, J.-J. Ahn, E. Hitz, and Y. S. Obeng, "Scanning Probe Microscopes for Subsurface Imaging," ECS Transactions, vol. 61, pp. 185-193, 2014. 


\title{
Models for Low Energy Electron Beam Induced Current Experiments in polycrystalline thin film photovoltaics
}

\author{
Paul M. Haney ${ }^{1}$, Heayoung P. Yoon ${ }^{1,2}$, Prakash Koirala ${ }^{3}$, Robert W. Collins ${ }^{3}$, Nikolai B. Zhitenev ${ }^{1}$ \\ 1. National Institute of Standards and Technology, Gaithersburg, MD, 20889, USA \\ 2. Maryland Nano-Center, University of Maryland, College Park, MD 20742, USA \\ 3. Department of Physics and Astronomy, University of Toledo, Toledo, OH, 43606, USA
}

\begin{abstract}
Electron beam induced current (EBIC) is a powerful technique which measures the charge collection efficiency of electron-hole pairs generated by an electron beam. EBIC offers sub-micron spatial resolution and is naturally suited to study polycrystalline materials. Ideally, an EBIC measurement reflects the spatially resolved quantum efficiency of the solar cell. However, critical analysis of low energy EBIC data obtained on CdTe-CdS solar cells indicates that the EBIC signal is not simply proportional to the collection probability of a working device. As a step towards more quantitative analysis of EBIC measurements of these materials, we develop models of the collection efficiency which account for surface recombination in depletion regions and screening of built-in fields from carrier accumulation. We discuss how these models can be applied to obtain quantitative data on grain boundaries in polycrystalline materials.
\end{abstract}

Index Terms - thin film photovoltaics, electron beam induced current, surface recombination.

\section{INTRODUCTION}

Quantitative determination of electronic properties at high spatial resolution is crucial for the development of highefficiency polycrystalline solar cells. Electron beam induced current (EBIC) is a powerful technique in which electron-hole pairs are created in proximity to an exposed surface, and the carrier collection efficiency is measured as a function of excitation position [1]. EBIC is a well-established tool to measure material properties such as the bulk minority carrier diffusion length and surface recombination. High-resolution, low electron beam energy EBIC has recently become a commonly used technique to image the response of polycrystalline solar cells such as CdTe, particularly to discriminate between the properties of grain boundaries and grain interiors. These studies demonstrate a clear correlation between device preparation, power conversion efficiency, and the contrast of grain boundaries in EBIC images [2,3]. Generally, properly treated, high efficiency devices result in bright grain boundaries, while low efficiency devices result in dark grain boundaries. This correlation is most pronounced in samples prepared by focused ion beam milling (FIB). Several techniques, such as Kelvin probe and atomic force microscopy, indicate that grain boundaries in $\mathrm{CdTe}$ are charged, and most likely undergo type inversion such that the grain boundary core is n-type (within the bulk p-type CdTe) [4]. This results in electrostatic fields near the grain boundary core which serve to separate carriers.
It is commonly assumed that an EBIC image corresponds to a spatially resolved map of the internal quantum efficiency. We argue that this cannot be the case, simply due to the discrepancy between the device short circuit current $J_{s c}$ and the $J_{\text {sc }}$ implied by an EBIC image. Referring to Fig. 1, the EBIC collection efficiency (which we denote by $\eta$ ) maximum is less than 0.13 . We estimate $10 \%$ relative uncertainty in the measured EBIC efficiency (all uncertainties are reported as one standard deviation). The dominant sources of uncertainty are from the beam current, and from the inhomogeneous material composition, which introduces uncertainty into the backscattering coefficient of the electron beam. Integrating the CdTe EBIC collection efficiency with the absorption profile of CdTe leads to a $J_{\mathrm{sc}}$ value of $1 \mathrm{~mA} / \mathrm{cm}^{2}$, compared to the device $J_{\mathrm{sc}}$ of $23.5 \mathrm{~mA} / \mathrm{cm}^{\wedge} 2$. The reduced collection efficiency is specific to CdTe: we've observed that single crystal $\mathrm{Si}$ solar cells prepared by cleaving exhibit a maximum EBIC collection efficiency of 1 .

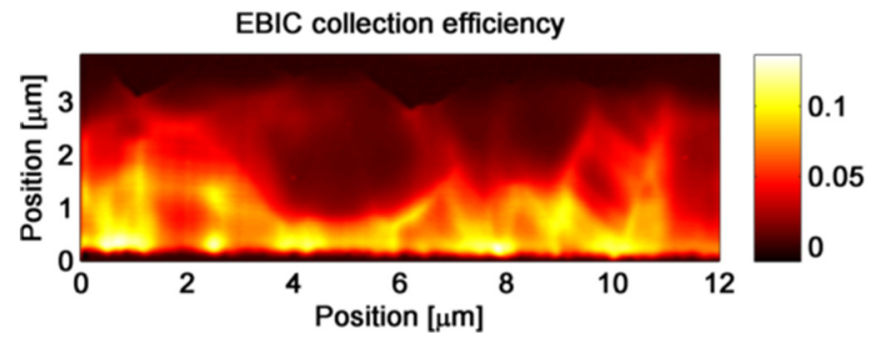

Fig. 1. EBIC collection efficiency versus beam position for a CdTe-CdS solar cell. The sample is prepared by focused ion beam milling. Note the collection efficiency varies between 0 and 0.13 .

Though the EBIC image does not represent a direct map of the internal quantum efficiency, the correlation between EBIC grain boundary contrast and device performance strongly suggests that the image reveals meaningful and important material characteristics. To help uncover the information contained in EBIC images of polycrystalline solar cells, we've developed models which account for the important features of experiments performed on polycrystalline samples.

The factors which are of significance to interpreting the collection efficiency in low electron beam energy EBIC measurements include: 1. The substantial (if not dominant) role of the surface, 2. The effect of built-in electric fields on the collection probability, and 3 . The possible screening of 
built-in fields due to the high carrier generation rate associated with electron beam excitations. In this work we describe the theoretical development of models which include these effects. The system is inherently nonlinear, requiring "brute force" numerical methods for the general solution. Our approach is to make approximations in order to extract simple analytical expressions which are amenable to fitting experimental data. We compare the analytical expressions to numerical simulation results to check the validity of the approximations employed. We discuss how these formulas may be applied to obtain quantitative information about grain boundaries from EBIC data.

\section{MODELS}

\section{A. The effect of surface recombination in depletion regions.}

In the first section we describe the model which accounts for surface recombination in depletion regions. Traditional models of EBIC assume perfect carrier collection in depletion regions - an assumption which is clearly violated by the data of Fig. 1. Two factors point to a dominant role of surface recombination on the collection efficiency in the depletion region: 1. The maximum collection efficiency in samples prepared with FIB is significantly less than in samples prepared by cleaving, indicating that the FIB process leads to significant surface damage 2. The maximum collection efficiency increases sharply as the beam energy is increased and carriers are generated further from the surface.

Fig. 2(a) shows the geometry of the numerical model we use to explore the influence of the surface on the collection efficiency in the depletion region. The drift-diffusion equations for electrons and holes are solved together with the Poisson equation. We assume Shockley-Read-Hall recombination due to a defect level positioned at midgap. The size of the system in the z-direction $\left(L_{z}\right)$ is chosen to be large enough to ensure the results are independent of its value. See the caption of Fig. 2 for all simulation parameters. We assume a Gaussian excitation centered at the beam position $x$ and below the surface a distance of $z_{0}=0.3 \times R_{B}$, where the excitation bulb size $R_{B}$ is taken to vary with the electron beam energy $E_{\text {beam }}$ as $R_{B}=R_{0} \times 0.043\left(\rho_{0} / \rho\right) \times$ $\left(E_{\text {beam }} / E_{0}\right)^{\wedge} 1.75$, where $\rho$ is the material mass density, $\rho_{0}=$ $1 \mathrm{~g} / \mathrm{cm}^{3}, E_{\text {beam }}$ is the electron beam energy, $E_{0}=1 \mathrm{keV}$, and $R_{0}=1 \mu \mathrm{m}$. The variance $\sigma^{2}$ of the Gaussian excitation varies with $R_{B}$ as $\sigma^{2}=\left(R_{B}^{2}\right) / 15$.

Fig. 2(b) shows the calculated EBIC linescan for $E_{\text {beam }}=1$ $\mathrm{keV}$ and 3 values of the surface recombination velocity. The maximum EBIC collection efficiency decreases with increasing $S$. We find the following expression for the EBIC efficiency describes the simulation results well:

$$
\eta=1-\left(\frac{D / z_{0}}{D / z_{0}+\mu E}\right)\left(\frac{S / 2}{S / 2+\mu E}\right)
$$

where $\mu$ is the carrier mobility (assumed equal for electron and holes), $D$ is the carrier diffusivity, $E$ is the magnitude of the electric field (which will depend on position), and $z_{0}$ is the distance of the excitation center from the surface. In (1), the first factor in parentheses represents the probability a charge located at a distance $z_{0}$ below the surface will diffuse to the surface. This is given by the ratio of the diffusion velocity to the sum of the diffusion velocity and drift velocity. The second term in parenthesis is the recombination probability for a charge located at the surface. This is given by the ratio of the recombination velocity to the sum of drift and recombination velocities. The position-dependent electric field $E(x)$ is used with (1) to predict EBIC lineshapes $\eta(x)$. The $E_{\text {beam }}$ dependence of $z_{0}$ results in the beam energy dependence of $\eta$. Figs. 2(b)-(e) demonstrate that this expression agrees well with the simulation data for a range of $S$ and $E_{\text {beam. }}$.

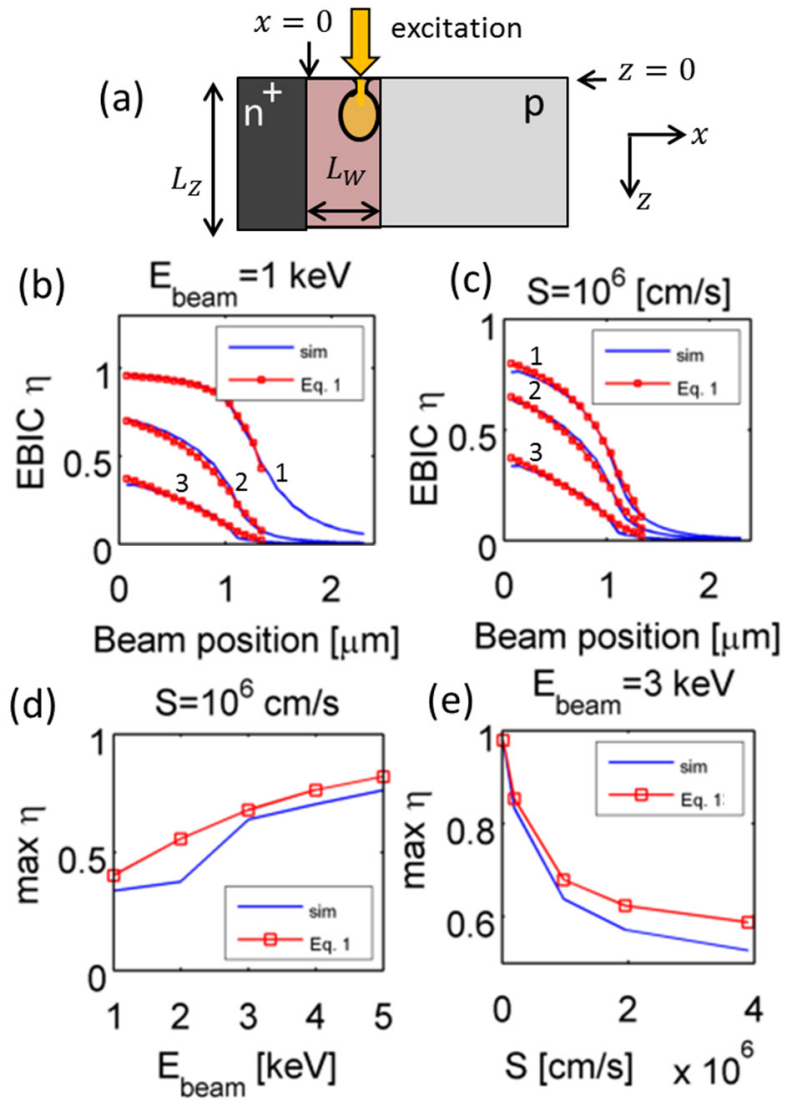

Fig. 2. (a) Model schematic of the 2-d numerical simulation of EBIC. (b) Simulation and analytical result for varying $S$, curves labeled 1, 2, and 3 correspond to $S$ values of $\left(2 \times 10^{4}, 2 \times 10^{5}, 10^{6}\right) \mathrm{cm} / \mathrm{s}$. (c) Simulation and analytical result for varying beam energy. Curves labeled 1,2 , and 3 correspond to electron beams of 5, 3, and $1 \mathrm{keV}$. (c) maximum EBIC versus beam energy for fixed S. (d) Maximum EBIC versus surface recombination velocity for fixed electron beam energy. Simulation parameters are: $E_{g}=1.5 \mathrm{eV}, L_{z}=15 \mu \mathrm{m}, N_{A}=$ $10^{15} \mathrm{~cm}^{-3}, N_{D}=5 \times 10^{16} \mathrm{~cm}^{-3}, \mu=10 \mathrm{~cm}^{2} /(\mathrm{V} \cdot \mathrm{s}), \tau=12 \mathrm{~ns}, \epsilon=$ 11 , and selective contacts: $S_{\text {min }}=0, S_{\text {maj }}=10^{6} \mathrm{~cm} / \mathrm{s}$.

An important feature of Eq. (1) is that the concavity of the lineshape in the depletion region is downward. In contrast, 
it's known that in the neutral region - where traditional models of EBIC apply - the concavity is upward [4]. The downward concavity in the depletion region follows from the monotonically decreasing magnitude of the electric field there. Fitting the concave downward portion of the EBIC lineshape at different beam energies and allows for an estimation of $S$ and $E(x)$. This procedure may be used to map out the field distribution of the p-n junction, and the fields surrounding isolated grain boundaries.

\section{B. Screening of internal fields due to high carrier generation rate}

We next describe a model which accounts for the screening of built-in fields due to the high generation rate associated with electron beam excitation. An electron with energy $E_{\text {beam }}$ generates approximately $E_{\text {beam }} /\left(3 \times E_{g}\right)$ free electron-hole pairs, where $E_{g}$ is the material band gap. The range of excitation is given by $R_{B}$, as described in the last section. For an electron beam current of $200 \mathrm{pA}$ and energy $E_{\text {beam }}=$ $5 \mathrm{keV}$, the generation rate density exceeds that of 1 sun illumination by a factor of $10^{5}$. This indicates that it is possible to drive the system into a nonlinear regime, by inducing large nonequilibrium carrier concentrations which screen the build-in fields.

Generally, screening is important when the total carrier generation rate $G$ exceeds the maximum current which is accommodated by the p-n junction. In a one-dimensional description, this maximum current is $q \mu N_{A} V_{b i} / L$, where $q$ is the electron charge, $\mu$ is the majority carrier mobility, $N_{A}$ is the doping, $V_{b i}$ is the built-in potential of the p-n junction, and $L$ is the absorber thickness. In this case, the maximum current is the product of the material conductivity with the built-in electric field. In an EBIC experiment, carriers are generated in a small region of three-dimensional space inside a material. This alters the electrostatics relative to the one-dimensional description, as described in detail in Ref. [6]. In three dimensions, the critical generation rate scales as $q \mu N_{A} V_{b i} L$.
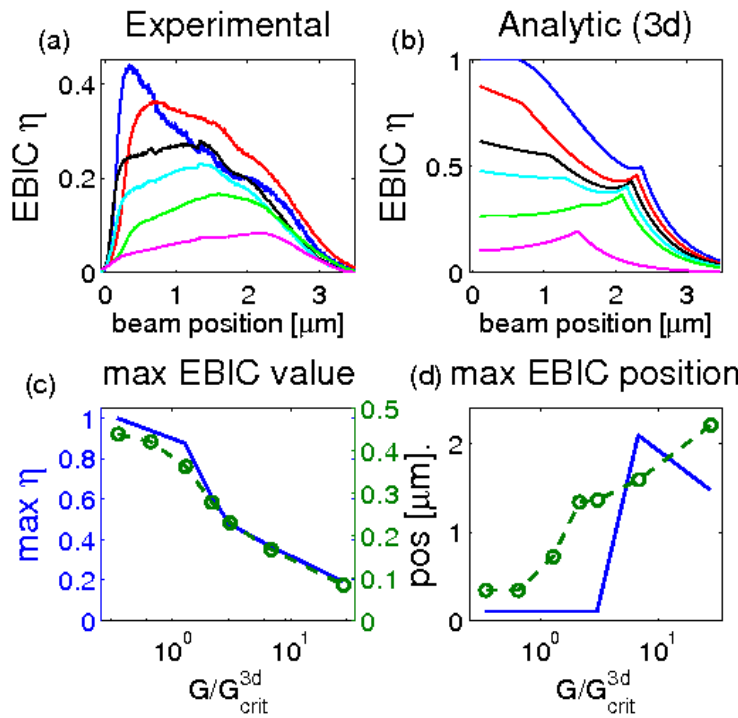

Fig. 3. (a) shows experimental EBIC profiles for CdTe with electron beam energy of $5 \mathrm{keV}$, and electron beam currents of (26, 97, 162, 231, 516, and 2110) pA (in blue, red, black, cyan, green, and purple, respectively). (b) shows the analytical model lineshape for the same set of beam current values, with $\mu N_{A}=1.2 \times 10^{14}(\mathrm{~cm}$. $\mathrm{V} \cdot \mathrm{s})^{-1}, V_{b i}=1.5 \mathrm{eV}$, and $L_{D}=500 \mathrm{~nm}$. (c) dashed green curve shows the experimental maximum EBIC efficiency as a function of total generation rate (scaled by critical generation rate, taken experimentally from letting $I_{\text {crit }}=70 \mathrm{pA}$.) Solid blue line is the same result for the analytical model. Note the experimental (analytic) y-axis is on the left (right). (d) dashed green curve shows the experimental position of the EBIC maximum, while the solid blue line is the analytical model.

We omit a detailed derivation of the form of the EBIC lineshape in the screened regime. To give a flavor of the model, we offer the following qualitative description of the important ingredients: We assume that the carriers accumulate and screen the built-in electric field. We denote the length scale over which fields are screened by $R^{*}$. We assume charges diffuse and recombine within the screened volume (a sphere of radius $R^{*}$ ), while charges which diffuse to the edge of the screened region are collected. Setting the total charge generated equal to the sum of the recombination and collected current leads to an implicit the equation for the screening length $R^{*}$, from which the EBIC collection efficiency may be determined. $R^{*}$ satisfies:

$$
\frac{G R^{*}}{\sinh \left(R^{*} / L_{D}\right)}-\frac{2 \pi \mu V_{b i} N_{A} L}{1-R^{*} / L}=0
$$

where $L_{D}$ is the diffusion length.

Fig 3(a) shows experimental EBIC lineshapes for increasing electron beam current. As before, we estimate $10 \%$ relative uncertainty in the measured EBIC efficiency amplitude. The uncertainty in the magnitude of the EBIC signal therefore has little influence on the uncertainty of the shape. This is because the length scale for this material inhomogeneity (e.g. allying) is much smaller than the electron beam spot size, so that this source of error is uniform across linescans. We estimate an uncertainty in the maximum position of $50 \mathrm{~nm}$ based on the discretization of the electron beam position in the linescan. Fig. 3(b) shows the corresponding lineshape derived from (2). The model provides the qualitative features of the EBIC lineshapes as one enters the screening regime: the maximum of the EBIC profile moves away from the p-n junction into the middle of the device, and the peak broadens out substantially. Additionally, the maximum value of the EBIC efficiency drops. By varying the beam current and studying the trend of these quantities, the presence of screening may be detected, and the EBIC lineshapes may be interpreted appropriately. The model also provides guidance for the onset of the screening regime: when the total generation rate exceeds $G_{c r i t}^{3 d}=q \mu N_{A} V_{b i} L$, screening effects become important. 


\section{CONCLUSIONS AND OUTLOOK}

To summarize, we present two models which apply to EBIC experiments on polycrystalline solar cells. Using the fact the collection efficiency is always below 1 , we develop a model which described carrier collection efficiency in depletion regions which may be applied to extract the structure of builtin fields near grain boundaries. Additionally, we develop a model that accounts for screening effects which may be important for thin film photovoltaic response to electron beam excitation.

\section{REFERENCES}

[1] J. I. Hanoka, and R. O. Bell, "Electron-Beam-Induced Currents in Semiconductors", Ann. Rev. of Mat. Sci., vol. 11, 353-380 (1981).

[2] C. Li, Y. Wu, J. Poplawsky, T. J. Pennycook, N. Paudel, W. Yin, S. J. Haigh, M. P. Oxley, A. R. Lupini, M. Al-Jassim, S. J. Pennycook, and Y. Yan, "Grain-Boundary-Enhanced Carrier
Collection in CdTe Solar Cells", Physical Review Letters, vol. 112, 156103-1-5 (2014).

[3] Heayoung P. Yoon, Paul M. Haney, Dmitry Ruzmetov, Hua Xua, Marina S. Leite, Behrang H. Hamadani, A. A. Talin, N. B. Zhitenev, "Local electrical characterization of cadmium telluride solar cells using low-energy electron beam", Sol. En. Mat. And Sol. Cells, vol. 117, 599-504 (2013).

[4] I. Visoly-Fisher, S. R. Cohen, K. Gartsman, A. Ruzin, and D. Cahen, "Understanding the Beneficial Role of Grain Boundaries in Polycrystalline Solar Cells from Single-GrainBoundary Scanning Probe Microscopy", Adv. Func. Mat., vol. 16, 649-660 (2006).

[5] W. Van Roosbroek, "Injected Current Carrier Transport in a Semi-Infinite Semiconductor and the Determination of Lifetimes and Surface Recombination Velocities", J. App. Phys. Vol. 26, 380-391 (1955).

[6] P. M. Haney, H. P. Yoon, P. Koirala, R. W. Collins, N. B. Zhitenev, "Electron beam induced current in the high injection regime", arXiv:1410.4435v1, (2014). 


\title{
Molecular Alignment and Rashba Splitting in Organometal Halide Perovskite $\mathrm{CH}_{3} \mathrm{NH}_{3} \mathrm{PbI}_{3}$ Absorbers
}

\author{
Junwen $\mathrm{Li}^{1,2}$ and Paul M. Haney ${ }^{1}$ \\ 1. Center for Nanoscale Science and Technology, National Institute of Standards and Technology, \\ Gaithersburg, MD 20899, USA \\ 2. Maryland Nano-Center, University of Maryland, College Park, MD 20742, USA
}

\begin{abstract}
Organometal halide perovskite $\mathrm{CH}_{3} \mathrm{NH}_{3} \mathrm{PbI}_{3}$ solar cells have witnessed unprecedented progress in power conversion efficiency, reaching more than $20 \%$ within 5 years. Despite this rapid progress, there remain open questions about the basic properties of these materials, such as the role of the polar molecule $\mathrm{CH}_{3} \mathrm{NH}_{3}$ (methylammonium), and the possible existence of ferroelectric ordering, and its role in device operation. Using first-principles density-functional theory, we investigated the effect of molecular alignment on the electronic structure. We find that the molecular alignment significantly modifies the near-gap states indirectly through the induced structural distortion of the $\left(\mathrm{PbI}_{6}\right)^{4-}$ octahedron. The reduction in symmetry due to this distortion, combined with strong spin-orbit coupling of $\mathrm{Pb}$, leads to a Rashba-like splitting of valence and conduction bands. This in turn leads to reduction of the degeneracy of the valence and conduction bands. These results imply that the electrical and optical properties are highly sensitive to ordering of the $\mathrm{CH}_{3} \mathrm{NH}_{3}$ dipole orientation.
\end{abstract}

Index Terms - organometal halide perovskite, molecular alignment, Rashba splitting.

\section{INTRODUCTION}

Organometal halide perovskites have recently attracted a great deal of attention because of their exceptional power conversion efficiency. The current record efficiency is more than $20 \%$, following an unprecedented pace of development since being first employed as photovoltaic absorbers in 2009 with an efficiency of $3.8 \%$ [1]-[2]. The high efficiency is attributed to the optimal band gap $(\approx 1.5 \mathrm{eV})$, high absorption coefficient, efficient charge transport properties, and long charge carrier diffusion length of over $100 \mathrm{~nm}$ for $\mathrm{CH}_{3} \mathrm{NH}_{3} \mathrm{PbI}_{3}$ and $1 \mu \mathrm{m}$ for mixed perovskite $\mathrm{CH}_{3} \mathrm{NH}_{3} \mathrm{PbI}_{3-\mathrm{x}} \mathrm{Cl}_{\mathrm{x}}$ [3]-[4]. In addition to the exceptional performance in power conversion efficiency, these perovskite materials exhibit many unusual characteristics, among which the anomalous hysteresis in $J-V$ curve is of great interest [5]. The hysteretic behavior leads to a large discrepancy in the cell efficiency between the forward and reverse bias scans. The source and significance of the hysteretic behavior are still unclear, with proposals such as trapping and de-trapping of carriers, ionic motion driven by the applied potential, and ferroelectric ordering of the material [5-7]. Ferroelectric domains have been experimentally observed directly using piezo force microscopy on solution-processed $\mathrm{CH}_{3} \mathrm{NH}_{3} \mathrm{PbI}_{3}$ perovskite thin films [8]. It has been proposed that the presence of ferroelectric domains would facilitate separation of electrons and holes, providing "ferroelectric highways" for efficient charge collection [9].

In this work, we focus on the effect of ferroelectric ordering on the electronic structure. Ferroelectric order breaks inversion symmetry. It's well known that the presence of strong spin-orbit coupling, combined with breaking of inversion symmetry leads to a Rashba splitting of the electronic states [10]. This alters the electronic structure near the band gap, which can have significant impact on the optical and electronic material properties.

The ferroelectric polarization is derived from two distinct features of the lattice structure. One is the alignment of the methylammonia molecules' dipole moments. The other is associated with the structural distortion of the inorganic framework. The Rashba splitting is a result of these two contributions. In this paper, we investigated the effect of molecular alignment on the electronic structure and found that the molecular alignment leads to large Rashba splitting through the alignment-induced structural distortion in the $\left(\mathrm{PbI}_{6}\right)^{4-}$ octahedron. Since the different molecular alignments have comparable energies, temperature may be used to tune the percentage of the ordering. We therefore, propose that the electronic structure and optical properties will change with varying temperatures if ferroelectric effects are present.

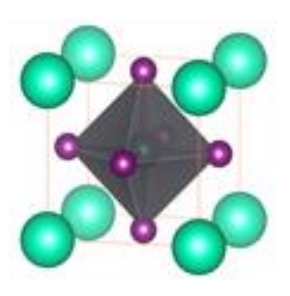

(a)

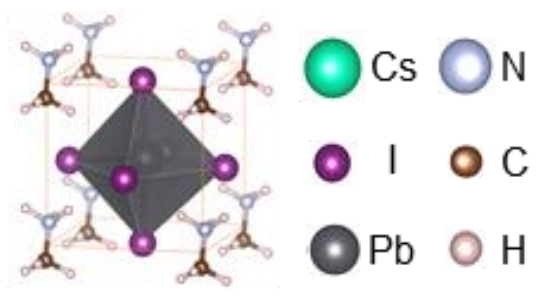

(b)
Fig. 1. Schematic of cubic perovskite (a) $\mathrm{CsPbI}_{3}$ and (b) $\mathrm{CH}_{3} \mathrm{NH}_{3} \mathrm{PbI}_{3}$. The polar $\mathrm{CH}_{3} \mathrm{NH}_{3}{ }^{+}$ions are aligned along $<001>$ direction. 


\section{ATOMIC STRUCTURES}

Experimentally, the perovskite crystals exhibit three different structural phases, namely cubic $(P m \overline{3} m)$, tetragonal $(I 4 / \mathrm{mcm})$, and orthorhombic (Pnma) depending on the temperature [11]. To focus on the effect of molecular alignment, we choose the cubic phase and align the molecules along $<001>$ direction which is of the lowest energy among several possible alignments [12]. The lattice constant and atomic positions within the cubic unit cell were optimized using local density approximation in the form of normconserving pseudopotentials as implemented in QuantumESPRESSO [13] with an energy cutoff $80 \mathrm{Ry}$ for the plane wave basis expansion and a $6 \times 6 \times 6$ grid for the Brillouin zone sampling. During relaxation, all atoms in the unit cell were allowed to move until the force on each atom is less than $0.5 \mathrm{eV} / \mathrm{nm}$. The lattice constant is calculated to be $\mathrm{a}=$ $0.621 \mathrm{~nm}$, in good agreement with the experimental result $(\mathrm{a}=$ $0.626 \mathrm{~nm} \mathrm{[14])} \mathrm{and} \mathrm{also} \mathrm{consistent} \mathrm{with} \mathrm{the} \mathrm{structural}$ determination with local density approximation which usually underestimates the lattice constant. Distortions of the $\left(\mathrm{PbI}_{6}\right)^{4-}$ octahedron are observed and the $\mathrm{Pb}$ atom shifts away from the body center by $0.019 \mathrm{~nm}$. For comparison, we also studied the $\mathrm{CsPbI}_{3}$ with undistorted octahedron. The lattice constant is determined to be $0.619 \mathrm{~nm}$, comparable to the experimental value of $0.629 \mathrm{~nm}[15]$.

\section{ELECTRONIC STRUCTURES}

We first consider the cubic $\mathrm{CsPbI}_{3}$ with undistorted octahedron as a reference system and show in Fig. 2 the total and projected density of states without spin-orbit coupling included. Near the Fermi level, the conduction band states are derived from the $p$ orbital of $\mathrm{Pb}$ while the valence band states are composed of the $\mathrm{Pb} s$ orbital and I $p$ orbital. The electronic energy states derived from the Cs atom are mostly located more than $2 \mathrm{eV}$ away from the Fermi level both within the valence and the conduction bands. $\mathrm{CH}_{3} \mathrm{NH}_{3} \mathrm{PbI}_{3}$ has similar orbital contributions near the Fermi level. So the electronic and optical properties in the visible range are dominated by the orbitals of $\mathrm{Pb}$ and I atoms. Fig. 3(a) depicts the electronic structure of $\mathrm{CsPbI}_{3}$ without spin-orbit coupling considered. Both the valence band maximum (VBM) and the conduction band minimum $(\mathrm{CBM})$ are located at the $R$ point [zone boundary, $(\pi / a, \pi / a, \pi / a)$ ]. The band structure of $\mathrm{CH}_{3} \mathrm{NH}_{3} \mathrm{PbI}_{3}$ is similar to that of $\mathrm{CsPbI}_{3}$, but with significant changes in the conduction band edges as shown in Fig. 3(b). The presence of aligned dipolar methylammonium and the octahedral distortion lift the degeneracy between $p_{x}, p_{y}$, and $p_{z}$ orbitals. The gap becomes slightly indirect, with nearly degenerate energy states for CBM at $R$ point.

Because of the heavy $\mathrm{Pb}$ atom, relativistic effects are expected to play an important role. We calculate the electronic structures of $\mathrm{CsPbI}_{3}$ and $\mathrm{CH}_{3} \mathrm{NH}_{3} \mathrm{PbI}_{3}$ with spin-orbit coupling included as shown in Fig. 3(c) and (d), respectively. In $\mathrm{CsPbI}_{3}$ adding spin-orbit coupling splits degenerate conduction band states $(L=1)$ into lower $J=1 / 2$ and upper $J=3 / 2$ bands, whereas spin-orbit coupling has little effect on the valence band derived from the $s$ orbital, leading to a $J=1 / 2 \mathrm{CBM}$ and $S=1 / 2$ VBM. The spin-orbit coupling also significantly reduces the band gap by $0.98 \mathrm{eV}$.

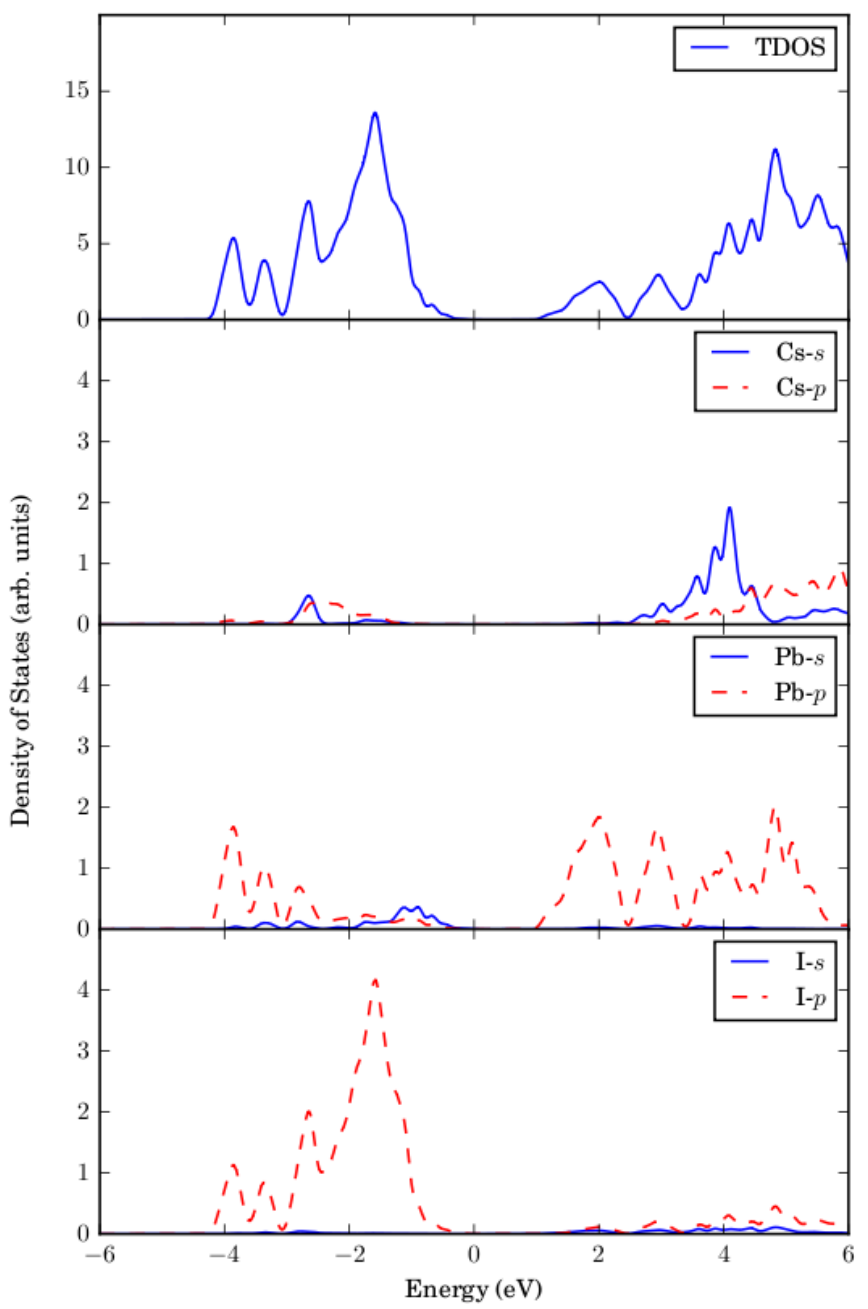

Fig. 2. Total and partial density of states projected onto $s$ and $p$ orbitals for $\mathrm{CsPbI}_{3}$. The Fermi level is set to zero.

The effect of spin-orbit coupling in $\mathrm{CH}_{3} \mathrm{NH}_{3} \mathrm{PbI}_{3}$ is more significant because of the ferroelectric polarization derived from the dipolar molecules alignment and ensuing structural distortion. Using the Berry phase approach, we find a polarization of $47.2 \mu \mathrm{C} / \mathrm{cm}^{2}$. As discussed in the introduction, the interplay between spin-orbit coupling and inversion symmetry breaking results in Rashba splitting. As shown in Fig. 3(d), the electronic structure exhibits larger Rashba splitting for the conduction band and in Fig. 4 we show the Rashba-type spin distribution on the energy contours of $170 \mathrm{meV}$ above the conduction band minimum in $k_{x}$ $k_{y}$ plane. The asymmetry in conduction band and valence band may have impact on the absorbance, and radiative 

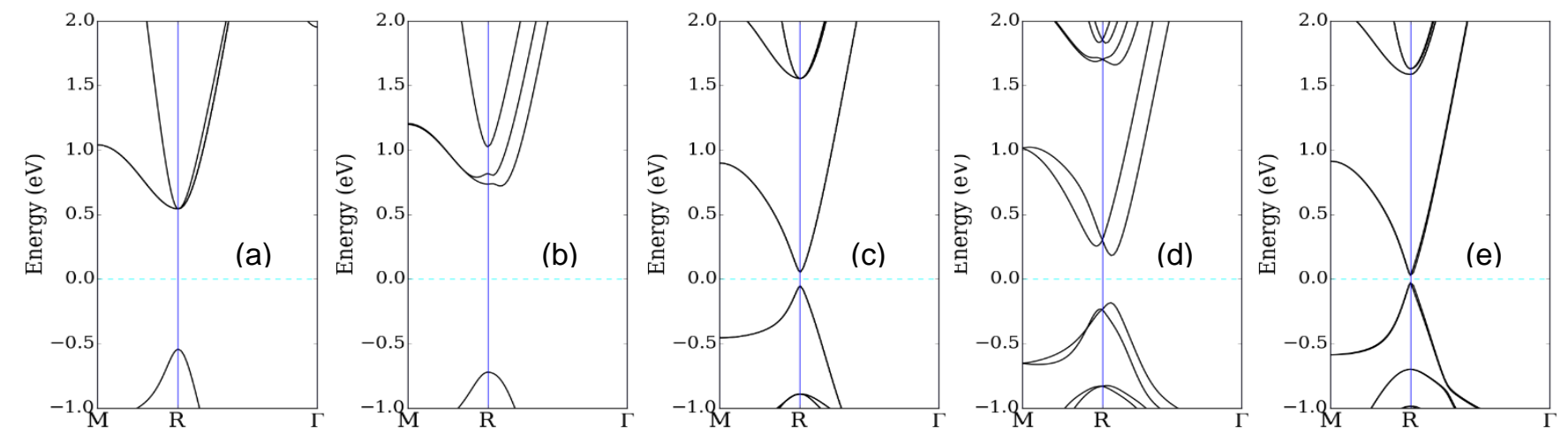

Fig. 3. Electronic structures of relaxed (a) cubic $\mathrm{CsPbI}_{3}$ and (b) $\mathrm{CH}_{3} \mathrm{NH}_{3} \mathrm{PbI}_{3}$ with ordered methylammonium ions and octahedral distortion, and without the consideration of spin-orbit coupling. (c) and (d) are the corresponding electronic structures with spin-orbit coupling included. (e) corresponds to the cubic $\mathrm{CH}_{3} \mathrm{NH}_{3} \mathrm{PbI}_{3}$ with ordered methylammonium ions and no octahedral distortion.

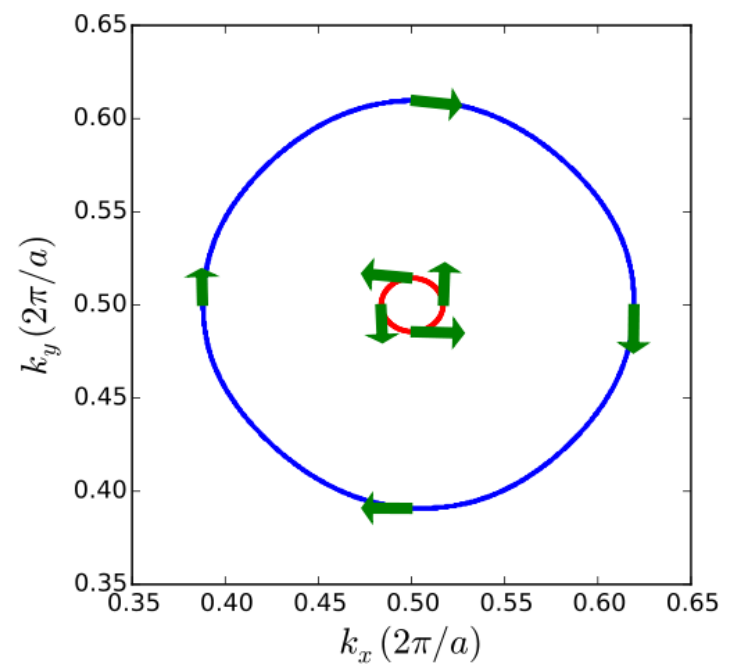

Fig. 4. In-plane spin distribution on the energy contours of $170 \mathrm{meV}$ above the conduction band minimum. Arrows indicate the in-plane orientation and the length is proportional to the modulus of the spin.

recombination of photo-excited electron and hole charge carriers.

In order to gain insight on the relative importance of dipolar ordering versus the distortion of the $\left(\mathrm{PbI}_{6}\right)^{4-}$ octahedron, we carried out simulations with a hypothetical model system in which we fixed the $\mathrm{Pb}$ in the body center and I atoms at face centers and aligned methylammonium along $<001>$ direction. The polarization is calculated to be $51 \mu \mathrm{C} / \mathrm{cm}^{2}$. Fig. 3(e) shows that the Rashba effect is nearly vanishing, suggesting that the octahedral distortion is the main cause of Rashba splitting. To understand this, we note that the Rashba splitting magnitude depends on the spin-orbit coupling strength and the degree of inversion symmetry breaking. In this material, the splitting is therefore determined by the asymmetry of the environment surrounding the $\mathrm{Pb}$ atom. In a symmetric octahedron with aligned dipoles, the screening of I ions leads to small change in the electrostatic potential around $\mathrm{Pb}$ atom and therefore reduced Rashba splitting. On the other hand, a distortion of the octahedron leads to a highly asymmetric environment for the $\mathrm{Pb}$ atom. For this reason, we conclude the alignment of the molecules is important for the band structure mostly due to the resulting distortion of the octahedron.

\section{SUMMARY}

In summary, we report on first-principles density-functional simulations on the effect of molecular alignment on the energy states near the Fermi level. We found that only the alignment of methylammonium ion dipoles alone does not significantly modify the near-gap states. However, accounting for the full relaxation of the atomic positions leads to an off-center displacement of the $\mathrm{Pb}$ atom which induces a much larger Rashba splitting. Therefore, the role of the methylammonium ions ordering on the electronic structure is indirect, and takes place through a structural change. Our finding suggests that engineering the octahedron directly or indirectly through tuning the molecules may be an effective route in tailoring the Rashba splitting and band gap nature. Additionally, these results suggest a strong dependence of optical properties, such as absorption, on the alignment of the methylammonium ions, and more generally on the symmetry of the octahedron. An observed dependence of the optical properties on temperature would be consistent with ferroelectric ordering, and would suggest that this effect plays an important role in the photovoltaic properties of this material.

\section{ACKNOWLEDGEMENT}

J. L. acknowledges support under the Cooperative Research Agreement between the University of Maryland and the National Institute of Standards and Technology Center for Nanoscale Science and Technology, Award 70NANB10H193, through the University of Maryland. 


\section{REFERENCES}

[1] A. Kojima, K. Teshima, Y. Shirai, and T. Miyasaka, "Organometal halide perovskites as visible-light sensitizers for photovoltaic cells," J. Am. Chem. Soc., vol. 131, no. 17, pp. 6050-1, May 2009.

[2] Best Research Cell Efficiency Chart. http://www.nrel.gov/ncpv/images/efficiency_chart.jpg.

[3] S. D. Stranks, G. E. Eperon, G. Grancini, C. Menelaou, M. J. P. Alcocer, T. Leijtens, L. M. Herz, A. Petrozza, and H. J. Snaith, "Electron-hole diffusion lengths exceeding 1 micrometer in an organometal trihalide perovskite absorber," Science, vol. 342, no. 6156 , pp. $341-4$, Oct. 2013.

[4] G. Xing, N. Mathews, S. Sun, S. S. Lim, Y. M. Lam, M. Grätzel, S. Mhaisalkar, and T. C. Sum, "Long-range balanced electron- and hole-transport lengths in organic-inorganic $\mathrm{CH}_{3} \mathrm{NH}_{3} \mathrm{PbI}_{3}$," Science, vol. 342, no. 6156, pp. 344-7, Oct. 2013.

[5] H. J. Snaith, A. Abate, J. M. Ball, G. E. Eperon, T. Leijtens, N. K. Noel, S. D. Stranks, J. T.-W. Wang, K. Wojciechowski, and W. Zhang, "Anomalous hysteresis in perovskite solar cells," $J$. Phys. Chem. Lett., vol. 5, no. 9, pp. 1511-1515, May 2014.

[6] Z. Xiao, Y. Yuan, Y. Shao, Q. Wang, Q. Dong, C. Bi, P. Sharma, A. Gruverman, and J. Huang, "Giant switchable photovoltaic effect in organometal trihalide perovskite devices," Nat. Mater., vol. 14, no. 2, pp. 193-198, Dec. 2014.

[7] C. C. Stoumpos, C. D. Malliakas, and M. G. Kanatzidis, "Semiconducting tin and lead iodide perovskites with organic cations: phase transitions, high mobilities, and near-infrared photoluminescent properties," Inorg. Chem., vol. 52, no. 15, pp. 9019-38, Aug. 2013.

[8] Y. Kutes, L. Ye, Y. Zhou, S. Pang, B. D. Huey, and N. P. Padture, "Direct observation of ferroelectric domains in solution-processed $\mathrm{CH}_{3} \mathrm{NH}_{3} \mathrm{PbI}_{3}$ perovskite thin films," J. Phys. Chem. Lett., vol. 5, no. 19, pp. 3335-3339, Oct. 2014.

[9] J. M. Frost, K. T. Butler, F. Brivio, C. H. Hendon, M. van Schilfgaarde, and A. Walsh, "Atomistic origins of highperformance in hybrid halide perovskite solar cells," Nano Lett., vol. 14, no. 5, pp. 2584-90, May 2014.

[10] L. Petersen and P. Hedegård, "A simple tight-binding model of spin-orbit splitting of $s p$-derived surface states," Surf. Sci., vol. 459, no. 1-2, pp. 49-56, Jul. 2000.

[11] Y. Kawamura, H. Mashiyama, and K. Hasebe, "Structural study on cubic-tetragonal transition of $\mathrm{CH}_{3} \mathrm{NH}_{3} \mathrm{PbI}_{3}$," J. Phys. Soc. Japan, vol. 71, no. 7, pp. 1694-1697, Jul. 2002.

[12] J. M. Frost, K. T. Butler, and A. Walsh, "Molecular ferroelectric contributions to anomalous hysteresis in hybrid perovskite solar cells," APL Mater., vol. 2, no. 8, p. 081506, Aug. 2014.

[13] P. Giannozzi, S. Baroni, N. Bonini, M. Calandra, R. Car, C. Cavazzoni, D. Ceresoli, G. L. Chiarotti, M. Cococcioni, I. Dabo, A. Dal Corso, S. de Gironcoli, S. Fabris, G. Fratesi, R. Gebauer, U. Gerstmann, C. Gougoussis, A. Kokalj, M. Lazzeri, L. MartinSamos, N. Marzari, F. Mauri, R. Mazzarello, S. Paolini, A. Pasquarello, L. Paulatto, C. Sbraccia, S. Scandolo, G. Sclauzero, A. P. Seitsonen, A. Smogunov, P. Umari, and R. M. Wentzcovitch, "QUANTUM ESPRESSO: a modular and opensource software project for quantum simulations of materials," J. Phys. Condens. Matter, vol. 21, no. 39, p. 395502, Sep. 2009.

[14] T. Baikie, Y. Fang, J. M. Kadro, M. Schreyer, F. Wei, S. G. Mhaisalkar, M. Graetzel, and T. J. White, "Synthesis and crystal chemistry of the hybrid perovskite $\left(\mathrm{CH}_{3} \mathrm{NH}_{3}\right) \mathrm{PbI}_{3}$ for solid-state sensitised solar cell applications," J. Mater. Chem. A, vol. 1, no. 18, p. 5628, Apr. 2013.

[15] D. M. Trots and S. V. Myagkota, "High-temperature structural evolution of caesium and rubidium triiodoplumbates," J. Phys. Chem. Solids, vol. 69, no. 10, pp. 2520-2526, Oct. 2008. 


\title{
Local Photocarrier Dynamics in CdTe Solar Cells Under Optical and Electron Beam Excitations
}

\author{
Heayoung P. Yoon ${ }^{1,2}$, Paul M. Haney ${ }^{1}$, Yohan Yoon ${ }^{1,2}$, Sangmin An ${ }^{1,2}$, James I. Basham ${ }^{1}$, \\ and Nikolai B. Zhitenev ${ }^{1}$
${ }^{1}$ National Institute of Standards and Technology, Gaithersburg, MD, 20889, USA, ${ }^{2}$ Maryland NanoCenter, University of Maryland, College Park, MD 20742, USA

\begin{abstract}
We investigate local carrier dynamics in $n$-CdS / $p$ CdTe solar cells, where the electron-hole pairs are generated by either near-field optical illumination or highly focused electron beam excitation. A focused ion milling process was used to prepare a smooth surface and cross-sections of the devices. The spatially resolved photocurrent images confirm high carrier collection efficiencies at grain boundaries. An analytical model was used to extract material parameters at the level of single grains. We find that the minority carrier diffusion length and device parameters extracted from the local measurement techniques are in excellent agreement, but can be different determined from macro-scale measurements.
\end{abstract}

\section{INTRODUCTION}

Polycrystalline thin film photovoltaic (PV) technologies have shown great promise for solar energy harvesting using inexpensive PV materials, currently reaching a power conversion efficiency of $21.0 \%$ for cadmium telluride (CdTe) and $21.7 \%$ for copper indium gallium selenide (CIGS) solar cells [1]. To achieve the maximum efficiency of $\approx 30 \%$ possible for these technologies, considerable efforts have been made to understand the physical mechanisms that limit cell performance. Large-area optical characterizations are wellestablished techniques that diagnose optical and electrical losses of PV devices. Among them, external quantum efficiency (EQE) measures the ratio of the collected charged carriers to the incident photons on a solar cell at different wavelengths, showing how effectively a photovoltaic device converts sunlight into electricity throughout the spectrum. Light absorption varies as a function of the wavelength of incident light (e.g., larger absorption volume at longer wavelengths) and thus the EQE data provides rich information about each layer and interface of the solar cell [2].

While extremely informative, the macro-scale ( $>1 \mathrm{~mm}$ ) optical data alone are not sufficient to understand the impact of microstructures $(<1 \mu \mathrm{m})$ on their operation and performance in CdTe or CIGS [3]. Local quantum efficiency can be measured by near-field scanning optical microscopy (NSOM). In this technique, local electron-hole pairs are generated by light injected through a small aperture $(<200 \mathrm{~nm}$ in diameter). Spatially resolved local quantum efficiency can be obtained by varying the wavelength of light. Alternatively, electron-beam induced current (EBIC) is a complementary technique that provides higher spatial resolution $(<20 \mathrm{~nm})$. In addition, this technique allows systematic controls of the carrier generation (three orders of magnitude) and of the size of interaction volume (tens of $\mathrm{nm}$ to a few $\mu \mathrm{m}$ ), well-suited for quantitative study. As in NSOM, the generated electronhole pairs are rapidly separated at Schottky barrier or $p-n$ junction before they recombine, and the carriers collected by the contacts produce a current. Although EBIC does not represent a direct map of the quantum efficiency, the correlation between EBIC grain boundary contrast and device performance strongly suggests that this technique reveals meaningful and important material characteristics $[4,5]$

In this work, we perform cross-sectional photocurrent measurements using optical and electron beam excitations. By fitting the line-scans using analytical models, materials parameters are extracted and compared to the corresponding macro-scale optical data. We discuss possible origin of the discrepancy between macro- and micro-scale properties.

\section{EXPERIMENTAL}

Standard semiconductor processes were used to fabricate Ohmic metal contacts on the surface of $p$-CdTe $/ n$-CdS junctions extracted from a commercial solar panel [4]. Crosssections of the devices were obtained by a focused ion beam milling (FIB) technique. In this process, a beam of $\mathrm{Ga}$ ions $\left(V_{b, G a}=30 \mathrm{keV} ; I_{b, G a}=2.5 \mathrm{nA}\right)$ is precisely controlled to etch away the target material, creating a smooth surface. The acceleration voltage and the beam current of the electron beam and the ion beam used in this work are nominal values. The measured fluctuation of the beam current is $<5 \%$. Topography and NSOM measurement were performed using a tapping mode atomic force microscopy (AFM) system. Multiple single mode lasers $(405 \mathrm{~nm}, 520 \mathrm{~nm}, 635 \mathrm{~nm}, 780$ $\mathrm{nm}$ ) were used. Acquisition of EBIC images at beam energies ranging from $1 \mathrm{keV}$ to $20 \mathrm{keV}$ was performed in a scanning electron microscopy (SEM) system. While the contact of $\mathrm{TCO} / n-\mathrm{CdS} /$ indium was grounded, a Tungsten probe tip controlled by a nano-manipulator was used as a current collector from patterned metal contact to $p$-CdTe. A custom designed sample stage was used to ensure high signal-to-noise ratio within the full range of signals for low- and high $\mathrm{keV}$ EBIC. 


\section{RESULTS AND DISCUSSION}

The baseline PV performance of the extracted CdTe device was evaluated. The measured power conversion efficiency was $\approx 12 \%$ with a short-circuit current $\left(J_{s c}\right)$ of $(23 \pm 0.5)$ $\mathrm{mA} / \mathrm{cm}^{2}$, an open-circuit voltage $\left(V_{o c}\right)$ of $(820 \pm 10) \mathrm{mV}$, and fill factor $(F F)$ of $(64 \pm 3) \%$, indicating that the device properties are mainly preserved after the extraction processes. Standard external quantum efficiency (EQE) profiles were collected under 1 sun illumination, shown in Figure 1 (a). In this optical measurement, a ratio of the number of collected carriers to the number of absorbed photons is recorded at each wavelength. A complete $\mathrm{QE}$ analysis is used to identify the optical and electronic losses reducing $J_{s c}$ and to extract the materials parameters. By fitting the variation of the $\mathrm{QE}$ from $650 \mathrm{~nm}$ to $800 \mathrm{~nm}$, we estimated the minority carrier diffusion length $\left(L_{n}\right)$ to be approximate $1 \mu \mathrm{m}$. Taking the average grain size of $\approx 1 \mu \mathrm{m}$ (Figure $1 \mathrm{~b}$ ), the typical beam $(\approx 2 \mathrm{~mm}$ in diameter) interacts with over $\approx 10^{6}$ grains.
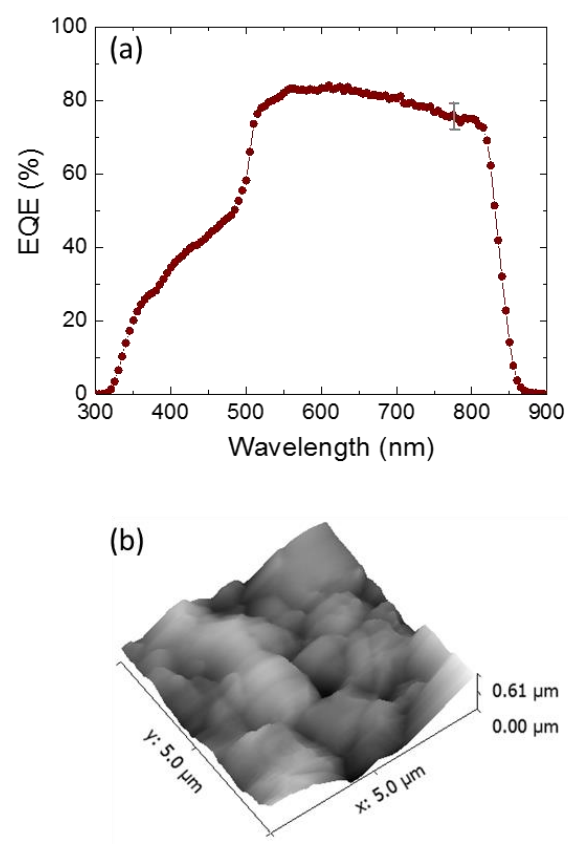

Fig. 1. (a) External quantum efficiency profile of a $n$-CdS / $p$ CdTe extracted from a commercial solar panel. The estimated minority carrier diffusion length $(650 \mathrm{~nm}$ to $800 \mathrm{~nm})$ is $\approx 1 \mu \mathrm{m}$. (b) An AFM image displays the peak-to-valley surface roughness of the $\mathrm{CdTe} \approx 0.6 \mu \mathrm{m}$. The typical grain size of the CdTe is $\approx 1 \mu \mathrm{m}$.

In order to correlate the macro-scale PV properties to microstructural properties, we perform near-field scanning optical microscopy. In contrast to the EQE, the electron-hole pairs are locally generated by the light injected through a small aperture $(<200 \mathrm{~nm}$ in diameter). Cross-sectional devices were used to map the local responses throughout the entire $p$ - $n$ junction region. The topography of the FIB milled section is very smooth (peak-to-valley roughness $<20 \mathrm{~nm}$ ).
Figure 2 (a) and (b) shows the photocurrent map at $405 \mathrm{~nm}$ and $635 \mathrm{~nm}$ illumination, respectively. The spatial resolution decreases at the higher wavelength, as expected, due to the increase in the absorption depth. Nonetheless, the bright contrast at the grain boundaries indicates high carrier collection efficiency, as proposed in previous works $[4,5]$. We fit the line-scan collected in a single grain bulk under the $635 \mathrm{~nm}$ illumination (Figure $2 \mathrm{c}$ ) and estimated $L_{n} \approx 0.6 \mu \mathrm{m} \pm$ $0.06 \mu \mathrm{m}$.

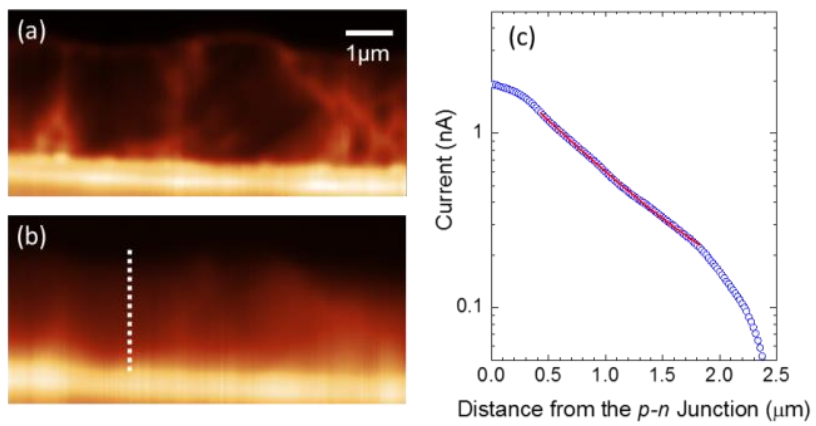

Fig. 2. Near-field scanning microscopy of a FIB prepared CdTe device. Photocurrent maps at $405 \mathrm{~nm}$ (a) and $635 \mathrm{~nm}$ (b). The estimated photon absorption depth is $100 \mathrm{~nm}$ and $400 \mathrm{~nm}$, respectively. (c) A line-scan profile at $635 \mathrm{~nm}$ illumination (white dot line in b). The red line represents the model fit to extract the minority carrier diffusion length.

The spectrally resolved photocurrent images are compared to the carrier collection maps obtained by EBIC. A series of cross-sectional EBIC images were obtained by varying the acceleration voltage of the electron beam (Figure 3). The strong bright contrast at the grain boundaries reflects high carrier collection efficiencies also seen in NSOM data (Figure $2 \mathrm{c})$. Qualitatively, the $5 \mathrm{keV}$ and $10 \mathrm{keV}$ EBIC images are similar to that of $405 \mathrm{~nm}$ and $635 \mathrm{~nm}$ NSOM maps, respectively. This correspondence indicates that the calculated photon absorption and electron penetration depths are in good agreement.
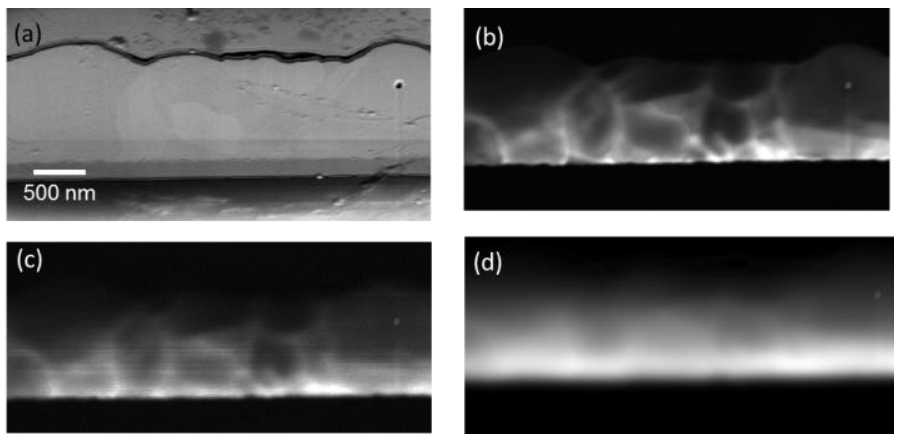

Fig. 3. (a) Cross-sectional SEM image on a FIB prepared device. Corresponding EBIC images at (b) $3 \mathrm{keV}$, (c) $5 \mathrm{keV}$, and (d) $10 \mathrm{keV}$. The calculated penetration depth in CdTe is $40 \mathrm{~nm}, 90 \mathrm{~nm}$, and 310 $\mathrm{nm}$ at $3 \mathrm{keV}, 5 \mathrm{keV}$, and $10 \mathrm{keV}$, respectively [7]. 
Both EBIC and NSOM techniques are surface sensitive owing to the carrier generation close to the exposed surface. To extract material parameters, an analytical modeling is required to de-convolute non-linear responses at different beam energies (or different wavelength) and complex signals arising from grain bulk and grain boundaries. Figure 4 (a) plots a series of EBIC line scans obtained on a large, single grain at different acceleration voltages. We estimate an uncertainty in the $p-n$ junction position of $50 \mathrm{~nm}$ based on the discretization of the electron beam position in the line scan. For quantitative comparison, we performed least-squares fitting of the individual EBIC line scans using an analytical model [6]. Figure 4 (b) plots extracted effective minority carrier diffusion lengths at different acceleration voltages, which is quite similar to that estimated from NSOM data (Figure $2 \mathrm{c}$ ). However, this local value $\left(L_{n} \approx 0.6 \mu \mathrm{m}\right)$ is smaller than the value of $L_{n}$ extracted from the macro-scale quantum efficiency profile $\left(L_{n} \approx 1 \mu \mathrm{m}\right)$, which would be attributed to influence of grain boundaries.

In conventional EBIC models, the carrier collection efficiency at $p$ - $n$ junction is $100 \%$ inside the depletion region where built-in potential is present [6]. On the contrary, we found that the EBIC efficiencies extracted in our devices are well below $100 \%$. The measured EBIC efficiency rapidly increases with the beam voltage, indicating that a strong recombination occurs near the surface. However, the EBIC efficiency remains below $60 \%$ even when the electron beam is injected far away from the surface. Such low EBIC efficiencies implies that some factors (e.g., recombination, screening of built-in field by generated carriers) not accounted in standard model play an important role. The source of the reduced EBIC efficiency in the depletion region is the subject of on-going work. Nevertheless, our model has shown that the diffusion length extracted in the neutral region is insensitive to enhanced recombination in the depletion region.

\section{CONCLUSIONS}

In summary, we present local photocurrent measurements of a cross-sectional CdTe solar cell that were obtained by nearfield optical illumination and by focused electron beam irradiation. The spatially and spectrally resolved efficiency maps confirm a higher carrier collection at grain boundaries. We estimate the minority carrier diffusion lengths of individual grains away from grain boundaries obtained from NSOM and EBIC data, showing an excellent agreement between the techniques. Local values $\left(L_{n} \approx 0.6 \mu \mathrm{m}\right)$ are smaller than the one $\left(L_{n} \approx 1 \mu \mathrm{m}\right)$ extracted from the macroscale quantum efficiency measurement which is attributed to the contribution of grain boundaries. We find that the maximum EBIC efficiency at the $p-n$ junction in the FIB prepared CdTe solar cell is significantly less than $100 \%$, implying that the standard EBIC model is insufficient for quantitative data processing. Development of analytical models which account for surface recombination in depletion regions and screening of built-in fields from carrier accumulation are in progress.
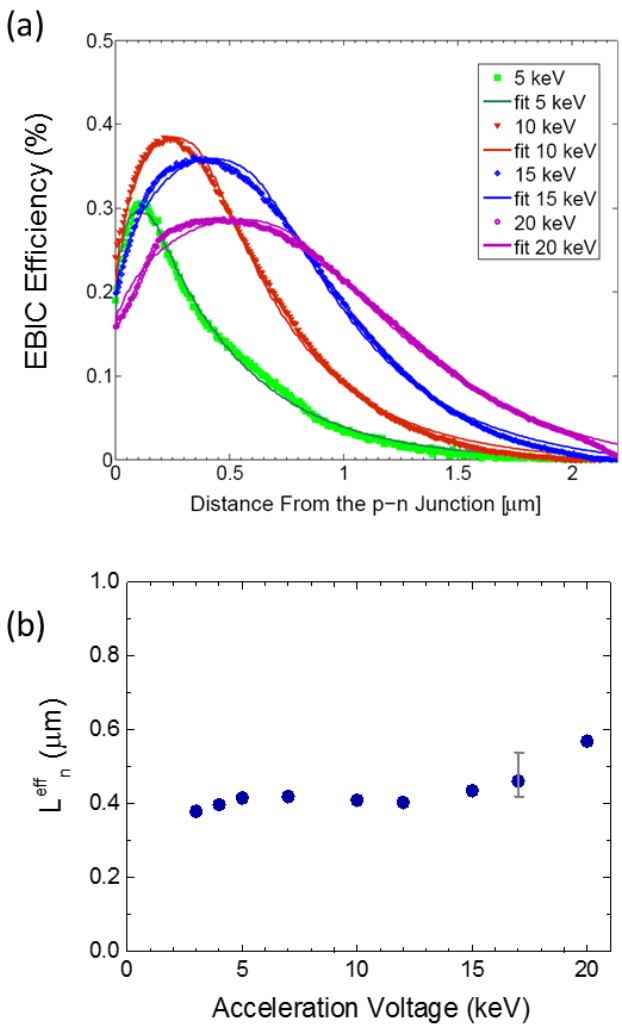

Fig. 4. (a) Solid are experimental EBIC line scans obtained for a large, single grain. (b) Estimated minority carrier diffusion lengths at different acceleration voltages.

\section{REFERENCES}

[1] Research Cell Efficiency Records (http://www.nrel.gov/ncpv/).

[2] S. S. Hegedus and W. N. Shafarman, "Thin-Film Solar Cells: Device Measurements and Analysis", Prog. Photovolt: Res. Appl., vol. 12, pp. 155-176, 2004.

[3] S. Kumar and K. Rao, "Physics and chemistry of CdTe/CdS thin film heterojunction photovoltaic devices: fundamental and critical aspects", Energy Environ. Sci., vol. 7, p.45, 2014

[4] H. Yoon et al., "Local electrical characterization of cadmium telluride solar cells using low-energy electron beam”, Sol. Eng. Mat. Sol. Cells, vol. 117, pp. 499-504, 2013.

[5] J. D. Poplawsky et al., "Direct Imaging of Cl- and Cu-Induced Short-Circuit Efficiency Changes in CdTe Solar Cells", Adv. Energy Mater., 1400454, 2014.

[6] P. M. Haney, H. P. Yoon, P. Koirala, R. W. Collins, N. B. Zhitenev, "Electron beam induced current in the high injection regime", arXiv:1410.4435v1, 2014.

[7] D. Drouin et al., "CASINO V2.42 - A Fast and Easy-touse Modeling Tool for Scanning Electron Microscopy and Microanalysis Users", Scanning 29, 2007, pp. 92-101. 


\section{LOCAL MEASUREMENTS OF PHOTOCURRENT AND BAND GAP IN CdTe SOLAR CELLS}

\section{Yohan Yoon,}

Center for Nanoscale Science and Technology, National Institute of Standards and Technology, Gaithersburg, MD 20899, USA

Maryland Nanocenter, University of Maryland, College Park, MD 20742, USA e-mail: yohan.yoon@ nist.gov Jungseok Chae,

Center for Nanoscale Science and Technology, National Institute of Standards and Technology, Gaithersburg, MD 20899, USA

Maryland Nanocenter, University of Maryland, College Park, MD 20742, USA

Aaron Katzenmeyer,

Center for Nanoscale Science and Technology, National Institute of Standards and Technology, Gaithersburg, MD 20899, USA

Maryland Nanocenter, University of Maryland, College Park, MD 20742, USA

\section{Heayoung Yoon,}

Center for Nanoscale Science and Technology, National Institute of Standards and Technology, Gaithersburg, MD 20899, USA

Maryland Nanocenter, University of Maryland, College Park, MD 20742, USA

Joshua Schumacher,

Center for Nanoscale Science and Technology, National Institute of Standards and Technology, Gaithersburg, MD 20899, USA

Sangmin An,

Center for Nanoscale Science and Technology, National Institute of Standards and Technology, Gaithersburg, MD 20899, USA

Maryland Nanocenter, University of Maryland, College Park, MD 20742, USA

\section{Andrea Centrone}

Center for Nanoscale Science and Technology, National Institute of Standards and Technology, Gaithersburg, MD 20899, USA

Nikolai Zhitenev,

Center for Nanoscale Science and Technology, National Institute of Standards and Technology, Gaithersburg, MD 20899, USA e-mail: nikolai.zhitenev@nist.gov

ABSTRACT: Polycrystalline thin film technology has shown great promise for low cost, high efficiency photovoltaics. To further increase the power efficiency, a better understanding of microstructural properties of the devices is required. In this work, we investigate the inhomogeneous electrical and optical properties using local excitation techniques that generate excess carriers by a near-field light illumination or by a focused electron beam irradiation. The spatially-resolved photocurrent images of n-CdS / p-CdTe devices obtained by both techniques show high carrier collection efficiencies at grain boundaries. A novel and complementary technique, photothermal induced resonance (PTIR), is also used to obtain absorption spectra and maps in the near-field over a broad range of wavelengths. In PTIR a wavelength tunable pulsed laser is used in combination with an atomic force microscope tip to detect the local thermal expansion induced by light absorption. Sub-micrometer thick lamella samples of CdTe solar cells are measured, and the variation of local band-gap is analyzed. We discuss the resolution and the sensitivity of the techniques in the range of photon energies close to the band gap.

Keywords: photothermal induced resonance (PTIR), CdTe solar cells, band-gap, scanning photocurrent microscopy (SPCM), near-field scanning optical microscopy (NSOM)

\section{INTRODUCTION}

Polycrystalline thin film photovoltaic (PV) technologies have shown great promise for solar energy harvesting using inexpensive PV materials, currently reaching a power conversion efficiency of $\approx 21 \%$ for cadmium telluride (CdTe) and $\approx 22 \%$ for copper indium gallium selenide (CIGS) solar cells [1]. To achieve the maximum efficiency of $\approx 30 \%$ theoretically possible for these technologies, considerable efforts have been made to understand the physical mechanisms that limit cell performance. To further understand current limitations, the impact of inhomogeneous variation of grains and grain boundaries (GBs) on charge transport needs to be analyzed. In general, grain boundaries can act as recombination centers, even if they are passivated during the post-annealing process (e.g., $\mathrm{CdCl}_{2}$ treatment) [2]. Inter-diffusion between $\mathrm{CdTe}$ and $\mathrm{CdS}$ can also induce additional recombination in the p-n junction or at the heterogeneous interfaces (e.g., grain to grain, grain to grain boundary) [3, 4]. In this work, we investigate the inhomogeneous electrical and optical properties using local excitation techniques that generate excess carriers by a near-field light illumination or by a focused electron beam irradiation. Furthermore, we present a complementary technique, photothermal induced resonance, which can directly measure the local variations of energy band-gap within CdTe grains and along the p-n junction.

\section{EXPERIMANTAL}

p-CdTe / n-CdS solar cells extracted from a commercial solar panel were used for scanning photocurrent microscopy electron beam induced current (EBIC) and photothermal induced resonance measurements. A thick $100 \mathrm{~nm}$ evaporated Au pad served as a top contact ( $\mathrm{Au} / \mathrm{p}-\mathrm{CdTe})$ and the common contact to the TCO layer was made using indium solder (indium / n$\mathrm{CdS} / \mathrm{SnO}_{2}$ ). Cross-sections of the samples for SPCM and EBIC and the lamella samples for PTIR were prepared by a focused ion beam (FIB). To achieve high spatial resolution $(<1 \mu \mathrm{m})$ in SPCM measurements, we adapted an optical fiber probe (200 $\mathrm{nm}$ in diameter) mounted on a tuning fork, typically used for near-field scanning optical microscopy (NSOM). EBIC measurements were performed in a scanning electron microscope (SEM) equipped with a nano-manipulator that was used to position an electrical probe on the contact to CdTe.

\section{RESULTS AND DISCUSSION}

\subsection{Scanning photocurrent microscopy (SPCM)}

Fig. 1 shows a cross-sectional SPCM (a; $405 \mathrm{~nm}$ illumination) and an EBIC image (b; $5 \mathrm{kV}$ excitation) of CdTe devices prepared by a FIB milling process. As 

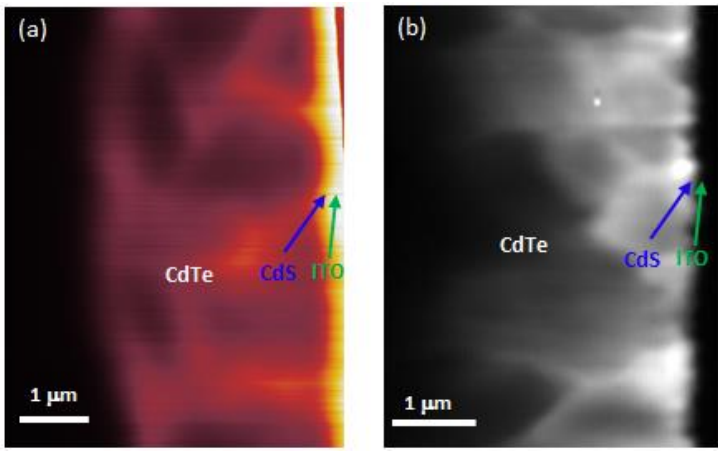

Fig. 1 Cross-sectional photocurrent images of FIB prepared p-CdTe/n-CdS devices. (a) SPCM image (laser wavelength: $405 \mathrm{~nm}$, diameter of NSOM probe: $200 \mathrm{~nm}$ ). The measured maximum photocurrent was $3.3 \mathrm{nA}$. (b) A 5 $\mathrm{kV}$ EBIC image (beam current: $300 \mathrm{pA}$ ).

expected, the measured photocurrent is large at the excitation near the $p-n$ junction and decreases away from the junction [5]. Both images show that the grain boundaries are indeed more efficient photocurrent collectors than the grain interiors (GIs). This property can be associated with the local electric field at GBs, possibly induced by the accumulation of chlorine $\left(\mathrm{Cl}^{-}\right)$[6] or by other charged defects. The band bending drives holes away from the grain boundary thus decreasing recombination. [7] Somewhat surprisingly, the photocurrent at GBs can be as strong or stronger than that at the $p$-n junction, making the identification of the depletion region difficult. We note that both a $405 \mathrm{~nm}$ laser beam and a $5 \mathrm{kV}$ electron irradiation have similar shallow absorption depth in CdTe $(\approx 100 \mathrm{~nm})$, and thus the generated free carriers are subject to high recombination at the surface or within a layer modified / damaged by ion milling. The interaction volumes are increasing at longer laser wavelengths and at larger electron beam voltages, thus the contrast between GBs and GIs becomes weaker due to the averaging of photocurrent over the excitation volume. To extract quantitative parameters, an accurate modeling is required to de-convolute the responses at different beam energies and/or different wavelengths and to explicitly account for recombination at or near the surface, which is an on-going study.

\subsection{Photothermal induced resonance (PTIR)}

To independently determine a compositional variation over the polycrystalline absorber and across the overall device structure, we explore the potential of a novel optical spectroscopic microscopy, PTIR, based on the photothermal effect [8]. The local expansion of the sample induced by the absorption of pulsed light from a tunable laser is detected by an AFM tip instantaneously (see Fig. 2). PTIR was recently extended to the visible and near-IR ranges [9]. In this work, we employ the PTIR technique to measure the local band-gap of CdTe lamella obtained by FIB-sectioning of a CdTe photovoltaic device. The bandgap transition of bulk CdTe absorber is expected to be $\approx 1.45 \mathrm{eV}$, while the local absorption may vary at interfaces (e.g., grain boundaries, metallurgical junction) due to inhomogeneous local compositions. For this study, we use a range of the energies near the CdTe band gap transition (around of $1.45 \mathrm{eV}$ ) to determine local absorption variation due to compositional variation in CdTe layer.
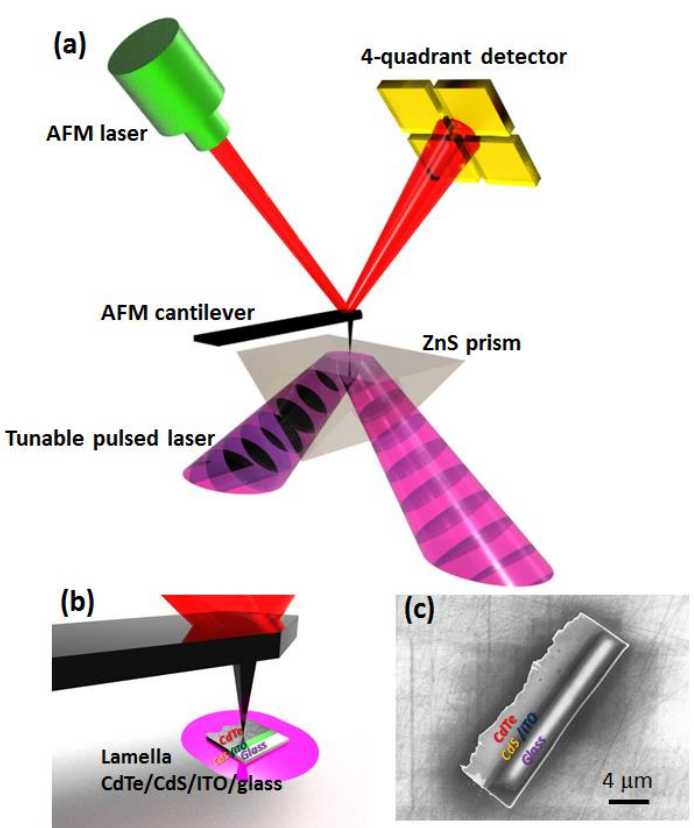

Fig. 2 Schematics of PTIR technique. (a) A visible laser illuminating from the side of the $\mathrm{ZnS}$ prism produces local expansion of the sample. (b) The local expansion induces the deflection of the AFM cantilever, which is detected by a 4-quadrant detector. (c) An SEM image of a FIB prepared lamella (CdTe/CdS/ITO/glass) on a $\mathrm{ZnS}$ prism.

We applied the PTIR technique to the FIB prepared lamella of CdTe sample that was transferred to a zinc sulfide $(\mathrm{ZnS})$ prism by a glass needle using a mircomanipulator. Fig 3 shows the topography, PTIR images $(1.45 \mathrm{eV}, 1.55 \mathrm{eV})$, and PTIR spectra $(1.2 \mathrm{eV}$ to $1.7 \mathrm{eV})$ for the $\mathrm{CdTe} / \mathrm{CdS} / \mathrm{ITO}$ (indium tin oxide)/glass lamella sample. PTIR images and spectra were acquired in the range of the energies of laser excitation close to CdTe band gap to explore the compositional variation through the absorber. As seen in the maps, the intensity of the PTIR signal varies over CdTe area: a larger signal is seen near CdTe surface (Fig. 3b) and, in some areas, in the vicinity of the p-n junction (blue lines in each image).
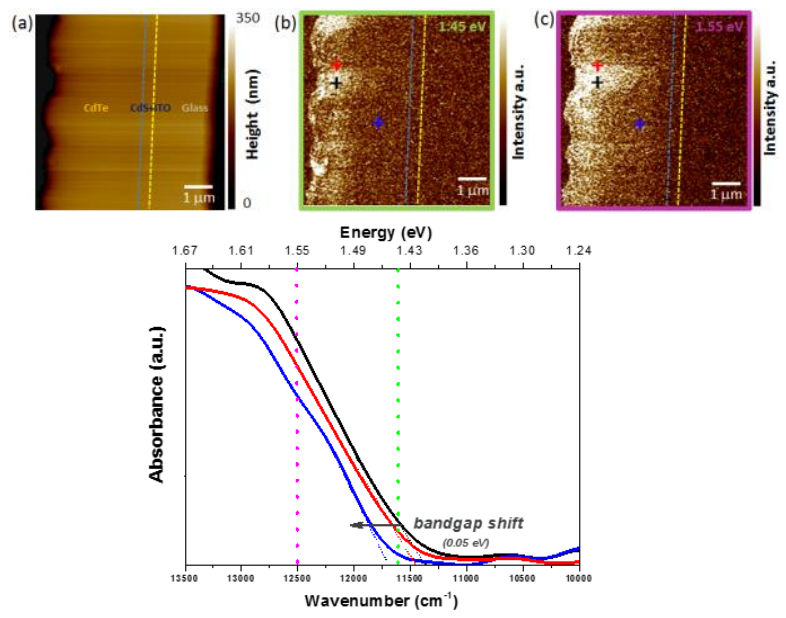

Fig. 3 PTIR data of a p-CdTe / n-CdS / ITO / glass lamella sample. (a) Topography, (b) $1.45 \mathrm{eV}$ PTIR image, and (c) $1.55 \mathrm{eV}$ PTIR image. (d) PTIR spectra collected at three different spots (color-coded locations denoted in the PTIR images) 
Thus, the PTIR technique enables imaging heterogeneous and homogeneous domains in CdTe with submicron spatial resolution. We interpret the origin of PTIR contrast as the variation of absorption caused by a band gap variation. The PTIR spectra in Fig. 3d clearly show a band gap shift $(0.045 \mathrm{eV})$ at the color-coded locations denoted in the PTIR images. The extrapolation lines (dashed lines) indicate optical band-gaps (Eg) of $\approx 1.412 \mathrm{eV}, \approx 1.426 \mathrm{eV}$ and $\approx 1.457 \mathrm{eV}$ for black, red, and blue lines, respectively. A possible reason for the band gap variation is sulfur diffusion from the CdS to the CdTe, resulting in the formation of $\mathrm{CdTe}_{1-\mathrm{x}} \mathrm{S}_{\mathrm{x}}$ [10]. Other possibility is that $\mathrm{CdCl}_{2}$ treatment affects the interior of CdTe grains via potential incorporation of electrically active impurities.

\section{CONCLUSIONS}

In summary, we have analyzed functional and structural properties of CdTe solar cells with two different techniques. In high-resolution photocurrent maps of crosssections of CdTe devices obtained by SPCM and EBIC techniques, grain boundaries showed higher carrier collection properties than grain interiors, and the photocurrent decreases away from the p-n junction. Spectroscopic PTIR measurements showed a variation of local absorption in a range of wavelengths near the CdTe band gap, implying an inhomogeneous composition of the CdTe absorber layer although the PTIR images don't show the contrast between GIs and GBs.

\section{REFERENCES}

[1] Research Cell Efficiency Records (http://www.nrel.gov/ncpv/).

[2] H. R. Moutinho, M. M. Al-Jassim, D. H. Levi, P. C. Dippo, and L. L. Kazmerski, J. Vac. Sci. \& Tech. A 16, 1251 (1998)

[3] M. K. Herndon, A. Gupta, V. Kaydanov, and R. T. Collins, Appl. Phys. Lett. 85, 3854 (2004)

[4] L. Kranz, J. Perrenoud, F. Pianezzi, C. Gretener, P. Rossbach, S. Buecheler and A. N. Tiwari, Sol. Energy Mater. Sol. Cells 105, 213 (2012)

[5] H. P. Yoon, P. M. Haney, D. Ruzmetov, H. Xu, M. S.

Leite, B. H. Hamadani, A. A. Talin, and N. B. Zhitenev, Solar Energy Mater. Sol. Cells 117, 499 (2013).

[6] I. Visoly-Fisher, S. R. Cohen, and D. Cahen, Appl. Phys. Lett. 82, 556 (2003)

[7] M. Gloeckler, J. R. Sites and W. K. Metzger, J. of Appl. Phys. 98 (11), 113704 (2005)

[8] B. Lahiri, G. Holland, A. Centrone, Small, 9439

(2013)

[9] A. Katzenmeyer, G. Holland, K. Kjoller, A. Centrone, submitted.

[10] Kumar, S. G.; Rao, K. S. R. K., Energy Environ. Sci.7, 45 (2014) 


\title{
Imaging in Liquids through Ultra-thin Membranes: A Comparative Analysis of Scanning Electron and Scanning Microwave Microscopies
}

\author{
Alexander Tselev ${ }^{1,2}$, Jeyavel Velmurugan ${ }^{3,4}$ and Andrei Kolmakov ${ }^{3 *}$ \\ ${ }^{1}$ Center for Nanophase Materials Sciences, Oak Ridge National Laboratory, Oak Ridge, TN 37831, USA \\ ${ }^{2}$ Institute for Functional Imaging of Materials, Oak Ridge National Laboratory, Oak Ridge, TN 37831 , \\ USA \\ ${ }^{3}$ Center for Nanoscale Science and Technology, National Institute of Standards and Technology, \\ Gaithersburg, MD 20899 \\ ${ }^{4}$ Maryland Nano Center, University of Maryland, College Park, MD 20742
}

The ability of the near-field microwave microscopy to image subsurface structures has been realized shortly after the invention of the technique [1-3]. Based on these capabilities, we proposed and successfully tested a novel concept of in situ near-field scanning microwave impedance microscopy (sMIM) for imaging of reactive and biological samples [4, 5]. In our approach, the samples of interest were separated from ambient by a few nanometer thick dielectric membranes transparent for microwave radiation. Different from prior examples of microwave imaging in liquids, where the probe had to be immersed in a liquid or exposed to aggressive vapors [6,7], our approach benefits from complete isolation of the probe and the rest of the microscope from reactive environment and therefore enables in situ imaging of previously inaccessible highly reactive, toxic, corrosive, or radioactive samples. Here we report on a comparative side-by-side study of imaging capabilities of liquid scanning electron microscopy (SEM) and liquid sMIM techniques using the same set of biological and inorganic samples. In particular, the sensitivity, spatial resolution, probing depth, scanning rate, and probe induced effects are compared.

Figure 1 demonstrates, the experimental setup and nanoscale images of electrochemical processes in liquids, using microwave near-field, formed at a sharp scanning probe. In particular, we demonstrate in situ real time imaging of metal (Ag) dendrites growth at electrode-liquid electrolyte interface during electrochemical plating reaction. The panel (d) shows low resolution SEM image of the same sample area as in (c). The contrast of the dendrites in secondary electron images confirms their higher $\mathrm{Z}$ number compared to the liquid electrolyte. The central part depicts the attempt to acquire higher resolution SEM image. The degradation of Ag deposit is due to electron beam induced oxidation of metal $\mathrm{Ag}$ and its dissolution by the electrolyte [8],[9]. Different to the liquid SEM and transmission electron microscopy, sMIM imaging is nondestructive, free from beam-induced artifacts and can be readily applied to electrochemical studies thanks to the extremely low energy deposited to the sample. Indeed, the energy of $3 \mathrm{GHz}$ microwaves is of the order of $\approx 10 \mu \mathrm{eV}$, which can affect neither electronic states nor chemical bonds in condensed matter.

The demonstrated spatial resolution of sMIM imaging in our experiment was $\approx 250 \mathrm{~nm}$. However, the resolution can be improved since it depends on parameters such as probe tip radius, membrane thickness and dielectric constants difference. Under optimal conditions, a resolution of $\approx 50 \mathrm{~nm}$ can be achieved for metallic objects with the commercially available probes with $\approx 50 \mathrm{~nm}$ tip apex radius. Such resolution is comparable to that demonstrated by SEM in liquids [10].

Kolmakov, Andrei; Tselev, Alexander; Velmurugan, Jeyavel. 
References:

[1] K. Lai, et al. Review of Scientific Instruments, vol. 78, 2007, p. 063702.

[2] C. Plassard, et al., Physical Review B, vol. 83, 2011, p. 121409.

[3] J. J. Kopanski, et al. ECS Transactions, vol. 61, 2014, pp. 185-193.

[4] A. Tselev and A. Kolmakov, Microwave Conference (EuMC), 2015 European, 2015, pp. 662-665.

[5] A. Tselev, et al. vol. 10 (3), 2016, pp 3562-3570.

[6] S. Kim, et al, Applied Physics Letters, vol. 86, 2005, p. 153506.

[7] M. Farina, et al, Microwave and Wireless Components Letters, IEEE, vol. 22, 2012, pp. 595-597.

[8] K. W. Noh, et al, Ultramicroscopy, vol. 116, 2012, pp. 34-38.

[9] N. M. Schneider, et al, The Journal of Physical Chemistry C, vol. 118, 2014, pp. 22373-22382.

[10] S. Thiberge, et al, Review of Scientific Instruments, vol. 75, 2004, pp. 2280-2289.

[11] The research was supported in part through Scientific User Facilities Division (ORNL), Office of Basic Energy Sciences, U. S. Department of Energy, U.S. Civilian Research and Development

Foundation and NIST-CNST/UMD-IREAP Cooperative Agreement

a)

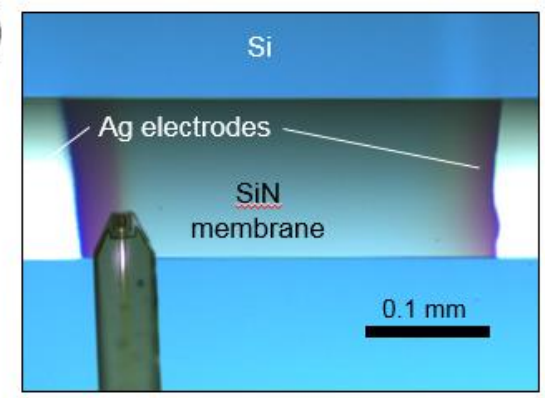

c)

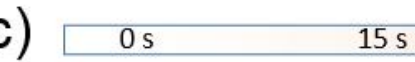

b)

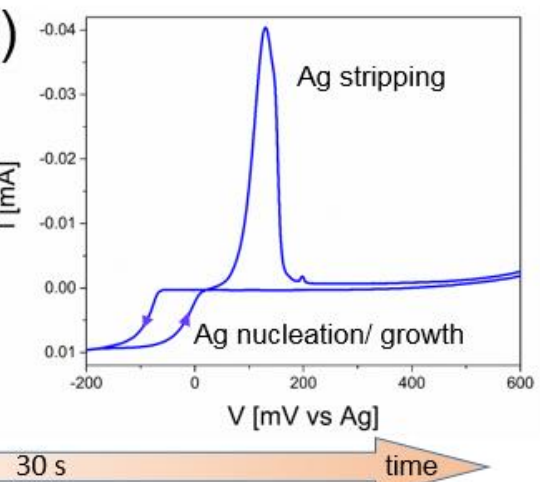

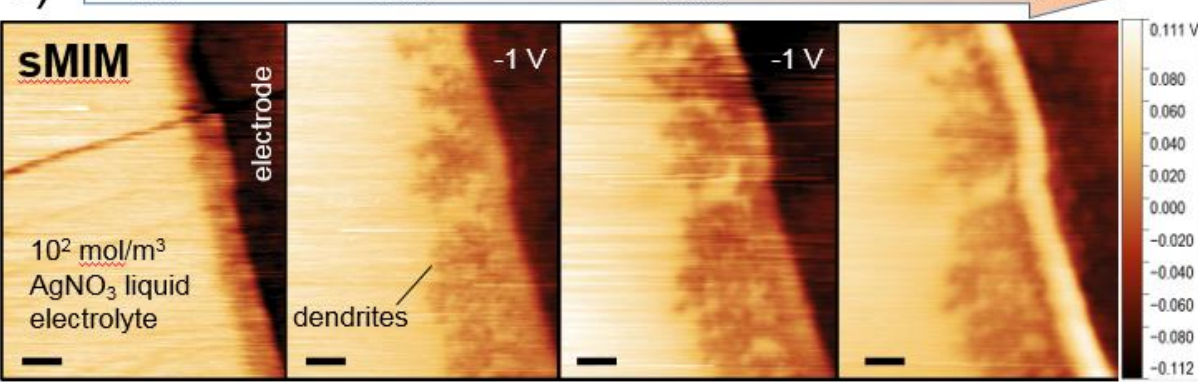

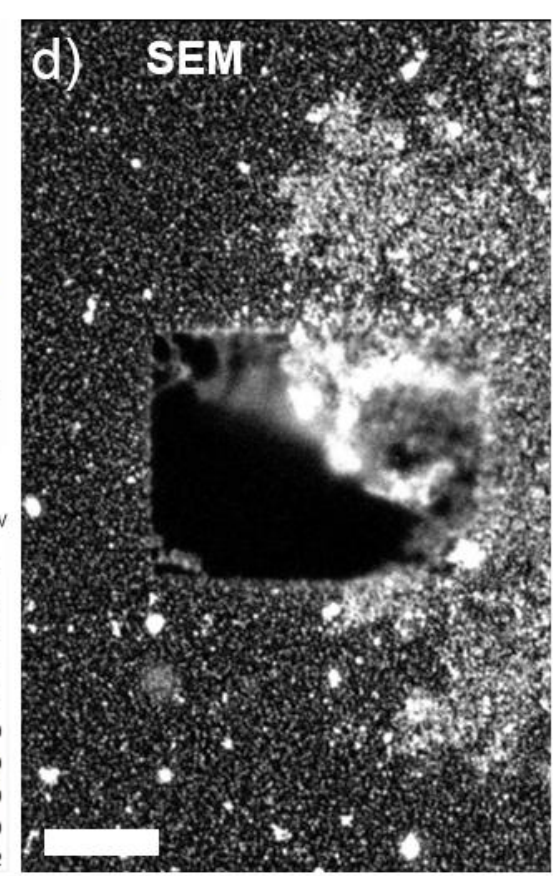

Figure 1. (a) Optical bright field top view of the electrochemical environmental cell for sMIM imaging in liquid electrolytes with sMIM probe scanning over the SiN membrane. (b) A cyclic voltammogram of Ag dendritic growth and stripping at/from Ag electrode of the environmental cell. (c) sMIM-C maps of electrochemical Ag dendritic growth on Ag cathode taken at open circuit condition induced by a sequence of the applied potential (-1 V, $15 \mathrm{~s}$ ) steps. (d) Comparative liquid SEM image of the same dendrites. An attempt to acquire high-resolution SEM image of the dendrite apex leads to beam induced dendrite oxidation and dissolution by electrolyte. Scale bars in (b) and (d) are $100 \mu \mathrm{m}$ and $2 \mu \mathrm{m}$ correspondingly. 


\title{
Bridging the Pressure Gap in Electron Beam Induced Current Microscopy: Imaging Charge Transport in Metal Oxide Nanowires under Atmospheric Pressures
}

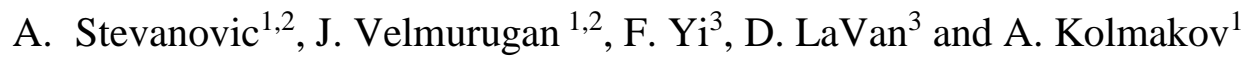 \\ 1. Center for Nanoscale Science and Technology, NIST, Gaithersburg, MD 20899 \\ 2. Maryland Nanocenter, University of Maryland, College Park, MD 20742 \\ 3. Material Measurement Laboratory, NIST, Gaithersburg, MD 20899
}

We investigate the charge transport in metal oxide semiconductor nanowires which are capable of converting solar light into electrical energy. The charge transport in semiconductors is influenced by electron donor or acceptor molecules adsorbed on a nanowire surface and by concentration of local defects affecting the work function and resulting in bending of the conduction and valence bands [1,2]. It is important to understand how this band bending in solid materials can be manipulated and optimized for the purposes of improving both photovoltaic (PV) cells and photocatalysts. In PV cells, band bending influences the charge transport along nanowires while in photocatalysis, the band bending governs the rates of photo-oxidation or photo-reduction reactions when electrons or holes are promoted to a surface. Therefore, the main objective is to characterize the dependence of charge transport through the nanowire and the chemical state of its surface.

We are developing a novel ambient pressure scanning electron microscopy (SEM) technique to study the interplay between surface reactivity and electronic properties of metal oxide nanowires such as $\mathrm{SnO}_{2}$, $\mathrm{TiO}_{2}$ and $\mathrm{ZnO}$. The main objective is to image electron beam induced current (EBIC) along a nanowire in the presence of oxidizing or reducing gas at atmospheric pressure. To bridge the "pressure gap" between high vacuum required for SEM operation and atmospheric pressure environment that houses the nanowire, we designed a measurement cell that is functional over a wide pressure range of $10^{-5} \mathrm{~Pa}$ to $10^{5} \mathrm{~Pa}$. An electron transparent $\mathrm{SiN}_{\mathrm{x}}$ membrane separates the two extremely different ambiences, atmospheric pressure region and high vacuum, allowing for both SEM and EBIC imaging as shown in Figure 1A. Figure $1 \mathrm{~B}$ shows the SEM image of a single $50 \mu \mathrm{m}$ long and $0.22 \mu \mathrm{m}(220 \mathrm{~nm})$ thick $\mathrm{SnO}_{2}$ nanowire stretched between two platinum electrodes.

Here, we present the first imaging of the charge transport along $\mathrm{SnO}_{2}$ nanowire excited by $10 \mathrm{keV}$ electron beam under both vacuum and atmospheric pressure environments. We found that EBIC measurements are sensitive to adsorbates on $\mathrm{SnO}_{2}$ surface and electrode contacts that induce changes in the electronic structure of the semiconductor affecting its conductivity. Figure 2 shows the change in contrast in EBIC images under (A) vacuum and (B) air at $10^{5} \mathrm{~Pa}$. The change in EBIC contrast when the nanowire is exposed to air shows that electron transport is significantly reduced (gray scale is less bright compare to the vacuum measurement) likely due to the adsorption of oxygen on $\mathrm{SnO}_{2}$ surface that acts like an electron acceptor molecule. In addition, the I-V measurements reveal that the conductivity of the nanowire under vacuum is much higher (about three orders of magnitude) than in air.

Kolmakov, Andrei; LaVan, David; Stevanovic, Ana; Velmurugan, Jeyavel; Yi, Feng. "Bridging the Pressure Gap in Electron Beam Induced Current Microscopy: Imaging Charge Transport in Metal Oxide Nanowires under Atmospheric Pressures."

Paper presented at Microscopy \& Microanalysis, Columbus, OH. July 24, 2016 - July 28, 2016. 
A.

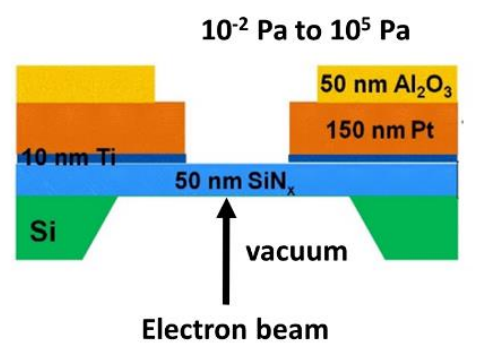

B.

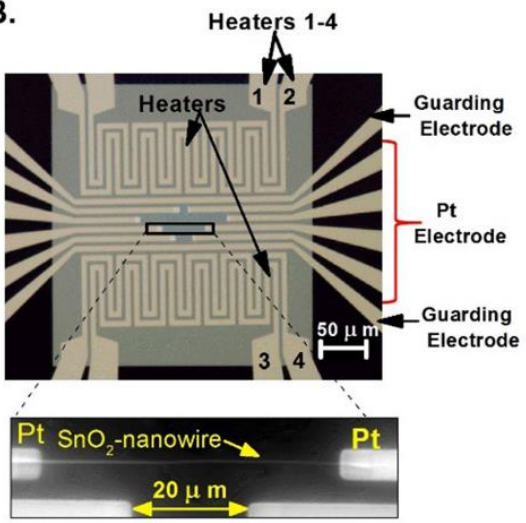

Figure 1. A. Fabrication of a micro cell - side view. A $50 \mathrm{~nm}$ thick $\mathrm{SiN}_{\mathrm{x}}$ electron transparent membrane (blue) which separates atmospheric from high vacuum conditions is deposited on the Si-chip. B. Electrodes layout. The micro sensor consists of Pt electrodes, Pt heaters and guarding electrodes [3]. The Pt electrodes can accommodate different nanowire lengths $(10 \mu \mathrm{m} ; 20 \mu \mathrm{m} ; 50 \mu \mathrm{m}$ and $100 \mu \mathrm{m})$ and the Pt heaters allow for uniform heating and cooling. The two guarding electrodes serve to block the electromagnetic field interference of the working heaters on electron beam induced current through a nanowire.
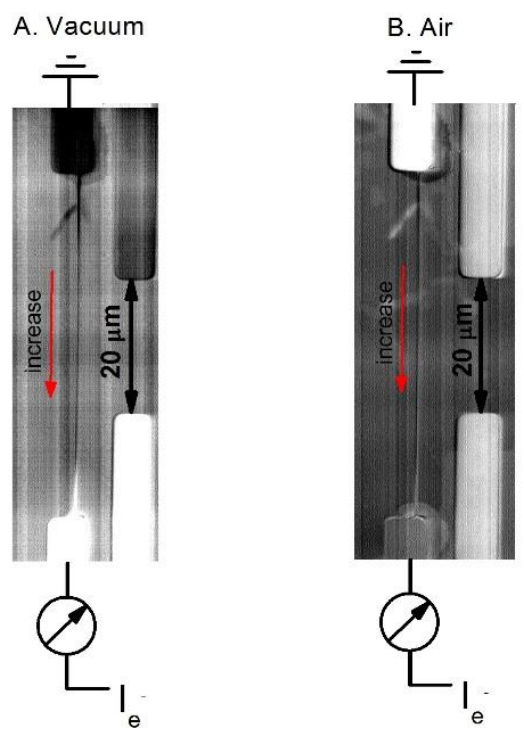

Figure 2. EBIC images of the charge transport through the $\mathrm{SnO}_{2}$ nanowire under A. vacuum and B. air at $10^{5} \mathrm{~Pa}$. Both images show increase in EBIC contrast as electron beam approaches the non-grounded electrode and electronically excited electrons in $\mathrm{SnO}_{2}$ are more collected there. However, the EBIC image recorded in air shows less contrast than the image recoded under vacuum. This decrease in charge transport in air can be attributed to the adsorption of $\mathrm{O}_{2}$ that takes away excited electrons from the nanowire.

\section{References:}

[1] A Stevanovic et al, J. Am. Chem. Soc. 134 (2011), p. 324-332

[2] A Stevanovic et al, J. Phys. Chem. C 117 (2013), p. 24189-24195

[3] F Yi et al, J. Microelectromech. S. 24 (2015), p. 1185-1192 


\title{
Li Diffusion in All-Solid-State Batteries Imaged Through Optical and Electron Transparent Electrodes
}

\author{
Alexander Yulaev ${ }^{1,2,3}$, A. Alec Talin ${ }^{4}$, Marina S. Leite ${ }^{2,5}$ and Andrei Kolmakov ${ }^{1}$ \\ ${ }^{1 .}$ Center for Nanoscale Science and Technology, NIST, Gaithersburg, MD, USA. \\ 2. Department of Materials Science and Engineering, University of Maryland, College Park, MD, USA. \\ 3. Maryland NanoCenter, University of Maryland, College Park, MD, USA. \\ 4. Sandia National Laboratories, Livermore, CA, USA \\ 5. Institute for Research in Electronics and Applied Physics, University of Maryland, College Park, USA.
}

The development of all-solid-state batteries has opened new scientific and engineering perspectives towards miniature standalone micro-electronics and medical devices [1]. All-solid-state batteries with thin-film solid electrolytes exhibit a high power-to-weight ratio and double or even triple energy density of liquid electrolyte batteries. In addition, solid batteries enable significant reduction of safety risks related to thermal runaway observed for liquid organic electrolytes [2]. Despite growing interest in the energy storage community dedicated to all-solid-state thin-film batteries, the electrochemical and electromechanical interfacial processes remain largely unexplored at the nanoscale. In particular, LiPON (lithium phosphorus oxynitride) is successfully used as a solid electrolyte for Li-ion batteries [3]. However, the factors that control the intercalation and homogeneity of Li transport in the solid electrolyte are still unknown. Balke et al. utilized electrochemical strain microscopy to study Li-ion transport through LiPON into $\mathrm{Si}$ anode at nanoscale level [4]. Ruzmetov et al. employed transmission electron microscopy (TEM) to probe a nanowire battery with a radial geometry to reveal the scaling limit of a LiPON layer [5].

Here, we report spatially- and depth-resolved study of Li intercalation into a thin-film solid electrolyte applying a combination of optical and electron microscopy. Varying the depth of electron interaction volume by changing energy of electron beam from $2 \mathrm{keV}$ to $15 \mathrm{keV}$, we selectively probe an electrolyte lithiated on one side at different depths. This approach can be used to reconstruct 3D diffusion pathways of Li similar to scanning electron microscopy (SEM) tomography. Our model thin-film all-solid-state Li battery consists of ultrathin silicon anode, LiPON as electrolyte, and metallic lithium as the positive electrode (Fig. 1). To make such a stack, $430 \mathrm{~nm}$ thick LiPON was sputtered onto $35 \mathrm{~nm}$ thick Si freestanding membrane (Fig. 1a). Micro-patterned $100 \mathrm{~nm}$ thick nickel layer was deposited onto electrolyte by e-beam evaporation through a stencil mask to serve as a diffusion barrier for Li intercalation. Using $\mathrm{Ni}$ as a stencil mask, spatially separated metal Li patches were created on LiPON via thermal evaporation (Fig. 1b). Lithium diffusion in LiPON layer was monitored using SEM imaging through the $35 \mathrm{~nm}$ thick Si membrane. Due to difference in electron yield from pristine and lithiated LiPON regions, the Li transport in the electrolyte can be mapped using different electron beam accelerating voltages. Fig. $2 \mathrm{a}$ was recorded at $2 \mathrm{kV}$ electron beam and, therefore, shows Li distribution close to surface $(\approx 40 \mathrm{~nm})$ region. At higher accelerating voltage $(15 \mathrm{kV})$, the electron probing depth spans up to $1.9 \mu \mathrm{m}$; consequently, (Fig. 2b) SEM image depicts Li distribution in electrolyte next to Ni layer. Comparative SEM image analysis reveals the difference in Li spatial distribution via specific choice of electron energy level (Fig. 2c). Monte-Carlo simulations of electron beam interaction in LiPON at $\mathrm{E}_{b}=2 \mathrm{keV}$ and $15 \mathrm{keV}$ supported the observed results. In addition, due to optical transparency of thin-film electrolyte and ultrathin Si anode, Li diffusion profiles at both sides of the battery can be visualized optically (Fig. 2d). We envision that depth selective electron microscopy of thin-film ion batteries will help elucidate the role of temperature, chemical and morphological inhomogeneity of the electrolyte in Li ion transport [6]. 


\section{References:}

[1] J. Bates, et al., Solid State Ionics, vol. 135, 2000, pp. 33-45.

[2] R. Huggins, "Advanced batteries: materials science aspects", (Springer Science \& Business Media), 2008.

[3] J. Bates, et al., Journal of Power Sources, vol. 43, 1993, pp. 103-110.

[4] N. Balke, et al., Nano letters, vol. 10, 2010, pp. 3420-3425.

[5] D. Ruzmetov, et al., Nano letters, vol. 12, 2011, pp. 505-511.

[6] This work has been supported in part by the NIST-CNST/UMD-IREAP Cooperative Agreement.
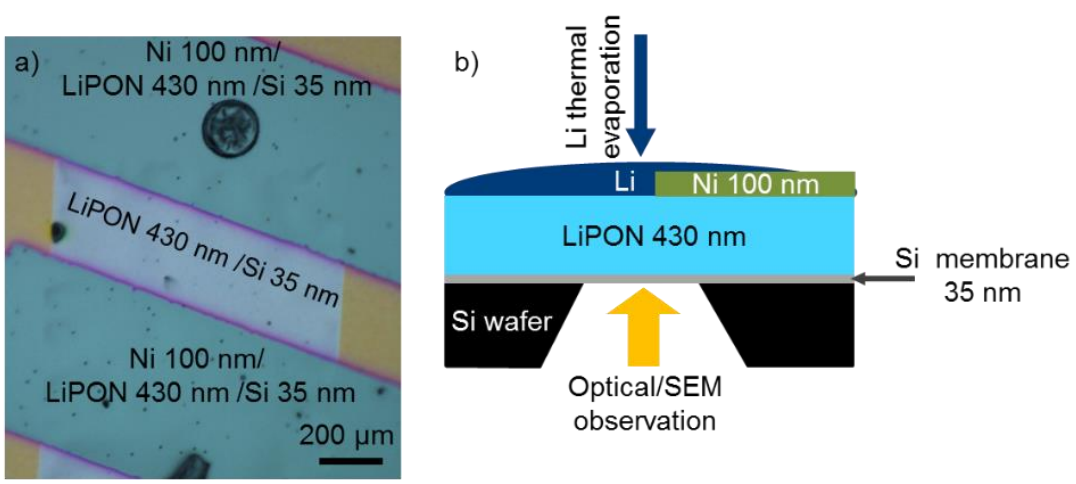

Figure 1. Sample structure: a) The optical image of the top side of the battery before Li evaporation; b) The schematics of the cross sectional view of the battery. Ultra-thin Si membrane is used as optical and electron transparent window.
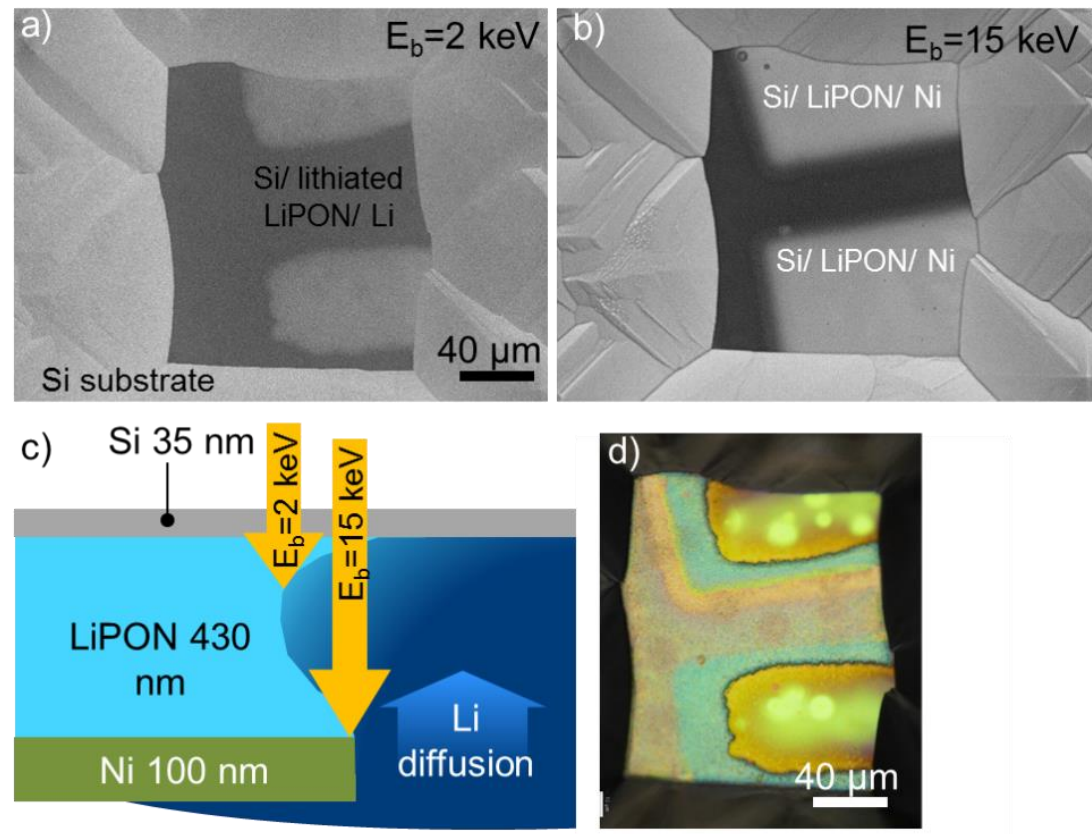

Figure 2. a), b) SEM images obtained at $E_{b}=2 \mathrm{keV}$ and $15 \mathrm{keV}$, respectively; bright areas in Si windows regions correspond to electron scattering from Ni electrode; dark regions represent electron yield from lithiated electrolyte; c) schematic explanation of SEM contrast formation at different energies of the electron beam; d) optical image: lithiated regions are seen through $35 \mathrm{~nm} \mathrm{Si}$ and LiPON layers. 


\section{Versatile fluidic cell for in-situ electrochemical measurements in SEM}

J. Velmurugan ${ }^{1,2}$, A. Stevanovic ${ }^{1,2}$, F. Yi $^{3}$, D. LaVan ${ }^{3}$ and A. Kolmakov ${ }^{1}$

1. Center for Nanoscale Science and Technology, NIST, Gaithersburg, MD 20899

2. Maryland Nanocenter, University of Maryland, College Park, MD 20742

3. Material Measurement Laboratory, NIST, Gaithersburg, MD 20899

Electrochemical techniques such as electrodeposition are crucial for fabrication of nanostructured materials. The details of cluster nucleation and growth at early stages of electrodeposition are extremely important for morphology and properties of the deposits. The growth mechanism is usually determined by analyzing global electrochemical data in conjunction with ex-situ images. Though this approach gives us a clear picture as a whole, it fails to reveal the evolution of islands morphology at nanoscale and its effects on reaction rate. Lately, in-situ transmission electron microscopy (TEM) in liquids has been used successfully to study the surface and interface processes and also to interpret the electrochemical data [1-3]. Surprisingly, there has been less research performed using liquid scanning electron microscopy (SEM) [4]. In this report, we describe novel chip and cell designs enabling us to study electrochemical processes in real time and as a function of temperature using SEM in liquids.

The cell is shown in figure 1 . The cell is based on a microfabricated Si chip equipped with $50 \mathrm{~nm}$ thick $\mathrm{SiN}_{\mathrm{x}}$ membrane, $150 \mathrm{~nm}$ thick Pt electrodes as working electrodes (WE) and heaters [5-6]. $30 \mathrm{~nm}$ layer of $\mathrm{Al}_{2} \mathrm{O}_{3}$ covers the top of electrodes and heaters and serves as an electrochemical protection layer. In conventional electrochemical experiments, a three- electrode geometry is usually preferred. This is because even if the counter electrode (CE) is designed to be larger than the working electrode, the current passing the cell will polarize the counter electrode. If a third electrode is used as a pseudo reference electrode (RE), the current drawn from it should be extremely small (e.g., a few fA) to avoid similar polarization issues. In addition, monitoring the voltage of the counter electrode proves to be useful even in three-electrode experiments to avoid large voltage sweeps that could damage the counter electrode or cause bubble formation in the electrolyte. Both the counter electrode $(\mathrm{Pt})$ and the reference electrode $(\mathrm{Ag})$ were wire bonded to the ceramic chip carrier. The ceramic microchip carrier pressed against a rubber gasket sits tightly on the top of a fluidic cell made of polyetheretherketone. For Ag electroplating, $100 \mathrm{~mol} / \mathrm{m}^{3}$ solution of $\mathrm{AgNO}_{3}$ in water was introduced through syringe and the cell was sealed.

Figure 2 shows the typical SEM images obtained during in-situ Ag electrodeposition on Pt electrode. The images detail the morphological evolution of silver dendrites that were prepared at $100 \mathrm{~mol} / \mathrm{m}^{3}$ silver nitrate aqueous solution. The growth of the dendritic nanostructure was carefully monitored in real time by a current measurement. As the applied deposition potential of $-0.5 \mathrm{~V}$, the initial silver nucleation experienced a morphological evolution and converted to some widespread dendrites. In the first few seconds, Ag start nucleating at several areas of Pt electrode. As the reaction proceeded individual dendrite length of about $5 \mu \mathrm{m}$ to $10 \mu \mathrm{m}$ start branching out from the electrode.

In conclusion, the proposed environmental cell is well suited for in-situ electrochemistry. The setup can be widely extended to investigate morphological transformations in biological systems and electrochemical phenomena like corrosion 


\section{References:}

[1] A. Radisic, P. M. Vereecken, J. B. Hannon, P. C. Searson, and F. M. Ross, Nano Letters, volume 6, no. 2 (2006), p. 238-242.

[2] N. de Jonge and F. M. Ross, Nature Nanotechnology, volume 6, no. 11 (2011), p. 695-704.

[3] J. M. Grogan, N. M. Schneider, F. M. Ross, and H. H. Bau, Journal of the Indian Institute of Science, volume 92, no. 2 (2012), p. 295-308.

[4] E. Jensen, C. Kobler, P. S. Jensen and K. Molhave, Ultramicroscopy, volume 129 (2013), p. 63-69

[5] F. Yi, W. Osborn, J. Betz, J and D.A. LaVan Microelectromechanical Systems, volume 24 (2015), 1185-1192.

[6] M. D. Grapes, T. LaGrange, L.H. Friedman, B. W. Reed, G. H. Campbell, T. P. Weihs and D. A. LaVan Review of Scientific Instruments, volume 85 (2014), 084902
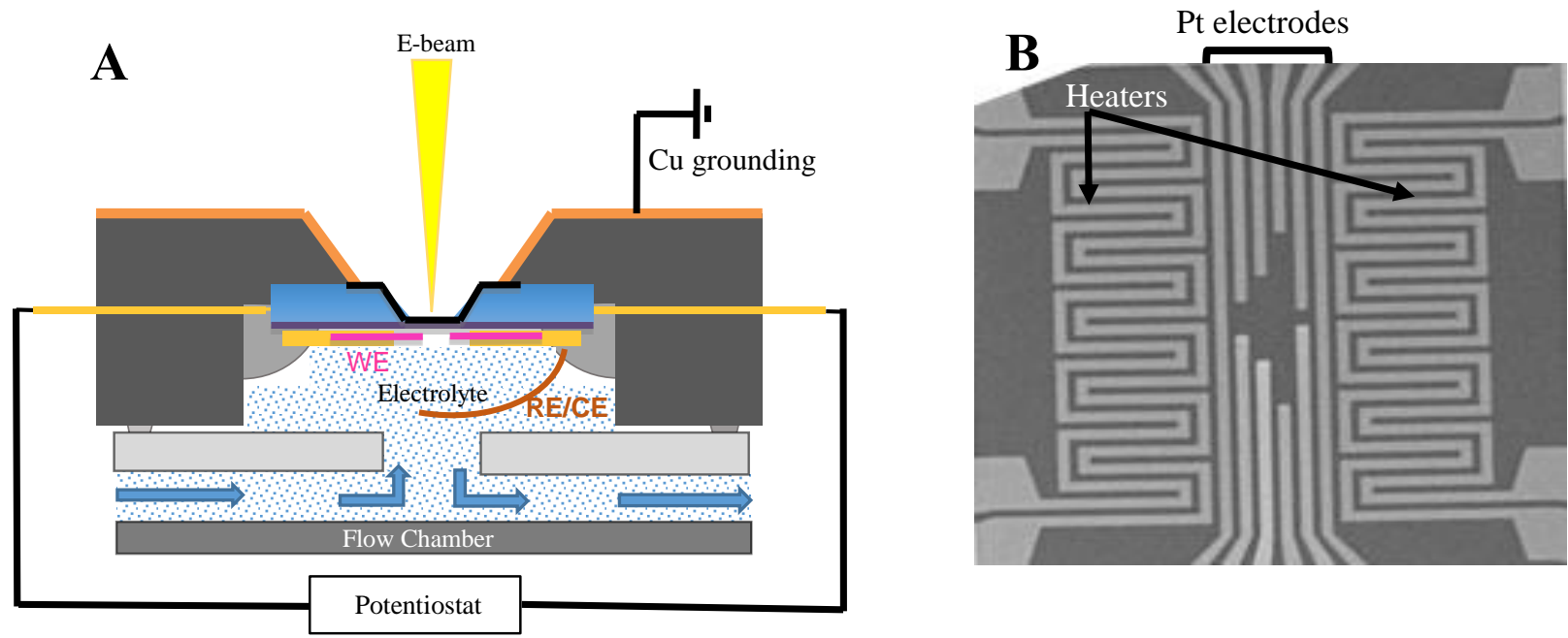

Figure 1: A. Microchip holder and liquid cell. The microchip carrier (dark grey) pressed against a rubber gasket sits tightly on the top of a liquid cell (gray). The chip carrier assembly consists of a Si-chip that hosts a metal oxide nanowire which can experience different gas environments (as shown in B in the circled area.) B. Design of a microchip. The microchip consists of Pt electrodes and Pt heaters. The Pt electrodes are the working electrodes in electrochemical process and the Pt heaters allow for uniform heating and cooling.
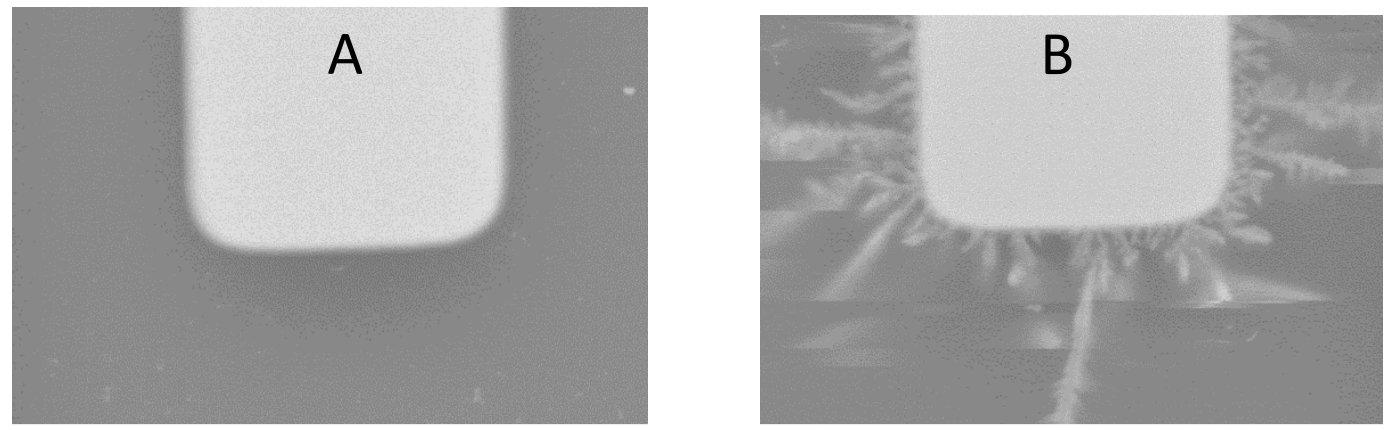

Figure 2: SEM images of the Ag dendrites electrochemically grown over at Pt electrode before (A) and after (B) potential hold at $-0.5 \mathrm{~V}$ vs $\mathrm{Ag} / \mathrm{AgCl}$ in $100 \mathrm{~mol} / \mathrm{m}^{3} \mathrm{AgNO}_{3}$ water solution through $50 \mathrm{~nm} \mathrm{SiN} \mathrm{membrane.}$ 


\section{Minority-carrier dynamics in semiconductors probed by two-photon microscopy}

\author{
Benoit Gaury \\ Center for Nanoscale Science and Technology \\ National Institute of Standards and Technology \\ Gaithersburg, MD 20899, USA \\ Maryland NanoCenter, University of Maryland \\ College Park, MD 20742, USA
}

\author{
Paul M. Haney \\ Center for Nanoscale Science and Technology \\ National Institute of Standards and Technology \\ Gaithersburg, MD, 20899, USA
}

\begin{abstract}
Two-photon time-resolved photoluminescence has been recently applied to various semiconductor devices to determine carrier lifetime and surface recombination velocities. So far the theoretical modeling activity has been mainly limited to the commonly used one-photon counterpart of the technique. Here we provide the analytical solution to a 3D diffusion equation that describes two-photon microscopy in the low-injection regime. We focus on a system with a single buried interface with enhanced recombination, and analyze how transport, bulk and surface recombinations influence photoluminescence decays. We find that bulk measurements are dominated by diffusion at short times and by bulk recombination at long times. Surface recombination modifies bulk signals when the optical spot is less than a diffusion length away from the probed interface. In addition, the resolution is increased as the spot size is reduced, which however makes the signal more sensitive to diffusion.

Index Terms-Two-photon microscopy, carrier lifetime, surface recombination velocity.
\end{abstract}

\section{INTRODUCTION}

The development of semiconductor devices such as photovoltaic solar cells requires quantitative characterization of materials parameters to improve their overall performances. While the minority-carrier lifetime may be the most influential parameter for photovoltaic devices, polycrystalline materials such as CdTe have many grains and grain boundaries whose contributions to recombination remains unclear. Timeresolved photoluminescence (TRPL) is a long-standing optical technique capable of probing bulk lifetime and surface recombination velocities of direct bandgap materials. A TRPL experiment consists of generating electron-hole pairs via a laser pulse, and collecting the radiatively emitted photons over time. The non-radiative recombination occurring in the bulk and at various interfaces influences the resulting photoluminescence (PL) intensity decay, so that adequate modeling of this decay can lead to values of bulk lifetime, diffusion constant and surface recombination velocities.

Two different setups have been used to realize TRPL experiments. The first, most common one, uses incident photons with energy larger than the semiconductor bandgap. The absorption of a single photon is sufficient to generate an electron-hole pair which leads to carrier generation that decays exponentially away from the sample surface. A second route taken in [1]- [2] uses photons with energy smaller

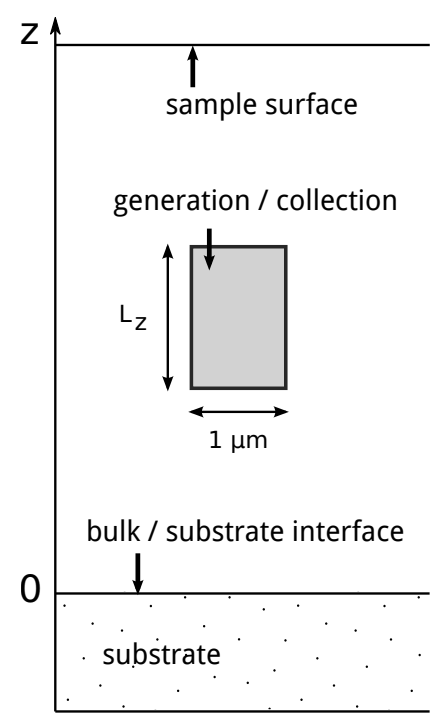

Fig. 1. Schematic of the sample probed by two-photon microscopy. The optical spot (gray area) has lateral size $1 \mu \mathrm{m}$ and axial size $L_{z}=3 \mu \mathrm{m}$. Generation and collection regions are taken identical.

than the semiconductor bandgap, that is in a spectral range where the material is normally transparent. The generation of an electron-hole pair now requires the absorption of two photons. Because this non-linear process is proportional to the square of the incoming photon flux, the generation of carriers occurs preferably at the focal volume of the optical setup. By changing the position of the sample with respect to this focal volume, electron-hole pairs can be generated far below the sample surface. Details on the operating principle can be found in the literature [3]- [4]. There exists an extensive literature on the modeling of one-photon TRPL [5]-[7], which assumes a carrier generation that decays exponentially away from the sample surface. The direct carrier generation below the surface taking place in a two-photon TRPL experiment requires new investigations.

In this manuscript we review our 3D model for two-photon TRPL (section $\Pi$ ), and we apply it to optical spots in the bulk and at the buried bulk/substrate interface of our model 
system described in Fig. 11. In section [III we focus on the impact of carrier diffusion and recombination on PL decays for a bulk measurement. We show how surface recombination changes PL intensities and discuss the resolution of the optical technique in section IV Throughout this paper, unless explicitly stated otherwise, we consider an optical spot shaped as a rectangular prism with lateral dimensions of $1 \mu \mathrm{m}$ and axial size $L_{z}=3 \mu \mathrm{m}$. We assume a uniform excitation of carriers and identical generation and collection regions. While these two regions may be in general different, this case can be obtained in an experimental setup using a confocal microscope.

\section{OPTICALLY INDUCED MINORITY-CARRIER TRANSPORT}

We start with our model for the transport of optically induced carriers in a p-type material, and introduce the general solution of the problem for the case of a single surface with enhanced recombination. This surface can describe a sample surface or a buried interface as described by our model system in Fig. [1] We refer to [8] and references therein for more details on the derivations.

The minority-carrier transport is described by the 3D timedependent diffusion equation

$$
\frac{\partial n}{\partial t}(\mathbf{r}, t)-D \Delta n(\mathbf{r}, t)+\frac{n(\mathbf{r}, t)}{\tau}=g(\mathbf{r}) \delta(t),
$$

and the boundary conditions determined by the surface recombination velocity $S$

$$
\begin{aligned}
& D \frac{\partial n}{\partial z}=S n, \quad z=0, \\
& n=0, \quad z \rightarrow+\infty,
\end{aligned}
$$

where $n(\mathbf{r}, t)$ is the electron density, $D$ is the electron diffusion constant, $\tau$ the bulk lifetime and $g(\mathbf{r}) \delta(t)$ is the carrier generation, taken to be instantaneous. Because we linearized the recombination rate [third term in Eq. (1)], Eq. (1) is valid only for small excited carrier densities (low-injection regime). We introduce the Green's function of the problem $G\left(\mathbf{r}, \mathbf{r}^{\prime}, t\right)$ that satisfies

$$
\frac{\partial G}{\partial t}\left(\mathbf{r}, \mathbf{r}^{\prime}, t\right)-D \Delta G\left(\mathbf{r}, \mathbf{r}^{\prime}, t\right)+\frac{G\left(\mathbf{r}, \mathbf{r}^{\prime}, t\right)}{\tau}=\delta\left(\mathbf{r}-\mathbf{r}^{\prime}\right) \delta(t) .
$$

Upon solving Eq. (4) with the boundary conditions Eqs. (2) and $(3)$, one finds $[\overline{8}]$

$$
\begin{aligned}
G\left(x, x^{\prime}, y, y^{\prime}, z, z^{\prime}, t\right)=\frac{e^{-t / \tau}}{2} \frac{e^{-\frac{\left(x-x^{\prime}\right)^{2}}{4 D t}}}{2 \sqrt{\pi D t}} \frac{e^{-\frac{\left(y-y^{\prime}\right)^{2}}{4 D t}}}{2 \sqrt{\pi D t}} \\
\times\left[\frac{e^{-\frac{\left(z-z^{\prime}\right)^{2}}{4 D t}}+e^{-\frac{\left(z+z^{\prime}\right)^{2}}{4 D t}}}{\sqrt{\pi D t}}\right. \\
\left.-2 \frac{S}{D} e^{\frac{S}{D}\left(z+z^{\prime}\right)+\frac{S^{2}}{D} t} \operatorname{erfc}\left(\frac{z+z^{\prime}}{2 \sqrt{D t}}+S \sqrt{\frac{t}{D}}\right)\right],
\end{aligned}
$$

where erfc is the complementary error function. The electron density follows by computing the convolution of the Green's function above with the carrier generation profile. Because the optical generation is assumed uniform, the electron density is obtained by simply integrating the previous Green's function over the volume of the spot $V_{\text {spot }}$

$$
n(\mathbf{r}, t)=\int_{V_{\text {spot }}} \mathrm{d} \mathbf{r}^{\prime} G\left(\mathbf{r}, \mathbf{r}^{\prime}, t\right),
$$

and similarly integrating the above density over the collection volume $V_{\text {spot }}$ yields the PL intensity

$$
I(t) \propto \int_{V_{\text {spot }}} \mathrm{d} \mathbf{r} n(\mathbf{r}, t) .
$$

The calculation of PL decays hence requires the integration of the Green's function Eq. (5) over all spatial arguments over the generation/collection volumes. We performed these integrations numerically for all the results presented in this paper.

In addition to being limited to the low-injection regime, our model does not include photon recycling [9] and space charge effects caused by local electric fields. Effects of differences in electron and hole mobilities as well as the high injection regime have been studied numerically [10].

\section{TRANSPORT AND LIFETIME EFFECTS IN BULK MEASUREMENTS}

We first consider the case of a bulk measurement, i.e. the generation/collection volumes are far from both the sample surface and the bulk/substrate interface. We analyze how the carrier recombination and diffusion away from the generation/collection region affect the PL intensity.

Fig. 2 shows the PL intensities obtained for (a) several values of the diffusion constant, keeping $\tau=1 \mathrm{~ns}$, and (b) several values of the bulk lifetime, keeping $D=25 \mathrm{~cm}^{2} / \mathrm{s}$. We compare these results to the limiting case of a point source whose PL decay reads [8]

$$
I_{b}(t) \propto \frac{e^{-t / \tau}}{8(\pi D t)^{3 / 2}} .
$$

While the PL intensities at short times $(t<\tau)$ are all identical
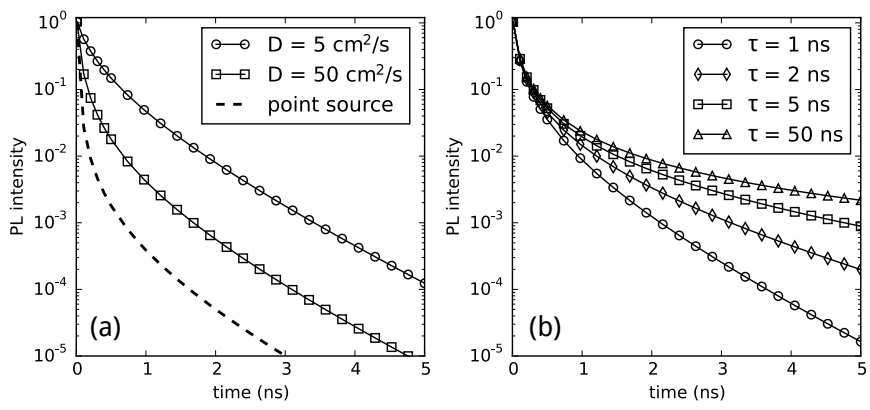

Fig. 2. Normalized PL intensities as a function of time for the optical spot in the bulk. (a) The diffusion constant $D$ is varied: $D=5 \mathrm{~cm}^{2} / \mathrm{s}$ (circles), $D=50 \mathrm{~cm}^{2} / \mathrm{s}$ (squares) with $\tau=1 \mathrm{~ns}$. The dashed line corresponds to Eq. (8). (b) The lifetime is varied: $\tau=1 \mathrm{~ns}$ (circles), $\tau=2 \mathrm{~ns}$ (diamonds), $\tau=5 \mathrm{~ns}$ (squares), $\tau=50 \mathrm{~ns}$ (triangles), with $D=25 \mathrm{~cm}^{2} / \mathrm{s}$. 
in Fig. 2(b), sharp drops occur as the diffusion constant, hence the diffusion velocity, is increased in Fig. 2(a). These drops reveal that charge carriers diffuse away from the generation spot and recombine outside of the collection volume, so that emitted photons are not collected. Note that the fast decay is not exponential but algebraic, as shown by Eq. (8) $\left(e^{-t / \tau} \approx 1\right)$. One can recover purely exponential decays at short times when collecting all emitted photons, which implies a collection region much larger than the diffusion length. Differences in generation and collection volumes can therefore be used as a knob to characterize carrier diffusion properties.

At long times $(t>\tau)$, Fig. 2(a) shows identical exponential decays, while the slopes of the decays decrease as the bulk lifetime is increased in Fig. 2(b). Eq. (8) shows that recombination exponentially reduces the PL intensity. At long times, this exponential decay (recombination) dominates the previously discussed algebraic decay (diffusion). This is seen in Fig. 2(b) for $\tau=1 \mathrm{~ns}$ and $\tau=2 \mathrm{~ns}$, while the traces for $\tau=5 \mathrm{~ns}$ and $\tau=50 \mathrm{~ns}$ are still in the diffusion dominated regime. As a result, experimentally, in the long time limit a mono-exponential fit should suffice to determine the bulk lifetime $\tau$. Comparisons with experimental data can be found in [8].

\section{SURFACE RECOMBINATION EFFECTS AND RESOLUTION OF 2-PHOTON TRPL}

We turn to calculations done for a generation/collection region at or close to the bulk/substrate interface. We analyze how the enhanced recombination at the interface changes the previous bulk PL decays, and discuss the resolution of the two-photon TRPL technique. Plots presented in this section were obtained with $\tau=1 \mathrm{~ns}$ and $D=25 \mathrm{~cm}^{2} / \mathrm{s}$. We denote $z$ as the distance of the bottom of the optical spot from the bulk/substrate interface.
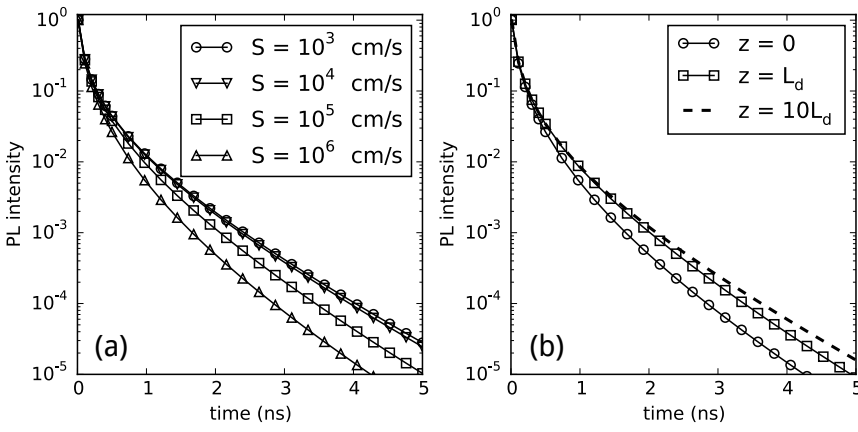

Fig. 3. Normalized PL intensities as a function of time for the optical spot around the bulk/substrate interface. (a) The spot is at the bulk/substrate interface $(z=0)$ and the surface recombination velocity varies: $S=10^{3} \mathrm{~cm} / \mathrm{s}$ (circles), $S=10^{4} \mathrm{~cm} / \mathrm{s}$ (down triangles), $S=10^{5} \mathrm{~cm} / \mathrm{s}$ (squares), $S=10^{6} \mathrm{~cm} / \mathrm{s}$ (up triangles). (b) The distance of the bottom of the optical spot is changed: $z=0$ (circles), $z=L_{d}$ (squares), $z=10 L_{d}$ (dashed line). Calculations were done with $S=10^{6} \mathrm{~cm} / \mathrm{s}$. For all plots $\tau=1$ ns and $D=25 \mathrm{~cm}^{2} / \mathrm{s}$.
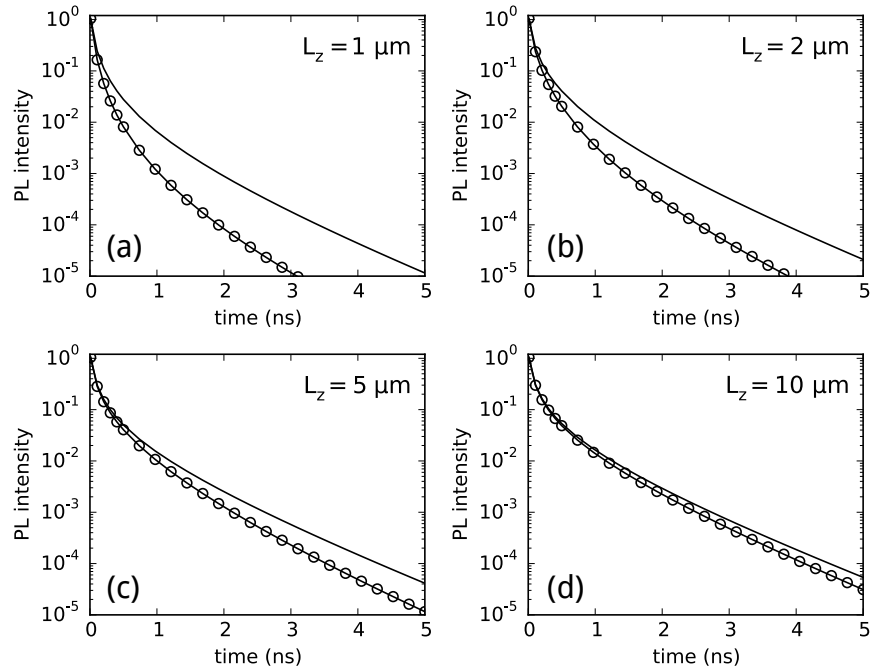

Fig. 4. Normalized PL intensities as a function of time when the axial size of the spot $\left(L_{z}\right)$ varies. (a) $L_{z}=1 \mu \mathrm{m}$, (b) $L_{z}=2 \mu \mathrm{m}$, (c) $L_{z}=5 \mu \mathrm{m}$, (d) $L_{z}=10 \mu \mathrm{m}$. The lines with symbols correspond to $S=5 \times 10^{5} \mathrm{~cm} / \mathrm{s}$ and the simple lines to $S=0$. All calculations were done with $\tau=1 \mathrm{~ns}$ and $D=25 \mathrm{~cm}^{2} / \mathrm{s}$.

Fig. 3 a) shows PL intensities for various surface recombination velocities when the generation/collection spot is at the bulk/substrate interface $(z=0)$. As expected the PL signal decreases more rapidly for greater values of $S$, as the recombination current is increased at the interface. PL decays at short times are still dominated by diffusion, as the fast decays for $t<1 \mathrm{~ns}$ are independent of the value of the recombination velocity. However, contrary to the previous bulk calculations, PL decays are not purely exponential for times $t>\tau$ as $S$ is increased. Consequently, a numerical fitting procedure relying on Eq. (5) together with known generation profile and collection volume is necessary to determine materials parameters.

We increased the distance of the generation/collection region from the bulk/substrate interface in Fig. 3 b). Calculations were done with $z=0$ (bottom of the spot at the interface), $z=L_{d}\left(L_{d}=\sqrt{D \tau}\right.$ : diffusion length) and $z=10 L_{d}$. The latter can be considered in the bulk of the system (i.e. no surface effects). We find that a displacement of the optical spot away from the interface by a diffusion length $\left(L_{d}=1.6 \mu \mathrm{m}\right)$ is enough to make the PL intensity insensitive to the probed interface.

In Fig. 4, we examine the variation in the PL decays as a function of the axial size of the generation/collection region. $L_{z}$ is varied from $1 \mu \mathrm{m}$ to $10 \mu \mathrm{m}$, and we compare the PL decays to the case $S=0$ (lines without symbols). The spot is at the bulk/substrate interface $(z=0)$. Increasing the spot size should reduce the effect of diffusion. However, because we only increase the axial dimension while keeping the lateral size $(1 \mu \mathrm{m})$ smaller than the diffusion length $(1.6 \mu \mathrm{m})$, there is still a rapid drop of the PL decay for $t<1 \mathrm{~ns}$. The drop is stronger for $L_{z}=1 \mu \mathrm{m}$, and the PL intensity is extremely 
reduced when the surface effects become visible, which may pose a challenge for experimental detection of photons. At times $t>\tau$ comparisons to the case $S=0$ show that the PL signals converge towards a single exponential decay as $L_{z}$ is increased. As expected the contribution of the bulk to recombination becomes dominant as the surface to volume ratio of the spot decreases.

\section{CONCLUSION}

Two-photon microscopy is a non-invasive and nondestructive optical technique that can probe subsurface materials parameters. Our 3D modeling allowed us to separate lifetime (recombination) from transport (diffusion) effects in PL intensities. In particular we found that the former influences decays at long times while the latter dominates short times. We have shown that the resolution of the technique for interfacial features is improved as the optical spot is confined at the bulk/substrate interface. However this comes with an increase of the diffusion effects causing a sharp drop in the PL decay at short times.

\section{ACKNOWLEDGMENT}

B. Gaury acknowledges support under the Cooperative Research Agreement between the University of Maryland and the National Institute of Standards and Technology Center for Nanoscale Science and Technology, Award 70NANB10H193, through the University of Maryland.

\section{REFERENCES}

[1] H. Wang, K. S. Wong, B. A. Foreman, Z. Y. Yang, and G. K. L. Wong, "One- and two-photon-excited time-resolved photoluminescence investigations of bulk and surface recombination dynamics in $\mathrm{ZnSe}$," J. Appl. Phys., vol. 83, no. 9, pp. 4773-4776, 1998.

[2] J. Ma, D. Kuciauskas, D. Albin, R. Bhattacharya, M. Reese, T. Barnes, J. V. Li, T. Gessert, and S.-H. Wei, "Dependence of the minoritycarrier lifetime on the stoichiometry of CdTe using time-resolved photoluminescence and first-principles calculations," Phys. Rev. Lett., vol. 111, p. 067402, 2013.

[3] E. S. Barnard, E. T. Hoke, S. T. Connor, J. R. Groves, T. Kuykendall, Z. Yan, E. C. Samulon, E. D. Bourret-Courchesne, S. Aloni, P. J. Schuck, C. H. Peters, and B. E. Hardin, "Probing carrier lifetimes in photovoltaic materials using subsurface two-photon microscopy," Sci. Rep., vol. 3, pp. 2098-2106, 2013.

[4] D. Kuciauskas, S. Farrell, P. Dippo, J. Moseley, H. Moutinho, J. V. Li, A. M. A. Motz, A. Kanevce, K. Zaunbrecher, T. A. Gessert, D. H. Levi, W. K. Metzger, E. Colegrove, and S. Sivananthan, "Charge-carrier transport and recombination in heteroepitaxial CdTe," J. Appl. Phys., vol. 116, pp. $123108-123115,2014$.

[5] M. Boulou and D. Bois, "Cathodoluminescence measurements of the minority-carrier lifetime in semiconductors," J. Appl. Phys., vol. 48, no. 11, pp. 4713-4721, 1977.

[6] G. W. t Hooft and C. van Opdorp, "Determination of bulk minoritycarrier lifetime and surface/interface recombination velocity from photoluminescence decay of a semiinfinite semiconductor slab," J. Appl. Phys., vol. 60, no. 3, pp. 1065-1070, 1986.

[7] R. K. Ahrenkiel and D. J. Dunlavy, "Minority-carrier lifetime in $\mathrm{Al}_{x} \mathrm{Ga}_{1-x}$ As," J. Vac. Sci. Tech. A, vol. 7, no. 3, pp. 822-826, 1989.

[8] B. Gaury and P. M. Haney, "Probing surface recombination velocities in semiconductors using two-photon microscopy," J. Appl. Phys., vol. 119, pp. $125105-125113,2016$.

[9] F. Stern and J. M. Woodall, "Photon recycling in semiconductor lasers," J. Appl. Phys., vol. 45, no. 9, pp. 3904-3906, 1974.

[10] A. Kanevce, D. Kuciauskas, D. H. Levi, A. M. Allende Motz, and S. W. Johnston, "Two dimensional numerical simulations of carrier dynamics during time-resolved photoluminescence decays in two-photon microscopy measurements in semiconductors," J. Appl. Phys., vol. 118 , no. 4, pp. 045 709-045 715, 2015. 


\title{
Subdiffraction optical motion transduction using a scalable plasmomechanical platform
}

\author{
Brian J. Roxworthy and Vladimir A. Aksyuk \\ Center for Nanoscale Science and Technology, National Institute of Standards and Technology, Gaithersburg, MD 20899, USA \\ Author e-mail address: brian.roxworthy@nist.gov
}

\begin{abstract}
We present a scalable platform for producing reconfigurable plasmonic devices. Using localized-gap plasmon resonators exhibiting large quality factors, we demonstrate sensitive, plasmon-based measurement of motion from a deep subdiffraction region.

OCIS codes: (250.5403) Plasmonics; (230.4685) Optical microelectromechanical devices; (220.4241) Nanostructure fabrication
\end{abstract}

Plasmonic devices and metasurfaces have had a broad impact across optical nanoscience, with applications including optical tweezers, holograms, and ultrathin optical elements [1,2]. Recently, there has been considerable interest in creating dynamic, mechanically reconfigurable plasmonic systems [3-5]. Such devices herald exciting opportunities for controlling light on the nanoscale, for instance, through the production of ultrafast and compact optical modulators [6]. Here, we present a new platform for creating these "plasmomechanical" devices, whereby gold plasmonic elements (rectangular blocks) are embedded directly into movable mechanical components (cantilever beams) and suspended above an underlying gold pad by a narrow gap (Fig. 1a). The gap, which is the critical feature defining plasmonic performance, is constructed "vertically" using a thin, planar Cr sacrificial layer that is selectively removed using wet-chemical etching; etching simultaneously releases the cantilevers from the substrate, allowing them to move. This method avoids complications associated with top-down milling processes, enabling repeatable production of large arrays of devices having precise, individual plasmonic gaps $\leq 15 \mathrm{~nm}$ in size. Using our platform, we demonstrate a new type of dynamic localized-gap plasmon (LGP) resonator, which is characterized by a high plasmonic quality factor and exceptionally large optomechanical coupling strength. We use these unique qualities of the LGP modes to achieve high-sensitivity transduction of mechanical motion from a deep subdiffraction region.

The basic concept features a mechanically coupled plasmonic resonator formed from a $350 \mathrm{~nm}$ long, $165 \mathrm{~nm}$ wide, $35 \mathrm{~nm}$ thick Au rectangular block embedded into a silicon nitride $\left(\mathrm{SiN}_{\mathrm{x}}\right)$ mechanical resonator and suspended above an underlying Au pad by a narrow gap. As the mechanical resonator - in this case, a $4 \mu \mathrm{m}$ long, $185 \mathrm{~nm}$ thick, singly clamped cantilever (Fig. 1b) - vibrates, the gap separating the block and pad is modulated. This, in turn, modulates the resonant optical frequency of the LGP mode. Consequently, the plasmomechanical device strongly couples the plasmonic resonance frequency and the local motion of the cantilever in a footprint determined by the block size.

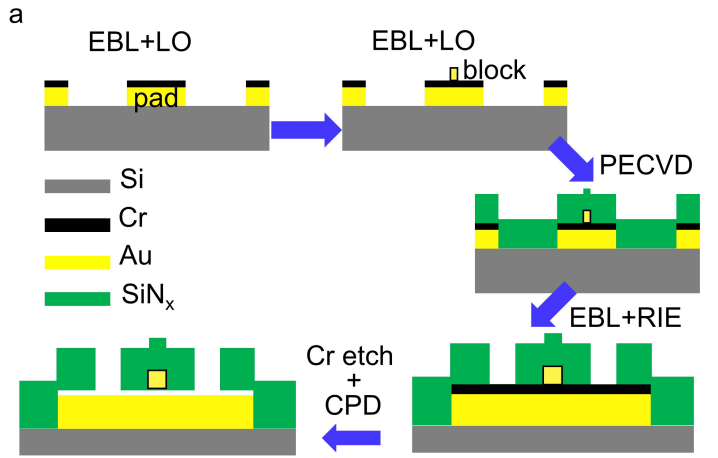

b

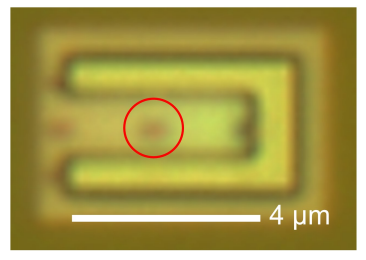

Fig. 1. a, Fabrication process flow for the plasmomechanical devices using electron beam lithography (EBL), metal evaporation and liftoff (LO), plasma-enhanced chemical vapor deposition (PECVD), reactive ion etching (RIE), and critical point drying (CPD). Black lines surrounding the Au block are included for clarity. b, Optical image of the $4 \mu \mathrm{m}$ long cantilever device with overlaid red circle showing the LGP resonator used for measurement.

The fabrication process (Fig. 1), which uses repeated aligned electron beam lithography (EBL) exposures and reactive ion etching (RIE) to pattern devices, has several unique features. First, devices are made with a very thin, $12 \mathrm{~nm} \mathrm{Cr}$ sacrificial layer placed atop the Au pad using metal evaporation and lift off (LO). This layer temporarily supports the Au blocks, formed by a second EBL+LO step, and therefore defines the gap in the plasmonic 
resonators. Second, we use low temperature $\left(180^{\circ} \mathrm{C}\right)$ plasma-enhanced chemical vapor deposition (PECVD) to deposit a conformal $\mathrm{SiN}_{\mathrm{x}}$ layer onto the pad-sacrificial layer-block stack, thereby embedding the block directly into the mechanical resonator and ensuring, after release using a $\mathrm{Cr}$ wet-etch and critical point drying, that the block travels with the cantilever. In addition to yielding thousands of functioning devices per chip, our process provides facile tunability of the plasmonic and mechanical geometries via simple modification of the EBL write pattern.
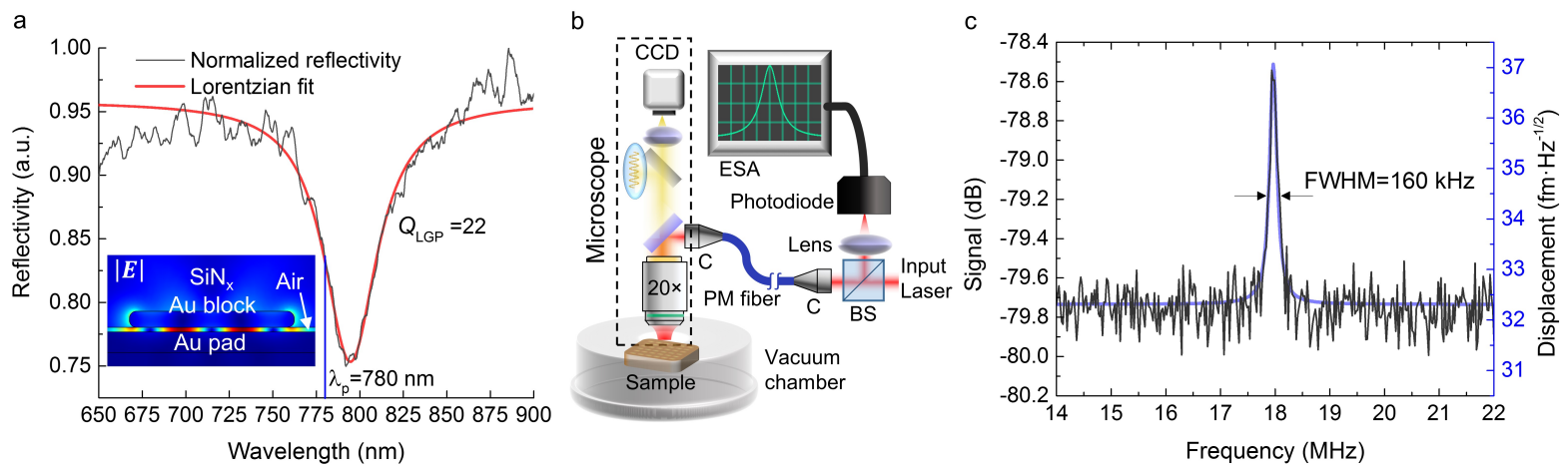

Fig. 2. a, Experimentally measured reflectivity for the LGP resonance; inset shows the normalized total electric field of the LGP mode on resonance, calculated using the finite-element method. b, Setup for motion measurement; collimator (C), 50:50 beamsplitter (BS), electronic spectrum analyzer (ESA), polarization maintaining (PM) optical fiber. c, Measured mechanical response of the device with Lorentzian fit (blue curve), showing a full-width at half-maximum (FWHM) of $160 \mathrm{kHz}$. Signal reference power is $1 \mathrm{~mW}$.

The LGP mode is a standing wave formed from counter-propagating gap plasmons that reflect from the ends of the upper block (Fig. 2a inset). We experimentally measure the LGP response of our device using a confocal optical spectroscopy setup. As shown in Fig. 2a, the LGP mode manifests as a distinct dip in the spectral reflectivity near a wavelength $(\lambda)$ of $790 \mathrm{~nm}$. The resonance has a quality factor $Q_{\mathrm{LGP}}=22$, which, owing to reduced radiation losses, is more than twice the value expected from dipolar antennas. From finite-element calculations, we find that for initial gaps $<15 \mathrm{~nm}$, the optomechanical coupling constant $\left(g_{\text {om }}\right)$, defined as the optical frequency shift of the LGP for a given change in gap size, ranges from $2 \mathrm{THz} \cdot \mathrm{nm}^{-1}$ to $4 \mathrm{THz} \cdot \mathrm{nm}^{-1}$. Such values are among the largest reported to date and more than $40 \times$ achieved in dielectric systems [7].

The combination of large $Q_{\mathrm{LGP}}$ and $g_{\mathrm{om}}$ makes the LGP resonators well-suited for transducing the motion of the overall plasmomechanical device. We measure the thermal motion of the cantilever by placing it in a vacuum chamber to reduce damping and probing the reflectivity of the LGP mode at wavelength $\lambda_{\mathrm{p}}=780 \mathrm{~nm}$ on the resonance shoulder (Fig. 2b). Motion of the device gap introduces a minute shift in the LGP resonance, which alters the reflectivity for the probe laser. We measure this amplitude-modulated reflectivity by imaging reflected light, collected with a 0.3 numerical aperture objective, onto a photodiode connected to a spectrum analyzer. The displacement power-spectral density of the cantilever motion is given in Fig. 2c, which shows a calibrated noise floor of $\approx 32 \mathrm{fm} \cdot \mathrm{Hz}^{-1 / 2} \pm 3.2 \mathrm{fm} \cdot \mathrm{Hz}^{-1 / 2}$, where the uncertainty corresponds to an estimated $10 \%$ error in determining the thermal displacement calibration factor. Transduction occurs from a region, corresponding to the $(350 \times 165) \mathrm{nm}^{2}$ plasmonic footprint, that is $150 \times$ smaller than the diffraction-limited focal spot of our microscope. This measurement represents a motion sensitivity $\approx 7 \times$ larger than previously demonstrated with comparable plasmomechanical devices.

\section{References}

1. $\quad$ N. Yu and F. Capasso, "Flat optics with designer metasurfaces," Nat. Mater. 13, 139-150 (2014).

2. B. J. Roxworthy, K. D. Ko, A. Kumar, K. H. Fung, E. K. C. Chow, G. L. Liu, N. X. Fang, and K. C. Toussaint, "Application of Plasmonic Bowtie Nanoantenna Arrays for Optical Trapping, Stacking, and Sorting," Nano Lett. 12, 796-801 (2012).

3. N. I. Zheludev and E. Plum, "Reconfigurable nanomechanical photonic metamaterials," Nat. Nanotechnol. 11, 16-22 (2016).

4. R. Thijssen, T. J. Kippenberg, A. Polman, and E. Verhagen, "Plasmomechanical Resonators Based on Dimer Nanoantennas.," Nano Lett. 15, 3971-3976 (2015).

5. B. J. Roxworthy, A. M. Bhuiya, X. Yu, E. K. C. Chow, and K. C. Toussaint, "Reconfigurable nanoantennas using electron-beam manipulation," Nat. Commun. 5, 4427 (2014).

6. B. S. Dennis, M. I. Haftel, D. a. Czaplewski, D. Lopez, G. Blumberg, and V. A. Aksyuk, "Compact nanomechanical plasmonic phase modulators," Nat. Photonics 9, 267-273 (2015).

7. M. Eichenfield, R. Camacho, J. Chan, K. J. Vahala, and O. Painter, "A picogram- and nanometre-scale photonic-crystal optomechanical cavity.," Nature 459, 550-555 (2009). 


\title{
Cantilever array with optomechanical read-out and integrated actuation for simultaneous high sensitivity force detection
}

\author{
Thomas Michels ${ }^{1,2}$, Ivo W. Rangelow ${ }^{2}$, and Vladimir Aksyuk ${ }^{1 *}$ \\ ${ }^{1}$ National Institute of Standards and Technology, Center for Nanoscale Science and Technology, Gaithersburg, Maryland 20899 \\ ${ }^{2}$ Department of Micro- and Nanoelectronic Systems, University of Technology, Ilmenau, Germany \\ * vladimir.aksyuk@nist.gov
}

\begin{abstract}
We present an on-chip cavity optomechanical cantilever array with integrated actuation, that combines high measurement bandwidth and very low displacement noise floor with compactness, robustness, small size, and potential for low cost batch fabrication inherent in micro- electro- mechanicalsystems (MEMS)
\end{abstract}

Keywords-optomechanics; cantilever array; integrated actuation

\section{INTRODUCTION}

Research and development of transducers based on cavity optomechanics is a topic of high interest particularly because these transducers enable measurement of mechanical motion down to the fundamental limit of precision imposed by quantum mechanics. We have developed an on-chip cavity optomechanical cantilever array that combines high bandwidth and high sensitivity with compactness, robustness, small size, and potential for low cost batch fabrication inherent in MEMS. The parallelization of multiple probes within one transducer array allows the simultaneous measurement of serial forces or mass detection [1].

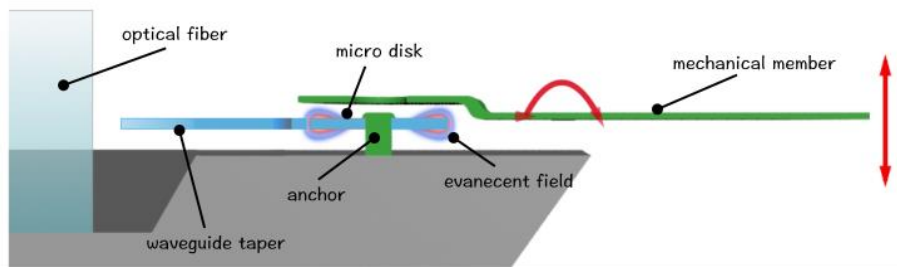

Fig. 1: Exemplary schematic of the transducer (not to scale) showing overhung cantilever on a torsional pivot as the mechanical device.

\section{FABRICATION}

Our fully-integrated, fiber-pigtailed cantilever array combines high sensitivity $\left(\approx 0.5 \mathrm{fm} \cdot \mathrm{Hz}^{-1 / 2}\right.$ to $\left.\approx 10 \mathrm{fm} \cdot \mathrm{Hz}^{-1 / 2}\right)$, high bandwidth optomechanical readout and built-in thermal actuation. We use a wafer-scale microfabrication process combining one e-beam patterning, six stepper, and three contact mask aligner lithography steps. These define the silicon nitride ( $\mathrm{SiN}$ ) cantilever, the single-crystal silicon-on- insulator (SOI) microdisk optical cavity with high optical Q (up to $\approx 2 \times 10^{6}$ ), SOI optical waveguides, and the patterned gold layer for bimorph actuation [2]. Back and front side anisotropic potassium hydroxide $(\mathrm{KOH})$ silicon etch allows the cantilever to hang over the edge of the silicon chip and to define v-grooves for single- mode optical fiber attachment. Two sacrificial silicon dioxide layers are removed by an isotropic hydrofluoric acid (HF) etch to free the mechanically movable structures. The fabrication process is shown in Fig. 3 on the example of a single cantilever device.

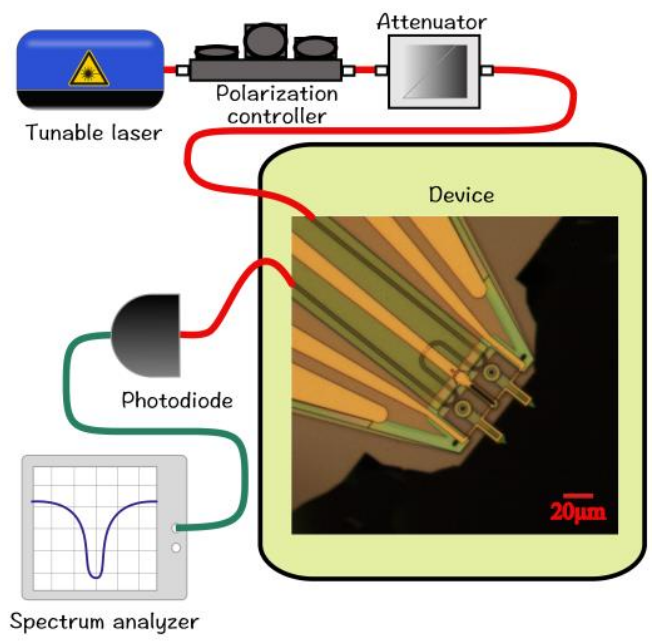

Fig. 2: Schematic of the detection setup with an embedded optical micrograph of the cantilever array. 

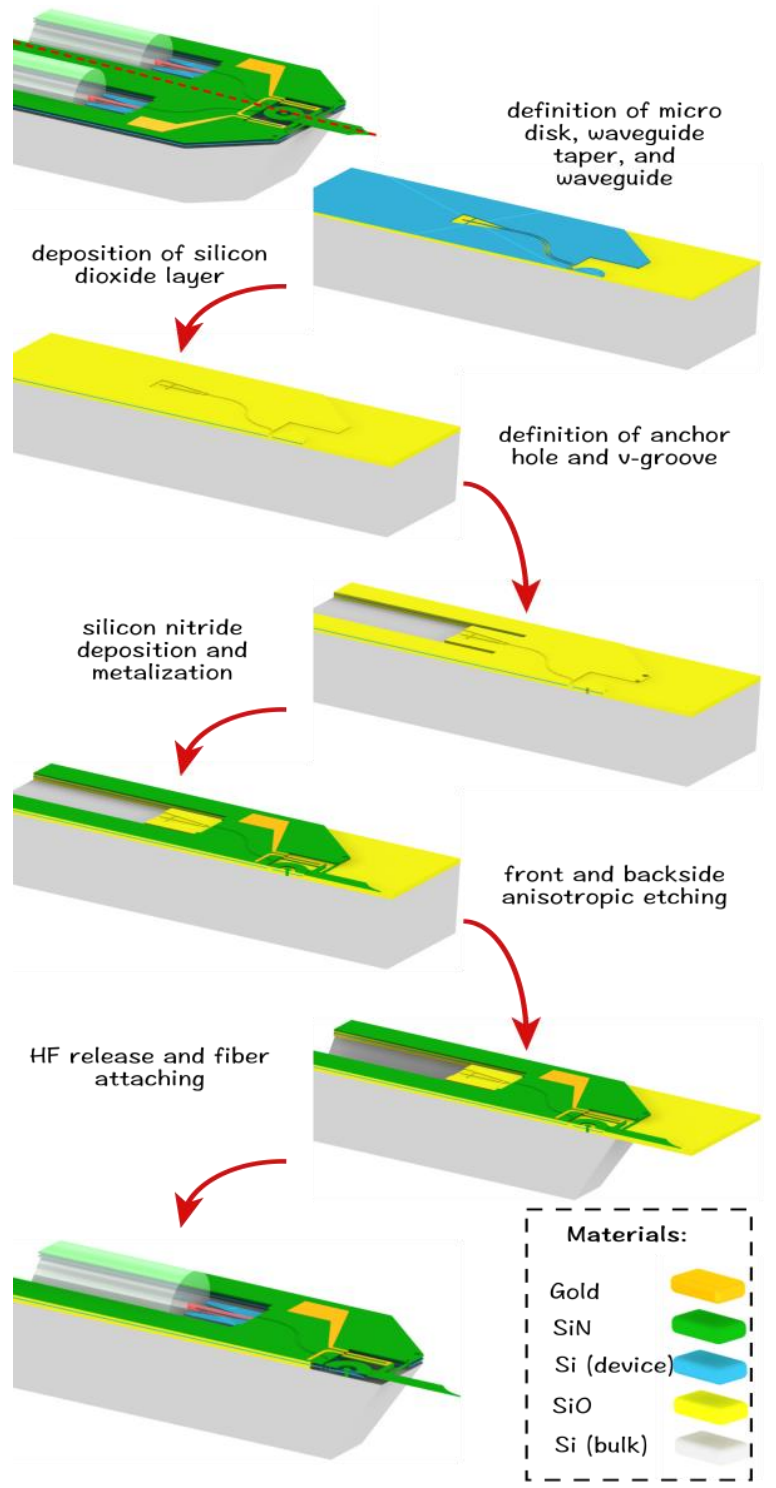

Fig. 3: Representation of the process flow for the transducer with integrated thermal actuation and overhanging tip. The image in the top left shows the whole device. The dashed red in indicates the path for the cross sectional views (a).

The $\mathrm{SiN}$ cantilever can be excited by an electrical signal supplied to an integrated thermal actuator. The cantilever is evanescently coupled to a high-Q optical whispering gallery mode of the optical microdisk cavity and the motion is detected by measuring the resonance frequency shift of the optical cavity mode. A schematic of the cantilever is shown in Fig. 1. The actuator can be used to individually address the cantilevers and dynamically move them as well as to tune the distance between the cantilever and the optical cavity, to change the sensitivity and range of measurement of the cantilever. One side of the cantilever overhangs the edge of the chip, where it can be easily coupled to a variety of off-chip samples and physical systems of interest. $\mathrm{A} \approx 10 \mathrm{um}$ long probe is currently designed to have a stiffness of $\approx 0.1 \mathrm{~N} / \mathrm{m}$ to $\approx 5 \mathrm{~N} / \mathrm{m}$ and a resonance frequency of $\approx 50 \mathrm{kHz}$ to $\approx 4 \mathrm{MHz}$. The design can be easily and broadly tailored for specific sensing applications.

The detection setup used to characterize the device is shown in Figure 2. Motion of the cantilever results in a frequency modulation of the optical cavity modes, which can be translated into an intensity modulation by probing these modes on the side of their resonance minima. The output signal is intensity-modulated in proportion to the mechanical motion, and is transduced by a photodetector before being sent to an electronic spectrum analyzer to reveal the spectrum of mechanical modes (Fig.4).

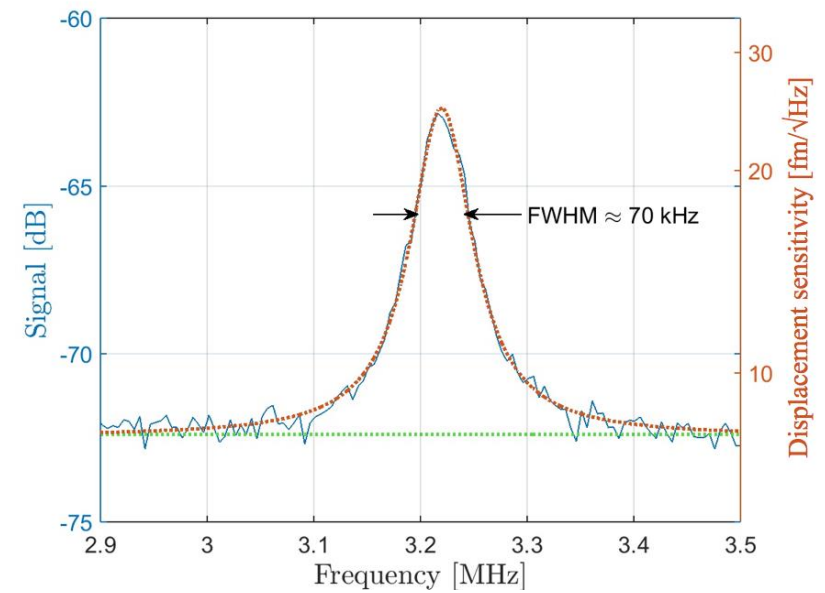

Fig. 4: Measured mechanical frequency noise spectrum of the cantilever transducer in vacuum with Lorentzian fit. The dotted green line indicates the background noise level. Signal power is reported relative to $1 \mathrm{~mW}$.

\section{CONCLUSION}

We have demonstrated the micro- and nanofabrication process and characterization of a novel type of fully- integrated cavity optomechanical cantilever array for high sensitivity force detection.

\section{REFERENCES}

[1] H. Miao, K. Srinivasan, and V. Aksyuk, "A microelectromechanically controlled cavity optomechanical sensing system," New J. Phys., vol. 14, no. 7, p. 075015 , Jul. 2012.

[2] T. Michels and I. W. Rangelow, "Review on Scanning Probe Micromachining and its Applications within Nanoscience," Vac. Sci. and Technol., pp. 1-21, Mar. 2014. 


\title{
1 Design and fabrication of neuroelectronic device containing gold
} 2 pyramid electrodes

\author{
3 Gymama Slaughter*a,b, Joel Tyson", , Matthew Robinson ${ }^{\mathrm{c}}$, Chen J. Zhang ${ }^{\mathrm{c}}$, Minhquan \\ 4 Tran $^{\mathbf{a}}$ \\ $5 \quad{ }^{a}$ Bioelectronics Laboratory, University of Maryland Baltimore County; bDepartment of Computer Science and \\ 6 Electrical Engineering, 1000 Hilltop Circle, Baltimore, MD, USA 21250; \\ $7 \quad{ }^{\mathrm{c}}$ Center for Nanoscale Science and Technology, National Institute of Standards and Technology Gaithersburg, MD, \\ 8 USA, 20899
}

\begin{abstract}
A neuroelectronic device containing an electrochemically deposited multi-walled carbon nanotube (MWCNT) sensitive layer was designed, fabricated and characterized. The neuroelectronic device consists of a flexible polyimide substrate bearing four shanks with 16 gold rectangular pyramid electrodes per shank and MWCNT sensitive layer. Each of the 16 gold pyramid electrodes with a top active area of $6 \mu \mathrm{m} \times 60 \mu \mathrm{m}$ and depth of $750 \mu \mathrm{m}$ are positioned with a vertical separation distance of $100 \mu \mathrm{m}$ on a shank width of $40 \mu \mathrm{m}$. Gold rectangular pyramid electrodes are selected as the recording electrodes to enhance the electroactive surface area. Electrochemical impedance spectroscopy (EIS) analysis of the resulting 16 gold pyramid electrodes on one of the shanks of the neuroelectronic device was characterized at the physiological relevant frequency of $1 \mathrm{kHz}$. The experimental results show that the gold electrodes are uniformly coated with MWCNTs sensitive layer. With no MWCNT coating, the impedance response at $1 \mathrm{kHz}$ could reach as high as $17.16 \mathrm{M} \Omega$ on average. Additionally, the MWCNT coated gold pyramid electrodes exhibited a significant reduction in electrode impedance at $1 \mathrm{kHz}$ to 45.73 $\mathrm{k} \Omega$ on average demonstrating MWCNT ability to lower the impedance of the electrodes by roughening the electrode surface.
\end{abstract}

Keywords: Microfabrication, polyimide, flexibility, neural probes, electrochemical impedance spectroscopy.

*Corresponding Author, E-mail: gslaught@umbc.edu

\section{Introduction}

29 Neuroelectronic devices used for the integration of neurons with electrodes in neurological studies have enabled a better understanding of the complex neurological processes in animals

31 and in humans. Electroencephalography (EEG), electrocorticography (ECoG), local field

32 potential (LFP) and single-unit recordings are the four main technologies employed to stimulate

33 and record electrical signal from targeted neural tissues, and are particularly useful for

34 elucidating the complex processes that contributes to the onset of neurodegenerative diseases ${ }^{1,2}$.

35 However, these technologies suffer from a number of limitations such as relatively low

36 resolution and are not suitable for use in recording brain activity from multiple sites deep within 
37 the brain. The application of micro- and/ or nano-electromechanical systems (MEMs) or (NEMs)

38 to the fabrication of LFPs or single-units recording electrodes have led to the development of

39 minimally-invasive high density neuroelectronic devices that are designed to penetrate the pia

40 and maintain intimate contact with neurons. Electrode characteristics such as size, electrical

41 impedance, and the electrode's ability to achieve intimate contact with a neuron play a key role

42 in differentiating LFPs from single-units recordings ${ }^{3}$.

43 The current metal stereotrodes, tetrodes and microwire arrays employed are only capable

44 of recording activity at their exposed distal tips and the number of recording electrodes that can

45 be incorporated in these devices are limited ${ }^{4}$. A key challenge that remains to be addressed is the

46 reduction in the probe size while maintaining a large density of recording electrodes in order to

47 minimize tissue damage, scarring and device encapsulation. An advantage of using silicon based

48 microelectronics and/ or MEMs based fabrication techniques is that it can effectively reduce the

49 probe size and address the limitations of conventional metal microwire based neuroeletronic

50 devices $^{5}$ in addition to the fact that it can be batch processed and yield reproducible planar or

51 three-dimensional electrode geometries ${ }^{6}$. However, these silicon-based electrodes are also prone

52 to host immune responses ${ }^{7,8}$ that eventually cause the recording signal to degrade during the

53 operation of the electrodes. Neuroelectronic devices based on polymers have been recently

54 demonstrated to offer an alternative to silicon based devices due to their mechanical (flexibility)

55 and chemical (biocompatible) ${ }^{9}$ properties. These devices can easily conform to the surrounding

56 brain tissue ${ }^{10}$, thus minimizing inflammation due to micromotion and tissue damage and improve

57 the device overall biocompatibility ${ }^{11,12}$.

58 In order to acquire high quality signal when implanted, it is important that the impedance

59 of the recording electrode is optimal for the recording and stimulation of brain cells or tissues. 
60 Neuroelectronic devices that exhibit high electrode impedance increases the electrode noise

61 profile, which further obscures recorded neuronal signals ${ }^{13}$. Therefore, it is important to employ

62 neuroelectronic devices with electrode sites that have low electrical impedances and high signal-

63 to-noise ratio to enable the acquisition of high quality recording signals from targeted neural

64 cells or tissues. Xiang et al demonstrated the reduction of gold electrode impedance

65 characteristics via the electrochemical deposition of multi-walled carbon nanotubes

66 (MWCNTs) $)^{14}$. The deposited MWCNTs provided a roughened sensitive layer that have been

67 shown to detect dopamine via cyclic voltametry ${ }^{14,15}$ and improve the quality of neuronal cell to 68 electrode adhesion ${ }^{16}$.

69 In this paper, we took advantage of the flexibility and biocompatibility of polymers to 70 design and fabricate polyimide based neuroelectronic device. Thin MWCNT coated gold 71 pyramid electrodes were employed to reduce the electrode impedance. The detailed design, 72 development and fabrication of such fully integrated neuroelectronic device is described and 73 discussed.

\section{Experimental Results and Discussion}

\section{$75 \quad 2.1$ Flexible Neuroelectronic Probe Design}

76 The flexible polyimide neurotronic probes are designed to use silicon substrate to provide initial

77 mechanical support, from which the flexible probe substrate is released and integrated with

78 interconnect cable. The 64-site flexible multi-shank neuroelectronic probes are constructed by

79 sandwiching the electrical interconnect traces in between two polyimide layers. These

80 interconnect traces connect the small electrodes sites to the large bond pads and is made from

81 chromium $(\mathrm{Cr})$ thin film and gold $(\mathrm{Au})$ thin film. The two polyimide layers consist of 1) an 
82 insulating layer of HD-4104 (HD Microsystems, USA) polyimide (PI1) to structurally connect

83 the electrodes sites and bond pads, but electrically isolate them and 2) a final passivation layer of

84 HD-8820 polyimide (PI2) to serve as the final structural material and passivation for the 85 interconnect traces. Figure 1 presents the probe architectural design where four shanks are 86 protruding from the wider base platform. The multi-shank probe is made up of two metallization 87 layers: the first metallization layer forms the exposed 64 electrode sites and bond pads and the 88 second metallization carries the insulated interconnect traces. The metallization layers are 89 separated by a thin PI1 film. This architecture enables the packing of 16 electrodes per shank. 90 The shank width is $40 \mu \mathrm{m}$, widening to $250 \mu \mathrm{m}$ at the base platform. The probe thickness is 91 approximately $8 \mu \mathrm{m}$. The shank length is $3 \mathrm{~mm}$ and the shanks are spaced at $7750 \mu \mathrm{m}$ with 16 92 electrode sites on each of shanks. The electrode sites have top area of $6 \mu \mathrm{m} \times 60 \mu \mathrm{m}$ and 93 thickness of $750 \mu \mathrm{m}$ and distributed with $100 \mu \mathrm{m}$ pitch. Additionally, the key design elements of 94 the probe structure are: the fine tip pyramidal shaped electrodes, $30^{\circ}$ tapered polyimide shanks 95 for penetration into neural tissue, and the narrowly spaced gold interconnect traces. 


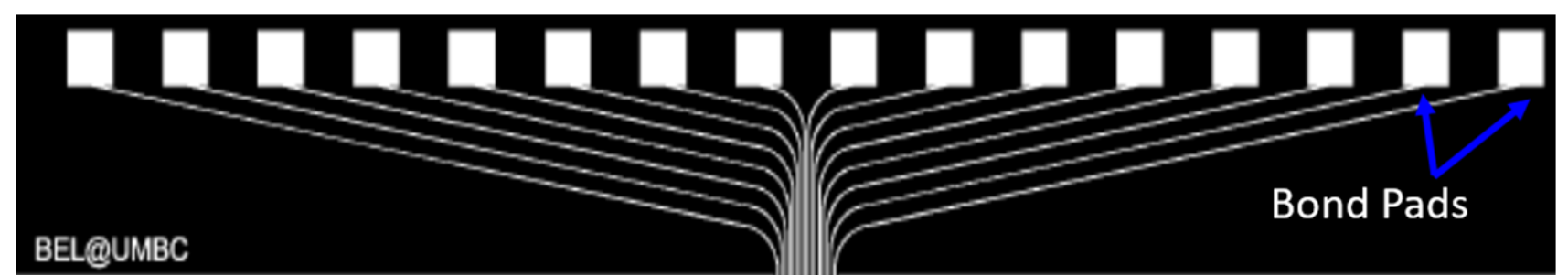

Routing Leads

Figure 1. Schematic design of the flexible neuroelectronic probe structure and CAD layout showing the 98 electrode sites, interconnect traces and bond pads. 
100 A flexible gold rectangular pyramid neuroelectronic probe platform is fabricated using flexible 101 biocompatible polyimide ${ }^{17}$. Figure 2 illustrates the fabrication sequence employed to translate the 102 gold pyramid electrodes into a flexible multi-shank neural probe in a cross-sectional view. Five 103 photolithographic masks are used to fabricate the probes on a 4-inch silicon wafer. The p-type 104 (100) silicon wafer is purchased from WRS Materials. The electrodes contain two metallization 105 layers. The first metallization forms the gold pyramid recording electrodes and bond pads and 106 the second metallization defines the interconnection traces. Polyimide (HD-4104) is used as the 107 insulation layer for the electric interconnection traces on the front side and are necessary to block 108 ionic current flow due to HD-4104 high electrical resistivity $\left(\sim 10^{16} \Omega \mathrm{cm}\right)^{18,19}$. Both negative 109 (HD-4104) and positive (HD-8820) polyimide are used as the dielectric and structural material 110 respectively for the probe manufacturing because together they provide better mechanical 111 stiffness and flexibility for probing neurons. 


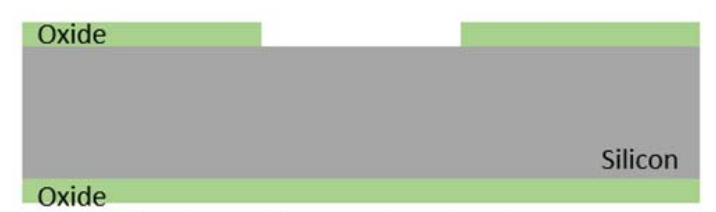

1) Oxide deposition and patterning

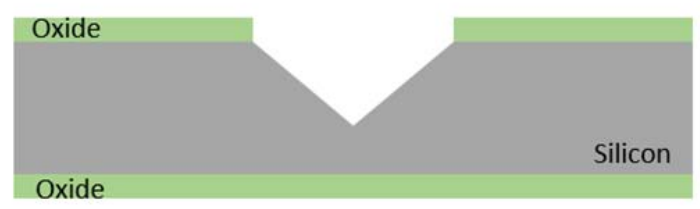

2) Silicon $\mathrm{KOH}$ etching to form V-grove

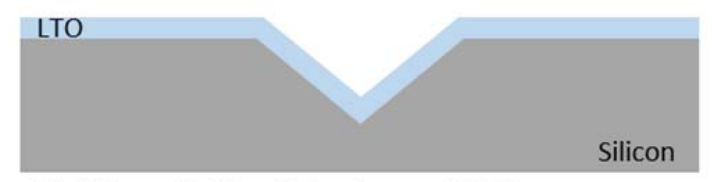

3) Silicon Oxide stripping and LTO deposition

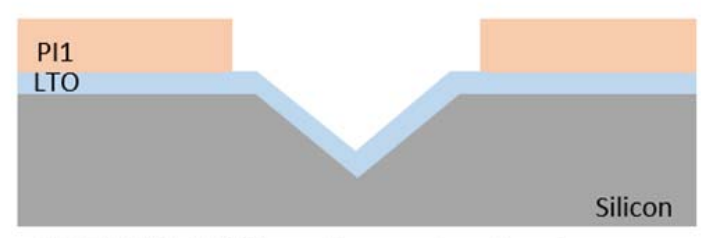

4) PI1(HD-4104) coating and patterning

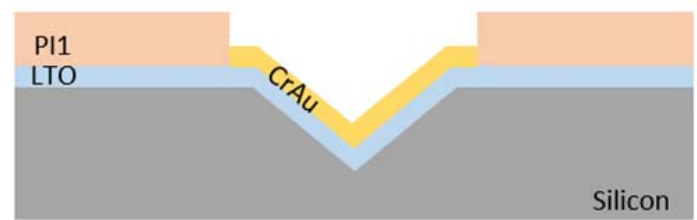

5) CrAu electrodes patterning and deposition

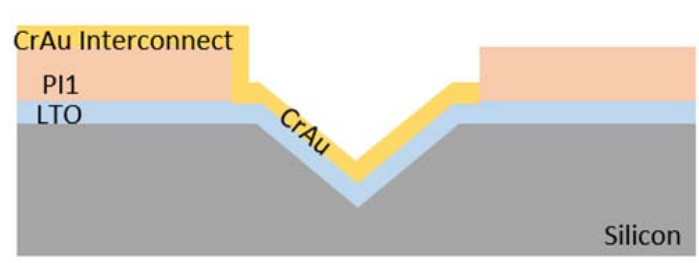

6) CrAu interconnect patterning and deposition

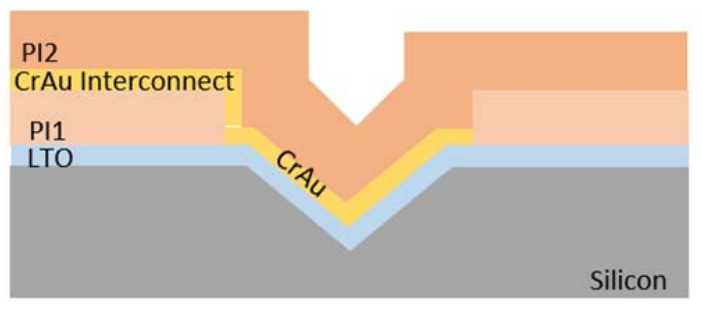

7) $\mathrm{PI}$ (HD-8820) coating and patterning

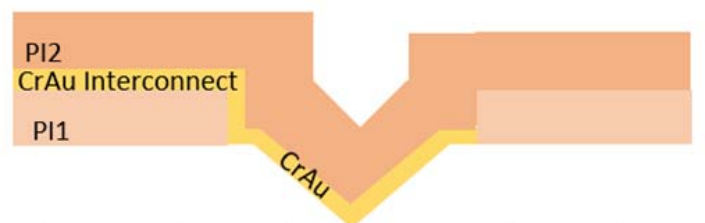

8) LTO etching and final device releasing from silicon
115 A $200 \mathrm{~nm}$ thermal oxide is grown on the silicon wafer to serve as a mask for anisotropic etching 116 of silicon using potassium hydroxide $(\mathrm{KOH})$. Photoresist patterning (standard S-1813 recipe 117 using MA6/MA8 mask aligner) is employed to create a mask for etching the $\mathrm{SiO}_{2}$ with buffer 118 oxide etchant. The silicon is then etched with $\mathrm{KOH}$ to form the inverted rectangular pyramid 119 cavity to enable the molding of the gold rectangular electrode sites inside the inverted pyramid 120 cavity. Upon stripping the $\mathrm{SiO}_{2}$ layer, a blanket $600 \mathrm{~nm}$ LTO is deposited and serves as the 
121 sacrificial layer for final release of the probe. HD-4104 is photolithographically patterned on the

122 silicon substrate to expose the inverted pyramid cavity and to define the bond pads as well as

123 serve as the insulation layer for the second metallization layer. After curing the polyimide, the

124 polyimide film measures approximately $2-4 \mu \mathrm{m}$ thick depending of the curing processing details.

125 NR9-6000PY is photolithograpically patterned on top of the polyimide to expose the electrodes

126 and associated bond pads. The first metallization layer composed of $20 \mathrm{~nm}$ chromium and 750

$127 \mathrm{~nm}$ is then deposited. The chromium layer is designed to provide adhesion between gold and

128 LTO. The gold electrodes and associated bond pads are e-beam evaporated to a thickness of 750

$129 \mathrm{~nm}$ from a $99.99 \%$ pure gold target. Gold $(\mathrm{Au})$ is selected as the electrode material due to its

130 chemical inertness, and compatibility with brain tissue ${ }^{20,21}$. The second metallization layer of

$131 \mathrm{Cr} / \mathrm{Au}(20 \mathrm{~nm} / 250 \mathrm{~nm})$ is e-beam evaporated and designed for the interconnection traces. The

132 interconnect traces are photoresist patterned and etched via ion milling to connect the electrode

133 sites to the bond pads. After interconnect traces fully patterned, a second polyimide layer HD-

1348820 is spin coated, patterned to define the outer geometry of the devices and insulate the

135 interconnect traces as well as serve as the structural material. This is then followed by the

136 complete etching of the $600 \mathrm{~nm}$ LTO layer in buffer oxide etchant for the final release of the

137 flexible neuroelectronic probe. The chrome surfaces on the electrode sites and bond pads are

138 then removed by carefully dipping the probe in a chromium etchant solution while monitoring

139 the etch process under a microscope. The chromium etched probes are cleaned with Isopropanol

140 and DI water.

$141 \quad 2.3$ Process development and integration challenges

142 The polyimide based neuroelectronic probe fabrication was carried out on a 4-inch silicon

143 substrates. Polyimide serves as structural, insulating, and passivating layers for the device. Both

Robinson, Matthew; Slaughter, Gymama; Tyson, Joel; Zhang, Chen.

"Neuroelectronic Device Process Development and Challenge."

Paper presented at Optical Microlithography, San Jose, CA. February 26, 2017 - March 2, 2017. 
144 negative and positive polyimides selected provide mechanical flexibility, chemical stability, and 145 biocompatibility. In addition, they both exhibit the lowest moisture uptake of $0.5 \%$ and are 146 expected to provide excellent biostability by minimizing possible failure due to moisture 147 absorption. As for the metallization layers, gold was chosen for its biocompatibility and process 148 compatibility. The characteristic pyramid profile of the electrode was achieved via anisotropic 149 etching of $5 \mu \mathrm{m} \times 50 \mu \mathrm{m}$ features patterned on the silicon wafer with $\mathrm{KOH}$. The bias introduced 150 by $\mathrm{KOH}$ in these features and the alignment marks resulted in several process challenges 151 downstream, wherein subsequent features were not perfectly aligned. To resolve this challenge, 152 it was important that the mask used (Step 1; Figure 2) was aligned flush with the wafer during 153 UV exposure to ensure minimum $\mathrm{KOH}$ bias. Additionally, the first polyimide layer PI1 (Step 4; 154 Figure 2) was designed to create a larger $6 \mu \mathrm{m} \times 60 \mu \mathrm{m}$ openings to account for this bias in the 155 electrode features and any undercutting that may occur during the wet-chemical etch process. 156 The $10 \mu \mathrm{m}$ routing leads, bond pads and electrode sites were formed on a single metallization 157 layer (Step 5; Figure 2). However, the wet etching of $\mathrm{Cr} / \mathrm{Au}$ resulted in an interface-activated 158 undercutting of the resist film and openings between some of the routing leads and the bond 159 pads. Although the use of $10 \mu \mathrm{m}$ routing leads with a wider opening toward the bond pads were 160 found to eliminate the openings observed between these features, the electrode site features were 161 still defective. To circumvent this problem, we employed e-beam lift-off process using negative 162 resist to eliminate the undercut problem.

Moreover, the thick polyimide/metal stack posed challenge for process integration. In 164 order to ensure continuous electrical flow between the pyramid gold electrode and bond pad, 165 blanket $\mathrm{Cr} / \mathrm{Au}$ deposition follow by ion milling was developed over the thick polyimide to form 166 the interconnect traces (Step 6; Figure 2). The interconnect traces were designed to overlap the 
$16710 \mu \mathrm{m}$ routing leads and the electrode sites. During the initial ion milling process development, 168 complete etching of the $\mathrm{Cr} / \mathrm{Au}$ was not achieved, thereby impacting the functionality of the final 169 device. A descum step using reactive ion etching (RIE) was adopted to remove any residual

170 photoresist thus enable the $\mathrm{Cr} / \mathrm{Au}$ to be fully etched. As a result, the ion milling process produced 171 interconnect traces with a width and spacing as narrow as $1 \mu \mathrm{m}$ on certain portions of the shank. 172 Final device was formed by releasing shanks from the silicon substrate using buffer oxide 173 etching. The final shank formed has thickness of $8 \mu \mathrm{m}$ and width of $40 \mu \mathrm{m}$. These shanks are 174 tapered at angle of $30^{\circ}$ and exhibit flexible properties. Thereby, flexible gold pyramid electrode 175 probes with a Young's Modulus of $8.5 \mathrm{GPa}$ are realized. The flexibility afforded by the probe 176 enables the shanks to bend when in contact with neural tissue, thus minimizing neural tissue 177 damage.

178 Scanning electron microscopy (SEM) micrographs of the 16-site electrodes per shank are 179 shown in Figure 3. A shank width of approximately $40 \mu \mathrm{m}$ accommodates 16 channels. The 180 thickness of the shank varied from $6-10 \mu \mathrm{m}$. The shank length of $3 \mathrm{~mm}$ is necessary to allow 181 access to specific target of neural clusters within the brain tissue. The probe tip has a sharp angle 182 of $30^{\circ}$ to enable easy penetration where the insertion force is concentrated at the end of the 183 probe. The shanks are placed $7750 \mu \mathrm{m}$ apart and all the electrode sites are positioned within a $1841.55 \mathrm{~mm}$ vertical coverage in order to increase the probability of recording neural signals from 185 multi-target neural clusters. The insert of Figure 3 depicts a close up view of the tip of the shank 186 and subsequently the electrode site, rectangular pyramid structure. This pyramidal structure 187 increases the conductive surface available for signal acquisition similar to that observed for 188 nano-composite surfaces [13]. 


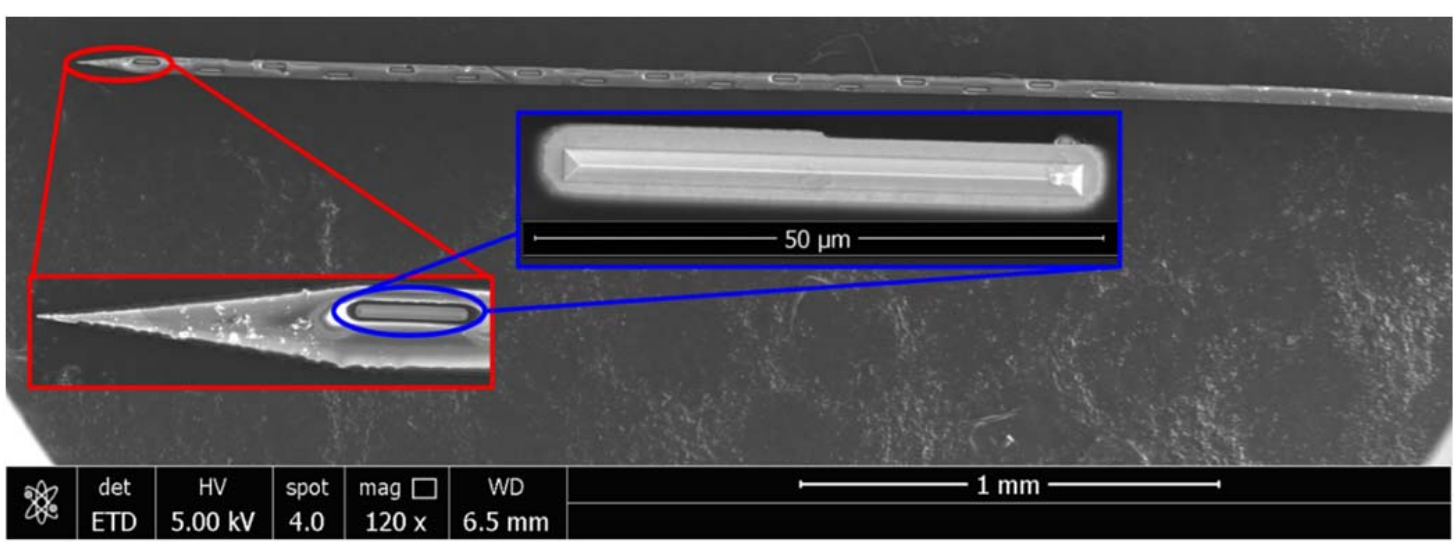

Figure 3. SEM micrographs of neuroelectronic probe shank with 16 recording electrode sites and submicron 200 interconnect traces sandwiched between two polyimide layers and insert views of shank tip and rectangular pyramid 201 shaped electrode.

The probes that are acceptable for testing are identified by visual inspection under a microscope, 204 and by electrochemical impedance spectroscopy (EIS) to assure electrical isolation of the 205 electrode sites. Optical images of the fabricated probes are shown in Figure 4. To isolate the 206 interconnect traces of the device from the aqueous environment, the traces were sandwiched 207 between two biocompatible polyimide layers with low liquid permeability. The polyimide layers 208 show very good adhesion. No visible delamination or defect due to adhesion is found between 209 the first polyimide (HD-4104) layer and the second polyimide (HD-8820) layer, nor between the 210 two polyimide layers and the gold electrode sites, interconnect traces and bond pads. The present 211 yield indicates that about $90 \%$ of the fabricated probes pass these initial tests and can be suitable 212 for impedance characterization and neural recording. As shown in Figure 4, the surface of the 213 gold interconnect traces and electrode sites are smooth and reflective, whereas the polyimide 214 surface appears dark due to light absorption by the polymer resin. 


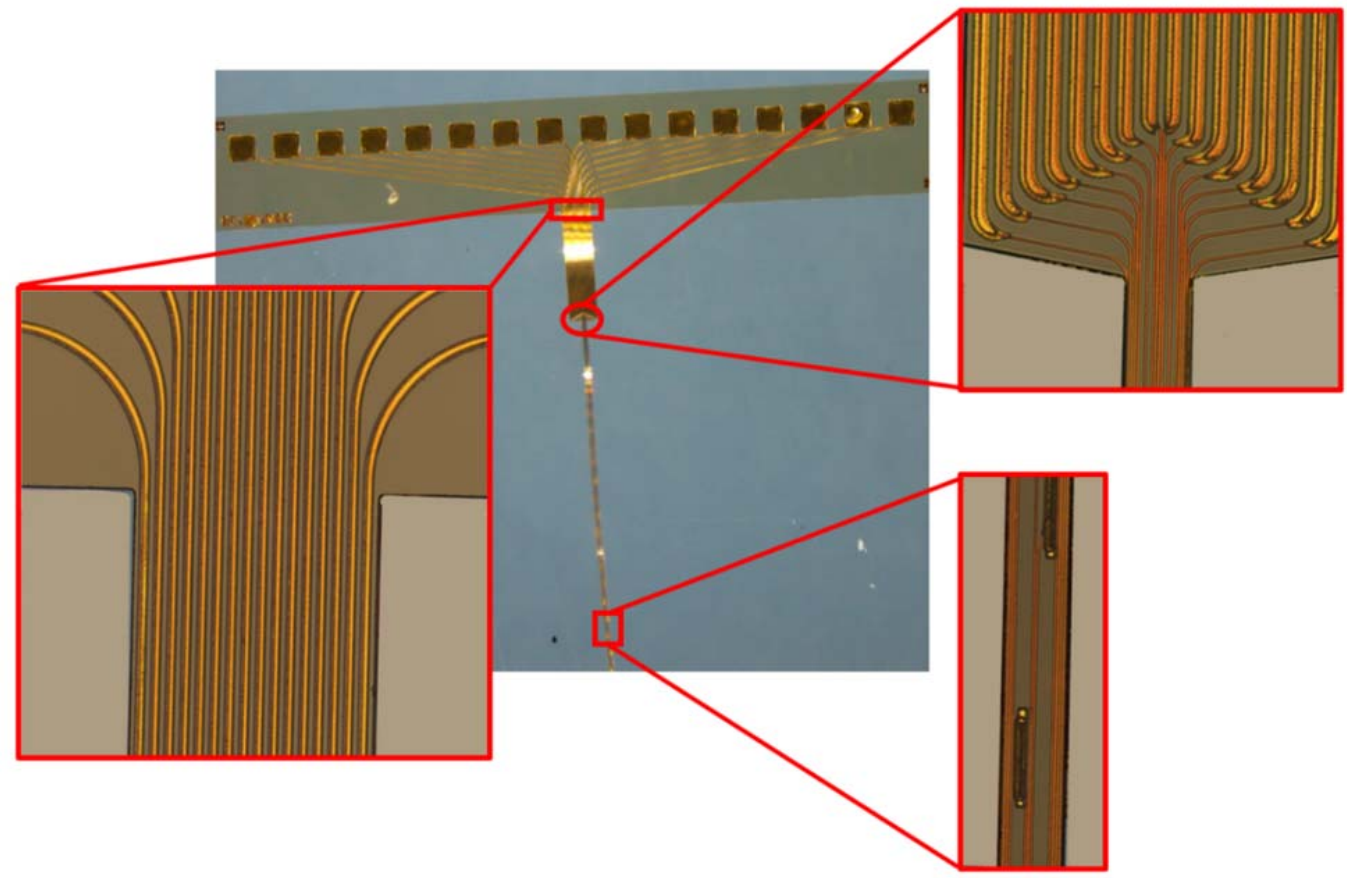

216 Figure 4. Flexible and optically transparent polyimide neuroelectronic probe accommodates associated bond pads,

217 interconnect traces $10 \mu \mathrm{m}$ to $1 \mu \mathrm{m}$ in two distinct metallization layers, and the traces are connected to the electrode 218 sites.

\section{2.4 Device Packaging}

220 The optical image in Figure 5 displays a clear view of the fabricated interconnects, bonding pads

221 and electrode sites on a single shank equipped with 16 gold recording electrode sites. For ease of 222 interfacing the flexible polyimide neuroelectronic probe to the measurement equipment, a printed 223 circuit board (PCB) consisting of interconnect lines is fabricated and connected to the probe 224 contact pads via tungsten wires $(\phi \approx 200 \mu \mathrm{m})$. The tungsten wires were manually affixed with 225 conductive silver wire glue to the bonding pads in order to create a secure electrical connection. 226 Finally, a group of jumper wires were soldered to the distal end of the PCB for further wire 227 extension. 


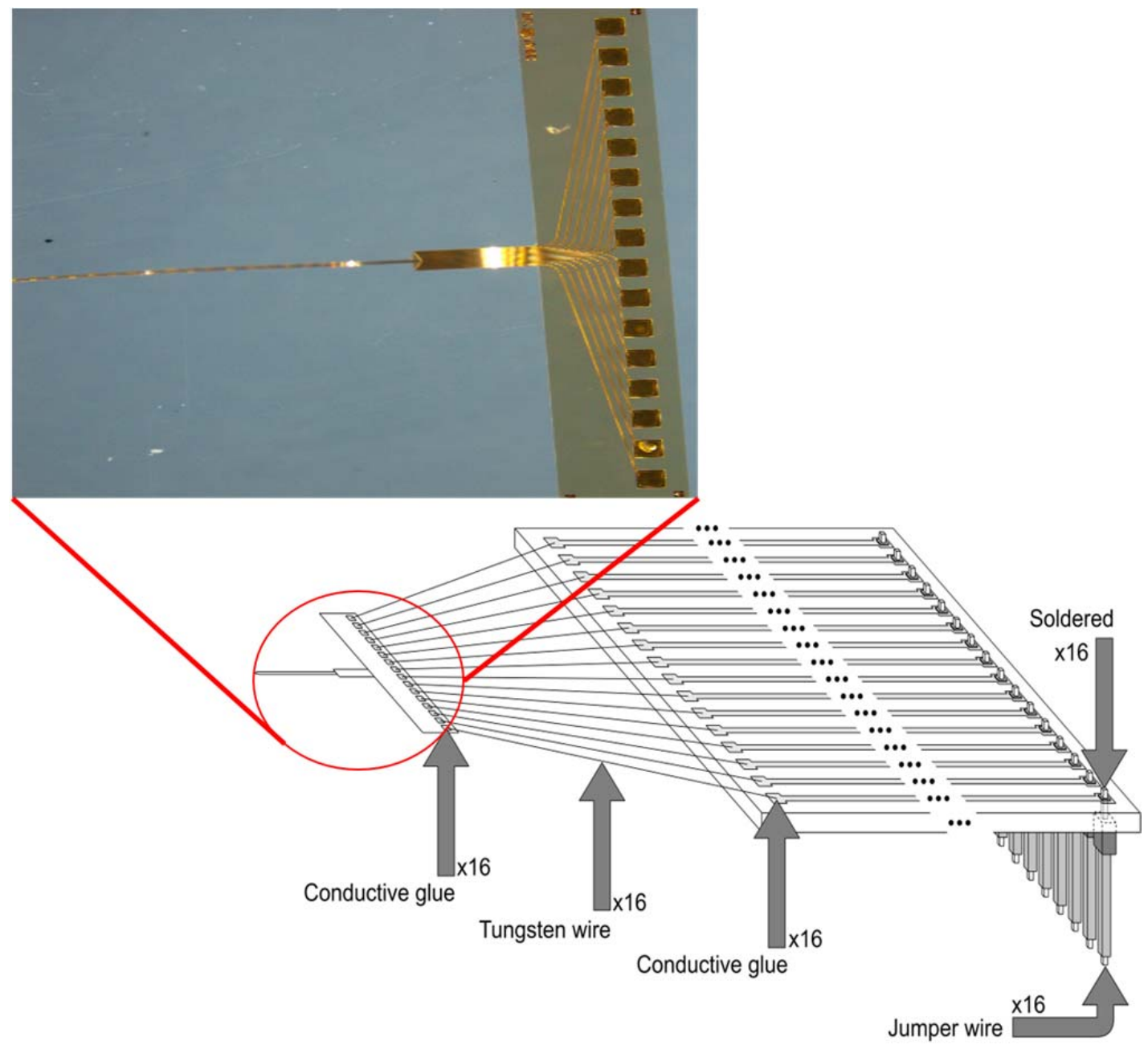

229 Figure 5: Flexible and optically transparent polyimide neural probe packaging.

\subsection{Electrochemical Impedance Spectroscopy}

231 The shank of probe was mounted in a test fixture and the probe was then immersed beyond 232 the uppermost electrode site in a phosphate buffer saline (PBS) to mimic the physiologic 233 environment at $37{ }^{\circ} \mathrm{C}$ and $\mathrm{pH}$ 7.4. The EIS experiment was performed with a potentiostat 234 PGSTAT204, Metrohm Autolab to determine the electrode site impedance before and after 235 MWCNT deposition. A three-electrode cell configuration with a $\mathrm{Ag} / \mathrm{AgCl}$ electrode as the 236 reference electrode and platinum electrode as the counter electrode was used. The probe was left 237 in solution for 45 minutes before acquiring each impedance recording in triplicates. The 
238 electrochemical impedance measurements were taken with a frequency range between $0.1 \mathrm{~Hz}-$

$23910 \mathrm{kHz}$ using a $10 \mathrm{mV}$ peak-to-peak waveforms in order to identify stabilized probe site

240 impedances. The electrode impedance was observed to be on average approximately $135 \mathrm{k} \Omega(\mathrm{n}=$

$24124)$ at $1 \mathrm{kHz}$. In addition, the impedance was observed to gradually increases with decreasing

242 frequency. The impedance values obtained for the gold electrode sites are attributed to the

243 pyramidal geometry of the recording site electrode. The impedance value is well below most

244 values reported for non-surface treated neural probes $^{22}$. Moreover, observed failed sites

245 (impedance out of range) are usually due to open interconnect traces or over etched traces. Post

246 failure SEM micrograph of probes appear to indicate open interconnect traces caused by

247 fabrication as the major cause for the failed sites.

$248 \quad 2.5$ Multi-walled carbon nanotube (MWCNT) deposition

249 The gold recording pyramid electrode surfaces were roughened with MWCNT in order to 250 increase the electrocatalytic surface area for cell adhesion and sensing, in addition to lowering 251 the overall electrode impedance. MWCNTs concentration of $1 \mathrm{mg} / \mathrm{mL}$ was prepared using gold 252 sulfite electrolyte solution. The prepared solution was sonicated for 1 hour prior to 253 electrochemical deposition. The probe shank was assembled in a test fixture and to isolate the 254 gold bond pads of the device from the liquid test fixture environment, the tungsten wires and the 255 upper section of the probe were encapsulated with a biocompatible epoxy low water 256 permeability. MWCNT were deposited on six electrodes subsequently using each of the 257 recording electrodes as the cathode. A platinum wire electrode was used as the anode. The anode 258 and cathode were then connected to a function generator (Agilent 33250A). A monophasic 259 voltage pulse of $1.2 \mathrm{~V}, 10 \mathrm{~Hz}$, at $50 \%$ duty cycle was applied for 1 minute. After the deposition 260 cycles, multiple layers of MWCNTs with gold nanoparticles were deposited on each of the gold 
261 recording electrode sites on the shank. The optical images of the flexible polyimide probe shank

262 tip pre and post-MWCNT deposition are shown in Figure 6. The surface of the rectangular gold

263 electrode appears smooth and reflective, whereas the MWCNTs coated electrode surface

264 appeared rough and dark.

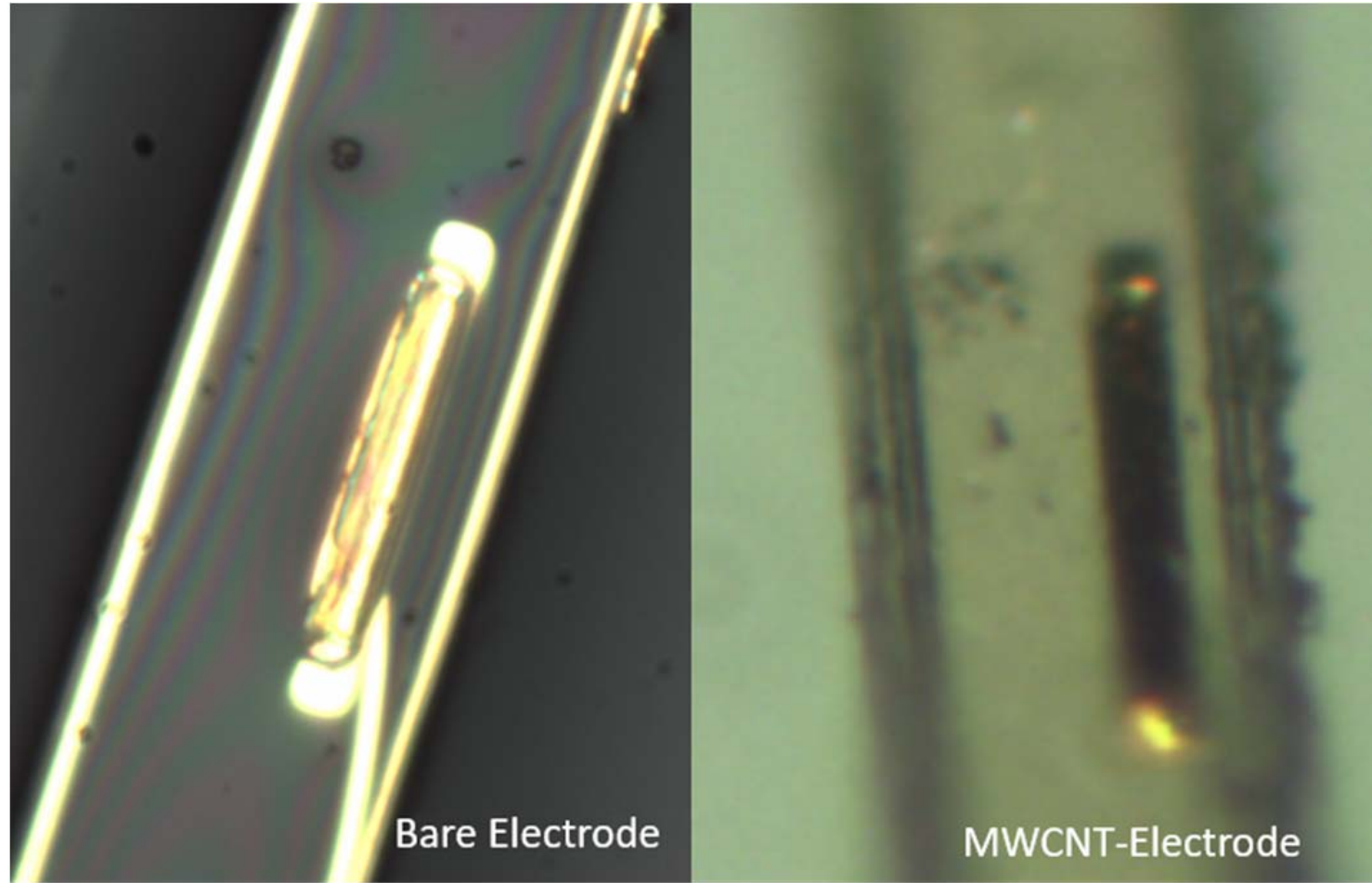

Figure 6: Recording electrode site before (left) and after (right) MWCNT deposition.

268 Figure 7 shows the comparison of the electrode impedance before and after MWCNT deposition.

269 The relatively large impedance value of $17.16 \mathrm{M} \Omega$ at $1 \mathrm{kHz}$ on average is attributed to the 270 electrode area being covered by a small amount of the biocompatible epoxy used to affix the 271 flexible probe shank to the borosilicate glass during the construction of the test fixture chamber.

272 The 60 seconds long deposition of MWCNTs resulted in a significant decrease in the overall 273 impedance. The average impedance at $1 \mathrm{kHz}$ after MWCNT deposition was $45.73 \mathrm{k} \Omega$. Clearly, 274 the MWCNTs contribute to the decrease of the electrodes electrical impedance. Thereby 
275 resulting in an enhancement of charge storage capability ${ }^{23}$. The observed average impedance 276 after MWCNT deposition is an acceptable impedance value for electrochemical sensing of 277 dopamine ${ }^{14}$.

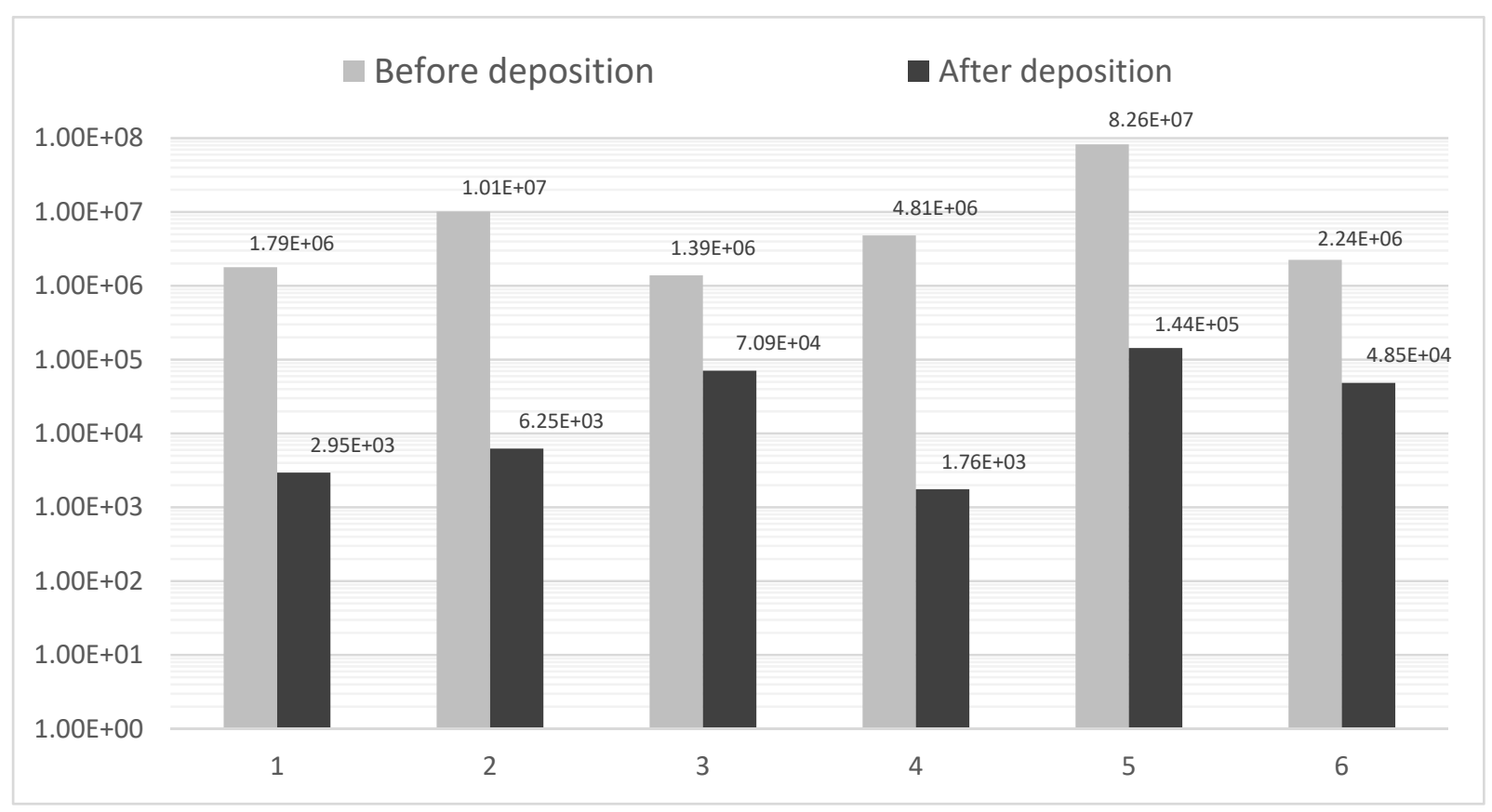

279 Figure 7. EIS measurement of six electrodes on a single probe shank before and after MWCNT deposition on a 280 logarithmic scale.

281 This work is the first to demonstrate the use of standard microfabrication processes to fabricate 282 multi-site neural probes with polyimide as supporting/structural and insulating/protective layers. 283 Polyimide is an excellent alternative because it exhibits high electrical resistivity, mechanical 284 flexibility, and biocompatibility properties. In addition, polyimide is known to not hydrate over 285 time, which will eventually improve the probes' usefulness for chronic neural recording. The 286 flexible neuroelectronic probe as described herein contains two polyimide layers as the insulting 287 and structural material. The probe is approximately $8 \mu \mathrm{m}$ thick and contains 64 pyramidal shaped 288 electrode sites. The device was successfully integrated and the process development challenges 289 were overcome by employing lift-off process to eliminate metal undercut, ion milling process to 
290 create good step coverage of $\mathrm{Cr} / \mathrm{Au}$ layer over the thick polyimide, and RIE descum process to 291 remove resist residual and resolve thin interconnect traces. The polyimide probe structure is 292 fabricated using standard microfabrication technology and does not require advanced CMOS 293 processing techniques. The flexibility afforded by the probe should increase the ruggedness of 294 the probe in various chronic neural recording applications. Additionally, we successfully 295 demonstrated the reduction of the gold recording electrodes' impedance via the electrochemical 296 deposition of MWCNTs. The observed impedance values at physiologically relevant frequency 1 $297 \mathrm{kHz}$ were on average $45.73 \mathrm{k} \Omega$ ), which is suitable for electrochemical sensing.

298 Disclosures

299 The authors have no conflicts of interest.

301 Acknowledgments

302 The authors acknowledge the financial support of the National Science Foundation, United 303 States (Award ECCS-\# 1342912). The infrastructure used for this work would not have been 304 possible without the significant contributions of the Center for Nanoscale Science and 305 Technology, National Institute of Standards and Technology Gaithersburg, MD, USA, and the 306 FEI SEM of the NanoImaging Center at the University of Maryland Baltimore County.

307 References

308 1. G.E. Slaughter, E. Bieberich, G.E. Wnek, K.J. Wynne, and A. Guiseppi-Elie, "Improving neuron-to309 electrode surface attachment via alkanethiol self-assembly: an alternating current impedance study," 310 Langmuir, 20(17), 7189-7200 (2004). 
311 2. M. HajjHassan, V. Chodavarapu, and S. Musallam, "NeuroMEMS: neural probe microtechnologies,"

312 Sensors, 8(10), 6704-6726 (2008).

313 3. M.Y. Chenget al., "A low-profile three-dimensional neural probe array using a silicon lead transfer 314 structure," Journal of Micromechanics and Microengineering. 23(9),095013 (2013).

315 4. S. Herwik et al., "Fabrication technology for silicon-based microprobe arrays used in acute and sub316 chronic neural recording," Journal of Micromechanics and Microengineering. 19(7), 074008 (2009).

317 5. J.F. Hetke and D.J. Anderson, D.J, "Silicon microelectrodes for extracellular recording" in Handbook 318 of neuroprosthetic methods, W.E. Finn and P.G. LoPresti, Eds., CRC Press, Boca Raton, chapter 7 3192003.

320 6. A.K. Koivuniemi, S.J. Wilks, A.J. Woolley, and K.J. Otto, "Multimodal, longitudinal assessment of 321 intracortical microstimualtion," Progr. Brain Res. 194, 131-144 (2011).

322 7. M.P. Ward, P. Rajdev, C. Ellison, and P.P. Irazoqui, "Toward a comparison of microelectrodes for 323 acute and chronic recordings," Brain research. 1282, 183-200 (2009).

324 8. J. Subbaroyan, D.C. Martin, and D.R. Kipke, "A finite-element model of the mechanical effects of 325 implantable microelectrodes in the cerebral cortex," Journal of neural engineering, 2(4), 103 (2005).

326 9. J. Tyson, M. Tran, and G. Slaughter, "Biocompatibility of a Novel Quad-Shank Neural Probe," Solid 327 State Electronics. 2017.

328 10. R.R. Richardson, J.A. Miller, and W.M. Reichert, "Polyimides as biomaterials: preliminary 329 biocompatibility testing," Biomaterials, 14(8), 627-635 (1993).

330 11. K.C. Cheung, P. Renaud, H. Tanila, and K. Djupsund, "Flexible polyimide microelectrode array for 331 in vivo recordings and current source density analysis," Biosensors and Bioelectronics, 22(8), 1783$3321790(2007)$

333 12. G. Rios, E.V. Lubenov, D. Chi, M.L. Roukes, and A.G. Siapas, "Nanofabricated Neural Probes for 334 Dense 3-D Recordings of Brain Activity," Nano letters, 16(11), 6857-6862 (2016).

335 13. F. Lisdat,and D. Schäfer, "The use of electrochemical impedance spectroscopy for biosensing," 336 Analytical \& Bioanalytical Chemistry, 391(5), 1555-1567 (2008). 
337 14. Z. Xiang et al., "Ultra-thin flexible polyimide neural probe embedded in a dissolvable maltose-coated 338 microneedle," Journal of Micromechanics and Microengineering, 24(6), 065015, (2015)

339 15. M.L. Huffman and B.J. Venton, "Carbon-fiber microelectrodes for in vivo applications," The Analyst, $340 \quad$ 134(1), 18-24 (2009).

341 16. N. Burblies, "Coatings of Different Carbon Nanotubes on Platinum Electrodes for Neuronal Devices: 342 Preparation, Cytocompatibility and Interaction with Spiral Ganglion Cells," Plos ONE, 11(7), 1-22 $343 \quad$ (2016).

344 17. G. Slaughter, D. Gupta, T. Kulkarni, and L.L. Morton, “A microfabricated low-cost Au nanotip 345 pyramidal electrode array using anisotropic etching for enhanced performance of a glucose 346 biosensor," Proc. SENSORS, IEEE 2015, 1-4 (2015).

347 18. G.T.A. Kovacs, "Introduction to the theory, design, and modeling of thin-film microelectrodes for 348 neural interfaces," in Enabling Technologies for Cultured Neural Networks, D.A. Stenger, T.

349 McKenna, Eds., Academic Publishers; New York, 121-165 (1994).

350 19. B. He, Neuron Engineering, Kluwer Academic, Norwell, MA, (2005).

351 20. R.L. White and T.J. Gross, "An evaluation of the resistance to electrolysis of metals for use in 352 biostimulation microprobes," IEEE Transactions on Biomedical Engineering, 6, 487-490 (1974).

353 21. S.S. Stensaas, and L.J. Stensaas, "Histopathological evaluation of materials implanted in the cerebral 354 cortex," Acta neuropathologica, 41(2), 145-155 (1978).

355 22. A.K. Ahuja, M.R. Behrend, J.J. Whalen, M.S. Humayun, and J.D. Weiland, "The dependence of 356 spectral impedance on disc microelectrode radius," IEEE Transactions on Biomedical Engineering, $357 \quad 55(4), 1457-1460(2008)$.

358 23. A.R. Rahman, J. Register, G. Vuppala, and S. Bhansali, "Cell culture monitoring by impedance 359 mapping using a multielectrode scanning impedance spectroscopy system (CellMap)," Physiological 360 Measurement, 29(6), S227-S239 (2008). 
363 Gymama Slaughter is currently an Associate Professor of Computer Engineering at the 364 University of Maryland Baltimore and Director of the Bioelectronics Laboratory. Dr. 365 Slaughter's research has been supported by the National Science Foundation for her diabetes

366 research that focuses on the design and development of self-powered glucose biosensor, 367 especially in relationship to monitoring blood glucose in diabetics. She is the recipient of the 368 National Science Foundation's prestigious CAREER AWARD.

370 Biographies and photographs for the other authors are not available.

\section{$\underline{\text { Caption List }}$}

375 Fig. 1 Schematic design of the flexible neuroelectronic probe structure and CAD layout showing 376 the electrode sites, interconnect traces and bond pads.

377 Fig. 2 Schematic illustration of the major processing steps in the fabrication of the 64-site probe.

378 Fig. 3 SEM micrographs of neuroelectronic probe shank with 16 recording electrode sites and 379 submicron interconnect traces sandwiched between two polyimide layers and insert views of 380 shank tip and rectangular pyramid shaped electrode.

381 Fig. 4 Flexible and optically transparent polyimide neuroelectronic probe accommodates 382 associated bond pads, interconnect traces $10 \mu \mathrm{m}$ to $1 \mu \mathrm{m}$ in two distinct metallization layers, and 383 the traces are connected to the electrode sites..

384 Fig. 5 Flexible and optically transparent polyimide neural probe packaging..

385 Fig. 6 Recording electrode site before (left) and after (right) MWCNT deposition.

386 Fig. 7 EIS measurement of six electrodes on a single probe shank before and after MWCNT 387 deposition on a logarithmic scale. 


\title{
Parametric Amplification of Acoustically-Excited Micromechanical Oscillators Using Fringing Electrostatic Fields
}

\author{
Stella Lulinsky*, Tsvi Schmilovich*, Bojan Rob Ilic ${ }^{* *}$ and Slava Krylov ${ }^{*}$ \\ "School of Mechanical Engineering, Tel Aviv University, Tel Aviv, ISRAEL \\ ***enter for Nanoscale Science and Technology, National Institute of Standards and Technology \\ Gaithersburg, MD, USA
}

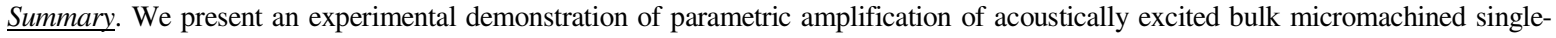
crystal silicon cantilevers. We used electrostatic actuation by fringing fields for the parametric pumping of vibrational oscillations. The omnidirectional acoustic pressure served as a non-contact source for linear harmonic driving. We show that acoustic actuation is a convenient and versatile tool for dynamic characterization of micromechanical devices. This excitation method is suitable for wafer level dynamic testing, and could serve as an alternative to commonly used piezoelectric actuators. Our results suggest that this amplification approach may have applications in a wide variety of micromechanical devices, including resonant sensors, microphones and hearing aids.

\section{Introduction}

Parametric amplification plays an important role in resonant micro and nanoelectromechanical (MEMS/ NEMS) sensors due to its ability to increase the amplitude of vibrations and squeeze noise, thereby improving sensitivity [1-4]. In MEMS/NEMS structures parametric pumping of an external harmonic signal was demonstrated using electrostatic [1,2], piezoelectric [3] and magnetic actuation. The use of electrostatic actuation for direct harmonic driving [2] introduces a nonlinearity that alters the spectral characteristics of the structure. While integration of piezoelectric materials allows linear actuation and high vibrational amplitudes [3], the method requires deposition of additional layers, complicating the fabrication process. These layers may also induce undesirable residual stresses. To achieve parametric amplification in conjunction with a commonly used linear harmonic forcing by an external piezoelectric actuator $[1,4]$, an additional electric signal is required at the electrode. In a laboratory environment, the piezo mounted devices cannot be electrically connected with micro manipulator probes. System testing requires wire bonding and packaging. Consequently, inertial excitation of micro structures is not suitable for wafer level testing.

In this work we use electrostatic forces associated with fringing fields for parametric pumping, and omnidirectional acoustic pressure as a source for non-contact linear harmonic mechanical excitation. In the MEMS/NEMS arena, investigations into the acoustic actuation were primarily motivated by the development of microphones and hearing aids [5]. Several groups have reported on resonant acoustic excitation of optical micro scanners [6] or micro cantilevers [7]. Here, we use acoustic fields for the mechanical excitation of a parametrically amplified device.

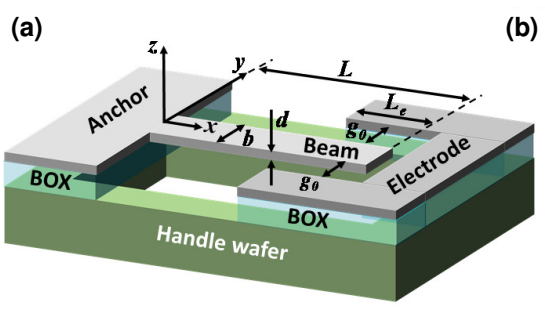

(b)

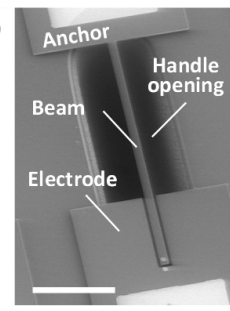

(c)

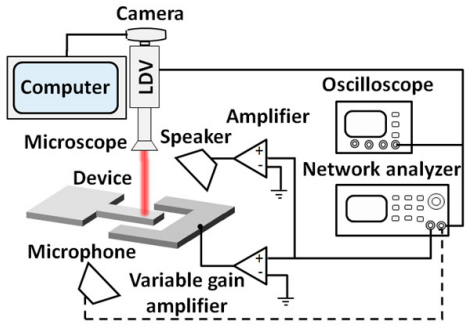

Fig. 1: (a) Schematics of the device. (b) Scanning electron micrograph of the fabricated device. The scale bar is $100 \mu \mathrm{m}$. Measured dimensions of the structure are $L=296.13 \pm 0.60 \mu \mathrm{m}, b=15.13 \pm 0.60 \mu \mathrm{m}, L_{e}=97.27 \pm 0.60 \mu \mathrm{m}, g_{0}=5.61 \pm 0.60 \mu \mathrm{m}$. The estimated measurement error was based on the pixel-to-micrometer conversion factor of the scanning electron micrographs and a one-pixel uncertainty in determining feature dimensions. (c) Schematics of the experimental setup.

\section{Device Architecture}

Our device [8] consists of a cantilever of a nominal (as deigned) length $L=300 \mu \mathrm{m}$, width $b=16 \mu \mathrm{m}$ and thickness $d=3 \mu \mathrm{m}$, Fig. 1(a). A planar side electrode of length $L_{e}=100 \mu \mathrm{m}$ is located symmetrically at two sides of the beam, at a distance $g_{0}=5 \mu \mathrm{m}$. To allow large unobscured vibrations in the out-of-plane $(z)$ direction, an opening was created in the substrate under the beam. Since both the cantilever and the electrode are fabricated from the same wafer layer, due to symmetry, the resultant electrostatic force is zero in the initially undeformed configuration. In the deformed state, the distributed electrostatic force, arising from asymmetries of the fringing fields, acts in a direction opposite to the beam's deflection and serves as a restoring force. Application of a time-dependent voltage to the electrode results in a modulation of an effective stiffness and in parametric amplification of the beam. Using deep reactive ion etching (DRIE), cantilevers and electrodes were fabricated from $\mathrm{a} \approx 3 \mu \mathrm{m}$ thick single crystal silicon device layer using a silicon on insulator wafer. DRIE was used also to etch a cavity within the handle wafer. The devices were released using a vapor hydrofluoric acid process. A fabricated device is shown in Fig. 1(b). 


\section{Experimental Setup}

The schematics of the experimental setup is illustrated in Fig. 1(c). The device was mounted on the chuck of a wafer prober. Using micro manipulator probes, the beam was set to ground with the electrodes set at a potential supplied by a voltage source. The cantilever was acoustically actuated by a speaker placed at a distance of $\approx 5 \mathrm{~cm}$ from the device. The sinusoidal, zero offset, voltage signal provided by a network analyzer was split into two channels. The first channel, connected to a fixed $\times 20$ gain amplifier, supplied to the speaker a peak-to-peak voltage signal $\left(V_{\text {spkr }}\right)$ ranging from $\approx 3 \mathrm{~V}$ to $\approx 10 \mathrm{~V}$. The second channel, connected to a variable gain (up to $\times 150$ ) voltage amplifier, supplied a signal to the electrode. The out-of-plane response of the beam was measured using a laser Doppler vibrometer (LDV) operated in a velocity acquisition mode. The output of the LDV was fed back into the network analyzer. The velocity time history of the LDV output was monitored by an oscilloscope. During our speaker calibration, the acoustic signal was monitored by a microphone. The measured resonant frequency of the speaker was $39.68 \mathrm{kHz} \pm 6.63 \mathrm{~Hz}$ (mean \pm one standard deviation). The uncertainties were estimated from a Lorentzian functional fit to the measured spectra.

\section{Results}

First, the electrode was disconnected from the voltage source and vibrations of the beam were induced acoustically. Two resonant peaks were registered, associated with the speaker and the cantilever resonances, Fig. 2(a). To distinguish between the spectral outputs of the speaker and the beam, the re-scaled response of the speaker was subtracted from the LDV output, Fig 2(a). Acoustically excited amplitudes of the cantilever were estimated using the velocity time history (insert in Fig. 2(a)). In our frequency tuning, experiment, a harmonic voltage of $V_{s p k r} \approx 5 \mathrm{~V}$ was applied to the speaker. The signal frequency was swept from $\approx 43 \mathrm{kHz}$ to $\approx 47 \mathrm{kHz}$ for $\approx 25 \mathrm{~s}$. Simultaneously, a steady-state dc voltage $V_{d c}$ was applied to the electrode. We demonstrate that by applying a steady dc voltage the fundamental beam frequency can be tuned up to $\approx 2.5 \%$, Fig. 2(b). Finally, voltage signals $V_{s p k r} \cos (\omega t)$ and $V_{a c} \cos (\omega t)$ were applied simultaneously to the speaker and to the electrode, respectively. In this scenario, the beam undergoes direct harmonic acoustic forcing and parametric excitation by the electrostatic force. Since the electrostatic force is proportional to the square of the voltage, the parametric signal is at the frequency of $2 \omega$ and the case of a degenerate parametric pumping [1-4] is realized. Results shown in Fig. 2(c) indicate that the application of the electrostatic force at the voltage values below the parametric resonance threshold $[3,4]$ allows amplification of the acoustically induced vibrations.
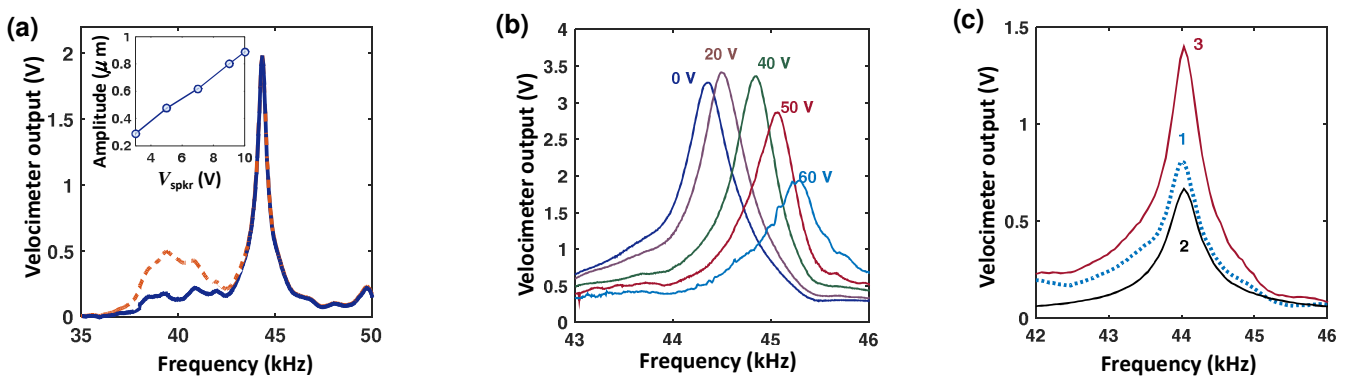

Fig. 2: (a) Frequency response of the beam excited by the speaker at $V_{s p k r} \approx 9 \mathrm{~V}$ before (dashed) and after (solid line) extraction of the microphone output. Two peaks at $39.61 \mathrm{kHz} \pm 6.63 \mathrm{~Hz}$ and $44.34 \mathrm{kHz} \pm 1.98 \mathrm{~Hz}$ correspond to the speaker's and to the beam's resonances, respectively. Insert shows the beam's resonant amplitude at different $V_{s p k r}$. The amplitude uncertainty, estimated by sinusoidal fitting to the time history data is $15 \mathrm{~nm}$. (b) Frequency response of the beam at different values $V_{d c}$ (numbers) of the voltage applied to the side electrode; speaker voltage is $V_{s p k r} \approx 5 \mathrm{~V}$. (c) Parametric amplification: 1 - acoustic excitation; 2 - electrostatic excitation; 3 - combined acoustic and electrostatic excitation. $V_{s p k r} \approx 600 \mathrm{mV}, V_{a c} \approx 30 \mathrm{~V}$.

\section{References}

[1] Rugar D., Grütter P. (1991) Mechanical parametric amplification and thermomechanical noise squeezing. Phys. Rev. Lett. 67: 699 - 702.

[2] Carr D. W., Evoy S., Sekaric L., Craighead H. G., Parpia J. M. (2000) Parametric amplification in a torsional microresonator. Appl. Phys. Lett. 77:1545- 1547 .

[3] Karabalin R. B., Masmanidis S. C., Roukes M. L. (2010) Efficient parametric amplification in high and very high frequency piezoelectric nanoelectromechanical systems. Appl. Phys. Lett. 97:183101-1 - 183010-3.

[4] Rhoads J.F., Miller N.J., Shaw S.W., Feeny B.F. (2008) Mechanical domain parametric amplification. J. Vib. Acoust. 130:061006-1 - 061006-7.

[5] Miles R. N., Cui W., Su Q. T., Homentcovschi D. A. (2015) MEMS low-noise sound pressure gradient microphone with capacitive sensing. J. Microelectromech. Syst. 24: $241-248$.

[6] Ahmida K. M., Ferreira L. Otávio S. (2004) Design and modeling of an acoustically excited double-paddle scanner. J. Micromech. Microeng. 14:13371344.

[7] Cetinkaya C., Ban L., Subramanian G., Akseli I. (2008) Multimode air-coupled excitation of micromechanical structures. IEEE Transact. Instr. Meas. 57:2457-2461.

[8] Linzon Y., Ilic B., Lulinsky S., Krylov S. (2013) Efficient parametric excitation of silicon-on-insulator microcantilever beams by fringing electrostatic fields, J. Appl. Phys. 113:163508-1 - 163508-11. 


\title{
OPEN-LOOP, SELF-EXCITATION IN A BISTABLE MICROMECHANICAL BEAM ACTUATED BY A DC ELECTROSTATIC LOAD
}

\author{
L. Medina ${ }^{1}$, R. Gilat ${ }^{2}$, B. R. Ilic $^{3}$, and S. Krylov ${ }^{1}$ \\ ${ }^{1}$ School of Mechanical Engineering, Faculty of Engineering, Tel-Aviv University, Ramat Aviv 69978, \\ Israel \\ ${ }^{2}$ Department of Civil Engineering, Faculty of Engineering, Ariel University, Ariel 44837, Israel \\ ${ }^{3}$ Center for Nanoscale Science and Technology, National Institute of Standards and Technology, \\ Gaithersburg, MD, 20899, USA
}

\begin{abstract}
We demonstrate an open-loop self-excitation response in a curved, bistable microelectromechanical beam under a time-independent electrostatic load. The self-excitation is triggered by placing a high value resistor in series with a beam that is on the verge of bistability. The voltage-deflection curve of such a beam contains an inflection point, where the slope of the curve is approximately zero. Our results show that actuation at a voltage corresponding to the inflection point induces stable self-sustained oscillations. We further observe that implementation of the same actuation scenario in a bistable beam, with the voltage-deflection curve containing two stable branches, does not lead to self-excitation.
\end{abstract}

\section{KEYWORDS}

Curved micromechanical beam; Electrostatic actuation; Self-excitation; Bistability; MEMS/NEMS

\section{INTRODUCTION}

Self-excited systems are able to maintain stable oscillations under steady, time-independent, loading. Due to its beneficial features, this effect, which is also of fundamental scientific interest, has been employed in various applications, including atomic force microscopy [1,2], sensors [3,4] and energy harvesting [5].

In the realm of micro- and nanoelectromechanical systems (MEMS/NEMS), self-induced oscillations can be achieved using several approaches. The most common methods employ a closed-loop control, where an actuation voltage change, based on the velocity of the system, introduces nonlinear virtual damping $[1,2,4]$. Closed-loop control, proposed by S. S. Lee [6], includes an active amplification change of the driving electronic circuit for enhancing amplitude oscillations of the acoustic transducer. In general, closed-loop control at the micro- and nano-scale requires implementation of complex feedback algorithms $[1,2,4]$ with demanding accuracy requirements for the position or velocity of the sensing transducer.

In contrast, open-loop devices take advantage of the structural intrinsic nonlinearities and interactions with their surroundings [3-8]. In these systems, self-induced oscillations are caused by parametric, configuration dependent forces. These forces result from nonlinear thermo- opto-mechanical coupling [7] or electrostatically-induced structural contact with an actuating electrode $[8,9]$. Contact based systems may suffer from inferior reliability and long-term performance stability.

In this work, we report on experiments where selfsustained oscillations are triggered in an open-loop driven micromechanical device. We used an electrostaticallyactuated, curved, bistable micromechanical beam. We show that self-excitation is an intrinsic property of such a beam, and can be achieved under appropriate conditions.

\section{EXPERIMENT}

\section{Experimental Procedure \& Equipment}

Curved microelectromechanical beams (Fig. 1) were fabricated from a highly doped, single-crystal silicon device layer using a silicon-on-insulator wafer [10,11]. The structures were lithographically defined and etched using a deep reactive ion etching process. The structures were released by dissolution of the sacrificial oxide with hydrofluoric acid. After a high-pressure critical-point drying process, the device dimensions were measured using an environmental scanning electron microscope.

Micromechanical beams were designed with nominal dimensions of $b=20 \mu \mathrm{m}, d=3.5 \mu \mathrm{m}, g_{0}=10 \mu \mathrm{m}$, $L=1000 \mu \mathrm{m}$ and nominal initial elevations of $h_{0}=0.5 \mu \mathrm{m}$ and $h_{0}=2 \mu \mathrm{m}$. Fabricated structural dimensions of the first beam were $d \approx 3.31 \mu \mathrm{m}, g_{0} \approx 10.97 \mu \mathrm{m}$ and $h \approx 2.12 \mu \mathrm{m}$. The second beam had a higher $h$ with system dimensions of $d \approx 3.71 \mu \mathrm{m}, g_{0} \approx 10.37 \mu \mathrm{m}$ and $h \approx 3.13 \mu \mathrm{m}$. We attribute the discrepancy between the nominal and measured dimensions to the fabrication-related scatter in the geometric parameters due to residual stress, as discussed in our previous work $[11,12]$.

Devices were mounted onto a wafer probing station and tested at room temperature under ambient air conditions. The electrical signal was generated and amplified by a factor of 20 using a signal processor and a high voltage amplifier, respectively. The in-plane motion of the beams was measured using an optical setup by video recording the beam's motion with a camera operating at a frame rate of $\approx 12 \mathrm{~Hz}$. The beam midpoint position $\left(w_{m}\right)$ corresponding to each video frame was determined using image processing technique [13]. 


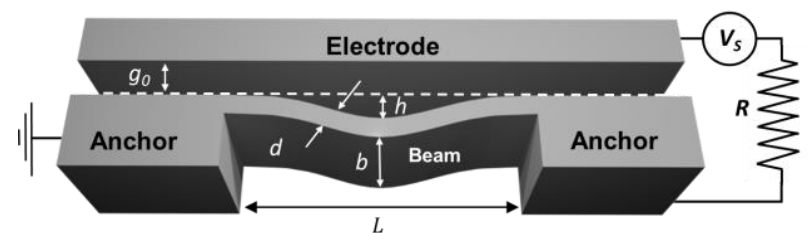

Figure 1: Schematics of a curved beam of length L, elevation $h$, thickness $d$, width $b$, beam to electrode distance $g_{0}$, series resistor $R$ and source voltage $V_{S}$.

\section{Results \& Discussion}

Curved, micromechanical beams are characterized by their equilibrium curves, as Figure 2 illustrates. The plots were obtained using a reduced order (RO) model for a specified beam geometry [12]. Figure 2 presents two possible equilibrium curve scenarios for a curved beam used in our experiments. Figure 2(a) shows a beam on the verge of bistability with two limit, zero-slope, points labeled as the inflection and pull-in points. Figure 2(b) shows an equilibrium curve for a bistable beam characterized by three limit points: snap-through, release and pull-in points.
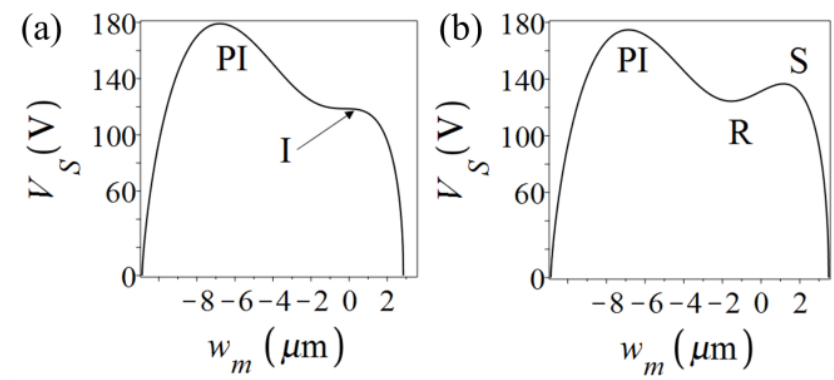

Figure 2: Reduced order model results: equilibrium curve for beams with $L=1000 \mu \mathrm{m}, \quad b=20 \mu \mathrm{m}, \quad d=3.23 \mu \mathrm{m}$, $g_{0}=10.88 \mu \mathrm{m}$. (a) $h=2.85 \mu \mathrm{m}$ showing two limit points, $a$ pull-in (PI) and an inflection point (I). (b) $h=3.5 \mu \mathrm{m}$ showing three limit points, snap-through $(\mathrm{S})$, release $(R)$ and pull-in (PI).

In our experiments, equilibrium curves were first obtained by applying a triangular voltage signal between the beam and the electrode. The maximum amplitude and duration of the signal were $\approx 30 \mathrm{~V}$ and $\approx 60 \mathrm{~s}$, respectively. A resistor $R \approx 10 \mathrm{k} \Omega$ was placed in series with the beam to limit current induced heating resulting from a possible pull-in collapse. Subsequently, static experiments with $R \approx 20 \mathrm{M} \Omega$ showed qualitatively similar beam motion with additional oscillations around the equilibrium curve. This procedure was carried out for two different beams. Figure 3 shows a response of a beam with $h \approx 2.12 \mu \mathrm{m}$. The measured equilibrium curve shows that the beam is on the verge of bistability. This implies that instead of having a snap-through point, the beam has an inflection point [12]. The results presented in Fig. 4 superimposed against the applied triangular actuation signal, further show a gradual increase of vibrational amplitudes with increasing voltage. At the applied load of $\approx 20 \mathrm{~V}$, vibrational amplitudes of $\approx 0.69 \mu \mathrm{m}$ were observed.
Based on the results of the above experiment, we applied the following trapezoidal voltage signal

$$
V_{S}(t)=V_{\max }\left(\frac{t}{T}(1-H(t-T))-\frac{t-2 T}{T} H(t-2 T)\right)
$$

where $t$ is time, $H(t)$ is the Heaviside step function. The maximum voltage signal was $V_{\max } \approx 20 \mathrm{~V}$, with time $T \approx 30 \mathrm{~s}$ for each of the three trapezoidal segments: linearly increasing ramp, constant and a linearly decreasing ramp. Figure 5 shows the beam response to the trapezoidal signal.

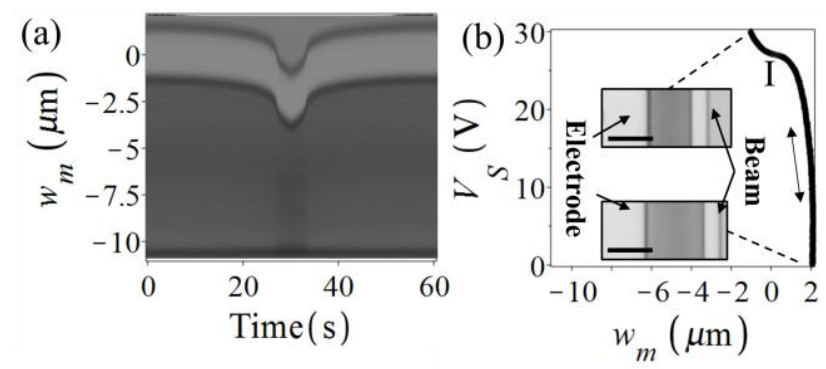

Figure 3: Experimental results: a quasi-static experiment with $R \approx 10 \mathrm{k} \Omega, L \approx 1000 \mu \mathrm{m}, b \approx 20 \mu \mathrm{m}, d \approx 3.32 \mu \mathrm{m}$, $h \approx 2.12 \mu \mathrm{m}$, and $g_{0} \approx 10.97 \mu \mathrm{m}$. (a) Direct visualization of the time history. (b) Static buckling curve. Insets represent snapshots of the initial position and maximum deflection corresponding to the highest voltage $V_{\max } \approx 30 \mathrm{~V}$. Scale bars represent $\approx 10 \mu \mathrm{m}$. The two-directional arrows represent the beam midpoint movement along the equilibrium curve. Label I denotes the inflection point.
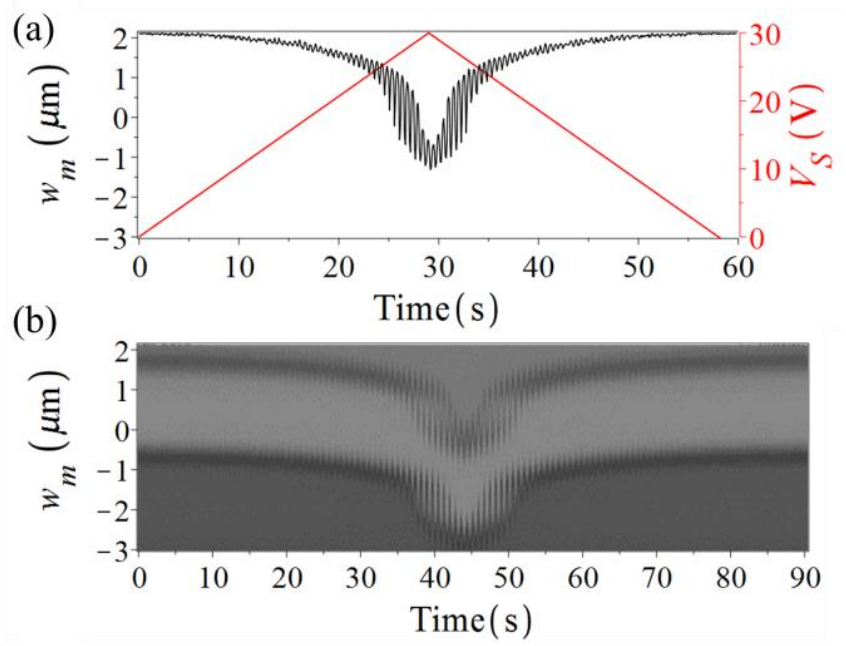

Figure 4: Experimental time history results for the beam with dimensions defined in Figure 3 with $R \approx 20 \mathrm{M} \Omega$ : (a) Time history of the beam mid-point motion (black) and the source voltage (red). (b) Direct visualization of the time history. 

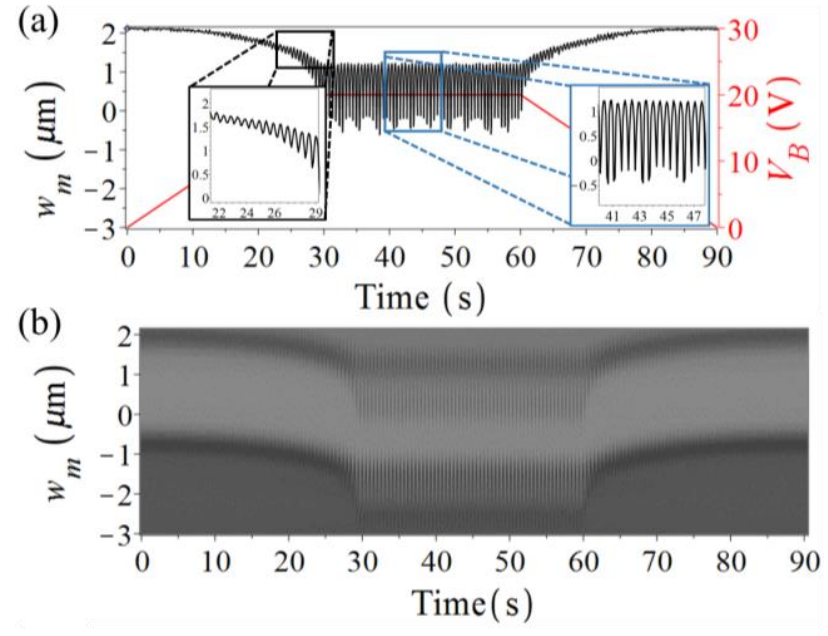

Figure 5: Experimental results of the beam from Fig. 3 with $R \approx 20 \mathrm{M} \Omega$. The voltage signal consisted a linearly increasing ramp, a constant region with $V_{\max } \approx 20 \mathrm{~V}$, and a decreasing ramp (red curve): (a) Time history. The black and blue framed insets represent zoomed-in figure data. (b) Direct visualization of the time history.
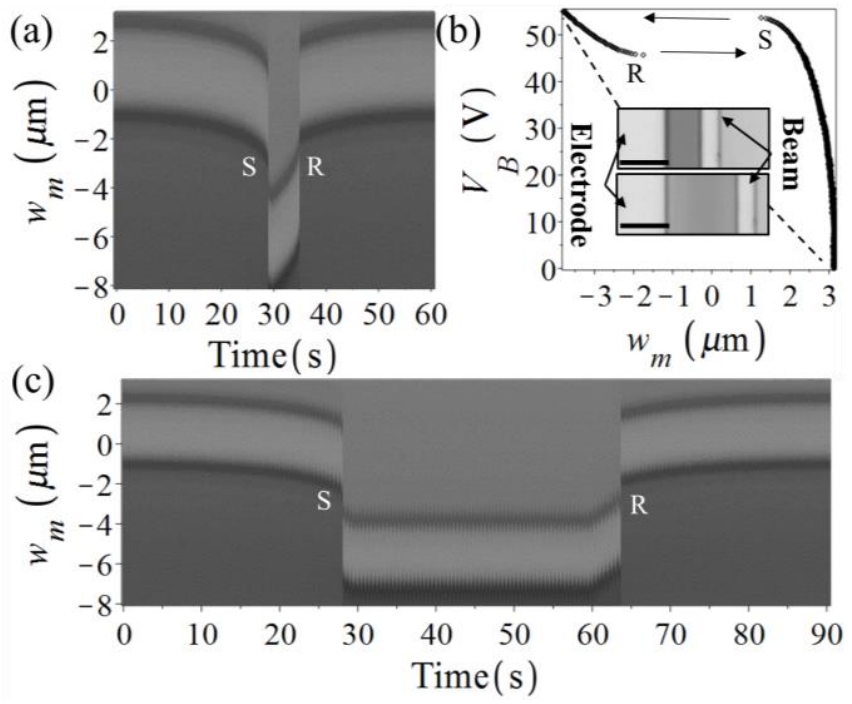

Figure 6: Results of experiments on a bistable beam with $L \approx 1000 \mu \mathrm{m}, \quad b \approx 20 \mu \mathrm{m}, \quad d \approx 3.71 \mu \mathrm{m}, \quad h \approx 3.14 \mu \mathrm{m}$, $g_{0} \approx 10.37 \mu \mathrm{m}$. (a) Direct visualization of the time history and (b) buckling curve with snapshots of the beam with $R \approx 10 \mathrm{k} \Omega$ under a triangular signal. Insets represent the initial position and maximum deflection corresponding to the highest voltage $V_{\max } \approx 55 \mathrm{~V}$. Scale bars represent $\approx 10 \mu \mathrm{m}$. The arrows represent the beam midpoint direction upon loading-unloading triangular signal. (c) Direct visualization of the response of the beam with $R \approx 20 \mathrm{M} \Omega$ to a trapezoidal voltage signal of amplitude $V_{\max } \approx 50 \mathrm{~V}$, and total duration of $3 T \approx 90 \mathrm{~s}$. $\mathrm{S}$ and $\mathrm{R}$ represent snap-through and release points.

We observed high amplitude oscillations in the region of constant bias $(\approx 30 \mathrm{~s}<t<\approx 60 \mathrm{~s})$. Figure 5 insets show the measured beam response during the linearly increasing voltage ramp (black frame inset) and in the region with $V_{\text {max }} \approx 20 \mathrm{~V}$ (blue frame inset). With the frame rate of $\approx 12 \mathrm{~Hz}$, even though the full time history of the oscillatory behavior is not fully apparent, the boundaries of the response are visible, yielding a maximum oscillatory amplitude of $\approx 1.66 \mu \mathrm{m}$.

To gain further insight into the conditions for the observed self-excitation, the behavior of a bistable [10] curved beam with $h \approx 3.13 \mu \mathrm{m}$ under a triangular signal with $T \approx 60 \mathrm{~s}$ was examined and is shown in Fig. 6 . For the case of $R \approx 10 \mathrm{k} \Omega$, the quasi-static response and the corresponding equilibrium curve, Fig. 6(a) and (b) show bistability with a clear distinction between the snap-through and release limit points. Figure 6(c) shows that a trapezoidal signal with $V_{\max } \approx$ $50 V$ and $R \approx 20 \mathrm{M} \Omega$ did not produce large amplitude self-excited oscillations. The observed oscillations had a maximum amplitude of $\approx 0.16 \mu \mathrm{m}$, which is smaller than the distance between the two stable equilibria of the bistable beam. These results imply that in order to induce selfoscillations, the difference between the snap-through and the release voltages should be small. This condition is satisfied when the beam is on the verge of bistability, where the snap and release points coincide.

\section{SUMMARY \& CONCLUSIONS}

Our work demonstrates a first instance of self-oscillatory behavior of curved, bistable, micromechanical beams electrostatically actuated by a single electrode in an open-loop configuration. We experimentally showed that self-induced oscillations occur when an additional, sufficiently large, resistance is added in series with the microelectromechanical beam. Our observations suggest that the development of high amplitude vibrations is possible when the curved beam is on the verge of bistability. This requirement allows vibrational shifting along the zero slope part of the equilibrium curve around an inflection point.

The self-excitation is attributed to the influence of a large resistor which limits the charging current and therefore increases the capacitor charging time. As the beam moves quasi-statically, wherein the rate of motion is much slower than the capacitor charging rate, the beam is in equilibrium at any point. Alternatively, when the beam approaches and passes the inflection point, where the slope of the voltage-displacement curve is close to zero, the rate of motion increases. Since the electric current, limited by the resistor, is not sufficient to charge the capacitor, the effective electrode voltage drops. As a result, the beam is in a non-equilibrium state, where the elastic restoring force is larger than the electrostatic force. This configuration corresponds to smaller beam deflections and decreased capacitance. The consequent voltage increase sets the beam to a non-equilibrium configuration, where the actuating electrostatic force is higher than the restoring mechanical force. This process repeats, thereby producing self-sustained vibrations. 


\section{ACKNOWLEDGEMENTS}

The devices were fabricated and tested at the Center for Nanoscale Science and Technology (CNST) at the National Institute of Standards and Technology and at the Tel Aviv University MEMS design and characterization lab (MDCL). The authors would like to thank Stella Lulinski in helping with the presented work. The research is supported by the Israel Science Foundation (ISF grant no. 1272/16), Israel Ministry of Science, Technology and Space and Ariel University.

\section{REFERENCES}

[1] Yabuno, H., Kaneko, H., Kuroda, M., \& Kobayashi, T. (2008). Van der Pol type self-excited micro-cantilever probe of atomic force microscopy. Nonlinear Dynamics, 54(1-2), 137-149.

[2] Lee, C., Itoh, T., \& Suga, T. (1999). Self-excited piezoelectric PZT microcantilevers for dynamic SFMwith inherent sensing and actuating capabilities. Sensors and Actuators A: Physical, 72(2), 179-188.

[3] Sung, S., Lee, J. G., Lee, B., \& Kang, T. (2003). Design and performance test of an oscillation loop for a MEMS resonant accelerometer. Journal of Micromechanics and Microengineering, 13(2), 246.

[4] Lee, Y., Lim, G., \& Moon, W. (2006). A self-excited micro cantilever biosensor actuated by PZT using the mass micro balancing technique. Sensors and Actuators A: Physical, 130, 105-110.

[5] Wang, Z., Li, Z., \& Lu, W. (2007, October). A new selfoscillation loop for MEMS vibratory gyroscopes. In 2007 7th International Conference on ASIC.

[6] Lee, S. S., \& White, R. M. (1996). Self-excited piezoelectric cantilever oscillators. Sensors and Actuators A: Physical, 52(1), 41-45.

[7] Aubin, K., Zalalutdinov, M., Alan, T., Reichenbach, R. B., Rand, R., Zehnder, A., Parpia, J. \& Craighead, H. (2004). Limit cycle oscillations in CW laser-driven NEMS. Journal of Microelectromechanical Systems, 13(6), 1018-1026.

[8] Bienstman, J., Vandewalle, J., \& Puers, R. (1998). The autonomous impact resonator: a new operating principle for a silicon resonant strain gauge. Sensors and Actuators A: Physical, 66(1), 40-49.

[9] Shmulevich, S., Hotzen, I., \& Elata, D. (2015, January). The electromechanical response of a self-excited MEMS Franklin oscillator. In IEEE MEMS 2015 28th International Conference (pp. 41-44). IEEE.

[10]Krylov, S., \& Dick, N. (2010). Dynamic stability of electrostatically actuated initially curved shallow micro beams. Continuum Mechanics and Thermodynamics, 22(6-8), 445-468.

[11] Krylov, S., Ilic, B. R., Schreiber, D., Seretensky, S., \& Craighead, H. (2008). The pull-in behavior of electrostatically actuated bistable microstructures. Journal of Micromechanics and Microengineering, 18(5), 055026.
[12] Medina, L., Gilat, R., \& Krylov, S. (2014). Symmetry breaking in an initially curved pre-stressed micro beam loaded by a distributed electrostatic force. International Journal of Solids and Structures, 51(11), 2047-2061.

[13] Medina, L., Gilat, R., Ilic, B., \& Krylov, S. (2014). Experimental investigation of the snap-through buckling of electrostatically actuated initially curved pre-stressed micro beams. Sensors and Actuators A: Physical, 220, 323-332.

\section{CONTACT}

*L. Medina; liormedi@post.tau.ac.il

Gilat, Rivka; Ilic, Bojan; Krylov, Slava; Medina, Lior. 


\title{
Measuring the Phase Transformation Kinetics Under Non-Equilibrium Conditions from Time Resolved High Resolution TEM Images
}

\author{
Renu Sharma ${ }^{1}$, Zahra Hussani ${ }^{1}$, Pin Ann Lin $^{1.2}$ and Bharath Natarajan ${ }^{1,2}$ \\ 1. Center for Nanoscale Science and Technology, National Institute of Standards and Technology, \\ Gaithersburg, MD 20899-6203. \\ 2. Maryland NanoCenter, University of Maryland, College Park, MD 20742, USA
}

The interaction of gases with a solid catalyst nanoparticle during catalysis is a non-equilibrium system that requires high spatial and temporal resolution measurements to elucidate underlying mechanisms. State-of-the-art environmental transmission electron microscopy (ETEM) enables in situ measurements of the dynamic changes occurring under reaction conditions [1,2]. These changes usually take place rapidly at the nanometer scale. In order to record them in real time, both the high image resolution and frame rates are needed. Recently, direct electron detection cameras, have enabled us to record atomic resolution images with ms time resolution but result in producing large video data sets (gigabytes per second). To follow the atomic-level changes occurring under reaction conditions over time, the structure and phase of the nanostructure under observation must be analyzed for each individual frame. It is laborious to analyze such large videos frame by frame. An automated image processing scheme (AIPS) is therefore desirable to increase the speed and reliability of the analysis. There are two major inherent problems for such an automated structural analysis: (a) individual frames are noisy due to the short frame acquisition time and (b) the sample drifts during video recording periods that can range from seconds to minutes. We have developed an automatic method to mitigate these problems using a combination of publicly available and NIST-developed algorithms to obtain structural information from the images extracted from videos. Here we present the kinetics of phase transformation in catalyst nanoparticles under non-equilibrium reactive environment.

We use an ETEM to record atomic resolution videos of catalyst nano particles under reaction conditions. The images are analyzed using fast Fourier transform (FFT) and image simulation to identify and confirm the structures of different phases present. Individual frames extracted from the videos are filtered and drift corrected, using publically available programs such as ImageJ [3], to obtain a stack of images for automated image processing scheme (AIPS). Atomic coordinates are obtained using template matching followed calculating centroid of each atomic column. Structure of phases present was then identified from the nearest neighbor distances to measure the reaction kinetics.

Carbide phase has been reported to form during the nucleation and growth of carbon nanotubes [4,5]. Here we show that the formation and decomposition of metal carbide is an intermediate step for the formation carbon-carbon bonds, i.e. nucleation of single wall carbon nanotube (SWCNT). Figure 1a shows an atomic resolution image of a catalyst nanoparticle during a SWCNT growth at $650{ }^{\circ} \mathrm{C}$ in 0.01 $\mathrm{Pa} \mathrm{C}_{2} \mathrm{H}_{2}$ gas pressure. Measured inter-atomic distances, i.e. nearest neighbor distances (Figure 1b) were used to identify the areas occupied by $\mathrm{Co}$ and $\mathrm{CoC}_{2}$ at any given time (Figure 1c). Figure 2 (left) shows a series of images extracted from the videos at different time showing the fluctuation in the two regions with time. We find that fluctuations in the measured carbon content (Figure 2, right) for either of the reactions is aperiodic and in anti-sync, i.e. SWCNT growth is negligible as the carbide region in the particle increase. On the other hand, the tube grows as the carbide region decrease. Such precise (0.07 $\mathrm{pm}$ ) measurement of interatomic distances can be used not only to measure phase transformation rates in 
a catalyst nanoparticle during the growth of SWCNT but also for other reactions such as reduction of iron oxide nanowires. Videos recorded using two different cameras have been analyzed. Details of the image processing scheme and its application will be presented.

\section{References:}

[1] R. Sharma, J. Mater. Res. 20 (2005) 1695.

[2] Sharma and Crozier in F Author in “Transmission Electron microscopy for nanotechnology", ed. Nan Yao \& Z.L Wang, (Springer Verlag and Tsinghua University press) p.531.

[3] https://imagej.nih.gov/ij/

[4] Yoshida et al, Nano Lett. 8 (2008) 2082

[5] Picher et al. Nano Lett. 14 (2014) 6104.
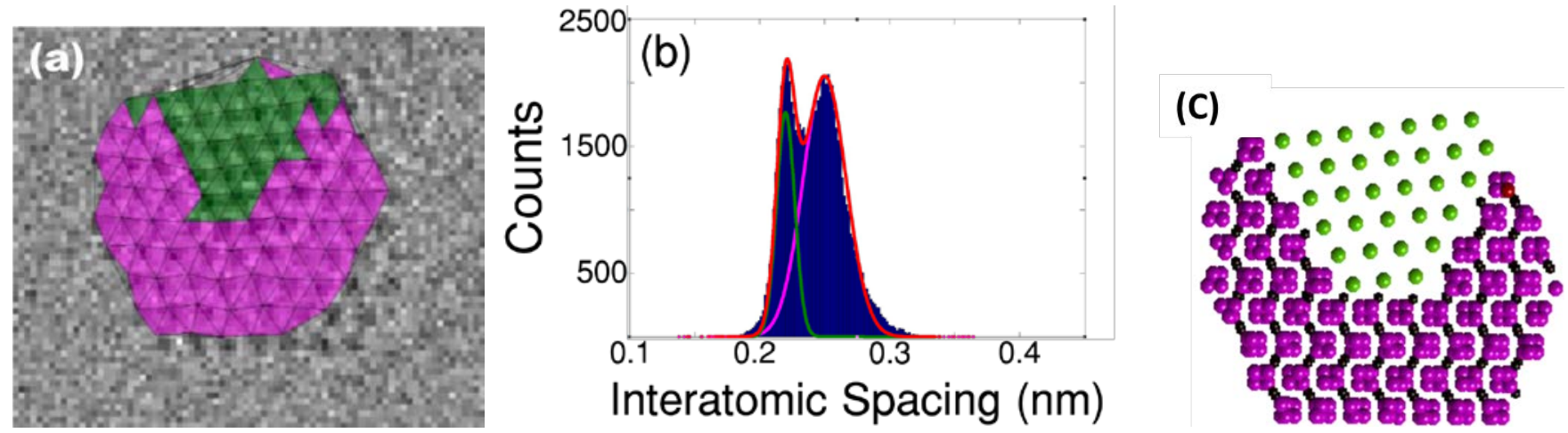

Figure 1. (a) Filtered high resolution image extracted from a video showing the existence of two phases, green and pink in a nanoparticle. (b) measured nearest neighbor distances are used to identify the green and pink regions as Co metal and Cobalt carbide structures, respectively. (a) Atomic model showing the relationship between the structures that was used for image simulation (not shown here).
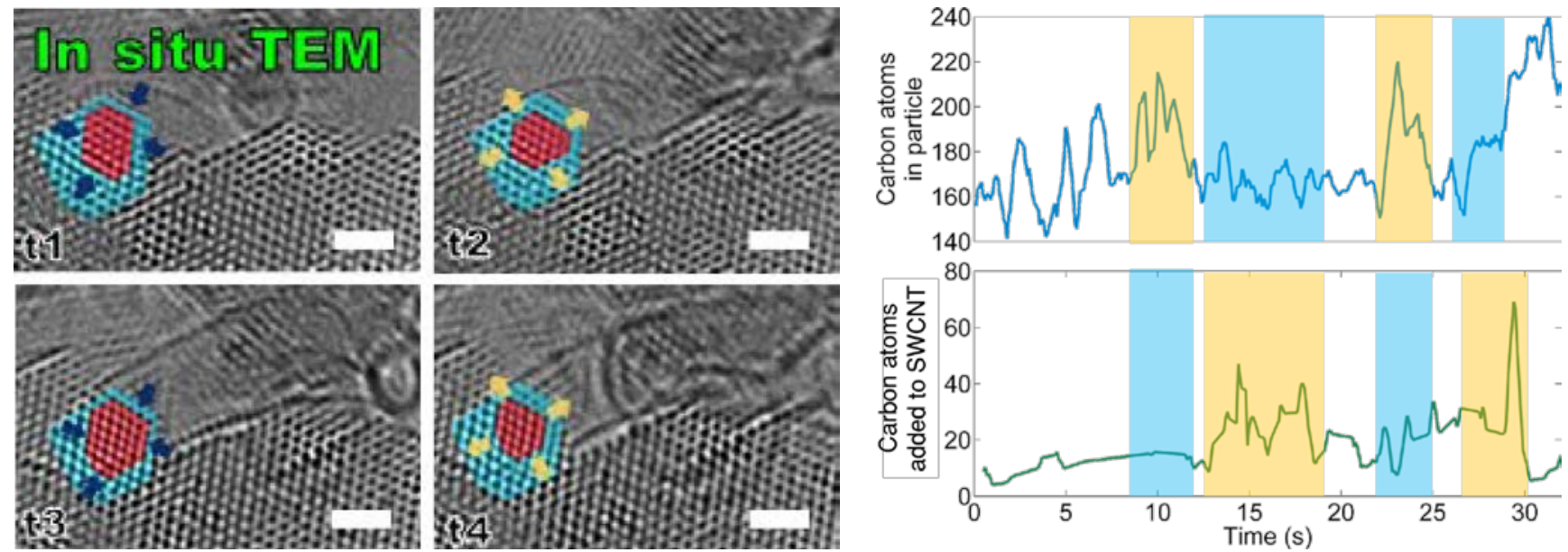

Figure 2. Sequence of 'unprocessed' time resolved images (left) showing the fluctuation in the area occupied by $\mathrm{Co}$ and $\mathrm{CoC}_{2}$ structures with time. The measured areas were used to calculate the fluctuation in the number of carbon atoms with time in the particle (top right) and compared with number of carbon atoms added to SWCNT during growth (bottom right). Areas shaded in blue and yellow in the graph show the increase in carbon content in carbide and SWCNT, respectively. 


\section{Physical Measurement Laboratory Work of researchers at professional conferences as reported in Fiscal Year 2017}




\title{
A Simple Model for the Prediction of AC-DC Difference of Multi- Junction Thermal Converters
}

\author{
Stefan Cular and Thomas E. Lipe \\ Quantum Measurement Division \\ National Institute of Standards and Technology \\ Gaithersburg, MD 20899-8172 USA \\ stefan.cular@nist.gov
}

\begin{abstract}
This paper describes a simple model for planar multi-junction thermal converters based on physical dimensions and properties of the converter chip and all wire bonds. The model was used to explain extremely low $(<700 \mu \mathrm{V} / \mathrm{V})$ AC-DC differences at $100 \mathrm{MHz}$. Two designs of multi-junction thermal converters (MJTC) were tested against the model and the results are presented.
\end{abstract}

Index Terms - AC-DC Difference, Planar Multi-junction Thermal Voltage Converters, MJTC, SPICE model.

\section{INTRODUCTION}

$\mathrm{AC}$ voltage is a unique measurement that requires a standard to compare the energy from an AC voltage to that of a DC voltage. Several notable designs of voltage converters have been used with ever decreasing AC-DC differences and uncertainties. Today, it is possible to know AC voltage to within $16 \mu \mathrm{V} / \mathrm{V}$ at $1 \mathrm{MHz}$; however, as the frequency increases to $100 \mathrm{MHz}$, the expanded uncertainty $(k=2)$ in the measurements increases to $0.18 \%$. There are many causes for the increasing uncertainty with increasing frequency that were previously accounted for through various physical models [14]. While all of these models focused on explaining the results, available literature does little to link physical design parameters to electrical models in a fashion that can readily be used to revise designs.

LTSPICE $^{1}$ is a common electrical engineering tool that permits quick simulations of electrical circuits. The advantages of using this tool are quick computations and ease of adding additional components such as capacitance for a bifilar resistor versus a straight geometry.

\section{MUlti-JunCtion THERMAL CONVERTER DESIGNS}

The premise for this work was based on knowing the physical dimensions and general layout of the multi-junction thermal voltage converter (MJTC) chip. Based on these, estimates for the resistance, capacitance and inductance were created using simple models and approximations.

The first design, referred to as coaxial, consists of a resistive element that is a straight line on the substrate. The signal and ground connections for the device are on opposite ends of the device. The second design, referred to as bifilar, consists of a U-shaped resistive heating element, with signal and ground connections at the same reference plane. Both general designs have been in use at the National Institute of Standards and Technology (NIST) for a number of years and detailed descriptions of the designs and AC-DC differences from $10 \mathrm{~Hz}$ to $100 \mathrm{MHz}$ are readily available in the literature $[5,6]$.

\section{COMPONENT CONTRIBUTIONS}

The model was based on an impedance for each of the various sections as shown in Fig. 1. For the practical purposes of this work, the typical coaxial connector was not included in the model, as the dimensions and materials are not readily changed; however, the authors acknowledge the connector does contribute to the AC-DC difference as described by Huang, et al. [2]. We identified the following seven conductors as contributors to the AC-DC difference: Wire from connector to carrier, Carrier, Wire bond from carrier to MJTC chip, MJTC

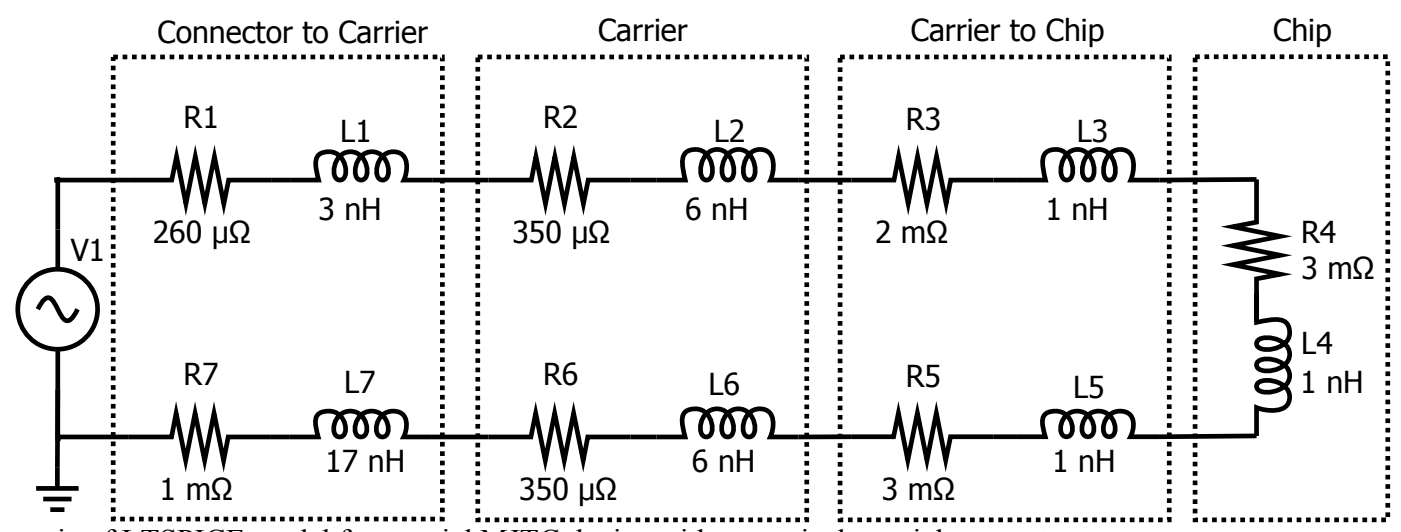

Fig. 1. Schematic of LTSPICE model for coaxial MJTC device without typical coaxial connector. 
chip, Wire bond from MJTC chip to carrier, Carrier, and Wire from carrier to connector. For each of the conductors, an inductance and a resistance were calculated using common material properties and measured dimensions. These values were then entered into the LTSPICE model as shown in Fig. 1.

\section{RESULTS}

The model was run from DC to $100 \mathrm{MHz}$, and the resulting ACDC difference values were calculated and compared with two actual devices that were built for each of the designs. Analysis of the data shown in Fig. 2 yields agreement within the expected measurement uncertainties highlighted in Table 1.

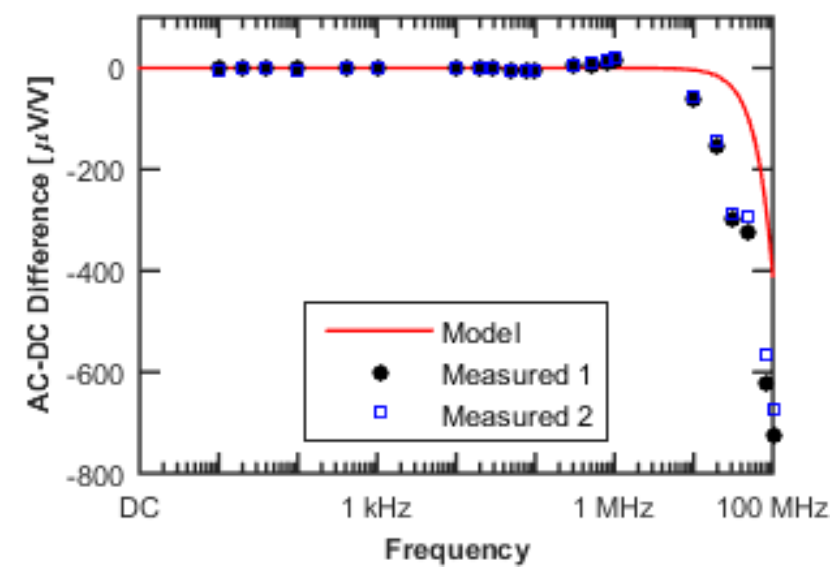

Fig. 2. AC-DC difference of coaxial MJTC as measured on two devices of the same configuration with comparison to LTSPICE model. Error bars are omitted from this plot for clarity.

Further testing of the model was conducted through implementation of a second design referred to as bifilar. In this design the resistive element of the MJTC forms a U-shape, that adds capacitance to all elements as the signal and ground paths are parallel. The results for the second design and comparison to two actual devices are shown in Fig. 3.

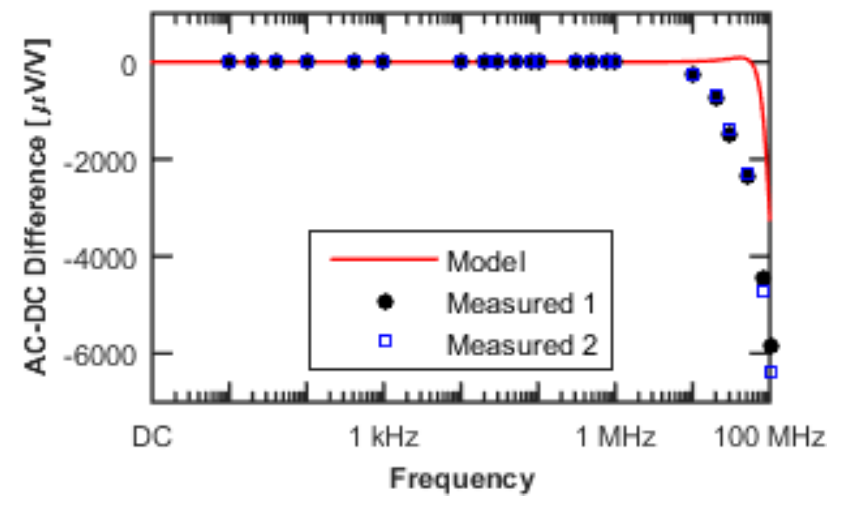

Fig. 3. AC-DC difference of bifilar MJTC as measured on two devices of the same configuration with comparison to LTSPICE model. Error bars are omitted from this plot for clarity.
Table 1. AC-DC difference measurement uncertainties for key frequencies.

\begin{tabular}{l|c|c|c|c} 
Frequency & $1 \mathrm{kHz}$ & $1 \mathrm{MHz}$ & $10 \mathrm{MHz}$ & $100 \mathrm{MHz}$ \\
\hline $\begin{array}{l}\text { Expanded } \\
\begin{array}{l}\text { Uncertainty }(k=2) \\
{[\mu \mathrm{V} / \mathrm{V}]}\end{array}\end{array}$ & 1.7 & 18 & 150 & 1800 \\
& & & &
\end{tabular}

\section{CONCLUSION}

A simple model for estimating the AC-DC difference of MJTC's from physical design parameters was created and compared to actual devices. The results indicate overall general agreement with experimental data for both coaxial and bifilar designed devices. The gained insight from the model can be used to further develop MJTC AC-DC difference standards.

\section{REFERENCES}

[1] J. R. Kinard and T.-X. Cai, "Determination of AC-DC Difference in the 0.1-100 MHz Frequency Range," IEEE Transactions on Instrumentation and Measurement, vol. 38, pp. 360-367, Apr 1989.

[2] D.-X. Huang, J. R. Kinard, and G. Rebuldela, "RF-DC Difference of Thermal Voltage Converters Arising from Input Connectors," IEEE Transactions on Instrumentation and Measurement, vol. 40, pp. 360$365,1991$.

[3] L. Scarioni, M. Klonz, D. Janik, H. Laiz, and M. Kampik, "High-Frequency Thin-Film Multijunction Thermal Converter on a Quartz Crystal Chip " IEEE Transactions on Instrumentation and Measurement, vol. 52, pp. 345-349, 2003.

[4] M. Halawa and N. Al-Rashid, "Performance of the Single Junction Thermal Voltage Converter at 1 MHz via Equivalent Circuit Simulation " The International Journal of Metrology, vol. April-June, pp. 40-45, 2009.

[5] T. E. Lipe, "50 ohm Multijunction Thermal Converters on Fused Silica Substrates," in Precision Electromagnetic Measurements (CPEM 2014), 2014 Conference on, Rio de Janeiro, 2014, pp. 44-45.

[6] T. E. Lipe, J. R. Kinard, Y. Tang, and J. E. Sims, "New technologies to improve AC-DC difference measurements at NIST," NCSLI Measure, pp. 52-58, 2014.

${ }^{1}$ Certain commercial software is identified in this paper in order to specify the experimental procedure adequately. Such identification is not intended to imply recommendation or endorsement by the National Institute of Standards and Technology, nor is it intended to imply that the software identified is necessarily the best available for the purpose. 


\title{
VISION EXPERIMENT II ON WHITE LIGHT CHROMATICITY FOR LIGHTING
}

\author{
Yoshi Ohno', Semin $\mathrm{Oh}^{2}$ \\ ${ }^{1}$ National Institute of Standards and Technology, Gaithersburg, Maryland, USA \\ ${ }^{2}$ Ulsan National Institute of Science and Technology, Ulsan, South Korea \\ ohno@nist.gov
}

\begin{abstract}
In response to a published discussion (Wei \& Houser, 2015) on the 2013 NIST experiment on white light chromaticity for lighting (Ohno \& Fein, 2014), the second experiment was conducted at NIST to determine the white light chromaticity perceived most natural in a simulated interior room environment using the NIST Spectrally Tunable Lighting Facility. The 2015 experiment was conducted with 21 subjects, for shift of Duv (chromaticity shifts above and below the Planckian locus), using the same procedures as the 2013 experiment but minimizing the changes in gamut area in each pair of lights compared so that only the effects of chromaticity would be evaluated. The results showed that the lights with $D_{\mathrm{uv}} \approx-0.015$, nearly the same as the results in 2013, appeared the most natural at all correlated color temperatures.
\end{abstract}

Keywords: colour rendering, white light, chromaticity, light source, Planckian locus, preference

\section{Introduction}

Traditionally the white chromaticity points of light sources for general lighting have been designed to be around the Planckian locus, as specified by the standards for fluorescent lamps (e.g., IEC, 1997, ANSI, 2001) as well as by the recent standard for solid state lighting products (ANSI, 2015). These specifications assumed that Planckian radiation for lower correlated color temperature (CCT) and daylight for higher CCT ranges would be natural white light, but these were not based on visual experimental data. In this paper, chromaticity shifts away from the Planckian locus (yellowish/pinkish shift) is expressed by Duv (Symbol: $D_{\mathrm{uv}}$ ), which is defined as the shortest distance from the chromaticity of the light to the Planckian locus on the CIE $\left(u^{\prime}, 2 / 3 v^{\prime}\right)$ coordinates, with plus sign for above and minus sign below the Planckian locus, as defined by (ANSI, 2015) and also described in a recent article (Ohno, 2013).

A recent study on a vision experiment (Rea \& Freyssinier, 2013) reported that perceived neutral white points are $D_{\mathrm{uv}} \approx-0.01$ at $2700 \mathrm{~K}$ to $3500 \mathrm{~K}, D_{\mathrm{uv}} \approx 0.00$ at $4000 \mathrm{~K}$, and $D_{\mathrm{uv}} \approx 0.005$ at $6500 \mathrm{~K}$ (though the report did not use Duv). This experiment was done with a lighting booth with white inner walls and no objects inside. Another experimental study (Dikel et al, 2014) showed that subjects viewing an office miniature set preferred white light illumination of Duv around -0.014 on average. In 2013, vision experiments were conducted at NIST for white points perceived most natural in a simulated real-size interior room environment using the NIST Spectrally Tunable Lighting Facility (STLF) (Miller et al 2009), with 18 subjects. The results showed that the lights with $D_{\mathrm{uv}} \approx-0.015$ on the average, much below the Planckian locus, appeared most natural (Ohno and Fein, 2014). This experiment used broadband spectra, and the gamut area for saturated object colors naturally increased as the Duv shifted to the negative direction.

Following this research, a discussion (Wei \& Houser, 2015) was published that the results of the NIST 2013 experiment might have been caused by the changes of gamut area and other color quality characteristics, rather than change of chromaticity. It was considered to be an important point to clarify. To address this question, another series of vision experiment was conducted at NIST in 2015 so that the effect of only chromaticity could be evaluated, with other effects such as color gamut or chroma saturation minimized.

\section{Method}

The experiment was conducted using the same facility (NIST STLF) and the same procedures as those used in 2013, with only light spectra modified. See (Ohno \& Fein, 2014) and (Miller et al 2009) for the details of the NIST STLF. 
Fig. 1 shows the curves of Color Rendering Index (CRI) $R_{\mathrm{a}}$, Color Quality Scale (CQS) $Q_{\mathrm{a}}, Q_{\mathrm{f}}$, and $Q_{g}$ (Davis \& Ohno, 2010), for the spectra used in the NIST 2013 experiment. $Q_{g}$ is the relative gamut area of the 15 test samples of CQS on $\left(a^{*}, b^{*}\right)$ coordinates of CIELAB color space. Fig. 2 shows a few examples of the gamut area plots at $3500 \mathrm{~K}$. As shown in these figures, the relative gamut area increases as the Duv changes in the negative direction, which is the reason for the discussion by Wei and Houser (2015). Increase of the gamut area at negative Duv is inevitable because the green region of the spectra must decrease to bring the chromaticity below the Planckian locus, which in turn increases the luminance of colored objects (compared to that with the reference illuminant at the same illuminance). This effect cannot be removed over the large range of Duv (-0.03 to 0.02). However, the 2013 experiment consisted of a number of comparisons of light pairs of small Duv differences, and it was possible with NIST STLF to adjust the light pairs so that the gamut area and chroma differences in each pair were made nearly equal.
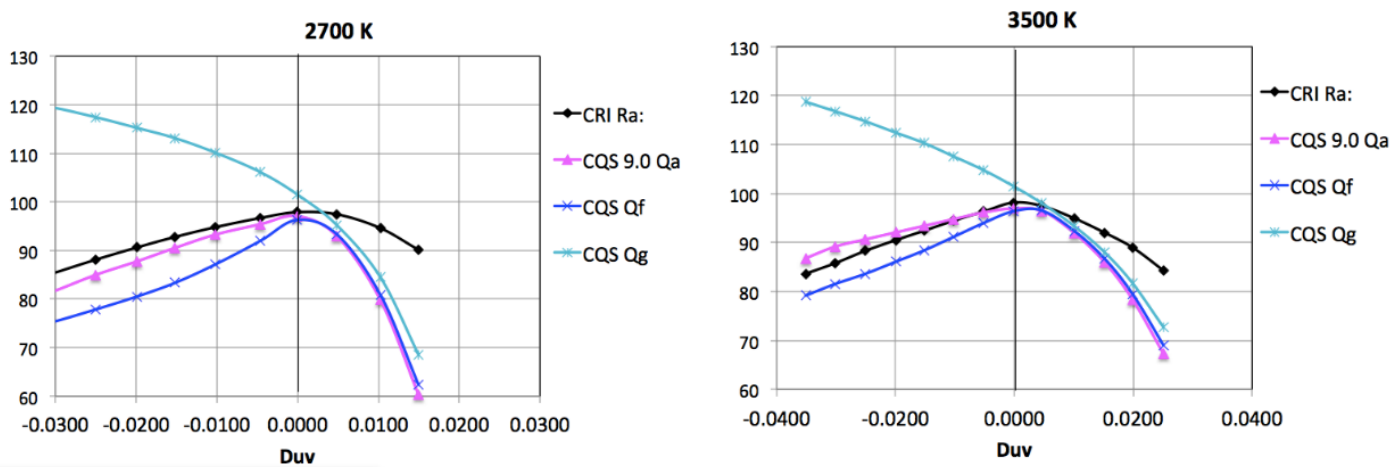

$4500 \mathrm{~K}$
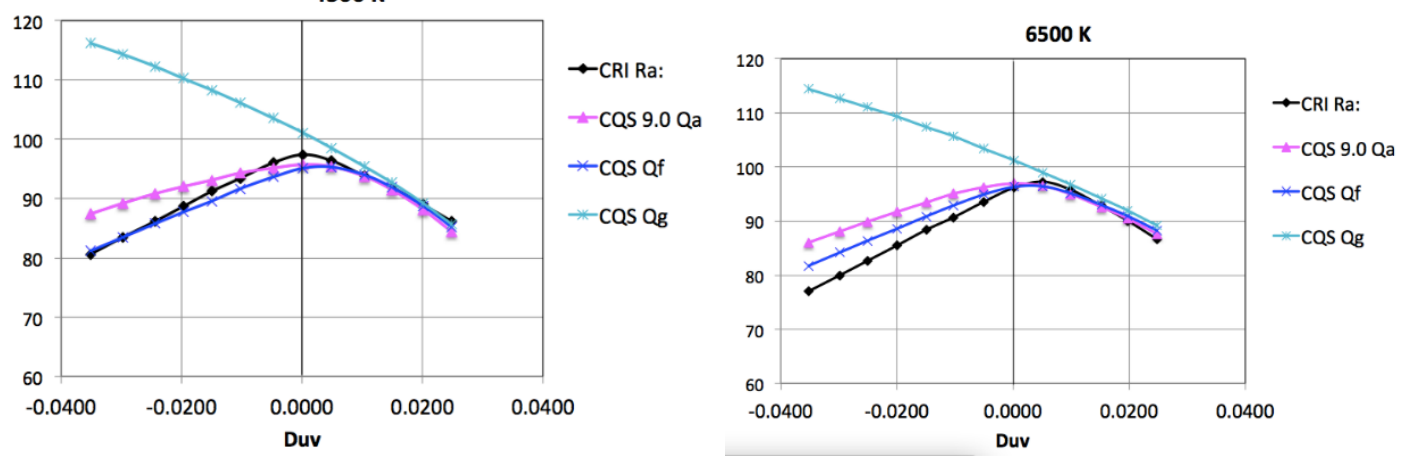

Figure $1-C R I$ and CQS color quality values of the lights used in the 2013 experiment.

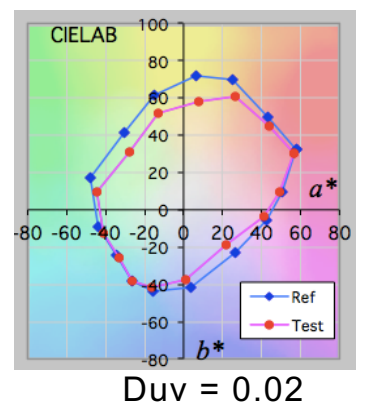

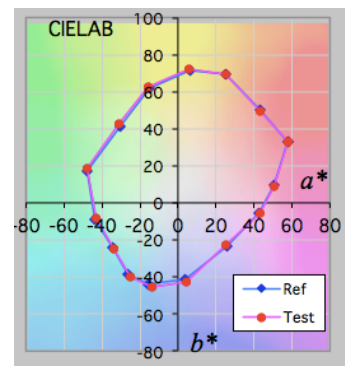

Duv $=0.000$

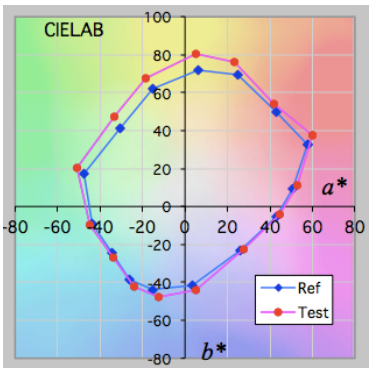

Duv $=-0.03$

Figure 2 - Gamut area plots of the CQS 15 samples of the lights at $3500 \mathrm{~K}$ used in the 2013 experiment. The blue curve is for the reference illuminant (Planckian) and the red curve is for the test light source.

For the 2015 experiment, the broadband spectra, similar to those used in 2013, were first prepared for each Duv point, and they were slightly modified and adjusted so that the gamut area $Q_{g}$ and the chroma difference $\Delta C^{*}$ ab for the red and green CQS samples would be as equal 
as possible in each pair of light. Figure 3 shows the plots of $Q_{g}$, and Figure 4 shows the chroma difference $\Delta C_{\mathrm{ab}}^{*}$ of the red and green test samples of CQS (between the test light and the reference illuminant). The thin solid lines in Figs. 3 and 4 are the same plots of the 2013 experiment data. The intention in 2015 experiment was to remove the slopes and make them as flat as possible between the lights in each pair. As it is difficult to make both of these completely flat, a higher priority was placed in minimizing the chroma differences in the red and green test samples, as these colors are most critical in perception of color quality.
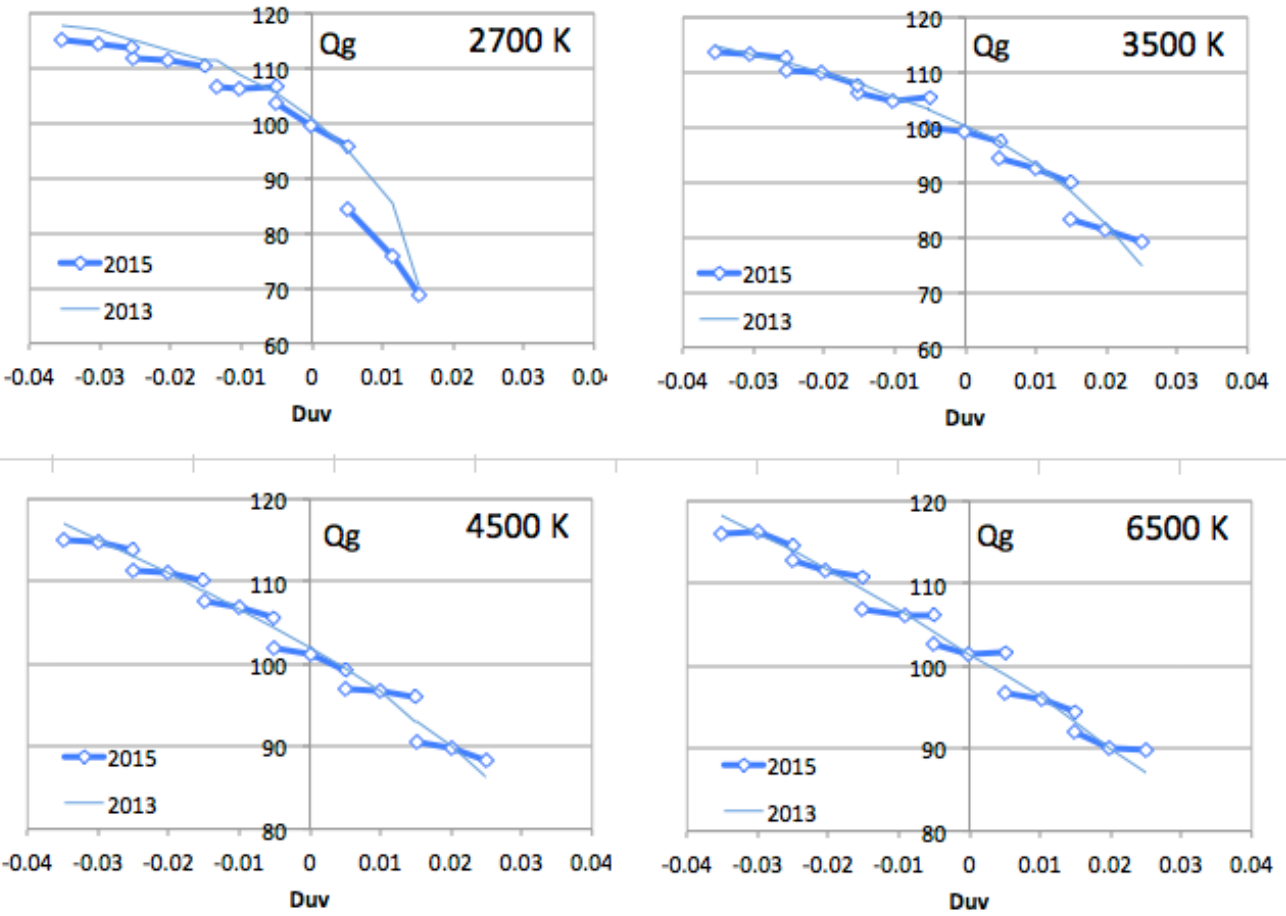

Figure 3 - Plots of the relative gamut area $Q_{g}$ in the 2015 experiment.

Thus, the remaining differences in the gamut area (Fig. 3) are mostly in the yellow-blue direction, which is much less distinctive in perception of color quality. For example, the average differences in $Q_{g}$ between two lights in each pair for the six Duv conditions at $4500 \mathrm{~K}$ was reduced from $\Delta C^{*}{ }_{a b}=5.1$ in 2013 to $\Delta C^{*}{ }_{a b}=1.5$ in 2015 . The average chroma differences $\Delta C^{*}{ }_{a b}$ for red and green samples between the lights in each pair were reduced from 1.4 (red) and 2.2 (green) in 2013 to 0.3 (for red and green) in 2015, at $4500 \mathrm{~K}$.

The resulting spectra of the lights used in the 2015 experiments are shown in Fig. 5. Each curve corresponds to each point on the graphs in Fig. 3 or Fig. 4.

21 subjects having normal color vision from 19 to 68 years of age participated in the experiment. The subjects were the summer students and employees at NIST, who were not experts on color or lighting. 

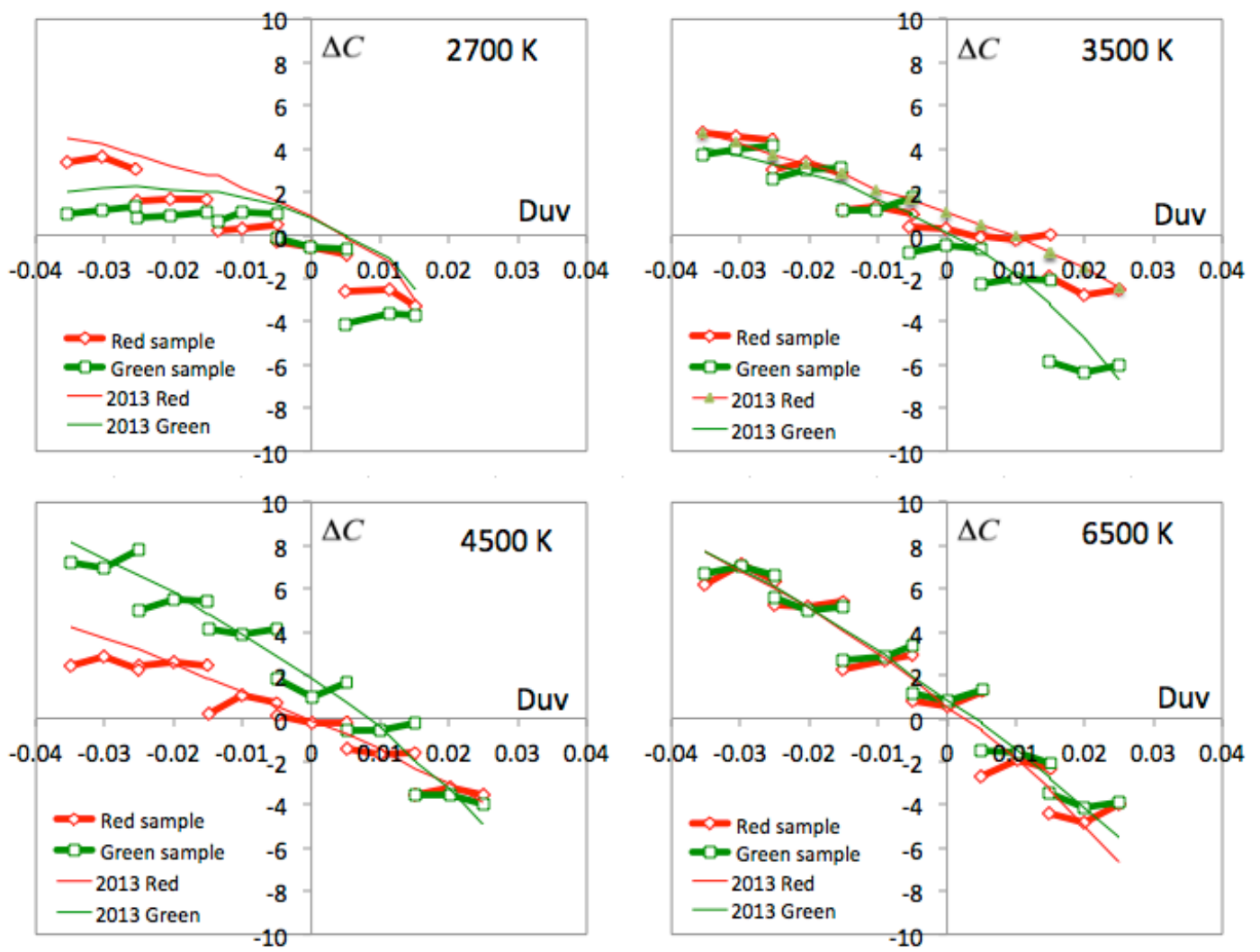

Figure 4 - Plots of the chroma differences $\Delta C^{*}$ ab of the red and green test samples of CQS in the 2015 experiment.
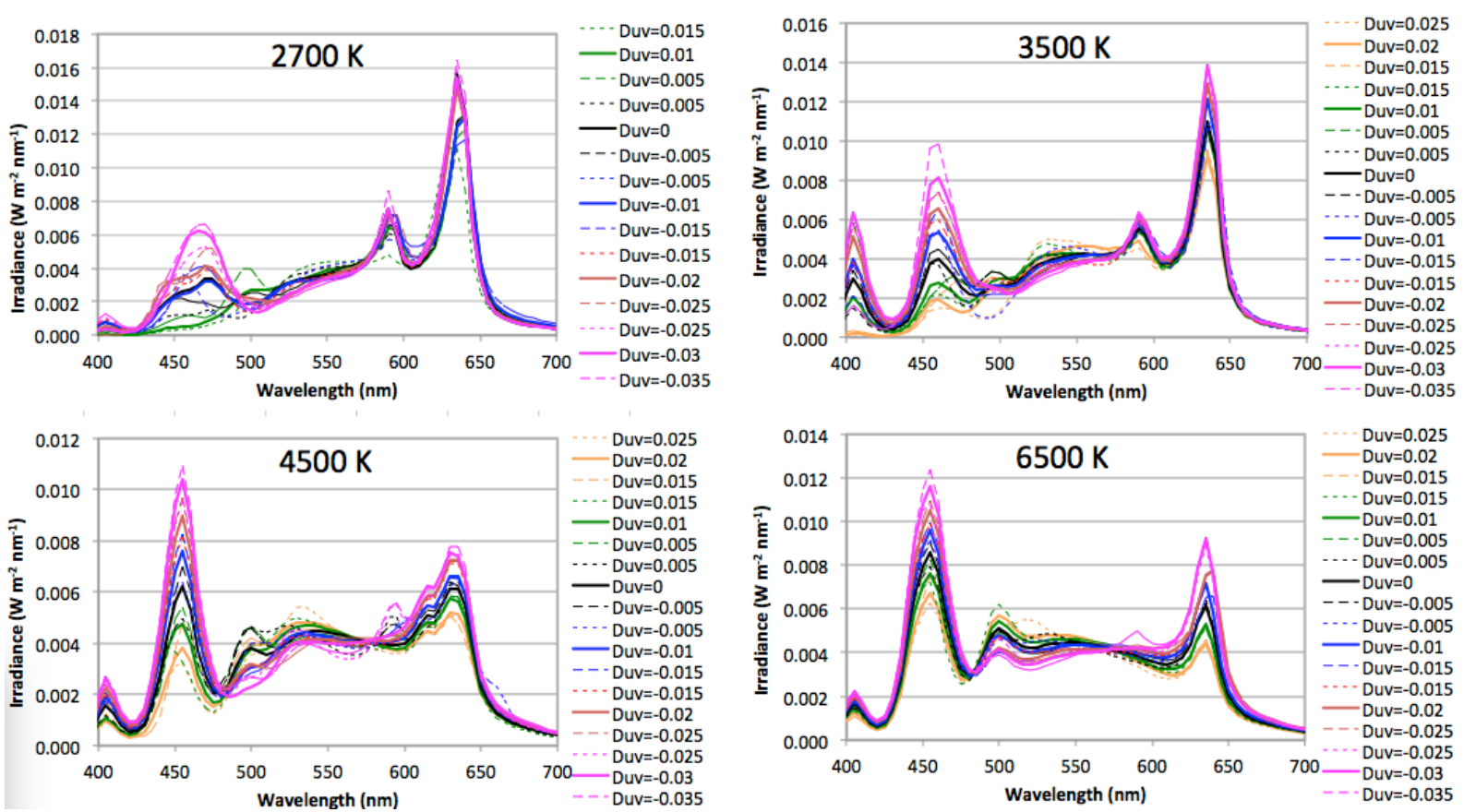

Figure 5 - Spectra of the lights used in the 2015 experiment. 


\section{Experimental Procedures}

The experiments were conducted generally at six different Duv levels $(0.02,0.01,0,-0.01$, $0.02,-0.03)$ and at four CCTs $(2700 \mathrm{~K}, 3500 \mathrm{~K}, 4500 \mathrm{~K}, 6500 \mathrm{~K})$ plus $2200 \mathrm{~K}$, at the illuminance level of $300 \mathrm{~lx}$. These experimental points are depicted in Fig. 6 (black circles). The highest Duv point at $2200 \mathrm{~K}$ had to be Duv $=0$, and for $2700 \mathrm{~K}$, Duv=0.01, because further points would be too close to the spectrum locus and nearly monochromatic. $2200 \mathrm{~K}$ was added in this experiment because the CCT categories of $2500 \mathrm{~K}$ and $2200 \mathrm{~K}$ have been added in the latest version of the ANSI specification (ANSI, 2015) and data at such very low CCTs were desired. However, this was decided at a later stage, this condition was run separate from the other four CCTs, using only six subjects, and no adjustment was made for gamut and chroma saturation (it was very difficult), thus the results for $2200 \mathrm{~K}$ was to connect to the 2013 results. The 2200 K experiment was done with 2700 K (2013 setting) again to verify the consistency with the 2013 experiment.

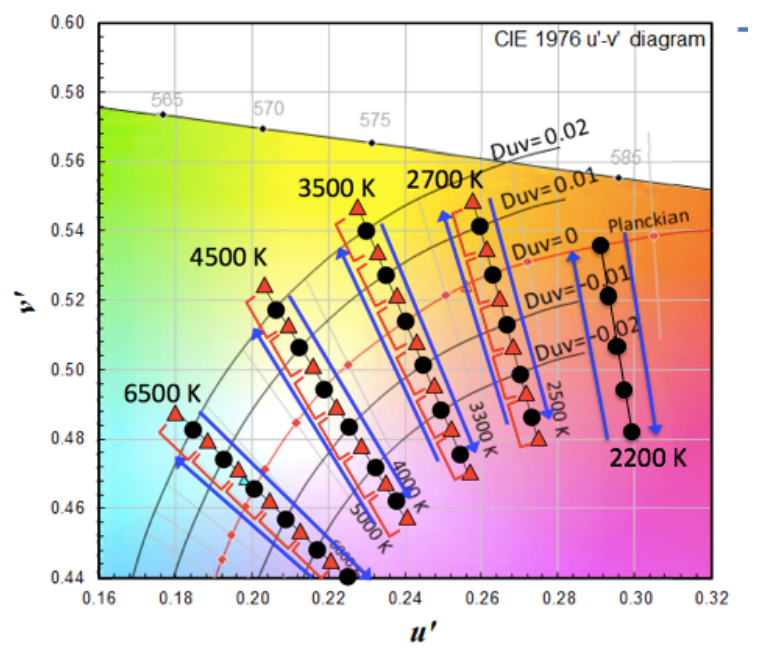
Figure 6 - The chromticity points for the experiment (black circules) and pairs of light
for visual comparison (red triangules)

Figure 7 shows the room settings of the NIST STLF. The subject sat on a couch placed at the open side of the cubicle so that he/she viewed the entire room, and was completely immersed in the lighting environment, and his/her full view was adapted to the illumination. For the subjects to be able to judge naturalness of light, common real objects in daily life were placed on the table. They were two dishes of real fruits and vegetables (red apple, yellowish apple, orange, green pepper, lettuce, tomato, banana, strawberries, and grapes). These were the same set of objects as in the 2013 experiment. The fruits and vegetables were replaced at a few days' intervals to keep them fresh. There was a mirror in front of the subject, and he/she could look at their face skin tone in the mirror, as well as their hands skin tone. Along the wall of the cubicle, there was a bookshelf with some books, artificial flowers, and two paintings hung on the side walls. The subjects were asked to make overall judgement of viewing the whole room, fruits and vegetables, and their skin tone. 

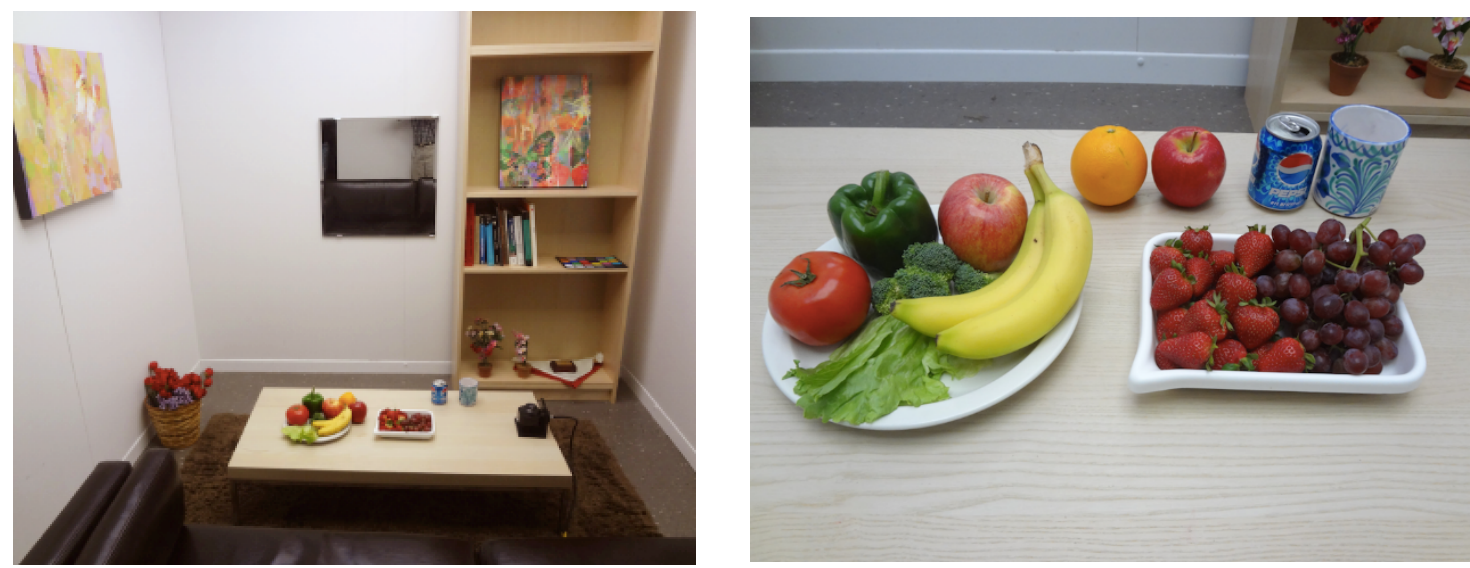

Figure 7 - The experimental settings of STLF - the room setting (left) and fruits and vegetables on the table (right).

The experimental run was made in the order from high Duv to low Duv (forward direction) and low Duv to high Duv (backward direction) at each CCT condition. The subject was adapted to the light for 5 min at the first Duv point in a CCT (either end of the Duv points) and adapted for 1 min at each subsequent Duv point. See (Ohno \& Fein 2014) explaining this adaptation time. After adaptation at each Duv point, a pair of lights (red triangles in Fig. 6), slightly above and below $( \pm 0.005)$ the adaptation point Duv (black circles in Fig. 6), were presented alternately, and subject chose which light looked "more natural". When starting from $D_{\mathrm{uv}}=0.02$, for example, a subject typically chose the light with lower Duv shift (toward Planckian) in the pair, which indicates that $D_{\mathrm{uv}}=0.02$ was too yellowish. At $D_{\mathrm{uv}}=-0.03$, the subject typically chose light with higher Duv shift (toward Planckian), which means $D_{\mathrm{uv}}=-0.03$ was too pinkish. As these comparisons were made from the high Duv end to the low Duv end, or vice versa, subject's response changes over at some point of Duv, and this crossover point of Duv is considered as the most natural point. The crossover point was determined at each run for each subject at each CCT condition. The results for the forward and the backward direction were averaged for each subject and each CCT to remove any effects of imperfect chromatic adaptation or sequential effect. In 2013, the whole experiment depicted in Fig. 4 repeated, but in 2015 it was not repeated due to the time limitation. The reproducibility of whole experiment in 2013 was checked that the results of repeated sessions were very consistent, and it was concluded that one session was sufficient.

The experiments were conducted in June to August 2015. Each run for six Duv points for one CCT took 12 to 15 minutes, so about 1 hour for four CCTs, and total about 2 hours for each subject. The order of CCT and the order of forward/backward was randomly set, and the same order was used for all subjects.

The chromaticities of these points were initially adjusted to be within \pm 0.0003 from the intended chromaticity, and were maintained within \pm 0.0006 (relative to Duv measured at 0.000 ) throughout the experiment. The illuminance of all lights were set to $300 \mathrm{Ix} \pm 1 \%$. The CCT, Duv, and illuminance was monitored before each experiment with each subject to verify that the colorimetric values are stable within the variations as above.

The color quantities of the lights were measured on the center of the table in the cubicle, using an array type spectroradiometer with a small integrating sphere input for cosine response, calibrated with a NIST spectral irradiance standard scale (NIST, 2011). The spectroradiometer measured spectra and illuminance on the table from the $2 \pi$ solid angle including light from the entire room including reflections from the walls and other objects as well as from the light source itself. The estimated expanded uncertainties $(k=2)$ of measurements varied depending on spectra, but in all cases, they were within 0.0012 in $u^{\prime}, 0.0011$ in v', 0.0009 in Duv, $24 \mathrm{~K}$ in CCT at $2700 \mathrm{~K}$ and $92 \mathrm{~K}$ at $6500 \mathrm{~K}$. The expanded uncertainty in the relative chromaticity measurement between each pair of light was 0.0002 in $u^{\prime}$ and 'v', which is the typical repeatability of the instrument. The expanded uncertainty of illuminance of the spectroradiometer is estimated to be $3 \%(k=2)$ for directional incident light, and its uncertainty 
for relative measurement was $0.1 \%$, which is the typical repeatability of the instrument. Also, when the spectrum is changed on STLF, the spectrum and color are switched instantly and stable immediately so that sequential comparison of lights is possible.

The spatial uniformity of color and illuminance in the room was evaluated using the broadband spectra shown in Fig. 5 at Duv $=0$ setting at all four CCTs. Figure 8 shows the layout of the STLF room and the 28 points measured on the table, floor, and the walls including two positions on the mirror. Measurements were made with a tristimulus colorimeter for chromaticity and illuminance. The uniformity of chromaticity over the table top at all CCTs was within \pm 0.0013 in $\left(u^{\prime}, v^{\prime}\right)$ and within \pm 0.0002 in Duv. The chromaticity of all 28 points at all CCTs were within 0.0044 in $\left(u^{\prime}, v^{\prime}\right)$ and within \pm 0.0010 in Duv from the average chromaticity on the table. The uniformity of illuminance on the table was within $\pm 13 \%$, on the floor $\pm 16 \%$, and on the walls \pm $15 \%$. The absolute illuminance on the floor was about $40 \%$ lower, and about $60 \%$ lower on the wall (vertical illuminance) than the average illuminance on the table.

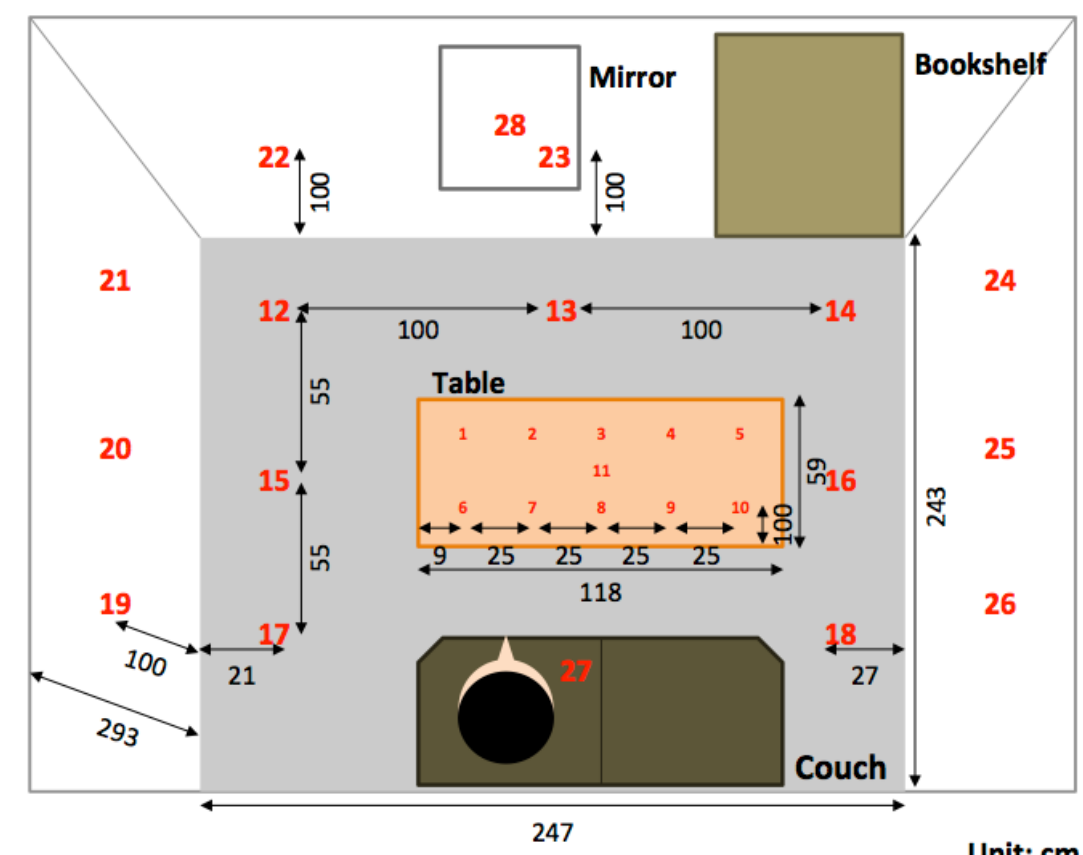

Figure 8 - STLF room layout and the points for uniformity measurements. The red fonts show measurement points.

\section{Results}

The data analysis was similar to that used in the 2013 experiment. See (Ohno \& Fein 2014) for the details. Figure 9 shows the results for the crossover points determined from the 2015 experiment for each subject. Each line in the figure is for each subject. Several straight lines around -0.03 are all at $D_{u v}=-0.03$ and actually overlapped with each other but lines are shown separately to indicate that there are several lines. Since -0.03 was the lowest Duv used this time, these subjects may have preferred even lower Duv lights.

The results for $2200 \mathrm{~K}$ are also included in this figure. Note that $2200 \mathrm{~K}$ results are only with six subjects, and the lines from $2200 \mathrm{~K}$ are connected to results at $2700 \mathrm{~K}$, which was run at the same time with $2200 \mathrm{~K}$. These six $2700 \mathrm{~K}$ points, as well as $2200 \mathrm{~K}$ lights, were not adjusted for gamut area and chroma saturation (as explained in section 3 ) and are different from other $2700 \mathrm{~K}$ results where gamut and chroma were adjusted.

The average of all subjects is plotted in the thick red line in Fig. 9. The average results of the 2015 experiment, together with the 2013 experiment, are shown in Fig. 10. The error bars are the standard deviations of the averages of all subjects. Table 1 shows these results numerically. Considering the standard deviations, the average results of the experiments in 2015 and 2013 
are practically the same. It was also shown that preference for $2200 \mathrm{~K}$ is similar to other CCTs, possibly with larger shifts, but this result is less reliable due to fewer number of subjects.

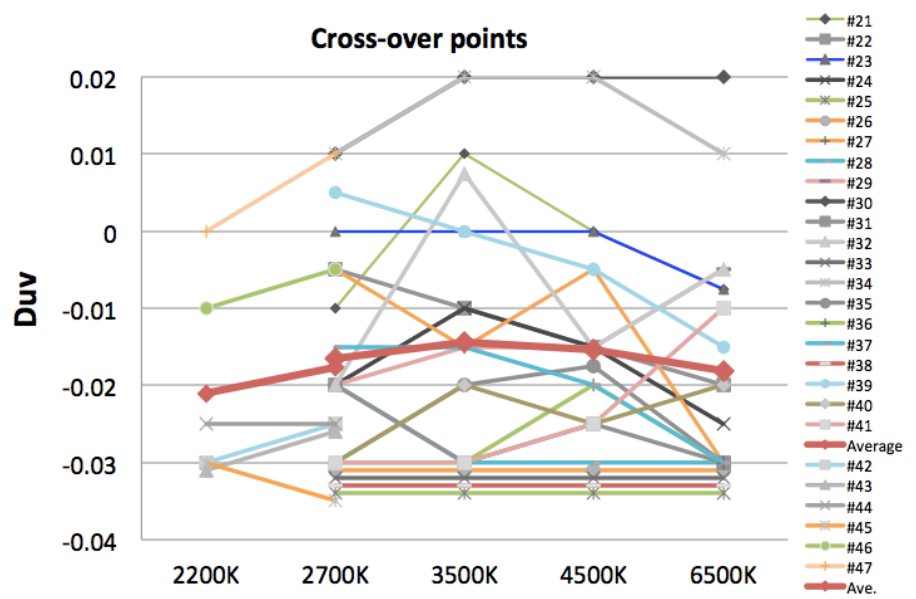

Figure 9 - The results of experiment (crossover Duv points) of individual subject.
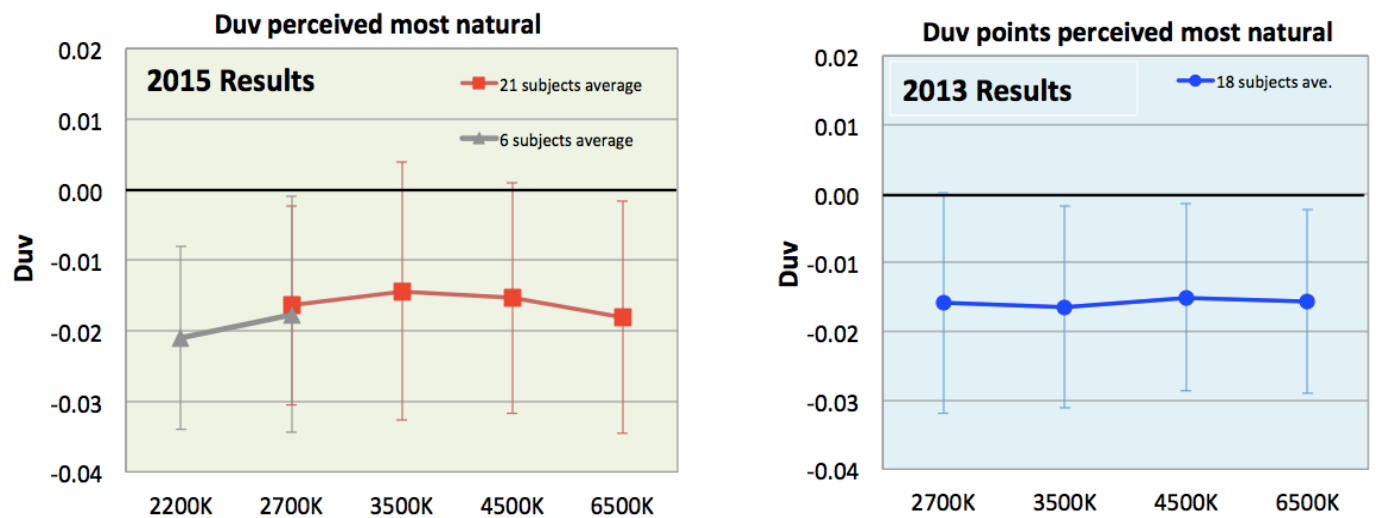

Figure 10 - The average results of the 2015 and the 2013 experiments

Table 1 - Average results of 2015 and 2013 - Duv values at $50 \%$ crossover points and standard deviations.

\begin{tabular}{|c|c|c|c|c|}
\hline \multirow[b]{2}{*}{ CCT } & \multicolumn{2}{|c|}{2015 Experiment } & \multicolumn{2}{|c|}{2013 Experiment } \\
\hline & $\begin{array}{c}\text { Duv at } 50 \% \\
\text { crossover } \\
\text { average }\end{array}$ & $\begin{array}{l}\text { Standard } \\
\text { deviation }\end{array}$ & $\begin{array}{c}\text { Duv at } 50 \% \\
\text { crossover } \\
\text { average }\end{array}$ & $\begin{array}{l}\text { Standard } \\
\text { deviation }\end{array}$ \\
\hline $2200 \mathrm{~K}^{*}$ & -0.021 & 0.013 & & \\
\hline $2700 \mathrm{~K}$ & -0.016 & 0.014 & -0.016 & 0.016 \\
\hline $3500 \mathrm{~K}$ & -0.014 & 0.018 & -0.017 & 0.015 \\
\hline $4500 \mathrm{~K}$ & -0.015 & 0.016 & -0.016 & 0.014 \\
\hline $6500 \mathrm{~K}$ & -0.018 & 0.016 & -0.016 & 0.013 \\
\hline
\end{tabular}

The results below are broken down into different conditions and different subject groups, though the number of subjects may not be sufficient for such analyses. Figure 11 shows the results. 
There are slight differences between forward and backward direction, which indicates that the adaptation time may not have been sufficient or there may have been some time sequential effects, but the differences are not significant. The differences in sex groups and age groups are also shown but it is uncertain whether these distinctions are significant due to insufficient number of subjects.
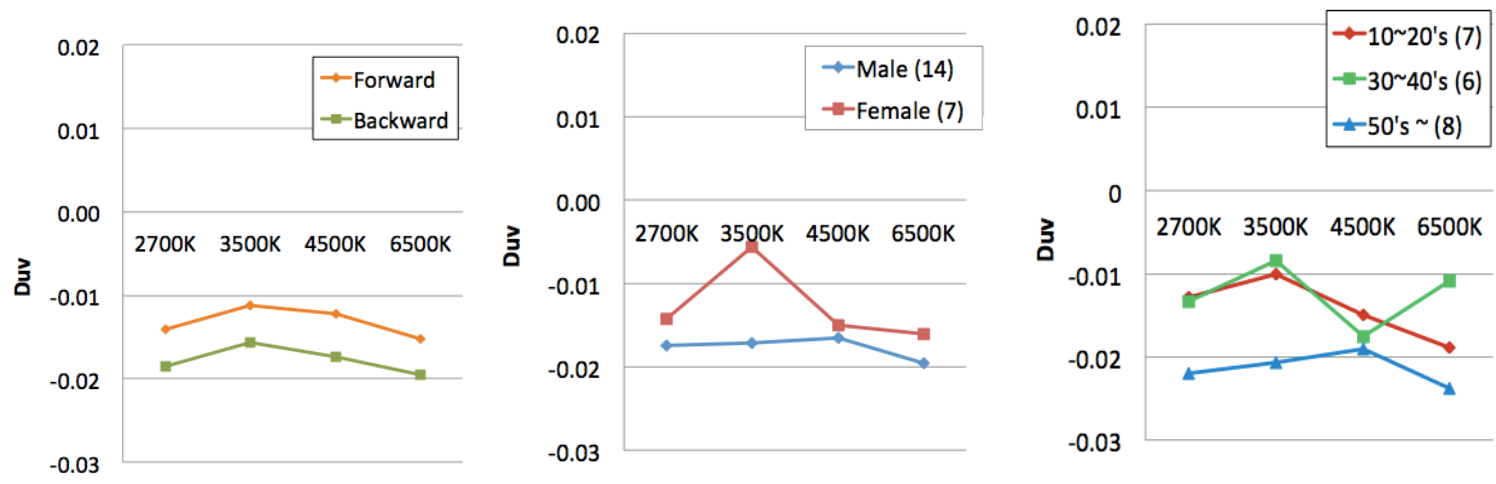

Figure 11 - Break down results for forward/backward, gender, and age groups.

In addition to the results presented above, subjects were also asked whether each presented light after adaptation was acceptable or not, before comparing the light pair. Figure 11 shows the results. All the curves make their bottom at around Duv -0.01 to -0.02 , which is consistent with the results of the preferred crossover points reported above. Also, these data show that positive Duv at low CCTs (2700 K and 2200$)$ are clearly disliked by many subjects, while this judgement is less sensitive at higher CCTs.
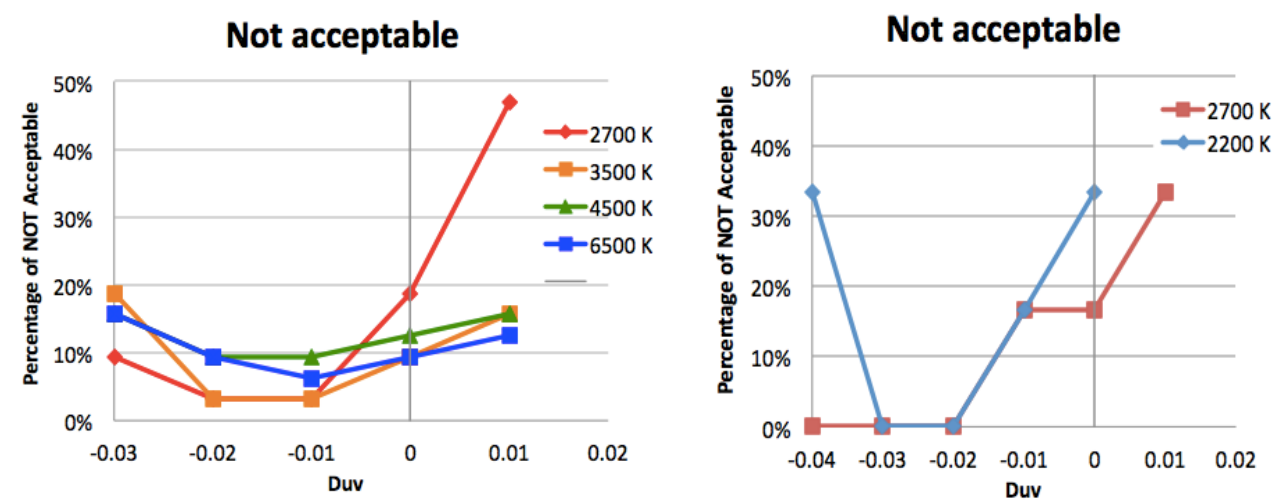

\section{Figure 11 - The percentage of subjects' response of "Not acceptable" for the light presented, after adaptation.}

\section{Conclusions}

The experiments conducted in 2015 clarified that there were no notable effects of changes in gamut area or chroma saturation in the results of the NIST 2013 experiments. It is considered that the preference for negative Duv is mainly due to the effects of light chromaticity, though the increase of gamut area may also be contributing. The two experiments at NIST verified that there is a general preference to the negative Duv lights for typical interior room environment and that the most preferred white light chromaticity, after sufficient chromatic adaptation, was found to be around Duv -0.015 and is consistent over all CCT ranges from $2200 \mathrm{~K}$ to $6500 \mathrm{~K}$. This preference, however, may be different for different applications. Also, these results are for the condition when subjects are fully adapted to each Duv point. A shift of -0.015 in Duv may be significant under the transient conditions when the occupants move from one space under 
such Duv to other spaces or vice versa. Field studies are desired on the preferred Duv levels in various real applications.

\section{References}

ANSI 2001. C78.376 Specifications for the Chromaticity of Fluorescent Lamps .ANSI, 2015. ANSI_NEMA_ANSLG, C78.377-2015 Specifications for the Chromaticity of Solid State Lighting Products.

Davis W. and Ohno, Y., 2010. Color Quality Scale," Optical Engineering, 033602, 49, 3, 033602-1 to 033602-16.

Dikel, E. E., Burns, G. J., Veitch, J. A., Mancini, S., and Newsham, G. R., 2014. Preferred Chromaticity of Color-Tunable LED Lighting, LEUKOS, 10:2, 101-115, 101-115.

IEC, 1997. IEC 60081, Double-capped fluorescent lamps - Performance specifications, Annex D.

Miller, C., Ohno, Y., Davis, W., Zong, Y., and Dowling, K. 2009. "NIST spectrally tunable lighting facility for color rendering and lighting experiments," in Proc. CIE 2009: Light and Lighting Conference. 5 pages (2009).

NIST 2011, NIST Special Publication 250-89 Spectral Irradiance Calibration.

Ohno, Y., 2013. Practical Use and Calculation of CCT and Duv, LEUKOS, 10:1, 47-55, DOI: 10.1080/15502724.2014.839020, 47-55.

Ohno, Y., Fein, M., 2014. Vision Experiment on Acceptable and Preferred White Light Chromaticity for Lighting, CIE x039:2014, 192-199.

Rea, M. S. and Freyssinier, J. P., 2013. White Lighting, Color Research and Application, 38- 2 Wei, M. and Houser, K., 2015. What is the cause of apparent preference for sources with chromaticity below the blackbody locus?, Leukos, DOI: 10.1080/15502724.2015.1029131. 


\title{
SOLID-STATE LIGHTING MEASUREMENT ASSURANCE PROGRAM SUMMARY WITH ANALYSIS OF METADATA
}

\author{
Miller, C.C., Nadal, M., Ohno, Y., Tsai, B. and Zong, Y. ${ }^{1}$ \\ ${ }^{1}$ National Institute of Standards \& Technology, Gaithersburg, MD USA \\ c.miller@nist.gov
}

\begin{abstract}
The National Institute of Standards and Technology (NIST) began to offer proficiency testing for Solid-State Lighting (SSL) products through a Measurement Assurance Program (MAP) in 2010. This article communicates the results of the first version of the MAP in which 118 worldwide laboratories participated. Statistical analysis of how the laboratories' measurements compared to NIST's measurements are presented. In general, all the laboratory results are within +/- $4 \%$ for total luminous flux and luminous efficacy measurements. The discussion provides reasons for any discrepancies or large uncertainty intervals found in the data. A major finding was that measurement differences of RMS current had a larger standard deviation and number of outliers than expected. Two possible explanations are (1) the discrepancies are due to issues with using 4-pole sockets, and (2) the large deviation is caused by some solid state lamps being sensitive to impedance and slew rate of $A C$ power supplies.
\end{abstract}

Keywords: e.g. Photometry, Proficiency Testing, Solid-State Lighting

\section{Introduction}

In January 2010, the National Institute of Standards and Technology (NIST) began to offer a Measurement Assurance Program (MAP) for solid-state lighting (SSL) products to customers of the National Voluntary Laboratory Accreditation Program (NVLAP) Energy-Efficient Lighting Products (EELP) program ${ }^{1}$ under the support of the United States Department of Energy (DOE). The MAP program provided proficiency testing complimenting laboratory accreditation to ensure that as SSL products became more prevalent, capable testing laboratories would be available to handle the volume of measurement work. At the request of the Energy Star program, in January 2011 the MAP was opened to any testing laboratories that wanted to participate, independent of accrediting body. As of December 2014, the first version of the MAP was closed with 118 participant laboratories representing 13 countries. The results of the comparison provide a snapshot of the capabilities of accredited laboratories worldwide. The participant laboratories include the United States (49 laboratories), China (45), Taiwan (9), Korea (4), Canada (3), the Netherlands, Brazil, Singapore, India, Malaysia, Hungary, Italy, and Germany.

Scope of the measurement assurance program covered the procedures described in Illuminating Engineers Society (IES) LM-79-08 ${ }^{2}$. The following properties/quantities were measured for each artifact: total luminous flux (Im), RMS voltage (V) and current (A), electrical active power (W), luminous efficacy $(\mathrm{Im} / \mathrm{W})$, chromaticity coordinates $(x, y)$, correlated color temperature (CCT) (K), and color rendering index (CRI) (Ra).

\section{Artifact Characteristics}

Six different types of lamp or luminaire were used in MAP as listed in Table 1. The F-lamp is a recessed ceiling luminaire (downlight) which was chosen because of its physical size (large enough to cause potential self-absorption concerns with sphere measurements) and because it has a feedback mechanism that measures the chromaticity of the emitted light (and light reflected into the luminaire) and adjusts the red light emitted to maintain a constant chromaticity. The L-lamp has a large remote phosphor that may cause potential selfabsorption concerns in a small sphere measurement system. The S-lamp has a sharp peaked current wave that has a maximum when the voltage is at a maximum which makes the measurement of the current and power factor of the lamp challenging. The R-lamp is a $30^{\circ}$ 
spot lamp which requires the laboratory to correct for angular non-uniformity in a sphere measurement or angular sampling frequency for a goniometric based measurement. The Ilamp is a simple incandescent halogen lamp used to evaluate the luminous flux scale of the laboratory. These five lamps are operated with $120 \mathrm{~V}$ of $60 \mathrm{~Hz}$ AC electricity. The sixth lamp, T-lamp, is a 24 inch under cabinet type lamp which is operated with $0.2250 \mathrm{~A}$ of constant current DC electricity with an approximate compliance voltage of $12 \mathrm{~V}$. The T-lamp has a high correlated color temperature near $7000 \mathrm{~K}$.

Table 1 - Test artifact identification, nominal CCT and rated electrical conditions

\begin{tabular}{|c|c|c|c|c|}
\hline Identifier & Type & $\begin{array}{c}\text { Nominal } \\
\text { CCT }\end{array}$ & $\begin{array}{c}\text { Rated } \\
\text { voltage } \\
\text { or current }\end{array}$ & $\begin{array}{c}\text { Rated } \\
\text { power }\end{array}$ \\
\hline F-lamp & Directional & $3500 \mathrm{~K}$ & $120 \mathrm{~V} \mathrm{AC}$ & $12 \mathrm{~W}$ \\
\hline L-lamp & Omnidirectional & $2700 \mathrm{~K}$ & $120 \mathrm{~V} \mathrm{AC}$ & $12.5 \mathrm{~W}$ \\
\hline S-lamp & Omnidirectional & $2800 \mathrm{~K}$ & $120 \mathrm{~V} \mathrm{AC}$ & $8 \mathrm{~W}$ \\
\hline R-lamp & Spot lamp & $3000 \mathrm{~K}$ & $120 \mathrm{~V} \mathrm{AC}$ & $8 \mathrm{~W}$ \\
\hline T-lamp & Undercabinet tube & $8000 \mathrm{~K}$ & $0.225 \mathrm{~A} \mathrm{DC}$ & $2.88 \mathrm{~W}$ \\
\hline I-lamp & Incandescent & $2900 \mathrm{~K}$ & $120 \mathrm{~V} \mathrm{AC}$ & $100 \mathrm{~W}$ \\
\hline
\end{tabular}

\section{MAP Structure and Analysis}

The Measurement Assurance Program was conducted as a star-type comparison. Along with the measurement results each laboratory provided information on how they conducted the measurements and what equipment was used. The difference between the results of the laboratories' measurements and NIST's measurements for each of the eight properties/quantities was calculated and categorized by lamp type. This analysis provides a 'snapshot' of the lighting measurement community's capability to measure solid-state lighting products and is presented in such a way that an individual laboratory's results cannot be identified. Individual laboratories have received formal reports describing their particular results. With those reports, individual laboratories can determine where their results fit into the overall capabilities of the lighting measurement community.

To determine whether the differences between measurements were normally distributed and therefore potentially coming from a random process, the values were ordered from smallest to largest and then plotted on a Normal Probability Plot (NPP). ${ }^{3}$ This method uses theoretical normally distributed values (called "normal order statistic medians") as a horizontal axis to plot against the observed measurement differences. If the observed differences are normally distributed, then the resulting graph will be linear to a certain significance determined by the correlation coefficient and the number of data points. ${ }^{4}$ For example, Fig. 1 shows the sequenced distribution of all the observed differences between laboratories' measurements and NIST's measurements of luminous flux, and Fig. 2 shows the NPP of the data in Fig. 1.

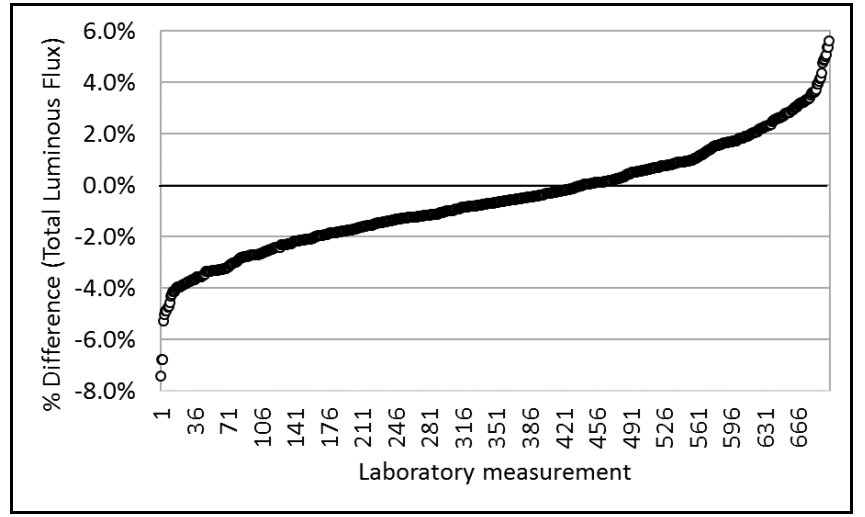

Figure 1 - The sequenced distribution of all the observed differences in luminous flux measurements 


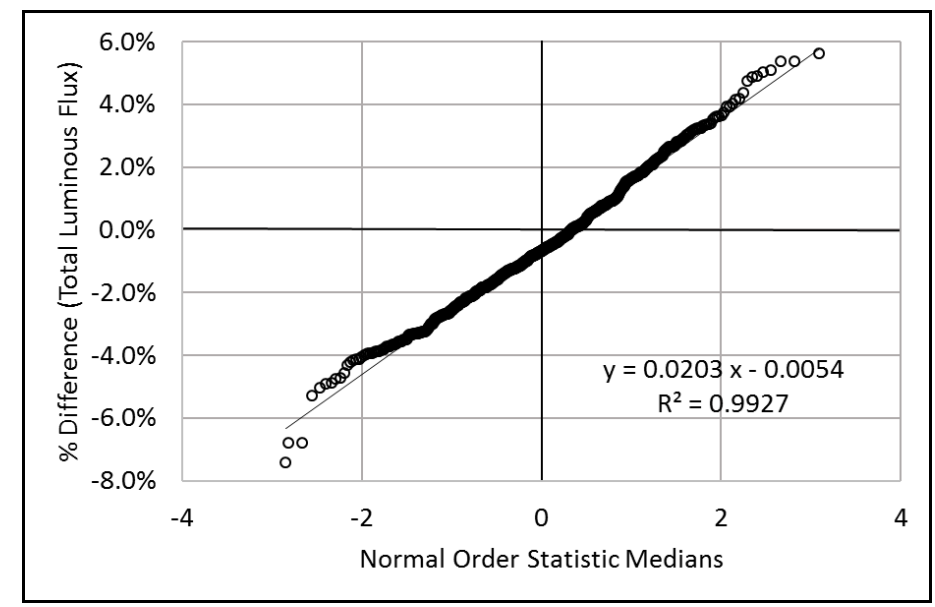

\section{Figure 2 - A Normal Probability Plot of all the observed differences in luminous flux} measurements which has been fit to a linear function

The NPP also provides the mean and standard deviation of the sequenced distribution as a result of the fit where the mean is estimated by the $y$-intercept and the standard deviation is approximated by the slope of the fit. The y-intercept of the graph shows how far the laboratories' measurements fall from NIST's measurements altogether. In this case, the intercept is -0.0054 meaning that in general, laboratories measured luminous flux about $0.54 \%$ lower than NIST. The standard deviation of the measured differences is $\pm 2.0 \%$. Another analysis step included is identifying any outliers in the data which were dealt with by using the method described in ASTM E 178-08.

\section{Results and Discussion}

\subsection{Luminous Flux}

Fig. 3 shows a compilation of the normal probability plots for the difference of luminous flux for each lamp type. The difference, NIST's measurement minus the laboratory's measurement, is shown on the vertical axis and the normal order statistic medians are shown on the horizontal axis. Table 2 lists the standard deviations of the distributions in the figures above them, the overall bias/offset of laboratories' measurements compared to NIST's measurements, the number of data points in the distributions, the correlation coefficients of each linear fit, and the critical values for each distribution. Not included in Fig. 3 or the results in Table 2 is one data point identified as an outlier. The measurement made by a laboratory on an $S$ type lamp may have been influenced by the $S$ type artifact instability. The $S$ type lamp in question was removed after this laboratory's measurement.

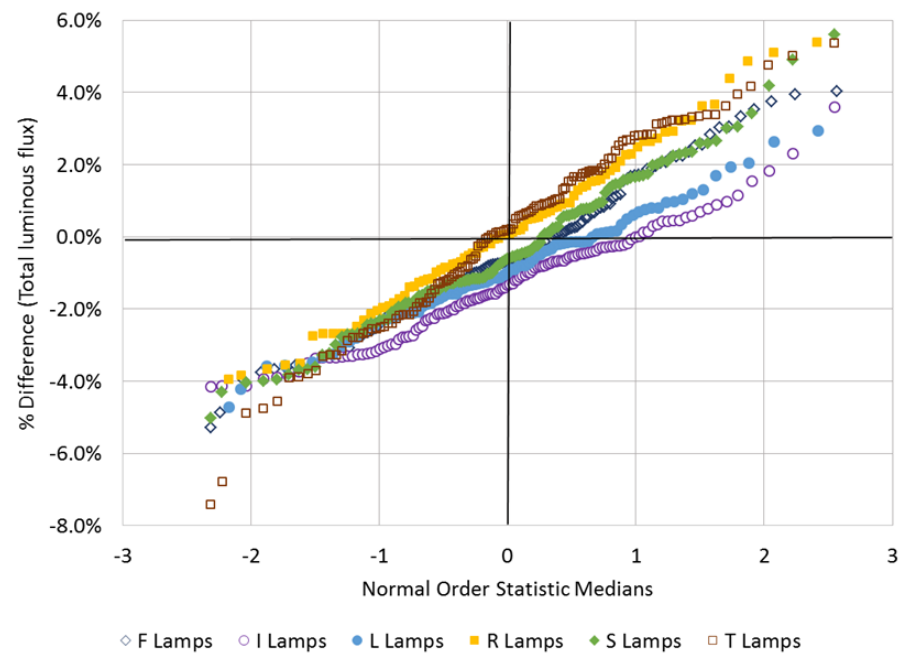

Figure 3 - Normal Probability Plots of the percent differences in luminous flux measurements between the laboratories and NIST for each lamp type 
Table 2 - Fit parameters for the luminous flux measurement differences for each lamp

\begin{tabular}{|c|c|c|c|c|c|}
\hline Lamp Type & $\begin{array}{c}\text { Standard } \\
\text { Deviation }\end{array}$ & Bias/Offset & $\begin{array}{c}\text { Number of } \\
\text { Points }\end{array}$ & $\begin{array}{c}\text { Correlation } \\
\text { Coefficient }\end{array}$ & Critical Value \\
\hline F & $1.93 \%$ & $-0.54 \%$ & 135 & 0.9947 & 0.9897 \\
\hline I & $1.47 \%$ & $-1.37 \%$ & 129 & 0.9920 & 0.9897 \\
\hline L & $1.53 \%$ & $-0.94 \%$ & 89 & 0.9975 & 0.9850 \\
\hline R & $2.12 \%$ & $0.15 \%$ & 88 & 0.9968 & 0.9850 \\
\hline S & $1.99 \%$ & $-0.43 \%$ & 129 & 0.9944 & 0.9897 \\
\hline T & $2.47 \%$ & $0.04 \%$ & 128 & 0.9921 & 0.9897 \\
\hline
\end{tabular}

The number of data points is larger than the number of laboratories participating because some laboratories used several measurement facilities (different spheres or sphere and goniometer). The $\mathrm{L}$ type and $\mathrm{R}$ type lamps were added after the initial roll out of the measurement assurance program. These lamps were found to be more stable than the initial lamps chosen.

For all the types of lamps the correlation coefficient is larger than the critical value; therefore, the hypothesis that the distributions do not come from a normal distribution cannot be rejected. The distribution of differences is well represented by a normal distribution which implies the data is generated by a random process. Using a sigma of 2 and the standard deviation of the fits, $95 \%$ of the measurements are within $\pm 4.0 \%$. The incandescent lamps which are operated on AC electricity are slightly better at $\pm 3.0 \%$, and the T type lamp which has a very high correlated color temperature is slightly worse at $\pm 5.0 \%$.

The bias shows the difference between the population and NIST. The average bias is $0.54 \%$ which means laboratories typically measure lamps with lower lumen values than NIST. This is somewhat expected because as the calibration chain becomes longer and older in time, incandescent lamps which maintain the scale decrease in luminous flux. The positive bias for the $R$ type lamp which is a spot lamp may be due to the angular non-uniformity of sphere responsivities. The larger negative bias for the I type lamp may be due to problems with 4pole sockets used in the laboratories.

\subsection{RMS Current}

Fig. 4 shows a compilation of the NPP for the difference of RMS current for each lamp type. Not included in Fig. 4 or the results in Table 3 are the data points listed in Table 4 which were identified as outliers. An unexpected result of the MAP1 was the large standard deviation and the number of outliers identified for the measurement of current. The 30 measurements identified as outliers included the results from 19 different laboratories. For all the types of lamps the correlation coefficient is smaller than the critical values; therefore, the hypothesis that the distributions come from a normal distribution is rejected. The $S$ type lamp was included in the MAP1 because of its current waveform and the standard deviation was twice any of the other type lamps. One laboratory was a consistent outlier and many times had the largest deviation; measuring a much larger current than NIST. This laboratory identified a wiring problem and has corrected the situation.

In Fig. 4 it visually appears that a fraction of the laboratories' measurements may result from a normal distribution while the standard deviation is larger than expected. In the wings of the distribution the deviation becomes larger quickly; therefore, there are two types of laboratories. The laboratories with the larger deviations may be due to wiring concerns where resistance, capacitance, or inductance out of the normally expected ranges is playing a role. NIST is conducting research to determine potential dependencies. Another possible explanation for the differences is improper implementation of using a 4-pole or Kelvin socket to eliminate junction potentials. If a laboratory is measuring the voltage drop across the lamp at a wiring junction outside of the sphere or perhaps at the power supply terminals, a voltage drop will be measured that includes the voltage drop across the lamp, at any junctions, and through the length of wire. The lamp will be operated at a lower than specified voltage. 


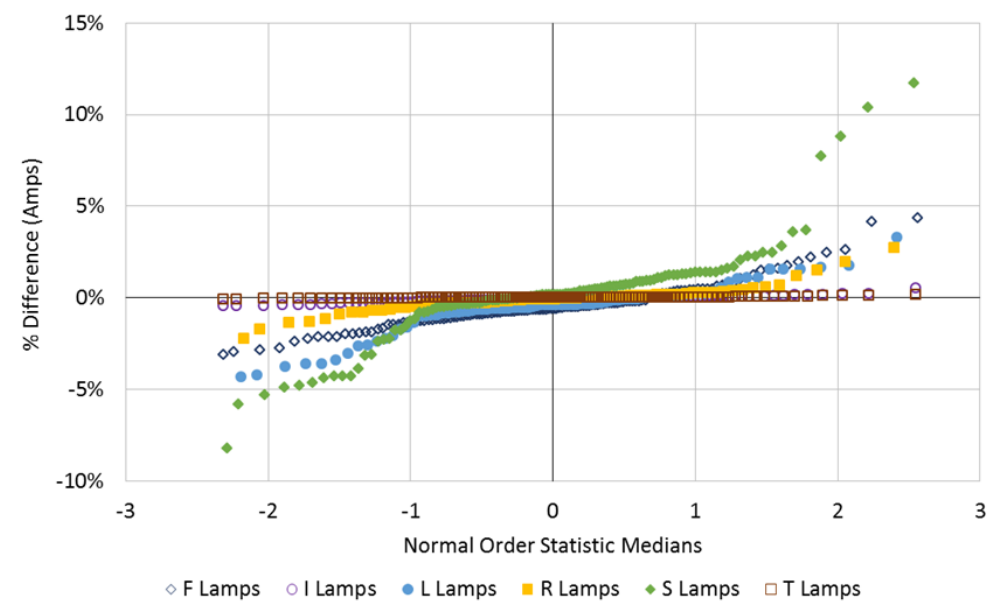

Figure 4 - Normal Probability Plots for the differences in RMS current measurements for each lamp type except the $T$ type lamp which is a difference in voltage measurements.

Table 3 - Fit parameters for the current measurement differences for each lamp.

\begin{tabular}{|c|c|c|c|c|c|}
\hline $\begin{array}{c}\text { Lamp } \\
\text { Type }\end{array}$ & $\begin{array}{c}\text { Standard } \\
\text { Deviation }\end{array}$ & Bias/Offset & $\begin{array}{c}\text { Number of } \\
\text { Points }\end{array}$ & $\begin{array}{c}\text { Correlation } \\
\text { Coefficient }\end{array}$ & Critical Value \\
\hline \multicolumn{5}{|l|}{} \\
\hline R & RS Current & 134 & 0.9581 & 0.9897 \\
\hline I & $1.18 \%$ & $-0.46 \%$ & 127 & 0.9791 & 0.9897 \\
\hline L & $0.15 \%$ & $-0.09 \%$ & 89 & 0.9405 & 0.9857 \\
\hline R & $1.31 \%$ & $-0.57 \%$ & 84 & 0.9156 & 0.9850 \\
\hline S & $0.65 \%$ & $-0.09 \%$ & 124 & 0.8937 & 0.9889 \\
\hline \multicolumn{7}{|l|}{} & $0.13 \%$ & 127 & 0.9573 & 0.9897 \\
\hline T & $2.59 \%$ & $0.02 \%$ & &
\end{tabular}

Table 4 - Measurement differences that were determined to be outliers.

\begin{tabular}{|c|c|c|c|c|c|}
\hline Lamp Type & Outlier 1 & Outlier 2 & Outlier 3 & Outlier 4 & Outlier 5 \\
\hline \multicolumn{5}{|c|}{ RMS Current } \\
\hline F & $18.7 \%$ & $9.68 \%$ & $5.76 \%$ & $-5.17 \%$ & \\
\hline I & $0.62 \%$ & $-0.58 \%$ & $-0.81 \%$ & $-0.93 \%$ & $-1.37 \%$ \\
\hline L & $4.55 \%$ & $-4.43 \%$ & & & \\
\hline R & $17.9 \%$ & $12.5 \%$ & $10.4 \%$ & $6.86 \%$ & $-3.82 \%$ \\
\hline \multirow{2}{*}{ S } & $20.9 \%$ & $19.1 \%$ & $18.1 \%$ & $17.9 \%$ & $14.5 \%$ \\
\cline { 2 - 6 } & $12.5 \%$ & $-13.1 \%$ & $-13.3 \%$ & $-13.8 \%$ & $-29.0 \%$ \\
\hline \multicolumn{7}{|l|}{ RMS Voltage } \\
\hline T & $11.4 \%$ & $4.22 \%$ & $3.84 \%$ & $3.58 \%$ & \\
\hline
\end{tabular}

\section{Conclusions and Future Work}

The results of the Measurement Assurance Program offered by NIST are a 'snapshot' of lighting testing laboratories' capabilities to measure total luminous flux (Im), RMS voltage (V) and current (A), electrical active power (W), luminous efficacy ( $\mathrm{Im} / \mathrm{W})$, chromaticity coordinates $(x, y), C C T(K)$, and CRI $(R a)$ according to IES LM-79-08. The results are for the measurements of 118 laboratories located worldwide between the years of 2010 and 2014 .

In general, independent of the lamp type, the laboratories that participated in MAP were able to measure the total luminous flux and the luminous efficacy within $\pm 4 \%$ (variance of the 
distribution, capturing $95 \%$ of the measurements). The laboratories were able to measure the active power within $\pm 1 \%(k=2)$ for most of the lamps. The $F$ type lamp which has an active feedback and the T type lamp which is a $12 \mathrm{~V}$ DC lamp (uncommon for many laboratories) have a larger spread.

The somewhat surprising result was the large spread for the measurement of RMS current, \pm $5 \%(k=2)$ for the $S$ type lamp. This large spread has motivated research in this area. One conclusion is that many laboratories may have issues with 4-pole sockets. A specific lamp has been included in the second version of the MAP to investigate 4-pole socket problems. Additionally, some of the early results reveal that a select set of solid state lamps are sensitive to the impedance and slew rate of the AC power supplies, which is not specified in LM-79. Additional research is required in this area to help the testing community reach more consistent results. The CCT, chromaticity coordinates and CRI results showed standard deviations that were on the order of the expected uncertainty of these measurements.

In January 2015, NIST started to offer a second version of the MAP (MAP 2) with different SSL artifacts meant to evaluate the laboratory's capabilities. The new version has a set of proficiency artifacts for a laboratory to measure, and the laboratory will be graded for passing or failing for each artifact. The MAP 2 artifacts were selected to allow the laboratory to diagnose potential deficiencies in its measurement system or to provide diagnostics to improve the lighting measurement standards. MAP 2 is expected to run for three years and is available to any testing laboratory for a service fee. MAP 2 has three options: A - SSL products, B - SSL products with 2 different 4 foot LED tubes, and C -SSL products along with 4 compact fluorescent lamps (CFLs) (with or without 4 foot LED tubes). Not every laboratory has the capability to measure lighting products 4 -foot-long, so the 4 foot tubes are not part of the proficiency test grading.

\section{References}

1 Miller, C.C., Crickenberger, J., NIST Handbook 150-1, 2010 Ed., National Voluntary Laboratory Accreditation Program - Energy Efficient Lighting Products, Dec. 2010.

2 Illuminating Engineering Society, LM-79-08, "Approved Method: Electrical and Photometric Measurements of Solid-State Lighting Products", 2008.

3 NIST. 2013. Engineering Statistics Handbook. 1.3.3.21 Normal Probability Plot. http://www.itl.nist.gov/div898/handbook/eda/section3/normprpl.htm, accessed 2016-0108.

${ }^{4}$ NIST. 2013. Engineering Statistics Handbook. 1.3.6.7.6. Critical Values of the Normal PPCC Distribution. http://www.itl.nist.gov/div898/handbook/eda/section3/eda3676.htm, accessed 2016-01-08.

${ }^{5}$ ASTM. 2008. Standard Practice for Dealing with Outlying Observations. West Conshohocken (PA): ATSM E178-08. 


\title{
Lambertian nature of tissue phantoms for use as calibrators in near infrared fluorescence imaging
}

\author{
Maritoni Litorja* ${ }^{\mathrm{a}}$, Simón Lorenzo ${ }^{\mathrm{b}}$, Banghe $\mathrm{Zhu}^{\mathrm{c}}$ and Eva Sevick Muraca ${ }^{\mathrm{c}}$ \\ ${ }^{a}$ National Institute of Standards and Technology, Gaithersburg, MD 20899 \\ ${ }^{\mathrm{b}}$ Dept. of Physics and Astronomy, Louisiana State University, Baton Rouge, LA 70803 \\ ${ }^{\mathrm{c}}$ Brown Foundation Institute for Molecular Medicine, \\ Univ. of Texas Houston Health Science Center, Houston, TX 77030
}

\begin{abstract}
The use of tissue phantoms as calibrators to transfer SI-referenced scale to an imager offers convenience, compared to other methods of calibration. The tissue phantoms are calibrated separately for radiance at emission wavelength per irradiance at excitation wavelength. This calibration is only performed at a single geometric configuration, typically with the detector normal to the sample. In the clinic however, the imager can be moved around, resulting in a geometric configuration different from the calibration configuration. In this study, radiometric measurements are made at different sample-imager angles to test whether the tissue phantoms are Lambertian and the angular limits to which the calibration values hold true.
\end{abstract}

Keywords: near infrared fluorescence imaging, molecular imaging, radiometry, calibration, tissue phantoms

\section{INTRODUCTION}

The acquired light signal in in vivo fluorescence imaging is intended to correlate to the presence, or if possible, quantity of a desired biological marker. Inside the tissue, the fluorescence emission is dependent on factors of biological (e.g., binding to desired marker), chemical (e.g. proticity, solvation, competitive energetic pathways), and physical (attenuation of excitation and emission radiation, scattering) origin. Once the emitted fluorescence exits the patient (e.g skin) surface into free space and of which a portion is collected by the imager situated at some distance away from the patient, it is a physical measurement of light involving primarily geometric optics. The measurement sources of uncertainty are the same as that of other measurements of a light source.

The imager is a light collector and its performance can be specified using light, independent of the molecular probe. Spectrally, the performance specification needs to be relevant to the molecular probe the imager is intended for use.

\subsection{Radiometric Calibration}

In order to quantify the amount of fluorescence emitted from the surface (patient), the imager's quantitative response to light (responsivity) has to be calibrated. Calibration is the process where the instrument acquires a scale by virtue of comparisons against a standard. It is an important task in the validation of diagnostic and therapeutic devices. It provides the daily user with confidence in the performance of the device to the specified task.

A photodetector such as the fluorescence imager can be calibrated to acquire a responsivity scale. That is, the imager's signal in digital counts can be correlated to the amount of light detected in units traceable to the International System (SI). Being traceable to the SI is highly advantageous because the SI is anchored in physical laws and maintained by an international system of laboratories which underpins the global scientific and commercial measurements. The fluorescence imager can be calibrated against a standard detector, a standard source, or through the use of a reference material.[1]

\subsection{Radiometric Units}

There are various units by which the quantity of light can be expressed. The choice of radiometric quantity and therefore units to use in a measurement is fit-for-purpose. For example, radiant flux (power) with units of watts ( $\mathrm{W}$ or Js ${ }^{-1}$ ) is an appropriate quantity only if the light to measure underfills the detector, such as in the case of measuring the power in a laser beam. Radiance with units of $\mathrm{W} \mathrm{m}^{-2} \mathrm{sr}^{-1}$ is typically a quantity associated with real light sources. Irradiance with 
units of $\mathrm{W} \mathrm{m}^{-2}$ is the appropriate unit to measure how much light is incident on a given surface area. Fig. 1 illustrates the difference between radiance $L$ and irradiance $E$.

Tissue phantoms consisting of quantum dots dispersed with titanium dioxide to mimic tissue optical scatterers in a polyurethane matrix have been shown to be robust enough to serve as near infrared fluorescence imager calibrators.[2] It is a good candidate as a reference material to use as transfer or working standard to carry SI-traceable scale for light measurements. Currently there are no transfer standard reference materials for use in the near infrared with radiometric units.
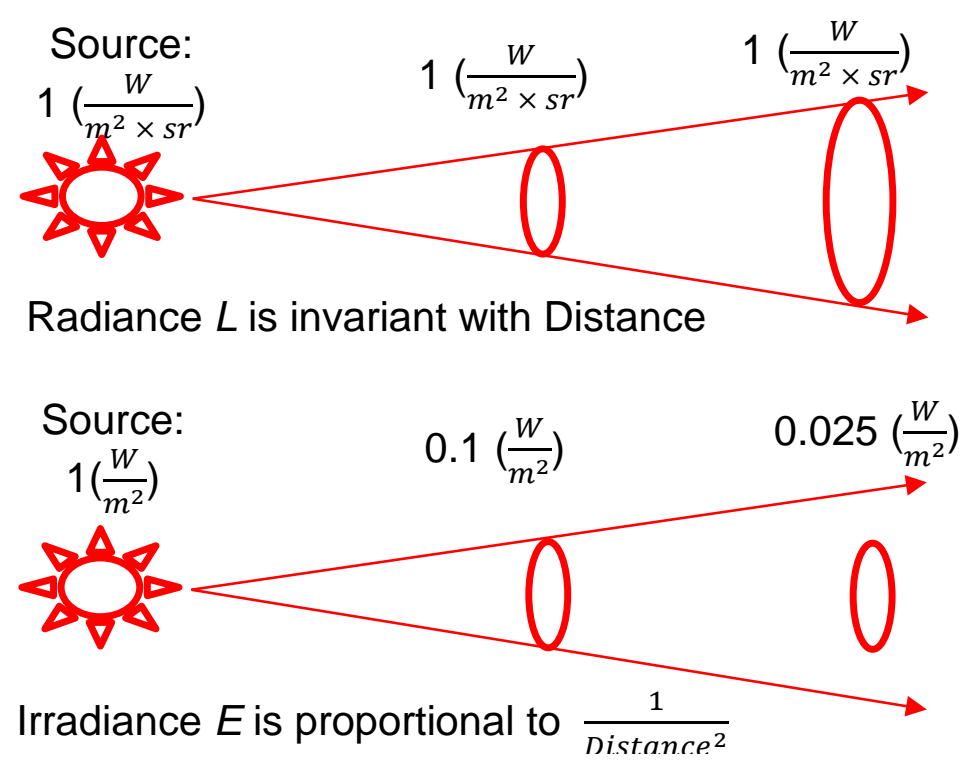

Figure 1. Illustration on the differences between radiance and irradiance variation with distance from source.

The spectral radiance $L_{\lambda e m}\left(\mathrm{~W} \mathrm{~m}^{-2} \mathrm{sr}^{-1}\right)_{\lambda e m}$ from the fluorescence emission of the tissue phantom is a function of the spectral irradiance of the excitation radiation $E_{\lambda e x}\left(\mathrm{~W} \mathrm{~m}^{-2}\right)_{\lambda e x}$ and the fluorescence characteristics $\mathrm{F}_{\lambda e m, \lambda e x}$ of the material

$$
L_{\lambda e m}=\mathrm{F}_{\lambda e m, \lambda e x} \cdot E_{\lambda e x}
$$

Here we use the special character $\mathrm{F}$ as an aggregate fluorescence yield factor for radiometry purposes, to distinguish it from the normally reported molar fluorescence yield of the pure fluorophore, which is an intrinsic optical property of the material. The factor $\mathrm{F}_{\lambda e m, \lambda e x}$ to be determined in a separate calibration procedure, will need to have units of spectral radiance at emission wavelength per spectral irradiance at excitation wavelength $\left(\mathrm{W} \mathrm{m}^{-2} \mathrm{sr}^{-1}\right)_{\lambda e m} /\left(\mathrm{W} \mathrm{m}^{-2}\right)_{\lambda e x}$. With the calibrated tissue phantom and the irradiance of the excitation radiation $E_{\lambda e x}$ measured at the time of image collection, the spectral radiance emitted $L_{\lambda e m}$ by the phantom can be known. Since the phantoms then act as light sources of known radiance, and the collection geometry are known $\left(\mathrm{m}^{2}\right.$ and $\mathrm{sr}$ ), the signal counts correlated to that radiance can then be converted to watts $(\mathrm{W})$. We have recently shown the procedure of transferring the calibration scale from a photodiode, to a laser input into an integrating sphere matching radiance of the tissue phantoms.[3] Since this procedure is not readily accessible to most laboratories, a convenient method is to have the tissue phantoms calibrated using a dedicated measurement station, using the choice of excitation and emission wavelengths for which the clinical imager will be used.

\subsection{Angular distribution of emission}

The optical properties of the tissue phantoms as radiator (fluorescence emission) needs to be studied as a matter of course for materials intended for use as reference standards. Ideal extended sources are assumed to be Lambertian, diffusely radiating in all directions. That is, the radiance measured at all angles with respect to the surface normal is constant; and that the irradiance measured at the observation plane follows Lambert's cosine law.[4] In reality, extended sources are not perfectly Lambertian or the angular range with respect to surface normal at which the radiance is constant is limited. 


\section{LABORATORY MEASUREMENTS}

\subsection{Measurements of Radiance}

The tissue phantom is a photoactivated light source, as opposed to an electrically driven light bulb, a common calibrator for radiometry. Since the tissue phantoms are intended to be used as radiance calibrators, we examine its properties as a radiator. The objective of the measurement is to compare the tissue phantom radiance with respect to distance to that of a typical lamp calibrator. Figure 2 illustrates the measurement setup in the laboratory for measuring radiance. The radiance from one tissue phantom is compared to that of a typical tungsten halogen lamp. The detector used to measure radiance is a NIST-calibrated silicon photodiode. The excitation radiation for the tissue phantom is supplied by a $785 \mathrm{~nm}$ laser, with the beam expanded using a Galilean telescope assembly such that the laser uniformly illuminated the whole surface of the tissue phantom. A field stop is placed in front of the tissue phantom to define the source area. An $830 \mathrm{~nm}$ bandpass filters is placed in the detection optical path to limit the band fraction detected. All optical components are placed on an optical rail. The detector assembly is placed at least $300 \mathrm{~mm}$ away from the source. The radiance is then measured at different source-detector distances. Baffles and shields comprising of black cloth, and black painted metal shields are used. The photodiode assembly includes a series of baffles to minimize stray light. An aperture is placed in front of the photodiode to define the collection area. The tissue phantom is replaced by a tungsten halogen calibrated lamp source and its radiance measured. Results from the variation of radiance with distance for the tungsten halogen lamp calibrator and the tissue phantom are shown in Figure 3. While the radiance level of the tissue phantom is

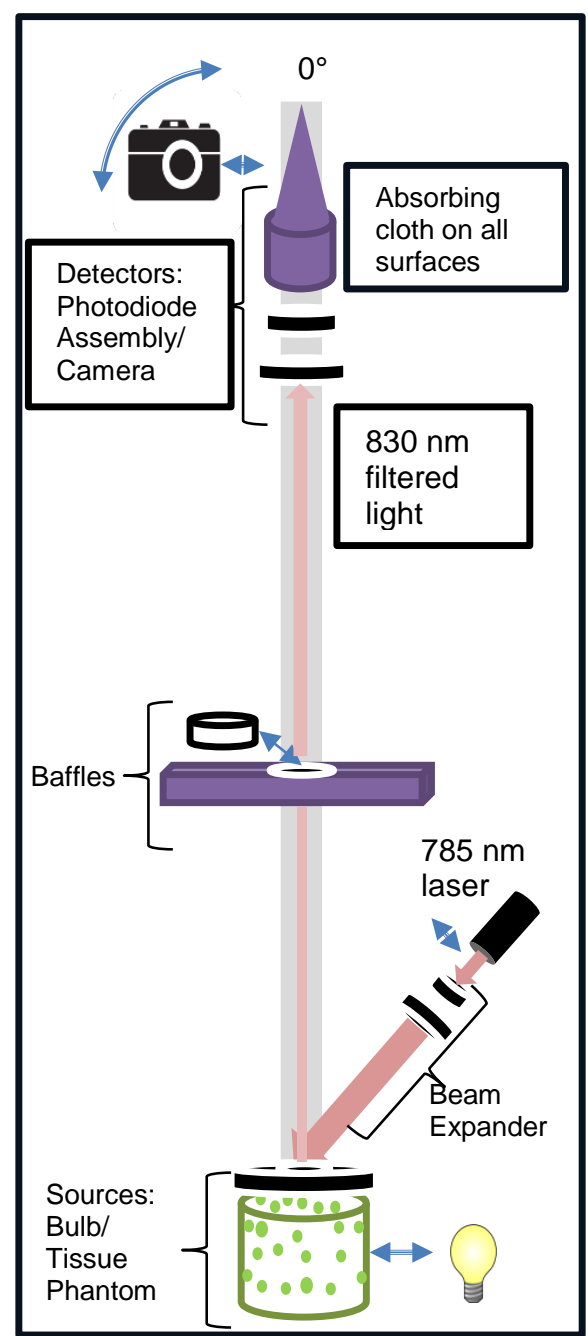

Figure 2. Illustration of the laboratory measurement setup for radiance. Distances are not drawn to scale.

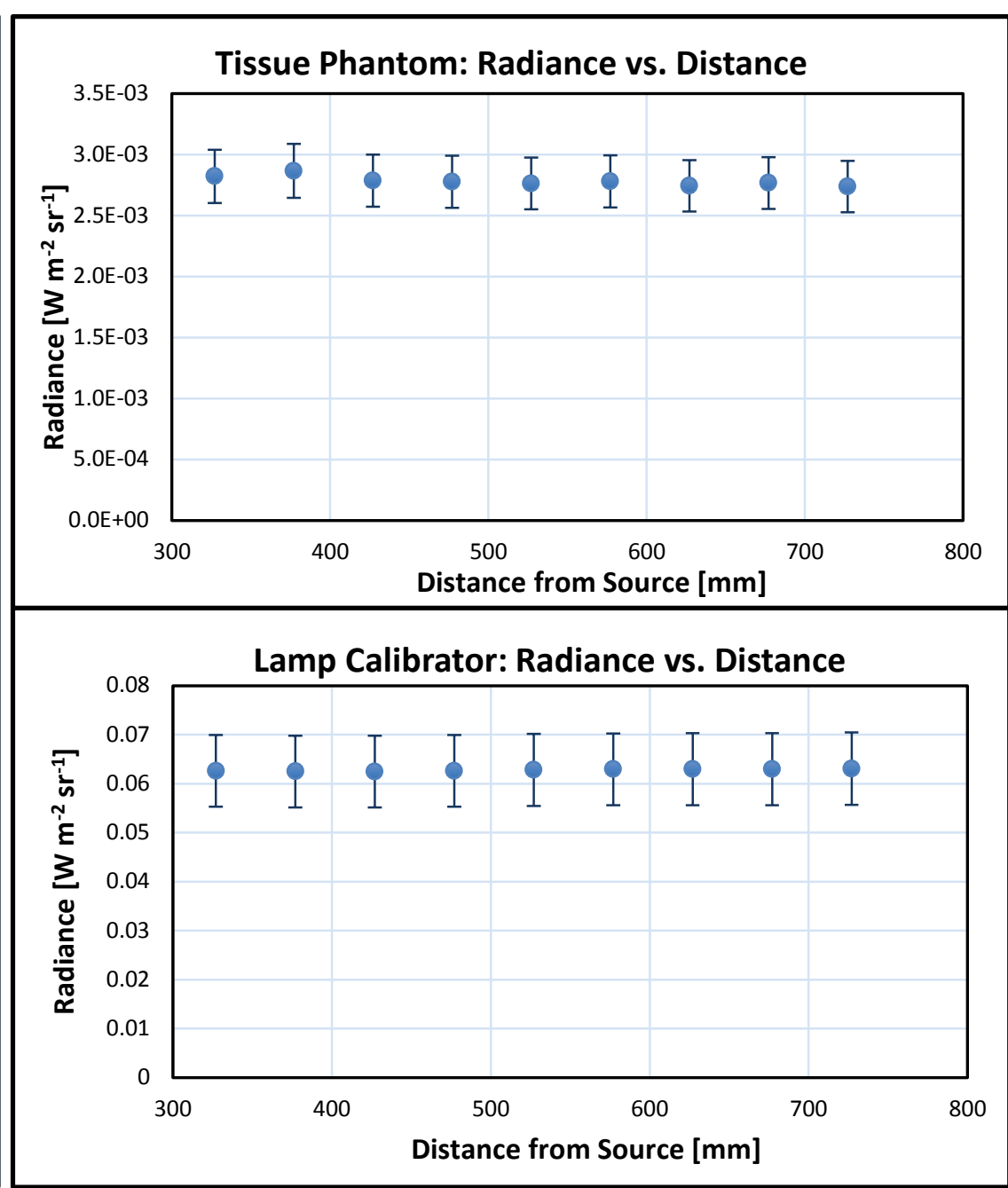

Figure 3. Radiance of tissue phantom (top) is constant with distance, similar to a source calibrator (bottom). 
much lower than a lamp calibrator, the value is constant with distance, as is desired in a calibrator. The radiance values have an estimated $1 \%$ relative uncertainty at coverage factor of $k=1$. The steps in estimating the standard uncertainty in radiance is discussed in Sec. 2.2.

It should be noted that a tungsten halogen lamp with its broadband spectral output is not an appropriate light source to calibrate the imager, even with a bandpass filter since the detector is sensitive over the entire visual range and the filter has a non-zero throughput outside of the specified $10 \mathrm{~nm}$ bandpass. The lamp introduces stray light into the camera. Moreover, unlike the tissue phantom, its radiance does not have any relationship to the $785 \mathrm{~nm}$ excitation radiation. In calibration terms, it is dissimilar to the radiance source being interrogated by the imager for its intended use.

\subsection{Uncertainty in Radiance Measurements}

The photocurrent generated at the photodiode by the collected light is converted to voltage, amplified and measured using a multimeter. The total radiance collected giving rise to this signal is

$$
L_{\lambda}=\frac{v}{s_{\Phi, \lambda} \cdot \Delta_{\lambda} \cdot G \cdot \Gamma}
$$

where $L_{\lambda}$ is the spectral radiance of the source with units of $\mathrm{W} \mathrm{m}^{-2} \mathrm{sr}^{-1} \mathrm{~nm}^{-1}$

$v$ is the voltage measured with units of $\mathrm{V}$

$s_{\Phi, \lambda}$ is the spectral flux (power) responsivity of the detector with units of $\mathrm{A} \mathrm{W}^{-1} \mathrm{~nm}^{-1}$

$\Delta_{\lambda}$ is the bandwidth of the filter with units of $\mathrm{nm}$

$G$ is the amplifier gain setting with units of $\mathrm{V} \mathrm{A}^{-1}$

$\Gamma$ is the throughput of the optical configuration, defined in this optical configuration as

$$
\Gamma=A_{d} \cdot \omega_{s} \cdot T
$$

$A_{d}$ is the receiving area of the detector with units of $\mathrm{m}^{2}$

$\omega_{s}$ is the solid angle of the source viewed by the detector with units of steradian (sr) and approximated as $\omega_{s}=\frac{A_{s}}{d^{2}} . \quad A_{s}$ is the area of the source and $d$ the distance between the source and detector at the optic axis (centers of source and detector planes). Note that $\omega_{d}=\frac{A_{d}}{d^{2}}$ since radiance is invariant with distance.

$T$ is the filter transmittance

To estimate the combined uncertainty $u_{c}^{2}\left(L_{830}\right)$ in radiance value $L_{830}$ computed from the measured voltage signal from the tissue phantom as measured by the photodiode with a filter, we follow the method of propagation of uncertainties [5] in Equation (4) or its relative form (Equation (5)) for the radiance measurement equation in Equation (2) and Equation (3).

$$
\begin{aligned}
& u_{c}^{2}\left(L_{\lambda}\right)=\sum_{i=1}^{m} c_{i}^{2} u^{2}\left(x_{i}\right)+2 \sum_{i=1}^{m-1} \sum_{j=i+1}^{m} c_{i} c_{j} u\left(x_{i}, x_{j}\right) \\
& u_{c}^{2}\left(L_{\lambda}\right)=L_{\lambda}^{2} \cdot\left[\sum_{i=1}^{m}\left(c_{i, r} \cdot \frac{u\left(x_{i}\right)}{x_{i}}\right)^{2}+2 \sum_{i=1}^{m-1} \sum_{j=i+1}^{m} c_{i, r} c_{j, r} \frac{u\left(x_{i}, x_{j}\right)}{x_{i} x_{j}}\right]
\end{aligned}
$$

$c_{i}=\frac{\partial L_{\lambda}}{\partial x_{i}}$ is the absolute sensitivity coefficient and $c_{i, r}$ the relative sensitivity coefficient to each variable

$x_{i}=$ the variables $v, s_{\Phi, 830}, \Delta_{\lambda}, G$ and the elements of $\Gamma$ which are $A_{d}, A_{s}$ and $d$

$u\left(x_{i}\right)=$ standard uncertainty of $x_{i}$

$u\left(x_{i}, x_{j}\right)$ is the covariance of $x_{i}$ and $x_{j}$ (assumed to be zero in this measurement)

The uncertainty of each variable is estimated and the value may be determined experimentally (Type A), such as the case with $v$ from replicate measurements and $\Delta_{\lambda}$ and $T$ from a spectrometric measurements. The uncertainty in the photodiode's spectral power responsivity $s_{\Phi, \lambda}$ comes from the calibration [6] and the uncertainty in gain $G$ is given by the manufacturer specification of the preamplifier used (Type B). The optical system throughput $\Gamma$ is the arrangement of lenses, filters, baffles, apertures, that collects incoming light that give rise to the signal. Many factors affect throughput. This includes the filter transmittance, stray light, as well as dimensional measurements of aperture areas, solid angle and distances. Table 1 is a partial list of possible sources of uncertainty in measuring radiance from the tissue phantom using the photodiode. The effort to reduce uncertainties requires careful cataloguing of possible sources and refining the measurement through better instrumentation and procedures. 
Evaluating the contribution of each variable to the combined uncertainty in the measurand is useful in prioritizing effort and resources to minimize the combined uncertainty. For instance, in Table 1, the major contributors to uncertainty in the radiance $L_{830}$ are the voltage signal measurements which are dominated by random (Type A) uncertainties, the filter bandwidth and the filter transmittance. The filter bandwidth and transmittance uncertainties can be reduced by finer spectroscopic measurements and the voltage signal uncertainty can be reduced by replicate measurements.

\begin{tabular}{|c|c|c|c|c|c|c|c|c|}
\hline Variable & Unit & Value & $\begin{array}{l}\text { Standard } \\
\text { Unc. }\end{array}$ & Type & $\begin{array}{c}\text { Relative } \\
\text { Sensitivity }\end{array}$ & $\begin{array}{c}\text { Relative } \\
\text { Unc }\end{array}$ & $\begin{array}{c}\text { Total Rel } \\
\text { Uncertainty }\end{array}$ & $\begin{array}{c}\text { Rel Variance } \\
\text { Fraction }\end{array}$ \\
\hline$x_{i}$ & & & $u\left(x_{i}\right)$ & & $c_{i, r}$ & $\left(u\left(x_{i}\right) / x_{i}\right)$ & $c_{i}^{2} \cdot\left(\frac{u\left(x_{i}\right)}{x_{i}}\right)^{2}$ & $\left(\frac{u\left(x_{i}\right)}{x_{i}}\right)^{2} /\left(\frac{u_{c}\left(L_{830}\right)}{L_{830}}\right)^{2}$ \\
\hline Signal $v$ & $\mathrm{~V}$ & 0.1199 & 0.006 & A,B & 1 & $5.0 \mathrm{E}-2$ & $2.5 \mathrm{E}-3$ & 0.43 \\
\hline$S_{\Phi, 830}$ & $\mathrm{~A} \mathrm{~W}^{-1}$ & 0.4411 & $0.1 \%$ & B & 1 & $1.0 \mathrm{E}-3$ & $1.0 \mathrm{E}-6$ & 0.00 \\
\hline$\Delta_{\lambda}$ & $\mathrm{nm}$ & 9.3 & $4.0 \mathrm{E}-1$ & A & 1 & $4.3 \mathrm{E}-2$ & $1.9 \mathrm{E}-3$ & 0.32 \\
\hline$G$ & $\mathrm{VA}^{-1}$ & $10^{9}$ & $1 \%$ & $\mathrm{~B}$ & 1 & $1.0 \mathrm{E}-2$ & $1.0 \mathrm{E}-4$ & 0.02 \\
\hline$d$ & $\mathrm{~m}$ & 0.577 & $2.1 \mathrm{E}-3$ & A & 2 & $3.6 \mathrm{E}-3$ & $5.3 \mathrm{E}-5$ & 0.01 \\
\hline$T$ & & 0.66 & 0.024 & A & 1 & $3.6 \mathrm{E}-2$ & $1.3 \mathrm{E}-3$ & 0.23 \\
\hline$\overline{A_{d}}$ & $\mathrm{~m}^{-2}$ & $1.1 \mathrm{E}-4$ & $4.4 \mathrm{E}-7$ & $\mathrm{~A}$ & 1 & $4.1 \mathrm{E}-3$ & $1.7 \mathrm{E}-5$ & 0.00 \\
\hline$A_{s}$ & $\mathrm{~m}^{-2}$ & $4.9 \mathrm{E}-5$ & $4.4 \mathrm{E}-8$ & $\mathrm{~A}$ & 1 & $9.0 \mathrm{E}-4$ & $8.0 \mathrm{E}-7$ & 0.00 \\
\hline \multicolumn{7}{|c|}{ Combined Rel Uncertainty } & 7.7E-2 & 1.00 \\
\hline$L_{830}$ & $\mathrm{Wm}^{-2} \mathrm{sr}^{-1}$ & $2.8 \mathrm{E}-3$ & & & & & & \\
\hline$u_{c}\left(L_{830}\right)$ & $\mathrm{Wm}^{-2} \mathrm{sr}^{-1}$ & $2.1 \mathrm{E}-4$ & & & & & & \\
\hline
\end{tabular}

Table 1 . The contributors to the uncertainty of a single radiance measurement from the tissue phantom fluorescence evaluated using the measurement Equation 2.

\subsection{Measurements of Irradiance at Off Normal Observation Angles}

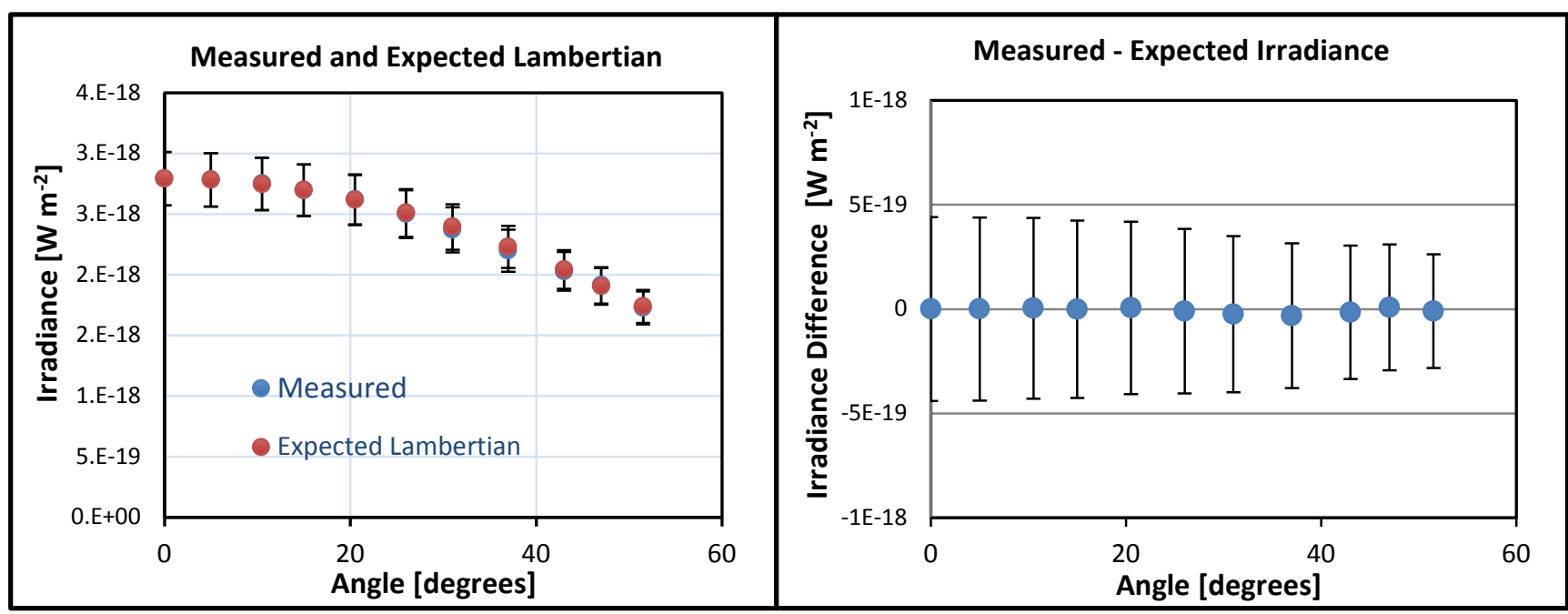

Figure 4. (Left)The measured and expected irradiances at off-normal angles if the source is Lambertian.(Right) The differences between the measured and expected irradiances are within the estimated uncertainties of $7 \%$ at $\mathrm{k}=1$.

The tissue phantoms' emittance distribution over a range of angles from the normal position was measured using both the photodiode and the camera, similar to the radiance measurements in Section 2.1. The irradiance measured at $632 \mathrm{~mm}$ from the source is shown in Figure 4 (left) along with the expected irradiance for a Lambertian radiator at those 
same angles computed from the irradiance measured at normal position $\left(0^{\circ}\right)$. The differences between the measured and computed irradiances are within the estimated uncertainties.

\section{DISCUSSION AND FUTURE WORK}

The measurement configuration used in this measurement is meant to mimic that of a clinical imager viewing the patient at a distance of about $600 \mathrm{~mm}$ and the excitation light incident at 45 degrees from the tissue phantom surface. We have shown in these few measurements that the tissue phantoms behave similarly to a lamp calibrator. Unlike a lamp calibrator with emission and over a wide spectral range, the tissue phantom is designed to have radiance output at the radiance level of the fluorophores emitting from inside the body and only at the desired spectral range. The choice of radiance as the radiometric quantity to ascribe to the tissue phantom enables the user to evaluate stray light issues with the collection geometry since radiance does not vary with distance. We have shown in Table 1 that the radiance measurement uncertainty is estimated to be at $7 \%$ at a coverage of $\mathrm{k}=1$. A similar procedure will be used to determine the uncertainty in irradiance of the excitation light incident on the surface to give the total uncertainty estimate for

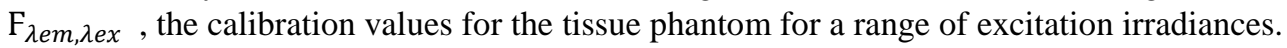

In order for the tissue phantoms to be useful as a reference material bearing radiometric units, its properties as a radiator need to be known. In this short experiment, we have verified that the tissue phantoms show evidence of being a Lambertian radiator; it does not show anomalous radiance at some angles as it would if the fluorescence emitted were somehow directional. This is not surprising given that the quantum dots are dispersed with highly scattering titanium dioxide. Calibration at a single position, typically at detection normal to the tissue phantom surface, is sufficient. If the tissue phantom is viewed at angles differing from surface normal, its radiance will be the same. Since the measurements were taken only at a single angle of incidence for the excitation light and a single viewing plane, this does not offer a complete picture of the hemispherical reflectance and fluorescence angular emission. Measurements using the NIST Robotic Optical Scatter Instrument (ROSI) [7], a goniometer where any combination of angles of incidence and viewing is possible, are planned; this will require some modifications to the instrument for fluorescence detection.

The tissue phantoms consisting of quantum dots dispersed with titanium dioxide in a polyurethane matrix offers a convenient way by which an optical calibration scale can be transferred from a reference detector (calibrated photodiode) to an imager. The calibrated tissue phantom can be presented to different imagers and each imager's response in arbitrary units can be converted to SI-traceable radiometric units.

\section{ACKNOWLEDGEMENT}

This material is based upon work supported by the NIST Summer Undergraduate Research Fellowship (SURF) Program. The authors also thank John Woodward for the filter transmittance measurements in the NIST SIRCUS facility.

\section{REFERENCES}

[1] Litorja, M., Urbas, A. and Zong, Y. "Radiometric calibration methods to consider in quantitative clinical fluorescence imaging measurements,” Proc. SPIE 9311, 931114 (2015).

[2] Zhu, B., Rasmussen, J. C. and Sevick-Muraca, E. M. "A matter of collection and detection for intraoperative and noninvasive near-infrared fluorescence molecular imaging: To see or not to see?” Med. Physics,41(2), 022105(2014).

[3] Zhu, B., Rasmussen, J., Litorja, M., Sevick-Muraca, E.M. "Determining the Performance of Fluorescence Molecular Imaging Devices using Traceable Working Standards with SI Units of Radiance,” IEEE Transactions on Medical Imaging, 35(3), 802-811(2016).

[4] McCluney, R., [Introduction to Radiometry and Photometry], Artech House, Boston and London, 13-15 (1994).

[5] Taylor, B.N. and Kuyatt, C.E. "Guidelines for Evaluating and Expressing the Uncertainty of NIST Measurement Results,” NIST TN 1297 (1994). Available at http://www.physics.nist.gov/Pubs

[6] Larason, T.C., and Houston, J.M. "NIST Measurement Services: Spectroradiometric Detector Measurements: Ultraviolet, Visible and Near-Infrared Detectors for Spectral Power,” NIST Special Publications 250-41 (2008).

[7] Patrick, H.J., Zarobila, C.J. and Germer, T.A., "The NIST Robotic Optical Scatter Instrument (ROSI) and its Application to BRDF Measurements of Diffuse Reflectance Standards for Remote Sensing,” Proc. SPIE 8866, 886615-1-12 (2013).

Litorja, Maritoni; Lorenzo, Simon; Sevick Muraca, Eva; Zhu, Banghe.

"Lambertian nature of tissue phantoms for use as calibrators in near infrared fluorescence imaging."

Paper presented at Molecular-Guided Surgery: Molecules, Devices, and Applications II (Conference 9696), San Francisco, CA. February 12, 2016 - February 18, 2016. 


\title{
Scattered-Light Analysis of Birefringent Coatings for Distributed Polarization Rotators
}

\author{
K. A. Sharma, ${ }^{* 1,2}$ T. A. Germer, ${ }^{3}$ C. Smith, ${ }^{2}$ J. D. Zuegel, ${ }^{2}$ J. B. Oliver, ${ }^{2}$ and T. G. Brown ${ }^{1}$ \\ ${ }^{I}$ The Institute of Optics, University of Rochester, 275 Hutchison Road, Rochester, NY 14627, USA \\ ${ }^{2}$ Laboratory for Laser Energetics, 250 East River Road, Rochester, NY 14623-1299, USA \\ ${ }^{3}$ Sensor Science Division, National Institute of Standards and Technology, 100 Bureau Drive, Gaithersburg, MD 20899, USA \\ *ksha@lle.rochester.edu
}

\begin{abstract}
Novel, birefringent thin-film coatings have been developed for improved irradiation uniformity by polarization smoothing in direct-drive fusion. Forward scatter distribution of $351 \mathrm{~nm}$ radiation is characterized and its operational impact analyzed.

OCIS codes: (290.0290) Scattering; (290.1483) BSDF, BRDF, and BTDF; (290.5855) Scattering, polarization
\end{abstract}

\section{Introduction}

To achieve spherical direct-drive implosions at the National Ignition Facility at Lawrence Livermore National Laboratory, beam-smoothing techniques, such as illumination with different polarization states, are employed to avoid beam modulation on target [1]. Efforts have been made at the Laboratory for Laser Energetics (LLE) to create a distributed polarization rotator (DPR) to accomplish this goal [2]. Although they have many benefits, birefringent thin films can have appreciable scatter from their microstructure, which can create amplitude modulation at the target, reducing the efficacy of the DPR. Collaboration between LLE and the National Institute of Standards and Technology (NIST) has begun to measure scattered light fields at $351 \mathrm{~nm}$. To help optimize DPR manufacturing, it is necessary to understand the source of the scattered radiation and the directions in which it radiates.

The birefringent coatings are fabricated using glancing angle deposition (GLAD), whereby material is deposited at very high angles onto a substrate. The coatings are deposited in alternating directions through the layer thickness - a method known as serial bideposition - to suppress column broadening and extinction, creating vertical columns of material with elliptical cross sections [3]. Figure 1(a) shows a typical scanning electron microscopy (SEM) image of a cross section. The optical properties of the coating will change based on the density of the columns, the material used, the ellipticity of the columns, and the angle at which the material is deposited [4]. The goal is to create a quarter-wave coating and to pattern that coating with alternating optical axes. The coating will be index-matched to the substrate to minimize inter-reflections. Currently, magnesium oxide (MgO) is the material of choice because of its refractive index, stress, and deposition properties.

(a)

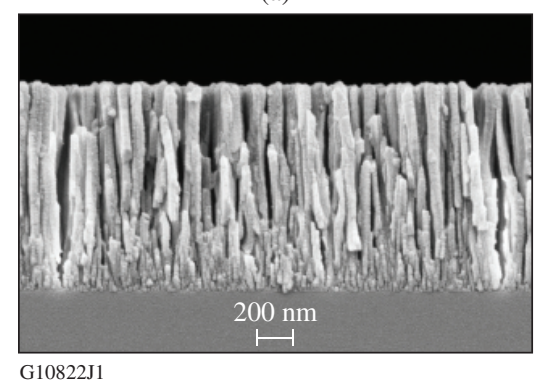

(b)

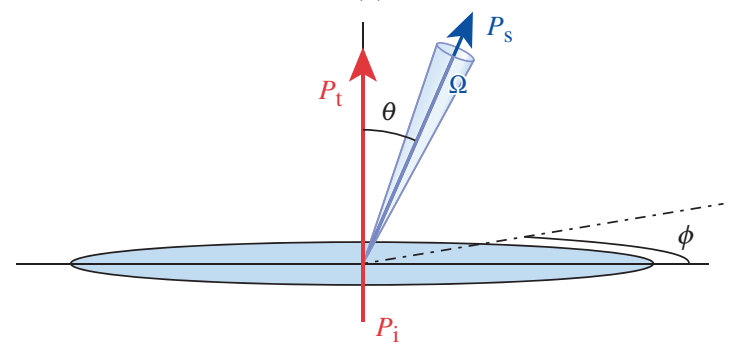

Fig. 1. (a) SEM image of an optic similar to Sample C and (b) the geometry of the scattered-light measurements.

\section{Scatter measurements}

A goniometric optical scatter instrument [5] was used to measure optical scatter and polarization effects of a variety of GLAD coatings. The instrument measures both the forward and backward scattered radiation. Figure 1(b) shows the geometry of the measurement for reflection and transmission.

The scatter was measured over many directions in both polar angle $\theta$ and azimuthal angle $\phi$. The reported bidirectional scattering distribution function (BSDF) is $f_{r}=P_{\mathrm{i}} /\left(P_{\mathrm{s}} \Omega \cos \theta\right)$ where the incident power is $P_{\mathrm{i}}$, the scattered power is $P_{\mathrm{s}}$, and the solid angle subtended by the detector is $\Omega$. Data were taken such that the incident power direction was normal to the substrate surface and the polarization was oriented $45^{\circ}$ to the deposition direction. These conditions match the geometry and polarization for which the DPR will be used. The BSDF can be integrated to yield the total hemispherical scatter level. 


\section{GLAD DPR scatter measurements}

Table 1 describes some of the manufacturing parameters of each sample measured. The main difference between Sample A and Sample B is the dipped sol-gel antireflective (AR) coating, which changed the retardance properties and likely contributed to the increase in scattered light. Figure 2 shows the transmitted BSDF as a function of projected cosine space. The center of each image corresponds to the normally transmitted beam, and the detector is blocked by parts of the instrument in the dark corner points. It is noted that the larger retardance of Part $\mathrm{C}$ did not correlate to more scatter, as originally expected. Overall, scatter in the backward hemisphere was very low (less $<1 \%$ ) and showed minimal directionality. The standard uncertainties in the BSDF and integrated measurements are expected to be below $2 \%$ of the stated values.

Table 1. Sample information and integrated scatter results.

\begin{tabular}{|c|c|c|c|c|c|}
\hline \hline Part & $\begin{array}{c}\text { Deposition } \\
\text { Angle }\end{array}$ & $\begin{array}{c}\text { Estimated Film } \\
\text { Thickness }\end{array}$ & Retardance & $\begin{array}{c}\text { Sol-gel antireflective } \\
\text { coating }\end{array}$ & $\begin{array}{c}\text { Transmitted } \\
\text { Scatter }\end{array}$ \\
\hline $\mathrm{A}$ & $73^{\circ}$ & $1.14 \mu \mathrm{m}$ & $55 \mathrm{~nm}$ & yes & $2.77 \%$ \\
\hline $\mathrm{B}$ & $73^{\circ}$ & $1.14 \mu \mathrm{m}$ & $89 \mathrm{~nm}$ & no & $1.65 \%$ \\
\hline $\mathrm{C}$ & $58^{\circ}$ & $1.15 \mu \mathrm{m}$ & $96 \mathrm{~nm}$ & no & $1.69 \%$ \\
\hline \hline
\end{tabular}

(a)

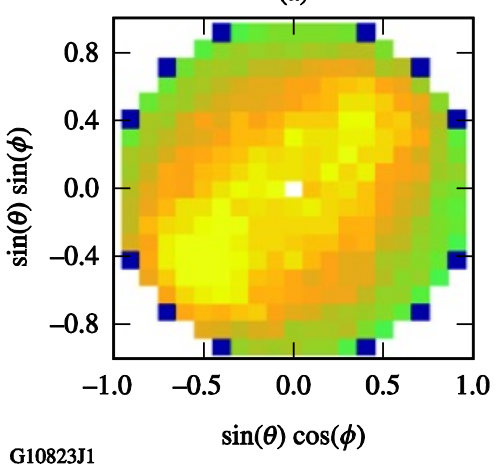

(b)

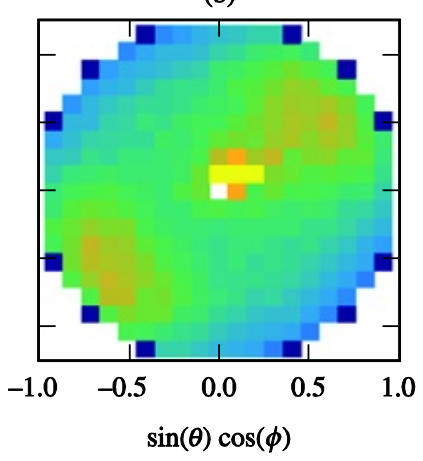

(c)

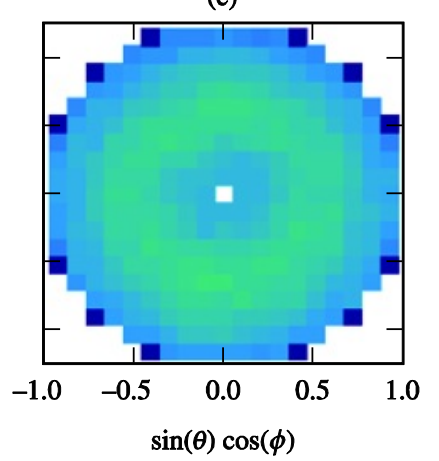

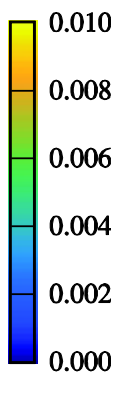

Fig. 2. BSDF in the forward, transmitted hemisphere projected on a 2-D plane for (a) Part A, (b) Part B, and (c) Part C. Data are shown in BSDF units $\left(\mathrm{sr}^{-1}\right)$, and the axes are defined by the direction cosines.

Future work will focus on ellipsometry of the birefringent MgO GLAD coatings and modeling of the interaction of the electric field with serial bideposition columnar structures. More exploration is needed to correlate scattered light with variations in deposition angle, film thickness, and retardance.

The authors acknowledge funding from the Frank J. Horton Fellowship at the Laboratory for Laser Energetics. This material is based upon work supported by the Department of Energy National Nuclear Security Administration under Award Number DE-NA0001944, the University of Rochester, and the New York State Energy Research and Development Authority. The support of DOE does not constitute an endorsement by DOE of the views expressed in this article.

\section{References}

[1] J. E. Rothenberg, "Polarization beam smoothing for inertial confinement fusion,” J. Appl. Phys. 87, 3654-3662 (2000).

[2] J. B. Oliver, T. J. Kessler, C. Smith, B. Taylor, V. Gruschow, J. Hettrick, and B. Charles, "Electron-beam-deposited distributed polarization rotator for high-power laser applications," Opt. Express 22, 23,883-23,896 (2014).

[3] M. M. Hawkeye, M. T. Taschuk, and M. J. Brett, Glancing Angle Deposition of Thin Films: Engineering the Nanoscale, Wiley Series in Materials for Electronic \& Optoelectronic Applications (Wiley, Chichester, United Kingdom, 2014), pp. 240-244.

[4] M. W. McCall, I. J. Hodgkinson, and Q. Wu, Birefringent Thin Films and Polarizing Elements, 2nd ed. (Imperial College Press, London, 2015).

[5] T. A. Germer and C. C. Asmail, "Goniometric optical scatter instrument for out-of-plane ellipsometry measurements," Rev. Sci. Instrum. 70, 3688-3695 (1999).

[6] J. B. Oliver, C. Smith, J. Spaulding, A. L. Rigatti, B. Charles, S. Papernov, B. Taylor, J. Foster, C. W. Carr, R. Luthi, B. Hollingsworth, and D. Cross, "Glancing-angle-deposited magnesium oxide films for high-fluence applications," to be published in Optical Materials Express. 


\title{
Stochastic Single Flux Quantum Neuromorphic Computing using Magnetically Tunable Josephson Junctions
}

\author{
Stephen E. Russek, Christine A. Donnelly, Michael L. Schneider, Burm Baek, Mathew R. Pufall, William H. Rippard, \\ Peter F. Hopkins, Paul D. Dresselhaus, Samuel P. Benz \\ NIST, Boulder CO
}

\begin{abstract}
Single flux quantum (SFQ) circuits form a natural neuromorphic technology with SFQ pulses and superconducting transmission lines simulating action potentials and axons, respectively. Here we present a new component, magnetic Josephson junctions, that have a tunablility and reconfigurability that was lacking from previous SFQ neuromorphic circuits. The nanoscale magnetic structure acts as a tunable synaptic constituent that modifies the junction critical current. These circuits can operate near the thermal limit where stochastic firing of the neurons is an essential component of the technology. This technology has the ability to create complex neural systems with greater than $10^{21}$ neural firings per second with approximately $1 \mathrm{~W}$ dissipation.
\end{abstract}

Keywords-single flux quantum, neuromorphic, magnetic Josephson junctions

\section{INTRODUCTION}

Single flux quantum (SFQ) logic[1-3] relies on voltage pulses generated by $2 \pi$ phase slips of the superconducting order parameter across a Josephson junction. These voltage pulses have a time-integrated amplitude given by the flux quantum $\phi_{0}=2.068 \times 10^{-15} \mathrm{Vs}$. Complex logic circuits have been fabricated using this technology and major efforts are ongoing to fabricate a new class of low power SFQ supercomputers.[4] Neuromorphic variations of SFQ logic have been proposed and fabricated.[5-7] These papers have highlighted the natural analogy between SFQ pulse trains and action potentials. However, key components of neural operation, such as neuromorphic synapses that are dynamically reconfigurable and system operation near the thermal limit, have not been addressed. Here, we introduce magnetic Josephson junctions based on reconfigurable magnetic nanoclusters, as a new component for neuromorphic SFQ. Further, we develop stochastic models required to characterize and design SFQ neural circuits which, to achiever ultra-low power, need to operate near the thermal limit. In addition to achieving ultra-low power, operating in the partially stochastic regime has been identified as important for efficient neuromorphic learning systems.[8]

Design tools and state-of-the-art fabrication facilities have been developed for SFQ logic circuits including fabrication on $200 \mathrm{~mm}$ wafers, planarized multilayer wiring and high junction uniformity.[9] These capabilities can be leveraged to develop high-complexity very fast neural systems that may be suitable for both application specific computing such as image analysis and, more importantly, for computations that require cognitive processes.

While SFQ logic circuits require a high degree of device uniformity, this constraint is greatly relaxed for neuromorphic circuits. Further, SFQ logic requires precise timing and clock distribution, at frequencies of $10 \mathrm{GHz}$ and above, which can be very challenging, while neuromorphic SFQ is asynchronous (although strongly correlated). Finally, SFQ circuits usually require significant bias currents, typically $100 \mu \mathrm{A}$ per device, which can lead to a prohibitively large total current when device counts are on the order of $10^{9}$. Stochastic SFQ works very close to equilibrium and does not require significant bias currents.

\section{STOCHASTIC JOSEPHSON JUNCTIONS}

A standard circuit model of a Josephson junction is shown in Fig. 1 along with the equations dictating the junction dynamics.[10] The supercurrent is given by $I_{s}=I_{c} \sin (\theta)$, where $\theta$ is the change of the phase of the superconducting order parameter across the junction, $V$ is the voltage across the junction which is proportional to the time derivative of $\theta, I_{c}$ is the critical current, $R_{n}$ is the normal state resistance of the device, $C$ is the junction capacitance, $I_{n}$ is the thermal noise term, and $I_{b}$ is the total current through the junction. Here, $I_{n}$ is assumed to be random Gaussian noise with rms amplitude of $\left(4 \mathrm{k}_{\mathrm{B}} T / R_{n} \tau\right)^{0.5}$, where $\mathrm{k}_{\mathrm{B}}$ is Boltzmann's constant, $T$ is the device temperature, and $\tau$ is the noise sampling time used in modeling. The resulting dynamics are the same as a driven damped pendulum. The energy barrier, the difference between the energy at $\theta=0$ and $\theta=\pi$, is given by $I_{c} \phi_{0} / 2 \pi$. For stochastic operation (as shown in the modelling below) we require $I_{c} \phi_{0} / k_{B} T \approx 10$ to 20 , with the exact value being an important circuit parameter. The damping is given by the McCumber parameter $\beta_{c}=2 \pi I_{c} R_{n}{ }^{2} C / \phi_{0}$. Most SFQ circuits require the junctions to be close to critical damping with $\beta_{c} \approx 1$.

Fig. 2 shows numerical solutions of the dynamical equations for a typical stochastic SFQ junction with an 


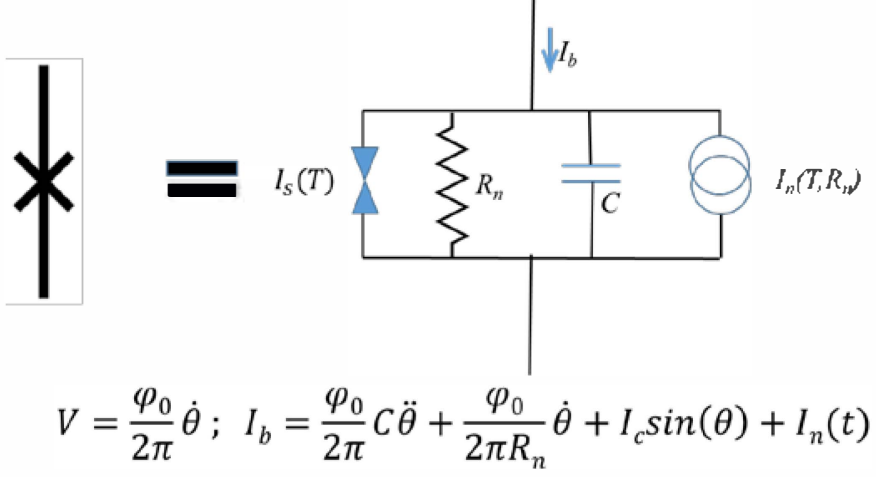

Figure 1 Circuit model of a Josephson junction, along with the corresponding dynamical equations.

operation temperature of $4 \mathrm{~K}$ and a critical current of $0.5 \mu \mathrm{A}$. Fig. $2 \mathrm{~b}$ shows the resting state spiking characteristic of the junction, while Figs. 2a, c show the spiking with small applied bias currents of $\pm 0.1 \mu \mathrm{A}$. Unlike real neural systems, the resting state can generate either positive or negative pulses and the pulse durations are $<100$ ps. We see that small bias currents can stimulate strong pulse trains. The bias current can be viewed as integrated inputs from other neural spike chains.

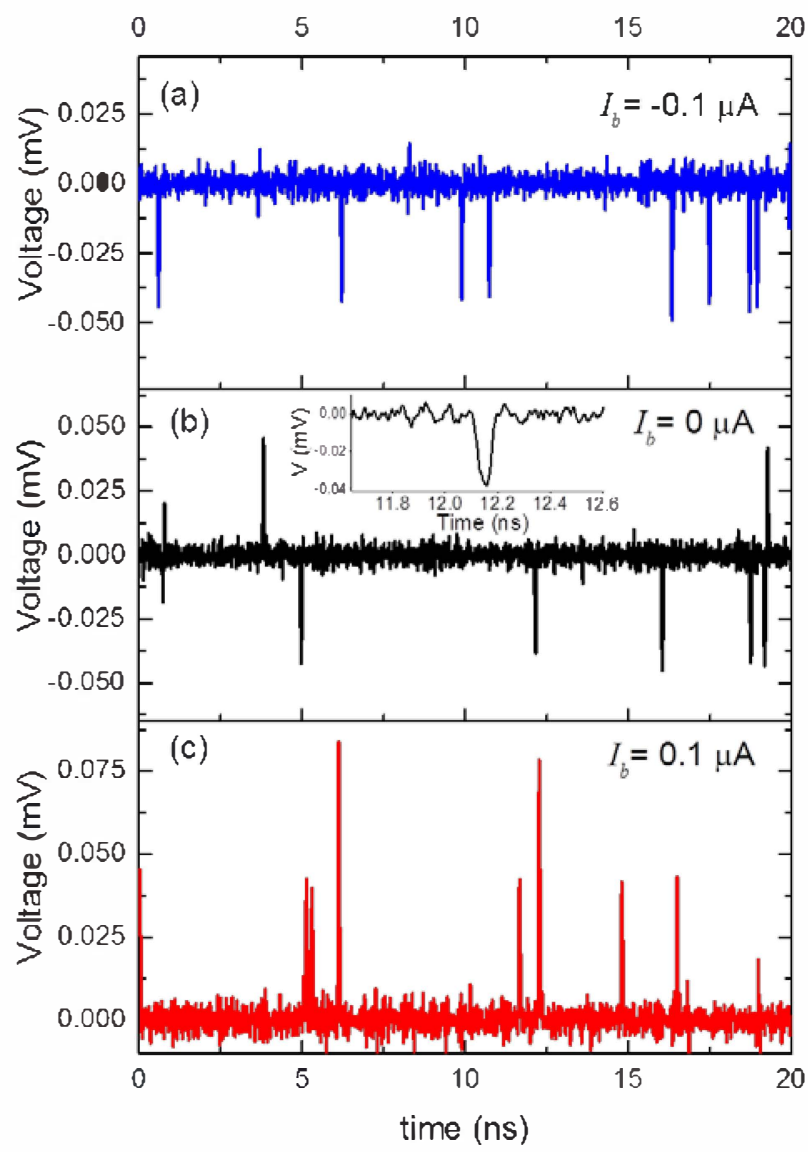

Figure 2 Simulations of spiking characteristics of a Josephson jucntion with $0.5 \mu \mathrm{m}$ diameter, $2 \mathrm{~nm}$ thick Si barrier, $I_{c}=0.5 \mu \mathrm{A}, R_{n}=400 \Omega, \mathrm{T}=4 \mathrm{~K}$. $I_{c} \phi_{0} / k_{B} T=18$ : a) $-0.1 \mu \mathrm{A}$ bias current, b) resting state spiking, c) spiking with $+0.1 \mu \mathrm{A}$ bias currents. The circuit bandwidth gives a pulse width near $50 \mathrm{ps}$.
Fig. 3 shows the spiking characteristic of two otherwise identical junctions with different critical currents, $I_{c}=0.5 \mu \mathrm{A}$ and $1.0 \mu \mathrm{A}$. Since the spiking rate is exponentially dependent on the critical current, a small change in critical current has a dramatic effect in the spiking characteristics. This type of $I_{c}$ change is what we hope to dynamically produce through the use of magnetically tunable junctions discussed in the next section.

The spiking rate shown in Fig. $3 \mathrm{~b}$ is approximately $10^{10}$ single flux quantum per second. The power dissipated for a single active SFQ neuron, the bias current times the average voltage, is therefore $10^{10} \mathrm{~s}^{-1} I_{b} \phi_{0} \approx 1 \times 10^{-11} \mathrm{~W}$. Hence, large scale neural circuits can be fabricated with up to $10^{11}$ neurons and $10^{21}$ firings per second that consume less than $1 \mathrm{~W}$ of power (this does not include the refrigeration power).

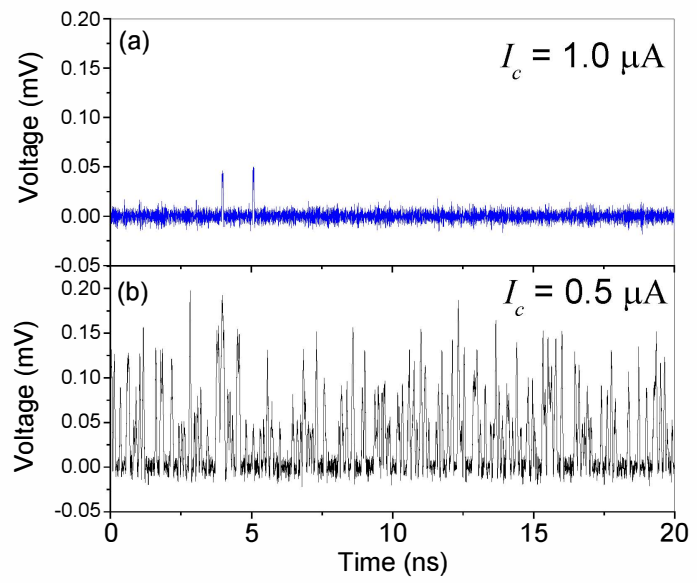

Figure 3 Simulations of spiking of a $0.5 \mu \mathrm{m}$ diameter $400 \Omega$ junction at $4.2 \mathrm{~K}$ for critical currents of a) $1.0 \mu \mathrm{A}$ and b) $0.5 \mu \mathrm{A}$ and a bias current of $0.3 \mu \mathrm{A}$. The $I_{c}=0.5 \mu \mathrm{A}$ junction shows spikes corresponding to 1,2 and 3 flux quanta.

\section{MAGNETIC JOSEPHSON JUNCTIONS}

Magnetic Josephson junctions have recently received a lot of attention due to their potential use as compact nonvolatile memory elements in SFQ circuits. It has been shown that by changing the magnetic state of a spin valve incorporated into a Josephson junction, one can modulate both the amplitude and phase of the supercurrent.[11-16] For carefully chosen thicknesses of the magnetic layers, the modulation of the critical current can be greater than $100 \%$.[17] Further, it has been shown that in small devices of less than $100 \mathrm{~nm}$ diameter, the magnetic state can be changed by current pulses though the device via spin transfer torque.[17, 18]

An existing drawback of the digital magnetic Josephson devices is that large currents are required to switch them. This is due to the fact that the magnetic layers have large moments and that each electron can only transfer $\hbar$ of angular momentum. A large number of electrons are required to transfer the required angular momentum, while only a small percent of the energy of the electrons is used to overcome the magnetic energy barrier. To make the magnetic Josephson 
junction have an analog $I_{c}$ variation and to make switching more efficient, we fabricate Josephson junctions with Mn clusters embedded in the Si barrier, as shown in Fig. 4. These Josephson junctions, with the exception of Mn doping, are very similar to the ones used in NIST Josephson voltage standards.[19,20]

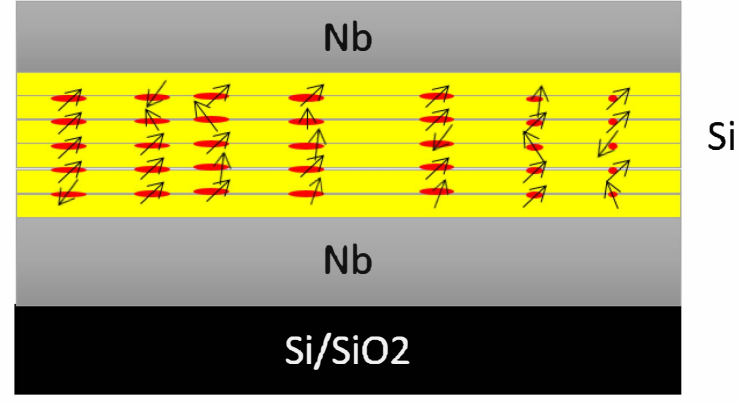

Figure 4. Schematic of magnetic Josephson junction with Mn clusters embedded in a Si barrier.

The junctions, as seen in Fig. 5, show nearly ideal currentvoltage characteristics for overdamped junctions, nearly ideal $I_{c}$ vs field characteristics, high uniformity as determined by measurements on large arrays, and high quality microwave response. The presence of the magnetic clusters can greatly suppress $I_{c}$ and it has been shown (to be reported in another publication) that $I_{c}$, as with other magnetic Josephson junction structures [11, 21-24], depends on the magnetic state. While the critical currents for the junctions shown here are quite large, for smaller junctions with dimensions of $100 \mathrm{~nm}$, which are appropriate for large scale neural networks, the critical currents will be on the order of a few microamps.

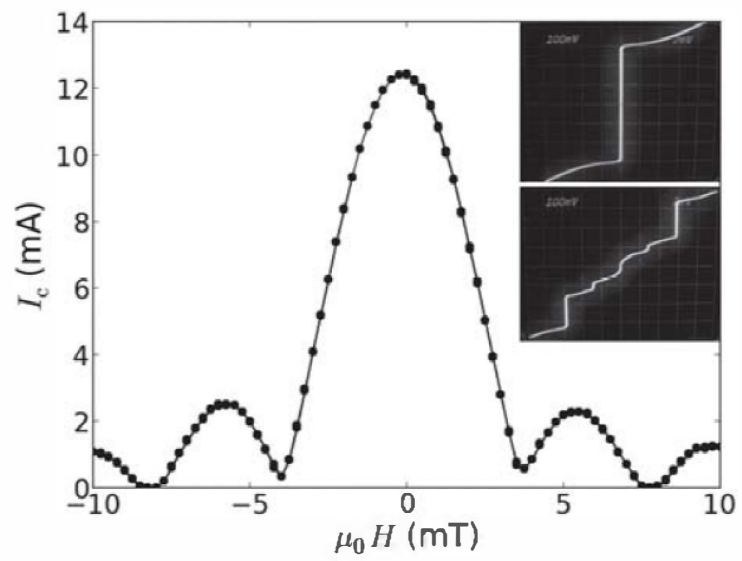

Figure 5 Critical current vs. magnetic field for a 3 um x $6 \mu \mathrm{m}$ Mn-doped Josephson junction.[23] The insets show the current-voltage curves for series array of 600 Mn-doped Josephson junctions with and without $4.7 \mathrm{GHz}$ microwaves applied.

The cluster size and switching energies are set by post deposition anneals. The anneals are done in a rapid thermal annealer at $573 \mathrm{~K}$ to $723 \mathrm{~K}$ for approximately $2 \mathrm{~min}$. Fig. 6 shows typical zero-field-cooled and field-cooled moment vs. temperature data. Below the blocking temperature, indicated by the vertical arrows, the Mn spins can be configured in either random or ordered states. Figure 6a shows a sample that was annealed at $573 \mathrm{~K}$ with a blocking temperature of $34 \mathrm{~K}$. Figure $6 \mathrm{~b}$ shows a sample that was annealed at $723 \mathrm{~K}$ with a blocking temperature of $240 \mathrm{~K}$. Figure 6 demonstrates the ability to readily change both the blocking temperature as well as the total moment by varying the annealing temperature. By varying the anneal time and temperature we have been able to vary the blocking temperature, $T_{B}$, from $10 \mathrm{~K}$ to $240 \mathrm{~K}$.

The lower the blocking temperature, the easier the magnetic clusters are to switch, with a tradeoff of loss of thermal stability. The switching energy for a magnetic cluster is given approximately by $E_{s} \approx \ln \left(t_{m} / t_{a}\right) \quad \mathrm{k}_{\mathrm{B}} T_{B}$, where $t_{m}$ is a measurement time, and $t_{a}$ is an attempt time. For a blocking temperature of $30 \mathrm{~K}, E_{s} \approx 70 \mathrm{meV}$. The cluster moment can be estimated from Langevin fits to moment versus field curves above the blocking temperature. These fits give the cluster moments of a few Bohr magnetons to several hundred Bohr magnetons, depending on the annealing conditions. A key element of these junctions is that the ratio of the switching energy to the angular momentum of the clusters is close to the ratio of energy to angular momentum of an electron, indicating that efficient energy and angular momentum transfer can occur.
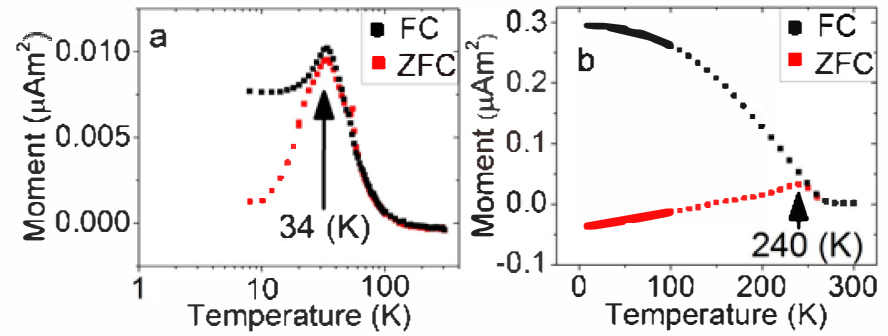

Figure 6 Zero field cooled (red) and field cooled (black) magnetic moment plots of a Mn-doped Josephson juntion showing hysteretic magnetic states below the blocking temperature, indicated by the vertical arrows.

\section{MAgnetic Jospehson Junction DeVice Model}

To develop complex neural circuits it is essential to have a device model that can be incorporated into large scale circuit simulations. We have modified existing Verilog A models of a standard Josephson junction to accommodate an internal magnetic order parameter. Fig. 7 shows the circuit model of a nanocluster magnetic Josephson junction, now with the supercurrent a function of the magnetic order parameter $m$, which in turn is a function of the integrated voltage history. The parameter $m$ is zero when the spins are disordered and 1 when they are completely aligned. Positive applied voltage pulses will drive the system to a disordered state with $m=0$, while negative pulses will drive the system to $m=1$.

Here we ignore the dependence of the phase on the magnetic order parameter, $\delta(m)=$ constant, and only allow the critical current to vary. As with the spin valve Josephson junctions, the critical current is maximized in the state with unaligned spins and minimized in the state with spins aligned. 
The temperature is a critical experimental parameter that can simultaneously scale all critical currents to allow evaluation of the performance with different levels of resting state activity. At present, this model is not validated by experimental data. It represents the simplest dependence of $I_{c}$ on $m$ to allow simulations to determine the utility of such a device in complex neural circuits.

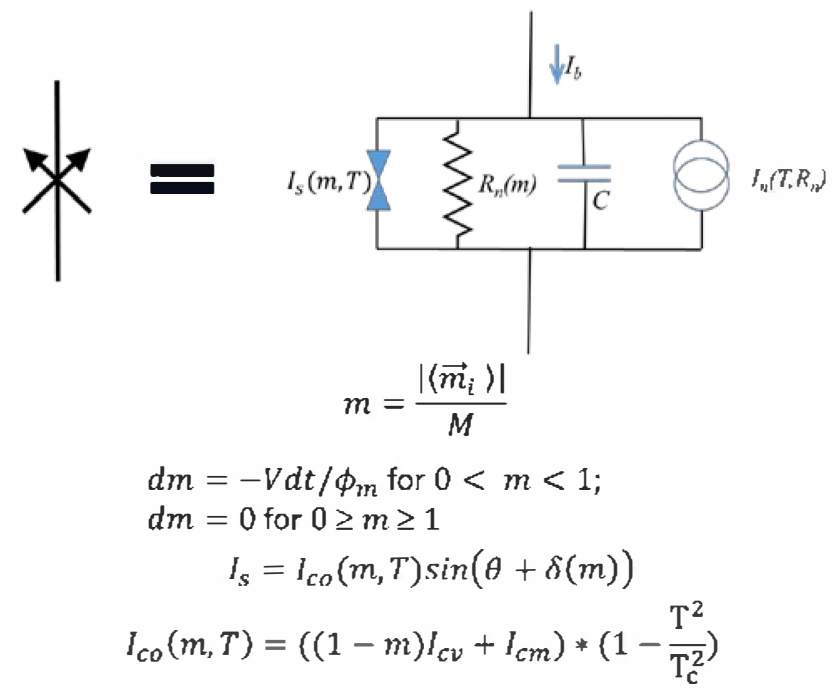

Figure 7 Circuit model of a nanocluster magnetic Josephson junction along with model equations describing dependence of $I_{c}$ on the magnetic order parameter.

Given this device model, the performance of devices and circuits can be modeled with SPICE as shown in Fig. 8. This simulation shows similar device response as seen in Fig.1, but now with dynamically variable critical current and done in SPICE, which is capable of extension to large scale circuit simulation. Fig. $8 \mathrm{~b}$ shows the dynamical response, voltage vs. time, of a magnetic cluster Josephson junction, at $T=0 \mathrm{~K}$, for large negative bias, zero bias, and large positive bias. The voltage oscillation amplitude is seen to first decrease with time in the negative current bias regime and then increase with time in the positive current bias regime. This corresponds to a time dependent decrease in the critical current during negative bias (negative input pulses) and an increase in critical current during positive bias. As shown in Fig. 3, this change in critical current can activate a magnetic Josephson junction or deactivate it. An input of negative pulses to a junction will increase $I_{c}$ causing it to fire more strongly, while a series of positive input pulses will raise $I_{c}$ preventing future firings.

\section{SUMMARY}

We have introduced nanocluster magnetic Josephson junctions as a potential synaptic component for SFQ neural circuits. We have demonstrated that in the stochastic limit the spiking behavior can be made very sensitive to small changes in critical currents that can be obtained by changing the magnetic structures in magnetic Josephson junctions. We have shown that operating in the stochastic limit can achieve much lower power and eliminate the need for large total bias currents. Finally, we have introduced stochastic circuit models that can be used in large scale SPICE simulations of SFQ neural circuits.

\section{ACKNOWLEDGMENT}

We thank the IARPA C3 program for providing inspiration and guidance for this work.
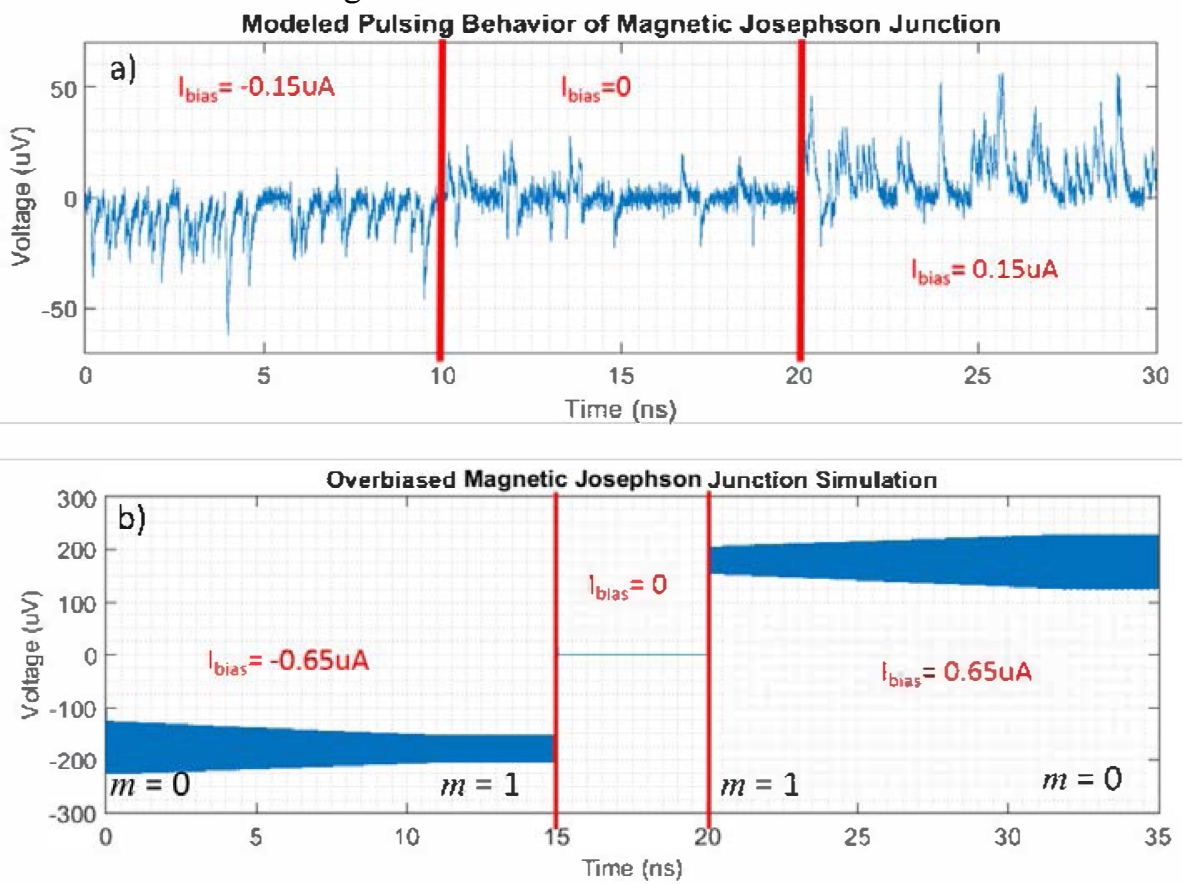

Figure 8 SPICE simulations showing voltage vs. time of a magnetic Josephson junction, with a critical current of $0.5 \mu \mathrm{A}$, when time dependent bias currents are applied. a) Stochastic spiking behavior for a junction at $\mathrm{T}=4 \mathrm{~K}$ with bias currents of $-0.15 \mu \mathrm{A}, 0 \mu \mathrm{A}$, and $0.15 \mu \mathrm{A}$. b) Oscillation behavior at $\mathrm{T}=0 \mathrm{~K}$, with applied bias currents larger than $I_{c}$ showing dynamic reconfigurablilty of the junction critical current. 


\section{REFERENCES}

1 Likharev, K.K., and Semenov, V.K.: 'RSFQ logic/memory family: a new Josephson-junction technology for subterahertz-clock-frequency digital systems', IEEE Transactions on Applied Superconductivity, 1991, 1, (1), pp. 3-28 Bunyk, P., Likharev, K., and Zinoviev, D.: 'RSFQ Technology: Physics and Devices', International Journal of High Speed Electronics and Systems, 2001, 11, (01), pp. 257-305

3 Dorojevets, M., Chen, Z., Ayala, C.L., and Kasperek, A.K.: 'Towards 32-bit Energy-Efficient Superconductor RQL Processors: The Cell-Level Design and Analysis of Key Processing and On-Chip Storage Units', IEEE Transactions on Applied Superconductivity, 2015, 25, (3), pp. 1-8 Holmes, D.S., Ripple, A.L., and Manheimer, M.A.: 'Energy-Efficient Superconducting Computing; Power Budgets and Requirements', IEEE Transactions on Applied Superconductivity, 2013, 23, (3), pp. 1701610-1701610

Hirose, T., Asai, T., and Amemiya, Y.: 'Pulsed neural networks consisting of single-flux-quantum spiking neurons', Physica C: Superconductivity and its Applications, 2007, 463-465, pp. 1072-1075 Onomi, T., Kondo, T., and Nakajima, K.: 'High-speed single flux-quantum up/down counter for neural computation using stochastic logic', Journal of Physics: Conference Series, 2008, 97, pp. 012187 Crotty, P., Schult, D., and Segall, K.: 'Josephson junction simulation of neurons', Phys Rev E Stat Nonlin Soft Matter Phys, 2010, 82, (1 Pt 1), pp. 011914 Neftci, E.O., Pedroni, B.U., Joshi, S., Al-Shedivat, M., and Cauwenberghs, G.: 'Stochastic Synapses Enable Efficient Brain-Inspired Learning Machines', Front Neurosci, 2016, 10, pp. 241

Tolpygo, S.K., Bolkhovsky, V., Weir, T.J., Johnson, L.M., Gouker, M.A., and Oliver, W.D.: 'Fabrication Process and Properties of Fully-Planarized Deep-Submicron Nb/Al2O3/Nb Josephson Junctions for VLSI Circuits', IEEE Transactions on Applied Superconductivity, 2015, 25, (3), pp. 1-12 Van Duzer, T., and Turner, C.W.: 'Principles of superconductive devices and circuits' (Elsevier, 1981. 1981) Bell, C., Burnell, G., Leung, C.W., Tarte, E.J., Kang, D.J., and Blamire, M.G.: 'Controllable Josephson current through a pseudospin-valve structure', Applied Physics Letters, 2004, 84, (7), pp. 1153-1155 Abd El Qader, M., Singh, R.K., Galvin, S.N., Yu, L., Rowell, J.M., and Newman, N.: 'Switching at small magnetic fields in Josephson junctions fabricated with ferromagnetic barrier layers', Applied Physics Letters, 2014, 104, (2), pp. 022602 Baek, B., Rippard, W.H., Benz, S.P., Russek, S.E., and Dresselhaus, P.D.: 'Hybrid superconducting-magnetic memory device using competing order parameters', Nature communications, 2014, 5, pp. 3888

14 Banerjee, N., Robinson, J.W.A., and Blamire, M.G.: 'Reversible control of spin-polarized supercurrents in ferromagnetic Josephson junctions', Nature communications, 2014, 5

15 Niedzielski, B.M., Gingrich, E.C., Loloee, R., Pratt, W.P., and Birge, N.O.: 'S/F/S Josephson junctions with singledomain ferromagnets for memory applications', Superconductor Science and Technology, 2015, 28, (8), pp. 085012 Gingrich, E.C., Niedzielski, B.M., Glick, J.A., Wang, Y., Miller, D.L., Loloee, R., Pratt Jr, W.P., and Birge, N.O.: 'Controllable 0-[pi] Josephson junctions containing a ferromagnetic spin valve', Nat Phys, 2016, advance online publication

Baek, B., Rippard, W.H., Pufall, M.R., Benz, S.P., Russek, S.E., Rogalla, H., and Dresselhaus, P.D.: 'Spin-Transfer Torque Switching in Nanopillar Superconducting-Magnetic Hybrid Josephson Junctions', Physical Review Applied, 2015, 3, (1) 1190-1216

Olaya, D., Dresselhaus, P.D., Benz, S.P., Herr, A., Herr, Q.P., Ioannidis, A.G., Miller, D.L., and Kleinsasser, A.W.: 'Digital circuits using self-shunted $\mathrm{Nb} / \mathrm{Nb}_{\mathrm{x}} \mathrm{Si}_{1-\mathrm{x}} / \mathrm{Nb}$ Josephson junctions', Applied Physics Letters, 2010, 96, (21), pp. 213510

Tang, Y., Ojha, V.N., Schlamminger, S., Ruefenacht, A., Burroughs, C.J., Dresselhaus, P.D., and Benz, S.P.: 'A 10 V programmable Josephson voltage standard and its applications for voltage metrology', Metrologia, 2012, 49, (6), pp. 635-643 superconductors through a ferromagnet: Evidence for a pi junction', Physical Review Letters, 2001, 86, (11), pp. 24272430 ferromagnetic layer: Negative coupling', Physical Review Letters, 2002, 89, (13) Blum, Y., Tsukernik, A., Karpovski, M., and Palevski, A.: 'Oscillations of the superconducting critical current in Nb-Cu$\mathrm{Ni}-\mathrm{Cu}-\mathrm{Nb}$ junctions', Physical Review Letters, 2002, 89, (18)

24 Baek, B., Benz, S.P., Rippard, W.H., Russek, S.E., Dresselhaus, P.D., Pufall, M.R., Rogalla, H., and Ieee: 'Magnetic barrier structures for superconducting-magnetic hybrid Josephson junctions', 2013 Ieee 14th International Superconductive Electronics Conference (Isec), 2013 


\title{
Color Quality Metrics - Recent Progress and Future Perspective
}

\author{
Y. Ohno \\ Sensor Science Division, National Institute of Standards and Technology \\ 100 Bureau Drive, Gaithersburg Maryland 20899 USA
}

\begin{abstract}
The color quality of light sources for lighting includes the effects of white light chromaticity, as well as color rendition characteristics, including color fidelity and the aspects related to color preference and perception. In this paper, the current standards and the latest research on white light chromaticity and color saturation preferences are overviewed. The characteristics of TM-30 Fidelity Index and Gamut Index from the Illuminating Engineering Society (IES) are examined in comparison to the Color Rendering Index (CRI). Current activities in International Commission on Illumination (CIE) related to these and future perspective of color quality metrics are discussed.
\end{abstract}

\section{INTRODUCTION}

It is critical for light-emitting diode (LED) sources for general lighting to have acceptable color quality suitable for intended applications. There are two primary aspects of color quality of lighting sources: white light chromaticity and color rendition. These two aspects are interrelated, and both of these characteristics are critical for general lighting. The chromaticity ranges for general lighting products have been well standardized for many years [1, 2]. However, new research is raising questions about whether the current chromaticity ranges are most appropriate for all lighting applications. The current standards and recent studies on white light chromaticity are overviewed and discussed in section 2. Color rendition is considered largely in two aspects - color fidelity and an aspect related to color preferences. The current standardized metric, Color Rendering Index (CRI) [3], is designed to evaluate color fidelity. However, color fidelity does not always correlate well with visual evaluations of the color quality of lighting, and thus there is a need for another metric that evaluates these aspects of color preference and perception. This issue is discussed in section 3 with the results of recent studies conducted at National Institute of Standards and Technology (NIST). TM30 [4] from the Illuminating Engineering Society of North America (IES) was recently published as a proposed new method for evaluating color rendition. This metric is discussed and compared to CRI in section 4, and the related CIE activities and future considerations are discussed.

\section{WHITE LIGHT CHROMATICITY STANDARDS AND RECENT RESERCH}

The chromaticity of light sources for general lighting is normally chosen to be around the Planckian locus or the daylight locus, which are generally considered as the center locations for white light, as specified in the existing standards $[1,2]$. Figure 1, for example, shows the American National Standards Institute (ANSI) chromaticity specifications for solid state lighting products [2]. While these standards are widely used, new research raises questions as to whether the current chromaticity ranges are most appropriate for all lighting applications. Shifts in chromaticity across the Planckian locus are expressed by the term, Duv (symbol $D_{\text {uv }}$ ), defined in the standard [2] as shown in Fig. 2, and its use is discussed in elsewhere [5].

A recent study on a vision experiment [6] reported that perceived neutral white points determined by the subjects were $D_{\mathrm{uv}} \approx-0.01$ at $2700 \mathrm{~K}$ to $3500 \mathrm{~K}, D_{\mathrm{uv}} \approx 0.00$ at

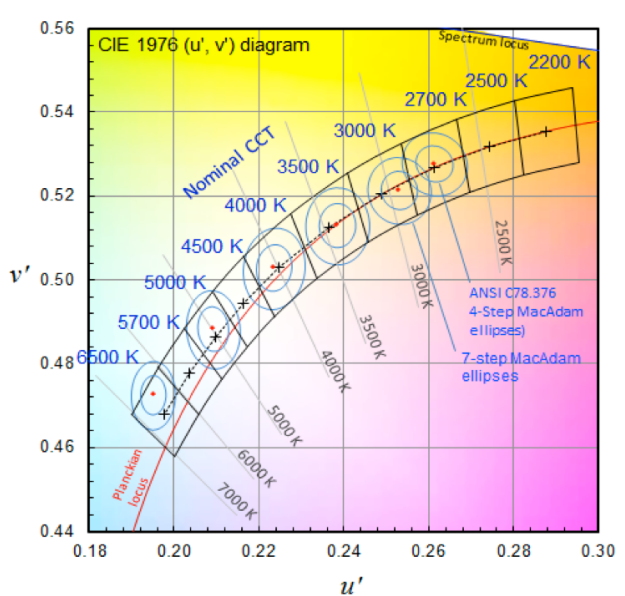

Fig. 1 The chromaticity specifications for solid-state lighting products by ANSI C78.377, 2015 revision [2] on the CIE (u', v') diagram. 
$4000 \mathrm{~K}$, and $D_{\mathrm{uv}} \approx 0.005$ at $6500 \mathrm{~K}$ (though the report did not use the Duv measure). This experiment was done with a lighting booth with white inner walls and no objects inside. Another experimental study [7] showed that subjects, viewing an office miniature, freely adjusted the chromaticity of illumination as they preferred, choosing points mostly below the Planckain locus, with an average Duv around -0.014 .

In 2013, vision experiments were conducted at NIST for white chromaticity points perceived most natural in a real-size interior room setting using the NIST Spectrally Tunable Lighting Facility (STLF) [8], participated by 18 subjects. The average results are plotted with the ANSI specifications in Fig. 3. The results showed that the lights with average $D_{\mathrm{uv}} \approx-0.015$, far below the Planckian locus, appeared most natural to the subjects [9]. The results from this experiment were verified with additional experiments conducted in 2015 [10] with 21 subjects, using modified spectra of lights, addressing the question raised by other researchers [11]. Further studies are desired to verify the applicability of these results in various real application conditions.

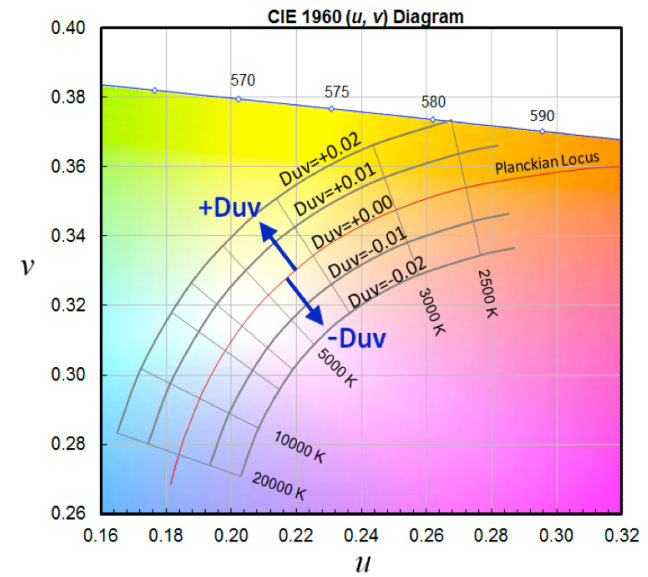

Figure 2. The scale of Duv shown on the CIE $1960(u, v)$ diagram.

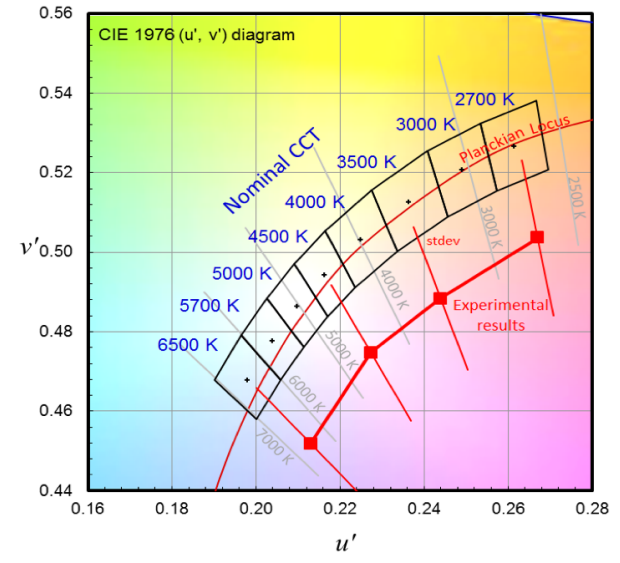

Figure 3. Average chromaticities judged to be most natural, plotted with ANSI C78.377 chromaticity specifications for SSL products.

\section{RECENT RESEARCH ON COLOR SATURATION PREFERENCE}

There have been many research articles about this topic, and it is generally well known that people prefer slightly over-saturated object colors in illuminated indoor scenes. Increased chroma, however, deviates from the fidelity, causing decreases in the CRI $R_{\mathrm{a}}$ score. To determine the level of chroma saturation that is generally most preferred, a large-scale vision experiment was conducted at NIST in 2014 using the NIST STLF [12]. The experiment was conducted with 20 subjects at correlated color temperatures (CCTs) of $2700 \mathrm{~K}, 3500 \mathrm{~K}, 5000 \mathrm{~K}$ (all $D_{\mathrm{uv}}=0$ ) and $3500 \mathrm{~K}$ with $D_{\mathrm{uv}}=-0.015$. There were four different viewing targets: mixed fruits and vegetables and the entire room, skin tone, red fruits/vegetables only, and green fruits/vegetables only. Pairs of lights were presented, one of which was always the neutral saturation (the same level as the CRI reference illuminant). Figure 4 shows the STLF room setting (left) and the nine different levels of chroma saturation used in the experiment (right). The right figure shows the plots of the 15 test color samples used in Color Quality Scale (CQS) [13]. Figure 5 shows the average results for all subjects and all CCT/Duv conditions for each viewing target. The vertical axis is the percentage that subjects preferred the light with a given chroma saturation (horizontal axis) over the neutral light $\left(\Delta C_{\mathrm{ab}}^{*}=0\right)$. The chroma saturation $\left(\Delta C_{\mathrm{ab}}^{*}\right)$ is calculated for the red test sample in the CQS. The results were unexpectedly consistent across all CCT/Duv conditions and for all targets, showing that the subjects most preferred the chroma increase $\Delta C_{\text {ab }}^{*}$ between 4 to 8 . The subjects' preference starts decreasing at larger saturations, as the objects start appearing unnatural. The subjects' preference at saturation level $\Delta C_{\mathrm{ab}}^{*} \approx 16$ (most saturated condition) is still comparable to that of the neutral saturation $\left(\Delta C_{\mathrm{ab}}^{*}=0\right)$. 

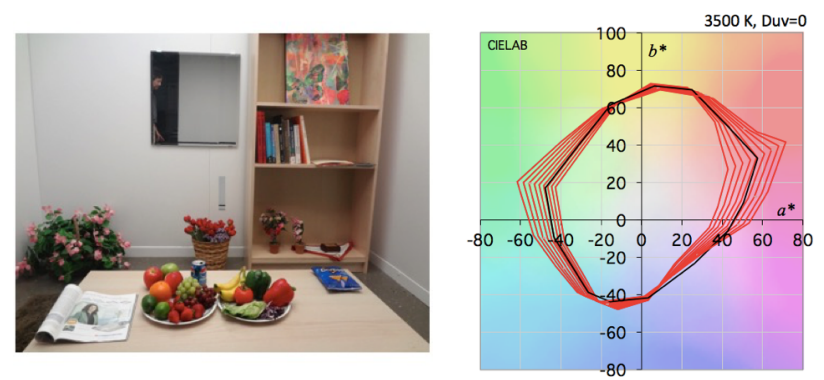

Figure 4. The experimental set up (left) and the CIELAB $\left(a^{*}, b^{*}\right)$ plots of the 15 CQS samples for the nine different saturation levels used for the NIST experiment $(3500 \mathrm{~K}$, $\left.D_{u v}=0\right)$.

Figure 6 shows the $3500 \mathrm{~K}$ results (average of all participants, all targets) plotted with the CRI $R_{\mathrm{a}}$ values and other metrics' values (discussed in section 4) for these lights. The $R_{\mathrm{a}}$ value sharply decreases as the chroma saturation increases from neutral. The CRI penalizes these preferred lights with increased chroma. For example, if $R_{\mathrm{a}} \geq 80$ is required in a regulation, many preferred lights (shaded area) are disqualified. This sharp slope results from the heavy weights in the red region in the $\mathrm{W} * \mathrm{U}^{*} \mathrm{~V}^{*}$ color space (see section 4 ). Further studies are desired on the effects for longerterm use of such preferred lights in real applications.

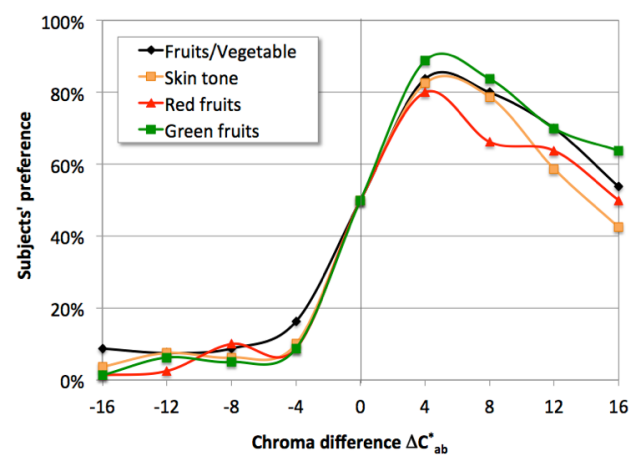

Figure 5. Subjects' preferences for all $C C T / D u v$ conditions for the different viewing targets, as a function of chroma difference from the reference illuminant.

\section{ANALYSES OF IES TM-30 COMPARED TO CRI}

The IES TM-30 [4] was developed to address the shortcomings of CRI experienced with LED light sources for general lighting [14]. A major problem is that CRI is a color fidelity metric and it does not assess other aspects related to color preference and perception of illuminated scenes. The CRI $R_{\mathrm{a}}$ scores do not correlate well with visual evaluation for preferred light sources as discussed in the previous sections. The earlier research, Color Quality Scale [13], attempted to address this issue, but could not be agreed in CIE as an update of CRI, mainly due to the difficulty in defining what it measures scientifically. The CIE then decided to take the direction to develop new metrics separately, one for color fidelity (dealt with by TC1-90) and another for the aspects other than color fidelity (dealt with by TC1-91). IES TM-30 was developed as a proposal from IES in this direction, to provide an improved color fidelity metric and a means to assess the aspects related to color preference. Thus, TM-30 is a two-metric system, consisting of Fidelity Index $R_{\mathrm{f}}$ and Gamut Index $R_{\mathrm{g}}$. The $R_{\mathrm{f}}$ is intended as an improvement of CRI $R_{\mathrm{a}}$ as a color fidelity metric. However, a color fidelity metric alone is not sufficient to assess all aspects of color rendition. Gamut Index is intended to provide preferencerelated information, though it does not directly indicate the degree of preference. A research article on the details of TM-30 is available [15].

One of the significant problems of CRI as a color fidelity metric is the very non-uniform object color space $\mathrm{W}^{*} \mathrm{U}^{*} \mathrm{~V}^{*}$ used to evaluate color differences of test color samples. Figure 7 shows how distorted the $\mathrm{W}^{*} \mathrm{U}^{*} \mathrm{~V}^{*}$ color space is for a $2700 \mathrm{~K}$ Planckian source and standard daylight (D65). As shown, this distortion is less prominent at higher CCTs. This distortion causes the color differences in the redgreen region (particularly red) to be weighted heavily and the yellow-green region very lightly 
weighted in CRI. This feature of CRI might be suitable for preference aspects, but is a significant problem as a color fidelity metric. CIE $1976 L^{*} a^{*} b^{*}$ (CIELAB) [16] is the current CIE standard object color space. CAM02UCS is the latest color space based on the color appearance model [17] and has been experimentally verified that the color differences calculated on this color space correlate well with the perceived color differences of objects of various colors, better than CIELAB.

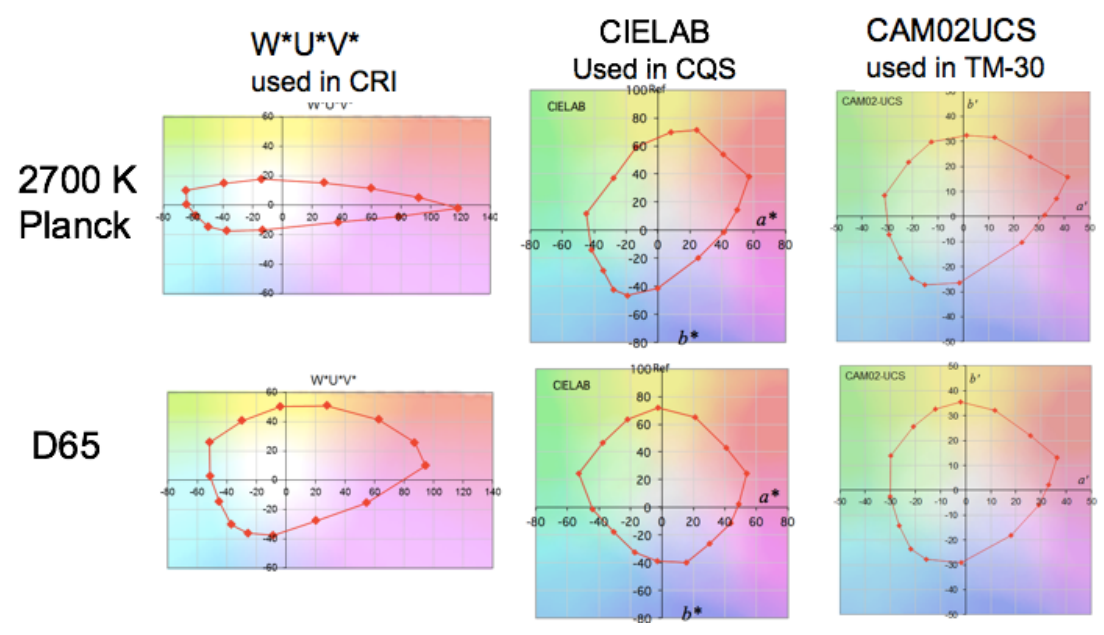

Figure 7. Two-dimensional plots of the 15 saturated color samples of CQS under $2700 \mathrm{~K}$ Planckian radiation and under D65 illumination on $W^{*} U^{*} V^{*}, C I E L A B$, and CAMO2UCS object color spaces.

Due to the nonuniformity of the color space, there are two cases of score deviation in CRI; (1) light sources that create relatively large chroma increases in yellow-blue direction (e.g., triphosphor fluorescent lamps and some phosphor-type LED lamps) tend to get higher CRI $R_{\mathrm{a}}$ score than $R_{\mathrm{f}},(2)$ light sources that create relatively large chroma increases in red-green directions (e.g., some RGB or RGBA LED sources) tend to get lower $R_{\mathrm{a}}$ scores than $R_{\mathrm{f}}$. Such examples of light source are shown in Fig. 8, where (a) and (b) are examples of case (1) above, and (c) is an example of case (2) above. Case (2) is also demonstrated in Fig. 6 above. Compared to CRI, TM-30 $R_{\mathrm{f}}$ is designed to evaluate the color differences of all hue colors evenly and more accurately as perceived by human observers.

(a) $R_{\mathrm{a}}=80, R_{\mathrm{f}}=75$
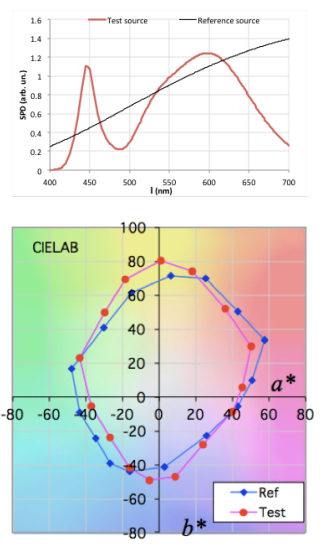

(b) $R_{\mathrm{a}}=92, R_{\mathrm{f}}=86$
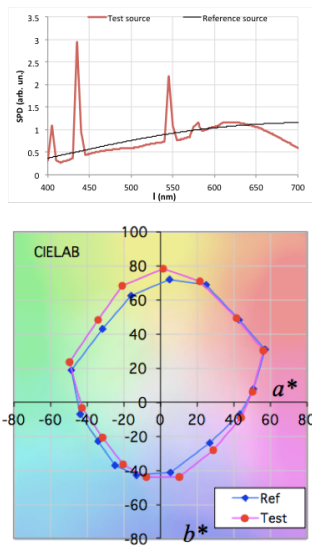

(c) $R_{\mathrm{a}}=69, R_{\mathrm{f}}=80$
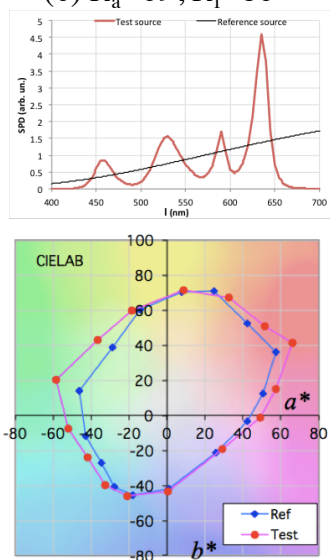

Figure 8. The spectral power distributions of three example light sources and the CIELAB plots of the CQS 15 samples for these sources - (a) a phosphor type LED lamp, (b) fluorescent lamp, both showing srong yellow-blue saturation, and (c) an RGBA LED source showing strong red-green saturation.

Another significant problem of CRI is the use of only eight medium-saturated test color samples, which can cause anomalies (unreasonably higher value than real performance) in the $R_{\mathrm{a}}$ score. 
Figure 9 shows the spectral reflectance curves of the test color samples of (a) CRI, (b) CQS, and (c) TM-30, plotted with an RGB LED source spectrum. This light source has unacceptably poor rendering of red objects $\left(R_{9}=-97\right)$ though it produces a generally acceptable value of $R_{\mathrm{a}}=82$. It is observed that the CRI test sample curves are fairly flat in the red region, which means the $R_{\mathrm{a}}$ score is less senstive to the location of the red peak, allowing a good $R_{\mathrm{a}}$ score in spite of poor red rendering. The TM-30 and CQS detect this problem and the effect is reflected in the score. The $Q_{\mathrm{f}}$ in the figure

(a) CRI test samples

$$
\begin{aligned}
& \text { Light } \\
& \text { source: } \\
& R_{\mathrm{a}}=82 \\
& R_{9}=-97 \\
& Q_{\mathrm{f}}=76 \\
& R_{\mathrm{f}}=76
\end{aligned}
$$
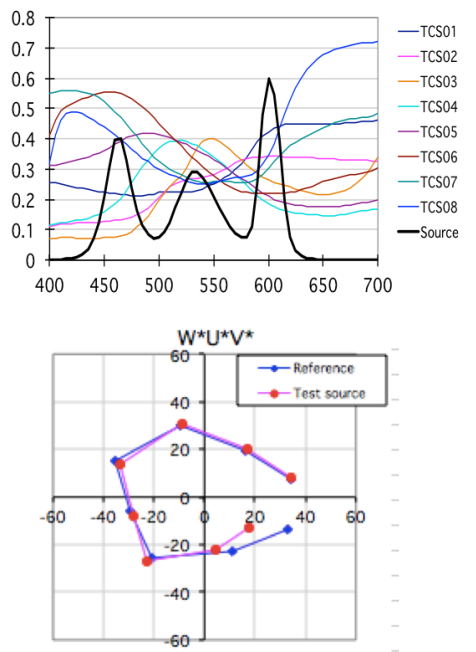

(b) CQS test samples
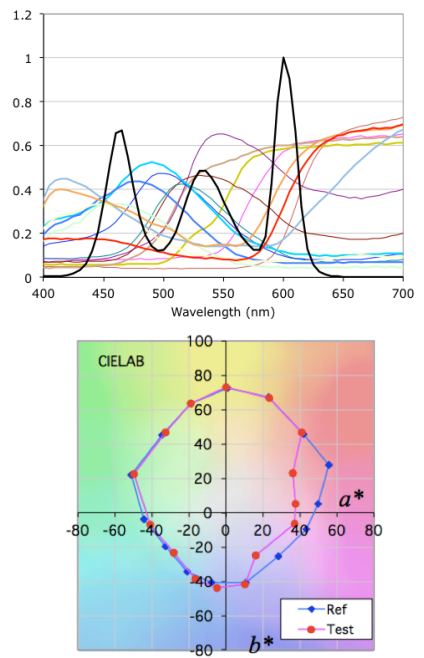

(c) TM-30 test samples
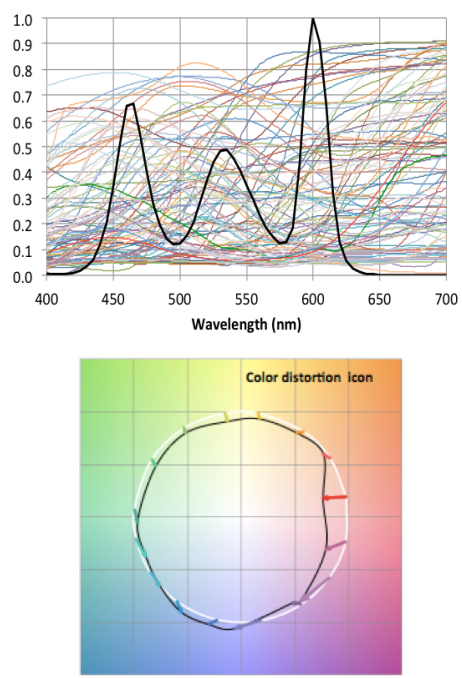

Figure 9. The spectral reflectance curves of the test color samples of (a) CRI, (b) CQS, and (c) $T M-30$, plotted with the spectral distribution of an RGB LED source, and color gamut presentations of the RGB source in each metric.

is the color fidelity scale in CQS [13].

Another example of an anomaly in CRI $R_{\mathrm{a}}$ is shown in Fig. 10, which shows a case of an RBG LED source optimized for high $R_{\mathrm{a}}$ score. The TM-30 Color Vector Graphics show significantly large hue shifts in the blue-green region, however, the CRI eight samples do not detect this problem.
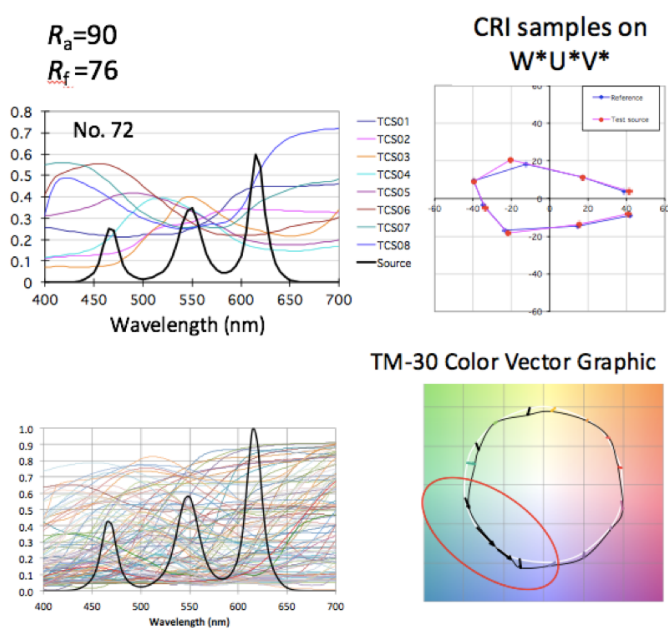

Figure 10. An example of $R G B$ source showing a large difference in value of $R_{a}$ and $R_{f}$.

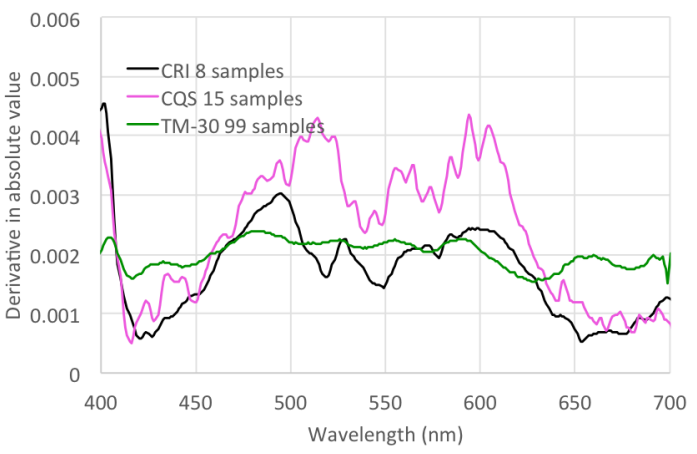

Figure 11. Average slope (absolute value) of spectral reflectance curves of all test color samples of CRI, $C Q S$, and TM-30 
The slopes of the spectral reflectance curves in Fig. 9 imply how sensitively the spectral component of the light source at that wavelength affects the calculation of the metric, though the effects are compounded with integration by the color matching functions. Figure 11 shows the average slopes (in absolute value) of all the test color samples of CRI, CQS, and TM-30 at each wavelength. The curves of CRI and CQS go down at near both ends (deep blue and deep red) of the spectral region, while TM-30 curves are kept fairly flat. This feature of TM-30 allows the metric to evaluate all (possible) object colors uniformly, while it is considered that CRI may not be able to evaluate accurately the colors of some objects having spectral reflectance curves changing rapidly in the deep red and deep blue region. Such colors are typically purple. This is the main reason that the $R_{\mathrm{f}}$ scores for narrow-band spectra that do not cover enough wide spectral region (e.g., triphosphor fluorescent lamps and RGBA LED sources) are lower than the $R_{\mathrm{a}}$ scores.

\section{CIE ACTIVITIES AND FUTURE PROSPECT}

The IES published the position statement of CRI (2015) [18], which indicates that TM-30 is not a required standard and is issued for evaluation by the international lighting community. The $R_{\mathrm{f}}$ metric was proposed for consideration in CIE TC1-90, which accepted it as the metric for the first TC draft and work is in progress. However, a color fidelity metric alone is not sufficient to evaluate all aspects of color rendition of light sources, and another metric to evaluate the aspect related to color preference is needed, and is being addressed in TC1-91. CIE also published a position statement on CRI and color quality metrics [19], which clarified these points. The TM-30 $R_{\mathrm{g}}$ (Gamut Index) is proposed for inclusion in CIE TC1-91 report, but the gamut area is not sufficient to address the aspects of general color preference. CIE is encouraging further research on this topic, and hopes to develop a recommendation of a more useful color preference-related metric in the future. Then, it is expected that two metrics (a color fidelity metric and a color preference-related metric) will be used to specify and evaluate light sources for general lighting.

\section{References}

[1] IEC 60081-1997, Double-capped fluorescent lamps - Performance specifications, Annex D.

[2] ANSI_NEMA_ANSLG, C78.377-2015 Specifications for the Chromaticity of Solid State Lighting Products.

[3] CIE 13.3: 1995, Method of measuring and specifying colour rendering properties of light sources.

[4] IES TM-30-15, IES Method for Evaluating Light Source Color Rendition.

[5] Y. Ohno, Y, Practical Use and Calculation of CCT and Duv, LEUKOS, 10:1, 47-55 (2013).

[6] M. S. Rea and J. P. Freyssinier, 2013, White Lighting, Color Research and Application, 38- 2.

[7] E. E. Dikel, G. J. Burns, J. A. Veitch, S. Mancini, and G. R. Newsham, Preferred Chromaticity of Color-Tunable LED Lighting, LEUKOS, 10:2, 101-115, 101-115, 2014.

[8] C. Miller et al, NIST spectrally tunable lighting facility for color rendering and lighting experiments, in Proc. CIE 2009: Light and Lighting Conference. 5 pages, 2009.

[9] Y. Ohno and M. Fein, Vision Experiment on Acceptable and Preferred White Light Chromaticity for Lighting, CIE x039:2014, pp. 192-199 (2014).

[10] Y. Ohno and S. Oh, Vision Experiment II on White Light Chromaticity for Lighting, CIE x042:2016, pp. 175-184 (2016).

[11] M. Wei and K. Houser, 2015. What is the cause of apparent preference for sources with chromaticity below the blackbody locus?, Leukos, DOI: 10.1080/15502724.2015.1029131.

[12] Y. Ohno, M. Fein, and C. Miller, Vision Experiment on Chroma Saturation for Color Quality Preference, Proc. $28^{\text {th }}$ Session of CIE, CIE 216: 2015, pp. 60-69 (2015).

[13] W. Davis and Y. Ohno, 2010. Color Quality Scale," Optical Engineering, 033602, 49, 3, 033602-1 to 033602-16.

[14] CIE 177: 2007, Colour rendering of white LED light sources.

[15] A. David et al, Development of the IES method for evaluating the color rendition of light, Optics Express Vol. 23, Issue 12, pp. 15888-15906 (2015).

[16] ISO 11664-4:2008(E)/CIE S 014-4/E:2007: Colorimetry - Part 4: CIE 1976 L*a*b* Colour Space

[17] CIE 159:2004: A Colour Appearance Model for Colour Management Systems: CIECAM02.

[18] IES PS-8-15 Color Rendering Index, https://www.ies.org/PDF/PositionStatements/PS-8-15.pdf.

[19] CIE Position Statement on CRI and Color Quality Metrics, http://www.cie.co.at/index.php?i_ca_id=981 (2015). 


\title{
Compliance-Free Pulse Forming of Filamentary RRAM
}

\author{
P. R. Shrestha ${ }^{\mathrm{a}, \mathrm{b}}$, D. Nminibapiel ${ }^{\mathrm{a}, \mathrm{b}}$, J.H. Kim ${ }^{\mathrm{a}}$, H. Baumgart ${ }^{\mathrm{b}}$ K.P. Cheung, and J.P. \\ Campbell ${ }^{\mathrm{a}}$ \\ aEngineering Physics Division, National Institute of Standards and Technology, \\ Gaithersburg, Maryland 20899, USA \\ bepartment of Electrical and Computer Engineering, Old Dominion University, Norfolk, \\ Virginia 23508, USA
}

\begin{abstract}
Despite the overwhelming effort to improve the efficacy of resistive random access memory (RRAM), the underlying physics governing RRAM operation have proven elusive. A survey of the recent literature almost universally indicates that the remaining glaring issues center around variability as well as endurance. The initial filamentary "forming" process is often linked to these problems. This work details our recent efforts to bring the forming process under control and the resulting improvements in RRAM viability in hafnia-based devices. We track the forming process via a "forming energy" metric that allows for filament optimization. By removal of all current compliance elements, and their associated parasitics, a targeted forming energy is achieved using ultrashort voltage pulses. By tailoring the forming energy, we show remarkable endurance window control.
\end{abstract}

\section{Introduction}

Recent memory research has focused on the development of transition metal oxide filamentary resistive random access memories (RRAM) $(1,2)$. While there are many published accounts detailing resistive switching in a variety of transition metal oxide material systems, hafnia-based systems are seemingly one of the most promising candidates $(3,4)$. Most explanations of filamentary RRAM involve discussions akin to traditional dielectric breakdown phenomena (5). The application of a relatively large voltage is thought to induce a breakdown event which "forms" a conductive filament through the dielectric region and induces a consequent resistance change. However, unlike dielectric breakdown, the effect in RRAM is not catastrophic. It is commonly observed that limiting the extent of the breakdown event results in a less robust conductive filament. Subsequent bipolar voltage application leads to cyclic dissolution and reforming of this conductive filament. This reversible switching between high and low conductance states defines the RESET and SET state variables of this non-volatile memory. Considering that the initial breakdown (forming event) greatly determines the switching characteristics, a substantial effort has been spent studying this initial electrical forming process $(6,7)$. A survey of the recent literature almost universally indicates that the remaining glaring RRAM issues center around variability as well as endurance. Perhaps the only consensus in the RRAM community is that the, inherently random, forming process is strongly linked to these variability and subsequent endurance concerns (8). 
Experimentally, forming is difficult to control due to the inrush of current associated with a large resistance change. The most common way of controlling the forming process is to introduce a resistive component in series with the RRAM element (9). This series resistance component (via resistor or transistor) introduces a current compliance limit which arrests the forming process by shifting the voltage drop from the RRAM element to the resistive compliance element. Our recent efforts show that even the most careful experimental inclusion of a series resistance element still introduces an unavoidable parasitic capacitance which invokes a serious forming variability $(10,11)$. During the fast transient forming process, the, often unintentional, parasitic capacitance discharges in an effort to exert a compliance limit. This discharge leads to a current level which briefly exceeds the compliance limit and is often referred to as overshoot (10). The current overshoot greatly alters the ability to terminate forming (i.e., a large current continues to flow through the filament for some uncontrollable time before the compliance element can clamp). This introduces a relatively large uncertainty in the forming energy and consequent variability in the filament (11).

In this work we show that the source of the overshoot variation is strongly linked not only to the overshoot amplitude, but also to the duration of the overshoot $(10,11)$. Some recent simulations show that the duration of the forming process is linked to the higher overshoot amplitude $(6,12)$. However, we demonstrate that these two factors (amplitude and duration) are independent by monitoring the effect of the current overshoot duration (fixed overshoot amplitude) on the first RESET current (10,11). Armed with this knowledge of the compliance-induced overshoot variability we propose a compliance-free forming process which allows for remarkable control of the forming energy via short voltage pulses (picosecond range)(11). This approach allows for an examination of the dissipated energy during the forming and SET/RESET processes and reveals how this energy plays a critical role (11). The SET/RESET cycling endurance of thus formed devices is shown to also be dependent on the SET/RESET energy (11). Multiple-pulse forming is also investigated as a method to further tighten the control of forming energy with promising endurance results (11).
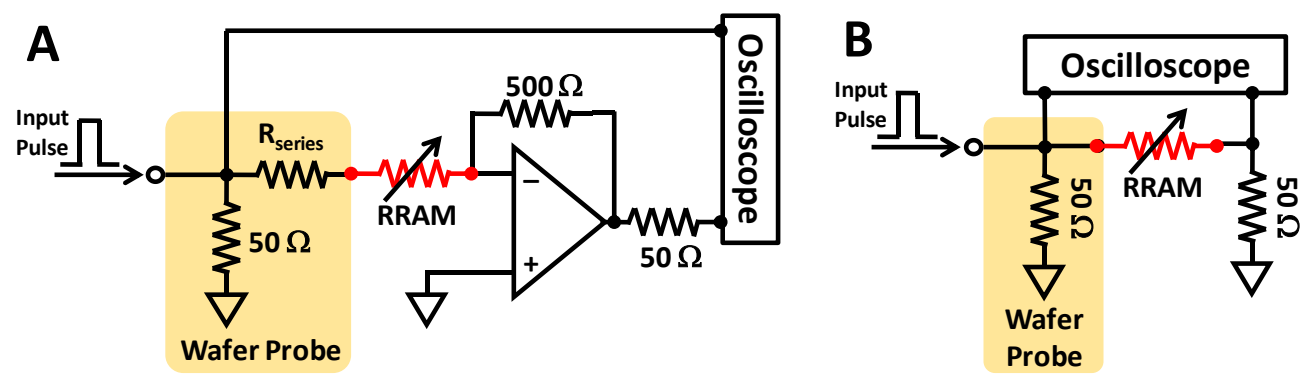

Figure 1. (a) Experimental set-up for forming of RRAM using series resistance for current compliance and a current amplifier to monitor the transient forming current. (b) Experimental setup for ultra-short voltage pulse forming. Note that there is no compliance element. All connections are consistent with proper high speed signal integrity. 


\section{Experimental Details}

The RRAM devices used in this study consist of TiN/Ti/ $\mathrm{HfO}_{2} / \mathrm{TiN}$ crossbar stacks ( 5.8 $\mathrm{nm} \mathrm{HfO}_{2}$ ). The Ti metal film was deposited on top of the metal oxide to create a sufficient degree of oxygen deficiency in the dielectric, which allows to perform forming at a reasonably low voltage. Both $\left(100 \times 100 \mathrm{~nm}^{2}\right.$ and $(50 \times 50) \mathrm{nm}^{2}$ device geometries were utilized in these studies. In order to better examine the forming process, a variety of experimental approaches are employed.

In an effort to understand the impact of current overshoot we employ an experimental set-up schematically shown in Fig. 1a. In this arrangement, the forming voltage is applied to the device through a series resistance $\left(\mathrm{R}_{\mathrm{s}}\right)$ compliance element. The current through the device is measured using a fast amplifier capable of sensing current with the resolution of $4 \mathrm{~ns}$ (10). The value of the series resistance is chosen to achieve a desirable compliance current during forming. This simple series resistor configuration is sufficient to understand the details of the effects of the overshoot during forming (10). The experimental parasitic capacitance is minimized as much as possible by minimizing signal path lengths. This is mostly accomplished via good signal integrity practices. For these measurements the series resistor is mounted on a PCB (printed circuit board) and placed as close to the device as possible as shown in inset of Fig. 1a. In this arrangement, the vast majority of the parasitic capacitance consists of the pad capacitance of the device $(<50 \mathrm{fF})$, and the capacitance of the trace connecting the probe to the device $(<0.4 \mathrm{pF})$. The total parasitic capacitance is thus less than $0.5 \mathrm{pF}$. Note that these parasitics are far less than that reported in most RRAM experiments. Forming is performed by applying a voltage pulse to the top electrode using a pulse generator. The bottom electrode is held at ground potential through the virtual ground of the current measuring amplifier. The input voltage and the current through the device are recorded using an oscilloscope. After forming, the device is RESET by applying a slow voltage sweep using a parameter analyzer. The maximum RESET current $\left(\mathrm{I}_{\max }\right)$ depends on the size and the resistivity of the formed filament (6). Thus, the obtained $I_{\max }$ is taken as an indicator to check for the variations in the formed filaments $(10,11)$.
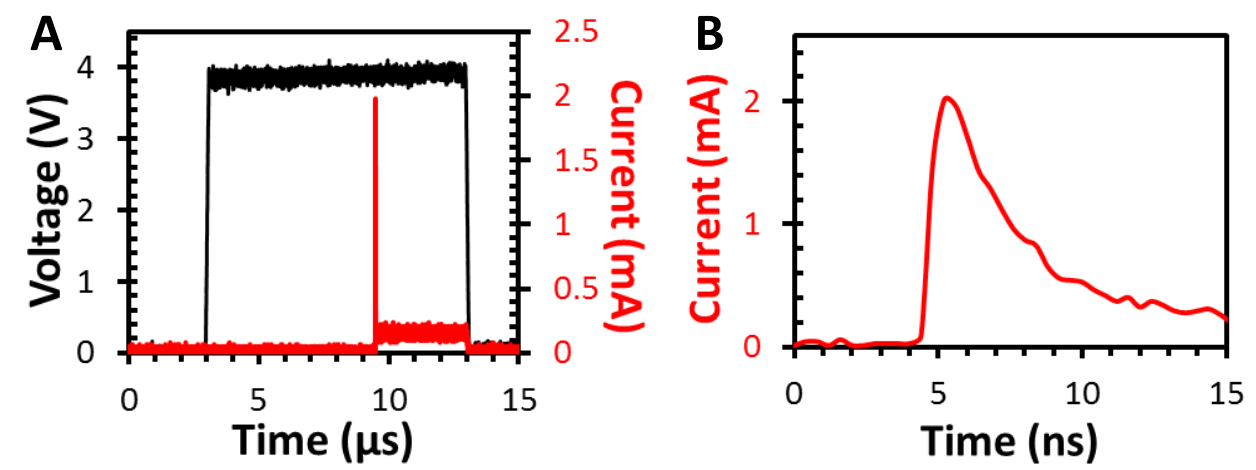

Figure 2. (a) Voltage and current output obtained during forming showing the large current overshoot before the compliance element can clamp. (b) Zoomed in image of the current overshoot in (a) showing that the overshoot lasts for a few ns. 

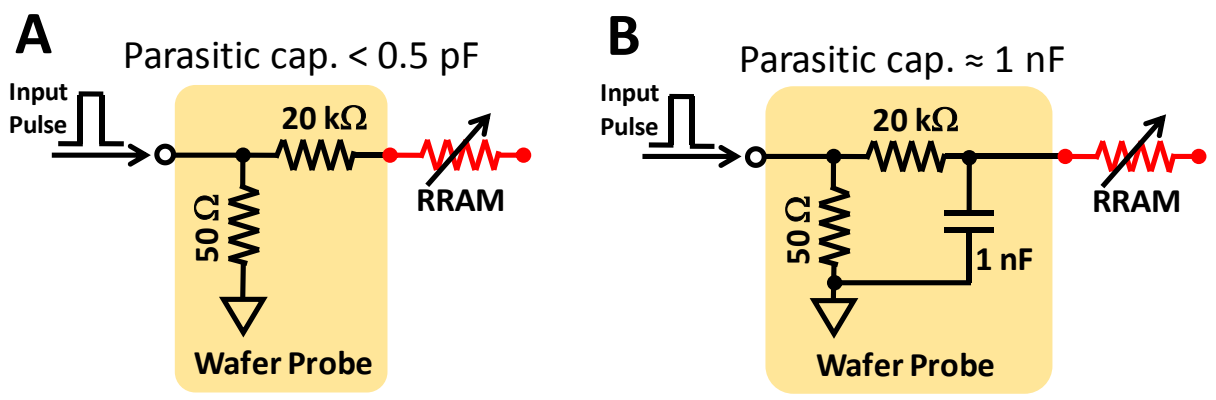

Figure 3. Schematic of our wafer probe used to deliver the forming voltage. The wafer probe consists of a circuit board which can accept the external series resistors (a) and parasitic capacitors (b). The distance between the probe top and the series resistor is $<1$ $\mathrm{cm}$. The unavoidable parasitic capacitance in (a) is dominated by the circuit board line capacitance.

In an effort to mitigate current overshoot and explore a means to better control the forming process, a slightly different experimental arrangement is also employed (Fig. 1b) for parts of this study. In this set-up impedance matching and return path control are of critical importance to ensure accurate forming characteristics. Note that there is no current limiting (compliance) element. The $50 \Omega$ termination at the probe provides a fast path to dissipate the charges stored by the parasitic elements, drastically minimizing any overshoot. In this manner, the forming current duration is entirely limited by the pulse duration which is kept very short (ns to ps). In other words, this compliance-free experimental implementation allows the overshoot amplitude to increase in an uncontrolled manner. However, the extent of this overshoot is highly controlled by the pulse duration. All post-forming RESET operations were achieved with a parametric analyzer negative voltage. $I_{\max }$ is then noted as a characteristic indicator of the quality of the formed filament.

\section{Results and Discussion}

\section{An Examination of the Current Overshoot}

Utilizing the experimental set-up in Fig. 1a, a typical input voltage and output current plot obtained during forming is shown in Fig. 2. The current level is very low before the device forms around $2.5 \mu \mathrm{s}$. Note the large transient overshoot current at forming followed by a settling to the compliance limited value. This current overshoot during forming is linked to higher $I_{\max }$ and likely more filament variability. In an effort to establish the illeffects of the overshoot due to parasitic capacitance, two experimental variations are employed (Figs. 3a and 3b). The parasitic capacitance in Fig. 3a is (as describe above) experimentally minimized to $<0.5 \mathrm{pF}$ and is mostly comprised of the wafer probe capacitance. Contrastingly, the parasitic capacitance of the second set-up (Fig 3b) is dominated by the addition of a $1 \mathrm{nF}$ external capacitor. This value ( $1 \mathrm{nF})$ was chosen to imitate common experimental arrangements (6). For both cases the parasitic capacitance is in parallel to the device. The amplitude of the forming voltage pulse is $+4 \mathrm{~V}$ for both cases. The width of the forming voltage pulse is $2 \mu \mathrm{s}$ for first case $(<0.5 \mathrm{pF})$ and $2 \mathrm{~ms}$ for the second case $(1 \mathrm{nF})$. The current transient during forming is then plotted for both set-ups as in Figs. $4 \mathrm{a}$ and $4 \mathrm{~b}$ respectively. It can be clearly seen that the time taken to reach the current 
compliance for each set-up is quite different. For the first case, the time to compliance is in the range of a few ns, whereas for the $1 \mathrm{nF}$ capacitor it is few $\mu \mathrm{s}(1000 \mathrm{x}$ longer).
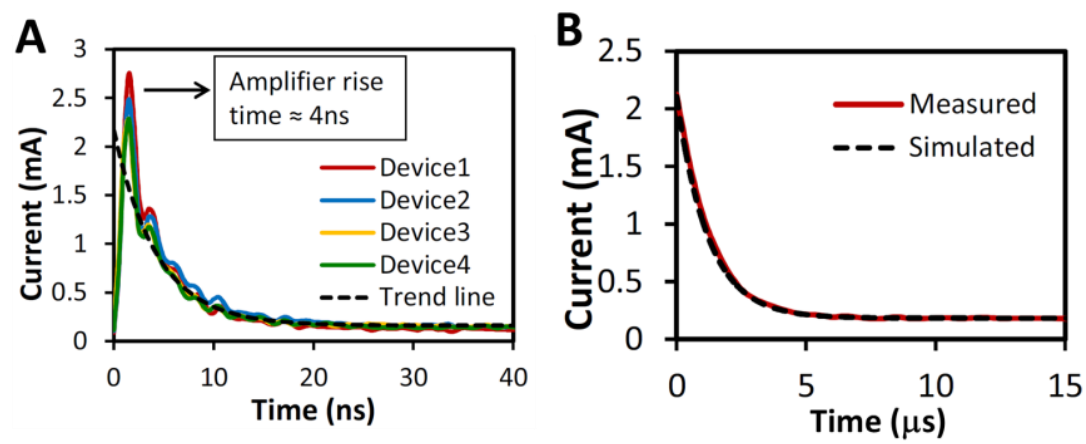

Figure 4. (a) Current transient during forming for the small parasitic capacitance of Fig. $3 \mathrm{a}$ and (b) the large $1 \mathrm{nF}$ parasitic external capacitance. Note that the time range in (a) is in ns further demonstrating the very low parasitic capacitance compared to (b) where the time is in the $\mu$ s range.

\begin{tabular}{|c|c|c|}
\hline 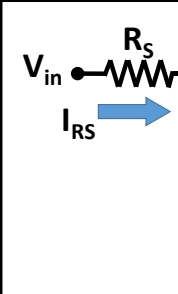 & 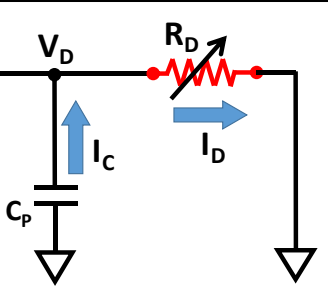 & $I_{D}=I_{R S}+I_{C}\left\{\begin{aligned} I_{D} & =\frac{V_{D}}{R_{D}} \\
I_{R S} & =\frac{V_{i n}-V_{D}}{R_{S}} \\
I_{C} & =C_{P} \frac{d V_{D}}{d t}\end{aligned}\right.$ \\
\hline $\begin{array}{l}V_{D}(t)= \\
\text { where } \tau=\end{array}$ & $\begin{array}{l}\left.\text { in }-\frac{R_{D}}{R_{S}+R_{D}} V_{\text {in }}\right) e^{\frac{-t}{\tau}} \\
R_{S} C_{P} \\
\left(\frac{R_{S} R_{D}}{R_{S}+R_{D}}\right) C_{P} \ldots \ldots \ldots \text { for } t \text { bef } t \text { foft }\end{array}$ & $\begin{array}{l}+\frac{R_{D}}{R_{S}+R_{D}} V_{\text {in }} \\
\text { re change in } \mathrm{R}_{\mathrm{D}} \\
\text { r change in } \mathrm{R}_{\mathrm{D}} \text { (compliance) }\end{array}$ \\
\hline
\end{tabular}

Figure 5. Circuit schematic of the RRAM device $\left(R_{D}\right)$ along with the series resistance ( $\left.\mathrm{R}_{\mathrm{S}}\right)$ and parasitic capacitance $\left(\mathrm{C}_{\mathrm{P}}\right)$ showing the origin of the current overshoot through the device. Also given are the equations for current through the device, $I_{D}$, and the voltage across the device, $\mathrm{V}_{\mathrm{D}}$.

The overshoot transients in Fig. 4 illustrate that during forming, the current peaks before it settles to the current compliance level set by the series resistance. The current peaking is due to the charge stored in the parasitic capacitor. A simple circuit schematic of the system is shown in Fig. 5 along with a simple circuit analysis of the current through the device during forming. $\mathrm{R}_{S}$ is the series resistance used to limit current through the device during forming. $R_{D}$ is the resistance of the device. $C_{P}$ is the parasitic capacitance. $V_{\text {in }}$ and $\mathrm{V}_{\mathrm{D}}$ are the input voltage and voltage across the device respectively. When the device is in the high resistance state $\left(\mathrm{R}_{\mathrm{D}}>\mathrm{G} \Omega\right)$ all the applied voltage $\left(\mathrm{V}_{\text {in }}\right)$ drops across the device. Therefore, voltage across the device $\mathrm{V}_{\mathrm{D}} \approx \mathrm{V}_{\mathrm{in}}$. When the filament forms, the device changes 
to a low resistance state $\left(\mathrm{R}_{\mathrm{D}}<10 \mathrm{k} \Omega\right)$ presumably very fast $(\mathrm{t}<130 \mathrm{ps})(11)$. In this low resistance state, the voltage across the device, $V_{D}$ may not decrease at the same rate as the resistance reduction. The rate depends strongly on the size of the parasitic capacitor. The voltage across the device is given by the equation for $\mathrm{V}_{\mathrm{D}}(\mathrm{t})$ in Fig. 5. This is derived by solving the differential equation given by the total current $I_{D}$ flowing through the device. From the equation for $\mathrm{V}_{\mathrm{D}}(\mathrm{t})$, it is clear that larger parasitic capacitance leads to slower discharges and therefore, longer overshoot current duration (bigger RC product in the exponential). This is easily verified via an examination of the current overshoot durations in Fig. 4. The derived equation for $\mathrm{V}_{\mathrm{D}}(\mathrm{t})$ was also used to simulate the current through the device. The simulated and measured current overshoots match well (Fig 4b).

Note that the current overshoot amplitude is quite similar for both parasitic capacitance cases. This may seem counterintuitive to recent reports $(6,12)$. But if the change in the resistance is abrupt such that the rate of change of resistance is faster than the discharge rate of the parasitic capacitance, then the maximum overshoot current is independent of the parasitic capacitance. This is true for both of the cases illustrated in Fig. 4. In the lower parasitic capacitance case, the amplitude of the overshoot current is about $2 \mathrm{~mA}$ as shown by the trend line Fig. 4a. The measured data below $4 \mathrm{~ns}$ is not reliable due to the amplifier ringing, therefore, we use an extrapolation to estimate the current amplitude. The amplitude given by the trend line in Fig. $4 \mathrm{a}$ is similar to that of the higher parasitic capacitance case ( $1 \mathrm{nF}$ shown in Fig. 4b). This result differs from earlier reports linking higher parasitic capacitance to higher overshoot amplitude $(6,12)$. This is the case only when the change in the resistance is slower compared to the discharge rate of the parasitic capacitance. One such slow change in resistance shown in (6) is similar to progressive breakdown in thin film dielectrics. Such reduced rate in change of resistance during forming was achieved using constant voltage forming (CVF) (6) with very low forming voltages.
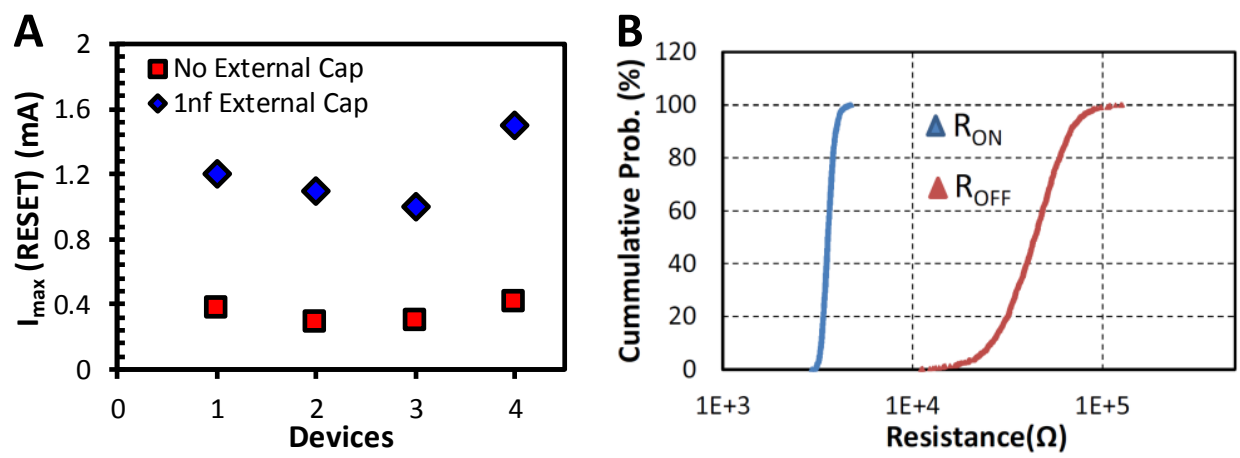

Figure 6. (a) Measured $I_{\max }$ required for $1^{\text {st }}$ RESET after forming with and without the 1 $\mathrm{nF}$ capacitor. The RESET currents required after forming with $1 \mathrm{nF}$ are higher compared to the devices formed without the $1 \mathrm{nF}$ capacitance. (b) 1000 cycles switching using $\mathrm{V}_{\mathrm{SET}}=1.5 \mathrm{~V}$ with the pulse width of $500 \mathrm{~ns}$ and $\mathrm{V}_{\text {RESET }}=-2 \mathrm{~V}$ with the pulse width of $250 \mathrm{~ns}$.

Utilizing the two experimental arrangements of Fig. 3, formed devices were subsequently RESET (low resistance to high resistance) by applying a negative voltage using a parameter analyzer (Fig. 6a). This $I_{\max }$ is lower for the minimum parasitic capacitance case as compared to the $1 \mathrm{nF}$ capacitance set-up which may indicate the 
formation of a filament of a smaller cross-section. After the first RESET, only the devices formed using the lower parasitic capacitance set-up were able to be successively switched between low and high resistance states (Fig. 6b) while the higher capacitance devices simply did not switch (forming was catastrophic).

It is important to note that the peak overshoot currents during forming are similar for both parasitic capacitance cases depicted in Fig 3. However, the duration of the current overshoot is much longer for the $1 \mathrm{nF}$ capacitance forming (Fig 4). This is an indication that the variations in $I_{\max }$ in Fig. 6a and device switching capabilities are due to the difference in the duration of the current overshoot, which has been observed to be critical to control the filament properties.

\section{The Forming Energy Paradigm}

The demonstrated independence of the magnitude and duration of the current overshoot provides a useful clue towards bringing the forming process under better control. As illustrated in the preceding section, increasing the overshoot duration while maintaining quite similar overshoot amplitudes led to undesirable filaments which were not switchable (high $\mathrm{I}_{\max }$ ). This strongly suggests that the product of the amplitude and duration (or integrated area under the overshoot current curve) is a more apt indicator of the forming process. Further scaling this product with the forming voltage leads to a forming energy metric with which to track the effectiveness of the forming process. The forming energy is taken as the product of the forming voltage, forming current, and time.
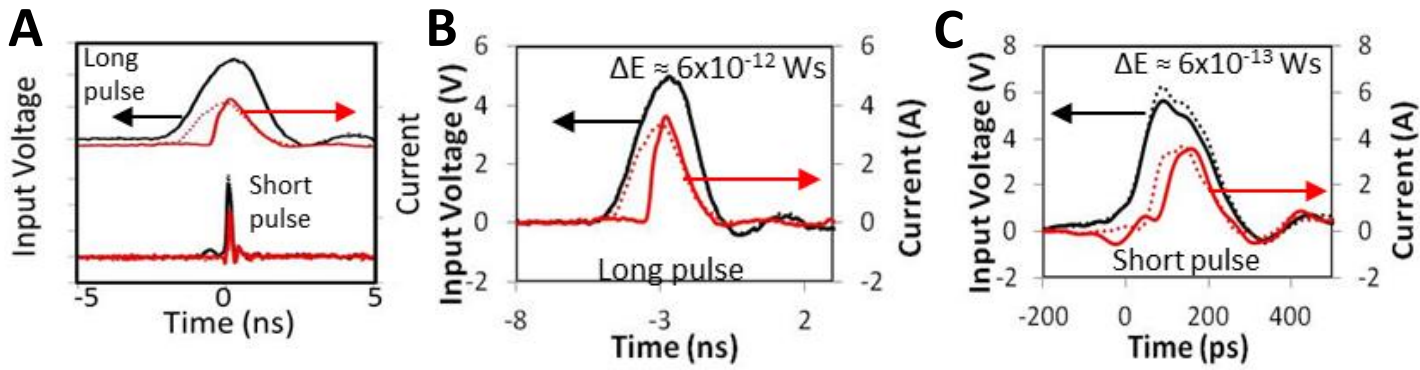

Figure 7. (a) Voltage and current vs. time for forming pulses with pulse durations of 2.5 ns and 130 ps. (b) details the $2.5 \mathrm{~ns}$ forming and (c) details the $130 \mathrm{ps}$ forming. The calculated energy difference $(\Delta \mathrm{E})$ for dotted (early forming) and the solid line (late forming) are also show. Note that long pulse forming $(\Delta \mathrm{E})(\mathrm{b})$ is almost 10x larger than for (c).

Recognition that the inclusion of a compliance element in the RRAM forming process will invariably introduce an unwanted (and uncontrolled) current overshoot might tempt one to suspend all hopes of forming control. However, it is exactly this recognition, coupled with the notion of a forming energy, which allowed us to gain substantially greater control on the forming process. Since there will be an inevitable current overshoot associated with the forming resistance change, then one can limit its duration by minimizing capacitance. In the ultimate incarnation of this approach, one can nearly eliminate the capacitance by also eliminating the compliance element and employing very short voltage pulses (Fig 1b). In this scenario, there is still a current overshoot which proceeds unchecked for the duration of the forming voltage pulse. However, limiting the duration of the forming voltage pulse 
limits the duration of the current overshoot and actually provides greater control in targeting a specific forming energy (and consequent $I_{\max }$ ). One can think of each pulse as providing a quantized portion of the total energy necessary to form. Narrower pulses provide a smaller quanta and therefore improve forming control.
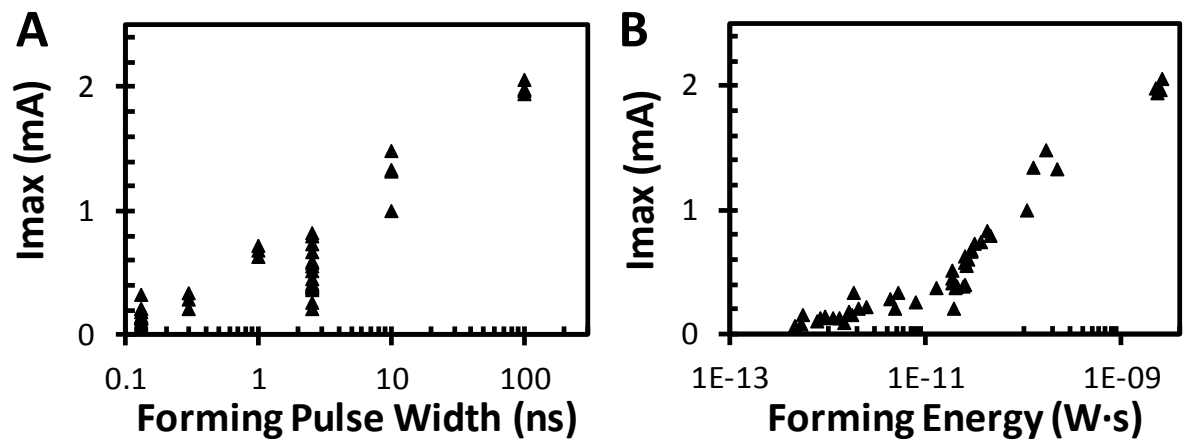

Figure 8. (a) $\mathrm{I}_{\max }$ current observed after pulsed forming with varying pulse duration. After forming, the devices are RESET using a parametric analyzer. It can be seen that the variation in $I_{\max }$ (with same pulse duration) is larger for longer durations. (b) $I_{\max }$ versus energy during forming. We observe a much tighter distribution (than in (a)) when the forming energy is carefully monitored.

\section{Compliance-Free Forming}

Representative compliance-free forming characteristics are shown in Fig. 7a for both slow (2.5 ns) and fast (130 ps) duration forming pulses. Sometimes forming occurs early in the pulse (Fig. 7 dotted lines), while other times forming occurs later in the pulse (Fig. 7 solid lines). In the case of early forming, the filament is subject to forming current for longer durations. This results in less desirable properties in formed filaments. Note that even in the $2.5 \mathrm{~ns}$ pulse duration case, there exists a significant randomness as to when the forming process occurs (within the $2.5 \mathrm{~ns}$ pulse). As shown in Fig. $7 \mathrm{~b}$ for the $2.5 \mathrm{~ns}$ pulse case, this randomness of the forming current transient can introduce substantial differences in the forming energy $\left(\Delta \mathrm{E}_{\text {forming }} \approx 6.1 \mathrm{pWs}\right)$ which results in poorer control of the forming process. However, reducing the pulse duration down to 130 ps greatly minimizes this forming energy randomness (Fig 7c) and brings the forming process into greater control ( $\Delta \mathrm{E}_{\text {forming }} \approx 0.65 \mathrm{pWs}$ ). While the within pulse randomness of the current transient is still present, its impact on the overall forming energy (and presumably filament variability) is greatly reduced. This is best illustrated by comparing the calculated difference between late and early forming for the two pulse cases (2.5 ns and $130 \mathrm{ps})$. This early/late forming uncertainty introduces almost an order of magnitude more energy uncertainty into the formed filament (Figs $7 \mathrm{~b}$ and 7c).

One might be tempted to ignore this early/late forming pulse variation and simply look at the product of the pulse duration and forming voltage (Fig 8a). However, proper accounting for the "within-pulse variability" (which was the impetus for forming energy evaluation) is seemingly required to bring the forming process under control. If one presumes that $I_{\max }$ is related to forming energy, then proper within-pulse variability accounting, drastically reduces forming variation (Fig. 8b). Fig. $8 \mathrm{~b}$ also suggests that any chosen $\mathrm{I}_{\max }$ is attainable via the application of the necessary pulses to net the target forming 
energy. In order to trust the forming energy values shown in Fig. 8b, there is a requirement to accurately measure the forming current. At very high speeds, the parasitic current that does not actually flow through the device becomes large (Fig. 9a) and must be removed. We employ a de-embedding procedure to remove these parasitic components from the measured forming current (Fig. 9b) (13). Using this approach, proper forming energies can be extracted.
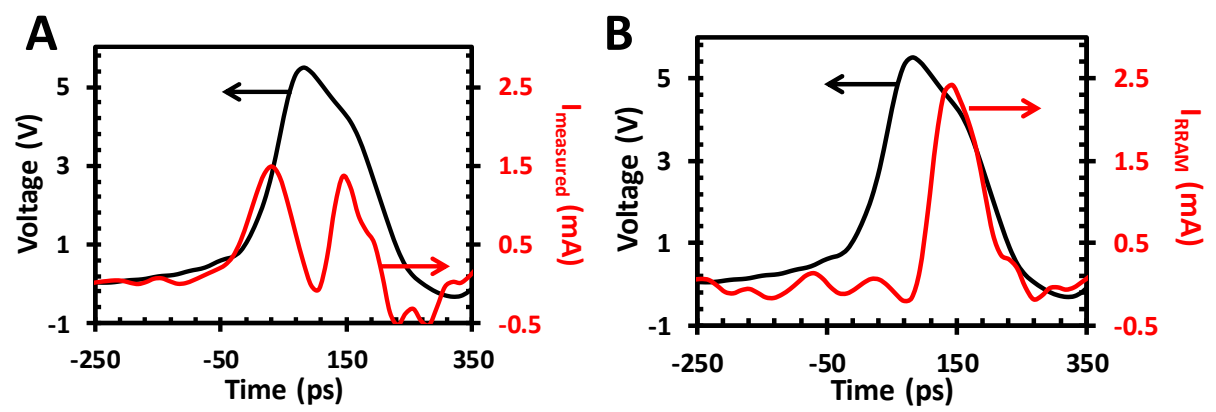

Figure 9. Measured voltage and current pulse before (a) and after (b) de-embedding the parasitic current components. This step is necessary to extract accurate forming energy.

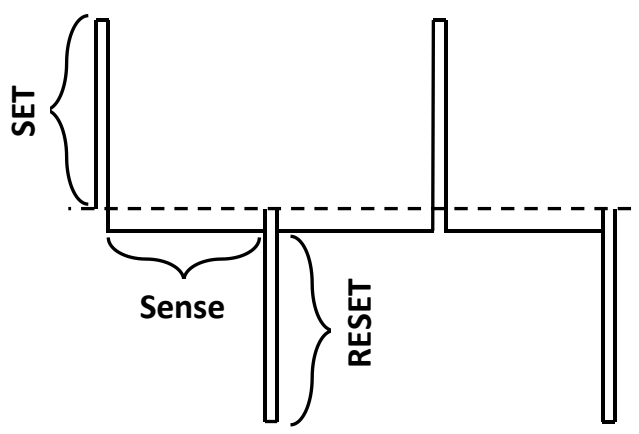

Figure 10. Input pulse train used to analyze the SET/RESET endurance. In this manner every single SET/RESET cycle can be measured.
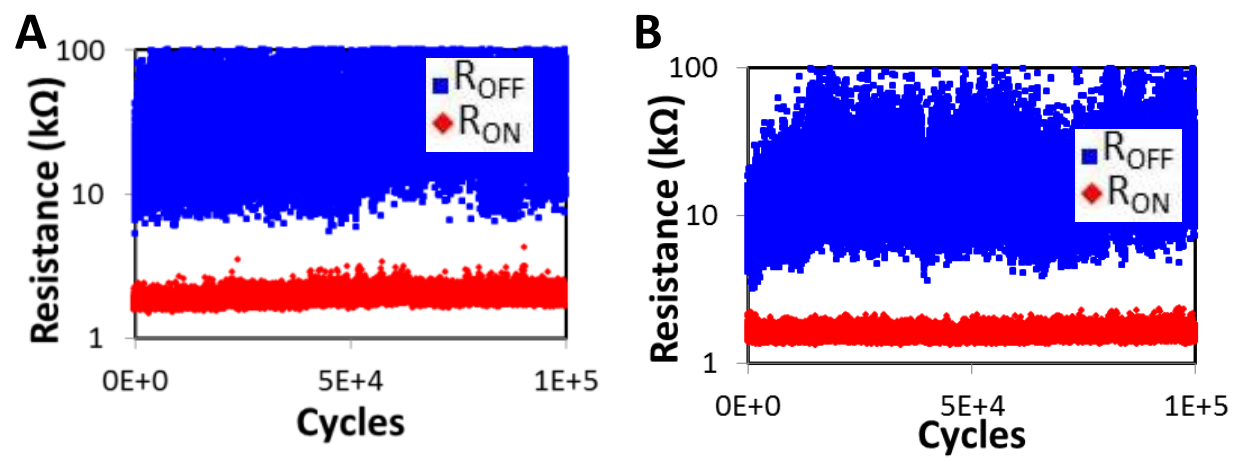

Figure 11. Reliable SET/RESET was obtained on devices formed using a "medium" energy. The switching duration $=2.5 \mathrm{~ns}$ and the forming energy was (a) $4.7 \times 10^{-11} \mathrm{Ws}$ $\left(\mathrm{I}_{\max }=790 \mu \mathrm{A}, \mathrm{V}_{\mathrm{ON}}=1.5 \mathrm{~V}, \mathrm{~V}_{\mathrm{OFF}}=-2 \mathrm{~V}\right)$ and $(\mathrm{b}) 2.67 \times 10^{-11} \mathrm{Ws}_{\mathrm{s}}\left(\mathrm{I}_{\max }=550 \mu \mathrm{A}, \mathrm{V}_{\mathrm{ON}}\right.$ $\left.=2.5 \mathrm{~V}, \mathrm{~V}_{\mathrm{OFF}}=-2.5 \mathrm{~V}\right)$. 
Using forming energy (fig. 8b) as a guide, we investigated the endurance of the pulse formed devices using the input pulse train schematically shown in Fig. 10. Typical SET/RESET operations involve a $2.5 \mathrm{~ns}$ pulse of several volts. The RRAM resistance values are sensed at $-200 \mathrm{mV}$. We note that devices with larger forming energies exhibited very poor switching behavior with most devices lasting only a few cycles (not shown). However, as the forming energies and the consequent $\mathrm{I}_{\max }$ is reduced to $790 \mu \mathrm{A}$ (Fig. 11a), and then to $550 \mu \mathrm{A}$ (Fig. 11b), we observe a steady increase of the endurance window and a drastic reduction in SET/RESET switching errors.
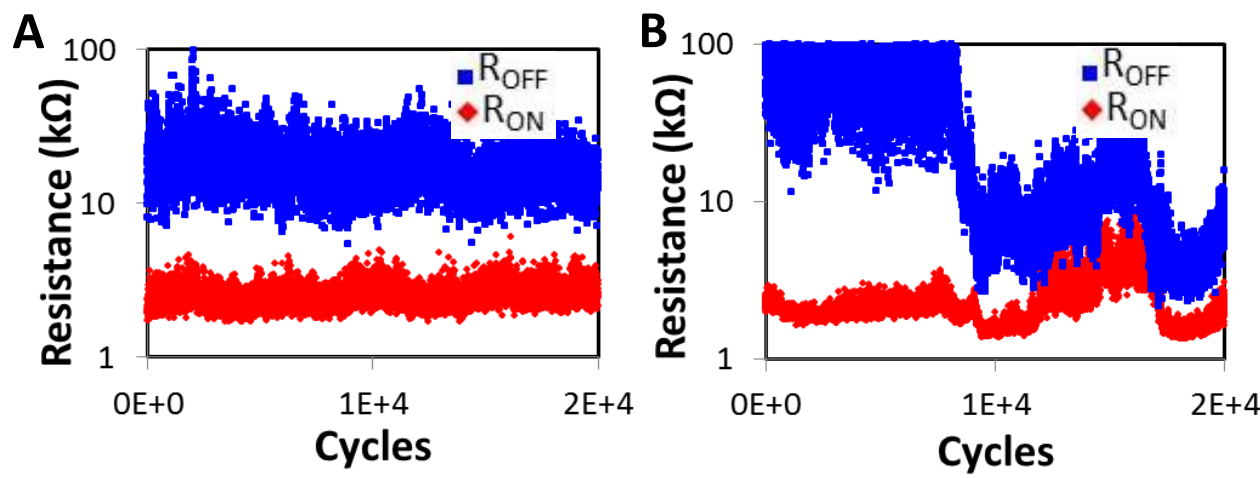

Figure 12. Dependence of cycling reliability on SET/RESET energy is shown by comparing (a) low energy $\left(\mathrm{V}_{\mathrm{ON}}=1.5 \mathrm{~V}, \mathrm{~V}_{\mathrm{OFF}}=-2 \mathrm{~V}\right.$, duration $\left.=2.5 \mathrm{~ns}\right)$ and (b) high energy $\left(\mathrm{V}_{\mathrm{ON}}=2.5 \mathrm{~V}, \mathrm{~V}_{\mathrm{OFF}}=-2.5 \mathrm{~V}\right.$, , duration $\left.=2.5 \mathrm{~ns}\right)$ SET/RESET.
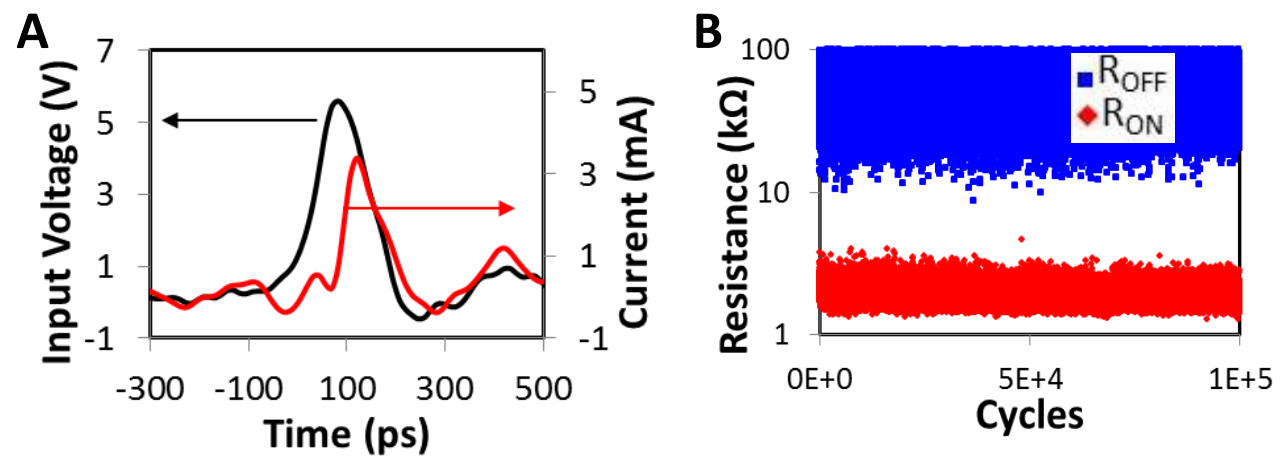

Figure 13. (a) First forming voltage and current plot for multiple pulse forming. After the first pulse shown in (a) 4 additional pulses of the same amplitude and duration were applied to the formed filament. (b) $10^{5} \mathrm{SET} / \mathrm{RESET}$ cycles for the multiple-pulse forming $\left(\mathrm{V}_{\mathrm{ON}}=2 \mathrm{~V}, \mathrm{~V}_{\mathrm{OFF}}=-2 \mathrm{~V}\right.$, duration $\left.=2.5 \mathrm{~ns}\right)$.

Since SET/RESET processes are composed of short pulses, the energy metric is useful here as well. Similar to forming energy, an endurance dependence on the SET/RESET energy (Fig. 12) is also observed. In fig. 12a $\left(\mathrm{I}_{\max }=900 \mu \mathrm{A}\right)$ a device was switched with lower energies $\left(\mathrm{V}_{\mathrm{ON}}=1.5 \mathrm{~V}, \mathrm{~V}_{\mathrm{OFF}}=-1.5 \mathrm{~V}\right.$, pulse duration $\left.=2.5 \mathrm{~ns}\right)$. In Fig. $12 \mathrm{~b}$ the same device was switched with higher energies $\left(\mathrm{V}_{\mathrm{ON}}=2.5 \mathrm{~V}, \mathrm{~V}_{\mathrm{OFF}}=-2.5 \mathrm{~V}\right.$, pulse duration $=$ $2.5 \mathrm{~ns}$ ). It is clear that the higher SET/RESET energy (Fig. 12b) temporarily increases the resistance window markedly, though it comes at the price of lower endurance. This strongly suggests that there is an additional SET/RESET energy influence which is linked to 
forming energy (i.e., a filament formed with a specific energy has a SET/RESET energy which complements its switching characteristics).

Even with short pulses, there is still a random forming or SET/RESET timing within the pulse duration. One solution to further tighten the timing, and therefore energy distribution, is to use multiple-pulses for forming. This approach is investigated by first forming the filament using a very short pulse (130 ps) (Fig. 13a) and then subjecting the device to an additional four pulses. The resulting endurance (Fig. 13b) shows a very promising improvement in endurance window. In general, one might be able trade much lower amplitude forming/SET/RESET voltage pulses for the requirement of using multiple pulses to accomplish each step. This could, in principle, allow one to tailor the forming/SET/RESET energies to maximize endurance. This collective line of thinking leads to the notion that the forming step should really be thought of as the last processing fabrication step which defines the identity of the final device.

\section{Conclusions}

In this work we have discussed the sources of variability associated with the forming process in RRAM devices and their impact on the endurance window. We have shown that the intentional inclusion of a compliance series resistance unintentionally introduces a parasitic component which leads to current overshoot. This current overshoot was shown to have deleterious effects on the RRAM characteristics and be a significant source of variability. Both the duration and amplitude of this current overshoot are shown to be independent and can each be experimentally treated in different fashions (10). Together, the product of the forming voltage and the duration and amplitude of the current overshoot define a forming energy (11). This forming energy was shown to be strongly correlated to the maximum RESET current, which is an indication of the viability of the formed filament $(10,11)$. We then introduced a method to completely remove the compliance element from the forming procedure and instead utilize very short voltage pulses to induce a forming event (11). Minimizing the duration of these forming pulses provides greater control of the forming energy and the consequent maximum RESET current. We demonstrated that selecting a target forming energy and pairing this energy with appropriate SET and RESET pulse "energies" leads to improved endurance. Furthermore, this compliance-free forming approach lends itself to multi-pulse forming which shows improved control of the forming energy and a commensurate improvement in endurance (11). Overall, our results strongly suggest that controlling the forming/SET/RESET energy is an effective method to achieve reliable RRAM endurance.

\section{References}

1. R. Waser, R. Dittmann, G. Staikov, et al., Adv Mater, 21, 2632 (2009).

2. H. S. P. Wong, H. Y. Lee, S. M. Yu, et al., Proceedings of the IEEE, 100, 1951 (2012).

3. F. Nardi, S. Larentis, S. Balatti, et al., Ieee T Electron Dev, 59, 2461 (2012).

4. B. Govoreanu, G. S. Kar, Y. Y. Chen, et al., IEEE International Electron Devices Meeting (IEDM), 729 (2011).

5. N. Xu, L. F. Liu, X. Sun, et al., Appl Phys Lett, 92 (2008).

6. A. Kalantarian, G. Bersuker, D. C. Gilmer, et al., IEEE International Reliability Physics Symposium (IRPS), 6C.4.1 (2012). 
7. G. Bersuker, D. C. Gilmer, D. Veksler, et al., J Appl Phys, 110, 124518 (2011).

8. F. Pan, S. Gao, C. Chen, et al., Mat Sci Eng R, 83, 1 (2014).

9. K. Kinoshita, K. Tsunoda, Y. Sato, et al., Appl Phys Lett, 93, 033506 (2008).

10. P. Shrestha, D. Nminibapiel, J. P. Campbell, et al., IEEE International Integrated Reliability Workshop Final Report (IIRW), 55 (2013).

11. P. R. Shrestha, D. Nminibapiel, J. H. Kim, et al., IEEE International Reliability Physics Symposium (IRPS), MY.10.1 (2014).

12. Y. Shimeng, G. Ximeng and H. S. P. Wong, in IEEE InternationalElectron Devices Meeting (IEDM), p. 26.1 .1 (2012).

13. P. Shrestha, D. Nminibapiel, J. P. Campbell, et al., International Symposium on VLSI Technology, Systems and Application (VLSI-TSA), TR82 (2014). 


\title{
Mueller matrix bidirectional reflectance distribution function measurements and modeling of textured silicon surfaces
}

\author{
Thomas A. Germer ${ }^{\mathrm{a}}$, Martin Foldyna ${ }^{\mathrm{b}, \mathrm{c}}$, Zuzana Mrazkova $^{\mathrm{b}, \mathrm{d}}$, \\ Guillaume Fischer ${ }^{\mathrm{c}}$, and Etienne Drahi ${ }^{\mathrm{c}, \mathrm{e}}$ \\ ${ }^{a}$ Sensor Science Division, National Institute of Standards and Technology, Gaithersburg, Maryland 20899 USA \\ bLPICM-CNRS (Laboratoire de Physique des Interfaces et des Couches Minces - Centre National de la Recherche Scientifique), \\ Ecole Polytechnique, Université Paris-Saclay, 91128 Palaiseau, France \\ 'IPVF (Institut Photovoltaïque d'Ile-de-France), 8 rue de la Renaissance, 92160 Antony, France \\ ${ }^{\mathrm{d} N a n o t e c h n o l o g y}$ Centre and Department of Physics, Technical University of Ostrava, 708 33, Ostrava-Poruba, Czech Republic

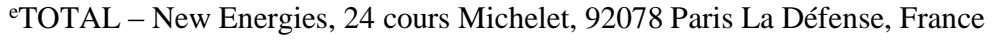

\begin{abstract}
Surface texturing plays an important role in trapping light in photovoltaic materials. Understanding and modeling diffuse scatter from various textured silicon surfaces should aid in increasing light trapping in these materials, as well as improving material characterization and inspection during manufacture. We have performed Mueller matrix bidirectional reflectance distribution function (BRDF) measurements from a variety of textured silicon surfaces. Simulations, using multiple reflection polarization ray tracing, reproduce many of the features in the data. Evidence for diffraction, however, can also be observed, suggesting that a purely ray-tracing approach is insufficient for accurately describing the scatter from these materials.
\end{abstract}

Keywords: BRDF, Mueller matrix, photovoltaics, pyramids, scattering, surface texture

\section{INTRODUCTION}

Surface texturing is often used to increase absorption of light in photovoltaic materials. [1] The texture serves two functions: it reduces the reflectance by allowing incident radiation to reflect multiple times, and it diffuses the radiation within the material, so that weakly absorbing radiation experiences an enhanced path length to improve absorption. Besides geometrically increasing the path length inside the material, obliquely propagating radiation will experience total internal reflection at the interfaces, further trapping the radiation. Yablonovitch determined that the path length enhancement afforded by enhanced trapping can be as high as $4 n^{2}$ for a random texture, where $n$ is the index of refraction. [2, 3]

Characterization of surface texturing is needed during solar cell fabrication and can be performed rapidly by optical scattering measurements. $[4,5]$ While intensity measurements alone may be sufficient to provide pass/fail determination, more rigorous measurements using polarimetry may yield secondary information that helps to identify process failure modes. However, unless one resorts to a signature-based or process-experience method, an understanding of the scattering mechanisms and how the texture affects the scatter distribution and polarization is needed.

In this study, we performed optical scattering measurements from textured silicon surfaces, in order to better understand the propagation of radiation from these surfaces. While one of the research goals is to better understand the optical trapping characteristics at long wavelengths, these were reflectance measurements performed at a wavelength where the material is opaque. Nonetheless, the results demonstrate the usefulness of light scattering for characterizing these surfaces.

We will describe the measurement techniques in Sec. 2. In Sec. 3, we describe the samples that we performed the measurements on. Section 4 describes the ray models used and how diffraction would be expected to play a role. The results, together with discussion, will be given in Sec. 5. Finally, in Sec. 6, we will make some concluding remarks. 


\section{MEASUREMENT METHODS}

\subsection{Instrument}

All of the measurements shown in this paper were performed at a wavelength of $633 \mathrm{~nm}$, using a HeNe laser and the Goniometric Optical Scatter Instrument (GOSI). [6] This laser-based scatterometer can measure the Mueller matrix bidirectional reflectance distribution function (BRDF) for nearly any pair of incident and viewing directions, including those out of the plane of incidence. The incident direction $\hat{\mathbf{k}}_{\mathrm{i}}$ is parameterized by spherical coordinates with polar angle $\theta_{\mathrm{i}}$ and azimuthal angle $\phi_{\mathrm{i}}$, while the viewing direction $\hat{\mathbf{k}}_{\mathrm{r}}$ is parameterized with a polar angle $\theta_{\mathrm{r}}$ and azimuthal angle $\phi_{\mathrm{r}}$. Restrictions include some directions with polar angles greater than $75^{\circ}$, dependent upon all four angles, because of the presence of a rotation stage, and for directions within $5^{\circ}$ of the retroreflection direction, because of the size of the receiver. The receiver has a precision aperture subtending approximately $\Omega=1.15 \times 10^{-4} \mathrm{sr}$, a focusing lens, a rotating retarder, a polarizer, and an integrating sphere. The integrating sphere has a Si photodiode and a photomultiplier tube. However, for the measurements described here, the scattering levels were high enough that only the Si photodiode was used. The incident laser beam is fixed and horizontal, while the receiver rotates in a horizontal plane. The BRDF, $f_{\mathrm{r}}$, is determined from

$$
\Phi_{\mathrm{r}}=f_{\mathrm{r}} \Phi_{\mathrm{i}} \Omega \cos \theta_{\mathrm{r}}
$$

where $\Phi_{\mathrm{r}}$ is the scattered radiant flux, and $\Phi_{\mathrm{i}}$ is the incident radiant flux, measured with the sample removed and the receiver in the incident beam. The measurement sequence consisted of two hemispherical scans, described in Sec. 2.2, and two retroreflective scans, described in Sec. 2.3.

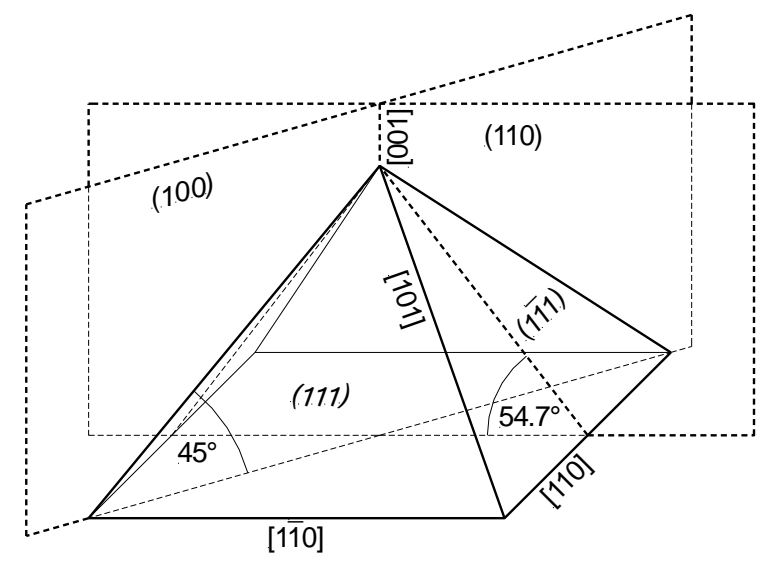

Figure 1. A pyramid on a (001) silicon surface, shown as solid lines, showing various directions (in brackets), planes (in parentheses), and angles. The two incident planes, (110) and (100), used in the measurements are shown with dashed lines.

\subsection{Hemispherical BRDF measurements}

Measurements of the BRDF were performed for two incident angles, $\theta_{\mathrm{i}}=5^{\circ}$ and $60^{\circ}$, where the incident plane is the (110) Si crystal plane, which includes the [110] and [001] crystal directions (see Fig. 1). The viewing directions were sampled on an evenly spaced grid in projected-cosine space: $\hat{k}_{\mathrm{r}, x}=\sin \theta_{\mathrm{r}} \cos \phi_{\mathrm{r}}$ and $\hat{k}_{\mathrm{r}, y}=\sin \theta_{\mathrm{r}} \sin \phi_{\mathrm{r}}$. The spacing of $\hat{k}_{\mathrm{r}, x}$ and $\hat{k}_{\mathrm{r}, y}$ were each 0.1 , so that the angle spacing was about $5.7^{\circ}$ near the surface normal $\left(\theta_{\mathrm{r}}=0\right)$ and larger at larger $\theta_{\mathrm{r}}$. Although this is a coarse grid, there were few sharp features in the scattering distribution, so it was sufficient to capture the details of the scattering behavior.

\subsection{Near-retroreflective BRDF measurements}

Measurements of the BRDF were also performed in a near-retroreflective geometry, simultaneously varying the incident and viewing directions. Since the detector cannot view the illuminated sample closer than about $5^{\circ}$ from the retroreflective direction without blocking the incident radiation, the incident direction was scanned from $\theta_{\mathrm{i}}=0^{\circ}$ to $-75^{\circ}$, 
while the receiver was scanned from $\theta_{\mathrm{r}}=-5^{\circ}$ to $70^{\circ}$, each in steps of $1^{\circ}$, maintaining a constant bistatic angle of $5^{\circ}$. The results are displayed as a function of the central angle $\theta=\left(\theta_{\mathrm{r}}-\theta_{\mathrm{i}}\right) / 2$. These measurements were carried out scanning in the (110) crystal plane, as described before, and in the (100) crystal plane, which includes the [010] and [001] crystal directions (see Fig. 1). Scanning in the (110) plane allows us to observe direct specular reflection from the faces of the pyramids, while scanning in the (100) plane allows us to irradiate and view the sample along the edges of the pyramids. While the measurements are performed in steps of $1^{\circ}$, the goniometer and sample angles are aligned to within $0.1^{\circ}$. Sharp retroreflective features are reported to a precision of $0.5^{\circ}$.

\subsection{Mueller matrix measurements}

All of the measurements mentioned in Secs. 2.2 and 2.3 were obtained as full Mueller matrix polarimetry. The polarization state of directional radiation is characterized by a four-element Stokes vector,

$$
\left(I_{\hat{\mathbf{a}}}+I_{\hat{\mathbf{b}}}, \quad I_{\hat{\mathbf{a}}}-I_{\hat{\mathbf{b}}}, \quad I_{\hat{\mathbf{a}}+\hat{\mathbf{b}}}-I_{\hat{\mathbf{a}}-\hat{\mathbf{b}}}, \quad I_{\mathrm{lcp}}-I_{\mathrm{rcp}}\right)^{T},
$$

where $\hat{\mathbf{a}}$ and $\hat{\mathbf{b}}$ are unit vectors chosen to be orthogonal to each other and to the direction of propagation $\hat{\mathbf{k}}$, so that $\hat{\mathbf{a}} \times \hat{\mathbf{b}}=$ $\hat{\mathbf{k}}$. The choice for $\hat{\mathbf{a}}$ and $\hat{\mathbf{b}}$ will be discussed below. The last element is the difference between left circular polarization (lcp) and right circular polarization (rcp). The $I_{q}$ indicates a temporally- and/or spatially-averaged intensity-like quantity (e.g., radiance, irradiance, radiant power, etc.), measured with an analyzer for polarization $q$. The choice of handedness and the signs of the second, third, and fourth elements is arbitrarily chosen, but is consistent, once that choice is made.

A Mueller matrix represents a linear relationship between two intensity-like quantities. In the context of the measurements here, the BRDF, given by Eq. (1), is the relationship between the incident radiant power, $\Phi_{i}$, a Stokes vector, and the scattered radiant power, $\Phi_{\mathrm{r}}$, also a Stokes vector, and is thus a Mueller matrix. The representation of that Mueller matrix, however, depends upon the basis used for defining the Stokes vectors. [7] The choice for $\hat{\mathbf{a}}$ and $\hat{\mathbf{b}}$ in Eq. (2) is relatively straightforward for specular reflection and for measurements in the plane of incidence, since it is natural to use vectors $\widehat{\mathbf{s}}$ and $\widehat{\mathbf{p}}_{j}$, respectively, where $\widehat{\mathbf{s}}$ is perpendicular to the plane of incidence, and $\widehat{\mathbf{p}}_{j}=\hat{\mathbf{k}}_{j} \times \widehat{\mathbf{s}}(j=\mathrm{i}, \mathrm{r})$. However, in general viewing geometries out of the plane of incidence, the choice of basis is not unique. Converting Mueller matrices measured in one basis to that measured in another is straightforward. At least three such bases, which we will denote spsp, plane, and xyxy, are typically used:

- For spsp, $\widehat{\mathbf{s}}_{\mathrm{i}}$ and $\widehat{\mathbf{p}}_{\mathrm{i}}$ are defined perpendicular and parallel, respectively, to the plane containing the surface normal and the incident direction (the incident plane) and used for the incident direction, while $\widehat{\mathbf{s}}_{\mathrm{r}}$ and $\widehat{\mathbf{p}}_{\mathrm{r}}$ are likewise defined by the plane containing the surface normal and the viewing direction (the viewing plane) and used for the viewing direction. The spsp basis has an ambiguity when either the incident direction or the scattering direction lies along the surface normal, creating a basis-induced singularity in this direction.

- The plane basis uses $\widehat{\boldsymbol{\sigma}}$ and $\widehat{\boldsymbol{\pi}}_{\mathrm{i}}$ for the incident radiation and $\widehat{\boldsymbol{\sigma}}$ and $\widehat{\boldsymbol{\pi}}_{\mathrm{r}}$ for the viewing direction, where $\widehat{\boldsymbol{\sigma}}$ is perpendicular to the scattering plane, defined by the incident and viewing directions, and $\widehat{\boldsymbol{\pi}}_{\mathrm{i}}$ and $\widehat{\boldsymbol{\pi}}_{\mathrm{r}}$ are each in the scattering plane $\widehat{\boldsymbol{\pi}}_{j}=\hat{\mathbf{k}}_{j} \times \widehat{\boldsymbol{\sigma}}$. The plane basis also has basis-induced singularities, namely when the incident and scattering directions are collinear. Thus, for reflective measurements, a singularity will occur in the retroreflective direction. This basis is the natural one for two different types of scattering: free space aerosols (not considered here) and simple facet scattering (discussed later). It is also the natural basis for the laboratory, since the scattering plane is fixed and horizontal.

- Finally, the xyxy basis uses vectors $\hat{\mathbf{y}}_{\mathrm{i}}$ and $\hat{\mathbf{x}}_{\mathrm{i}}$ for the incident radiation and $\hat{\mathbf{y}}_{\mathrm{r}}$ and $\hat{\mathbf{x}}_{\mathrm{r}}$ for the scattered radiation, where

$$
\begin{aligned}
\hat{\mathbf{y}}_{j} & =\widehat{\mathbf{s}}_{j} \cos \phi_{j}-\widehat{\mathbf{p}}_{j} \sin \phi_{j}, \\
\widehat{\mathbf{x}}_{j} & =\widehat{\mathbf{s}}_{j} \sin \phi_{j}+\widehat{\mathbf{p}}_{j} \cos \phi_{j},
\end{aligned}
$$

which removes the basis-induced singularity along the surface normal that exists in the spsp basis. The xyxy basis is the natural laboratory basis for conoscopic, back-focal plane imaging, microscopes.

All measurements reported here were obtained as full Mueller matrices using a $4 \times 4$ measurement scheme with four incident polarization states and four polarization analysis states. These states were each achieved by rotating a retarder with one of its axes at $-45^{\circ},-15^{\circ}, 15^{\circ}$, and $45^{\circ}$ with respect to the axis of an adjacent polarizer. The data reduction 
matrix, which relates the sixteen measurements to the sixteen elements of the Mueller matrix, was determined using the scheme described by Compain, et al., [8] using eighteen rotations of a Glan polarizer and reflection from an aluminum mirror at thirteen incident angles. The method, requiring only a single polarizer at $45^{\circ}$ and a single retarder/diattenuator, was performed with much higher redundancy to improve the calibration and to provide statistics that indicate the uncertainties in subsequent measurements. The standard deviation of all of the matrix elements from their nominal values was 0.004 . The mean transmittance of the polarizer was found to be 0.479 with a standard deviation of 0.009 . Mueller matrix BRDFs in this paper are displayed normalized. That is, the upper left element, $f_{\mathrm{r}, 00}$, is shown in the natural units for BRDF, $\mathrm{sr}^{-1}$, while the other elements are shown normalized, that is $m_{i j}=f_{\mathrm{r}, i j} / f_{\mathrm{r}, 00}$.

The Gil-Bernabeu depolarization index is a measure of the degree to which a medium depolarizes radiation and is related to the Mueller matrix $\mathbf{M}$ by [9]

$$
P_{\mathrm{D}}=\sqrt{\left[\operatorname{tr}\left(\mathbf{M}^{\mathrm{T}} \mathbf{M}\right)-M_{00}^{2}\right] /\left(3 M_{00}^{2}\right)}
$$

If $P_{D}=1$, the medium is non-depolarizing, while for $P_{D}=0$, the medium is a total depolarizer. A lack of depolarization suggests that only one scattering path is contributing to the signal, while existence of depolarization suggests that multiple, incoherent paths are contributing to the signal.

\section{SAMPLES}

Three silicon samples were measured for this study. Figure 2 show a scanning electron microscope (SEM) image for each sample. Figure 2(a) shows an as-cut silicon surface, that obtained after sawing and with no polishing or etching. No visible saw marks could be observed on the surface. Figures 2(b) and 2(c) show pyramidal silicon, created by alkaline etching $(\mathrm{KOH})$ of silicon with two different additives. The sample shown in Fig.2(b) had isopropyl alcohol (IPA) as an additive, while Fig. 2(c) used a commercial surfactant (RENA monoTEX ${ }^{1}$ ). These samples will be referred to as as-cut, IPA, and RT, respectively. The IPA sample has pyramids with linear dimensions about two to three times larger than those on the RT sample.

(a) As-cut silicon

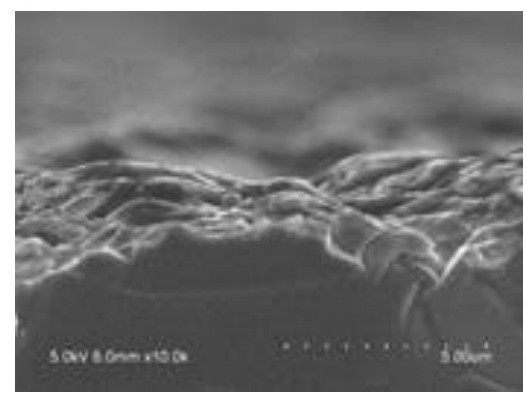

(b) IPA

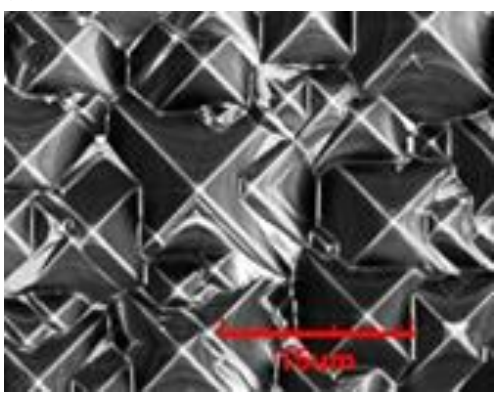

(c) RT

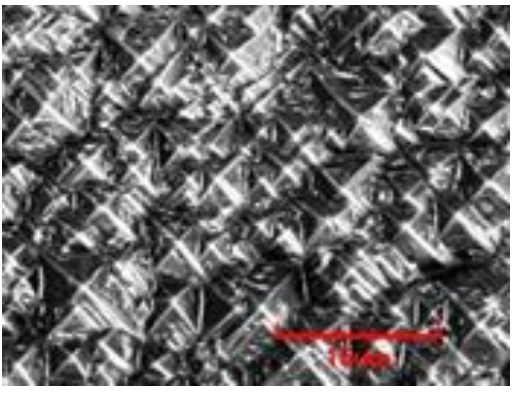

Figure 2. SEM images of the three samples used in this study: (a) as-cut silicon, (b) silicon etched with KOH and an IPA additive, and (c) silicon etched with $\mathrm{KOH}$ and a commercial surfactant additive. The scales of the three images are different; the bars correspond to $5 \mu \mathrm{m}, 15 \mu \mathrm{m}$, and $15 \mu \mathrm{m}$, respectively. In (a) the image is a side view, while in (b) and (c), the images are top view.

${ }^{1}$ Certain commercial equipment, instruments, or materials are identified in this paper to foster understanding. Such identification does not imply recommendation or endorsement by the authors, their employers, or their sponsors, nor does it imply that the materials or equipment identified are necessarily the best available for the purpose. 


\section{MODELING METHODS}

\subsection{Single reflection facet model}

The single reflection facet model for light scattering by a very rough surface has been commonplace in the scattering literature. The attractiveness of the model is that it is very simple to evaluate, as the BRDF is given by [10,11]

$$
f_{r}=\frac{P\left(\tan \theta_{n}\right)}{4 \cos \theta_{\mathrm{i}} \cos \theta_{\mathrm{r}} \cos ^{4} \theta_{\mathrm{n}}} R,
$$

where $P(\zeta)$ is the slope distribution function, $\theta_{\mathrm{n}}$ is the polar angle of a facet that specularly reflects from the incident direction to the viewing direction, and $R$ is the reflectance of such a facet. The reflectance $R$ is a non-depolarizing Mueller matrix that is derived from the Jones matrix for specular reflection and is most naturally expressed in the plane basis. In the retroreflection geometry, the normalized Mueller matrix should be diag $(1,1,-1,-1)$, as expected for normal incidence reflection. Many models have been derived from Eq. (5), differing by having different functional forms for $P(\zeta)$. The single reflection facet model does not treat multiply reflected rays.

\subsection{Monte Carlo ray tracing}

The scattering by pyramidal silicon, at least at normal incidence, is dominated by multiple reflective events. Monte Carlo ray tracing code was developed for modeling the scatter and absorption from rough silicon surfaces. The code generates random realizations of one or more interfaces, directs rays at a specified incident direction, and accumulates the Mueller matrix in a global basis for each path until the ray is either reflected or transmitted from the material, or if it has lost all but a specified fraction of its original energy, or if it has interacted with a surface over a specified number of times. At each interface, the Mueller matrices for reflection and transmission are calculated in a local basis. A uniform random number generator is used to decide which path (reflection, transmission, or absorption by any films on the surface) is chosen, and the normalized Mueller matrix is multiplied by the ray's accumulated Mueller matrix. Between surface interactions, the distance traveled is used with the absorption coefficient to reduce the energy in the ray. If the ray reflects or transmits outside the material, a virtual collector accumulates the Mueller matrix. The collector discretizes the directional spectrum by collecting proportional power onto four nearest grid points in proportion to the inverse distance of the ray from the grid points. The grid points can be on an even solid angle, projected solid angle, or angle grid and the number of grid points can be chosen as needed. The total absorbed energy for unpolarized incident light is also accumulated for each layer. Any number of surfaces can be included, but for the simulations described here, since silicon does not transmit at $633 \mathrm{~nm}$, only one was needed.

A wide variety of random and deterministic surfaces can be generated on a discretized grid. The surfaces can be randomized by Fourier decomposition, multiplication by random phases, and inverse Fourier restoration, if desired. Gaussian smoothening can be applied, as well, by a similar algorithm. Multiple surface functions can be added to one another. A number of surface generators specific to pyramids were developed. The one used here randomly places the top of pyramids at uniform random lateral positions and exponentially-distributed depths and chooses the highest point of all of the overlapping pyramids to determine the surface function. All surfaces are designed to be periodic, so that no vertical edge artifacts exist. One or more thin films can be applied to any of the interfaces, and their inclusion affects the reflection and transmission coefficients and absorption. Typically, 80 to 120 realizations of the surface are generated, each with 50 pyramids, and $10^{4}$ rays used for each realization.

\subsection{Diffraction}

The preponderance of aligned triangular facets on the surface are expected to behave like triangular apertures. The diffraction from these triangles can be estimated by Fourier transformation of the triangular function and the result are fans extending perpendicular to the edges when the radiation is incident normal to the triangle. [12] The full-width at halfmaximum of the fans is inversely proportional to the length $L$ of the edges, roughly $0.88 \lambda / L$ in radians. At non-normal incidence, the directions that diffraction from an edge occurs can be calculated by requiring that the projection of the incident direction onto the edge is the same as the projection of the scattered direction on that edge. That is, these directions form the locus of solutions to

$$
\hat{\mathbf{k}}_{\mathrm{i}} \cdot \hat{\mathbf{v}}=\hat{\mathbf{k}}_{\mathrm{r}} \cdot \hat{\mathbf{v}},
$$

where $\hat{\mathbf{v}}$ is a unit vector along the edge. These diffracted directions satisfy 


$$
\hat{\mathbf{k}}_{\mathrm{r}}=\hat{\mathbf{v}}\left(\hat{\mathbf{k}}_{\mathrm{i}} \cdot \hat{\mathbf{v}}\right)+(\widehat{\mathbf{u}} \cos \beta+\widehat{\mathbf{w}} \sin \beta) \sqrt{1-\left(\hat{\mathbf{k}}_{\mathrm{i}} \cdot \hat{\mathbf{v}}\right)^{2}},
$$

where $\widehat{\mathbf{u}}$ and $\widehat{\mathbf{w}}$ are unit vectors perpendicular to $\widehat{\mathbf{v}}$ and to each other, and $\beta$ parameterizes the locus. Retro-reflection (that is, when $\hat{\mathbf{k}}_{\mathrm{r}}=-\hat{\mathbf{k}}_{\mathrm{i}}$ ) from that edge will occur when $\hat{\mathbf{k}}_{\mathrm{i}} \cdot \hat{\mathbf{v}}=0$, that is, when the incident radiation is perpendicular to the edge.

The angle $\alpha_{\mathrm{e}}$ that the edge of a square pyramid makes with respect to the mean surface plane is related to the angle $\alpha_{\mathrm{f}}$ of the pyramid face by $\sqrt{2} \tan \alpha_{\mathrm{e}}=\tan \alpha_{\mathrm{f}}$. A pyramid on a (001) surface of silicon and having faces consisting of (111) faces will have those faces oriented $\alpha_{\mathrm{f}}=\arctan \sqrt{2} \approx 54.7^{\circ}$ from the surface normal and edges oriented at $\alpha_{\mathrm{e}}=$ $45^{\circ}$.

Diffraction from edges may also be reflected in opposing faces. The angle at which a ray diffracts from an edge to be retro-reflected from a face, when the incident direction is in the (100) plane, is

$$
\cos \alpha_{\mathrm{r}}=\frac{2 \cos ^{2} \alpha_{\mathrm{f}}}{\sqrt{-\frac{7}{2}+6 \cos 2 \alpha_{\mathrm{f}}-\frac{1}{2} \cos 4 \alpha_{\mathrm{f}}+2 \sec ^{2} \alpha_{\mathrm{f}}}} .
$$

(a) $\theta_{\mathrm{i}}=5^{\circ}$

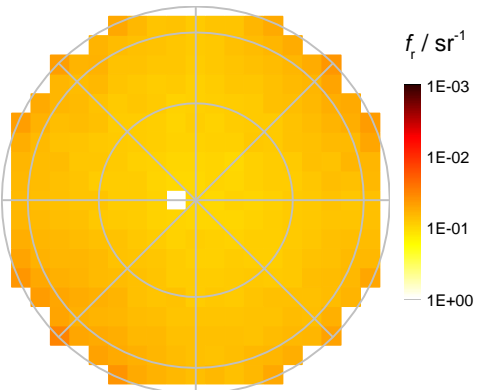

(b) $\theta_{\mathrm{i}}=60^{\circ}$

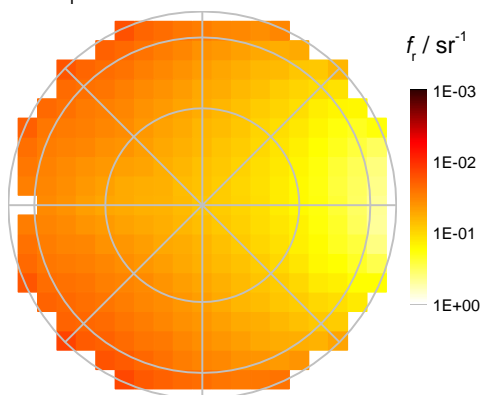

Figure 3. The BRDF measured from the as-cut silicon sample shown in direction-cosine space for incident angles (a) $5^{\circ}$ and (b) $60^{\circ}$. The plane of incidence is a horizontal slice through the center. The missing point in each frame is due to the receiver blocking the incident beam. A polar grid is superimposed on the BRDF to guide the eye.

\section{RESULTS AND DISCUSSION}

\subsection{As-cut sample measurements}

Figure 3 shows the results for the hemispherical BRDF measurements for the as-cut silicon sample, with incident angles of $\theta_{\mathrm{i}}=0^{\circ}$ and $60^{\circ}$. The BRDF shows few features, only a broad forward scattering lobe when $\theta_{\mathrm{i}}=60^{\circ}$. There is a slight asymmetry to the scattering that is roughly aligned with the crystal axes, but a little off, presumably due to the saw direction, a propensity for a specific cleavage facet, or both. Since the reflectance $\rho=\pi\left\langle f_{r}\right\rangle$ when points are sampled uniformly in direction-cosine space, the reflectance of the surface can be determined from these data to be about 0.24 and 0.25 at $0^{\circ}$ and $60^{\circ}$, respectively.

Figure 4 shows the normalized Mueller matrix for the as-cut silicon sample and $\theta_{\mathrm{i}}=60^{\circ}$ for the three different bases. By showing the Mueller matrix with the three bases, we demonstrate why a specific basis may be particularly advantageous for a specific scatterer. In this case, the results in the plane basis are predominantly of the form

$$
\left(\begin{array}{cccc}
1 & m_{01} & 0 & 0 \\
m_{01} & 1 & 0 & 0 \\
0 & 0 & m_{22} & m_{23} \\
0 & 0 & -m_{23} & m_{22}
\end{array}\right)
$$


This form is expected when the material is non-depolarizing and when the Jones matrix is diagonal. Furthermore, the $m_{23}$ and $m_{32}$ elements are weak when the phase between the Jones matrix elements is small. Thus, in the context of this measurement, the plane basis is the natural basis for the scattering from this sample. As for the other bases, there is a singularity in the Mueller matrix in the spsp basis that dominates the appearance of that matrix. No singularities are observed in the xyxy basis set, but the form of the matrix is more complicated than Eq. (9). The singularity that exists in the plane basis, seen along the plane of incidence, to left of the center of the graphs, in the retroreflective direction, is at a point where the Mueller matrix is very nearly diagonal (the off-diagonal elements all show nearly white in this region), and is very weak for this sample. While weak, it is most observable in the $m_{13}$ and $m_{31}$ elements, and to an even lesser degree, the $m_{23}$ and $m_{32}$ elements.

(a) plane

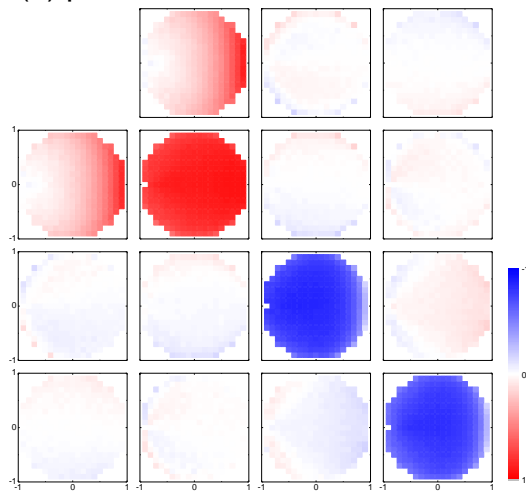

(b) xyxy

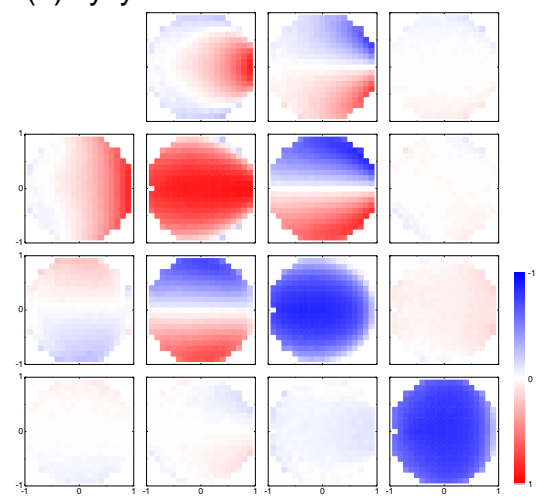

(c) $\operatorname{spsp}$

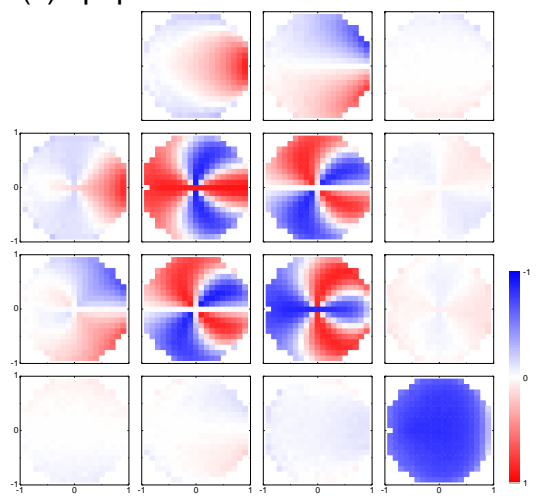

Figure 4. The normalized Mueller matrix for scattering from the as-cut silicon sample for three different bases measured with an incident angle $\theta_{\mathrm{i}}=60^{\circ}$. The bases are (a) plane, (b) xyxy, and (c) spsp.

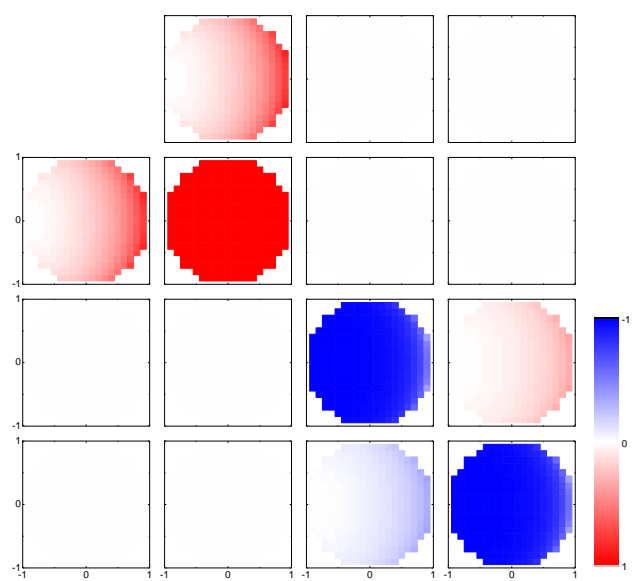

Figure 5. The simulated normalized Mueller matrix elements in the plane basis for a rough silicon surface in the single reflection microfacet approximation with $\lambda=633 \mathrm{~nm}$ and $\theta_{\mathrm{i}}=60^{\circ}$. A $20 \mathrm{~nm}$ Bruggeman effective medium approximation $1: 1$ mix of silicon and vacuum was included in the simulation.

That the plane basis nearly diagonalizes the Jones matrix is not universal to surface scattering. However, it is the natural basis for the single reflection facet scattering model. In Fig. 5, we show a simulation using the facet model, where we have included a thin effective medium approximation layer, which is necessary to simulate the observed behavior in $m_{23}$ and $m_{32}$. It is worth noting that, within the facet approximation, the Mueller matrix is not a function of the slope distribution. The agreement between the simulation (Fig. 5) and the measurement [Fig. 4(a)] is reasonable. As mentioned above, the small deviations and especially the observation of the weak singularity in elements $m_{13}, m_{31}, m_{23}$, and $m_{32}$ suggest that other effects, such as multiple scattering and diffraction, play a minor role. 
Figure 6 shows the near-retroreflection measurement results for the as-cut silicon sample. It is observed that the scatter is higher and has a broad shoulder in the (110) plane, in comparison to measurements in the (100) plane. Whether these results are indicative of residual saw marks or whether they are due to a preference for facets aligned along the (111) cleavage plane is unknown. The shoulder in the (110) retroreflection measurements, while being centered near the $54.7^{\circ}$ angle, is too broad to make this assignment conclusive.

Figure 6 also shows the block-diagonal elements of the Mueller matrix in retroreflection. The off-block-diagonal elements are all zero within the expected uncertainties and are not shown. If the retroreflection signal were completely dominated by single reflection, the Mueller matrix would indicate normal incidence reflection and be diag $(1,1,-1,-1)$. While the matrix is close to this, especially in the (110) plane, clear deviations can be observed. These deviations are probably from the minor role that multiple scattering and diffraction are expected to play.
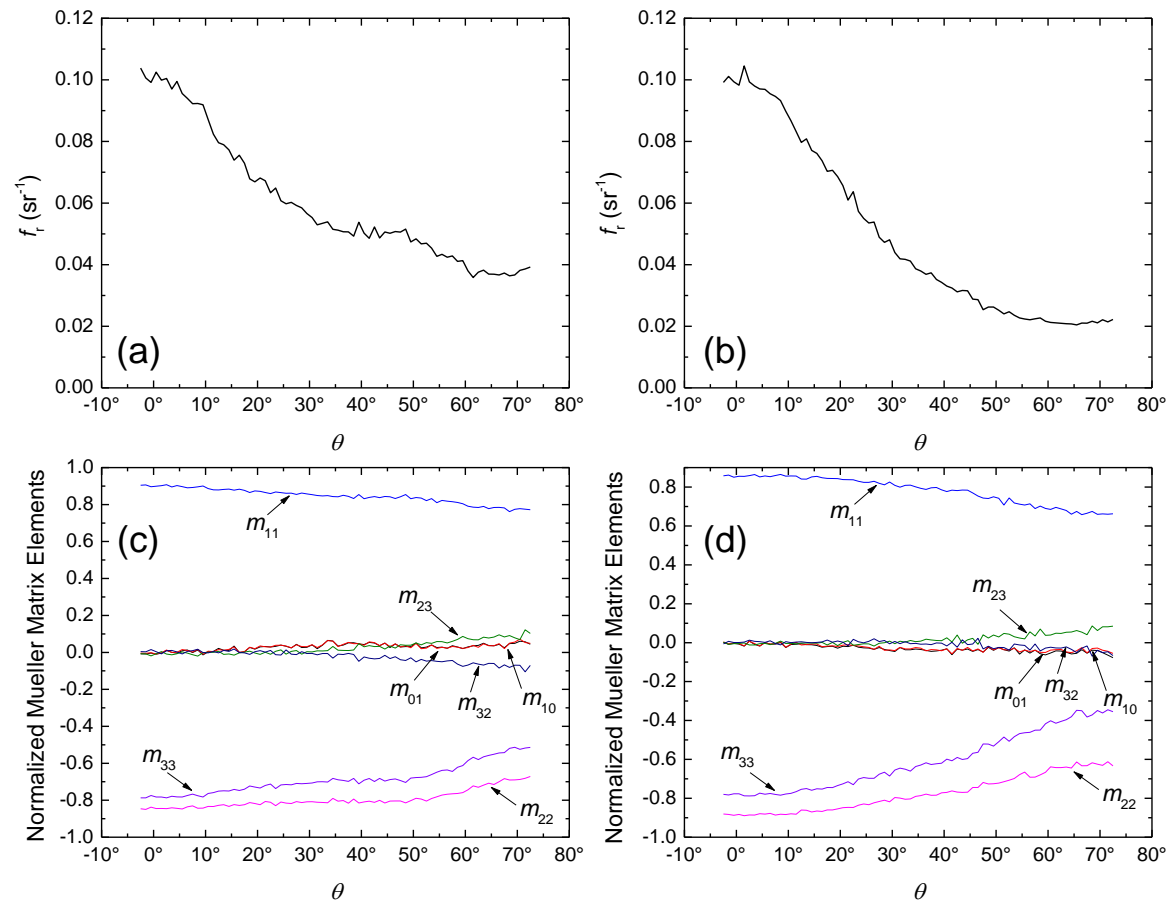

Figure 6. The results from near-retroreflection measurements from the as-cut silicon sample as functions of the center angle: the BRDF measured in (a) the (110) plane, and (b) the (100) plane, and the normalized Mueller matrix elements measured in (c) the (110) plane, and (d) the (100) plane. Only the block-diagonal Mueller matrix elements are shown; the off-block elements are very close to zero.

While the plane basis is particularly interesting for the as-cut silicon sample, the block-diagonal behavior in that basis was unique to that sample. In the remainder of this paper, we will only show data in the xyxy basis, since it has the fewest basis-induced artifacts. No diagonalization in the plane basis was observed for the pyramidal silicon samples.

\subsection{Pyramidal silicon sample measurements}

Figure 7 shows the unpolarized BRDF (the $f_{\mathrm{r}, 00}$ Mueller matrix element) for the pyramidal silicon samples for $5^{\circ}$ and $60^{\circ}$ incident angles. Both samples show significantly more structure than the as-cut sample. At $\theta_{\mathrm{i}}=5^{\circ}$ the IPA sample shows four clear peaks. These four peaks, marked A, B, and C, arise from double reflections from the faces of neighboring pyramids (see key on right of Fig. 7). The breaking of the symmetry (that the two out-of-plane peaks, marked C, move to the right, while the two in-plane peaks, marked A and B move to the left, as shown) is predicted by the multiple reflection model, and the peaks are roughly where they are expected. However, close inspection reveals that they are closer together than expected from (111) facets. At $\theta_{\mathrm{i}}=60^{\circ}$, there is a very strong back reflection (shown saturated in the figure at D) that is expected from the direct reflection from the pyramid faces. In the forward scattering direction, just off the plane of 
incidence, there are two distinct, but relatively weak peaks marked $\mathrm{C}$, which correspond to a double reflection and which evolved from the out-of-plane peaks observed at $\theta_{\mathrm{i}}=5^{\circ}$. Most striking in the $\theta_{\mathrm{i}}=60^{\circ}$ data is the band of radiation in outof-plane directions starting from the feature D. This latter band can be best assigned to diffraction from the edges of the triangular faces of the pyramids. Superimposed on the $\theta_{\mathrm{i}}=60^{\circ}$ data in Fig. 7 are curves showing the expected directions from such diffraction. Also, the expected locations of other doubly reflected rays (E and F) are shown with the key to their assignments. The angles used for calculating the diffraction curves and the locations of the rays are taken from the retroreflection measurements described below.

(a) IPA $5^{\circ}$

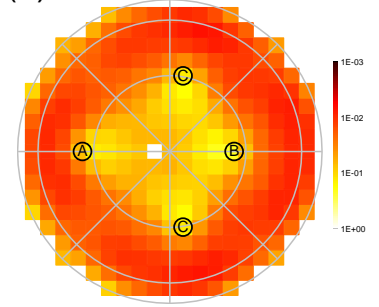

(c) IPA $60^{\circ}$

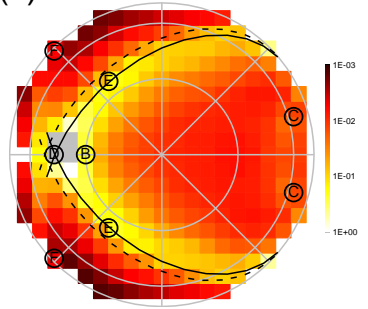

(b) RT $5^{\circ}$

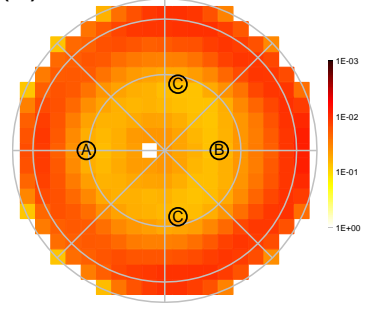

(d) RT $60^{\circ}$

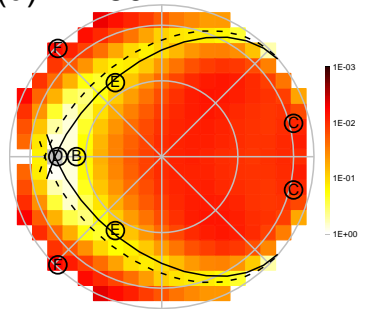

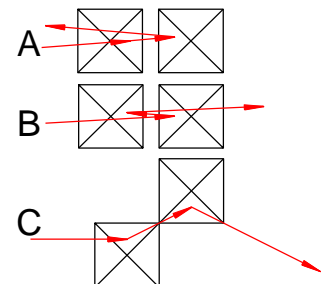

D

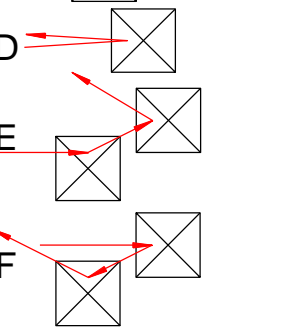

Figure 7. The BRDF measured from the pyramidal silicon samples shown in direction-cosine space for (a) and (c) the IPA sample and (b) and (d) the RT sample. The incident angles were (a) and (b) $\theta_{\mathrm{i}}=5^{\circ}$ and (c) and (d) $\theta_{\mathrm{i}}=60^{\circ}$. The plane of incidence is a horizontal slice through the center. Superimposed upon the BRDF data are expected locations for diffraction from edges with angles (solid curves) $42.5^{\circ}$ and (dashed curves) $45.5^{\circ}$, and (circles with letters, key on the right) single and double reflections. Also superimposed on the BRDFs are polar grids to guide the eye.

Figure 8 shows the near retro-reflection behavior of the pyramidal silicon samples. In the (110) plane, a sharp peak is observed for the IPA sample, centered on the angle $\theta=52.5^{\circ}$. This peak can be readily assigned to the normal reflection of pyramid faces. That this angle differs from the orientation of $(111)$ crystal faces $\left(54.7^{\circ}\right)$ is consistent with other findings that the faces of the pyramids formed by alkali etching are close to, but not exactly, (111) faces. [4, 13] There is also a broad peak near $12^{\circ}$; this peak is believed to be a combination of a number events, including a three-reflection, enhanced backscatter event.

When measuring the near-retro-reflection from the IPA sample in the (100) plane, shown in Fig. 8(b), there are two distinct peaks at $36.5^{\circ}$ and $42.5^{\circ}$. These features cannot be correlated with any expected reflections from (111) or near(111) facets. Instead, we find that they require diffraction to understand. In particular, the peak at $\theta=42.5^{\circ}$ matches well to the edge angle of the pyramids, which would be $42.7^{\circ}$ for pyramids having faces oriented at $52.5^{\circ}$. Thus, the $\theta=42.5^{\circ}$ feature is assigned to diffraction from the pyramid edges. The $\theta=36.5^{\circ}$ feature is likely to be a combination of diffraction from an edge and reflection from a pyramid face. Such a reflection and diffraction combination depends strongly on the pyramid angle $\alpha_{\mathrm{f}}$ [see Eq. (8)]. Thus, for $\alpha_{\mathrm{f}}=52.5^{\circ}$ (the value determined for the pyramid angle above), 53.2 ${ }^{\circ}$, and $54.7^{\circ}$ (the ideal pyramid), retro-reflection will occur at $\theta=32.8^{\circ}, 36.5^{\circ}$ (the value from above), and $45^{\circ}$, respectively. Thus, we believe that this assignment lies within the anticipated resolution of the measurement ( $\approx 1^{\circ}$ due to sampling spacing).

We notice that the intensity of the $36.5^{\circ}$ feature is higher than the $42.5^{\circ}$ feature. Because it is a multiple scattering event, i.e., a face reflection and an edge diffraction, it is subject to enhanced backscattering. [14] That is, in the retroreflection direction, the path lengths of the forward and reversed paths coincide, so that the fields coherently add. Thus, even though the extra reflection might be expected to reduce the reflectance, the backscattering effect might be enhancing it. 

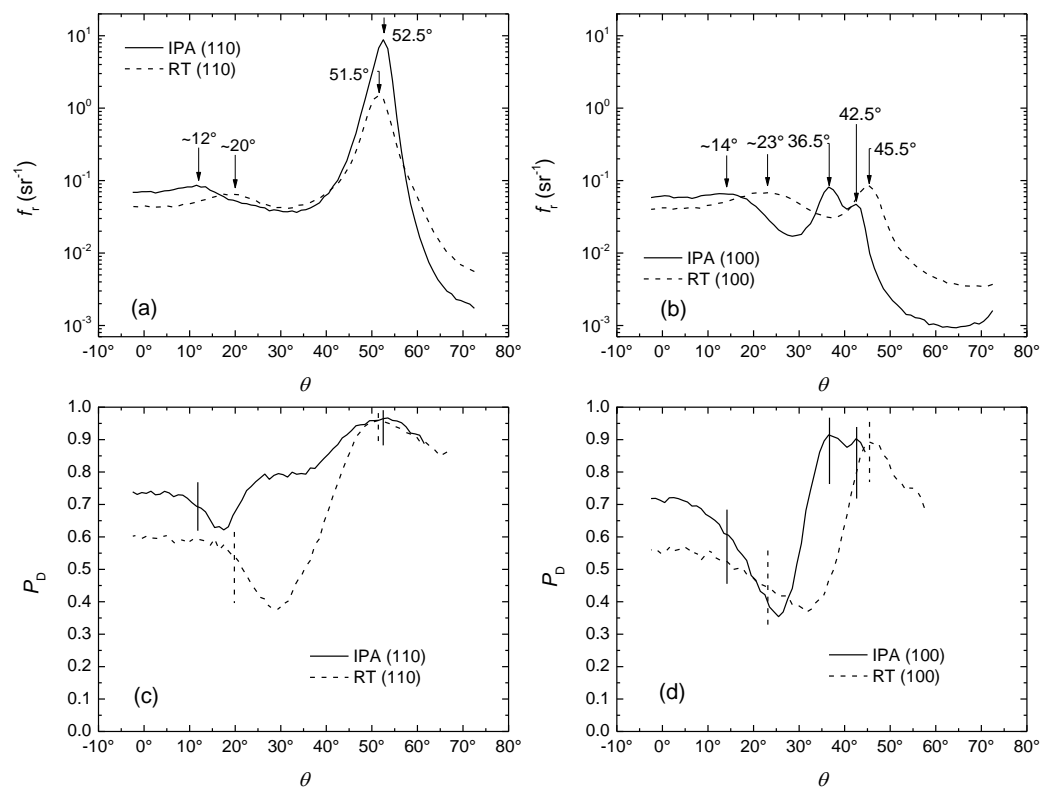

Figure 8. The results from near-retroreflection measurements from the (solid) IPA sample and (dashed) RT sample as functions of the center angle, with (a) and (b) the BRDF and (c) and (d) the depolarization index, measured in (a) and (c) the (110) plane and (b) and (d) the (100) plane. Vertical marks in (c) and (d) are at the same angles as the feature labels in (a) and (b), respectively.

(a)

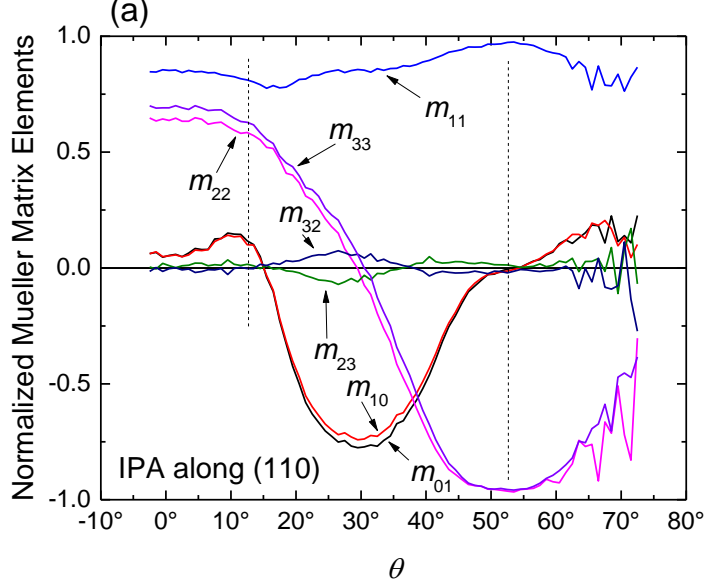

(b)

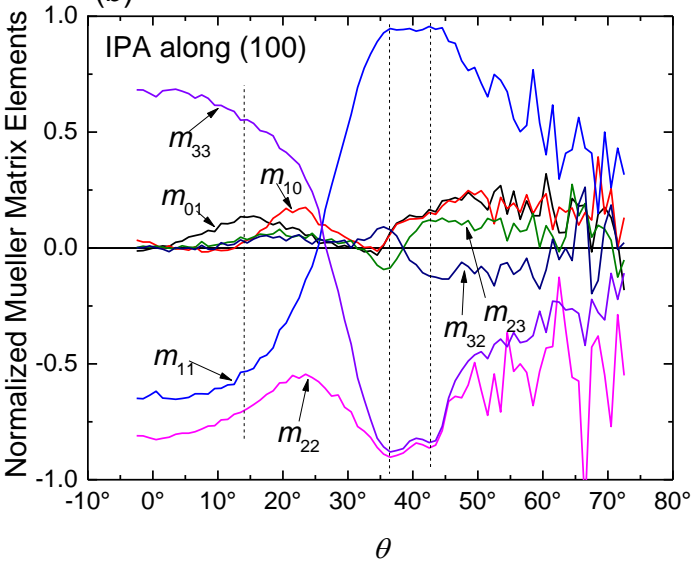

Figure 9. Normalized Mueller matrix elements measured for the IPA sample in the near-retroreflection geometery in (a) the (110) plane and (b) the (100) plane. The vertical marks are aligned with features observed for the IPA sample in Fig. 8.

Figure 8 also shows the depolarization index $P_{\mathrm{D}}$ for the near-retroreflection measurements. At the locations of the assigned higher angle peaks mentioned above, the depolarization index is high, as expected for simple deterministic scattering events. At the lower angle peak, the depolarization index is significantly lower and does not peak at the same angles as peaks in the intensity, suggesting that the origin of these features is significantly more complicated. Figure 9 shows the block diagonal Mueller matrix elements for the same data shown in Fig. 8. For measurements in the (110) plane, the matrix has approximately the behavior observed in Eq. (9) (the off-block-diagonal matrix elements are zero and not shown). The feature at $52.5^{\circ}$ shows values close to $\operatorname{diag}(1,1,-1,-1)$, consistent with normal incidence reflection from the pyramid facets. For measurements in the (100) plane, the matrix is still block diagonal. While the matrix at both $36.5^{\circ}$ and $42.5^{\circ}$ features follow the form of Eq. (9), the elements $m_{23}$ and $m_{32}$ flip sign between them. At the lower angle 
features, however, there is a marked difference between $m_{10}$ and $m_{01}$. Such asymmetries in the Mueller matrix have been observed in the past in the presence of significant depolarization and indicate the presence of depolarization before or after the other scattering events. [15]

Figures 7 and 8 also show the hemispherical BRDF and the near-retroreflection measurements for the RT sample. The unpolarized BRDF shows a number of differences from the IPA sample. At $\theta_{\mathrm{i}}=5^{\circ}$, the four peaks observed for the IPA sample have disappeared and been replaced by a broad doughnut, while at $\theta_{\mathrm{i}}=60^{\circ}$, the feature assigned to diffraction from the pyramid edges is also broader and has shifted to higher polar angles. The broadening of the features is not surprising, since from Fig. 2 the pyramid dimensions are much smaller.

In the near-retroreflection measurements, the RT sample has somewhat different results than the IPA sample. In the (110) plane, the main peak is significantly reduced in intensity and shifted to a slightly lower angle, $\theta=51.5^{\circ}$, while the low angle peak has shifted to a higher angle, about $20^{\circ}$. In the (100) plane, there are only two features at about $23^{\circ}$ and $45.5^{\circ}$. It is difficult to reconcile the assignments of these angles with simple pyramids. The feature at $45.5^{\circ}$ is very close to the value expected for a (111)-faceted pyramid. Furthermore, the diffraction curve observed in the hemispherical data is closer to that expected for $45.5^{\circ}$ edges [see dashed curves in Fig. 7(d)]. This suggests that the edges are well aligned with the [101] direction. However, the direct retro-reflection suggests that the faces have a shallower angle than the (111) faces. These results suggest that the RT sample pyramids are more complex, perhaps having multiple facets, such as octagonal pyramids, or even curved faces. Such features have been observed in the past.[13, 16]

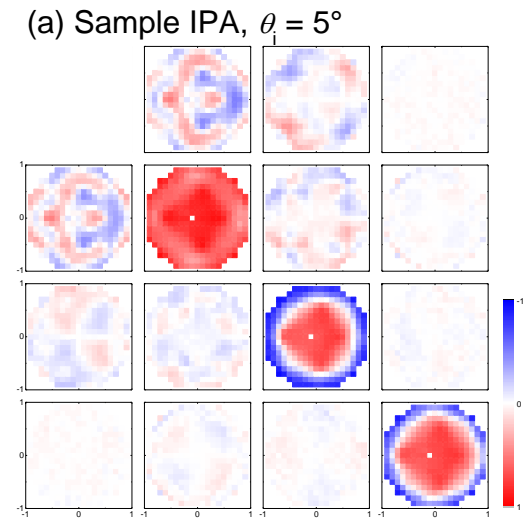

(c) Sample RT, $\theta_{\mathrm{i}}=5^{\circ}$

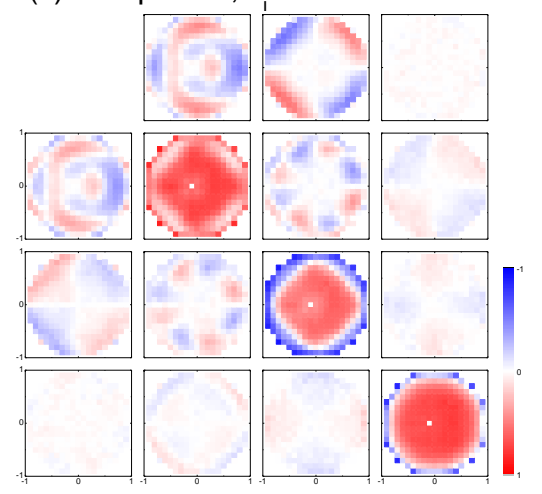

(b) Sample IPA, $\theta_{\mathrm{i}}=60^{\circ}$

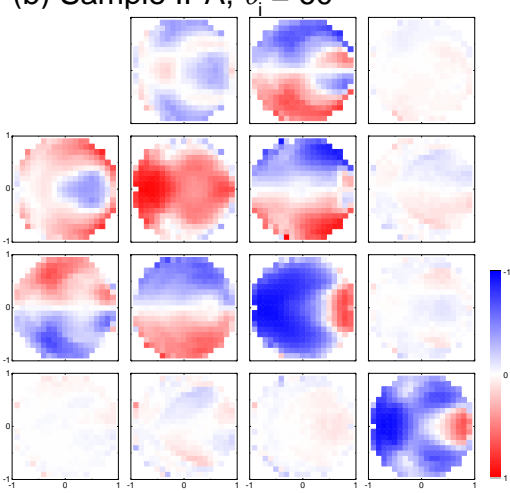

(d) Sample RT, $\theta_{\mathrm{i}}=60^{\circ}$

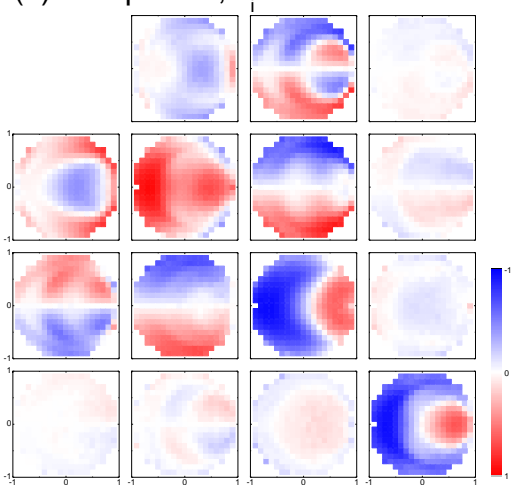

Figure 10. Normalized Mueller matrix in the xyxy basis measured for (a) and (b) sample IPA and (c) and (d) sample RT. The incident angles were (a) and (c) $5^{\circ}$ and (b) and (d) $60^{\circ}$.

Figure 10 shows the normalized Mueller matrices for both the IPA and the RT samples for both incident angles. As mentioned above, we use the xyxy basis to show the matrices, since there are no basis-induced artifacts and we did not observe any evidence for diagonalization by using the plane basis. Both samples show quite a bit of structure, which is not surprising, given the number of different reflection and diffraction features that are present. In general, the two samples 
have similar Mueller matrices for each angle, although those for the RT sample are much smoother and less distinct than those of the IPA sample. It is interesting to note that, while one has difficulty observing the double-reflection peaks in the unpolarized BRDF of the RT sample, one can see vestiges of those peaks in the Mueller matrix for $\theta_{\mathrm{i}}=5^{\circ}$.

Modeling was carried out to attempt to simulate the observed Mueller matrices. Since the models described above do not include diffraction, it is difficult to simulate the Mueller matrices over the hemisphere. However, we made a number of approximations that attempt to mimic the effects of diffraction. In particular, if we start with a pyramidal structure, smoothening the surface will cause the edges to round off in a manner that will reflect in directions that have a similar requirement as Eq. (5). Secondly, by adding some random roughness, we can simulate the natural broadening that will occur due to diffraction from the finite size of the pyramid faces. Figure 11 shows the results of these simulations. In must be stressed that these are not rigorous models and are just attempts to empirically capture the behavior of the observed Mueller matrices. While some features in the Mueller matrix are mimicked well, there are a number of features that were difficult to replicate. In particular, it is found that features in the last row and last column (except where they coincide, $m_{33}$ ), appear to have the wrong sign. While an effective medium layer was used to simulate subwavelength roughness (otherwise these elements would be negligible), it was found that the sign of these elements could only be simulated if the effective medium had a higher index than the bulk material. While a poor result may be expected in those scattering directions that are dominated by diffractive effects, it is somewhat surprising that the modeling does not replicate the measured behavior at the double-reflection features labeled C in Fig. 7(c).

(a)

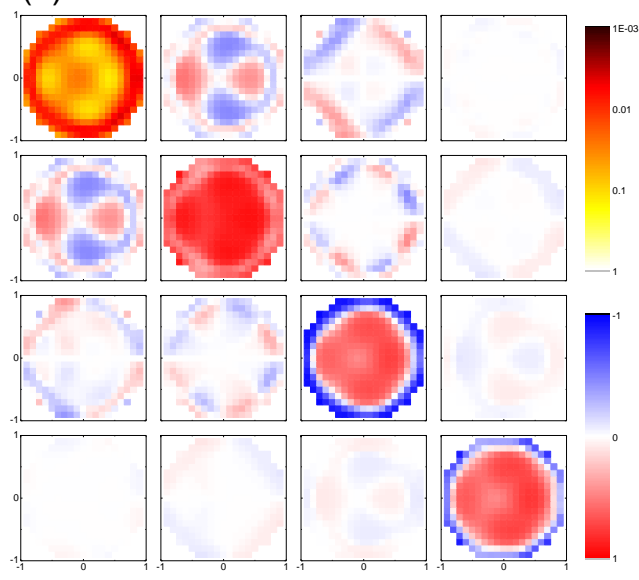

(b)

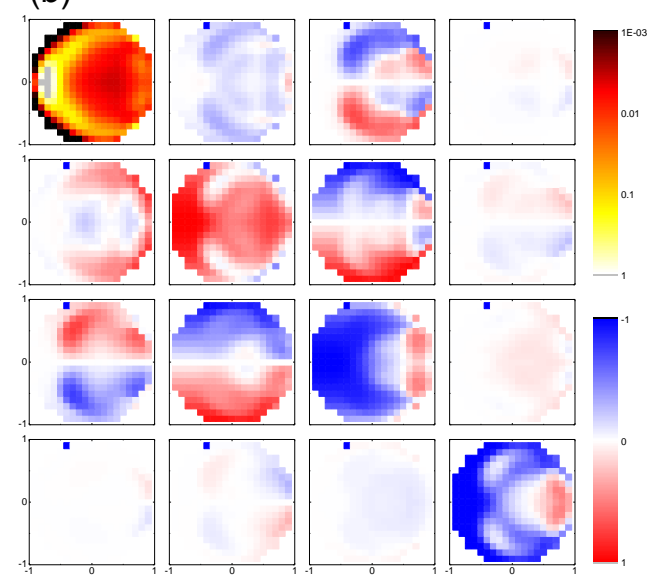

Figure 11. Modelled BRDF and normalized Mueller matrix for a pyramidal silicon as described in the text. The incident angles were (a) $5^{\circ}$ and (b) $60^{\circ}$.

\section{CONCLUSIONS}

These measurements demonstrate some of the strengths as well as weaknesses of using light scattering to characterize rough surfaces. The additional information provided by the Mueller matrix helps to identify the sources of light scattering and guides the researcher into applying an appropriate model. Additionally, the use of full hemispherical scans highlights anisotropic features in the material. Retroreflection measurements can be used to infer the slope distribution of the surface, and polarimetry provides a verification or rejection of that interpretation. In the case of the textured silicon surfaces measured in this study, some details of the surface pyramids were able to be deduced from the light scattering measurements. However, the results shown here also demonstrate the limitations of the scattering models. In particular, it was found that diffraction plays an important role in these materials. It is clear that models need to be developed that can be applied to large scale roughness, but which also include the diffractive nature of scattering. That is particularly difficult in these materials, since not only the lateral distances are large, the vertical distances are, too. The information gained from these measurements should prove useful for the development of photovoltaics. 


\section{REFERENCES}

[1] P. Campbell and M. A. Green, "Light trapping properties of pyramidally textured surfaces," J. Appl. Phys. 62, 243249 (1987).

[2] E. Yablonovitch, "Statistical ray optics," J. Opt. Soc. Am. 72, 899-907 (1982).

[3] E. Yablonovitch and G. D. Cody, "Intensity Enhancement in Textured Optical Sheets for Solar Cells," IEEE Trans. Electron Devices ED-29, 300-305 (1982).

[4] E. Forniés, C. Zaldo and J. M. Albella, "Control of random texture of monocrystalline silicon cells by angleresolved optical reflectance," Solar Energy Materials and Solar Cells 87, 583-593 (2005).

[5] H. Mäckel, D. M. Cambre, C. Zaldo, J. M. Albella, S. Sánchez, C. Vázquez, I. Sánchez and M. Á. Vásquez, "Characterization of monocrystalline silicon texture using optical refelctance patterns," in 23rd European Photovoltaic Solar Energy Conference and Exhibition, Valencia, Spain, pp. 1160-1163 (2008).

[6] T. A. Germer and C. C. Asmail, "Goniometric optical scatter instrument for out-of-plane ellipsometry measurements," Rev. Sci. Instr 70, 3688-3695 (1999).

[7] N. Trouve, E. Colin-Koeniguer, P. Fargette and A. De Martino, "Influence of Geometrical Configurations and Polarization Basis Definitions on the Analysis of Bistatic Polarimetric Measurements," IEEE Transactions on Geoscience and Remote Sensing 49, 2238-2250 (2011).

[8] E. Compain, S. Poirier and B. Drevillon, "General and self-consistent method for the calibration of polarization modulators, polarimeters, and Mueller-matrix ellipsometers," Appl. Opt 38, 3490-3502 (1999).

[9] J. J. Gil and E. Bernabeu, "Depolarization and polarization indices of an optical system," Optica Acta 33, 185-189 (1986).

[10] D. E. Barrick, "Rough surface scattering based on the specular point theory," IEEE Trans. Ant. Prop AP-16, 449454 (1968).

[11] K. E. Torrance and E. M. Sparrow, “Theory of off-specular reflection from roughened surfaces,” J. Opt. Soc. Am 57, 1105-1114 (1967).

[12] R. C. Smith and J. S. Marsh, “Diffraction patterns of simple apertures," J. Opt. Soc. Am. A 64, 798-803 (1974).

[13] J. D. Hylton, A. R. Burgers and W. C. Sinke, "Alkaline Etching for Reflectance Reduction in Multicrystalline Silicon Solar Cells," J. Electrochem. Soc 151, G408-G427 (2004).

[14] Y. N. Barabanenkov, Y. A. Kravtsov, V. D. Ozrin and A. I. Saichev, "Enhanced Backscattering in Optics," Progress in Optics 29, 65-197 (1991).

[15] T. A. Germer and H. J. Patrick, "Mueller matrix bidirectional reflectance distribution function measurements and modeling of diffuse reflectance standards," in Polarization Science and Remote Sensing V, edited by J. A. Shaw and J. S. Tyo, Proc. SPIE 8160, 81600D (2011).

[16] S.-S. Tan, M. L. Reed, H. Han and R. Boudreau, "Mechanisms of Etch Hillock Formation," J. Microelectromech. Sys. 5, 66-72 (1996). 


\title{
FINE-TUNING ELECTRICAL FLOW RATE SENSING IN DEFORMABLE CHANNELS
}

\author{
Pengfei Niu, Brian J. Nablo, Kiran Bhadriraju and Darwin R. Reyes \\ BioMEMS and Microsystems, Engineering Physics Division, PML, NIST, USA
}

\begin{abstract}
Here we present conclusive proof that when using electrical impedance to measure volumetric flow rate in polydimethylsiloxane (PDMS) microchannels a considerable fraction of the change in impedance, due to change in flow rate, is caused by the variation in bulk solution resistance rather than the disruption of the Helmholtz double layer. The variation in solution resistance is caused, in part, by the channel deformation under pressure. Placing electrodes near the microchannel inlet produces higher sensitivity due to greater channel deformation than those at the outlet. This finding is of paramount importance for the future design of impedance-based flow sensors.
\end{abstract}

KEYWORDS: Flow sensor, Flow rate, Channel Deformation, Electrical Impedance, PDMS

\section{INTRODUCTION}

Electrical monitoring of fluid flow within a microchannel is very attractive due to its relatively ease in fabrication, compactness, integration in microchannels and their non-invasiveness measurement process [1]. To develop highly sensitive and accurate impedance-based flow sensors, it is of paramount importance to understand what are the processes occurring between the two electrodes when changing flow rate, an issue that is not been well understood.

\section{EXPERIMENTAL}

Three pairs of parallel coplanar $12.5 \mu \mathrm{m}$ wide $\mathrm{Ti} / \mathrm{Au}(10 / 90 \mathrm{~nm})$ electrodes with varying gap distances $(0.37 \mathrm{~mm}, 1.00 \mathrm{~mm}, 3.70 \mathrm{~mm})$ were patterned on glass slides via standard lift-off processes. Microchannels (L x W x H: $45 \mu \mathrm{mm}$ x $420 \mu \mathrm{m}$ x $28 \mu \mathrm{m}$ ) were made of PDMS by soft lithography. The plasma-oxidized microchannel was bonded to the patterned glass with the electrodes positioned perpendicular to flow direction. The volumetric flow rates of phosphate buffer saline (PBS) solution in microchannels were controlled by a syringe pump (Harvard Apparatus) [2]. The impedance between two electrodes at different flow rates were measured with a Modulab potentiostat (Solartron Analytical). The deformation of microchannels with flow rate was measured by confocal microscopy after staining the channel walls with dead red stain (LIVE/DEAD cell imaging kit, Thermo Fisher).

\section{RESULTS AND DISCUSSION}

We observed a dependence of the electrical impedance on fluid flow rate (Fig. 1a) that could not be solely due to a disruption of the Helmholtz double layer. At low frequencies $(<1 \mathrm{kHz})$, where the total impedance is governed by the electrode/solution interface impedance, there is no considerable dependence of impedance on flow rate. In contrast, the greatest flow rate dependence was observed above $1 \mathrm{kHz}$, where the solution resistance governs the total impedance. Confocal imaging (Fig. 1b) of the channel cross-section clearly indicates a significant enlargement of the channel's cross-section area as the flow rate is increase to $100 \mu \mathrm{L} / \mathrm{min}$. In fact, the cross-sectional area correlated directly to the fluid flow rate with the greatest deformation observed closer to the channel inlet $\left(\mathrm{P}_{1}\right)$, where the pressure is higher. The solution resistance between two electrodes can be defined as $R=\rho \cdot L / S$, where $\rho$ is the solution resistivity, S is the cross section area of the PDMS channel and L is the separation between the two electrodes. A greater variation in the cross section area results in a larger change in solution resistance. Consequently, impedance sensors positioned closest to the inlet $\left(\mathrm{P}_{3}\right)$ will have a greater sensitivity to flow rate than those downstream $\left(\mathrm{P}_{4}\right)($ Fig. $1 \mathrm{c})$, which is demonstrated by the sensitivity differences at $\mathrm{P}_{3}$ and $\mathrm{P}_{4}$. Widening the electrode gap increases the contribution of solution resistance, thus further improving the sensitivity (Figure 1d).

By separating the contribution of solution resistance $\left(R_{S}\right)$ and electrode/solution interface impedance $\left(I Z I-R_{S}\right)$ from the total impedance value (Table 1), a clear relationship between flow rate and electrode/solution interface impedance is not apparent. By contrast, the real part impedance ( $\left.Z^{\prime}\right)$ decreases with flow rate. Moreover, this sensor demonstrates better performance when the real part of the impedance is greater than the imaginary component (i.e., the electrode/solution interface is not the dominant component of the impedance). 

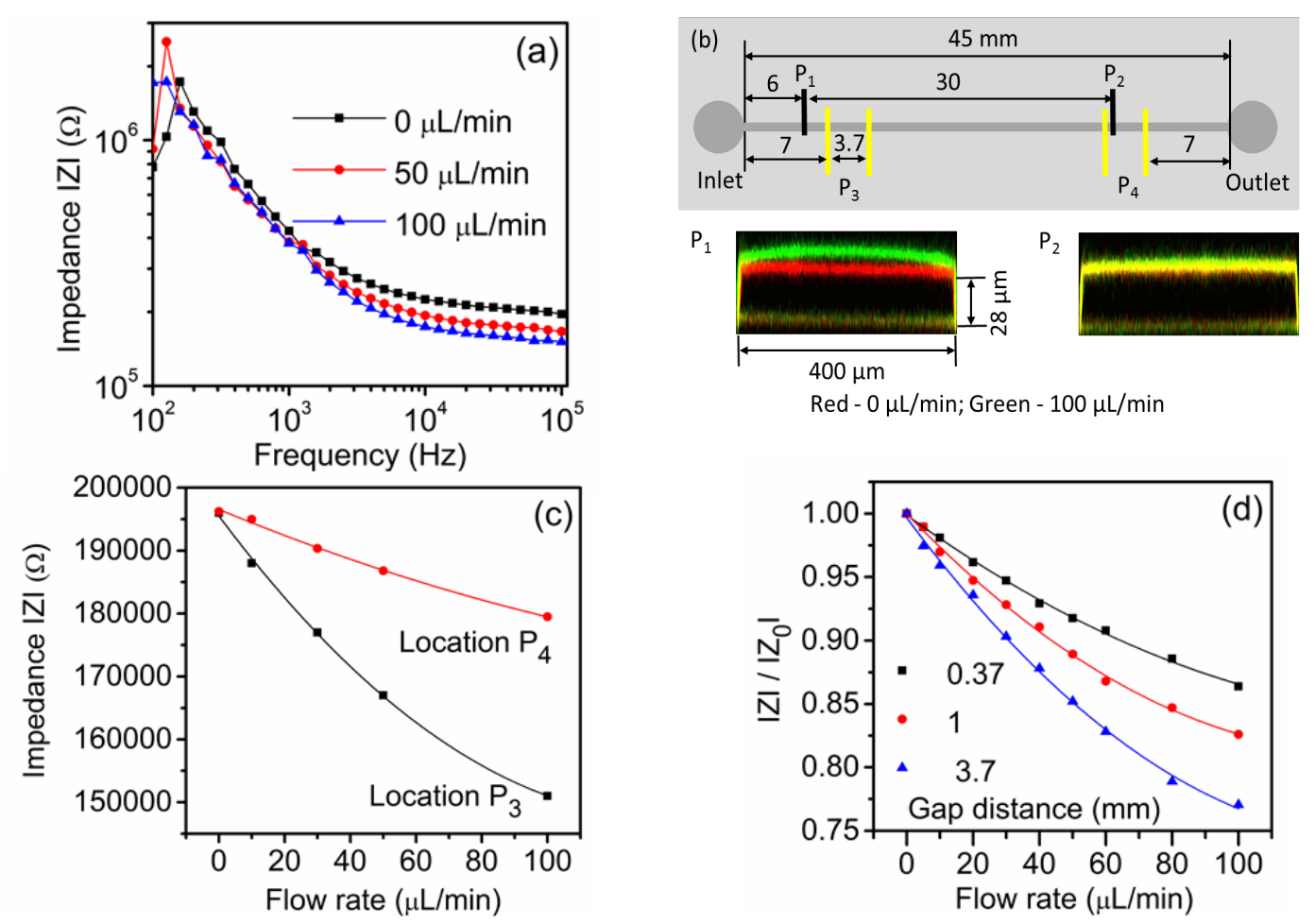

Figure 1. (a) Typical electrical impedance spectra of $1 X$ PBS recorded on sensor design of $12.5 \mu m$ wide $x$ $3.70 \mathrm{~mm}$ gap distance, positioned at location $P_{3}$ under flow rates of 0,50 and $100 \mu \mathrm{L} / \mathrm{min}$. (b) Cross section profile under $1 X$ PBS flow rates of 0 and $100 \mu \mathrm{L} / \mathrm{min}$, the aspect ratio was adjusted for presentation. (c) Influence of electrode position in the channel on sensor's sensitivity to flow rate. (d) Influence of gap distance on sensor's performance. The plotted impedance was measured at frequency of $100 \mathrm{kHz}$.

Table 1. Contribution of electrodelelectrolyte interface impedance in the total impedance (IZI- $\left.R_{S}\right)$ at various frequencies on sensor design (12.5 $\mu \mathrm{m}$ wide $\times 3.70 \mathrm{~mm}$ separation) in a $1 X$ PBS solution $(Z=k \Omega, R=k \Omega)$.

\begin{tabular}{|c|c|c|c|c|c|c|c|c|c|c|c|c|c|}
\hline \multirow{2}{*}{$\begin{array}{c}\text { Flow rate } \\
(\mu \mathrm{L} / \mathrm{min})\end{array}$} & \multirow{2}{*}{$\begin{array}{l}\mathrm{R}_{\mathrm{S}} \text { solution } \\
\text { resistance }\end{array}$} & \multicolumn{4}{|c|}{$2512 \mathrm{~Hz}$} & \multicolumn{4}{|c|}{$10000 \mathrm{~Hz}$} & \multicolumn{4}{|c|}{$100000 \mathrm{~Hz}$} \\
\hline & & $Z^{\prime}$ & $Z^{\prime \prime}$ & $|Z|$ & $|Z|-R_{S}$ & $Z^{\prime}$ & $Z^{\prime \prime}$ & $|Z|$ & $|\mathrm{ZI}| \mathrm{R}_{\mathrm{S}}$ & $Z^{\prime}$ & $Z^{\prime \prime}$ & $|Z|$ & $I Z I-R_{S}$ \\
\hline 0 & 187 & 252 & 151 & 293 & 106 & 218 & 55 & 225 & 38 & 192 & 39 & 196 & 9 \\
\hline 10 & 178 & 242 & 140 & 280 & 102 & 209 & 51 & 216 & 38 & 184 & 36 & 188 & 10 \\
\hline 30 & 173 & 228 & 126 & 261 & 88 & 197 & 48 & 203 & 30 & 173 & 33 & 177 & 4 \\
\hline 50 & 162 & 220 & 136 & 259 & 97 & 187 & 50 & 194 & 32 & 164 & 31 & 167 & 5 \\
\hline 100 & 146 & 197 & 138 & 241 & 95 & 168 & 48 & 174 & 28 & 149 & 26 & 151 & 5 \\
\hline
\end{tabular}

\section{CONCLUSIONS}

Flow derived microchannel deformation, which brings about the changes of bulk solution resistance between two electrodes, plays a decisive role in the impedance changes over flow rates. Those parameters that can increase the contribution of solution resistance, such as diluting solution concentration and widening electrode gap distance, will be critical when designing sensors with better performance.

\section{ACKNOWLEDGEMENTS}

This research was performed in part at the NIST Center for Nanoscale Science and Technology.

\section{REFERENCES}

[1] J. Collins and A. P. Lee, Microfluidic flow transducer based on the measurement of electrical admittance, Lab chip, 4, 7-10, 2004.

[2] Certain commercial equipment, instruments, or materials are identified in this paper to foster understanding. Such identification does not imply recommendation or endorsement by the National Institute of Standards and Technology, nor does it imply that the materials or equipment is necessarily the best available for the purpose.

\section{Contact}

*Darwin R. Reyes; phone: +1-301-975-5466; darwin.reyes@nist.gov 


\title{
Extended data analysis of bilateral comparisons with air and natural gas up to $5 \mathrm{MPa}$ Bodo Mickan ${ }^{1}$, Jean-Pierre Vallet ${ }^{2}$, Chunhui $\mathrm{Li}^{3}$ and John Wright ${ }^{4}$
}

\author{
${ }^{1}$ Physikalisch-Technische Bundesanstalt (PTB), Germany \\ ${ }^{2}$ CESAME EXADEBIT S.A., France \\ ${ }^{3}$ National Institute of Metrology (NIM), China \\ ${ }^{4}$ National Institute for Standards and Technology (NIST), USA \\ ${ }^{1}$ E-mail:bodo.mickan@ptb.de
}

\begin{abstract}
In 2015, the PTB, LNE-LADG, NIM and NIST performed informal bilateral comparisons using six critical venturi nozzles (CFVN). The goal of the comparisons was to prove the equivalence of reference standards for gas flow using pressurized air and natural gas at pressures from $0.1 \mathrm{MPa}$ to $5 \mathrm{MPa}$. The evaluation of the data utilized a function for the discharge coefficient $c_{\mathrm{D}}$ of critical nozzles as a function of the Reynolds number and real gas characteristics. The fitting function covers both laminar and turbulent boundary layer operating ranges of a nozzle with a single equation. The summary of all results of this comparison series shows that the proposed function for the discharge coefficient $c_{\mathrm{D}}$ can represent single measurement values within $0.1 \%$ with a $95 \%$ confidence level.
\end{abstract}

The approach represents the results reported by participants using functions based on physical understanding of the transfer standards. The paper documents the full equivalence of all traceabilities involved in these comparisons. The comparison database includes not only measurement sets of 2015 but also measurements done by PTB in other years with the same nozzles. Hence, qualitative statements on the long term reproducibility could inform the analysis on multilateral equivalence of the participants. Linked bilateral comparisons can effectively expose discrepant labs and perhaps give insight on the long term drift of primary standards.

\section{Introduction}

In the past 15 years, the National Metrology Institutes (NMIs) and Designated Institutes (DIs) made substantial efforts to establish a system of intercomparisons to support the quality of calibration results under the Mutual Recognition Arrangement (MRA) organised within the Bureau International des Poids et Mesures (BIPM, www. bipm.org).

Besides high level key comparisons, many NMIs or DIs in the field of fluid flow continue to do informal comparisons, performed bilaterally and efficiently at a less sophisticated technical level. These comparisons can provide a database to make the calibration capabilities evident.

The comparisons discussed herein were organised ad-hoc in 2015 between 1) PTB and LNE-LADG ${ }^{1}$, 2) PTB and NIM, and 3) NIM and NIST using a total of six critical nozzles. See Table 3 for definitions of acronyms. A special characteristic of the database generated within this series of measurement is the wide range of pressure (0.1 $\mathrm{MPa}$ to $5 \mathrm{MPa}$ ) and the different gas species (air and natural gas) used for the calibrations. Furthermore, PTB could provide datasets of calibrations for five of the nozzles performed in previous years and based on consistent traceability to the same primary standards.

Hence, a large database was available and it was the central challenge to make use of all these data to determine the degrees of equivalence between the participants. Therefore, it was necessary to introduce new approaches based on the application of functions to represent the values of $c_{\mathrm{D}}$ reported by the NMIs.

\section{Overview of the transfer standards and the NMIs}

The transfer standards for the comparison measurements were critical nozzles of different throat diameters. Both types of nozzles defined by the ISO 9300 [1], toroidal and cylindrical, have been used. To keep the comparisons simple, less expensive, and low risk regarding damage or loss of equipment, only the nozzles were transported among the labs. Consequently, all auxiliary equipment (pressure, temperature, and composition sensors) necessary for the complete determination of the discharge coefficient $c_{\mathrm{D}}$ were provided by each participating laboratory. Each participant included the uncertainty of the auxiliary instruments in their

\footnotetext{
${ }^{1}$ LNE-LADG is the Laboratoire National de Métrologie et d'Essai, Laboratoire Associé de Débitmétrie Gazeuse. CESAME EXADEBIT is designated institute of LNE-LADG.
} 
uncertainty of the reported $c_{\mathrm{D}}$ values. Table 1 and Figure 1 give an overview to the six nozzles and the mass flow range covered. From these data, it is apparent that at mass flows of approximately $100 \mathrm{~kg} / \mathrm{h}$, there is indirect linkage from NIST through NIM and PTB to LNE-LADG via three or more CFVNs. Our goal is to advance the analysis of this bilateral comparison database.

Table 2 lists the NMIs and their primary standards that are relevant for the comparisons discussed here. All NMIs have already established CMCs in the BIPM database, but $\mathrm{CMC}$ revisions are in process:

- $\quad \mathrm{NIM}$ has increased the operating pressure to 2.5 $\mathrm{MPa}$ [3].

- LNE has completely re-built their $p V T t$ system and is using air [6].

- PTB offers direct calibrations of customer devices with their primary standard for high pressure natural gas.

It is one intention of this paper to provide evidence regarding these ongoing changes of the related $\mathrm{CMC}$ entries.

Table 1: Nozzles used in the comparisons.

Table 1: Nozzles used in the comparisons.
\begin{tabular}{|c|c|c|c|c|c|c|}
\hline CFVN & $\boldsymbol{d}[\mathbf{m m}]$ & Form & NIST & NIM & PTB & LNE \\
\hline NIST $2.5 \mathrm{~mm}$ & 2.5 & toroidal & $\mathrm{X}$ & $\mathrm{X}$ & & \\
\hline HD-17b & 2.156 & toroidal & & $\mathrm{X}$ & $\mathrm{X}$ & \\
\hline HD-9b & 4.9452 & toroidal & & $\mathrm{X}$ & $\mathrm{X}$ & \\
\hline HD-5b & 6.9882 & toroidal & & $\mathrm{X}$ & $\mathrm{X}$ & \\
\hline TF 65 & 10.007 & cylindrical & & & $\mathrm{X}$ & $\mathrm{X}$ \\
\hline TF200 & 17.396 & cylindrical & & & $\mathrm{X}$ & $\mathrm{X}$ \\
\hline
\end{tabular}

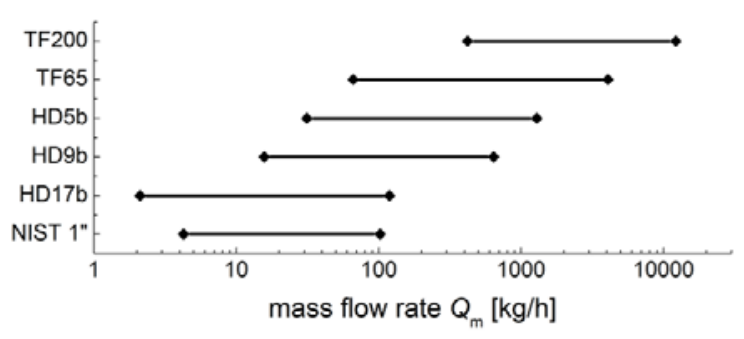

Figure 1: Ranges of mass flow covered by the different nozzles within the comparison campaigns.

Table 2: List of the NMIs and their primary standards.

\begin{tabular}{|l|l|l|l|l|}
\hline NMI & Gas used & $\begin{array}{l}\text { Primary } \\
\text { standard }\end{array}$ & Ref. & $\begin{array}{l}\text { Maximum } \\
\text { pressure } \\
\text { [MPa] }\end{array}$ \\
\hline NIST & Air & $p V T t$ & {$[2]$} & 0.7 \\
\hline NIM & Air & $p V T t$ & {$[3]$} & 2.5 \\
\hline PTB & Air & Bell prover* & {$[4]$} & 0.8 \\
& Natural gas & piston & {$[5]$} & 5.6 \\
& & prover** & {$[6]$} & 4 \\
\hline LNE & Air & $p V T t$ & {$[6 / 2$} \\
\hline
\end{tabular}

* Working standards were used for the calibrations in all measurements with air above $100 \mathrm{kPa}$.

** Working standards were used for the calibrations in all measurements with natural gas before 2015 .

\section{On the uncertainties in comparisons and the degree of equivalence}

The Working Group for Fluid Flow (WGFF) in the Consultative Committee for Mass and Related Quantities at the BIPM, released a guideline [7] for CMC and Calibration Report Uncertainties in 2013. According to the role of the BIPM, the statement about the uncertainty in a CMC entry in the BIPM database has to include the repeatability $u_{\text {repeat,BED }}$ of a Best Existing Device (BED) under test in addition to the base uncertainty of the laboratory's reference standard:

$$
u_{C M C}^{2}=u_{\text {base }}^{2}+u_{\text {repeat }, B E D}^{2}
$$

The Transfer Standard (TS) used in an intercomparison will have a different repeatability performance $u_{\text {repeat,TS }}$ than the laboratory's BED. Consequently, the uncertainty reported by a laboratory $u_{\text {Lab,reported }}$ in a comparison is given by:

$$
\begin{gathered}
u_{\text {Lab,reported }}^{2}=u_{C M C}^{2}-u_{\text {repeat }, B E D}^{2}+u_{\text {repeat }, T S}^{2} \\
=u_{\text {base }}^{2}+u_{\text {repeat }, T S}^{2} .
\end{gathered}
$$

In practice, transfer standards have a certain long term instability $u_{\mathrm{TS} \text {,stab }}$ which is not represented by the $u_{\text {repeat,TS }}$ and has to be determined by repeated calibrations covering a long period. Therefore the value $u_{\text {Lab,applied }}$ finally used for the calculation of the degree of equivalence includes this additional contribution to the uncertainty:

$$
u_{\text {Lab,applied }}^{2}=u_{\text {base }}^{2}+u_{\text {repeat }, T S}^{2}+u_{T S, \text { stab }}^{2} .
$$

The (bilateral) degree of equivalence is the central outcome of the comparisons and is expressed here as the normalized difference between the values reported by two laboratories:

$$
E_{N, L a b 1, L a b 2}=\frac{c_{D, L a b 1}-c_{D, L a b 2}}{k \sqrt{u_{\text {Lab1,applied }}^{2}+u_{L a b 2, a p p l i e d}^{2}}},
$$

where the combined uncertainty of both participants is the normalization factor using the expansion factor $k=2$ so that we get the $95 \%$ coverage interval. The critical level for $\operatorname{abs}\left(E_{\mathrm{N}}\right)$ is therefore 1. Exceeding the level of 1 indicates the non-equivalence of the calibration results.

In the past, some effort was necessary to make sure that all participants performed their measurements under very similar working conditions. Specifically the operating pressure, flow, and fluid composition or properties had to be similar for all participants because the indication performance of our transfer standards is dependent on these operating conditions. Hence, the comparisons were evaluated in a so-called point-to-point (P2P) manner, meaning that only values were compared which were generated at very similar operating conditions for all participants. The database generated within the series of measurements documented in this paper does not fulfill the requirements for such a $\mathrm{P} 2 \mathrm{P}$ evaluation. Therefore, we have chosen to pursue an approach that determines functions that which represent the data of the participating labs. 
Least Squares Fit (LSF) functions are practical solutions, but require the consideration of the following topics to avoid misinterpretation of the results:

- Correlation among data measured with common traceability (data out of one lab), ${ }^{2}$

- Bias effects due to imbalances regarding number of data provided by the labs (the results can be biased towards the larger datasets),

- Choice of the fit function. The structure of the fit function shall have sufficient complexity to cover the characteristics of the true relationship (a demonstrated physical model) but shall also provide results based on a sufficient statistical significance.

- Extrapolation to very different conditions of flow and composition increases uncertainty.

Our approach here was to use a function which shall represent the general behavior of the dependency of our measurand, the discharge coefficient $c_{\mathrm{D}}$ of a critical nozzle as a function of the Reynolds number $R e$. This first approximation $c_{\mathrm{D} \text {, base }}=f(R e)$ is determined by means of a weighted LSF with data from all available labs for each nozzle. The uncertainties $u_{\text {Lab,reported }}$ were used for the weights but we did not consider any correlation among the data (LSF with uncorrelated data). As a consequence, the result for $C_{\mathrm{D} \text {,base }}$ is definitely biased in multiple ways and the resulting uncertainty of the fit result is strongly underestimated. Therefore, a more sophisticated approach is needed before using this function to produce a comparison reference value.

But we can make use of $c_{\mathrm{D} \text {,base }}$ to remove a common base line from all single measurement values:

$$
\Delta c_{D, \text { Lab }}=c_{D, \text { meas }}-c_{D, \text { base }} .
$$

In a second step, the remaining single data $\Delta c_{\mathrm{D}, \mathrm{Lab}}$ are approximated again by means of a LSF:

$$
\Delta c_{D, f i t, L a b}(R e)=\operatorname{LSF}\left(\Delta c_{D, L a b, i}, R e_{i}\right)
$$

The complexity of functions being necessary to represent the $\Delta c_{\text {D,Lab }}$ is dramatically reduced when the first estimation $C_{\mathrm{D} \text {,base }}$ is quite close to the real behavior of $C_{\mathrm{D}}(R e)$. For this database, it was possible to apply polynomials of first order in most cases and higher order only in a few cases (the maximum was third order in one case).

The LSF processing of the data provides finally the parameters for the fitted function and the variancecovariance matrix of these parameters. With this we generated the values of the fitted curve $\Delta c_{\mathrm{D}, \mathrm{fit}, \mathrm{Lab}}(R e)$ and the related uncertainty to this value $u_{\text {conff,fit }}(R e)$ for a new set of Reynolds number in the Reynolds range covered by two laboratories. At each "new" Reynolds number we applied then the conventional approach for the P2P determination of the degree of equivalence Equation (9).
We did not perform extrapolation beyond the range of Reynolds number provided by a participating laboratory.

Please note that the outcome of LSF for the uncertainty of the fitted value at confidence level $u_{\text {conf,fit }}(R e)$ has similar meaning as the standard uncertainty of the mean which is background for the $u_{\text {repeat,Ts }}$ reported by the lab. Furthermore, we assume that critical nozzles are very stable artifacts and it is not necessary to include a value for $u_{\mathrm{TS}, \text { stab }}$ for the short duration of these comparisons. Hence, we assumed the following simplifying relations for all evaluations documented here:

$$
\begin{gathered}
u_{\text {repeat }, T S}^{2} \Rightarrow u_{\text {conf , fit }}^{2} \text { and } u_{T S, \text { stab }}^{2} \Rightarrow 0 \\
u_{\text {Lab, fitted }}^{2}=u_{\text {Lab,applied }}^{2}=u_{\text {base }}^{2}+u_{\text {conf, fit }}^{2} .
\end{gathered}
$$

The bilateral degree of equivalence is finally:

$$
E_{N, L a b 1, L a b 2}=\frac{\Delta c_{D, f i t, L a b 1}-\Delta c_{D, \text { fit,Lab2 }}}{k \sqrt{u_{L a b 1, \text { fitted }}^{2}+u_{L a b 2, \text { fitted }}^{2}}} .
$$

\section{Definition of $C_{D}=f(R e)$ covering wide Reynolds range}

The discharge coefficient $c_{\mathrm{D}}$ depends mainly on the Reynolds number of the flow through the nozzle. Looking to the characteristics of this dependency we have to distinguish two main cases, when the boundary layer of the flow inside the nozzle is laminar $\left(c_{\mathrm{D}, \text { lam }}\right)$ and when the boundary layer is turbulent $\left(c_{\mathrm{D}, \text { turb }}\right)$ :

$$
\begin{gathered}
c_{D}=s_{a}\left(a+b_{\text {lam }} \cdot \operatorname{Re}^{-0.5}\right)+s_{e}\left(a+b_{\text {turb }} \cdot \operatorname{Re} e^{-0.139}\right), \\
s_{a}=0.5\left\{1-\tanh \left[k_{u} \log \left(\operatorname{Re} / \operatorname{Re}_{\text {tr }}\right)\right]\right\} \\
s_{e}=0.5\left\{1+\tanh \left[k_{u} \log \left(\operatorname{Re} / \operatorname{Re} e_{\text {tr }}\right)\right]\right\} .
\end{gathered}
$$

with

The parameter $a$ represents the impact of the inclination of the isotach lines (lines of equal speed) at the nozzle throat [9] to the discharge coefficient $c_{\mathrm{D}}$ and $b_{\text {lam }}$ as well as $b_{\text {turb }}$ indicate the dependency of $c_{\mathrm{D}}$ on Reynolds number in the case of laminar or turbulent boundary layers respectively (for discussion regarding the exponent 0.139 for turbulent boundary layers please refer e.g. to [10]).

It is common to bridge the laminar and turbulent boundary layer transition with one function for the whole Reynolds range by means of a transition function given by the two terms $s_{\mathrm{a}}$ and $s_{\mathrm{e}}$. Note that $s_{a}+s_{e} \equiv 1$. The parameter $R e_{\text {tr }}$ defines the middle point of the transition and $k_{\mathrm{u}}$ the "sharpness" of the transition (the larger $k_{\mathrm{u}}$, the more "sudden" the transition occurs).

\footnotetext{
${ }^{2}$ Correlation among data can be included in application of LSF in principle [8] if we would have the prior information about the
}

structure and level of correlations. However this approach needs the prior analysis of the uncertainty budgets . 
Equation 10 defines our base function for $C_{\mathrm{D}}$ for a particular nozzle versus all Reynolds numbers which we call in the following, $\boldsymbol{C}$ D,base. We will use the database from the participating labs to determine the free parameters in Equation (10) for each nozzle. In many cases, the fitted values for some of the free parameters (and particularly $b_{\text {turb }}$ ) are more difficult and less reliable because of a lack of comparison data in the turbulent regime. Also, the sensitivity to the parameter $k_{\mathrm{u}}$ is weak. Therefore, some reduction of the independent parameters is applied:

- the parameter $k_{\mathrm{u}}$ is fixed arbitrarily to the value $k_{\mathrm{u}}=5.5$ for all nozzles, and

- we introduce a fixed relationship between $b_{\text {lam }}$ and $b_{\text {turb. }}$

Based on previous theoretical and numerical studies [10], the following fixed relation between the parameters $b_{\text {lam }}$ and $b_{\text {turb }}$ can be used:

$$
b_{\text {turb }}=0.003654 \cdot b_{\text {lam }}^{1.736} \text {, }
$$

removing $b_{\text {turb }}$ from the list of parameters to be determined for each nozzle.

The measurements from our bilateral comparisons cover air and natural gas up to a pressure of $5 \mathrm{MPa}$. Therefore, real gas effects are significant and an inclusion of gas characteristics as a function of composition and pressure is necessary ${ }^{3}$. For the parameter $b_{\text {lam }}$ we made use of the theoretical work of Geropp [11]. Geropp showed that the parameter $b_{\text {lam }}$ has dependencies on the isentropic exponent $\kappa$, the Prandtl-number Pr, the difference of the wall temperature to ideal condition $\Delta T_{\text {Wall, }}$, and the radius of curvature of the nozzle inlet near the throat $R_{\text {throat }}$. Explicit equations for these dependencies are found in reference [11]:

$$
b_{\text {lam }}=G\left(\kappa, \operatorname{Pr}, \Delta T_{\text {Wall }}\right) \cdot R_{\text {throat }}^{0.25} .
$$

Up to now, we have no detailed knowledge about $\Delta T_{\text {Wall }}$ therefore we are ignoring the influence of heat transfer on $b_{\text {lam }}$ and assume always $\Delta T_{\text {Wall }}=0$.

To utilize the relation given by Equation (12) for our application, we use a parameter $b_{\text {lam }, 0}$ which is assumed to be valid for the specific gas parameters $\kappa=1.4$ and $\mathrm{Pr}$ $=1$. This yields:

$$
\frac{b_{\text {lam }}(\kappa, P r)}{b_{\text {lam }, 0}}=\frac{G(\kappa, P r)}{G_{\kappa=1.4, P r=1}}=C_{b}(\kappa, P r) .
$$

Hence, for a certain value of $b_{\mathrm{lam}, 0}$ we can apply the related $b_{\text {lam }}$ specific for each gas and each pressure when we calculate $c_{\mathrm{D} \text {,base }}$.

For the parameter $a$, representing the impact of the inclination of the isotach lines, we apply a similar approach. The parameter $a$ also depends on the isentropic exponent $\kappa$ and the radius of curvature at nozzle throat $R_{\text {throat: }}$

$$
a=H\left(\kappa, R_{\text {throat }}\right) .
$$

An explicit equation for this dependency is given e.g. by [9].

Analogous to the approach for $b_{\text {lam }}$, we use a parameter $a_{0}$ assumed to be valid for $\kappa=1.4$ and which is related to the actual parameter for a specific, actual $\kappa$ according to:

$$
\frac{a}{a_{0}}=\frac{H\left(\kappa, R_{\text {throat }}\right)}{H_{\kappa=1.4, R_{\text {trroat }}}}=C_{a}\left(\kappa, R_{\text {throat }}\right),
$$

with

$$
R_{\text {throat }}=\left(\frac{b_{\text {lam }, 0}}{G_{\kappa=1.4, P r=1}}\right)^{4} .
$$

Hence, the determination of the function $C_{\mathrm{D} \text {,base }}$ for one nozzle needs now the determination of three parameters $\left\{a_{0}, b_{\text {lam }, 0}, R e_{\text {tr }}\right\}$ instead of the original five.

The characteristics of our definition of $c_{D}$,base have important consequences for our purpose here. The relationships of Equation (13) and Equation (15) introduce systematic dependency on the fluid parameters isentropic exponent $\kappa$ and the Prandtl number Pr. These parameters differ significantly for different gases as well as for different pressures of the same gas (because of real gas effects). An illustration is given for the nozzles investigated here in the Appendix, Figure 7 to Figure 11. The $c_{\mathrm{D} \text {,base }}$ in these graphs is calculated using the same $a_{0}$ and $b_{\text {lam }, 0}$ for both air and natural gas (NG). But at the high Reynolds numbers, i.e. at the higher pressures, the difference for $C_{\mathrm{D} \text {,base }}$ between air and natural gas is approximately $0.075 \%$ which is of the same order as the expanded uncertainty of the single values. It would definitely distort the results for comparisons using air and natural gas if we ignored this behaviour.

\section{Results for the Degree of Equivalence $E_{N}$}

All data sets of the nozzles have been treated with the principles given above in Sections 3 and 4. The related database from the six nozzles can be seen in graphical form in the Appendix, Figure 7 to Figure 11. The database is available from the authors upon request.

Figure 2 and Figure 3 below illustrate the $\Delta c_{\mathrm{D}}$ values for one of the six nozzles (HD-9b) and its final $E_{\mathrm{N}}$ curves. The $E_{\mathrm{N}}$ curves in Figure 3 and elsewhere in this paper have been determined with data sets that are pair-wise independent regarding their traceability; so e.g. NIM (2015 air) with PTB (2015 air) but also with PTB (2015 NG) and with PTB (2006 air). To avoid introducing uncertainty due to extrapolation, the $E_{\mathrm{N}}$ values are calculated only for the range of Reynolds numbers where the data sets overlap.

\footnotetext{
3 This should not be confused with the influence of real gas characteristics which is taken into account by the critical flow factor $C^{*}$, see e.g. [12].
} 


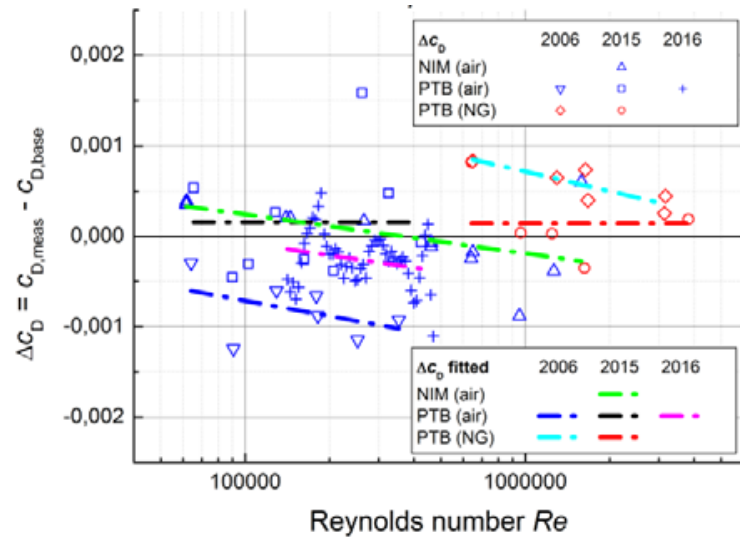

Figure 2: Differences $\Delta c_{\mathrm{D}}$ of single $C_{\mathrm{D}}$ values against the $C_{\mathrm{D}}$, base function for the nozzle HD-9b. The $\Delta c_{\mathrm{D}}$ for each measurement series are approximated by a polynomial $\Delta c_{\mathrm{D}}$,fit. Please refer to Figure 9 in the Appendix for plot of $c_{D}$,meas and $C_{\mathrm{D}}$,base.

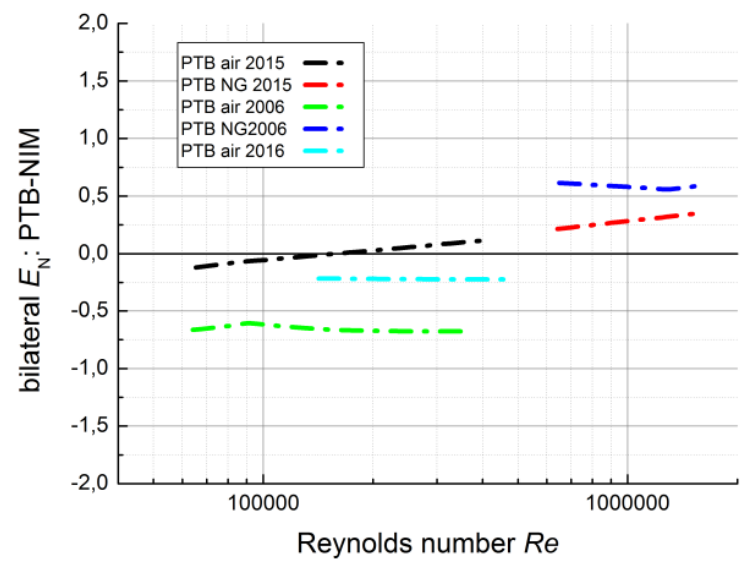

Figure 3: Bilateral $E_{\mathrm{N}}$ PTB - NIM using nozzle HD-9b. $E_{\mathrm{N}}$ is calculated according to Equation (9).

The $E_{\mathrm{N}}$ curves in Figure 3 for the bilateral equivalence between PTB and NIM are all significantly within \pm 1 and vary approximately \pm 0.5 against an average. This indicates the overall stability of the measurment process including the reference standards of participants and the transfer standard over all the years from 2006 to 2016 !

Figure 4 shows the $E_{\mathrm{N}}$ curves for all the the data for all nozzles. To maintain readability, the curves are distiguished by colour based on the bilateral comparison pairs.

All $E_{\mathrm{N}}$ are within \pm 1 and the pair-wise equivalence between PTB/NIM, PTB/LNE, and NIM/NIST is evident.

After the calculation of the $E_{\mathrm{N}}$ curves and the approval of bilateral equivalence we can ask about the multilateral equivalence. The multilateral equivalence is here evaluated qualitatively ${ }^{4}$ using the fact that the bilateral comparisons are establishing a chain between NISTNIM-PTB-LNE in which NIM and PTB can be used as the link between the other two participants.

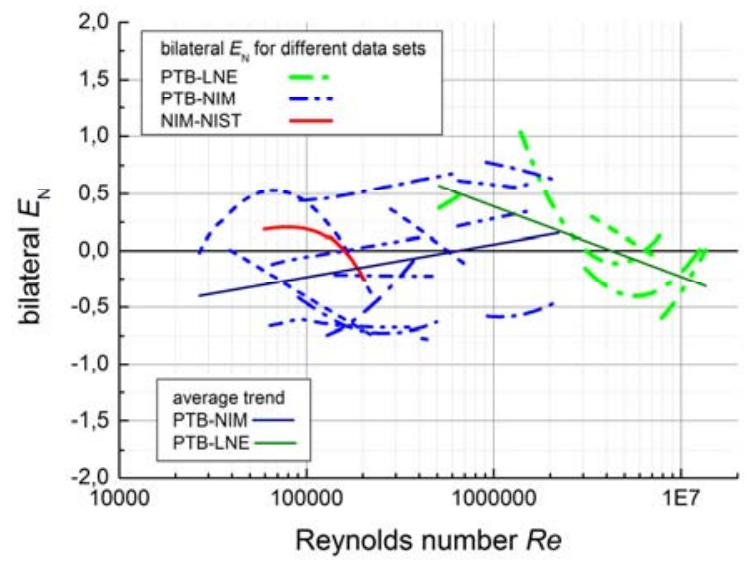

Figure 4: Bilateral $E_{\mathrm{N}}$ PTB-LNE, PTB-NIM and NIM-NIST for all nozzles and all datasets.

Looking to Figure 4, we can conclude first that there is only a slight trend in $E_{\mathrm{N}}$ with respect to Reynolds number for all compared data sets between NIM and PTB. Secondly, considering the data PTB-LNE, we can see a trend starting from about +0.6 going down to -0.3 . Both trends are getting rather close together in the overlapping range. Hence, we can conclude that also for NIM-LNE we can expect equivalence in the operation range where both NIM and LNE can provide calibrations.

For NIST-NIM we had only one data set and the line of $E_{\mathrm{N}}$ for this case is also reasonably close to the overall trend line for NIM-PTB.

\section{Evaluation of all single $c_{D}$ values using $C_{D}$,base}

In the following, we will use the overall function $c_{\mathrm{D}}$,base in the sense of a comparison reference value, although formally we are not allowed to name it so as already mentioned above in Section 3. Hence, we will talk about the normalised differences $\Delta c_{\mathrm{D}} / U_{\text {Lab,reported }}$ (Figure 6) which have the same philosophy behind them but may slightly differ from the exact values for $E_{N}$. However we can assume that the difference is small and probably $\Delta c_{\mathrm{D}} / U_{\text {Lab,reported }}$ will be overestimated because an existing bias is increasing the values for at least one of the participants.

Figure 5 shows the relative differences $\Delta c_{\mathrm{D}, \text { rel }}$ against the $C_{\mathrm{D} \text {,base }}$ functions for all measurements. It can be seen that the majority of the values for $\Delta c_{\mathrm{D} \text {,rel }}$ are approximately in a range of $0.1 \%$. There are only a few values between $60 \mathrm{~kg} / \mathrm{h}$ and $200 \mathrm{~kg} / \mathrm{h}$ that exceed this limit significantly (up to $+0.3 \%{ }^{5}$ ).

\footnotetext{
5 These values belong to the measurements with nozzle HD-5b at PTB in 2015. This is the only case where we have to assume instability of ${ }^{4}$ The trend lines in Figure 4 are given only for orientation because we
have not quantified the uncertainties of these average lines. 


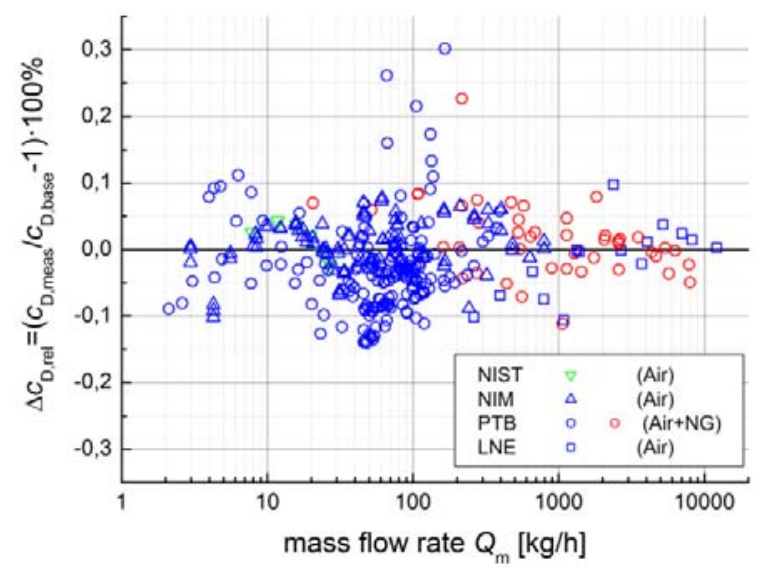

Figure 5: Differences $\Delta c_{\mathrm{D}}$ of single $C_{\mathrm{D}}$ values (relative in \%) against the $C_{D}$,base functions for all nozzles and all measurement series.

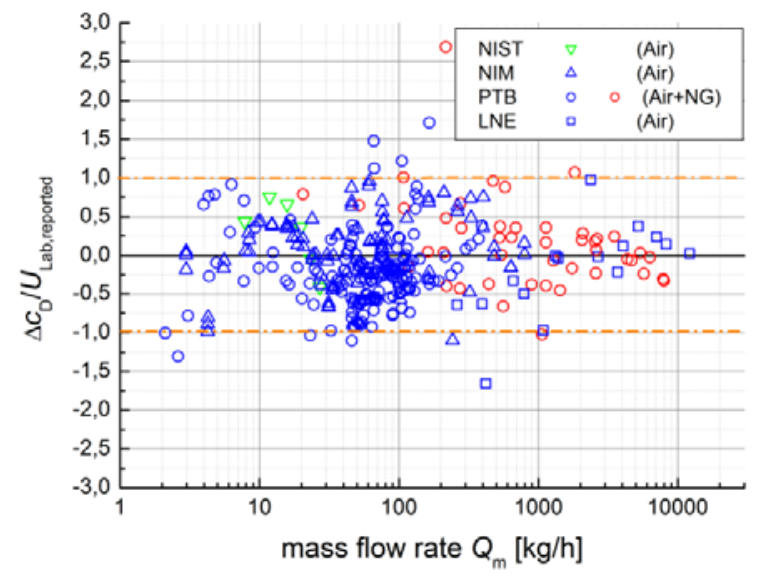

Figure 6: Normalised differences $\Delta c_{\mathrm{D}} / U_{\text {Lab,reported }}$ as shown in Figure 5 using the related uncertainties reported by the laboratories $(k$ $=2$ ) for all nozzles and all measurement series.

The normalised differences $\Delta c_{\mathrm{D}} / U_{\mathrm{Lab}, \text { reported }}$ are shown in Figure 6. The overall histogram statistics for these data are shown in the Appendix, Figure 12, and indicate that $95 \%$ of the values are within $\pm 1 .^{6}$

Additionally we can conclude that the functionality behind $C_{\mathrm{D} \text {,base }}$ is sufficient to represent a single experimental datum at the level of $0.1 \%$ because otherwise the normalised difference is highly unlikely to give $\operatorname{abs}\left(E_{\mathrm{N}}\right)$ values in the manner shown here.

\section{Conclusions}

First of all, we conclude from these comparisons that there is equivalence between all the measurement capabilities involved. These results support of the following improved CMCs: 1) LNE with their new $p V T t-$ facility [6] with pressure up to $4 \mathrm{MPa}$ and uncertainties of $\left.U_{\text {base }} \geq 0.1 \%, 2\right)$ NIM with their $p V T t$ [3] up to $2.5 \mathrm{MPa}$

the nozzle itself because the values both of the independent measurement series with air and natural gas show these changes and higher scatter than usual. We could not detect the physical reason; during the repeated measurements in 2016 all values were consistent again. and $U_{\text {base }}=0.08 \%$ and 3) PTB's capability to calibrate sonic nozzles with their High Pressure Piston Prover with $U_{\text {base }}=0.072 \%$.

Functions were successfully introduced into the evaluation of the comparison database. This approach was necessary because the database did not allow the application of the conventional approach of point-topoint evaluation. The procedure to first subtract a base line $\left(c_{\mathrm{D}}\right.$,base $)$ from the reported results is very helpful; it reduces the complexity for the representations of data of each laboratory.

It has to be emphasized hereby that the additional contributions of uncertainties caused by the transfer standard to the final values of $E_{\mathrm{N}}$ are reduced because the value for the uncertainty of the fit $u_{\text {conffit }}$ is replacing the

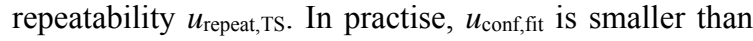
the repeatability at one point due to the higher degrees of freedom and is also representing some parts of the $u_{\mathrm{TS} \text {,stab }}$. Reducing the uncertainty due to the transfer standard is important advantage of this approach for future comparisons because it enhances our ability to assess CMCs based on the comparison results (see for this [13]).

Last but not least, the application of a generalised function to represent the database of discharge coefficients was demonstrated and with the set of overall results it was shown that this function can reflect the experimental data at a precision level of $0.1 \%$ with $95 \%$ confidence. Furthermore, the function includes additional real gas effects on the boundary layer that were used to correct significant differences (up to $0.075 \%$ ) between application with air and natural gas at high pressures (2 $\mathrm{MPa}$ to $5 \mathrm{MPa})$.

Table 3. Definitions of Acronyms.

\begin{tabular}{|l|l|}
\hline BED & Best Existing Device \\
\hline BIPM & Bureau International des Poids et Mesures \\
\hline CFVN & Critical flow venturi nozzle \\
\hline CMC & Calibration Measurement Capability \\
\hline DI & Designated Institute \\
\hline $\begin{array}{l}\text { LNE- } \\
\text { LADG }\end{array}$ & $\begin{array}{l}\text { Laboratoire National de Métrologie et } \\
\text { d'Essai, Laboratoire Associé de } \\
\text { Débitmétrie Gazeuse }\end{array}$ \\
\hline MRA & Mutual Recognition Arrangement \\
\hline NG & Natural gas \\
\hline NIM & National Institute of Metrology, China \\
\hline NIST & $\begin{array}{l}\text { National Institute for Standards and } \\
\text { Technology }\end{array}$ \\
\hline NMI & National Metrology Institute \\
\hline PTB & Physikalisch-Technische Bundesanstalt \\
\hline WGFF & Working Group for Fluid Flow \\
\hline
\end{tabular}




\section{References}

[1] International Standards Organization, Measurement of Gas Flow by Means of Critical Flow Venturi Nozzles, ISO 9300: 2005(E).

[2] J. D. Wright and A. N. Johnson, NIST Lowers Gas Flow Uncertainties to $0.025 \%$ or Less, NCSL International Measure, 5, pp. 30 to 39, March, 2010.

[3] C. Li, L. Cui, C. Wang: The new pVTt facility in NIM, Proceedings of the $13^{\text {th }}$ Int. Conference on Flow Measurement FLOMEKO 2013, Paris, France 2013.

[4] B. Mickan, R. Kramer, and M. Strunck, Highest Precision for Gas Meter Calibration Worldwide: The High Pressure Gas Calibration Facility pigsarTM with Optimized Uncertainty, $7^{\text {th }}$ ISFFM, Anchorage, Alaska, 2009.

[5] B. Mickan, R. Kramer: Experiences with sonic nozzles used for different gases and wide range of pressure and temperature conditions, Proceedings of $7^{\text {th }}$ International Symposium on Fluid Flow Measurement, Anchorage, Alaska, 2009.

[6] J. P. Vallet, G. Miault, C. Windenberger, P. Manrot, P. Kervevan, The conception and the construction of a new High Pressure Primary Facility for Gas, Proceedings of $8^{\text {th }}$ International Symposium on Fluid Flow Measurement, Colorado Springs, Colorado, 2012.

[7] WGFF Guidelines for CMC and Calibration Report Uncertainties, http://www.bipm.org/utils/en/pdf/cc m-wgff-guidelines.pdf, 2013.

[8] M. G. Cox, P. M. Harris: The evaluation of key comparison data using key comparison reference curves, Metrologia 49, 437-445, 2012.

[9] I. M. Hall, Transonic Flow in Two-Dimensional and Axially-Symmetric Nozzles, Quarterly Journal of Mechanics and Applied Mathematics, Vol. XV, Pt. 4, pp. 487-508, 1962.

[10] B. Mickan, R. Kramer, D. Dopheide: Determination of Discharge Coefficient of Critical Nozzles Based on their Geometry and the Theory of Laminar and Turbulent Boundary Layers; $6^{\text {th }}$ ISFFM, Queretaro, Mexico, 2006.

[11] Geropp, D., Laminare Grenzschichten in Überschalldüsen, Deutsche Luft- und Raumfahrt, Forschungsbericht 01 TM 8603-AK/PA 1, 1987.

[12] J.P. Vallet, C. Windenberger, F. Vulovic, and R. Bourd, Improvement of Thermodynamic Calculations Used for the Flow Rate of Sonic Nozzles, Proceedings of the $10^{\text {th }}$ Int. Conference on Flow Measurement FLOMEKO 2000, Salvador, Brasil, 2000.

[13] J. D. Wright, B. Toman, B. Mickan G. Wübbeler, O. Bodnar, C. Elster, Pass / Fail / Inconclusive Criteria for Inter Laboratory Comparisons, Proceedings of $9^{\text {th }}$ International Symposium on Fluid Flow Measurement, Arlington, Virginia, 2015.

\section{In Memoriam of Jean-Pierre Vallet}

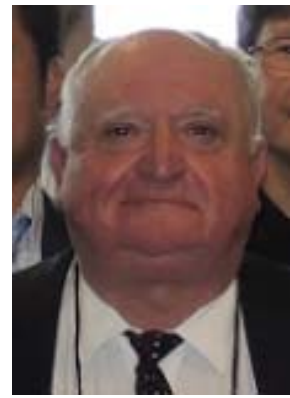

With great sorrow, we received the message that our friend and colleague Jean-Pierre Vallet passed away on July 5, 2016. Jean-Pierre was a kind and generous friend to many of us and his death is a big loss for the flow community.

Critical flow nozzles and their application in flow metering was one of his central topics over all the years, always encouraging cooperative research on his favorite flow meter. Besides advancing technical issues, he also had tremendous abilities to identify strategic needs and to pursue them with long lasting energy.

But in all these long years of his active participation in our community, it was not only his ambition to bring forward our topics in fluid flow that impressed us, but even more, his great joie de vivre, ability to enjoy life itself with a deep sense for real friendship and good lifestyle. With this, we will keep him always in our memory as a great example of a fulfilled life. 


\section{Appendix: Graphical presentation of all measurement results}

Results of $c_{\mathrm{D}}$ values determined by the participants and the $c_{\mathrm{D} \text {,base }}$ function (see Section 4) based on a LSF are given in Figure 7 to Figure 11. We refrain from showing the error bars for the single measurement values to keep the graphs readable.

Figure 12 shows the histogram statistics for data in Figure 6.

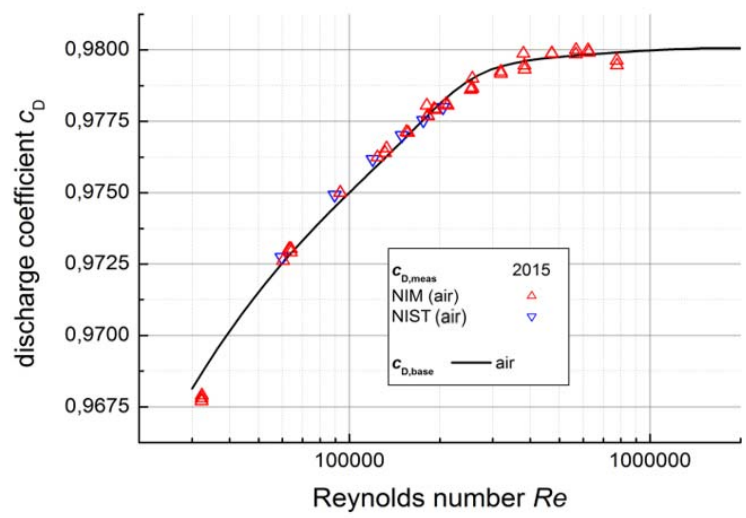

Figure 7: Data for nozzle NIST $2.5 \mathrm{~mm}$.

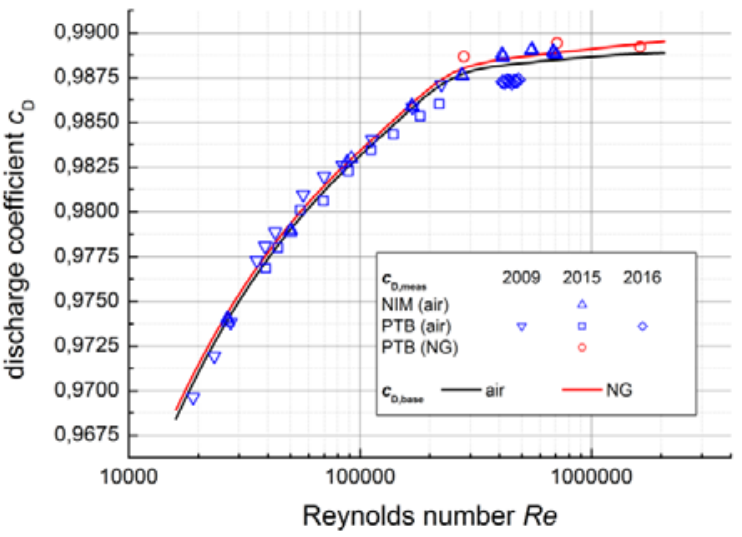

Figure 8: Data for nozzle HD-17b.

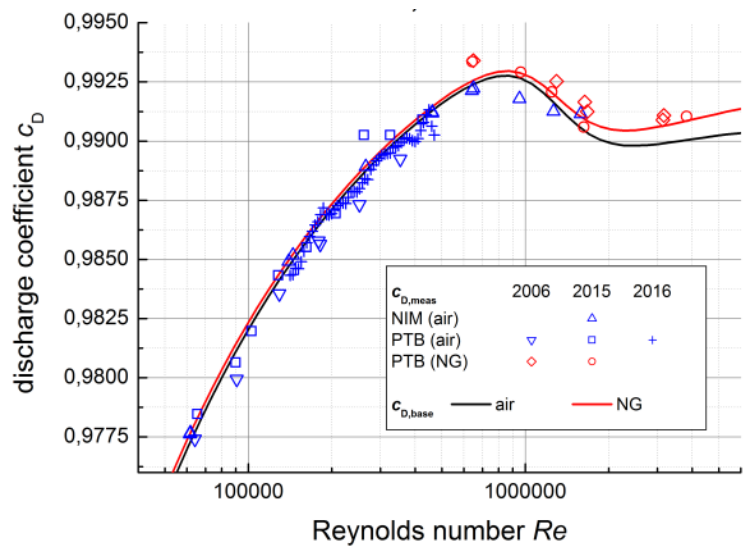

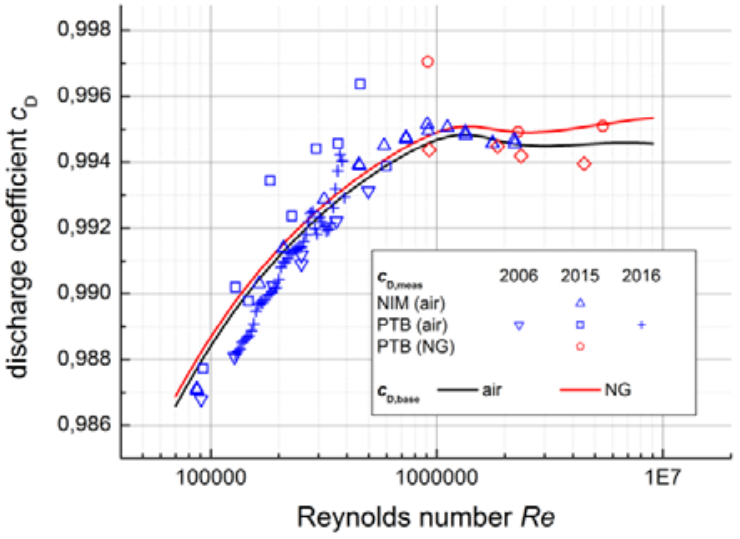

Figure 10: Data for nozzle HD-5b.

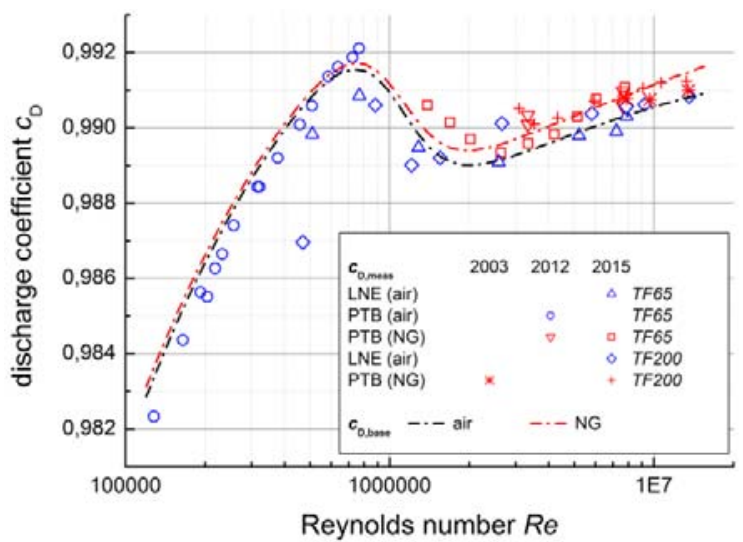

Figure 11: Data for nozzles TF65 and TF200. Please note that only one parameter triplet $\left\{a_{0}, b_{\mathrm{lam}, 0}, R e_{\mathrm{tr}}\right\}$ has been applied for both nozzles.

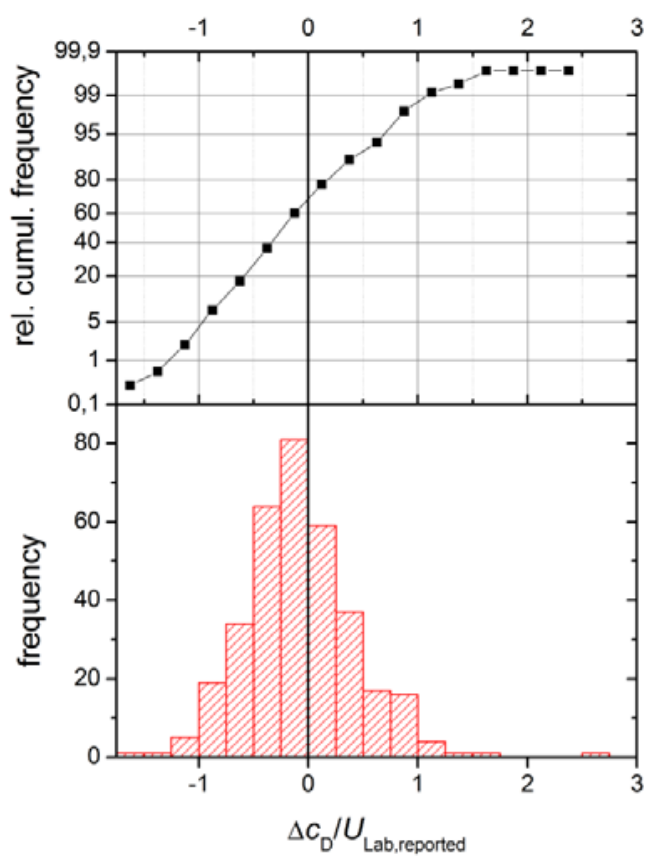

Figure 12: Histogram statistics on the data shown in Figure 6.

Figure 9: Data for nozzle HD-9b. 


\title{
Back Pressure Ratio and the Transonic Resonance Mechanism of Low Unchoking in Critical Flow Venturis
}

\author{
J. D. Wright ${ }^{1}$, B. W. Sims' ${ }^{2}$ R. J. McKee ${ }^{3}$, K. A. Gillis ${ }^{1}$, \\ A. N. Johnson ${ }^{1}$, M. S. Carter ${ }^{2}$, and Ida I. Shinder ${ }^{1}$ \\ ${ }^{1}$ National Institute of Standards and Technology, 100 Bureau Dr, Gaithersburg, MD, USA \\ ${ }^{2}$ Flow Systems Inc., 220 Bunyan Ave, Berthoud, CO, USA \\ ${ }^{3}$ Robert McKee Engineering LLC, Everett, WA, USA \\ E-mail (J. D. Wright): john.wright@nist.gov
}

\begin{abstract}
Reliable Critical Flow Venturi (CFV) operation requires sonic velocity at the throat of the device. The maximum ratio of exit pressure to inlet pressure that ensures this sonic velocity is referred to as the maximum back pressure ratio (MBPR). Being able to accurately predict the MBPR for a specific CFV as well as design a CFV to have a high MBPR allows diverse application and confidence in CFV flow measurements. At Reynolds numbers based on throat diameter below 50 000, CFVs can display "low unchoking" and the standard equation over-reports the flow by $1 \%$ or more.

We show that MBPR for a particular, $0.8 \mathrm{~mm}$ throat diameter CFV using dry air, argon, helium, and sulfur hexafluoride is well correlated by the "fully expanded jet Mach number". Sound detected by microphones placed up and downstream from a CFV show high correlation between low unchoking and the presence of powerful transonic resonances (oscillations at audio frequencies in pressure and the position of a lambda shock in the CFV diffuser) described by Zaman et al. We propose a mechanism in which pressure fluctuations from the transonic tones lead to intermittent unchoking of the CFV throat.

This paper also presents unchoking test results from 270 unchoking tests on 79 CFVs with a wide range of throat diameters, Reynolds numbers, diffuser lengths, half angles, and gases. Correlations for avoiding low unchoking and predicting MBPR for broader application are presented that incorporate the necessary effects due to Re, diffuser length, diffuser area ratio, and specific heat ratio. We advocate inclusion of these correlations in documentary standards.
\end{abstract}

\section{Introduction}

Critical nozzles are widely used as transfer standards to compare gas flow calibration capabilities or as working standards for calibrating other flow meters. Their advantages include stable calibration over long periods of time and a well understood physical model. Critical nozzles have the disadvantage of a large pressure drop relative to other gas flow meters, but this is improved by adding a diffuser for pressure recovery downstream from the throat. ISO and ASME [1, 2] standard designs for a critical flow venturi (CFV) use a conical diffuser with half angle $\theta$ between 2.5 and 6 degrees. Detailed drawings and definitions are provided in the standards. Using nomenclature defined at the end of this paper, the standard equation for mass flow is

$$
\dot{m}=\frac{C_{\mathrm{d}} C_{\mathrm{R}}^{*} P_{0} A^{*} \sqrt{M_{\mathrm{m}}}}{\sqrt{R T_{0}}} .
$$

Equation 1 is only valid when the critical nozzle or CFV has small enough pressure ratio $P_{\mathrm{e}} / P_{0}$ so that the gas velocity reaches the speed of sound at the throat, i.e. "choked" condition. The value of the discharge coefficient $C_{\mathrm{d}}$ is often based on a flow calibration against a reference standard, but can be calculated within $0.05 \%$ from theory [3]. If the CFV is not

\footnotetext{
${ }^{1}$ The change in pressure upstream from the CFV under test could be used instead of the change in $C_{\mathrm{d}}$ that is normally plotted to
}

choked, the standard mass flow equation will over-report the flow. The pressure ratio $P_{\mathrm{e}} / P_{0}$ across the CFV that produces choked flow depends on the CFV geometry, Reynolds number, and the gas species. The largest $P_{\mathrm{e}} / P_{0}$ value at which Equation 1 gives reliable flow values (within a specified tolerance) is called the maximum back pressure ratio (MBPR). The goals of this work are to (1) provide guidance on the MBPR necessary for choked flow for ISO/ASME standard CFV geometries and (2) develop better physical understanding of the relationship between the CFV shape and the MPBR.

Choked flow is a limiting condition where the fluid velocity reaches the speed of sound at the throat such that the mass flow will not increase with a further decrease in the downstream pressure [2]. In an unchoking test, a CFV is put in series with a flow reference (usually a second, smaller diameter CFV placed upstream) and the exit pressure $P_{\mathrm{e}}$ from the CFV under test is incrementally increased by closing a throttling valve until changes in the discharge coefficient of the CFV under test using Equation 1 are observed with respect to the reference flow meter [4]. When the change in $C_{\mathrm{d}}$ of the CFV under test is larger than a specified tolerance, the MBPR is noted and not exceeded during future operation. ${ }^{1}$

indicate unchoking: when less of the throat area is at $M=1$, the presure increases to maintain conservation of mass flow through the meters in series. 
Compressible flow theory, assuming a calorically perfect gas (constant specific heats), one-dimensional (1-D), adiabatic, and inviscid flow predicts that the throat pressure ratio or MBPR necessary for choked flow without a diffuser is:

$$
\left(\frac{P^{*}}{P_{0}}\right)_{\mathrm{crit}}=\left(\frac{2}{\gamma+1}\right)^{\gamma /(\gamma-1)}
$$

where $\gamma$ is the specific heat ratio, $c_{P} / c_{V}, P_{0}$ is the stagnation pressure upstream from the critical flow venturi or nozzle, and $P^{*}$ is the pressure at the CFV throat [5]. For dry air, $\left(P^{*} / P_{0}\right)_{\text {crit }}=0.53$, i.e. if the pressure at the throat is $53 \%$ of the inlet pressure or less, the gas velocity will match the speed of sound at the throat, the CFV will be choked, and the flow is independent of the downstream pressure. Note that it is usually impractical to measure $P^{*}$ in CFVs, especially when they have a small throat diameter. The exit pressure $P_{\mathrm{e}}$ is measured in the plenum downstream from the CFV diffuser exit and the pressure ratio $P_{\mathrm{e}} / P_{0}$ is used instead of $P^{*} / P_{0}$ to assure a CFV is used in a choked condition.

The purpose of a CFV diffuser is to raise the pressure of the gas as it moves from the throat to the exit and thereby allow the CFV to be used for reliable flow measurement over a wider range of pressures. When the CFV diffuser is performing well, shock structures in the diffuser provide pressure recovery, $P_{\mathrm{e}}$ can be larger than $P^{*}$, and Equation 1 gives reliable flow values for a large range of $P_{\mathrm{e}} / P_{0}$ values. MBPR > 0.95 is reported for some CFVs in air [6]. Research has shown that Equation 1 gives reliable flows for standard diffuser geometries for $R e>1 \times 10^{5}[6,7]$.

It is widely understood that the concept of choked flow and Equation 1 is an idealization and that in fact the flow is not sonic (i.e., not at the speed of sound) across the entire area of the throat because of the boundary layer near the wall. Nonetheless, researchers $[8,9]$ were surprised to observe "low unchoking", i.e. that the flow through some CFVs departed significantly from Equation 1 at pressure ratios well below the values from Equation 2. An example is shown in Figure 1.

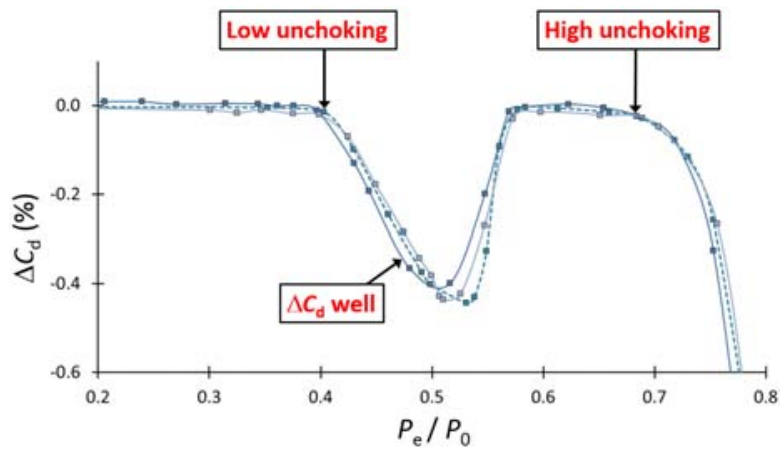

Figure 1. A plot of percent change in CFV discharge coefficient in air versus pressure ratio for CFV A14, $d=$ $0.8 \mathrm{~mm}$, diffuser length $=8.4 d$, and $\theta=2.5$.

Figure 1 presents three sets of low unchoking data at nominally the same flow conditions for CFV “A14”, a CFV that we will use as an example often in this paper. For air, the percent change in $C_{\mathrm{d}}$ should be zero for $P^{*} / P_{0}<0.53$, but for $R e=10080\left(P_{0}=100 \mathrm{kPa}\right)$, there is a $\Delta C_{\mathrm{d}}$ "well" as deep as $0.4 \%$ for $P^{*} / P_{0}$ between 0.4 and 0.58 .

Prior researchers of low unchoking have studied a variety of CFV designs that meet the ISO and ASME standards, testing various throat diameters, conical diffuser designs, pressure ratios, and gas species. Diffuser geometry is characterised by length and half-angle or exit to throat area ratio $A_{\mathrm{e}} / A^{*}$.

Nearly every researcher of low unchoking has speculated that it is caused by the interaction of shocks in the diffuser with the boundary layer at the throat and diffuser entrance and possible flow separation. The "shock + boundary layer" explanation is supported by the absence of a $\Delta C_{\mathrm{d}}$ well in quadrant nozzles. (A quadrant nozzle ends at the throat and has no diffuser.) The sharp edge at the exit of a quadrant nozzle establishes a centered Prandtl-Meyer expansion fan that thins the boundary layer. Consequently, quadrant nozzles have a thinner boundary layer than a CFV with a diffuser [10]. Moreover, the favourable pressure gradient from the Prandtl-Meyer expansion fan reduces the chance of boundary layer separation: a shock cannot occur until after the Prandtl-Meyer expansion fan, further downstream. The few CFVs with a step increase in diameter downstream from the throat that have been tested also do not show low unchoking presumably because of the consistent conditions at the intersection of the throat boundary layer and any shocks in the diffuser $[7,11]$.

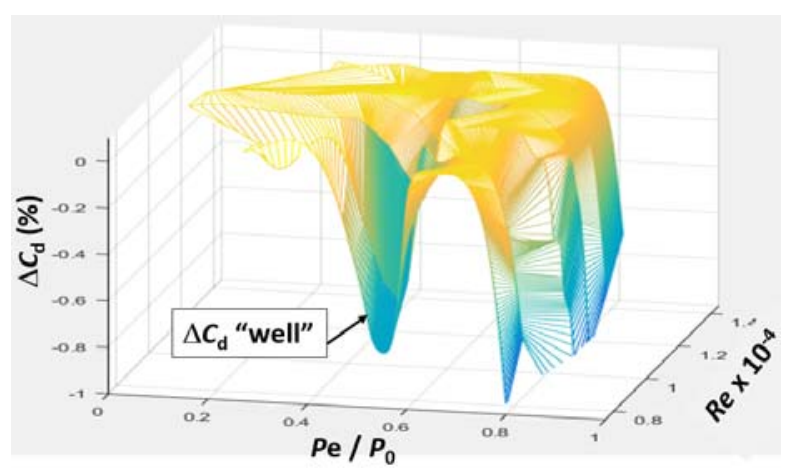

Figure 2. A surface plot of the change in discharge coefficient versus pressure ratio and Reynolds number for CFV A14 in air. At lower $R e$, the $\Delta C_{\mathrm{d}}$ well is deeper, but the range of pressure ratios over which it occurs does not increase much.

For a given gas, low unchoking is also more prevalent and severe at lower Reynolds numbers, as shown in Figure 2. At low $R e$, momentum forces are small, the boundary layer in the throat and diffuser are significant portions of the cross sectional area and the diffuser is more prone to boundary layer separation. The boundary layer thickness in the diffuser affects the boundary layer thickness in the throat, as studied by Ding et al. [12]. For CFV A14 under the conditions that it was tested herein $(R e \cong 10,000)$, the cross sectional area due to the boundary layer displacement thickness was approximately $3 \%$ of the throat area. 
Research by Carter et al. [7, 13] strived to better understand the source of low unchoking and established experimental guidelines for the MBPR that will give reliable CFV performance for particular diffuser geometries. They have coined the term "diffuser performance inversion" instead of the original term "premature unchoking" to highlight that low unchoking is related to the performance of the diffuser and that a CFV is always unchoked to some degree due to the presence of a subsonic boundary layer. Part of the objection to the term premature unchoking is that it seems likely that there is still a portion of the throat that is moving at $M=1$, at least some of the time. In this paper, we use the terms low unchoking and high unchoking (Figure 1).

It should be emphasized that the $\Delta C_{\mathrm{d}}$ due to low unchoking is only significant at the $0.01 \%$ level for Reynolds numbers below $1 \times 10^{5}$. This may occur when using CFVs smaller than $2 \mathrm{~mm}$. Furthermore, even CFVs much smaller than 2 $\mathrm{mm}$ are reliable flow sensors if plots like Figures 1 or 2 are available and followed. Also, empirical studies of standardized geometries (which show that long diffusers are less susceptible to low unchoking) are a practical approach.

\section{Proposed mechanism for low unchoking: transonic resonance}

The shock structures of conical diffusers have been visualized and studied extensively by prior researchers. The shocks depend on the geometry of the diffuser (half angle, length, surface roughness), the gas specific heat ratio, the Reynolds number (and the boundary layer thickness), and whether the boundary layer is laminar or turbulent. A review of the prior literature reveals a progression of shock conditions as $P_{\mathrm{e}} / P_{0}$ varies that we depict in Figure 3.

Figure 3a illustrates the CFV at low pressure ratios. The flow expands via a Prandtl-Meyer fan that originates at the change in curvature between the toroidal and conical sections of the diffuser. A plot of the pressure versus streamwise location is also shown at the bottom of Figure 3a. The favourable pressure gradient downstream of the throat leads to a thinning of the boundary layer and helps keep it attached to the diffuser wall.

Figure 3b illustrates an oscillating shock within the diffuser that generates what Zaman et al. [14] call "transonic resonance”. Zaman and Papamoschou et al. [15] demonstrated that a lambda shock (a combination of a normal and an oblique shock) forms in the diffuser over a range of pressure ratios and that the shock oscillates in a piston-like manner. Papamoschou et al. measured shock oscillations in a 2-D diffuser via the coherence of highfrequency pressure measurements made at taps on opposite sides of the diffuser. Zaman et al. measured peaks in the acoustic spectra from a microphone placed downstream from CFVs with various diffuser geometries. Zaman et al. referred to these peaks as "transonic tones" or "x-tones". The existence of transonic tones correlates with researchers noting an audible change in the sound emanating from a CFV depending upon whether it is choked are not [16].
Transonic resonance occurs only when the shock is within the diffuser. External shocks cause screech tones at low pressure ratios [14].

The time-averaged position of the lambda shock moves upstream (closer to the throat) as $P_{\mathrm{e}} / P_{0}$ is increased. The pressure rise across the lambda shock produces a strong, oscillating adverse pressure gradient that triggers boundary layer separation. The oscillating lambda shock causes periodic changes in the boundary layer thickness and periodic flow separations. We hypothesize that when the pressure oscillations from the lambda shock resonances are sufficiently powerful and close to the CFV throat (or $P_{\mathrm{e}} / P_{0}$ is sufficiently high), the sonic core flow is disturbed in a periodic manner as well, causing low unchoking, as depicted in Figure 3c.

This explanation agrees with von Lavante's [17] observation from a computational fluid dynamic simulation of a CFV: “At approximately, $\left[P_{\mathrm{e}} / P_{0}\right]=0.4$, it displays a clear sign of low unchoking, again, due to unsteady movement of the lambda shock upstream all the way through the throat.”

The nature of the disturbance of the sonic core by transonic resonance is not known, but we propose two possible mechanisms: 1) the sonic core is periodically changing in size due to periodically thickening boundary layer and separation in the diffuser and 2) the drum-like motion of the lambda shock within the diffuser in the axial direction produces periodic pressure oscillations immediately downstream from the throat that lead to movement and area changes of the sonic core and/or the sonic core choking and unchoking in a periodic manner.

Figure 3d depicts the situation at higher pressure ratios where the shocks are non-existent or so weak, symmetric, and stable that they no longer cause flow separation near the throat. The lack of a strong shock within the diffuser means that the adverse pressure gradient is weak and separation is less likely. For these conditions, the sonic core is stable at the geometric throat position and $\Delta C_{\mathrm{d}}$ returns to near zero.

In section 4, we will present acoustic spectra along with low unchoking test data that show strong correlation between the transonic tones and low unchoking.

Accurately predicting the location, period, and pressure rise of the shocks depicted in Figures $3 b$ and $3 c$ and the occurrence of separation is a yet unsolved topic of research. Therefore, it is presently impractical to predict the onset of low unchoking for a given CFV, gas species, and pressure condition. However, in later sections, we assemble experimental results to guide users regarding what pressure ratios are required for reliable flow measurements from standard CFV designs. 


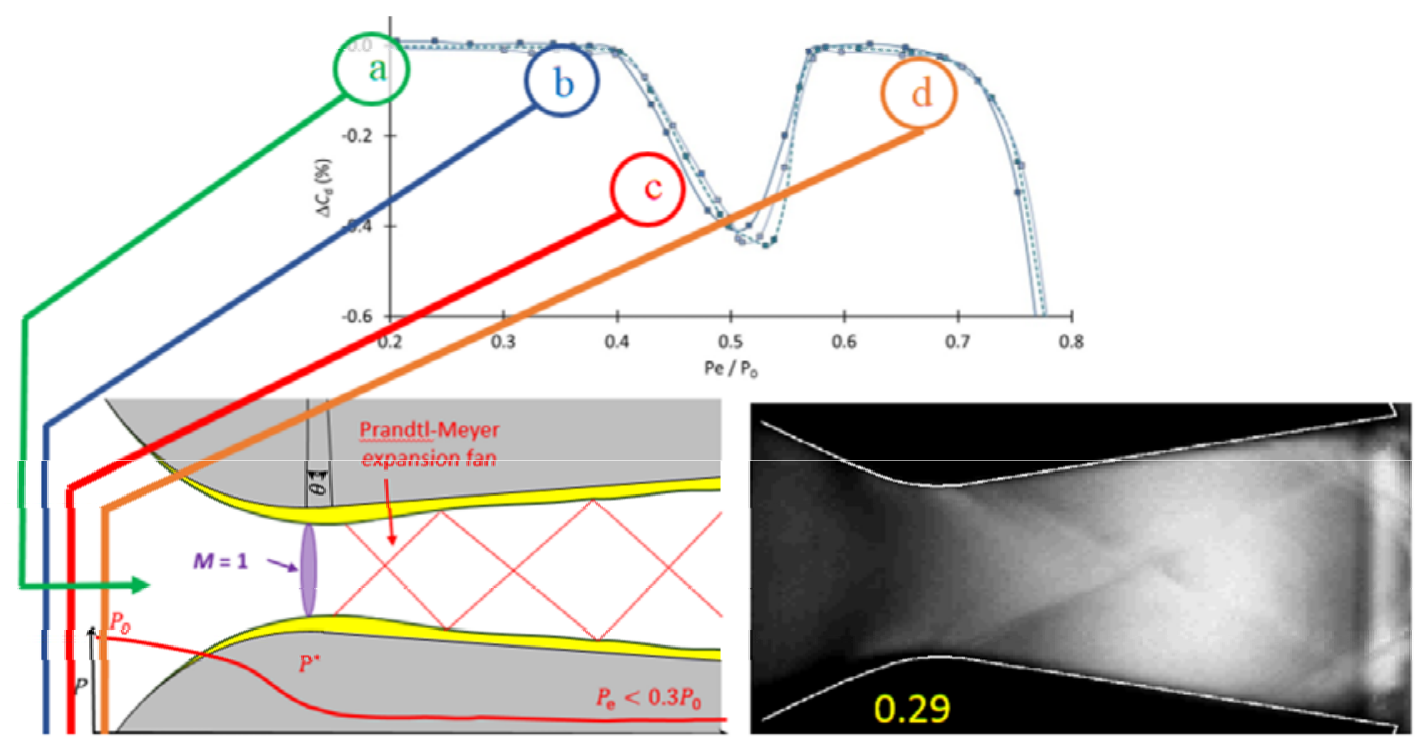

a) Prandtl-Meyer expansion fan causing favorable pressure gradient in diffuser, attached boundary layer near throat, stable sonic core at geometric throat. Various shock structures may be present at the diffuser outlet (under, perfectly, or over expanded).
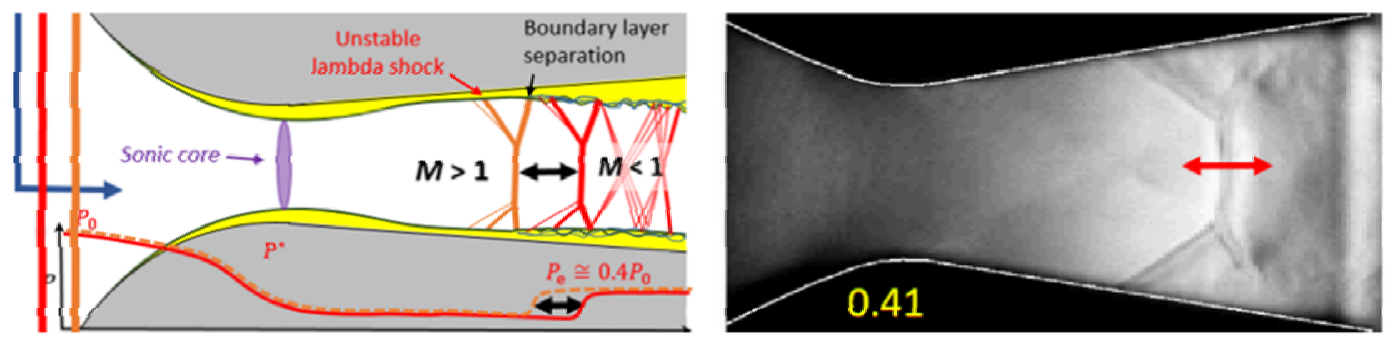

b) Transonic resonance, oscillating lambda shock, strong adverse pressure gradient at shock, boundary layers separated, stable sonic core at the geometric throat.
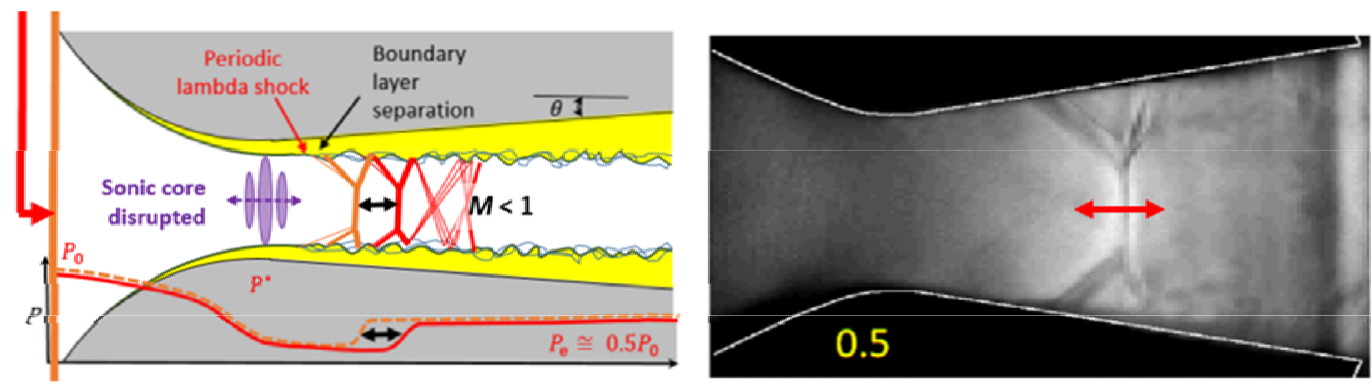

c) Transonic resonance, oscillating lambda shock, strong adverse pressure gradient at shock, boundary layers separated, disrupted sonic core.
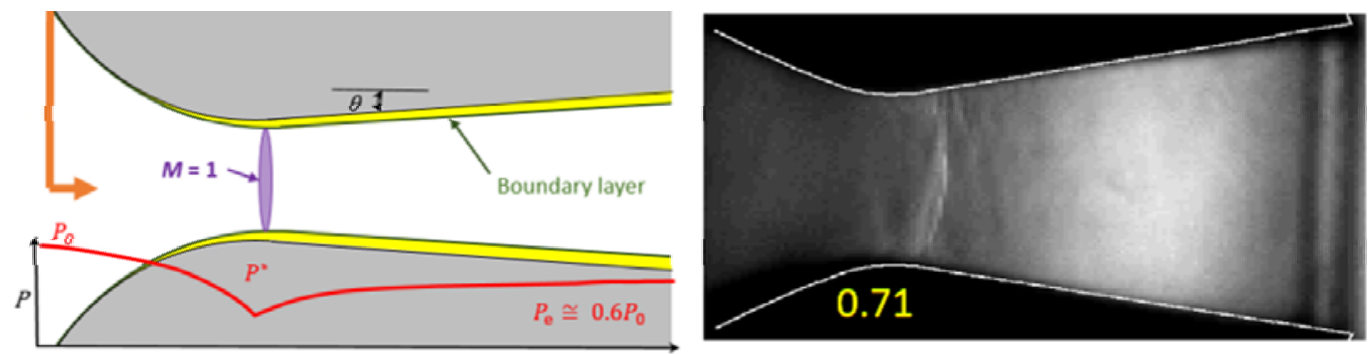

d) CFV just choked, no strong shocks, weak adverse pressure gradient in the diffuser, boundary layers attached, stable sonic core at the geometric throat.

Figure 3. Left) Schematic shock structure and boundary layer interactions and their role in low unchoking. Right) Schlieren photograp hs of a 2-D CFV from Hunter [18], labelled with their $P_{\mathrm{e}} / P_{0}$ values. Note that the dark vertical lines at the CFV exit are apparently not shocks: they are present in all of the images. 


\section{Characteristics of transonic tones}

In this section, we review the work of Hunter [18], Zaman [14], and Papamoschou [15] and their many colleagues to better understand the flow phenomena in a nozzle diffuser and the characteristics of transonic resonances.

Zaman states that transonic resonances are longitudinal acoustic modes of the diffuser that are modified by the flow. The conical diffuser forms a $1 / 4$ wave resonator with modes that have an acoustic pressure antinode at the position of the lambda shock and a node near the diffuser exit. Thus, the lowest-frequency (fundamental) resonance occurs when the wavelength of sound is about 4 times the distance between the shock and the diffuser exit: higherfrequency resonances occur at odd multiples of the fundamental. Zaman compares the lambda shock to the head of a drum. He said the resonance amplitude "is not well defined and sensitive to e. g. the surface finish.” When the lambda shock is in the diffuser (Figures $3 \mathrm{~b}$ and $3 \mathrm{c}$ ), as $P_{\mathrm{e}} / P_{0}$ increases, the distance between the lambda shock and the diffuser exit increases (the shock moves towards the throat), thereby the resonance wavelength increases and the frequency decreases. This behaviour is the opposite of screech tones which increase in frequency with increasing $P_{\mathrm{e}} / P_{0}$.

Zaman presented correlations that allow prediction of the transonic resonance frequency as a function of the diffuser length, half angle, the speed of sound, and the fully expanded jet Mach number, $M_{\mathrm{j}}$ defined by

$M_{\mathrm{j}} \equiv\left[\left[\left(\frac{P_{0}}{P_{\mathrm{e}}}\right)^{\frac{\gamma-1}{\gamma}}-1\right] \frac{2}{\gamma-1}\right]^{1 / 2}$

$M_{\mathrm{j}}$ is the Mach number that would be present at the exit of the diffuser for an adiabatic, isentropic, and inviscid flow (no boundary layer and no shocks), for a perfect gas, with constant specific heats. Zaman's correlation worked well for diverse geometries, axisymmetric, 2-D, and annular, i.e., similar transonic phenomena occur in various $\mathrm{CFV}$ geometries.

Both Zaman and Papamoschou found the lambda shock at much smaller area ratios $A / A^{*}$ than predicted by 1-D theory, i.e. closer to the throat.

Figure 4a shows Hunter's centerline pressure data for a range of $P_{\mathrm{e}} / P_{0}$ 's, labelled with the corresponding schematics from Figure 3. Successful diffuser performance and pressure recovery, without sonic core disruption occur at high $P_{\mathrm{e}} / P_{0}$ 's (labelled Fig. 3d). Intermediate pressure ratios that show low unchoking in CFV A14 have large, oscillating pressure gradients near the throat (Fig. 3c). For the lower pressure ratios labelled Fig. 3b and 3a, the shocks are near the exit, and low unchoking is not present (at least for CFV A14).
Papamoschou [15] found that the oscillating lambda shock "creates a 'back pressure' much higher than the theory predicts" for a stationary shock. Figure 4b shows the pressure measured with a probe on the centerline, deconvoluted to remove lags from the sensor's time constant, to give a better idea of the real pressure fluctuations.

Zaman found that the transonic resonance only occurred when the nozzle surface was smooth and that the tones disappeared if the boundary layer was tripped by small protrusions or pieces of tape upstream of the lambda shock location. This suggests that a CFV with a stepped diffuser, i.e. a small step increase in the diffuser diameter, would not have transonic tones.
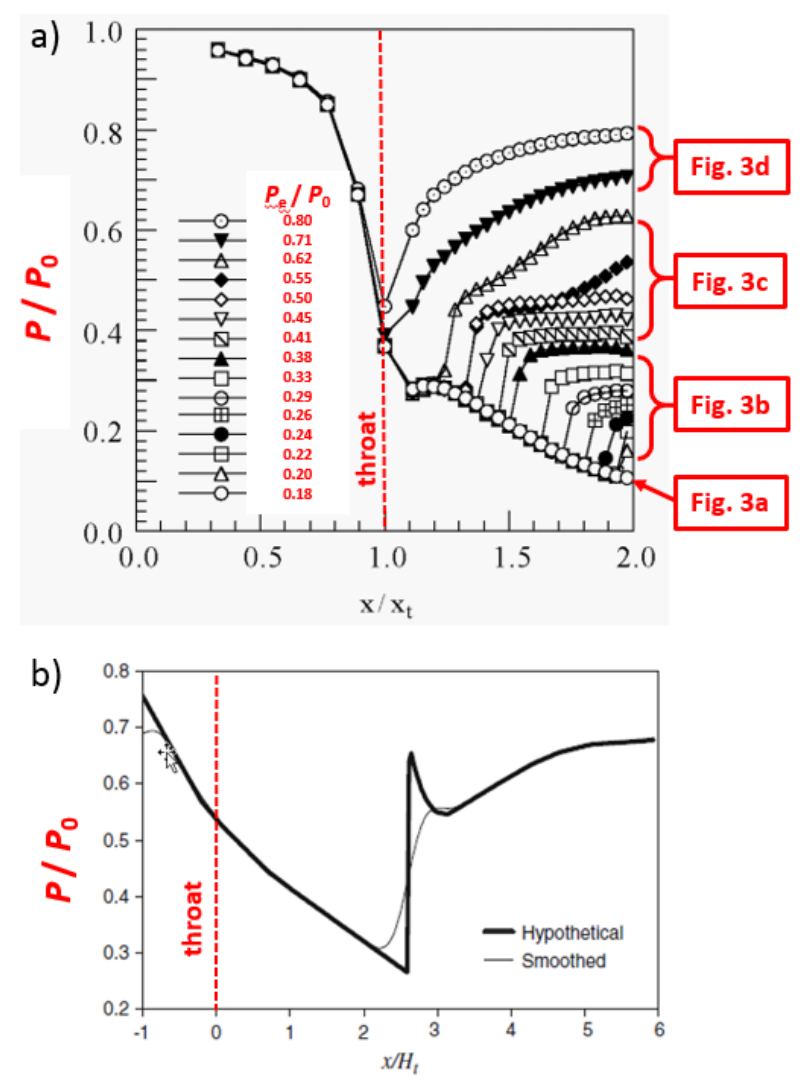

Figure 4. Centerline pressure in 2-D diffusers, with our annotations in red font. a) from Hunter [18] for $R e=3.2 \times$ $10^{6}, H_{t}=14 \mathrm{~mm}, L=53.8 \mathrm{~mm}, \theta=11^{\circ}$, and $\left.\mathbf{b}\right)$ from Papamoschou [15], showing underestimation of pressure changes due to sensor time response for a trumpet shaped diffuser at $R e=2.1 \times 10^{5}, L=117 \mathrm{~mm}$.

\section{Acoustic spectra for CFV A14}

We installed two microphones, one upstream and one downstream from CFV A14. The CFV was installed with $2.2 \mathrm{~cm}$ approach and exit tubes and the microphones were installed via $1.0 \mathrm{~cm}$ inside diameter Tees (Figure 5). Using a spectrum analyser, we measured the acoustic frequency spectrum that was present for various pressure ratios traversing the low unchoking phenomenon in dry air. Strong acoustic resonances and their harmonics were detected at pressure ratios correlated with low unchoking. 
Using the manufacturer specified diffuser length and half angle, the frequencies of the resonances were $2 \%$ to $13 \%$ lower than those predicted by Zaman's correlation (Table 2).

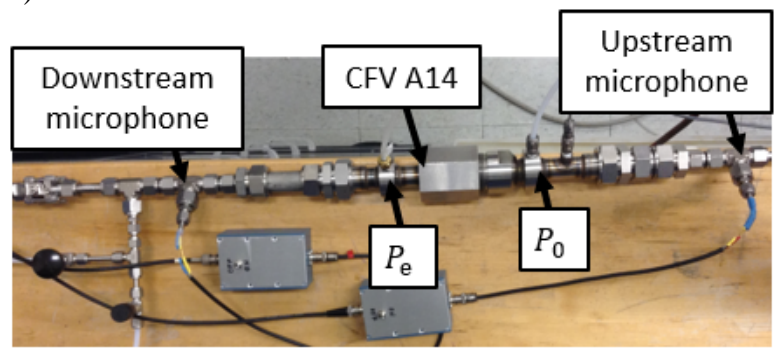

Figure 5. Positions of CFV A14, pressure sensors and microphones for acoustic spectra.

Figure 6 shows spectra from the downstream microphone for $P_{\mathrm{e}} / P_{0}$ ranging from 0.3 to 0.6 . The fundamental frequency was nominally $14 \mathrm{kHz}$ and the $2^{\text {nd }}$ and $3^{\text {rd }}$ harmonics are also visible in Figure $6 \mathrm{a}$ at $\cong 28 \mathrm{kHz}$ and $\cong 42 \mathrm{kHz}$. For the fundamental mode, resonance peaks $>$ $100 \mathrm{~Pa}^{2}$ were present for pressure ratios between 0.35 and 0.57 . Figure $6 \mathrm{~b}$ zooms in on the fundamental mode outlined by the dashed box and uses a linear scale to make their relative power more apparent.
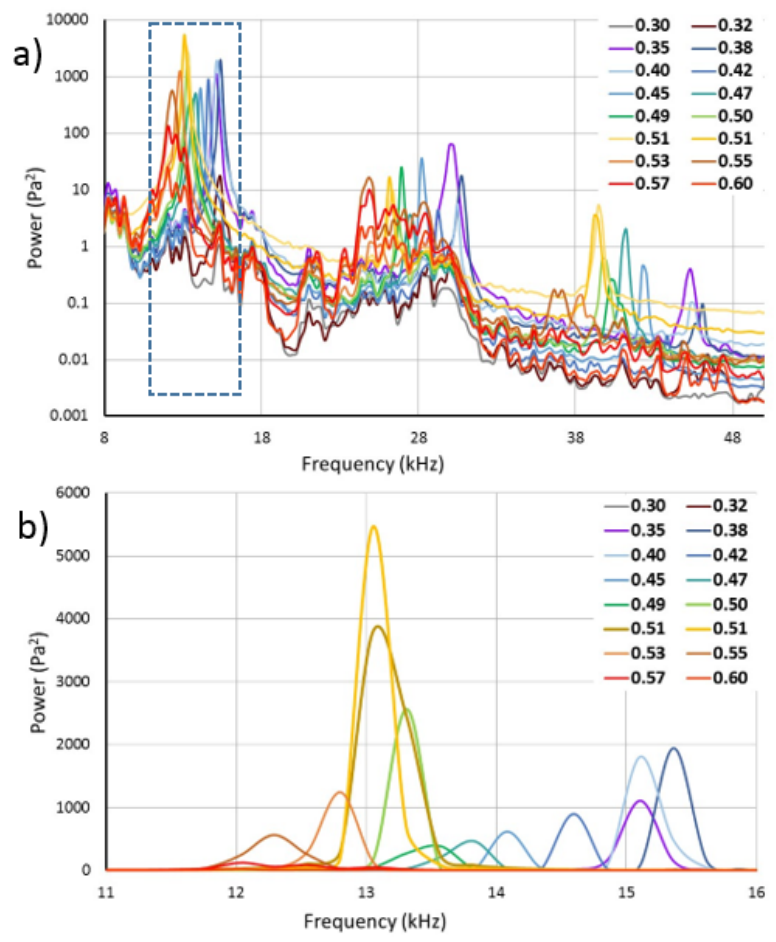

Figure 6. Acoustic spectra from the downstream microphone for various pressure ratios.

Figure 7 shows spectra from the upstream microphone. The resonance peaks are weaker and are present over a narrower and higher range of pressure ratios.
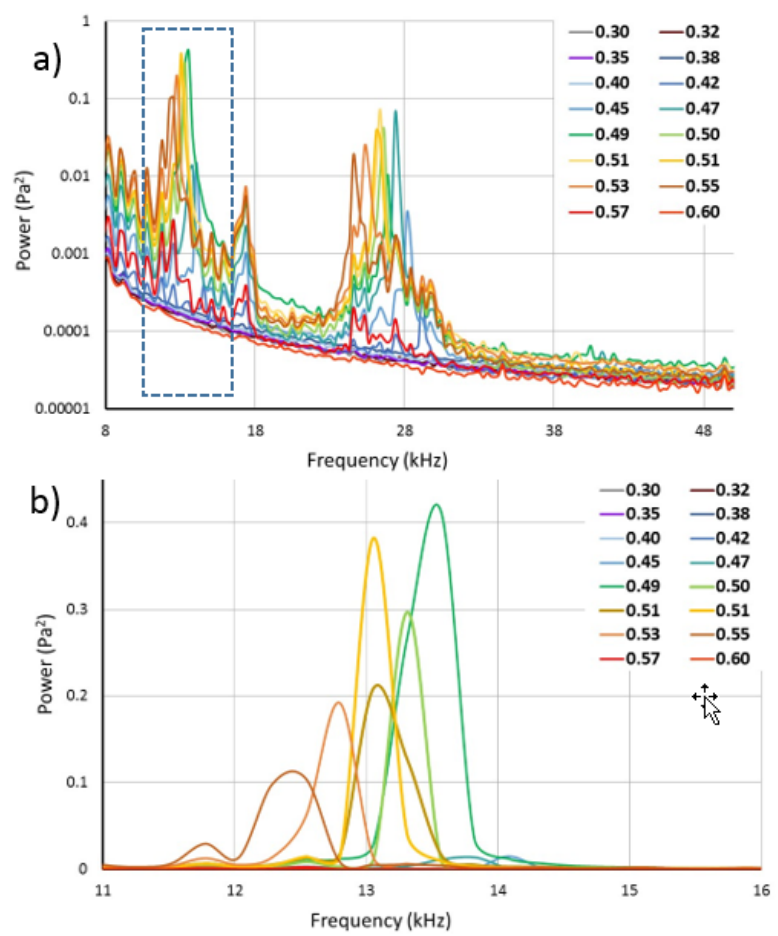

Figure 7. Acoustic spectra from the upstream microphone.

Figure 8a shows the frequency of the fundamental transonic tones versus $P_{\mathrm{e}} / P_{0}$ for both the up and downstream microphones, along with the $\Delta C_{\mathrm{d}}$ data gathered in unchoking measurements conducted at the same time. Data from two experiments are presented to assess reproducibility. The upstream and downstream frequencies match, as shown in Figure 8a. The frequency of the tones decreases with increasing pressure, matching the behaviour of transonic resonance described by Zaman (as opposed to screech tones).

Figure $8 \mathrm{~b}$ plots the acoustic power versus $P_{\mathrm{e}} / P_{0}$ for the two sets of data in air. The largest values of acoustic power from the downstream and upstream microphones and the minimum of the $\Delta C_{\mathrm{d}}$ well coincide at $P_{\mathrm{e}} / P_{0} \cong 0.5$. The transonic tones are not detected on the upstream side of the CFV until $P_{\mathrm{e}} / P_{0}=0.45$, well into the $\Delta C_{\mathrm{d}}$ well and their power is approximately three orders of magnitude lower than on the downstream side. There are strong transonic tones downstream at $P_{\mathrm{e}} / P_{0}=0.38$ where $\Delta C_{\mathrm{d}}=0$, while at $P_{\mathrm{e}} / P_{0}=0.45$ the power from the downstream microphone is relatively low, but unchoking is significant.

Based on a simple model (the ratio of cross sectional area at the pipe where the downstream microphone was installed to the area of the throat), we estimate the acoustic pressure fluctuations to be 170 times stronger at the CFV throat than at the microphone, or as much as $13 \mathrm{kPa}$ on the downstream side.

A possible explanation for the data in Figure 8a is that for low unchoking to occur, the oscillating pressure component downstream from the throat must be sufficiently large to cause the throat to be intermittently sonic or subsonic, as illustrated in Figure 9. 

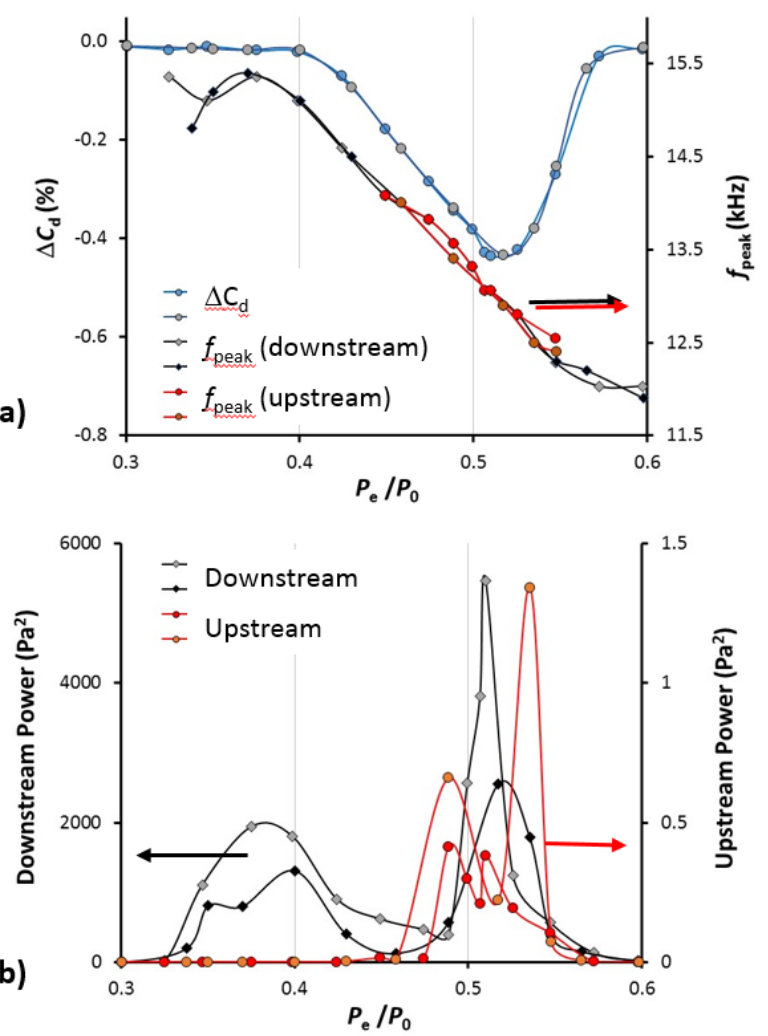

Figure 8. a) $\Delta C_{\mathrm{d}}$, resonant frequencies, and b) acoustic power from the two microphones, versus pressure ratio.

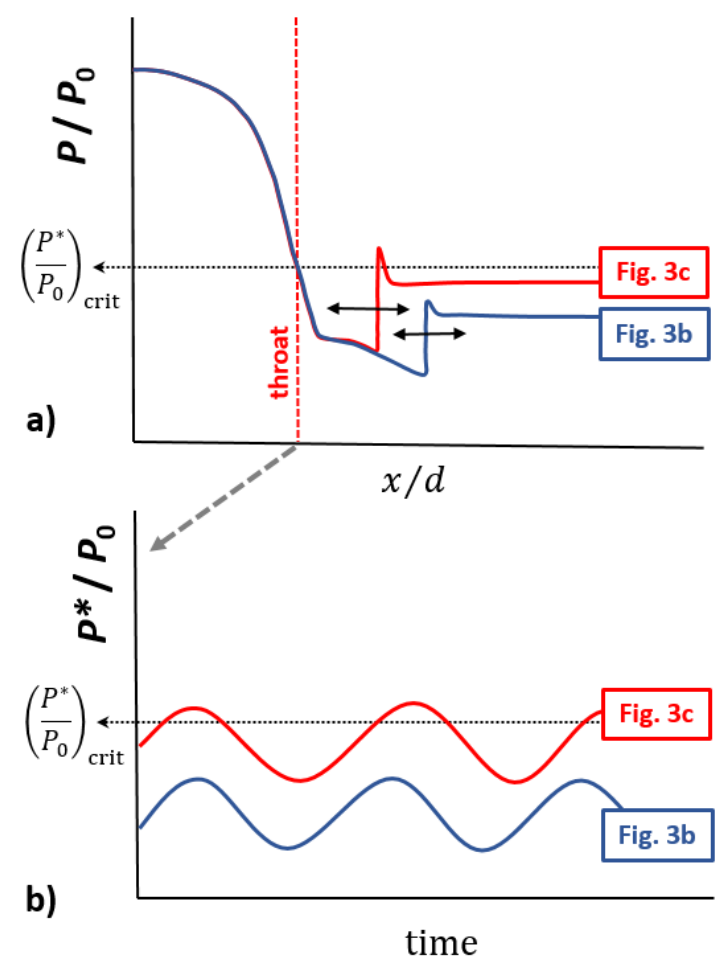

Figure 9. Illustration of how transonic pressure fluctuations could cause an intermittent sonic core because pressure ratio at the throat periodically exceeds $\left(P^{*} / P_{0}\right)_{\text {crit }}$, a) plotted versus position and $\mathbf{b}$ ) at the throat versus time.

\section{Low unchoking of CFV A14 for gases with various specific heat ratios}

Many aspects of the flow through a CFV depend on the gas specific heat ratio, $\gamma$, for instance, the angle of oblique shocks and the pressure rise across a normal shock. The fully expanded jet Mach number $M_{\mathrm{j}}$ used to characterize the transonic tones is also a function of $\gamma$. Figure 10a shows $\Delta C_{\mathrm{d}}$ versus $P_{\mathrm{e}} / P_{0}$ for CFV A14 using sulfur hexafluoride, dry air, helium, and argon.

In this experiment, we designed the test conditions to provide the same boundary layer thickness at the CFV throat for all of four gases to see if the low unchoking plots would match. CFD simulations of a CFV performed by Johnson [19] show that the boundary layer thickness depends on both $R e$ and $\gamma$. (The specific heat ratio affects the temperature in the boundary layer and larger $\gamma$ causes a thicker boundary layer.) Based on Figure 3.12 in reference [19], we performed the unchoking tests at the Reynolds numbers (or $P_{0}$ values) listed in Table 1, appropriate to achieve a displacement thickness that comprised $3 \%$ of the throat area for each gas.

Table 1. Test conditions to produce same displacement thickness in $d=0.8 \mathrm{~mm}$ CFV A14 for various gases.

\begin{tabular}{|c|c|c|c|}
\hline Gas & $\boldsymbol{\gamma}[-]$ & $\boldsymbol{R} \boldsymbol{e}[-]$ & $\boldsymbol{P}_{\mathbf{0}}[\mathbf{k P a}]$ \\
\hline $\mathrm{SF}_{6}$ & 1.10 & 9400 & 37.6 \\
\hline Dry air & 1.40 & 10080 & 100.5 \\
\hline $\mathrm{Ar}$ & 1.67 & 10500 & 104.9 \\
\hline $\mathrm{He}$ & 1.67 & 10500 & 287.1 \\
\hline
\end{tabular}

Figure 10a shows the results from eight low unchoking tests for the four gases plotted versus $P_{\mathrm{e}} / P_{0}$ (same air data as Figure 1) and Figure 10b uses the inverse of the Mach jet number as the abscissa. The onset of low unchoking is better correlated by the jet Mach number than by $P_{\mathrm{e}} / P_{0}$. Differences in the depth of the $\Delta C_{\mathrm{d}}$ wells remain in both plots $(0.19 \%)$, despite controlling for equal boundary layer thicknesses across the four gases. In another experiment where all four gases were tested at the same Reynolds numbers (not shown here), the depths of the $\Delta C_{\mathrm{d}}$ wells varied by $0.32 \%$, supporting the idea that the boundary layer thickness (not Reynolds number alone) is important in the low unchoking phenomenon. The two gases with the same specific heat ratios (helium and argon) show nearly the same low unchoking behaviour. We note that the bottom of the $\Delta C_{\mathrm{d}}$ wells is at $M_{\mathrm{j}} \cong 1$, which corresponds to the critical back pressure given by Equation 2 for each gas. 

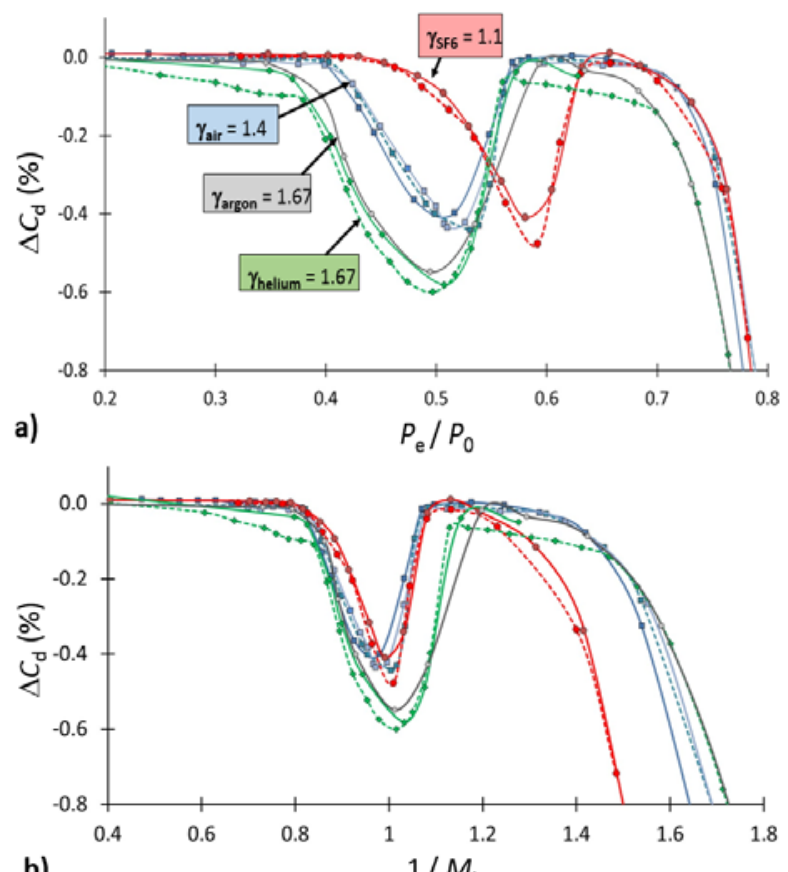

b)

$1 / M_{\mathrm{j}}$

Figure 10. Change in discharge coefficient versus a) pressure ratio and $\mathbf{b}$ ) inverse jet Mach number, for various gases in CFV A14.

Acoustic spectra were also gathered for A14 flowing sulfur hexafluoride and helium. The spectra were qualitatively similar to those presented herein for air, but the frequency of the tones was quite different. The frequency of the transonic resonances, measured and predicted using Zaman's correlation are listed in Table 2.

Table 2. Resonant tone frequency at bottom of $\Delta C_{\mathrm{d}}$ well, measured and calculated following Zaman [14].

\begin{tabular}{|c|c|c|}
\hline Gas & $\begin{array}{c}\text { Measured } \boldsymbol{f}_{\text {peak }} \\
{[\mathbf{k H z}]}\end{array}$ & $\begin{array}{c}\text { Calculated } \boldsymbol{f}_{\text {peak }} \\
{[\mathbf{k H z}]}\end{array}$ \\
\hline $\mathrm{SF}_{6}$ & 5.38 & 5.25 \\
\hline Dry air & 13.1 & 11.6 \\
\hline $\mathrm{He}$ & 34.8 & 30.4 \\
\hline
\end{tabular}

\section{Avoiding low unchoking}

We have learned much about the mechanism of low unchoking and can make reasonable predictions of the frequency of transonic tones, but not their power. So, theoretically predicting the onset of low unchoking for an arbitrary geometry is not yet practical. Several practical approaches are available for designing CFVs that do not or are less likely to exhibit low unchoking:

1) Reduce the diffuser length to $1 d$, per ISO 9300 and ASME MFC-7, and operate these CFVs at back pressures below the critical pressure given by Equation 2,

2) Add a diffuser step just downstream of the CFV throat to ensure stability of the shock structure,

3) Extend the diffuser length to $15 d$ or more to provide a sufficiently low pressure gradient to suppress low unchoking per proven performance of similar CFVs,
4) Use the correlation equation given below, generated from unchoking data to design a CFV that will not demonstrate low unchoking at the minimum operational Reynold Number.

1) Reduction of the CFV diffuser length to $1 d$, the minimum length allowable by the ISO and ASME [1, 2] CFV standards, has been shown, in the vast majority of tested cases, to eliminate low unchoking. A $1.575 \mathrm{~mm}$ CFV with a $\theta=4^{\circ}$ and a $5.3 d$ long diffuser demonstrated low unchoking when tested. The diffuser was then shortened to $1 d$ and re-tested at a similar Reynolds number, and no low unchoking was observed, as shown in Figure 11. The disadvantage of using this method to avoid low unchoking is the loss of pressure recovery in the diffuser resulting in a much lower maximum back pressure ratio than could be attained with a longer diffuser.

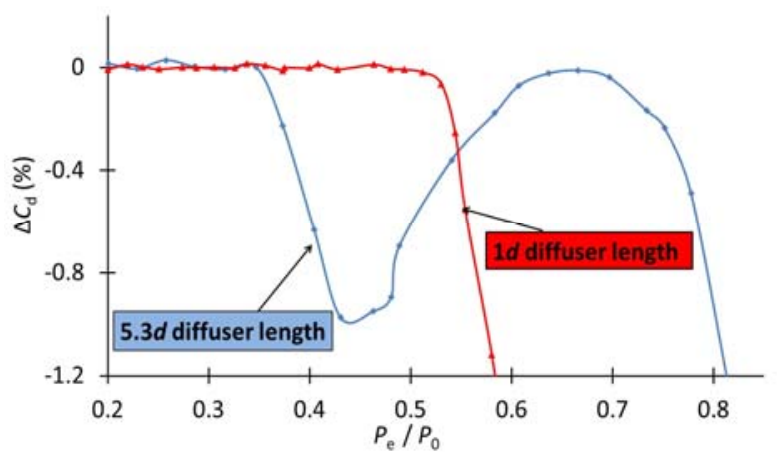

Figure 11. Elimination of low unchoking with $1 d$ diffuser length.

2) An increasing diameter "step" in the diffuser has been shown to prevent low unchoking. Two CFVs of similar geometry, except one with a diffuser step, were tested and the results can be seen in Figure 12. The work of Xu et al. [20] shows that "crest structures" as small as $2 \mathrm{~nm}$ near the throat cause large differences in low unchoking behaviour in a $d=200 \mu \mathrm{m}$ CFV. We conclude that in small CFVs, the boundary layer trip should be a step of increasing diameter, not a raised surface. The step should be located approximately $1 d$ from the throat of the CFV and result in a diffuser cross-sectional area increase of about $1 \%$.

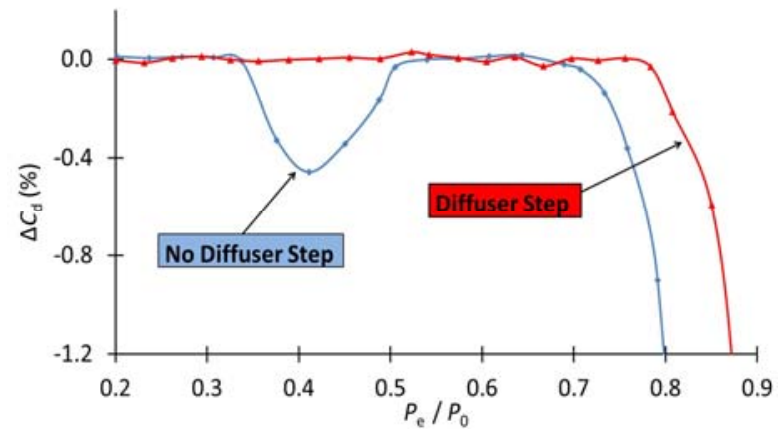

Figure 12. Elimination of low unchoking with a diffuser step.

3) Data from a range of small CFVs with $10 d$ and $15 d$ diffusers show that for the longer diffusers, low unchoking was not observed for $R e>3000$ [11]. Such long diffusers reduce the pressure gradients in the flow where the shock 
structure occurs and thereby avoid the occurrence of low unchoking.

4) Data from 270 unchoking tests on 79 CFVs with throat diameters from $0.39 \mathrm{~mm}$ to $12.7 \mathrm{~mm}, R e=2800$ to $240000, \theta=2.5$ to 6 degrees, $L=4.9 d$ to $20 d$, and $\gamma=$ 1.09 to 1.67 were studied regarding the occurrence of low unchoking (Figure 13). Throat diameters were corrected from the assumed values to match the $C_{\mathrm{d}}$ equation provided by the ISO Standard [1] to reduce error in Reynolds numbers. Diffuser length and Reynolds number were found to be the most significant factors correlated to the occurrence of low unchoking. While some data show magnitude and back pressure location variations in low unchoking due to the specific heat ratio, no significant correlation between occurrence and lack of occurrence was found. The apparent sensitivity of low unchoking to surface finish and imperfections (which was not measured) probably lead to some scatter in the results. To avoid low unchoking it is recommended that diffuser length, $L$ (in throat diameters) should conform to

$$
L / d \geq \max \left(5.0,11.89-1.556 \times 10^{-4} R e\right),(4)
$$

valid for $\operatorname{Re}>2800,1.09<\gamma<1.67$, and $2.5^{\circ}<\theta<$ $6^{\circ}$.

Equation 4 is designed to take into account expected surface finish variations from manufacturing. It is conservative, correctly avoiding $100 \%$ of occurrences of low unchoking in the test data. This is shown in Figure 13 as all low unchoking events occur to the left of the red line. The equation provides recommended diffuser length for a specified minimum operation Reynolds number.

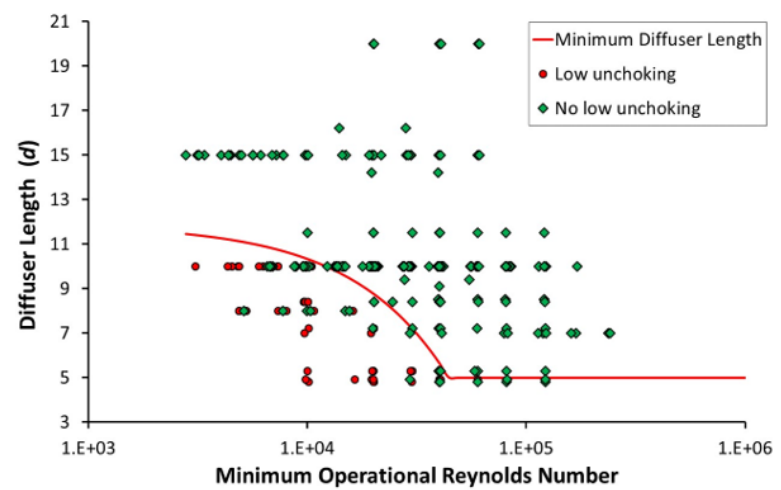

Figure 13. Diffuser length versus Reynolds number plot for avoidance of low unchoking.

\section{Correlation Equation for predicting MBPR}

Using the data from all the unchoking tests that didn't display low unchoking, a correlation equation for predicting maximum back pressure ratio was developed. MBPR was found to be influenced most strongly by Reynolds number but was also sensitive to throat diameter, diffuser length, and diffuser half angle. The correlation equation was designed to be conservative, predicting MBPR values that are equal to or lower than $95 \%$ of the collected data.

$$
\begin{array}{r}
M B P R=1.0305-\frac{19.49}{R_{e}^{0.5}}+3.247 \times 10^{-2} \ln \left(\frac{d}{25.4}\right)- \\
3.316 \times 10^{-2} \theta+2.354 \times 10^{-3} \frac{L}{d}
\end{array}
$$

where $d$ is in mm. Equation 5 is valid for $2800<R e<$ $240000,1.09<\gamma<1.67,2.5^{\circ}<\theta<6^{\circ}$, and $L / d>5$.

Table 3. Example values for MBPR correlation equation.

\begin{tabular}{|c|c|c|c|c|}
\hline $\begin{array}{c}\boldsymbol{d} \\
{[\mathrm{mm}]}\end{array}$ & $\begin{array}{c}\boldsymbol{L} / \boldsymbol{d} \\
{[-]}\end{array}$ & $\begin{array}{c}\theta \\
{\left[{ }^{\circ}\right]}\end{array}$ & $\begin{array}{c}\boldsymbol{R e} \\
{[-]}\end{array}$ & $\begin{array}{c}\text { MBPR } \\
{[-]}\end{array}$ \\
\hline 0.41 & 11 & 3 & 6835 & 0.59 \\
\hline 0.56 & 11 & 4 & 9455 & 0.60 \\
\hline 0.79 & 10 & 4 & 13395 & 0.64 \\
\hline 1.12 & 9 & 5 & 19100 & 0.64 \\
\hline 1.60 & 8 & 5 & 27456 & 0.68 \\
\hline
\end{tabular}

\section{Summary and conclusions}

We have shown strong correlation between low unchoking and the presence of a transonic resonance described by Zaman [14], Papamoschou [15], and others. We propose that low unchoking is due to interactions of the transonic resonance with the boundary layer and the sonic core at the throat (Figure 3c). This explanation matches the ideas of prior experimental and computational researchers and accounts of audible tones during low unchoking.

The mechanisms for low unchoking and transonic resonance are interactions between acoustics and fluid mechanics that are still incompletely understood. But a complete physical model will likely include: 1) acoustic modes of a cone, 2) oscillatory pressures due to movement of the lambda shock in the diffuser, and 3) the boundary layer thickness in the throat and separation in the diffuser. Flow separation occurs downstream from lambda shocks and may trigger the shock oscillations.

Low unchoking is strongly dependent on the Reynolds number, establishing that a thick boundary layer is a prerequisite to the problem. In this study we found that the depth of the $\Delta C_{\mathrm{d}}$ wells better matches across various gas species when boundary layer thicknesses are matched rather than just $R e$. We also found that the jet Mach number correlates the onset of low unchoking across gases with different specific heat ratios (Figure 10b).

We project from our new understanding of transonic resonance in CFVs and some experimental evidence (Figure 12) that there is a practical remedy for low unchoking: a "stepped diffuser”, i.e., a diffuser with a small step increase in diameter near the geometric throat. Zaman states, "The resonance ceases when the shock location has moved sufficiently downstream when the flow can no longer support the $1 / 4$ wave" [14]. A step 1) reduces the boundary layer thickness (see Ding et al. [12]), 2) trips the boundary layer from laminar to turbulent (helping it remain attached like the dimples on a golf ball) and 3) anchors a Prandtl-Meyer expansion fan and forces normal shocks further downstream, away from the throat. We plan to 
perform more experiments with stepped diffusers to check that they eliminate low unchoking for various diffuser geometries and still deliver the desired pressure recovery. We also want to study the nature of the higher harmonics of the transonic resonance and why the jet Mach number collapses low unchoking results for different gas species.

Finally, we presented practical correlations that allow CFV users to avoid low unchoking for CFVs that meet the ISO and ASME standard geometries and recommend that ISO and ASME include the correlations in future CFV standards.

\section{Acknowlegements}

This work was partially sponsored by the US Department of Defense Calibration Coordiantion Group, Physical / Mechanical Project \# 654. We also thank Iosif Shinder and our NIST SURF student, Sam McClung for Figure 2.

Nomenclature
\begin{tabular}{|l|l|}
\hline$A^{*}=\pi d^{2} / 4$ & $\begin{array}{l}\text { CFV throat area at reference } \\
\text { temperature }\end{array}$ \\
\hline$A_{\mathrm{e}}$ & CFV diffuser exit area \\
\hline$C_{\mathrm{d}}=\dot{m}_{\mathrm{ref}} / \dot{m}_{\mathrm{CFV}}$ & $\begin{array}{l}\text { Experimental CFV discharge } \\
\text { coefficient }\end{array}$ \\
\hline$C_{P}$ & Constant pressure specific heat \\
\hline$C_{V}$ & Constant volume specific heat \\
\hline$C_{\mathrm{R}}^{*}$ & $\begin{array}{l}\text { Real gas critical flow factor } \\
\text { (calculated from a thermodynamic } \\
\text { database [21]) }\end{array}$ \\
\hline
\end{tabular}

1 International Standards Organization, Measurement of Gas Flow by Means of Critical Flow Venturi Nozzles, ISO 9300, $1^{\text {st }}$ edition,1990, $2^{\text {nd }}$ edition, 2005.

2 American Society of Mechanical Engineers, Measurement of Gas Flow by Means of Critical Flow Venturis and Critical Flow Nozzles, ASME MFC-7-2016.

${ }^{3}$ Johnson, A. N. and Wright, J. D., Comparison between Theoretical CFV Models and NIST's Primary Flow Data in the Laminar, Turbulent, and Transition Flow Regimes, ASME Journal of Fluids Engineering, vol. 130, July, 2008.

${ }^{4}$ Nakao, S.-I. and Takamoto, M., Choking Phenomena of Sonic Nozzles at Low Reynolds Numbers, Flow Meas. Instrum., 11, pp. 285 to 292, 2000.

${ }^{5}$ Anderson, J. D., Modern Compressible Flow, $3^{\text {rd }}$ ed., McGraw Hill, 2004.

${ }^{6}$ Hillbrath, H. S., Dill, W. P., and Wacker, W. A., The Choking Pressure Ratio of a Critical Flow Venturi, J. Eng. Ind., Ser. B, 97, no. 4, pp. 1251 to 1256, 1975.

${ }^{7}$ Carter, M. S., Sims, B. W., and McKee, R. J., Choking Pressure Ratio Guidelines for Critical Flow Venturis and the Study of Diffuser Pressure Distribution, Proceedings of the $9^{\text {th }}$ International Symposium on Fluid Flow Measurement, Arlington, Va, USA, April 14 to 17, 2015.

${ }^{8}$ Britton, C. L. and Caron, R. W., Unchoking Pressure Ratio for Critical Flow Venturis, FEDSM97-3004, Proceedings of the ASME Fluids Engineering Summer Meeting, Vancouver, Canada, 1997.

\begin{tabular}{|c|c|}
\hline$d$ & CFV throat diameter \\
\hline$f_{\text {peak }}$ & $\begin{array}{l}\text { Frequency of the highest } \\
\text { amplitude sound in acoustic } \\
\text { spectrum }\end{array}$ \\
\hline$H_{t}$ & Height of throat in a 2-D nozzle \\
\hline$L$ & Diffuser length \\
\hline MBPR & Maximum back pressure ratio \\
\hline$M_{\mathrm{m}}$ & Gas molar mass \\
\hline$M$ & Mach number \\
\hline$\dot{m}_{\mathrm{ref}}$ & $\begin{array}{l}\text { Mass flow measured with a } \\
\text { reference flow standard }\end{array}$ \\
\hline$\dot{m}_{\mathrm{CFV}}$ & $\begin{array}{l}\text { Mass flow through a CFV } \\
\text { calculated by theoretical or } \\
\text { analytical means }\end{array}$ \\
\hline$P^{*}$ & $\begin{array}{l}\text { Stagnation pressure at the CFV } \\
\text { throat }\end{array}$ \\
\hline$P_{0}$ & $\begin{array}{l}\text { Stagnation pressure at the CFV } \\
\text { inlet }\end{array}$ \\
\hline$P_{\mathrm{e}}$ & $\begin{array}{l}\text { Stagnation pressure at the CFV } \\
\text { exit }\end{array}$ \\
\hline$R e=\frac{4 \dot{m}}{\pi d \mu_{0}}$ & $\begin{array}{l}\text { Reynolds number, using the throat } \\
\text { diameter as length scale, } \mu_{0} \text { is the } \\
\text { dynamic viscosity based on } P_{0} \\
\text { and } T_{0}\end{array}$ \\
\hline$R$ & Universal gas constant \\
\hline$T_{0}$ & $\begin{array}{l}\text { Stagnation temperature in the CFV } \\
\text { approach pipe }\end{array}$ \\
\hline$\gamma$ & $\begin{array}{l}\text { Ratio of constant pressure and } \\
\text { constant volume specific heats, } \\
=c_{P} / c_{V}\end{array}$ \\
\hline$\theta$ & CFV diffuser half-angle \\
\hline
\end{tabular}

${ }^{9}$ Ishibashi, M., Study of the Standard of Gas Flow Rate Using Sonic Nozzles and Its Application Techniques, NRLM Tsukuba, 1996.

${ }^{10}$ Ishibashi, M. and Takamoto, M., Methods to Calibrate a Sonic Nozzle and Flowmeter Using Reference Critical Nozzles, Flow Meas. Instrum., 11, pp. 293 - 304, 2000.

${ }^{11}$ von Lavante, E., Zachcial, A., Zeitz, D., Dietrich, H., and Nath, B., Effects of Various Geometric Parameters on Flow Behavior in Sonic Nozzles, Proceedings of FLOMEKO, Salvador, Brazil, Paper F6, 2000.

${ }^{12}$ Ding, H., Wang, C., Zhao, Y., Influence of Divergent Section on Flow Fields and Discharge Coefficient of ISO 9300 Toroidal-Throat Sonic Nozzle, Flow Meas. Instrum., 40, pp. 19 to 27, 2014.

${ }^{13}$ Carter, M. S., Sims, B. W., Britton, C. L. and McKee, R. J., Choking Pressure Ratio Guidelines for Small Critical Flow Venturis and the Effects of Diffuser Geometry, Proceedings of FLOMEKO, Paris, France, Paper B3.4, 2013.

${ }^{14}$ Zaman, K. B. M. Q., Dahl, M. D., Bencic, T. J. and Loh, C. Y., Investigation of a 'Transonic Resonance' with Convergent-Divergent Nozzles, J. Fluid Mech., 463, pp. 313 to 343, 2002.

${ }^{15}$ Papamoschou, D., Zill, A., and Johnson, A., Supersonic Flow Separation in Planar Nozzles, Shock Waves, 19, 171, July 2009.

${ }^{16}$ Ishibashi, M., Super-Fine Structure in the Critical FlowRate of Critical Flow Venturi Nozzles, FEDSM2002- 
31079, Joint US-European Fluids Engineering Conference, Montreal, Canada, 2002.

17 von Lavante, E., Winzosch, F. and Brinkhorst, S., Detailed Study of Flow Structure in CFVN and its Effects on the Flow Rate, Proceedings of FLOMEKO, Paris, France, Paper A3.5, 2013.

18 Hunter, C. A., Experimental, Theoretical, and Computational Investigation of Separated Nozzle Flows, $34^{\text {th }}$ AIAA/ASME/SAE/ASEE Joint Propulsion Conference and Exhibit, Cleveland, Ohio, USA, July 13 to 15, 1998.

19 Johnson, A. N., Numerical Characterization of the Discharge Coefficient in Critical Nozzles, Ph.D. Thesis,
Pennsylvania State Univ., University Park, Pennsylvania, USA, 2000.

${ }^{20}$ Xu, M., Kauth, F., Mickan, B., and Brand, U., Traceable Profile and Roughness Measurements Inside Micro Sonic Nozzles with the Profilscanner, $17^{\text {th }}$ International Congress of Metrology, 13004, 2015,

http://dx.doi.org/10.1051/metrology/20150013004.

${ }^{21}$ Lemmon E. W., Huber M. L., and McLinden M. O., NIST Standard Reference Database 23: Reference Fluid Thermodynamic and Transport Properties-REFPROP, Version 9.0 National Institute of Standards and Technology, Standard Reference Data Program, Gaithersburg, 2007. 


\title{
Ring Resonator Thermometry
}

\author{
Nikolai Klimov ${ }^{1,2}$ and Zeeshan Ahmed ${ }^{1 *}$ \\ ${ }^{I}$ Thermodynamic Metrology Group, Sensor Science Division, Physical Measurement Laboratory, NIST, Gaithersburg, MD 20899 \\ ${ }^{2} J o i n t$ Quantum Institute, University of Maryland, College Park, MD 20742 \\ "zeeshan.ahmed@nist.gov
}

\begin{abstract}
We report on our study of temperature response of ring resonator based sensors and their interchangeability over a wide temperature range. Our results suggest that with a proper fabrication process control the interchangeability in photonic thermometers can be on a $0.2^{\circ} \mathrm{C}$ tolerance band.
\end{abstract}

Keywords - ring resonator, photonic thermometer, temperature sensors

1. Introduction. Temperature measurements play a central role in all aspects of modern life including manufacturing, medicine and environmental engineering controls [1,2]. Despite the ubiquity of thermometers, the underlying technology, resistance measurement of a thin metal film or wire, has been slow to advance [3,4]. Though resistance thermometers can routinely measure temperature with uncertainties as low as $10 \mathrm{mK}$, they are sensitive to environmental variables, such as humidity and mechanical shock, which causes the resistance to drift over time requiring frequent off-line, expensive, and time consuming calibrations [3]. In recent years there has been considerable interest in developing photonic devices as an alternative to resistance thermometers [5-7]. Extending the concepts from Kim et al. [8], we demonstrated that silicon ring resonator devices show temperature sensitivity of $\approx 77 \mathrm{pm} / \mathrm{K}$ with a noise floor of $\approx 80 \mu \mathrm{K}$ [9]. Recently, we undertook a systematic survey of ring resonator parameter space that aimed to optimize the device performance while achieving consistent results $[10,11]$. Our results suggest that consistently high performance temperature sensors are obtained from the zone of stability (ring waveguide width $>600 \mathrm{~nm}$, air gap $\approx 130 \mathrm{~nm}$ and ring radius $>10 \mu \mathrm{m}$ ) such that quality factors are consistent $\approx 10^{4}$ and the temperature sensitivity is consistently in the range of $80 \mathrm{pm} / \mathrm{K}$ to $85 \mathrm{pm} / \mathrm{K}$ range [11].

In this work, we extend upon our previous work and examine the temperature dependent response of evanescently coupled ring resonator devices over the temperature range of $20^{\circ} \mathrm{C}$ to $135^{\circ} \mathrm{C}$. Our results demonstrate that for an individually calibrated sensor the fit error varies from $0.02{ }^{\circ} \mathrm{C}$ to $0.11^{\circ} \mathrm{C}$. A comparison of the same device fabricate across different chips in the same batch reveals a significant variation in temperature response, though our results do suggest that with better device fabrication process control it may be possible to achieve device inter-changeability on a $0.2{ }^{\circ} \mathrm{C}$ wide band.

2. Experimental. The photonic device consists of a ring resonator coupled to a straight-probe waveguide, with $610 \mathrm{~nm} \times 220 \mathrm{~nm}$ cross-section designed to assure a single-mode propagation of the transverse-electric (TE) light at $1550 \mathrm{~nm}$. For the three devices examined, the structural parameters (ring waveguide width/air gap/ring radius) were centered in the zone of stability (Device A: $610 \mathrm{~nm} / 130 \mathrm{~nm} / 15 \mu \mathrm{m}$; Device B: $610 \mathrm{~nm} / 130 \mathrm{~nm} / 11 \mu \mathrm{m}$; Device C: $610 \mathrm{~nm} / 140 \mathrm{~nm} / 20 \mu \mathrm{m}$ ). The photonic chip was fabricated using standard CMOS (complementary metal oxide semiconductor) technology using a silicon-on-insulator (SOI) wafer with a $220 \mathrm{~nm}$ thick layer of silicon on top of a 2 $\mu \mathrm{m}$ thick buried oxide layer. The fabrication of silicon devices itself was performed at $\operatorname{LeTI}^{1}$ (Laboratoire d'Electronique et de Technologie de l'Information, France) facility. Grating couplers where utilized as a means for efficient free space coupling of light in/out of the photonic device. Coupling losses were approximately $4 \mathrm{~dB}$ per coupler.

In our experiments, a tunable extended cavity laser (TLB-6700) was used to probe the ring resonator. A trace laser power was immediately detected from the laser output for wavelength monitoring (HighFinesse WS/7) while the rest, after passing through the photonic device via grating couplers was detected by a large sensing-area power meter (Newport, model 1936-R). The photonic chip was mounted on a 3-axis stage (Newport) in a temperature controlled enclosure.

3. Results and Discussion. Over the temperature range of $20{ }^{\circ} \mathrm{C}$ to $135^{\circ} \mathrm{C}$ the photonic ring resonators show a mean linear slope of $83.6 \mathrm{pm} /{ }^{\circ} \mathrm{C}$ and quality factor of $10^{4}$, indicating excellent temperature sensitivity. As shown in Figure 1, the sensor response when individually fitted to a quadratic equation effectively minimizes the fit residuals. This result is in agreement with our previous examination of silicon waveguide Bragg (Si WBG) thermometer [12]. The standard

\footnotetext{
1 Disclaimer: Certain equipment or materials are identified in this paper in order to specify the experimental procedure adequately. Such identification is not intended to imply endorsement by the National Institute of Standards and Technology, nor is it intended to imply that the materials or equipment identified are necessarily the best available.
} 
deviation of fit residual $\left(\sigma_{R}\right)$ varies from $0.02{ }^{\circ} \mathrm{C}$ to $0.11^{\circ} \mathrm{C}$ (Figures 1 and 2) showing an apparent bimodal distribution for $\sigma_{R}$. Bimodal distribution in $\sigma_{\mathrm{R}}$ would suggest a systematic defect or structural variance likely due to fabrication

variations. We note that the fit error contains contributions from the error in peak center estimation as well as fitting the quadratic function to the overall data. Based on our previous work with Si WBG [12], we anticipate the total fit error will dominate the combined expanded uncertainty of any ring resonator based thermometer. Our results suggest that, currently, ring resonator devices could offer measurement uncertainties that may be competitive with a wide range of resistance thermometers including platinum resistance thermometers.

We have examined the feasibility of device interchangeability in photonic thermometers. In this analysis, for each device, the fitted polynomial coefficients taken

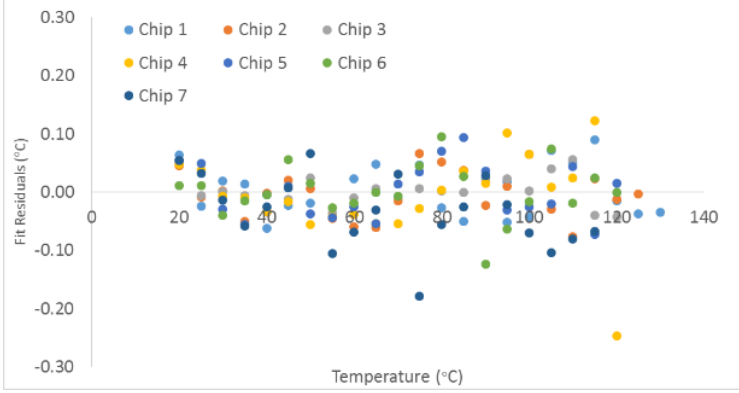

Figure 1: Residuals from quadratic fit to device $\mathrm{C}$ do not show any systematic structure suggesting a quadratic fit is sufficient to describe the temperature behavior of photonic ring resonators. from one chip were used to fit the data for the same device from other chips that were randomly selected from the entire batch. The residuals thus calculated allow us to determine how closely different devices from the same batch perform. As shown in Figure 2, the residuals for device A and B show significant variance. On the other hand, for device $\mathrm{C}$ the residuals for 6 out of 7 chips are less than $0.1{ }^{\circ} \mathrm{C}$. These results suggest that with a better fabrication process control, photonic thermometers could achieve the same level of inter-changeability that is available with resistance thermometers.
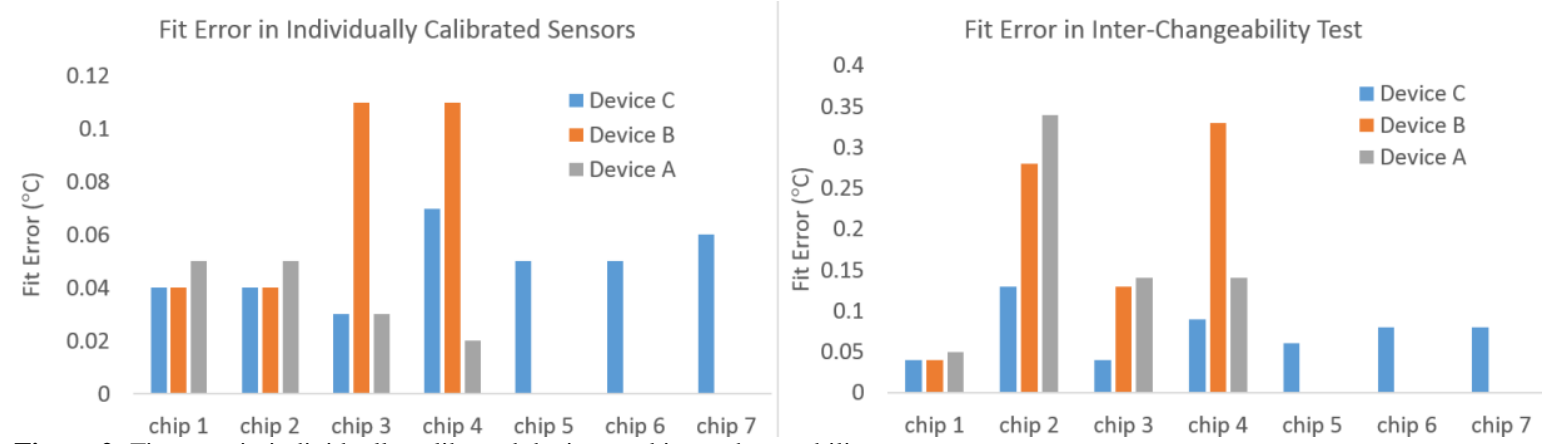

Figure 2: Fit errors in individually calibrated devices and inter-changeability test.

4. Summary. We examined the response of ring resonator devices over the temeprautre range of $20{ }^{\circ} \mathrm{C}$ to $135{ }^{\circ} \mathrm{C}$. Our results indicate the temeprautre response of an individual device shows fit residuals of $\approx 0.05{ }^{\circ} \mathrm{C}$. However, a cross-device comparison reveals significant variation between devices and chips that arises due to routine fabrication errors. Our results suggest that with proper device fabrication process control interchangeability in photonic thermometers can be winin a $0.2{ }^{\circ} \mathrm{C}$ tolerance band.

\section{References:}

[1] R. Salomir, F. C. Vimeux, J. A. de Zwart, N. Grenier, and C. T. W. Moonen, "Hyperthermia by MR-guided focused ultrasound: Accurate temperature control based on fast MRI and a physical model of local energy deposition and heat conduction," Magnetic Resonance in Medicine 43, 342-347 2000 .

[2] Z. Ahmed, S. G. Chou, K. Siegrist, and D. F. Plusquellic, "State-resolved THz spectroscopy and dynamics of crystalline peptide-water systems," Faraday Discussions 150, 175-192 2011.

[3] G. F. Strouse, "Standard Platinum Resistance Thermometer Calibrations from the Ar TP to the Ag FP," NIST Special Publication 250-281 2008.

[4] R. Price, "The Platinum resistance Thermometer," Platinum Metals Rev. 3, 78-87 1959.

[5] Z. Ahmed, J. Filla, W. Guthrie, and J. Quintavall, "Fiber Bragg Gratings Based Thermometry," NCSLI Measure 10, $24-272015$.

[6] P. C. Liacouras, G. Grant, K. Choudhry, G. F. Strouse, and Z. Ahmed, "Fiber Bragg Gratings Embedded in 3D-printed Scaffolds," NCSLI Measure J. Meas. Sci 10, 50-52 2015.

[7] S. J. Mihailov, "Fiber Bragg Grating Sensors for Harsh Environments," Sensors 12, 1898-1918 2012.

[8] G.-D. Kim, H.-S. Lee, C.-H. Park, S.-S. Lee, B. T. Lim, H. K. Bae, and W.-G. Lee, "Silicon photonic temperature sensor employing a ring resonator manufactured using a standard CMOS process," Opt. Express 18, 22215-22221 2010.

[9] H. Xu, M. Hafezi, J. Fan, J. Taylor, G. F. Strouse, and Z. Ahmed, "Ultra-Sensitive Chip-Based Photonic Temperature Sensor Using Ring esonator Structures," Optics Express 22, 3098-3104 2014.

[10] N. N. Klimov, M. Berger, and Z. Ahmed, "Towards Reproducible Ring Resonator Based Temperature Sensors," Sensors \& Transducers Journal 191, 63-66 2015.

[11] N. N. Klimov, M. Berger, and Z. Ahmed, "Characterization of Ring Resonator Structures for Applications in Photonic Thermometry," in Advance Photonics Congress, 2015),

[12] N. N. Klimov, S. Mittal, M. Berger, and Z. Ahmed, "On-chip silicon waveguide Bragg grating photonic temperature sensor," Optics Letters 40, 3934-3936 2015. 


\title{
Elucidating the Electronic Properties of Colloidally-Synthesized 2D Nanoelectronic Device Components
}

\author{
Adam J. Biacchi, Son T. Le, Brian G. Alberding, Joseph A. Hagmann, Edwin J. Heilweil, Curt A. Richter, and \\ Angela R. Hight Walker \\ Nanoelectronics Group, Engineering Physics Division, National Institute of Standards and Technology (NIST), \\ Gaithersburg, MD, USA. \\ adam.biacchi@nist.gov
}

2D nanostructures are an important class of materials that has seen an explosion of interest in recent years due to their unique optoelectronic properties in conjunction with their morphology, which lends itself well for incorporation into nanoscale devices. To date, the vast majority of nanoscale 2D materials are synthesized by mechanical exfoliation or gas phase deposition techniques. Alternatively, colloidal-based solution syntheses offer a scalable and cost-efficient means of producing nanomaterials in high yield. While much progress has been made in engineering nanostructured materials in solution, it still remains a substantial challenge to fully characterize their electronic properties. The difficulty therein is largely due to their small dimensions (typically $5-100 \mathrm{~nm}$ in size), which usually require the formation of polycrystalline colloidal assemblies or films prior to performing carrier transport measurements. However, such a strategy does not allow for the deconvolution of the intrinsic carrier transport within individual crystals from the transfer between separate colloids.

Here we present the synthesis of 2D post-transition metal chalcogenide nanomaterials and a thorough investigation of the inherent electronic properties of individual crystals. First, we detail the development of a novel solution chemistry-based synthetic approach to produce relatively large, nearlymonodisperse, tin(II) sulfide (SnS) nanoribbons (Fig. 1) and nanosheets. Next, we describe the chemical and structural characterization of these nanomaterials, and how they are processed from solution to fabricate back-gated, single-crystalline, solid-state devices (Fig. 2). Finally, we interrogate their electronic properties by a combination of multi-point contact probe transport measurements and time-resolved terahertz spectroscopy. These studies allow for the direct determination of the materials' carrier concentration, carrier mobility, resistivity, and majority carrier type, which have been rarely reported for individual colloidally-synthesized nanostructures.

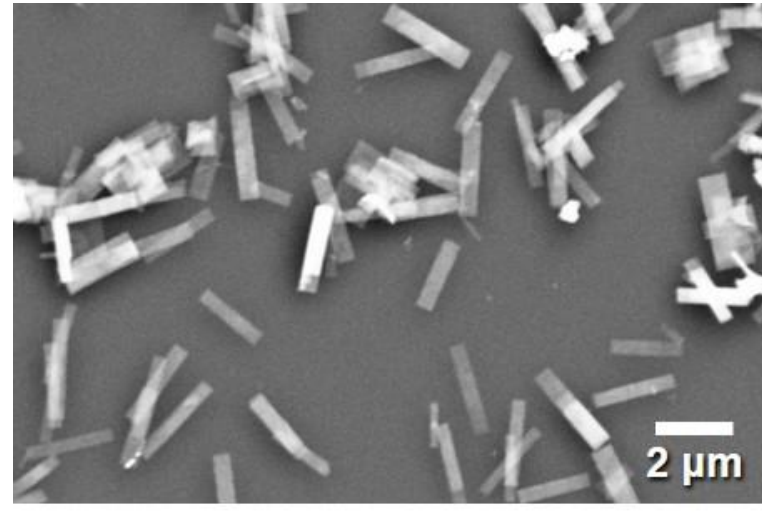

Fig 1. SEM Image of Solution-Synthesized 2D SnS Semiconductor Nanoribbons.

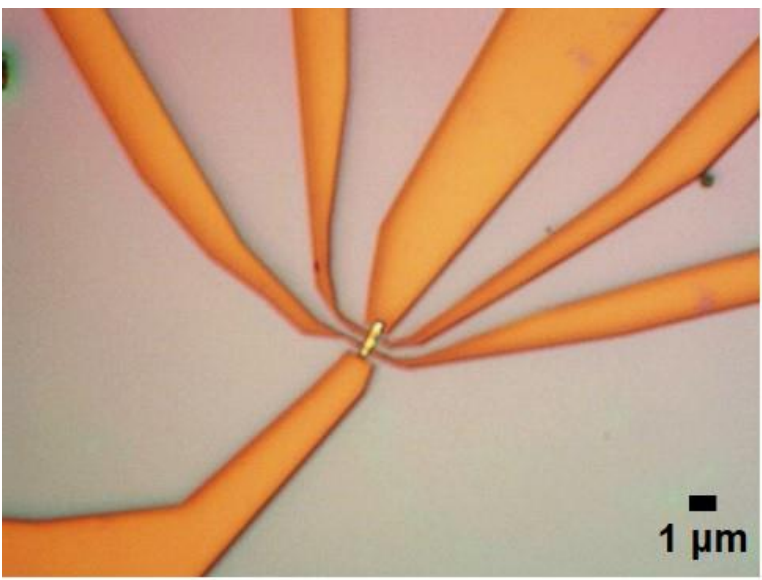

Fig 2. Optical Microscope Image of a Device Fabricated from a Single Solution-Synthesized 2D SnS Nanoribbon. 


\title{
DIRECT MEASUREMENT OF DISSIPATION IN PHONONIC CRYSTAL AND STRAIGHT TETHERS FOR MEMS RESONATORS
}

\author{
Vikrant J. Gokhale ${ }^{1,2}$ and Jason J. Gorman ${ }^{1}$ \\ ${ }^{1}$ National Institute of Standards and Technology, Gaithersburg, MD, USA \\ ${ }^{2}$ Electrical Engineering and Computer Science, University of Michigan, Ann Arbor, MI, USA
}

\begin{abstract}
This paper presents optical measurements of the dynamic strain profiles along the tethers of microelectromechanical resonators and relates them to mechanical quality factor $(Q)$. Such measurements allow for the quantification of tether dissipation and fair comparison between various tether designs. Our experiments present the first systematic comparison between the best-performing conventional tethers with one-dimensional phononic crystal (PnC) tethers for silicon bulk acoustic resonators, and demonstrate more than $3 \times$ improvement in $Q$ when the PnC tethers are used. The spatial decay rate of the mechanical strain profile along the tethers correlates well with the measured $Q$. This work is the first to demonstrate one-dimensional PnC tethers for electrostatic bulk acoustic resonators.
\end{abstract}

\section{INTRODUCTION}

The search for a clear and well-defined relationship between elastic energy dissipation and the quality factor $(Q)$ of a mechanical resonator is complicated by the number of dissipation mechanisms that exist in any real vibrating microelectromechanical system (MEMS). These dissipation mechanisms can be categorized as intrinsic mechanisms, such as phonon [1], electron [2], and thermoelastic damping [3], and extrinsic mechanisms, such as viscous damping [4], interface loss, and tether loss [5]. The situation is further compounded by the fact that there has been very limited success in acquiring direct, independent measurements of individual mechanisms of energy loss. Instead, most studies rely on analytical relationships, computational models, and empirical fitting to approximate the underlying physics.

Here, we focus on one of the design-dependent extrinsic energy loss mechanisms: tether loss. Tether loss (also known as anchor, clamping, or acoustic radiation loss) is the result of strain energy transmitted from the driven resonator through the supporting tethers and lost to the anchoring substrate. There have been efforts to model this loss analytically for some canonical geometries [6], to use computational methods to predict the loss $[5,7]$, and to reconcile these models with experimental data. There have also been efforts to reduce tether loss by designing efficient tethers (quarter-wave tethers, one-dimensional (1-D) or 2-D phononic crystal $(\mathrm{PnC})$ tethers $[8,9])$ or by modifying the resonator itself to better confine strain energy away from the tethers [10]. These efforts have relied on theoretical models and measurements of aggregate resonator $Q$ without measuring the actual energy flux through the tether.
This paper provides the first direct measurements of the strain energy dissipation profiles along the tethers of mechanical microresonators and clearly demonstrates the difference between efficient and inefficient designs. We use an optical reflection technique that relies on photoelastic modulation of reflected light due to the harmonic strain in the resonator [11] to measure the spatially resolved strain profile on the resonator surface and along the tether. We compare data for a set of conventional and 1-D PnC tether designs for identical silicon bulk acoustic resonators (SiBARs) and verify that the energy lost through the PnC tethers is lower and well correlated with higher resonator $Q$.

\section{EXPERIMENTAL DETAILS}

\section{Resonator and Tether Design}

Tether loss affects all mechanically supported vibrating devices. The photoelastic measurement used in this work to characterize tethers can be broadly applied to a variety of materials, designs, and frequency ranges. We have selected width-extensional mode SiBARs because they have a wellknown electrostatic actuation model, simple design, repeatable fabrication, well-known material properties, and crucially, the ability to make monolithic, single material, lowloss resonators with no material interfaces losses. The SiBARs presented in this work were all fabricated on the same wafer, and have the same nominal dimensions $(80 \mu \mathrm{m}$ $\times 25.5 \mu \mathrm{m} \times 10 \mu \mathrm{m}, L \times w \times t$ ), with an actuation gap of $\approx 500 \mathrm{~nm}$ on each side. The width is the primary frequency determining dimension. Each SiBAR has a different design for its symmetric tethers. We use four conventional tether designs with lengths equal to $\lambda / 8, \lambda / 4,3 \lambda / 8$, and $\lambda / 2$, where $\lambda=w \times 2$ is the acoustic wavelength (Fig.1a).

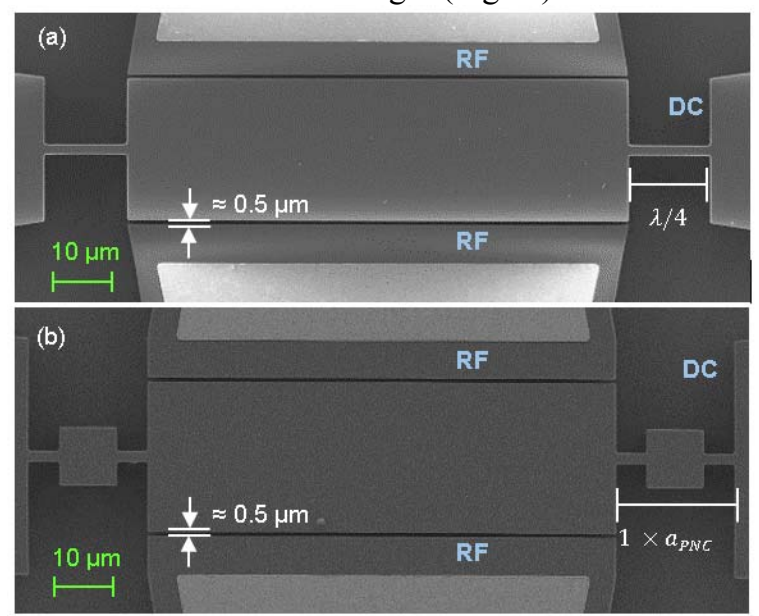

Figure 1: Scanning electron microscope (SEM) image of identical SiBARs $(80 \mu \mathrm{m} \times 25.5 \mu \mathrm{m} \times 10 \mu \mathrm{m})$ with (a) straight tethers of length $\lambda / 4$, and (b) 1-period PnC tethers. 


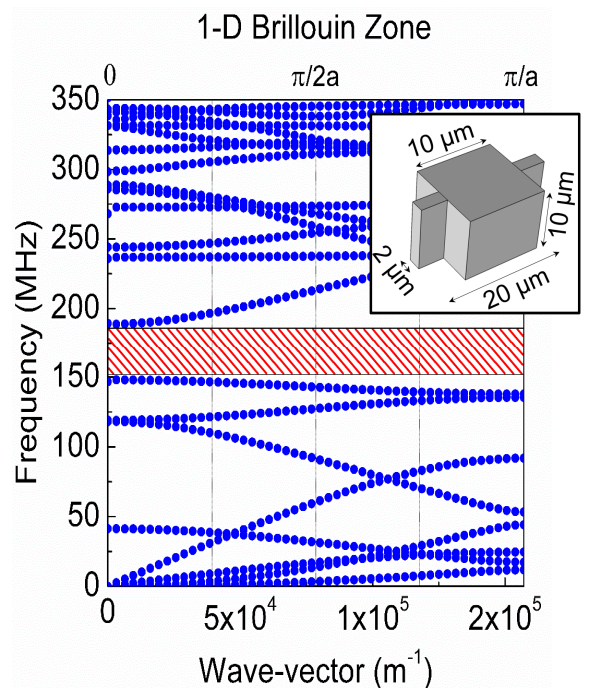

Figure 2: The $1^{\text {st }}$ Brillouin zone for a 1-D phononic crystal unit cell (dimensions in the inset schematic). This PnC design has a wide phononic bandgap (in red) in the frequency range $149 \mathrm{MHz}$ to $188 \mathrm{MHz}$. a = unit cell length

We also designed a 1-D tether comprised of repeated PnC unit cells (Fig. 1b) with an ideal phononic bandgap between $149 \mathrm{MHz}$ to $188 \mathrm{MHz}$ (Fig. 2), easily blocking the SiBAR fundamental resonance frequency, which is expected to be $\approx 164 \mathrm{MHz}$ from analytical calculations. To test the dependence of the bandgap on the number of unit cells in a real resonator, $\mathrm{PnC}$ tethers with 1,3 and 5 unit cells are used.

\section{Fabrication Process}

Standard MEMS fabrication processes are used to make the SiBARs [11]. The process starts with a silicon-on-insulator (SOI) wafer (10 $\mu \mathrm{m}$ device layer, $2 \mu \mathrm{m}$ buried oxide layer). Metal electrodes are patterned using lift-off and electronbeam evaporation. The resonator, tethers, and actuation gap are defined using optical lithography and etched using deep reactive ion etching (DRIE). The resonators are released by etching the buried oxide using vapor hydrofluoric acid. The structure of the resonator and tethers is fully monolithic and made only of single crystal silicon.

\section{Measurement Principle \& Experimental Setup}

Driving the resonator into harmonic motion using $\mathrm{DC}+\mathrm{RF}$ electrostatic actuation causes a harmonic change in the strain at every point on the resonator surface. This periodic strain modulates the refractive index of the material due to the photoelastic effect, and thus modulates the reflection amplitude of a normally incident probe laser. A simplified schematic of the experimental set-up is shown in Fig. 3. An intensity-stabilized He-Ne laser is collimated and focused onto the device with a $20 \mathrm{X}$ microscope objective $(N A=0.42$, spot size $\approx 2 \mu \mathrm{m}$ ). The reflected signal, measured by a photodetector and network analyzer, provides information about the mechanical motion, including the $Q$. The amplitude variation as the laser is scanned along the resonator surface enables reconstruction of the strain profile in the plane [11].

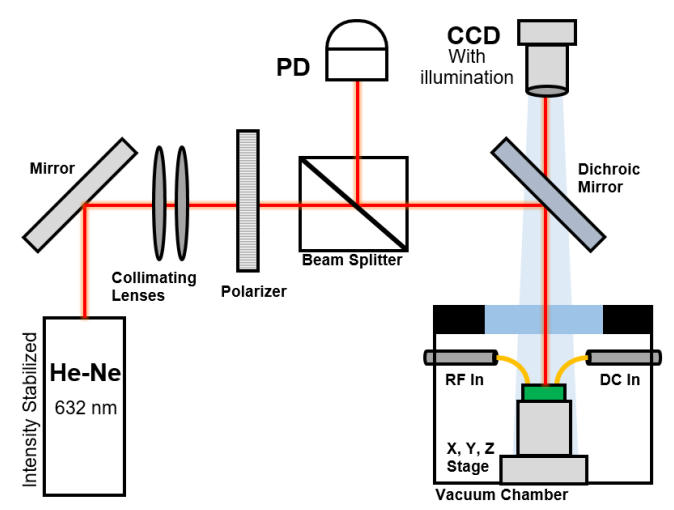

Figure 3: A simplified schematic depicting the measurement setup for photoelastic modulation measurements of in-plane dynamic strain in MEMS resonators, tethers and anchors.

This technique can be used to measure the strain profile along the tethers and on the anchors/substrate. As the tethers are not being actively driven into motion, we expect that the strain decays as we move further from the driven resonator. The spatial rate of decay of the mechanical strain along the length of the tethers is a measure of the tether loss, independent of the other dissipation mechanisms in the environment or the body of the resonator itself. It is expected that an efficient tether design has a high decay rate, and does not allow significant transmission of strain energy to the anchors. Thus, this new technique can directly compare resonator tethers and determine the most efficient designs.

\section{EXPERIMENTAL RESULTS}

\section{Spectral Response}

The SiBARs are actuated electrically $(+21 \mathrm{~V} \mathrm{DC},+10 \mathrm{dBm}$ $\mathrm{RF})$. The average resonance frequency was $167.041 \mathrm{MHz}$ $(\sigma=0.039 \%)$. The spectral response of three representative devices is shown in Fig.4, showing the influence of the tether design on the performance of otherwise identical resonators.

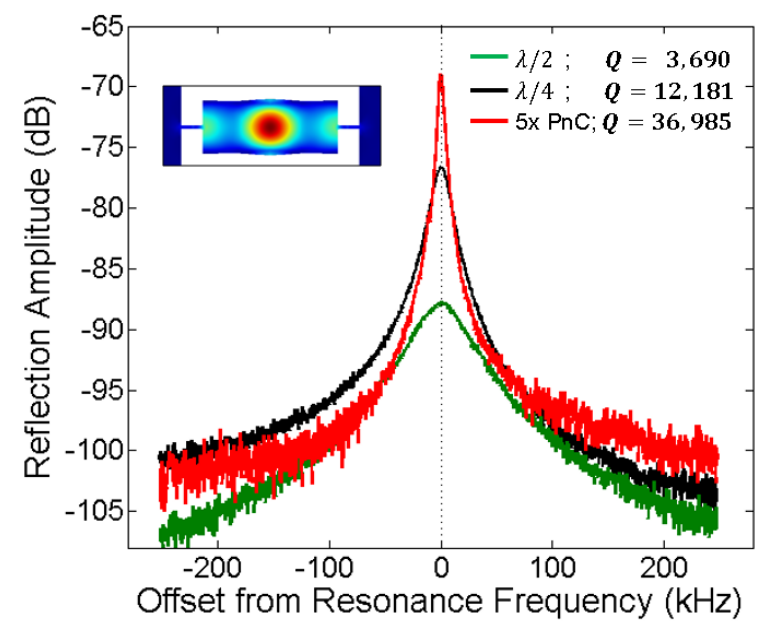

Figure 4: Photoelastic reflection response for three identical SiBARs with different tether configurations. The PnC tethers result in a significantly sharper and stronger mechanical resonance. Inset: Vibrational mode shape of the SiBAR at resonance, showing in-plane strain. 


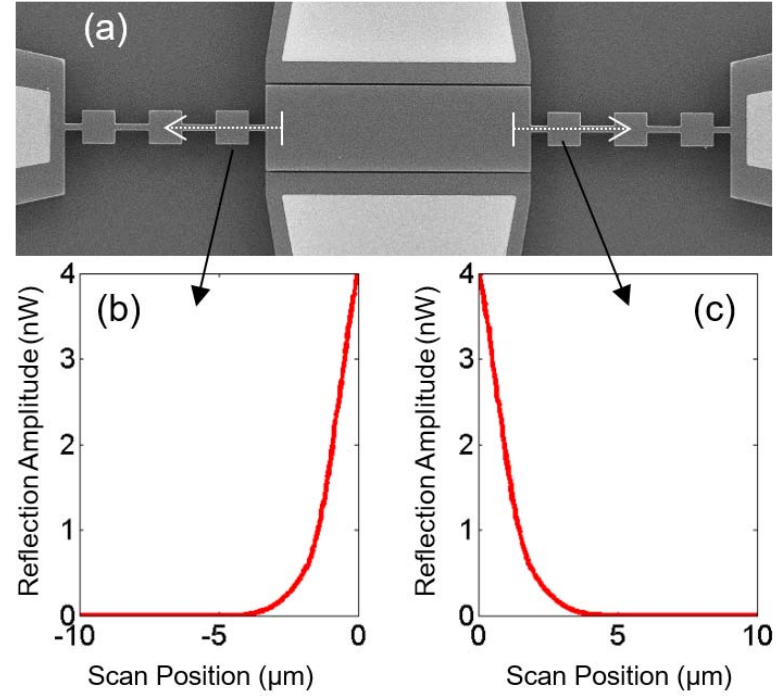

Figure 5: (a) SEM image of a 3-period SiBAR showing the scan vectors for both tethers, with measured reflection amplitude profiles for the (b) left and (c) right tethers.

\section{Tether Profiles}

The SIBAR was scanned relative to the probe laser along the length of the tethers using a positioner stage. The spatial rate of decay of the reflection signal along both tethers of each SiBAR was measured, with at least two data sets per tether. To minimize any effect of positioning error, scans were started $5 \mu \mathrm{m}$ before the root of the tether (on the body of the resonator) and moved towards the anchors. Scans were automated and the positioner stage moved in steps of $\approx 30$ nm. Feedback control was implemented to maintain the root mean square (RMS) position error of the stage to under \pm 2 $\mathrm{nm}$ per step. The reflection amplitudes measured at the network analyzer are proportional to the actual strain. Fig. 5 shows a 3-period PnC tether SiBAR with the scan directions indicated, and the measured profiles along both tethers.

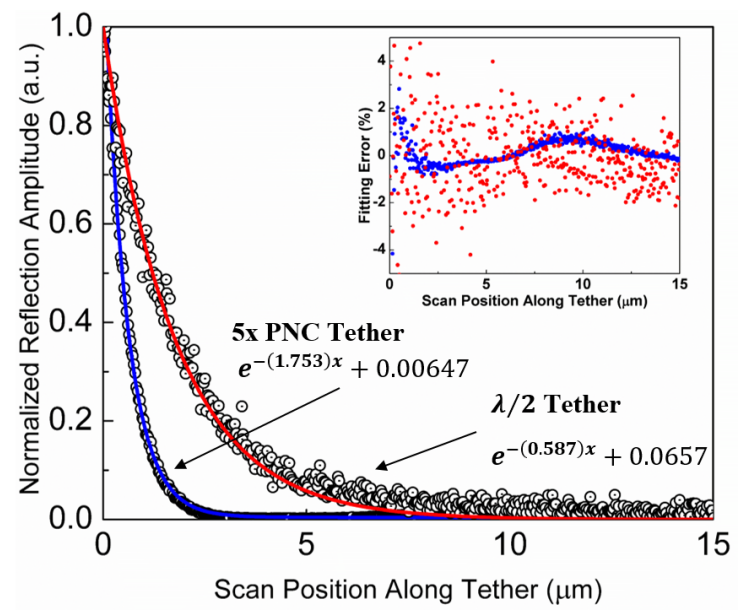

Figure 6: Tether profiles (normalized to full span of each data set) and best-fit exponential curves of the form $\left(\mathrm{y} / \mathrm{y}_{0}=\mathrm{e}^{-\beta \mathrm{x}}+\mathrm{c}\right)$ for two extreme tether designs: $a \lambda / 2$ beam tether and a 5-period PnC tether. Values of $\beta$ are in $\mu m^{-1}$. Inset: The fitting error is less than 5\% over the entire range.
Each dataset has been fitted to an exponential curve of the form $y / y_{0}=e^{-\beta x}+c$, using a robust least squares algorithm, where $\beta\left(\mu \mathrm{m}^{-1}\right)$ is the spatial rate of decay. The fitting algorithm searches for the minimum residual fitting error and optimizes starting position and size of a sliding data window to eliminate potential positioning offset errors. Fig. 6 shows two extreme tether strain profiles and best-fit exponential curves. For the purposes of comparison, each data set is normalized to span the full scale between the maximum value at the root of the tether and the minimum value at the system noise floor. The comparison clearly shows the sharp decay for the 5-period PnC tether $(Q=36,985)$ in contrast to the slow decay for the $\lambda / 2$ beam tether $(Q=3,690)$.

\section{TETHER LOSS AND QUALITY FACTOR}

The measured values of mechanical $Q$ and the best-fit values of $\beta$ are plotted in Fig. 7 as a function of the tether design (length or number of periods). For the conventional straight tethers, the relation between length of the tether and the acoustic wavelength is critical, with the $\lambda / 4$ tether significantly outperforming the $\lambda / 2$ tether. This experimentally verifies established results based on transmission theory for acoustic/electromagnetic waves. At the same time, we see that the 1-D PnC tethers outperform even the $\lambda / 4$ tethers significantly, yielding higher $Q$ and sharper damping curves.

For an infinite chain of PnC units, one should see perfect isolation, and an abrupt drop in strain levels at the root of the tether. In a finite PnC chain, we expect sharp, but not abrupt changes in strain levels. As the number of unit cells is increased (which better approximates an infinite phononic crystal), we should expect better performance from the tether. Some evidence of this effect has been demonstrated in the past $[8,12]$. Only a slight improvement is found in the present data for PnC tethered SiBARs. This is potentially due to the fact that the PnC tethered SiBARs measured here are limited by intrinsic phonon damping in the Akhieser regime [1], and any increase in $Q$ due to better tether design would be suppressed by phonon damping (Fig. 8).

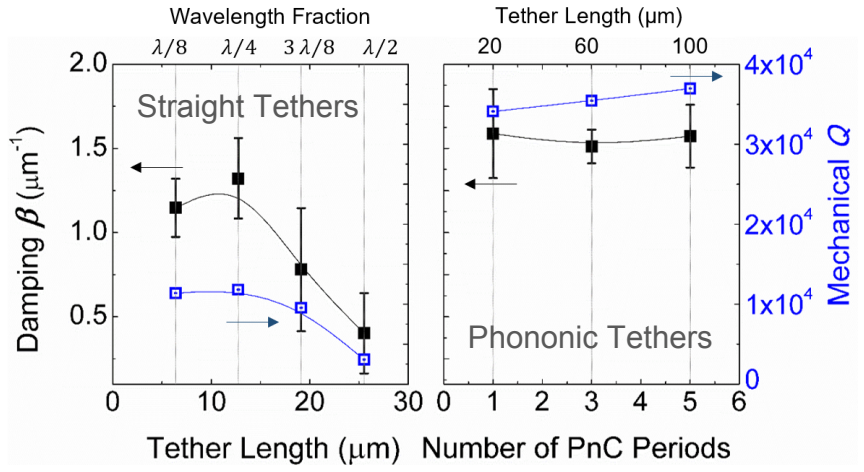

Figure 7: Measured $Q$ (blue open squares) and best-fit $\beta$ (black closed squares) of straight and PnC tethers. Values of $\beta$ are averages of 4 data-sets each (two per tether); error bars show one standard deviation. PnC tethers show consistently better $Q$ (lowest $Q$ value for PnC tethers is 2.9 times better than the highest $Q$ value for straight tethers). 


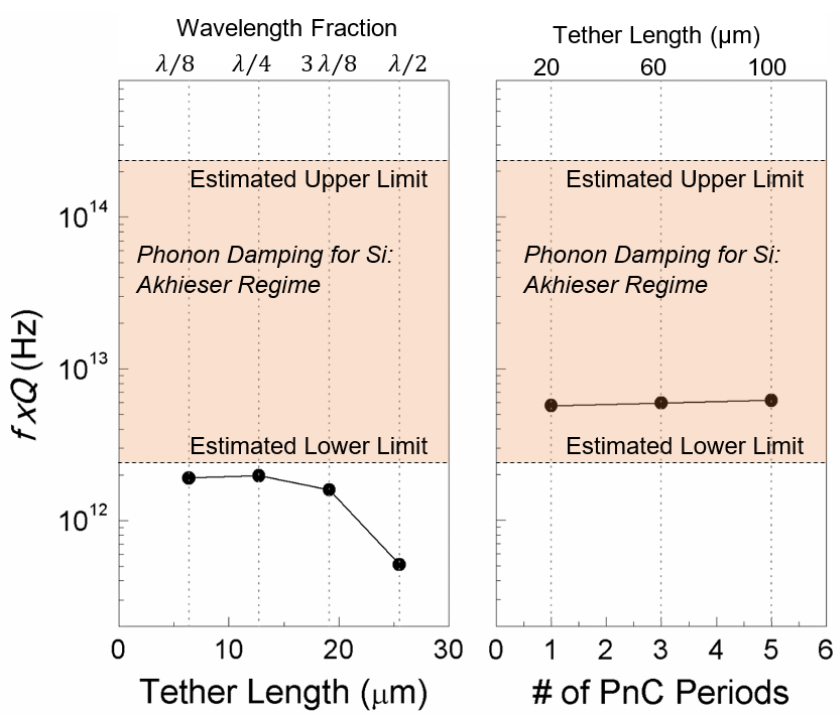

Figure 8: Measured $f \times Q$ values indicate that for the same resonator design and fabrication, phononic tethers outperform conventional beam tethers. Further, the PnC tethered resonators are potentially only limited by Akhieser loss. Akhieser damping limit estimates derived from [1].

\section{CONCLUSION}

We have demonstrated the first implementation of 1-D PnC tethers for single-material SiBARs. Significant improvement in the mechanical $Q$ has been systematically demonstrated using PnC tethers as compared to even the best straight beam tethers. The results indicate that the SiBARs are limited by tether loss or phonon loss; by eliminating tether loss using PnC tethers, the resonators appear to be phonon scattering limited. These SiBARs can be used as high-quality test platforms for gaining a better experimental understanding of phonon loss in silicon, as well as other materials, and be implemented over a wide frequency range.

The scanning strain measurement technique presented here enables spatially resolved visualization of the in-plane dynamics of MEMS resonators and their supporting tethers and anchors in ways that aggregate measurements of electrical parameters cannot accomplish. It provides the ability to measure tether loss profiles directly and independently, and correlate the behavior of various tether designs with the mechanical $Q$ of the resonator. Future research shall aim to achieve quantitative, calibrated strain measurements and an analytical relation between the decay rate and $Q$. The ability to separate and evaluate tether loss experimentally, especially for in-plane strain, has broader implications for design optimization and verification of a wide range of mechanically suspended devices including resonators, accelerometers, and gyroscopes.

\section{ACKNOWLEDGEMENTS}

This work was supported by DoC/NIST Award \# 70 NANB14H253 and performed in part in the NIST Center for Nanoscale Science and Technology Nanofab.

\section{REFERENCES}

[1] S. Ghaffari, S. A. Chandorkar, S. Wang, E. J. Ng, C. H. Ahn, H. Vu, et al., "Quantum Limit of Quality Factor in Silicon Micro and Nano Mechanical Resonators," Scientific Reports, vol. 3, p. 3244, 2013.

[2] V. J. Gokhale and M. Rais-Zadeh, "Phonon-electron interactions in piezoelectric semiconductor bulk acoustic wave resonators," Scientific Reports, vol. 4, p. 5617 , 2014.

[3] A. Duwel, R. N. Candler, T. W. Kenny, and M. Varghese, "Engineering MEMS Resonators With Low Thermoelastic Damping," IEEE/ASME Journal of Microelectromechanical Systems vol. 15, pp. 14371445, 2006.

[4] M. Bao and H. Yang, "Squeeze film air damping in MEMS," Sensors and Actuators A: Physical, vol. 136, pp. 3-27, 2007.

[5] A. Frangi, M. Cremonesi, A. Jaakkola, and T. Pensala, "Analysis of anchor and interface losses in piezoelectric MEMS resonators," Sensors and Actuators A: Physical, vol. 190, pp. 127-135, 2013.

[6] Z. L. Hao, A. Erbil, and F. Ayazi, "An analytical model for support loss in micromachined beam resonators with in-plane flexural vibrations," Sensors and Actuators APhysical, vol. 109, pp. 156-164, 2003.

[7] D. S. Bindel and S. Govindjee, "Elastic PMLs for resonator anchor loss simulation," International Journal for Numerical Methods in Engineering, vol. 64, pp. 789. $818,2005$.

[8] H. Zhu and J. E. Y. Lee, "Design of Phononic Crystal Tethers for Frequency-selective Quality Factor Enhancement in AIN Piezoelectric-on-silicon Resonators," Procedia Engineering, vol. 120, pp. 516519, 2015.

[9] L. Sorenson, J. L. Fu, and F. Ayazi, "One-dimensional linear acoustic bandgap structures for performance enhancement of AlN-on-Silicon micromechanical resonators," in 16th International Solid-State Sensors, Actuators and Microsystems Conference (Transducers '11), 2011, pp. 918-921.

[10] J. Zou, C.-M. Lin, and A. P. Pisano, "Quality factor enhancement in Lamb wave resonators utilizing butterfly-shaped AIN plates," in IEEE International Ultrasonics Symposium, 2014, pp. 81-84.

[11] V. J. Gokhale and J. J. Gorman, "Dynamic characterization of in-plane bulk acoustic resonators using high-sensitivity optical reflection measurements," in Solid-State Sensors, Actuators, and Microsystems Workshop, Hilton Head Island, 2016, pp. 145-148.

[12]D. Feng, D. Xu, G. Wu, B. Xiong, and Y. Wang, "Phononic crystal strip based anchors for reducing anchor loss of micromechanical resonators," Journal of Applied Physics, vol. 115, p. 024503, 2014.

\section{CONTACT}

*Jason J. Gorman, email: gorman@nist.gov 
Invited Paper

\title{
Enriching and purifying silicon epilayers for quantum information
}

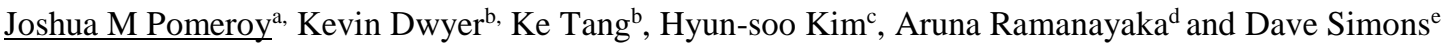 \\ ${ }^{\text {a } Q u a n t u m ~ M e a s u r e m e n t ~ D i v i s i o n, ~ N a t i o n a l ~ I n s t i t u t e ~ o f ~ S t a n d a r d s ~ a n d ~ T e c h n o l o g y, ~ U S A, ~ j o s h u a . p o m e r o y @ n i s t . g o v, ~}$ \\ ${ }^{\mathrm{b}}$ Dept. of Materials Science and Engineering, Univ. of Maryland, USA, ${ }^{\mathrm{c}}$ Dept. of Electrical and Computer \\ Engineering, Univ. of Maryland, USA, d Joint Quantum Institute, Univ. of Maryland, USA, ${ }^{e}$ Materials Measurement \\ Science Division, National Institute of Standards and Technology, USA.
}

High quality, enriched silicon contains an exceptionally low density of defects and unpaired electron and nuclear spins that allow candidate qubits (single donors or quantum dots) to exhibit very long dephasing times compared to silicon with a natural abundance of isotopes[1]. Unfortunately, high quality enriched silicon is not a readily available resource. Only a few niche supplies exist and the advancement of silicon based quantum information is limited by access to these. As quantum information has made progress, efforts to increase supply have been made, but these are also hampered by sparse evidence for determining what level of enrichment is sufficient for eliminating the effects of nuclear spin dephasing.

In this talk, I will describe very highly enriched silicon films that are grown epitaxially on $\operatorname{Si}(100)$ substrates. We have been refining the quality of these films with the aim of experimentally determining the relationship between important metrics, like coherence time, and the physical mechanisms limiting them, like nuclear spin density or chemical impurity density. At present, we have suppressed the minor isotope fraction in these films to $\approx 10^{-7}$, grown the films epitaxially with high crystalline quality and are successively reducing contaminant densities (mostly light gas components.) As the quality of the films has improved, we have begun making electronic test devices from this silicon, in particular, diodes, capacitors and transistors on the way to realizing single and multiple quantum dot devices.

The extreme suppression of the minor isotopes is accomplished through the use of ionized mass separation in a high vacuum environment. An ionized beam of essentially monoisotopic ${ }^{28} \mathrm{Si}$ is generated by 1) introducing natural abundance silane gas into an ionization cell, 2) producing a plasma of ionized silicon and silane fragments, 3) extracting and collimating an ion beam from the cell, 4) passing the ion beam through a mass analyzer composed of a magnetic field and a small aperture, 5) refocusing and transporting the monoisotopic beam into the ultra-high vacuum (UHV) growth chamber and 6) decelerating the ions onto the sample for gentle, epitaxial growth[2]. Once the sample is removed from UHV we can perform a wide range of analyses, including secondary ion mass spectroscopy (SIMS) to evaluate the distribution of isotopes in the sample. An example of a SIMS depth profile for one of our samples is shown in Figure 1. During SIMS, the sample is successively sputter eroded from left to right (surface to substrate) while progressively measuring the number of secondary ions for the three silicon isotopes. After an initial surface transient, the measured isotope fractions become stable at values of $\approx 1.27$ x $10^{-7}$ for ${ }^{29} \mathrm{Si}$ and $\approx 5.2 \times 10^{-8}$ for ${ }^{30} \mathrm{Si}[3]$. Once the sputter depth reaches the sample substrate the isotope fractions are observed to return to the natural abundance values. In analyzing the possible contamination sources limiting the enrichment, we find that the minor isotope contamination is not a consequence of the mass selectivity, but due to silane diffusion. Through further analysis of the pressure and temperature dependence, we have developed a predictive model for the enrichment as a function of these parameters that we expect to use targeting specific levels of enrichment from natural abundance down to the levels shown in Figure 1, i.e., $\approx 10^{-7}$.

In order to achieve the goals of very long coherence times, a high level of enrichment alone is not sufficient, but must also be accompanied by

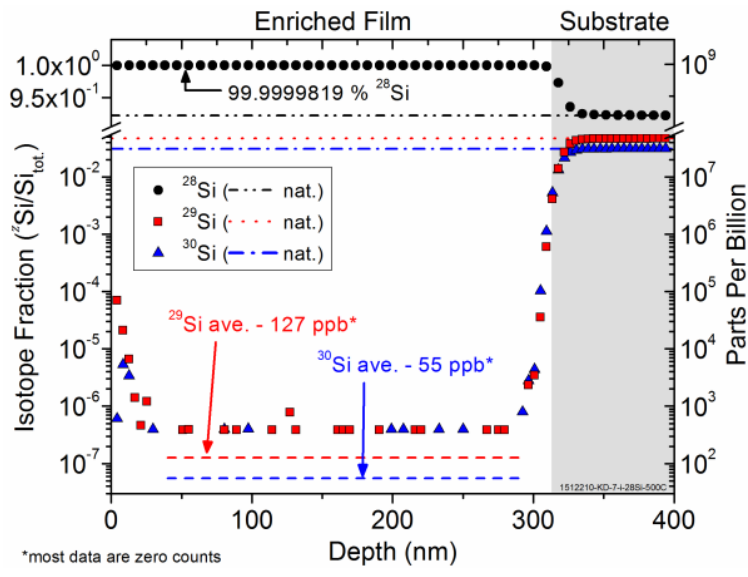

Figure 1- Secondary Ion Mass Spectroscopy (SIMS) depth profile showing nearly complete suppression of the minor isotopes of silicon in this highly enriched film. 
excellent crystalline quality and very low densities of chemical impurities. In order to assess these properties, we have conducted a number of additional analyses, including in situ scanning tunneling microscopy of every substrate and film. Shown in Figure 2 is a transmission electron microscopy (TEM) image taken on an enriched silicon sample with less than $10^{-6}$ contribution from the minor isotopes. The image is from the interface region between the substrate at the lower left and the enriched film toward the upper right. The enriched film seems to show a more "mottled" overall contrast, but exhibits a crystalline orientation identical to the substrate. We perform rigorous assessments of the chemical composition by using SIMS and selecting a suite of likely targets, e.g., targets known to the semiconductor industry to be deleterious. Results from SIMS analysis of this type is shown in Figure 3 for silicon and 22 other elements, including heavy metals, transition metals, alkalis and light gasses. Of these, only light gasses and aluminum was detected. We attribute the aluminum to auto-doping that has since been eliminated and note that the light gasses have since been reduced by almost a factor of 100 with further improvements in progress expected to improve more than 100 times more.

As we move forward with this project, we are fabricating and measuring electrical test devices while also developing the techniques to produce arbitrary targeted levels of enrichment. The fabrication of test devices has met with challenges in growing high quality oxide isolation layers, which we attribute to a self-limited growth arising from the excess nitrogen. None-the-less, we have plans to mitigate these difficulties even while we seek to substantially reduce the nitrogen. Additionally, we are pursuing collaborations to conduct electron spin resonance (ESR) studies of small numbers of donors in our

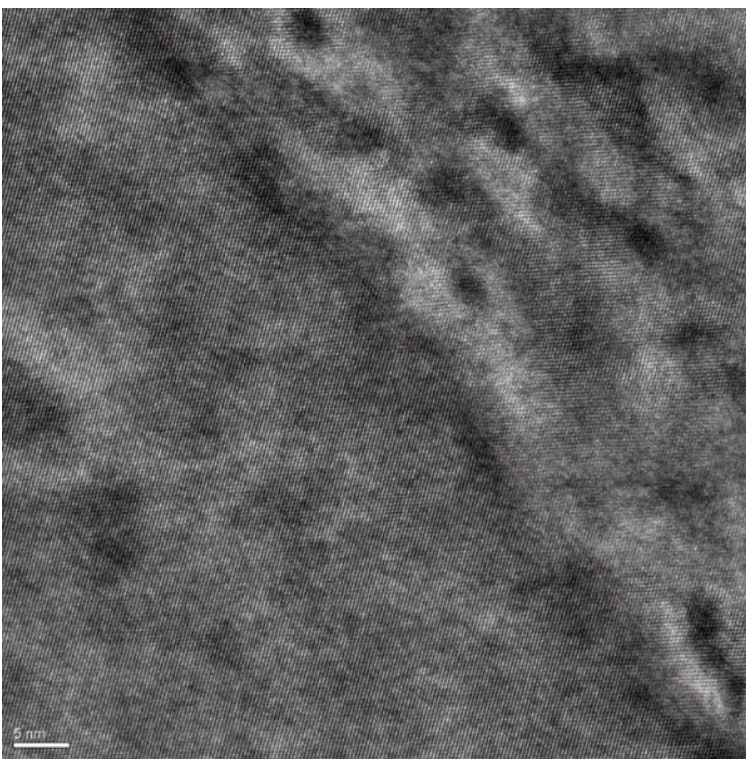

Figure 2- Transmission Electron Microscope (TEM) image of a highly enriched silicon film (upper right) grown on a natural abundance Si(100) substrate (lower left).

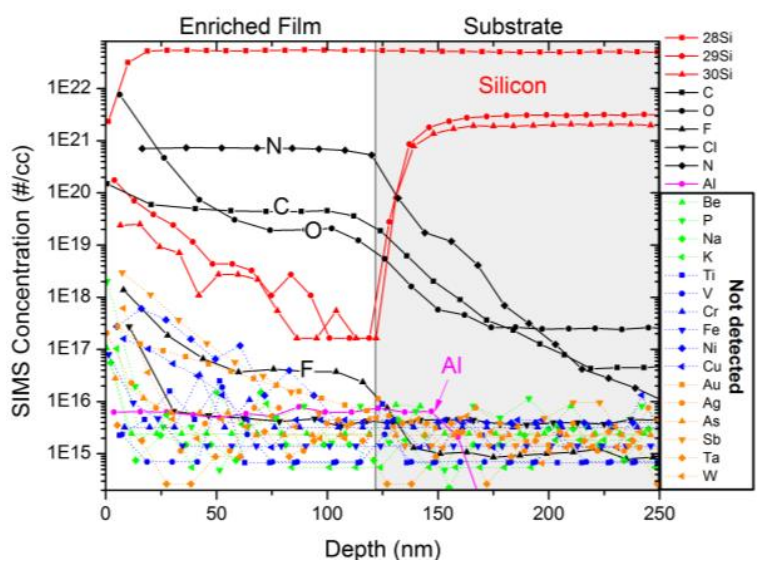

Figure 3-SIMS measurements for a wide range of target contaminants. The dominant elements found are from incorporated light gasses. highly enriched silicon to directly correlate the coherence properties with the materials and electrical properties independently measured.

\section{References}

1. Steger, M., et al., Quantum Information Storage for over 180 s Using Donor Spins in a Si-28 "Semiconductor Vacuum". Science, 2012. 336(6086): p. 1280-1283.

2. $\quad$ Pomeroy, J.M., et al., Hyperthermal ion beam system optimized for studying the effects of kinetic energy on thin-film growth. Review of Scientific Instruments, 2002. 73(11): p. 3846-3852.

3. Dwyer, K.J., et al., Enriching 28 Si beyond $99.9998 \%$ for semiconductor quantum computing. Journal of Physics D: Applied Physics, 2014. 47(34): p. 345105. 


\title{
Assessing Scanning Electron Microscopy Stereophotogrammetry Algorithms with Virtual Test Samples
}

\author{
John S. Villarrubia, Vipin N. Tondare, and András E. Vladár \\ Engineering Physics Division, Physical Measurement Laboratory, NIST, Gaithersburg, MD 20899-8212, USA ${ }^{\dagger}$
}

\section{INTRODUCTION}

Planar memory and logic devices have always had functional dependences on in-plane dimensions of their structures, but non-planar devices have added another dependence: on vertical dimensions. For example, the size of the conduction gate channel of a FinFET (a field effect transistor with raised channels, called fins, between source and drain) depends on the height of the fin. Optical and electron microscopy images have only two spatial dimensionsin the lateral plane. Their third dimension is an intensity. However, images from different viewing angles may be combined. Features extended along the insensitive vertical axis in one image will have a component in the sensitive lateral plane in one or more of the others. This permits in principle reconstruction of the 3D shape via stereophotogrammetry. Application of stereophotogrammetry to scanning electron microscopy (SEM) was described by Piazzesi in $1973 .^{1}$

Since SEMs now have spatial resolution near $1 \mathrm{~nm}$, the question naturally arises whether SEM-based stereo methods are sufficiently accurate for 3D nanometrology needs. Apart from the usual question of measurement errors that affect the inputs (e.g., the SEM images and the coordinates and angular viewpoints assigned to them) and how these errors then propagate to the result, there is the question to which we here address ourselves: whether important errors result from assumptions and approximations within the reconstruction software itself. For example, reconstruction of the position of a point $\mathrm{A}$ on the sample generally requires identifying its corresponding homologous image points $A, A^{\prime}$, etc., in two or more different views. Identification may be based on similarity of appearance as determined by correlation, but this is an approximation since appearance changes partly due to electron beam/sample interaction effects for which the reconstruction software does not account. Filtering may also be used to reduce errors caused by noise in the images. Filtering errors will also propagate to some extent into the reconstruc-

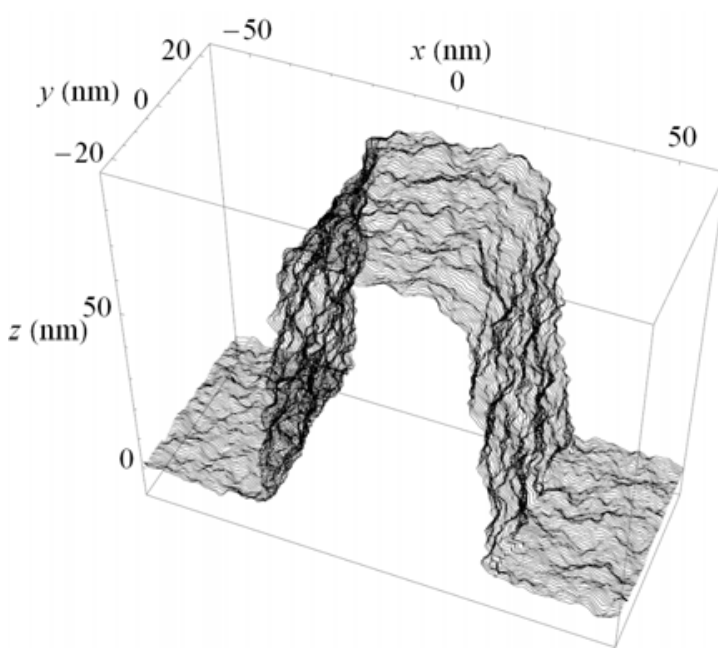

FIGURE 1. Line drawing of the roughest of our 3 virtual samples. The line is oriented to show the right edge. The left edge is obscured by absence of hidden line removal. tion. Because of effects like these, even in the ideal case of error-free inputs, we might expect reconstruction errors. Also, of course, different strategies that might be selected by the algorithm developer to handle these or other issues may differ in their effectiveness.

†. Contributions of the National Institute of Standards and Technology are not subject to copyright in the United States. 

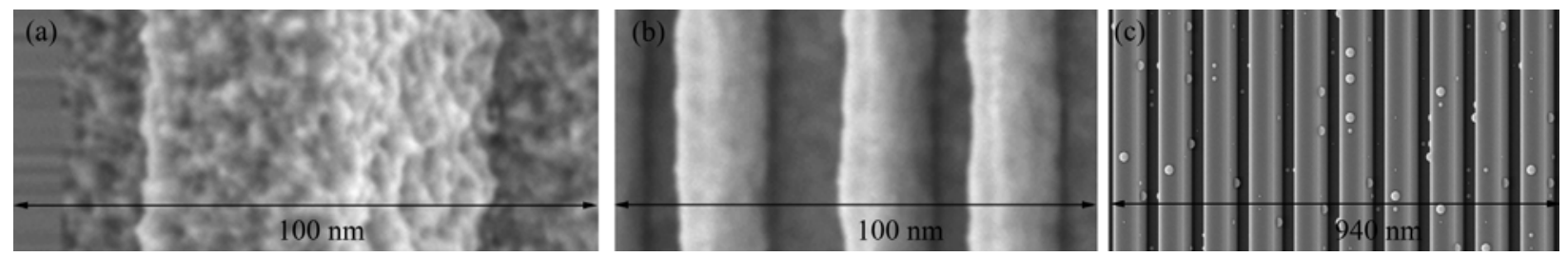

FIGURE 2. Simulated SEM images of 3 virtual samples. The samples were imaged with tilts at $5^{\circ}$ increments from $-85^{\circ}$ to $85^{\circ}$ from the normal (axis of rotation along the lines). The views shown here are at $20^{\circ}$.

Developers of most algorithms can test them by applying them to test problems with known answers. We construct such problems by starting with virtual samples. A virtual sample is a mathematical object, which is completely known. The virtual samples are "imaged" at varying tilt angles with an SEM simulator. These images are then input to commercially available stereo SEM reconstruction software, and the software's output is compared to the known virtual sample to assess errors.

\section{PROCEDURES}

We produce images of the virtual samples with the JMONSEL simulator. ${ }^{2}$ The simulator employs models of electron elastic scattering, secondary electron generation, and scattering at boundaries to compute electron yield vs. position, capabilities that have been used for model-based metrology that agrees with transmission electron microscopy and critical dimensions small angle x-ray scattering measurements to better than $1 \mathrm{~nm}^{2}$

We report results here for three virtual samples with height, $h$, width at mid-height, $w_{\text {mid }}$, sidewall angle, $\theta$, and top corner radii, $r$. One was a near-trapezoidal line $\left(h=80 \mathrm{~nm}, w_{\text {mid }}=50 \mathrm{~nm}, \theta=3^{\circ}, r=10 \mathrm{~nm}\right)$ around which a rough skin was wrapped. The sample is shown in Fig. 1 and its image in Fig. 2a. It has $1 \mathrm{~nm}$ root mean square (RMS) roughness and a $15 \mathrm{~nm}$ correlation length. Some results from this sample were given in an earlier report. ${ }^{3}$ The second sample (image in Fig. 2 b) had the same RMS roughness and similar design but with a longer roughness correlation length $(30 \mathrm{~nm})$ and multiple smaller lines $\left(h=30 \mathrm{~nm}, w_{\text {mid }}=10 \mathrm{~nm}, \theta=2^{\circ}\right.$, $r=2 \mathrm{~nm}$ ) separated alternately by $18 \mathrm{~nm}$ - and $26 \mathrm{~nm}$-wide trenches. The final sample (image in Fig. 2c) was the smoothest. It consisted of an array of 10 lines $\left(h=60 \mathrm{~nm}, w_{\text {mid }}=60 \mathrm{~nm}, \theta=3^{\circ}, r=10 \mathrm{~nm}\right)$ on $100 \mathrm{~nm}$ pitch. The sample was decorated with hemispherical bumps on the trench floor, line tops, and line sides.

The images along with the tilt angles are input to the reconstruction software, which produces a number of different outputs, among them a profile across the sample.

\section{RESULTS}

Profiles across the middle of the Fig. 2a sample are shown in Fig. 3. The rough black line is the true profile. The smoother green and blue ones were outputs of two commercial software packages. For this very rough structure, the two packages made errors of $1.4 \mathrm{~nm}$ or less in the average height and less than $1 \mathrm{~nm}$ for the average width. These errors are small enough that we can not confidently attribute them to reconstruction error. They could be due to modeling or sampling errors (the latter because the software did not

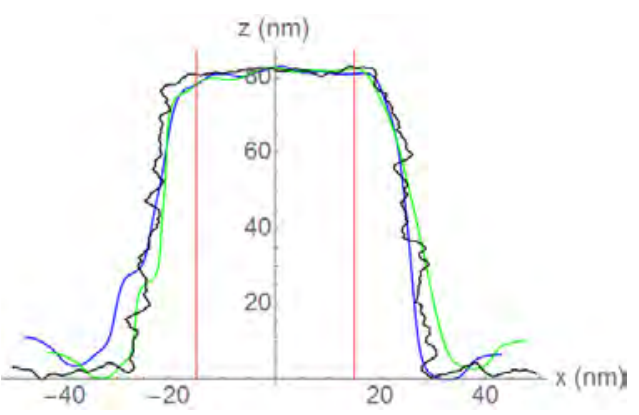

FIGURE 3. Profiles across the Fig. 1 sample from two different commercial software packages (the smoother blue and green lines) superimposed on the true profile (rougher black line). reconstruct the feature all the way to the edge of the image, so sampled less than the full profile). As we mentioned, the reconstructed profiles are obviously smoother than the true profile. This means the reconstruction is not useful for assessing surface roughness of this sample.

The Fig. 2a sample was the roughest of the three. At the other extreme, the Fig. 2c sample has line tops and trench floors that are completely flat planes except for a sprinkling of hemispherical bumps. These proved difficult for all 
the tested algorithms. Even the best of the reconstructions had the average line almost $40 \%$ shorter than its true $60 \mathrm{~nm}$ height, and although the lines are-apart from the hemispherical markers—all the same height, the reconstructed heights varied with standard deviation of more than $8 \mathrm{~nm}$. Interesting in this context is that we could determine the height from the input images along a slice through one of the small hemispheres with much smaller error than this $(\sim 1 \mathrm{~nm})$ by using some simple geometry and a calculator. The line shapes in this case were also strongly distorted and about $10 \%$ too narrow. The sample with intermediate roughness (Fig. 2b) also suffered from significant errors on reconstruction, with heights smaller by $4 \mathrm{~nm}$ in the wider trench and $8.7 \mathrm{~nm}$ in the narrower trench than the true value of $30 \mathrm{~nm}$. On this sample, six pairs of homologous points identified by eye on line tops and in the trenches produced reconstructed heights that averaged only $2.5 \mathrm{~nm}$ too low with standard deviation $0.4 \mathrm{~nm}$.

\section{DISCUSSION AND CONCLUSION}

Roughness serves a useful function for stereo reconstruction algorithms. It provides a non-periodic surface texture that facilitates identification of homologous points in images from different viewing angles. Two of the samples we examined represent extremes relative to the amount of roughness one would ordinarily encounter in integrated circuit production. The sample of Fig. 1 and Fig. 2a is very rough whereas that of Fig. 2c is very smooth. The best of the tested commercial software algorithms reconstructed the very rough sample with height and width errors of about $1 \mathrm{~nm}$. (This excludes errors due to vibration, noise, angular positioning, etc., for which the reconstruction algorithm bears no responsibility. In a real measurement, these other errors would of course be additional.) Errors on the very smooth sample, on the other hand, were a significant fraction of feature size. The disparate performance at these two extremes motivated a test with a sample (Fig. 2b) intermediate between the two, with roughness bearing a greater resemblance to that expected in practice. Errors in this case were again a significant fraction of feature size, e.g., height errors of $13 \%$ to $29 \%$ of the true height.

Manual line height determinations using simple geometry and homologous points identified by eye outperformed the automated algorithms on the smooth and moderately rough samples. This demonstrates that the larger errors are not inherent in these kinds of samples, but must rather be due to less than full use of available information by the tested algorithms. That is, a different algorithm design could perform better. Absence of test problems with known solutions has heretofore been an obstacle to algorithm development. Test problems based on simulated images of samples with known shape can be useful in this respect.

\section{REFERENCES}

1. G. Piazzesi, “Photogrammetry with the scanning electron microscope,” J. Phys. E: Sci. Instrum. 6, $392-397$ (1973).

2. J. S. Villarrubia, A. E. Vladár, B. Ming, R. J. Kline, D. F. Sunday, J. S. Chawla, and S. List, "Scanning electron microscope measurement of width and shape of $10 \mathrm{~nm}$ patterned lines using a JMONSEL-modeled library,” Ultramicroscopy 154, 15-28 (2015), http://dx.doi.org/10.1016/j.ultramic.2015.01.004

3. J. S. Villarrubia, V. N. Tondare, and A. E. Vladár, "Virtual rough samples to test 3D nanometer-scale scanning electron microscopy stereo photogrammetry,” Proc. SPIE 9778, 977809-1 (2016).

\section{KEYWORDS}

Monte Carlo SEM modeling, nanometer-scale dimensional metrology, scanning electron microscopy, stereophotogrammetry

Tondare, Vipin; Villarrubia, John; Vladar, Andras.

"Assessing Scanning Electron Microscopy Stereophotogrammetry Algorithms with Virtual Test Samples."

Paper presented at 2017 International Conference on Frontiers of Characterization and Metrology for Nanoelectronics,

Monterey, CA. March 21, 2017 - March 23, 2017. 


\title{
Comparison of specular gloss values from a spectrally resolved five-axis goniometer and a reference goniophotometer
}

\author{
Renée Charrière ${ }^{1,2}$ and Maria Nadal ${ }^{1}$ and Clarence Zarobila ${ }^{1}{ }^{1}{ }^{1}$ National Institute of Standards and Technology; Gaithersburg, MD, \\ United States; ${ }^{2}$ Ecole Nationale Supérieure des Mines de Saint-Etienne, SMS EMSE, CNRS UMR5307, Laboratoire Georges Friedel; \\ Saint-Etienne, France
}

\begin{abstract}
The present work consists in developing a procedure which will allow comparison of gloss values obtained from angularly and spectrally resolved Bidirectional Reflectance Distribution Function (BRDF) measurements and gloss values measured with a national reference goniophotometer. The gloss measurements will be performed on the Reference Goniophotometer for Specular Gloss Calibration available at the Sensor Science Division of the National Institute of Standards and Technology (NIST), whereas the BRDF measurements will be conducted on the Five-Axis Goniometer for Color \& Appearance also available at the same division of NIST.
\end{abstract}

\section{Introduction}

The appearance of a product is important for many industries, for example automotive, cosmetics, paper, printing, packaging, coatings, plastics, steel industries, etc., as this is frequently one of the most critical parameters affecting customer decision. Gloss is the second most relevant visual attribute of a surface, second to color. While the perceived color of an object originates from the wavelength distribution of the reflected light, gloss originates from its angular distribution.

A specular glossmeter measures the flux reflected from a specimen in comparison to the flux reflected from a standard under the same standardized measurement conditions. The theoretical primary standard is specified to be a perfect polished material with refractive index of 1.567 at $589.3 \mathrm{~nm}$ [1]. In 1939, ASTM adopted the $60^{\circ}$ geometry in a standard method, i.e., ASTM method D523 [2]. Two additional measurement procedures were incorporated into ASTM D523 in 1951, with the addition of a $20^{\circ}$ geometry test method for the evaluation of high gloss finishes and an $85^{\circ}$ geometry test method for the evaluation of low gloss surfaces. Although specular glossmeters have been used in industry for decades, their limited capabilities have been recognized for a long time. The current specular gloss measurement standards fail to distinguish between type of materials or degree of specular gloss [3]. For example, Vienot \& Obein compared the gloss of a piece of tire and of a black inked paper [4]. Though both samples reflected almost the same level of lux and have similar specular gloss values, the difference in visual gloss was obvious and all the observers were able not only to correctly rank the gloss, but also to identify the material of which the sample was made of. A study of the angular distribution of the reflected light revealed that the shape of the specular peak was very different between the two samples and suggested that the visual system extracts information not only from the magnitude of the peak but also from the shape of the peak.
Two facilities available at the Sensor Science Division of the National Institute for Standards and Technology (NIST) were used in this work. These facilities are the Five-Axis Goniometer for Color \& Appearance [5] and the Reference Goniophotometer for Specular Gloss Calibration [6]. The Five-Axis Goniometer is currently used to measure the Bidirectional Reflectance Distribution Function (BRDF) of gonio-apparent materials, that is, materials that change their appearance as a function of illumination and viewing directions. The Reference Goniophotometer for Specular Gloss Calibration is an aging facility that provides the calibration of specular gloss standards at the specular geometries of $20^{\circ}, 60^{\circ}$ and $85^{\circ}$. The aim pursued here is to perform specular gloss measurements on the Five-Axis Goniometer. This will first allow decommissioning of the present gloss facility and eliminate the need for its upgrade and maintenance. This will also allow to perform angular resolved gloss measurements, which will give us the opportunity for new characterization features of glossy samples.

\section{Definition of gloss}

The specular gloss $G_{s p l e}\left(\theta_{0}\right)$ of a sample, illuminated with light around the direction $\theta_{0}$ from the normal of the sample surface is defined by :

$$
G_{s p l e}\left(\theta_{0}\right)=G_{p-s t d}\left(\theta_{0}\right) \frac{\rho_{V, s p l e}\left(\theta_{0}\right)}{\rho_{V, p-s t d}\left(\theta_{0}\right)} .
$$

$G_{p-s t d}\left(\theta_{0}\right)$ is the specular gloss of a primary standard. $\rho_{V, s p l e}\left(\theta_{0}\right)$ and $\rho_{V, p-s t d}\left(\theta_{0}\right)$ are respectively the specular luminous reflectance of the sample and the specular luminous reflectance of the primary standard.

\section{Definition of the specular luminous reflectance}

Let's now define the term "specular luminous reflectance". Let's consider a light source with a spectral power density $\varphi(\lambda)$ at the wavelength $\lambda$ measured in Watts $(\mathrm{W} / \mathrm{nm})$. The luminous flux in lumen ( $\mathrm{lm}$ ) related to $\Phi(\lambda)$ is defined by:

$$
\Phi_{V}=K_{M} \int_{\lambda} \varphi(\lambda) V_{\lambda}(\lambda) d \lambda
$$

where $K_{M}=683 \mathrm{~lm} / \mathrm{W}$ is the maximum spectral luminous efficacy for photopic vision and $V_{\lambda}(\lambda)$ is the Commission Internationale de l'Eclairage (CIE) 1924 photopic luminosity function. The specular luminous reflectance $\rho_{V}\left(\theta_{0}\right)$ of a surface is defined by the ratio:

$$
\rho_{V}\left(\theta_{0}\right)=\frac{\Phi_{V, r}\left(\theta_{0}\right)}{\Phi_{V, i}}
$$


$\Phi_{V, r}\left(\theta_{0}\right)$ is the luminous flux reflected from the surface integrated over all incidence and reflection directions. A direction is defined by two angles $\theta$ and $\phi . \theta$ and $\phi$ are respectively the polar and azimuthal angles in a spherical coordinate system with a zenith direction merged with the normal to the sample surface. Incidence directions are denoted with the subscript $i$ and observation directions with the subscript $r$. We have then:

$$
\Phi_{V, r}\left(\theta_{0}\right)=K_{M} \int_{S} \iint_{\theta_{i}, \phi_{i}} \iint_{\theta_{r}, \phi_{r}} \int_{\lambda} L_{i}\left(\theta_{i}, \phi_{i}, \lambda\right) B R D F\left(\theta_{i}, \phi_{i}, \theta_{r}, \phi_{r}, \lambda\right) V_{\lambda}(\lambda) \cos \left(\theta_{i}\right) \sin \left(\theta_{i}\right) \cos \left(\theta_{r}\right) \sin \left(\theta_{r}\right) d \lambda d \theta_{i} d \phi_{i} d \theta_{r} d \phi_{r} d S,
$$

where $\operatorname{BRDF}\left(\theta_{i}, \phi_{i}, \theta_{r}, \phi_{r}, \lambda\right)$ is the spectral BRDF of the surface and $L_{i}\left(\theta_{i}, \phi_{i}, \lambda\right)$ is the spectral radiance of the light illuminating the surface. $S$ is the illuminated area of the surface. The dependency in $\theta_{0}$ is not explicit in the right part of equation 4 but affects $L_{i}\left(\theta_{i}, \phi_{i}, \lambda\right)$, the domain of integration of the angles $\left(\theta_{r}, \phi_{r}\right)$ and the surface $S$. The specular luminous reflectance is indeed measured in a specular geometry. $L_{i}\left(\theta_{i}, \phi_{i}, \lambda\right)$ will thus be a peakshape function centered around the direction $\theta_{0}$ from the normal of the sample surface. The illuminated surface $S$ will be an elliptical surface with its major axis proportional to $\frac{1}{\cos \left(\theta_{0}\right)}$. The domain of integration of the angles $\left(\theta_{r}, \phi_{r}\right)$ will be centered around the direction of the specular reflection on the sample, that is in a direction symmetrical to the incident light direction relatively to the sample surface normal.

For a perfectly flat material with an homogeneous refractive index $n(\lambda)$, the BRDF simplifies to [7]:

$$
\operatorname{BRDF}\left(\theta_{i}, \phi_{i}, \theta_{r}, \phi_{r}, \lambda\right)=\frac{\rho_{F R}\left(\theta_{i}, n(\lambda)\right)}{\cos \left(\theta_{r}\right) \sin \left(\theta_{r}\right)} \delta\left(\theta_{i}-\theta_{r}\right) \delta\left(\phi_{i}-\phi_{r} \pm \pi\right),
$$

where $\rho_{F R}\left(\theta_{i}, n(\lambda)\right)$ is the Fresnel reflection coefficient for an incidence angle $\theta_{i}$ and a refractive index $n(\lambda)$. Gloss values are ideally measured with non-polarized light, so the Fresnel reflection coefficient to be considered here is for non-polarized light. $x \rightarrow \delta(x)$ is the Dirac delta function. In that case, the luminous reflected flux by the surface becomes:

$$
\Phi_{V, r}\left(\theta_{0}\right)=K_{M} \iiint \int_{S, \theta_{i}, \phi_{i}, \lambda} L_{i}\left(\theta_{i}, \phi_{i}, \lambda\right) \rho_{F R}\left(\theta_{i}, n(\lambda)\right) V_{\lambda}(\lambda) \cos \left(\theta_{i}\right) \sin \left(\theta_{i}\right) d \lambda d \theta_{i} d \phi_{i} d S .
$$

$\Phi_{V, i}$ is the luminous flux incident on the surface:

$$
\Phi_{V, i}=K_{M} \int_{\lambda} \varphi_{i}(\lambda) V_{\lambda}(\lambda) d \lambda
$$

with $\varphi_{i}(\lambda)$ being the spectral power density of the light incident on the surface. Note that we have the following relationship:

$$
\iiint_{S, \theta_{i}, \phi_{i}} L_{i}\left(\theta_{i}, \phi_{i}, \lambda\right) \cos \left(\theta_{i}\right) \sin \left(\theta_{i}\right) d \theta_{i} d \phi_{i} d S=\varphi_{i}(\lambda) .
$$

ASTM D523 precisely specifies the geometries through which the luminous reflectance has to be measured. The angular field apertures for the source and the receiver in the incidence plane (respectively $\Theta_{i}^{I P}$ and $\Theta_{r}^{I P}$ ) and perpendicular to the incidence plane (resp. $\Theta_{i}^{P I P}$ and $\Theta_{r}^{P I P}$ ) are defined, according to figure 1 , in a plane which is conjugated with the "real" source field aperture through the source and receiver lenses. Note that the definition presented in figure 1 is valid only for so called "parallel beam glossmeters", that is glossmeters where the beam incident on the sample would be perfectly collimated in case of a point light source. The values of the angles $\Theta_{i}^{I P}, \Theta_{i}^{P I P}, \Theta_{r}^{I P}$ and $\Theta_{r}^{P I P}$ for the three standards incidence angles $20^{\circ}, 60^{\circ}$ and $85^{\circ}$ and the corresponding tolerances are given in table 1 . The tolerance on the angular values $20^{\circ}, 60^{\circ}$ and $85^{\circ}$ is $0.1^{\circ}$.

Table 1. ASTM D523 source image and receiver angular apertures specifications and tolerances.

\begin{tabular}{|c|c|c|}
\hline Apertures & $\begin{array}{c}\text { Parallel to the } \\
\text { plane of } \\
\text { incidence }\left[{ }^{\circ}\right]\end{array}$ & $\begin{array}{c}\text { Perpendicular to } \\
\text { the plane of } \\
\text { incidence }\left[{ }^{\circ}\right]\end{array}$ \\
\hline & $2 \Theta_{i}^{I P}$ & $2 \Theta_{i}^{P I P}$ \\
\hline Source image & $0.75 \pm 0.25$ & $2.5 \pm 0.5$ \\
\hline $20^{\circ}$ receiver & $2.8 \pm 0.05$ & $2 \Theta_{r}^{I P}$ \\
\hline $60^{\circ}$ receiver & $4.4 \pm 0.1$ & $3.6 \pm 0.1$ \\
\hline $85^{\circ}$ receiver & $4 \pm 0.3$ & $6 \pm 0.3$ \\
\hline
\end{tabular}

ASTM D523 specifies also that "the obtained results should not differ significantly from those obtained with a source-filter combination that is spectrally corrected to yield CIE luminous efficiency with CIE source C"'. That is, by referring to our previous notations, we should obtain the same results as if $\varphi_{i}(\lambda)$ were proportional to the spectral power density $C(\lambda)$ of the CIE illuminant C.

\section{Definition of the primary standard specular gloss value}

Gloss values are calculated relatively to a reference standards. The theoretical reference standard for gloss measurements is specified to be a highly polished plane black glass with an index of refraction at the wavelength of the sodium D line $\left(\lambda_{D}=589.3\right.$ $\mathrm{nm})$ equal to $n_{t h-s t d}\left(\lambda_{D}\right)=1.567$. The gloss value for this theoretical standard is set to 100 for the three standard specular geometries $20^{\circ}, 60^{\circ}$ and $85^{\circ}$. Primary standards are used since these specifications are not found in real materials. The gloss value $G_{p-s t d}\left(\theta_{0}\right)$ of the primary standard illuminated with light around the direction $\theta_{0}$ from the normal of its surface is defined by the following equation:

$$
G_{p-s t d}\left(\theta_{0}\right)=100 \frac{\rho_{F R}\left(\theta_{0}, n_{p-s t d}\left(\lambda_{D}\right)\right)}{\rho_{F R}\left(\theta_{0}, n_{t h-s t d}\left(\lambda_{D}\right)\right)},
$$

where $n_{t h-s t d}\left(\lambda_{D}\right)$ is the refractive index of the theoretical standard at the wavelength $\lambda_{D}$.

Note that an alternative definition for $G_{p-s t d}\left(\theta_{0}\right)$ can be used, by replacing the Fresnel reflection coefficient $\rho_{F R}\left(\theta_{0}, n_{p-s t d}\left(\lambda_{D}\right)\right)$ by the measured specular luminous reflectance of the primary standard. 


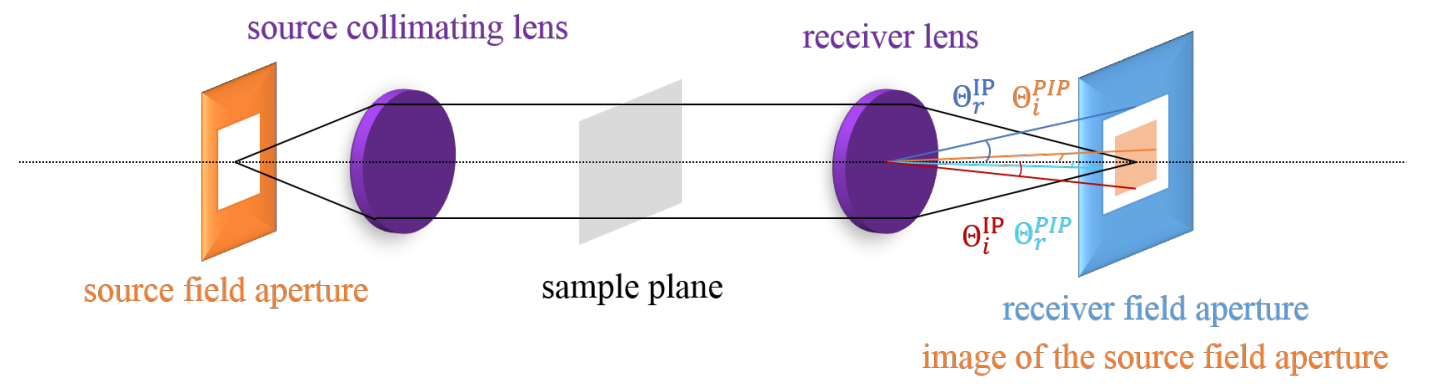

Figure 1. Diagram of the optical system of a parallel beam glossmeter showing the definitions of the angular source ( $\left.\theta_{s}\right)$ and receiver $\left(\theta_{r}\right)$ field apertures.

\section{Proposed Goniometer modifications}

A detailed description of the original optical system for the Five-Axis Goniometer is given in reference [5]. Figure 2 shows the proposed optical layout on the Five Axis Goniometer in order to be able to measure specular gloss according to ASTM D523. A high power broadband Laser Driven Light Source (LDLS) was chosen as the illuminating source making it possible to measure low gloss samples. Broadband white light is generated by a continuous laser focused in a xenon plasma. The white light is then collected through an elliptical reflector. This reflector has a hole in order to allow the continuous laser to reach the plasma. Consequently the cross-section of the beam generated by the LDLS source has a doughnut shape in the far field, even after a multimode $910 \mu \mathrm{m}$ fiber. A set of two microlens arrays in imaging configuration is added on the beam path in order to improve the homogeneity of the beam cross-section.

The conjugation ratio for the source aperture is equal to 1 as it is imaged in the receiver field aperture plane through two 150 $\mathrm{mm}$ focal length lenses in $f-\infty-f$ configuration. In order to follow ASTM D523 angular specifications (see table 1), the source and receiver apertures are rectangular, with angular dimensions specified in table 2 .

Table 2. Source and receiver apertures dimensions. Each dimension is equal to the focal length of the receiver lens $(150 \mathrm{~mm})$ multiplied by the tangent of the angular value specified in table 1 .

\begin{tabular}{|c|c|c|}
\hline Apertures & $\begin{array}{c}\text { Parallel to the } \\
\text { plane of } \\
\text { incidence }\end{array}$ & $\begin{array}{c}\text { Perpendicular to } \\
\text { the plane of } \\
\text { incidence }\end{array}$ \\
\hline Source & $1.96 \mathrm{~mm}$ & $6.55 \mathrm{~mm}$ \\
\hline $20^{\circ}$ receiver & $4.71 \mathrm{~mm}$ & $9.43 \mathrm{~mm}$ \\
\hline $60^{\circ}$ receiver & $11.53 \mathrm{~mm}$ & $30.74 \mathrm{~mm}$ \\
\hline $85^{\circ}$ receiver & $10.48 \mathrm{~mm}$ & $15.72 \mathrm{~mm}$ \\
\hline
\end{tabular}

A beam-splitter and a fiber-coupled spectrometer will be added in the beam path to correct for power fluctuations of the illuminating beam. The beam reflected by the sample will go through the receiver aperture into an integrating sphere. A fiber used at one port of the integrating sphere brings the beam into a spectrometer.

\section{Experimental computation of the gloss value}

The gloss value is measured by simultaneously acquiring the monitor beam and the spectrum given by the receiver spectrometer for both the test sample and the primary standard. Let's denote respectively by $\mathscr{S}_{m}^{\text {sple }}(\lambda)$ and $\mathscr{S}_{r}^{\text {sple }}(\lambda)$ the spectra given by the monitor and the receiver spectrometers when measuring the sample and by $\mathscr{S}_{m}^{p-s t d}(\lambda)$ and $\mathscr{S}_{r}^{p-s t d}(\lambda)$ the spectra given by the monitor and the receiver spectrometers when measuring the primary standard.

Therefore, for the monitor signal:

$$
\mathscr{S}_{m}^{x}(\lambda)=\mathscr{R}_{m s p}(\lambda) T_{m f}(\lambda) T_{L 60}(\lambda) R_{G P}(\lambda) \phi_{i}^{x}(\lambda)
$$

where $x$ superscript is either sple or $p-s t d . \mathscr{R}_{m s p}(\lambda)$ is the spectral responsivity of the monitor spectrometer in counts/W, $T_{m f}(\lambda)$ is the spectral transmittance of the fiber bringing the light into the monitor spectrometer, $T_{L 60}(\lambda)$ is the spectral transmittance of the $60 \mathrm{~mm}$ focal lens achromat injecting the light into the monitor fiber and $R_{G P}(\lambda)$ is the spectral reflectance of the glass plate extracting a part of the beam incident on the sample into the monitor arm (see figure 2). $\phi_{i}^{x}(\lambda)$ is the spectral power density of the light incident either on the sample or on the primary standard. Note that without any temporal fluctuations of the light source, we should have $\phi_{i}^{\text {sple }}(\lambda)=\phi_{i}^{p-s t d}(\lambda)$ and consequently $\mathscr{S}_{m}^{\text {sple }}(\lambda)=\mathscr{S}_{m}^{p-s t d}(\lambda)$. Equation 10 neither takes into account potential non-linearity of the spectrometer responsivity nor potential wavelength shift due to non-perfect calibration of the spectrometer.

For the receiver spectrum of the test sample, we have:

$\mathscr{S}_{r}^{\text {sple }}(\lambda)=\mathscr{R}_{r s p}(\lambda) T_{r f}(\lambda) \mathscr{R}_{i s}(\lambda) T_{L 150}(\lambda) T_{G P}(\lambda) \int_{S} \iiint \int_{\theta_{i}, \phi_{i}, \theta_{r}, \phi_{r}} L_{i}^{s p l e}\left(\theta_{i}, \phi_{i}, \lambda\right) B R D F^{s p l e}\left(\theta_{i}, \phi_{i}, \theta_{r}, \phi_{r}, \lambda\right) \cos \left(\theta_{i}\right) \sin \left(\theta_{i}\right) \cos \left(\theta_{r}\right) \sin \left(\theta_{r}\right) d \theta_{i} d \phi_{i} d \theta_{r} d \phi_{r} d S$

$\mathscr{R}_{r s p}(\lambda)$ is the spectral responsivity of the receiver spectrometer in counts/W, $T_{r f}(\lambda)$ is the spectral transmittance of the fiber bringing the light into the receiver spectrometer, $\mathscr{R}_{i s}(\lambda)$ is the spectral responsivity of the integrating sphere (this is a global factor including all geometrical considerations related to the integrating sphere and the injection of the light into the receiver fiber), $T_{L 150}(\lambda)$ is the spectral transmittance of the $150 \mathrm{~mm}$ focal lens achromat bringing the light reflected by the sample into the 


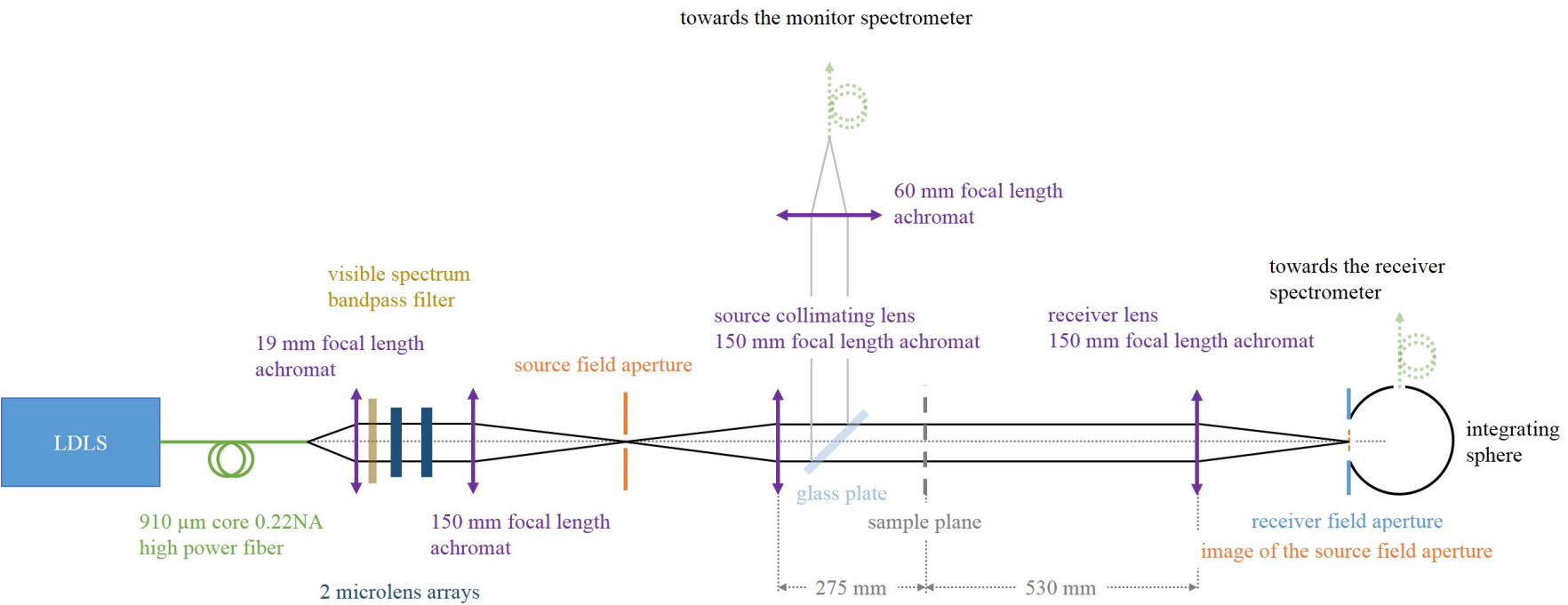

Figure 2. Diagram of the proposed optical system to measure gloss on the Five-Axis Goniometer. Note that distances are not to scale on the diagram. The distance between the two lenses surrounding the two microlens arrays is about $15 \mathrm{~mm}$ and the distance between the two microlens arrays is equal to the focal lens of the microlenses $(5.1 \mathrm{~mm})$.

integrating sphere and $T_{G P}(\lambda)$ is the spectral transmittance of the glass plate. All other notations are the same as in equation 4.

The equation for the receiver spectrum of the primary standard is more simple than for the test sample. The primary standard used at NIST is an optical quality barium crown glass BaK50 with an index of refraction at the wavelength $\lambda_{D}$ equal to $n_{p-s t d}\left(\lambda_{D}\right)=1.5677$ [6]. The front surface of the primary standard were polished to a roughness of $0.6 \mathrm{~nm}$. For this reason, the primary standard can be considered as a perfectly flat sample and it's BRDF can be simplified according to equation 5. We thus have:

$$
\mathscr{S}_{r}^{p-s t d}(\lambda)=\mathscr{R}_{r s p}(\lambda) T_{r f}(\lambda) \mathscr{R}_{i s}(\lambda) T_{L 150}(\lambda) T_{G P}(\lambda) \int_{S} \iint_{\theta_{i}, \phi_{i}} L_{i}^{p-s t d}\left(\theta_{i}, \phi_{i}, \lambda\right) \rho_{F R}\left(\theta_{i}, n_{p-s t d}(\lambda)\right) \cos \left(\theta_{i}\right) \sin \left(\theta_{i}\right) d \theta_{i} d \phi_{i} d S
$$

A direct measurement of both monitor and receiver spectra with the incident beam striking directly on the receiver arm (that is without any interaction with a sample) is necessary to get rid of the spectral responsivities of the various optical elements (lenses, glass plate, fibers, spectrometers, integrating sphere...) present on the path of the beam either in the monitor arm or in the receiver arm. Let's denote respectively by $\mathscr{S}_{m}^{0}(\lambda)$ and $\mathscr{S}_{r}^{0}(\lambda)$ the spectra given by the monitor and the receiver spectrometers when the incident beam is striking directly the receiver arm. We have:

$\left\{\begin{array}{l}\mathscr{S}_{m}^{0}(\lambda)=\mathscr{R}_{m s p}(\lambda) T_{m f}(\lambda) T_{L 60}(\lambda) R_{G P}(\lambda) \phi_{i}^{0}(\lambda) \\ \mathscr{S}_{r}^{0}(\lambda)=\mathscr{R}_{r s p}(\lambda) T_{r f}(\lambda) \mathscr{R}_{i s}(\lambda) T_{L 150}(\lambda) T_{G P}(\lambda) \phi_{i}^{0}(\lambda)\end{array}\right.$

where $\phi_{i}^{0}(\lambda)$ is the Power Spectral Density of the light incident on the sample plane. Note that as the monitor and the receiver signals are acquired at the same time, computing the ratio $\mathscr{Q}_{0}(\lambda)=\frac{\mathscr{S}_{r}^{0}(\lambda)}{\mathscr{S}_{m}^{0}(\lambda)}$ allows to get rid of the spectral responsivity of the various elements present on the beam paths excluding all source temporal fluctuations.

To compute the gloss value of the sample from the experimentally measured quantities, we first compute, as a function of wavelength, the following quantities:

$$
\left\{\begin{array}{l}
\frac{\mathscr{Q}_{\text {sple }}(\lambda)}{\mathscr{Q}_{0}(\lambda)}=\frac{\mathscr{S}_{r}^{\text {sple }}(\lambda)}{\mathscr{S}_{m}^{\text {sple }}(\lambda)} \frac{1}{\mathscr{Q}_{0}(\lambda)} \\
\frac{\mathscr{Q}_{p-s t d}(\lambda)}{\mathscr{Q}_{0}(\lambda)}=\frac{\mathscr{S}_{r}^{p-s t d}(\lambda)}{\mathscr{S}_{m}^{p-s t d}(\lambda)} \frac{1}{\mathscr{Q}_{0}(\lambda)}
\end{array} .\right.
$$

The choice has been made on our optical design neither to try to generate a spectral power density of the light incident on the sample close to the CIE illuminant $\mathrm{C}$ nor to simulate a $V_{\lambda}(\lambda)$ responsivity for the receiver. Custom made filters are quite expensive and don't allow any flexibility on the experimental setup. To determine the gloss value of the sample, the specular luminous reflectances of the sample and the primary standard are computed from the experimentally measured quantities. Computing the ratios $\mathscr{Q}_{\text {sple }}$ and $\mathscr{Q}_{p-s t d}$ allows to get rid of temporal fluctuations of the source between the measurements of the sample and the primary standard. These normalized quantities are independent of the spectral power density of the illuminating source. While neglecting all chromatic aberrations of the optical elements, angular and spectral variations of the radiance of the incident light can also be considered as uncoupled. We can thus write $L_{i}^{x}\left(\theta_{i}, \phi_{i}, \lambda\right)=\tilde{L}_{i}\left(\theta_{i}, \phi_{i}\right) l^{x}(\lambda)$, where $l^{x}(\lambda)$ includes spectral variations as well as potential temporal variations of the source. Then equation 14 becomes: 


$$
\left\{\begin{array}{l}
\frac{\mathscr{Q}_{\text {sple }}(\lambda)}{\mathscr{Q}_{0}(\lambda)}=\frac{\int_{S} \iiint_{\theta_{i}, \phi_{i}, \theta_{r}, \phi_{r}} \tilde{L}_{i}\left(\theta_{i}, \phi_{i}\right) B R D F^{s p l e}\left(\theta_{i}, \phi_{i}, \theta_{r}, \phi_{r}, \lambda\right) \cos \left(\theta_{i}\right) \sin \left(\theta_{i}\right) \cos \left(\theta_{r}\right) \sin \left(\theta_{r}\right) d \theta_{i} d \phi_{i} d \theta_{r} d \phi_{r} d S}{\int_{S} \iint_{\theta_{i}, \phi_{i}} \tilde{L}_{i}\left(\theta_{i}, \phi_{i}\right) \cos \left(\theta_{i}\right) \sin \left(\theta_{i}\right) d \theta_{i} d \phi_{i} d S} \\
\frac{\mathscr{Q}_{p-s t d}(\lambda)}{\mathscr{Q}_{0}(\lambda)}=\frac{\int_{S} \iint_{\theta_{i}, \phi_{i}} \tilde{L}_{i}\left(\theta_{i}, \phi_{i}\right) \rho_{F R}\left(\theta_{i}, n_{p-s t d}(\lambda)\right) \cos \left(\theta_{i}\right) \sin \left(\theta_{i}\right) d \theta_{i} d \phi_{i} d S}{\int_{S} \iint_{\theta_{i}, \phi_{i}} \tilde{L}_{i}\left(\theta_{i}, \phi_{i}\right) \cos \left(\theta_{i}\right) \sin \left(\theta_{i}\right) d \theta_{i} d \phi_{i} d S}
\end{array}\right.
$$

The gloss value of the sample is then computed by the following formula:

$$
G_{\text {sple }}^{\text {exp }}\left(\theta_{0}\right)=100 \frac{\rho_{F R}\left(\theta_{0}, n_{p-s t d}\left(\lambda_{D}\right)\right)}{\rho_{F R}\left(\theta_{0}, n_{t h-s t d}\left(\lambda_{D}\right)\right)} \frac{\sum_{\lambda_{i}} V_{\lambda}\left(\lambda_{i}\right) C\left(\lambda_{i}\right) \frac{\mathscr{Q}_{s p l e}\left(\lambda_{i}\right)}{\mathcal{L}_{0}\left(\lambda_{i}\right)}}{\sum_{\lambda_{i}} V_{\lambda}\left(\lambda_{i}\right) C\left(\lambda_{i}\right) \frac{\mathscr{Q}_{p-s t d}\left(\lambda_{i}\right)}{\mathscr{Q}_{0}\left(\lambda_{i}\right)}},
$$

The wavelengths $\lambda_{i}$ range is from $380 \mathrm{~nm}$ to $780 \mathrm{~nm}$ by increments of either $5 \mathrm{~nm}$ or $10 \mathrm{~nm}$.

\section{Comparison between BRDF and gloss mea- surements}

The BRDFs of two black glass samples with different gloss levels have been measured with the Five-Axis Goniometer for the visible spectrum $(380 \mathrm{~nm}$ to $780 \mathrm{~nm})$. Note that the BRDF measurements were made with the goniometer's original optical system [5], not the proposed modification shown in figure 2. These samples are denoted NIST-SG\#6 and NIST-SG\#7. The NIST BaK50 gloss primary standard has been also measured with the Five-Axis Goniometer to calibrate the response of the goniometer. The gloss values of these samples, measured with the Reference Goniophotometer for Specular Gloss Calibration are presented in table 3. Note that the uncertainties for the gloss measurements using the Reference Goniophotometer are in the order of $0.8 \%$ to $1.5 \%$ and the uncertainties for the gloss measurements using the Five-Axis Goniometer are in the order of $2 \%$ to $3 \%$.

Table 3. Gloss values measured with the Reference Goniophotometer for Specular Gloss Calibration of the black glass samples NIST-SG\#6 and NIST-SG\#7 and of the NIST BaK50 gloss primary standard at incidence angles of $20^{\circ}$ and $60^{\circ}$.

\begin{tabular}{|c|c|c|}
\hline Sample name & $\begin{array}{c}\text { Gloss value for } \\
\text { incidence angle } \\
20^{\circ}\end{array}$ & $\begin{array}{c}\text { Gloss value for } \\
\text { incidence angle } \\
60^{\circ}\end{array}$ \\
\hline NIST-SG\#6 & 70.8 & 91.2 \\
\hline NIST-SG\#7 & 8.9 & 48.2 \\
\hline BaK50 & 100.8 & 100.6 \\
\hline
\end{tabular}

For the work with the Five-Axis Goniometer, we will first focus on the wavelength of $500 \mathrm{~nm}$. Figure 3 represents BRDF measurements in the incidence plane at $20^{\circ}$ and $60^{\circ}$ incidence angles for the two black glass samples NIST-SG\#6 and NIST-SG\#7 as well as for the BaK50 NIST gloss primary standard. Both the Full Width at Half Maxiumum (FWHM) and the maximum value of the BRDF are linked to the gloss value of the sample (see table 4). As observed by Leloup et al. in reference [8], the width of the BRDF doesn't depend much on the incidence angle, whereas, in accordance with the Fresnel equations giving the reflectance factor of a perfectly flat and homogeneous surface, the maximum value of the BRDF increases with the incidence angle.

Table 4. BRDF maximum and FWHM for the samples NISTSG\#6, NIST-SG\#7 and the BaK50 NIST gloss primary standard at incidence angles of $20^{\circ}$ and $60^{\circ}$.

\begin{tabular}{|c|c|c|c|c|}
\hline $\begin{array}{c}\text { Sample } \\
\text { name }\end{array}$ & $\begin{array}{c}\text { BRDF } \\
\text { maximum } \\
\text { for } \\
\text { incidence } \\
\text { angle } 20^{\circ}\end{array}$ & $\begin{array}{c}\text { FWHM of } \\
\text { BRDF } \\
\text { peak for } \\
\text { incidence } \\
\text { angle } 20^{\circ}\end{array}$ & $\begin{array}{c}\text { BRDF } \\
\text { maximum } \\
\text { for } \\
\text { incidence } \\
\text { angle } 60^{\circ}\end{array}$ & $\begin{array}{c}\text { FWHM of } \\
\text { BRDF } \\
\text { peak for } \\
\text { incidence } \\
\text { angle } 60^{\circ}\end{array}$ \\
\hline $\begin{array}{c}\text { NIST- } \\
\text { SG\#6 }\end{array}$ & $22.9 \mathrm{sr}^{-1}$ & $2.7^{\circ}$ & $102.3 \mathrm{sr}^{-1}$ & $2.7^{\circ}$ \\
\hline $\begin{array}{c}\text { NIST- } \\
\text { SG\#7 }\end{array}$ & $2.4 \mathrm{sr}^{-1}$ & $6.8^{\circ}$ & $23.1 \mathrm{sr}^{-1}$ & $5^{\circ}$ \\
\hline BaK50 & $30.8 \mathrm{sr}^{-1}$ & $2.6^{\circ}$ & $125.7 \mathrm{sr}^{-1}$ & $2.5^{\circ}$ \\
\hline
\end{tabular}

The BaK50 primary standard has a highly polished surface and the shape of the curve given by the goniometer is not related to the sample surface properties but characterizes the response of the goniometer. Experimentally, the BRDF is computed on the Five-Axis Goniometer by the following formula [5]:

$$
\operatorname{BRDF}\left(\theta_{i}, \phi_{i}, \theta_{r}, \phi_{r}, \lambda\right)=\frac{\Phi_{R}\left(\theta_{i}, \phi_{i}, \theta_{r}, \phi_{r}, \lambda\right)}{\Phi_{i}(\lambda) \cos \left(\theta_{r}\right) \Omega},
$$

where $\Phi_{R}\left(\theta_{i}, \phi_{i}, \theta_{r}, \phi_{r}, \lambda\right)$ is the flux reflected by the sample collected by the receiver. $\Phi_{i}(\lambda)$ is the flux incident on the sample, measured when the incident beam is striking directly the receiver. $\Omega$ is the receiver collection solid angle. The Fresnel equations give us the values of the ratio $\frac{\Phi_{R}\left(\theta_{i}, \phi_{i}, \theta_{r}=\theta_{i}, \phi_{r}=\phi_{i}+180^{\circ}, \lambda\right)}{\Phi_{i}(\lambda)}$ from the refractive index values of the sample. Thus, in case when the shape of the experimental BRDF is limited by the goniometer response, multiplying the maximum value of the BRDF by $\cos \left(\theta_{r}\right) \Omega$ allows us to retrieve the Fresnel reflectance factor. The refractive index values of the BaK50 primary standard are given in reference [6]. For this sample, the values of the Fresnel reflectance factor and the product of the maximum value of the measured BRDF by $\cos \left(\theta_{r}\right) \Omega$ can thus be compared (see table 5 ). Note that the goniometer source is supposed to be perfectly nonpolarized and the Fresnel reflectance factors are thus computed for non-polarized light.

As observed on figure 3 and in table 4, the FWHM value of the BRDF of the NIST-SG\#6 sample is very close to that of the BaK50 standard, whereas the FWHM value of the BRDF of the NIST-SG\#7 sample is about twice as higher. The shape of the NIST-SG\#6 BRDF is thus mainly related to the goniometer response, whereas the shape of the NIST-SG\#7 BRDF is clearly influenced by the surface properties of the sample. In the case 

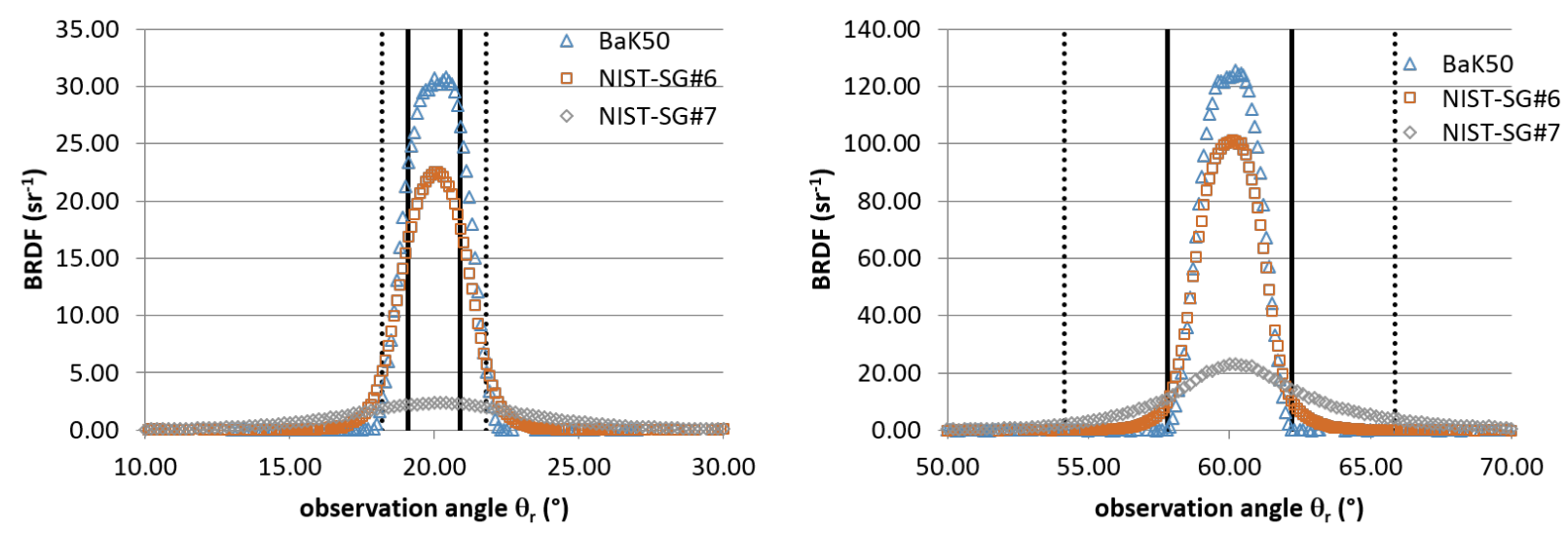

Figure 3. BRDF measurements in the incidence plane at $20^{\circ}$ (left) and $60^{\circ}$ (right) incidence angles of two black glass samples with different gloss levels for a wavelength of $500 \mathrm{~nm}$. The red curves represent the BRDFs of the sample NIST-SG\#6 and the gray curves the BRDFs of the sample NIST-SG\#7. The blue curves represent the measurements of the BaK50 NIST gloss primary standard. The black solid vertical lines delimit the ASTM D523 angular receiver aperture specifications in the incidence plane for $20^{\circ}$ and $60^{\circ}$ geometries. The black dashed vertical lines delimit the ASTM D523 angular receiver aperture specifications perpendicular to the incidence plane for $20^{\circ}$ and $60^{\circ}$ geometries.

Table 5. Products of the maximum value of the measured BRDFs by $\cos \left(\theta_{r}\right) \Omega$ at incidence angles of $20^{\circ}$ and $60^{\circ}$ for the samples NISTSG\#6, NIST-SG\#7 and the BaK50 NIST gloss primary standard. Are also indicated the non-polarized Fresnel reflectance factors computed from the BaK50 refractive index values.

\begin{tabular}{|c|c|c|}
\hline Sample name & $\begin{array}{c}B R D F_{\max } \cos \left(\theta_{r}\right) \Omega \\
\text { for incidence angle } \\
20^{\circ}\end{array}$ & $\begin{array}{c}B R D F_{\max } \cos \left(\theta_{r}\right) \Omega \\
\text { for incidence angle } \\
60^{\circ}\end{array}$ \\
\hline NIST-SG\#6 & 0.0381 & 0.0924 \\
\hline NIST-SG\#7 & 0.0021 & 0.0385 \\
\hline BaK50 & 0.0513 & 0.1135 \\
\hline BaK50 Fresnel & 0.0500 & 0.1012 \\
\hline
\end{tabular}

where the shape of the measured BRDF doesn't characterize the surface properties of the sample, the first equation of the equation array 15 has to be replaced by:

$$
\frac{\mathscr{Q}_{p-s t d}(\lambda)}{\mathscr{Q}_{0}(\lambda)}=\frac{\int_{S} \iint_{\theta_{i}, \phi_{i}} \tilde{L}_{i}\left(\theta_{i}, \phi_{i}\right) \rho_{F R}\left(\theta_{i}, n_{s p l e}(\lambda)\right) \cos \left(\theta_{i}\right) \sin \left(\theta_{i}\right) d \theta_{i} d \phi_{i} d S}{\int_{S} \iint_{\theta_{i}, \phi_{i}} \tilde{L}_{i}\left(\theta_{i}, \phi_{i}\right) \cos \left(\theta_{i}\right) \sin \left(\theta_{i}\right) d \theta_{i} d \phi_{i} d S},
$$

where $n_{\text {sple }}(\lambda)$ is the refractive index of the sample at the wavelength $\lambda$. By neglecting also the variations of the Fresnel reflectance factor of both the sample and the primary standard with $\theta_{i}$, the equation 16 can be then approximated by:

$G_{s p l e}^{\text {exp }}\left(\theta_{0}\right)=100 \frac{\rho_{F R}\left(\theta_{0}, n_{p-s t d}\left(\lambda_{D}\right)\right)}{\rho_{F R}\left(\theta_{0}, n_{t h-s t d}\left(\lambda_{D}\right)\right)} \frac{\sum_{\lambda_{i}} V_{\lambda}\left(\lambda_{i}\right) C\left(\lambda_{i}\right) \rho_{F R}\left(\theta_{0}, n_{s p l e}\left(\lambda_{i}\right)\right)}{\sum_{\lambda_{i}} V_{\lambda}\left(\lambda_{i}\right) C\left(\lambda_{i}\right) \rho_{F R}\left(\theta_{0}, n_{p-s t d}\left(\lambda_{i}\right)\right)}$.

Let first assume that, for both NIST-SG\#6 and NIST-SG\#7 samples, the previous approximation is valid and that the product of the maximum value of the BRDF by $\cos \left(\theta_{r}\right) \Omega$ gives us the Fesnel reflectance factor of the samples. We thus calculate the gloss value of these samples according to equation 19. The results are presented in table 6. For NIST-SG\#6 sample, the calculated gloss values compared well with the measured values listed in table 3 particularly for the incidence angle of $60^{\circ}$. For NIST-SG\#7 sample, we observe that the calculated $20^{\circ}$ gloss value is in good agreement with the value measured with the Reference Goniophotometer for Specular Gloss Calibration, but not the calculated value for the $60^{\circ}$ geometry. In the case where the shape of the BRDF is clearly limited by the angular resolution of the goniometer, gloss values computed from the maximum of the BRDF are in good agreement with the values measured with the Reference Goniophotometer for Specular Gloss Calibration. For the NISTSG\#7 sample a good agreement is obtained at $20^{\circ}$ that is in the case where the ASTM D523 angular receiver specifications include an area where the BRDF is nearly equal to is maximum value (see figure 3 ).

Table 6. Gloss values of NIST-SG\#6 and NIST-SG\#7 samples computed according to equation 19 assuming the maximum of the measured BRDF is directly related to the Fresnel reflectance factor of the sample.

\begin{tabular}{|c|c|c|}
\hline Sample name & $\begin{array}{c}\text { Gloss value } \\
\text { computed from } \\
\text { BRDF maximum for } \\
\text { incidence angle } 20^{\circ}\end{array}$ & $\begin{array}{c}\text { Gloss value } \\
\text { computed from } \\
\text { BRDF maximum for } \\
\text { incidence angle } 60^{\circ}\end{array}$ \\
\hline NIST-SG\#6 & 75.6 & 90.9 \\
\hline NIST-SG\#7 & 8.3 & 22.5 \\
\hline
\end{tabular}

To compute properly the gloss value of the NIST-SG\#7 sample from its BRDF, equations 15 and 16 need to be used. These equations require to know the variations of the BRDF with the five variables $\theta_{i}, \phi_{i}, \theta_{r}, \phi_{r}$ and $\lambda$. We will neglect the variations of the BRDF with $\theta_{i}$ and $\phi_{i}$. First BRDF measurements have been done only in the incidence plane. To compute the integration of the BRDF over the geometrical extent defined by the ASTM D523 receiver aperture specifications, the out of plane values of the BRDF have be numerically computed assuming a rotational symmetry 

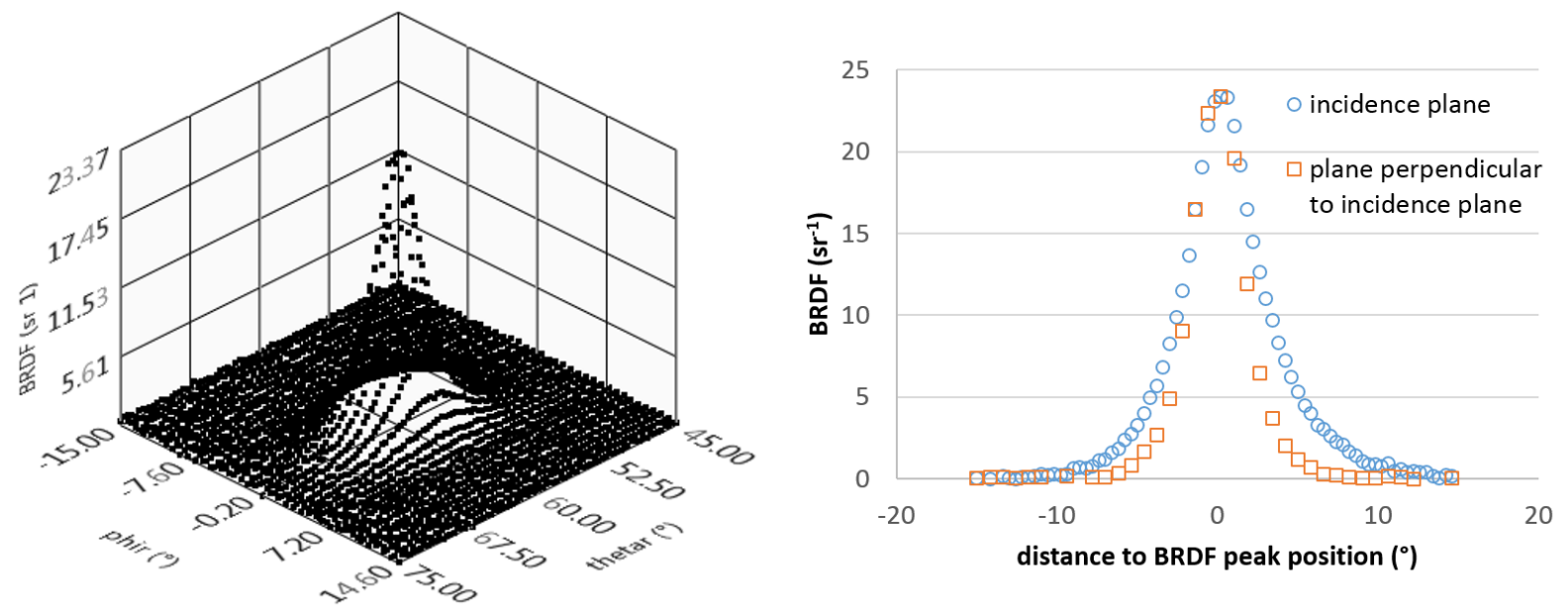

Figure 4. Three dimensional measurement of the BRDF of the NIST-SG\#7 sample (left) for an incidence angle of $60^{\circ}$. Comparison between the BRDF profiles of the NIST-SG\#7 sample extracted from the three dimensional measurement in the incidence plane and perpendicular to the incidence plane (right).

of the BRDF. Note that the integration domain is a rectangle in the $\left(\theta_{r}, \phi_{r}\right)$ plane with side lengths defined in table 1 . Table 7 shows the gloss values computed this way for both NIST-SG\#6 and NIST-SG\#7 samples. We observe that for the NIST-SG\#6 sample at the incidence angle of $60^{\circ}$ the gloss value is still close to the value measured with the Reference Goniophotometer for Specular Gloss Calibration (see table 3) whereas the gloss value for the incidence angle of $20^{\circ}$ is not the expected value. For the NIST-SG\#7 sample the gloss value at the incidence angle of $20^{\circ}$ is also not the expected value. Nevertheless the value at $60^{\circ}$ is closer to the expected value than the value computed from the BRDF maximum value (see table 6 ).

Table 7. Gloss values of NIST-SG\#6 and NIST-SG\#7 samples computed according to equations 15 and 16 assuming the shape of the measured BRDF characterizes the surface properties of the sample.

\begin{tabular}{|c|c|c|}
\hline Sample name & $\begin{array}{c}\text { Gloss value } \\
\text { computed from } \\
\text { BRDF integration } \\
\text { for incidence angle } \\
20^{\circ}\end{array}$ & $\begin{array}{c}\text { Gloss value } \\
\text { computed from } \\
\text { BRDF integration } \\
\text { for incidence angle } \\
60^{\circ}\end{array}$ \\
\hline NIST-SG\#6 & 18.1 & 89.0 \\
\hline NIST-SG\#7 & 3.0 & 61.8 \\
\hline
\end{tabular}

The gloss values listed on the table 7 have been computed by assuming a rotational symmetry of the BRDF. To study the influence of this assumption on the gloss value a three dimensional measurement of the BRDF of the NIST-SG\#7 sample for an incidence angle of $60^{\circ}$ have been performed (see figure 4). We observe that the profiles of the BRDF in the incidence plane and perpendicular to the incidence plane are slightly different. The gloss value computed from this three dimensional BRDF measurement is closer to the value measured with the Reference Goniophotometer for Specular Gloss Calibration than the value computed from in plane BRDF measurements and assuming a rotational symmetry of the BRDF (see tables 8 and 7).
Table 8. Comparison between the gloss value of NIST-SG\#7 sample at an incidence angle of $60^{\circ}$ computed according to equations 15 and 16 from a three dimensional BRDF measurement and its gloss value at the same incidence angle measured with the Reference Goniophotometer for Specular Gloss Calibration.

\begin{tabular}{|c|c|}
\hline $\begin{array}{c}\text { Gloss value } \\
\text { computed from 3D } \\
\text { BRDF } \\
\text { measurement }\end{array}$ & $\begin{array}{c}\text { Gloss value } \\
\text { measured with the } \\
\text { Reference } \\
\text { Goniophotometer } \\
\text { for Specular Gloss } \\
\text { Calibration }\end{array}$ \\
\hline 53.3 & 48.2 \\
\hline
\end{tabular}

\section{Conclusion and perspectives}

The preliminary results presented in this paper show that a good agreement can be obtained between gloss measurements performed on a device dedicated to gloss calibration and following ASTM D523 specifications (the Reference Goniophotometer for Specular Gloss Calibration) and BRDF measurements, particularly for high (around 90) or low (around 10) gloss values and in the case where the maximum value of BRDF is used to evaluate the Fresnel reflectance factor of the sample. The way to compute the gloss value from the BRDF is highly dependent on the relationship between the width of the angular device response and the angular response of the studied material. In the case where the shape of the measured BRDF if limited by the device response, the maximum value of the BRDF is linked to the gloss value of the sample. On the contrary, when the shape of the measured BRDF is linked to the surface properties of the material, an integration of the BRDF over the receiver geometrical extent defined by ASTM D523 need to be performed. In this case, computing the gloss value from a three dimensional BRDF measurement seems to give more accurate results. These results need to be confirmed by additional measurements on samples describing a more extended and detailed scale of gloss values. Intermediate cases where the shape of the BRDF is linked partially to the device response and partially to the sample response will certainly need to decorrelate both influences before computing the gloss value. 
We have also proposed modifications to the existing FiveAxis Goniometer that should enable its use as a direct replacement for the Goniometer for Specular Gloss Calibration. In the future, we plan to test these modifications and hopefully demonstrate the suitability of the modified instrument for providing gloss calibrations.

\section{References}

[1] W. Budde, "The Calibration of Gloss Reference Standards," Metrologia, vol. 16, pp. 89-93, 1980.

[2] “ASTM D523-14 Standard Test Method for Specular Gloss," ASTM International, 2014.

[3] F. B. Leloup, G. Obein, M. R. Pointer, and P. Hanselaer, "Toward the soft metrology of surface gloss: A review," Color Research and Application, vol. 39, no. 6, pp. 559-570, 2014.

[4] F. Vienot and G. Obein, "Is gloss recognized as a surface property?," in Proceedings of MS 2004, 1st International Workshop on Materials and Sensations, pp. 77-82, 2004.

[5] G. Obein, R. Bousquet, and M. E. Nadal, "New NIST reference goniospectrometer," in SPIE Proceedings, vol. 5880, International Society for Optics and Photonics, aug 2005.

[6] M. E. Nadal, E. A. Early, and E. A. Thompson, "Specular Gloss," NIST Special Publication, vol. SP250-70, 2006.

[7] T. A. Germer, J. C. Zwinkels, and B. K. Tsai, eds., Spectrophotometry: Accurate Measurement of Optical Properties of Materials. Academic Press, 2014.

[8] F. Leloup, P. . Hanselaer, J. Versluys, and S. Forment, "BRDF AND GLOSS MEASUREMENTS," in Proceedings of the CIE expert symposium on visual appearance, CIE (Commission International de l'Eclairage), 2006.

\section{Author Biography}

Renee Charriere has completed a PhD in Optics and Quantum Mechanics in 2011 in the French Aerospace Lab (Office National dEtudes et de Recherches Arospatiales) and Paris VI University. As postdoctoral fellow she worked on the optical characterization and modeling of nanostructured surfaces with complex visual appearance. She is now assistant professor at the French Laboratory Georges Friedel in Saint-Etienne and is currently guest researcher at National Institute of Standards and Technology in USA.

Charriere, Andree; Nadal, Maria; Zarobila, Clarence.

"Comparison of specular gloss values from a spectrally resolved five-axis goniometer and a reference goniophotometer." 


\title{
Time and frequency from electrical power lines
}

\author{
Jonathan E. Hardis, National Institute of Standards and Technology (NIST) \\ Blair Fonville and Demetrios Matsakis, U.S. Naval Observatory (USNO)
}

\section{BIOGRAPHIES}

Dr. Jonathan Hardis is a Senior Scientific Advisor within the Physical Measurement Laboratory at NIST. Among his current responsibilities, he serves as a liaison on positioning, navigation, and timing (PNT) issues. Previously, he worked in the NIST laboratories developing photometric measurement and consensus standards. Dr. Hardis received a Ph.D. in Physics from the University of Chicago and an S.B. in Physics from MIT. Email: jonathan.hardis@,nist.gov

Blair Fonville is a Senior Electrical Engineer for Time Services at the U.S. Naval Observatory. He received his M.S. degree in Electrical Engineering from The University of Alabama in 2003. His work at the observatory has involved the characterization of noise processes and precision measurement of phase delays through communication systems equipment, such as the Two-Way Satellite Time and Frequency Transfer system and the Global Positioning System (GPS). Email: blair.fonville@,usno.navy.mil

Dr. Demetrios Matsakis is Chief Scientist for Time Services at the U.S. Naval Observatory (USNO). He received his undergraduate degree in Physics from MIT. His Ph.D. was from U.C. Berkeley, and his thesis, under Charles Townes, involved building masers and using them for molecular radio astronomy and interferometry. Hired at the USNO in 1979, he measured Earth rotation and orientation using Connected Element Interferometry and Very Long Baseline Interferometry (VLBI). Beginning in the early 1990s, he started working on atomic clocks and in 1997 was appointed Head of the USNO's Time Service Department. He has over 100 publications, has served on many international commissions, and for three years was President of the International Astronomical Union's Commission on Time. Email: demetrios.matsakis@usno.navy.mil

\begin{abstract}
Due to the efforts of Henry Warren, inventor of the Telechron electric clock, electric power companies have been a source of time and frequency reference for the public for over a hundred years. However, advances in technology and changes in the electric power industry have generated a movement within the industry to end the time-reference service. Power systems in the U.S. operate at a nominal $60 \mathrm{~Hz}$, but in actual operation they accumulate significant phase error. It must be deliberately backed out to keep synchronous clocks on time - a procedure known as Time Error Correction (TEC). Today, many electric clocks still depend on the power system as the reference oscillator-that is, are synchronous-while others use other time references, such as local quartz oscillators and networked time servers - a benefit of the Internet of Things. Little is known about the overall impact of TEC on timekeeping in modern times. The Blackout of 2003 spawned a new regulatory structure for the electric power industry to improve reliability, and as an unanticipated side effect, a decision process that would most likely eliminate TECs was set in motion. The specific proposal is to retire regulatory standards designated $B A L-004$ and $W E Q-006$. We review the relevant structure and governing bodies of the U.S. power grids, and report on the current procedural status of these standards. In addition, we review possible scenarios for the future of the power system as an elapsed-time reference absent TEC. For this, we include analysis of the electric power at USNO, as measured over five years. The TECs appear in the data; an analysis with the industry-supplied record of the TECs indicates that without them a time deviation of about $71 / 2$ minutes would have occurred on the Eastern Interconnection (grid) between the daylight saving time switches of March 2016 and November 2016.
\end{abstract}

\section{INTRODUCTION}

Had this meeting on precision time been held 100 years ago, we surely would have discussed Henry Warren's latest invention. For on February 5, 1917, Mr. Warren — founder of the Warren Clock Company-filed for a U.S. patent on the first synchronous electric motor suitable for turning the gears and hands of a clock [1]. His company, and the clocks it produced, ultimately adopted the trademark name "Telechron."

U.\$. Government work not protected by U.S. copyright

Fonville, Blair; Hardis, Jonathan; Matsakis, Demetrios.

"Time and frequency from electrical power lines."

Paper presented at 48th Annual Precise Time and Time Interval Systems and Applications Meeting, 
As the electric power industry began to develop in the late $19^{\text {th }}$ century, various types of electric clocks were introduced. Some synchronized to the Master Clock at USNO through signals sent out nationwide over Western Union telegraph. Others were from the Self Winding Clock Company of Brooklyn, New York, which used electricity as a continual source of energy. However, prior to the Telechron clock, there was no clock that used the frequency of the power system as a timing reference.

At first, the synchronous electric clock hit a snag- $60 \mathrm{~Hz}$ electric power wasn't exactly $60 \mathrm{~Hz}$ after all. As Warren himself noted, "As a time-keeper the device was a failure. It was off as much as 10 or 15 minutes a day. But it was a success so far as checking the accuracy of alternations, or waves, was concerned. [2]" Seeing another opportunity, Warren developed a device to compare power line frequency to a precision pendulum clock - the Type "A" Master Clock. On October 23, 1916, Boston Edison became the first electric company to adopt a Warren Type "A" Master Clock as their frequency standard, and by 1947, Warren Master Clocks regulated over $95 \%$ of the electric lines in the United States [3]. As an unforeseen benefit, once different electric utilities had sufficiently synchronized generation, it became possible to form the first electric power grids.

Telechron began as a master/slave clock synchronization system. A master clock at the power company would be used to ensure the accuracy of the $60 \mathrm{~Hz}$ frequency, while Telechron secondary clocks could be sold inexpensively in the mass market. As the electric power industry developed and grew in the early $20^{\text {th }}$ century, they realized that they could also sell their service as a "correct time" service [4]. In modern parlance, timekeeping was a "killer app."

General Electric Company was quick to recognize the potential of the Telechron system, and in 1917 purchased a halfinterest in the Warren Clock Company. (GE acquired full ownership in 1943, and it was merged into GE in 1951 [5].) Those of us of a certain age remember the Telechron trademark on many GE products. However, many other companies came to rely on $60 \mathrm{~Hz}$ power as a frequency reference as well [6].

Dependence on a $60 \mathrm{~Hz}$ power source was not limited to synchronous motors. Digital display clocks that became commonplace beginning in the 1970s could also rely on the $60 \mathrm{~Hz}$ frequency as a reference. They would count the power line cycles electronically, rather than electromechanically. (Of course, battery powered clocks and some plug-in clocks rely on quartz crystal oscillators.)

Today, there is diversity in the way consumer clocks maintain their time accuracy. Quartz crystal oscillators are ubiquitous, and modern circuits improve accuracy through automatic compensation for temperature changes. Digital televisions receive time data from broadcasters, as do smartphones from cell sites. For devices appropriately situated to receive other broadcast signals, time is available from NIST Radio Station WWVB and GPS. For computers and the increasing number of devices on "the internet of things," time is readily available from network servers. Nonetheless, many electric clocks in our homes and offices - standalone, and integrated into appliances and time-sensitive devices (e.g., event recorders, lawn sprinklers) - still use the $60 \mathrm{~Hz}$ reference, and we take for granted that these clocks will maintain their time setting, at least to within a few seconds. However, after 100 years, the era of your power company providing a correct-time service may be coming to an end.

\section{TIME ERROR CORRECTION}

The Warren master clocks were not only frequency references, they also compared the integrated number of periods of both the pendulum clock and the power line. Their primary function, after all, was to steer the many secondary clocks to the time of the master one. However, in recent decades, electric power companies have had better frequency references. Precise synchronization of frequency across many generators is a key to forming power grids. And while $60.000 \mathrm{~Hz}$ is the normative goal, as a practical matter the frequency will vary with shifting loads and changing amounts of generation. A rising demand for electricity is felt by the turbine generators as an increased countertorque, which slows their rotation slightly until a feedback system applies more input power - and vice versa. Indeed, mismatched frequencies within a grid are indicative of unbalanced generation and load.

Even though the system is controlled to maintain constant frequency, phase noise is inevitable. Over time, there can be a significant accumulation of phase error, which is reflected as time error on synchronous clocks. In modern practice, the electric power industry monitors this time error, and once it reaches a threshold-10 seconds in the Eastern U.S., 5 seconds in the West, and at operator discretion in most of Texas - a procedure called manual Time Error Correction (TEC) is initiated to back it out. While the details vary a bit between the three major "Interconnections" (power grids) in the U.S.-Eastern, Western, and ERCOT (Texas) - in essence a central authority in each Interconnection monitors the Time Error and can issue an order for all producers in that Interconnection to target a different frequency: $59.980 \mathrm{~Hz}$ to retard synchronous clocks, or $60.020 \mathrm{~Hz}$ to advance them. For each hour of operation at these frequencies (a $20 \mathrm{mHz}$ offset), synchronous clocks will nominally gain or lose 1.2 seconds (subject to variation due to the vagaries of generation and load). 

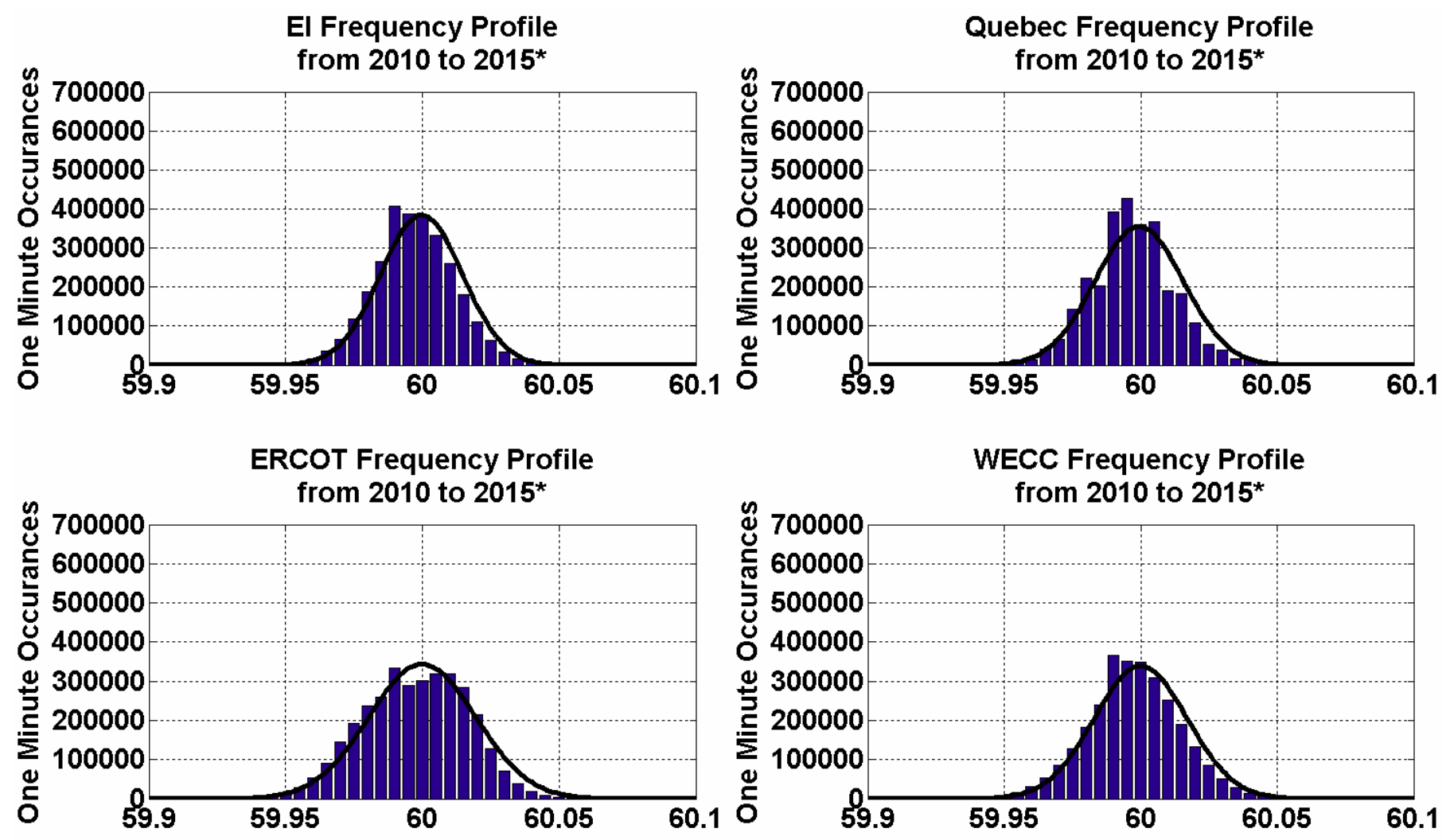

Figure 1. Histograms of one-minute average frequencies, in hertz, from the beginning of 2010 through June 2015 , for the four major North American Interconnections. Source: North American Electric Reliability Corporation, Ref. [25].

This is why synchronous electric clocks are so amazingly consistent over long periods of time. It isn't that the power system is itself a more stable oscillator than, say, a quartz one; it's because there is an invisible hand that intervenes to keep these clocks on time.

\section{STANDARDS IN THE BULK POWER INDUSTRY}

A defining event for the electric power industry was the Blackout of 2003, which affected an estimated 50 million people in Ontario and seven U.S. Northeast and Midwest states [7]. Congress, determined that such a thing should never happen again, established a new regulatory regime for Reliability Standards in Sec. 1211 of the Energy Policy Act of 2005. The Federal Energy Regulatory Commission (FERC) was given new oversight authority. FERC was required to select a nongovernmental "Electric Reliability Organization" to develop and enforce such standards, which FERC would then consider for adoption as binding Federal regulation. In 2006, the North American Electric Reliability Corporation (NERC) was chosen to fulfill this role.

Put another way, NERC acts as a voluntary consensus standards organization, in many ways similar to ANSI and ASTM. Standards intended to ensure the reliability of the bulk power system are developed through open participation and transparent process, study and voting. Once NERC has voted to adopt a new standard or to revise an old one, the document is submitted to FERC, which conducts a notice-and-comment rulemaking. This may result in a standard being legally enforceable, with stiff penalties for violations. Conversely, FERC may retire a standard if NERC so requests.

In addition to NERC, a second non-governmental organization, the North American Energy Standards Board (NAESB), develops voluntary consensus standards for the industry. While NERC standards are reliability-oriented, NAESB standards are business-oriented. "[NAESB] serves as an industry forum for the development and promotion of standards which will lead to a seamless marketplace for wholesale and retail natural gas and electricity, as recognized by its customers, business community, participants, and regulatory entities [8]." 
Both NAESB and NERC have adopted industry standards for Time Error Correction. NAESB standard WEQ-006 [9] requires, among other things, that all three Interconnections conduct TECs, what their respective trigger thresholds should be, and that balancing authorities within the Interconnections should participate [10]. NERC standard BAL-004 requires, among other things, that "The Interconnection Time Monitor shall monitor Time Error and shall initiate or terminate corrective action orders in accordance with the NAESB Time Error Correction Procedure [11-12]."

FERC has adopted both of these standards into regulations. BAL-004 was made enforceable in 2007 [13]. WEQ-006 was readopted most recently in 2014 (Version 3) [14], and a pending rulemaking would update the regulation to Version 3.1 [15]. However, in 2007 an unanticipated side effect arose. While the Interconnection Time Monitor had previously been an innocuous volunteer position, now non-compliance with the letter of $B A L-004$ could lead to a penalty of up to $\$ 325,000$. Perhaps not surprisingly, "[t]he entities [that] have been serving as 'volunteers' don't want to continue to serve in this role if they are subject to sanctions for non-compliance [16]."

\section{FIRST ATTEMPT TO RETIRE TIME ERROR CORRECTION}

To address this situation, NERC developed a revision to BAL-004 (BAL-004-1), and in 2009, they petitioned FERC to adopt it [17]. One of the proposed changes was to remove the requirement to initiate TECs in accordance with NAESB's procedure (WEQ-006). NERC argued that time error was not a reliability issue, and therefore there should not be a requirement in a reliability standard to conduct TECs. In taking this action, the industry had taken its first step back from its tradition of reliably providing correct time as well as power.

As the proceeding further developed, NERC raised a new argument that TEC was actually deleterious to reliability. "While it is expected that actual frequency will deviate to a certain extent from scheduled frequency (and indeed the Bulk Power System has been designed to allow for such variability), intentionally moving away from a scheduled frequency target of 60 Hertz serves no reliability purpose and effectively 'wastes' the safety margins designed into the system by the engineers who planned it [18]." Furthermore, TEC was characterized as an unnecessary relic from a bygone era. "[I]s Time Error Correction still a needed and valuable service, particularly in light of the data showing that Time Error Corrections appear to be placing the reliability of the Bulk Power System at greater risk? NERC's Balancing Authority Controls Standard Drafting Team, which is responsible for the redrafting of $B A L-004$, believes it is not. While proceeding cautiously, that team is tentatively recommending that the practice of Time Error Corrections be halted [19]." NERC filed a Motion to Defer Action on the proposed revision to regulation, given that "....research and analysis regarding Time Error Correction is ongoing, that NERC and its stakeholders are exploring the possibility of implementing a Field Test to evaluate elimination of Time Error Corrections, and that the results of such a field test may lead to the withdrawal of NERC's request for the approval of BAL004-1 and the retirement of BAL-004-0 [20].”

Ultimately, in 2012, the NERC Board of Trustees rescinded approval of BAL-004-1. "While it was clear that eliminating Time Error Corrections would likely have no negative impact on reliability that was insurmountable, it also became clear that the potential for other problems was largely undefined and not well understood. Following exhaustive discussion and debate over several months, it was ultimately determined by the OC [Operating Committee] at their March 6-7, 2012 meeting that NERC should discontinue its pursuit of the elimination of Manual Time Error Corrections [21]." Further, NERC petitioned FERC to withdraw its proposal for any regulatory changes to BAL-004 [22]. In their petition, NERC reported that they had determined the proposed changes to be "unnecessary."

\section{CURRENT ATTEMPT TO RETIRE TIME ERROR CORRECTION}

The issue was revived in February 2015, when a Periodic [Standards] Review Team within NERC recommended that BAL004-0 be retired and that manual Time Error Correction be eliminated as a continent-wide NERC standard [23]. Strictly, this was not a call to end the practice of TECs, as had been the case before, but merely a recommendation to retire the formally documented operating procedure under which TECs had been conducted.

This recommendation worked its way through the NERC standards development process, which included three rounds of comments and two rounds of balloting [24]. A white paper prepared in advance of the last round of comments [25] made several arguments in favor of the proposal. The principal one was a carefully worded variant of previous themes: "The practice of using manual TEC to place the Interconnection closer to the settings for automatic underfrequency load shedding does not support or enhance reliability. Therefore, BAL-004-0 should be retired." That is, the $20 \mathrm{mHz}$ frequency offset of TEC did not support reliability because it put the operating point closer to the threshold of Underfrequency Load Shedding 
(UFLS), also known as the Frequency Relay Limit (FRL). In the Eastern Interconnection, the FRL is $59.7 \mathrm{~Hz}$ [26], thus the margin is reduced from $300 \mathrm{mHz}$ to $280 \mathrm{mHz}$. (The FRL is not to be confused with the Frequency Trigger Limits (FTL) at $59.95 \mathrm{~Hz}$ and $60.05 \mathrm{~Hz}$, which if exceeded for five minutes, merely causes notification messages to be generated.)

The white paper continued with other arguments, which included: TEC is a strictly commercial service that does require a mandatory and enforceable Reliability Standard. There is no documentation that TEC has been important since 1976. Quartz oscillators provided a more reliable and less expensive method to keep accurate time. GPS is even better. Grid frequency is not the appropriate source for alignment to official time. Manual TEC is occurring less frequently. Newer versions of other reliability standards, $B A L-003-1$ and $B A L-001-2$, will maintain the grid closer to an average of $60 \mathrm{~Hz}$, so TEC will be redundant. (The authors do not necessarily concur with these arguments. For example, while BAL-003-1 and BAL-001-2, may result in smaller frequency deviations from $60 \mathrm{~Hz}$, it is not clear how they could control the average frequency, which would be the key to substituting for TECs.)

On November 2, 2016, the NERC Board of Trustees voted to file requests for the retirement of Reliability Standard BAL-004 with applicable regulatory authorities (FERC in the U.S and the National Energy Board in Canada) [27]. The petition to FERC was filed a week later [28]. On January 18, 2017, FERC approved the petition [29].

Approval for retirement of $B A L-004$ is contingent on the retirement of WEQ-006. On February 2, 2016, NERC filed a formal request with NAESB asking them to retire WEQ-006 [30]. This request was received favorably [31] and has been circulated for comment [32]. If approved within NAESB, it is likely that this will also result in a petition to FERC. A limited period for public comment would follow.

\section{USNO DATA MONITORING}

For situational awareness, the USNO began monitoring the time offset of the electrical power supplied by its local utility in 2011, by using a counter to measure the time intervals between the on-second marks of UTC(USNO) and the next upwardmoving zero volt crossing of the incoming alternating electrical current. Since 2011, we have accumulated over 63 million observations at 2 second intervals. An independent system was also in operation from January 9, 2013 to October 20, 2014, with 5.5 million observations at 1 second intervals.

In this measurement scheme, $y$ is a measured time interval $\left(0 \mathrm{~s} \leq y \leq \mathrm{t}_{\text {cycle, }}\right.$, where $\left.\mathrm{t}_{\text {cycle }}=1 /(60 \mathrm{~Hz}) \cong 0.0167 \mathrm{~s}\right)$. Figure 2 shows an example of these data. The phase wraps stem from the fact that the power line frequency, $f_{\mathrm{PL}}$, is not exactly $60 \mathrm{~Hz}$, and therefore in a period of length $\triangle \mathrm{UTC}$ seconds between two measurements there can be one zero-crossing more or less than $60 \mathrm{~s}^{-1} \Delta \mathrm{UTC}$ zero-crossings. ( $\triangle \mathrm{UTC}=2 \mathrm{~s}$ for the results reported here.)

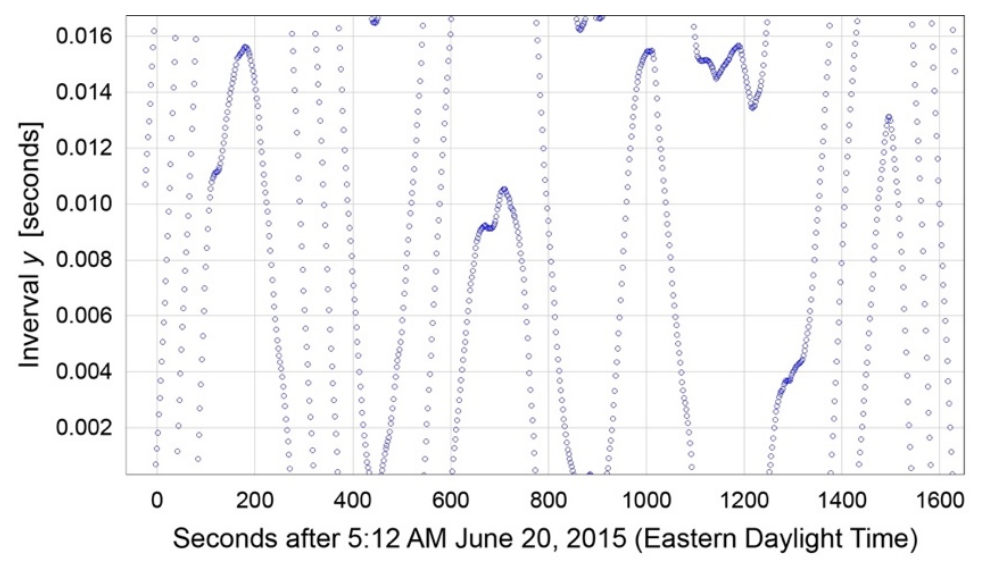

Figure 2. Typical USNO monitor data.

The difference between the $y$ of consecutive measurements, $\Delta y$, is expected to be near $0 \mathrm{~s}$ or $\mathrm{t}_{\text {cycle }}$, since the irregularities of the zero-crossing times makes them wander ahead of or behind the integer-second times of UTC(USNO). Approximately

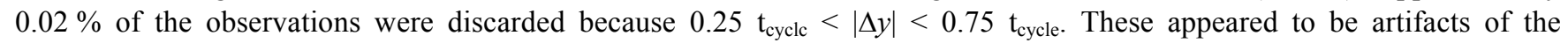
measurement system, which was not designed for this purpose. 
The instantaneous frequency of the power line can be computed from the measurements as:

$$
f_{\mathrm{PL}}=\frac{\left(60 \mathrm{~s}^{-1}\right) \Delta \mathrm{UTC}+N}{\Delta \mathrm{UTC}+\Delta y},
$$

where $N$, the number of cycle slips, is $0,-1$, or $+1 . N$ is chosen so as to bring the inferred $f_{\mathrm{PL}}$ closest to $60 \mathrm{~Hz}$, which is equivalent to making $\Delta y+N \mathrm{t}_{\text {cycle }}$ closest to $0 \mathrm{~s}$.

The wrap-corrected frequencies (Fig. 3) were used to infer the time $t_{\mathrm{PL}}$ by integrating up from the first datum (Fig. 4); a user's clock would integrate from the device's last reset against UTC(NIST) or UTC(USNO).

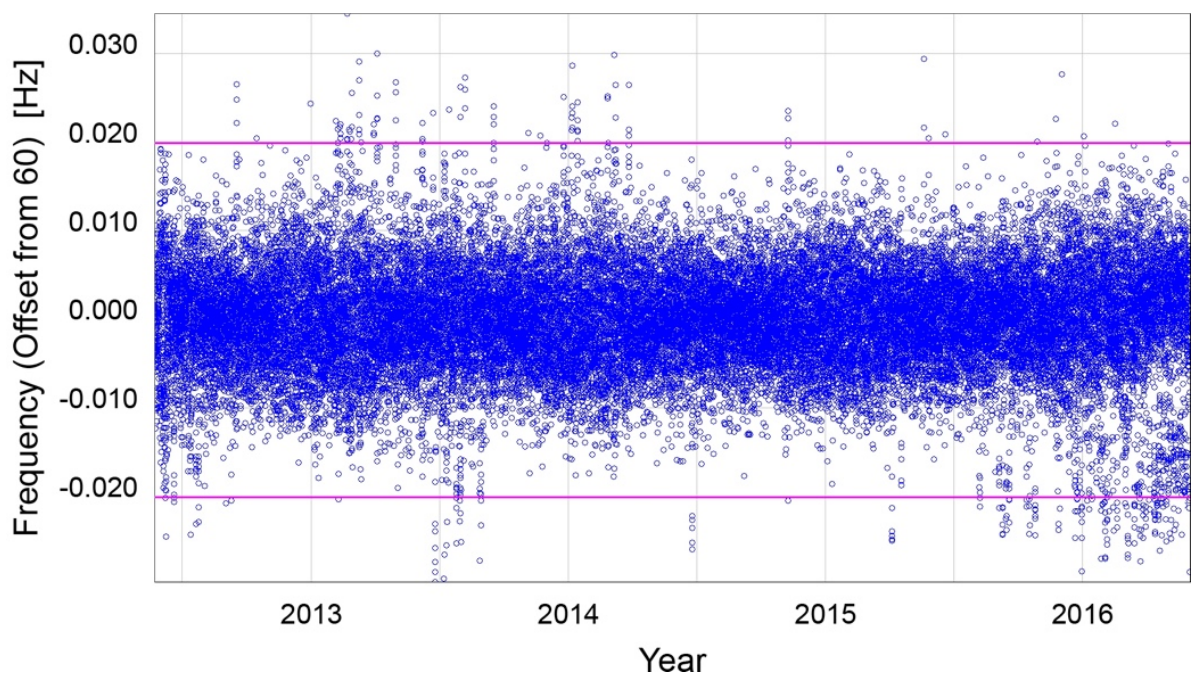

Figure 3. The frequency of the electric power provided to the USNO minus its nominal $60 \mathrm{~Hz}$ value. Frequencies outside the region enclosed by the horizontal bars likely indicate times of TECs.

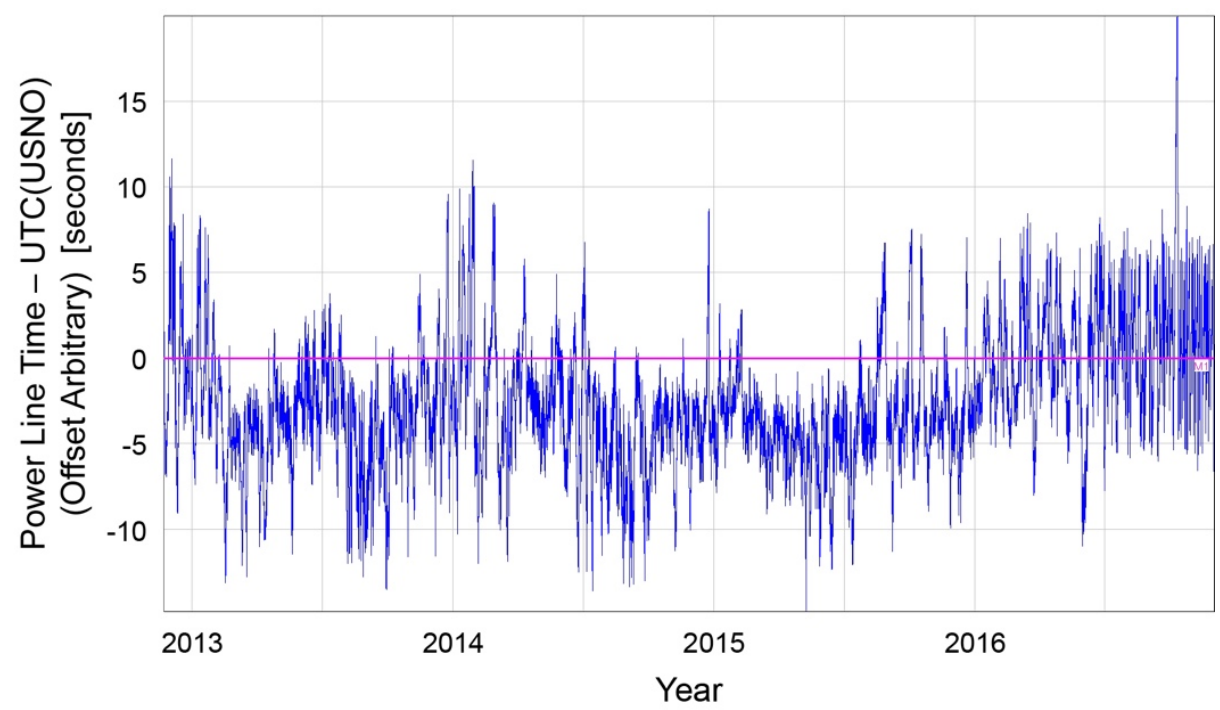

Figure 4. Power Line Time - UTC(USNO), as observed at the USNO, 1-minute averages. This is the time error that an ideal clock would display if it had been started on-time at the beginning of the dataset. In practice, clocks that do not provide a single-touch one-hour advancement or retardation feature are reset by the user at the beginning and end of Daylight Saving Time. 
On the U.S. Eastern Interconnection, TECs are implemented in the form of intentional $0.02 \mathrm{~Hz}$ adjustments of the frequency, over periods of up to 14 hours. They are initiated when the offset from UTC reaches approximately 10 seconds and halted when the offset has been reduced to about 6 seconds. These can be seen as outliers in the frequency data, although they are not distinct in a histogram of the hourly frequency deviations from $60 \mathrm{~Hz}$ (Fig. 5). The inference that these frequency outliers were intentional TEC insertions was largely confirmed by data kindly supplied by the NERC Resources Subcommittee, although one frequency outlier (Fig. 6) was apparently due to other causes.

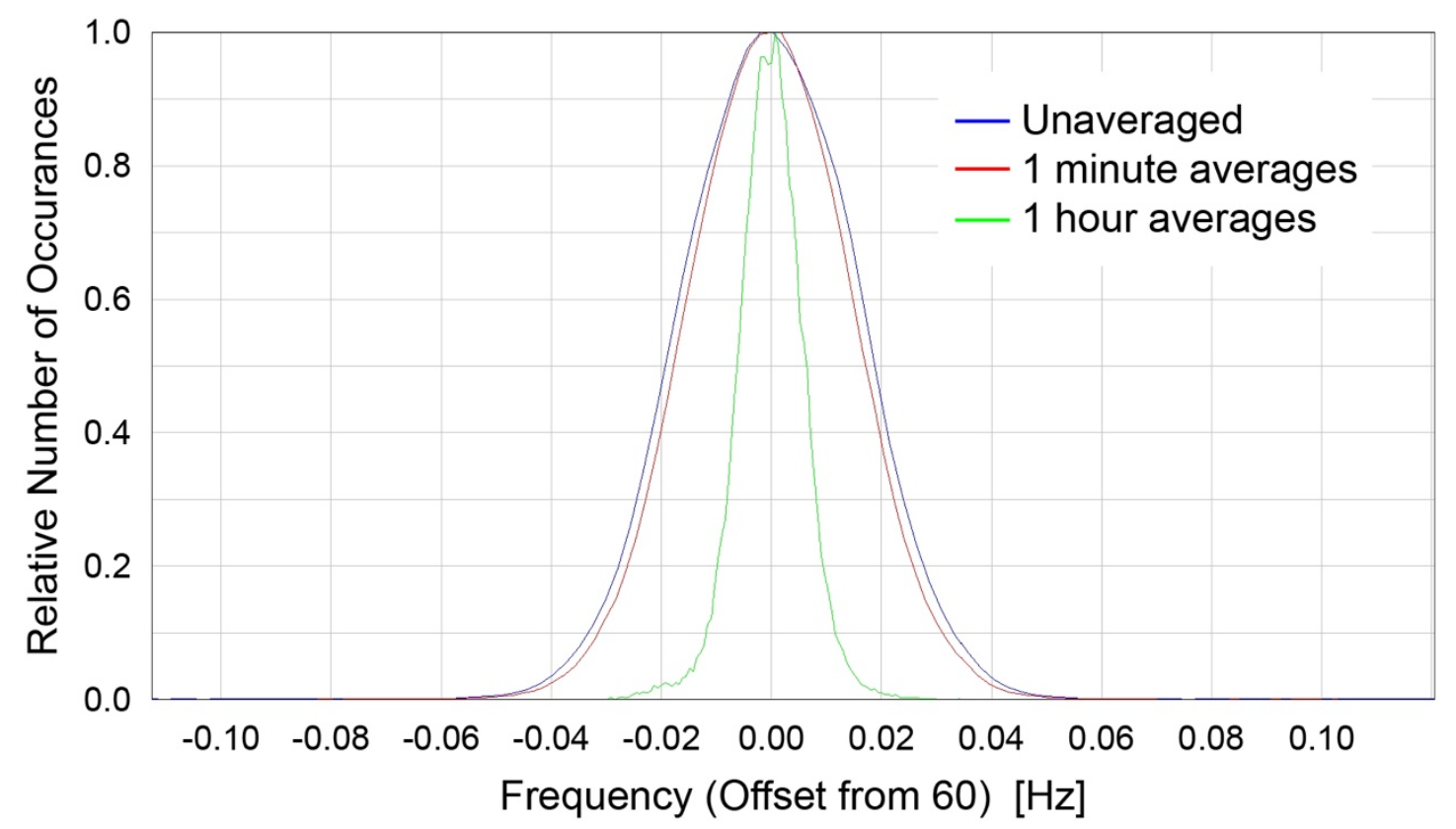

Figure 5. Histogram of Power Line Frequencies as observed at the USNO, averaged over different periods.

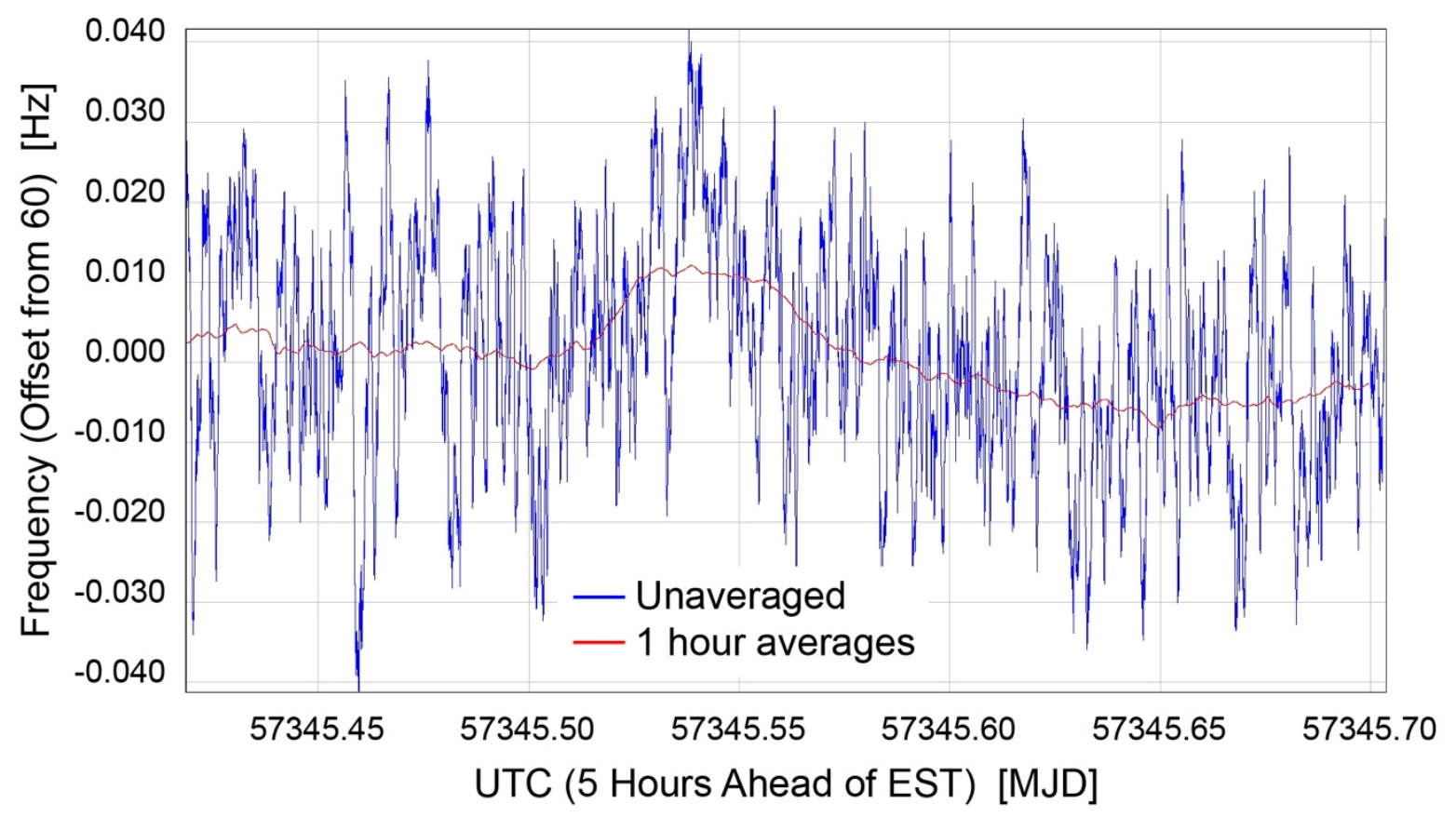

Figure 6. A frequency variation not associated with a recorded TEC. The time advanced $>4$ seconds over this period. 
In order to ensure the accuracy of our remaining analysis, we confine ourselves to the data since January 1, 2014. Figure 7 shows the cumulated time corrections of the TECs whose intentional insertion was confirmed. It shows that a clock reset for Daylight Saving Time (DST) in March 2016 would have been over 7 minutes off when Standard Time was re-implemented in November, unless it had been reset in the period. In many but not all sections of the U.S., such resets can also be forced a few times a year by power interruptions.

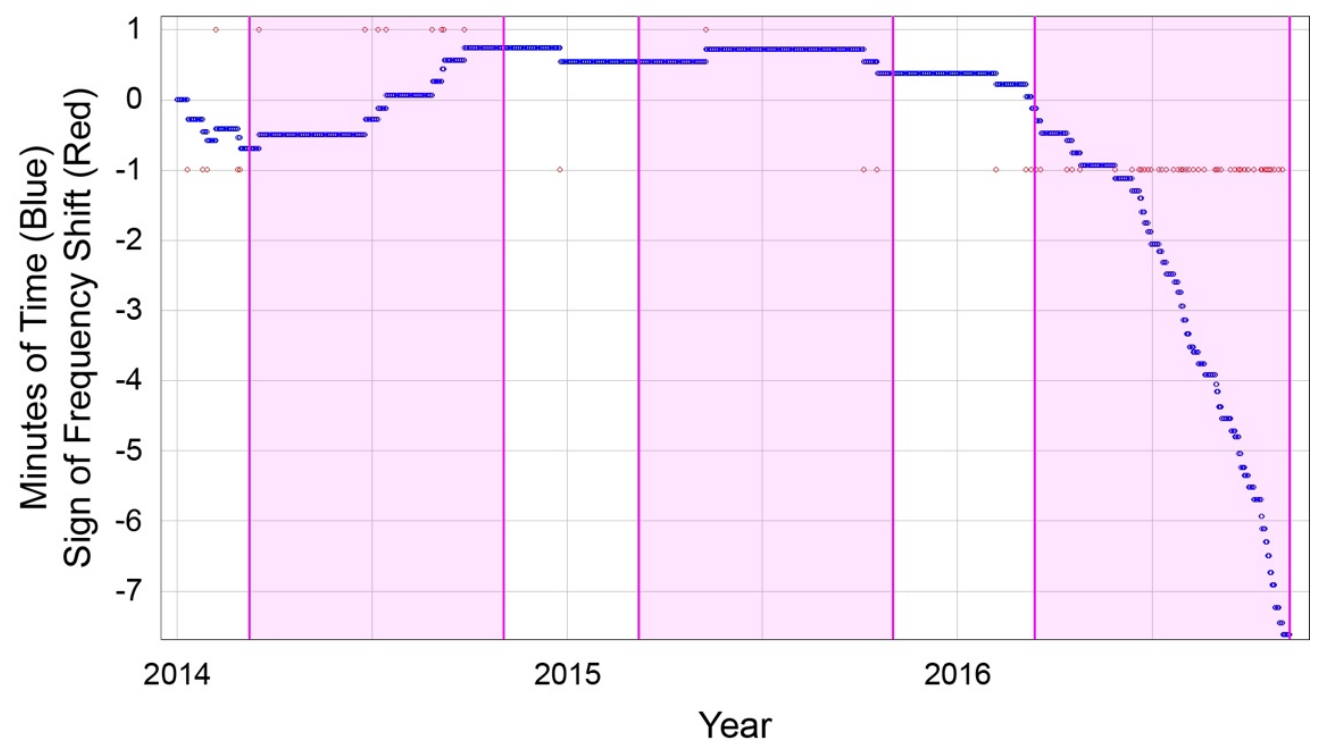

Figure 7. Cumulative effect of TECs. The large number and consistent sign of the corrections in the summer of 2016 imply that the power line frequency would have been slightly higher than $60 \mathrm{~Hz}$ most of the time. The shaded areas indicate periods of DST; presumably clocks dependent on the power line for time would be reset at the beginning and end of DST.

Figure 8 shows the effect of removing the TECs from real data, and Fig. 9 is the Allan Deviation of the uncorrected and TEC-removed data over the period of Fig. 8. As would be expected, the great improvement in long-term stabilities brought about by TECs also brings about a slight degradation of frequency stability on hourly to daily timescales.

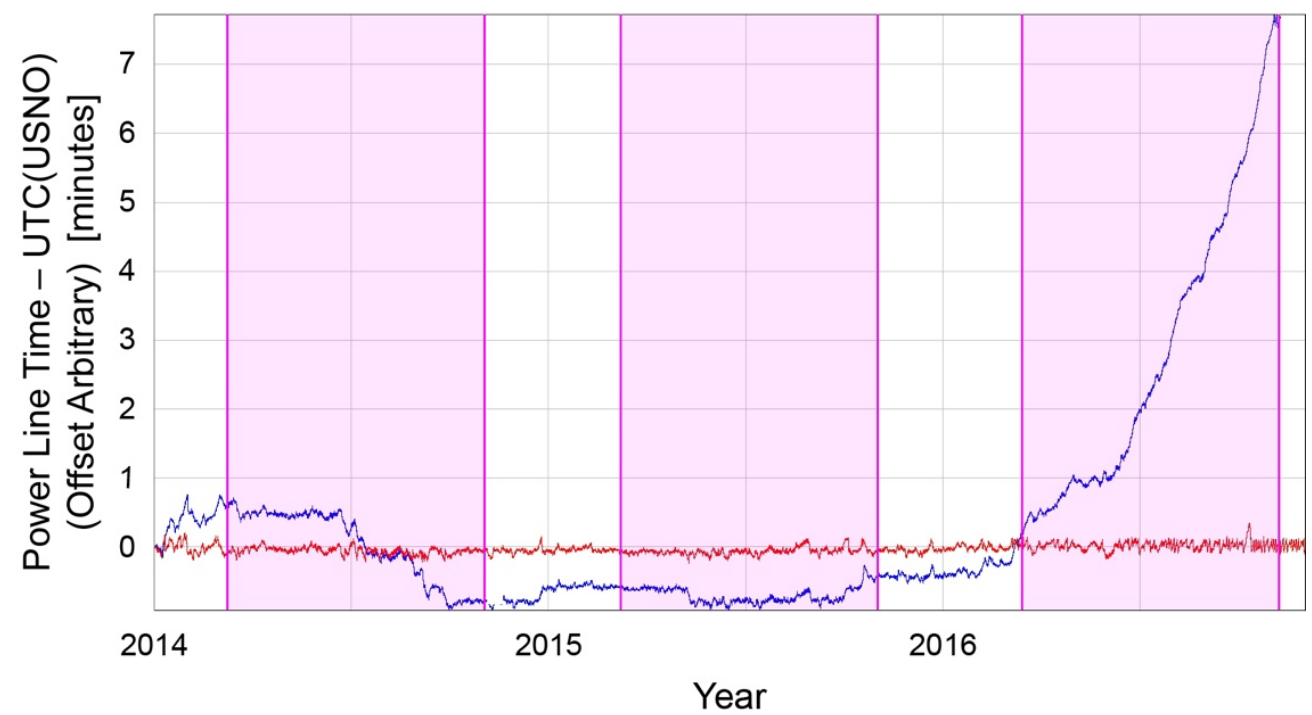

Figure 8. Power Line Time as observed (red) and on a pro forma basis without TECs (blue) by subtracting the actual TECs. The shaded areas indicate periods of Daylight Saving Time. 


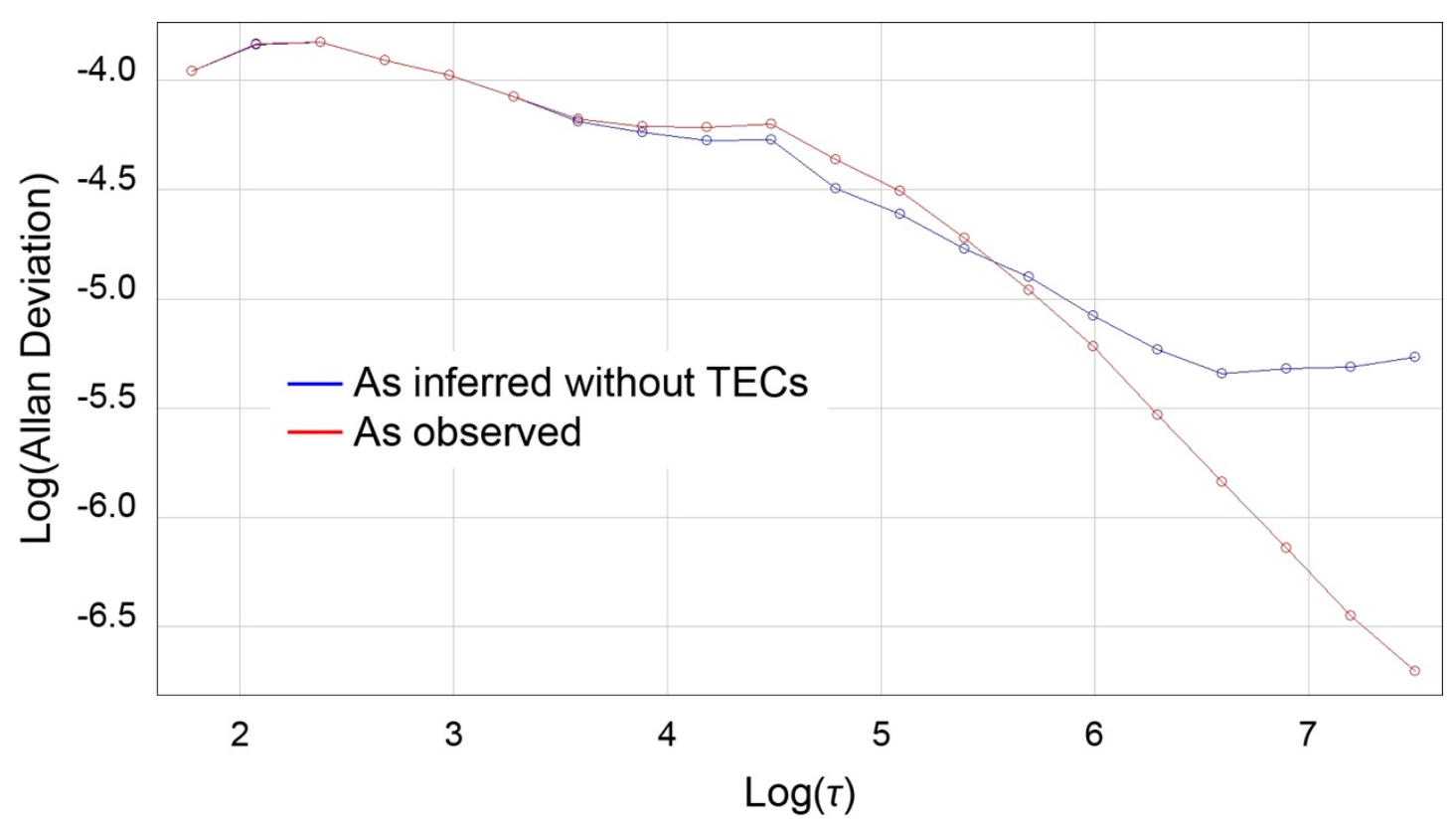

Figure 9. The Allan Deviation of Power Line time as observed, and as inferred without TECs.

\section{EXTREMELY HYPOTHETICAL STEERING STRATEGIES}

While it is unlikely that the power authorities would adopt any form of steering to replace the TECs, we have considered two families of steering strategies, without consideration of the practical implications for implementation. One family of strategies involves proportional steering, in which the frequency is routinely adjusted by the sum of a frequency gain times the frequency offset and a phase gain times the phase offset [33]. Another family of steering strategies, which we shall term "triggered" steering, is a generalization of the current practice on the Eastern Interconnection to act only when the time exceeds a certain trigger value. The frequency is then adjusted by a fixed amount $(0.02 \mathrm{~Hz})$ until the phase is brought back to a specified fraction of the trigger value.

In both cases, a model is needed in order to extract the phase and frequency from the raw data; this is a function of the jitter in the data. Using the TEC-removed dataset, it was found that a model which computes the frequency from the difference between each datum and one two minutes earlier was a good choice; therefore this section uses frequencies as determined by subtracting adjacent two-minute averages.

Figure 10 is the result of simulations to show the effect of increasing or decreasing the magnitude of the TEC by a power of 10 on a triggered steering strategy. TECs reduced to $0.002 \mathrm{~Hz}$ would result in only a 2.8 second time shift per day, which is not enough to keep up with the frequency variations observed last summer. However, the plots show little difference between TEC frequency offsets of $0.02 \mathrm{~Hz}$ and $0.2 \mathrm{~Hz}$, and those two curves are similar to the actual data observed at the USNO (Fig. 4). Since the red curve (for TEC of $0.02 \mathrm{~Hz}$ ) is an attempt to mimic the actual procedure that was followed, the difference between it and the observed data may be ascribed to the different measurement locations as well as the difference between a not-fully automated control system and the theoretically exact one of the model.

Fonville, Blair; Hardis, Jonathan; Matsakis, Demetrios.

"Time and frequency from electrical power lines."

Paper presented at 48th Annual Precise Time and Time Interval Systems and Applications Meeting,

Monterey, CA. January 30, 2017 - February 2, 2017. 


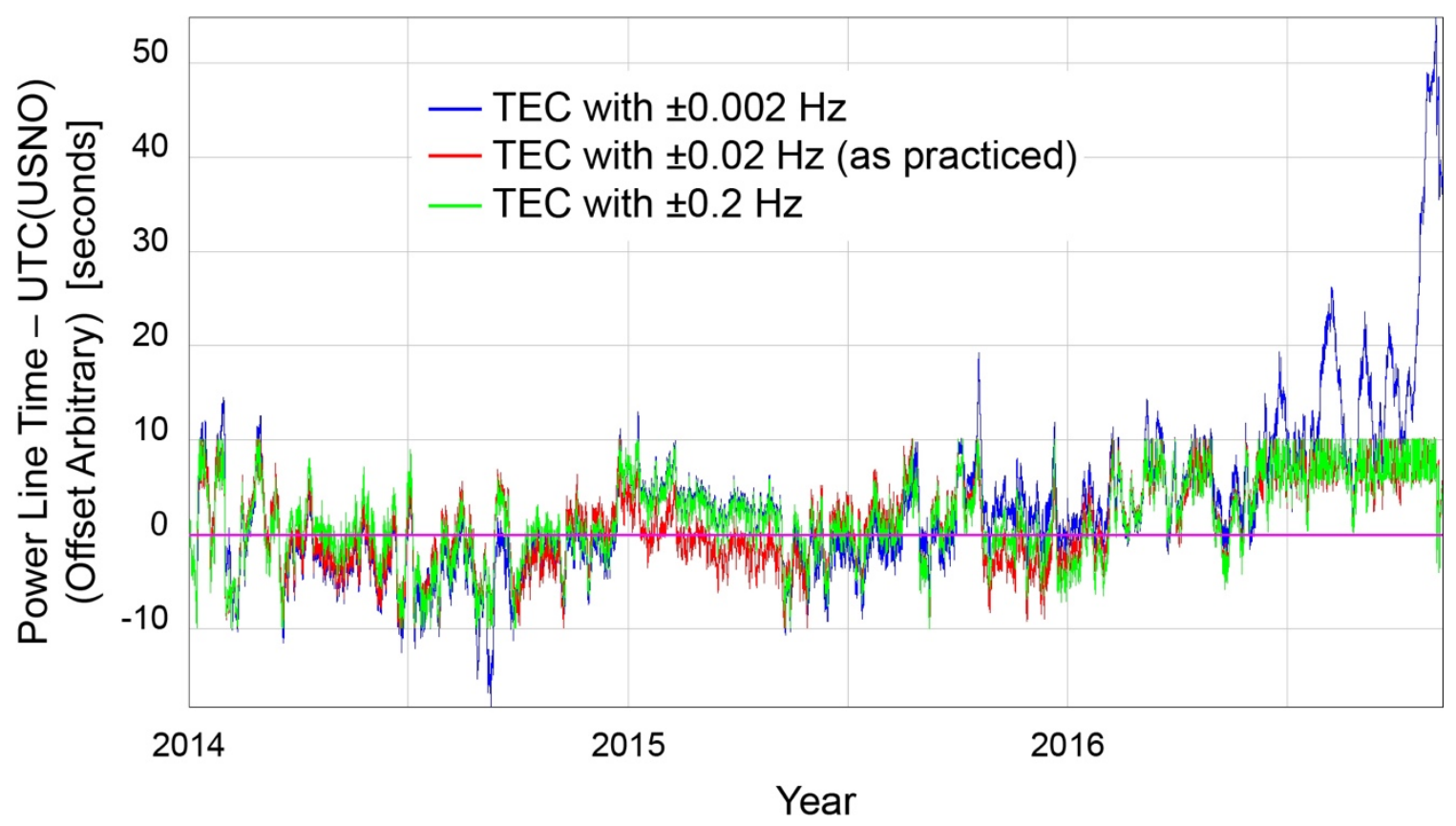

Figure 10. Simulations of Power Line Time with TECs practiced as currently (with $0.02 \mathrm{~Hz}$ offsets), with smaller frequency offsets $(0.002 \mathrm{~Hz})$, and with larger frequency offsets $(0.2 \mathrm{~Hz})$. The large variation in the blue curve in the last half of 2016 implies that the average frequency over that period (before TECs were applied) differed from $60 \mathrm{~Hz}$ by more than $0.002 \mathrm{~Hz}$.

Figure 11 shows the effect of changing the threshold value for triggering a TEC, and Table 1 summarizes the associated TECs. Most of these TECs, of course, would have been in the summer of 2016.

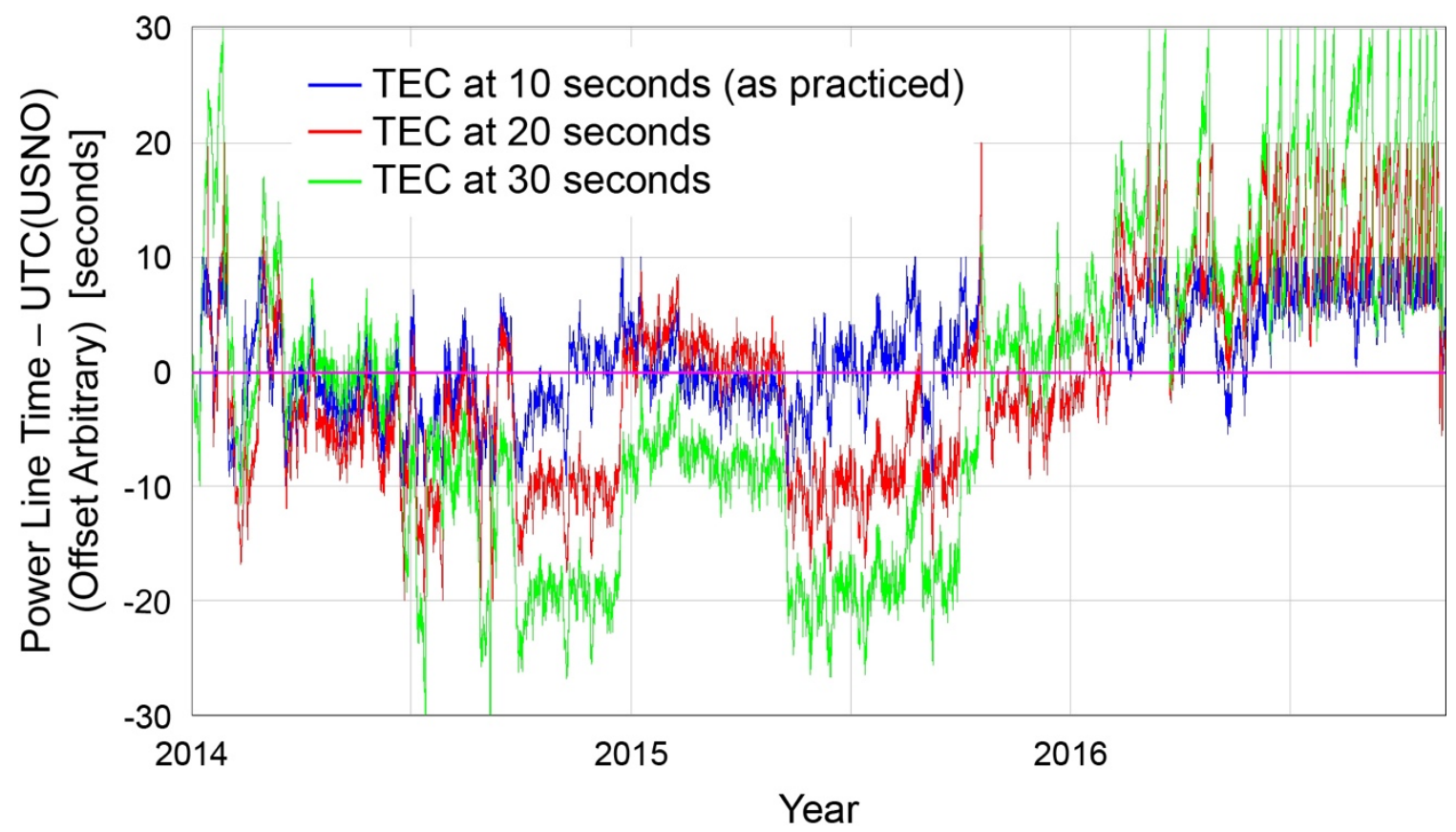

Figure 11. Simulation of Power Line Time if TECs were initiated when the time offset exceeded 10, 20, or 30 seconds. 


\begin{tabular}{|r|c|c|r|r|}
\hline Strength & Trigger & Termination & Duration & \multicolumn{1}{|c|}{$\begin{array}{c}\text { TEC- } \\
\text { Hours }\end{array}$} \\
\hline $\mathrm{Hz}$ & Seconds & Seconds & \multicolumn{1}{c|}{ Hours } & $\begin{array}{c}\text { Hours } \times \\
\mathrm{Hz}\end{array}$ \\
\hline 0.002 & 10 & 6 & 515.7 & 1.0 \\
\hline 0.02 & 10 & 6 & 579.5 & 11.6 \\
\hline 0.2 & 10 & 6 & 57.1 & 11.4 \\
\hline 0.02 & 20 & 6 & 499.2 & 10.0 \\
\hline 0.02 & 30 & 6 & 467.8 & 9.4 \\
\hline 0.02 & 30 & 10 & 465.9 & 9.3 \\
\hline
\end{tabular}

Table 1. Total duration of steering in simulations varying the strength of the TEC, or the offsets required to initiate or terminate a TEC event.

A proportional steering method can be characterized by a phase gain $g_{\mathrm{x}}$ and a frequency gain $g_{\mathrm{y}}$. The frequency steer is given by:

$$
\Delta f=g_{x} x+g_{y} y
$$

where $x$ is the offset in Power Line Time from UTC and $y$ is its dimensionless frequency. By way of illustration, in the computer code used for this section, time deviation $x$ was expressed in seconds and the frequency deviation $y$ was expressed in units of seconds/day, as the deviation of the frequency from $60 \mathrm{~Hz}$ (divided by $60 \mathrm{~Hz}$ ).

While the gains can be given any values, oscillatory behavior is minimized in a critically damped situation [33], which in our simple model reduces the number of free parameters to just one: the desired recovery time after a fluctuation or disturbance. As noted in the reference, the critically damped gains are given by:

$$
\begin{aligned}
& g_{x}=\frac{1}{\Delta t}\left(1-\mathrm{e}^{-\frac{\Delta t}{\mathrm{~T}_{\mathrm{c}}}}\right) \\
& g_{y}=2 \sqrt{\Delta \operatorname{tg}_{x}}-\Delta \operatorname{tg}_{x},
\end{aligned}
$$

where $\Delta t$ is the time interval between data points, and $\mathrm{T}_{\mathrm{c}}$ is the recovery time.

In Fig. 12 we compare the observed value of Power Line Time with a simulation in which 2 minute averages were steered in a critically damped situation with a time constant of one day. The subsequent figure is a histogram of the frequency steers called for by proportional steering with our approximate model for how the power grid actually behaved. 


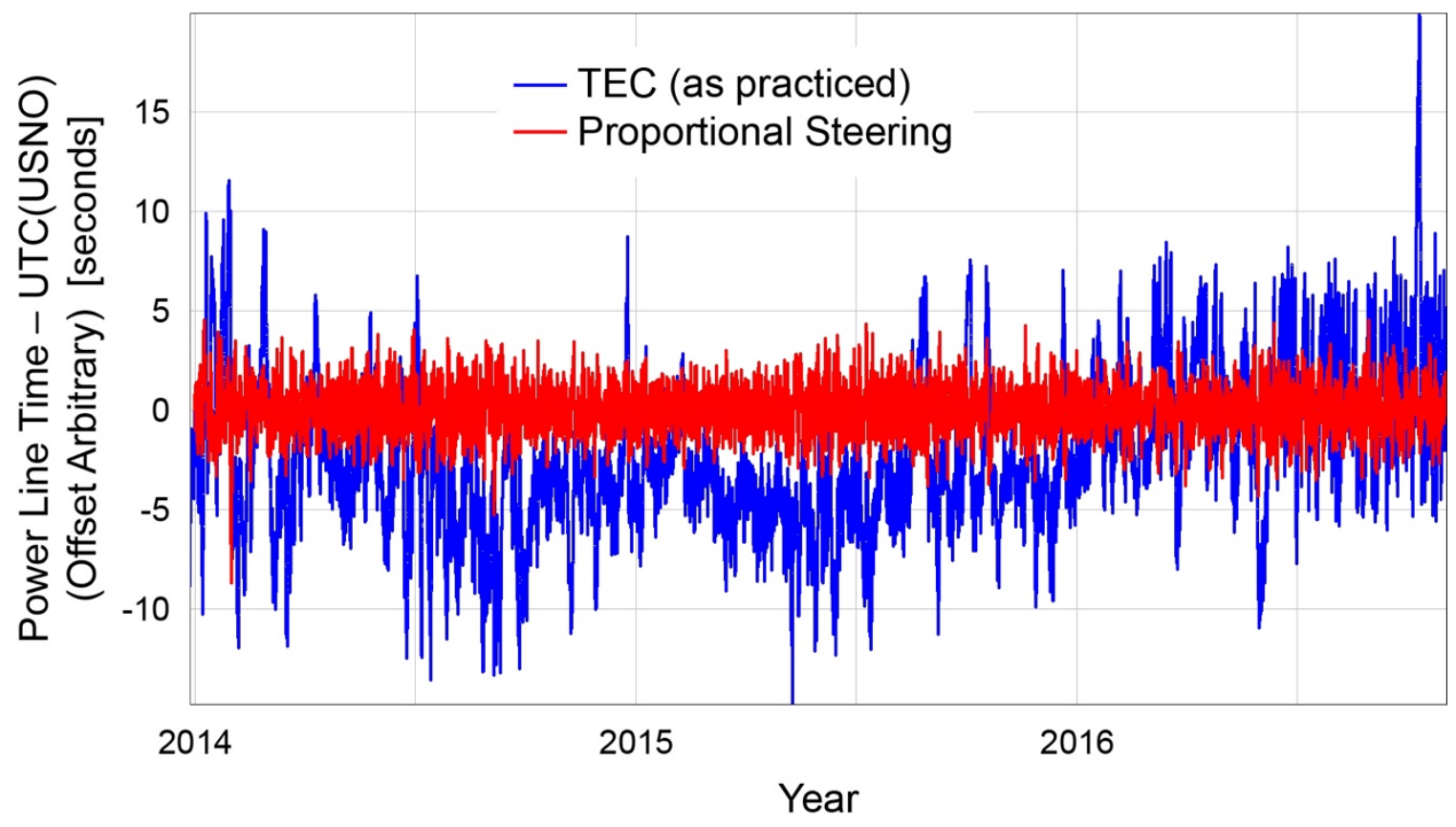

Figure 12. Comparison of a simulated proportional steering strategy (red) with a triggered strategy (blue)

As is evident in Fig. 13, the much larger number of proportional steers enables them to be much smaller in magnitude than the TECs, which appear only at $+0.02 \mathrm{~Hz}$ and $-0.02 \mathrm{~Hz}$.

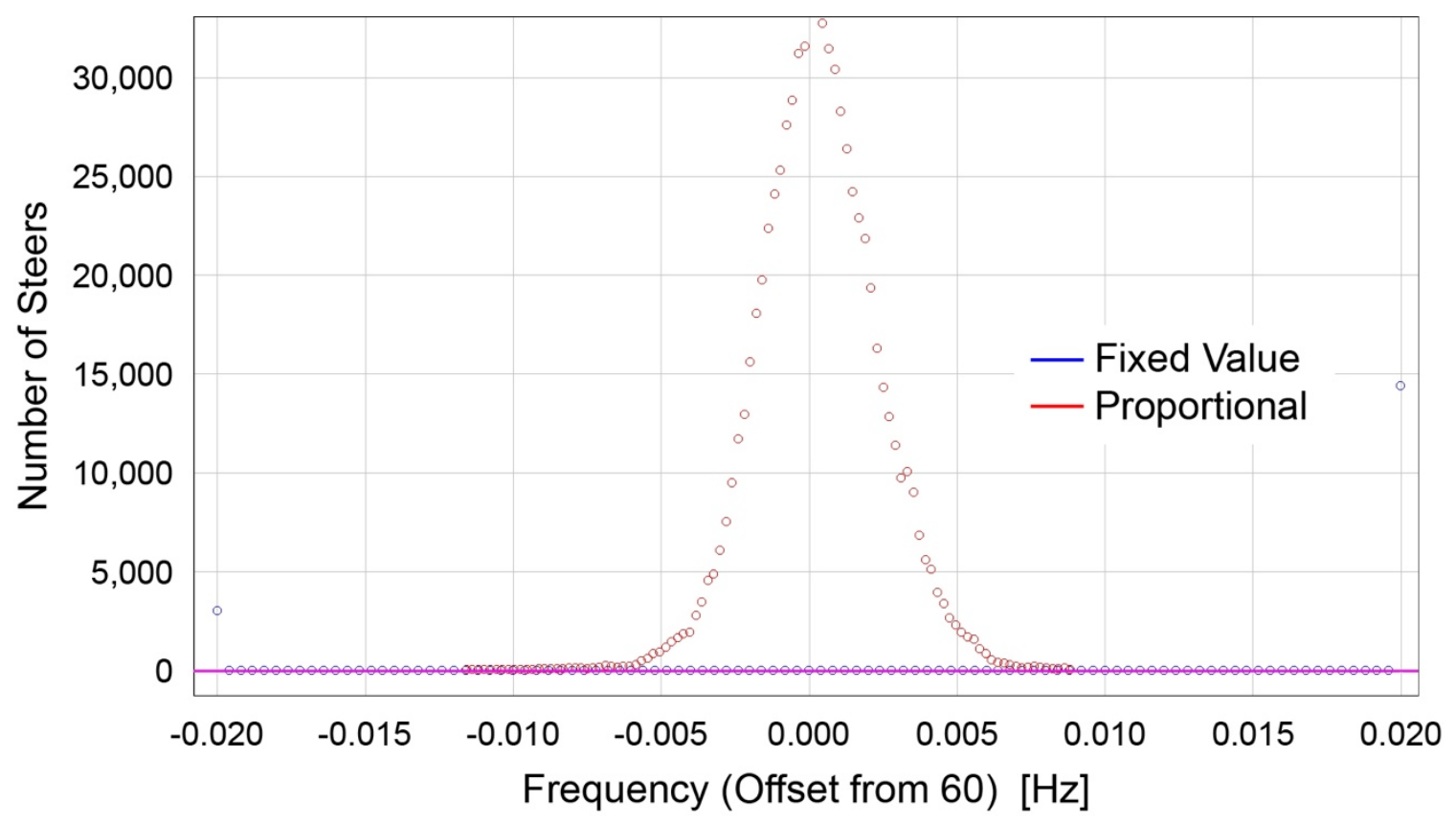

Figure 13. Histogram of steers of the proportional steering strategy (red) with a model of the current triggered system (blue). 


\section{ACKNOWLEDGMENTS}

We thank Terry Bilke (Midcontinent Independent System Operator) and Don Badley (Northwest Power Pool) for many helpful discussions and the NERC Resources Subcommittee for detailed information about recent TECs.

\section{DISCLAIMERS}

The purpose of this paper is to inform about contemporaneous issues affecting time keeping and time dissemination. Any opinions, recommendations, findings, and conclusions do not necessarily reflect the views or policies of NIST, USNO, or the United States Government. Neither the National Institute of Standards and Technology (U.S. Department of Commerce) nor the U.S. Naval Observatory (U.S. Department of Defense) take any positions with respect to the merits of these issues before other Federal agencies and other decision-making bodies. Certain commercial equipment, products, and services are identified in this paper in order to describe the context adequately. Such identification is not intended to imply recommendation, endorsement, or criticism by NIST or USNO, nor is it intended to imply that the equipment, products, or service identified are or were necessarily the best available for the purpose.

\section{REFERENCES}

[1] U.S. Patent No. 1,283,433, “Self Starting Synchronous Motor,” Issued October 29, 1918; online at http://patft.uspto.gov/ netacgi/nph-Parser?patentnumber=1283433

[2] Steve Leacu, “The Master of Time," Ashland Directions, February 2012; online at http://www.ashlandhistsociety.com/ Pages/TheMasterofTime.aspx (Visited February 9, 2017).

[3] Henry Ellis Warren, A Biographical Memoir, reprinted from “The Encyclopedia of American Biography,” a publication of The American Historical Company, Inc., New York; online at http://www.telechron.com/telechron/warren_bio.pdf

[4] Correct Time-A New Central-Station Service (Experience of the Philadelphia Electric Company in Merchandising Secondary Telechron Clocks...), Electrical World, February 20, 1926; online at http://www.telechron.com/telechron/central_station.pdf

[5] Henry Ellis Warren, A Biographical Memoir, Id.; The business and trademark were sold to Timex in 1979.

[6] As one example, the Hammond Clock Company of Chicago also made synchronous clocks. When the clock business didn't work out well, they shifted to making a musical instrument that used the $60 \mathrm{~Hz}$ as a frequency reference- the Hammond organ. See, e.g., https://en.wikipedia.org/wiki/Hammond_Clock_Company (Visited February 9, 2017).

[7] U.S.-Canada Power System Outage Task Force, Final Report on the Implementation of the Task Force Recommendations, Natural Resources Canada and the U.S. Department of Energy, September 2006; online at https://energy.gov/sites/prod/files/oeprod/DocumentsandMedia/BlackoutFinalImplementationReport\%282\%29.pdf

[8] https://www.naesb.org/aboutus.asp (Visited February 9, 2017)

[9] NAESB WEQ [Wholesale Electric Quadrant] Manual Time Error Correction Business Practice Standards - WEQ-006, Version 003, July 31, 2012. Outdated, 2005 version online at https://www.naesb.org/pdf2/weq_bklet_011505_tec_mc.pdf. Current version (3) available for a fee from NAESB (https://www.naesb.org/contactus.asp) and for public inspection at FERC (https://www.ferc.gov/resources/pub-ref-rm.asp).

[10] A "Balancing Authority" is a subdivision of an Interconnection that is responsible for resource planning to ensure a realtime balance between generation and load within its area of responsibility. For a map showing the Interconnections and balancing authorities within the U.S., please see: $\underline{\text { http://www.eia.gov/todayinenergy/detail.php?id=27152 }}$

[11] NERC Standard BAL-004-0 — Time Error Correction; online at http://www.nerc.com/files/BAL-004-0.pdf

[12] The Western Interconnection also utilizes an automatic TEC system to reduce the number of manual TECs. It is governed by a separate standard, "WECC Standard BAL-004-WECC-02 - Automatic Time Error Correction; online at https://www.wecc.biz/Reliability/BAL-004-WECC-2\%20From\%20NERC\%20site\%209-26-2016.pdf

Fonville, Blair; Hardis, Jonathan; Matsakis, Demetrios.

"Time and frequency from electrical power lines."

Paper presented at 48th Annual Precise Time and Time Interval Systems and Applications Meeting, 
[13] Mandatory Reliability Standards for the Bulk-Power System, 72 FR 16416 (April 4, 2007), online at https://www.gpo.gov/fdsys/pkg/FR-2007-06-18/pdf/E7-11685.pdf ; FERC Stats. \& Regs. \ 31,242 (2007) (Order No. 693), online at https://www.ferc.gov/whats-new/comm-meet/2007/031507/e-13.pdf

[14] Standards for Business Practices and Communication Protocols for Public Utilities, 79 FR 56939 (Sept. 24, 2014), online at https://www.gpo.gov/fdsys/pkg/FR-2014-09-24/pdf/2014-22601.pdf; FERC Stats. \& Regs. ๆ 31,359 (2014) (Order No. 676-H), online at https://www.ferc.gov/whats-new/comm-meet/2014/091814/e-5.pdf

[15] Standards for Business Practices and Communication Protocols for Public Utilities, Federal Energy Regulatory Commission, Notice of proposed rulemaking, 81 FR 49580 (July 28, 2016), Docket No. RM05-5-025; online at https://www.gpo.gov/fdsys/pkg/FR-2016-07-28/pdf/2016-17841.pdf . As of this writing, final action has not yet occurred.

[16] Petition of the North American Electric Reliability Corporation for Approval of BAL-004-1 Reliability Standard, FERC Docket No. RM06-16-000, March 11, 2009; online at http://elibrary.ferc.gov/idmws/common/opennat.asp?fileID=11965142, at Exhibit B.

[17] Id, passim.

[18] Comments of the North American Electric Reliability Corporation in Response to Notice of Proposed Rulemaking, Time Error Correction Reliability Standard, Docket No. RM09-13-000, April 28, 2010; online at http://elibrary.ferc.gov/idmws/ common/opennat.asp?fileID $=12332343$

[19] Id., p. 12.

[20] Motion of North American Electric Reliability Corporation to Defer Action on Time Error Correction Reliability Standard, Docket No. RM09-13-000, August 20, 2010; online at http://elibrary.ferc.gov/idmws/common/opennat.asp? $\underline{\text { fileID }=12417263}$

[21] Agenda of the NERC Board of Trustees, August 16, 2012; online at http://www.nerc.com/gov/bot/Agenda\%20Minutes \%20and\%20Highlights\%20DL/2012/0-BOT08-12a-complete.pdf , at Agenda Item 7d, p. 41 in the PDF file

[22] Notice of Withdrawal of the North American Electric Reliability Corporation of BAL-004-1 - Time Error Correction, Docket No. RM09-13-000, October 24, 2012; online at http://elibrary.ferc.gov/idmws/common/opennat.asp? fileID=13095024

[23] North American Electric Reliability Corporation, "Time Error Correction and Reliability White Paper: Recommendation of the Balancing Authority Reliability-based Controls 2 Periodic Review Team to Retire BAL-004-0 - Time Error Correction," February 25, 2015 (from metadata); online at http://www.nerc.com/pa/Stand/Project 20101422 Phase 2 of BARC BAL004 DL/Project_2010-14.2.2_BARC-BAL-004_White_Paper-20150225.pdf

[24] Please see the project page at http://www.nerc.com/pa/Stand/Pages/Project-20101422-Phase-2-Balancing-AuthorityReliabilitybased-Controls-BAL0042.aspx for the procedural history and supporting documents. (Visited February 9, 2017)

[25] North American Electric Reliability Corporation, "Time Error Correction and Reliability White Paper: Recommendation of the Balancing Authority Reliability-based Controls 2.2 Standard Drafting Team to Retire BAL-004-0 Time Error Correction," September 24, 2015 (from metadata); online at http://www.nerc.com/pa/Stand/Project 20101422 Phase 2 of BARC BAL004 DL/BAL-004-0_White_Paper_Clean_09242015.pdf

[26] North American Electric Reliability Corporation, "Eastern Interconnection Frequency Initiative," presentation of January 28-29, 2015, chart "Intelligent Alarm Review Threshold" at p. 15 of PDF file; online at http://www.nerc.com/ comm/OC/Resources Subcommittee RS 2013/RS Meeting Presentations_January_28-29_2015.pdf

[27] North American Electric Reliability Corporation, Agenda and Minutes of the November 2, 2016 meeting of the Board of Trustees, Item 5f; online at http://www.nerc.com/gov/bot/Agenda highlights and Mintues 2013/Board_November_2_2016 _Agenda_Pkg_Final.pdf

[28] Before the Federal Energy Regulatory Commission, Petition of the North American Electric Reliability Corporation for the Retirement of Reliability Standard BAL-004-0, November 10, 2016; online at http://www.nerc.com/FilingsOrders/us/ NERC Filings to FERC DL/BAL-004 Petition for Retirement.pdf 
[29] Federal Energy Regulatory Commission, Letter of January 18, 2017 to North American Electric Reliability Corporation, Docket No. RD17-1-000; online at https://elibrary.ferc.gov/IDMWS/common/OpenNat.asp?fileID=14468241

[30] North American Energy Standards Board, Request for Retirement of a NAESB Business Practice Standard, Model Business Practice or Electronic Transaction, Request No. R16002; online at https://naesb.org/pdf4/r16002.doc

[31] Recommendation to NAESB Executive Committee, Re: R16002, November 9, 2016; online at https://www.naesb.org/ pdf4/weq_2016_ap1d_r16002_rec.docx

[32] NAESB WEQ Request for Formal Comments, Re: R16002, November 9, 2016; online at https://www.naesb.org/pdf4/ weq_110916 reqcom.doc

[33] P. A. Koppang, “State space control of frequency standards,” Metrologia 53(3), R60-R64 (2016); doi:10.1088/0026$\underline{1394 / 53 / 3 / R 60}$ 


\title{
Improvements in Accelerometer Calibration at NIST Using Digital Vibrometry
}

\author{
Bev Payne, Richard A. Allen and Colleen E. Hood \\ National Institute of Standards and Technology (NIST)
}

\begin{abstract}
Improvements in shaker design and laser vibrometers have provided more precise calibration of accelerometers at NIST. Calibrations are performed at frequencies from less than $1 \mathrm{~Hz}$ to $20 \mathrm{kHz}$ with improved uncertainty and improved efficiency compared to the former systems. The vibrometer mounting hardware is designed for easier and repeatable optical alignment at multiple unique reference positions on the shaker's mounting table. Calibrations using this system, which is now used for the NIST routine calibration service, show excellent agreement with traditional NIST calibrations obtained using the laser interferometer methods that were developed at NIST. The legacy NIST laser-interferometer system uses a shaker with a beryllium mounting table insert, while the vibrometerbased system uses multiple shakers to cover the desired frequency range. For high frequencies (10 Hz to $20 \mathrm{kHz})$, a shaker with a ceramic mounting table is used; this ceramic table is designed to reflect the laser beam without requiring a mirror. For low frequencies $(<10 \mathrm{~Hz})$, a shaker with an aluminum mounting table is used. This system is described and compared with the previous calibration system. Comparison results for accelerometer measurements using the two measurement systems are presented.
\end{abstract}

\section{INTRODUCTION}

Recent improvements in shaker design and the introduction of the laser vibrometer have greatly improved the capacity for routine accelerometer calibrations with much lower uncertainties. This paper will show that the traditional NIST laser interferometer calibration systems are in good agreement with the newer laser vibrometer system at NIST. Both systems were independently used to calibrate two laboratory-type accelerometers and the measurements were found to be in excellent agreement over a range of $100 \mathrm{~Hz}$ to $10 \mathrm{kHz}$.

\section{METHODS FOR PRIMARY ACCELEROMETER CALIBRATION}

\section{RECIPROCITY}

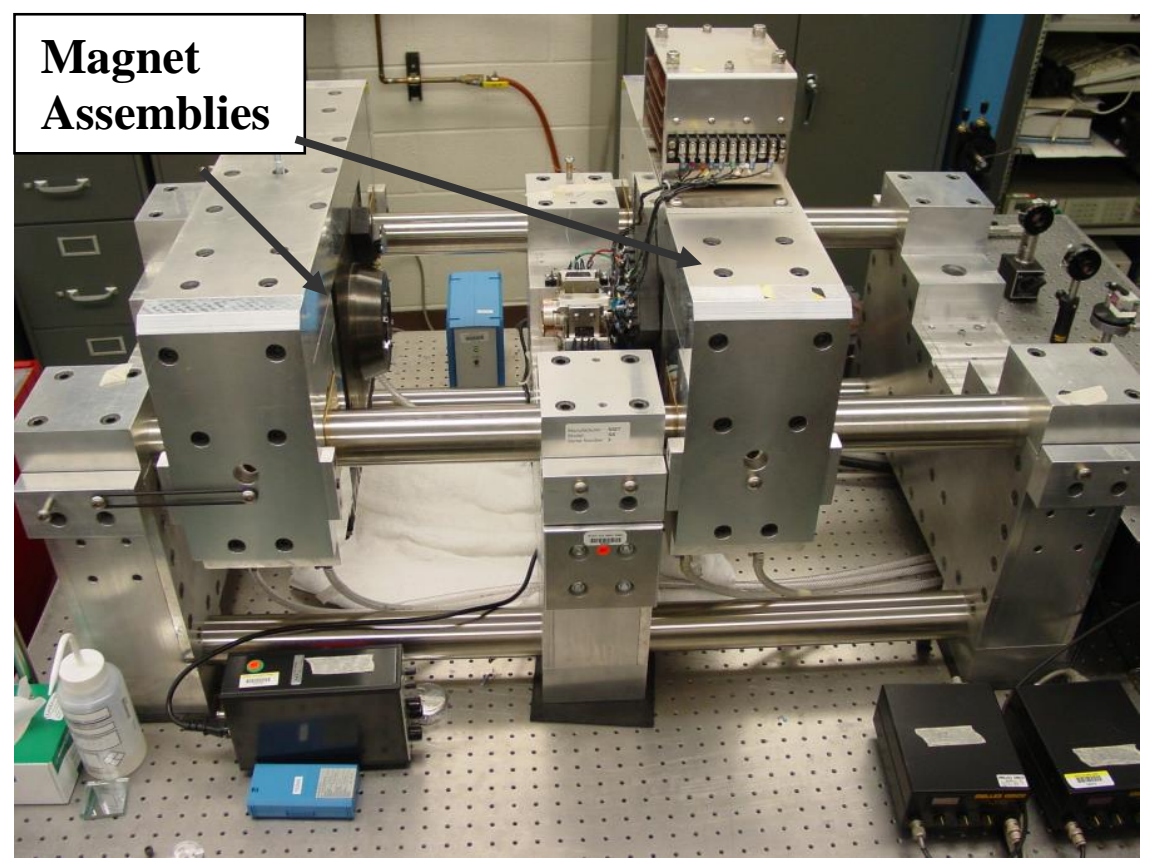

Figure 1a Dual coil shaker for reciprocity and laser interferometry calibrations; dual magnets mounted on air bearings, shown with left magnet retracted to allow access 


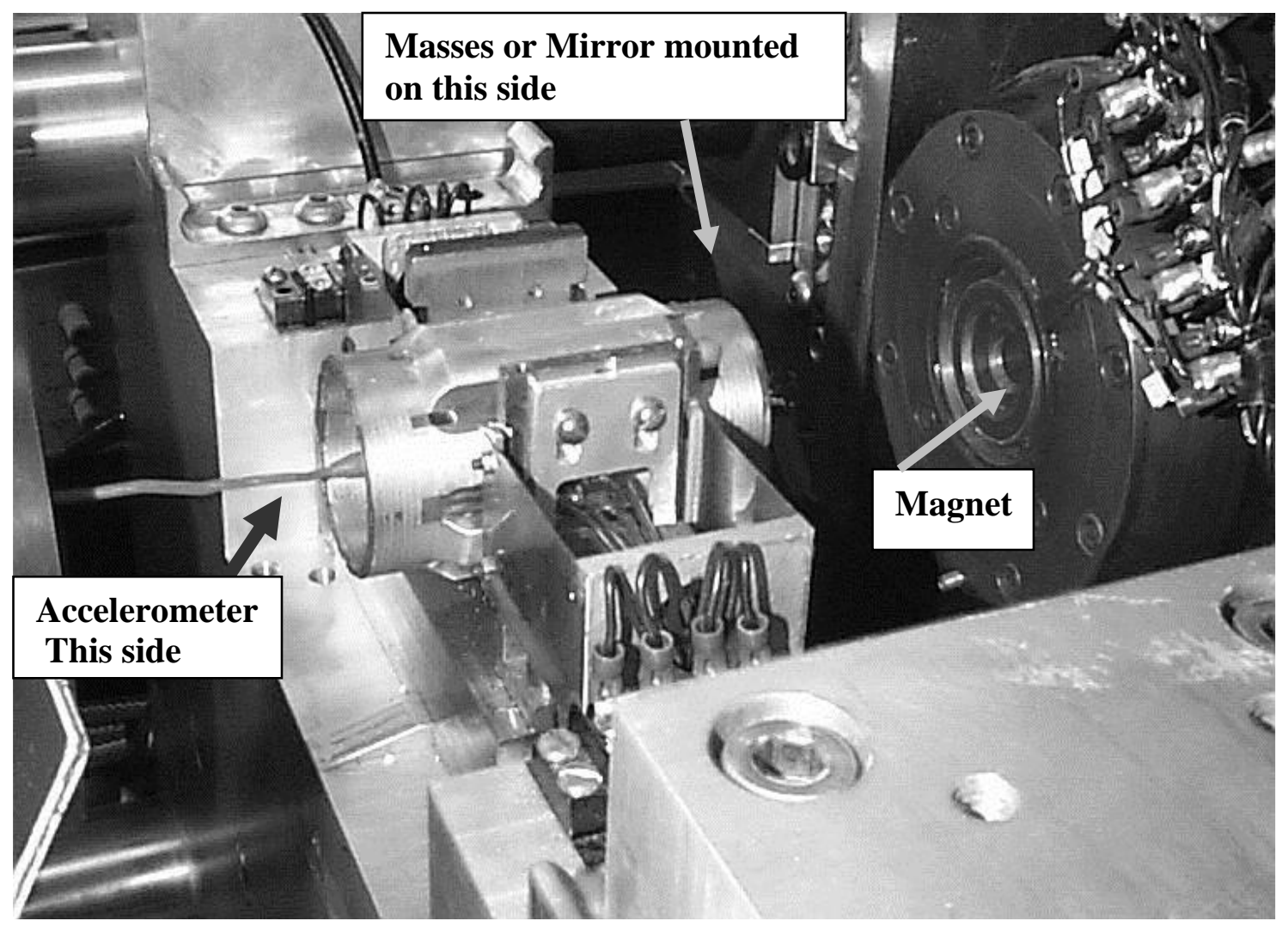

Figure 1b Shaker showing close-up of dual-coil moving element, both magnets retracted for access to the moving element

Reciprocity calibration is documented in ISO 16063-12 as a primary calibration method [1]. It does not involve the use of optical equipment of any kind. The unit of mass is recognized as the fundamental unit of reference for this method. A set of small, calibrated masses are placed (one at a time) on the shaker with the attached or imbedded accelerometer to be calibrated. Measurements of the transfer admittance between the primary drive coil (current) and the accelerometer (voltage) are made with each of the masses. The admittance is a function of the mass value. A second set of measurements is performed between the accelerometer and the driver coil of the shaker (now acting as a velocity coil) to determine the voltage ratio, while the shaker is excited with a secondary shaker (or a secondary coil if the shaker has two coils). With these two sets of data, accelerometer sensitivity can be calculated.

NIST has used a dual-coil shaker (Figure 1) for reciprocity calibrations to avoid the problems of connecting a secondary shaker to the primary shaker [2]. The dual-coil shaker is also designed to be compatible with laser interferometer calibrations for cross validation. Reciprocity requires shakers with very low distortion and cross-axis motion to provide quality accelerometer calibrations. Research has shown that reciprocity calibration is equivalent to optical calibration methods within limits based on the quality of the shakers. [3]. 
Figure 2 shows results from a comparison of reciprocity and fringe counting calibrations (described below) of an accelerometer at $100 \mathrm{~Hz}$, ten data points for each method. By carefully holding the temperature of the reciprocity system constant, one can get lower than $0.1 \%$ difference in sensitivity

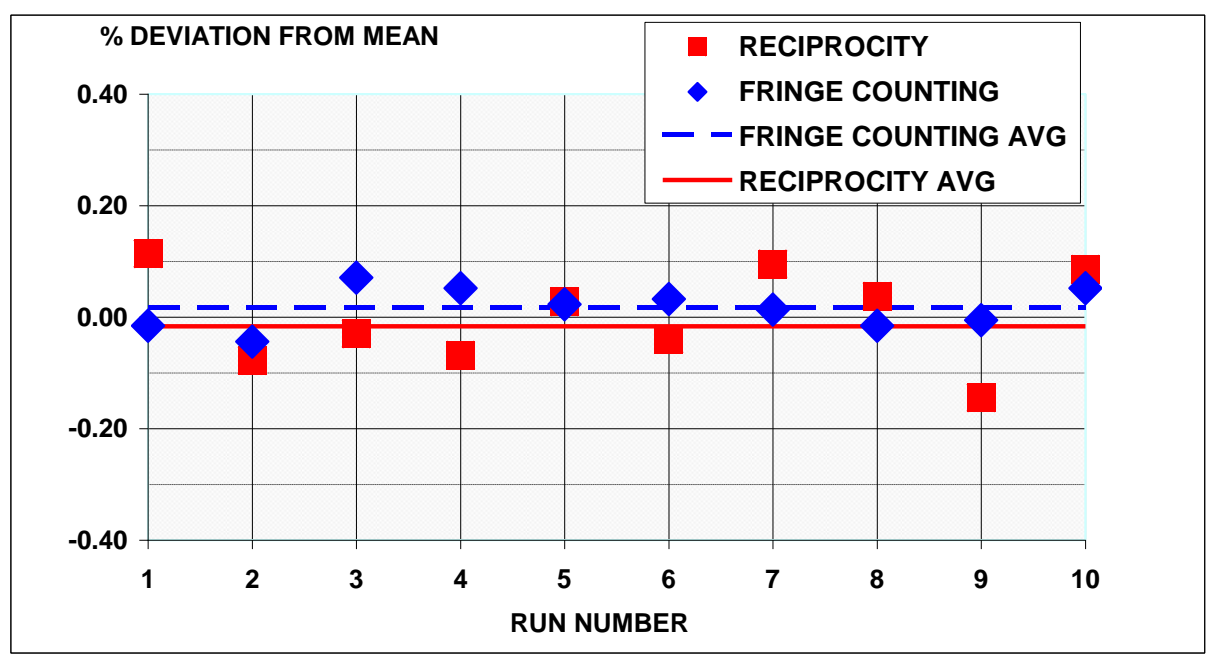
for the two methods [3]. Figure 2. Reciprocity and fringe counting calibration differences

\section{FRINGE COUNTING}

Fringe-counting calibration is documented in ISO 16063-11, Method 1, as a primary calibration method [4]. It uses a laser interferometer as shown in Figure 3. Figure 3 shows the schematic for a simple singlecoil shaker where the accelerometer is mounted in the center of the shaker table and the reflecting mirror is attached as close to the accelerometer as practical, without contacting the accelerometer.

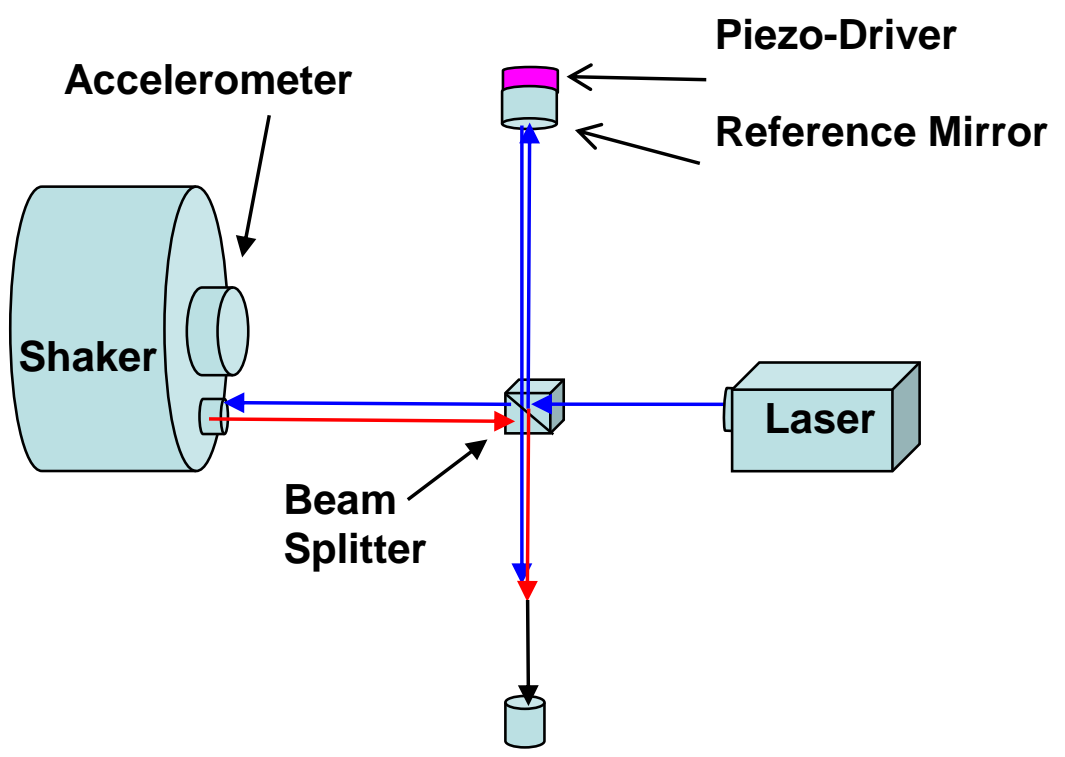

Figure 3. Laser interferometer for fringe-counting and minimum point calibrations

Some shakers now have mounting tables designed to reflect light from any point on the mounting table. For the dual-coil shaker shown in Figure 1, there are two mounting tables facing in opposite directions. A flat mirror is mounted on the shaker table located on the right side of the moving element, opposite the table upon which the accelerometer is mounted. This shaker design allows optical access to the shaker mounting tables through a center hole in the magnet (bottom photo in Figure 1). This shaker system was described by Payne and Steffen [2]. The acceleration, in meters per second squared, is given by

$$
A=\lambda v \pi^{2} f^{2} / 2
$$


where $\lambda$ is the wavelength of He-Ne laser light in meters, $v$ is the integral number of fringe counts per vibration cycle, and $f$ is frequency in hertz [4].

\section{MINIMUM-POINT METHOD}

Minimum Point calibration is documented in ISO 16063-11, Method 2, as a primary calibration method [4]. It uses the same interferometer as the fringe-counting interferometer, Figure 3. The minimum-point method is based on the determination of displacement corresponding to the zero crossings of the Bessel function of the first kind and first order, $J_{1}$. In order to reject spectral components present in the output of the photo-detector other than those corresponding to the fundamental frequency of the vibration, the output of the photo-detector is filtered using a narrow band-pass filter centered at the fundamental frequency of the vibration. The amplitude of vibration is then adjusted until the null corresponding to the desired zero crossing of $J_{1}$ is obtained. Peak displacement amplitudes of the first six zero-crossings of $J_{1}$ for a He-Ne laser $(\lambda=632.8 \mathrm{~nm})$ are listed in Table 1 . For minimum-point calibrations at each tested frequency, the number of the zero crossing was selected to obtain displacements corresponding to accelerations in the range of $50 \mathrm{~m} / \mathrm{s}^{2}$ to $200 \mathrm{~m} / \mathrm{s}^{2}$ based on the capacity of the shaker.

Table 1. Peak Displacement Amplitudes for the First Six Zero Crossings

\begin{tabular}{|c|c|}
\hline Zero Crossing No. & Displacement $(\mathrm{nm})$ \\
\hline 0 & 0.00 \\
\hline 1 & 192.95 \\
\hline 2 & 353.29 \\
\hline 3 & 512.31 \\
\hline 4 & 670.95 \\
\hline 5 & 829.42 \\
\hline
\end{tabular}

Once the displacement, $d$, is determined, the acceleration is calculated using the following equation:

$$
A=(2 \pi f)^{2} d
$$

The sensitivity of the accelerometer is then obtained by dividing the accelerometer output voltage by the acceleration, $A$.

As the above method is very time consuming and difficult to automate, NIST has used the Fringe Disappearance Method for many years. This method which is related to the Minimum Point Method above, and consists of finding the first zero-crossing of the Bessel function of first kind of order zero $\mathrm{J}_{0}(4 \pi \mathrm{d} / \lambda)$. At this displacement the phenomenon of fringe disappearance occurs, and a constant intensity illumination is observed on the photodetector. The implementation of this method is described by Robinson and Payne [5]. 


\section{SINE-APPROXIMATION METHOD}

Sine-approximation calibration is documented in ISO 16063-11, Method 3, as a primary calibration method [4]. Using the setup shown in Fig. 4, calibration data can be obtained using the dual-coil shaker for frequencies up to $1 \mathrm{kHz}$ using fringecounting, and up to $5 \mathrm{kHz}$ using the minimum-point method by monitoring the output from either Detector 1 or Detector 2. At frequencies higher than $5 \mathrm{kHz}$, the sine-approximation method is needed because of smaller displacement.

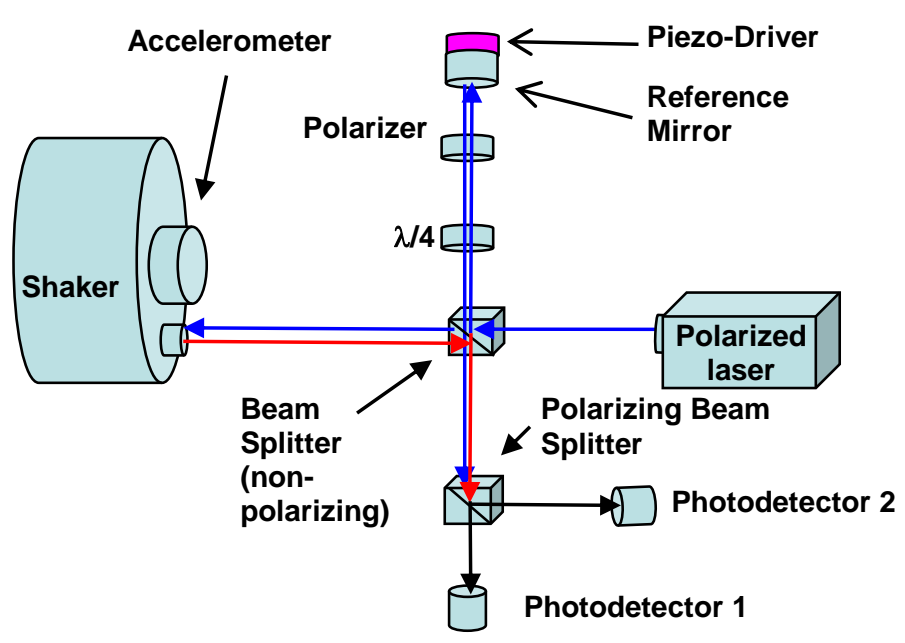

Figure 4. Interferometer for sine approximation

Sine approximation is most useful at higher frequencies where calibrations at smaller displacements must be made due to amplitude limitations in electro-dynamic shakers. In actual practice the sine-approximation method gives good results over a wide frequency range. Typically for the dual-coil shaker shown above (Fig. 1) the sine-approximation method has been used at NIST for calibration of accelerometers at accelerations of $50 \mathrm{~m} / \mathrm{s}^{2}$ to $100 \mathrm{~m} / \mathrm{s}^{2}$ from $100 \mathrm{~Hz}$ to $10 \mathrm{kHz}$, and fringe-counting has been used for frequencies of $100 \mathrm{~Hz}$ and below. This range of acceleration is used to minimize the heating effects on the shaker.

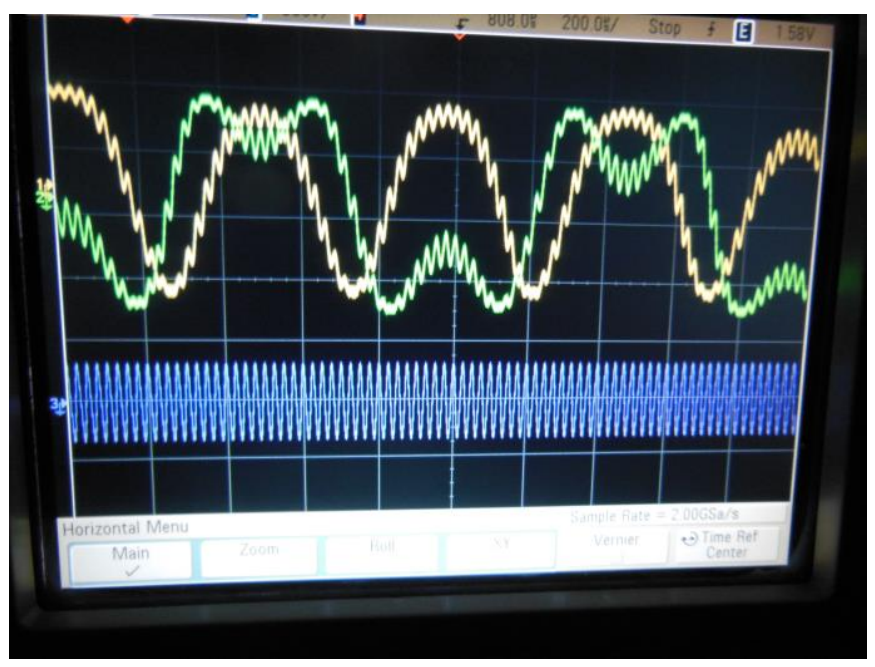

Figure 5. Photo-detector signals in quadrature with $1 \mathrm{kHz}$ modulation on reference mirror. Blue (bottom) trace is accelerometer output.

The sine-approximation method is also used for calibrations that use piezo-electric shakers designed and used at NIST for frequencies of $5 \mathrm{kHz}$ and higher. The small piezo-driver shown in Fig. 4 is used to modulate the reference mirror of the interferometer so that calibrations may be obtained at smaller displacements. Figure 5 shows an oscilloscope display of the two photodetector signals in quadrature with $1 \mathrm{kHz}$ modulation on the reference mirror. The lower trace shows the accelerometer signal. 


\section{CALIBRATION USING DIGITAL VIBROMETRY}

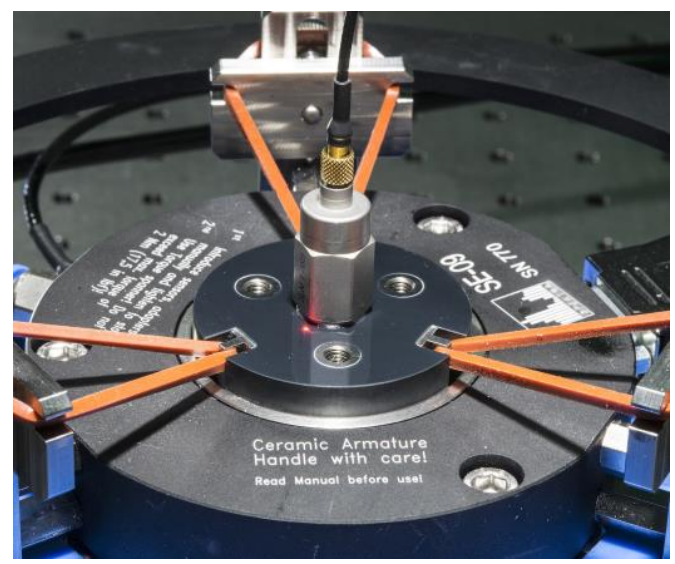

Figure 6. Ceramic shaker for calibration by laser vibrometry. Note the vibrometer laser spot next to accelerometer.

The vibrometer is mounted directly above the accelerometer on a suspended mount on an isolation platform. The vibrometer mounting hardware includes a positioning goniometer to allow for alignment of the position of the laser at multiple, repeatable, and unique reference positions on the shaker's mounting table. Measurements are made at multiple - a minimum of three locations around the accelerometer and this data is averaged to obtain final calibration results. Figures $7 \mathrm{a}$ and $7 \mathrm{~b}$ show calibration measurements for two different single-ended accelerometers, comparing the results using the NIST laser sine-approximation method that uses the dual-coil shaker described above with results on the new laser vibrometer system. The vibrometer is

programmed to also use Method 3, sine-approximation for calibration.
Improvements in shaker design and laser vibrometers have provided more precise calibration of accelerometers. Calibrations can be performed at frequencies from below $1 \mathrm{~Hz}$ to greater than $20 \mathrm{kHz}$ with improved uncertainty and improved efficiency. Figure 6 shows a commercial ceramic shaker with low distortion and low cross-axis motion, with an accelerometer mounted for calibration.

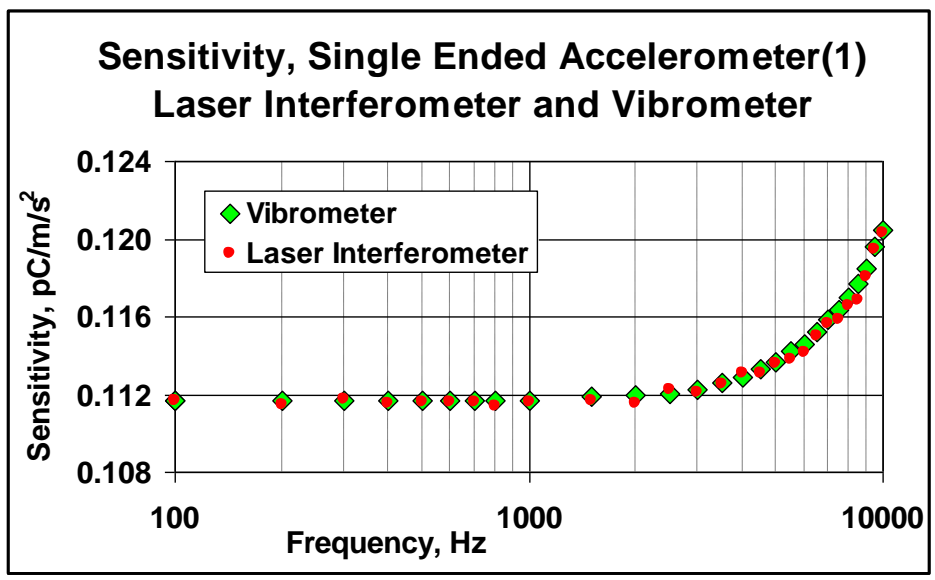

\section{Difference (\%) Laser Interferometer, Vibrometer} Single Ended Accelerometer (1)

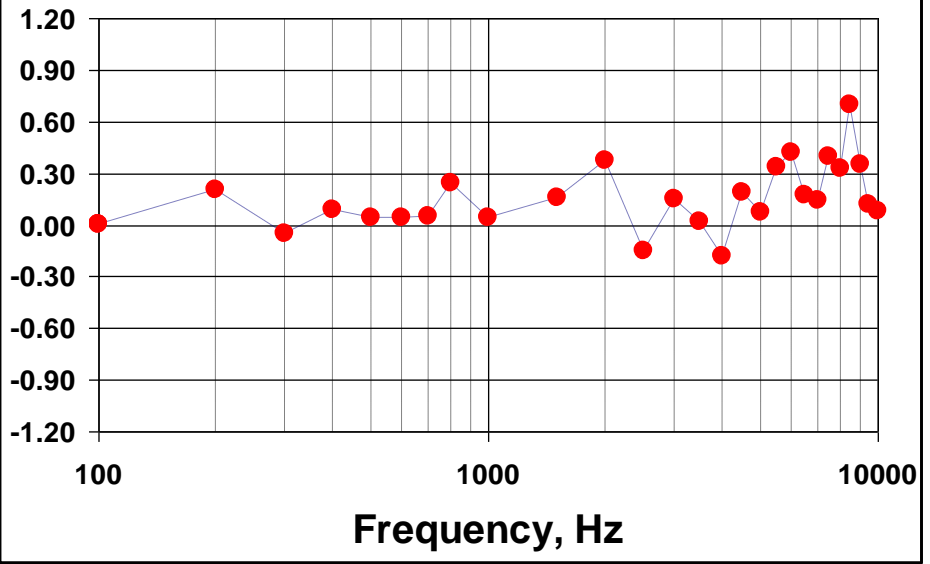

Figure 7a. Calibration results for single ended Accelerometer 1. 

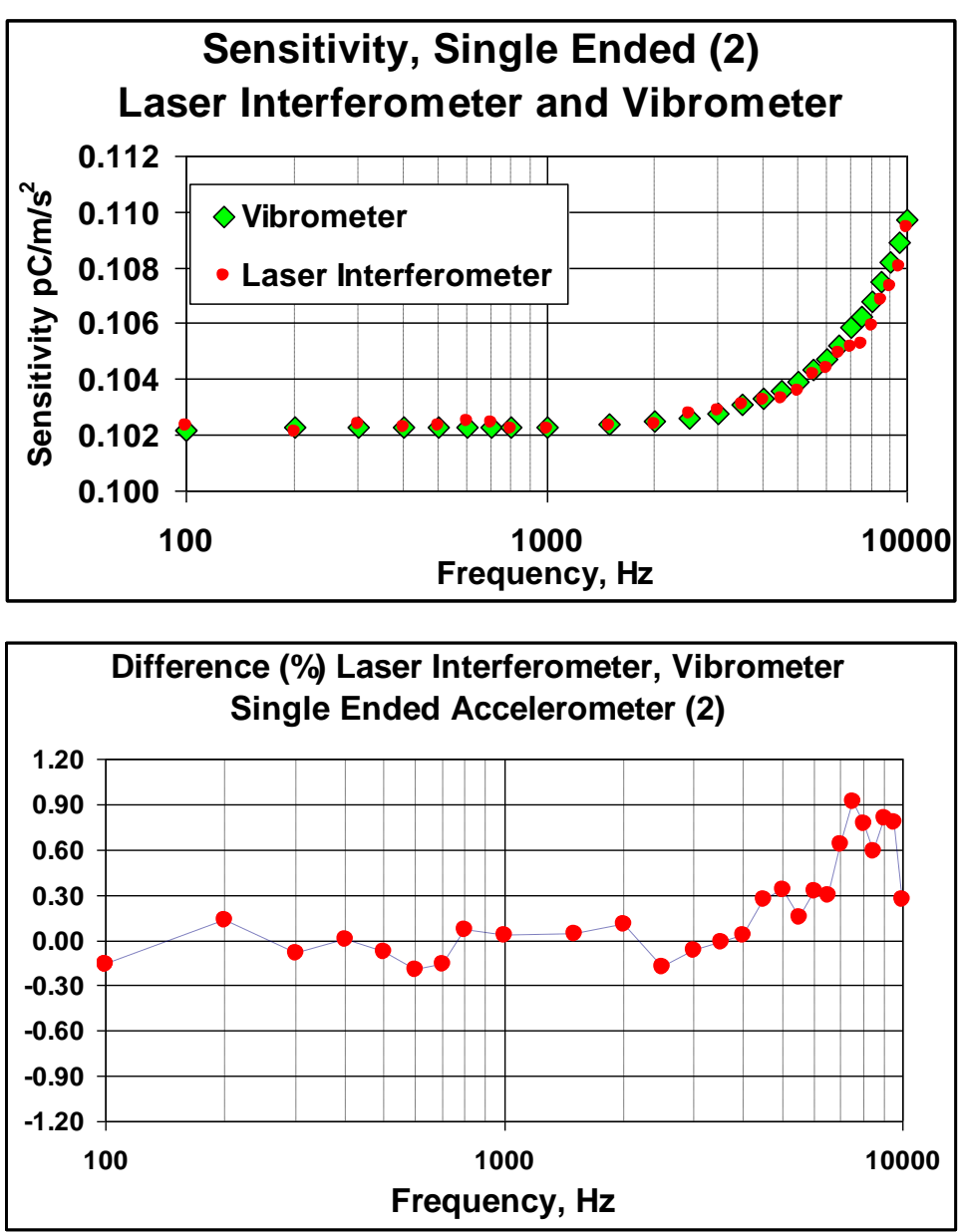

For the test results shown in Figure 7, the combined relative uncertainty was calculated in accordance with methodologies described in the Guide to the Expression of Uncertainty in Measurement [6] using Type A and Type B evaluations of uncertainty components, including those contained in ISO 16063 Part 11 on the calibration of vibration and shock transducers [6]. Using a coverage factor of 2, the estimated expanded relative uncertainty, $U$, at $1 \mathrm{kHz}$ is $0.3 \%$.

Figure 7b. Calibration results for single ended Accelerometer 2. 


\section{SUMMARY}

The updated vibration calibration system, using ISO 16063-11, Method 3, sine approximation implemented by means of the heterodyne (laser vibrometer) provides many advantages for routine calibration of high precision accelerometers, including

1. Calibration over a wide amplitude and frequency range, $10 \mathrm{~Hz}$ to $20 \mathrm{kHz}$ on the high frequency shaker, and $<0.5 \mathrm{~Hz}$ to $40 \mathrm{~Hz}$ for a low frequency shaker;

2. Automated digital system for ease of use;

3. Accommodates single-ended and 'back-to-back' sensors as well as end and side cable connections;

4. Calibration system provides for easy rotation of vibrometer to conveniently allow calibration at multiple points on the shaker table or on the top surface of the "back-toback' accelerometers; and

5. Significantly reduces calibration time and cost to customers.

This configuration also can measure the phase, and NIST is working to develop an uncertainty budget for this quantity. Once this is complete, phase will be reported alongside the sensitivity in accelerometer calibrations.

\section{REFERENCES}

1 Standard ISO 16063-12, "Primary vibration calibrations by Reciprocity," International Organization for Standardization, Geneva Switzerland, 1999.

2 B. Payne and J. Steffen, "Primary Calibration of Accelerometers by Optical Methods, NIST and USAF," Proceedings SAVIAC, $81^{\text {st }}$ Annual Shock \& Vibration Symposium, Oct 25-29, 2010, Orlando, FL

3 B. Payne, and D. Evans, "Comparison of Results of Calibrating the Magnitude of the Sensitivity of Accelerometers by Laser Interferometry and Reciprocity," Metrologia, 1999, 36, 391-394

4 International Standard ISO 16063-11,"Primary vibration calibrations by laser interferometry," International Organization for Standardization, Geneva Switzerland, 1999.

5 D.C. Robinson, M.R. Serbyn, B. F. Payne, A Description of NBS Calibration Services in Mechanical Vibration and Shock, NBS Technical Note 1232, 1987.

6. "Guide to the Expression of Uncertainty in Measurement", $2^{\text {nd }}$ Ed., International Organization for Standardization, Geneva, Switzerland, ISBN 92-10188-9 (1995). 


\title{
ANALYSYS AND PROTOCOL FOR CHARACTERIZING INTRINSIC PROPERTIES OF THREE-AXIS MEMS ACCELEROMETERS USING A GIMBAL ROTATED IN THE GRAVITATIONAL FIELD
}

\author{
Michael Gaitan, Muhammad Yaqub Afridi, and Jon Geist
}

National Institute of Standards and Technology, USA, gaitan@ nist.gov

\begin{abstract}
Three-axis MEMS accelerometers are typically characterized in terms of their cross-sensitivity matrix. We present an analysis and protocol to characterize them in terms of their intrinsic properties, which we define as the responsivity of each accelerometer along its axis of maximum response in a gravitational field and the angles between each of these axes. An analysis and test protocol is developed to determine these properties using a gimbal to rotate each axis of the device orthogonally to the gravitational field. We propose that this approach is better suited for laboratory inter-comparisons.
\end{abstract}

Keywords: MEMS, Microelectromechanical Systems, Accelerometer, Intrinsic, Calibration

\section{INTRODUCTION}

The MEMS and Sensors Industry Group (MSIG) has been engaged in the development of standards for defining sensor performance that are unique to MEMS technologies since 2009. The production of MEMS-based sensors, including accelerometers, gyroscopes, magnetometers, and pressure sensors, has been increasing dramatically since their introduction into automobiles and smart phones. The industry group recognized the inconsistency of how device manufacturers defined performance in data sheets was potentially leading to loss of efficiency, adoption by customers, and time to market as new generations of devices became available.

MISG worked with the IEEE Standards Association to publish IEEE P2700 Standard for Sensor Performance Parameter Definitions [1] in 2014. This standard defines accelerometer sensitivity, for example, as the change in acceleration input corresponding to one least significant bit change in output. MEMS-based devices typically provide digital output of the measurand, and this fundamental characteristic is what sets apart the IEEE P2700 standard for other similar standards.

With performance parameters defined, the next step is to develop testing protocols to measure them. MEMS device manufacturers typically calibrate their accelerometers by flipping them over, and often called the flip test. Three-axis accelerometers require a more refined approach as can be seen in [2]. The results are typically represented by a crosssensitivity matrix.

$$
\mathcal{P}=\left[\begin{array}{lll}
\rho_{x x} & \rho_{x y} & \rho_{x z} \\
\rho_{y x} & \rho_{y y} & \rho_{y z} \\
\rho_{z x} & \rho_{z y} & \rho_{z z}
\end{array}\right]
$$

where R is the cross-sensitivity matrix and,

$$
\left[\begin{array}{l}
a_{x} \\
a_{y} \\
a_{z}
\end{array}\right]=\left[\begin{array}{lll}
\rho_{x x} & \rho_{x y} & \rho_{x z} \\
\rho_{y x} & \rho_{y y} & \rho_{y z} \\
\rho_{z x} & \rho_{z y} & \rho_{z z}
\end{array}\right]\left[\begin{array}{l}
R_{x}-O_{x} \\
R_{y}-O_{y} \\
R_{z}-O_{z}
\end{array}\right]
$$

$a_{\mathrm{x}}, a_{\mathrm{y}}, a_{\mathrm{z}}$, represent the acceleration vector, $R_{\mathrm{x}}, R_{\mathrm{y}}, R_{\mathrm{z}}$, represent the output of each of the accelerometers, and $O_{\mathrm{x}}$, $O_{\mathrm{y}}, O_{\mathrm{z}}$, represent each of the offsets of the accelerometers.

This representation is useful for practical applications but may not be useful for measurement inter-comparisons. The cross-sensitivity matrix has dependence on how the device is mounted since the output of each of the axes of the three-axis accelerometer are modeled to be aligned with the coordinate system of the measurement instrument and it is further assumed that they are perfectly orthogonal to each other.

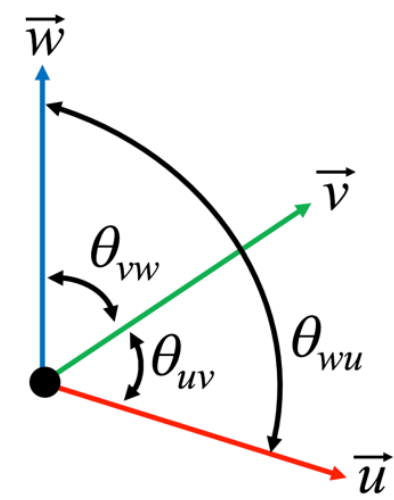

Figure 1 Model of a three-axis accelerometer with axes $\mathrm{u}, \mathrm{v}$, and $\mathrm{w}$ corresponding to the $\mathrm{x}, \mathrm{y}, \mathrm{z}$ coordinate system. 


\section{INTRINSIC PARAMETERS}

We define parameters as intrinsic to signify that they are independent to the orientation in which the device is mounted in a package, device, or measurement apparatus. We model the 3-axis accelerometer as having 3 axes that each point in their direction of maximum response and are not assumed to be perfectly orthogonal, as depicted in Figure 1.

The vectors $\boldsymbol{u}, \boldsymbol{v}$, and $\boldsymbol{w}$ correspond to the magnitude and direction of the responsivity for each of the three accelerometers. The variables $\theta_{u v}, \theta_{v w}$, and $\theta_{w u}$ represent the angles between the vectors $\boldsymbol{u}, \boldsymbol{v}$, and $\boldsymbol{w}$, respectively.

Next, the three-axis accelerometer is assumed to be mounted inside of a package or, for example, a product such as a smart phone, which we will hence forth refer to as the device under test (DUT), as depicted in Figure 2.

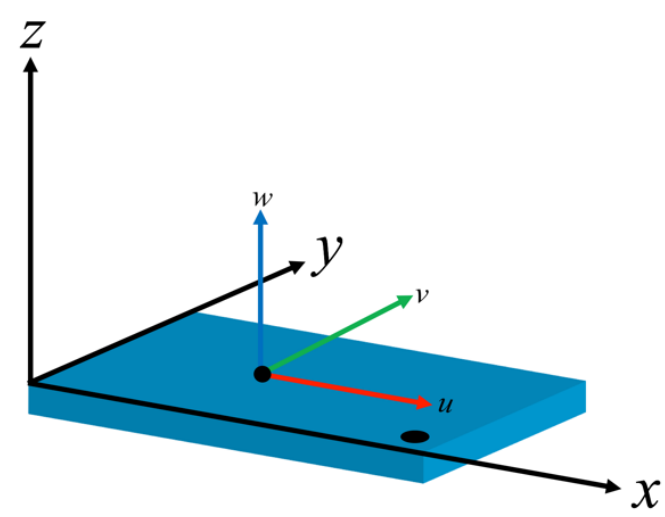

Figure 2 Model of the device under test (DUT) that incorporates the three-axis accelerometer as depicted in Figure 1.

The three-axis accelerometer is not expected to be perfectly aligned to the $\mathrm{x}, \mathrm{y}$, and $\mathrm{z}$ axes of the Cartesian coordinate system defined by the DUT. It follows from the diagram above that the response of the $\mathrm{u}, \mathrm{v}$, and $\mathrm{w}$ accelerometers can be written as:

where the unit vectors $\mathbf{i}, \mathbf{j}, \mathbf{k}$ point in the direction of the $\mathrm{x}, \mathrm{y}$, $\mathrm{z}$ directions of the Cartesian coordinate system.

\section{ANALYSIS AND PROTOCOL}

We present a protocol to determine the responsivities of each of the accelerometers $\mathrm{u}, \mathrm{v}$, and $\mathrm{w}$, and the angles between them, $\theta_{u v}, \theta_{v w}$, and $\theta_{w u}$. Each axis of the DUT (x', $\left.y^{\prime}, z^{\prime}\right)$ is aligned to be perpendicular to the direction of the gravitational field while the device is rotated around that
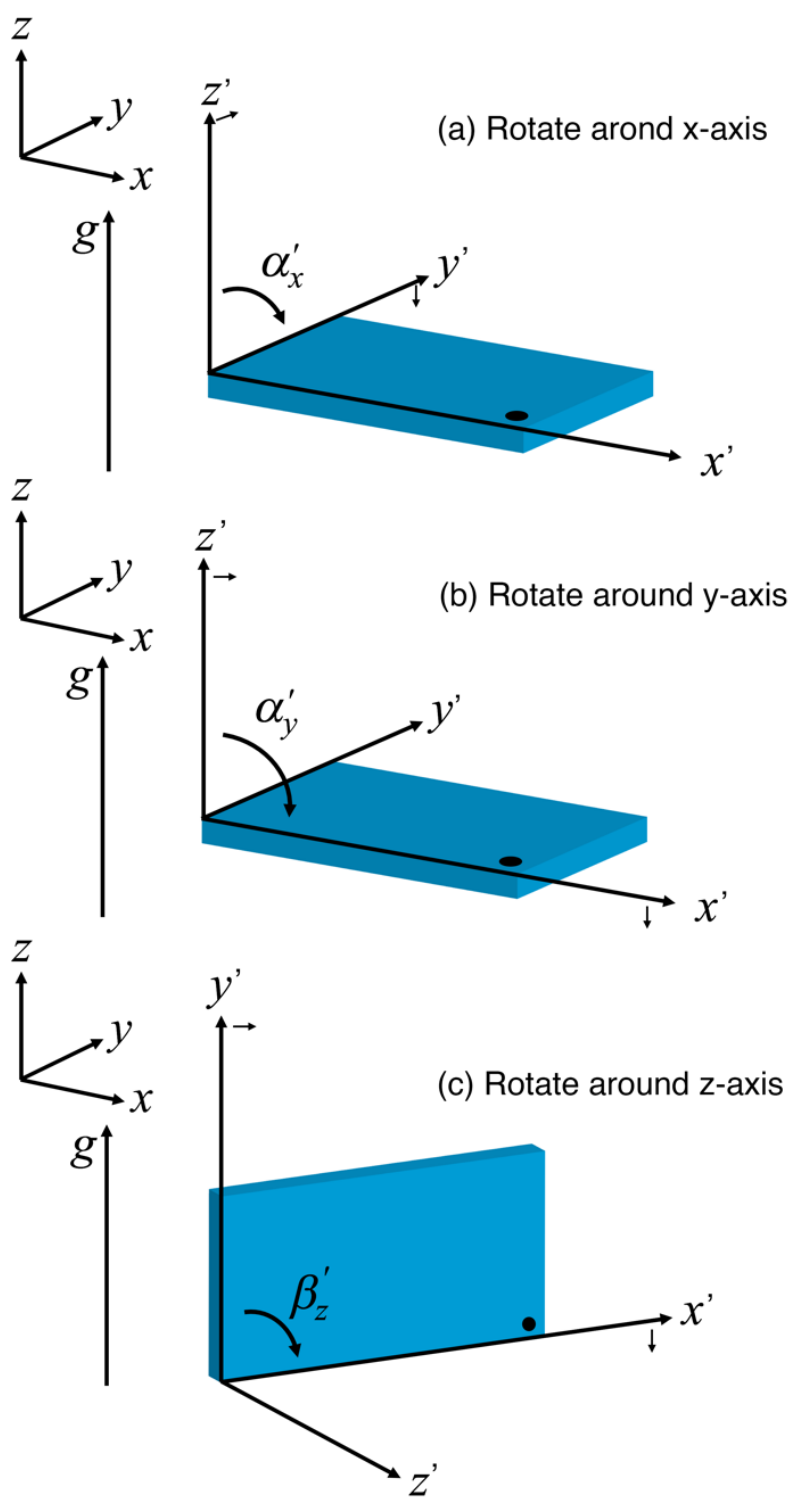

Figure 3 Protocol for rotation of the accelerometer around each axis of the device under test (DUT). The vector $\mathbf{g}$ represents the magnitude and direction of the earth's gravitational field.

axis using a gimbal. This procedure is depicted in Figure 3 as having 3 parts: (a) rotation around the $x$ axis, (b) rotation around the $y$ axis, and (c) rotation around the $\mathrm{z}$ axis of the DUT.

$$
\begin{aligned}
& \boldsymbol{u}=u_{x} \mathbf{i}+u_{y} \mathbf{j}+u_{z} \mathbf{k} \\
& \boldsymbol{v}=v_{x} \mathbf{i}+v_{y} \mathbf{j}+v_{z} \mathbf{k} \\
& \boldsymbol{w}=w_{x} \mathbf{i}+w_{y} \mathbf{j}+w_{z} \mathbf{k}
\end{aligned}
$$

Now, considering $R_{u}, R_{v}, R_{w}$ to be the output measured from each of the accelerometers, the rotation of the DUT around each axis as described previously and the resulting measurements tabulated in increments of $360^{\circ} / \mathrm{N}$ for $\mathrm{n}=1$ $\ldots \mathrm{N}$ results [3] in the following set of equations: 


$$
\begin{aligned}
& \mathrm{R}_{\mathrm{u}}\left(\alpha_{\mathrm{xn}}\right)=\mathrm{u}_{\mathrm{y}} \mathrm{g} \sin \left(\alpha_{\mathrm{xn}}\right)+\mathrm{u}_{\mathrm{z}} \mathrm{g} \cos \left(\alpha_{\mathrm{xn}}\right)+\mathrm{O}_{\mathrm{ux}} \\
& \mathrm{R}_{\mathrm{v}}\left(\alpha_{\mathrm{xn}}\right)=\mathrm{v}_{\mathrm{y}} \mathrm{g} \sin \left(\alpha_{\mathrm{xn}}\right)+\mathrm{v}_{\mathrm{z}} \mathrm{g} \cos \left(\alpha_{\mathrm{xn}}\right)+\mathrm{O}_{\mathrm{vx}} \\
& \mathrm{R}_{\mathrm{w}}\left(\alpha_{\mathrm{xn}}\right)=\mathrm{w}_{\mathrm{y}} \mathrm{g} \sin \left(\alpha_{\mathrm{xn}}\right)+\mathrm{w}_{\mathrm{z}} \mathrm{g} \cos \left(\alpha_{\mathrm{xn}}\right)+\mathrm{O}_{\mathrm{wx}} \\
& \mathrm{R}_{\mathrm{u}}\left(\alpha_{\mathrm{yn}}\right)=\mathrm{u}_{\mathrm{x}} \mathrm{g} \sin \left(\alpha_{\mathrm{yn}}\right)+\mathrm{u}_{\mathrm{z}} \mathrm{g} \cos \left(\alpha_{\mathrm{yn}}\right)+\mathrm{O}_{\mathrm{uy}} \\
& \mathrm{R}_{\mathrm{v}}\left(\alpha_{\mathrm{yn}}\right)=\mathrm{v}_{\mathrm{x}} \mathrm{g} \sin \left(\alpha_{\mathrm{yn}}\right)+\mathrm{v}_{\mathrm{z}} \mathrm{g} \cos \left(\alpha_{\mathrm{yn}}\right)+\mathrm{O}_{\mathrm{vy}}
\end{aligned}
$$

$$
\mathrm{R}_{\mathrm{w}}\left(\alpha_{\mathrm{yn}}\right)=\mathrm{w}_{\mathrm{x}} \mathrm{g} \sin \left(\alpha_{\mathrm{yn}}\right)+\mathrm{w}_{\mathrm{z}} \mathrm{g} \cos \left(\alpha_{\mathrm{yn}}\right)+\mathrm{O}_{\mathrm{wy}}
$$$$
\mathrm{R}_{\mathrm{u}}\left(\beta_{\mathrm{zn}}\right)=\mathrm{u}_{\mathrm{x}} \mathrm{g} \sin \left(\beta_{\mathrm{zn}}\right)+\mathrm{u}_{\mathrm{y}} \mathrm{g} \cos \left(\beta_{\mathrm{zn}}\right)+\mathrm{O}_{\mathrm{ux}}
$$$$
\mathrm{R}_{\mathrm{v}}\left(\beta_{\mathrm{zn}}\right)=\mathrm{v}_{\mathrm{x}} \mathrm{g} \sin \left(\beta_{\mathrm{zn}}\right)+\mathrm{v}_{\mathrm{y}} \mathrm{g} \cos \left(\beta_{\mathrm{zn}}\right)+\mathrm{O}_{\mathrm{vx}}
$$$$
\text { R }(R)-w r \sin (R) \perp w r a n c(R) \perp \cap
$$

where $\mathrm{O}_{\mathrm{u}}, \mathrm{O}_{\mathrm{v}}, \mathrm{O}_{\mathrm{w}}$ are the offsets of the $\mathrm{u}, \mathrm{v}, \mathrm{w}$ accelerometers, respectively,

$$
O_{u}=\sqrt{O_{u x}^{2}+O_{u y}^{2}+O_{u z}^{2}}
$$

(5)

Equation (4) represents 9 sets of $\mathrm{N}$ equations, which can be solved for the unknowns $\mathbf{u}, \mathbf{v}, \mathbf{w}$, and $\mathbf{O}$ by standard least squares methods to determine the intrinsic properties of the three-axis accelerometer.

The maximum responsivities of the three accelerometers, are determined separately by,

$$
\begin{aligned}
& u=|| \overrightarrow{\mathrm{u}}||=\sqrt{u_{x}^{2}+u_{y}^{2}+u_{z}^{2}} \\
& v=\|\vec{v}\|=\sqrt{v_{x}^{2}+v_{y}^{2}+v_{z}^{2}} \\
& w=\|\vec{w}\|=\sqrt{w_{x}^{2}+w_{y}^{2}+w_{z}^{2}}
\end{aligned}
$$

and the angles between the accelerometer axes are determined by,

$$
\begin{aligned}
& \theta_{u v}=\arccos \left(\frac{u_{x} v_{x}+u_{y} v_{y}+u_{z} v_{z}}{u v}\right) \\
& \theta_{v w}=\arccos \left(\frac{v_{x} w_{x}+v_{y} w_{y}+v_{z} w_{z}}{v w}\right) \\
& \theta_{w u}=\arccos \left(\frac{w_{x} u_{x}+w_{y} u_{y}+w_{z} u_{z}}{w u}\right)
\end{aligned}
$$

\section{CONCLUSION}

An analysis and protocol was presented to determine the intrinsic properties of a three-axis accelerometer, which we define as the responsivity of each accelerometer along its axis of maximum response in a gravitational field and the angles between each of these axes. The often used crosssensitivity matrix is dependent on how the device is mounted on the measurement instrument and, though it has been found useful in practical applications, we propose that the intrinsic properties that we present here are more useful for laboratory inter-comparisons for SI realization of acceleration units.

\section{REFERENCES}

[1] Standard for Sensor Performance Parameter Definitions, IEEE Standard P2700-2014.

[2] M. Pedley, "High-Precision Calibration of a Three-Axis Accelerometer", FreeScale Application Note AN4388 Rev. 2.0, (2015).

[3] J. Geist, M. Y. Afridi, C. D. McGray, and M. Gaitan, Gravity-based characterization of three-axis MEMSaccelerometers in terms of intrinsic accelerometer parameters, NIST Journal of Research, in press. 


\title{
Reference Materials to Enable Precise and Accurate Imaging with Electrical Scanning Probe Microscopes
}

\author{
J. J. Kopanski, L. You, J. Corona, and Y. S. Obeng \\ National Institute of Standards and Technology \\ Engineering Physics Division \\ 100 Bureau Dr., stop 8120 \\ Gaithersburg, MD 20899-8120
}

\section{INTRODUCTION}

Electrical scanning probe microscopes (eSPMs), such as the scanning Kelvin force microscope (SKFM), scanning capacitance microscope (SCM), or various scanning microwave microscopes (SMMs) are sensitive to the electric field between the sample and tip. Interpretation of measurements with these techniques can be confounded due to unknown tip shape and volume of interaction with the sample. Any two-terminal electrical measurement of electric field, capacitance, resistance, or inductance depends on the shape of the electrodes at each terminal. For simple onedimensional metal-insulator capacitors, $\mathrm{C}=\varepsilon_{0} \varepsilon_{\mathrm{i}} \mathrm{A} / \mathrm{t}_{\mathrm{i}}$ where $\mathrm{C}$ is capacitance, $\varepsilon_{\mathrm{o}}$ is the dielectric constant, $\varepsilon_{\mathrm{i}}$ the insulator dielectric constant, $A$ is the device area, and $t_{i}$ is the insulator thickness. For one-dimensional resistors, $R=\rho L / A$, where $\mathrm{R}$ is the resistance, $\rho$ the material resistivity, and $\mathrm{L}$ the device length. Without knowing the device geometries, the material electrical properties (in these examples, $\varepsilon_{\mathrm{i}}$ and $\rho$ ) cannot be deduced regardless of how accurately the capacitance or resistance is measured. For complex electrode shapes varying in three dimensions, such as eSPMs, direct extraction of material properties is impossible without detailed information about the shape of the electrodes.

This work describes simulation, design and preliminary measurements of reference materials with precisely known geometries that will allow precisely calculable electric field gradients at a sub-micrometer scale to be generated. These structures have two intended uses: 1) as reference materials to determine the actual spatial resolution and accuracy of various eSPM techniques sensitive to electric field with different conductive tip technologies; and 2) as an electrical tip shape profiler. Electrical tip shape may vary significantly from physical tip shape. Knowledge of the electrical tip shape can then be fed back into image analysis software to improve the spatial resolution and accuracy of electric field measurements.

\section{COMSOL MODEL OF ELECTRICAL SPMS}

The COMSOL ${ }^{1}$ MultiPhysics simulation software was used to simulate the electric field for candidate test structures. The COMSOL AC/DC Module combined with the RF Module allows simulation of high-frequency eSPMs such as SCM and SMM [1]. Many types of scanning probe microscope tips are integrated with conducting cantilevers and other conducting structures. For electrical measurements, all conducting parts of the probe assembly must be considered. We developed a complete COMSOL model of the SPM tip assembly to include the tip, the cantilever, the tilt of the cantilever with respect to the sample, and an arbitrary test structure (consisting of metal layers at defined bias voltages, ground planes, and dielectrics), Fig. 1. This model allows us to simulate the measured electrical potential between the defined test structure electrode and the defined probe electrode as a function of the terminal tip location.

${ }^{1}$ Certain commercial equipment, instruments, or materials are identified in this paper in order to adequately specify the experimental procedure. Such identification does not imply recommendation or endorsement by NIST, nor does it imply that the materials or equipment used are necessarily the best available for the purpose.

Kopanski, Joseph; Obeng, Yaw; You, Lin. 


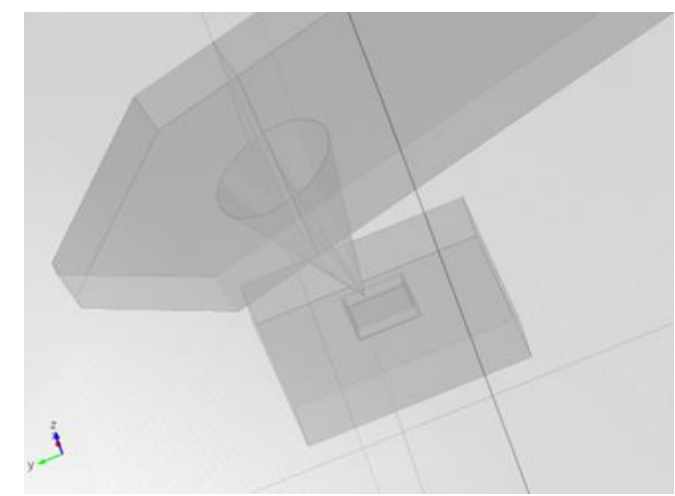

Figure 1: COMSOL generated image of the simulation geometry. A round base, conical tip $(10 \mu \mathrm{m}$ in height $)$, the conductive cantilever, and a two-line test structure are shown.

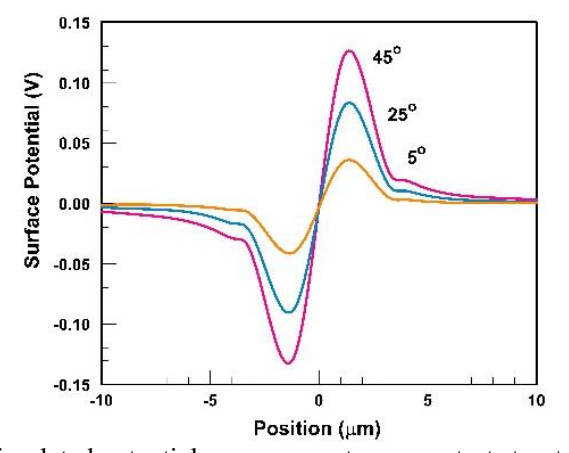

Figure 2: Simulated potential measurement across a test structure consisting of two lines, with the left line at negative potential and the right line at positive potential. The width of the response is related to the top cone width, while the slope of the response at the boundaries is inversely proportional to cone angle $\left(5^{\circ}, 25^{\circ}\right.$ or $\left.45^{\circ}\right)$.

A basic eSPM tip shape of a round cone is parameterized by the terminal tip radius, $r_{\text {tip }}$, the tip cone angle, $\Theta$, the tip height (terminal tip to cantilever distance), $h$, and the top cone radius, $r_{\text {top }}$. For a perfect cone normal to the sample surface, the tip would be completely described by a single value of these four parameters (with $r_{\text {top }}=h \tan (\Theta)$ ). This model can be extended to simulate real eSPM tips that are not perfect cones (many are pyramidal), include some degree of asymmetry, and are used with their principle axis of symmetry tilted with respect to the sample surface. The physics of the tip-test structure interactions requires a dense mesh near the terminal tip, but also consideration of the long range contribution of all the parts of the test structure and the larger cantilever assemble. Many runs of the COMSOL model were require to determine a set of boundary conditions adequate to efficiently simulate the measurement while maintaining a realistic spatial domain and calculation time.

\section{ELECTRICAL TIP PROFILER DESIGN STUDY}

COMSOL simulations of the measured potential between a conical tip and simple test structures (generating an abrupt boundary in surface potential) revealed that both the cone angle and the top radius effect the measured surface potential in systematic ways, Fig. 2. The top radius broadens the measured response in direct proportion to the width of the tip as it crosses the boundary (Fig. 3a). Likewise, the slope of the response is inversely proportional to the cone angle at the point of maximum signal change (Fig. 3b). These results suggest a method of determining the tip parameters and asymmetry of an unknown tip from measurements on a test structure. If we consider the tip as an n-sided pyramid, with each side having an independent slope, then we can measure the width of the top of the pyramid and the slope of the side when that side cross the boundary in potential generated by the test structure. We suspect that most tips can be well characterized by four scans across the boundary with a $45^{\circ}$ rotation between scans. While this approach will not be able to identify convex or concave tip sides, it will determine an equivalent tip shape (that is, the model tip parameters that produce a response equivalent to the tip under interrogation.) If the height of the tip is known by other methods, a complete equivalent tip shape can be determined and a figure of merit (measured field / actual field) for electrical profiling specified. The measured electrical tip parameters can then be used in a reverse model to deduce actual surface potential arising from structures whose geometry is not well known.

\section{ELECTRIC FIELD MEASUREMENTS}

The simulations have allowed us to design test structures capable of determining the electrical tip shape of eSPM tips. A simple biased line is sufficient if: 1) The line width is greater than the top radius of the tip, 2) regions beyond the active area are covered with a ground place, and 3) spacing of the active region to the ground plane is kept small. From a practical viewpoint a square test structure is preferable. With his geometry, the desired four angles of attack can be acquired from two images, one with the cantilever sides orthogonal to the directions of scan and a second with the sample-cantilever geometry rotated by $45^{\circ}$. A series of designed square test structures using this approach are shown in Fig. 4. As a preliminary test of our model, we imaged a wide aluminum line [2] with SKFM using a Point Probe Plus conducting eSPM tip. These tips have a symmetric orientation (left-to-right across the cantilever) and an asymmetric orientation (front-to-back along the cantilever). Figure 5 shows two line scans 
across the aluminum-to-oxide interface. When scanned with the symmetric tip profile parallel to the $\mathrm{Al}$ line, a symmetric contact potential difference response is obtained. When scanned with the asymmetric tip profile parallel to the $\mathrm{Al}$ line, an asymmetric response is obtained with the highly asymmetric side of the tip generating a kink in the CPD response as it passes over the Al-to-oxide interface.

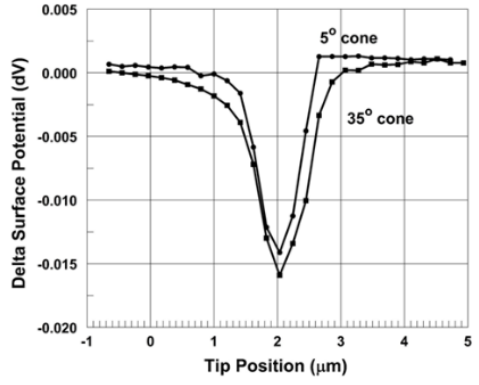

Figure 3a: Simulated potential measurement across a test structure consisting of a single biased line, surrounded by grounded planes on either side for conical tips with $5^{\circ}$ and $35^{\circ}$ cone angles. The broadening of the response provides a measurement of the top cone radius.

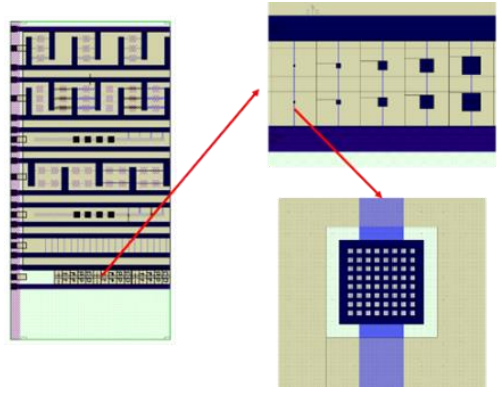

Figure 4: Section of test chip design with progressive enlargements showing the location of various sized 2-D electric tip profiler artefacts. Largest magnification shows buss structure, mid magnification shows different size 2D tip profilers, and highest magnification shows details of vias within a single profiler structure.

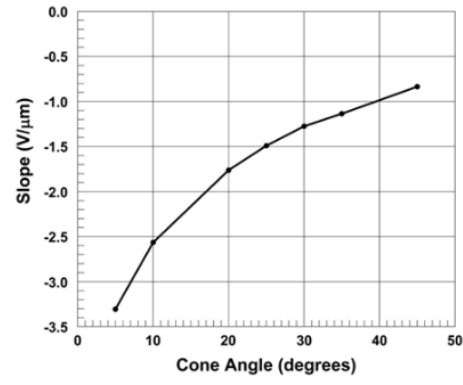

Figure 3b: Summary of the slopes at the boundary of simulated potential measurements across a test structure consisting of a single biased line, surrounded by grounded planes on either side for conical tips with cone angles of $5^{\circ}, 10^{\circ}, 20^{\circ}, 30^{\circ}, 35^{\circ}$, and $45^{\circ}$. Measured slope is proportional to cone angle.

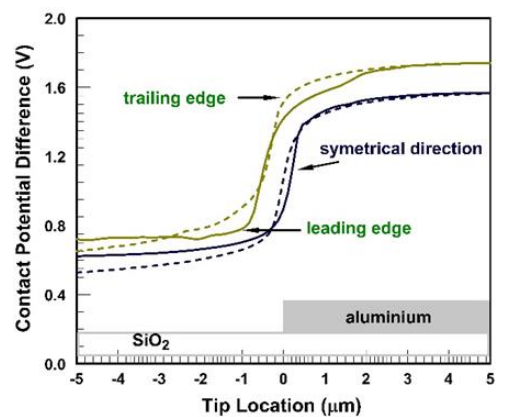

Figure 5: Measured surface potential across a biased metal line extracted from a SKFM image of a single metal line. The tip location data from the leading edge has been flipped and aligned with the trailing edge data for easier comparison. In the symmetric (purple lines) direction the tip (and the measured response) is broader. In the asymmetric direction, the response differs depending on whether the leading edge or trailing edge encounters the Al-to- $\mathrm{SiO}_{2}$ boundary first. Symmetric data was offset for clarity

\section{CONCLUSIONS}

In order to obtain accurate electric field based eSPM measurement results, or even to evaluate accuracy, it is necessary to have common calibration standards, ideally leading to SI traceability. COMSOL simulations show the electrical tip shape influences electric field based eSPM measurements in systematic ways. SKFM measurements with real tips on simple structures verify that such influences are real and can be experimentally measured. We have designed test structures that will amplify these effects and allow the tip electrical shape to be extracted.

\section{REFERENCES}

1. J. J. Kopanski, L. You, J. Li, J. Ahn, and Y. S. Obeng, ECS Transactions 72(2), pp. 131 -138 (May 2015).

2. L. You, J.J. Ahn, E, Hitz, J. Michelson, Y. Obeng, and J. J. Kopanski, Proc. 2015 IEEE Intl. Conf. Microelectronic Test Structures, pp. 235-239, Tempe, AZ (March 23-26, 2015).

\section{KEYWORDS}

Electric field measurement, scanning Kelvin force microscope, scanning probe tip profiler, reference materials

Kopanski, Joseph; Obeng, Yaw; You, Lin

"Reference Materials to Enable Precise and Accurate Imaging with Electrical Scanning Probe Microscopes."

Paper presented at the 2017 International Conference on Frontiers of Characterization and Metrology for Nanoelectronics,

Monterey, CA. March 21, 2017 - March 23, 2017. 


\title{
STUCK IN A MOMENT: A VIEW FROM THE MIRE
}

\author{
Patrick Egan, Jack Stone, Jacob Ricker, and Jay Hendricks \\ National Institute of Standards and Technology \\ 100 Bureau Drive, Gaithersburg, MD 20899
}

\section{INTRODUCTION}

The next-generation pressure standards will be realized via gas density and the equation of state. One way to access the density is through a measurement of gas refractivity, underpinned by the theoretical calculations that predict the relationship between density and refractivity. At present, calculations with sufficient accuracy that link refractivity to density are only available for helium. To measure helium refractivity we employ interferometry to make ultraprecise measurements of the optical length of gas-filled cells and cavities. The refractivity of helium at atmospheric pressure is about $3.2 \times 10^{-5}$ : to measure this to $10^{-6}$ fractional uncertainty-our goal for a pascal realization-would require the measurement of a $15 \mathrm{~cm}$ optical length with $4.5 \mathrm{pm}$ accuracy.

We are pursuing two approaches to the measurement of refractivity: a Fabry-Perot (FP) cavitybased system with four interferometers, which we call a variable-length optical cavity (VLOC), and a cell-based heterodyne interferometer, which we call a monolithic interferometer for refractometry (MIRE). Both these optics have been built to precise geometric constraints by silicate-bonding.

The VLOC will measure refractivity as the difference between two $15 \mathrm{~cm}$ displacements, one in vacuum and one in helium (where gas pressure and temperature are kept constant during the motion). In actuality we have three interferometers in vacuum surrounding a central interferometer in helium. The main challenge was to minimize the Abbe error: the Abbe offset in this measurement is the deviation of the central interferometer mode from the geometric center of the outer three interferometer modes. In order to minimize the Abbe error, the VLOC has been built with an Abbe offset of less than $70 \mu \mathrm{m}$. This feat means that keeping the Abbe error below $1 \mathrm{pm}$ will require measurement and control of angle to better than 20 nrad, which is relatively straightforward given the picometer resolution of FP interferometry. Another feature of the VLOC is that it relies on the flatness of an optical flat to ensure parallel beams in multiple optical cavities and minimize errors as- sociated with beam walk across imperfect optical surfaces. This requires that local surface slopes have a variation ideally held to less than $1 \mu \mathrm{rad}$, where the slopes of interest are on a spatial scale of the cavity mode size (sub-millimeter). Many high-precision optics-including optics with better than $\lambda / 100$ figure-may not achieve this specification.

In the case of the MIRE, refractivity will be measured as the change in pathlength through a gas cell, as the cell is filled from vacuum to some pressure. One benefit of a $25 \mathrm{~cm}$ multipass gas-cell compared to a $15 \mathrm{~cm}$ FP cavity is that it has less stringent requirements on the accuracy and stability of the length metrology, and the fact that the experiment can be performed without motion is a great simplification; on the other hand, a heterodyne interferometer has much lower resolution, and cell window thinning caused by changing pressure can contribute a systematic error of several hundred parts per million. To cancel this systematic error we plan to make refractivity measurements through cells of different lengths but with almost identical material properties, borehole and window geometries, and beam incidence. This last requirement-beam incidencehas motivated us build an interferometer that has four beams parallel to two planes within $\pm 85 \mu \mathrm{rad}$.

This proceeding describes the features of both optics,-VLOC and MIRE-the geometric requirements that had to be met to achieve our desired measurement precision, and the steps we have taken to build the optics and satisfy these geometric requirements.

\section{VLOC}

A general overview assembly of the VLOC is shown in Fig. 1, and the principle behind the measurement is described in more detail in Ref. [1]. At the heart of the device are four Fabry-Perot (FP) cavities, formed on two optics, a parallel mirror plate and a concave mirror assembly. A photograph of the optics is shown in the inset of Fig. 1: three outer FP cavities in vacuum surround an inner FP cavity which is to be sealed in a bellows and filled with helium (the bellows is not shown 


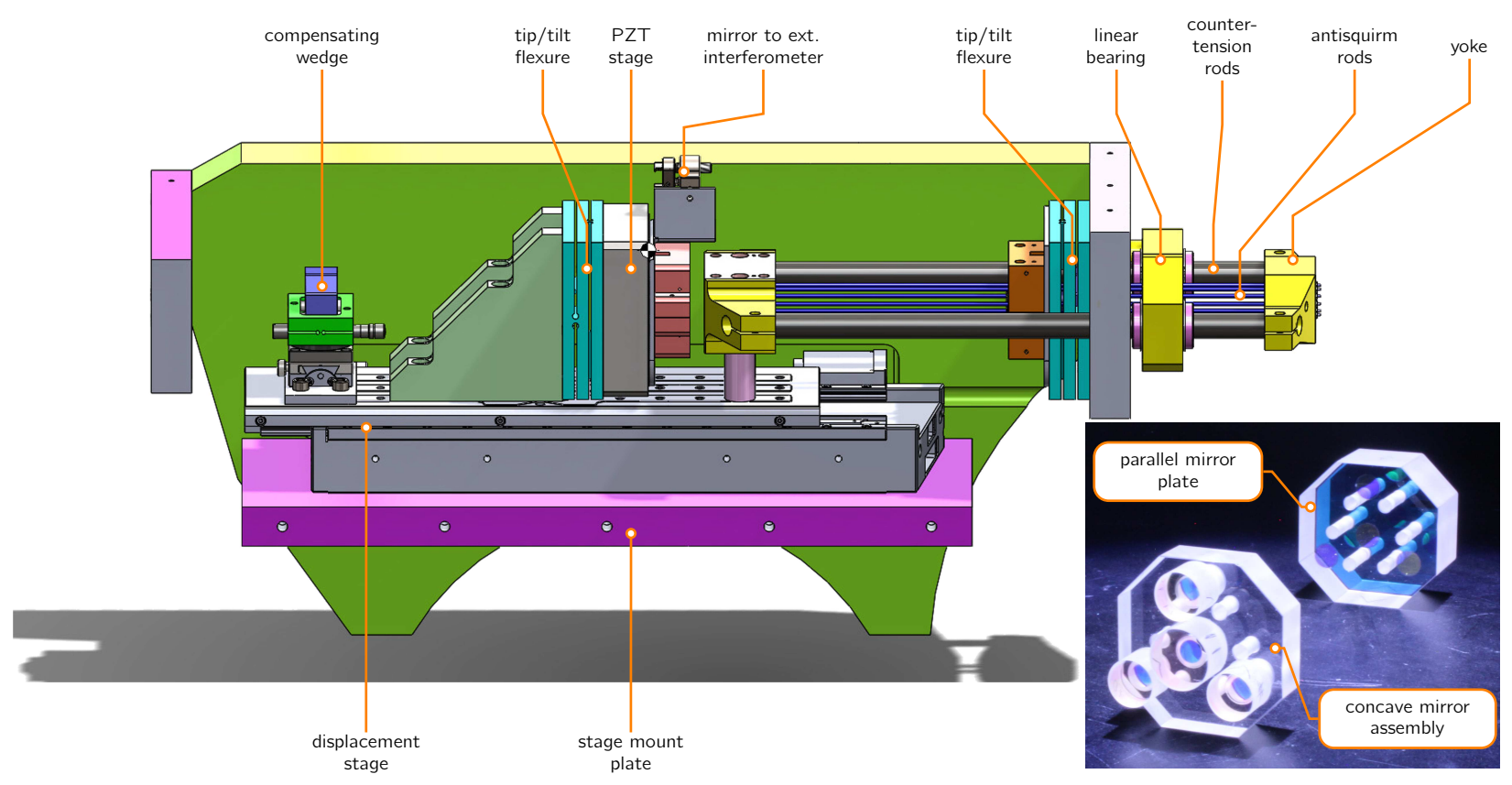

FIGURE 1. The VLOC apparatus consists of a fixed parallel mirror plate inside the tip/tilt flexure at right, and a moving concave mirror assembly inside the PZT stage at left. The displacement stage gives $15 \mathrm{~cm}$ of travel, while the PZT stage makes fine adjustments to maintain angular alignment below 20 nrad at the opposite ends of the travel. The inset photograph shows the interferometer optics: concave mirror assembly and parallel mirror plate. The concave mirror assembly has four mirrors silicate-bonded to precise geometric constraint. The span of the octagons are $38 \mathrm{~mm}$.

in Fig. 1). The octagonal shape of the VLOC optics owes its origin to alternative design scenarios that envisioned the bellows sealed to the glass with an o-ring and clamped at four corners; the design we are currently pursuing has the bellows transitioned to glass, and the glass monolithically bonded to the octagon. The six $3.2 \mathrm{~mm}$ diameter holes around the center of the octagons are for (tensioned) bellows antisquirm rods: in the mechanicals of Fig. 1, these holes are redundant on the concave mirror assembly.

The concave mirror assembly is housed in the PZT stage which sits on a linear translation stage. In the typical mode of operation the inner cavity will be filled to about $10^{5} \mathrm{~Pa}$ of helium, and its length is to be changed from $15 \mathrm{~cm}$ to $30 \mathrm{~cm}$ by the translation stage. (The pressure of helium inside the bellows will be generated by a piston gage: the pressure will be unknown, but constant to $10^{-6} \cdot p$ independent of the volume change.) The outer cavities in vacuum will measure the displacement $\Delta L$, and also monitor pitch/yaw errors in the motion: the PZT stage will be adjusted to correct these motion errors to below 10 nrad. The inner cavity in helium will measure a change in optical length of $n \Delta L$, and thus the difference between the displacements in the outer and inner cavities is a measure of refractivity $n-1$. This measure of helium refractivity at a known temperature can be used to realize the pascal, as discussed for example in Refs. [2, 3]. In other words, we will use the measured helium refractivity to determine the unknown pressure generated by the piston gage.

These refractivity measurements are expected to happen in the near future. In addition to pitch/yaw at sub-10 nrad, we will also be monitoring straightness/flatness at sub- $1 \mu \mathrm{m}$ : the metrology supporting this apparatus is a large undertaking and will not be described here. The purpose of this section is to describe the characteristics of the interferometer optics at the heart of the VLOC.

\section{Parallel mirror plate}

The parallel mirror plate consists of four mirrored portions, $(6.5 \pm 0.5) \mathrm{mm}$ in diameter, coated on a $20 \mathrm{~mm}$ thick, flat substrate. The HR coating was specified as $99.7 \%$ at $633 \mathrm{~nm}$ and $1542 \mathrm{~nm}$. (The mirrored portions were masked so that the bellows to glass transition can be bonded and sealed 
to bare glass on the parallel mirror plate.) Since the mirrors are deposited directly on the plate, the plate had to be wedged at 9 mrad to avoid parasitic reflections entering the cavities (an extra precaution in addition to the AR coating on the back-surface). Because we intend to translate the concave mirror assembly by $\Delta L=15 \mathrm{~cm}$, motion errors during translation will inevitably cause beam walk across the parallel mirror plate, and if the mirrored portions are out-of-parallel by $\phi$, this beam walk effectively introduces an alignment error between the interferometers approximately $n \Delta L\left(\frac{\phi^{2}}{2}+\theta \phi\right)$ [1]. Here, $\theta$ is the misalignment of the central mode direction with the direction of the displacement of the moving mirrors, and $\theta+\phi$ is the misalignment of one of the outer inteferometers. Typically, $\theta$ is much larger than $\phi$. It will vary with position due to the stage straightness (about $2 \mu \mathrm{m}$ ) and due to roll ( $30 \mu \mathrm{rad})$, so that it is typically larger than $7 \mu \mathrm{rad}$ regardless of how well overall alignment is achieved. The modes of the three outer interferometers are on a "bolt circle" of $13 \mathrm{~mm}$ radius, and the mode diameter is about $0.3 \mathrm{~mm}$ : the specification requested of the manufacturer was that all four mirrored regions were to be parallel to $1 \mu \mathrm{rad}$, and that surface spatial variations were to be less than $1 \mathrm{~nm} / \mathrm{mm}$. It is notable that a $\lambda / 100$ surface flatness would not necessarily satisfy this unique parallelism requirement. The manufacturer achieved the $1 \mu \mathrm{rad}$ parallelism before coating, but stresses induced by the masked coatings changed surface form. The parallel mirror plate was subsequently measured at NIST with a Fizeau interferometer. In Fig. 2 we show surface flatness for each of the outer mirrors relative to the central mirror. The slope of the coatings are within $2 \mu \mathrm{rad}$ of parallel (ie, $\phi=2 \mu \mathrm{rad}$ ) to one another, which means that misalignment errors between central and outer interferometers should contribute less than $2 \mathrm{pm}$ error to the displacement measurement. (Interestingly and not apparent in Fig. 2, is that the slope of the coatings relative to the substrate is $15 \mu \mathrm{rad}$, and the height of the mirror stacks are about $6.5 \mu \mathrm{m}$.)

\section{Concave mirror assembly}

The concave mirror assembly is a complicated optic. Its main requirement was to maintain a very stable relationship between the inner and outer mirrors, and also to keep the Abbe offset below $100 \mu \mathrm{m}$. For the symmetric case, this is equivalent to saying that the centers of curvature of the three outer mirrors must be located on a circle whose center is concentric within $100 \mu \mathrm{m}$ of

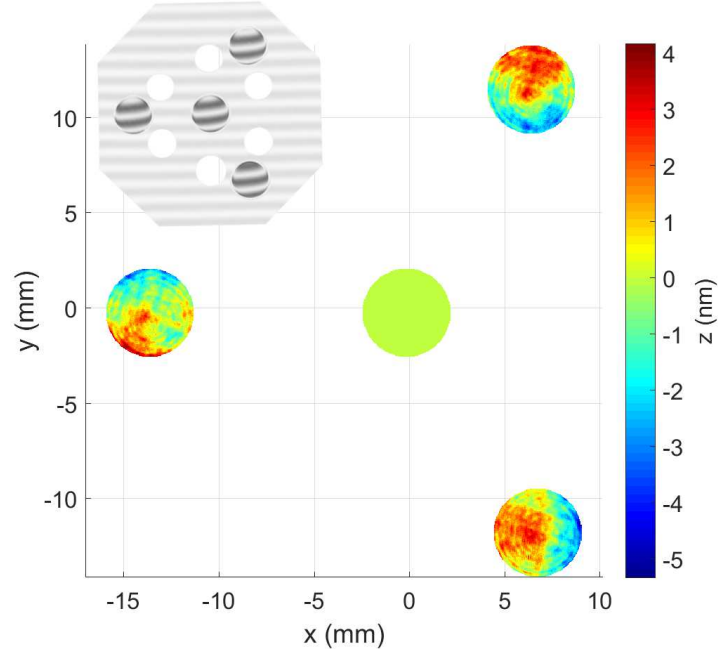

FIGURE 2. Profile of the four HR-coated regions of the parallel mirror plate: The slopes of the three outer coated areas relative to the central coated area are within $2 \mu \mathrm{rad}$. Inset: fringes on the Fizeau flatness interferometer.

the center of curvature of the central mirror. To our knowledge it is not presently feasible to polish multiple concavities into one substrate and meet this $100 \mu \mathrm{m}$ concentricity requirement, so positioning and bonding four separate mirrors to one baseplate seemed the viable solution. Additionally, the central interferometer (and the bond between the optics and the bellows-to-glass stub) needs to be leak-tight, and any bonding technique must be ultrastable. These requirements led us to conclude that silicate-bonding [4] was the best (if not only) solution.

The optic consists of a $10 \mathrm{~mm}$ thick baseplate (parallel window) with four $6 \mathrm{~mm}$ holes drilled through it to allow beam transmission. The four concave mirrors were face-bonded to the parallel window using silicate-bonding, following the LIGO recipe [5]: we used a $4: 1$ ratio of distilled water to sodium-silicate solution, approximately $0.8 \mu \mathrm{L} / \mathrm{cm}^{2}$ solution per bond area, and all surfaces were flat to $\lambda / 10$. Our approach of face-bonding four separate concave mirrors to a flat baseplate means that a flat annulus must remain on the substrate into which the concavity is polished. It is also desirable that the annulus of the central mirror be as large as possible to minimize leak rates. These requirements must be put in context to a size constraint of $38 \mathrm{~mm}$-square which was placed on our baseplate by the aperture of the PZT stage: all this meant that our inner mirror substrate had a diameter of $13.5 \mathrm{~mm}$ and 

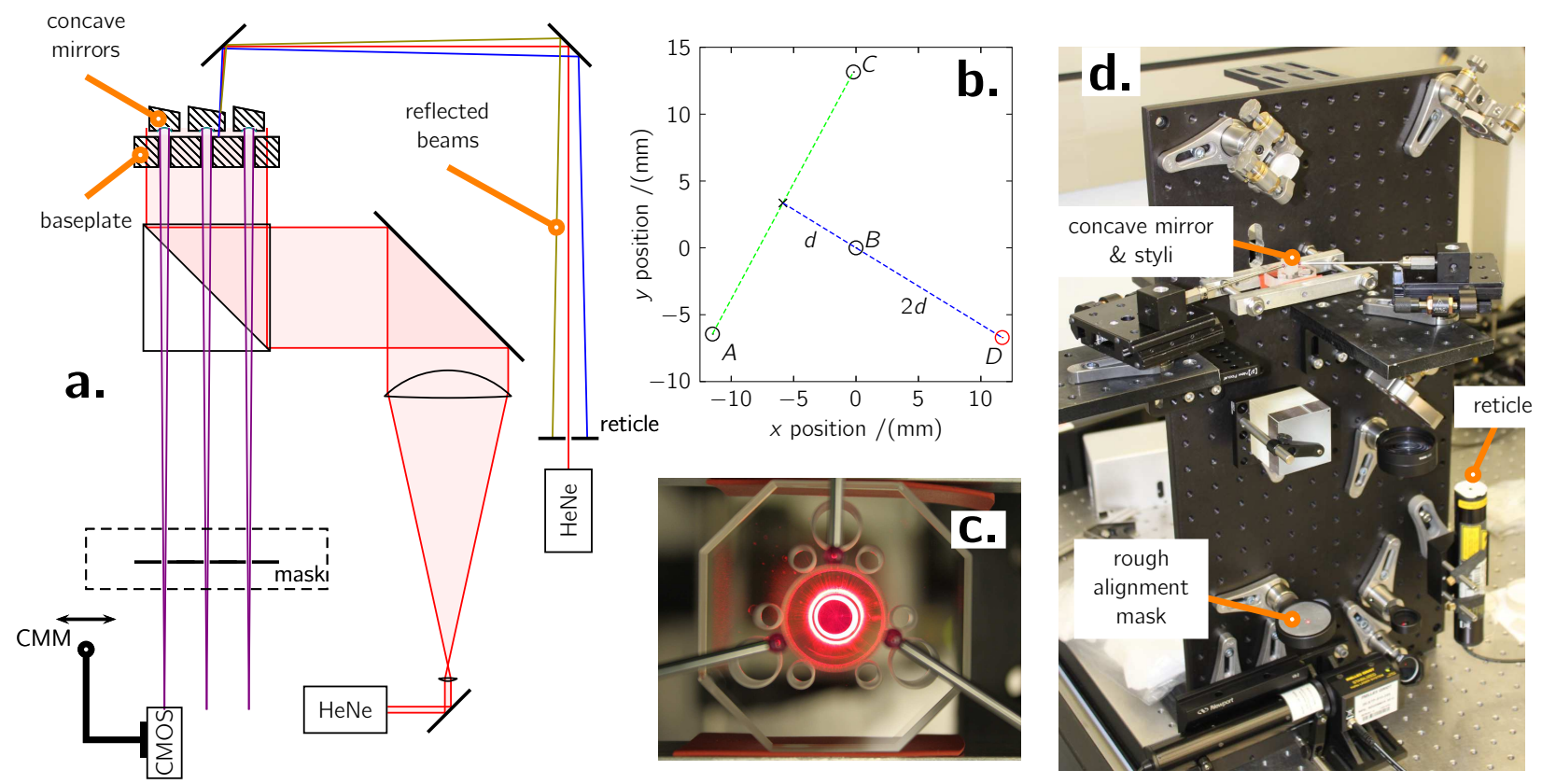

FIGURE 3. (a) Schematic of the jig used for positioning and bonding the concave mirror assembly. (b) The position of the third outer mirror $D$ was found as a projection of the distance $2 d$ through the center $B$ from the midpoint of the line $A C$ : the points $A, B, C, D$ refer to the locations of the mirror focal points. (c) Photograph of center mirror located by ruby styli while bonding. For scale, the diameter of the center mirror is $13.5 \mathrm{~mm}$. (d) The actual jig being used for bonding with the rough alignment mask. To bond the final mirror, the mask was removed, and a camera attached to the ram of a CMM probed the mirror focal points.

the outer mirrors had diameters of $10.5 \mathrm{~mm}$; we specified a diameter on the extent of the concave polish of $(7 \pm 1) \mathrm{mm}$. The sagitta of a $0.5 \mathrm{~m}$ arc extending $7 \mathrm{~mm}$ is only $3 \mu \mathrm{m}$, and not only was our tolerance on polish depth/diameter challenging, the best that could be done with centering the polish relative to the mirror substrates was $\pm 1 \mathrm{~mm}$. The lack of concentricity in the concave mirrors meant that we could not use the mirror substrate as a dimensional reference: we had to position and bond the mirrors while looking at their actual focal points. Also note: The lack of concentricity between the concave polish and the coating mask meant that the focal point was the only point at which we could reliably gage the relative locations of the four interferometer modes. (The coating stack was also undersized relative to the concave polish, so as to permit face bonding between the baseplate and outer annulus of the mirror substrate.)

The principle behind assembling the optic was to nudge the mirrors around so that the locations of their focal points met the near zero-Abbe offset requirement. For nudging the mirrors we used three ruby styli mounted on manual translation stages, shown in Fig. 3(c). In practice and for the most reliable bonding, the assembly proceeded by first bonding the inner mirror and two of the outer mirrors in a cleanroom with rough alignment, and then bonding the last outer mirror in a lab with a coordinate-measuring machine (CMM) that told us precisely where the focal point had to be. Our assembly jig, shown in Figs. 3(a) and (d), worked as follows: We used a $\times 300$ beam expander and HeNe laser to produce a collimated beam of about $100 \mathrm{~mm}$ diameter, and this extra large beam was directed to the baseplate. (As mentioned previously, the separation between the outer mirrors is $26 \mathrm{~mm}$, so $50.8 \mathrm{~mm}$ optics offered a large enough aperture.) When a concave mirror was placed on the baseplate, its coating reflected a very small part of the large collimated beam and focused it to a point at its focal length. For coarse positioning of the first three mirrors in the cleanroom, we used an aluminum plate as a rough alignment mask, which had $0.2 \mathrm{~mm}$ diameter holes drilled in locations where the centers of curvature were nominally supposed to be; we bonded the inner and two outer mirrors so that three beams reflected and focused from these mirrors passed through the holes in the mask. 
This rough alignment and bonding was judged by eye, and is shown in process in Fig. 3(d).

For ease and convenience, we did not aim to achieve an equilateral triangle with the focal points of our first three coarsely positioned mirrors, and therefore lacking symmetry, the zeroAbbe position of the third outer mirror does not follow the concentric circle geometry previously mentioned: instead, as in Fig. 3(b), the third outer mirror was positioned a precise distance along a line from the mid-point of the line between the two outer mirrors projected through the point of the central mirror. When positioning the final mirror the coarse alignment mask was removed, and we probed the focal point of beams reflected from the mirrors with a camera mounted to a CMM. [For reference, an analogous setup with a camera on a CMM is shown in Fig. 4(c).] The positional uncertainty of the CMM was $2 \mu \mathrm{m}$. We used the centroid of the camera image as the reference, and moved the camera (ram of CMM) so that the centroid of each beam was referenced to the same pixel location: we had reproducibility of $6 \mu \mathrm{m}$ locating the focal points of the three coarsely positioned mirrors. As mentioned previously, there is a lack of concentricity between the concave polish and coated region, which meant that the relative separations of the reflected beams changed as a function of distance (typically less than $1.7 \mu \mathrm{m} / \mathrm{mm}$ ) and we had to translate the camera at the focal plane: we were confident of locating the focal plane to $3 \mathrm{~mm}$. Once the focal points of the three coarsely placed mirrors were found, the position of the third outer mirror was calculated, the camera moved to that calculated point, and the mirror was nudged to position and bonded so that its focal point was at the reference position on the camera. We achieved an Abbe offset of $62 \mu \mathrm{m}$.

A minor complication in the concave mirror assembly is that the concave mirrors are polished into wedged substrates to avoid parasitic cavities. Since the concave mirror assembly is the moving part of the VLOC, beam alignment and coupling into the cavities would be translation dependent. To mitigate this problem, we employ the compensating plate shown in Fig. 1, which has a wedge nearly identical to that of the concave mirror substrates but rotated in azimuth so that the two prisms effectively cancel. However, effective cancellation of the beam deviation demanded that we also ensure the rotation of each mirror- wedge orientation-was nominally the same. To achieve this, we used a second laser as a beam pointer, also shown in Fig. 3(a) and (d). For the bonding of each mirror, we directed the beam pointer so that it passed through the outer edge of the mirror substrate. This resulted in two reflections: one from the wedged back-surface of the mirror and one from the baseplate. (In actuality, the reflection from the baseplate overlapped the reflection from the front-surface of the mirror substrate-and this became a single reflection after bonding.) We positioned a reticle in front of the pointing laser: the reticle was simply a line on a piece of paper placed on the laser head (ie, the reflection was deliberately directed almost back into the laser tube). Our premise was that if each mirror was rotated such that the two reflections end up on the line of the reticle, then the wedge of each mirror was orientated the same. Our premise relied on the fact that the baseplate is flat, and that the reticle did not rotate between the bonding of each mirror. However, the approach was independent of the angle and relative location of the incident beam.

\section{MIRE}

MIRE is based on the classical Tanaka-style interferometer [6], in which we have a gas cell in the measurement arm and a vacuum cell in the reference arm. We actually implement this in a multipass scheme with a triple-cell, where either the central cell or the two outer cells can serve as a reference arm while the other serves as the measurement arm, as in Fig. 4(a). The body of the triple-cell is a single piece of borosilicate crown glass with three holes through it, and at each end a single piece of glass is silicate-bonded forming windows for the three holes: knowledge of cell length is a prerequisite for accurate refractivity measurements, and the thin and repeatable $(100 \pm 50) \mathrm{nm}$ bond interface allowed us to accurately measure cell length on a CMM before the windows were bonded. Unlike the VLOC, which is designed in such a manner that it can be operated at constant pressure, the critical measurand for the MIRE is a change in optical pathlength when gas is admitted to the measurement arm. Effects of pressure distortions [7] must be taken into account by building at least two such triplecells, one short and one long. This is a simpler alternative than a possible approach based on a variable length cell, analogous to the VLOC but with a moving window replacing the moving mirror, as discussed next. 
In most refractometers such as the MIRE, the reference and measurement beams pass through the same thickness of glass windows. This arrangement will largely compensate various effects including thermal variations of the windows and changing pathlengths that will occur if the window tilts. The compensation also will eliminate the effect of any uniform wedge in window thickness, which might otherwise cause an unacceptably large variation in pathlength accompanying even microscopic changes in where the beam passes through the window. However, the compensation will be imperfect due to non-flatness of window surfaces, and the situation for a variable length cell with a moving window then becomes entirely analogous to the problem of beam-walk across a non-flat mirror coating in the VLOC, where variations in surface slopes of the optical element between the positions of the measurement and reference beams will give rise to errors. As a practical matter the problem is somewhat more difficult to remedy for a cell-based approach than for the VLOC. For this reason and for reasons of mechanical simplicity, we completely abandon the moving window scheme with its requirement of having equal glass paths independent of cell length. Our approach is based on two (or more) fixed-length cells, where the window thicknesses will ideally be matched at the micrometer level but need not be matched at the picometer level! The critical measurement is not a change in pathlength for a known displacement but changes in pathlength when gas is admitted to the short and long cell. Under these circumstances the requirement that the window optical pathlength is independent of cell length is replaced by a requirement that, for any given fixed length, the variation of the window pathlength with cell pressure is the same for the short and long cells. This can be assured if the window does not move transversely to the beam when pressure is changed (beam-walk), and if window thinning due to the pressure change is the same for both cells. The second requirement is more demanding than the first but can be met if the dimensions and geometry of the windows are near-identical, if the mechanical and optical properties of the windows are near-identical (glass made from the same melt), and if the beams pass through all windows at approximately the same position relative to the underlying holes. This last requirement means that beams must be parallel to each other so that they pass through the same window position in cells of different length. We now describe how the interferometer was built, and the steps we have taken to ensure similar beam incidence between long and short triple-cells.

For the MIRE apparatus stability is a chief concern, and our requirement is rather challenging: a thermodynamic measurement like refractivity has a timescale of several hours $(0.1 \mathrm{mHz})$, and we need the interferometer to be stable below $100 \mathrm{pm}$ during the measurement. By comparison, if extrapolated to $0.1 \mathrm{mHz}$, the stability requirement of the LISA Pathfinder interferometer is $5 \mathrm{~nm}$ [8]. We follow the LISA approach for interferometer stability, and have all optics silicatebonded [4] to a $42 \mathrm{~cm} \times 15 \mathrm{~cm} \times 2.5 \mathrm{~cm}$ fused silica baseplate. A photograph and schematic of the baseplate part of the interferometer are shown in Fig. 4(a) and (b). All bonding surfaces were optically polished to $\lambda / 10$ flatness: the baseplate has $\lambda / 10$ local flatness over $6.5 \mathrm{~cm}^{2}$, and $3 \lambda$ global flatness. Most optics were bonded directly to the baseplate (fiber launch, polarizing-beamsplitters, mirrors, and Jamin-beamsplitter); whereas others were face-bonded to one another (polarizer and waveplates to polarizing-beamsplitters, collimating lenses to the fiber launch, and wedge prismpairs to a mount block). The face-bonding was done with uncoated surfaces, and we typically observe reflections less than $0.03 \%$ from the bond interface.

Fiber-launching (beam-pointing) began by inserting flat-polished and antireflection (AR) coated fiber ferrules into $20 \mu \mathrm{m}$ clearance holes which set the $22 \mathrm{~mm}$ separation between the beams. The ferrules were epoxied into this tight-fit, and the depth of insertion was adjusted to collimate the beams at $2.1 \mathrm{~mm}$ diameter. In process we found a shear plate and viewer the most practical method of judging collimation, and we subsequently used a wavefront sensor to measure beam divergences of $0.190 \mathrm{mrad}$ and $0.254 \mathrm{mrad}$, which are close to the 0.192 mrad of perfect collimation. Two aspheric lenses (plano-convex) were face-bonded along their $1 \mathrm{~mm}$ outer annulus to the fiber launch block, and were nudged into place with three ruby styli on micrometer stages to set the beams to be parallel. (The collimating lenses are AR-coated, and this outer annulus was the one bond that involved a coated surface.) We configured a CMM to translate a camera parallel to the polished baseplate surface, and beam-pointing was adjudged by looking at the image-centroid at near and far positions. See 

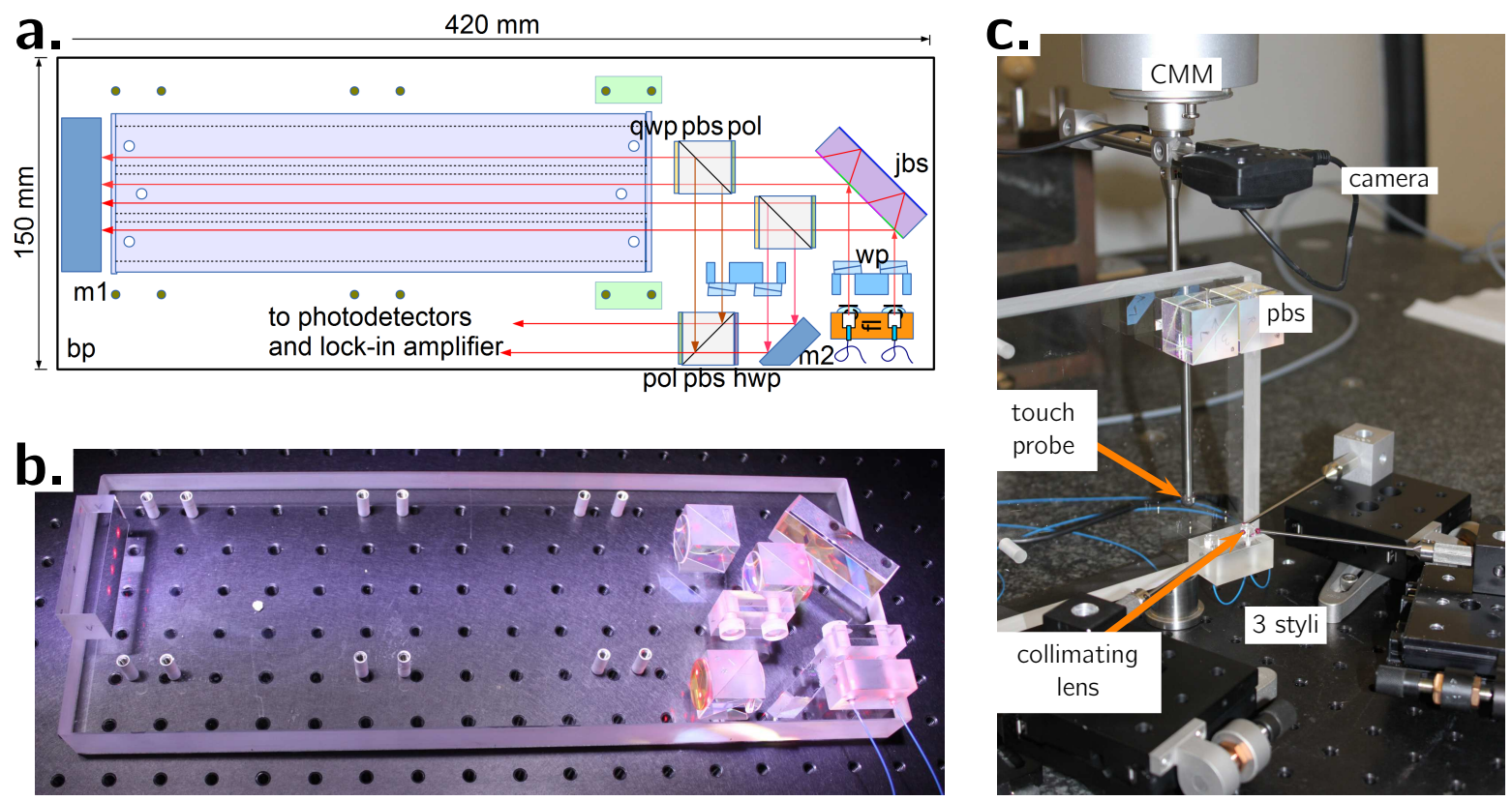

FIGURE 4. (a.) Schematic of the interferometer, with components drawn to scale. (b.) Photograph of MIRE, without gas triple-cell. Threaded invar inserts are epoxied into the baseplate to clamp the inlet/outlet o-ring flanges to the triple-cell. (c.) Setup to align beam-pointing. The collimating lens is positioned by three styli on translation stages. The camera on the CMM is vertically translated by about $20 \mathrm{~cm}$ : the camera measures the beam centroid at near and far positions. A touchprobe is used to build a coordinate system so that the camera runs parallel to a desired plane (eg, the plane of the polished baseplate).

Fig. 4(c) for how this setup looked in practice. We achieved beams out-of-parallel to one another by $(220 \pm 15) \mu \mathrm{rad}$. This residual error in beam alignment necessitated the 9 mrad wedge prism-pairs to further correct beam-pointing, a point we will return to momentarily.

After the fiber launch the two beams are separated into four by a Jamin-beamsplitter, which consists of alternating $0.25 \%$ and $50 \%$ reflectivecoated portions on its front surface and a $99.5 \%$ reflective-coating on its back surface; the parallelism on the Jamin-beamsplitter was $10 \mu \mathrm{rad}$, and its thickness sets the $12 \mathrm{~mm}$ reflected beam separation. The four beams reflected from the Jamin-beamsplitter are polarized with a $10^{5}$ extinction ratio, and a quarter-waveplate set the polarization state such that more than $99.3 \%$ of the beam returning from the fold-mirror was reflected by the polarizing-beamsplitter. The polarizingbeamsplitters are epoxy-free, and the transmitted beam deviation through the assemblies was as large as $250 \mu \mathrm{rad}$; which is why the aforementioned lens bonding was done with the beamsplitters temporarily in place, as shown in Fig. 4(c). The bonding surface of the fold-mirror and Jamin- beamsplitter were polished within $100 \mu$ rad of perpendicular to the coated surfaces. Two $0.4 \mathrm{~mm}$ diameter aperture jigs were located relative to the straightedge of the baseplate and temporarily tacked in place at the extremities between the Jamin-beamsplitter and eventual location of the fold-mirror: the Jamin-beamsplitter was nudged into position and bonded so as to maximize the intensity passing through both apertures on a large-area photodetector. At this point of the build, we returned to the CMM and locked-in the final beam-pointing with the wedge prismpairs. The CMM was configured to run parallel to the polished surface and straightedge of the baseplate: referencing beam alignment to both these planes is advantageous for reproducible positioning of triple-cells of different length. We achieved beam alignment parallel to these two planes within $\pm 85 \mu \mathrm{rad}$. The resolution of our beam-pointing setup was about $50 \mu \mathrm{rad}$, as determined by the $6 \mu \mathrm{m}$ reproducibility of the image centroid, the few micrometers form of the planes to which the CMM was configured to run parallel, and the $15 \mathrm{~cm}$ travel of the CMM axis. In hindsight, we believe an improvement factor of two to three could be gained with a more clever ar- 
rangement on the CMM (ie, more travel) and with wedge prism-pairs closer to $1 \mathrm{mrad}$ (our choice of $9 \mathrm{mrad}$ was based on what is available off-theshelf).

The aforementioned aperture jigs were once again tacked in place and referenced to the straightedge, and the fold mirror was nudged into position and bonded so as to maximize the intensity returning through the two apertures (as observed reflected by the polarizing-beamsplitters); this setup ensured that alignment of the returning beams were referenced to the baseplate straightedge within $100 \mu \mathrm{rad}$; subsequent positioning of the triple-cells (of different lengths) can initially be achieved by caliper measurements referenced to this straightedge, and reproducibility ensured by hard-stops (ie, spheres epoxied in place). The result of these efforts is that when we measure gas refractivity in triple-cells of different lengths, we are confident beam incidence is the same for all window pairs to within $0.2 \mathrm{~mm}$. After the beams are returned through the triple-cell and reflected from the beamsplitters, they are combined in a final halfwaveplate-beamsplitter-polarizer optic and sent to photodetectors, outside the vacuum chamber in which the MIRE sits. Before interference, we employed another wedge prismpair to optimize visibility: these prism-pairs are a minor weakness of the design, because the glass pathlength imbalance introduces a temperature dependent drift of about $26 \mathrm{pm} / \mathrm{mK}$; during refractivity measurements we typically have sub$\mathrm{mK}$ temperature stability. Differential phase is measured with a lock-in amplifier. As of writing, MIRE is demonstrating sub- $50 \mathrm{pm}$ stability over $15 \mathrm{~h}$ qupmy whwwhwip (min: $-44 \mathrm{pm}$, max: $45 \mathrm{pm}$, 600 -s averaging), which in a $12.5 \mathrm{~cm}$ long triplecell would correspond to a fractional error of less than $2 \times 10^{-6}$. $p$ for a pascal realization at $200 \mathrm{kPa}$. We are in the process of taking refractivity data in triple-cells of different lengths, and these measurements will be the subject of a future article.

\section{OUTLOOK}

We have described the precision engineering behind optics at the heart of two ultraprecise refractometers built at NIST, the VLOC and MIRE. The refractometers will be used to realize the pascal via measurements of gas density and the equation of state. We described some of the unique features of the VLOC optic, among which is a $2 \mu \mathrm{rad}$ parallelism between mirrored regions $26 \mathrm{~mm}$ apart, a $2 \mathrm{~nm} / \mathrm{mm}$ local flatness, and an
Abbe offset of $62 \mu \mathrm{m}$. The critical feature of MIRE is that, while keeping the build quasi-monolithic and ultrastable, two pairs of beams launched from two fibers have been aligned to two planes within $\pm 85 \mu \mathrm{rad}$ of parallel.

At the present time, MIRE is running and taking data, and the experiment will soon be described in more detail. The VLOC apparatus is nearing completion, and initial characterization tests are expected to begin late 2017 .

\section{REFERENCES}

[1] Stone J, Egan P, Hendircks J, Strouse G, Olson D, Ricker J, et al. Metrology for Comparison of Displacements at the Picometer Level. In: Precision Engineering and Nanotechnology V. vol. 625 of Key Engineering Materials. Trans Tech Publications; 2014. p. 79-84.

[2] Schmidt JW, Gavioso RM, May EF, Moldover MR. Polarizability of Helium and Gas Metrology. Physical Review Letters. 2007 Jun;98:254504.

[3] Puchalski M, Piszczatowski K, Komasa J, Jeziorski B, Szalewicz K. Theoretical determination of the polarizability dispersion and the refractive index of helium. Physical Review A. 2016 Mar;93:032515.

[4] Gwo DH. Ultra-precision bonding for cryogenic fused-silica optics. In: Heaney JB, Burriesci LG, editors. Cryogenic Optical Systems and Instruments VIII. vol. 3435. SPIE; 1998. p. 136-142.

[5] Armandula $\mathrm{H}$, van Veggel M. Silicate bonding procedure (hydroxide-catalysis bonding). LIGO Scientific Collaboration; 2010. Document \#: E050228-v2.

[6] Tanaka M, Yamagami T, Nakayama K. Linear interpolation of periodic error in a heterodyne laser interferometer at subnanometer levels. IEEE Transactions on Instrumentation and Measurement. 1989 Apr;38(2):552-554.

[7] Birch KP, Downs MJ, Ferriss DH. Optical path length changes induced in cell windows and solid etalons by evacuation. Journal of Physics E. 1988;21(7):690-692.

[8] Audley H, Danzmann K, Marín AG, Heinzel G, Monsky A, Nofrarias M, et al. The LISA Pathfinder interferometry: hardware and system testing. Classical and Quantum Gravity. 2011;28(9):094003. 


\title{
Assessing Quantitative Optical Imaging for Realizing In-die Critical Dimension Metrology
}

\author{
Bryan M. Barnes, Mark-Alexander Henn, Hui Zhou, Martin Y. Sohn, \\ and Richard M. Silver \\ Engineering Physics Division, National Institute of Standards and Technology, \\ Gaithersburg, MD 20899-8212 USA
}

\section{INTRODUCTION}

The need for accurate critical dimension (CD) measurements as dimensions decrease for semiconductor manufacturing is now more acute with the ubiquitous challenge of reconstructing 3-D nanostructures. While optical methods are uniquely positioned as the high-throughput solution for process control due to their relative high speed and low cost, methods for sidestepping optical diffraction limits must be further advanced to meet industry needs. Our group at the National Institute of Standards and Technology (NIST) has recently demonstrated the capability of measuring finite sets of features of $15 \mathrm{~nm}$-scale CD within a high-magnification imaging platform using $\lambda=450 \mathrm{~nm}$ light. This measurement technique requires a thorough understanding of the tool, rigorous electromagnetic modeling, as well as tailoring of the metrology target. This paper briefly reviews these recent accomplishments, target optimizations, and ongoing research into the challenges and extensibility of this scatterfield microscopy methodology.

\section{QUANTITATIVE PARAMETRIC FITTING VIA IMAGING}

Comparisons between experiment and simulation are made successfully in scatterometry for arrays of lines that are often larger than the incident spot size with pitches smaller than the wavelength. Much smaller line arrays (Fig. 1a) that would fit within the field-of-view of a microscope are desirable such targets will yield multiple scattering frequencies. These frequencies are inseparable within the real-space image, greatly complicating experiment-tosimulation comparisons. A new measurement approach has recently been published ${ }^{1}$ that describes the fitting of simulation to experiment for intensity profiles obtained through-focus; two key components are highlighted here. First, the comparison is enhanced by correcting known errors from the instrument through Fourier Domain Normalization. When simulating the scattered field, the individual scattering frequencies are separable for each incident plane wave. By mapping the empirical imperfections of the tool through tool functions ${ }^{2}$ (Fig. 1b), these known imperfections can be mapped upon each of the simulated, discretized Fourier components. By combining modified plane wave simulations to represent the finite aperture of the experiment, the resultant simulated image more closely takes on the imperfections of the experimental data.

Second, after investigating the possible Type "B" error components for the experimental data, potential error sources were determined to have correlated effects among the nominally independent measurements (the intensities at each pixel). These relationships were included within the covariance matrix (Fig. 1c) before performing the linear regression between simulated and experimental data to determine not only the best model parameter values but also their parametric uncertainties. Selected comparisons between Fourier Domain Normalized simulations and experimental profiles at several focus positions are shown in Fig. 1d. 

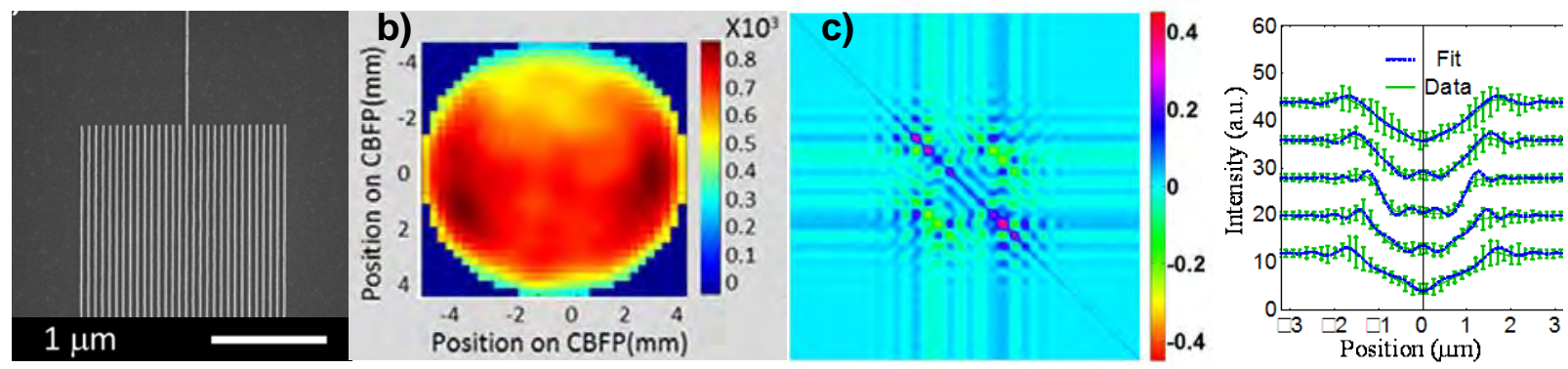

FIGURE 1. a) Scanning electron micrograph of a 30-line metrology target b) Example of an illumination path tool function (in arbitrary units) for one linear polarization. An aperture was rastered in the conjugate to the back focal plane (CBFP) of the objective lens and the light measured near the sample plane. Tool functions for the collection path were also required. c) Illustration of one part of an off-diagonal covariance matrix. d) Simplified example of theory to experiment fitting at five focus heights with multiple error bars removed for visual clarity. Actual fits were performed at 21 focus positions and two polarizations. Panels a), c) from Ref. 1, b) from Ref. 2.

\section{OPTIMIZATION VIA SIMULATIONS}

The metrology target in Fig. 1a has a patterned area of about $2 \mu \mathrm{m}$ width and $6 \mu \mathrm{m}$ length, excluding the protrusion for atomic force microscopy measurements. The total required area is also dependent upon the proximity of nearby objects, especially to the left and right of the target; objects should be at least $10 \lambda$ away from the sides of the target as shown in Fig. 2a. This observation is informed by simulations at $\lambda=450 \mathrm{~nm}$ for Ref. 1 of the target using rigorous coupled-wave analysis (RCWA), which implies a periodic structure. These 30-line targets were modeled as a repeating pattern of a finite set of lines surrounded by relatively vast unpatterned regions. The domain size was increased until the scattering from the target converged in amplitude, which occurred when the target was optically isolated from its periodic copies for a $10 \mu \mathrm{m}$ domain. Simulations at $193 \mathrm{~nm}$ reinforce this $10 \lambda$ observation.

The specific target shown in Fig. 2a is impractically sized then for insertion into the active region of a device. Through simulation, however, optimization of the size and design of the target has been performed in addition analysis of the number of arrayed lines and number of focus measurements required to yield acceptable parametric uncertainties ${ }^{3,4}$. The simulation study, unlike the fitting above, assumed a two-parameter model (height and midwidth) for simplicity and also application of a finite-element (FEM) Maxwell's equations solver to facilitate both twoand three-dimensional targets. It has been shown that using combinations of reduced line lengths and numbers of lines can potentially lead to a four-fold decrease in target area as compared to cutting-edge scatterometry targets ${ }^{4}$. In fact, as little as 10 lines can lead to uncertainties comparable to the ones obtained from measuring the larger 30-line target, see Fig. $2 \mathrm{~b}$. The restriction to less focus positions in the measurement can furthermore reduce the total time needed to acquire the necessary image data. Figure 2c shows little difference between taking images at four different focus positions and eleven focus positions. For favorable quantification of even smaller deep-subwavelength features, these optimization methods should be repeated to enable more thoughtful choices in geometrical layout and experimental design.
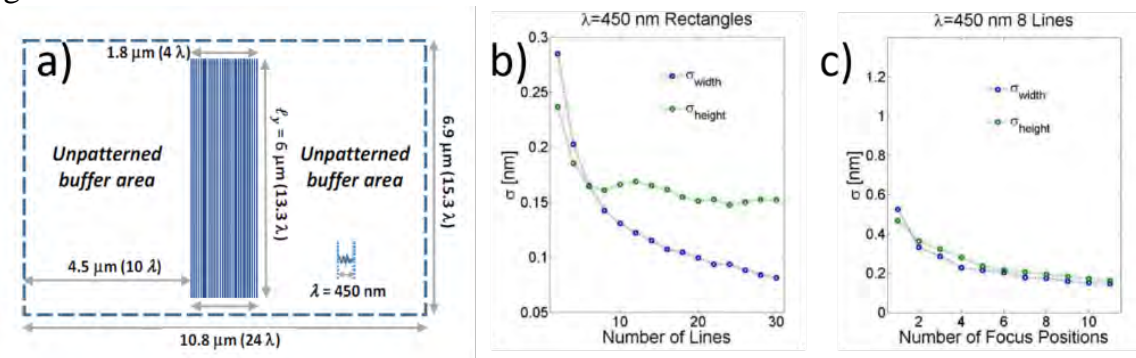

FIGURE 2. a) Initial proposed in-die target design (to scale) based upon the measured target from Ref. 1 . The target has 30 lines with $60 \mathrm{~nm}$ pitch and line length $\left(l_{y}\right)$ of $6 \mu \mathrm{m}$. A three-dimensional electromagnetic scattering model was used to determine the minimum line length for maintaining accuracy. b) Dependence of the estimated uncertainties on the number of lines. c) Dependence of the estimated uncertainties on the number of focus positions. Panel a) reprinted from Ref. 3. 


\section{CONSIDERATION OF POSSIBLE SYSTEMATIC BIASES}

With increasing dependence upon accurate electromagnetic modeling for determining CDs, the limitations and simplifications assumed in these modeling methods become more relevant and may yield systematic biases in such measurement-to-model fits and yield erroneous parametric values. Two of the most prominent issues are the limitations due to the finiteness of the line arrays and the presence of roughness (Fig. 3a) in the measured targets. In order to reduce computation time and memory resources, arrays of lines are often assumed to be "infinite" along one spatial direction for modeling purposes, allowing the use of two-dimensional modeling codes. To test this assumption for the relatively short line lengths of proposed "in-die" targets, three-dimensional FEM-based simulations were compared against FEM-based two-dimensional solutions, shown in Fig. 3b. As shown, the simulated images diverge from the infinite model with decreasing line length, which if unchecked would lead to a systematic bias. The second source of systematic errors is the over-simplification of assuming perfectly smooth lines, whereas all actual arrays have some degree of line-edge width variation and roughness (LEWR). Simulation of such rough edges increases the computational challenges significantly, Fig. 3a shows an example of a rough grid used in simulations. The effect of the rough lines on the imaging can be observed in Figs 3c-d. A systematic bias that exceeds the expected random noise is introduced.

For both the finite vs. infinite comparison and the LEWR evaluation, two different ways to confront these problems are presented. The first one is to simply adapt the measurement set-up, i.e. choose measurement (including target) configurations for which the effects are minimal, while the second is to attempt to include resultant systematic biases into the actual modeling process.
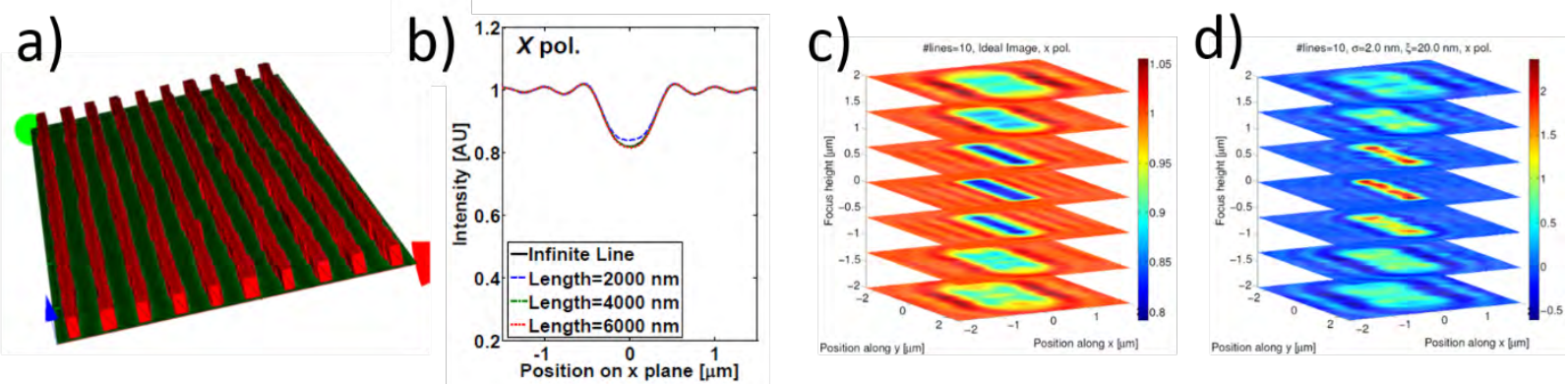

FIGURE 3. a) Single realization of a rough 10-line structure. b) Comparison between the profiles of the infinite and finite lines for three different line lengths $l_{y}$ for a 10 -line target at $\lambda=450 \mathrm{~nm}$. c) Images of the ideal, i.e. non-perturbed 10-line structure at several focus positions. d) Relative difference between the image of ideal and rough 10 line structures at several focus positions.

\section{REFERENCES}

1. J. Qin, R. M. Silver, B. M. Barnes, H. Zhou, R. G. Dixson and M. A. Henn, Light-Sci. Appl. 5, e16038 (2016).

2. J. Qin, R. M. Silver, B. M. Barnes, H. Zhou and F. Goasmat, Appl. Opt. 52 (26), 6512-6522 (2013).

3. B. M. Barnes, M. A. Henn, M. Y. Sohn, H. Zhou and R. M. Silver, " Proc SPIE 9778, 97780Y (2016).

4. M.-A. Henn, B. M. Barnes, H. Zhou, M. Sohn and R. M. Silver, Opt. Lett. 41 (21), 4959-4962 (2016).

\section{KEYWORDS}

computational microscopy; light scattering; metrology; quantitative nanoscale microscopy; sub-nanometer uncertainties; metrology; scattering measurements; three-dimensional microscopy; linewidth; spatial frequency 


\title{
Evaluating the Effects of Modeling Errors for Isolated Finite 3-D Targets
}

\author{
Mark-Alexander Henn, Bryan M. Barnes, and Hui Zhou \\ Engineering Physics Division, National Institute of Standards and Technology, 100 Bureau \\ Drive MS 8212, Gaithersburg, MD, USA 20899-8212
}

\begin{abstract}
Optical 3-D nanostructure metrology utilizes a model-based metrology approach to determine critical dimensions (CDs) that are well below the inspection wavelength. Our project at the National Institute of Standards and Technology is evaluating how to attain key CD and shape parameters from engineered in-die capable metrology targets. More specifically, the CDs are determined by varying the input parameters for a physical model until the simulations agree with the actual measurements within acceptable error bounds.

As in most applications, establishing a reasonable balance between model accuracy and time efficiency is a complicated task. A well-established simplification is to model the intrinsically finite 3-D nanostructures as either periodic or infinite in one direction, reducing the computationally expensive 3-D simulations to usually less complex 2-D problems. Systematic errors caused by this simplified model directly influence the fitting of the model to the measurement data and are expected to become more apparent with decreasing lengths of the structures. In this paper we investigate these effects, and will report experimental set-ups, e.g., the used illumination numerical apertures and focal ranges, that can increase the validity of the 2-D approach.
\end{abstract}

Keywords: electromagnetic simulation, sensitivity and uncertainty evaluation, through-focus three-dimensional field

\section{INTRODUCTION}

Optical critical dimension (OCD) metrology has in the recent years been demonstrated to be a reliable, cheap, and quick non-destructive metrology technique for semiconductor devices [1] that is capable of determining critical dimensions (CDs). Hybridization techniques that combine OCD with alternative measurements such as scanning electron microscopy (SEM) [2-6], can help to further reduce the parametric uncertainty. Innovations in structured illumination [7], tool characterization and Fourier domain normalization [8], and systematic error estimation has made it possible to use image-based methods [9] to determine CDs as small as $16 \mathrm{~nm}$ with parametric uncertainties less than $1 \mathrm{~nm}$.

Yet, accurate in-die metrology remains a challenging task for OCD, and additionally the question remains if the placement of a scatterometry target within the active area is even practical. From a manufacturing point of view, it is desirable to limit the size of the in-die targets. However, their size also has an influence on the viability of the OCD measurements. In a recent paper published by our group, we have addressed how a reduction of the number of lines from 30 to 2, i.e., the reduction of the dimensions of the metrology target in one direction, affects the accuracy of the CD measurements [10]. It was shown that a finite array of 12 lines is enough to guarantee a reasonable accuracy. Also, faster data acquisition is possible by reducing the focus positions to four and by considering only one polarization without significantly increasing the parametric uncertainties.

In this paper we want to additionally investigate how the reduction of the line lengths influences CD measurements. Scatterfield microscopy is one of many OCD methodologies that are model-based, and often the employed physical models assume infinitely long lines in order to keep the costs low in terms of computation time and memory consumption. Any 3-D structure will have a slightly different scattering behavior than infinitely long

Further author information: (Send correspondence to Mark-Alexander Henn)

Mark-Alexander Henn: E-mail: mark.henn@nist.gov

Barnes, Bryan; Henn, Mark Alexander; Zhou, Hui.

"Evaluating the Effects of Modeling Errors for Isolated Finite 3-D Targets."

Paper presented at SPIE Advanced Lithography 2017: Metrology, Inspection, and Process Control for Microlithography XXXI,

San Jose, CA. February 27, 2017 - March 2, 2017. 
lines, and this difference is expected to increase with decreasing line length. Whether this introduces a systematic error that influences the measured CDs can only be answered by a thorough simulation study, assessing and addressing observed systematic bias in the parametric fitting. Although the fitting is image-based, this study can therefore also be understood as a report on the validity of the infinite line model if applied to finite scatterometry targets.

\section{OPTICAL CRITICAL DIMENSION METROLOGY}

This section gives a short introduction into the principles, methodology, and terminology of OCD. Readers that wish to learn about OCD in greater detail should consult Refs. [9, 11]. Optical critical dimension metrology is an indirect measurement method; since the measurement data

$$
\mathbf{y}=\left(y_{1}, \cdots, y_{m}\right)^{T}
$$

contains only indirect information about the quantity of interest, e.g., the spacial intensity distribution for light with a given wavelength scattered by the measurand, we need to analyze the data in terms of an inverse problem $[12,13]$ if we want to determine the dimensions of the measurand. A common approach to solve an inverse problem is to set it up as a regression problem. Given a model function

$$
\mathbf{f}: \mathbb{R}^{n} \rightarrow \mathbb{R}^{m}, \mathbf{f}(\mathbf{p})=\left(f_{1}(\mathbf{p}), \cdots, f_{m}(\mathbf{p})\right)^{T}
$$

that maps the parameters, or critical dimensions, $\mathbf{p}=\left(p_{1}, \cdots, p_{n}\right)^{T}$, that we want to determine (e.g., the height, the width, etc.) to an approximation of the measurement data, the regression problem amounts to minimizing the difference between the modeled and the measured data. In other words, we solve for the parameter vector $\hat{\mathbf{p}}$ that minimizes the so-called $\chi^{2}$ function

$$
\chi^{2}(\mathbf{p})=\|\mathbf{f}(\mathbf{p})-\mathbf{y}\|^{2}=(\mathbf{f}(\mathbf{p})-\mathbf{y})^{T} \mathbf{V}^{-1}(\mathbf{f}(\mathbf{p})-\mathbf{y}) .
$$

In this formulation we implicitly assume that the measurement data $\mathbf{y}$ is a noisy realization of the model, such that we have an additive error model where

$$
\mathbf{y}=\mathbf{f}(\mathbf{p})+\boldsymbol{\epsilon} .
$$

We assume that the error can be modeled as a zero-mean multivariate normally distributed random variable, i.e., $\boldsymbol{\epsilon} \sim \mathcal{N}(\mathbf{0}, \boldsymbol{\Sigma})$. The matrix $\mathbf{V} \in \mathbb{R}^{m \times m}$ in Eq. 2 is usually chosen to be an estimate of the covariance matrix $\boldsymbol{\Sigma}$. Once we have found an optimal $\hat{\mathbf{p}}$, its parametric uncertainties can be estimated by calculating

$$
\operatorname{Cov}(\hat{\mathbf{p}})=\left(\mathbf{J}(0)^{T} \mathbf{V}^{-1} \mathbf{J}(0)\right)^{-1}
$$

with $\mathbf{J}(0)$ being the Jacobian matrix of the model function at $\hat{\mathbf{p}}[14,15]$.

\section{SIMULATION SETUP}

The simulation setup we used in this paper resembles the actual experimental setup of the $193 \mathrm{~nm}$ microscope at NIST [16] and is similar to the configuration in our recent publication [10]. The measured target is assumed to be a non-periodic line array that consists of 10 silicon lines on a silicon substrate $(n=0.85, k=2.61)$ with each of the lines having a nominal width of $20 \mathrm{~nm}$, a height of $35 \mathrm{~nm}$ and a sidewall angle (SWA) (measured between the substrate and the edge of the line) of $90^{\circ}$ (see Fig. 1). The pitch between the lines is $60 \mathrm{~nm}$. The lines are either assumed to be infinitly long in the groove direction, called the 2-D model, or assumed to have a finite length $l_{y}$, called the 3-D model, see Fig. 2. The electromagnetic scattering of the grating interacting with the incoming radiation has been modeled using the FEM-based software package JCMsuite [17].*

${ }^{*}$ Certain commercial materials are identified in this paper in order to specify the experimental procedure adequately. Such identification is not intended to imply recommendation or endorsement by the National Institute of Standards and Technology (NIST), nor is it intended to imply that the materials are necessarily the best available for the purpose.

Barnes, Bryan; Henn, Mark Alexander; Zhou, Hui.

"Evaluating the Effects of Modeling Errors for Isolated Finite 3-D Targets."

Paper presented at SPIE Advanced Lithography 2017: Metrology, Inspection, and Process Control for Microlithography XXXI, San Jose, CA. February 27, 2017 - March 2, 2017. 
Subsequent imaging of the scattering structures requires taking only the far field data into account. Here, the Fourier transform corresponding to the returning part of the total field is determined and the Fourier spectrum is used as input to propagate the field and calculate the images at different focus positions. In order to account for a finite illumination numerical aperture (INA) we treat the light in the illumination path as the sum of plane waves originating from different points in a plane that is conjugate to the back focal plane (CBFP) of the objective lens. The distribution of the plane waves in the CBFP along with the circles corresponding to the different INAs is given in Fig. 1. The number of plane waves ranges from 9 for the smallest INA of 0.2 to 145 for the largest INA of 0.7. Each plane wave takes about $70 \mathrm{~s}$ to calculate for the infinite lines, i.e., using the 2-D model and up to $70 \mathrm{~min}$ for the finite lines, i.e., using the full 3-D model. The total calculation time has in all cases been reduced by making use of the symmetry of the target geometry and the illumination set up.

The images were calculated for a collection numerical aperture of 0.95 at 11 equally spaced focus positions between $-1 \mu \mathrm{m}$ to $1 \mu \mathrm{m}$ relative to the surface of the substrate, for two orthogonal polarization states: $\mathrm{X}$ polarization, i.e., light polarized perpendicular to the lines, and Y polarization where the light is polarized along the lines, see coordinate system in Fig. 2. The sampling rate in the $y$ direction corresponds to a pixel size of 25 $\mathrm{nm}$ with an overall range of $3 \mu \mathrm{m}$ between $-1.5 \mu \mathrm{m}$ to $1.5 \mu \mathrm{m}$ relative to the center of the structure. Together with the above specifications this configuration leads to an individual data set that consists of 2640 data points (11 focus positions, 2 polarization states and 120 pixels for each image).

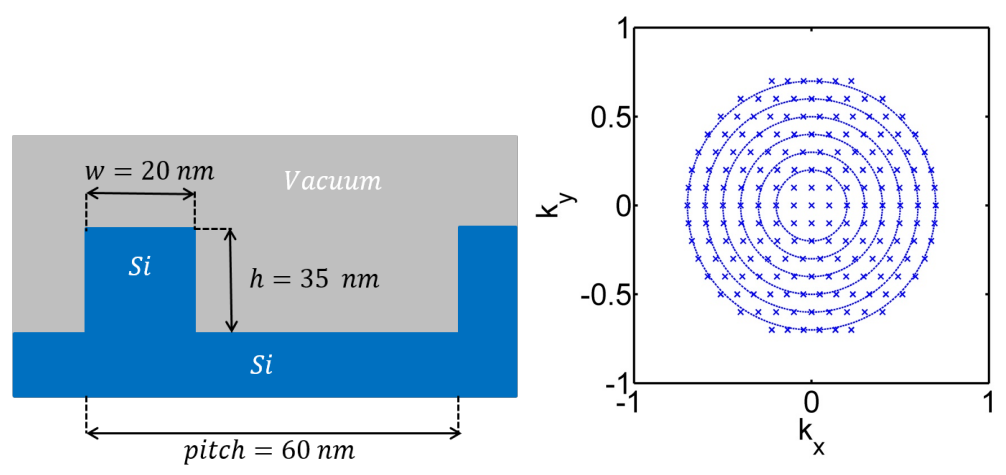

Figure 1. Parametrization (left), and distribution of plane waves in k-space (right). Circles depict the INAs from 0.2 to 0.7 .

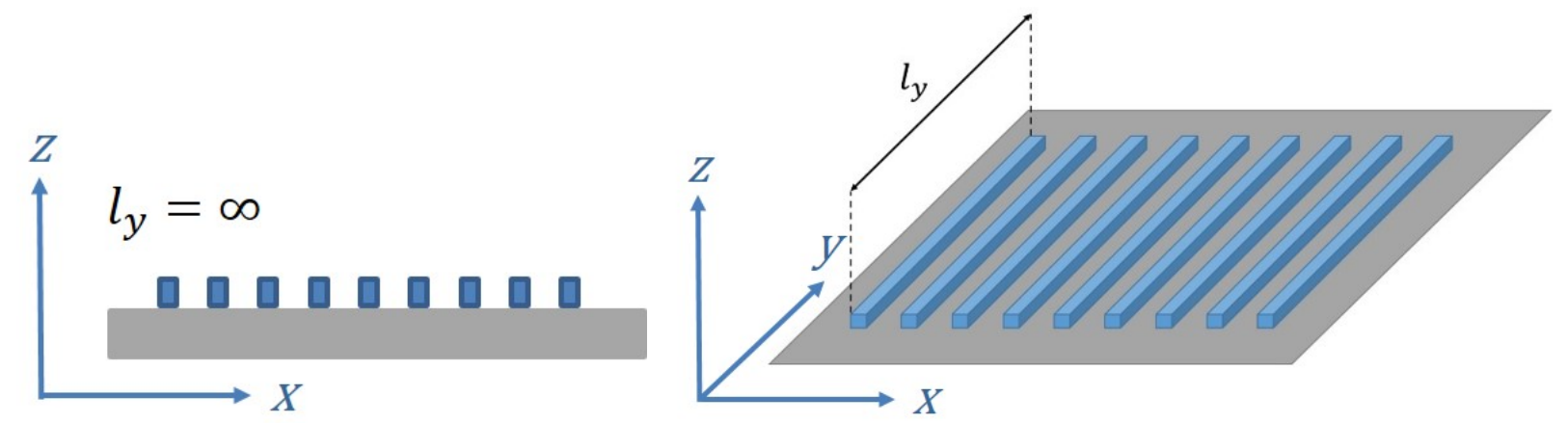

Figure 2. Comparison of the 2-D and the 3-D models

\section{SIMULATION STUDY RESULTS}

We start our studies with a quantitative comparison between the images based on an infinite line array and a finite line array, for three different lengths $l_{y} \in\{1 \mu \mathrm{m}, 2 \mu \mathrm{m}, 4 \mu \mathrm{m}\}$ similar to the comparison in [10]. The measure

Barnes, Bryan; Henn, Mark Alexander; Zhou, Hui.

"Evaluating the Effects of Modeling Errors for Isolated Finite 3-D Targets."

Paper presented at SPIE Advanced Lithography 2017: Metrology, Inspection, and Process Control for Microlithography XXXI, 
of difference that is being used is the root-mean-square deviation, i.e., the norm $\frac{\left\|\mathbf{f}_{\text {finite }}-\mathbf{f}_{\text {infinite }}\right\|}{\sqrt{m}}$, with $m$ being the dimensions of $\mathbf{f}_{\text {finite }}$ and $\mathbf{f}_{\text {infinite }}$, respectively. A plot of the results can be found in Fig. 3 . Note, that we calculate the RMSD for six different INAs from 0.2 to 0.7 , while the figure only shows three for clarity. A decrease of the RMSD with increasing INA can be observed, and, less surprising, a decrease of the RMSD with increasing line length $l_{y}$.
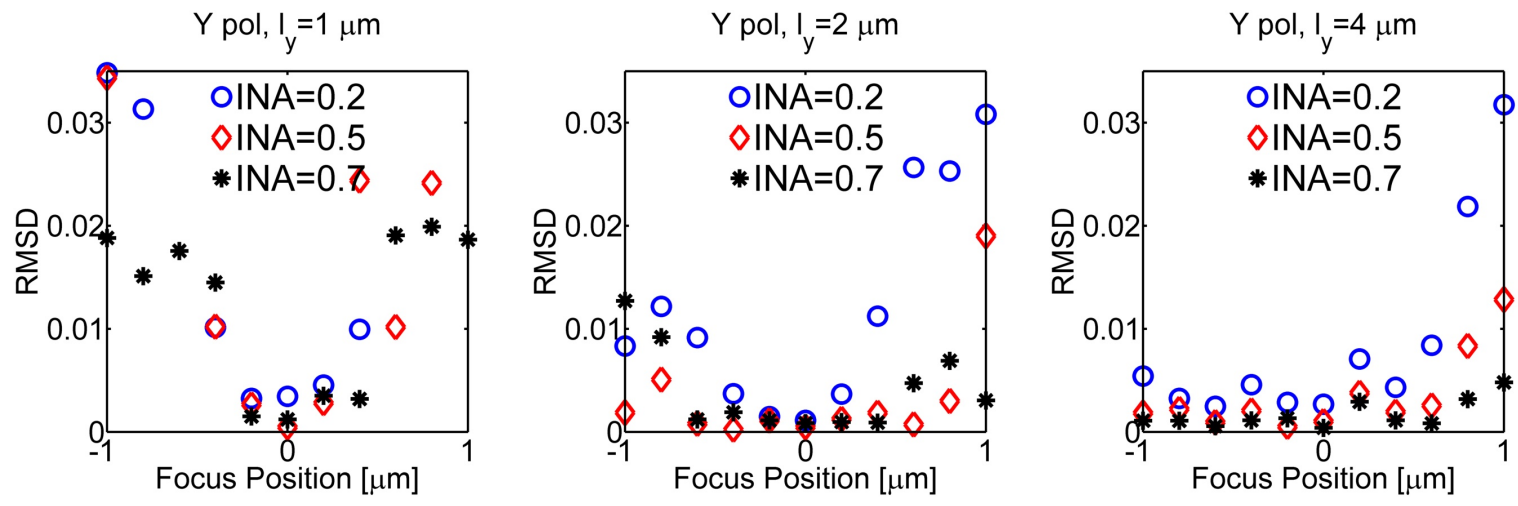

Figure 3. RMSD as a function of INA and focus position

Next, we investigate what errors on the parametric estimations, if any, are introduced if we fit the data from the finite lines that were generated using the 3-D model, with the model function $\mathbf{f}$ (see Eqs. 1 and 2), that is based on the 2-D model. Even though using two different models to generate and fit the data should prevent us from committing inverse crimes [13], we additionally perturb each data set by adding a random error to further reduce the risk of obtaining overly optimistic results. For the perturbation we use white noise with $\sigma^{2}=(0.01)^{2}$, that is a standard deviation of one percent relative to the incident intensity $I_{0}$.

This data is then used as input data for our 2-D-model-based metrology algorithms, and estimated parametric values $\hat{\mathbf{p}}$ along with the $1.96 \cdot \hat{\sigma}$ estimated parametric uncertainty values based on Eq. 3 are reported. Solutions that cover the actual parametric values, i.e., solutions such that

$$
p_{0} \in[\hat{p}-1.96 \cdot \hat{\sigma}, \hat{p}+1.96 \cdot \hat{\sigma}],
$$

are called consistent solutions. This procedure is repeated 50 times with different realizations of the white noise for each combination of $l_{y}$, INA, and polarization. The results for one combination of $l_{y}$, INA, and polarization are shown in Fig. 4. Consistent solutions are marked as orange patches, solutions that yield estimates that are smaller than the nominal values appear as black patches, solutions that yield estimates that are larger than the nominal values appear as white patches. Note, that in this example the estimated heights are systematically shifted towards a value that is smaller than the nominal value of $35 \mathrm{~nm}$, and that the $1.96 \cdot \hat{\sigma}$ error bars do not cover this value and are thus inconsistent solutions.

The experimental conditions in terms of chosen INA, focal range, polarization, etc. are then varied in order to find combinations that yield a sufficient number of consistent solutions. Based on a total of 50 data sets the number of solutions that do not meet this criterion, i.e., the number of solutions that are inconsistent, should be around 2-3.

\subsection{Influence of INA, Line Length, and Polarization}

Before looking at how the number of consistent solutions changes as a function of INA, line length, and polarization, note that there is a clear dependency of the estimated parametric uncertainties on the INA, as can be seen in Fig. 5. The larger the INA, the larger the parametric uncertainties, both for the width and the height. This is in accordance with previous reports that edge-based methods traditionally improve the accurancy by increasing the INA, but the opposite is true for model-based approaches [18].

Barnes, Bryan; Henn, Mark Alexander; Zhou, Hui. 

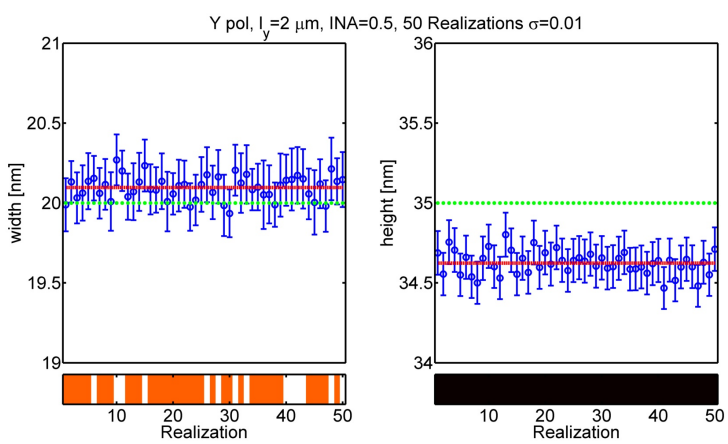

Figure 4. Example of consistency plot

The comprehensive results of this study are presented in Fig. 6. Two things can be observed: There is a clear trend indicating that the number of consistent solutions increases with increasing line length $l_{y}$. This is not surprising since we have already seen in Fig. 3 that the differences between the 2-D and 3-D images decrease with increasing line length, i.e., the systematic error should be reduced with increasing line length. More notable, however, is a significant difference between the number of consistent solutions for the two different polarization states. Using light that is polarized along the lines ( $\mathrm{Y}$ polarized), increases the number of consistent solutions significantly for this geometry across all INA values. A specific INA that maximizes this number can not be clearly defined.
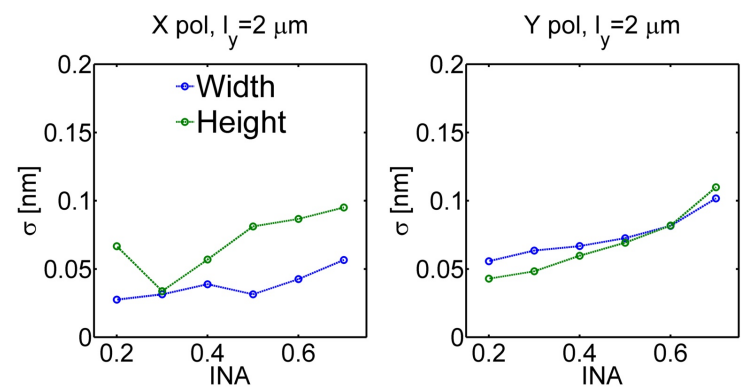

Figure 5. Parametric uncertainties as a function of INA
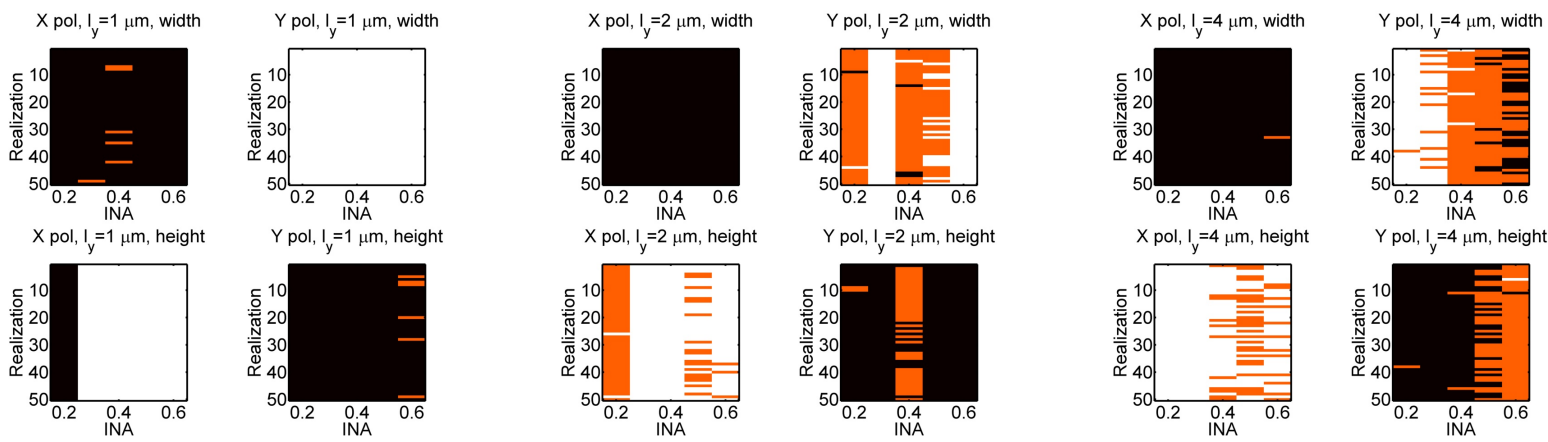

Figure 6. Dependency of consistency on INA and $l_{y}$

\subsection{Influence of Kernel Length}

Until this point, the input data consisted of a slice through the very center of the finite line array. Figure 7 (a) however shows a clear change in the intensity profile as one moves along the lines in the $y$ direction, which 
cannot occur in the infinite long line array case. The effect of an averaging of the image along the $y$ direction to minimize potential systematic bias due to pixel selection also requires investigation. The pixel size along the $y$ direction is assumed to be the same as in $x$ direction: $25 \mathrm{~nm}$. The kernel length $l_{k}$ ranges from 1 to 11 pixels, or $25 \mathrm{~nm}$ to $275 \mathrm{~nm}$ see Fig. 7(b). Not shown, averaging the images does not help to increase the number of consistent solutions for the $1 \mu \mathrm{m}$ or the $2 \mu \mathrm{m}$ long line array. It does help to increase the number of consistent solutions for the $4 \mu \mathrm{m}$ line though, as can be seen in Fig. 8. Note that there is a distinct maximum for a kernel length $l_{k}=175 \mathrm{~nm}$, or 7 pixels for all INAs studied here.
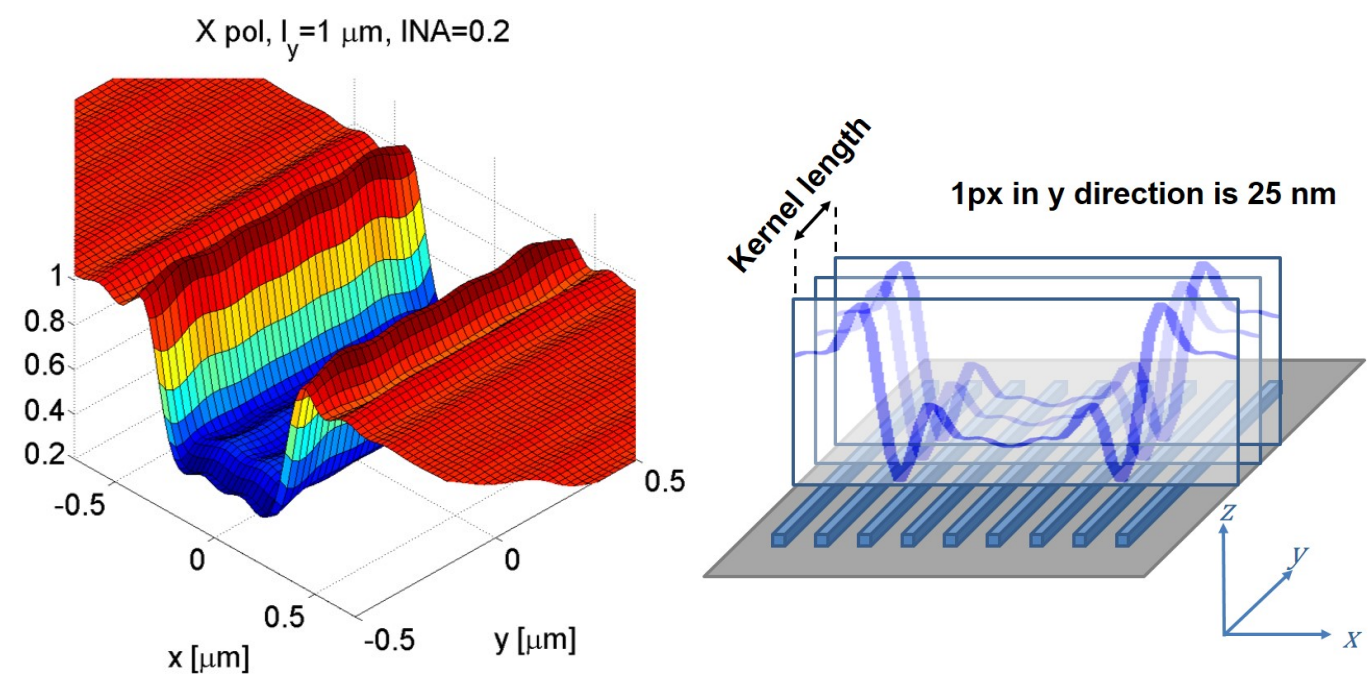

Figure 7. Example of 2-D image $(z=0 \mu \mathrm{m})$ (left), and principle of averaging (right)
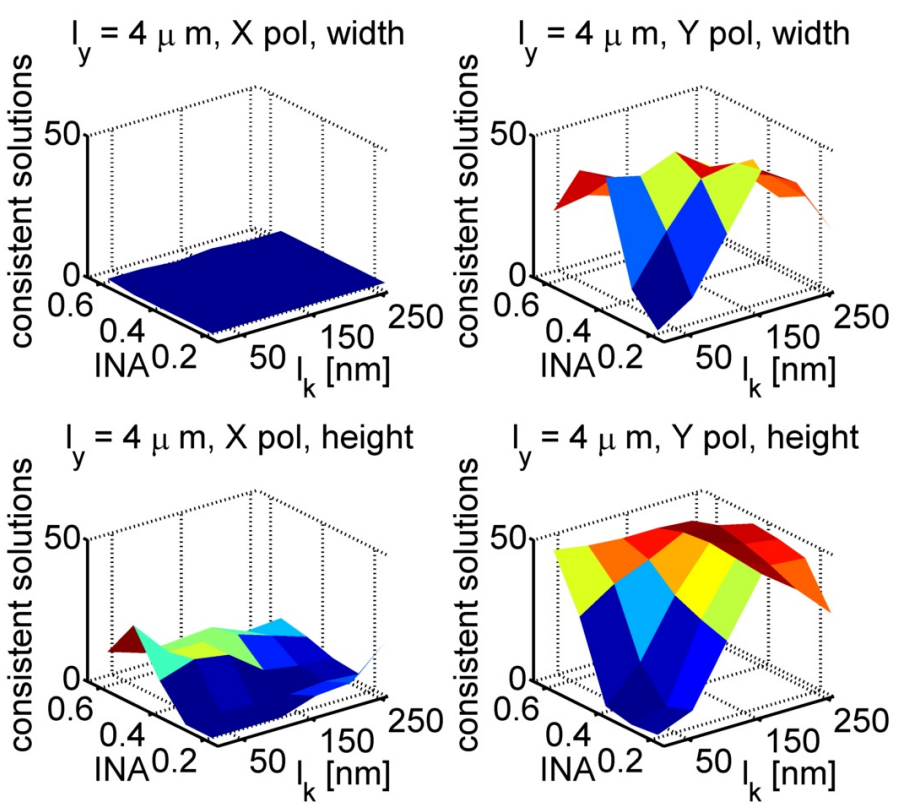

Figure 8. Effect of averaging for $l_{y}=4 \mu \mathrm{m}$

\subsection{Influence of Focal Range}

Having established and justified the width of the kernel $l_{k}$, how the number of consistent solutions changes with focal range can now be investigated. As mentioned earlier the full simulated data set consists of 11 images taken 
over a focal range of $2 \mu \mathrm{m}$. Figure 3 has shown that there is a focus dependency for the RMSD, such that the RMSD increases the further away from the surface the substrate we focus. In order to evaluate how this behavior effects the parametric estimators we reduce the focal range in two steps. First we use the full data set, in the next step we reduce the data to the images corresponding to a focus position $|z| \leq 0.4 \mu \mathrm{m}$, that is 5 images, and finally we use only the single image corresponding to a focus position on the surface of the substrate $(z=0 \mu \mathrm{m})$. Since the results so far imply that the differences between the 2-D and 3-D models are more pronounced using $\mathrm{X}$ polarized light, and hence the number of consistent solution is significantly smaller than for using Y polarized light, we will from now on restrict ourselves here to data taken in Y polarization.

The results for the $2 \mu \mathrm{m}$ line array are presented in Fig. 9 below. Reducing the focal range helps to dramatically increase the number of consistent solutions, the same can be observed for the $1 \mu \mathrm{m}$ and $4 \mu \mathrm{m}$ targets. This is of course partly due to the larger estimated parametric uncertainties: As we reduce the data by a factor of 11 going from the full data set and 11 focus position to a single data set, the uncertainties increase by a factor of $\sqrt{11}$. However, as Fig. 10 demonstrates, the reduction of the focal ranges actually reduces the systematic offset of the parametric estimations from the nominal values.

$\mathrm{I}_{\mathrm{y}}=2 \mu \mathrm{m},|\mathrm{z}| \leq 1.0 \mu \mathrm{m}$, width

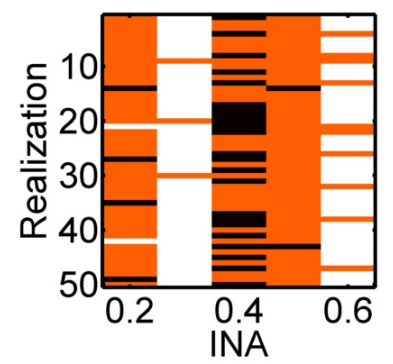

$I_{y}=2 \mu \mathrm{m},|z| \leq 1.0 \mu \mathrm{m}$, height

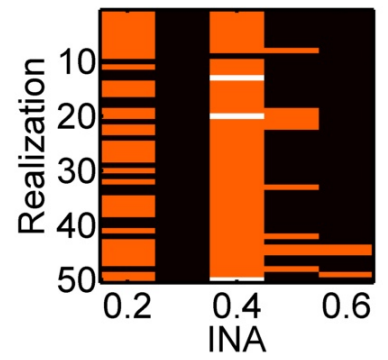

$\mathrm{I}_{\mathrm{y}}=2 \mu \mathrm{m},|\mathrm{z}| \leq 0.4 \mu \mathrm{m}$, width

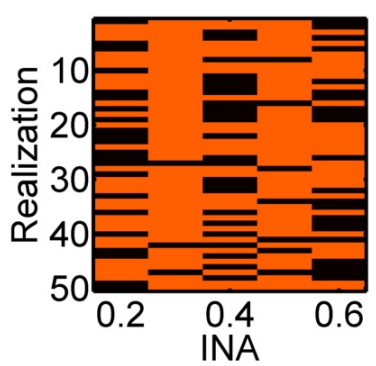

$\mathrm{I}_{\mathrm{y}}=2 \mu \mathrm{m},|\mathrm{z}| \leq 0.4 \mu \mathrm{m}$, height

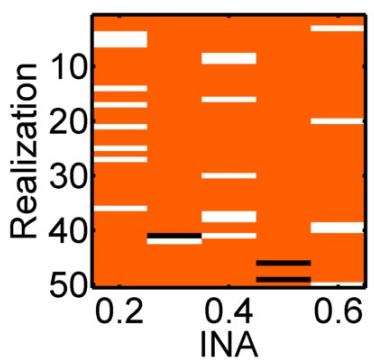

$\mathrm{I}_{\mathrm{y}}=2 \mu \mathrm{m}, \mathrm{z}=0 \mu \mathrm{m}$, width

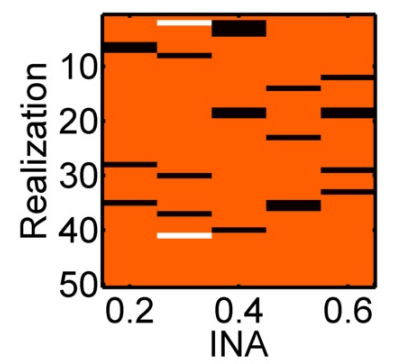

$\mathrm{I}_{\mathrm{y}}=2 \mu \mathrm{m}, \mathrm{z}=0 \mu \mathrm{m}$, height

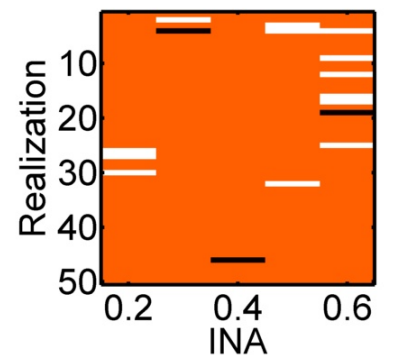

Figure 9. Dependency of consistency on focal range, all results for Y polarization

Having seen that limiting ourselves to a single image at the substrate can help to obtain more reliable results, we investigate if it matters where the single image has been taken. Or stated differently: Is there a dependence of the estimated parameters on the focus position $z$ ? From Fig. 11 we can see that using an image that is been taken too far out of focus, i.e., an image with $|z| \geq 0.4 \mu \mathrm{m}$ may lead to wrong estimates. Single images taken within this range are shown to yield consistent results with reasonable parametric uncertainties. Furthermore collecting through focus images within $|z| \leq 0.4 \mu \mathrm{m}$ for the purposes of data fitting will reduce the possible systematic effect on the parameters due to the finiteness of the lines and decrease parametric uncertainties further.

\section{DISCUSSION AND CONCLUSION}

In the present paper we have studied the impact of modeling errors on the OCD model-based metrology using an inspection wave length of $\lambda=193 \mathrm{~nm}$. In particular we have investigated what kind of systematic errors 

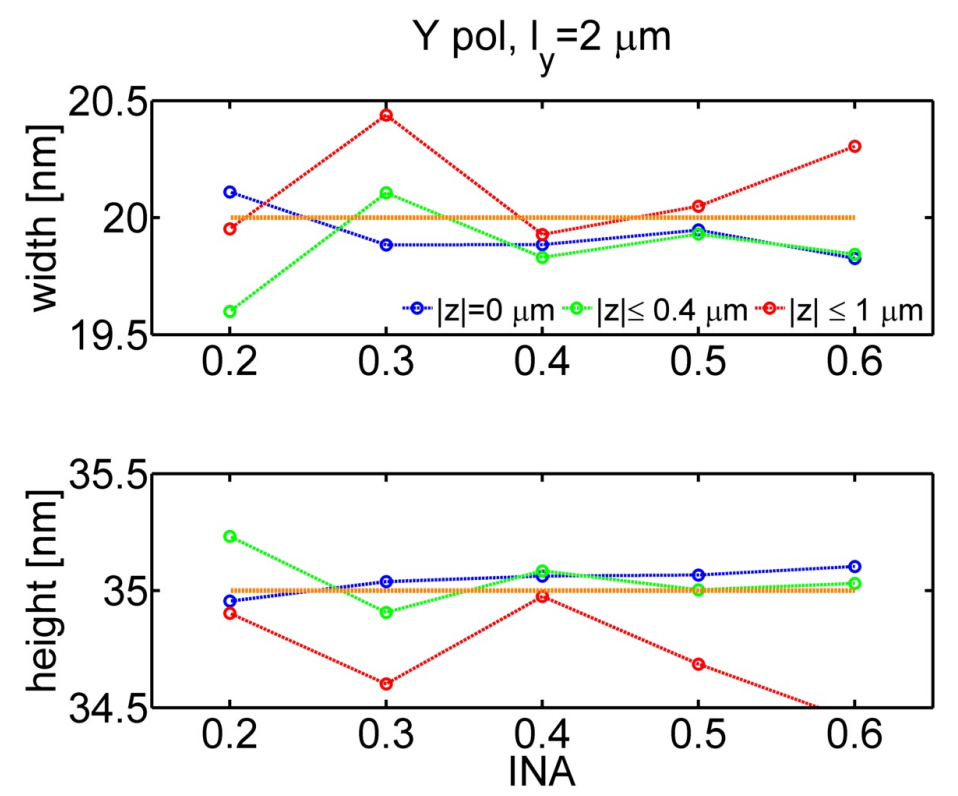

Figure 10. Offset from nominal values with changing focal range

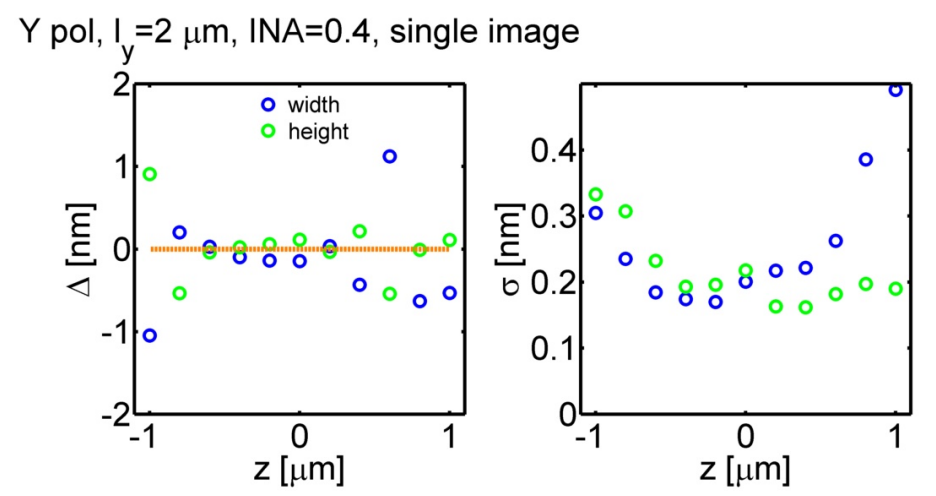

Figure 11. Means and uncertainties for single profiles

might be introduced if the critical dimensions of finite line arrays with lenghts below $4 \mu \mathrm{m}$ are determined using a physical model that assumes the lines to be infinitely long, a model we call the 2-D model.

For these line lengths, using the 2-D model has introduced a significant systematic error to the CD estimates that can not be accounted for in a straightforward manner. Its effects were reduced by carefully tailoring the experimental conditions. Several experimental parameters have been varied, such as the length of the line array, the INA, the polarization, the focus range, etc., in order to find a setup that would minimize the effect of the modeling error and yield reliable reconstructions of the parametric estimates. Reliable parametric estimates in this context have been defined as estimates that cover the nominal values within their $1.96 \cdot \sigma$ confidence intervals.

A strong dependence of the number of consistent solutions on the length of the line array, the polarization, and the focal range has been observed, while the influence of the chosen INA on the estimates has been demonstrated to be negligible. The 2D-model for this finite set of features at $\lambda=193 \mathrm{~nm}$ has been proven to be able to give reliable results if the inspection light has been polarized along the lines (Y polarized), and the focus range has been limited to about $0.8 \mu \mathrm{m}$.

Barnes, Bryan; Henn, Mark Alexander; Zhou, Hui.

"Evaluating the Effects of Modeling Errors for Isolated Finite 3-D Targets."

Paper presented at SPIE Advanced Lithography 2017: Metrology, Inspection, and Process Control for Microlithography XXXI, 


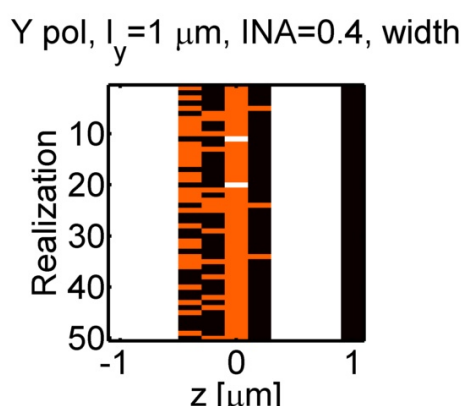

$Y$ pol, $I_{y}=1 \mu \mathrm{m}$, INA $=0.4$, height

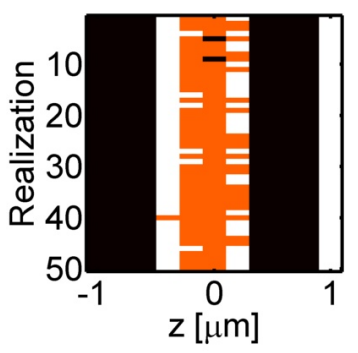

$Y$ pol, $I_{y}=2 \mu \mathrm{m}, I N A=0.4$, width

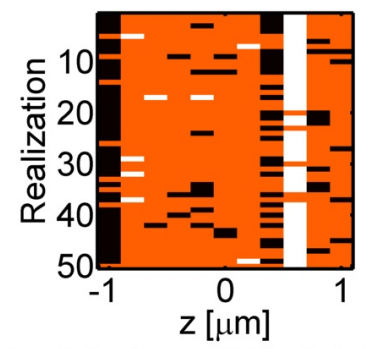

$Y$ pol, $I_{y}=2 \mu \mathrm{m}$, INA $=0.4$, height

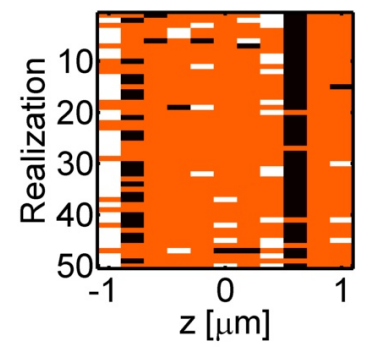

$Y$ pol, $I_{y}=4 \mu m, I N A=0.4$, width

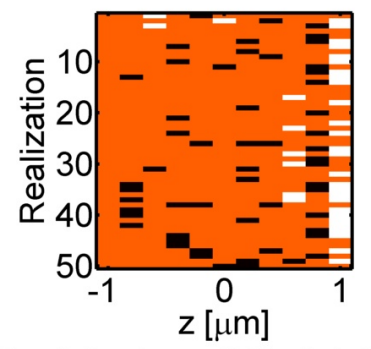

$Y$ pol, $I_{y}=4 \mu \mathrm{m}, \mathrm{INA}=0.4$, height

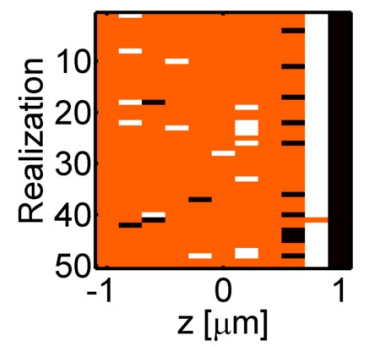

Figure 12. Dependency of consistency on focus position $z$ for a single image

With the knowledge derived from this study it should be possible to reliably determine the CDs of in-die metrology targets as short as $2 \mu \mathrm{m}$ that is about 10 times the wave length $\lambda=193 \mathrm{~nm}$ of the inspection light, without using a way more computational expensive full 3-D model, by optimizing the experimental conditions.

\section{References}

[1] A. J. den Boef, "Optical wafer metrology sensors for process-robust CD and overlay control in semiconductor device manufacturing," Surface Topography: Metrology and Properties 4, 2016.

[2] R. M. Silver, B. M. Barnes, H. Zhou, N. Zhang, and R. G. Dixson, "Angle-resolved optical metrology using multi-technique nested uncertainties," Proc. SPIE 7390, 2009.

[3] N. Rana and C. Archie, "Hybrid reference metrology exploiting patterning simulation," Proc. SPIE 7638, 2010.

[4] A. Vaid, B. B. Yan, Y. T. Jiang, M. Kelling, C. Hartig, J. Allgair, P. Ebersbach, M. Sendelbach, N. Rana, A. Katnani, et al., "A holistic metrology approach: hybrid metrology utilizing scatterometry, CD-AFM, and CD-SEM," Proc. SPIE 7971, 2011.

[5] N. F. Zhang, R. M. Silver, H. Zhou, and B. M. Barnes, "Improving optical measurement uncertainty with combined multitool metrology using a Bayesian approach," Appl. Opt. 51, 2012.

[6] M.-A. Henn, B. M. Barnes, N. F. Zhang, H. Zhou, and R. M. Silver, "The effect of systematic errors on the hybridization of optical critical dimension measurements," Proc. SPIE 9526, 2015.

[7] R. M. Silver, T. Germer, R. Attota, B. M. Barnes, B. Bunday, J. Allgair, E. Marx, and J. Jun, "Fundamental limits of optical critical dimension metrology: a simulation study," Proc. SPIE 6518, 2007.

[8] J. Qin, R. M. Silver, B. M. Barnes, H. Zhou, and F. Goasmat, "Fourier domain optical tool normalization for quantitative parametric image reconstruction," Appl. Opt. 52, 2013.

[9] J. Qin, R. M. Silver, B. M. Barnes, H. Zhou, R. G. Dixson, and M.-A. Henn, "Deep-subwavelength Nanometric Image Reconstruction using Fourier Domain Optical Normalization," Light Sci Appl 5, 2016.

Barnes, Bryan; Henn, Mark Alexander; Zhou, Hui.

"Evaluating the Effects of Modeling Errors for Isolated Finite 3-D Targets."

Paper presented at SPIE Advanced Lithography 2017: Metrology, Inspection, and Process Control for Microlithography XXXI, 
[10] M.-A. Henn, B. M. Barnes, H. Zhou, M. Y. Sohn, and R. M. Silver, "Optimizing the nanoscale quantitative optical imaging of subfield scattering targets," Optics Letters 41(21), 2016.

[11] B. M. Barnes, M.-A. Henn, M. Y. Sohn, H. Zhou, and R. M. Silver, "Enabling quantitative optical imaging for in-die-capable critical dimension targets," Proc. SPIE 9778, 2016.

[12] A. Tarantola and B. Valette, "Generalized nonlinear inverse problems solved using the least squares criterion," Reviews of Geophysics 20, 1982.

[13] J. Kaipio and E. Somersalo, Statistical and Computational Inverse Problems, Springer Science \& Business Media, Berlin, 2006.

[14] T. A. Germer, H. J. Patrick, R. M. Silver, and B. Bunday, "Developing an uncertainty analysis for optical scatterometry," Proc. SPIE 7272, 2009.

[15] R. M. Al-Assaad and D. M. Byrne, "Error analysis in inverse scatterometry. I. Modeling," JOSA A 24(2), 2007.

[16] M. Y. Sohn and R. M. Silver, "193 nm scatterfield microscope illumination optics," Proc. SPIE 9293, 2014.

[17] S. Burger, L. Zschiedrich, J. Pomplun, and F. Schmidt, "JCMsuite: An Adaptive FEM Solver or Precise Simulations in Nano-Optics," Integrated Photonics and Nanophotonics Research and Applications, p. ITuE4, 2008.

[18] R. M. Silver, R. Attota, M. Stocker, M. Bishop, L. Howard, T. A. Germer, E. Marx, M. Davidson, and R. Larrabee, "High-resolution optical metrology," Proc. SPIE 5752, 2005.

Barnes, Bryan; Henn, Mark Alexander; Zhou, Hui.

"Evaluating the Effects of Modeling Errors for Isolated Finite 3-D Targets."

Paper presented at SPIE Advanced Lithography 2017: Metrology, Inspection, and Process Control for Microlithography XXXI, San Jose, CA. February 27, 2017 - March 2, 2017. 


\title{
Assessing the wavelength extensibility of optical patterned defect inspection
}

\author{
Bryan M. Barnes*, Hui Zhou, Mark-Alexander Henn, Martin Y. Sohn, and Richard M. Silver \\ Engineering Physics Division, National Institute of Standards and Technology, \\ 100 Bureau Drive MS 8212, Gaithersburg, MD, USA 20899-8212
}

\begin{abstract}
Qualitative comparisons have been made in the literature between the scattering off deep-subwavelength-sized defects and the scattering off spheres in free space to illustrate the challenges of optical defect inspection with decreasing patterning sizes. The intensity scattered by such a sphere (for diameters sized well below the wavelength) is proportional to its diameter to the sixth power, but also scales inversely to the fourth power of the wavelength. This paper addresses through simulation the potential advantages of applying shorter wavelengths for improved patterned defect inspection. Rigorous finite-difference time-domain 3-D electromagnetic modeling of the scattering from patterned defect layouts has been performed at five wavelengths which span the deep ultraviolet $(193 \mathrm{~nm})$, the vacuum ultraviolet $(157 \mathrm{~nm}$ and $122 \mathrm{~nm})$, and the extreme ultraviolet $(47 \mathrm{~nm}$ and $13 \mathrm{~nm})$. These patterned structures and defects are based upon publicly disclosed geometrical cross-sectional information from recent manufacturing processes, which then have been scaled down to an $8 \mathrm{~nm}$ Si linewidth. Simulations are performed under an assumption that these wavelengths have the same source intensity, noise sources, and optical configuration, but wavelengthdependent optical constants are considered, thus yielding a more fundamental comparison of the potential gains from wavelength scaling. To make these results more practical, future work should include simulations with more process stacks and with more materials as well as the incorporation of available source strengths, known microscope configurations, and detector quantum efficiencies. In this study, a $47 \mathrm{~nm}$ wavelength yielded enhancements in the signal-to-noise by a factor of five compared to longer wavelengths and in the differential intensities by as much as three orders-of-magnitude compared to $13 \mathrm{~nm}$, the actinic wavelength for EUV semiconductor manufacturing.
\end{abstract}

Keywords: defect metrology, extreme ultraviolet, EUV, vacuum ultraviolet, VUV, deep ultraviolet, DUV, defect inspection, finite-difference time-domain, simulation, simulated imaging

\section{INTRODUCTION}

A core principle of optical patterned defect inspection is that with decreased dimensions, "killer" pattered defects also scale in size proportionally [1-3]. A heuristic comparison has been made in the literature [3] between the scattering off deep-subwavelength-sized defects and the scattering off spheres in free space, the latter of which can be analytically solved using Mie's Theory [4]. This theory reduces to the Rayleigh approximation for a particle with diameter $d<<\lambda$, the inspection wavelength, such that the scattered intensity $I$ is given by

$$
I=\left(\frac{1+\cos ^{2} \theta}{2 R^{2}}\right)\left(\frac{2 \pi}{\lambda}\right)^{4}\left(\frac{|\tilde{n}|^{2}-1}{|\tilde{n}|^{2}+2}\right)^{2}\left(\frac{d}{2}\right)^{6} I_{0} \quad \text { for } d \ll \lambda,
$$

where $\tilde{n}$ is the complex index of refraction $(\tilde{n}=n+i k), \theta$ is the angle of incidence, $R$ is the distance of the observer from the sphere, and $I_{0}$ is the incident intensity. Figure 1 illustrates the exponential decrease in scattered intensity for reduced diameters below $50 \mathrm{~nm}$ and the exponential increase in scattered intensity for reduced wavelengths shorter than $200 \mathrm{~nm}$. Each plot in Fig. 1 optimistically assumes that no other variables in the scattering calculation in Eqn. 1 change except for a single parameter of interest.

Although Eqn. 1 may suggest trends for the intensities scattered by a defect, there are several key caveats. First, if the wavelength is scaled down such that $d \cong \lambda$ then Eqn. 1 is no longer valid and the full Mie Theory would be required. Second, even if $d \gg \lambda$, one must also consider variations in the index of refraction. The $d^{6}$ trend shown in Fig. 1 would be mostly unaffected except for the very smallest diameters, as it has been shown that nanoparticles with

*bryan.barnes@nist.gov; phone 1301 975-3947; fax 1301 975-4396; www.nist.gov

Barnes, Bryan; Henn, Mark Alexander; Silver, Richard; Sohn, Martin; Zhou, Hui.

"Assessing the wavelength extensibility of optical patterned defect inspection."

Paper presented at SPIE Advanced Lithography 2017: Metrology, Inspection, and Process Control for Microlithography XXXI, San Jose, CA. February 20, 2017 - March 23, 2017. 
Relative Mie Scattering, $\lambda=193 \mathrm{~nm}$

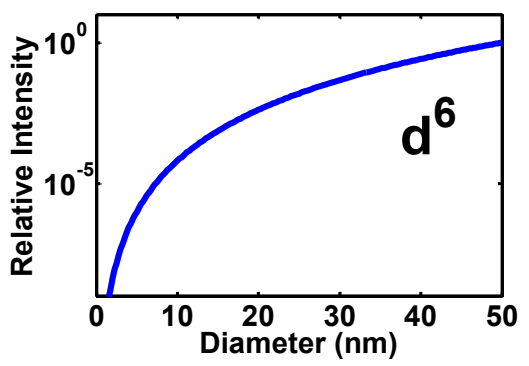

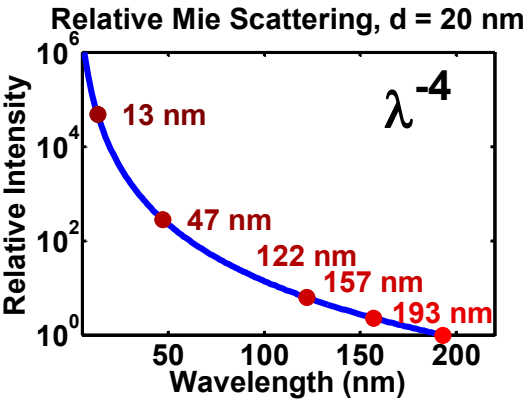

Figure 1. Trends in relative scattered intensity of a sphere in free space as functions of diameter, $d$, and wavelength, $\lambda$.

$d<5 \mathrm{~nm}$ may have a size-dependent index of refraction [5]. The $\lambda^{-4}$ trend pictured in Fig. 1 would be altered significantly due to the variations in the optical constants as pictured in Fig. 2. This figure shows the wavelength dependence of the optical constants $n, k$ for four materials in use for semiconductor manufacturing: crystalline Si (Si-c) [6], amorphous $\mathrm{Si}(\mathrm{Si}-\mathrm{a})$ [7], hafnium oxide $\left(\mathrm{HfO}_{2}\right)$ [8-10], and silicon dioxide $\left(\mathrm{SiO}_{2}\right)$ [11]. Third, Eqn. 1 is defined for a sphere in free space, and this simple system cannot account for the complex interactions among the defect, the patterned layout, and the substrate.
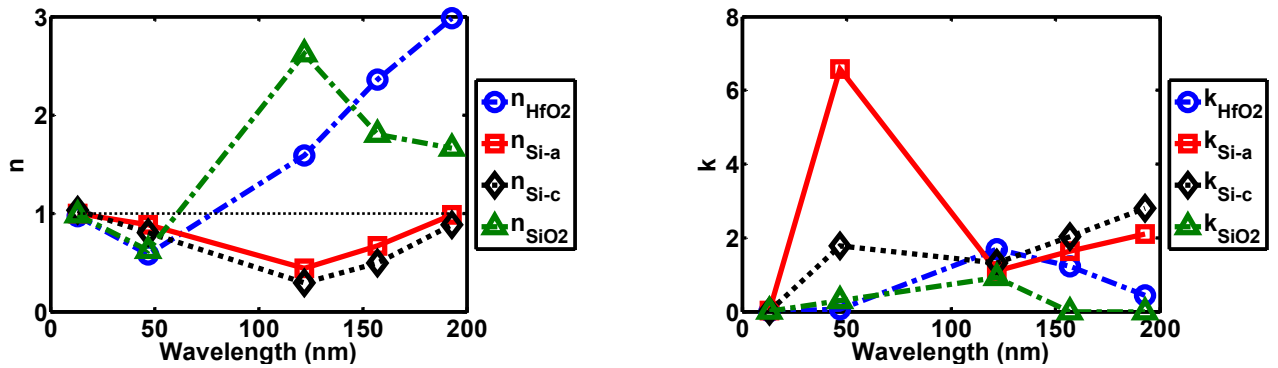

Figure 2. Optical constants $n$ and $k$ for four key materials used in semiconductor manufacturing, as found in the literature.

Unlike for defect size, this equation cannot be considered as a heuristic model for the effects of wavelength scaling. To develop a fundamental understanding of the effects of reduced wavelength, electromagnetic simulations with realistic patterned layouts and defects are required to determine the qualitative and quantitative effects of reduced wavelengths upon defect inspection. In this paper, a simulation study is presented comparing defect scattering at five different wavelengths using an in-house developed finite-difference time-domain (FDTD) [12] Maxwell's equations solver. A defect metric is developed that is applicable across these five wavelengths. This signal-to-noise based metric will allow direct comparisons of the fundamental performance across these wavelengths and will illustrate the potential gains and challenges of using shorter wavelengths.

\section{SIMULATION METHODOLOGY}

\subsection{Shared geometric simulation inputs}

Individual FDTD calculations simulate a single plane wave incident upon the sample with polar and azimuthal angles defined as illustrated in Fig. 3(a). The Cartesian coordinate system is tied to the orientation of the layout as illustrated in that same panel. Simulations are performed with the plane wave linearly polarized either perpendicular to or within the plane of incidence, and these results are used to calculate the effects of linearly polarized light that is oriented with respect to the sample (e.g., X polarization, Y polarization). The simulation geometries are based upon public information about recent manufacturing processes $[13,14]$ while reducing dimensions such that silicon lines are $8 \mathrm{~nm}$; areas and heights are scaled accordingly. The nominal patterning for all simulations is of Si fins with conformal side coatings and a significant layer of silicon oxide between these fins that is also coated. The four materials used are identified in Fig, 3(b) in the $x z$ cross-sectional view, the optical constants for these materials appear as Fig. 2, and the five wavelengths are shown at right in Fig. 1. Figure 3(c) shows the $x y$ view of the unit cell (UC) used for this simulation study. Calculations were performed for three cases: first, with no defects in the simulation domain; second, with one bridging defect that connects the ends of two fins together; and third, with one bridging defect that connected 
(a)

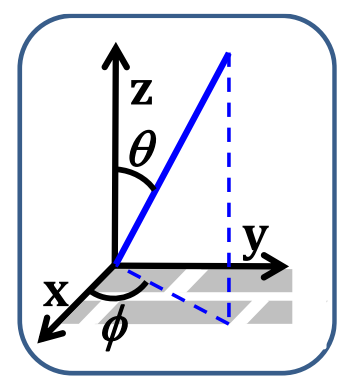

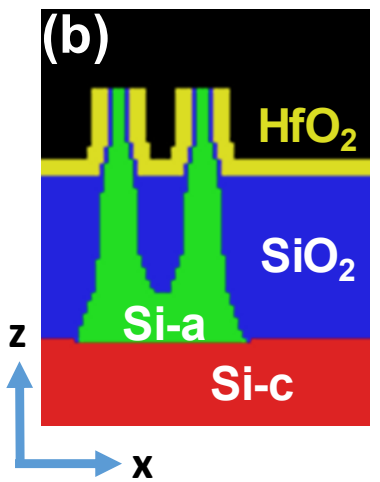

(c)

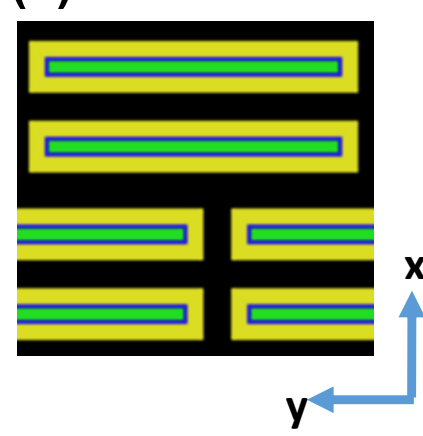

(d)

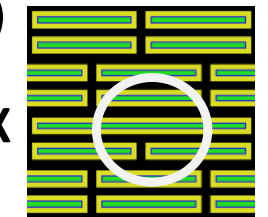

Figure 3. (a) Coordinate system for simulated incident plane wave linear polarization axis and angle of incidence, both polar $\theta$, and azimuthal, $\phi$. (b) $y z$ cross-section of fin pair modeled upon a transmission electron micrograph in Ref. [14]. Materials shown were chosen from analysis of the public literature. (c) $x y$ cross-section through the fins. Image is of one unit cell (UC). (d) $x y$ cross-sections showing bridge defects "Bx" and "By" within a 2 UC $\times 2$ UC area. Note, the length of the "Bx" bridge runs along the $y$ direction in our coordinate system, and the length of "By" runs along the $x$ direction.

adjacent lines together. These bridging defects are commonly referred to by their SEMATECH ${ }^{1}$ naming scheme, "Bx" and "By" defects, and are illustrated in Fig. 3(d).

\subsection{Essential variations specific to multi-wavelength defect modeling}

As in previous reports from our group on the simulations of defect structures using FDTD [2, 15, 16], the patterned structures have been treated as periodic for the purposes of calculation. For these simulations, there is a periodicity in the $x y$ directions and perfectly matching layers (PMLs) are placed only at the bottom and top of the simulation domain. As the size of the unit cell in Fig. 3(c) is $168 \mathrm{~nm} \times 180 \mathrm{~nm}$, the placement of one unit cell (UC) containing a defect in the simulation domain is insufficient to model a single defect. There will be defect-dependent interactions with the defect's multiple periodic copies. Therefore, the single defect UC is placed with an array of non-defect UCs as illustrated in Fig. 4.

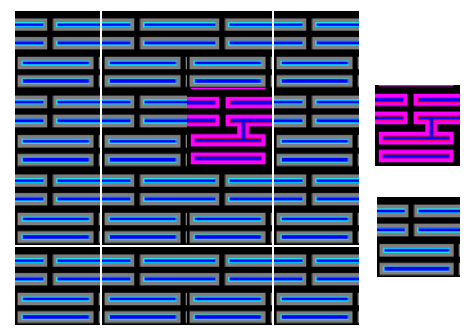

\section{Defect Unit Cell}

\section{No-Defect Unit Cell}

Figure 4. For $\lambda=13 \mathrm{~nm}$, the simulation domain was populated with by a $4 \times 4$ array of unit cells (UC) as defined in Fig. 2(c), with only one UC containing a defect to guarantee at least $10 \lambda$ separation between the edges of the simulation domain. Domain sizes as functions of wavelength are provided in Table 1.

The $x y$ dimensions of the simulation domain determine the lengths between the periodic copies of the "isolated" defect. To ensure a minimal amount of interaction among these copies, our convergence testing (not shown) has determined that this distance should be at least $10 \lambda$, and therefore in performing a multi-wavelength study, the domain size must be relatively large for the longest wavelength can be made smaller for shorter wavelengths. The simulation domain lengths and widths for these five wavelengths are provided in Table 1 in nanometers, in wavelengths, and in unit cells.

${ }^{1}$ Certain commercial materials are identified in this paper in order to specify the experimental procedure adequately. Such identification is not intended to imply recommendation or endorsement by the National Institute of Standards and Technology, nor is it intended to imply that the materials are necessarily the best available for the purpose. 
Table 1. FDTD domain size, cubic grid size, and numerical precision as functions of wavelength. Cubic grid size corresponds to the length of one side of a cube used in the simulations, with $\Delta s=2 \mathrm{~nm}$ leading to a cube $8 \mathrm{~nm}^{3}$ in volume. Single precision and double precision are common, alternative names for 32 bytes and 64 bytes, respectively.

\begin{tabular}{|c|c|c|c|c|c|c|c|c|c|c|}
\hline$\lambda$ & \multicolumn{4}{|c|}{ Domain Size } & \multicolumn{3}{c|}{ Width } & Grid Size & Floating Point \\
$\mathbf{( n m})$ & $(\mathbf{U C})$ & $(\mathbf{n m})$ & $(\boldsymbol{\lambda})$ & $(\mathbf{U C})$ & $(\mathbf{n m})$ & $(\boldsymbol{\lambda})$ & $(\mathbf{n m})$ & $(\boldsymbol{\lambda})$ & $(\mathbf{n m})$ & $\begin{array}{c}\text { Precision } \\
(\mathbf{b y t e s})\end{array}$ \\
\hline 13 & 4 & 672 & 51.7 & 4 & 720 & 55.4 & 300 & 23.1 & 1 & 64 \\
\hline 47 & 8 & 1344 & 28.6 & 8 & 1440 & 30.6 & 300 & 6.4 & 2 & 64 \\
\hline 122 & 8 & 1344 & 11.0 & 7 & 1260 & 10.3 & 300 & 2.5 & 2 & 32 \\
\hline 157 & 10 & 1680 & 10.7 & 9 & 1800 & 11.5 & 300 & 1.9 & 2 & 32 \\
\hline 193 & 12 & 2016 & 10.4 & 11 & 1980 & 10.3 & 300 & 1.6 & 2 & 32 \\
\hline
\end{tabular}

Another essential element of this FDTD simulation work is the wavelength dependence of the cubic grid size, $\Delta s$. The in-house FDTD code utilizes a uniform cubic grid size throughout the simulation domain. Convergence testing (not shown) and computational constraints were considered in establishing the grid size. It was confirmed that the cubic grid size could not be less than $\lambda / 10$. As will be shown in the following section, the scattered intensities from the $13 \mathrm{~nm}$ wavelength simulations can be much lower than that of the other wavelengths. To better enable convergence to a steady-state solution, the FDTD code was operated with a floating-point data type that is 64 bytes long for extreme ultra-violet (EUV) wavelengths, $13 \mathrm{~nm}$ and $47 \mathrm{~nm}$, while longer wavelengths were calculated using a 32-byte long floating-point data type. The lower precision allows for an improvement in calculation speed by a factor of four to eight when applicable; limited comparisons between 32-byte and 64-byte simulations for the longer wavelengths, not shown, yielded negligible differences.

\subsection{Simulation study parameters}

The following section will describe the data processing steps applied to the FDTD outputs after the defect simulations, illustrated using one example from the simulation study. The full set of simulation study parameters that were varied were the type of defect (none, "Bx", or "By"), the linear polarization (X or Y), the incident polar and azimuthal angles, and the incident wavelength. Calculations were performed at as many as thirteen angles for each combination of wavelength, polarization, and defect type, as presented in Section 4.

\section{DATA PROCESSING METHODOLOGY}

Several data processing steps are required to move from the computationally expensive electromagnetic simulations to a proper quantification of the impact of these parameters upon defect detectability. To better illustrate these procedures, one example comparison at these five wavelengths is presented in this section. This comparison follows the treatment for a single combination of incident angle, linear polarization, and defect type. Specifically, a "By" bridging defect illuminated at normal incidence with $\mathrm{X}$ polarization is shown.

\subsection{Imaging and differential imaging examples}

As noted in the Introduction, each of these five wavelengths can have optical source strengths that can differ greatly in photon flux. Other variations include the applicability and quality of the optical elements. For example, refractive elements are not available below $122 \mathrm{~nm}$ thus reflective elements dominate work in the EUV. This study acknowledges that substantive differences exist today for microscopes at shorter wavelengths, but this work concentrates on the fundamental scattering capabilities of these structures for enhancing defect detection as a function of wavelength. Therefore, imaging of the scattered fields from the ideal patterned structure and those containing defects is performed assuming ideal Fourier optics with a large collection numerical aperture of 0.95 NA. Figure 5 shows side-by-side comparisons of the images of the simulation domain with a "By" bridge defect along with the differential image formed by subtracting the no-defect image from the image with the defect. Qualitatively, these data demonstrate an increase in defect-based scattering intensity as the wavelength decreases to $47 \mathrm{~nm}$, but poor intensities at $13 \mathrm{~nm}$. This trend is not unexpected, as $13 \mathrm{~nm}$ reflects poorly at normal incidence for many materials. 


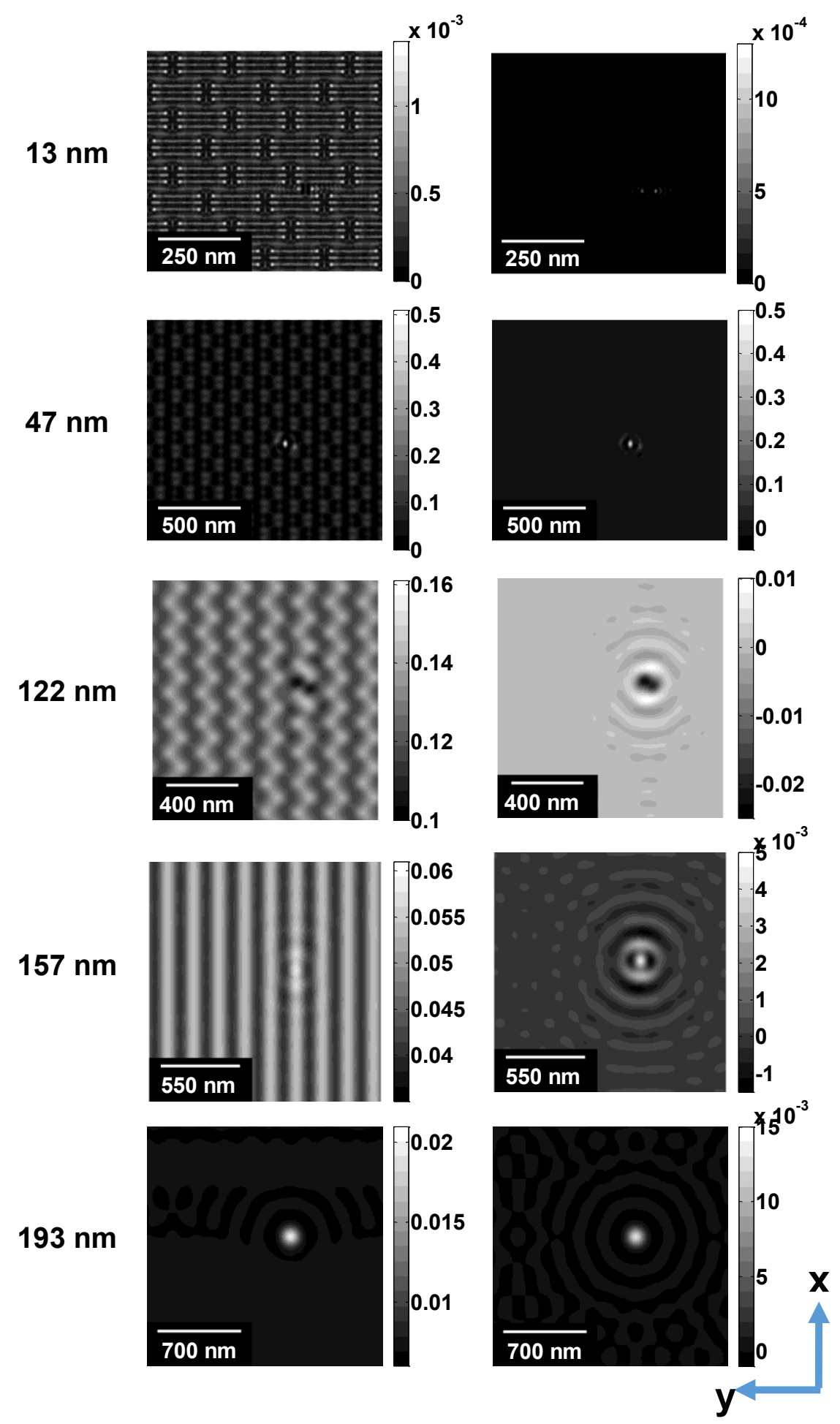

Figure 5. (left) Simulated images and (right) differential images of a "By" bridging defect assuming X polarization and plane wave illumination at normal incidence $(\theta, \phi)=\left(0^{\circ}, 0^{\circ}\right)$. Differential images are formed by subtracting a nodefect simulated image with the same polarization and incident angle from the simulated image containing the defect. Images at $\lambda=13 \mathrm{~nm}$ are resolved. Higher scattering frequencies exist for $47 \mathrm{~nm}$ through $157 \mathrm{~nm}$, while only the defect scattering exceeds the DC component at $193 \mathrm{~nm}$. 


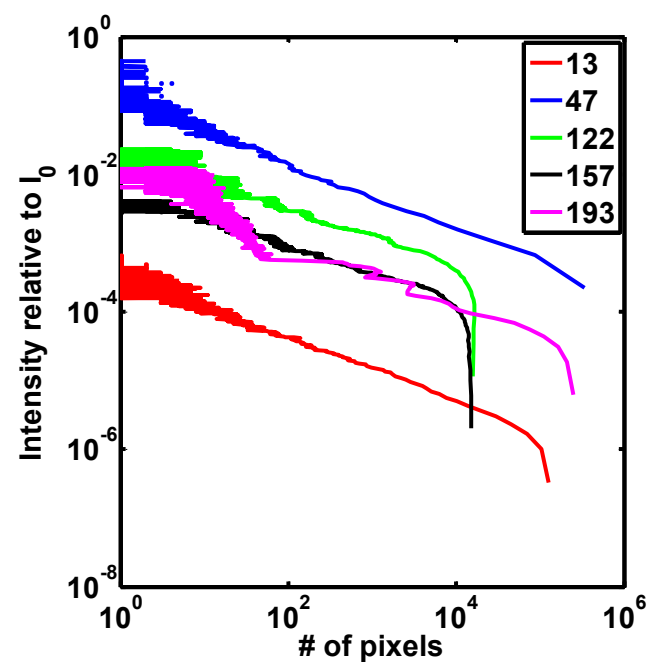

Figure 6. Differential image intensity as a function of the number of pixels in the differential image. Data were obtained by converting each differential image in Fig. 5 to its absolute value and then taking a histogram of the absolute value differential image (AVDI) using 300 bins that evenly divided the full intensity range of each AVDI. The weakest signal appears for the $13 \mathrm{~nm}$ AVDI, the strongest for the $47 \mathrm{~nm}$ AVDI.

The intensity ranges of the five differential images in Fig. 5 vary greatly with wavelength. The simplest analysis is to compare the peak intensity against zero intensity or the peak-to-valley intensity range, but these rely on one or two pixels out of an image with thousands of pixels. One method for visualizing the scattering variation using all the pixels in these images with wavelength is shown in Fig. 6. Here, the intensity distributions of the absolute value of the differential images (AVDI) are plotted with respect to the number of pixels in the image detecting such an intensity. These data are acquired from performing a binning of the pixels in the AVDI (e.g., a histogram). Figure 6 shows that there are very large numbers of pixels in each of these AVDI that show little to no practical intensity difference due to the defect, or stated more simply no "defect signal". Likewise, there is a relatively small number of pixels that exhibit a relatively strong defect signal.

Notable comparisons in Fig. 6 are among the intensity ranges captured by 100 pixels or less, which are the strongest indicators of the presence of a defect in the AVDI. While the intensity distribution for $193 \mathrm{~nm}$ straddles portions of the distribution of $122 \mathrm{~nm}$ (and to a lesser extent, $157 \mathrm{~nm}$ ), there is a clear ordering in these intensity distributions with respect to wavelength. The most sensitive wavelength is $47 \mathrm{~nm}$, followed by $122 \mathrm{~nm}, 193 \mathrm{~nm}$, and $157 \mathrm{~nm}$, with $13 \mathrm{~nm}$ the least sensitive to the "By" defect using X polarization at normal incidence. The intensity differences between the $47 \mathrm{~nm}$ response and the $13 \mathrm{~nm}$ response are about three orders-of-magnitude for these conditions.

\subsection{Applying noise}

The data above are presented without the addition of noise sources. The most thorough analysis of simulated defect images requires the inclusion of realistic noise sources. It is preferable to incorporate known sources of wafer noise such as line edge roughness (LER) into the modeling itself, which falls outside the scope of this paper. In addition, the analysis is aided by the proper treatment of process noise, but public information on process noise is lacking. While detectors are vulnerable to a variety of noise sources (e.g., thermal), one noise source is of particular interest for detectors with low photon flux, their inherent Poisson noise. One can make an estimate of the Poisson noise, also known as shot noise, at the detector, which depends upon the number of photons per pixel at the sensor (e.g., chargecoupled device (CCD) camera). The fullest treatment of Poisson noise would be wavelength-dependent, as the various sources have different photon fluxes. A credible lower bound can be established however from estimating the photon flux of a $13 \mathrm{~nm}$ wavelength source. Wojdyla et al. have reported a value of 64 photons per pixel per exposure in their actinic EUV mask metrology [17]. Conservatively estimating that the signal measured might have been on the order of $1 \%$, the incident intensity, $I_{0}$, may be approximated as 6400 photons per pixel. This value was applied to the determination of the Poisson noise for each individual pixel. In Fig. 7, the noise for the $13 \mathrm{~nm}$ wavelength image has a Poisson distribution as the scattering intensities are relatively small. On the other hand, the noise distribution for the $47 \mathrm{~nm}$ wavelength image is Gaussian, as Poisson noise has a Gaussian distribution for large photon count values. 

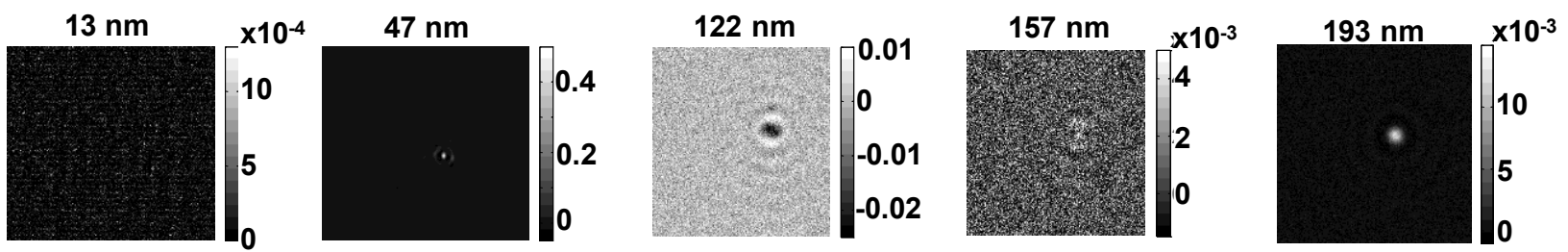

Figure 7. Differential images after the application of Poisson noise, also called shot noise, on both the "By" defect image and the no-defect image for each wavelength.

From Fig. 7, the defect is readily obscured for the $13 \mathrm{~nm}$ wavelength and the $157 \mathrm{~nm}$ wavelength defect is somewhat suppressed as well.

\subsection{Multi-wavelength defect metric}

The goal of the study is to quantify the defect detectability as functions of incident angle, polarization, defect type, and wavelength. Thus, a defect metric is required that is applicable across the five wavelengths that is independent of domain size and cubic grid size. Utilizing the absolute value of the differential image (AVDI), an ideal candidate for this defect metric is a signal to noise ratio, defined here as

$$
S N R=\frac{I_{\text {signal }}}{\sigma_{\text {noise }}},
$$

where $I_{\text {signal }}$ corresponds to the total intensity gathered due to the defect while $\sigma_{\text {noise }}$ is the standard deviation of the intensities at all pixels falling below certain thresholds; the latter is not to be confused with $\sigma$, the standard deviation of the ADVI, as it is a $5 \sigma$ threshold is initially used to differentiate the pixels with large intensities due to the defect from those pixels with a smaller signal from the defect or the applied noise. In Fig. 8, two examples are provided which illustrate the process of determining this signal-to-noise ratio. At left in Fig. 8 are images showing only those pixels with intensities greater than $5 \sigma$. The upper right panel is from the $\lambda=47 \mathrm{~nm}$ simulation and features three regions of interest, while the lower left panel shows the intensity-thresholded $\lambda=122 \mathrm{~nm}$ image, which yields not only a central optical response from the defect but also several pixels of noise. An area threshold is required to separate this random noise from the defect signal. As the wavelength decreases, one might reasonably expect the optical scattering volume from a sub-resolved object to decrease, but for this study a simple area threshold of $A_{\min }=1000 \mathrm{~nm}^{2}$ was sufficient. The center column of Fig. 8 shows the images after the removal of areas below $A_{\text {min }}$. These remaining pixels, colored in green on the right side of Fig. 8, are averaged to determine $I_{\text {signal }}$. The standard deviation of the
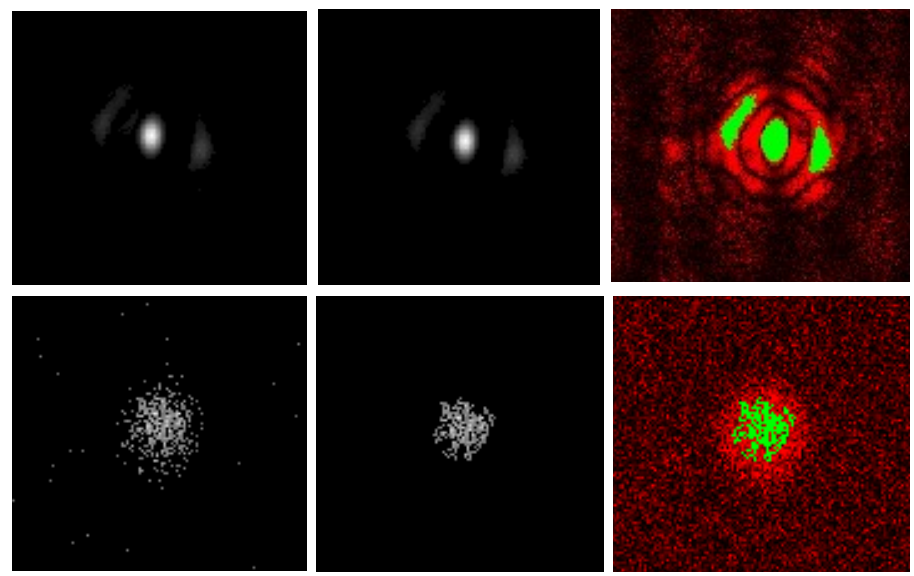

Figure 8. Application of intensity and area thresholding to separate a defect signal from its noise. Top row is data at $\lambda=47 \mathrm{~nm}$, bottom row is $\lambda=122 \mathrm{~nm}$. The left column shows pixels with intensities exceeding $5 \sigma$. The center column shows the exclusion of areas less than a constant minimum area $A_{\text {min. }}$. The right column shows the differential image due to the defect in green with the noise in red, permitting a signal to noise ratio to be determined from a single differential image. 
pixel intensities for the pixels in red determines $\sigma_{\text {noise }}$. For each combination of incident angle, polarization, defect type, and wavelength a signal to noise ratio can be evaluated.

\section{SIMULATION STUDY RESULTS}

\subsection{Comparisons of incident angle using polar plotting}

To effectively present the trends in the defect metric data, the SNR is plotted in this paper using polar plotting as illustrated in Fig. 9. In this figure, the SNR at thirteen angles (noted with circles) are shown. Interpolation is made among the points to illustrate the effects of angle-resolved illumination on the defect detectability of the "By" defect using $X$ polarization at $\lambda=47 \mathrm{~nm}$. As shown, a polar angle $\theta=15^{\circ}$ yields the optimal signal to noise with only a minor difference apparent between $\phi=30^{\circ}$ and $\phi=60^{\circ}$.

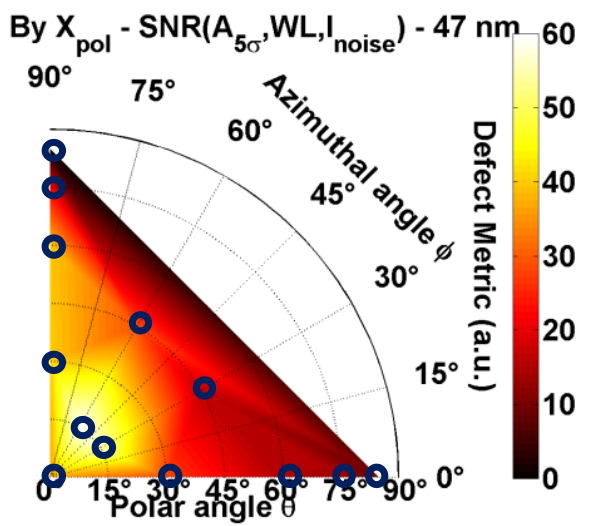

Figure 9. Polar plot of the signal to noise defect metric for the "By" defect illuminated using X-polarized, $47 \mathrm{~nm}$ wavelength illumination at 13 angles noted by circles.

\subsection{Defect metric results across the five wavelengths}

Figures 10 and 11 yield the full results of this simulation study, which yielded over 240 values for the signal to noise defect metric. Each value summarizes a combination of wavelength, defect type, polarization, and incident angle. Each figure is organized as a $2 \times 5$ array of polar plots to span these combinations.

The clearest observation in this fundamental study is that $\lambda=47 \mathrm{~nm}$ is the optimal wavelength for defect detection, outperforming $\lambda=122 \mathrm{~nm}$ by a factor of five or more. Minimal gains are observable decreasing the wavelength from $\lambda=193 \mathrm{~nm}$ to $\lambda=122 \mathrm{~nm}$, but the most pronounced results are from the $\lambda=47 \mathrm{~nm}$ simulations. Note, the domain size in Table 1 for $\lambda=47 \mathrm{~nm}$ was relatively large. There were two separate simulations studies at $\lambda=47 \mathrm{~nm}$ : the first was with a smaller domain and the second with this larger domain to greatly reduce the possibility that these gains are from periodic copies of the defects. These results are independent of these domain sizes.

The lack of appreciable signal at $13 \mathrm{~nm}$ should be noted as it was anticipated that larger angles of incidence, that is smaller grazing angles, might have yielded comparable defect detectability. The optical constants $n(\lambda)$ as shown in Fig. 2 are near or at unity at $13 \mathrm{~nm}$, while $k(\lambda)$ is near zero as well at that wavelength. As optical constants for key semiconductor materials are better characterized in the future, additional simulation study may be warranted.

Another observation that has been noted in our prior work is that there is not a single combination of angle of incidence and polarization that is optimal for these defect types. Across the wavelengths, the "Bx" defect in Fig. 10 is better observed using the Y polarization while the "By" defect in Fig. 11 is better detected using X polarization. The bridging directions of these two defects are orthogonal to each other and in both cases, the polarization that optimizes detection runs parallel to the bridging direction of the bridge. There is an azimuthal dependence at $\lambda=47$ $\mathrm{nm}$ for the "Bx" defect with Y polarization, but in general the polar angle seems more important at these wavelengths. 


\section{Bx Defect}

$X$ pol.

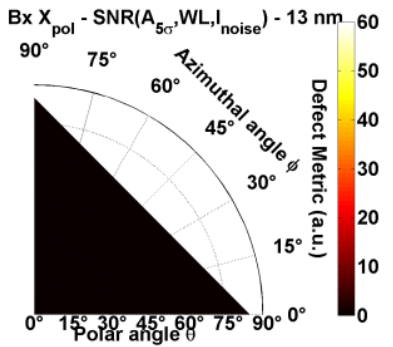

$47 \mathrm{~nm}$

$122 \mathrm{~nm}$

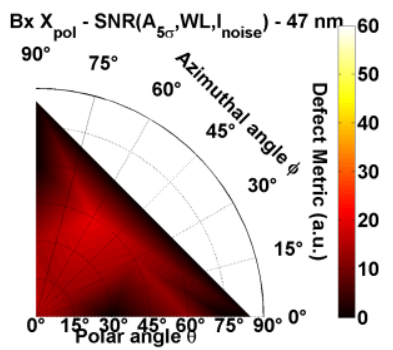

$B \times X_{p o l}-\operatorname{SNR}\left(A_{5 \sigma}, W L, I_{n o i s e}\right)-122 \mathrm{~nm}_{60}$

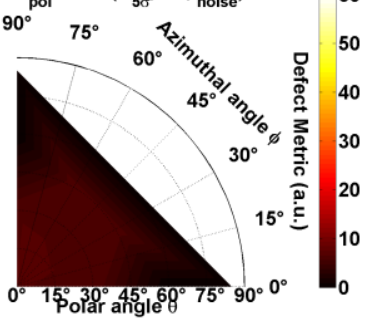

$B \times X_{\text {pol }}-\operatorname{SNR}\left(A_{5 \sigma}, W L, I_{n o i s e}\right)-157 \mathrm{~nm}_{60}$

$157 \mathrm{~nm}$

$193 \mathrm{~nm}$
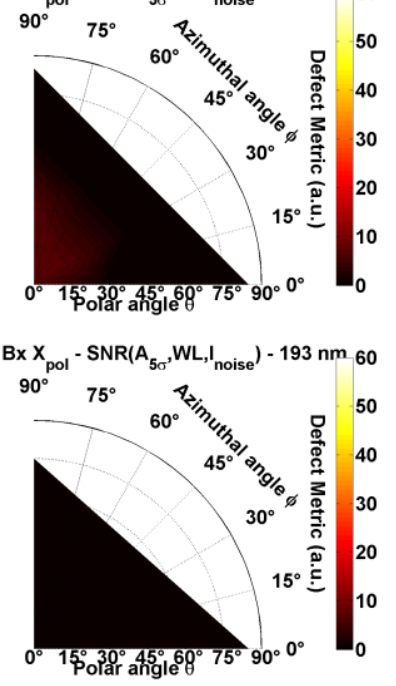

$B \times X_{p o l}-\operatorname{SNR}\left(A_{5 \sigma}, W L, I_{\text {noise }}\right)-193 \mathrm{~nm}_{60}$
Y pol.
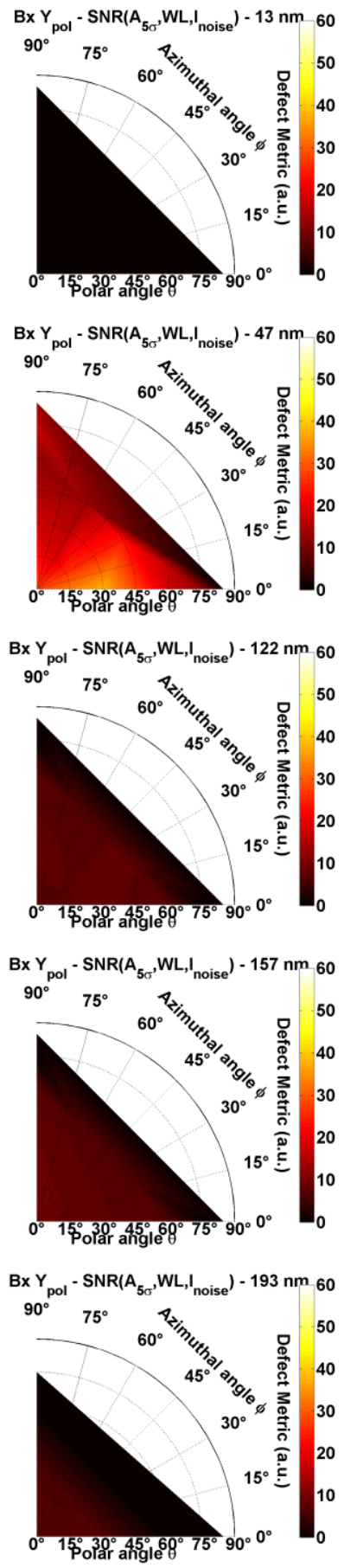

Figure 10. Signal to noise defect metric, plotted in polar plots showing the effects of polar and azimuthal angle, as functions of polarization and wavelength for the "Bx" defect. All plots in Fig. 10 and 11 are on the same color scale. With this defect metric, the $\lambda=47 \mathrm{~nm}$ yields the greatest defect detectability. Detectability is improved for all wavelengths $\lambda \geq 47 \mathrm{~nm}$ if the linearly polarized illumination is aligned with the direction of the defect. 


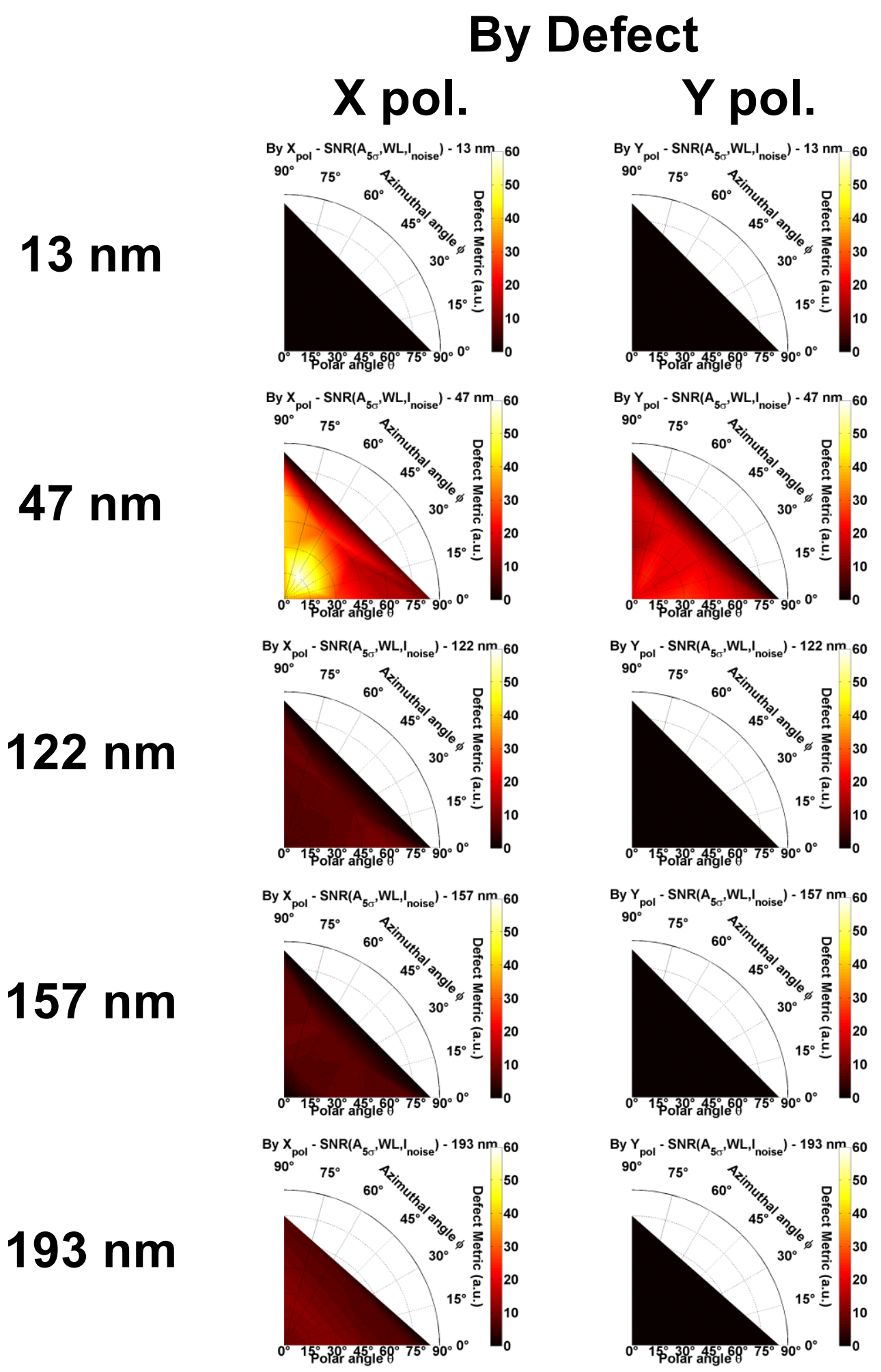

Figure 11. Signal to noise defect metric, plotted in polar plots showing the effects of polar and azimuthal angle, as functions of polarization and wavelength for the "By" defect. All plots in Fig. 10 and 11 are on the same color scale. With this defect metric, the $\lambda=47 \mathrm{~nm}$ yields the greatest defect detectability. Detectability is improved for all wavelengths $\lambda \geq 47 \mathrm{~nm}$ if the linearly polarized illumination is aligned with the direction of the defect. 


\section{CONCLUSIONS}

A simulation study has been performed at five wavelengths spanning from the DUV, through the VUV, and into the EUV to determine the fundamental improvements in patterned defect detection that may be realized by reducing the inspection wavelength. This study establishes that up to a factor of five improvement may be realized in signal to noise ratio from the adoption of $\lambda=47 \mathrm{~nm}$ as an inspection wavelength. A shorter EUV wavelength, $13 \mathrm{~nm}$, was illsuited for defect inspection, yielding differential intensities as much as three orders of magnitude smaller than from simulations using $\lambda=47 \mathrm{~nm}$.

These results, based on the basic physics, indicate how defect identification trends with wavelength into the EUV. For example, the optical constants $n$ and $k$ for $13 \mathrm{~nm}$ are at or near 1 and 0 , respectively, and a much smaller optical signal is backscattered. The much larger values of $k$ at $\lambda=47 \mathrm{~nm}$ as well as the metallic behavior of $n$ at this wavelength contribute to its notably strong response. In addition, the defect is resolved at $\lambda=13 \mathrm{~nm}$, thus there is little optical interaction between the scattering due to the defect and the scattering due to the ideal patterned structure. For longer wavelengths, the underlying patterns and defects are unresolved and their scattering interacts, and further work should be performed to define the positive effects of such interactions.

Optimized combinations of incident angle, linear polarization, bridge direction, and wavelength were determined for the two bridge defects. Following the SEMATECH naming scheme, the "Bx" bridge (running along the $y$ direction) is enhanced by Y polarization while the "By" bridge (running along the $x$ direction) is enhanced by $\mathrm{X}$ polarization. This result agrees well with our previous studies of intentional defect arrays at larger critical dimensions.

Although additional work remains to rigorously include the challenges faced at each of these wavelengths, this study reveals the potential gains of reducing the inspection wavelength in patterned defect inspection. To make these results more practical, additional, credible elements need to be considered, such as more process stacks, more materials, available source strengths, microscope configurations, and detector quantum efficiencies and noise.

\section{REFERENCES}

[1] C. Hess, and L. H. Weiland, "Issues on the size and outline of killer defects and their influence on yield modeling," IEEE/SEMI 1996 Advanced Semiconductor Manufacturing Conference and Workshop. 423428 (1996). http://dx.doi.org/10.1109/ASMC.1996.558102

[2] R. M. Silver, B. M. Barnes, Y. Sohn et al., "The Limits and Extensibility of Optical Patterned Defect Inspection," Proc. SPIE, 7638, 76380J (2010). http://dx.doi.org/10.1117/12.850935

[3] T. F. Crimmins, "Defect metrology challenges at the $11 \mathrm{~nm}$ node and beyond," Proc. SPIE, 7638, 76380H (2010). http://dx.doi.org/10.1117/12.846623

[4] M. I. Mishchenko, "Gustav Mie and the fundamental concept of electromagnetic scattering by particles: A perspective," J. of Quant. Spectro. Rad. Trans., 110(14-16), 1210-1222 (2009). http://dx.doi.org/10.1016/j.jpsrt.2009.02.002

[5] M. Tian, M. Li, and J. C. Li, "Effect of size on dielectric constant for low dimension materials," Physica B: Condensed Matter, 406(3), 541-544 (2011). http://dx.doi.org/10.1016/j.physb.2010.11.034

[6] D. F. Edwards, [Silicon (Si)*] Academic Press, Boston(1985). http://dx.doi.org/10.1016/B978-0-08054721-3.50029-0

[7] H. Piller, [Silicon (Amorphous) (a-Si) ] Academic Press, Boston(1985). http://dx.doi.org/10.1016/B978-008-054721-3.50030-7

[8] D. Franta, D. Nečas, I. Ohlídal et al., "Dispersion model for optical thin films applicable in wide spectral range," Proc. SPIE, 9628, $96281 \mathrm{U}$ (2015).

[9] Q.-J. Liu, N.-C. Zhang, F.-S. Liu et al., "Structural, electronic, optical, elastic properties and Born effective charges of monoclinic HfO2 from first-principles calculations," Chinese Physics B, 23(4), 047101 (2014). http://dx.doi.org/10.1088/1674-1056/23/4/047101

[10] E. Filatova, A. Sokolov, J.-M. André et al., "Optical constants of crystalline $\mathrm{HfO}_{2}$ for energy range 140930 eV," Appl. Opt., 49(14), 2539-2546 (2010). http://dx.doi.org/10.1364/AO.49.002539

[11] H. R. Philipp, [Silicon Dioxide $\left(\mathrm{SiO}_{2}\right)$, Type $\alpha$ (Crystalline)] Academic Press, Boston(1985). http://dx.doi.org/10.1016/B978-0-08-054721-3.50039-3 
[12] A. Taflove, "Application of the Finite-Difference Time-Domain Method to Sinusoidal Steady-State Electromagnetic-Penetration Problems," IEEE T. Electromag. Compat., EMC-22(3), 191-202 (1980). http://dx.doi.org/10.1109/TEMC.1980.303879

[13] A. Raghunathan, S. Bennett, H. O. Stamper et al., "13nm gate Intentional Defect Array (IDA) wafer patterning by e-beam lithography for defect metrology evaluation," Microelec. Engr., 88(8), 2729-2731 (2011). http://dx.doi.org/10.1016/j.mee.2011.02.109

[14] S. Natarajan, M. Agostinelli, S. Akbar et al., "A 14nm logic technology featuring $2^{\text {nd }}$-generation FinFET, air-gapped interconnects, self-aligned double patterning and a $0.0588 \mu \mathrm{m}^{2}$ SRAM cell size." 3.7.1-3.7.3 (2014). http://dx.doi.org/10.1109/IEDM.2014.7046976

[15] B. M. Barnes, F. Goasmat, M. Y. Sohn et al., "Enhancing $9 \mathrm{~nm}$ Node Dense Patterned Defect Optical Inspection using Polarization, Angle, and Focus," Proc. SPIE, 8681, (2013). http://dx.doi.org/10.1117/12.2012250

[16] B. M. Barnes, F. Goasmat, M. Y. Sohn et al., "Effects of wafer noise on the detection of 20-nm defects using optical volumetric inspection," J. Micro-Nanolitho. MEMS MOEMS, 14(1), 9 (2015). http://dx.doi.org/10.1117/1.jmm.14.1.014001

[17] A. Wojdyla, A. Donoghue, M. P. Benk et al., "Aerial imaging study of the mask-induced line-width roughness of EUV lithography masks," Proc. SPIE, 9776, 97760H (2016).

http://dx.doi.org/10.1117/12.2219513 


\title{
Characterization of the Dimensions of Internal Gap in a Glass Artifact Using a Laser Triangulation Probe
}

\author{
Speaker: W. Ren \\ Engineering Physics Division \\ National Institute of Standards and Technology \\ Gaithersburg, MD 20899 \\ Authors: W. Ren, T. Doiron, J. Stoup, E. Stanfield \\ National Institute of Standards and Technology
}

\section{Introduction}

The National Institute of Standards and Technology (NIST) Dimensional Metrology Group (DMG) develops and delivers unique and critical high-value dimensional measurements that promote industry innovation, ensure product quality, and are not commercially available. In some cases, we need to use multiple measuring systems to provide our customers with the needed measurement results. This paper is the story of one of these measurements that involved a number of different instruments and is typical of the complexity of many of our customer requests. Recently, NIST provided a measurement solution for characterizing the gap width of a new biotechnology absorbance spectrometry standard reference material (SRM). This SRM is a reduced path length validation absorption standard for Ultraviolet (UV) visible spectroscopic measurements. The SRM consists of a series of very small rectangular glass containers called cuvettes and has been developed for the biotechnology community's daily measurements of samples where the sample amount available is very limited: typically, samples include DNA, RNA, or an antibody. Identification of the exact substance by spectrometry measurements is based on how the substance absorbs light. The dimension of the gap between the inside walls of the cuvette is a critical characterization, therefore measurement of the gap width with low uncertainty is required.

\section{Dimensional measurement challenge \\ 2.1 Challenge}

The Standard Reference Materials (SRMs) are transparent cuvettes made of fused quartz glass, $45 \mathrm{~mm}$ long and $10 \mathrm{~mm}$ wide, with gaps of three different widths, $2 \mathrm{~mm}, 1 \mathrm{~mm}$, and $0.5 \mathrm{~mm}$. An example of a cuvette is shown in Figure 1. Due to geometric imperfections during manufacture and assembly, the gap dimension does vary as a function of location (length), thus a rigorous characterization is needed. Dimensional Metrology Group (DMG) has several high-accuracy Coordinate Measuring Machines (CMMs) with a variety of probe sizes and configurations. However, as the gap width becomes smaller, the required probe diameter becomes smaller and subsequently the maximum probe shaft length becomes shorter in order to maintain required probe stiffness. The large aspect ratio of gap width-to-length thus presents a challenge for DMG using conventional touch-probe CMMs. 


\subsection{The limitation of CMM touch probe}

NIST's M48 ${ }^{1}$ CMM is recognized for its unparalleled accuracy over its measurement volume [1][2]. The $2 \mathrm{~mm}$ cuvette can be measured over its entire length by the M48 CMM using a probe with a $1.5 \mathrm{~mm}$ diameter tip and a $40 \mathrm{~mm}$ long shaft. This arrangement can easily provide the sub-micrometer level expanded uncertainties $(k=2)$ required for the measurement. However, for the smaller cuvettes, the narrow opening limited the size of the tip that could be used. In addition, the smaller diameter tips require a very thin shaft with a much shorter useable length. The restricted probe length limited the accessible region to $1.25 \mathrm{~mm}$ of the $34 \mathrm{~mm}$ length desired. The CMM cuvette measurement also required the data to be collected at multiple probing forces. The thin glass walls of the cuvette may bend under the probing force and the elastic deformation of the glass needed to be characterized. The CMM data was extrapolated to an undeformed result for the comparison with the non-contact system.

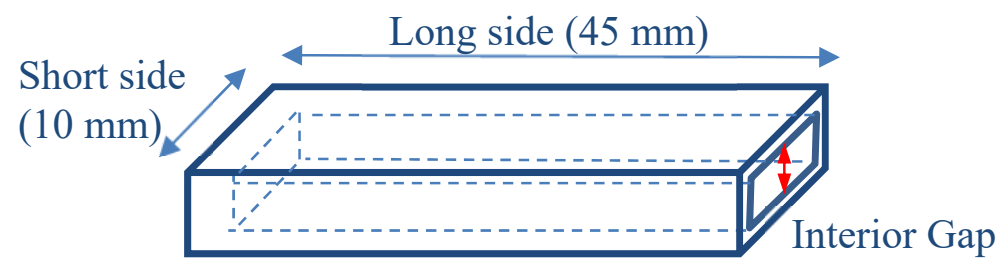

Figure 1. An example of cuvette

\section{A Non-contact measurement technique}

\subsection{Introducing Laser Triangulation Probe}

A laser triangulation probe is a non-contact measurement technology and is sometimes preferred over contact-probe technology when measuring delicate or hard to reach surfaces. The basic principle of triangulation is shown in Figure 2. A semiconductor laser emits the laser beam to the target. The light scattered from the surface of the target (seen as a small spot of light) is focused by the lens and forms an image on the light-receiving element which is either a CCD (charge-coupled device)/CMOS (complementary metaloxide semiconductor) array or a PSD (position sensitive detector) element. The position of the beam spot on the receiving element varies with the distance to the target. This variation is evaluated and converted into a measurement of target position [3].

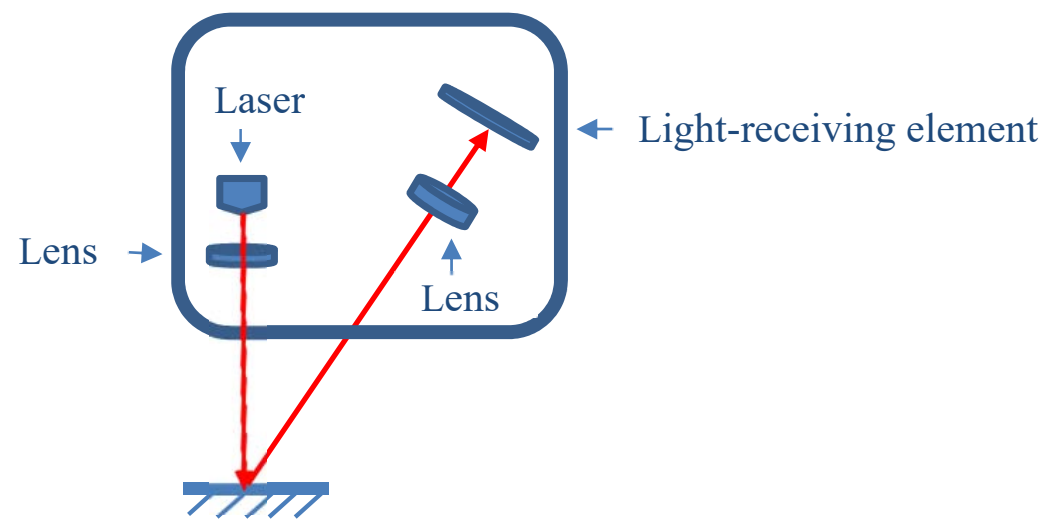

Figure 2. The principle of the triangulation probe 
${ }^{1}$ Certain commercial equipment, instruments, or materials are identified in this paper in order to specify the experimental procedure adequately. Such identification is not intended to imply recommendation or endorsement by the National Institute of Standards and Technology, nor is it intended to imply that the materials or equipment identified are necessarily the best available for the purpose.

We have used the triangulation probe for a number of projects where the measurement artifacts were not transparent and thus only one surface was measured. The gap measurement here, using a laser triangulation probe, is based on the location difference between spots generated at 2 of the 4 glass-air interfaces, specifically the two inner interfaces. The concept seems straight-forward but, in reality, deciphering which 2 spots represent the inner interfaces and extracting the distance between them was not simple.

\subsection{System setup and measurement process}

When measuring a diffuse-reflective target, we mount the laser triangulation probe head straight down and perpendicular to an X-Y stage as shown in Figure 2. The incident light hits the target and the reflected light intensity is uniform in all directions, and the lightreceiver element can detect the target "image" and calculate the displacement of the target. However, the cuvette is made of fused quartz glass and is a specular-reflective target with most of the light being reflected at an angle equal to the incident angle. The light-receiver sensor in the triangulation probe head does not receive an adequate signal from the transparent surfaces if the probe head is mounted as in Figure. 2. Therefore, a different measurement setup for the triangulation probe head is introduced as shown in Figure 3. The probe head is mounted on a precision linear stage, along the $Y$ axis of our setup, with the head tilted at an angle of 20 degrees, which is half of the original light receiving angle for this instrument configuration. The cuvettes with different gap sizes are laid on a small adjustable Z-axis vertical stage, which sits on a high precision air bearing linear stage, with its motion aligned in the $\mathrm{X}$ direction. The $\mathrm{X}$-axis stage is moved at a speed of $100 \mathrm{~mm} / \mathrm{s}$ during measurement, with the speed remaining reasonably constant.

Doiron, Theodore; Ren, Wei; Stanfield, Eric; Stoup, John. 


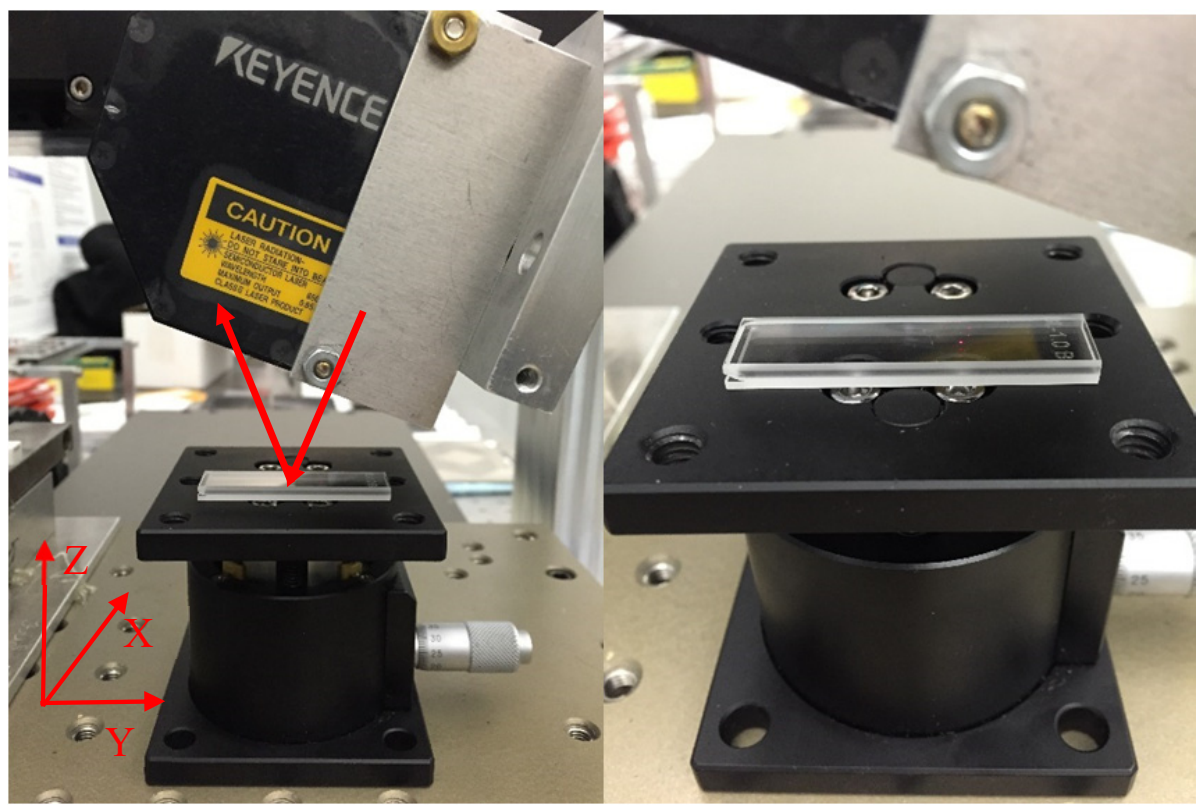

Figure 3. Triangulation probe setup for measuring a cuvette. The incident and reflected beam as a red line shows in the figure above.

The cuvette is mounted so that its short side is parallel to the $\mathrm{X}$ axis. We align the cuvette by stepping the laser spot along the $\mathrm{Y}$ axis near the edge and ensuring that the edge is at the same $\mathrm{X}$ position at different locations along the $\mathrm{Y}$ axis. A careful alignment is important for measuring the cuvette due to the offset calculation performed as part of the data analysis. A detailed explanation will be presented in section 3.3. The triangulation probe head is mounted in such a way that the incident and reflected beam form a triangle that lies in the $\mathrm{YZ}$ plane.

The measurement procedure is as follows. We first acquire a scan along the $\mathrm{X}$ axis at a $\mathrm{Y}$ position that is $0.5 \mathrm{~mm}$ from the open end of the cuvette. We compute the gap width for each position along the $\mathrm{X}$ axis and compute the average of multiple runs. We then step the probe another $0.5 \mathrm{~mm}$ along the $\mathrm{Y}$ axis, acquire a new scan along the $\mathrm{X}$ axis, and compute the average gap width, again from multiple runs. We repeat this process all the way down to $34 \mathrm{~mm}$ from the open end of the cuvette. From all the data, we obtain the gap width as a function of the location along the long side of the cuvette. The gap thickness is calculated using the multiple reflections obtained when the laser source is directed at each of the four cuvette surfaces.

Knowing that the laser beam is refracted when it passes through the cuvette, Snell's law tells us that the amount of refraction can be related to the angle of the laser beam [4]. If we were interested in measuring the thickness of cuvette wall, the influence of refractive index of glass would need to be calculated. However, given that we are interested in measuring the gap between the glass plates we know both beams are refracted by exactly the same amount during each pass through the glass and the effects cancel as shown in Figure 4. 


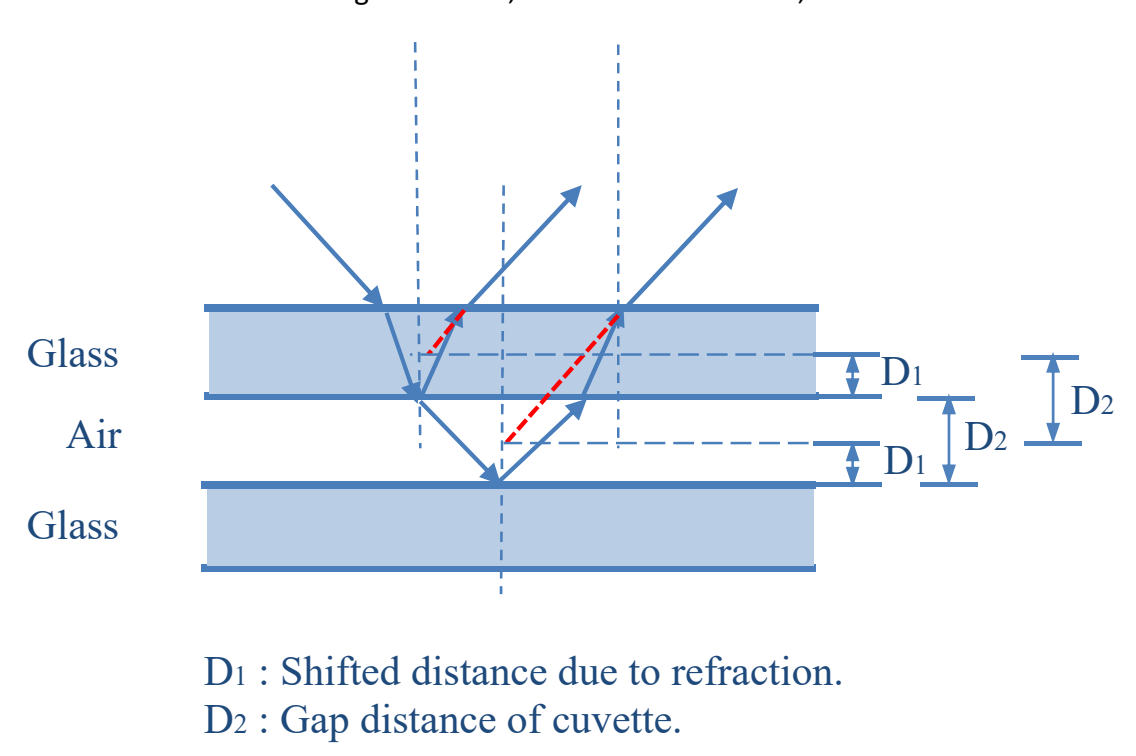

Figure 4. Beam diagram for thickness measurement. The reflections from the top and bottom surfaces are omitted.

\subsection{System validation}

The system is validated by comparing the triangulation probe results with M48 CMM measurements made on a $2 \mathrm{~mm}$ cuvette, since the $2 \mathrm{~mm}$ cuvette can be measured over its entire length by the M48 CMM. Two aspects of the data are compared - the variation of the gap dimension as a function of location and the magnitude of the gap dimensions. The data in Figure 5 show that the variation of the gap dimension as a function of location is the same using both measurement techniques, thus verifying that the gap dimension is changing due to cuvette geometry and is not a function of the measurement technique. However, a systematic offset exists between the gap dimension reported by the two methods which is most likely related to the not precisely known angle between the laser and the cuvette surface. Instead of determining this angular error and correcting for it independently, we instead correct the triangulation results by this constant offset, effectively using the triangulation probe as a comparison measurement tool. This validation demonstrates that the laser triangulation probe method can be used as a comparison method but it does not free us from the need for reference measurements for gap dimension on the smaller cuvettes, since the magnitude of the correction will vary with gap size and the angle the laser makes with the cuvette surface. 


\section{$2 \mathrm{~mm}$ Cuvette}

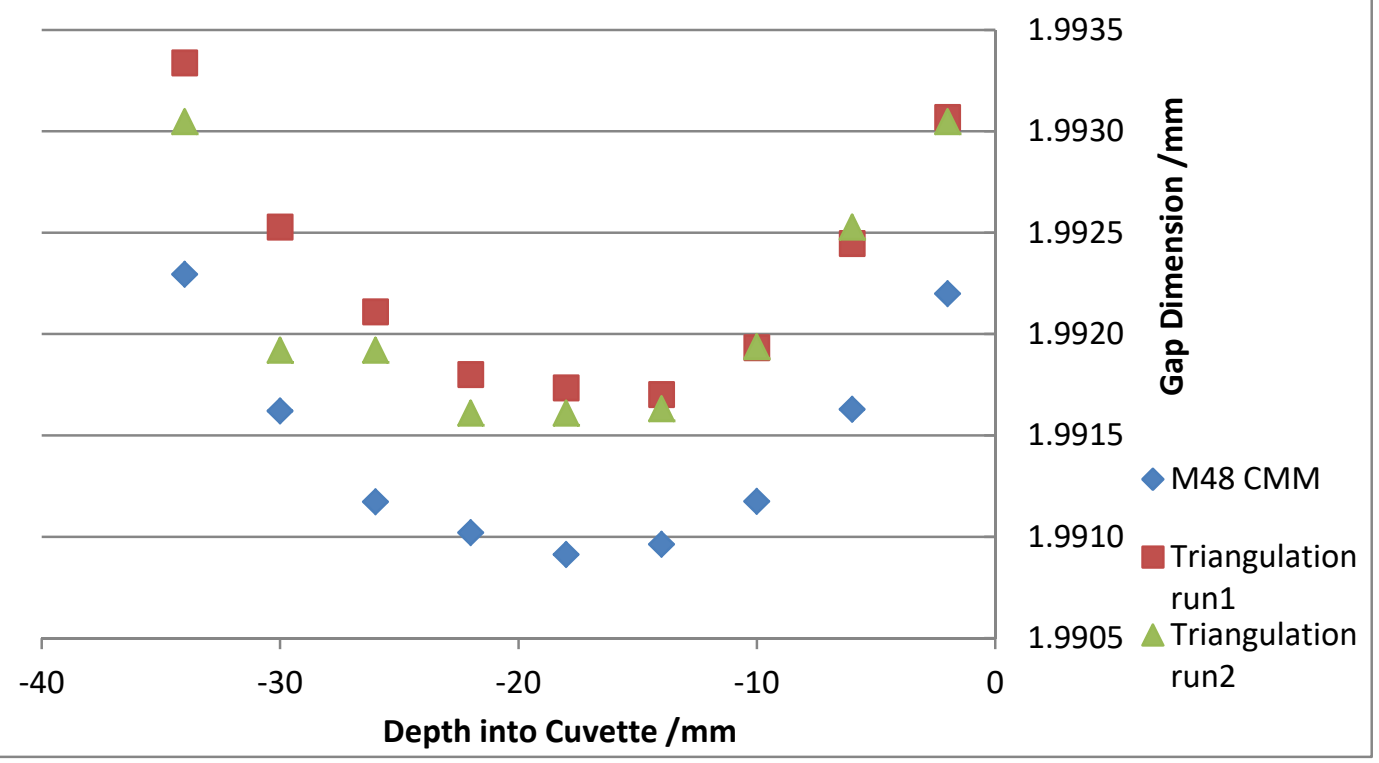

Figure 5. Measurement results for $2 \mathrm{~mm}$ cuvette

\subsection{Measurement of Smaller Cuvettes}

For the smaller cuvettes, we use the largest CMM probe that will fit in the gap to maximize the depth within the cuvette that can be measured with the M48 CMM. For the $0.5 \mathrm{~mm}$ cuvette, we used a $300 \mu \mathrm{m}$ diameter probe, the smallest effective probe that the CMM can use without shaft breakage. As concluded from the system validation, the M48 measurements serve as the reference for making corrections to the triangulation probe results for the two smaller cuvettes. This is not done without some concern for the validity of the corrected data due to the limited depth of overlap between the M48 and triangulation probe measurements. With this concern, we incorporated an additional check by using the Mitutoyo ${ }^{1}$ UMAP ULTRA350 Dual-Probe Micro-Feature CMM [5]. This special purpose CMM has multiple probes, one with a $100 \mu \mathrm{m}$ diameter probe on an $80 \mu \mathrm{m}$ diameter shaft that is $10 \mathrm{~mm}$ long and a second probe with a $30 \mu \mathrm{m}$ diameter probe on a $20 \mu \mathrm{m}$ diameter shaft that is $2 \mathrm{~mm}$ long. For this measurement, the larger of the two UMAP probes was used.

Figure 6 shows the measurement results for all three processes and the extent of overlap, limited in the case of the physical probes, by the probe shaft length. The M48 measures the gap to a depth of $1.25 \mathrm{~mm}$ from open end and the UMAP measures the gap to a depth of $8.5 \mathrm{~mm}$ from the open end. Comparison of the results in Figure 6 show that all three systems see a similar variation in gap geometry, which supports applying a systematic correction to the triangulation probe results using the M48 results as a reference. Upon closer examination to the data presented in Figure 7, the slope of triangulation probe and the M48 data match within $29 \mathrm{~nm} / \mathrm{mm}$ in the overlap region. Although the UMAP data shows an increasing gap dimension as a function of depth like the other results, the slope is not nearly as close of a match. This observation is not a surprise in that the UMAP probe often exhibits errors that are function of depth due to sidewall-probe stem 
interactions that result from the shaft having a diameter only $10 \mu \mathrm{m}$ smaller than the probe. As more and more of the shaft is exposed to the part sidewall, the interaction forces increasingly affect the trigger point. This trend could be removed by measuring the $2 \mathrm{~mm}$ cuvette with the UMAP probe then extracting the depth dependent correction using the M48 data, but the $2 \mathrm{~mm}$ cuvette was no longer available by the time the decision was made to include the UMAP. Ultimately, we concluded that all comparisons were useful and we could confidently use the triangulation data for measuring the smaller cuvettes, correcting systematic differences based on overlap measurements with the M48, even though the overlap region was only a small fraction of the gap depth.

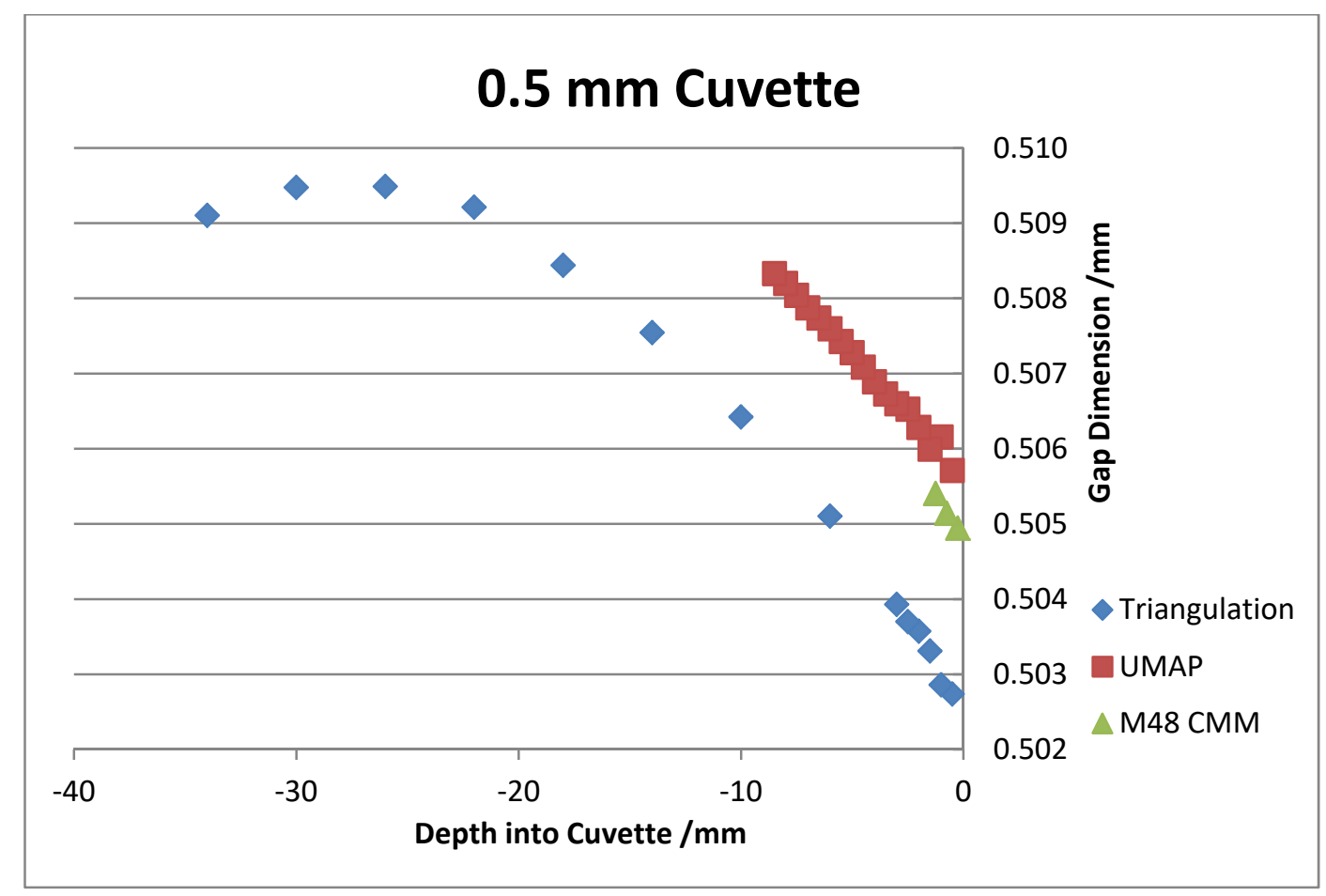

Figure 6. Measurement results for $0.5 \mathrm{~mm}$ cuvette

Doiron, Theodore; Ren, Wei; Stanfield, Eric; Stoup, John. 


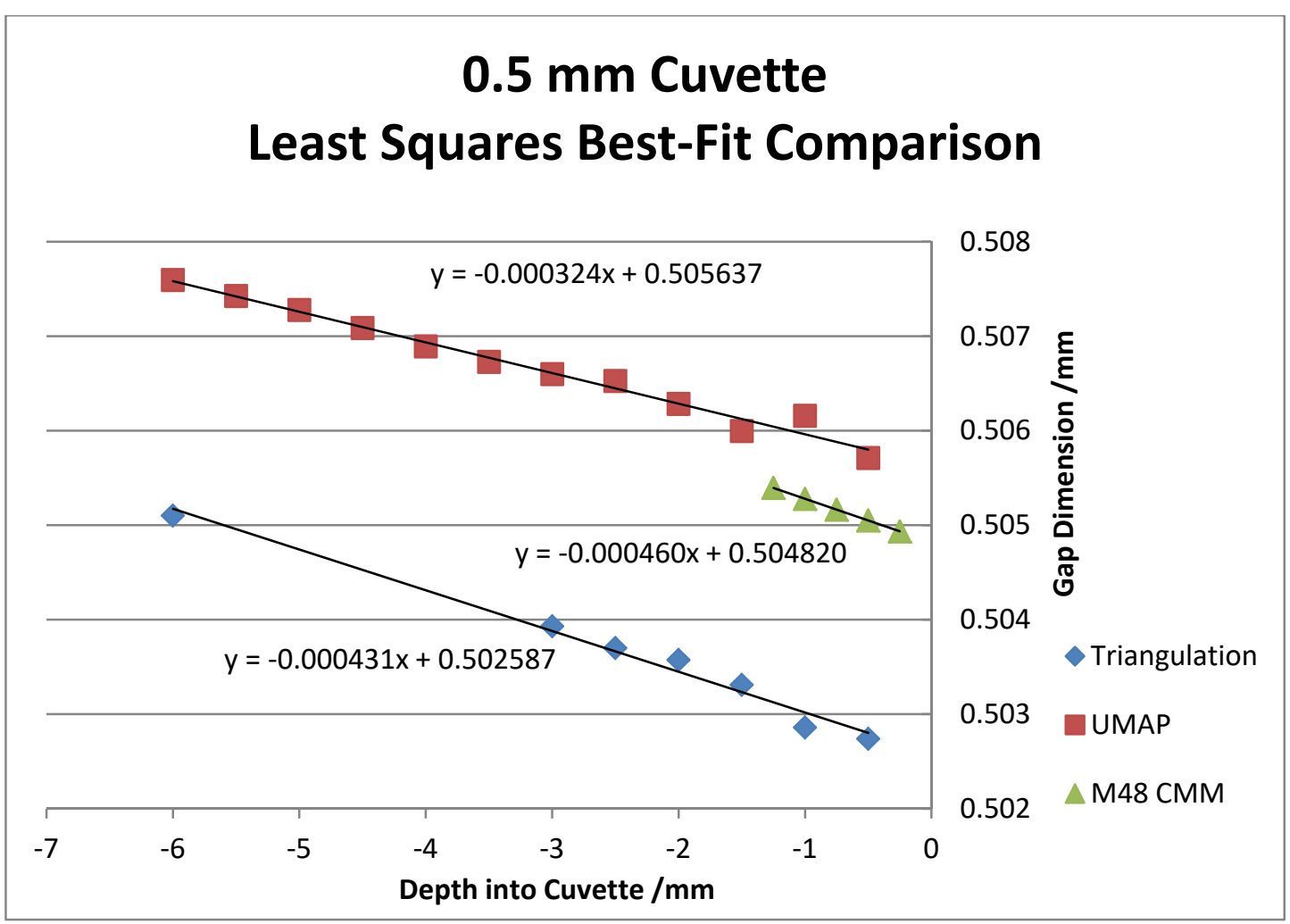

Figure 7. Linear plots for $0.5 \mathrm{~mm}$ cuvette

\subsection{Data Analysis and Uncertainty}

Both the $1 \mathrm{~mm}$ and $0.5 \mathrm{~mm}$ cuvettes were measured using two different methods, a high accuracy M48 CMM and a laser triangulation probe. Because of the small gap widths, the CMM could only measure down $1.25 \mathrm{~mm}$ into these cuvette openings. The laser triangulation probe collected 3,800 points across the width of the cuvette, and each collection of 3,800 points was referred to as a line or profile. The cuvette was translated along the $\mathrm{Y}$ direction, the long dimension of the cuvette, and the line scan was repeated at 14 different levels, $0.5 \mathrm{~mm}$ to $34 \mathrm{~mm}$ from the open end of the cuvette. The accessible depths were then measured with the M48 CMM and used to correct the small bias in the laser triangulation probe data. The bias corrections were based on the average gap dimension across the width.

The short-term reproducibility of the laser triangulation probe is $0.1 \mu \mathrm{m}$ and, from the comparison data between the M48 and the triangulation measurement system, the standard error of each comparison correction is $0.3 \mu \mathrm{m}$, which includes the triangulation probe repeatability. We will call this $\mathrm{u}_{1}$. The M48 CMM, which is used as the reference for these comparison measurements, has a standard uncertainty of $0.1 \mu \mathrm{m}$, which we will call $u_{2}$. Other sources of error are negligible. Combining these standard uncertainties, $u_{1}$ and $u_{2}$ in quadrature yields a combined standard, $u_{\mathrm{c}}$, of $0.32 \mu \mathrm{m}$. With a coverage factor of $k=2$, the expanded uncertainty, $U$, then becomes $\pm 0.64 \mu \mathrm{m}$. 


\section{Conclusion}

Although the laser triangulation probe is not an absolute measurement, we devised a creative approach using multiple measurement techniques at our disposal to extend our measurement capabilities to solve a critical measurement request. This method used the laser triangulation probe as a comparator with the M48 CMM as the reference based on the overlap where the two processes could measure the same measurand. As the primary means of validation for the approach to measure $0.5 \mathrm{~mm}$ and $1 \mathrm{~mm}$ cuvettes, we used comparison measurements on an near identical item, the $2 \mathrm{~mm}$ cuvette, for which both technologies could measure the entire item. In addition, out of an abundance of caution, we also used a third measurement system, the Mitutoyo UMAP ULTRA350 Microfeature CMM, to give support to the notion that we could extrapolate our correction from a very small overlap between the two methods on the $0.5 \mathrm{~mm}$ and $1.0 \mathrm{~mm}$ cuvettes to the remaining $90 \%$ or more of the measurement results produced by the laser triangulation probe. The high degree of accuracy of the cuvette gap measurements, resulted in an expanded uncertainty for the SRM's absorbance as a function of pathlength of less than $0.08 \%$, which exceeded expectations.

Lastly, it is important to note that the results reported represent the average gap across the width of the cuvette face at the specified depth. The data from both the M48 and the laser triangulation probe revealed that there was significant geometry variation across the width. The reported uncertainty assumes that the laser spot during the absorbance spectrometry measurements is relatively well centered on the cuvette face. If this is not the case, the user would need to incorporate additional uncertainty to cover geometry based variability caused by misalignment relative to the center where the reference measurements and uncertainty are valid.

\section{Acknowledgments}

We would like to acknowledge Bala Muralikrishnan, Meghan Shilling, Daniel Sawyer and Eugene Song for reviewing the manuscript and suggesting many useful changes and corrections.

\section{References}

[1] J. Stoup and T. Doiron, The Accuracy and Versatility of the NIST M48 Coordinate Measuring Machine, Proc. SPIE Vol. 4401 (2001).

[2] J. Stoup, Measuring Step Gauges Using the NIST M48 CMM, NCSLi Conference Proceedings (2011).

[3] Keyence Corporation, Measurement Application Guide for Thickness/Width Measurement.

[4] P. Mihas, Developing Ideas of Refraction, Lenses and Rainbow Through the Use of Historical Resources, Science \& Education (2008).

[5] E. Stanfield, B. Muralikrishnan, T. Doiron, A. Zheng, S. Orandi and D. Duquette, Two applications of small feature dimensional measurements on a coordinate measuring machine with a fiber probe, Measurement Science and Technology, Vol. 24, No. 10 (2013). 
Metrology Outreach and Training: A Fulbright Experience in Mexico

Authors:

Georgia L. Harris, U.S. National Institute of Standards and Technology (NIST), Office of Weights and Measures (OWM)

Flora E. Mercader Trejo, Universidad Politécnica de Santa Rosa Jáuregui (UPSRJ)

Adriana Veraza Arellano, Universidad Politécnica de Santa Rosa Jáuregui (UPSRJ)

Salvador Echeverria Villagomez, Centro Nacional de Metrologia (CENAM)

Abstract

2016 was an exciting year for international collaboration on metrology education and training. This paper provides a 3-part look at how a Fulbright Specialist grant supported collaboration between the United States and Mexico. Part 1 describes the experience of Georgia Harris (NIST) as a Fulbright Specialist, from the application process to the implementation activities in Mexico and shares insight about lessons learned and benefits to NIST. In Part 2, Flora Mercader and Adriana Veraza describe the application process within the University to obtain approvals for the grant, the implementation process, how additional parties were engaged for participation, some immediate benefits, some expected long-term impacts, and lessons learned. Part 3 includes Salvador Echeverria's description of CENAM's involvement in the courses conducted at the University as well as the sessions held at CENAM, immediate benefits that were observed, and provides insight for ongoing collaboration for metrology education and training in Mexico. Recommendations and additional ideas for international collaboration and future work on measuring the impact of collaborative efforts are proposed.

\section{Introduction}

The Fulbright Specialist Program is managed by the United States Department of State, Bureau of Educational and Cultural Affairs Exchange Programs. The Fulbright Program is an international educational exchange program sponsored by the U.S. government and is designed to increase mutual understanding between the people of the United States and the people of other countries. "The Fulbright Specialist Program sends U.S. faculty and professionals to serve as expert consultants on curriculum, faculty development, institutional planning, and related subjects at academic institutions abroad for a period of two to six weeks. The Fulbright Specialist Program allows overseas universities, cultural centers, non-governmental organizations, and other institutions abroad to develop collaborative projects which host a leading U.S. academic or professional at their institution to work on diverse, short-term collaborative projects focused on education and training. These projects support critical priorities identified by the host institutions and supported by U.S. embassies and binational Fulbright Commissions abroad." 1

\footnotetext{
${ }^{1}$ Fulbright Specialist Program details are available online at: https://exchanges.state.gov/us/program/fulbrightspecialist-program. For details about applying to become a Fulbright Specialist, you can visit:

https://fulbrightspecialist.worldlearning.org/eligibility-specialists/ (2017-04-16).
} 
Requests for U.S. National Institute of Standards and Technology (NIST) to conduct training at the University were initially received by Ms. Carol Hockert, Chief of the Office of Weights and Measures while attending a Centro Nacional de Metrologia (CENAM) Symposium (CENAM Simposio) session in Mexico in 2014. After discussions related to the normal costs of organizing the NIST Fundamentals of Metrology ${ }^{2}$ seminar, both in terms of the logistics/operational costs to NIST and the travel/per diem costs of the attendees, the discussions stalled due to excessive costs of providing the program on site in Mexico.

The idea to use the Fulbright Specialist Program as a collaborative tool was initially raised during discussions Ms. Georgia Harris had at the American Society for Engineering Education (ASEE) conferences where staff managing the Fulbright Specialist Program were recruiting potential experts for international collaboration opportunities. Ms. Harris then suggested to Dr. Flora Mercader, Universidad Politécnica de Santa Rosa Jáuregui (UPSRJ), the idea of coordinating the requested training through a Fulbright Specialist Program. The difference between the Fulbright Scholar and Fulbright Specialist program is that the former is a student and the latter is a professional expert providing instruction and consultation. One major advantage of the program is that of cost-sharing and funding sources.

The activities described in this paper were funded through a mixture of financial sources, including the Fulbright Specialist Grant awarded to Ms. Harris which covered flight expenses to and from Mexico; the U.S. National Institute of Standards and Technology (NIST), which covered staff time and course materials; the Universidad Politécnica de Santa Rosa Jáuregui (UPSRJ), which covered all in-country expenses associated with transportation, food, and lodging, plus the local coordination of logistics, space, and materials; and the Centro Nacional de Metrologia (CENAM), which hosted a coordinated effort for portions of the sessions held at CENAM. In addition, course equipment and standards were provided by Sartorius Corporation, Troemner, LLC, and Ms. Harris. Because Ms. Harris was working for NIST at the time of the grant/project, she was ineligible to receive the per-diem allotment that is available to other grantees.

\section{Fulbright Specialist Perspectives}

\subsection{Application}

Basic eligibility requirements are available on the Fulbright Specialist program website. But in general, applicants must be U.S. citizens, have significant experience in their respective field, as demonstrated by professional, academic, or artistic achievements, and able to travel overseas for 14 to 42 days (length varies depending on project). The application process consists of an on-line application, including curriculum vitae (CV) and submission of two letters of reference.

Once the application has been submitted by one of several annual deadlines, there is a peerreview process that assesses: professional qualifications (professional standing and other

\footnotetext{
${ }^{2}$ Fundamentals of Metrology, seminar descriptions are available here: English: https://www.nist.gov/pml/weightsand-measures/fundamentals-metrology. Spanish: https://www.nist.gov/pml/weights-and-measures/fundamentos-demetrolog\%C3\%ADa
} 
achievements), demonstrated capacity for leadership within their field, suitability for Specialist Grant activity, alignment of candidate's background and skills with their objectives for the program outlined in the candidate's application essays, feasibility of the candidate to successfully engage in eligible grant activities, quality of the submitted references, cross-cultural communication skills and adaptability, quality of previous international/intercultural experiences, and the likely overall benefit to stakeholders, including potential to contribute to a host institution, benefit to the home institution (NIST in this case), and potential for advancement of knowledge in the applicant's field of study and personal qualities to successfully complete a project designed by a host institution abroad and to serve as a cultural ambassador for the United States. A successful peer-review process places the applicant on the Fulbright Applicant Roster. Once a project and grant are approved, the applicant is then awarded a "Fulbright Specialist Grant.”

The grant authorization describes the status, for example: "Georgia Harris has been selected by the J. William Fulbright Foreign Scholarship Board for a FULBRIGHT SPECIALIST GRANT under the provisions of the Mutual Educational and Cultural Exchange Act of 1961 (as amended), the FULBRIGHT PROGRAM. This grant is administered by the Bureau of Educational and Cultural Affairs, United States Department of State with the cooperation of the Institute of International Education/Council for International Exchange of Scholars (IIE/CIES).”

The application process, is simple and straightforward.

\subsection{Project Development (Statement of Work)}

Host institutions are required to lead the project development process. This ensures that expertise requested through the Fulbright Specialist Program meets a need that the institution has selfidentified as a critical area for its growth and development. For the activities described here, the collaboration and discussions about potential projects had already taken place. So, while potential Fulbright Specialists are permitted to explore collaborations with potential host institutions, host institutions are under no obligation to consider such requests. Host institutions who ultimately elect to name a Fulbright Specialist candidate on their project proposal must include a strong justification for the need to work with this candidate and for all proposed activities.

The proposed statement of work built on potential courses that Ms. Harris has previously taught and that the University determined would be of greatest value in Mexico. Ms. Harris provided the related course descriptions and learning objectives for the project proposal to ensure that the host university needs were met with materials that could be successfully delivered.

\subsection{Planning for Materials, Logistics, Equipment, and Standards}

The courses that were selected by UPSRJ and included in the Project Proposal were designed to be conducted at alternative facilities and locations. Therefore, logistics details and host instructions were readily available. The most challenging aspect associated with the advance coordination of logistics was communication regarding the materials needed for a successful training event. A preliminary list of measuring equipment, standards, and other instructional 
materials were provided to UPSRJ. When most aspects of the equipment and standards were identified as not being readily available, alternative sources were sought. Small hand tools, like micrometers, pocket balances, and items to be measured (in this case pennies), were shipped with the training materials from NIST. UPSRJ provided a local computer printer for printing draft calibration certificates during the course, and was made available for use by the students.

All course notebook and handouts, along with printing instructions, were sent electronically to the University in advance of the sessions to be printed by the University and then provided to the students on-site. This process of providing electronic media has worked well for the Fulbright Specialist at other training events previously conducted in Colombia. Additional resources were hand-carried, such as multiple copies of a USB “technical library.”

A few incidental instructional resources, such as easels to hold flip chart paper were not readily available at UPSRJ or for purchase at the local office supply store, but could have been shipped with other supplies from NIST since this type of material is routinely shipped for conduct of offsite training events. Having some experience with international shipments, there was some concern about materials not being received in a timely manner. However, the shipping to and from the training site worked extremely well and all materials that were shipped arrived on time and without damage or incident. Materials returned to the U.S. were also returned without undue delay or problems.

\subsection{Conducting the Fundamentals of Metrology Course and Lessons Learned}

Dr. Mercader was the primary technical contact with the UPSRJ for collaboration. She arranged campus access on the Sunday prior to beginning of the first course to the classroom and the laboratory, which were then set-up. Advance access to the campus classroom and laboratory alleviated some of the stress associated with teaching a course in an unknown location. Even though everything was available and arrived on time, flexibility and creativity were called for on the first day. Everyone arrived to find that the facility was experiencing an electrical power outage! The host was quickly able to acquire an electric generator to power the instructor laptop and projector to initiate the course on time!

One of the key observations for the course and this audience is that the Fundamentals of Metrology curriculum is normally framed around operating a calibration or testing laboratory per the ISO/IEC 17025 documentary standard ${ }^{3}$. Because the training participants were professors and instructors of metrology, most were not currently working in a calibration laboratory, which is why the documentary standard was assigned as pre-reading. Several participants previously worked at CENAM or in industry calibration laboratories and were familiar with these concepts. Participants of this course are usually required to pass a mathematics pre-test. This requirement was waived for this audience of engineering professors, which did not result in any problems for the successful completion of the course. Several of the usual examples that are provided in the Fundamentals of Metrology course, such as "internal auditing” were shifted to "internal auditing to accreditation requirements” to share the ideas and concepts. Several minor shifts and exceptions were made due to this audience being primarily engineering professors. Instead of

\footnotetext{
3 ISO/IEC 17025: 2005, General Requirements for the Competence of Testing and Calibration Laboratories.
} 
teaching how something would be applied in a calibration or testing laboratory, emphasis was placed on how to teach the content to subsequent students who would be applying the material in a training laboratory and ultimately on-the-job.

The other challenge that required flexibility and creativity were related with teaching using English language. Having better Spanish language skills would have been a definite advantage. However, the engineering professor participants as well as their engineering programs are considered bi-lingual (Spanish/English) and the course was intentionally taught in English so that participants would have the instructional experience from an English speaker, using their primary language. The course is normally divided into small groups. During introductions, the instructor made an initial assessment of English language skills to help divide students into their teams. At least one highly proficient English speaker was on each team to ensure that the debriefing process associated with each activity would be successful. Most activities and handson measurements were conducted in the student's preferred language with summaries and debriefing conducted in English.

\subsection{Conducting Project Based Learning Train the Trainer Seminar and Lessons Learned}

The second major course conducted as a part of the Fulbright Specialist project was a Project Based Learning Train the Trainer seminar that focused on application to Metrology instruction for engineering professors. Ms. Harris has previously conducted Train the Trainer events at a CENAM Symposium and at several metrology training events within the United States. As a part of engineering outreach efforts, Ms. Harris has presented hands-on metrology training concepts to engineering professors at the previous American Society for Engineering Education (ASEE) conferences ${ }^{4}$. The 2.5 day seminar combined perspectives of adult learning, Train the Trainer activities, and outreach to engineering professors, and focused on the use of the ADDIE Instructional Design (ID) model $^{5}$, developing effective course learning objectives, designing hands-on practical exercises for teaching metrology concepts, and sharing ideas for practical hands-on activities among the participants.

Additional benefit could be gained in future sessions by obtaining the reference documents and instructional models that professors may be required to use when developing engineering curricula. The learning objective development process is standard, but the ADDIE model, while often used for instructional design efforts, is not always the method used in all universities. A preliminary web search determined that the ADDIE model is in common use among universities and is also used in the NIST training program, which is why the model was presented; however, several other participants noted that they were required to use alternative models in their work environments.

\footnotetext{
${ }^{4}$ Incorporating Adult Learning Methods and Project Based Learning in Laboratory Metrology Courses, Georgia L. Harris, ASEE Annual Conference and Exposition, 2013.

${ }^{5}$ The ADDIE Instructional Design (ID) model is a framework for designing and developing educational and training programs, originally developed in 1975 by Florida State University's Center for Educational Technology (Branson, Rayner, Cox, Furman, King, Hannum 1975; Watrson, 1981) for the U.S. Army. “ADDIE” stands for the five steps: Analyze, Design, Develop, Implement, and Evaluate.
} 
The integration and application of adult learning and project-based learning (PBL) concepts as a part of these course provided an opportunity for each professor to experience and observe the impact of an alternative to a traditional lecture-based course. Significant feedback was received from the participants on the value of experiencing the ideas in action and participating in the technical courses themselves. This concept is routinely integrated in NIST Office of Weights and Measures courses, resulting in significant improvement of students applying concepts on the job, rather than just gaining knowledge or familiarity.

During the events held in Mexico, it was possible to observe the level of comprehension of engineering professors related to metrology; they also provided feedback that the course provided them with extensive insight in metrology that was greater than their previous experience provided. Several professors reported that while they have been working in metrology and engineering for many years, they gained additional useful insight about teaching metrology concepts. Even though many of the professors had a background in metrology, little hands-on measurement exercises have been integrated into the engineering curricula. In other cases, applying metrology to engineering is much further ahead of many U.S. engineering programs. For example, Mexico has numerous vocational and higher education organizations providing courses in metrology, including the integration of metrology within engineering programs. Because most of the participating professors were from recently established universities, they now have opportunities to create and integrate metrology concepts and handson activities into their curricula before the programs become entrenched in lecture based methods and become less flexible. That is, there is a tendency to leave a course as it is designed to minimize the additional effort that could be required if incorporating major changes, or even continual improvement, both of which might require accreditation body or governmental assessments of curriculum changes.

\subsection{Outreach to University Students and Observations}

Two key outreach sessions were provided to UPSRJ students to increase their awareness of measurement science career opportunities and leverage the metrology expertise of the Fulbright Specialist while on campus. The first technical topic explored metrological traceability and included a discussion of "who's who" in the world of metrology. About 25 or 30 third and fourth year engineering students participated in this session. The students were engaged in the topic and asked insightful application questions. About 80 first year engineering students participated in the second technical session, "How Do You Know Your Measurements are Right?” Concepts such as traceability, calibration, and uncertainty, using a theme of temperature were presented. The presentation highlighted Mexican women metrology scientists and engineers as well as several women students currently studying within the UPSRJ industrial metrology engineering program. Much world-wide Science, Technology, Engineering, and Mathematics (STEM) outreach is targeted to young women to encourage them to pursue and stay in technical fields. Identifying suitable role models and available mentors is critical to demonstrating that women can be successful in STEM careers. While targeting young women was not a specific goal of the Fulbright Specialist project proposals, it is a key aspect of the outreach work conducted by both Dr. Mercader and Ms. Harris. 
It was extremely rewarding to observe the excitement among the engineering students about metrology! It was refreshing to observe a high number of young women enrolled in the program, as well as the overall interest expressed by outreach session attendees. Students who approached Ms. Harris after the sessions were primarily interested in hearing more about metrology careers.

\subsection{Participating at CENAM Sessions and Insights}

Dr. Mercader coordinated with Dr. Echeverria to have Ms. Harris participate in several technical sessions at CENAM with the final plan and agenda coordinated during the Fulbright Specialist project visit to Querétaro. The two sessions previously presented to students at UPSRJ ("How Do You Know Your Measurements are Right?” and “Essentials of Metrological Traceability”) were presented at CENAM, with the idea that the content could be used as a model for agency scientific staff to conduct local outreach with students (rather than as technical content focused on the participants themselves).

Of most interest during the CENAM visit was a session on Best Practices in Metrology Instruction, with additional participants from CENAM (Ismael Castelazo, General Director of Technology Services), UPSRJ (Flora Mercader), and The Metrology School (Hugo Hernández), which was attended by about 50 CENAM staff and students. The presenters during the Best Practices session primarily shared details about their programs and courses. Rather than talk about NIST training activities, personal insights about "five best practices" were shared as had previously been discussed with Dr. Echeverria. The Fulbright Specialist expressed personal opinions and experiences regarding the five best practices:

1. Instructors should participate in instructor professional development, such as in the concepts of adult education and instructional design (a technical PhD alone is insufficient).

2. Course design must include the integration and alignment of learning objectives, activities, and student assessments. Students must be assessed on stated objectives and learning methodology must be consistent with expectations.

3. Adult learning processes and concepts need to be used at the university and postuniversity level (i.e., andragogy versus pedagogy).

4. Ongoing evaluation is required for all course content and educational activities to ensure continual improvement and to remain current. For example, a course developed on ISO Guide 25: 1990 would not be applicable for a course that implements ISO/IEC 17025:2005.

5. Metrology training and education programs benefit from some level of accreditation to raise the quality bar and professionalism.

\subsection{Final Report Required}

After a Fulbright Specialist completes the project, a final project report is required to be submitted. It includes an opportunity to identify lessons learned and benefits for all the participants. Information, much like was is in this paper, was submitted as a part of the final report. Of specific interest is the intent to conduct ongoing collaboration and evaluate the longterm impact of the Fulbright Specialist project. 


\subsection{Benefits to NIST}

Collaboration in metrology education and training helps fulfill NIST objectives by maintaining international leadership, ensuring the acceptance and use of international and U.S. documentary standards, and dissemination of NIST measurement procedures and metrology training methods, thus helping to ensure a solid measurement foundation in academia, government, and industry throughout the Americas. Part of the Office of Weights and Measures (OWM) mission is to "improve the accuracy of measurements, enhance consumer protection, foster fair competition, and facilitate economic growth and trade through technical activities that promote uniformity in national and international legal metrology laws, regulations, standards, test procedures, and enforcement." Much of the OWM efforts include publication of measurement procedures and training on those procedures for the weights and measures and metrology communities. Many NIST documents were referenced and used during the events.

The OWM training program is accredited by the International Association for Continuing Education and Training (IACET) which requires use of adult-learning methodologies. Having participants in the courses, who are metrology professionals as well as university professors, provided unique feedback and insights to the Fulbright Specialist that will be applied when updating OWM metrology courses. Gaining experience working with professors, and finding out their unique challenges in academia, will also help with ongoing effort and metrology outreach work that is being coordinated with professors at U.S. schools of higher education through association with NCSL International and ASEE.

\section{Universidad Politécnica de Santa Rosa Jáuregui (UPSRJ) Perspectives}

Universidad Politécnica de Santa Rosa Jáuregui (UPSRJ) is a higher education institution with public funding founded in 2011. UPSRJ is part of a network of Polytechnic Universities in Mexico with a high degree of specialization in the field of engineering. The academic program of Industrial Metrology Engineering was designed at UPSRJ, thus becoming the first University in Mexico to offer this educational option which has remarkable industrial requirements. To ensure that the professors who teach in this program are kept up-to-date and aware of the importance of metrology, the University is constantly looking for associations with institutions and experts who can contribute their experience and knowledge to the program. The interaction of UPSRJ students and teachers with foreign experts is also a way to expand the internationalization program on campus.

\subsection{Application for a Fulbright Specialist Project}

In 2012, Dr. Flora Mercader, (UPSRJ) and Ms. Georgia Harris, (NIST) met at the Metrology Symposium organized by the National Metrology Institute of Mexico (Centro Nacional de Metrologia, CENAM Simposio). Both identified that they are carrying out activities to strengthen the teaching of metrology to future generations. Several opportunities for collaboration were considered. However, the Fulbright Specialist program was identified as a potential cost-sharing opportunity for the University to have short-term collaborative projects with highly experienced specialists. 
The application process to obtain the expert was simple and straightforward The success of the program was that we could determine exactly who the expert would be, which made the process much easier. Dr. Mercader Trejo and Ms. Adriana Veraza contacted Dr. Jackal Talelorn at La Comisión México-Estados Unidos para el Intercambio Educativo y Cultural (COMEXUS), which manages the Fulbright program in Mexico. Dr. Talelorn explained the application process and the deadlines for delivery of the application format. We told him about Ms. Harris, the expert we had in mind. Once he checked that Ms. Harris was among the pool of registered experts, he explained the process.

\subsection{Logistics and Challenges at UPSRJ}

An estimate of costs and expenses of the program was made during the planning phase. Once U.S. Fulbright Program replied that the project was possible with the requested expert, the project was presented in detail with the budget and dates to Mr. Enrique Sosa Gutiérrez, UPSRJ Rector, and Orfelinda Torres Rivera, the academic director, explaining the scope and impact to teachers and students and for the University itself. The academic directors and the rector together decided to present a proposal date, which was presented to Ms. Harris to initiate event scheduling to finally make the project a reality.

Dr. Mercader and Ms. Harris developed the scope of work, which presented high level detail of the program content and requited resources required for the visit. All the presentations, conferences, and courses would be combined in an event called "Metrology Seminar." The activities of the seminar are described in the following table. Dr. Mercader and Ms. Harris reviewed and selected courses and topics that would be of benefit to the participants in Mexico.

Table 1. Fulbright Specialist Program Metrology Seminar Components.

\begin{tabular}{|l|c|c|}
\hline \multicolumn{1}{|c|}{ Activity } & 2016 Dates & Participants \\
\hline COURSE "Fundamentals of Metrology” & $\begin{array}{c}\text { Oct 31 to Nov } \\
07\end{array}$ & $\begin{array}{c}\text { 4 Professors from UPSRJ, 1 from UNAQ, 1 } \\
\text { UTSJR, 1 UPG, 1 UPQ, 1 ITC, and 2 from } \\
\text { CENAM }\end{array}$ \\
\hline $\begin{array}{l}\text { CONFERENCE "Essential Elements of } \\
\text { Metrological Traceability" }\end{array}$ & Nov 07 & $\begin{array}{c}\text { Second and third year students from the } \\
\text { Industrial Metrology Engineering Program }\end{array}$ \\
\hline $\begin{array}{l}\text { COURSE "Project Based Learning Applied to } \\
\text { Metrology Instruction" }\end{array}$ & Nov 08 to 10 & $\begin{array}{c}\text { Professors form UPSRJ and guests from } \\
\text { different institutions }\end{array}$ \\
\hline $\begin{array}{l}\text { CONFERENCE "How do you know your } \\
\text { measurements are right?" }\end{array}$ & Nov 10 & $\begin{array}{c}\text { First year students from the Industrial } \\
\text { Metrology Engineering Program }\end{array}$ \\
\hline $\begin{array}{l}\text { CONFERENCE "Essential Elements of } \\
\text { Traceability and Risks; How do you know your } \\
\text { measurements are right”? and Best Practices in } \\
\text { Metrology Education and Training Panel" }\end{array}$ & Nov 11 & CENAM \\
\hline
\end{tabular}

Ms. Harris provided a detailed list of the materials, equipment, standards, and teaching resources that would be needed to effectively conduct the seminars in Mexico, and the University then conducted an inventory of materials. The project preparation before the specialist's arrival was a challenge because the UPSRJ currently does not have all the required course laboratory equipment. Ms. Harris and Dr. Mercader contacted research centers and institutions to request 
support for equipment loans. UPSRJ is a recently created institution, founded less than 6 years ago. Because the organization is in the development stage, facilities and equipment infrastructure is deficient. One of the biggest challenges UPSRJ faced was having the necessary equipment, standards, and materials for the metrology seminar. One way to solve it was to collaborate with training attendees to bring typical course equipment and calibration artifacts, which included certified mass standards, thermos-hygrometers, and certificate gage blocks. In addition, Ms. Harris contacted companies that supply analytical balances and mass standards, (such as Sartorius, Troemner LLC) exploring the possibility of lending or donating balances, mass standards, and other laboratory resources for the seminar. Sartorius gave us a positive answer and provided 4 analytical balances with the characteristics that were needed for the seminar. Sartorius staff were also available on site to talk to the participants and students of the University before/after the sessions and during breaks. Another very important contribution was the donation of three sets of OIML Class $F_{2}$ mass standards (100 g to $1 \mathrm{mg}$ ) with NVLAP accredited calibration certificates from Troemner, LLC.

Another challenge for the university was the space and materials to teach the courses. Many of the materials normally used to teach the respective seminars were not available and the room configurations were not ideal. However, despite having the students and the specialist move to several rooms on campus, the sessions were still successful. Once the university staff moved other scheduled courses to alternative locations, the group could minimize the use of multiple classrooms and could use a regular classroom that was located very near the laboratory.

The International Department, headed by Ms. Veraza, oversaw preparing the lodging, food, transportation, and course materials prior to the arrival of Ms. Harris. This aspect was less challenging and worked very smoothly. The necessary course material for the participants was sent electronically in advance for preparation of notebooks and handouts for the participants. The University also generally prefers to conduct courses in a sustainable manner, with less "paper" and requested Ms. Harris to consider not using printed course materials. However, Ms. Harris responded that the course activities required review and mark-ups of some of the materials, designed to emulate the typical process performed in an operational laboratory, and had not designed the course to meet sustainability goals.

\subsection{Collaboration with additional Universities, Industry, and CENAM}

To extend the impact of Ms. Harris' visit as a Fulbright Specialist, UPSRJ invited professors from other universities and institutions. The invitation was extended to institutions that are part of the Network for Innovation and Research in Metrology for the Automotive Industry (RIIMSA), which is coordinated by UPSRJ. The following additional seven RIIMSA member institutions were represented through their staff: Centro Nacional de Metrología (CENAM), Centro Regional de Optimización y Desarrollo de Equipo (CRODE), Universidad Politécnica de Guanajuato (UPG), Centro de Ingeniería y Desarrollo Industrial (CIDESI),Centro de Investigación y Asistencia Técnica del Estado de Querétaro (CIATEQ),Universidad Politécnica de Querétaro (UPQ), Universidad Aeronáutica en Querétaro (UNAQ),Universidad Tecnológica de San Juan del Río (UTSRJ). 
Additionally, participants from the following three institutions attended: Universidad Anahuac Campus Querétaro, Universidad Tecnológica de Querétaro (UTEQ), Instituto Tecnológico de Celaya (ITC). It is important to emphasize that CENAM support was essential for this event to be successfully developed and implemented. Good relationships were also developed between Sartorius, Troemner and the university due to the donations of equipment and standards.

\subsection{Visit Logistics}

The University oversaw transporting the specialist during the entire visit, from Ms. Harris' arrival at the airport until her return. Accommodation and meals were also covered by UPSRJ. For the seminar, University staff were aware of the lectures and courses for any need that arose. Regarding laboratory practices, Dr. Mercader served as an assistant to Ms. Harris, providing guidance to the professors who were attending the course.

\subsection{Immediate Benefits}

Several immediate benefits were achieved:

- Greater collaboration among Queretaro regional universities and metrology institutions interested in improving their capabilities in teaching metrology through networking and team activities conducted during the courses.

- UPSRJ obtained a good supply of equipment and calibration artifacts useful for hands-on metrology practices, through the donation of balances, calipers, gage blocks, weight sets, pocket balances.

- Metrology and adult learning concepts were improved for training and developing professors who are teaching metrology topics.

- Increased metrology exposure for university students who are studying engineering in industrial metrology to see the importance and impact of measurement science.

- Educational tools and models that can be used to improve the metrology curricular contents through project-based learning methods were developed.

\subsection{Expected Long-term Benefits}

One of the most important benefits of these courses was the opportunity to positively impact how metrology is taught for future generations. This is one of the UPSRJ's priority objectives. Through the training that was provided, professors are already implementing actions to improve the metrology teaching methods both at the University and in at the other institutions that participated in the seminar. Following the seminar, several instructors have contacted Ms. Harris to request clarification and additional resources related to those provided during the course sessions.

\subsection{Lessons Learned and Insights Gained}

The primary lesson learned from this great experience was that there are many ways to overcome obstacles and barriers to effective metrology instruction - especially those due to the lack of equipment and standards and limited classroom spaces. Ms. Harris’s availability prior to, during, and after the seminar provided for a resoundingly successful event. 


\section{CENAM Experience}

\subsection{Role and function of CENAM in the project.}

CENAM, the Centro Nacional de Metrologia, is the National Metrology Institute (NMI) of Mexico. As such, it is the representative of Mexico in the International Committee on Weights and Measures (CIPM) and signatory of the Mutual Recognition Arrangement (CIPM MRA) which is the framework through which NMIs demonstrate the international equivalence of their measurement standards and declare the calibration and measurement certificates they issue. CENAM was created by the Federal Law on Metrology and Standardization (1992) which assigns to the Center, among others, the following functions:

Federal Law on Metrology and Standardization (LFMN) of Mexico, Chapter V (Centro Nacional de Metrología), Article 30 (Functions) states CENAM:

I. To be the primary Laboratory of the National Calibration System.

IV. To promote and develop research and technological development activities in different fields of metrology, as well as to collaborate in the development of human resources with the same objective.

VIII. To organize and participate, if convenient, in congresses, seminars, conferences, courses and other type of events related to metrology.

IX. To sign agreements with research and institutions that have capabilities to develop primary standards or high precision instruments, as well as education institutions that can offer specializations in metrology.

As can be seen, CENAM has the mandate by law to collaborate with Higher Education Institutions for developing competencies regarding metrology. In addition, the high value that collaboration with NIST and the UPSRJ provide to this mandate, has been clear since CENAM's founding.

The long-standing collaboration and support received by CENAM from NIST scientists and specialists have been always recognized. In this case, the interaction with Ms. Georgia Harris and Ms. Carol Hockert through the NCSL International ${ }^{6}$ Learning and Development Committees, has led to very fruitful results. An indicator of this is the number of papers published in the NCSLI Workshop and Symposium by members of the Mexican community, most times promoted by Ms. Georgia Harris in her work as leader of the NCSLI Learning and Development Committees.

In the Mexican scene, the fact that the training program in metrology and quality developed by the UPSRJ was the pioneer in the educational field, makes this university an ideal partner to fulfill the function and the objectives in terms of human resources development in metrology as outlined earlier.

\footnotetext{
${ }^{6}$ NCSL International (NCSLI) - formerly National Conference of Standards Laboratory. More information about NCSLI can be viewed at www.ncsli.org.
} 
The Metrology Outreach and Training program, promoted by the UPSRJ and supported by the Fulbright Specialist Program of the U.S. Department of State, has been seen by CENAM as a great opportunity that has demonstrated its benefits.

\subsection{Coordinating an Event at CENAM}

The coordination of the event at CENAM was very straightforward, because Ms. Georgia Harris has much experience, both technical and logistical, in these types of events, with the specifics of the event regarding cultural adaptation and CENAM logistics where well cared for by Dr. Mercader and her team. Details of the planning process were provided in section 2.7. The final selection of activities for the CENAM journey were defined in common agreement among Ms. Harris, Dr. Mercader and Dr. Echeverria. This was as follows:

Table 2. Fulbright Specialist Program Metrology Seminar Program in CENAM, Nov 11, $2017 .$.

\begin{tabular}{|l|c|c|}
\hline \multicolumn{1}{|c|}{ Activity } & Objective & \multicolumn{1}{|c|}{ Participants } \\
\hline $\begin{array}{l}\text { TALK: How do you know your } \\
\text { measurements are right? }\end{array}$ & $\begin{array}{c}\text { To realize, for different types of } \\
\text { public, the role of measurement } \\
\text { uncertainty in their own } \\
\text { activities. }\end{array}$ & $\begin{array}{l}\text { Instructor: Ms. Georgia Harris. } \\
\text { Participants: PhD Students of the } \\
\text { Autonomous University of Querétaro. } \\
\text { Metrologists and scientists of } \\
\text { CENAM. }\end{array}$ \\
\hline $\begin{array}{l}\text { TALK: Essential Elements of } \\
\text { Metrological Traceability. }\end{array}$ & $\begin{array}{c}\text { To provide elements for } \\
\text { judgement regarding } \\
\text { measurement traceability and its } \\
\text { implications, including risk. }\end{array}$ & $\begin{array}{c}\text { Instructor: Ms. Georgia Harris. } \\
\text { Participants: Metrologists and } \\
\text { scientists of CENAM. Metrologists } \\
\text { and staff of secondary laboratories. }\end{array}$ \\
\hline $\begin{array}{l}\text { PANEL: Best Practices in } \\
\text { Metrology Education and Training. }\end{array}$ & $\begin{array}{c}\text { Coordinator: Dr. Ismael Castelazo, } \\
\text { Co reflect and share experiences } \\
\text { in the exploration of new } \\
\text { approaches of metrology } \\
\text { education and training. }\end{array}$ & $\begin{array}{c}\text { Panelists: Ms. Georgia Harris, NIST. } \\
\text { Dr. Flora Mercader, UPSRJ. } \\
\text { Mr. Hugo Hernandez, Metrology } \\
\text { School }\end{array}$ \\
& $\begin{array}{c}\text { Participants: CENAM metrologists } \\
\text { and University Teachers. }\end{array}$ \\
\hline $\begin{array}{l}\text { TALK "Project Based Learning } \\
\text { Applied to Metrology Instruction" }\end{array}$ & $\begin{array}{c}\text { Instructor: Ms. Georgia Harris } \\
\text { To share principles and best } \\
\text { practices related to the NIST } \\
\text { approach for metrology instruction: } \\
\text { five best practices }\end{array}$ & $\begin{array}{c}\text { Participants: CENAM metrologists } \\
\text { hat offer training. } \\
\text { University Teachers related to the } \\
\text { field. }\end{array}$ \\
\hline
\end{tabular}

\subsection{Inviting students and staff}

The invitation to students and staff was straightforward. Students that attended the CENAM event were mainly from the Autonomous University of Queretaro that are in postgraduate programs (MS and PhD) in different fields of engineering. Many of them have undertaken the course of "Measurement in the Research Process" given by Dr. Echeverria. The topic of "How do you know your measurements are right" was immediately interesting for them. From that, the topic of "Essential Elements for Metrological Traceability” followed for logical association.

Another group with great interest in most topics regarding teaching, training and learning was that of metrologists who, among their functions, provide courses and training in metrology. 
Naturally, most of them were from CENAM, because that was the main interest of the Center, but also metrologists from other calibration laboratories attended with interest.

Overall there were more than 50 attendants, besides the speakers and organizers, in the various sessions, and their evaluations of the event were "excellent" and "very useful".

\subsection{Creating a Panel to discuss Best Practices in Metrology Education and Training}

Metrologists that provide different types of education and training are very interested in exploring new training techniques. In Mexico, most metrologists are experts in some specific discipline, or in some of them, but not necessarily experts in education or training. The possibility of having Ms. Harris in Mexico, with a significant expertise in education, training, learning and development in metrology, offered the best opportunity to share her experience with others interested in this important function. The other panelists also offered important experiences. Dr. Ismael Castelazo presented aspects of the CENAM experience in continuous education in metrology. CENAM has followed mostly traditional ways of teaching, but has a long experience from its beginning (1994) of about 50 courses a year in different themes of metrology. It is important for CENAM to identify the most valuable of learning experiences and nurture them with other experiences as those presented in the panel. Dr. Flora Mercader, with a strong experience in metrology at CENAM and abroad, has been one of the architects of the Metrology Program at the UPSRJ and has led very important projects in this institution, such as the one reported in this paper. Mr. Hugo Hernández has been also a champion of metrology education and, after his experience in CENAM, he is now director of the company Metrology School which has innovative training-learning methods and is providing much for metrology education in Mexico. CENAM works with a variety of education and training providers such as UPSRJ, other universities, and The Metrology School (a private company) to expand the metrology impact within Mexico.

The contributions of these different perspectives created a very interesting kaleidoscope of inspiring images, all related to metrology education and training, mostly emphasizing the aspects of learning and development, as promoted by Ms. Georgia Harris for several years.

\subsection{Immediate Benefits}

For the group of students that attended the first two parts of the event, one immediate benefit is that they got tools for a better assessment of the role metrology plays in their research and development activities. After the event, they were given the homework of applying the discussed principles to their projects and they presented their essays the following week of the event. The results were very satisfying for them and useful for their projects.

For the group of metrologists-teachers that participated in the panel and the last session, one immediate benefit was a deep reflection on the techniques they are using to promote learning and development of competences, knowledge, skills, and attitudes in their trainees. They declared the 'five best practices' presented by Ms. Harris are a most useful tool to assess their teaching strategy and this will change their approach. 
These best practices are described in section 2.7; but, in short they can be named:

1. Professionalize the instruction activity.

2. Align course design with methodology, learning objectives, activities and assessments.

3. Consider andragogy, the learning process of adults vs pedagogy, for children.

4. Apply ongoing evaluation of course design to remain current.

5. Professionalism and quality are better if accreditation of training programs is applied.

Some of the attendants immediately evaluated themselves in each of the best practices and are willing to address the points in which they are weak.

\subsection{Expected Long-term benefits}

For CENAM, the panel and the session about "five best practices" promoted an ongoing reflection about the role this Center must play in promoting learning and development in metrology in the whole country. The best practices must be followed, and a group is addressing this issue to define a strategy.

Another aspect being discussed is that of "Training Trainers", that must go together with the construction of alliances and a network of instructors. The strategy to be defined must consider essential aspects of resources, logistics and the whole system to make it sustainable.

\subsection{Lessons learned and insights gained}

A main lesson learned from the collaboration described in this paper is that of open innovation. The interchange achieved with Ms. Harris and the different specialists from other organizations, UPSRJ, CENAM, Metrology School, UAQ, and secondary laboratories are, precisely, an example of open innovation regarding education and training in metrology. It is believed that this type of approach has great potential for further developments and this is being discussed in CENAM in its new strategic planning.

Another lesson is that very good results can be achieved by open collaboration among diverse institutions, sharing resources, work load, knowledge and experience. CENAM is grateful to the Fulbright Specialist Program, to NIST and the UPSRJ for having shared with us in this project.

\section{Recommendations}

Opportunities for international collaboration in metrology education and training can be sponsored and supported through various avenues, such as through the Fulbright Specialist program. University students from Mexico might apply to participate as Fulbright Scholars at NIST. Professional metrology instructors can apply to become Fulbright Specialists as a cost sharing mechanism. There are opportunities for U.S. technical experts to consider future collaboration using this mechanism. However, Fulbright Specialists (grantees) are restricted from receiving a grant more often than once every two years.

Regardless of funding sources, much can be gained from international collaboration in metrology education. Lessons learned and insights can enable improved international uniformity in 
professional metrology education, training, and understanding for the entire metrology community. In general, fewer resources are applied to metrology education and training than technical research and realization of measurements, so those with an interest and passion in educational subjects can and should share ideas and resources to make a difference for the next generation. Projects between the Fulbright Specialist and UPSRJ and CENAM on developing supporting materials and publication references are also being pursued.

Future opportunities to measure the impact of international collaboration should also be considered. Developing published case studies that demonstrate the impact and benefit of international collaboration, metrology education and training, and metrology outreach can help support and defend the need and impact of ongoing efforts on these topics.

\section{Disclaimer}

Certain commercial products are identified in this paper to foster understanding. Such identification does not imply recommendation by the National Institute of Standards and Technology, nor does it imply that the products identified are necessarily the best available for the purpose. 


\title{
Nanoelectronics Dimensional Metrology: Understanding the Differences between Secondary and Backscattered Electron Imaging ${ }^{1}$
}

\author{
Michael T. Postek, András E. Vladár, John S. Villarrubia \\ Engineering Physics Division \\ Physical Measurement Laboratory \\ National Institute of Standards and Technology \\ Gaithersburg, MD 20899
}

\section{INTRODUCTION}

In many fields of research and production, a great deal of dimensional metrology, characterization and process control is accomplished using scanning electron microscopes (SEM). The accuracy of these SEM measurements has always been important but is often overshadowed by two other main measurement drivers: throughput and precision. It is slow and often tedious to achieve high degree of accuracy and, so it is often ignored, especially in production where measurements must be made very quickly. However, the accuracy of a measurement is becoming more important as the frontiers of nanoelectronics are being explored, and sub-10 nm semiconductor device structures are routinely produced. Hence, the metrology error budget has shrunk, and has become truly atomic scale. This presentation will discuss new measurement, signal collection and modeling methods applied to sub-10 nm metrology being pursued for all types of semiconductor nanostructures, nanomaterials and nano-enabled materials to ultimately achieve the needed accurate measurements.

\section{SEM MEASUREMENTS}

The quality of the SEM measurements depends on how the acquired image is influenced by vibration, drifts, sample contamination and charging. In addition, accounting for specimen-electron beam interactions and their contributions to the acquired image must also be considered. New acquisition methods and successful mitigation of the previously mentioned environmental and instrument-induced effects can alleviate some of the imaging uncertainties. However, another key element is the application of advanced electron beam-solid state interaction modeling such as the NIST JMONSEL model [2] to interpret and account for the physics of the signal generation and help to understand and minimize the various contributions to measurement inaccuracy.

\section{EXPERIMENT}

The first part of this work involved a fundamental comparison of secondary (SE), backscattered (BSE) and low-loss (LLE) electron signals acquired on a new instrument that was equipped with SE, high-angle BSE and energy- filtered LLE detectors. Early work indicated that the BSE and LLE signal could be advantageous to help to understand the metrology of semiconductor structures [3]. The LLE image is produced by high-pass energy filtered BSE. These have undergone only minimal inelastic interactions with a sample and therefore, carry high-resolution information, specific to sample geometry [4-7]. LLE imaging is difficult because the collected signal is produced not only by the most energetic, but also a small minority of backscattered electrons. Early work pointed to a potential measurement difference between the SE and the BSE signals [8], it was difficult to obtain the needed information because of the resulting poor signalto-noise ratio and other instrument-specific geometric limitations. A measurement difference between the 
two signal modes was documented and was as large as $100 \mathrm{~nm}$ on the relatively large lines studied. Later, technology improvements facilitated the collection of conventional BSE and SE electron signal with a microchannel-plate electron detector. This approach was shown to be advantageous at low landing energies because of its improved geometry and signal collection capabilities [9]. In that early work, on different samples, collection and comparison of BSE and SE images of line structures again demonstrated that comparative threshold-based width measurements of the BSE images yielded values as much as $10 \mathrm{~nm}$ larger for the SE measurements than the BSE measurements on nominal $1.0 \mu \mathrm{m}$ gold lines. Due to the enhanced emission of low-energy (typically less than $10 \mathrm{eV}$ ) electrons at the sides and corners, there are common circumstances in which the SE intensity increases more abruptly at an edge than the BSE intensity. If width assignments are based on an intensity threshold, as is often the case, SE images would then be interpreted as showing a wider, larger feature than the BSE image. It was anticipated that the LLE signal would provide results similar to the BSE results, but with higher edge fidelity. A Hitachi SU 8230 FESEM [10], equipped with an in-lens, high-angle energy-filtered BSE detector, was used to compare the SE, BSE and LLE signals for dimensional measurements of the NIST RM 8820 magnification calibration sample [11]. The design of the field emission SEM incorporating a new in-lens energy filtered detector improves the LLE and BSE signal-to-noise ratio and reduces the geometrical limitations of the early LLE detectors.

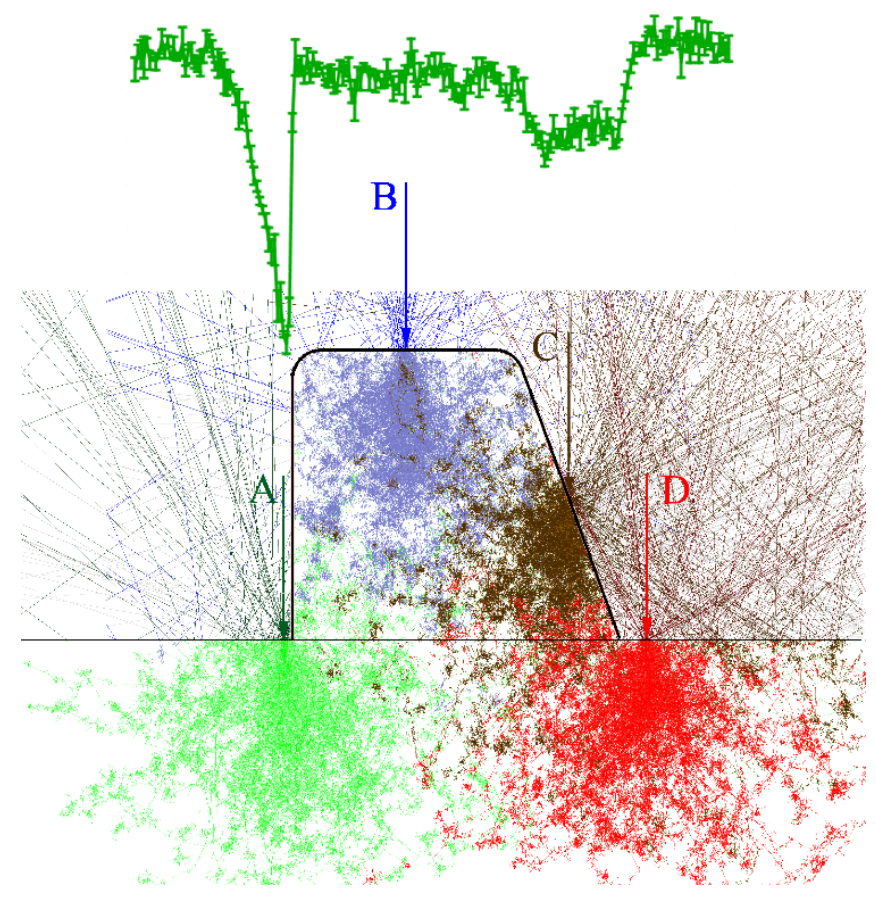

FIGURE 1. Model relationship of LLE signal to sample geometry. The upper (green) curve shows the modeled low-loss signal intensity from the line with near-vertical (left) and sloped wall (right) cross section shown in the lower portion. Electron trajectories at 4 landing positions, labeled A-D are superimposed on the sample geometry.

\section{JMONSEL MODELING}

In the second part of the work, we continued the analysis of the dimensional measurements using simulations with JMONSEL (Figure 1), an electron microscope simulator. JMONSEL indicated, as expected, that the nanometer- scale differences observed on this sample can be explained by the different convolution effects of a beam with finite size on signals with different symmetry (the SE signal's characteristic peak vs. the BSE or LLE signal's characteristic step). But this effect is too small to explain 
the $10 \mathrm{~nm}$ to $100 \mathrm{~nm}$ discrepancies that had been observed in earlier work on the different samples [8,9]. Additional modeling then indicated that those discrepancies could be explained by considering the much larger sidewall angles of the earlier samples, coupled with the different response of SE vs. BSE/LLE linescans and measurement algorithms to those wall angles. Clearly, serious measurement errors would be encountered in such cases if measurement algorithms were applied blindly without considering the underlying physics provided by applying model-based metrology.

\section{CONCLUSION}

In summary, this paper has: (1) demonstrated, for the first time, by simultaneous imaging that the previously observed bias between SE and LLE/BSE images is indeed real, not just an artifact of charging, drift, detector positioning, or some other instrument - or measurement-related error; (2) documented the measurement variation inherent in algorithm choice both on modeled and experimental data; (3) clearly pointed out that modeling of the image formation is necessary for highly accurate measurements, and (4) explained the previously observed mysterious size difference in the measurements with a simple phenomenological model and by a more complete Monte Carlo model.

\section{REFERENCES}

1. Contribution of the National Institute of Standards and Technology; not subject to copyright.

2. J. S. Villarrubia et al., Ultramicroscopy 154:15 (2015).

3. M. T. Postek, et al., SCANNING 23(5): 298 (2001).

4. O. C. Wells, Appl. Phys. Lett. 16(4):151 (1970).

5. O. C. Wells, Appl. Phys. Lett. 19(7): 232: (1971).

6. O. C. Wells, Scan. Electron Microscopy, 1, IITRI Chicago, 43, (1972).

7. O. C. Wells, Appl. Phys. Lett. 49(13): 764: (1986).

8. M. T. Postek, et al., SCANNING 10:10-18, (1988).

9. M. T. Postek, et al., Rev. Sci. Instruments, 61(12):3750, (1990).

10. Certain commercial equipment is identified in this report to adequately describe the

experimental procedure. Such identification does not imply recommendation or endorsement by the National Institute of Standards and Technology, nor does it imply that the equipment identified is necessarily the best available for the purpose.

11. https://www-s.nist.gov/srmors/view_detail.cfm?srm $=8820$

\section{ACKNOWLEDGEMENT}

The authors would like to thank Mr. Atsushi Muto and Hitachi High Technologies America for their assistance and use of the instrumentation used in this paper.

\section{KEYWORDS}

scanning electron microscope, SEM, JMONSEL, modeling, metrology, secondary, backscattered, low-loss electron

Postek, Michael; Villarrubia, John; Vladar, Andras. 


\title{
UV scale calibration transfer from an improved pyroelectric detector standard to field UV-A meters and $365 \mathrm{~nm}$ excitation sources
}

\author{
G. P. Eppeldauer ${ }^{1}$, V. B. Podobedov, and C. C. Cooksey, \\ National Institute of Standards and Technology \\ 100 Bureau Drive, Gaithersburg, MD, USA 20899
}

\begin{abstract}
Calibration of the emitted radiation from UV sources peaking at $365 \mathrm{~nm}$, is necessary to perform the ASTM required $1 \mathrm{~mW} / \mathrm{cm}^{2}$ minimum irradiance in certain military material (ships, airplanes etc) tests. These UV "black lights" are applied for crack-recognition using fluorescent liquid penetrant inspection. At present, these nondestructive tests are performed using $\mathrm{Hg}$-lamps. Lack of a proper standard and the different spectral responsivities of the available UV meters cause significant measurement errors even if the same UV-365 source is measured. A pyroelectric radiometer standard with spectrally flat (constant) response in the UV-VIS range has been developed to minimize errors. The response curve of this standard determined from spectral reflectance measurement, is converted into spectral irradiance responsivity with $<0.5 \%(k=2)$ uncertainty as a result of using an absolute tie point from a Si-trap detector traceable to the primary standard cryogenic radiometer. The flat pyroelectric radiometer standard can be used to perform uniform integrated irradiance measurements from all kinds of UV sources (with different peaks and distributions) without using any source standard. Using this broadband calibration method, yearly spectral calibrations for the reference UV (LED) sources and irradiance meters is not needed. Field UV sources and meters can be calibrated against the pyroelectric radiometer standard. with significantly decreased uncertainties.
\end{abstract}

Keywords: UV responsivity scale, LED-365 irradiance, broadband UV measurement, integrated radiometric quantities, LED integrated irradiance, UV-LED measurement, flat-response UV meter, pyroelectric UV standard

\section{INTRODUCTION}

The International Committee on Illumination (CIE) recommended UV-A action spectrum has a rectangular shape that can be realized only with large spectral mismatch errors. Significant measurement errors are obtained when the measured sources are changed (have different spectral distributions). Applying spectral mismatch correction factors in different applications, makes the measurement procedure and evaluation complicated.

Instead of using the traditional CIE recommended source-based or detector-based calibration methods, a broadband UV measurement procedure is applied here for standardization to perform simple uniform measurements with low uncertainty. The broadband UV measurement procedure has been discussed earlier $[1,2]$.

It is necessary to perform the ASTM required $1 \mathrm{~mW} / \mathrm{cm}^{2}$ minimum irradiance [3] from UV sources peaking at 365 $\mathrm{nm}$ when ship and airplane components are examined in non-destructive military material tests. UV "black lights" are applied for the crack-recognition using fluorescent liquid penetrant inspection. At present, these nondestructive tests are performed using the $365 \mathrm{~nm}$ excitation line of Hg-lamps. In order to phase out mercury, LED-365 irradiance sources have been developed within this work to substitute the traditionally used UV lamps. In the UV meters used for this inspection, the CIE standardized UV-A function is realized using optical filters and Si photodiodes. The realized spectral responsivities of the available UV-meter models are different producing significant measurement errors even if the same UV-365 source is measured.

\footnotetext{
${ }^{1}$ george.eppeldauer@nist.gov; phone 1301 975-2338; fax 1301 869-5700
} 
A pyroelectric radiometer standard with spectrally flat (constant) response in the UV-VIS range has been developed at NIST to perform uniform UV irradiance measurements with low uncertainty. The organic-black coated radiometers have been used as reference detectors in this VIS-IR range for a few years and the responsivity changes were less than the scale realization uncertainty. The relative response of this organic-black coated detector was determined from spectral reflectance measurements. The relative curve was converted into a spectral irradiance responsivity function using an absolute tie point from a Si-trap detector traceable to the primary standard cryogenic radiometer.

The flat pyroelectric radiometer standard can be used to perform uniform integrated irradiance measurements from all kinds of UV sources (with different peaks and distributions) without using any source standard. Using this broadband calibration method, yearly spectral calibrations for the reference UV (LED) sources and irradiance meters [2] is not needed. Field UV meters (including existing commercial meters) may be calibrated against the pyroelectric radiometer standard for broadband (integrated) irradiance responsivity when both measure the same UV source. In this case, spectrally flat response for the field meter is not required. Using the broadband procedure, the UV measurement uncertainties can be significantly decreased.

\section{UV MEASUREMENT PROBLEM}

The realizations of the rectangular shape action spectrum for two commercial UV-A meters are illustrated in Fig. 1 [4].

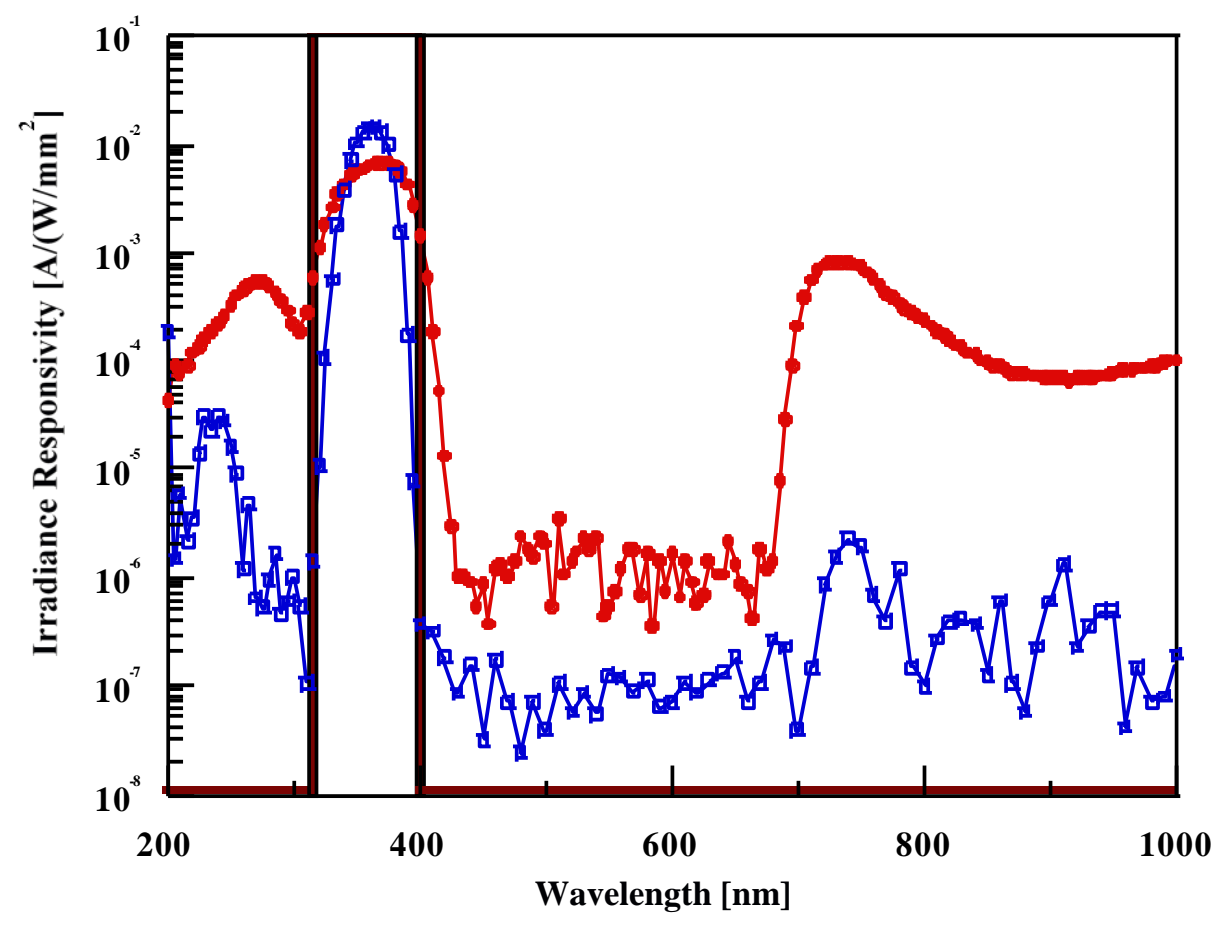

Fig. 1. The CIE standardized UV-A (rectangular-shape) function with two realized spectral responsivity functions.

The solid dots show an average realization and the open-squares represent an improved realization. It can be seen that both realizations have large spectral mismatch errors relative to the CIE (rectangular-shape) standard function. The measurement errors may increase to several times more than ten percent when the standard lamp type (e.g. an FEL lamp) is different than the test lamps e.g. mercury, deuterium, or xenon.

A reasonable calibration (correction) factor for broad-band UV measurements cannot be assigned based on the CIE UV-A standard responsivity function. Also, the spectral power distribution of the excitation 365-nm sources 
has not been standardized which is necessary if the realized spectral responsivity function of the UV meter is different from the standard function. The wavelength range of the presently used CIE standard function is too broad for the required tests where 365-nm excitation sources are used.

The goal of the present work is to make the broadband UV measurements uniform and to lower the broadband UV (with a typical emissivity peak of $365 \mathrm{~nm}$ ) irradiance measurement uncertainties. To achieve this goal, first a new procedure for standardized measurements was developed using reference UV excitation sources and meters [1, $2]$ and then spectrally flat reference meters were developed to simplify the procedure and using only a broadband pyroelectric detector based calibration without the need for a reference (standard) source.

\section{SOLUTION OF PROBLEM USING FLAT METER}

In the previously suggested measurement procedure [1,2], the UV LED source(s) is measured with a broadband meter. In the discussed example, an LED-365 irradiance source was measured with a UV irradiance meter. The measured output signal of the meter was equal to the spectral product of the source distribution and the meter response function. The requirement from the broadband measurement procedure is to obtain invariance in the measured signal (at the output of the meter) for changes in both the LED source (peak and spectral-width) and the spectral-shape of the meterresponse. To perform uniform broadband measurements, the spectral response of the meter must be broader than the distribution of the measured source(s) and the source distribution(s) must be within the spectral response function of the meter for all the expected source(s) and meter changes.

In the discussed example, to obtain the invariance in the measured (output) signal, the spectral response of the meter was selected to be close to constant in a spectral range equal to or wider than the widest source-distribution of the 365$\mathrm{nm}$ source to be measured. Using the broadband procedure, output signals can be measured with differences (errors) less than the required measurement uncertainty when different $365-\mathrm{nm}$ sources (with different peak wavelengths and spectral widths) are measured.

Recently developed low-NEP pyroelectric detectors [5] are excellent candidates to measure not only UV but also other kinds of LEDs in the spectral range where the pyroelectric detector has close to constant spectral response. Using the flat-response reference pyroelectric meter, the calibration procedure can be simplified, spectral calibrations for the standards are not required, source standards will not be needed, and selection of the field test meters is not necessary.

\section{INTEGRATED IRRADIANCE MEASUREMENT}

LED-365 irradiance sources and spectrally "flat" UV irradiance meters have been developed to implement the UV broadband measurement procedure for non-destructive testing of metal parts [3]. In this application example, the excitation irradiance source peaks at a nominal wavelength of $365 \mathrm{~nm}$. Typically, the purchased/applied high power LEDs have a few $\mathrm{nm}$ shift in their peak wavelength. In the suggested broadband measurement procedure, the integrated irradiance from a test UV LED source can be measured [6, 8]. Two different versions of the calibration steps (depending primarily on the spectral flatness of the meter) were also discussed earlier [7].

The measurement equation that describes the output signal of the reference meter for irradiance measurement mode can be written as

$$
i_{r e f}=\int_{\lambda} E(\lambda) s_{r e f}(\lambda) d \lambda
$$

where $E(\lambda)$ is the spectral irradiance of the calibrated (standard) UV-LED source, $s_{\text {ref }}(\lambda)$ is the spectral irradiance responsivity of the reference meter, and $\lambda$ is the wavelength.

Using the ASTM standardized requirement, the integrated irradiance can be determined in the reference plane of the meter, $40 \mathrm{~cm}$ away from the source: 


$$
\bar{E}=\frac{i_{r e f}}{\bar{s}_{r e f}}
$$

where the integrated irradiance responsivity of the reference meter is:

$$
\bar{s}_{r e f}=\frac{i_{r e f}}{\int E(\lambda) d \lambda}
$$

After these reference-level calibration steps, the reference meter (with the known integrated responsivity) can be taken to a field laboratory where the field-level calibration steps can continue the reference calibration steps [7].

When transferring the integrated irradiance responsivity to field UV meters, spectral response measurement of the test (field) UV meters is not needed. Also, a source standard will not be needed if the reference meter has a known constant spectral responsivity for the wavelength range where the measured LED(s) emits optical radiation. In this case, the output signal of an irradiance meter divided by the constant irradiance responsivity will result in the integrated irradiance from the measured LED. Similarly, when the response of the reference meter is not constant but an average responsivity can be determined for (most of the) measured radiation, a source standard will not be needed [7].

\subsection{Pyroelectric UV irradiance responsivity standard}

The procedure for broadband UV calibrations and measurements can be simplified when UV meters with spectrally constant response are used. Calibration of the reference meter for constant irradiance responsivity is enough to measure the integrated irradiance (or another radiometric quantity) of a test LED source(s). When using these spectrally "flat" standard meters, use of a standard source is not needed. In this detector-based calibration, the only standard is the meter with the known constant spectral responsivity. When the spectral flatness (the relative curve shape) is known, one absolute tie point can be enough to convert the relative response function into absolute (e.g. to obtain the constant spectral irradiance responsivity).

A spectrally "flat" UV meter can be made with either filtered quantum detector (like silicon detector and input optical filters) or pyroelectric detector. For these "flat" meters, Eq. 1 can be applied. Since $S_{\text {ref }}(\lambda)=s=$ constant, the output signal of the reference meter will be

$$
i_{\text {ref }}=i=s \int_{\lambda} E(\lambda) d \lambda
$$

and the integrated irradiance will be

$$
\bar{E}=\frac{i}{s}
$$

where the unit of $i$ is $\mathrm{A}$, the unit of $s$ is $\mathrm{A} \mathrm{cm}^{2} / \mathrm{W}$, and the obtained unit for $\bar{E}$ is $\mathrm{W} / \mathrm{cm}^{2}$.

Using a pyroelectric detector, the deviation from a spectrally constant response can be an order of magnitude smaller than using a filtered Si photodiode [7]. Also, the wavelength coverage of a pyroelectric detector with the flat response will be much wider.

Reference-level integrated irradiance calibrations are discussed below when using a pyroelectric "flat" irradiance meter standard. This detector can measure radiant power down to $1 \mu \mathrm{W}$ with a signal-to-noise ratio $(\mathrm{S} / \mathrm{N})$ of 100 . An organic black absorbing coating is applied on the front-surface of the pyroelectric detector. The picture of the recently developed pyroelectric UV standard meter is shown in Fig. 2. 


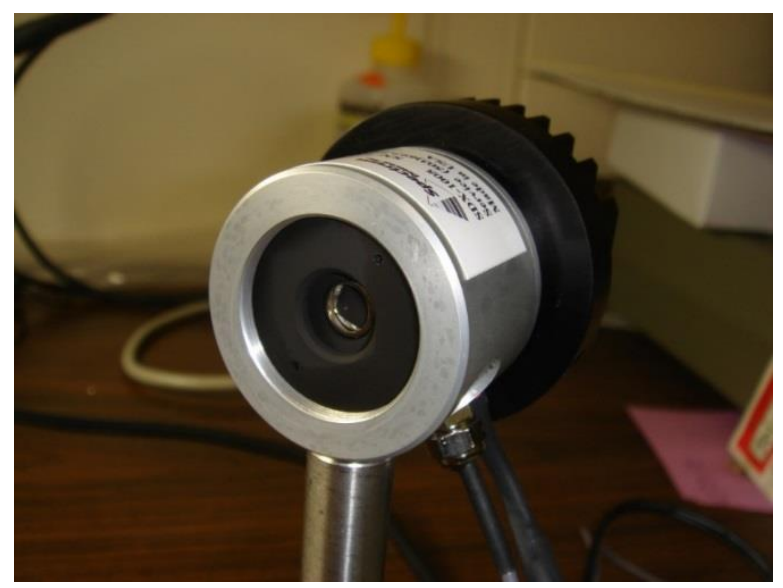

Fig. 2. Picture of a temperature controlled hybrid pyroelectric UV detector standard of low NEP. The $\mathrm{LiTaO}_{3}$ crystal is covered with organic-black coating.

As determined from spectral reflectance measurements, where both the specular and diffuse components are measured using a sphere, the spectral response of the reference pyroelectric detector can deviate $+/-0.1 \%$ from constant between $330 \mathrm{~nm}$ and $400 \mathrm{~nm}$. The deviation from constant can be a dominant uncertainty component of the integrated irradiance measured by the pyroelectric detector after its calibration. The here suggested broadband calibration needs a shorter calibration time and it is less expensive than the presently applied spectral calibration techniques. As a result of the simplified broadband calibration and scale transfer, the expected combined uncertainty for the reference integrated irradiance measurement is about $0.5 \%(k=2)$ which is significantly lower that the about $5 \%(k=2)$ uncertainty achieved using the previously discussed methods.

The spectral irradiance responsivity calibration of a pyroelectric detector in the UV takes a few steps including the realization/transfer of the absolute tie point in the visible or near-IR range, measurement of absorptance of the black coating on the top of the pyroelectric crystal, measurement of the integrated irradiance from a stable UV source and validation of this measured integrated irradiance from an independent irradiance measurement of the same UV source, in our case against an FEL lamp standard [7].

The measurements, described below, are the first time calibration of the irradiance responsivity of a pyroelectric detector. The measurements of the low-NEP pyroelectric detector were performed in AC mode using a lock-in amplifier. The upper roll-off frequency of the detector current meter was about $100 \mathrm{~Hz}$ and the chopping frequency was $10.5 \mathrm{~Hz}$. A beam geometry (without using any integrating sphere) at the output of a monochromator [8] was used in the near-IR range. The irradiance responsivity from the near-IR was extended to the UV range based on the close to constant spectral absorptance (relative response) curve derived from spectral reflectance measurements of the black coating. For this scale extension, the output of the pyroelectric detector was compared to the output of a silicon trapdetector transfer-standard for which the irradiance responsivity was known in the visible range. The detector substitution was made in the collimated radiation from a LED irradiance source peaking at $660 \mathrm{~nm}$. In Fig. 3, the absorptance curve of the organic black coating is shown in the range of $0.25 \mu \mathrm{m}$ to $0.8 \mu \mathrm{m}$ together with the absolute tie point at $660 \mathrm{~nm}$. The absorptance, which is proportional to the response, is equal to $1-\mathrm{R}$ if the transmittance is negligibly small (where the reflectance is $\mathrm{R}$ ). The tie point converts this relative response curve into absolute spectral irradiance responsivity. Based on the small changes in this relative curve, the irradiance responsivity of the pyroelectric detector was taken as a constant $910 \mathrm{VW}^{-1} \mathrm{~cm}^{2}$ between $0.25 \mu \mathrm{m}$ and $0.75 \mu \mathrm{m}$. The uncertainty budget of the spectral irradiance responsivity is shown in Table 1. The expanded $(k=2)$ irradiance responsivity uncertainty between $0.25 \mu \mathrm{m}$ and $0.75 \mu \mathrm{m}$ is $0.5 \%$. The max-to-min response change was $+/-0.12 \%$ between $300 \mathrm{~nm}$ and 660 $\mathrm{nm}$ and $+/-0.18 \%$ between $0.25 \mu \mathrm{m}$ and $0.75 \mu \mathrm{m}$.

A second tie point is also shown at $365 \mathrm{~nm}$ where the LED-365 excitation sources were calibrated against an FEL lamp standard [7]. The uncertainty of the FEL source-based spectral irradiance calibrations was $1.6 \%(k=2)$. 
The less than $+/-1 \%$ deviations from constant up to $2 \mu \mathrm{m}$ makes it possible to apply these broadband (integrated) irradiance LED measurements for the VIS-NIR range [9] as well.

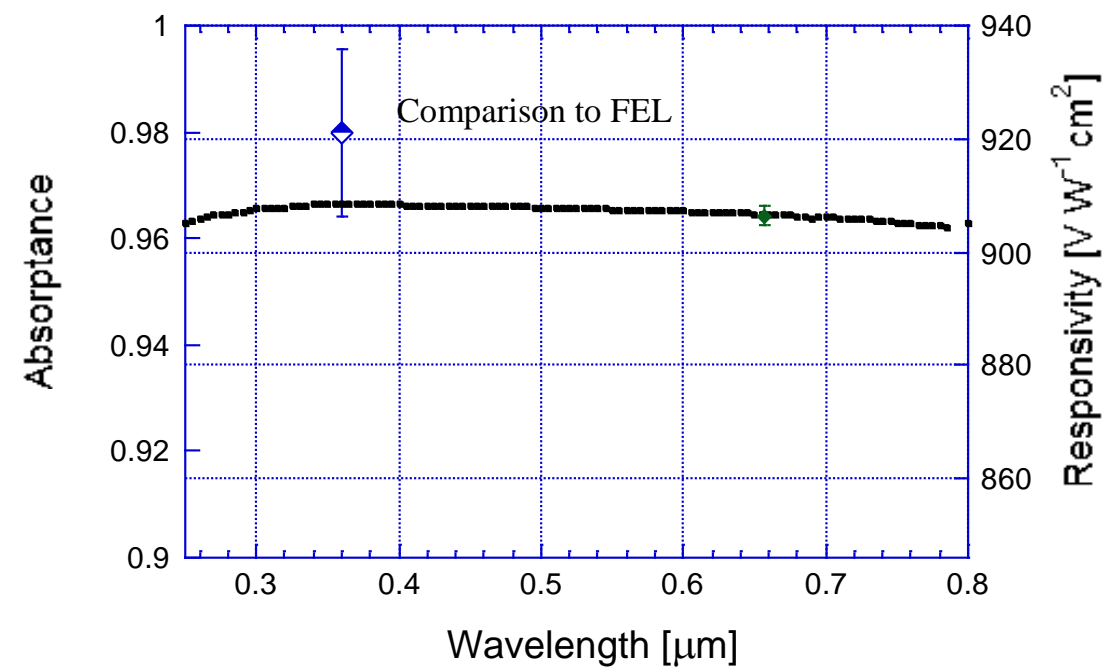

Fig. 3. Spectral absorption (1-reflectance) curve of the organic black coated pyroelectric detector standard. The irradiance responsivity tie (absolute) point against a Si-trap detector is shown at the $660 \mathrm{~nm}$ peak of the transfer LED.

Table 1. Uncertainty budget of the spectral irradiance responsivity of the flat pyroelectric detector standard for the $250 \mathrm{~nm}$ to $750 \mathrm{~nm}$ wavelength range.

\begin{tabular}{|l|c|}
\hline $\begin{array}{l}\text { Relative uncertainty } \\
\text { components }\end{array}$ & {$[\%]$} \\
\hline$\Delta \lambda$ & 0.03 \\
\hline Distance & 0.04 \\
\hline Target spot non-uniformity & 0.10 \\
\hline Spectral response change & 0.18 \\
\hline Output signal ratio & 0.10 \\
\hline Reference Si-trap & 0.10 \\
\hline Combined $(\boldsymbol{k}=\mathbf{1})$ & $\mathbf{0 . 2 5}$ \\
\hline Expanded $(\boldsymbol{k}=\mathbf{2})$ & $\mathbf{0 . 5}$ \\
\hline
\end{tabular}

\section{INTEGRATED IRRADIANCE MEASUREMENTS WITH TEST UV METERS}

Utilization of the pyroelectric detector-based (standard) radiometer allows to simplify the calibration of the filtered $\mathrm{Si}$ detector and field UV (test) irradiance meters. The field UV meters should have a broad enough spectral response to measure the integrated irradiance from a UV source. Since these test meters are calibrated against the standard meter using detector-substitution when measuring the same source, the spectral flatness of these test meters is not an important issue. Before calibrating a test meter, the integrated irradiance responsivity of the pyroelectric radiometer is to be determined as described above. 
In the following scale transfer, the test-meter can be substituted for the reference pyroelectric meter of known constant irradiance responsivity and the signal ratio, when they are measuring the same source, can be used to determine the "flat" irradiance responsivity of the test-meter. The integrated irradiance from the source will be equal to the ratio of the test-meter's output signal divided by the "flat" irradiance responsivity of the test-meter. The "flat" irradiance responsivity of the test meter is

$$
r_{\mathrm{t}}=\mathrm{k} s
$$

where $\mathrm{k}$ is a correction factor obtained as the ratio of the test-meter output-voltage to the output-voltage of the reference meter when both measure the same irradiance. The constant irradiance responsivity of the reference (standard) meter is $s$. This responsivity, as mentioned above, is usually determined in a primary level calibration laboratory. The following simple transfer-calibrations can be performed in field calibration places. The integrated irradiance measured by the test meter will be

$$
\bar{E}=\frac{U_{t}}{k s}
$$

where $U t$ is the output voltage of the test irradiance meter.

Continuing our previous UV-365 source measurement examples, the integrated irradiance responsivity of any field radiometer (including existing commercial meters) may be calibrated against a calibrated filtered UV-365 (transfer standard) radiometer if the same source is used what was used earlier for the calibration of the filtered UV-365 transferstandard irradiance meter. Also, in this case, spectral flatness for the field detector responsivity function is not required. However, if a different source is measured in the field, then the filtered UV-365 transfer-standard meter should be calibrated for that source first.

\subsection{Pyroelectric detector based broadband calibration of UV meters for non-destructive testing}

The broadband calibration can substitute the earlier used spectral calibrations for the previously developed LED-365 irradiance sources and the UV irradiance meters. Figure 4 illustrates a field calibration setup for broadband scale transfer from the flat pyroelectric detector standard to the previously developed UV-365 irradiance meters. The calibrated UV-365 irradiance meters are used for non-destructive material testing.

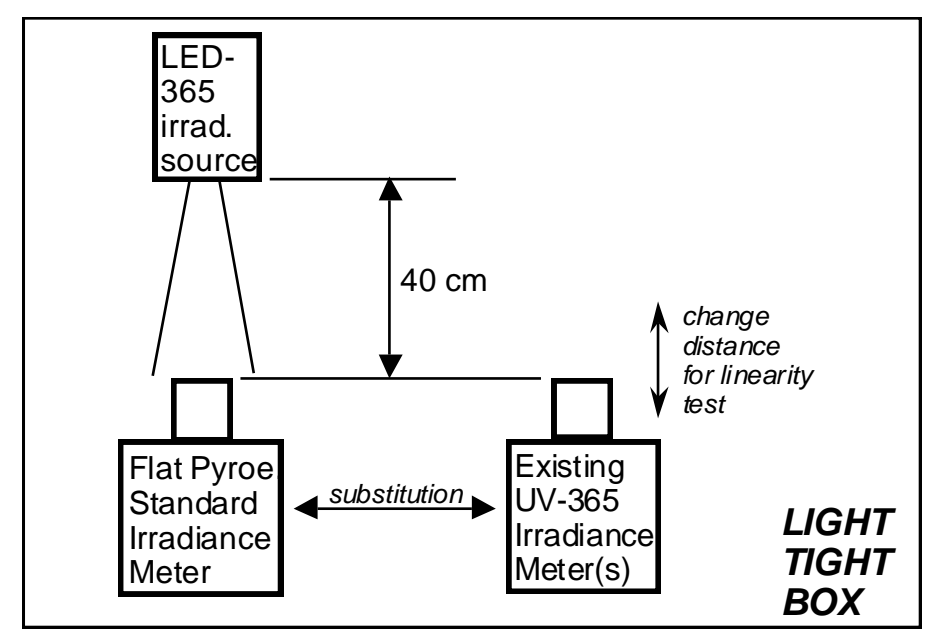

Fig. 4. Setup for broadband scale transfer from the flat pyroelectric standard to previously developed UV-365 irradiance meters. 


\section{CONCLUSIONS}

A broadband UV measurement procedure has been developed at NIST to calibrate UV sources for integrated irradiance. An irradiance meter standard has been developed for the UV range using a spectrally flat-response pyroelectric detector of low-NEP. A spectrally constant UV absorptance function with an irradiance tie point has been realized for the $250 \mathrm{~nm}$ to $660 \mathrm{~nm}$ wavelength range. This new detector-based UV irradiance responsivity scale has a $0.25 \%(k=1)$ uncertainty. The flat pyroelectric detector standard was used to calibrate existing filtered Si UV irradiance meters. The filtered Si meters measured the integrated irradiance of both the recently developed reference LED-365 excitation sources and field UV-365 sources. The broadband UV measurement procedure can be applied for all kinds of LEDs or groups of LEDs without using any source standard. The pyroelectric detector standard can be used to directly calibrate either UV irradiance meters and/or UV excitation (irradiance) sources. The broadband measurement procedure was successfully applied for fluorescent crack-recognition when using liquid penetrant inspection in non-destructive material testing.

\section{References}

1. G. P. Eppeldauer, Standardization of broad-band UV measurements for $365 \mathrm{~nm}$ LED sources. J. Res. Natl. Inst. Standards and Technology, 2012. 117: p. 96-103.

2. G. P. Eppeldauer, Standardization of broadband UV measurements. UVNews 9, Newsletter of the Thematic Network for Ultraviolet Measurements of the European Metrology Research Program of EURAMET, 2013. Published by Aalto University, Helsinki, Finland (9/January): p. 34-37.

3. $\quad$ ASTM-E1417, Liquid Penetrant Examination Standard

4. T. C. Larason, C. L. Cromer, "Sources of Error in UV Radiation Measurements," Journal of Research of the National Institute of Standards and Technology, 106 (\#4), 649-656 (2001).

5. G. P. Eppeldauer, J. Zeng, and H. W. Yoon, Low NEP pyroelectric radiometer standards. SPIE Proc., 2008. 6940: p. 694036-1 to 694036-8.

6. G. P. Eppeldauer, T. C. Larason, and H. W. Yoon, Standardization of UV LED measurements. Proc. SPIE, 2015. 9571(14th International Conf. on Solid State Lighting and LED-based Illumination Systems): p. 957105-1 to 957105-12.

7. G. P. Eppeldauer, C. C. Cooksey, H. W. Yoon, L. M. Hanssen, V. B. Podobedov, R. E. Vest, U. Arp, and C. C. Miller, Broadband radiometric LED measurements, Fifteenth International Conference on Solid State Lighting and LED-based Illumination Systems, edited by M. H. Kane, N. Dietz, I. T. Ferguson, Proc. of SPIE Vol. 9954, 99540J-1 to 99540J-15, 2016. The DOI for the paper is http://dx.doi.org/10.1117/12.2237033.

8. G. P. Eppeldauer, et al., Calibration procedure for UV-365 integrated irradiance measurements. CIE 28th Session Proc., 2015. Manchester, GB.

9. V. B. Podobedov, et al., Calibration of spectral responsivity of IR detectors in the range from $0.6 \mathrm{~mm}$ to $24 \mathrm{~mm}$. SPIE Proceedings, 2016. 9819 (Infrared Technology and Applications XLII, 98190P (May 20, 2016); doi:10.1117/12.2228384).

Cooksey, Catherine; Eppeldauer, George; Podobedov, Vyacheslav. 


\title{
Optimizing image-based patterned defect inspection through FDTD simulations at multiple ultraviolet wavelengths
}

\author{
Bryan M. Barnes*, Hui Zhou, Mark-Alexander Henn, Martin Y. Sohn, and Richard M. Silver \\ Engineering Physics Division, National Institute of Standards and Technology, \\ 100 Bureau Drive MS 8212, Gaithersburg, MD, USA 20899-8212
}

\begin{abstract}
The sizes of non-negligible defects in the patterning of a semiconductor device continue to decrease as the dimensions for these devices are reduced. These "killer defects" disrupt the performance of the device and must be adequately controlled during manufacturing, and new solutions are required to improve optics-based defect inspection. To this end, our group has reported [Barnes et al., Proc. SPIE 1014516 (2017)] our initial five-wavelength simulation study, evaluating the extensibility of defect inspection by reducing the inspection wavelength from a deep-ultraviolet wavelength to wavelengths in the vacuum ultraviolet and the extreme ultraviolet. In that study, a $47 \mathrm{~nm}$ wavelength yielded enhancements in the signal to noise (SNR) by a factor of five compared to longer wavelengths and in the differential intensities by as much as three orders-of-magnitude compared to $13 \mathrm{~nm}$. This paper briefly reviews these recent findings and investigates the possible sources for these disparities between results at $13 \mathrm{~nm}$ and $47 \mathrm{~nm}$ wavelengths. Our in-house finite-difference time-domain code (FDTD) is tested in both two and three dimensions to determine how computational conditions contributed to the results. A modified geometry and materials stack is presented that offers a second viewpoint of defect detectability as functions of wavelength, polarization, and defect type. Reapplication of the initial SNR-based defect metric again yields no detection of a defect at $\lambda=13 \mathrm{~nm}$, but additional image preprocessing now enables the computation of the SNR for $\lambda=13 \mathrm{~nm}$ simulated images and has led to a revised defect metric that allows comparisons at all five wavelengths.
\end{abstract}

Keywords: defect metrology, extreme ultraviolet, EUV, vacuum ultraviolet, VUV, deep ultraviolet, DUV, defect inspection, finite-difference time-domain, simulation, simulated imaging

\section{INTRODUCTION}

Semiconductor devices are the integral components of the ubiquitous electronic devices encountered in daily life. These devices (e.g., computer memory, central processing units (CPUs), etc.) are manufactured using multiple steps such as deposition, photolithography, and etching, with billions of transistors fabricated for a single device. There are several key parameters to monitor within these manufacturing processes. Monitoring the positioning offset of one photolithographically patterned layer with respect to the previous patterned layer is called overlay metrology, which is measured optically to sub-nanometer resolution using either scatterometry or imaging techniques. Measuring the width of the smallest features within these devices is called critical dimension (CD) metrology, and CDs can be measured by using scanning electron microscopy (SEM) or measured optically using model-based scatterometry. In manufacturing, currently, several devices are fabricated simultaneously on a $300 \mathrm{~mm}$ diameter silicon wafer, and sampling strategies are employed within a high-volume manufacturing environment to determine variations in overlay and CD across this wafer.

Meanwhile, this patterned layer must also be inspected for deviations from the nominal pattern that arise from manufacturing faults. Optics-based defect metrology specifically identifies locations on these wafers at which the printed pattern deviates from the designed pattern, specifically deviations that would lead to device failure due to shorted or broken electrical connections. Furthermore, if the undesirable changes in manufacturing conditions are systematic, then other devices on subsequent wafers may also have the same defects that continue to result in failed devices until the root cause is determined and resolved. These defects are called killer defects and the defining size characteristics of these defects grow smaller as the device sizes and critical dimensions continue to decrease. Stated differently, what previously may

*bryan.barnes@nist.gov; phone 1301 975-3947; fax 1301 975-4396; www.nist.gov 
have been a tolerable patterning imperfection can become a killer defect as densities increase and CDs decrease in modern manufacturing.

The industrial methods of choice for inspecting for defects are optical, with some approaches opting for image-based solutions while other approaches favor detecting changes in light scattered off the sample. Optical methods yield the high speeds, the inherently parallel detection, and non-destructive measurements required for defect inspection, even as the defects remain unresolved. Typically, optical methods detect and locate defects for subsequent inspection and fault analysis using an SEM review.

The complex optical scattering interactions among patterned layouts and the layers upon which they are patterned defy simple explanation but important trends have been identified. It is well-understood in the industry, as shown in the literature [1], that with decreased dimensions, these killer patterned defects will scatter far less light thus complicating inspection. To heuristically describe the challenge of the optical inspection with these ever-decreasing defect sizes, comparisons have been made to Mie scattering. It is known that the intensity $I$ scattered off spheres scales as $I \propto d^{6} / \lambda^{4}$ for a sphere with a diameter $d$ and an inspection wavelength $\lambda$ where $d<<\lambda$ [2]. Again, defect scattering is not directly analogous to the scattering from spheres as the intended pattern and its substrate will also scatter and reflect light as well. However, the implications from this model are that the optical scattering from defects will be extremely difficult as dimensions continue to decrease, with CDs presently $14 \mathrm{~nm}$ and smaller.

From this model, however, decreasing the inspection wavelength offers a potential remedy to this acute manufacturing challenge. In a recent proceedings paper [3], we presented our initial results from a five-wavelength simulation study of the optical scattering from patterned defects in a fin layout, with wavelengths spanning the deep ultraviolet (DUV), the vacuum ultraviolet (VUV), and the extreme ultraviolet (EUV). Such fins are the basis of current three-dimensional Fin Field Effect Transistors (FinFETs). Simulations have been performed using a finite-difference time-domain (FDTD) [4] Maxwell's equations solver. As with our prior work [5, 6], the roles of incident angle, defect orientation, and polarization have been explored using the difference images resulting from subtracting an image without a defect from an image with a defect. This study establishes that up to a factor of five improvement over longer wavelengths may be realized in signal to noise ratio from the adoption of $\lambda=47 \mathrm{~nm}$ as an inspection wavelength. A shorter EUV wavelength, $13 \mathrm{~nm}$, was illsuited for defect inspection, yielding differential intensities as much as three orders of magnitude smaller than from simulations using $\lambda=47 \mathrm{~nm}$. Wavelength-dependent optical constants are key to understanding these results. At $\lambda=47 \mathrm{~nm}$, the materials exhibit metal-like optical constants while $n$ and $k$ for $\lambda=13 \mathrm{~nm}$ approach 1 and 0 , respectively, for many materials. In addition, the defects are resolved at $\lambda=13 \mathrm{~nm}$ which leads to a highly localized signal that does not exceed the magnitude of the noise applied to the simulated images. A summary of this initial work is presented as Section 2 in this paper.

This proceedings paper explores possible computation-related causes for the disparity between the simulation results at $13 \mathrm{~nm}$ and $47 \mathrm{~nm}$ wavelengths, illustrating the inherent requirements upon FDTD modeling at multiple ultraviolet wavelengths for optimizing patterned defect metrology. It was noted in Ref. [3] that additional work remained to make the results more practical, such as the addition of line edge roughness and simulations with more process stacks. Our inhouse developed FDTD code is tested quantitatively in two dimensions (2-D) simulating the reflection from a substrate with the computed polarization- and angle-resolved reflectivities then compared against values obtained using the wellknown Fresnel equations. This test allows a qualitative check of the results obtained thus far at $\lambda=13 \mathrm{~nm}$ and $\lambda=47 \mathrm{~nm}$. A modified fin geometry is introduced that better represents industrially relevant design and allows further testing of the FDTD code in three-dimensions (3-D) and further validation of the observed trends. Two important simulation parameters, the cubic grid size $\Delta s$ and the simulation domain size are varied to seek conditions that will yield greater consistency in the simulation results. While defects at $\lambda=13 \mathrm{~nm}$ are again not readily apparent when applying the initial defect metric upon on preliminary defect simulations using this geometry, methods for improving the preprocessing of the differential image and modifying the defect metric are presented that yield comparisons across the full wavelength range.

\section{REVIEW OF INITIAL SIMULATION STUDY}

Methods, considerations, and results of our group's initial simulation study at five wavelengths $(13 \mathrm{~nm}, 47 \mathrm{~nm}, 122 \mathrm{~nm}$, $157 \mathrm{~nm}$, and $193 \mathrm{~nm}$ ) are summarized in this section. Full details appear in a recent proceedings paper [3]. 


\subsection{Simulation study methodology}

The goal of this initial study has been to quantify defect detectability as functions of incident angle, polarization, defect type, and wavelength. Finite-difference time-domain (FDTD) calculations of the electromagnetic field scattered from the patterned layout and its defects can yield images obtained through modeling of the Fourier optics at each of the five simulation wavelengths. The patterned layout is based upon public information about recent manufacturing processes $[7,8]$ while scaling features to make the silicon lines $8 \mathrm{~nm}$ wide, and are shown schematically in Fig. 1(a) and 1(b), with defects defined in Fig. 1(c). These bridging defects are commonly referred to by their SEMATECH ${ }^{1}$ naming scheme: "Bx" and "By" defects. The defects are also $8 \mathrm{~nm}$ wide silicon lines, less than $40 \mathrm{~nm}$ in length and conformally coated. Incident angles are indexed using polar and azimuthal angles, $\theta$ and $\phi$, as defined in Fig. 1(d). Simulations are performed with the plane wave illumination linearly polarized either perpendicular to or within the plane of incidence, and these results are used to calculate the effects of linearly polarized light that is oriented with respect to the sample patterning (e.g., $\mathrm{X}$ polarization, Y polarization). The materials used in this example are listed in Fig. 1(a) and their wavelength-dependent optical constants appear as Table 1.

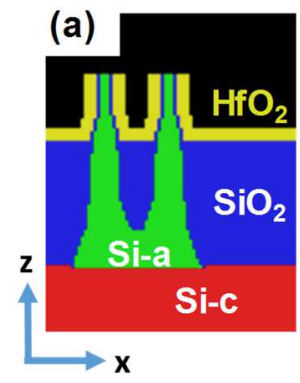

(b)

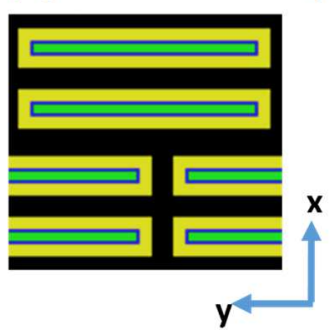

(c)

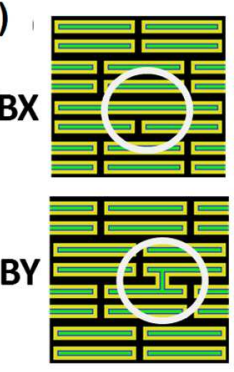

(d)

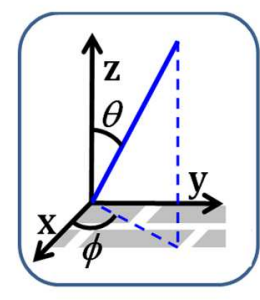

Figure 1. (a) $x z$ cross section of fin pair modeled upon a transmission electron micrograph in Ref. [8]. Materials shown were chosen from analysis of the public literature. (b) $x y$ cross section through the fins. Image is of one unit cell (UC). (c) $x y$ cross-sections showing bridge defects "Bx" and "By" within a 2 UC $\times 2$ UC area. Note, the length of the "Bx" bridge runs along the $y$ direction in our coordinate system, and the length of "By" runs along the $x$ direction. (d) Coordinate system for simulated incident plane wave linear polarization axis and angle of incidence, both polar $\theta$, and azimuthal, $\phi$.

Table 1. Optical constants $n$ and $k$ for four key materials used in semiconductor manufacturing, as found in the literature.

\begin{tabular}{|c|c|c|c|c|c|c|c|c|}
\hline $\begin{array}{c}\lambda \\
(\mathrm{nm})\end{array}$ & \multicolumn{2}{|c|}{$\mathrm{a}-\mathrm{Si}$} & \multicolumn{2}{c|}{$\mathrm{c}-\mathrm{Si}$} & \multicolumn{2}{c|}{$\mathrm{SiO}_{2}$} & \multicolumn{2}{c|}{$\mathrm{HfO}_{2}$} \\
\cline { 2 - 9 } & $n$ & $k$ & $n$ & $k$ & $n$ & $k$ & $n$ & $k$ \\
\hline 13 & 1 & 0.02 & 1.03 & 0.00113 & 0.98 & 0.01 & 0.97 & 0.0157 \\
\hline 47 & 0.88 & 6.58 & 0.803 & 1.78 & 0.62 & 0.30 & 0.58 & 0.06 \\
\hline 122 & 0.44 & 1.10 & 0.295 & 1.32 & 2.62 & 0.93 & 1.59 & 1.69 \\
\hline 157 & 0.67 & 1.63 & 0.49 & 2.04 & 1.80 & 0 & 2.36 & 1.22 \\
\hline 193 & 0.98 & 2.11 & 0.88 & 2.80 & 1.66 & 0 & 2.98 & 0.43 \\
\hline Refs. & \multicolumn{3}{|c|}{$[9]$} & \multicolumn{3}{|c|}{$[10]$} & {$[11-13]$} & \multicolumn{3}{c|}{$[14]$} \\
\hline
\end{tabular}

A guiding consideration in the simulation of defects is that the patterned structure can often be treated as periodic for the purposes of calculation. Periodicity removes possible unintended effects upon the scattered field from inadequate construction of perfectly matching layers (PMLs) around the sides the simulation domain, which are especially problematic

${ }^{1}$ Certain commercial materials are identified in this paper in order to specify the experimental procedure adequately. Such identification is not intended to imply recommendation or endorsement by the National Institute of Standards and Technology, nor is it intended to imply that the materials are necessarily the best available for the purpose. 
when using several oblique angles of incidence at five different wavelengths. However, it is possible for defects to interact optically with their periodic copies if the domain size is too small. To minimize this source of error, one defect-containing unit cell (UC) should be placed within a buffer of non-defect containing UCs that will fill the rest of the simulation domain. In this initial study, the domain size was scaled such that the $x$ and $y$ domain sizes were at least $10 \lambda$ in length with a constant $z$ size of $320 \mathrm{~nm}$. The simulation domain lengths and widths for these five wavelengths are provided in Table 2 .

For this study, obtaining numerical convergence for the simulations using $\lambda=13 \mathrm{~nm}$ proved difficult when using a 32 byte long floating-point data type, as the amplitude of the scattered field was on the order of the numerical noise in that simulation. For the $13 \mathrm{~nm}$ and $47 \mathrm{~nm}$ wavelengths, a 64-byte long floating-point data type was used instead, lengthening simulation times and increasing memory requirements. Also, this implementation of the FDTD uses equally sized cubic grids throughout the simulation domain to minimize potential sources of error from adaptive meshing; the cubic grid size, $\Delta s$, was set to yield integer widths of the fin dimensions while also maintaining $\Delta s<\lambda / 10$. These values also appear in Table 2.

Table 2. FDTD domain size and cubic grid size. Cubic grid size corresponds to the length of one side of a cube used in the simulations, with $\Delta s=2 \mathrm{~nm}$ leading to a cube $8 \mathrm{~nm}^{3}$ in volume.

\begin{tabular}{|c|c|c|c|}
\hline$\lambda(\mathrm{nm})$ & $x(\mathrm{~nm})$ & $y(\mathrm{~nm})$ & $\Delta s(\mathrm{~nm})$ \\
\hline 13 & 672 & 720 & 1 \\
\hline 47 & 1344 & 1344 & 1 \\
\hline 122 & 1344 & 1260 & 2 \\
\hline 157 & 1680 & 1800 & 2 \\
\hline 193 & 2016 & 1980 & 2 \\
\hline
\end{tabular}

Shown in Fig. 2 is a representative example of a simulated image and a simulated difference image. The image in Fig. 2 corresponds to $\lambda=13 \mathrm{~nm}$, normal incidence, X polarization imaging a "By" defect. The difference image is obtained by simulating the same patterned structure without the defect and subtracting its image from the image of the defect. In the absence of noise, these defects are observable. Noise was later added to this analysis through the inherent Poisson noise of the detectors, an important concern for detectors with low photon flux. This noise depends upon the number of photons per pixel at the sensor. A conservative estimate was made in Ref. [3] that yielded 6400 photons per pixel for each measurement, independent of wavelength. Using this value allowed the application of Poisson noise to each individual pixel. Representative images with added noise appear in Fig. 3.
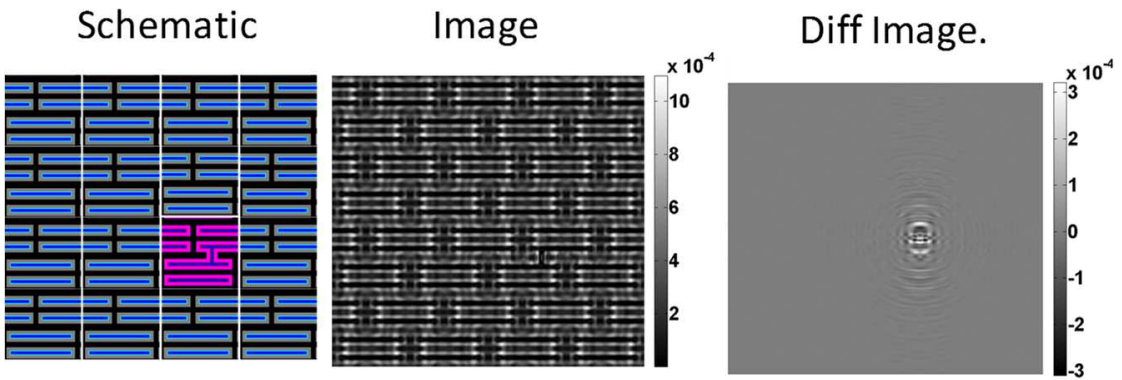

Defect Unit Cell
No-Defect Unit Cell

Figure 2. Schematic, simulated image, and difference image for a "By" defect. At $\lambda=13 \mathrm{~nm}$, the simulation domain was populated with by a $4 \times 4$ array of unit cells (UC) with one UC containing a defect, guarantee at least $10 \lambda$ separation between the edges of the simulation domain. Wavelength-dependent domain sizes are provided in Table 2. The image is simulated assuming normal incidence and $\mathrm{X}$ linear polarization. The difference image is determined by subtracting the image of a pattern with no defect (not shown) from this image with the defect shown. 


\subsection{Defect metric definition}

A primary concern for such a simulation study that considers five different wavelengths is the establishment of a defect metric that is applicable for all wavelengths, domain sizes, and cubic grid sizes. Utilizing the absolute value of the differential image (AVDI), an ideal candidate for this defect metric is a signal to noise ratio, defined here as

$$
S N R=\frac{I_{\text {signal }}}{\sigma_{\text {noise }}}
$$

where $I_{\text {signal }}$ corresponds to the total intensity gathered due to the defect while $\sigma_{\text {noise }}$ is the standard deviation of the intensities at all pixels falling below certain thresholds; the latter is not to be confused with $\sigma$, the standard deviation of the AVDI, as it is a $5 \sigma$ threshold is initially used to differentiate the pixels with large intensities due to the defect from those pixels with a smaller signal from the defect or the applied noise. In Fig. 3, two examples are shown to illustrate the calculation of the signal-to-noise ratio. The first column shows the application of this $5 \sigma$ thresholding on the AVDI. With noise, some of this signal comes from the optical response from the defect and from outlying pixels that exceed the threshold. The second column demonstrates how an area threshold can separate this random noise from the defect signal. In this initial simulation study, a simple area threshold of $A_{\min }=1000 \mathrm{~nm}^{2}$ was sufficient. In the third column, pixels colored in green are averaged to determine $I_{\text {signal }}$. The standard deviation of the pixel intensities for the red pixels determines $\sigma_{\text {noise }}$. For a given combination of incident angle, polarization, defect type, and wavelength a signal to noise ratio can be calculated.

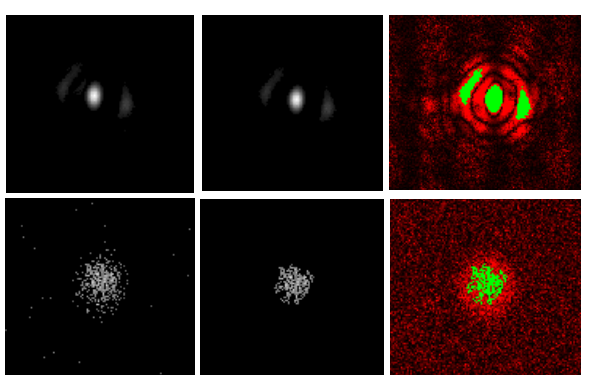

Figure 3. Application of intensity and area thresholding to separate a defect signal from its noise. The top and bottom rows are examples from $\lambda=47 \mathrm{~nm}$ and $\lambda=122 \mathrm{~nm}$, respectively. The left column shows pixels with intensities exceeding $5 \sigma$. The center column shows the exclusion of areas less than a constant minimum area $A_{\min }$. The right column shows the differential image due to the defect in green with the noise in red, permitting a signal to noise ratio to be determined from a single differential image.

\subsection{Comparisons among wavelengths, polarizations, incident angles, and defect types}

The key SNR results from the simulation study are presented as Fig. 4. SNR is plotted using polar plotting with interpolation that is based upon simulation results from 13 different angles of incidence. This presentation allows for comparisons among wavelength, defect type, polarization, and incident angle. For example, angle-resolved illumination positively affects the defect detectability of the "By" defect using X polarization at $\lambda=47 \mathrm{~nm}$, with a polar angle of $\theta=15^{\circ}$ and azimuthal angle of either $\phi=30^{\circ}$ or $\phi=60^{\circ}$ both yielding optimized signal to noise values for this defect.

From these data, $\lambda=47 \mathrm{~nm}$ is the optimal wavelength for defect detection, outperforming $\lambda=122 \mathrm{~nm}$ by a factor of five or more. Minimal gains are observable decreasing the wavelength from $\lambda=193 \mathrm{~nm}$ to $\lambda=122 \mathrm{~nm}$. A lack of appreciable signal even at large polar angles of incidence (i.e., small grazing angles) at $13 \mathrm{~nm}$ should be noted as it was anticipated that these might have yielded defect detectability. The optical constants $n(\lambda)$ as shown in Table 1 are near or at unity at $13 \mathrm{~nm}$, while $k(\lambda)$ is near zero at that wavelength. As noted in our prior work, there is not a single combination of angle of incidence and polarization that is optimal for these defect types. In Fig. 4 the "Bx" defect is shown with the $\mathrm{Y}$ polarization while the "By" defect is shown with the $\mathrm{X}$ polarization. The bridging directions of these two defects are orthogonal to each other and in both cases, the polarization that optimizes detection runs parallel to the bridging direction (i.e. its longer axis.) 


\section{Bx Defect Y pol.}
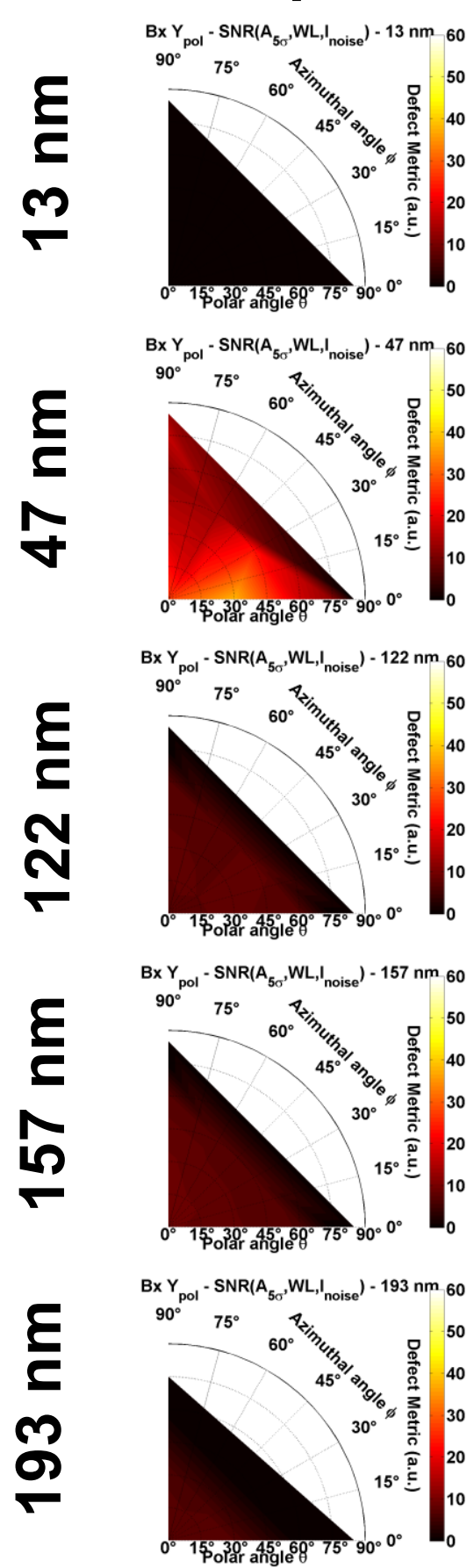

\section{By Defect $X$ pol.}

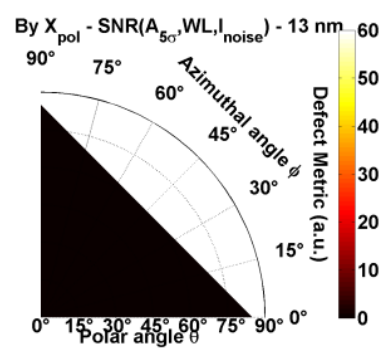

By $X_{\text {pol }}-\operatorname{SNR}\left(A_{5 \sigma}, W L, I_{\text {noise }}\right)-47 \mathrm{~nm} 60$
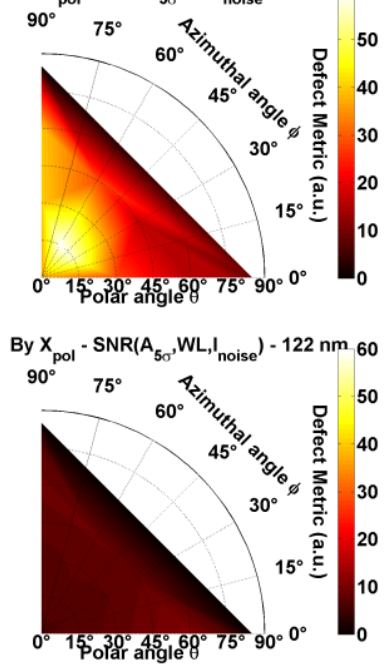

By $X_{\text {pol }}-\operatorname{SNR}\left(A_{50}, W L, I_{\text {noise }}\right)-157 \mathrm{~nm}_{60}$

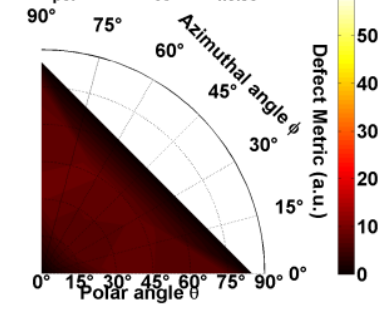

By $X_{\text {pol }}-\operatorname{SNR}\left(A_{50}\right.$, WL, $\left.{ }_{\text {noise }}\right)-193 \mathrm{~nm} 60$

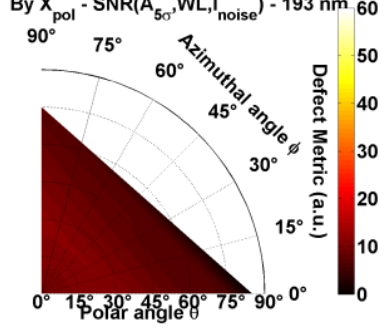

Figure 4. Signal to noise defect metric, plotted in polar plots showing the effects of polar and azimuthal angle, as functions of polarization and wavelength for the "Bx" defect, Y polarization (left) and the "By" defect, X polarization (right). For each column, the linearly polarized illumination is aligned with the direction of the defect, improving detectability for $\lambda \geq 47 \mathrm{~nm}$. With this defect metric, $\lambda=47 \mathrm{~nm}$ yields the greatest defect detectability. 


\section{ASSESSING FDTD PERFORMANCE ACROSS MULTIPLE WAVELENGTHS}

Two trends were identified in the initial simulation study: a five-fold increase in the SNR for $\lambda=47$ nm compared to longer wavelengths, and a decrease in the differential intensity of about three orders-of-magnitude for $\lambda=13 \mathrm{~nm}$ compared to $\lambda=47 \mathrm{~nm}$. This section addresses how computational factors intrinsic to our implementation of a FDTD Maxwell's equations solver influence these trends. The optical constants shown in Table 1 vary greatly among the wavelengths, and the accuracy of the code across this wavelength range should be investigated at multiple angles, either to verify the accuracy of the code or to estimate possible systematic bias in the results. It is essential to understand the interplay among the cubic grid size, the domain size, computational capabilities, and simulation wavelength upon simulation accuracy.

\subsection{Testing of two-dimensional FDTD using Fresnel equations}

The initial simulation study has calculated images of patterned structures and their defects using the geometry shown in Fig. 1 and the wavelength-dependent optical constants in Table 1. The scattering from these features and the reflectivity of the constituent materials are inseparable within images, but an understanding of the FDTD-calculated reflectivity of these materials can be gained through comparisons against Fresnel equations. This first assessment here compares twodimensional FDTD calculations of reflectivity from an unpatterned surface with the known polarization-dependent reflectivity of these materials. These simulations are functions of angle of incidence, optical properties, wavelength, and cubic grid size.
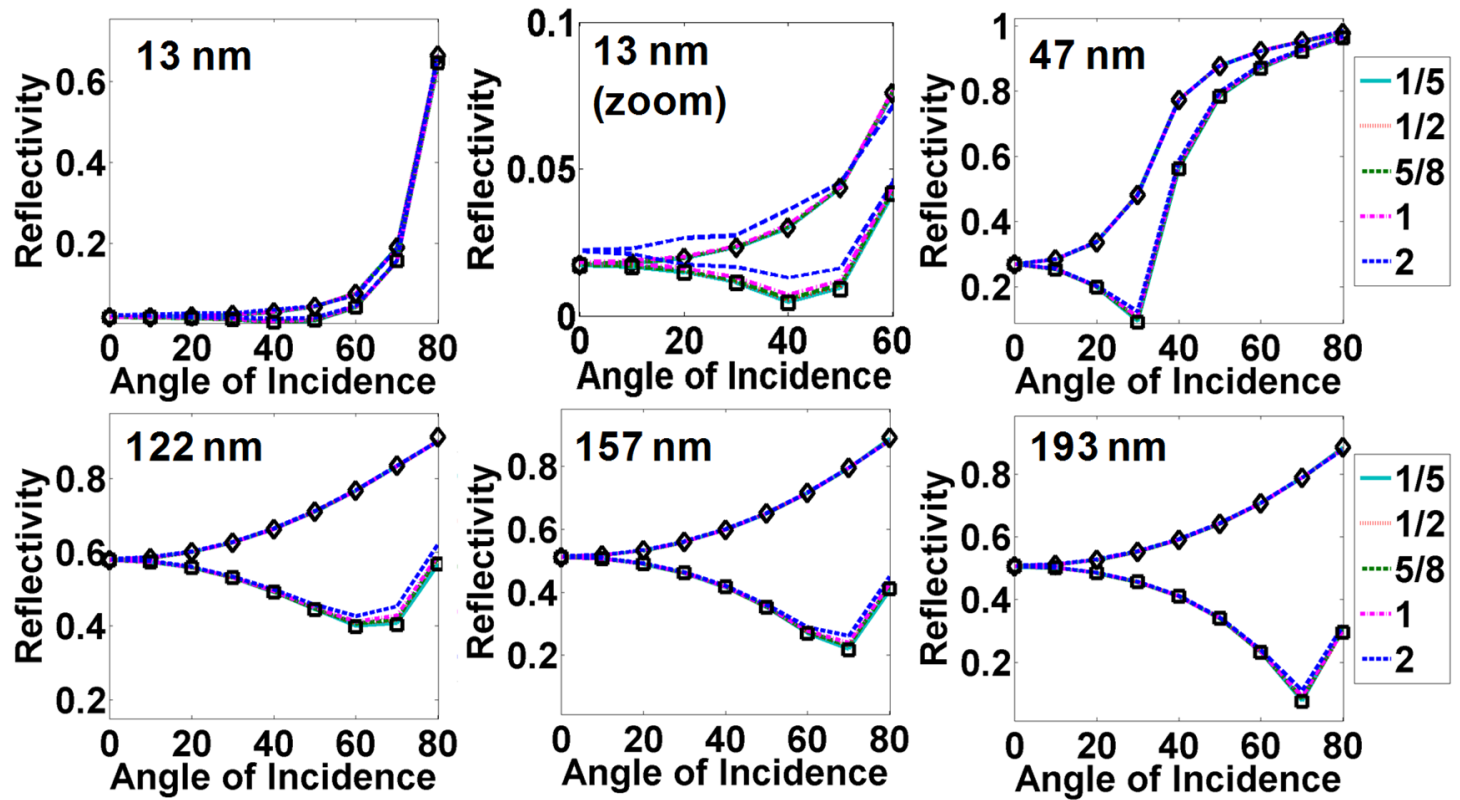

Figure 5. Reflectivity of $\mathrm{HfO}_{2}$ as calculated using Fresnel equations (markers) and FDTD (lines) in a two-dimensional configuration, using one of five cubic grid sizes $\Delta s: 1 / 5 \mathrm{~nm}, 1 / 2 \mathrm{~nm}, 5 / 8 \mathrm{~nm}, 1 \mathrm{~nm}$, and $2 \mathrm{~nm}$. The $s$ polarization is the set of curves with diamond markers, the $p$ polarization is the set of curves with square markers.

Figure 5 shows the reflectivity from $\mathrm{HfO}_{2}$ for the five simulation wavelengths. This material is presented first as a $\mathrm{HfO}_{2}$ film $8 \mathrm{~nm}$ thick coats the sides of the fins and the top of the $\mathrm{SiO}_{2}$ in Fig. 1. For each wavelength, multiple values of $\Delta s$ are calculated, with five presented here: $0.2 \mathrm{~nm}, 0.5 \mathrm{~nm}, 0.625 \mathrm{~nm}, 1 \mathrm{~nm}$, and $2 \mathrm{~nm}$. Each panel shows the Fresnel equation values for $s$ polarization (diamond markers) and for $p$ polarization (square markers). In each panel, the FDTD yields more accurate answers for the $s$ polarization than for the $p$ polarization across the range of incident angles and cubic grid sizes. For the $p$ polarization, cubic grid size reductions are required to approach the known values. Discrepancies in the FDTD simulation are most apparent at those angles at which the reflectivity for the $p$ polarization is lowest. This result follows naturally from the polarization of the light. The $s$-polarized illumination has its electric field vector parallel to the interface between the vacuum and the $\mathrm{HfO}_{2}$ and maintains its orientation at the interface. In contrast, the $p$-polarized illumination has its electric field vector in the plane of incidence and thus is at some angle to the interface of the vacuum and the $\mathrm{HfO}_{2}$. 
Quantifying the percent error between the FDTD and the Fresnel equations is one possible method for identifying potential systematic errors in the three-dimension patterned defect simulation studies across several wavelengths given the $\mathrm{HfO}_{2}$ coating in the initial simulation geometry. The initial simulation study used $\Delta s=1 \mathrm{~nm}$ for wavelengths of $13 \mathrm{~nm}$ and $47 \mathrm{~nm}, \Delta s=2 \mathrm{~nm}$ for $122 \mathrm{~nm}, 157 \mathrm{~nm}$, and $193 \mathrm{~nm}$, and it is these grid sizes that are compared. For the $s$ polarization, the percent error is less than $1 \%$ for those cubic grid sizes for all incident angles and all wavelengths. The results for the $p$ polarization must be described in more detail, as can be inferred from the " $13 \mathrm{~nm}$ (zoom)" panel of Fig. 5 . For $\Delta s=$ $1 \mathrm{~nm}$ and $\lambda=13 \mathrm{~nm}$, the largest percent error as a function of incident angle is about $55 \%$ for both $\theta=40^{\circ}$ and $\theta=50^{\circ}$. (The percent error drops down to about $25 \%, 20 \%$, and $5 \%$ for $\Delta s=5 / 8 \mathrm{~nm}, 1 / 2 \mathrm{~nm}$, and $1 / 5 \mathrm{~nm}$, respectively.) The FDTD is over-estimating the reflectivity for $p$-polarized light systematically. For $\lambda \geq 47 \mathrm{~nm}$, the $p$-polarization FDTD reflectivity has percent errors near $1 \%$ for most of the angles of incidence, but each wavelength has angles at which the percent errors increase notably. The maximum percent error for the chosen grid sizes for $\mathrm{HfO}_{2}$ is almost $20 \%$ at $\theta=30^{\circ}$ for $\lambda=47 \mathrm{~nm}, 12 \%$ at $\theta=70^{\circ}$ for $\lambda=122 \mathrm{~nm}, 21 \%$ at $\theta=70^{\circ}$ for $\lambda=157 \mathrm{~nm}$, and $45 \%$ at $\theta=70^{\circ}$ for $\lambda=193 \mathrm{~nm}$.

This 2-D FDTD evaluation suggests possible systematic bias in the accuracy of the images in the initial simulation study for images formed using $p$-polarized light. This corresponds to X polarization for $\phi=0^{\circ}$ and Y polarization for $\phi=90^{\circ}$, for example. However, this bias cannot account for the three-orders-of-magnitude change in the difference image intensity between $\lambda=13 \mathrm{~nm}$ and $\lambda=47 \mathrm{~nm}$. It is more likely that the difference signal is highly localized at $\lambda=13 \mathrm{~nm}$ and does not strongly interact optically with the intended pattern. For $\lambda \geq 47 \mathrm{~nm}$, the defects and pattern are not resolvable and the combination of the pattern and the defect ultimately determine the scattering. As to the five-fold increase in signal-tonoise for $\lambda=47 \mathrm{~nm}$ compared to the longer wavelengths, note that the "By" defect has its strongest SNR in Fig. 4 at $\theta=15^{\circ}$ at both $\phi=30^{\circ}$ and $\phi=60^{\circ}$, a polar angle at which the FDTD reflectivity is within $1 \%$ of the known values and is thus sufficiently stable. Although there is some minor difference in Fig. 4 in the SNR between $\phi=0^{\circ}$ and $\phi=90^{\circ}$ for the "Bx" defect at $\theta=30^{\circ}$ and Y polarization, we have not yet established how a $20 \%$ variation in the actual reflectivity could result in such a SNR increase, and in addition high SNR values are found for several other angle combinations for $\lambda=47 \mathrm{~nm}$.

For completeness, reflectivity comparisons for the three other materials in the stack in Fig. $1\left(\mathrm{SiO}_{2}, \mathrm{a}-\mathrm{Si}\right.$, and c-Si) are provided at the end of this paper as Fig. 10. The FDTD and the $s$ polarization again compare favorably while the grid size must be reduced to obtain better agreement between the $p$ polarization FDTD results and the known Fresnel equation values. While discrepancies in accuracy exist, none of these appear to impact the findings of the initial simulation study.

Even as these discrepancies yield insight into systematic errors within these multi-wavelength FDTD calculations, they cannot fully take into consideration scattering interactions with a patterned layout. To test scattering interactions, the full three-dimensional FDTD code must be used with a defined pattern of interest.
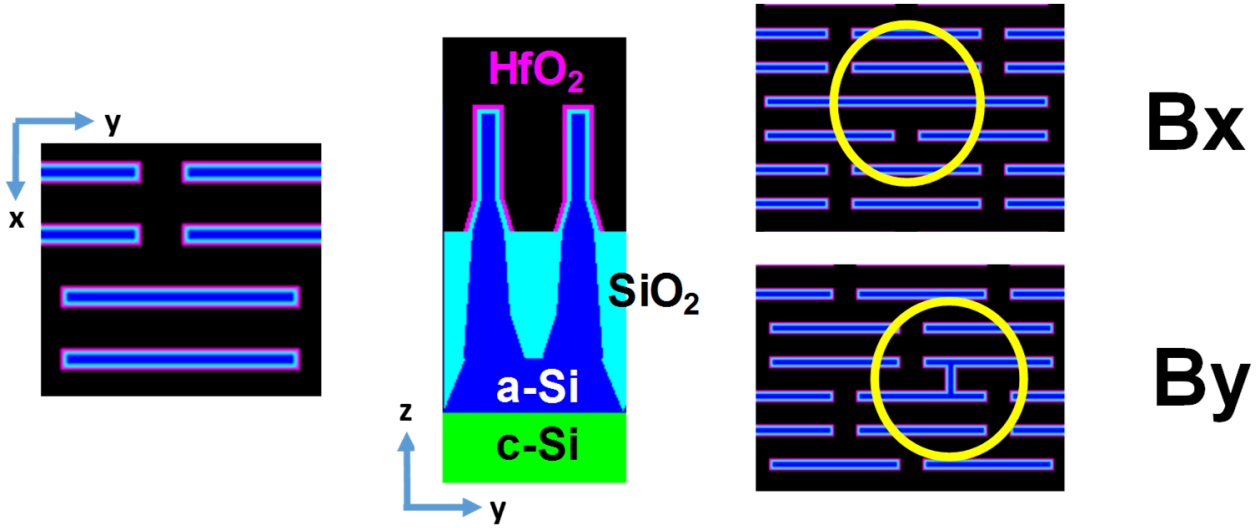

Figure 6. (left) $x y$ cross-section through a fin layout modified relative to Fig. 1. Image is of one unit cell (UC). (center) $y z$ cross-section of these fins, showing a $6 \mathrm{~nm}$ wide $\mathrm{Si}$ fin width in the fin with two conformal layers of $\mathrm{SiO}_{2}$ and $\mathrm{HfO}_{2}$, each $2 \mathrm{~nm}$ in thickness. (right) $x y$ cross-sections showing bridge defects "Bx" and "By." As before, the length of the "Bx" bridge runs along the $y$ direction in our coordinate system, and the length of "By" runs along the $x$ direction. 


\subsection{Modifying the simulation geometry}

The results of the initial simulation study should be viewed as specific to that materials stack and layout geometry. To further test trends in the scattering of patterned defects at multiple wavelengths, the simulation geometry has been altered as shown in Fig. 6. While the changes between the two figures may seem minor, several improvements may affect the scattered images. Foremost, the thickness of the $\mathrm{HfO}_{2}$ conformal layer has been reduced from $8 \mathrm{~nm}$ to $2 \mathrm{~nm}$ and the placement of the $\mathrm{HfO}_{2}$ has been localized to the fins only. There is no longer a conformal layer covering the $\mathrm{SiO}_{2}$ layer. In addition, both the sides and tops of the fins are now covered with the conformal layers. The fin height has been increased from $42 \mathrm{~nm}$ to $52 \mathrm{~nm}$ relative to the $\mathrm{SiO}_{2}$ layer and the $\mathrm{Si}$ fin width decreased from $8 \mathrm{~nm}$ to $6 \mathrm{~nm}$ to incorporate trends in the industry towards higher-aspect ratio fins [15]. The defects are also $6 \mathrm{~nm}$ wide silicon lines, less than $40 \mathrm{~nm}$ in length and conformally coated. Furthermore, the $(x, y)$ coordinates specifying the vertices for the polygons defining these structures have been adjusted such that these coordinates could be evenly divisible by $2 \mathrm{~nm}$, as much as practicable, to minimize possible rounding errors when using values of $\Delta s$ as large as $2 \mathrm{~nm}$. Rounding errors were reduced by reducing the fin pitch from $42 \mathrm{~nm}$ to $40 \mathrm{~nm}$ and changes in the unit cell size from $168 \mathrm{~nm} \times 180 \mathrm{~nm}$ previously to $160 \mathrm{~nm} \times 180$ nm. Optical constants are unchanged.

\subsection{Competing simulation requirements across multiple wavelengths}

The 2-D tests of our in-house FDTD code showed improved accuracy with smaller cubic grid sizes. A case has been made in the initial simulation study for maintaining simulation domains for which the extent in $x$ and $y$ are at least $10 \lambda$. However, cubic grid size and domain size are not the only considerations in performing these multiwavelength simulations.

The computing cluster upon which these simulations were performed has in general a maximum of $64 \mathrm{~GB}$ of memory per computing node. In Fig. 9, the total memory requirements of the $13 \mathrm{~nm}$ simulations are displayed to illustrate these constraints. Several combinations of large domain size and small cubic grid size cannot be realized within these memory requirements. In addition, simulations with $\lambda=47 \mathrm{~nm}$ have slightly larger memory requirements to account for the larger values of $k$ at this wavelength, such that the simulation domain must be slightly reduced in the $z$ axis to accommodate the combinations in Fig. 9. A similar assessment for the single precision (32-byte long floating-point data type) simulations for the VUV and DUV wavelengths leads to simulation domains as large as $10 \times 10$ for $\Delta s=1 \mathrm{~nm}, 6 \times 6$ for $2 / 3 \mathrm{~nm}$ grids, and $4 \times 4$ for $1 / 2 \mathrm{~nm}$ grids.

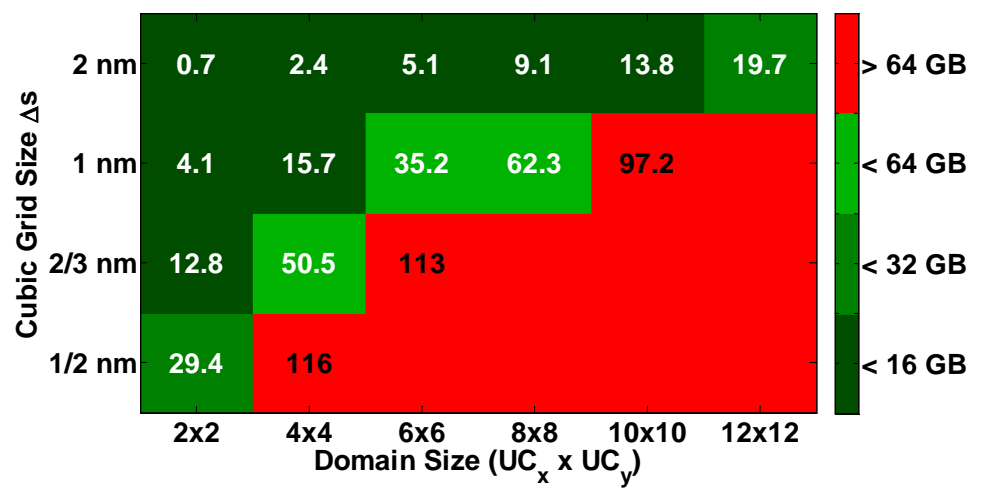

Figure 7. Memory requirements for performing FDTD simulations at $\lambda=13 \mathrm{~nm}$ with our in-house code on our local cluster, which in general has a limit of $64 \mathrm{~GB}$ total memory per node. Two simulations at $2 \times 2,1 / 2 \mathrm{~nm}$ can be run on a single node as each is less than $32 \mathrm{~GB}$, while a $4 \times 4,2 / 3 \mathrm{~nm}$ simulation requires the entire node.

These requirements directly challenge capabilities to maintain $10 \lambda$ spacing on the domain size for longer wavelengths for grid sizes below $\Delta s=2 \mathrm{~nm}$. Figure 8 shows the mean intensity of the "Bx" defect image with Y polarization using the $122 \mathrm{~nm}, 157 \mathrm{~nm}$, and $193 \mathrm{~nm}$ simulation wavelengths as a function of domain size and cubic grid size. Use of a $2 \mathrm{~nm}$ grid, as was done at these wavelengths for the initial study, would yield an error in the mean intensity of no greater than approximately $5 \%$ compared to $\Delta s=1 \mathrm{~nm}$ at all domain sizes. As expected, the mean intensity becomes more consistent as the domain size is increased. To allow for $10 \lambda \times 10 \lambda$ domain sizes, these domain sizes had been set for the initial simulation study to be $8 \times 7,10 \times 9$, and $12 \times 11$ for $122 \mathrm{~nm}, 157 \mathrm{~nm}$, and $193 \mathrm{~nm}$ wavelengths, respectively. Note that decreasing these domain sizes to $6 \times 6$ would lead to errors of less than $1 \%$ in mean intensity for these three wavelengths. 
Given these results and the known constraints, the results of the simulation study in Section 4 will feature $\Delta s=2 / 3 \mathrm{~nm}$ domain sizes of $4 \times 4$ for the EUV wavelengths and $6 \times 6$ for the VUV and DUV. In addition to improving the accuracy of the FDTD results for this defined geometry, reducing the cubic grid size will also allow the incorporation of line edge roughness (LER). Using $10 \%$ of the $\mathrm{CD}$ as a guideline for defining the three-sigma value of the $\mathrm{LER}, 3 \sigma_{\mathrm{LER}}=0.6 \mathrm{~nm}$ and this roughness will be better represented by $\Delta s=2 / 3 \mathrm{~nm}$ than by $\Delta s=1 \mathrm{~nm}$. Cubic grid sizes of $\Delta s=0.5 \mathrm{~nm}$ and smaller are much less viable given our computational constraints as the maximum domain sizes available for $\lambda>13 \mathrm{~nm}$ are insufficient to yield accurate differential imaging results.
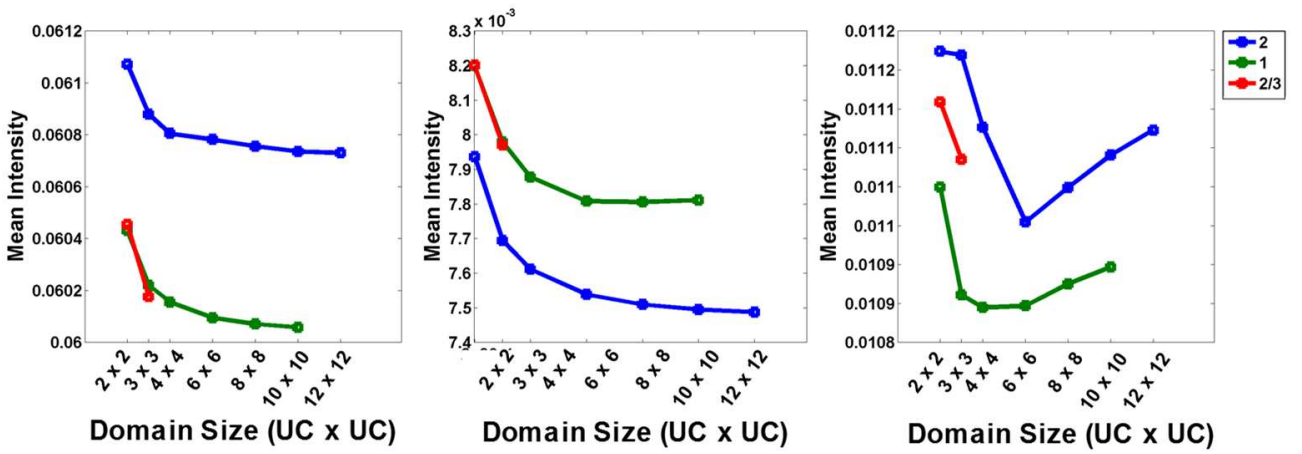

Figure 8. Mean intensity of the simulated "Bx" defect imaged using Y polarized light at normal incidence as functions of domain size and three cubic grid sizes $\Delta s$ for the vacuum- and deep-ultraviolet wavelengths.

\section{IMPROVING COMPARISONS AMONG WAVELENGTHS AND POLARIZATIONS}

Motivation for and preliminary results from our group's current simulation study of the structure defined in Fig. 6 at the five wavelengths are presented below, with comparisons made with the initial simulation study in Section 2 . This current simulation study is ongoing and full results will be presented in a subsequent publication.

\subsection{Simulation study methodology}

Goals for this new study are to quantify the defect detectability as functions of incident angle, polarization, defect type, and wavelength for patterns with and without line edge roughness (LER) and to validate trends observed in the initial simulation study. The addition of line edge roughness produces "wafer noise" which is extraneous signal in the difference images due to nuisance, non-killer deviations from the ideal patterning. Simulations with and without LER are to be performed to ascertain the wavelength dependence of the sensitivity to wafer noise and its effects on defect detectability. The same two bridging defects in Fig. 6, "Bx" and "By", are to be augmented with simulations of shorter non-bridging defects as well as line breaks. Definitions for the incident angles and polarizations persist from Fig.1, and the materials and their wavelength-dependent optical constants from Table 1 are once again used. For consistency and for improved convergence at $13 \mathrm{~nm}$, the EUV wavelengths of $13 \mathrm{~nm}$ and $47 \mathrm{~nm}$ are to be simulated with a 64-byte long floating-point data type with the longer wavelengths calculated with a 32-byte long floating-point data type.

This study should add to our understanding of inspection capabilities at EUV wavelengths. It is important to determine if the trends in the initial study persist with minor changes in geometry, a re-arrangement of key conformal layers, and a smaller cubic grid size. Questions persist regarding the general trends in the EUV wavelengths: whether $13 \mathrm{~nm}$ is ill-suited for defect inspection and whether $47 \mathrm{~nm}$ wavelength is a clear improvement over longer wavelengths.

\subsection{Comparisons between geometries for illumination at normal incidence}

Preliminary simulation results from the current study are reported here and are limited to normal incidence illumination. Data from the initial study are presented as well for the two bridging defects in common, the "Bx" and "By" defects. Both are treated with the same defect metric as defined in Section 2.2 using a signal to noise ratio calculated after applying a $5 \sigma$ threshold upon the absolute value of the differential image as well as an area threshold of $A_{\min }=1000 \mathrm{~nm}^{2}$. Results are shown in Table 3.

Considering first the current study, the defect is not recognized in not only for $\lambda=13 \mathrm{~nm}$ but also for $\lambda=193 \mathrm{~nm}$ and for "By" at $\lambda=122 \mathrm{~nm}$. The lack of a definitive signal at $193 \mathrm{~nm}$ may be related to the reduction in the simulation domain 
size, or the difficulties in separating the signal from the noise may be due to the small cubic grid size, as the images are produced with the pixel size matching the cubic grid size (e.g., $2 / 3 \mathrm{~nm}$ ). Comparing the initial study to the current study, the dominance of $\lambda=47 \mathrm{~nm}$ is reaffirmed with exceptionally strong signals for the "By" defect with $\mathrm{X}$ polarization. However, there is only a factor of two difference between the "Bx", Y polarization SNR for $\lambda=47 \mathrm{~nm}$ in the current study.

Table 3. Comparison between the initial and current simulation studies using the initial signal to noise defect metric defined in Section 2.2. All values are for normal incidence illumination $N / A$ indicates that no $I_{\text {signal }}$ was isolated due to the defect.

\begin{tabular}{|c|c|c|c|c|}
\hline \multirow{2}{*}{$\begin{array}{c}\lambda \\
(\mathrm{nm})\end{array}$} & \multicolumn{2}{|c|}{ Initial Simulation Study } & \multicolumn{2}{c|}{ Current Simulation Study } \\
\cline { 2 - 5 } & "Bx" defect, X pol. & "By" defect, X pol. & "Bx" defect, Y pol. & "By" defect, X pol. \\
\hline 13 & N/A & N/A & N/A & N/A \\
\hline 47 & 27.3 & 36.6 & 16.3 & 51.5 \\
\hline 122 & 7.1 & 6.4 & 7.8 & N/A \\
\hline 157 & 8.7 & $N / A$ & 7.2 & 6.5 \\
\hline 193 & 6.8 & 9.7 & $N / A$ & N/A \\
\hline
\end{tabular}

As to the loss of defect detectability at $\lambda=13 \mathrm{~nm}$, the 2D FDTD assessment in Section 3 did show almost an order-ofmagnitude difference in the reflectivity of $\mathrm{HfO}_{2}$ between $\lambda=13 \mathrm{~nm}$ and $\lambda=47 \mathrm{~nm}$, but this alone cannot describe the absence of defects at $13 \mathrm{~nm}$ here. In Ref. [3], it was stated that "[a]s the wavelength decreases, one might reasonably expect the optical scattering volume from a sub-resolved object to decrease, but for this study a simple area threshold of $A_{\min }=1000 \mathrm{~nm}^{2}$ was sufficient." This statement was made after assessing all the absolute value of the differential images (AVDIs) after the application of the Poisson noise, and as $13 \mathrm{~nm}$ wavelength images showed no clear defect-based signal, this area-based threshold was applied for the images for $\lambda=47 \mathrm{~nm}$ and longer wavelengths.

Having now reduced the likelihood that the lack of apparent defects using $13 \mathrm{~nm}$ was due to a systematic error in the FDTD or a particularly unfavorable materials stack, the focus now shifts to the application of the defect metric itself and its improvement for the challenge of capturing the signal to noise at $\lambda=13 \mathrm{~nm}$ and the equitable comparison of defect inspect across all five wavelengths.

\subsection{Preprocessing of differential image data and modifying the defect metric}

Calculating a defect metric using an AVDI is our standard practice and has been beneficial for four of the five wavelengths in both simulation studies here. It is anticipated that the area over which a difference would appear due to a $13 \mathrm{~nm}$ defect signal would be much smaller than those for the other defects and that it may reasonably have been lost with the application of Poisson noise. Additional processing of the AVDI would not tease out this small signal, so additional image processing has now been applied to the difference image before the application of the absolute value.

The amplitude of the Poisson noise is on par with the difference in intensity due to the defect. The mean intensity of the applied Poisson noise is zero, while the defect yields a non-zero difference in the absence of noise. To better isolate the defect signal, some averaging of the Poisson noise is required. Resizing the image effectively bins several of the smaller pixels to yield 1 larger pixel; areas of the image where the defect dominates will still have a non-zero mean while the noise will begin to average out. Figure 9 shows the effect of averaging upon the $13 \mathrm{~nm}$ differential image for the "Bx" defect with Y polarization. The final pixel size should be the same for direct comparison and will be $2 \mathrm{~nm}$, the largest grid size used in either study. Thus, the 13 and $47 \mathrm{~nm}$ images in the initial study $(\Delta s=1 \mathrm{~nm})$ are rescaled by a factor of $1 / 2$, and all images in the current study are rescaled by a factor of $1 / 3(\Delta s=2 / 3 \mathrm{~nm})$. Thus, results here are for simulated images with pixels that are $2 \mathrm{~nm} \times 2 \mathrm{~nm}$. Note for such pixel sizes, much work remains to make these results more practical.

The presence of a small but identifiable region in the rescaled AVDI for $13 \mathrm{~nm}$ requires an immediate reassessment of our existing quantitative defect metric. With this modification, the defects at $13 \mathrm{~nm}$ are identified areas of the defect's differential image that are greater than $40 \mathrm{~nm}^{2}$, far less than the previous threshold of $A_{\min }=1000 \mathrm{~nm}^{2}$. Determining the optimal defect metric for these data is still a work in progress, as changes are very likely required when incorporating additional incidence angles, additional defect types, and LER. Therefore, for this publication, the defect metric is treated 

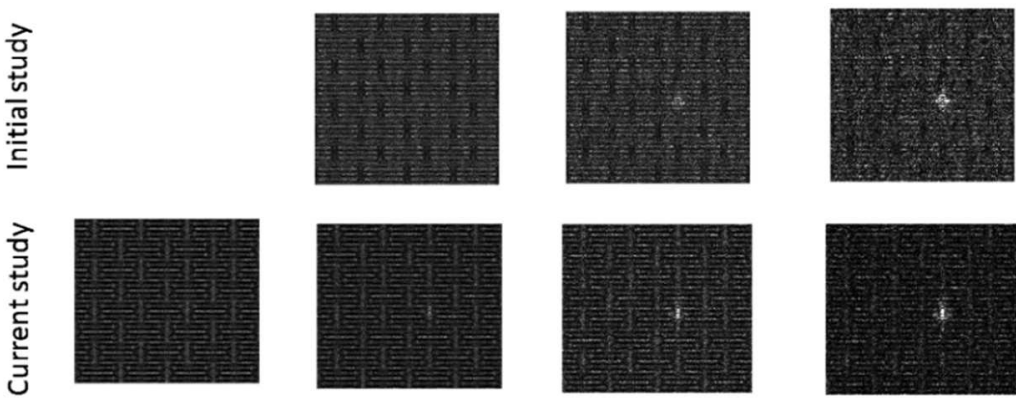

2/3 nm

pixel size

$1 \mathrm{~nm}$

pixel size

$2 \mathrm{~nm}$

pixel size
$4 \mathrm{~nm}$ pixel size

Figure 9. Changes in the AVDI with rescaling. The top row is for data from the original study with cubic grid size of $1 \mathrm{~nm}$, the bottom row for the current simulation study with cubic grid size of $2 / 3 \mathrm{~nm}$. The defect from the original study was not detected even with scaling to a $2 \mathrm{~nm}$ pixel size, but was for the current study due to this scaling to $2 \mathrm{~nm}$.

as provisional. We envision applying machine learning techniques to better define the defect metric as more images are simulated.

For the moment, however, an intermediate threshold can be proposed using the comparative values of the largest contiguous area identified in the $5 \sigma$ thresholding for the five wavelengths and two defect types as shown in Table 4 . For wavelengths greater than $13 \mathrm{~nm}$, these largest areas exceed the previous $A_{\min }$ threshold. It must be determined if a wavelength-dependent area threshold is required or whether it is adequate to simply set $A_{\text {min }}=40 \mathrm{~nm}^{2}$. Based upon analyses of the largest area identified in each differential image and upon a qualitative, visual check of the differential images, the provisional defect metric is defined for these initial results as a $5 \sigma$ threshold with an adjusted $A_{\min }$. Lowering the threshold to $A_{\min }=40 \mathrm{~nm}^{2}$ or defining it as $A_{\min }(\lambda)=3 \lambda$ both encompass the values listed in Table 4, but proper definition and further work are require to exclude as much of the noise as possible as well as capturing other defect-generated areas in the AVDI. For this provisional defect metric however, $A_{\min }(\lambda)=3 \lambda$ was used. Note, it is yet to be determined whether this new defect metric adequately identifies wafer noise as background and not as defect signal.

There are SNR values for most every entry in Table 4 except for the "Bx" defect and Y polarized light at $\lambda=13 \mathrm{~nm}$ that was shown in Fig. 9. However, $13 \mathrm{~nm}$ wavelength and $\mathrm{X}$ polarization (not shown) was detectable with SNR $=6.7$ with a largest area of $48 \mathrm{~nm}^{2}$. Thus, the defect metric has been adjusted to accommodate the entire wavelength range. The VUV and DUV results show the effects of image scaling. For the initial study, the images are not scaled at all and the SNR ratios are the same as in Table 3. For the current study, scaling the image by a factor of $1 / 3$ has brought out not only the defect at $\lambda=13 \mathrm{~nm}$ but also the defects at $\lambda=122 \mathrm{~nm}$ and $\lambda=193 \mathrm{~nm}$.

Table 4. Comparison between the initial and current simulation studies using the provisional defect metric defined in Section 4.3. All values are for normal incidence illumination. $N / A$ indicates that no $I_{\text {signal }}$ was isolated due to the defect thus no SNR.

\begin{tabular}{|c|c|c|c|c|c|c|c|c|}
\hline $\begin{array}{c}\lambda \\
(\mathrm{nm})\end{array}$ & \multicolumn{3}{|c|}{ Initial Simulation Study } & \multicolumn{4}{c|}{ Current Simulation Study } \\
\cline { 2 - 9 } & "Bx" defect, Y pol. & \multicolumn{2}{|c|}{ "By" defect, X pol. } & \multicolumn{2}{c|}{ "Bx" defect, Y pol. } & \multicolumn{2}{c|}{ "By" defect, X pol. } \\
\cline { 2 - 9 } & $\begin{array}{c}\text { Largest Area } \\
\text { above 5 } \sigma \\
\left(\mathrm{nm}^{2}\right)\end{array}$ & $\begin{array}{c}\text { Defect } \\
\text { Metric }\end{array}$ & $\begin{array}{c}\text { Largest Area } \\
\text { above 5 } \\
\left(\mathrm{nm}^{2}\right)\end{array}$ & $\begin{array}{c}\text { Defect } \\
\text { Metric }\end{array}$ & $\begin{array}{c}\text { Largest Area } \\
\text { above 5 } \sigma \\
\left(\mathrm{nm}^{2}\right)\end{array}$ & $\begin{array}{c}\text { Defect } \\
\text { Metric }\end{array}$ & $\begin{array}{c}\text { Largest Area } \\
\text { above 5 } \sigma \\
\left(\mathrm{nm}^{2}\right)\end{array}$ & $\begin{array}{c}\text { Defect } \\
\text { Metric }\end{array}$ \\
\hline 13 & 32 & $N / A$ & 56 & 7.6 & 72 & 6.9 & 196 & 7.8 \\
\hline 47 & 1800 & 41.5 & 376 & 53.7 & 2844 & 23.7 & 1480 & 57.8 \\
\hline 122 & 4148 & 7.1 & 2052 & 6.4 & 6500 & 14.1 & 10096 & 10.2 \\
\hline 157 & 10220 & 8.7 & 12 & $N / A$ & 10588 & 14.4 & 13732 & 13.1 \\
\hline 193 & 5356 & 6.8 & 13684 & 9.7 & 2588 & 6.4 & 15388 & 9.4 \\
\hline
\end{tabular}


The scaling does not have an appreciable effect on the comparisons between the simulation studies. The SNR for the "Bx" defect in the current study is still is largest at $\lambda=47 \mathrm{~nm}$ by only around a factor of two, where the initial value is almost a factor of five larger at normal incidence. This trend will be watched as more angles are simulated. Like the initial simulation study, the "By" defect at normal incidence has a SNR that is between 4 and 5 times larger than the SNR of any other wavelength. Note that challenges remain in understanding these preliminary results, as the minimum area threshold differs significantly between the simulation studies as the threshold has been lowered to allow all five wavelengths to be accounted for by the provisional defect metric. Many additional checks are required and additional work is to be conducted to complete this current study and to place comparisons with the initial simulation study into proper context.

\section{CONCLUSIONS}

Simulation studies encompassing five wavelengths that range from the DUV to the EUV are being performed to determine the extensibility of defect metrology for ever-decreasing "killer" defects with sub-10 nm critical dimensions. An initial, full simulation study has recently been reported by our group and has been summarized here. In this initial study, $\lambda=47 \mathrm{~nm}$ showed better defect sensitivity by a factor of five as determined through a defined defect metric. This result is in stark contrast with the simulations for $\lambda=13 \mathrm{~nm}$, as the difference image intensities for $\lambda=13 \mathrm{~nm}$ are about three orders-of-magnitude less than those for $\lambda=47 \mathrm{~nm}$ for the initial defined fin geometry.

In response to this initial study, a thorough FDTD consistency study has been presented as a check of these results and to quantify any possible sources of systematic bias in the simulations or other calculation steps that might affect the signal to noise. By comparing two-dimensional FDTD simulations of unpatterned substrates as functions of cubic grid size, angle of incidence, and wavelength, it was demonstrated that $s$-polarized reflectivities for our simulation conditions and in-house FDTD formulation are within $1 \%$ of those determined analytically using Fresnel equations. For $p$-polarized reflectivities, there is a clear improvement in the consistency of the results with decreased grid sizes. Certain angles at each wavelength would lead to deviations in the FDTD-determined reflectivity as high as $55 \%$ for $\lambda=13 \mathrm{~nm}$ using $\Delta s=1 \mathrm{~nm}$. The simulation accuracy of the full three-dimensional simulations is to be questioned, but these systematic deviations which always over-report the reflectivity do not appear to be the root cause of the differential intensity disparity between $\lambda=13 \mathrm{~nm}$ and $\lambda=47 \mathrm{~nm}$. In addition, increased SNR at $\lambda=47 \mathrm{~nm}$ in the initial study is observable in simulations at angles of incidence for which the $p$-polarized reflectivity is sufficiently stable, with less than $1 \%$ error. Therefore, it is not believed that these systematic biases in accuracy have affected the results of the initial study.

A modified three-dimensional geometry was introduced featuring higher aspect ratios and significant changes in the conformal layers. Whereas $\mathrm{HfO}_{2}$ was the predominant material at the vacuum interface in the initial study, both $\mathrm{HfO}_{2}$ and $\mathrm{SiO}_{2}$ would be at on top of this materials stack. This geometry has been simulated using a variety of cubic grid sizes and domain sizes to determine the effects of changing these parameters on the mean intensity scattered from the patterned surfaces. Better consistency can be achieved by decreasing the cubic grid size, although this may practically necessitate the reduction of the domain size. Errors in the mean intensity due to shrinking the domain size proved to be less than $10 \%$, but reducing the domain size to below $10 \lambda \times 10 \lambda$ requires additional study. FDTD consistency studies favored reducing the cubic grid size.

An additional factor, our desire to enable a second simulation study featuring line-edge roughness further motivates simulations of this new geometry with cubic grid sizes less than $1 \mathrm{~nm}^{3}$. Preliminary results without LER have been presented and similar to the initial study, defects could be not be seen in the simulated, Poisson-noise added differential images for the $13 \mathrm{~nm}$ wavelength. However, additional preprocessing of the differential image enables isolation of the defect-based signal at this EUV wavelength. This result allows and requires the defect metric to be reassessed to accommodate all five simulation wavelengths. Applying a modified defect metric, the $\lambda=47 \mathrm{~nm}$ seems nearly as effective at detecting the "By" bridge defect as in the initial simulation study, although results for the "Bx" defect were less clear. Much work is to be performed to optimize the defect metric and to balance the simulation domain sizes, the cubic grid sizes, the computational resources, and this improved defect metric to more accurately and confidently address optimizing image-based patterned defect inspection. 

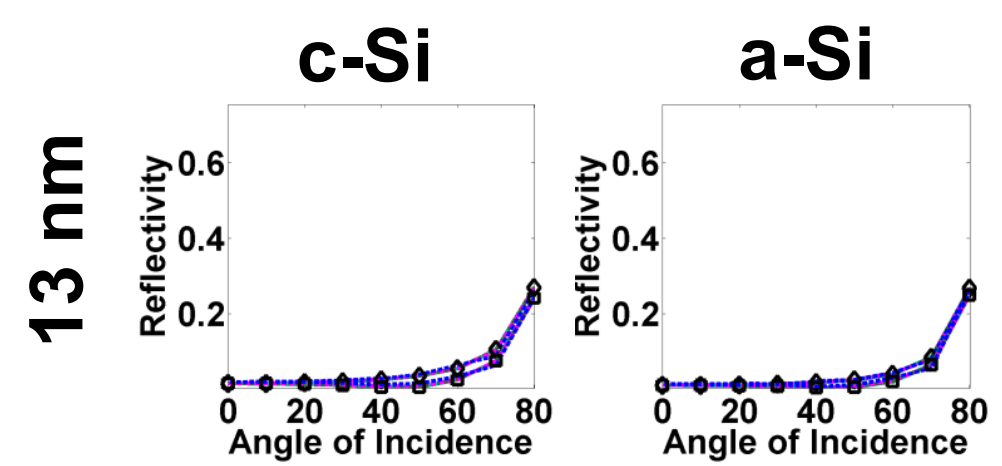

$\mathrm{SiO}_{2}$
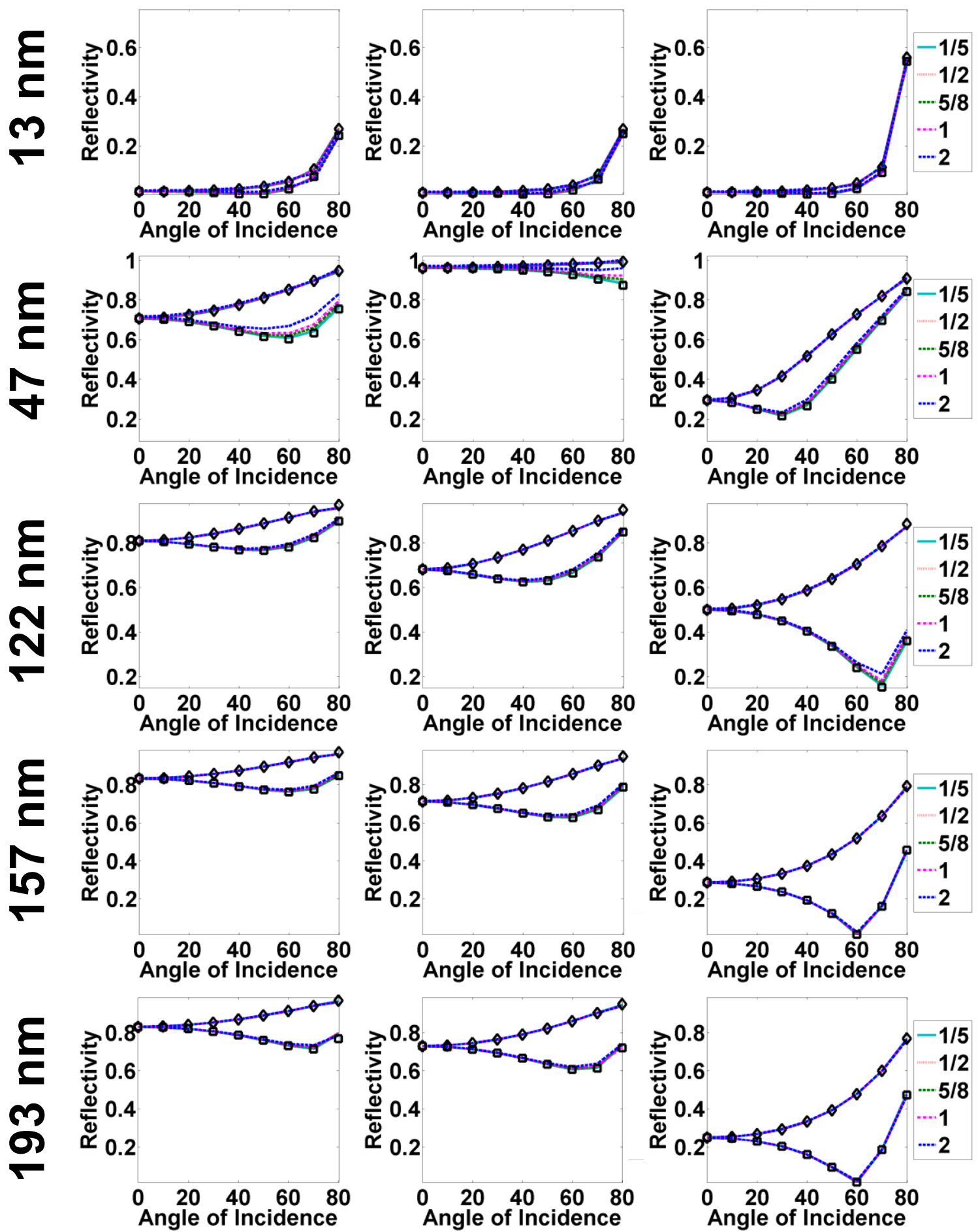

Figure 10. Reflectivity of $\mathrm{SiO}_{2}$, a-Si, and c-Si as calculated using Fresnel equations (markers) and FDTD in a two-dimensional configuration, using one of five cubic grid sizes $\Delta s: 1 / 5 \mathrm{~nm}, 1 / 2 \mathrm{~nm}, 5 / 8 \mathrm{~nm}, 1 \mathrm{~nm}$, and $2 \mathrm{~nm}$. The $s$ polarization is the set of curves with diamond markers, the $p$ polarization is the set of curves with square markers. 


\section{REFERENCES}

[1] T. F. Crimmins, "Defect metrology challenges at the $11 \mathrm{~nm}$ node and beyond," Proc. SPIE, 7638, 76380H (2010). http://dx.doi.org/10.1117/12.846623

[2] M. I. Mishchenko, "Gustav Mie and the fundamental concept of electromagnetic scattering by particles: A perspective,” J. of Quant. Spectro. Rad. Trans., 110(14-16), 1210-1222 (2009). http://dx.doi.org/10.1016/j.jqsrt.2009.02.002

[3] B. M. Barnes, H. Zhou, M.-A. Henn et al., "Assessing the wavelength extensibility of optical patterned defect inspection." Proc. SPIE, 10145, 1014516 (2017). http://dx.doi.org/10.1117/12.2262191

[4] A. Taflove, "Application of the Finite-Difference Time-Domain Method to Sinusoidal Steady-State Electromagnetic-Penetration Problems," IEEE T. Electromag. Compat., EMC-22(3), 191-202 (1980). http://dx.doi.org/10.1109/TEMC.1980.303879

[5] B. M. Barnes, F. Goasmat, M. Y. Sohn et al., "Effects of wafer noise on the detection of 20-nm defects using optical volumetric inspection,” J. Micro-Nanolitho. MEMS MOEMS, 14(1), 9 (2015).

http://dx.doi.org/10.1117/1.jmm.14.1.014001

[6] B. M. Barnes, F. Goasmat, M. Y. Sohn et al., "Enhancing 9 nm Node Dense Patterned Defect Optical Inspection using Polarization, Angle, and Focus,” Proc. SPIE, 8681, 86810E (2013). http://dx.doi.org/10.1117/12.2012250

[7] A. Raghunathan, S. Bennett, H. O. Stamper et al., "13nm gate Intentional Defect Array (IDA) wafer patterning by e-beam lithography for defect metrology evaluation," Microelec. Engr., 88(8), 2729-2731 (2011). http://dx.doi.org/10.1016/j.mee.2011.02.109

[8] S. Natarajan, M. Agostinelli, S. Akbar et al., "A $14 \mathrm{~nm}$ logic technology featuring $2^{\text {nd }}$-generation FinFET, airgapped interconnects, self-aligned double patterning and a $0.0588 \mu \mathrm{m}^{2}$ SRAM cell size." 2014 IEEE Int'1 Electron Devices Meeting (IEDM), 3.7.1-3.7.3 (2014). http://dx.doi.org/10.1109/IEDM.2014.7046976

[9] H. Piller, [Silicon (Amorphous) (a-Si) ] Academic Press, Boston(1985). http://dx.doi.org/10.1016/B978-0-08054721-3.50030-7

[10] D. F. Edwards, [Silicon (Si)*] Academic Press, Boston (1985). http://dx.doi.org/10.1016/B978-0-08-0547213.50029-0

[11] E. Filatova, A. Sokolov, J.-M. André et al., "Optical constants of crystalline HfO2 for energy range 140-930 eV," Applied Optics, 49(14), 2539-2546 (2010). http://dx.doi.org/10.1364/AO.49.002539

[12] Q.-J. Liu, N.-C. Zhang, F.-S. Liu et al., "Structural, electronic, optical, elastic properties and Born effective charges of monoclinic HfO2 from first-principles calculations," Chinese Physics B, 23(4), 047101 (2014). http://dx.doi.org/10.1088/1674-1056/23/4/047101

[13] D. Franta, D. Nečas, I. Ohlídal et al., "Dispersion model for optical thin films applicable in wide spectral range," Proc. SPIE, 9628, 96281U (2015).

[14] H. R. Philipp, [Silicon Dioxide $\left(\mathrm{SiO}_{2}\right)$, Type $\alpha$ (Crystalline)] Academic Press, Boston (1985). http://dx.doi.org/10.1016/B978-0-08-054721-3.50039-3

[15] B. Bunday, E. Solecky, A. Vaid et al., Proc. SPIE "Metrology capabilities and needs for $7 \mathrm{~nm}$ and $5 \mathrm{~nm}$ logic nodes." Proc. SPIE, 10145, 101450G (2017). http://dx.doi.org/10.1117/12.2260870 


\title{
AN OVERVIEW OF ACTIVITIES AT NIST TOWARDS THE PROPOSED ASTM E57 3D IMAGING SYSTEM POINT-TO-POINT DISTANCE STANDARD
}

\author{
Prem Rachakonda*, Bala Muralikrishnan*, Meghan Shilling*, Daniel Sawyer*, \\ Geraldine Cheok ${ }^{*}$ \\ *Dimensional Metrology Group, \\ Engineering Physics Division \& \\ National Institute of Standards and Technology, \\ Gaithersburg, MD \\ ISensing and Perception Systems Group, \\ Intelligent Systems Division, \\ National Institute of Standards and Technology, \\ Gaithersburg, MD
}

\section{INTRODUCTION}

Terrestrial laser scanners (TLSs) are a class of 3D imaging systems that measure the 3D coordinates of an object in their work volume. They capture the 3D coordinates using a ranging unit mounted on two rotation stages that are orthogonal to each other as illustrated in Figure 1. They are used for a variety of applications, e.g., large scale assembly, surveying, forensics, reverse engineering etc. The Dimensional Metrology Group (DMG) at the National Institute of Standards \& Technology (NIST), along with various other organizations has been involved in the development of a documentary standard for 3D imaging instruments that acquire data in spherical coordinate system. NIST led this effort and possesses unique expertise for this task. This expertise comes from NIST's prior experience on standardization activities related to laser trackers which also use spherical coordinate system and have very similar error sources.

TLSs were being used at NIST in several research projects starting in the early 2000s. It soon became clear that there was need to evaluate the instruments and determine the uncertainty of the measurements obtained. In this context, NIST organized three workshops between 2003 and 2006 which convened instrument manufacturers, end users and organizations like NIST to determine the needs of all the stake holders [1,2]. During these workshops the participants agreed upon the need for standard terminology, artifacts and standardized protocols that are needed for evaluating TLSs. Based on these workshops, an ASTM subcommittee on 3D imaging systems was established in 2006, and a working group started work on evaluating the fundamental measurement of 3D imaging systems, their range. In 2015, this working group published the ASTM E2938-15 standard for 3D imaging systems to evaluate relative-range. [3].

The scope of the ASTM E2938-15 standard was limited to the evaluation of the relative-range of $3 \mathrm{D}$ imaging systems that acquire data in both the spherical and nonspherical coordinate systems. As this standard was being balloted, another working group was established in 2013 [4]

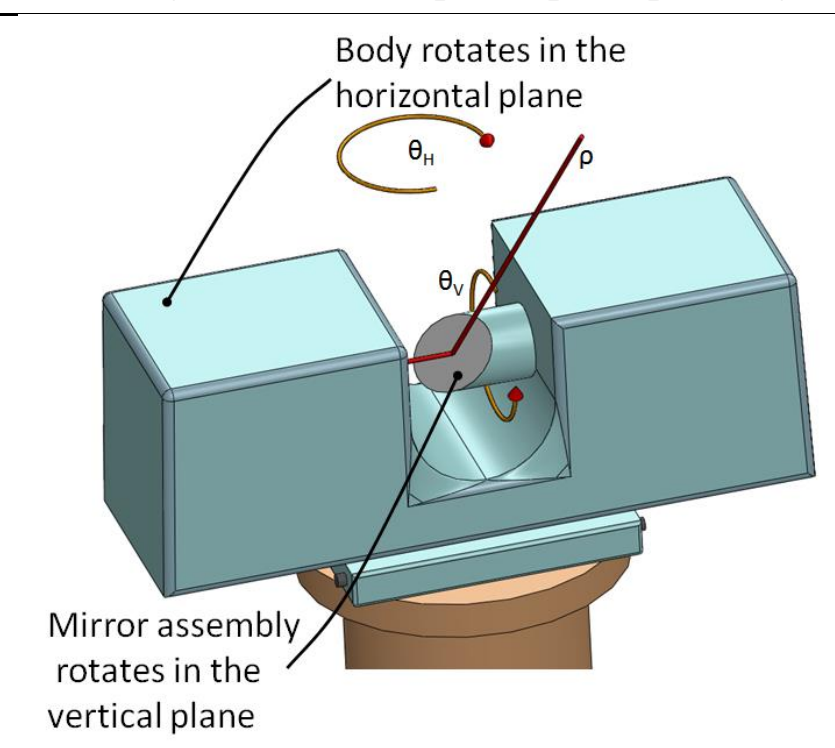

Figure 1: Schematic of a terrestrial laser scanner (TLS) within the E57.02 sub-committee that started addressing the performance evaluation of these instruments over their entire work volume. The proposed standard titled "Standard test method for 
evaluating the point-to-point distance measurement performance of spherical coordinate 3D imaging systems in the medium range" was submitted for balloting in the spring of 2017.

Challenges in developing the proposed standard included the lack of commercially available high quality targets, methods to obtain ground truth measurements and information about the sources of error. Other challenges included target mounting methods, data collection and data post-processing algorithms and to develop the performance criteria. The methods in the proposed standard were realized and tested at NIST before being incorporated into the document.

This paper will present an overview of the work, the procedures and recommendations for evaluating TLSs that found consensus among the members of the working group and were included in the proposed standard.

\section{ASTM STANDARD FOR POINT-TO-POINT DISTANCE EVALUATION}

\subsection{Motivation for the new standard}

At the outset of this work and as of the writing of this paper, no standard exists for evaluating TLS systems over their entire work volume. TLS manufacturers typically specify the instrument performance based on non-standard targets using characteristics and data throughput rates that are not consistent. Therefore, it is difficult for an end user to make an informed decision about their TLS purchase for technical, acceptance or warranty related purposes. As the ASTM E2938-15 standard only evaluates the relative-range performance, the end user does not have a standardized way to evaluate the overall instrument performance. Such an evaluation is require d because most real-world applications involve measurements that span the entire instrument work volume.

Another motivation for the proposed standard is the specific application/requirement from the industry. For example, one large scale US manufacturer approached NIST and was interested in determining the performance of their TLSs from technical and commercial perspectives. In this context, NIST worked with them under a cooperative research \& development agreement (CRADA). Under this agreement, various concepts were explored to understand the issues with evaluating such instruments. Two other TLS manufacturers collaborated with NIST to evaluate their instruments as NIST was in the process of developing TLS performance evaluation procedures.

\subsection{Development of the standard and the run-off meeting at NIST}

To address the lack of standardization of TLSs, an ASTM working group was constituted under the leadership of NIST to develop this new standard. The participants included manufacturers, experts, end users from the industry, and researchers from NIST and the National Research Council (NRC) of Canada. The mode of development involved bi-weekly internet based teleconference meetings that started in the summer of 2013 and extended into the spring of 2017. Participants of these meetings discussed a variety of topics, ideas, solutions, tested them at their facilities to evaluate their feasibilities and presented their findings. A substantial amount of raw data and information was exchanged in this process. Almost all the methods considered for this standard were tested before incorporation into the proposed standard.

In the spring of 2016, a four-day run-off meeting was held at NIST to evaluate the initial proposed methods. Five TLS manufacturers, representing most of the TLS market share, participated in this run-off meeting and tested their instruments using the proposed standard. The purpose of this run-off meeting was to verify whether the tests could be successfully performed by a variety of instruments and to obtain feedback in terms of feasibility and efficiency. After the tests concluded, the manufacturers gave considerable feedback that resulted in several major changes

Cheok, Geraldine; Muralikrishnan, Balasubramanian; Rachakonda, Prem; Sawyer, Daniel; Shilling, Katharine.

"An Overview of Activities at NIST Towards the Proposed ASTM E57 3D Imaging System Point-to-point Distance Standard."

Paper presented at 33rd Annual Coordinate Metrology Society Conference, Snowbird, UT. July 17, 2017 - July 21, 2017. 
to the proposed standard. Some of the changes include reducing the number of tests while maintaining the rigor of the evaluation and retaining the use of the flat plate target for the relativerange tests. Details of this run-off meeting were documented by Muralikrishnan et al. $[5,6]$.

\subsection{Scope of the proposed standard and realization of the tests}

The primary sources of errors in TLS measurements were deemed to be due to the instrument construction, target characteristics and data processing. Though environmental conditions could affect the results, they were not considered as sources of errors in the proposed standard. This is due to the fact that the tests were performed within rated operating conditions, and the environmental effects are addressed in the test uncertainty. Subsequently, the scope of the standard was restricted and the rationale is detailed in the next few sub-sections.

\subsubsection{Type of instruments and the measurand}

\subsubsection{Spherical coordinate system instruments}

There are many commercial systems that capture 3D data using different technologies (e.g., flash lidar, TLSs etc.). The ASTM E2938-15 standard included the evaluation of all such instruments. The scope of this new proposed standard however was limited to systems that acquire data in a spherical coordinate frame. This was primarily done for the following reasons.

1. Researchers at NIST have been involved in documentary standards for other instruments like laser trackers (e.g., ASME B89.4.19 and ISO 10360-10 standards) that use spherical coordinate systems which are similar in construction to TLSs. The sources of errors for such instruments were extensively studied and NIST researchers understood the issues with spherical coordinate 3D imaging systems.

2. The large volume 3D imaging systems, typically required for surveying and manufacturing assembly, use a spherical coordinate frame for acquiring data. Other instrument designs require performance tests that are sensitive to their error sources.

\subsubsection{Derived point to derived point distance evaluation}

TLSs capture data without the necessity of a cooperative target. They measure the distance of an object/target based on the reflected light from the object/target. Depending on the properties of the target surface, the noise in a single point measurement can be large. More importantly, most TLSs cannot measure only a single point as they are meant to operate in the scanning mode. To enable a standardized method of comparison and evaluation of these instruments, the concept of a derived point was introduced. A derived point is a point computed using multiple measured points on a target surface. It is a point that corresponds to the 3D point cloud of an object and may be one geometric parameter of that object. E.g., the center of a sphere derived from a scan of a sphere or the apex of a pyramid obtained from intersecting the planes of a pyramid. Use of a derived point enables the characterization of instrument construction errors by suppressing the effect of noise associated with a single point.

\subsubsection{Targets}

Though TLSs do not require a cooperative target, like a laser tracker, they can still benefit from using specialized targets for obtaining consistent, high quality data required for performance evaluation. The characteristics of the target such as its geometry, color, reflectivity and the associated data processing methods affect the results. The data quality can be improved by choosing a target that minimizes target induced errors. A variety of targets were considered for this process and were studied in detail at NIST [7,8]. Some of these targets included flat plate 
targets, contrast/checkerboard targets and spheres. Of these, metallic aluminum spheres that were media blasted to give a dull matte finish were found to perform well for some of the proposed tests (two-face and non-ranging point-to-point distance tests) and vapor blasted aluminum plates for relative-range tests. Some of the reasons for choosing these targets will be discussed next.

\subsubsection{Spheres}

Spheres can be suitable targets for evaluating 3D imaging instruments as their geometry appears the same regardless of the direction of the scan. They are typically used for registering multiple scans when a single scan cannot cover the entire region of interest. There are however issues pertaining to the data quality that influence their use in performance evaluation. The derived point for a sphere is its center, and determining the center of a sphere from TLS data is challenging. Recently, Rachakonda et al. [9] studied sphere data sets from several TLSs extensively and proposed novel algorithms and data segmentation techniques to obtain the sphere center. Due to various sources of errors from both the TLS and the sphere target, determining a sphere center is fraught with uncertainty. This is important because the target induced errors may incorrectly be attributed to the instrument construction.

It was observed that a major component of the error in determining the sphere center is in the ranging direction of the TLS [9]. Hence using such targets for relative-range evaluation would lead to a larger test uncertainty. However, sphere targets are suitable for non-ranging point-topoint distance tests that involve measuring targets at approximately the same distance. Sphere targets are also suitable for two-face tests because any ranging direction error introduced due to the spherical geometry is common-mode between the front-face and back-face measurements and is therefore not of any consequence. For these reasons, spherical targets were chosen for all the non-ranging length tests.

To enable a reference length measurement for the point-to-point distance tests (described in the later sections) a special custom sphere was commercially procured. This target, referred to as the "integration sphere" (Figure 2), is a partial sphere fabricated with a kinematic nest inside and a scannable surface on the outside. A 1.5 in $(38.1 \mathrm{~mm})$ diameter sphere or spherically mounted retroreflector (SMR) mounted in this nest is concentric with the center of the outer partial sphere. These "integration spheres" allow the measurement of their centers using a single measurement from a laser tracker.

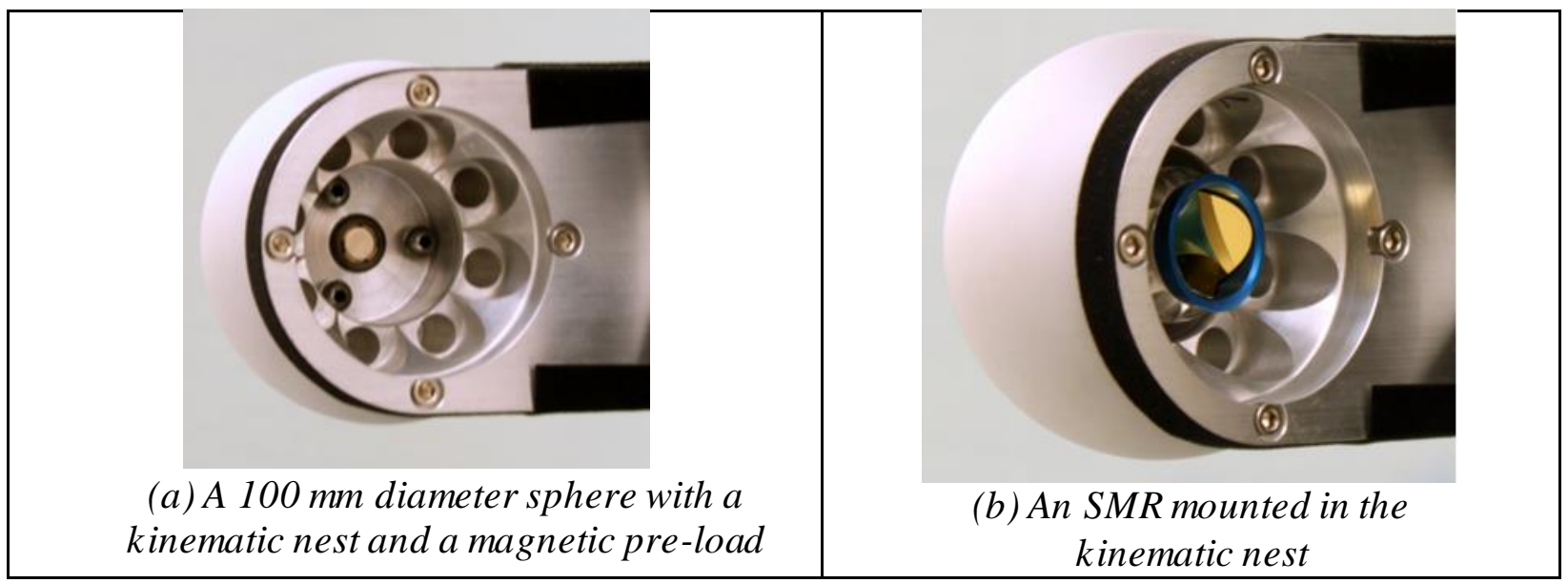

* Disclaimer: Commercial equipment and materials may be identified in order to adequately specify certain procedures. In no case does such identification imply recommendation or endorsement by the National Institute of Standards and Technology, nor does it imply that the materials or equipment identified are necessarily the best available for the purpose. 
Figure 2: An "Integration sphere" used for point-to-point distance evaluation of TLSs

\subsubsection{Flat plates \& hybrid targets}

The ASTM E2938-15 standard mandates the use of planar targets for relative-range tests. The recommendations from that standard have been adopted into this proposed standard based on several tests and studies performed at NIST [10]. In this process, several planar targets were designed and are presented in the appendices of the proposed standard. These targets allow for more efficient realization of the test. These implementations are not mandatory in the proposed standard, but have been included as methods for consideration by the user.

The derived point for such a planar target is its geometric center. Measuring the geometric center of a planar target with both the reference instrument (RI) and the instrument under test (IUT) can be challenging. Any misalignment of the target with respect to the line joining the RI and IUT will result in an abbe error which may be incorrectly attributed to the instrument construction. Various designs of planar targets were explored to minimize such errors due to the alignment process. These targets are depicted (front and back) in Figure 3 and are described next:

(a) Flat plate target with SMRs on the back: This target, depicted in Figure 3a, is of approximate dimensions $300 \mathrm{~mm} \times 300 \mathrm{~mm} \times 40 \mathrm{~mm}$ and has a media blasted front surface with a flatness of $\approx 20 \mu \mathrm{m}$ on the front and five kinematic nests to mount SMRs on the back. The front scannable surface was designed per the ASTM E2938-15 standard and the back surface was designed to aid in the alignment process using the RI. Because there are no fiducials on the front surface to locate a point on the plane, different segmentation and data processing methods may result in a different point being calculated as the derivedpoint of the plate. If the plate is not carefully aligned, and this derived-point does not coincide with the point measured by the RI, then there is an error associated with the measurement that appears as relative-range error, but is in fact due to the test setup.

(b) Plate-sphere artifact with $100 \mathrm{~mm}$ diameter "integration sphere" at the center: This artifact, depicted in Figure 3b, was designed so as to enable the RI and the IUT to measure a common point in space, thus overcoming the alignment problem that affects the target in Figure 3a. The flat plate is of approximate dimensions $450 \mathrm{~mm} \times 450 \mathrm{~mm} \times 40 \mathrm{~mm}$. A flat plate target results in a derived point with low uncertainty in the ranging direction, but not in the non-ranging direction. A sphere on the other hand results in reliable measurements in the non-ranging direction, but not in the ranging direction. A plate-sphere artifact combines these two geometries and overcomes the inadequacies of either one and results in a better estimate of the target derived point. When an "integration sphere" is used, obtaining the reference value becomes easier. Though this target design was promising, it had a drawback in that the sphere was too small to be measured at longer distances $(>20$ m) with some TLSs.

(c) Plate-sphere artifact with $200 \mathrm{~mm}$ diameter sphere at the center: This artifact, depicted in Figure 3c, was designed to overcome the issue of the $100 \mathrm{~mm}$ diameter sphere not being measurable by some TLSs. A larger $200 \mathrm{~mm}$ diameter "integration sphere" was used and additional recesses were added around this sphere to minimize the multi-path scattering that affects the determination of the sphere center. The size of the flat plate was approximately $600 \mathrm{~mm} \times 600 \mathrm{~mm} \times 40 \mathrm{~mm}$. This target was scanned by five different scanners during the run-off meeting held at NIST. Though the results were satisfactory, the participants in the meeting expressed concern about the large angle of sweep required to scan this artifact and the lack of data/features at the center of the artifact. A large angle of

Cheok, Geraldine; Muralikrishnan, Balasubramanian; Rachakonda, Prem; Sawyer, Daniel; Shilling, Katharine 
sweep introduces errors caused by the angular encoders into a relative-range test and the results will not be representative of the relative-range performance of the IUT.

(d) Flat plate target with fiducials: To allay the concerns expressed by the participants in the run-off meeting about the previous target design, a new target was designed. The flat plate target depicted in Figure 3a was modified to include two $100 \mathrm{~mm}$ diameter "integration sphere" targets to lower the uncertainty associated with derived point calculation. The addition of these two spheres help to reliably segment the data associated with the flat plate without obstructing the planar region of interest. Such a target design could be used with larger diameter "integration spheres" to enable IUT measurements at longer distances.

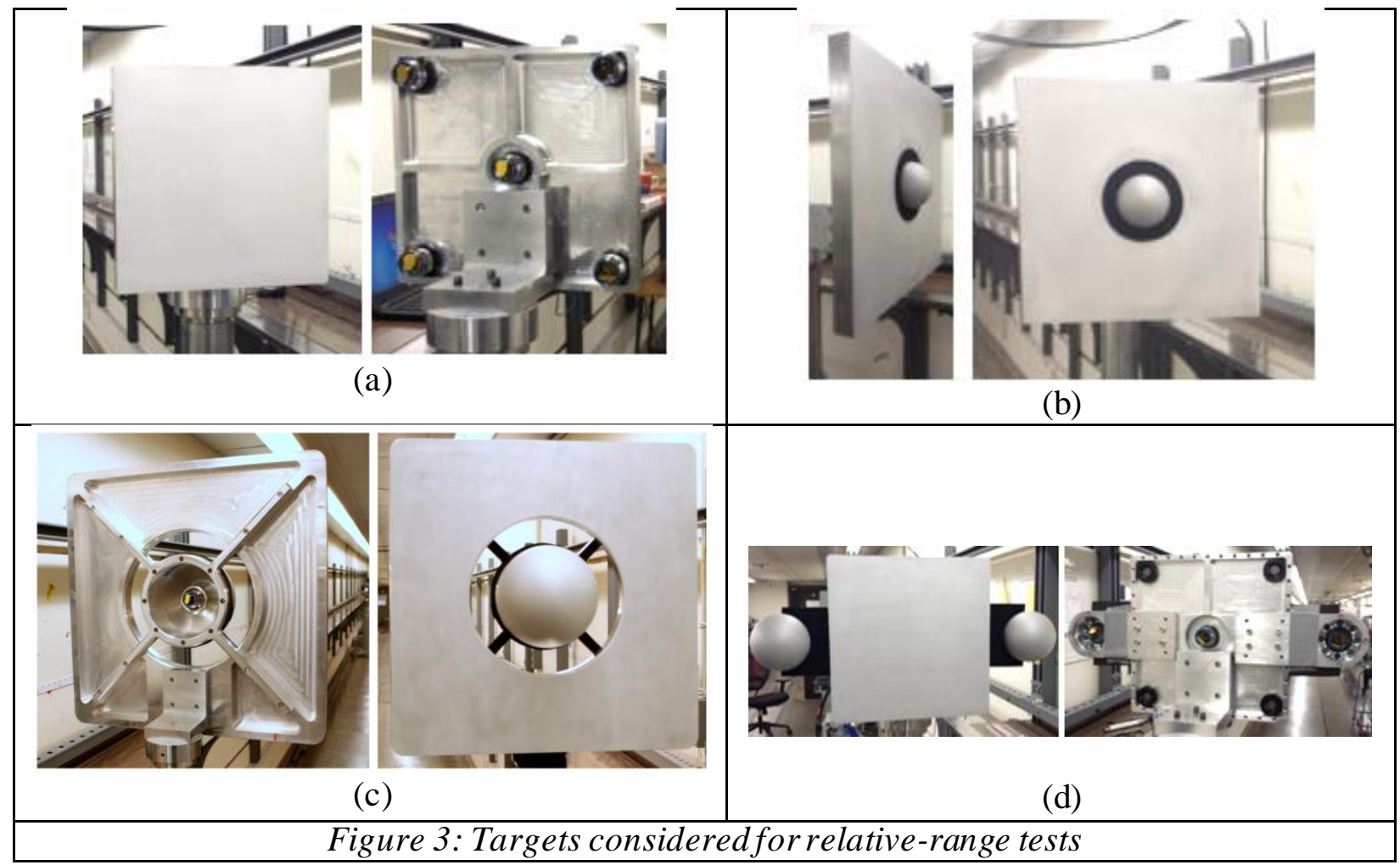




\subsection{Test me thods, positions and their imple mentation}

The scope of the proposed standard is to evaluate the instrument in its entire work volume. The number of point-to-point distances in a TLS's work volume is infinite and it will be practically impossible to test an instrument for all possible point-to-point distances. To limit the number of positions, it was important to identify the instrument related errors that contribute most to the performance of an instrument. To understand this, Muralikrishnan et al. [11] identified 18 sources of error that affect TLS performance and developed a detailed error model. All these sources of error are a result of the construction of the instrument, i.e., the imprecision in either fabrication or assembly of these instruments. Examples of these error sources are unintended beam \& mirror offsets and tilts, angular encoder related errors, etc. The error model provides an insight into the positions at which certain systematic errors are more prominent than others. The test positions in the proposed standard are based on this error model. It should be noted that any test position may reveal the effect of a combination of error sources and the contribution of each of those error sources may be mathematically deduced [12]. However, such an exercise is not the purpose of the proposed documentary standard.

The purpose of this error model in the context of the standardization activity is to reduce the number of test positions while maintaining sensitivity to all the sources of errors. Based on the error model and the feedback from

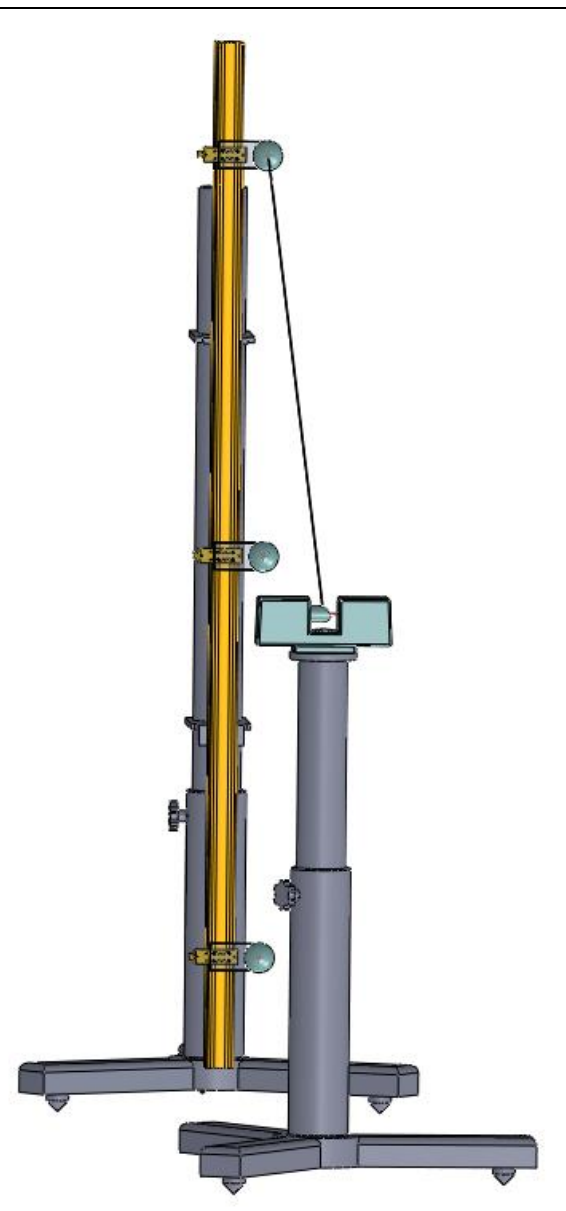

Figure 4: Setup to perform twoface tests the manufacturers, the proposed standard mandates 32 tests, namely 12 two-face tests and 20 pointto-point distance tests. The point-to-point distance tests include eight symmetric tests, six asymmetric tests, one inside test, three relative-range tests and two user-defined tests. The two user-defined tests may be performed at the discretion of the end-user in collaboration with the manufacturer. The next sub-sections will describe these tests in detail.

\subsubsection{Two-face tests}

Two face tests are designed for instruments that can scan both in the frontsight and backsight mode. These modes enable a TLS to rotate its head only $180^{\circ}$ to obtain a $360^{\circ}$ view by combining scans obtained from both the sides. Not all instruments may have such a mode, however for instruments that have this capability, the two-face test is mandatory per the proposed standard. These tests are sensitive to only 12 of the 18 sources of errors described in [11] and all the 12 sources of error manifest as angular errors in the derived point measurement.

To perform these tests, three spheres are mounted on a vertical column in such a way that one sphere is at an elevation angle $\phi=0^{\circ}$ (level with the origin of the scanner). The second sphere is placed at an $\phi=45^{\circ} \pm 10^{\circ}$ and the third sphere is placed at $\phi=-45^{\circ} \pm 10^{\circ}$. This is illustrated in Figure 4. The derived point of a single sphere measured using the IUT's frontsight mode will be compared to the derived point of the same sphere measured by the IUT's backsight mode. No 
reference length measurement is needed as the stationary spheres are assumed to be not affected by their mounting or environment in the short duration of the test. In essence, a zero-distance length is being measured by scanning the same sphere twice, once using the frontsight and the second time using the backsight of a TLS.

To perform these tests the scanner is placed at a distance $d$ from the target, with its elevation angle at $\phi$ and an azimuth angle $\theta$. The various values of $d, \phi$ and $\theta$ are listed in Table 1 . Here, the elevation angle is measured with respect to the horizontal plane.

\begin{tabular}{|c|l|c|c|}
\hline \multicolumn{4}{|c|}{ Table 1: Test positions for two-face tests } \\
\hline Test & $\begin{array}{l}\text { Distance of target } \\
\text { from IUT, } d\end{array}$ & $\begin{array}{l}\text { Elevation angle } \\
\phi \text { of target }\end{array}$ & $\begin{array}{l}\text { Azimuth } \\
\text { angle } \theta\end{array}$ \\
\hline TF1 & Not more than 10 m & $45^{\circ} \pm 10^{\circ}$ & $0^{\circ}$ \\
\hline TF2 & Not more than 10 m & $0^{\circ} \pm 10^{\circ}$ & $0^{\circ}$ \\
\hline TF3 & Not more than 10 m & $-45^{\circ} \pm 10^{\circ}$ & $0^{\circ}$ \\
\hline TF4 & Not more than 10 m & $45^{\circ} \pm 10^{\circ}$ & $90^{\circ}$ \\
\hline TF5 & Not more than 10 m & $0^{\circ} \pm 10^{\circ}$ & $90^{\circ}$ \\
\hline TF6 & Not more than 10 m & $-45^{\circ} \pm 10^{\circ}$ & $0^{\circ}$ \\
\hline TF7 & At least 20 m & $45^{\circ} \pm 10^{\circ}$ & $0^{\circ}$ \\
\hline TF8 & At least 20 m & $0^{\circ} \pm 10^{\circ}$ & $0^{\circ}$ \\
\hline TF9 & At least 20 m & $-45^{\circ} \pm 10^{\circ}$ & $90^{\circ}$ \\
\hline TF10 & At least 20 m & $45^{\circ} \pm 10^{\circ}$ & $90^{\circ}$ \\
\hline TF11 & At least 20 m & $0^{\circ} \pm 10^{\circ}$ & $90^{\circ}$ \\
\hline TF12 & At least 20 m & $-45^{\circ} \pm 10^{\circ}$ & \\
\hline
\end{tabular}

Though they are not comprehensive tests, two-face tests offer the following advantages compared to the point-to-point distance tests described in the next sub-section.

1. Simple setup (as illustrated in Figure 4).

2. No reference length measurements are required.

3. Simple sphere targets may be used. They do not need to be "integration spheres".

4. Two-face tests are quicker to perform than the point-to-point distance tests.

Any significant deviations from the expected values could point to instrument related errors. This may also indicate that some of the point-to-point distance test results may have a similar outcome and these deviations are best addressed before proceeding with further testing.

\subsubsection{Point-to-point distance tests}

The purpose of the point-to-point distance tests is to evaluate the IUT by measuring the distance between two targets and comparing it with a reference length. The reference length is obtained using an RI that can measure the reference length with an uncertainty value that is at least four times lower than that of the IUT. In the tests performed at NIST, the RI was a laser tracker and the target was an "integration sphere" for the non-ranging tests and a flat plate target for the relative-range tests.

\subsubsection{Non-ranging point-to-point distance tests}

The setup to perform most of these measurements could be either a grid of "integration spheres", a scale bar with two spheres at the ends, or any other setup where the distances can be realized and oriented in the required position with respect to the IUT. These tests are sensitive to all the 18 sources of error and give a near-complete picture of the instrument performance. 

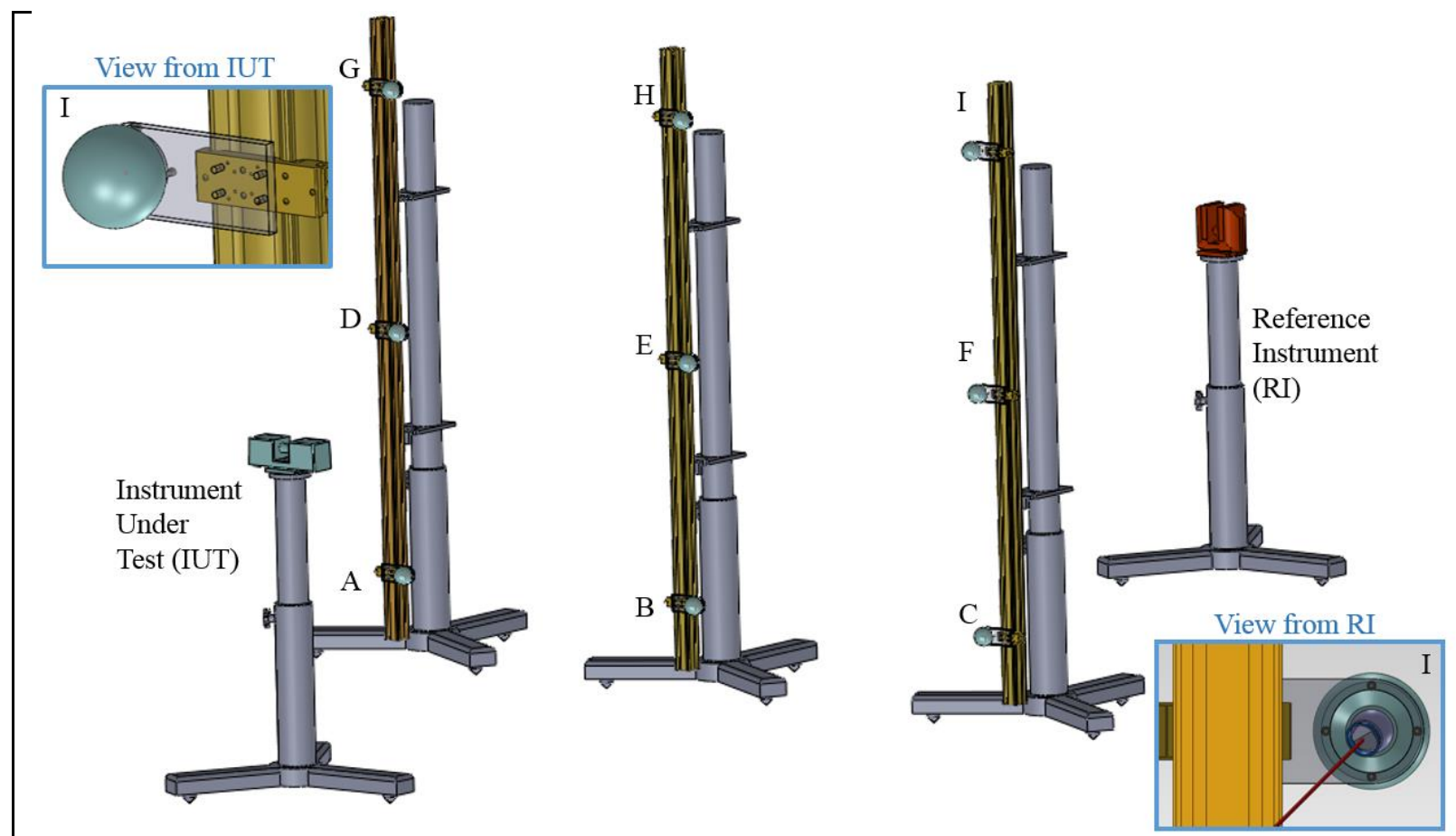

Figure 5: Setup to implement the most of the point-to-point distance tests

A test facility was set up at NIST to implement the procedures that could realize these tests. Per the proposed standard these tests can be implemented in any manner, but the setup depicted in Figure 5 was chosen to be implemented at NIST for the following reasons:

1. Commercial availability of the "integration sphere" made it easier to determine reference lengths by measuring only a single point, compared to probing the sphere at multiple points and deducing its center (SMR walking method [13]).

2. An implementation using scale bars, though simple, may introduce additional sources of uncertainty as the entire artifact is moved from one position to another.

3. An implementation using a grid of spheres mounted on a wall will require the use of the SMR walking method (or other methods) to obtain the reference lengths, which is a cumbersome process.

4. The DMG at NIST has environment controlled laboratories that are large enough to accommodate this grid setup where the RI can measure the reference length from one side of the grid and the IUT can scan the spheres from the other side. The large space is necessitated by the fact that the "integration spheres" are partial spheres and have only a limited line-of-sight to an SMR mounted inside it.

The point-to-point distance tests involve a series of symmetric and asymmetric length measurements. The various target positions are labeled in Figure 5 and the test distances and positions are listed in Table 2. Here, $\alpha$ is the angle subtended by the test length at the IUT's origin, and $\theta$ is the IUT's azimuth angle. These tests assume that the IUT's elevation angle $\phi \approx 0^{\circ}$ with the sphere at the center of the grid (sphere E in Figure 5) before scanning any sphere.

\begin{tabular}{|l|l|l|}
\hline \multicolumn{3}{|c|}{ Table 2: Test positions for non-ranging point-to-point distance tests } \\
\hline Test & \multicolumn{1}{|c|}{ Description } & Azimuth angle $\theta$ \\
\hline PP1 & $\alpha$ at least $80^{\circ}$ while measuring DF & $0^{\circ}$ \\
\hline PP2 & $\alpha$ at least $80^{\circ}$ while measuring DF & $90^{\circ}$ \\
\hline
\end{tabular}




\begin{tabular}{|l|l|l|}
\hline PP3 & $\alpha$ at least $80^{\circ}$ while measuring BH & $0^{\circ}$ \\
\hline PP4 & $\alpha$ at least $80^{\circ}$ while measuring BH & $90^{\circ}$ \\
\hline PP5 & $\alpha$ at least $80^{\circ}$ while measuring CG & $0^{\circ}$ \\
\hline PP6 & $\alpha$ at least $80^{\circ}$ while measuring CG & $90^{\circ}$ \\
\hline PP7 & $\alpha$ at least $80^{\circ}$ while measuring AI & $0^{\circ}$ \\
\hline PP8 & $\alpha$ at least $80^{\circ}$ while measuring AI & $90^{\circ}$ \\
\hline PP9 & $\alpha$ at least $40^{\circ}$ while measuring either ED or EF & $0^{\circ}$ \\
\hline PP10 & $\alpha$ at least $40^{\circ}$ while measuring either ED or EF & $90^{\circ}$ \\
\hline PP11 & $\alpha$ at least $40^{\circ}$ while measuring either EB or EH & $0^{\circ}$ \\
\hline PP12 & $\alpha$ at least $40^{\circ}$ while measuring either EB or EH & $90^{\circ}$ \\
\hline PP13 & $\begin{array}{l}\alpha \text { at least } 40^{\circ} \text { along the azimuth and at least } 40^{\circ} \text { along the } \\
\text { elevation directions while measuring either DH or HF }\end{array}$ & $0^{\circ}$ \\
\hline PP14 & $\begin{array}{l}\alpha \text { at least } 40^{\circ} \text { along the azimuth and at least } 40^{\circ} \text { along the } \\
\text { elevation directions while measuring either DH or } \mathrm{HF}\end{array}$ & $90^{\circ}$ \\
\hline PP15 & See details for inside test & \\
\hline
\end{tabular}

The inside test is a test illustrated in Figure 6, and involves two spheres placed at a certain distance $\left(\mathrm{d}_{0}\right)$ from the IUT in such a way that they are equidistant and collinear from the IUT, but diametrically on opposite sides of the IUT. The spheres are also placed in such a way that the IUT scans it at a mean elevation angle of $0^{\circ}$.

While the azimuth angles listed in Table land Table 2 are nominally $0^{\circ}$ and $90^{\circ}$, they can be any pair of angles that are $90^{\circ}$ apart to within $\pm 10^{\circ}$.

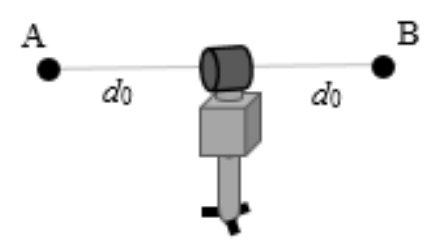

Figure 6: Setup to implement one of the point-to-point distance tests called the "Inside test"

\subsubsection{Relative-range tests}

The procedures from ASTM E2938-15 required to perform the relative-range evaluation were retained in the new proposed standard, but the mandated number of tests was increased to three, as illustrated in Figure 7. For each length test, a plate target is placed at a location close to the IUT and is measured by both the IUT and RI (e.g., position A in Figure 7). The target is then moved away from the IUT to a second location, collinear with the IUT and the previous location (e.g., any position B through D in Figure 7). The target is scanned again at this location by both the IUT and RI. The scan data is processed to obtain the test length and the RI measurements are processed to obtain the reference length. The positioning of the RI with respect to the targets and IUT depends on the design of the target [8]. This process is repeated to perform tests for two other lengths. 


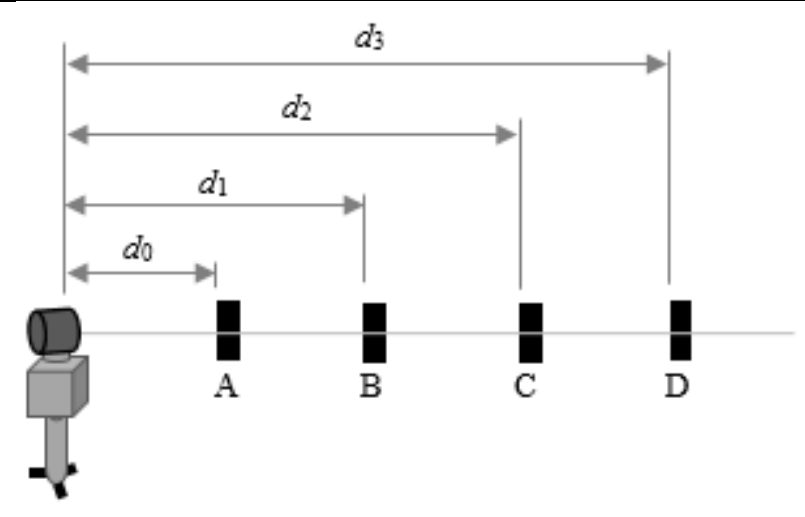

Figure 7: Relative-range tests

Since the non-ranging point-to-point distance tests use spheres, using spheres and other geometries for relative-range tests was explored [14]. Though these targets performed acceptably for a single distance, they involve systematic errors when used at multiple distances. The spherical geometry introduces a range dependent error in the ranging direction due to the squishing/flaring effect [8,9] which does not reflect the actual performance of the instrument. For this reason, planar targets were retained as the targets to be used in these tests.

\subsection{Data processing}

Data processing methods used to obtain the derived points in the proposed standard may introduce errors that may be incorrectly attributed to the instrument. The two geometries that are used in the proposed standard are spheres and planes. The methods to obtain the derived point for both the geometries involve a cleanup procedure where the data

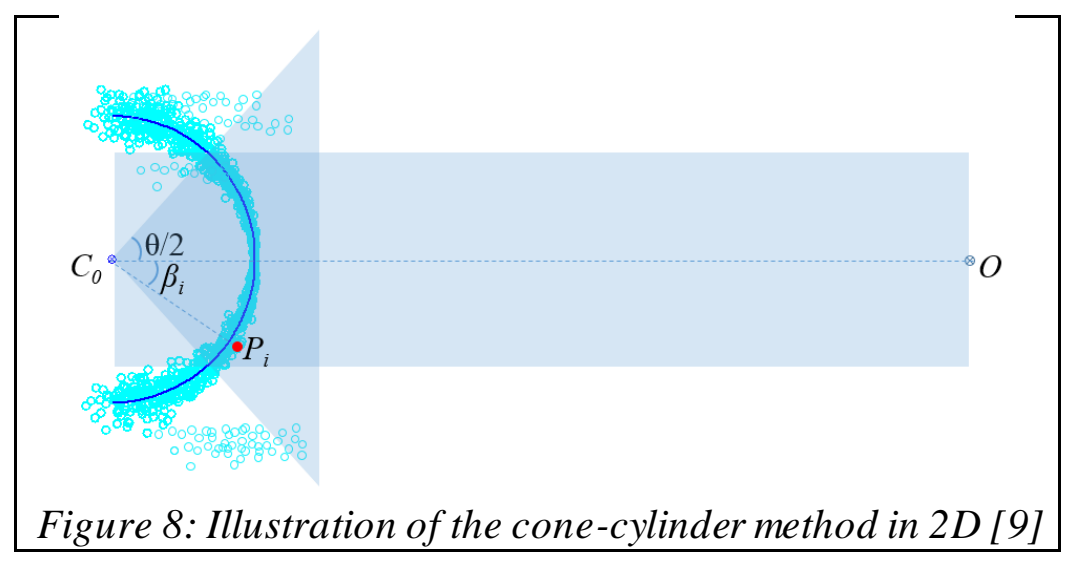
corresponding to the object of interest (plane or sphere) is separated from its surroundings.

The procedure for obtaining the derived point of a flat plate is the same as described in ASTM E2938-15[3]. The procedure to obtain the derived point of a sphere is described in detail by Rachakonda et al. [9] and was tested against thousands of datasets from several instruments. This process uses an iterative cone-cylinder exclusion routine, illustrated in Figure 8. In this method, an initial center of the sphere is calculated and is then refined by iteratively excluding the points outside a conical and cylindrical region constructed around the sphere data. The detailed procedures of obtaining the derived point is beyond the scope of this paper, however the recommendations from the studies by Rachakonda et al. [9] have been incorporated into the proposed standard. Some of these recommendations for a sphere target are listed below:

a) Calibration: The radius of the sphere target along with its uncertainty shall be determined through a calibration procedure. 
b) Target size: The minimum sphere target size should be specified by the IUT manufacturer, and shall be sufficient to yield a minimum of 300 points after point selection process (cone-cylinder method) described in the proposed standard.

c) Circularity: The circularity of the sphere target shall not exceed $20 \%$ of the smallest point-to-point distance maximum permissible error (MPE) of the IUT.

d) Segmentation: The derived-point coordinate of the sphere is refined through a "conecylinder" method described in the proposed standard.

\subsection{Metrics for evaluation}

To evaluate the instrument, the datasets are reduced to derived points and certain metrics are proposed for each of these tests. These metrics are described next and are in a coordinate system that is coincident with the IUT's origin.

\subsubsection{Two-face tests}

The two-face error is the root-sum-square of the components along the azimuth and elevation directions of the target and is given by:

$$
E_{t w o-f a c e}=r_{1} \sqrt{\left(\phi_{1}-\phi_{2}\right)^{2}+\left[\left(\theta_{1}-\theta_{2}\right) \cos \left(\phi_{1}\right)\right]^{2}}
$$

where, $\left(r_{1}, \theta_{1}, \phi_{1}\right)$ is the derived-point of the target measured in the frontsight and $\left(r_{2}, \theta_{2}, \phi_{2}\right)$ is the derived point of the target measured in the backsight (both in a spherical coordinate system).

\subsubsection{Point-to-point distance tests}

For all the point-to-point distance tests (both non-ranging and the relative-range tests), the metric adopted is the error in the measured distance. This is the difference between the distance measured by the IUT and that measured by the RI and is given by:

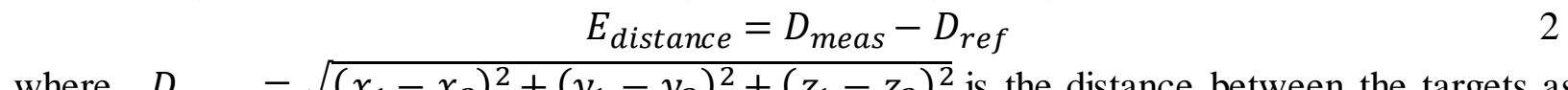
Where, $D_{\text {meas }}=\sqrt{\left(x_{1}-x_{2}\right)^{2}+\left(y_{1}-y_{2}\right)^{2}+\left(z_{1}-z_{2}\right)^{2}}$ is the distance between the targets as
measured by the IUT and $D_{\text {ref }}=\sqrt{\left(X_{1}-X_{2}\right)^{2}+\left(Y_{1}-Y_{2}\right)^{2}+\left(Z_{1}-Z_{2}\right)^{2}}$ is the distance between the same targets as measured by the RI. Here, $\left(x_{1}, y_{1}, z_{1}\right) \&\left(x_{2}, y_{2}, z_{2}\right)$ are the coordinates of the derived-point of the targets measured by the IUT and $\left(X_{1}, Y_{1}, Z_{1}\right) \&\left(X_{2}, Y_{2}, Z_{2}\right)$ are the coordinates of the derived point of the same target measured by the RI.

For the relative-range tests, the dispersion of the residuals is also calculated for reporting. This value is the root-mean-square of the residual distance $\left(q_{i}\right)$ to the plane representing the target, calculated using $N$ points, and is given by:

$$
R M S=\sqrt{\frac{\sum_{i=1}^{N} q_{i}^{2}}{N}}
$$

\section{SUMMARY AND CONCLUSION}

The working group within the ASTM E57 subcommittee on Test Methods developed a new standard for evaluating the ability of spherical coordinate, 3D imaging systems to measure derived point to derived point distance within the instrument work volume. The working group consisted of instrument manufacturers, stakeholders, subject matter experts and researchers. Many test methods were considered and tested rigorously with many TLSs to ensure that the tests can be reasonably performed and objective metrics can be calculated. Several papers [5-14] have been 
published by NIST researchers which document the issues regarding TLS performance evaluation. Decisions were made on many issues such as: a) number and types of test methods, b) targets to be used for these tests and c) methods and considerations to process the data. This paper gives an overview of all the work that was performed that culminated in the proposed standard that is presently being balloted in the ASTM E57 committee.

\section{ACKNOWLEDGEMENTS}

The authors would like to thank Mr. Luc Cournoyer of NRC (Canada) and all the participants of the ASTM E57.02 working group who contributed to this effort.

\section{REFERENCES}

[1] Cheok, G., "NISTIR 7266: Proceedings of the 2nd NIST LADAR performance evaluation workshop - March 15 - 16, 2005"

[2] Cheok, G., "NISTIR 7357: Proceedings of the 3rd NIST workshop on the performance evaluation of 3D imaging systems - March 2 - 3, 2006"

[3] ASTM E2938-15: "Standard test method for evaluating the relative-range measurement performance of 3D imaging systems in the medium range" https://www.astm.org/cgibin/resolver.cgi?E2938-15 (Retrieved May 23, 2017)

[4] ASTM E57.02 - "Test Methods", WK 43218: New test methods for evaluating the performance of medium-range, spherical coordinate 3-D imaging systems for point-to-point distance measurements.

[5] Muralikrishnan, B., Rachakonda, P., Shilling, M., Lee, V., Blackburn, C., Sawyer, D., Cheok, G., Cournoyer, L., "NISTIR 8152: Report on the May 2016 ASTM E57.02 instrument runoff at NIST, Part 1 - Background information and key findings".

[6] Muralikrishnan, B., Rachakonda, P., Shilling, M., Lee, V., Blackburn, C., Sawyer, D., Cheok, G., Cournoyer, L., "Report on the May 2016 ASTM E57.02 instrument runoff at NIST, Part 2 - NIST realization of test procedures and uncertainties in the reference lengths".

[7] Muralikrishnan, B., Shilling, M., Rachakonda, P., Ren, W., Lee, V., Sawyer, D., "Toward the development of a documentary standard for derived-point to derived-point distance performance evaluation of spherical coordinate 3D imaging systems", Journal of Manufacturing Systems, Vol. 37, Part 2, October 2015 pp550-557

[8] Rachakonda, P., Muralikrishnan, B., Shilling, M., Cheok, G., Lee, V., Blackburn, C., Everett, D., "Targets for relative range error measurement of 3D imaging systems", Journal of the CMSC, Vol. 12, No. 1, Spring 2017

[9] Rachakonda, P., Muralikrishnan, B., Cournoyer, L., Cheok, G., Lee, V., Shilling, M., Sawyer, D., "Methods and considerations to determine sphere center from terrestrial laser scanner point cloud data", (submitted to Measurement Science and Technology in March 2017)

[10] Muralikrishnan, B., Rachakonda, P., Lee, V., Shilling, M., Sawyer, D., Cheok, G., Cournoyer, L., "Relative range error evaluation of terrestrial laser scanners using a plate, a 
sphere, and a novel dual-sphere-plate target", (accepted for publication by Measurement in June 2017)

[11] Muralikrishnan, B., Ferrucci, M., Sawyer, D., Gerner, G., Lee, V., Blackburn, C., Phillips, S., Petrov, P., Yakovlev, Y., Astrelin, A., Milligan, S., and Palmateer, J., "Volumetric performance evaluation of a laser scanner based on geometric error model", Precision Engineering, 40, pp. 139-150, 2015

[12] Wang, L., Muralikrishnan, B., Wang, L., Rachakonda, P., Sawyer, D., "Determining geometric error model parameters of a terrestrial laser scanner through Two-face, Lengthconsistency, and Network methods", Measurement Science and Technology, Vol. 28, No.6, 2017.

[13] Rachakonda, P., Muralikrishnan, B., Lee, V., Sawyer, D., Phillips, S., and Palmateer, J., “A method of determining sphere center to center distance using laser trackers for evaluating laser scanners", Proceedings of the Annual Meeting of the ASPE; Boston, MA; 2014.

[14] Rachakonda, P., Muralikrishnan, B., Shakarji, C., Lee, V., Sawyer, D., "Evaluation of the range performance of laser scanners using non-planar targets", Proceedings of the Annual Meeting of the ASPE; Austin, TX; 2015.

Cheok, Geraldine; Muralikrishnan, Balasubramanian; Rachakonda, Prem; Sawyer, Daniel; Shilling, Katharine. 


\title{
A Photonic MEMS Accelerometer with a Low-Finesse Hemispherical Microcavity Readout
}

\author{
Yiliang Bao, Feng Zhou, Thomas W. LeBrun, and Jason J. Gorman \\ National Institute of Standards and Technology, Gaithersburg, MD USA \\ gorman@nist.gov
}

\begin{abstract}
This paper describes the design, fabrication, and testing of a photonic MEMS accelerometer that uses a hemispherical microcavity to transduce the motion of the proof mass. The cavity design provides stable operation that is relatively tolerant of misalignment errors. The prototype sensor is shown to have optical resonances that closely match the expected Airy function model.
\end{abstract}

Keywords-Hemispherical microcavity; Fabry-Pérot; accelerometer; photonic MEMS.

\section{INTRODUCTION}

The design and testing of a new photonic MEMS accelerometer that uses Fabry-Pérot interferometry (FPI) to transduce acceleration will be described. FPI is a compelling candidate for transduction in accelerometers since it is highly accurate and sensitive and requires minimal surface area compared to capacitive, piezoresistive, and piezoelectric techniques. FPI has been studied for MEMS accelerometers by several groups [1-4]. However, these designs have focused on plane-parallel cavities, which suffer from poor optical coupling stability and lower effective finesse. The design presented in this paper uses a hemispherical cavity with high quality mirrors, resulting in significantly more stable sensing compared to the plane-parallel cavities found in previous work. The fabrication process for this design allows for the inclusion of high reflectivity coatings for optical cavities with high finesse but only uncoated low-finesse cavities will be discussed here.

\section{ACCELEROMETER DESIGN}

The basic accelerometer concept was previously described in [5] and is shown in Fig. 1. The accelerometer consists of two silicon chips that are fabricated separately and then bonded together to build the sensor. The chips are assembled on a standard fiber-optic mount to provide simple optical coupling into the cavity. The first chip contains a concave micromirror and the other chip contains a proof mass, which is suspended by silicon nitride beams along both the top and bottom edges. This structural design ensures that the higher order modes (rocking modes) are well separated from the fundamental mode (piston mode). Finite element analysis (FEA) shows that the resonance frequency of the first higher mode is at least 10 times higher than that of the fundamental mode, as shown in Fig. 2.

A variety of suspended proof masses have been fabricated with lengths ranging from $1 \mathrm{~mm}$ to $5 \mathrm{~mm}$. To simplify the
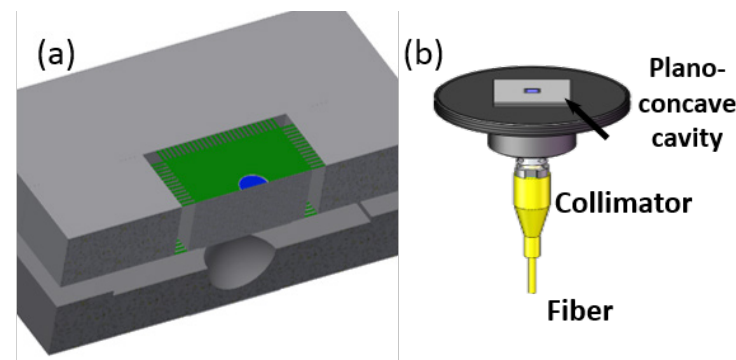

Fig. 1. Accelerometer design. (a) Exploded cross-sectional view showing the suspended proof mass and hemispherical microcavity, (b) fiber-optic sensor packaging.

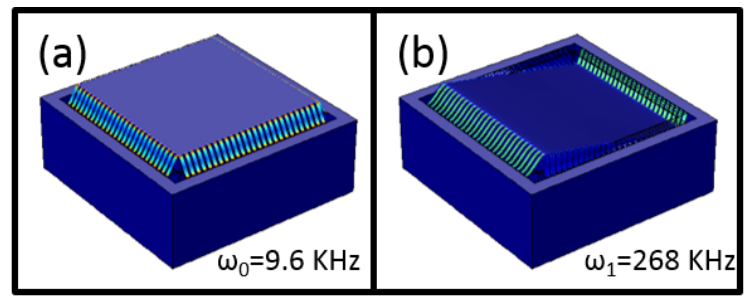

Fig. 2. Proof mass vibrational modes calculated using COMSOL Multiphysics. (a) Fundamental piston mode, (b) first rocking mode.

parameter variations, all designs use a $20 \mu \mathrm{m}$ beam width, 1.5 $\mu \mathrm{m}$ beam thickness, and $500 \mu \mathrm{m}$ proof mass thickness. The fundamental resonant frequency of the proof mass is set by varying the beam length from $40 \mu \mathrm{m}$ to $110 \mu \mathrm{m}$ and the proof mass width.

\section{FABRICATION}

The hemispherical mirror is etched using HNA etching [5] and a silicon nitride hard mask with circular apertures. The radius of curvature and depth of the fabricated hemispherical mirrors are optimized to achieve the best mode matching insensitivity to fabrication variations while achieving a reasonable reduction in the power density on the mirrors, thereby mitigating stability issues related to laser heating. This is done by tuning the ratio of the depth to the radius of curvature to approximately 0.5 . A typical mirror is shown in Fig. 3. The surface roughness is approximately $1 \mathrm{~nm}$ RMS, characterized using both an optical profilometer and AFM. The fabrication process for the suspended proof mass is shown in Fig. 4, which requires a complex etch that combines DRIE and $\mathrm{KOH}$ to release the proof mass and beams. A typical suspended proof mass is shown in Fig. 5. 


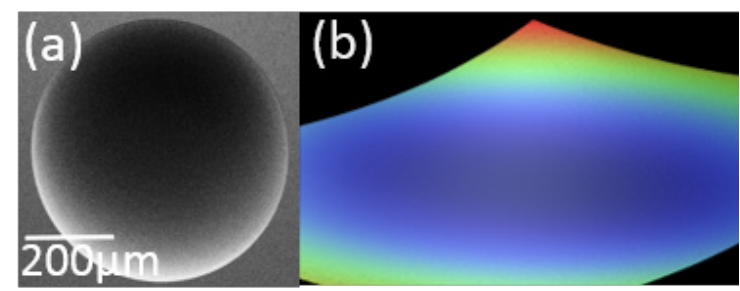

Fig. 3. Etched hemispherical mirror: (a) SEM top view, (b) 3D image from optical profilometer.

(a)

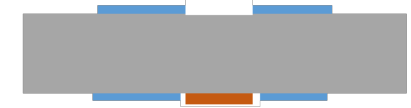

(b)

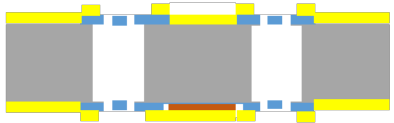

(c)

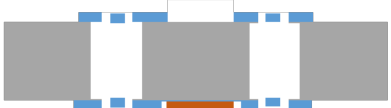

$\mathrm{Si}$

$\mathrm{Si}_{3} \mathrm{~N}_{4} \quad \square \mathrm{Ti} / \mathrm{Au}$

AR

Fig. 4. Fabrication process for the suspended proof mass: (a) low-stress $\mathrm{Si}_{3} \mathrm{~N}_{4}$ is patterned on silicon wafer and AR coating is deposited using a liftoff process, (b) Au mask is patterned using liftoff process for masking during $\mathrm{KOH}$ etch. The beams are patterned using RIE for $\mathrm{Si}_{3} \mathrm{~N}_{4}$. DRIE is used to etch through the $\mathrm{Si}$ and $\mathrm{KOH}$ releases the beams. (c) Au mask is removed.

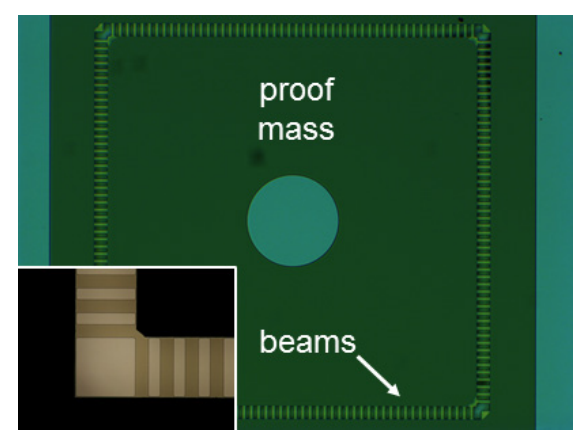

Fig. 5. Top view of a fabricated suspended proof mass where the inset shows a close-up of the beams.

\section{RESULTS}

The two chips have been bonded using an acrylic adhesive but future sensors will be assembled using fusion bonding, which will provide better thermal stability. The bonded chip is aligned on the fiber coupled package, as shown in Fig. 1(b), to form a portable accelerometer. The motion of the proof mass is measured by detecting shifts in the optical resonances of the cavity. A tunable laser is used to measure the transmission signal of the optical cavity as a function of the laser wavelength. Figure 6 shows the response of one optical cavity, where the transmitted and reflected signals are fit to an Airy function in order to extract the displacement information of the cavity length. On resonance, the cavity length change, $d z_{m}$, is translated into a wavelength change, $d \lambda$, as $d z_{m} / z_{m}=d \lambda / \lambda$. By evaluating the optical spectrum at the Airy function's point of maximum slope, we can determine the linearized transmission displacement sensitivity. Preliminary results for this cavity yield displacement and acceleration sensitivity of $1.8 \mathrm{mV} / \mathrm{nm}$ and $4.86 \mathrm{mV} / \mathrm{g}$, respectively. Additional performance results for the accelerometer will be reported in the final presentation, including noise floor and shaker tests.
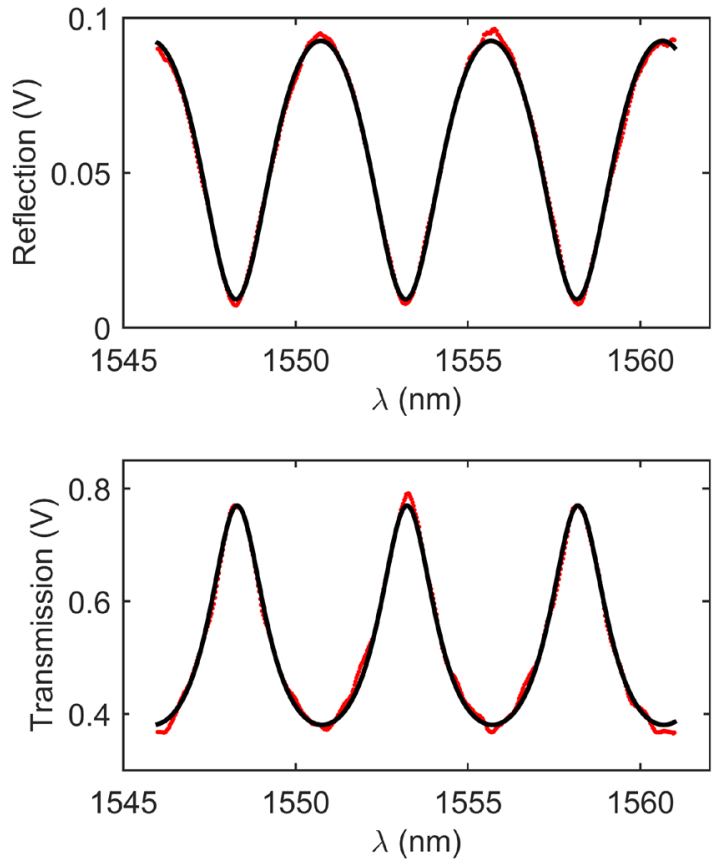

Fig. 6. Reflected and transmitted intensity of the hemispherical optical cavity as a function of laser wavelength. Red dots are the measured signal and the black lines are the fits to the standard Airy function model.

\section{ACKNOWLEDGMENT}

This research was performed in part in the NIST Center for Nanoscale Science and Technology Nanofab.

\section{REFERENCES}

[1] R.L. Waters and M.E. Aklufi, "Micromachined Fabry-Perot interferometer for motion detection," Appl. Phys. Lett., vol. 81, pp. 3320-3322, 2002.

[2] M.A. Perez and A.M. Shkel, "Design and demonstration of a bulk micromachined fabry-pérot $\mu$ g-resolution accelerometer," IEEE Sens. J, vol. 7, pp. $1653-1662,2007$.

[3] M.D. Pocha, G.A. Meyer, C.F. McConaghy, S.P. Swierkowski, J.D. Wolfe, "Miniature accelerometer and multichannel signal processor for fiberoptic Fabry-Pérot sensing," IEEE Sens. J., vol. 7, pp. 285-292, 2007.

[4] E. Davies, D.S. George, M.C. Gowera, and A.S. Holmes, "MEMS FabryPérot optical accelerometer employing mechanical amplification via a Vbeam structure," Sens. Actuators, A, vol. 215, pp. 22-29, 2014.

[5] Y. Bao, et al., "An optomechanical accelerometer with a high-finesse hemispherical optical cavity," IEEE Int. Symp. Inertial Sensors and Systems, Laguna Beach, CA, 2016, pp. 105-108. 


\title{
Bound Electron Nonlinearity Beyond the Ionization Threshold
}

\author{
J. K. Wahlstrand, ${ }^{1,2}$ S. Zahedpour, ${ }^{1}$ and H. M. Milchberg ${ }^{1, *}$ \\ ${ }^{1}$ Institute for Research in Electronics and Applied Physics, University of Maryland, College Park, MD 20742 \\ ${ }^{2}$ Engineering Physics Division, National Institute of Standards and Technology, Gaithersburg, MD 20899 \\ Author e-mail address: milch@umd.edu
}

\begin{abstract}
We measure the residual bound electron nonlinear polarizability well above the ionization threshold in a range of gases, and find that it scales approximately linearly with laser intensity.

OCIS codes: (020.4180) Multiphoton processes; (260.3230) Ionization; (190.3270) Kerr effect
\end{abstract}

Quantitative understanding and accurate simulations of femtosecond filamentation require a well understood optical nonlinearity, including the optical Kerr effect and the ionization induced plasma response. In the noble gases and air, the Kerr effect, which includes bound electronic, rotational, and vibrational contributions [1,2], has been carefully measured. In contrast, knowledge of the ionization-induced nonlinearity relies on relatively indirect measurements, performed in very low pressure gas samples [3,4], which have no time resolution. In addition, there remain persistent questions about the nature of the nonlinearity at ionizing intensities: is there a significant higher-order Kerr effect [5]? Here, we present all-optical absolute measurements of the optical nonlinearity in $\mathrm{Ar}, \mathrm{Kr}, \mathrm{Xe}, \mathrm{N}_{2}$, and $\mathrm{O}_{2}$ at intensities ranging from well below to well above the ionization threshold.

Our measurement technique, which uses chirped supercontinuum pulses to probe the nonlinear phase shift induced by a pump pulse as both propagate through a thin gas target [6], has recently been updated to yield $2 \mathrm{D}+1$ nonlinear phase shift information (2D transverse space plus time). Typical results are shown in Fig. 1. We observe a positive phase shift during the early portion of the pump pulse due to the Kerr effect and an accumulating negative response from ionization.
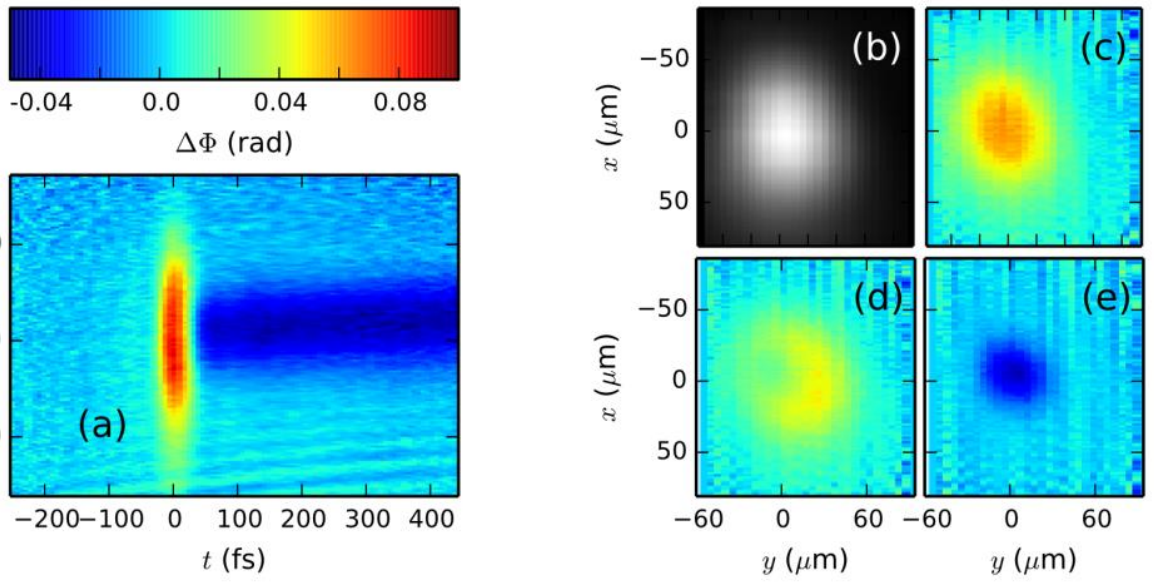

Fig. 1. Experimental results in Ar for peak pump intensity of $95 \mathrm{TW} / \mathrm{cm}^{2}$ and pulsewidth $42 \mathrm{fs}$. (a) Measured spatiotemporal phase shift $\Delta \Phi\left(x, y_{0}, t\right)$. (b) An image of the pump spot at the gas target. (c) The phase shift $\Delta \Phi(x, y, t=-14 \mathrm{fs})$, showing mostly the Kerr response. (d) Phase shift $\Delta \Phi(x, y, t=+25 \mathrm{fs})$, showing the Kerr response on the wings and the growing plasma contribution in the center of the beam. (e) Phase shift $\Delta \Phi(x, y, t=+100 \mathrm{fs})$, showing the dominant plasma contribution after the pump pulse. The peak of the pump pulse defines zero for the $x, y$, and $t$ coordinates.

Our results are qualitatively similar to what we have reported previously at high intensities [2]. However, those measurements showed effects from pump and probe refraction, and degraded time resolution [6]. Here, we use a larger (x2.5) pump spot, we have optimized the time resolution by reducing the probe chirp and improving the spectral resolution [6], and we measure the full 2D+1 phase shift by measuring the phase shift across the entire probe spot. The latter allows us to Fresnel propagate the experimental results to account for slight misalignment of the imaging system.

Under the assumption that probe refraction can be neglected, we can assign an intensity value to each (x,y) point. Conversion of the measured phase shift to the nonlinear refractive index is possible because the interaction length through the target is known through a separate interferometry measurement [1]. The ionization yield as a function of peak intensity extracted from our measurements is shown in Fig. 2, as is a fit of the data to $\sigma I^{m}$ (dashed lines). 

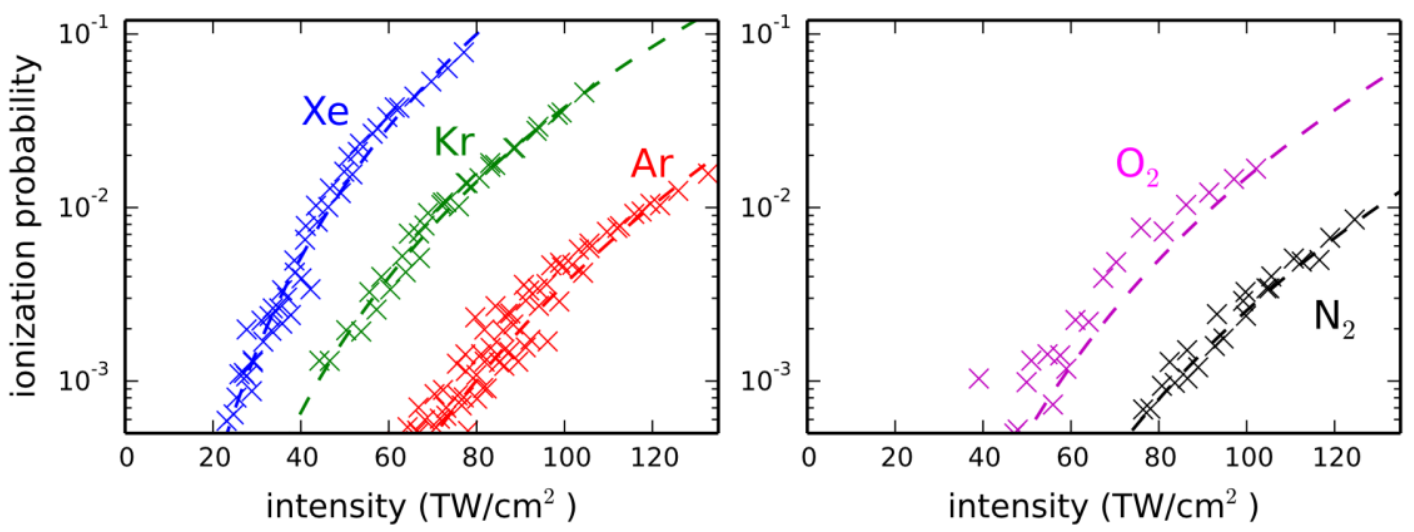

Fig. 2. Ionization probability vs. intensity in $\mathrm{Ar}, \mathrm{Kr}, \mathrm{Xe}, \mathrm{N}_{2}$, and $\mathrm{O}_{2}$. Ionization yield (x) as a function of peak intensity for (a) $\mathrm{Ar}$ (red), $\mathrm{Kr}$ (green), $\mathrm{Xe}$ (blue); (b) $\mathrm{N}_{2}$ (black) and $\mathrm{O}_{2}$ (magenta).

The phase shift during the pump pulse has contributions from both the Kerr effect and ionization. The time dependent phase shift is shown in Fig. 3 for Ar. The total phase shift is consistent with the simple addition of these two contributions. Also shown in the figures are fits of the data to $\Delta n(t)=\Delta n_{K} e^{-t^{2} / \tau^{2}}+\Delta n_{p} \operatorname{erf}\left[t /\left(\tau / m^{1 / 2}\right)\right]$. It is seen that the approximately linear dependence of $\Delta n_{K}$ on intensity measured at low intensities [2] continues at high intensity. The Kerr component may actually be slightly higher than the low intensity slope. Further investigations of this effect are underway.
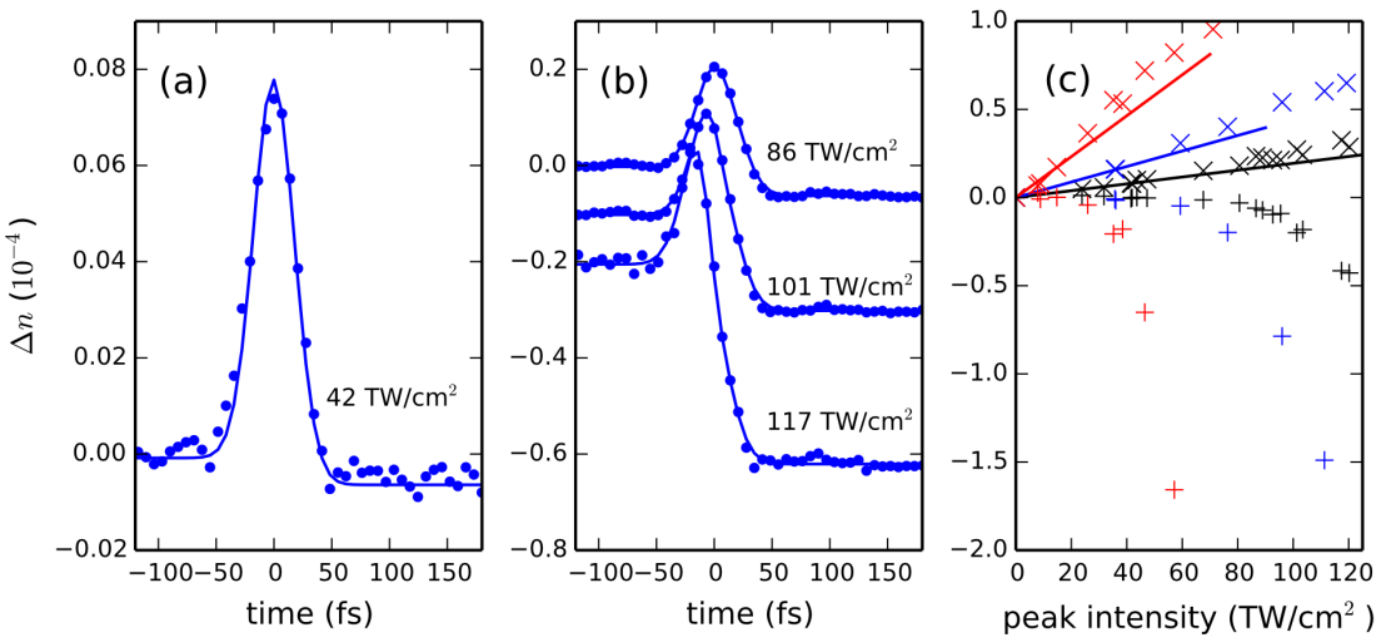

Fig. 3. Response during pump pulse. (a) The nonlinear refractive index as a function of time for peak intensity of $42 \mathrm{TW} / \mathrm{cm}^{2}$, below the threshold for ionization, and a fit to a Gaussian with FWHM 42 fs. (b) Nonlinear refractive index as a function of time and fits to the standard model (Kerr effect plus ionization) for Ar. The curves have been offset vertically for clarity. (c) Plot of Kerr index change $(x)$ and plasma index change (+) from fits as a function of intensity in $\mathrm{Ar}, \mathrm{Kr}$, and $\mathrm{Xe}$.

[1] J. K. Wahlstrand, Y.-H. Cheng, and H. M. Milchberg, "Absolute measurement of the transient optical nonlinearity in $\mathrm{N}_{2}, \mathrm{O}_{2}, \mathrm{~N}_{2} \mathrm{O}$, and $\mathrm{Ar}$," Phys. Rev. A 85, 043820 (2012).

[2] J. K. Wahlstrand, Y.-H. Cheng, and H. M. Milchberg, "High field optical nonlinearity and the Kramers-Kronig relations," Phys. Rev. Lett. 109, 113904 (2012).

[3] S. F. J. Larochelle, A. Talebpour, and S. L. Chin, “Coulomb effect in multiphoton ionization of rare-gas atoms,” J. Phys. B: At. Mol. Opt. Phys. 31, 1215-1224 (1998).

[4] A. Talebpour, J. Yang, and S. L. Chin, "Semi-empirical model for the rate of tunnel ionization of $\mathrm{N}_{2}$ and $\mathrm{O}_{2}$ molecule in an intense Ti:sapphire laser pulse," Opt. Commun. 163, 29-32 (1999).

[5] M. Richter, S. Patchkovskii, F. Morales, O. Smirnova, and M. Ivanov, "The role of the Kramers-Henneberger atom in the higher-order Kerr effect," New J. Phys. 15, 083012 (2013).

[6] J. K. Wahlstrand, S. Zahedpour, and H. M. Milchberg, "Optimizing the time resolution of supercontinuum spectral interferometry," J. Opt. Soc. Am. B 33, 1476-1481 (2016). 


\title{
INTEGRATED OPTICAL WAVEGUIDES FOR IN SITU MICROFLOW MEASUREMENT
}

\author{
James R. Hands and Gregory A. Cooksey* \\ Sensor Science Division, National Institute of Standards and Technology, Gaithersburg, MD, USA
}

\begin{abstract}
The need for quantitative microscale chemical and biological experiments has resulted in the increasing utilization of microfluidics with integrated sensors, allowing such architectures to synergistically enhance function and performance [1]. This work focuses on the design and characterization of optofluidic devices that easily integrate optical fibers into microfluidic waveguides. We demonstrate optical components that transmit and collect light from substances in microflows, such as fluorescent liquids. Optimization of the components for splitting light into multiple paths, focusing light into the microchannel, and collecting fluorescence emission while excluding excitation light is described. An integrated system is demonstrated for real-time and continuous flow-monitoring using caged fluorophores. These tools provide new methods to probe dynamic fluid properties and to measure samples using microfluidic cytometry.
\end{abstract}

KEYWORDS: Optofluidics, microfluidics, fluorescence, flow meter

\section{INTRODUCTION}

Microfluidic devices are widely used for investigations in chemistry, biology, and physics because they enable unique fluid transport regimes, dynamic control of small fluid volumes (femtoliters to nanoliters), and highthroughput experiments. Integration of on-chip optical components provides even greater benefit to miniaturization, portability, and speed of sensitive and multimodal measurement capabilities [2]. We aim to incorporate optical sensing elements into microfluidic devices in order to accurately and reproducibly measure microflows and characterize the optical properties of materials in flow using sensitive chemical and biological assays. Application areas include cytometry, drug delivery, and diagnostic assays. A key objective is to create optofluidic devices that can perform rapid, high quality measurements in both industrial and distributed/point-of-use applications.

\section{EXPERIMENTAL}

(Identification of commercial products does not imply recommendation/endorsement by the National Institute of Standards and Technology (NIST); materials/equipment used may not necessarily be best for purpose). Photolithography was conducted at the Center of Nanoscale Technology (CNST) at NIST. Chrome masks (Heidelberg Instruments DWL-2000) were made to create topographic features onto SU8 (Microchem) masters (Suss MicroTech MA8). Optofluidic devices were cast in poly(dimethylsiloxane) (PDMS) (Sylgard 184, Dow Corning) against masters [3-4]. Optical waveguides were filled with optical adhesive (NOA 88, Norland) followed by insertion of optical fibers (Thor Labs) and UV curing. A $10 \mathrm{~mW}$ tungsten light source and fiber-coupled LEDs (ThorLabs) were used with a power meter (2936-R, Newport) to deliver and measure light, respectively. Flows were monitored in series with the syringe pump with a commercial flow meter (Sensirion). Flows within the optofluidic devices were visualized with fluorescein and CMNB-caged fluorescein (Sigma Aldrich).
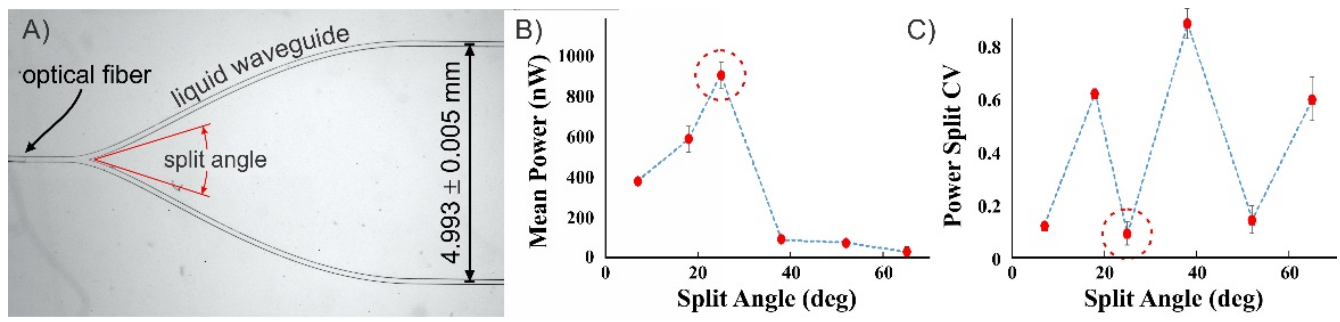

Figure 1: A) Image of an optofluidic device showing optical fiber coupled into a bifurcating waveguide that crosses a microfluidic channel (vertical) at two points. B) The mean power achieved at various split angles. C) The variation in power through the branches of the split normalized to the mean. Dashed red circles indicate the best conditions for power and coefficient of variation (CV). We show means and standard deviations from measurements of 3 replicate devices. 


\section{RESULTS AND DISCUSSION}

Using a single light source to interrogate multiple points along a flow path is advantageous for simplifying fabrication and aiding reproducibility. A range of branching angles $\left(5^{\circ}\right.$ to $\left.65^{\circ}\right)$ was tested to optimize power delivered through the split. Both total power (mean) and power variation between the branches were optimized at a $25^{\circ}$ split angle, where mean power of $900 \mathrm{nW}$ was observed with deviation of less than $5 \%$ between the branches (Figure 1).

To focus light from the waveguides across the microchannel, lenses with varying radii of curvature (Rc) were placed on the ends of the waveguides (flat [no Rc], 250, 175, 100, 75, and $50 \mu \mathrm{m}$; smaller curvatures were not practical given the width of the waveguides in these tests). A lens with $50 \mu \mathrm{m}$ Rc led to the highest power transmission from a fiber-coupled light-emitting diode $(11 \mu \mathrm{W}$ of the $22 \mu \mathrm{W}$ input) across the flow channel, which is in good agreement with what is expected from refractive index differences between NOA 88 (1.56) and PDMS (1.42). The shape of the illumination was imaged by exciting fluorescein in the flow channel with $488 \mathrm{~nm}$ light (Figure 2). We optimized collection of emitted light $(520 \mathrm{~nm}$ ) from fluorescein by placing a collection waveguide at an angle of 35 degrees from the nominal excitation direction, which is in the expected "shadow" of excitation light reflected from the wall of the microchannel.
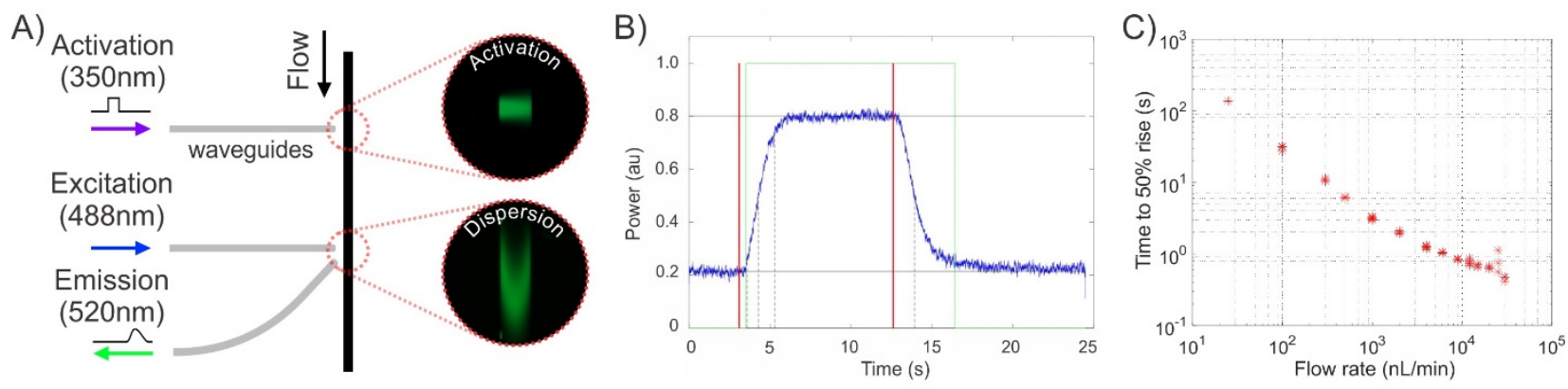

Figure 2: Use of optofluidic components to make a flow meter. A) CMNB caged fluorescein in the flow is activated by crossing a UV light pulse. A fluorescence image shows the activated "stripe" of fluorescein. Dispersion of the fluorescent stripe is shown by the fluorescence image at a point down the flow channel. Fluorescence emission is excited and collected by a pair of waveguides at a fixed distance downstream. B) A recording of the measured fluorescence by the power meter is shown following a long (saturated) UV activation upstream (between the red marks). C) Measurement of the time for the fluorescence to reach $50 \%$ of maximum is shown for various flow rates (triplicate for each flow rate).

We demonstrate use of the photonic elements to realize a continuous flow meter (Figure 2). Ultraviolet light delivered to an upstream waveguide was used to activate caged fluorescein, which "marks" the flow. Dispersion of the dye downstream is measured by collecting the emitted fluorescence when the dye crosses a waveguide carrying excitation light (488nm). Measurements were found to be reproducible with CV's lower than $9 \%$ over approximately 3 orders of magnitude in flow, from $20 \mathrm{~nL} / \mathrm{min}$ to about $20 \mu \mathrm{L} / \mathrm{min}$.

\section{CONCLUSION}

We have demonstrated optofluidic devices that can efficiently deliver and collect light across microfluidic channels. These devices were combined to create a microflow meter. These systems are useful for measuring dynamic fluid properties and provide new routes to characterization of particle and cells in microfluidic cytometry.

\section{REFERENCES}

[1] M. Ahrens, St. Klein, B. Nestler, C. Damiani. Measurement and Science Technology. 25, pp 1-9, 2014

[2] X. Fan, I. M. White. Nature Photonics, 5, pp 591-597, 2011

[3] J. C. McDonald, D. C. Duffy, J. R. Anderson, et al. Electrophoresis. 21(1), pp 27-40, 2000

[4] G. A. Cooksey, C. G. Sip, A. Folch. Lab Chip. 9(3), pp 417-426, 2009

\section{CONTACT}

${ }^{*}$ Gregory A. Cooksey; gregory.cooksey@nist.gov 
AVS 2016; Thin Film Division

TF13 Self-assembled Monolayers, Polymer Brushes and Organic Multi-layers

Integration of Redox-Active Diruthenium-based Molecular Layer onto Electrodes for Memory Device Applications

\author{
S. Pookpanratana, ${ }^{1}$ H. Zhu,,${ }^{1,2}$ J. W. F. Robertson, ${ }^{1}$ S. N. Natoli, ${ }^{3}$ E. G. Bittle, ${ }^{1}$ C.A. Richter, ${ }^{1}$ T. \\ Ren, ${ }^{3}$ Q. Li, ${ }^{2}$ and C. A. Hacker ${ }^{1}$ \\ ${ }^{1}$ Engineering Physics Division, National Institute of Standards and Technology \\ ${ }^{2}$ Department of Electrical and Computer Engineering, George Mason University \\ ${ }^{3}$ Department of Chemistry, Purdue University
}

Attaching and integrating electrochemically-active molecules to a variety of different surfaces is of importance for applications in catalysis, memory devices, and molecular electronics. With the increasing demand for personal electronics, growth in Flash-based memory has increased dramatically. However, the dimensional scaling of memory components faces many critical material limitations. A critical component to the memory device is the floating gate or charge trapping layer. To scale the charge trapping layer to nanometer dimensions, one approach is to use a discrete charge storage layer that is based on organic molecules. ${ }^{1,2,3}$ Reduction-oxidation (redox) active organic molecules hold potential for memory devices due to their nanoscale dimensions, potential for high charge density, and synthetic flexibility that could be tailor-made for specific electronic functionality.

Here, we investigated the potential of diruthenium-bearing organometallic molecules as the charge trapping layer for memory devices. Diruthenium-bearing organometallic molecules display multiple redox states, ${ }^{4}$ which makes them ideal to incorporate within non-volatile memory devices. Monolayer assembly is performed in a stepwise fashion by first forming azide-terminated monolayer on $\mathrm{SiO}_{2}$ by using azidoundecyl trimethoxysilane followed by a $\mathrm{Cu}$-catalyzed azidealkyne cycloaddition click reaction to attach diruthenium $\left(\mathrm{Ru}_{2}\right)$ compounds (note: $\mathrm{SiO}_{2}$ serves as the tunneling layer). ${ }^{5}$ Infrared spectroscopy and X-ray photoelectron spectroscopy confirmed the $\mathrm{Ru}_{2}$ attachment. Ultraviolet photoelectron spectroscopy identified the occupied electronic levels of the hybrid organic-inorganic surfaces before and after click reaction. Voltammetric measurements on $\mathrm{Ru}_{2}$-terminated $\mathrm{SiO}_{2} / \mathrm{Si}$ and $\mathrm{Au}$ electrodes confirm that the $\mathrm{Ru}_{2}$ is still electrochemically-active with accessible electronic states integrated on both surfaces.

To complete the memory capacitor device, an $\mathrm{Al}_{2} \mathrm{O}_{3}$ layer (serving as a charge blocking layer) was deposited by atomic layer deposition over the molecular layer followed by a metal Pd gate. The impact of different $\mathrm{Ru}_{2}$ compounds on the electronic structure and electrochemical properties of the electrodes and properties of the memory devices will be compared. Our results will provide future design considerations and limitations for molecular-integrated memory devices.

1. T. Shaw et al., IEEE T. Electron. Dev. 58 (3), 826-834 (2011).

2. D. Beckmeier and H. Baumgärtner, J. Appl. Phys. 113 (4), 044520 (2013).

3. H. Zhu, et al., Appl. Phys. Lett. 103 (5), - (2013).

4. W.-Z. Chen and T. Ren, Inorg. Chem. 45 (20), 8156-8164 (2006).

5. S. Pookpanratana, et al., Langmuir 30 (34), 10280-10289 (2014).

Bittle, Emily; Hacker, Christina; Li, Qiliang; Natoli, Sean; Pookpanratana, Sujitra; Ren, Tong; Richter, Curt; Robertson, Joseph; Zhu, Hao.

"Integration of Redox-Active Diruthenium-based Molecular Layer onto Electrodes for Memory Device Applications."

Paper presented at AVS 63rd International Symposium, Nashville, TN. November 6, 2016 - November 11, 2016. 


\title{
Should the repeatability of the instrument under test be included in test uncertainty?
}

\author{
Speaker/Author: Craig M Shakarji \\ Physical Measurement Laboratory \\ National Institute of Standards and Technology \\ 100 Bureau Dr., MS 8210 \\ Gaithersburg, Maryland 20899, USA \\ Phone: (301) 975-3545, Email: craig.shakarii@nist.gov \\ Author: Steven D Phillips \\ Physical Measurement Laboratory \\ National Institute of Standards and Technology \\ 100 Bureau Dr., MS 8210 \\ Gaithersburg, Maryland 20899, USA \\ Phone: (301) 975-3565, Email: steven.phillips@nist.gov
}

\begin{abstract}
Should the repeatability of the instrument under test be included in test uncertainty? The answer-which surprises many - is no, at least for the range of cases documented in this paper. This has been an area of confusion even among experts around the world. Some ramifications of this confusion have been known cases where companies have had to greatly and artificially inflate their accuracy specifications for their measuring instruments in order to meet decision rule requirements for acceptance testing.

The purposes of this paper are to (1) Clearly identify a large set of common instrument testing/calibration situations where instruments are verified to their accuracy specifications where the repeatability of the instrument under test should not be included, (2) Take the reader through a detailed tutorial path to gain understanding as to why it must be that such a component should not be included in those cases, and (3) Identify four common mistakes that lead even experts to incorrectly include the repeatability component: These being (i) misunderstanding that there are two measurands involved in testing, (ii) misunderstanding the difference between the test value uncertainty and uncertainty in the overall test, (iii) misunderstanding the role of test value uncertainty by envisioning its use in subsequent measurements made by the tested instrument, and (iv) misapplying the inclusion of the repeatability of the calibrating system performing the test to include the instrument under test.
\end{abstract}

\section{Introduction}

The question of whether the repeatability of the instrument under test should be included in test uncertainty has caused confusion in the metrological world since the publication of the Guide to the Expression of Uncertainty in Measurement (GUM) [1] and likely earlier still. In "normal" measurements, i.e., on objects that are not indicating instruments, the repeatability of the measuring instrument must be included in the uncertainty evaluation somehow-even if it is incorporated as part of another component with another name. But when an 
indicating instrument is being tested, and the measurand is the error in the instrument's measurement, how should the repeatability of the instrument under test be handled?

The answers to just what does and does not get included in test uncertainty is complicated by the fact that there are various types of testing with fundamentally different test measurands. Without going into further detail, we simply state that it is too large a subject for this paper to address all possible testing scenarios. However, there is a large swath of instrument testing per national and international standards that will be addressed here. For these, at least, the repeatability of the instrument should not be included in the test value uncertainty.

In 2016, Salsbury presented an NSCL International paper [2] highlighting some aspects of the recently published standard on test uncertainty related to dimensional metrology ISO 14253-5 [3], which included the fact that - for scope of testing relevant to that standard - the repeatability of the instrument under test is not included in the uncertainty of an individual test value. However, why the repeatability is not included involves subtle concepts that need explanation in light of confusion even among experts. The purpose of this paper is to take the reader through a course of thought to help explain why the repeatability should not be included in these cases.

For the purposes of this paper, we assume an indicating instrument has an accuracy specification defined by a documentary standard and stated by a maximum permissible error (MPE) that is a constant across its rated operating conditions. (An MPE specified by a non-constant function over its rated operating conditions is a straightforward extension of the concepts in this paper, but the constant MPE is used to keep examples and concepts simpler.) The rated operating conditions typically span several measuring conditions (e.g., range of ambient temperatures during measurement, range of allowable measurands, etc.).

Section 2 will further delineate the scope of testing we are considering in this paper. It describes a test protocol based on a system of a finite number of spot-checks. Section 3 then reasons from two example cases that the variation in time of the errors of the instrument under test have no bearing on the uncertainty of any individual test value for one of the spot-checks; thus, the repeatability of the instrument under test is not included as a component of the test value uncertainty. Our conclusions are given in Section 4.

\section{A careful delineation of the scope of testing considered}

Much of the confusion can be cleared up by a careful delineation of the scope of the testing being discussed. In our discussions in this paper we are assuming the following:

1) There is a written test protocol that has been agreed upon that includes sufficient definitions and instructions that - when executed - produces an unambiguous overall pass or fail. What action is taken as a result of an overall pass or fail result depends on the agreement. For example, in an acceptance test, a buyer and seller have agreed on the sale of a measuring instrument contingent upon the instrument's passing a particular documented test

2) The test protocol produces multiple (or possibly even just one) individual pass/fail test results, which then produce the overall test result of the test protocol. For example, a test protocol may require 35 measurements, each repeated 3 times for a total of 105 individual tests. If every one of them results in a pass, then the overall test result is a pass, and if any one (or more) individual test fails, then the overall test result is a fail.

3) Each individual test consists of obtaining a test value and comparing that test value against a threshold value. An uncertainty associated with the test value is taken into account by means of a decision rule to 
produce an unambiguous pass or fail result for the individual test. For example, an instrument under test has a claimed maximum permissible error (MPE) and (following the test protocol) an individual test value is obtained by having the instrument perform a measurement of a calibrated object. The test value is (in this example) the estimated error (the measured value produced by the instrument under test minus the calibrated value). The test value is then compared against the MPE where - in the comparison-the uncertainty associated with the test value is taken into account by means of a predetermined decision rule.

The reader should take care to note the difference between the two concepts of the overall test and the individual tests that are contained within it. This difference may seem straightforward, but the careful distinction is important. This is because the test uncertainty discussed for this type of test is the uncertainty associated with the test value from an individual test. It is not the uncertainty associated with the overall test outcome. For this reason, the label "test value uncertainty" is more specifically descriptive than "test uncertainty" for the cases currently under consideration. Thus (as will be discussed later) the test value uncertainty is not a measure of how thoroughly the overall test interrogates an instrument but rather what uncertainty is associated with an individual test value. And various test values within an overall test could have different test value uncertainties.

Once the test outcomes are known for the individual tests, it is a matter of counting to determine if the overall test is a pass or fail, which is a step that incurs no additional uncertainty.

The type of testing contained in this scope has been employed in numerous national and international documentary standards. Some dimensional metrology examples include various ANSI/ASME B89 standards and the ISO 10360 series of standards on various coordinate measuring technologies. ISO 14253-5 [3] covers the topic of test uncertainty for the area of verification of dimensional metrology instrumentation, and the concepts covered in this paper are consistent with that standard.

\section{Reasoning from two example cases}

In this section, we use two example cases to guide our thinking through the concepts of the overall test, the test values, and the test value uncertainty, as well as to address the question of whether the repeatability of the instrument under test should be included in the test value uncertainty. The first example involves the testing of a laboratory that has been equipped with an environmental control system that regulates the temperature in the room and the measurand is the room's temperature. The second example involves a coordinate measuring machine $(\mathrm{CMM})$ being tested against its accuracy specifications where the measurand is the CMM's measurement error. The second example is the most relevant to the topic of this paper (since it involves the testing of an indicating measuring instrument) but the first has been specifically chosen because we readily grasp the concept that seeking to measure the temperature at different times involves different measurands. We immediately know that the temperature outside at noontime is a different measurand than the temperature outside at midnight. We use this first example even though it does not involve the testing of a measuring instrument.

\section{Example 1 involving temperature measurements}

Consider an example where a company has been contracted to install an environmental control system in a laboratory such that the temperature within the room to always be kept between $19{ }^{\circ} \mathrm{C}$ and $21{ }^{\circ} \mathrm{C}\left(\mathrm{i} . e ., 20{ }^{\circ} \mathrm{C} \pm 1\right.$ ${ }^{\circ} \mathrm{C}$ ). The work has been completed, but before the payment is made the room must pass a temperature test-per the agreement - according to the specific test protocol identified. 
Before going further, we apologize to the experts who specialize in environmental control; this is a completely invented example of a test protocol for the simple purpose of guiding our thinking on a general issue. Having said that, suppose this fictitious test protocol states that the temperature (at some well-defined point in the room) shall be measured 24 times in the span of about 24 hours. Each temperature reading shall be taken at some instance of time in the interval between 10 minutes before and 10 minutes after the hour (e.g., between 9:50 and 10:10, between 10:50 and 11:10, etc.). ${ }^{1}$ While the test protocol allows the exact time of measurement (within the interval) to be up to the tester, the tester cannot use any test-specific information (e.g., watching the temperature) and can only arbitrarily pick some instant of time within the interval to take a reading. For each of these temperature readings, the measured temperature value is compared to see if it is indeed within the threshold $20{ }^{\circ} \mathrm{C} \pm 1{ }^{\circ} \mathrm{C}$. This comparison (as part of the written protocol) must be done, taking into account a decision rule of guarded acceptance [4] (also known as stringent acceptance [5]), such that the temperature and its entire expanded uncertainty interval (say at the $k=2$ level) must be wholly contained within the specified threshold interval. Thus, the overall test consists of 24 individual tests, each of which must pass for the overall test to pass. ${ }^{2}$

Now suppose such a test were executed, and the thermometer chosen to perform the test is known to produce each measurement result with a $k=2$ expanded uncertainty of $0.01{ }^{\circ} \mathrm{C}$. The tester who (for some reason) manually records the temperature (and who stays awake for 24 hours!) makes use of an imprecise clock on the wall to determine when to take a temperature reading. Since (we will suppose) it is known the wall clock could be incorrect by a maximum of two minutes, the tester records the temperature at some instance between 8 minutes before and 8 minutes after each hour (per the time on the clock) recording the wall clock time at each measurement. See fig. 1 showing an example of the temperature graph over the 24 hours and the 24 measurement results. The 24 expanded uncertainty intervals (of $0.01{ }^{\circ} \mathrm{C}$ each) about the measured values are not shown, but are smaller than the dots representing the readings. The tester concludes that all 24 individual tests have passed (using an expanded test value uncertainty of $0.01{ }^{\circ} \mathrm{C}$ for every test value) and thus also concludes that the overall test result is a pass.

\section{Deviation from $20^{\circ} \mathrm{C}$}

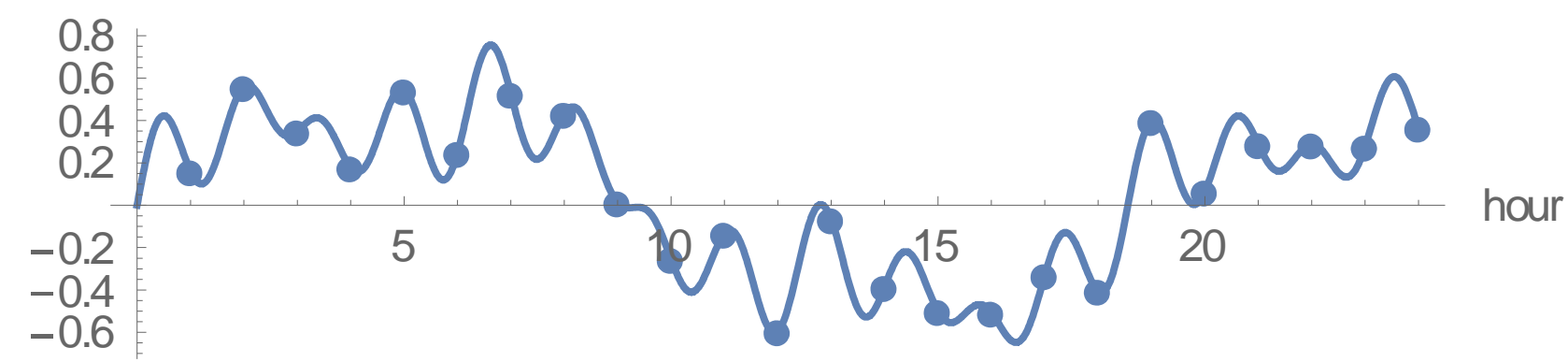

Fig. 1. An example graph showing the actual temperature variation and the points of temperature readings

We will now imagine the tester is interrogated on the matter and is called into question for not including the proper repeatability component in the test uncertainty. The first objection is that a component of the test uncertainty should be the standard deviation of the 24 measured temperatures and that such "repeatability" should be included in each uncertainty. In this example, this would increase the $(k=2)$ expanded test value uncertainty from 0.01 ${ }^{\circ} \mathrm{C}$ to roughly $0.5{ }^{\circ} \mathrm{C}$ with the consequence that the overall test would not pass since the acceptance zone of the decision rule, which is maximum allowed temperature reduced by the coverage interval (VIM 2.36, [6]) also

\footnotetext{
${ }^{1}$ While fine for our fictitious example, a cyclical test like this would have a weakness, since the air system could also be similarly cyclical.

${ }^{2}$ In reality, temperature readings can be taken so easily that a test protocol would not be written with such sparse sampling.
} 
known as the uncertainty interval [4], would not contain some of the observed temperature values. (We note that if the number of samples were increased, the standard deviation would not change much, meaning that the overall test would still not pass even if there were thousands of added readings, none of which exceeded $0.8^{\circ} \mathrm{C}$.)

The tester (correctly) rebuts that each test involves its own measurand. The temperature at hour 1 is a different measurand than the temperature at hour 2 just like we all know the temperature outside at 1:00 pm is a different quantity than the temperature at 2:00 pm. The fact that the quantity (temperature) varies with time has no bearing on the ability to measure the temperature at one specific time. If it did, then one could not measure the temperature on a summer day with much accuracy at all since the temperature would be so different next winter!

When we remember that each of the 24 tests is treated as an individual, isolated test with its own measurand this objection may seem like nonsense to the reader (and it should) but that is partly because this example has been carefully crafted to highlight the fallacy. In fact, this exact mistake is often made due to the fact that the concept of test uncertainty in other contexts is more subtle. The authors are aware of at least one case where this mistake had led to confusion in the marketplace for certain instrument specifications. And although the confusion is being cleared up for the case mentioned, we are emphasizing that this is no mere academic problem and that much confusion remains.

The objector concedes that point but now tries now another approach: "You indicate that at time 11:03 am you took a temperature reading. In fact, you were using an inaccurate clock. Due to the maximum two-minute clock error possible, your measurement could have actually taken place anytime between 11:01 and 11:05. Thus, you need to take into account the variation in temperature that can occur over a couple-of-minute time period in your test value uncertainty. This variation would be much greater than your claimed $0.01{ }^{\circ} \mathrm{C}$ test value uncertainty." This objection demands that repeatability over a shorter time period be included in the test uncertainty.

This objection is answered again by the tester who indicates that the test protocol allows for any time within the stated interval to be an acceptable time to take a reading. Therefore, even though the tester does not know what that time exactly was, it was certainly a time within the acceptable interval and therefore an allowed point in time to take the reading. The tester insists that the temperature one minute or two minutes before or after the actual measurement time is a different quantity and has no bearing on the accuracy of the recorded temperature even if the tester does not know precisely when it was. So, while it is true that the tester does not know very well what the temperature was at the true time of 11:03, the tester certainly knows that at some instance in time between 11:01 and 11:05 the temperature was at its recorded value with a $0.01{ }^{\circ} \mathrm{C}$ expanded uncertainty.

The objector tries a third time and indicates that for some short period of time, the temperature is constant enough to be considered a single quantity having an essentially unique value. During that time, the variation of temperature readings - if they were taken in rapid succession — would be a repeatability that needs to be included. The tester replies in agreement, but indicates that that repeatability is the repeatability of the thermometer, which is already taken into account by the $0.01{ }^{\circ} \mathrm{C}$ expanded uncertainty attributed to the thermometer readings.

The objector then tries lastly to argue that 24 measurements (one per hour) is entirely too small a number to ensure that the temperature of the room never exceeded the required limits. It is argued that the number should be more like 2400 or 24000 . And since 24 measurements are too few, uncertainty needs to be taken into account to reflect the lack of knowledge of the variation of the temperature in the times between readings. To this the tester sympathizes that there is in fact a lack of knowledge of the temperature of the room between readings. The tester even goes as far as to agree that it would be a more robust test if the test protocol should have included more 
individual tests. However, those issues are ones that have to be considered in the writing of the test protocol and in the agreement made. Once the agreement has been made that the overall pass or fail would be determined by the 24 temperature readings, the "rules of the game" have been set, and the overall test is simply comprised of however many individual tests are in the agreed-to protocol. The accuracy of any individual temperature reading cannot be dependent on how many other readings will be taken afterwards. A good test protocol should weigh the benefits of how many individual tests are performed against the cost and time burden incurred in performing the tests.

\section{Example 2 involving CMM testing}

The use of the contrived temperature example will help us in analyzing a more pertinent example of a CMM. Suppose a buyer and seller have agreed on the purchase of a CMM that has an accuracy specification (MPE) of $1 \mu \mathrm{m}$ for any point-to-point length in any position within its measurement volume as defined in detail in ISO 10360-2 [7]. We also suppose that, for this example, an agreement was made that the purchase is contingent on the CMM - once installed - passing an acceptance test as document in the test protocol ISO 10360-2. While that standard includes various testing, for this example we will restrict ourselves to the fact that the overall " $E_{0}$ " test requires that the CMM make 35 measurements, each repeated 3 times for a total of 105 measurements of calibrated test lengths (e.g., gage blocks) of various sizes and in various positions. Each measured value is compared with the calibrated length to estimate the error of the CMM measurement. These test values are compared with the MPE, taking into account the test uncertainty using the same decision rule described in the previous example.

For our specific example, we will assume that a CMM with claimed MPE of $1 \mu \mathrm{m}$ has been installed and that the 105 measurements have been carried out using calibrated test lengths (e.g., gage blocks) each of which has an expanded $(k=2)$ uncertainty in their lengths of $0.1 \mu \mathrm{m}$. (This $0.1 \mu \mathrm{m}$ is assumed to account for all things affecting the length as presented to the CMM, e.g., the calibration uncertainty, the nonrigid fixturing of the test length, etc.) The test was performed, and indeed, each test value was within the MPE, even taking into account the $0.1 \mu \mathrm{m}$ uncertainty per the decision rule. The test protocol yields an overall test result of "pass" because each individual test result was a "pass."

The objections and their responses that arose during the temperature example apply here. We do not blindly inflate the test value uncertainty by taking the standard deviation of the 105 test values observed. Nor do we inflate the uncertainty due to imperfect knowledge of the exact operating conditions at the time of testing, provided they are within the rated operating conditions (remembering that the MPE is constant across the rated operating conditions in this case). Nor do we include an uncertainty component to take into account the lack of coverage, due to the agreement made.

However, this example of the CMM is different from the temperature example in this regard: for the case of the temperature measurements, repeated measurements taken over a short time period would yield little observed variation, but the $3 \mathrm{CMM}$ measurements taken on the same gage block in the same position can have significant variation, even over the short term. This variation should still not be taken into account in the test value uncertainty, since the measurement error the CMM commits at time $\mathrm{t} 1$ is a different measurand than the error the CMM commits at time $t 2$, even if the times $\mathrm{t} 1$ and $\mathrm{t} 2$ are close together. Just like in the temperature example, the fact that the temperature was significantly different at 1:00 than at 2:00 did not affect the test value uncertainty, so also the fact that the CMM error at a point in time (t1) is different than it is a few seconds later does not affect the test value uncertainty for the error at time $t 1$. 
The fact that the measurands are different at times 11 and $\mathrm{t} 2$ may need further explanation, since it is easy to misunderstand the measurand in this case. The test measurand - the quantity we intend to measure - in this case is the error the CMM makes when making a particular measurement. In order to ascertain this error, the CMM is asked to perform a measurement on a gage block, for example. We thus have two measurands involved. The key measurand we are interested in is the error the CMM makes. The other measurand (the sub-measurand, if you will) is the length of the gage block. When two successive measurements are made at close times $\mathrm{t} 1$ and $\mathrm{t} 2$, we agree that the "sub-measurand" has not changed, i.e., the length of the gage block hasn't measurably changed. However, the error the CMM makes from t 1 to 22 can change dramatically. Indeed, they are two different measurands. When seeking to ascertain the error the CMM makes at $\mathrm{t} 1$, we are seeking a different quantity than when we are seeking the error the CMM makes at time t2.

The previous example involving temperatures can help us here. We readily saw that the temperature at one time was a different quantity than the temperature at a later time. If we think of the CMM as a black box that produces an error value at the press of a button, it becomes easier to see that the error at $\mathrm{t} 1$ is a different measurand than that at $\mathrm{t} 2$. If I press the button to view the CMM error at time $\mathrm{t} 1$ and then again at time $\mathrm{t} 2$, I would expect different numbers (since CMM errors normally have a seemingly random component to them). They are different measurands akin to the case where we readily realize that the temperature at 1:00 and at 2:00 are different measurands. In the CMM case, it does not matter that times $\mathrm{t} 1$ and $\mathrm{t} 2$ are close together, since the error the CMM makes can change significantly over a very short time.

If it appears that the physical phenomenon being measured at times $\mathrm{t} 1$ and $\mathrm{t} 2$ has not changed, it is probably because it is the "sub measurand" (the length of the gage block) that is incorrectly being considered. The measurand at hand is the error the CMM makes, and the inner workings of the CMM are in different states at times $\mathrm{t} 1$ and $\mathrm{t} 2$. Thus, one measurand was the error the instrument makes when its inner workings were in the state that they were in at time $t 1$ and the other measurand is the error the instrument makes when its inner workings are in the state they were in at time 2 . The fact that we cannot describe and do not even know the states of the inner workings does not matter. This is akin to the case of the temperature reading earlier: even though a temperature reading was taken at approximately 11:03, the tester could not say exactly when it was except that it was an allowable time per the test protocol. If the tester had to put the measurand into words, it would be "the temperature at the defined location in the room at whatever the actual time was when the reading was taken." The measurand sounds oddly defined, but it is perfectly sound and analogous to the inability to know or express the state of the instrument in the CMM case.

As in the case of the temperature, the repeatability of the thermometer was included in the test uncertainty (it was already incorporated into the $0.01{ }^{\circ} \mathrm{C}$ expanded uncertainty). Likewise, we would include the repeatability of the gage block length presented to the CMM (which is very small and already included in the $0.1 \mu \mathrm{m}$ expanded uncertainty).

We further note that even though the test value uncertainty was $0.1 \mu \mathrm{m}$ in every case, this value is not intended to and in fact does not represent the accuracy of the CMM when it is used in subsequent measurements. That accuracy is more correctly conveyed by the MPE of $1 \mu \mathrm{m}$.

The writers of the ISO 10360-2 standard purposefully included certain testing configurations that are known by experts to be likely to reveal the largest errors of the CMM. They also included some user-selectable configurations, which means a CMM manufacturer does not know a priori all the locations and orientations that 
will be tested. ISO 23165 [8] covers the subject of test uncertainty in the specific context of CMM testing per ISO 10360-2 and is consistent with the concepts discussed here.

\section{Conclusions}

The subject of test uncertainty is complex and efforts have been made in this paper to reduce issues to simplest cases. Even with such efforts, the concepts remain subtle but we have demonstrated that -at least for the scope of testing considered here - the repeatability of the instrument under test should not be included in test uncertainty. We have reduced example cases to guide through the reasoning for this, and sought to highlight areas where confusion can likely occur. In the end, the thinking has been consistent with published international standards that have been written for the kind of testing considered in the scope of this paper.

\section{References}

1. JCGM 100:2008, Evaluation of measurement data - Guide to the expression of uncertainty in measurement (GUM)

2. Salsbury, James, G., Understanding the Test Measurand and the Profound Impact on Calibration, Verification, and Uncertainty, NCSL International Workshop and Symposium, 2016.

3. ISO 14253-5:2015, Geometrical product specifications (GPS) -- Inspection by measurement of workpieces and measuring equipment -- Part 5: Uncertainty in verification testing of indicating measuring instruments

4. JCGM 106:2012, Evaluation of measurement data - The role of measurement uncertainty in conformity assessment

5. ISO 14253-1, Geometrical product specifications (GPS) -- Inspection by measurement of workpieces and measuring equipment -- Part 1: Decision rules for proving conformity or nonconformity with specifications.

6. JCGM 200:2012, International Vocabulary of Metrology - Basic and General Concepts and Associated Terms (VIM 3rd edition)

7. ISO 10360-2:2009 Geometrical product specifications (GPS) -- Acceptance and reverification tests for coordinate measuring machines (CMM) -- Part 2: CMMs used for measuring linear dimensions

8. ISO/TS 23165:2006, Geometrical product specifications (GPS) -- Guidelines for the evaluation of coordinate measuring machine $(\mathrm{CMM})$ test uncertainty 


\title{
Low-NEP pyroelectric detectors for calibration of UV and IR sources and detectors
}

\author{
G. P. Eppeldauer ${ }^{1}$, V. B. Podobedov, L. M. Hanssen, and C. C. Cooksey, \\ National Institute of Standards and Technology \\ 100 Bureau Drive, Gaithersburg, MD, USA 20899
}

\begin{abstract}
Pyroelectric radiometers with spectrally constant response have been developed at NIST with the cooperation of a few detector manufacturers. The new devices have noise-equivalent-power (NEP) values less than $1 \mathrm{nW} / \mathrm{Hz}^{1 / 2}$ sufficiently low for use at the output of regular monochromators. Their response flatness is an order of magnitude better than that of filtered Si detectors and can be used to realize simple and low-uncertainty responsivity scales for the UV and IR wavelength ranges. For the first time, the UV irradiance responsivity of a pyroelectric detector has been determined. Based on spectral reflectance measurements of the black coating of the pyroelectric detector, the relative spectral response was determined between $0.25 \mu \mathrm{m}$ and $30 \mu \mathrm{m}$. The relative response was then converted into spectral power and irradiance responsivities using absolute tie points from a silicon-trap-detector in the VIS range. In addition to the UV irradiance responsivity scale realization, the flat response between $1.6 \mu \mathrm{m}$ and $2.6 \mu \mathrm{m}$ was utilized and a constant irradiance responsivity was realized and applied as a reference scale for the Spectral Irradiance and Radiance Responsivity Calibrations with Uniform Sources (SIRCUS) facility of NIST. The spectral power responsivity of the low-NEP pyroelectric detector is the internal standard of the NIST VIS-IR detector calibration facility for the $0.6 \mu \mathrm{m}$ to $24 \mu \mathrm{m}$ wavelength range. The pyroelectric standard is used to calibrate other types of detectors for spectral responsivity using detector substitution. The flat-response interval of the pyroelectric standard, calibrated for irradiance responsivity, was also used to measure the integrated irradiance from UV LED sources without using any source standard. The broadband radiometric measurements can be applied to IR LEDs emitting low fluxes between $750 \mathrm{~nm}$ and $4300 \mathrm{~nm}$. All pyroelectric detector based calibrations were performed with expanded uncertainties of about $2 \%(k=2)$.
\end{abstract}

Keywords: flat-response UV detector, pyroelectric detector standard, spectral irradiance responsivity, UV responsivity scale, broadband radiometric measurement, broadband UV LED measurement, UV-VIS-IR integrated irradiance, flat-response IR detector

\section{INTRODUCTION}

Low noise-equivalent-power (NEP) pyroelectric detectors with close to constant spectral response have been developed with the cooperation of a few detector manufacturers to extend the NIST responsivity scale from the visible (VIS) to the ultraviolet (UV) and infrared (IR) regions [1], and to perform broadband measurements of UV and IR sources (including LEDs). The low-NEP was needed to obtain high signal-to-noise ratios at the output of the regular monochromator of the NIST VIS-IR detector calibration facility and to measure the integrated irradiance from sources with weak output flux (like deep-UV LEDs). The spectral reflectance-based [2,3] irradiance responsivity determination of the pyroelectric detectors in the IR range makes it possible to significantly decrease the responsivity calibration-time at the SIRCUS tunable-laser applied responsivity-calibration facility [4]. The relative response curve obtained from the spectral reflectance measurements is converted into a spectral responsivity function using absolute tie point(s) traceable to the primary standard cryogenic radiometer [4].

1 george.eppeldauer@nist.gov; phone 1301 975-2338; fax 1301 869-5700

Cooksey, Catherine; Eppeldauer, George; Hanssen, Leonard; Podobedov, Vyacheslav.

"Low-NEP pyroelectric detectors for calibration of UV and IR sources and detectors."

Paper presented at International Society for Optics and Photonics (SPIE) Optical Engineering and Applications Symposium, San Diego, CA. August 8, 2017 - August 9, 2017. 
The low-NEP made it possible to make a first-time irradiance responsivity measurement with a pyroelectric detector. The realization of a low-uncertainty irradiance responsivity scale is described here that extends the Si trap detector implemented [4] irradiance responsivity scale simply and fast into the UV and IR. Using the newly developed pyroelectric detector standards, the spectral range of two NIST spectral responsivity calibration facilities (the VIS-IR detector calibration facility and the SIRCUS) could be extended. Based on the new pyroelectric detector standards, these facilities can be used for the calibration of UV, VIS, and IR test detectors. Using the low-NEP pyroelectric irradiance meter with the flat spectral response, a simple and accurate broadband measurement procedure has been introduced for the measurement of UV and IR sources including LEDs.

\section{FLAT SPECTRAL RESPONSE SELECTION}

The response flatness of selected pyroelectric detectors can be an order of magnitude better than that of filtered $\mathrm{Si}$ detectors and can be used to extend the reference responsivity scale of a silicon-trap-detector to both the UV and IR ranges. The recently developed new-generation pyroelectric detectors have low NEPs and flat response functions and they can be used at the output of regular monochromators. The low-NEP is also important for broadband measurement of low-output-flux sources like deep-UV LEDs.

The here discussed radiometric quality pyroelectric detectors with flat spectral response and low-NEP were developed with NIST cooperation using two different research agreements. First, the NEP of organic-black coated hybrid pyroelectric detectors was decreased by Gentec-EO USA to an average of $5 \mathrm{nW} / \mathrm{Hz}^{1 / 2}$ [5]. This detector does not have any protecting (sealing) window. The response flatness of the 1 st generation organic-black coated pyroelectric detectors is shown in Fig. 1. The responsivity changes are close to $+/-1 \%$ relative to the linear-fit made for the seven spectral responsivity functions between $2 \mu \mathrm{m}$ and $14 \mu \mathrm{m}$.

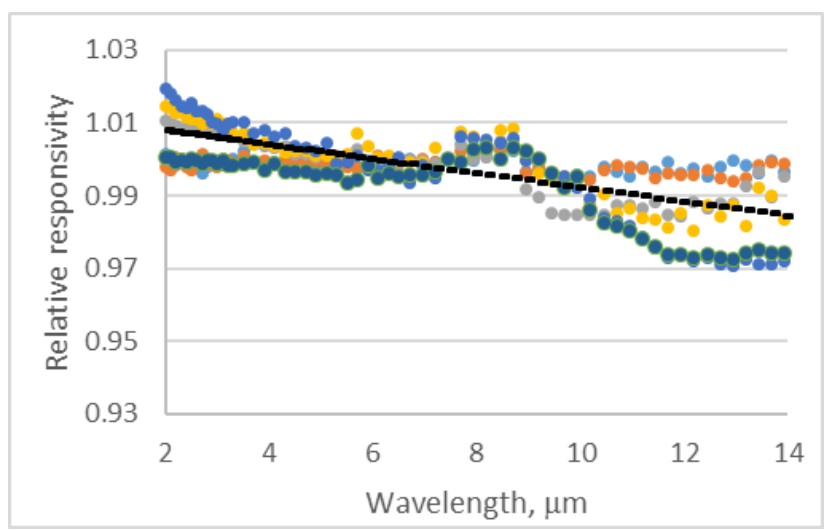

Fig. 1. Variation of spectral responsivity (normalized) for seven organic black coated pyroelectric detectors. The dotted black line represents a least-square linear fit.

To minimize the response deviation from a constant value, the gold black coatings of the pyroelectric detectors were studied. The coatings were deposited on thin glass plates. First, the effect of the coating thickness to the response change was analyzed. Figure 2 shows the measured spectral reflectance factors of the organic black coatings of 30 $\mu \mathrm{m}, 40 \mu \mathrm{m}$, and $50 \mu \mathrm{m}$ thicknesses. The measurements were made using a commercial spectrophotometer fitted with an integrating sphere. Each sample was mounted on the sample port of the sphere, so that the incident beam illuminated the sample (at an 8-degree angle with respect to the sample normal) and the reflected flux was collected within the integrating sphere where it was detected. The reflectance factor for this total reflectance measurement is the ratio of reflected flux to incident flux relative to the ratio of a perfectly reflecting diffuser. A flat reflectance was obtained above $1200 \mathrm{~nm}$ when the thickness of the coating was at least $50 \mu \mathrm{m}$. The reproducibility of the measured reflectance factor values was $6 \%$ and the reproducibility of thickness comparison was $3 \%$.

Figure 3 depicts the spectral diffuse transmittance of the organic black coatings. These measurements were collected by mounting each sample at the entrance port of the integrating sphere of the spectrophotometer. The incident beam

Cooksey, Catherine; Eppeldauer, George; Hanssen, Leonard; Podobedov, Vyacheslav. 
illuminated the sample (at a 0-degree angle with respect to the sample normal) and the transmitted flux was collected within the integrating sphere where it was detected. The diffuse transmittance is the ratio of the transmitted flux to the incident flux. The figure shows that the thickness of the organic black coating must be close to $50 \mu \mathrm{m}$ to get a negligible diffuse transmittance for the measured overall wavelength range. The negligible transmittance means that any light that's reflected is being absorbed, so Eq. 1 below, $A=1-\mathrm{R}$ is valid. It wouldn't be valid for the $30 \mu \mathrm{m}$ and $40 \mu \mathrm{m}$ coatings.

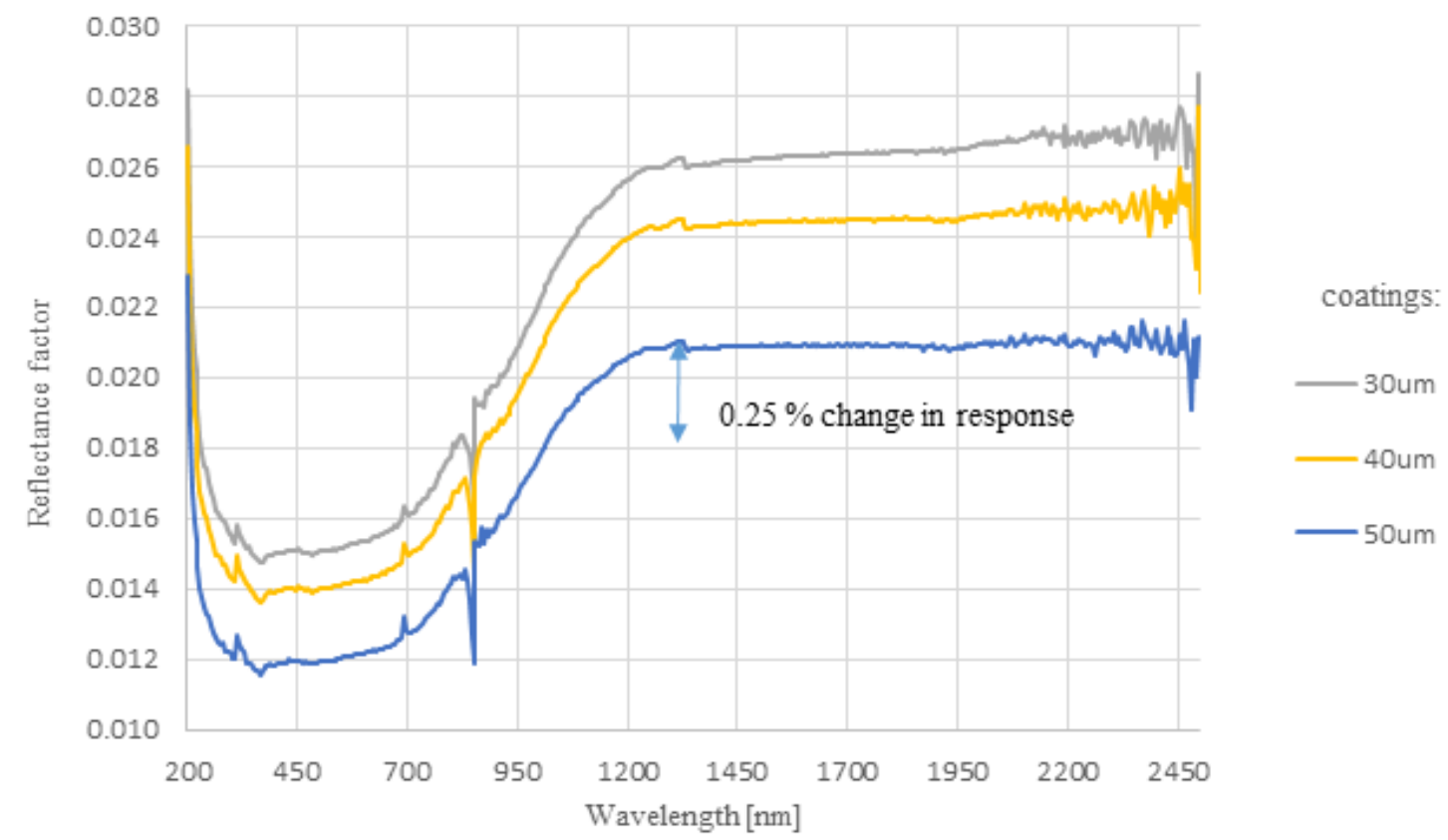

Fig. 2. Reflectance factor versus wavelength of organic-black coatings of different thickness in the wavelength range from $200 \mathrm{~nm}$ to $2500 \mathrm{~nm}$.

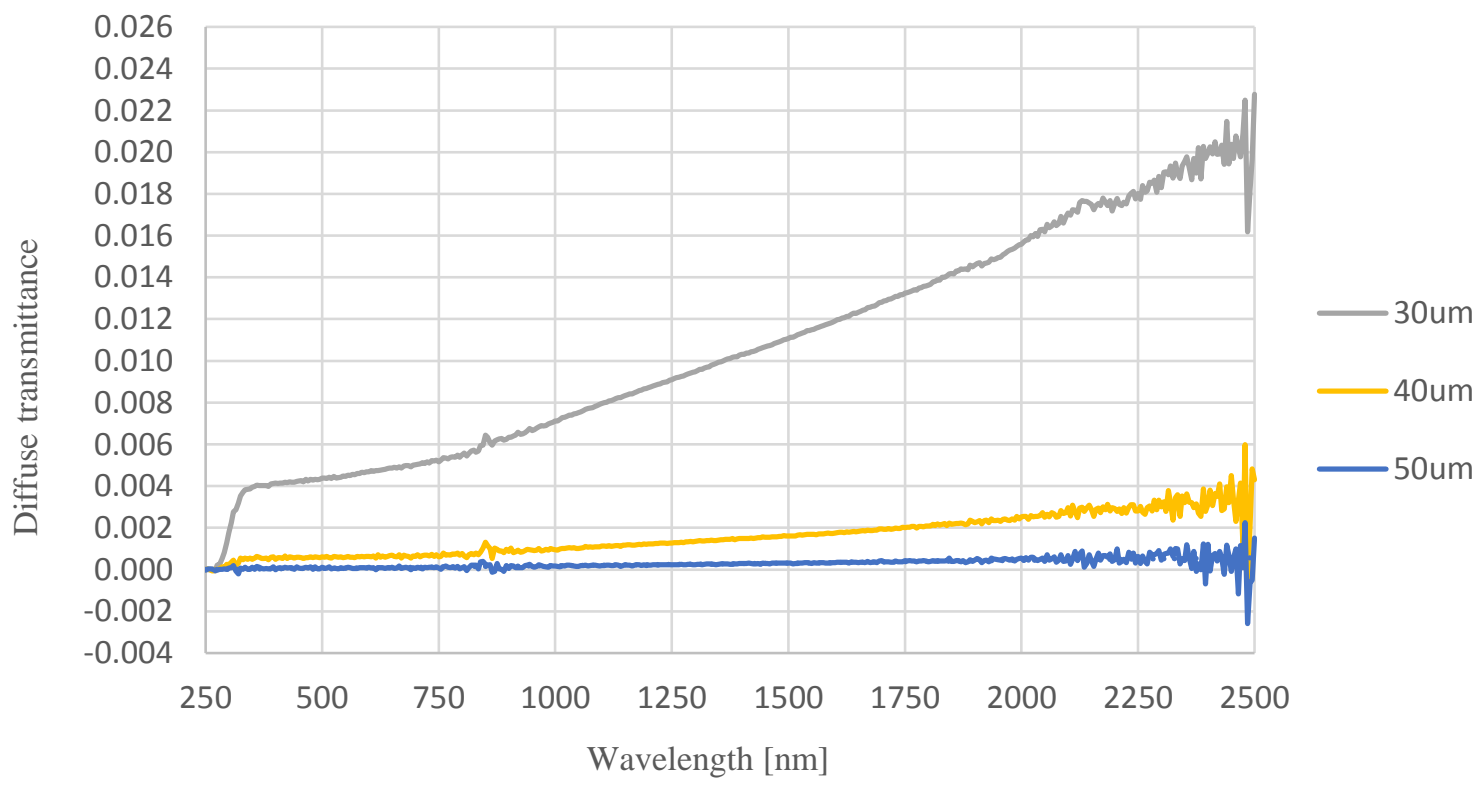

Fig. 3. Spectral diffuse transmittance for organic black coatings of three different thickness.

Cooksey, Catherine; Eppeldauer, George; Hanssen, Leonard; Podobedov, Vyacheslav.

"Low-NEP pyroelectric detectors for calibration of UV and IR sources and detectors."

Paper presented at International Society for Optics and Photonics (SPIE) Optical Engineering and Applications Symposium, San Diego, CA. August 8, 2017 - August 9, 2017. 
The spectral responsivity of the pyroelectric radiometer can be given in $\mathrm{V} / \mathrm{W}$ for radiant power measurements and in $\mathrm{V} \mathrm{cm} / \mathrm{W}$ for irradiance measurements. The here discussed hybrid pyroelectric detectors are connected to $10^{10} \mathrm{~V} / \mathrm{A}$ (fixed) gain current-to-voltage converters. In this hybrid design, both the detector and the converter are located in the same shielded metal can. On the detector surface, the diameter of the spot produced by the incident radiation in power mode measurements is $2.4 \mathrm{~mm}$. The spot is positioned into the center of the 5-mm detector. The total power in the incident beam can be measured with low uncertainty if the spatial nonuniformity of the detector-response is low. Figure 4 shows response scans along the orthogonal $\mathrm{X}$ and $\mathrm{Y}$ axes in the detector plane at a wavelength of $1.32 \mu \mathrm{m}$. The response change within the plateau is close to $1 \%$ which is comparable to the expanded uncertainty of the power responsivity determinations of the discussed pyroelectric detectors. The spatial non-uniformity of response may be larger in irradiance mode measurements where the detector is overfilled by the uniform field of the incident radiation.

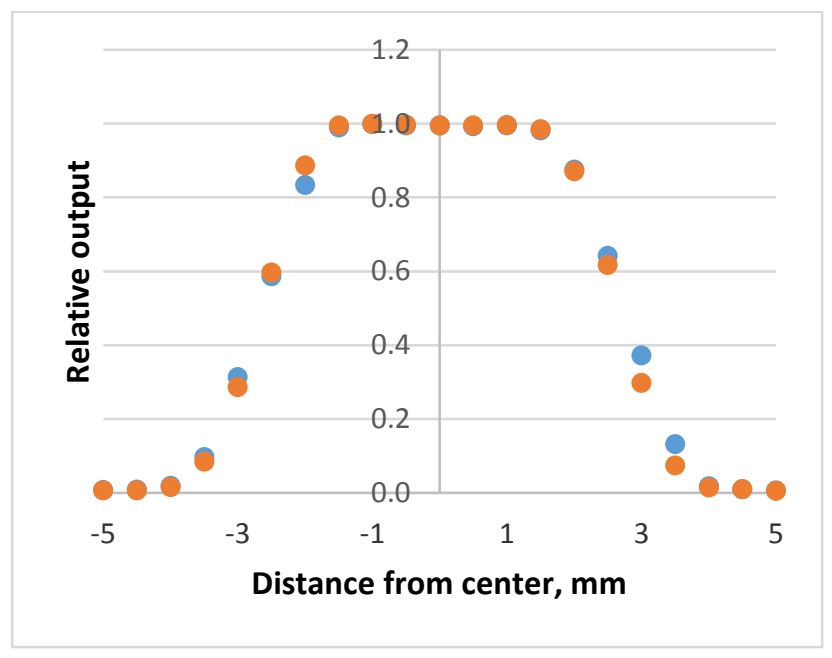

Fig. 4. Spatial uniformity of response of a 5-mm diameter organic-black-coated pyroelectric detector at $1.32 \mu \mathrm{m}$. The blue and orange markers represent orthogonal $\mathrm{X}$ and $\mathrm{Y}$ scans through the detector center taken with $0.5 \mathrm{~mm}$ increments. The scanning spot size was $2.4 \mathrm{~mm}$.

A UV-VIS-NIR irradiance responsivity scale as realized using an organic-black coated pyroelectric detector between $250 \mathrm{~nm}$ and $2000 \mathrm{~nm}$ is shown in Fig. 5. The deviation from a constant responsivity value is $+/-0.68 \%$ in this wavelength range. The uncertainty of the irradiance responsivity tie point at $660 \mathrm{~nm}$ (derived from a Si trap-detector) was combined with a few additional uncertainty components as discussed earlier [16]. Such components are distance measurement, wavelength error, target spot spatial nonuniformity, and signal (ratio) measurement errors. The response deviation from constant $(0.18 \%)$ was included for the $250 \mathrm{~nm}$ to $750 \mathrm{~nm}$ wavelength range. The spectral reflectance $\mathrm{R}$ of the black coating was measured with a few percent uncertainty. As shown in Fig. 2, R is between 0.02 and 0.03 (for the close to flat reflectance values), resulting in a spectral absorptance of

$$
A=1-R
$$

with an uncertainty of $\sim 0.1 \%$. The overall $0.5 \%(k=2)$ irradiance responsivity uncertainty (see Table 6 below) was utilized in the above UV-VIS range. This irradiance responsivity uncertainty, which is a few times smaller than the uncertainties obtained with traditional spectral responsivity calibrations (as discussed in Section 3), can be propagated to $2500 \mathrm{~nm}$.

For power responsivity calibration of the pyroelectric detector, the tie point can be derived from the power responsivity of the same Si trap-detector (where the irradiance responsivity was derived from) also at $660 \mathrm{~nm}$ but both detectors are to be underfilled by the incident-beam from the $660 \mathrm{~nm}$ LED. 


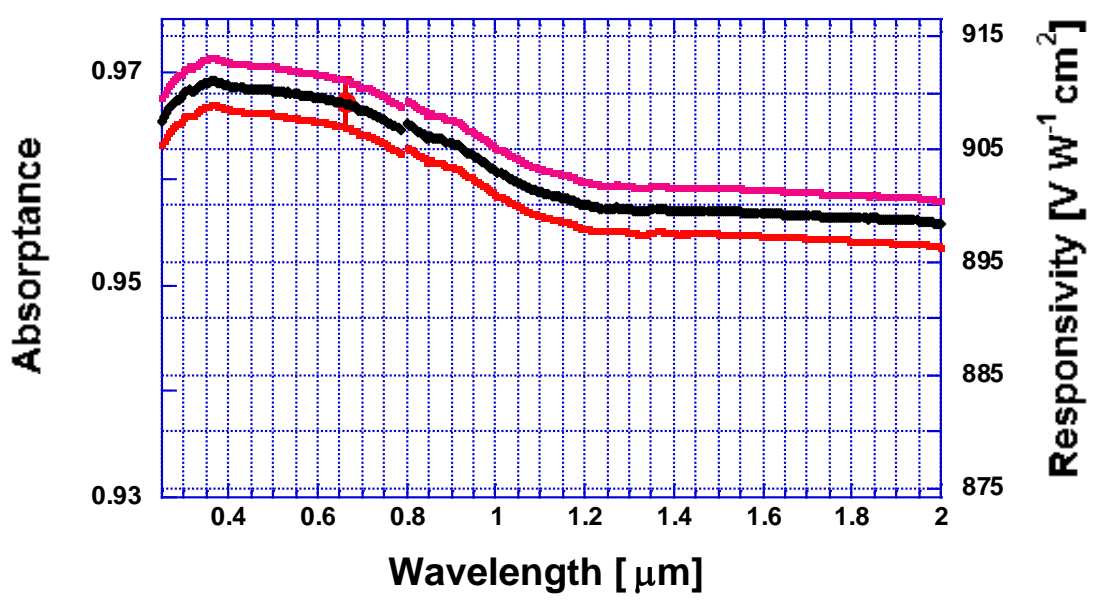

Fig. 5. Absorptance and irradiance responsivity of an organic-black-coated hybrid pyroelectric detector.

The $0.5 \%(k=2)$ irradiance responsivity uncertainty is propagated here to $2000 \mathrm{~nm}$.

The flat response was extended to the long-wave IR using $2^{\text {nd }}$ generation low-NEP organic-black-coated pyroelectric detectors. As shown in Fig. 6, the spectral power responsivity of the calibrated two pyroelectric detectors (\#1 and \#2) is close to constant between $0.85 \mu \mathrm{m}$ and $21 \mu \mathrm{m}$. The applied linear curve fits are shown with solid lines. The change in the flatness is $0.2 \%$ for the shown wavelength range. The $2.1 \%(k=2)$ responsivity error bars obtained from the power responsivity calibrations are larger than the max-to-min (individual) responsivity deviations from the constant value. The advantage of the constant response is that the relative spectral response calibrations (the spectral reflectance measurements) will not be needed when the detector to be calibrated is taken from the same batch where the measured (flat) detector was taken from.

Also, the integrated irradiance (discussed below) of infrared LEDs with peaks between $750 \mathrm{~nm}$ and $4300 \mathrm{~nm}$ needs to be measured. When using pyroelectric detectors with flat (relative) response function, only one or two absolute tie points are needed for both the near-IR and the SW-IR ranges. The tie points applied here were derived from sphereinput InGaAs [6] and sphere-input extended-InGaAs transfer standard detectors [7] calibrated against the cryogenic radiometer. The low-NEP for the pyroelectric detector is important because the output radiant flux is a few $\mathrm{mW}$ in the near-IR and 0.2-0.3 $\mathrm{mW}$ in the SW-IR.

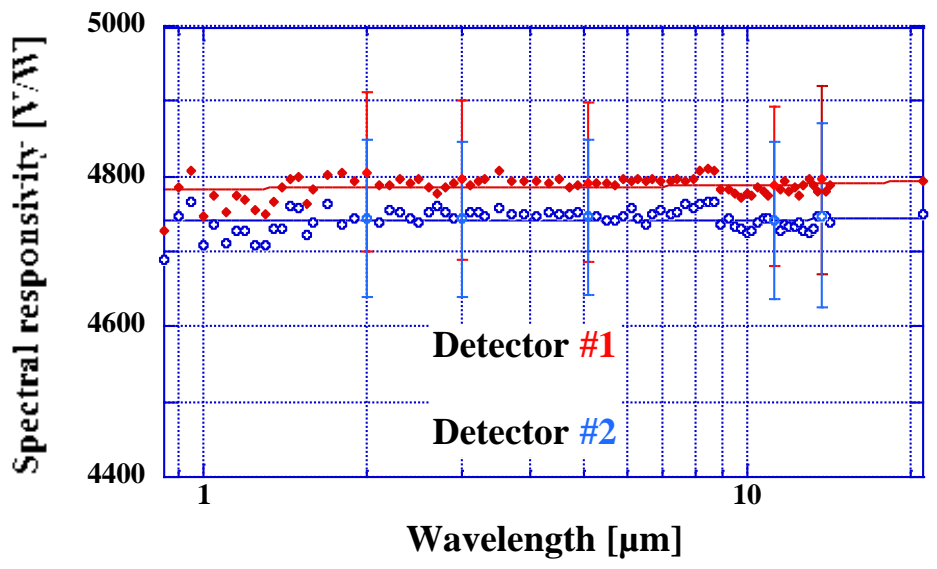

Fig. 6. Flat spectral power responsivity of $2^{\text {nd }}$ generation organic black coated pyroelectric detectors. in the $0.85 \mu \mathrm{m}$ to $21 \mu \mathrm{m}$ wavelength range. Linear curve fits are shown with solid lines. 
The main characteristics of two Gentec-EO organic-black-coated pyroelectric detectors are shown in Table 1. The responsivity primarily depends on the crystal thickness and the value of the feedback resistor. The applied feedback resistor of the current-to-voltage converter (inside of the hybrid package) was $10 \mathrm{G} \Omega$ for all organic-black coated detectors. The feedback capacitance was between $0.1 \mathrm{pF}$ and $0.2 \mathrm{pF}$. The $3 \mathrm{~dB}$ upper roll-off frequency was tuned to about a decade higher than the applied signal chopping frequency (which was selected between $10.5 \mathrm{~Hz}$ and $4 \mathrm{~Hz}$ ). As a result of the above described pyroelectric detector improvement with the NIST cooperation, Gentec EO has introduced a new hybrid pyroelectric detector, model STEP-45 [8]. This model was built with a $100 \mu \mathrm{m}$ thick $\mathrm{LiTaO}_{3}$ crystal. The detector was covered with an organic-black paint coating. This 5-mm detector has similar characteristics to the above discussed low-NEP hybrid pyroelectric detectors of flat-response.

Table 1. Main characteristics of two Gentec-EO organic-black-coated pyroelectric detectors.

\begin{tabular}{|c|c|c|c|c|}
\hline $\begin{array}{c}\text { Detector } \\
\#\end{array}$ & $\begin{array}{c}\text { Diameter } \\
{[\mathrm{mm}]}\end{array}$ & $\begin{array}{c}\text { Crystal } \\
\text { thickness } \\
{[\mu \mathrm{m}]}\end{array}$ & $\begin{array}{c}3 \mathrm{~dB} \text { roll-off } \\
\text { frequency } \\
{[\mathrm{Hz}]}\end{array}$ & $\begin{array}{c}\text { Responsivity } \\
{[\mathrm{V} / \mathrm{W}]}\end{array}$ \\
\hline 1 & 5 & 100 & 100 & $\begin{array}{c}5059 \\
\text { (at } 785 \mathrm{~nm})\end{array}$ \\
\hline 2 & 5 & 50 & 109 & $\begin{array}{c}16985 \\
\text { (at } 1.32 \mu \mathrm{m})\end{array}$ \\
\hline
\end{tabular}

As an alternate solution for flat-response pyroelectric detector based calibrations, InfraTec GmbH [8] developed the very-low-NEP Model LIE-651 custom pyroelectric detector with NIST cooperation. The characteristics of this detector were evaluated [9]. Using a $25-\mu \mathrm{m}$ thick pyroelectric chip and a feedback resistor of $100 \mathrm{G} \Omega$, the NEP was decreased to about $0.3 \mathrm{nW} / \mathrm{Hz}^{1 / 2}$. This detector had a silver-black coating with a diffuse reflection of less than $1 \%$ from about $2 \mu \mathrm{m}$ to $25 \mu \mathrm{m}$ due to the repeated coatings. Sealing windows of $\mathrm{ZnSe}, \mathrm{AgBr}, \mathrm{KBr}$, or $\mathrm{CaF}_{2}$ can modify the spectral coverage of the detector. The window behind the $5-\mathrm{mm}$ aperture was also needed to protect the metal black coating and to decrease the noise pickup. A low-loss feedback capacitor of $1.2 \mathrm{pF}$ was used against excessive noise and gain peaking. The upper roll-off frequency was about $1 \mathrm{~Hz}$. At the $4 \mathrm{~Hz}$ to $10.5 \mathrm{~Hz}$ chopping frequencies the signal and the noise changed in the same extent versus frequency.

The spectral power responsivity is shown in Fig. 7 between $1.6 \mu \mathrm{m}$ and $15.5 \mu \mathrm{m}$ when the detector is equipped with a $\mathrm{ZnSe}$ window. The flat irradiance responsivity is utilized as a reference scale between $1.6 \mu \mathrm{m}$ and $2.6 \mu \mathrm{m}$ at the NIST SIRCUS facility where tunable laser sources are used for spectral responsivity calibration of detectors, instead of using a monochromator. The close to constant responsivity was important for the SIRCUS facility to avoid the long and expensive tunable-laser used spectral irradiance responsivity calibrations. Instead, the fast and accurate monochromator based spectral reflectance calibrations with the flat pyroelectric response are utilized. The selected flat responsivity of the SIRCUS reference pyroelectric detector, equipped with a $\mathrm{CaF}_{2}$ window, is shown in Fig. 8 between $1.6 \mu \mathrm{m}$ and $2.6 \mu \mathrm{m}$. The response change in this interval was less than $+/-0.35 \%$.

The responsivity uncertainty of a pyroelectric detector from a monochromator-based responsivity calibration is typically 3 times higher (about $2 \%, k=2$ ) than the peak-to-peak change in the shown spectral responsivity function. Utilizing the lower uncertainty of the flat relative spectral response determination from spectral reflectance measurements and using an irradiance responsivity tie point from an InGaAs reference detector at about $1.6 \mu \mathrm{m}$, the spectral irradiance responsivity uncertainty can be significantly decreased in this SW-IR wavelength range.

Cooksey, Catherine; Eppeldauer, George; Hanssen, Leonard; Podobedov, Vyacheslav. 


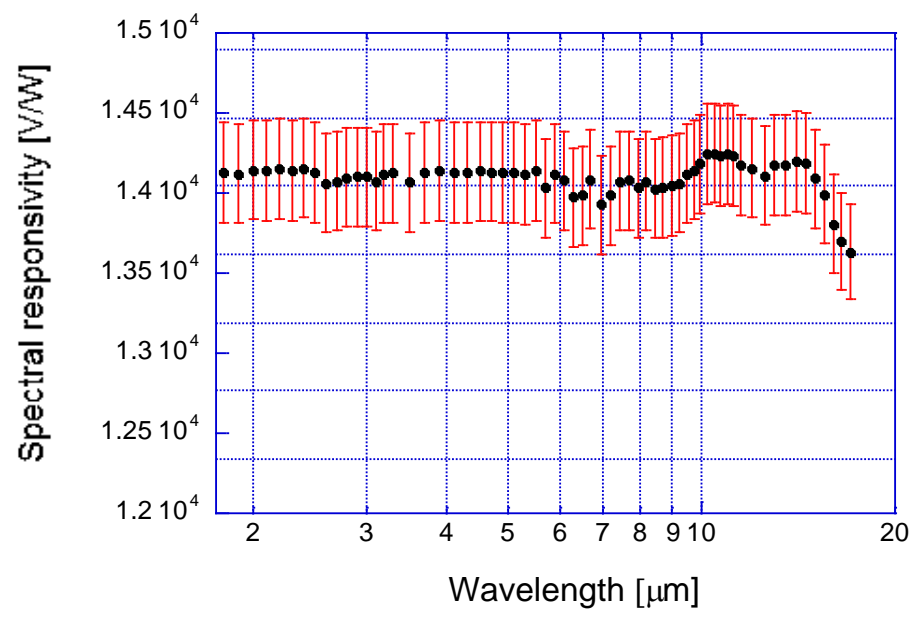

Fig. 7. Flat response of the InfraTec custom Model LIE-651 pyroelectric detector between $1.75 \mu \mathrm{m}$ and $15.5 \mu \mathrm{m}$ when the detector is equipped with a $\mathrm{ZnSe}$ window.

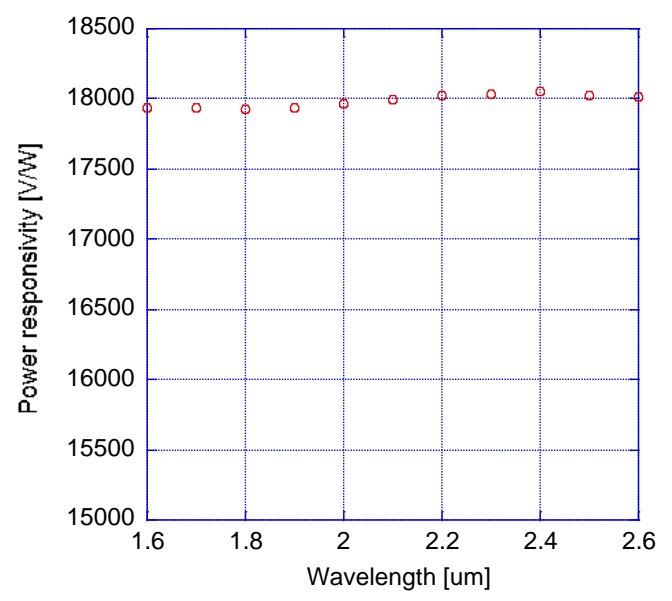

Fig. 8. SW-IR responsivity of a custom InfraTec pyroelectric detector when equipped with a $\mathrm{CaF}_{2}$ window.

The response change is $+/-0.35 \%$ between $1.6 \mu \mathrm{m}$ and $2.6 \mu \mathrm{m}$.

The NEPs of custom InfraTec LIE-651 pyroelectric radiometers measured with different time constants of a lock-in amplifier are shown in Table 2.

Table 2. NEPs of a 5-mm diameter InfraTec LIE-651 custom pyroelectric meter using different lock-in time constants.

\begin{tabular}{|c|c|}
\hline Time constant [s] & NEP [W] \\
\hline 0.1 & $3.1 \mathrm{E}-10$ \\
\hline 0.3 & $1.9 \mathrm{E}-10$ \\
\hline 1 & $9.9 \mathrm{E}-11$ \\
\hline 3 & $6.5 \mathrm{E}-11$ \\
\hline
\end{tabular}

Cooksey, Catherine; Eppeldauer, George; Hanssen, Leonard; Podobedov, Vyacheslav.

"Low-NEP pyroelectric detectors for calibration of UV and IR sources and detectors." 
The spatial nonuniformity of response of the 5-mm diameter custom InfraTec pyroelectric detector was measured with a 2-mm diameter scanning beam. As shown in Fig. 9, the max-to-min change of response during the orthogonal XY scans was $1.5 \%$ within a $2-\mathrm{mm}$ diameter spot around the center.

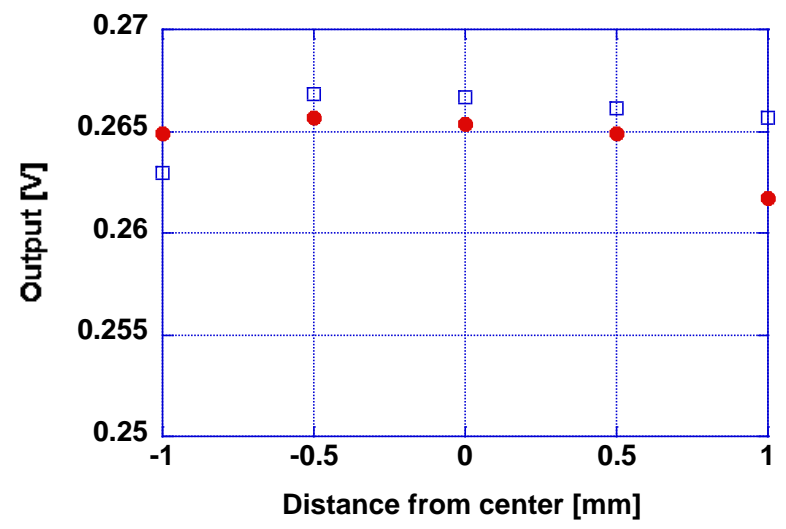

Fig. 9. Spatial nonuniformity of response of a custom LIE-651 pyroelectric detector from orthogonal XY scans around the detector-center using a 2-mm scanning beam.

\section{SPECTRAL CALIBRATIONS BASED ON LOW-NEP PYROELECTRIC DETECTORS}

While the flat spectral response can make spectral responsivity calibrations faster and more accurate than use of traditional calibrations, the flat-response for spectral responsivity calibrations is not a must. In traditional responsivity calibrations, the test detector is substituted for the standard detector (of known spectral responsivity) and the responsivity from the standard detector is transferred to the test detector while measuring the output signal from both detectors for the same incident radiation. Figure 10 shows the non-flat spectral power responsivity of an organic-black coated hybrid pyroelectric detector standard. The tie points were derived from a sphere-input ext-InGaAs transfer standard [7] at $2 \mu \mathrm{m}$ and a dome-input pyroelectric standard [10] at $10.6 \mu \mathrm{m}$. The uncertainty budget is shown in Table 3.

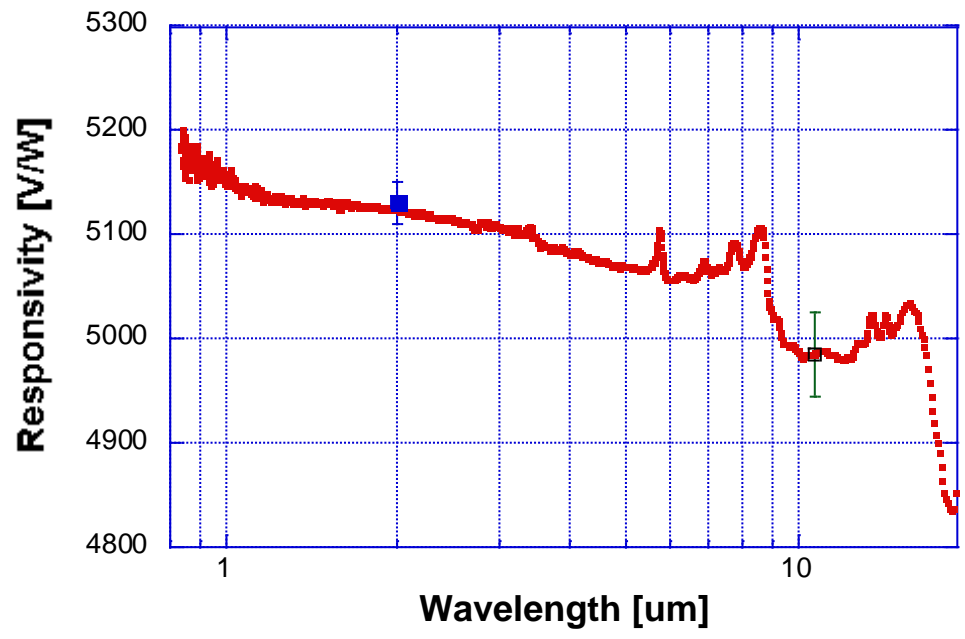

Fig. 10. Reference power responsivity scale of an organic-black coated hybrid pyroelectric detector standard.

Cooksey, Catherine; Eppeldauer, George; Hanssen, Leonard; Podobedov, Vyacheslav.

"Low-NEP pyroelectric detectors for calibration of UV and IR sources and detectors." 
Table 3. Spectral power responsivity uncertainty budget for the hybrid $5 \mathrm{~mm}$ OB-coated pyroelectric standard.

\begin{tabular}{|l|l|l|l|}
\hline Uncertainty components & Type A, \% & Type B, \% & Combined, \% \\
\hline Sphere Ext-InGaAs $(2 \mu \mathrm{m})$ & 0.4 & & \\
\hline Dome pyro $(10.6 \mu \mathrm{m})$ & 0.8 & & \\
\hline Pyroelectric radiometer gain & & 0.04 & \\
\hline Pyroe. temperature dependence & & 0.04 & \\
\hline Radiometer frequency dependence & & 0.08 & \\
\hline Spatial non-uniformity of resp. & & 0.5 & \\
\hline Pyroe noise dominated uncertainty & & 0.5 & \\
\hline Expanded combined uncertainty $(2 \mu \mathrm{m})$ & & & 0.82 \\
\hline Expanded combined uncertainty $(10.6 \mu \mathrm{m})$ & & & 1.06 \\
\hline Relative response from reflectance data & 0.5 & & \\
\hline Overall expanded $(k=2)$ at $2 \mu \mathrm{m}$ & & & 1.0 \\
\hline Overall expanded $(k=2)$ at $10.6 \mu \mathrm{m}$ & & & 1.2 \\
\hline
\end{tabular}

When the test detector has a flat response it may not need a traditional spectral calibration. If the response deviation from a constant value is equal to or less than the uncertainty of the relative spectral response determination, the calibration of the flat-response detector can be simplified. It is enough to derive a few absolute responsivity tie points from a standard detector. However, as discussed below, the flat detector-response is required when an integrated radiometric quantity (like the broadband irradiance) from a source (with broad spectral distribution) is to be measured.

Table 4 shows a summary of traditional spectral responsivity calibrations of large area IR detectors. The large area is needed for power-mode calibrations to underfill the test-detector with the incident beam. Small area or spatially nonuniform detectors should be calibrated in irradiance measurement mode to obtain low responsivity uncertainties. In irradiance mode, the detectors are overfilled with the uniform field of the incident radiation.

The spectral irradiance responsivity determination in Fig. 5 , used a large $(5 \mathrm{~mm})$ pyroelectric detector. This realized irradiance responsivity scale covers the $250 \mathrm{~nm}$ to $2000 \mathrm{~nm}$ wavelength interval and its uncertainty is $0.5 \%(k=2)$ for the overall wavelength interval. This low irradiance-responsivity uncertainty can be utilized in the UV-VIS-SWIR wavelength range in spectral responsivity (such as monochromator-based) calibrations. The range can be extended to $2.5 \mu \mathrm{m}$.

In broadband measurements (discussed below) where different source distributions are measured, the flat spectral response is a must. In this case, the use (extension) of the wavelength interval is limited for the response-interval where the response is constant (in the wavelength range of the emitted radiation). The wavelength limit is obtained where the response-deviation from constant becomes equal to the uncertainty of the responsivity (tie point) calibration.

Table 4. Examples for spectral power responsivity calibrations of different IR test detectors at the VIS-IR detector calibration facility [11].

\begin{tabular}{|l|c|c|}
\hline \multicolumn{1}{|c|}{ Test detector } & $\begin{array}{c}\text { Calibrated spectral } \\
\text { responsivity }\end{array}$ & $\begin{array}{c}\text { Expanded uncertainty } \\
{[\%]}\end{array}$ \\
\hline $\mathrm{LN}_{2}$ cooled PC-MCT & $1.7-12.2 \mu \mathrm{m}$ & 2.3 \\
\hline $\mathrm{InSb}$ & $1.6-5.2 \mu \mathrm{m}$ & 1.2 \\
\hline Low-NEP test pyroelectric & $0.6-24 \mu \mathrm{m}$ & $2.3 ;$ above $19 \mu \mathrm{m}: 2.5$ \\
\hline
\end{tabular}

Cooksey, Catherine; Eppeldauer, George; Hanssen, Leonard; Podobedov, Vyacheslav.

"Low-NEP pyroelectric detectors for calibration of UV and IR sources and detectors." 
The responsivity uncertainty in irradiance mode is higher than in power mode. Table 5 shows the expanded uncertainty budget (with the dominating components) of the irradiance responsivity transfer from an extended-InGaAs transfer standard to a $7 \mathrm{~mm}$ InSb working standard for the $1.95 \mu \mathrm{m}$ to $2.35 \mu \mathrm{m}$ interval. The $2.5 \%(k=2)$ uncertainty is roughly twice as high than the $1.2 \%(k=2)$ power responsivity uncertainty shown in Table 4 . The $2.5 \%(k=2)$ uncertainty was extended to $5.2 \mu \mathrm{m}$ based on the agreement of the spectral power responsivities derived from the sphere-input extInGaAs and the pyroelectric transfer standards [7].

Table 5. Spectral irradiance responsivity uncertainty budget for a $7 \mathrm{~mm} \mathrm{InSb}$ detector.

\begin{tabular}{|l|l|l|l|}
\hline Uncertainty factor & Type A [\%] & Type B [\%] & Combined [\%] \\
\hline Ext-IGA irradiance responsivity & 1.23 & & \\
\hline Distance & & 0.66 & \\
\hline Ext-IGA signal noise and drift & & 1.5 & \\
\hline InSb signal noise & & 1.5 & \\
\hline Expanded combined uncertainty $(k=2)$ & & & 2.5 \\
\hline
\end{tabular}

\section{FLAT-RESPONSE PYROELECTRIC STANDARD FOR BROADBAND MEASUREMENTS}

The organic-black coated pyroelectric-detector-based UV-VIS-NIR irradiance responsivity scale, shown in Fig. 5 (above), was utilized in the earlier developed broadband measurement procedure [12,13]. The output signal measured by a pyroelectric radiometer is equal to the spectral product of the source distribution and the meter response function. The requirement from the broadband measurement procedure is to obtain invariance in the measured signal (at the output of the meter) for changes in both the source (peak and spectral-width) and the spectral-shape of the meterresponse. To perform uniform broadband measurements, the spectral response of the meter must be broader than the spectral distribution of the measured source(s) and the source distribution(s) must be within the spectral response function of the meter for all the expected source(s) and meter changes. This is the reason that the above discussed flatresponse pyroelectric meters are excellent candidates for broadband (integrated) radiometric measurements. The measurement equation using a flat-response pyroelectric meter is:

$$
i=s \int_{\lambda} E(\lambda) d \lambda
$$

where $s$ is the constant spectral responsivity of the meter, $E(\lambda)$ is the spectral irradiance of the source to be measured, $\lambda$ is the wavelength, and $i$ is the measured output signal (current) of the flat-response irradiance-meter. The integrated (broadband) irradiance (from the source) is the ratio of the measured output current divided by the constant irradiance responsivity of the meter:

$$
\bar{E}=\frac{i}{s}
$$

where the unit of $i$ is $\mathrm{A}$, the unit of $s$ is $\mathrm{A} \mathrm{cm}^{2} / \mathrm{W}$, and the obtained unit for $\bar{E}$ is $\mathrm{W} / \mathrm{cm}^{2}$.

Use of the pyroelectric irradiance-measuring detector standard with the flat-response between $250 \mathrm{~nm}$ and $760 \mathrm{~nm}$, simplified the broadband measurement procedure and uniform integrated irradiance measurements from all kinds of UV and VIS sources (with different peaks and distributions) can be made without using any source standard. The deviation of the meter-response from constant in this spectral range was less than the responsivity uncertainty of the 660-nm irradiance responsivity (absolute) tie point (see the UV-VIS-NIR irradiance responsivity scale realization in Fig. 5). Using this flat-response pyroelectric standard, yearly spectral calibrations of the reference UV (lamp or LED) sources and irradiance meters is not needed [14]. Field UV meters (including existing commercial UV irradiance meters) can be calibrated against this irradiance measuring pyroelectric radiometer standard for integrated irradiance responsivity when both measure the same UV source. Using this calibration transfer, spectrally flat response for the field UV meters is not required. Applying the flat-response pyroelectric detector standard in the broadband measurement procedure, the UV measurement uncertainties (lamps or LEDs) were significantly decreased. 
The flat-response pyroelectric detectors, in Figs. 7 and 8, with NEP $\sim 0.3 \mathrm{nW} / \mathrm{Hz}^{1 / 2}$ can be used for scale transfer from sphere-InGaAs transfer standard to the flat-response pyroelectric detector or to directly measure the integrated irradiance from mid-IR LEDs that have low output flux of $0.2 \mathrm{~mW}$ to $3 \mathrm{~mW}$ between $1.55 \mu \mathrm{m}$ and $4.3 \mu \mathrm{m}$ [15].

\subsection{UV LED measurements}

The above described low-NEP pyroelectric detector with the flat-response between $250 \mathrm{~nm}$ and $760 \mathrm{~nm}$ was used to measure UV LEDs. This low-NEP detector can measure radiant power down to $1 \mu \mathrm{W}$ with a signal-to-noise ratio $(\mathrm{S} / \mathrm{N})$ of 100 [14]. For UV LED measurements, the spectral irradiance responsivity was determined first. This is the first-time calibration of the irradiance responsivity for a pyroelectric detector.

The uncertainty budget of the spectral irradiance responsivity for the organic black coated (hybrid) pyroelectric detector-standard is shown in Table 6 . The expanded irradiance responsivity uncertainty between $0.25 \mu \mathrm{m}$ and $0.76 \mu \mathrm{m}$ was $0.5 \%(k=2)$.

When measuring LEDs using the above pyroelectric radiometer, lower uncertainties can be obtained with the discussed radiometric broadband (integrated) irradiance measurements than with traditionally used $\mathrm{V}(\lambda)$-based illuminance measurements, especially in the blue and red. Using this broadband radiometric measurement, spectral information about the measured source will not be obtained.

As a result of the introduced broadband calibration procedure, the close to $0.5 \%(k=2)$ combined uncertainty for the reference level integrated irradiance measurement is significantly lower than the $5 \%(k=2)$ uncertainty performed using the previously applied source and detector spectral calibrations based method [16].

The less than $+/-1 \%$ responsivity deviations from constant up to $2 \mu \mathrm{m}$ makes it possible to apply broadband (integrated) irradiance LED measurements for the VIS-NIR range [9] as well.

Table 6. Uncertainty budget of spectral irradiance responsivity of the flat-response pyroelectric detector from $250 \mathrm{~nm}$ to $760 \mathrm{~nm}$.

\begin{tabular}{|l|l|}
\hline Relative uncertainty components & {$[\%]$} \\
\hline$\Delta \lambda$ & 0.03 \\
\hline Distance & 0.04 \\
\hline Target spot non-uniformity & 0.10 \\
\hline Spectral response change & 0.18 \\
\hline Output signal ratio & 0.10 \\
\hline Reference Si-trap & 0.10 \\
\hline Combined $(\boldsymbol{k}=\mathbf{1})$ & $\mathbf{0 . 2 5}$ \\
\hline Expanded $(\boldsymbol{k}=\mathbf{2})$ & $\mathbf{0 . 5}$ \\
\hline
\end{tabular}

UV LEDs peaking at $265 \mathrm{~nm}, 275 \mathrm{~nm}, 285 \mathrm{~nm}, 365 \mathrm{~nm}$, and $400 \mathrm{~nm}$ were measured for integrated irradiance using the flat-response pyroelectric standard discussed in Table 6. The high output-flux LEDs were used with forwardcurrent control and heat-dissipation using efficient heat sinks. For the LEDs of lower output flux, a UV-lens with fnumber of $f / 1$ was attached to the LED mount for beam collimation. Table 7 shows the measured integrated irradiance from six UV LEDs measured with the flat-response organic-black pyroelectric standard at a source-to-detector separation of $40 \mathrm{~cm}$. The measurement uncertainties were less than $1 \%(k=2)$. 
Table 7. Integrated irradiance form six UV LEDs measured with the flat-response organic-black coated (hybrid) pyroelectric detector at a distance of $40 \mathrm{~cm}$. The measurement uncertainties are less than $1 \%(k=2)$.

\begin{tabular}{|l|l|}
\hline ILT E275P $(1 \mathrm{~mW}$ flux $)$ & $\bar{E}=0.71 \mu \mathrm{W} / \mathrm{cm}^{2}$ at $19.8 \mathrm{~mA}$ \\
\hline Thorlabs M265L3 $(10 \mathrm{~mW})$ & $\bar{E}=80 \mu W / \mathrm{cm}^{2}$ at $300 \mathrm{~mA}$ \\
\hline Thorlabs M285L4 $(45 \mathrm{~mW})$ & $\bar{E}=0.44 \mathrm{~mW} / \mathrm{cm}^{2}$ at $325 \mathrm{~mA}$ \\
\hline Tenzi 365 & $\bar{E}=2.20 \mathrm{~mW} / \mathrm{cm}^{2}$ at $400 \mathrm{~mA}$ \\
\hline LED Engin LZ1 $365(1 \mathrm{~W})$ & $\bar{E}=48.6 \mathrm{~mW} / \mathrm{cm}^{2}$ at $1.0 \mathrm{~A}$ \\
\hline Old blue LED $400 \mathrm{~nm}$ & $\bar{E}=0.14 \mathrm{~mW} / \mathrm{cm}^{2}$ at $400 \mathrm{~mA}$ \\
\hline
\end{tabular}

\section{CONCLUSIONS}

As a result of several-year research and development work, pyroelectric detectors with low-NEP and spectrally constant response have been developed. The low NEP was required to use the pyroelectric detectors at the output of regular monochromators with signal-to-noise ratios high enough for low uncertainty detector calibrations and measurements. The flat-response was needed to simplify spectral responsivity scale realizations for the UV and IR wavelength ranges. For the first time, pyroelectric detectors were applied for irradiance responsivity calibrations and measurements. Based on spectral reflectance measurements of the black coatings, a UV-VIS-NIR irradiance responsivity scale with an uncertainty of $0.5 \%(k=2)$ was realized. This uncertainty is a few times lower than the uncertainty of traditional spectral responsivity calibrations. Using the flat-response of the pyroelectric irradiancemeasuring detector standard between $250 \mathrm{~nm}$ and $760 \mathrm{~nm}$, uniform integrated irradiance measurements from all kinds of UV and VIS sources (with different peaks and distributions) were performed without using any source standard. The response-deviation of the pyroelectric standard from constant in this spectral range was less than the responsivity uncertainty of the scale realization. Using the irradiance measuring pyroelectric standard, VIS, UV, and deep UV LEDs could be measured. The uncertainty of the pyroelectric detector based UV LED measurements was three times lower than that of traditional FEL lamp based spectral determinations. When measuring VIS LEDs using the constantresponse pyroelectric meter, lower measurement uncertainties can be obtained than with traditionally used $\mathrm{V}(\lambda)$-based photometric measurements, especially in the blue and red. However, the broadband measurement does not include the spectral distribution of the measured source. The flat-repose of the low-NEP pyroelectric detectors was extended to $21 \mu \mathrm{m}$ and utilized for reference spectral irradiance responsivity calibrations between $1.6 \mu \mathrm{m}$ and $2.6 \mu \mathrm{m}$ where the response deviation from constant is only $+/-0.35 \%$. Using again spectral reflectance based irradiance responsivity scale realization, the scale realization can be accurate, short, and simple. The low-NEP pyroelectric radiometers with the flat-response were used for irradiance responsivity transfer from a sphere-input InGaAs transfer-standard radiometer and they also measured integrated irradiance from LEDs in the UV and also in the near-to-mid IR ranges.

\section{References}

1. Larason, T. C. and Houston, J. M., Spectroradiometric Detector Measurements: Ultraviolet, Visible, and Near-infrared Detectors for Spectral Power, NIST Special Publication 250-41, NIST Printing Office, US Department of Commerce, (2008).

2. Barnes, P. Y., Early, E. A., Parr, A. C., NIST Measurement Services: Spectral Reflectance, NIST Special Publication 250-48, NIST Printing Office, US Department of Commerce, (1998).

3. Eppeldauer, G. P., et al., Spectral irradiance responsivity measurements between $1 \mu \mathrm{m}$ and $5 \mu \mathrm{m}$, SPIE Proc. 5543, p. 248-257, (2004).

4. Brown, S. W., Eppeldauer, G. P., Lykke, K. R., Facility for spectral irradiance and radiance responsivity calibrations using uniform sources, Appl. Opt. Vol. 45, p. 8218-37, (2006).

5. $\quad$ Eppeldauer, G. P., Zeng, J. and Yoon, H. W., Low-NEP pyroelectric radiometer standards, Proc. SPIE Vol. 6940, 6940361 to $694036-8,(2008)$.

6. Eppeldauer, G. P., Yoon, H. W., Zong, Y., Larason, T. C., Smith, A and Racz, M., Radiometer standard for absolute responsivity calibrations from $950 \mathrm{~nm}$ to $1650 \mathrm{~nm}$ with $0.05 \%(k=2)$ uncertainty, Metrologia, Vol. 46, S139-S145, (2009).

7. Eppeldauer, G. P. and Podobedov, V. B., Infrared spectral responsivity scale realization and validations, Appl. Opt. Vol. 51, No. 25, p. 6003-6008, (2012). 
8. Disclaimer, Certain commercial equipment, instruments, or materials are identified in this paper to foster understanding. Such identification does not imply recommendation or endorsement by NIST, nor does imply that the equipment are necessarily the best available for the purpose.

9. Hoppe, S., Stegbauer, H-J., Neumann, N., Pyroelectric IR reference detector with outstanding spectral electro-optic properties, Gassensor Workshop, Fraunhofer IPM, Germany, March 16, (2017).

10. Eppeldauer, G. P., Zeng, J., Hanssen, L. M., Development and calibration of pyroelectric radiometer standards at NIST, SPIE Proc. Vol. 6201, p.620119-1 to 620119-12, (2006).

11. Podobedov, V. B., et al., Calibration of spectral responsivity of IR detectors in the range from $0.6 \mathrm{~mm}$ to $24 \mathrm{~mm}$. SPIE Proceedings, 2016. 9819 (Infrared Technology and Applications XLII, 98190P, May 20, (2016); doi:10.1117/12.2228384).

12. Eppeldauer, G. P., Standardization of broad-band UV measurements for $365 \mathrm{~nm}$ LED sources. J. Res. Natl. Inst. Standards and Technology, 117: p. 96-103, (2012)

13. Eppeldauer, G. P., Standardization of broadband UV measurements. UVNews 9, Newsletter of the Thematic Network for Ultraviolet Measurements of the European Metrology Research Program of EURAMET. Published by Aalto University, Helsinki, Finland (9/January): p. 34-37, (2013).

14. Eppeldauer, G. P., Cooksey, C. C., Yoon, H. W., Hanssen, L. M., Podobedov, V. B., Vest, R. E., Arp, U. and Miller, C. C., Broadband radiometric LED measurements, Fifteenth International Conference on Solid State Lighting and LEDbased Illumination Systems, edited by M. H. Kane, N. Dietz, I. T. Ferguson, Proc. of SPIE Vol. 9954, 99540J-1 to 99540J-15, (2016). The DOI for the paper is http://dx.doi.org/10.1117/12.2237033.

15. Hamamatsu Photonics, http://www.hamamatsu.com/jp/en/4014.html

16. Eppeldauer, G. P., Podobedov, V. B., and Cooksey, C. C., UV scale calibration transfer from an improved pyroelectric detector standard to field UV-A meters and $365 \mathrm{~nm}$ excitation sources, SPIE Proc. 10181, p. 10181OW-1 to 10181OW$8,(2017)$.

Cooksey, Catherine; Eppeldauer, George; Hanssen, Leonard; Podobedov, Vyacheslav.

"Low-NEP pyroelectric detectors for calibration of UV and IR sources and detectors."

Paper presented at International Society for Optics and Photonics (SPIE) Optical Engineering and Applications Symposium, San Diego, CA. August 8, 2017 - August 9, 2017. 


\section{COMPUTATIONAL INVESTIGATIONS FOR A NEW, CONSTRAINED LEAST- SQUARES DATUM DEFINITION FOR CIRCLES, CYLINDERS, AND SPHERES}

\author{
Craig M Shakarji \\ Physical Measurement Laboratory \\ National Institute of Standards and Technology \\ Gaithersburg, Maryland 20899 \\ craig.shakarii@nist.gov
}

\author{
Vijay Srinivasan \\ Engineering Laboratory \\ National Institute of Standards and Technology \\ Gaithersburg, Maryland 20899 \\ vijay.srinivasan@nist.gov
}

\section{ABSTRACT}

For engineering drawings and CAD definitions, the problem of a suitable datum definition for datum features of circles, spheres, and cylinders has been sought by standards writers over decades. The maximum-inscribed and minimum-circumscribed definitions that have often been used have known problems relating to stability in many common, industrial cases. Examples of these problem cases include cylindrical datum features having an hourglass shape, barrel shape, or the shape of a tapered shaft and circular or spherical datum features that are dimpled. For these problematic cases, many resort to using a least-squares fit whose diameter is scaled to be just inside (or just outside) the datum feature. However, we show this shifted least-squares solution has its own drawbacks.

This paper investigates a new datum definition based on a constrained least-squares criterion. The use of this definition for datum planes has already elegantly solved the problem of providing a full contact solution when that solution is stable, while providing a balanced, stable solution in the case of rocker conditions. With that success as motivation, we now investigate using this definition for circles, spheres, and cylinders.

We demonstrate that the constrained least-squares is an excellent choice for several known problematic cases. This datum definition maintains stability in cases where the maximum-inscribed fits are not unique and thus not stable. Yet they also maintain close adherence to the maximum-inscribed solution when such solutions are stable. We also show that the constrained least-squares solution has clear benefits over the shifted least squares solution.

This is the first computational investigation into the behavior of the constrained least-squares as a possible datum definition for these features. While not being fully comprehensive, these initial findings indicate that the constrained least-squares appears to be a safe and advantageous datum definition choice and provide substantial optimism that results in future investigated cases will be pleasing as well.

\section{BACKGROUND AND INTRODUCTION}

In the world of Geometric Dimensioning and Tolerancing (GD\&T), datums are used extensively to locate and orient tolerance zones [1-7]. Given an (imperfect) datum feature on a real workpiece, a datum is a mathematically perfect geometry associated with that datum feature. An example of a datum plane associated with a datum feature is shown in Fig. 1. Some commonly used datums are planes, circles, cylinders, and spheres.

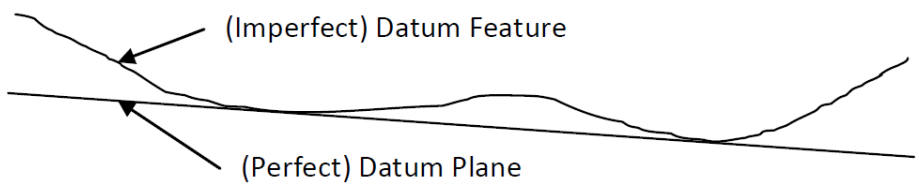

Fig. 1. Deriving a datum plane from a datum feature.

A decades-old problem in the field is creating rigorous definitions that determine which datums should be associated 
with given datum features [8]. Apart from standardization, there are several different yet reasonable approaches by which a datum can be established from a datum feature. For example, given a planar feature, one could associate a least-squares planar datum (which in the case of Fig. 1 would pass through the material), or a least-squares plane shifted to be just outside the material (which in the case of Fig. 1 would contact the datum feature at one point), or a one-sided, full-contact fit, which corresponds to the datum shown in Fig. 1. For planes, the full-contact fit is described primarily in [9] (but one can also see [10-11]).

But a datum definition that is perhaps the most sought has also historically been most elusive to define mathematically. In fact, it has been difficult to define just in words, but is somewhat captured by the following properties:

1) The datum should lie outside the material

2) The datum should contact the datum feature fully (unless that datum is not stable)

3) The datum should be stable in the sense that two datum features that are nearly the same should produce datums that are nearly the same.

It is the tension between (2) and (3) that produce the difficulty. In the case of datum planes, the ASME Y14.5 Standard [4-5] defines the datum plane as the full-contacting plane (as a planar surface resting on a surface plate) unless it is a "rocker" condition, which is handled separately. Another example of wording seeking to balance (2) and (3) is that some editions of ISO 5459 [6] indicate that a cylindrical datum given a datum feature is the maximum-inscribed (or minimum circumscribed) cylinder unless that is unstable, in which case the mobility is to be equalized to the surface. An example case is shown in Fig. 2.

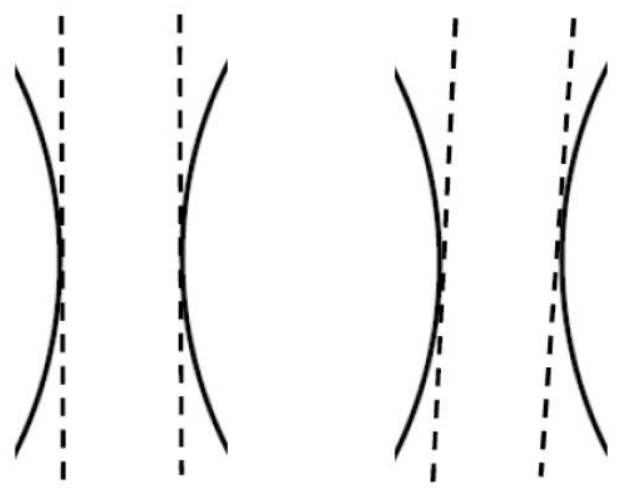

Fig. 2. The maximum-inscribed cylinder (2D cross section shown) is not stable for the hourglass shape, since both inscribed cylinders shown have nearly the same diameter.

${ }^{1}$ The ISO 5459 revision Draft International Standard (DIS) has included the constrained least-squares definition as its default datum definition for all geometries.
The difficulty of defining the desired datum is seen in the fact that when a threshold is given to define an unstable case, property (3) ends up being violated, because two similar datum features could be produced, each on other sides of the threshold, producing very different datums.

In the case of planes, a mathematical datum definition has recently been found that pleasingly solves the problem [12-13]. It is based on a constrained least-squares datum definition. This is different from a shifted least-squares solution and has the property that it makes full three-point contact with the surface when such full contact is not a rocker condition. When a rocker condition exists, the solution balances the rock, contacting only two points or one point as needed. In the case of Fig. 1, for example, the datum definition would provide the full contact datum shown. However, if the datum feature (in 3D) resembled and inverted pyramid, the datum feature would balance at only one point, the apex. If the datum feature were a concave, rectangular shape, meaning only the four corners are points of possible contact, the datum would make two diagonal points of contact and balance between the other two (like a four legged chair that rocks on two legs). In summary, a planar datum definition has been recently found that elegantly solves the elusive, decades-old datum problem.

The success of the planar problem has naturally led to questions about applying such a definition to other geometries. In this paper we investigate-largely through computational solutions - the behavior of applying a constrained least-squares definition to circles, spheres, and cylinders ${ }^{1}$.

\section{PROBLEMS WITH THE FULL-CONTACT SOLUTION}

For the planar case, the full contact solution has been rigorously defined by using a constrained $L_{1}$ association [9]. The definition produces a pleasing result in cases like Fig. 1, but is unstable and undesirable for the cases of a "V", as shown in Fig. 3.

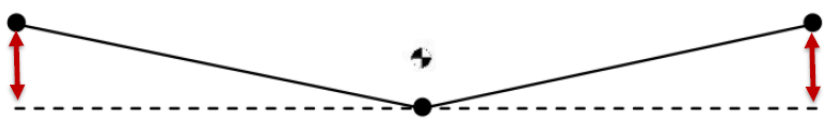

Fig. 3. The full-contact $\left(L_{1}\right)$ planar datum will not balance with one point of contact as shown, but will coincide with the longer edge or the other of the datum feature, making it unstable.

For the cases of circles, spheres, and cylinders, the full contact solution corresponds to the maximum-inscribed or minimum-circumscribed definitions. For example, in the case of a circle, given a $2 \mathrm{D}$ set of data points, it is readily seen that the maximum-inscribed circle must contact at least three points. (If it did not, and only contacted two points, then another circle must 
exist having greater diameter that is still inscribed, which can be seen by shifting the two-contact point circle's center perpendicular to the line connecting the two contact points.) In the continuous case, the full contact solution can contact two points-consider the maximum-inscribed circle of an ellipse. The same full contact applies to the cases of spheres and cylinders. However, just as in the planar case, these full contact solutions are unstable. Take, for example, the case of fitting a maximum-inscribed circle to the shape shown in Fig. 4.

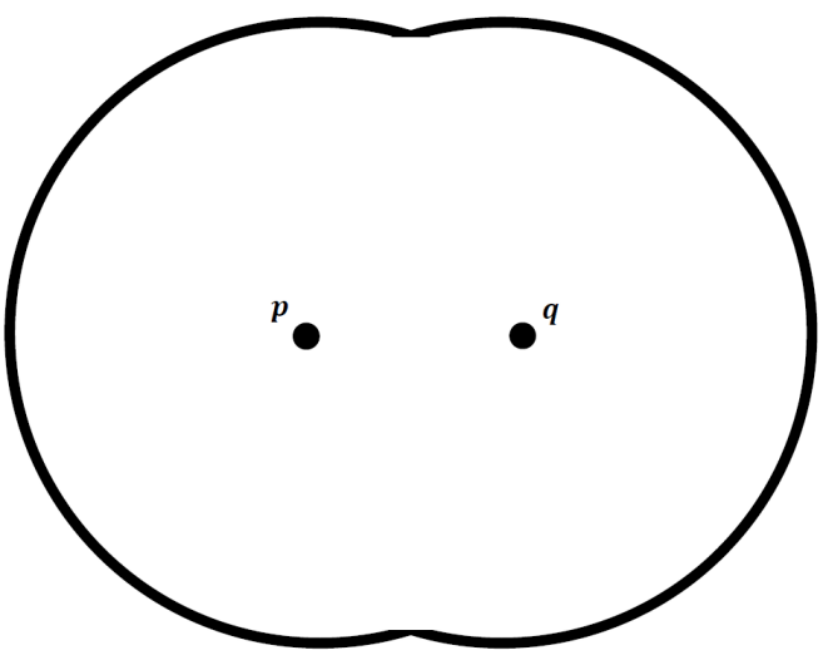

Fig. 4. A shape having two maximum-inscribed circles, one centered at $\boldsymbol{p}$ and one at $\boldsymbol{q}$.

The case of Fig. 4 is unstable in that the slightest change of shape can alter the center of the solution between the two centers shown. This has similarities to the planar datum case, where the full-contact solution varied in orientation between the two edges with slight changes of the datum feature (Fig. 3).

This problem with maximum-inscribed circles occurs more often in practice than a reader might realize. First, it can arise as a result of a simple combination of a two- and four-lobed form. For instance, the polar equation

$$
r(\theta)=10+(0.02) \sin (4 \theta)+(0.01) \sin (2 \theta)
$$

has two maximum-inscribed circles.

For another case, imagine a workpiece where the primary datum feature is a plane and the secondary datum feature is a cylinder nominally perpendicular to the plane. Then the secondary datum problem is actually a circular datum problem arising from projecting the cylinder into the primary datum plane. If the datum feature were sampled poorly, the following case would arise (shown in Fig. 5) where two centers of maximum inscribed circles exist. Interestingly, neither center is the one desired.

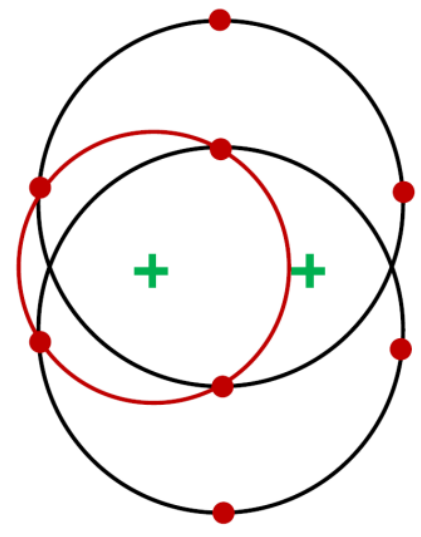

Fig. 5. A practical, unstable case showing two center locations possible. The misalignment of the cylinder projected into the plane is exaggerated for clarity.

Another example where the case of Fig. 4 can be seen is with discrete sampling. Imagine sampling a perfect circle except that two points on opposite sides of the center are slightly closer to the center than the others. This dimpled effect also creates an instability in the solution similar to what is depicted in Fig. 4. This effect can also occur with spheres.

The issue for cylinders is even more problematic and more common. The reason is that the instability shown in Fig. 2 did not arise as a result of non-uniqueness. Mathematically, the unique maximum-inscribed fit is shown on the left. But the inscribed cylinder on the right has a significantly different orientation with almost no change in diameter, causing computational instability. This instability has plagued coordinate metrology software as documented in [14] and it continues to the present time.

Other cases of instability are seen in the minimumcircumscribed cylinder of a barrel shaped datum feature (Fig. 6) and either the minimum-circumscribed or maximum-inscribed cylinders of a taper-shaped datum feature (Fig. 7 shows the inscribed case).

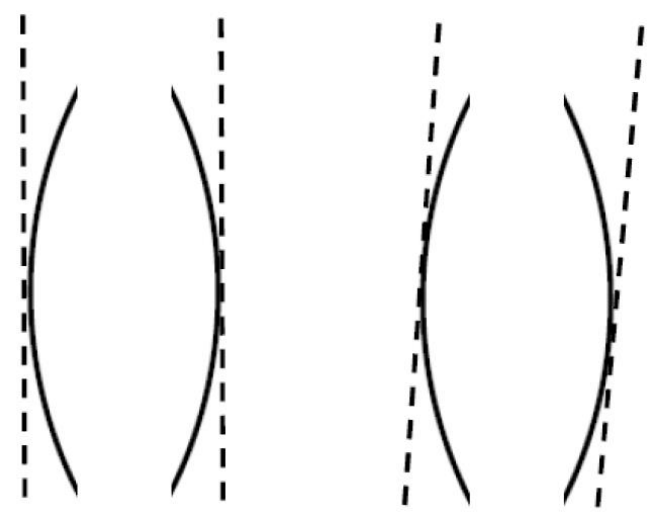

Fig. 6. The minimum-circumscribed cylinder (2D cross section shown) is not stable for the barrel shape, since both 
circumscribed cylinders shown have nearly the same diameter.

Fig. 7. The maximum-inscribed cylinder (2D cross section shown) is not stable for the taper shape, since rotating the axis as shown changes the diameter very little.

\section{THE CONSTRAINED LEAST-SQUARES DEFINITION FOR THE PLANAR DATUM CASE}

The problems that arise from the full contact solution were elegantly solved in $[12,13]$ by using the definition of a constrained least squares (called a constrained $L_{2}$ association in [12]). Figure 8 shows two typical cases where, on the left, one would seek to balance the rocking condition, and on the right, one would seek for the datum plane to be stably flush with the edge of the datum feature. This is what the constrained leastsquares solution does automatically.

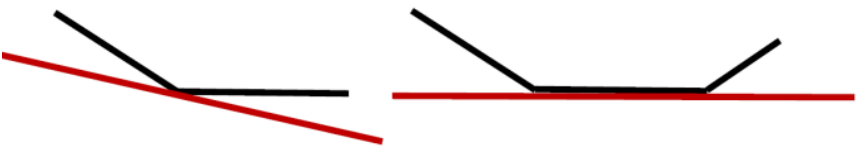

Fig. 8. Two typical cases of datum features with the associated constrained $L_{2}$ datums shown. The balanced

plane would not move from its stable state. It would remain flush with the edge of the datum feature until the line segment on the right grew long enough to make a rocker condition, at which point the $L_{2}$ constrained datum would smoothly begin to roll to the right to balance the rocker.

In contrast, the shifted least-squares solution would achieve a flush mating with the datum feature (as pictured on the right of Fig. 8) for only an instant. That is, as the line segment on the right began to be extended, there would only be one length that resulted in a flush mating. This contrast shows the fascinating feature of the constrained $L_{2}$, which stays flush with the datum feature - even while the line segment extends - until it reaches such a length that a rocking condition exists, like shown in Fig. 9.

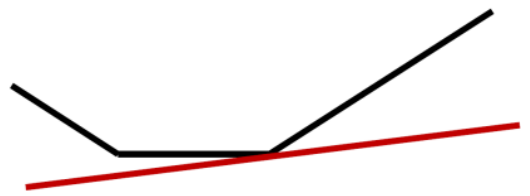

Fig. 9. The line segment on the right is long enough for the constrained $L_{2}$ datum to treat it as a rocking condition and separate from the flush contact it had in the right hand picture of Fig. 8. rocking case is on the left and the stable, flush case is on the right.

For the rocker condition pictured on the left side of Fig. 8, if the line segment on the right were made longer, the constrained $L_{2}$ datum plane would roll to the right smoothly. For the stable case pictured on the right side of Fig. 8, if the line segment on the right were made somewhat longer, the $L_{2}$ constrained datum

The constrained least-squares definition in this 2D case automatically chose between one and two points of contact in keeping the three properties desired as listed in Section 1. Similar behavior occurs in the 3D case where the datum plane makes one, two, or three points of contact with the datum feature, automatically and smoothly transitioning between these cases for varying datum features.

Since the instabilities that exist with the full contact solutions for the circle, sphere, and cylinder cases bear some similarity to the planar case, the hope is that the constrained least-squares criterion would also solve the problem of meeting all three desired properties for these cases as well.

\section{THE CONSTRAINED LEAST-SQUARES DEFINITION FOR CIRCULAR DATUMS}

Given an approximately circular curve, $C$, and a circle centered at $\left(x_{0}, y_{0}\right)$ with radius, $r$, then the least-squares objective function is given by $\int_{C} d^{2}(\boldsymbol{p}) d c$, where $d(\boldsymbol{p})$ represents the distance from point $\boldsymbol{p}$ (on $C$ ) to the circle (Fig. 10). With discrete sampling of points, we approximate the objective function

$$
\int_{C} d^{2}(\boldsymbol{p}) d c \approx \sum_{i=1}^{N} d^{2}\left(\boldsymbol{p}_{i}\right) \Delta L_{i},
$$

where $\boldsymbol{p}_{i}$ are the $N$ sampled points, one in each subdivision of length $\Delta L_{i}$. If $d$ represents the signed distance to the circle (positive indicating that the point is outside the circle) then the constraint can be written - for the maximum-inscribed case - as $d(\boldsymbol{p}) \geq 0$, for all points $\boldsymbol{p}$.

The objective functions for the minimum-circumscribed case, as well as for cases of spheres and cylinders are similar. 
Formulas for the distance calculations can be found in [14]. In this paper, our sampling was always uniform to allow the removal of the weighting factor $\Delta L_{i}$ from the objective function.

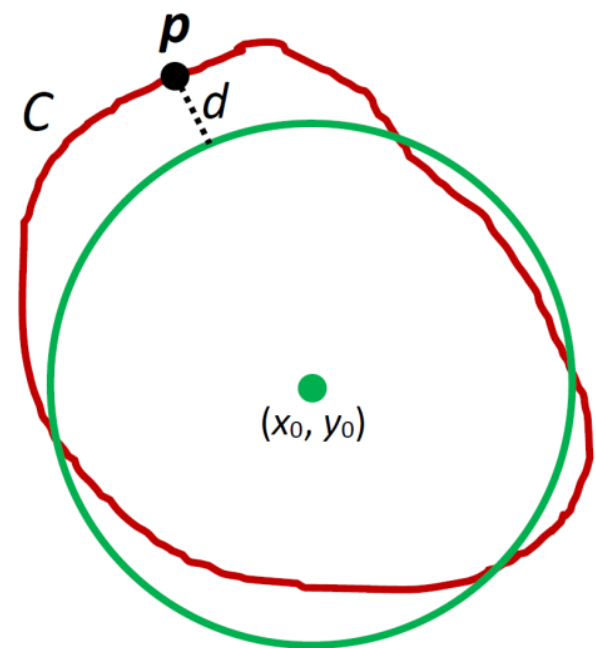

Fig. 10. Defining the objective function for fitting a circle to a curve, $C$.

We developed maximum-inscribed and constrained leastsquares algorithms in order to investigate the behavior of the constrained least-squares in contrast to the maximum-inscribed fits. We begin with the "peanut shaped" case pictured in Fig. 11, where the maximum-inscribed fit skips from side to side depending on the slightest random perturbation. One such solution is shown on the left side of Fig. 11. In contrast, the constrained least-squares solution remained near the center as shown on the right side of Fig. 11.
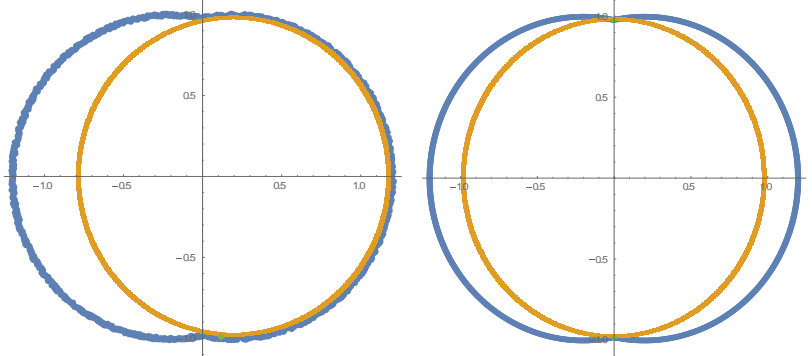

Fig. 11. The same set of data points fit with a maximuminscribed fit (left) and a constrained least-squares fit (right).

To give a quantitative feel for the behavior of these two fits, we used the dimpled problem mentioned in Section 2, for which the fits behave similarly to that shown in Fig. 11. We generated 40 uniformly spaced data points on a $20 \mathrm{~mm}$ diameter circle centered at the origin and allowed those points to vary in the radial direction uniformly randomly up to $\pm 10 \mu \mathrm{m}$. For each data set we also added two dimple points at $(0.0,9.97)$ and $(0.0,-9.97)$ (i.e., $30 \mu \mathrm{m}$ dimples) and fit the resulting data sets with both the maximum-inscribed and constrained least-squares functions. This procedure was repeated 500 times and the histograms for the $x$-coordinate of both fits are shown in Fig. 12.

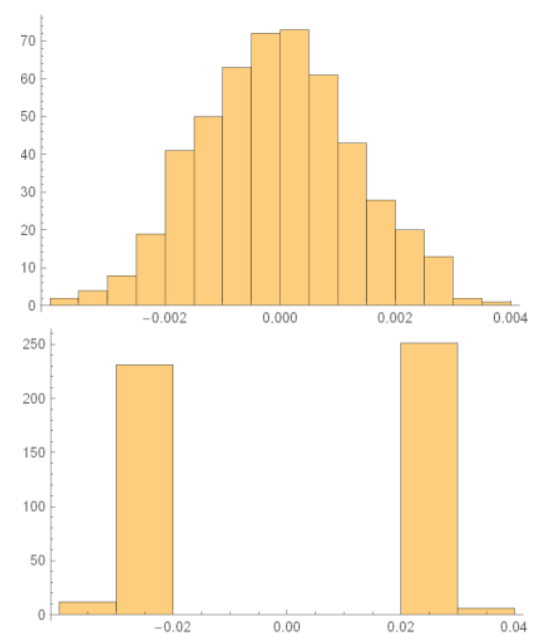

Fig. 12. Histograms showing the $x$-coordinate of the center location of fit circles (top) for the constrained least-squares fit and (bottom) for the maximum-inscribed fit. Units are $\mathrm{mm}$, but the horizontal scale differs by a factor of 10 between the histograms.

As expected, the maximum-inscribed fit has a bimodal distribution for the $x$-coordinate while the constrained leastsquares does not. But quantitatively, the standard deviation of the $x$-coordinate for the constrained least-squares is $1.3 \mu \mathrm{m}$ vs. $25 \mu \mathrm{m}$ for the maximum-inscribed fit, making them differ by a factor of nearly 20 . The important lesson is not the precise numbers themselves, for different contrived cases would yield different numbers, except to note that the constrained leastsquares is simply much more stable (by more than an order magnitude in the case shown here).

But one might then ask what is sacrificed with regard to the diameter of the fits. Interestingly, the mean diameter for the maximum-inscribed fit was $19.94006 \mathrm{~mm}$ vs. $19.94000 \mathrm{~mm}$ for the constrained least-squares. This means that the remarkable increase in stability came at the diameter "cost" of only 60 nanometers - an amount certainly negligible in light of the size of form deviations in this example case.

Before moving on to other geometries, we will make one observation about the constrained least-squares vs. the oftenused shifted least squares. For datum features having symmetric deviations (like shown in Fig. 11) the shifted least squares may work reasonably well. But for nonsymmetrical cases the shifted least squares can significantly underperform the constrained least squares.

Take, for example, eight uniformly spaced points on the $20 \mathrm{~mm}$ circle we have been considering. Assume all the points lie exactly on the circle except for the topmost point, which has coordinates $(0.0,10.01)$, meaning that for some reason this point 
is off the circle by $10 \mu \mathrm{m}$. Clearly this point will not affect the maximum-inscribed circle, which will be centered at the origin and have diameter $20 \mathrm{~mm}$. However, the shifted least-squares fit has its center at $(0,0.0025)$ and a diameter of 19.9965 - thus a $2.5 \mu \mathrm{m}$ shift in the center and a $3.5 \mu \mathrm{m}$ decrease in the diameter. By contrast, the constrained least-squares fit is centered at $(0,0.00037)$ with a diameter of 19.9995 , making only a $0.37 \mu \mathrm{m}$ shift in center and a $0.5 \mu \mathrm{m}$ decrease in diameter. These differences from the maximum-inscribed fit are lower by a factor of about seven from the shifted least squares fit. While shifted least-squares is often used in practice due to its stability in some cases, it seems evident thus far that excellent stability can be achieved without such departure from the full-contact solution.

Lastly we note that given a set of data points, the shifted least-squares fit generally contacts only one point. This alone implies an inefficiency. For this inscribed circle case, the full contact (i.e., maximum-inscribed) solution always contacts three points, even at the cost of sacrificing stability. The constrained least-squares contacts three points or sometimes two, depending on the nature of the form deviations, allowing it to maintain stability.

\section{THE CONSTRAINED LEAST-SQUARES DEFINITION FOR SPHERICAL DATUMS}

The case of spheres is very much like that of circles, extended by one dimension. We do not show numerical results, which are similar to those shown in Fig. 12. But we do take time to note some differences that the extra dimension brings. First, the dimpled problem considered in the case of circles also exists in the case of spheres, but with some extra possibilities. When two dimples are present, the maximum inscribed sphere's center shifts away from the nominal center (similar to Fig. 11) but instead of shifting only left or right as in Fig. 11, it can shift at any angle within the plane perpendicular to the line formed by the dimples. It is also possible to have three dimples (say, on a great circle) that force the full contact solution to one of two sides.

Given a set of data points for this inscribed sphere case, the full contact (i.e., maximum-inscribed) solution always contacts four points, even at the cost of sacrificing stability. The constrained least-squares contacts four points or sometimes three or sometimes even two, depending on the nature of the form deviations, allowing it to maintain stability. And similar to the $2 \mathrm{D}$ case, stability is gained with minimal cost to the diameter of the constrained least-squares fit.

The drawbacks that existed with the shifted least-squares on circles are present with similar magnitude in the case of spheres. Again, the constrained least-squares solution significantly mitigates these effects. Similar to the eight data point case with circles, a set of 14 points was created on a $20 \mathrm{~mm}$ sphere: six points were located at $\pm 10 \mathrm{~mm}$ on the axes and eight were located at $(10 \mathrm{~mm})\left( \pm \frac{\sqrt{3}}{3}, \pm \frac{\sqrt{3}}{3}, \pm \frac{\sqrt{3}}{3}\right)$. The exception was that the "north pole" point was shifted up $10 \mu \mathrm{m}$, making that point $(0,0,10.01)$.
The maximum inscribed fit was unaffected by the shifted point. Its center was $(0,0,0)$ and its diameter was $20 \mathrm{~mm}$. The shifted least squares fit was centered at $(0,0,0.0021)$ with a diameter of $19.9975 \mathrm{~mm}$ - thus a $2.1 \mu \mathrm{m}$ shift in the center and a $2.5 \mu \mathrm{m}$ decrease in the diameter. By contrast, the constrained least-squares fit was centered at $(0,0,0.00004)$ with a diameter of 19.9995 , making only a $0.4 \mu \mathrm{m}$ shift in center and a $0.5 \mu \mathrm{m}$ decrease in diameter. These differences from the maximuminscribed fit are lower by a factor of more than five from the shifted least squares fit.

\section{THE CONSTRAINED LEAST-SQUARES DEFINITION FOR CYLINDRICAL DATUMS}

As discussed earlier and as shown in Figs. 2, 6, and 7, the maximum inscribed and minimum circumscribed cylindrical functions suffer from instabilities for cases that are quite common. Besides the examples shown, a few individual discrete points that are located closer to the axis than the others can cause similar dimpling effects as discussed in the case of circles. This means that instabilities can arise from the actual form of the workpieces or from measurement errors affecting individual points.

In 2002 it was shown that some commercial software has had problems in the past with maximum-inscribed and minimum-circumscribed fits for cylinders [15]. Anecdotal evidence suggests some of the problems persist. A contributing factor to this problem may be the objective function.

To illustrate the problem and to show why the constrained least-squares fit is so much more stable in this case, we will graph the objective functions for the maximum-inscribed tapered case (Fig. 7), the other unstable cases being similar.

We consider a cylindrical bore $20 \mathrm{~mm}$ in diameter and $100 \mathrm{~mm}$ in length. We will impose a tapered form such that the top of the cylinder is exactly $20 \mathrm{~mm}$ in diameter and the bottom is $20.1 \mathrm{~mm}$ in diameter. For simplicity we sample only two rings of points - one at the top and one at the bottom (the results being similar if more rings were sampled). We will graph the objective functions for the maximum-inscribed and constrained leastsquares cases. If the axis of the tapered cylinder were along the z-axis, we will view the objective functions for inscribed cylinders having axes that pass through $(0,0,100)$ and $(\mathrm{x}, 0)$ where $x$ runs from -0.1 to 0.1 (see Fig. 13). 


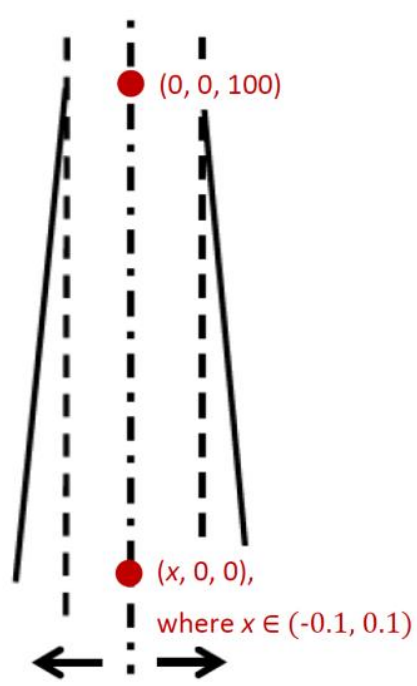

Fig. 13. The setup for the investigation into the objective functions for maximum-inscribed and constrained leastsquares. All units in $\mathrm{mm}$.

The optimal solution for both objective functions occurs when $x=0$. The radius of the cylinder is $10 \mathrm{~mm}$ at that point. To illustrate the difference in the objective functions, we graph them together. We have scaled the constrained least-squares objective function to have the same value (of $10.0 \mathrm{~mm}$ ) for the optimal case of $x=0$. We also plot the square root of the sum-of-squares instead of the sum-of-squares to properly make the results have the same units and thus be a fair comparison. The results are shown in Fig. 14.

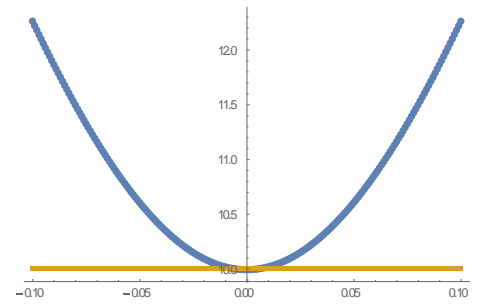

Fig.14. The maximum-inscribed objective function (nearly horizontal) and the constrained least-squares objective

function (appearing parabolic) plotted for values of $x$ ranging from $-0.1 \mathrm{~mm}$ to $0.1 \mathrm{~mm}$. All units are $\mathrm{mm}$.

As seen in the graph, the objective function for the maximum-inscribed cylinder is extremely shallow (and looks flat to the eye). An algorithm searching for a minimum would have to distinguish between objective function values that differ by extremely small amounts even for significant changes in orientation.

We note an important distinction between this type of instability and those discussed in the circle and sphere cases. In those cases, the instabilities described would have existed even if computations were made with arbitrarily high precision. In this case, the instability arises as a result of numerical issues. Nonetheless, even the effects of these instabilities are significantly diminished by the use of the constrained leastsquares objective function.

The constrained least-squares datum definition easily computed the desired solution in all of the example cases depicted in Figs. 2, 6, and 7.

\section{CONCLUSIONS}

Given the pleasing results of the constrained least-squares datum definition in the planar case, we were compelled to look at the behavior of this datum definition for other cases, namely of circles, spheres, and cylinders. Since this is the first investigation (e.g., little was mentioned for instance about minimum circumscribed cases) we recognize that the investigation is not finished. However, we investigated many of the known, major problem areas of instabilities of some other datum definitions, and the constrained least-squares definition has been shown to perform excellently in all of them. It seems to agree very closely with the full contact solution when that fit is stable, and it also maintains desired stability with little cost (i.e., with little change of diameter compared to the full contact fit). These initial findings indicate that the constrained least-squares appears to be a safe datum definition choice and provide substantial optimism that results in future investigated cases will be pleasing as well.

\section{REFERENCES}

[1] Srinivasan, V., "Reflections on the role of science in the evolution of dimensioning and tolerancing standards," Proceedings of the Institution of Mechanical Engineers, Part B: Journal of Engineering Manufacture, Vol. 227, No. 1, pp. 3-11, 2013. DOI: $10.1177 / 0954405412464012$

[2] Tandler, W. "All Those Datum Things" Inside Metrology, Quality Digest, Quality Digest Magazine, 13 February 2008.

[3] Tandler, W. "Establishing Datum Reference Frames," Inside Metrology, Quality Digest, 12 March 2008.

[4] ANSI/ASME Y14.5.1M-2009 "Dimensioning and Tolerancing," The American Society of Mechanical Engineers, New York.

[5] ANSI/ASME Y14.5.1M-1994 "Dimensioning and Tolerancing," The American Society of Mechanical Engineers, New York.

[6] ISO 5459:2011. "Geometrical product specifications (GPS) - geometrical tolerancing-datums and datum systems." Geneva: International Organization for Standardization, 2011.

[7] Zhang, Xuzeng, and Roy, Utpal "Criteria for establishing datums in manufactured parts" Journal of Manufacturing Systems, 12(1), pp 36-50, 1993. 
[8] Hopp, T. H., 1990, “The Mathematics of Datums," ASPE Newsletter, September 1990, American Society for Precision Engineering, Raleigh, NC. A reprint is available at:

http://www.mel.nist.gov/msidlibrary/doc/hopp90.pdf

[9] Shakarji, C. M., and Srinivasan, V., "Datum Planes Based on a Constrained L1 Norm," ASME Journal of Computing and Information Science in Engineering, 15(4), 2015.

[10] Shakarji, C. M., and Srinivasan V., "An improved L1 based algorithm for standardized planar datum establishment," DETC2014-35461, Proceedings, ASME 2014 International Design Engineering Technical Conferences and Computers and Information in Engineering Conference, Buffalo, NY, 2014.

[11] Shakarji, C. M., and Srinivasan V., "Theory and Algorithms for L1 Fitting Used for Planar Datum Establishment in Support of Tolerancing Standards," DETC2013-12372, Proceedings, ASME 2013 International Design Engineering Technical Conferences and Computers and Information in Engineering Conference, Portland, OR, 2013.

[12] Shakarji, C. M., and Srinivasan V., "A Constrained L2 Based Algorithm for Standardized Planar Datum Establishment," IMECE2015-50654, Proceedings, ASME 2015 International Mechanical Engineering Congress and Exposition Conference, Houston, TX, 2015.

[13] Shakarji, C. M., and Srinivasan V., "Theory and Algorithm for planar datum establishment using constrained total least squares," Proceedings, CIRP Conference on Computer Aided Tolerancing, Gothenburg, Sweden, 2016.

[14] Shakarji, C. M., "Least-squares fitting algorithms of the NIST algorithm testing system," J. Res. Natl. Inst.

Stand. Technol., 103, pp. 633-641, 1998.

[15] Shakarji, C. M., "Evaluation of One- and Two-sided Geometric Fitting Algorithms in Industrial Software," Proceedings, ASPE Annual Conference, St. Louis, MO, 2002. 


\section{DETC2017-67143}

\section{Optimality Conditions for Constrained Least-Squares Fitting of Circles, Cylinders, and Spheres to Establish Datums}

\author{
Craig M. Shakarji \\ Physical Measurement Laboratory \\ National Institute of Standards and Technology \\ Gaithersburg, MD 20899, U.S.A. \\ craig.shakarji@nist.gov
}

\author{
Vijay Srinivasan \\ Engineering Laboratory \\ National Institute of Standards and Technology \\ Gaithersburg, MD 20899, U.S.A. \\ vijay.srinivasan@nist.gov
}

\section{Abstract}

This paper addresses the combinatorial characterizations of the optimality conditions for constrained least-squares fitting of circles, cylinders, and spheres to a set of input points. It is shown that the necessary condition for optimization requires contacting at least two input points. It is also shown that there exist cases where the optimal condition is achieved while contacting only two input points. These problems arise in digital manufacturing, where one is confronted with the task of processing a (potentially large) number of points with three-dimensional coordinates to establish datums on manufactured parts. The optimality conditions reported in this paper provide the necessary conditions to verify if a candidate solution is feasible, and to design new algorithms to compute globally optimal solutions.

\section{Introduction}

Datums are important for the specification and verification of product geometries before, during, and after manufacturing [1-7]. Traditionally, datums were established on a manufactured part using physical devices such as surface plates, expanding and closing vises, expanding mandrels, and contracting chucks [8]. In the current era of digital manufacturing, one also faces the task of establishing datums by fitting mathematical objects such as planes, circles, cylinders, and spheres to a cloud of points, which may number in the millions, sampled on a manufactured part using coordinate measuring systems (CMS). Standards development organizations such as ISO (International Organization for Standardization) and ASME are now responding to this trend by moving beyond supporting merely analog (i.e., physical) inspection devices to more general standards definitions that also support such digital (i.e., coordinate measurement) technologies. Experts in ISO/TC 213 and ASME Y14 standards committees are now engaged in defining the proper fitting criteria that simulate in the digital world what has been practiced in the physical world thus far.

Mathematically and computationally, fitting can be viewed as an optimization problem. Two recent papers [9, 10] addressed the problem of fitting primary and secondary datum planes using constrained total least-squares. Another recent paper [11] reported some promising results in the use of constrained leastsquares fitting for circles, cylinders, and spheres to establish datums based on computational investigations.

This paper builds on the insights gained from [11], and addresses some of the theoretical aspects of such non-linear optimization problems. It will address only the combinatorial characterizations of the optimality conditions. Such optimality conditions provide two practical benefits [12]. First, they provide the necessary conditions that any solution to the non-linear optimization problem must satisfy; this comes in handy in (partially) verifying the correctness of a solution. Second, these optimality conditions suggest the design of new algorithms to find a global solution to the non-linear optimization problem; such algorithms may take advantage of modern advances in graphics processing units (GPUs) that are entering regular use in engineering and manufacturing.

The rest of the paper is organized as follows. Section 2 briefly reviews previously known optimality conditions for fitting datum planes to set the stage for non-linear datum features that are the main focus this paper. Section 3 describes the circle, cylinder, and sphere fitting problems as optimization problems in general, and summarizes the combinatorial characterizations of the optimality conditions under various optimization criteria. Then Section 4 summarizes a recent work on computational investigations of constrained least-squares fitting of circles, cylinders, and spheres. The main contribution of the paper appears in Section 5, where it is rigorously proved that the optimality conditions for constrained least-squares fitting involve a minimum of two contact points. Section 6 summarizes 
the results of the paper and offers some directions for future research.

\section{Optimality Conditions for Fitting Datum Planes}

Consider the problem of specification and verification involving datum planes. Figure 1(a) shows how a designer may graphically present the specification of position tolerancing of a cylindrical hole in a part with respect to a system of primary and secondary planar datums. Figure 1(b) illustrates how such a system of primary and secondary datum planes may be established on a manufactured instance of the part.

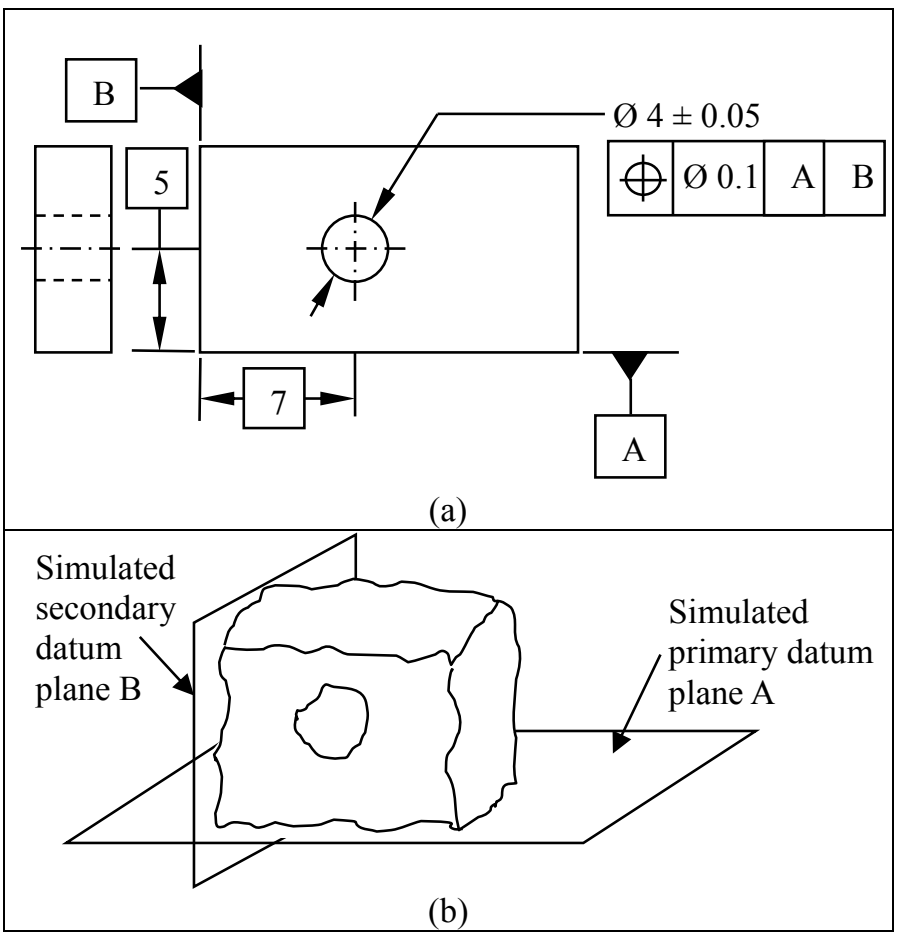

Figure 1. A simple example of (a) specification during design of a part, and (b) establishment of a system of primary and secondary datum planes on a manufactured instance of the part. The secondary datum plane $\mathrm{B}$ is required to be perpendicular to the primary datum plane $\mathrm{A}$.

In the current era of digital manufacturing, a manufactured part, such as the one shown in Fig. 1(b), can be scanned by a CMS to generate a large number of discrete points each with three-dimensional Cartesian coordinates. Those points corresponding to the datum feature A can then be processed to fit a primary datum plane $\mathrm{A}$, and similarly for a secondary datum plane B. The optimization problem for performing the plane fitting can be defined in several ways, depending on the choice of the objective function. In all cases, the datum planes are required to lie outside the material of the manufactured part while remaining as close to the part as possible.

Three of the more popular strategies for datum plane fitting have been explored exhaustively $[9,10,13-22]$, and are summarized in Table 1. (For the sake of simplicity, a tertiary datum is not shown in Fig.1.) In all these cases, the distances mentioned are the distances of the points measured perpendicular to the plane that is being fitted. In the first strategy, designated as $\mathrm{CL}_{1}$, the 3-2-1 combinatorial combination of the (minimum number of) contact points on the primary, secondary, and tertiary datum planes is in harmony with the engineering folklore of planar datum system establishment $[1,22] . \mathrm{CL}_{1}$ is also known as fitting under constrained $\mathrm{L}_{1}$-norm [20-22]. In the second strategy, designated as $\mathrm{CL}_{2}$, the combinatorial nature of the contact condition can vary depending on the configuration of the input data points $[9,10] . \mathrm{CL}_{2}$ is the fitting under constrained $\mathrm{L}_{2}$-norm; it is also known as the constrained total least-squares fitting. In the third strategy designated as $\mathrm{SL}_{2}$, only one point can be guaranteed to contact each of the datum planes $[16,18]$. $\mathrm{SL}_{2}$ is just the shifted, unconstrained least-squares fitting.

Table 1. Summary of minimum number of contact points for three datum plane fitting strategies.

\begin{tabular}{|c|l|c|c|c|}
\hline Designation & Optimization Problem & $\begin{array}{c}\text { Primary } \\
\text { Datum }\end{array}$ & $\begin{array}{c}\text { Secondary } \\
\text { Datum* }\end{array}$ & $\begin{array}{c}\text { Tertiary } \\
\text { Datum* }\end{array}$ \\
\hline $\mathrm{CL}_{1}$ & $\begin{array}{l}\text { Minimize the sum of } \\
\text { distances, subject to the } \\
\text { constraint that the datum } \\
\text { plane lies outside the } \\
\text { material. }\end{array}$ & 3 & 2 & 1 \\
\hline $\mathrm{CL}_{2}$ & $\begin{array}{l}\text { Minimize the sum of } \\
\text { squared distances, subject } \\
\text { to the constraint that the } \\
\text { datum plane lies outside } \\
\text { the material. }\end{array}$ & 3,2, or 1 & 1 \\
\hline $\mathrm{SL}_{2}$ & $\begin{array}{l}\text { Minimize the sum of } \\
\text { squared distances, } \\
\text { without any constraint. } \\
\text { Then shift the datum } \\
\text { plane to the outer most } \\
\text { point(s) of the material. }\end{array}$ & 1 & 1 & 1 \\
\hline
\end{tabular}

The numbers of contact points shown in Table 1 are the minimum numbers when the input points are in 'general position' - that is, there are no 'degenerate cases' such as four or more input points being co-planar. In those degenerate cases, it is possible that all the fitting strategies may result in contact points that exceed the minimum numbers shown in Table 1. However, even when the input points are in general position, the $\mathrm{CL}_{2}$ fitting strategy may result in 1,2 , or 3 contact points for the primary datum plane, and 1 or 2 contact points for the secondary datum plane.

While all the results summarized in this section have been already reported exhaustively elsewhere, it is worth recalling a few facts that are relevant to the rest of the paper. The ISO and ASME standards committees, which examined these three (and a few more, including minimum-zone) plane fitting strategies, are in favor of the $\mathrm{CL}_{2}$ fitting because it combines the mechanical stability of $\mathrm{CL}_{1}$ and the numerical stability of $\mathrm{SL}_{2}$. Also, the combinatorial characterizations of the optimality conditions that are presented in Table 1 as the minimum number of contact points have an important computational consequence. New 
algorithms for computing the fitted planes have been designed to exploit these conditions to generate a compact set of feasible solutions and to find a globally optimal solution [9, 10, 20-22].

\section{Fitting Circles, Cylinders, and Spheres}

Now consider the problem of fitting circles, cylinders, and/or spheres to sets of input points to establish datums. Figure 2 shows a simple example of how a designer may graphically present the specification of cylindrical datums (as primary and secondary datums) to position a pattern of holes in a part. Figs. 2(b) and 2(c) show how such primary and secondary datums may be established on manufactured instances of the part.

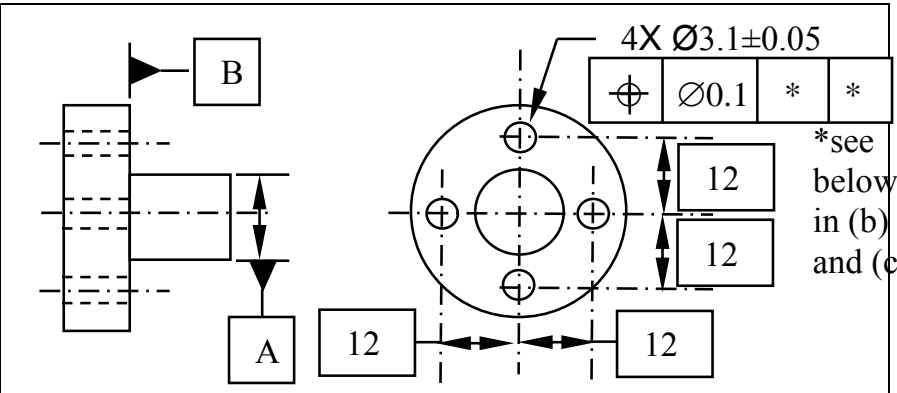

(a)

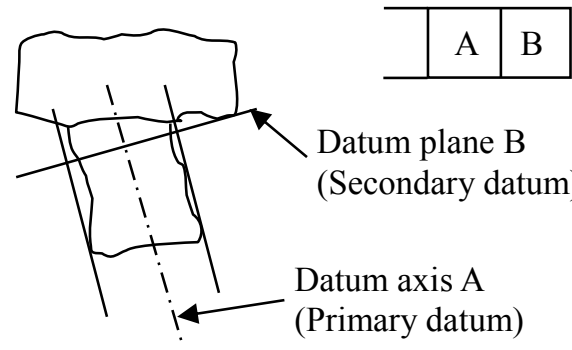

(b)

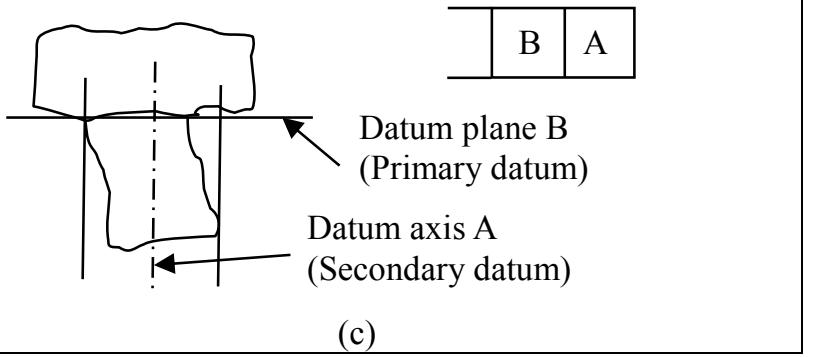

(c)

Figure 2. A simple example of (a) specification during design of a part, and (b, c) establishment of systems of primary and secondary datums involving cylindrical features on manufactured instances of the part.

In Fig. 2(b) the primary datum is the axis of a cylinder fitted to a set of points on the manufactured part that correspond to the datum feature A indicated in Fig. 2(a). In this case there is no constraint on the fitting, other than that the cylinder should enclose all the input points; in other words, it is a circumscribing cylinder. Then a secondary planar datum is fitted to the set of points on the manufactured part that correspond to the datum feature B indicated in Fig. 2(a). Here the constraint is added that the secondary datum plane $\mathrm{B}$ must be perpendicular to the primary datum axis A.

In Fig. 2(c) the specifications of the primary and secondary datums are reversed. The primary datum is a plane fitted to a set of points on the manufactured part that corresponds to datum feature B indicated in Fig. 2(a), following the procedure outlined in Section 2. Then a cylinder is fitted to the set of points on the manufactured part that corresponds to datum feature A indicated in Fig. 2(a). Here the additional constraint is that the fitted cylindrical axis, which is the secondary datum, must be perpendicular to the datum plane $\mathrm{B}$.

Figure 2 also illustrates an important fact. While fitting a cylinder is important in all cases that involve cylindrical datum features, one may want to fit a circle to a set points on a plane as in Fig. 2(c). In practice, fitting the cylinder in Fig. 2(c) is accomplished by first projecting all the relevant input points on to the primary datum plane $\mathrm{B}$ and then fitting a circle to these projected points.

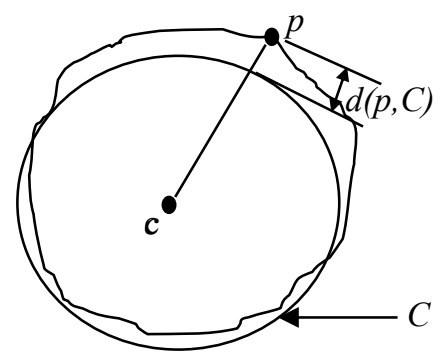

(a)

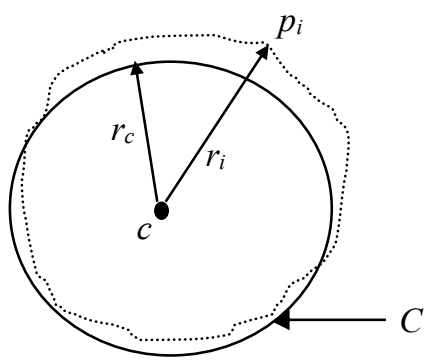

(b)

Figure 3. Fitting a circle $C$ with center $c$ to (a) an arbitrary, closed curve and (b) to a set of discrete points sampled on that curve.

With this in mind, this paper treats the circle fitting problem at par with those of fitting cylinders and spheres. In fact, it will be shown that theoretical arguments about circle fitting carry nicely to those about cylinder and sphere fitting as well. Figure 3 illustrates some of the notations and elements involved in fitting circles. In Fig 3(a) a circle $C$ with center $c$ is fitted to an arbitrary, closed curve. The distance of any point $p$ on this curve 
to the circle $C$ is the perpendicular distance between $p$ and $C$, shown as $d(p, C)$.

Table 2. Summary of combinatorial characterizations of the optimality conditions for fitting circles, cylinders, and spheres.

\begin{tabular}{|c|c|c|c|}
\hline & Designation & Optimization Problem & $\begin{array}{l}\text { Minimum } \\
\text { number of } \\
\text { contact } \\
\text { points }\end{array}$ \\
\hline \multirow{5}{*}{ 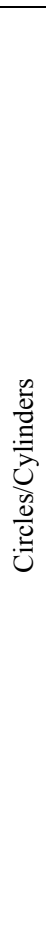 } & $\mathrm{MIC} / \mathrm{MIC}_{\mathrm{y}}$ & $\begin{array}{l}\text { Maximum inscribing } \\
\text { circle/cylinder: Maximize the } \\
\text { radius so that the circle/cylinder } \\
\text { keeps all the input points outside. }\end{array}$ & 3 \\
\hline & $\mathrm{MCC} / \mathrm{MCC}_{\mathrm{y}}$ & $\begin{array}{l}\text { Minimum circumscribing } \\
\text { circle/cylinder: Minimize the } \\
\text { radius so that the circle/cylinder } \\
\text { keeps all the input points inside. }\end{array}$ & 3 or 2 \\
\hline & $\mathrm{SL}_{2} \mathrm{C} / \mathrm{SL}_{2} \mathrm{C}_{\mathrm{y}}$ & $\begin{array}{l}\text { Shifted (offset) least-squares } \\
\text { circle/cylinder: Minimize the sum } \\
\text { of the squares of the distances to } \\
\text { the circle/cylinder. Then change } \\
\text { the radius so that the } \\
\text { circle/cylinder contacts the } \\
\text { outermost point(s). }\end{array}$ & 1 \\
\hline & $\mathrm{CL}_{2} \mathrm{IC} / \mathrm{CL}_{2} \mathrm{IC}_{\mathrm{y}}$ & $\begin{array}{l}\text { Constrained least-squares } \\
\text { inscribing circle/cylinder: } \\
\text { Minimize the sum of the squares of } \\
\text { the distances to the circle/cylinder, } \\
\text { subject to the constraint that the } \\
\text { circle/cylinder inscribes (keeps } \\
\text { outside) all the input points. }\end{array}$ & $2 *$ \\
\hline & $\mathrm{CL}_{2} \mathrm{CC} / \mathrm{CL}_{2} \mathrm{CC}_{\mathrm{y}}$ & $\begin{array}{l}\text { Constrained least-squares } \\
\text { circumscribing circle/cylinder: } \\
\text { Minimize the sum of the squares of } \\
\text { the distances to the circle/cylinder, } \\
\text { subject to the constraint that the } \\
\text { circle/cylinder circumscribes } \\
\text { (keeps inside) all the input points. }\end{array}$ & $2 *$ \\
\hline \multirow{5}{*}{$\begin{array}{l}0 \\
\frac{0}{0} \\
\frac{\pi}{2} \\
\text { की }\end{array}$} & $\mathrm{MIS}_{\mathrm{p}}$ & $\begin{array}{l}\text { Maximum inscribing sphere: } \\
\text { Maximize the radius so that the } \\
\text { sphere keeps all the input points } \\
\text { outside. }\end{array}$ & 4 \\
\hline & $\mathrm{MCS}_{\mathrm{p}}$ & $\begin{array}{l}\text { Minimum circumscribing sphere: } \\
\text { Minimize the radius so that the } \\
\text { sphere keeps all the input points } \\
\text { inside. }\end{array}$ & 4,3 , or 2 \\
\hline & $\mathrm{SL}_{2} \mathrm{~S}_{\mathrm{p}}$ & $\begin{array}{l}\text { Shifted (offset) least-squares } \\
\text { sphere: Minimize the sum of the } \\
\text { squares of the distances to the } \\
\text { sphere. Then change the radius so } \\
\text { that the sphere contacts the } \\
\text { outermost point(s). }\end{array}$ & 1 \\
\hline & $\mathrm{CL}_{2} \mathrm{IS}_{\mathrm{p}}$ & $\begin{array}{l}\text { Constrained least-squares } \\
\text { inscribing sphere: Minimize the } \\
\text { sum of the squares of the distances } \\
\text { to the sphere, subject to the } \\
\text { constraint that the sphere inscribes } \\
\text { (keeps outside) all the input points. }\end{array}$ & $2 *$ \\
\hline & $\mathrm{CL} 2 \mathrm{CS}_{\mathrm{p}}$ & $\begin{array}{l}\text { Constrained least-squares } \\
\text { circumscribing sphere: Minimize } \\
\text { the sum of the squares of the } \\
\text { distances to the sphere, subject to } \\
\text { the constraint that the sphere } \\
\text { circumscribes (keeps inside) all the } \\
\text { input points. }\end{array}$ & $2 *$ \\
\hline
\end{tabular}

* indicates new results reported in this paper.
In practice, a discrete set of points (also called input points) will be sampled on the arbitrary, closed curve as shown in Fig. 3 (b). Then the distance of any input point $p_{i}$ to a circle $C$ is just the difference between the radii $r_{i}$ and $r_{c}$ shown in Fig. 3(b). That is, $d\left(p_{i}, C\right)=\left|r_{i}-r_{c}\right|$. A circle $C$ can be fitted to the set of input points by reducing the distances of the input points to the circle using some appropriate criteria.

The circle fitting problem can then be posed as an optimization problem involving the input points and the parameters of the fitted circle (e.g., the coordinates of its center $c$ and radius $r_{c}$ ). In fact, the same definition and parameters hold for the sphere fitting problem and the cylinder fitting problem (where the 'center' is replaced by the 'axis of the cylinder'). With these preliminaries, the combinatorial characterizations of the optimality conditions for various fitting problems involving circles, cylinders, and spheres are summarized in Table 2 . The input to each of these optimization problems is a discrete set of points. The starred items shown in the last column of Table 2 are new, and are proved in Section 5 of this paper. All the other items in the last column of Table 2 are quite well known in research literature and engineering practice $[8,15]$.

It is worth noting that shifting (offsetting) the radius for $\mathrm{SL}_{2} \mathrm{C}, \mathrm{SL}_{2} \mathrm{C}_{\mathrm{y}}$, and $\mathrm{SL}_{2} \mathrm{~S}_{\mathrm{p}}$ in Table 2 does not change the center or axis, and therefore has no effect on the established datum itself based on unconstrained least-squares fitting. Also worth noting is the absence of minimum-zone fittings of circles, cylinders, and spheres from Table 2. While the minimum-zone fittings are important for the specification and verification of form tolerances such as roundness and cylindricity $[1,3,15]$, they are not relevant for datum establishment.

The optimality conditions for MIC and $\mathrm{MIS}_{\mathrm{p}}$ shown in Table 2 have inspired the design of some elegant algorithms to find global solutions. These involve nearest-neighbor Voronoi diagrams of the input points and their dual in the form of Delaunay triangles and Delaunay tetrahedra [23]. Since Delaunay triangles/tetrahedra can be derived from convex hulls (albeit in spaces with one dimension higher), clever algorithms can be designed to compute MIC and $\mathrm{MIS}_{\mathrm{p}}$ within tractable computational complexity. Some of these algorithms can exploit the increasingly ubiquitous GPUs to speed up the computation.

In the same vein, the optimality conditions for MCC and $\mathrm{MCS}_{\mathrm{p}}$ shown in Table 2 can be used to design algorithms to find globally optimal solutions. These algorithms involve furthestneighbor Voronoi diagrams, which can also be derived from convex hulls in higher dimensions [23].

\section{Computational Investigation of Constrained Least- squares Fitting}

Buoyed by the initial success of the constrained leastsquares fitting of planes to establish planar datums, a computational investigation of the constrained least-squares fitting for circles, cylinders, and spheres was conducted recently [11]. This empirical study used a general-purpose solver for the constrained non-linear optimization problems of $\mathrm{CL}_{2} \mathrm{IC}, \mathrm{CL}_{2} \mathrm{IC}_{\mathrm{y}}$, and $\mathrm{CL}_{2} \mathrm{IS}_{\mathrm{p}}$. The results were compared to the solutions of more commonly used fittings $\mathrm{MIC}, \mathrm{MIC}_{\mathrm{y}}$, and $\mathrm{MIS}_{\mathrm{p}}$, and were found 
to exhibit several beneficial properties, such as the one described below.

Consider the case of a set of sampled points on a 'pinched' circle shown dotted in Fig. 4. The solid circle shows a constrained (inscribing) least-squares circle $\left(\mathrm{CL}_{2} \mathrm{IC}\right)$ fitted to these input points. The MIC problem to the same set of input points will provide two solutions, one centered at $c_{l}$ on the left half of the pinched circle and the other centered at $c_{r}$ on the right half of the pinched circle. Since the datums critically depend on the location of these centers (and axes for cylinders), it is quite disconcerting that even a small perturbation of the input points can cause violent changes in the centers of the MIC solutions. The center of the $\mathrm{CL}_{2} \mathrm{IC}$ solution, on the other hand, is very stable in the sense that small changes in the input points will lead only to small changes in the location of the datum.

Results from the computational investigations for constrained least-squares fitting of cylinders and spheres reproduced the same beneficial effects found for circles [11]. These computational investigations looked only into the inscribing circles, cylinders, and spheres. However, the results were sufficiently encouraging to prompt a theoretical investigation of the constrained least-squares fitting problem for the non-linear datum elements. A first step towards this theoretical investigation is taken in the next section.

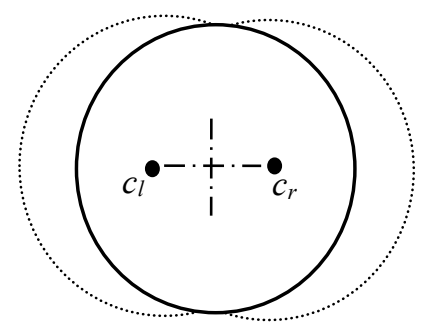

Figure 4. Fitting a constrained (inscribed) least-squares circle to a set of input points.

\section{Optimality Conditions for the Constrained Least- squares Fitting}

The combinatorial characterizations of the optimality conditions are proved in this section by considering the circles first. It is then shown that the equations, statements, and proofs can be easily extended to the cases of spheres and cylinders. Section 5.1 proves that there should be at least one contact point for optimality using a strategy of growing and shrinking concentric circles. The results are extended in Section 5.2, where proofs are provided that there should be at least two contact points for optimality using a strategy of growing and shrinking co-tangential circles. Then Section 5.3 presents cases that involve only two contact points at the optimal condition. The possibility of having more than two contact points for optimality is explored in Section 5.4 using a strategy of growing and shrinking co-chordal circles.
To set the stage for proving these results, consider a set of $n$ input points and three concentric circles with center $c$ in a plane as shown in Fig. 5. The radial distance of any point $p_{i}$ from $c$ is indicated as $r_{i}$. The radius of any arbitrary circle with an arbitrary center $c$ is indicated as $r_{c}$. Also, let $C_{I}$ be any (small) inscribing circle centered at $c$ and $C_{C}$ be any (large) circumscribing circle centered at $c$. Since their radii can be arbitrarily small or large, such circles $C_{I}$ and $C_{C}$ can always be found. (Note that an inscribing circle keeps all the input points outside of it, and a circumscribing circle keeps all the input points inside of it.)

With these notations, the optimization problems for constrained least-squares fitting of circles can be posed as

subject to

$$
\min _{c, r_{c}} \sum_{i=1}^{n}\left(r_{i}-r_{c}\right)^{2}
$$

$$
\begin{aligned}
& r_{c} \leq r_{i}, \forall i \text { for } \mathrm{CL}_{2} \mathrm{IC}, \\
& r_{c} \geq r_{i}, \forall i \text { for } \mathrm{CL}_{2} \mathrm{CC} .
\end{aligned}
$$

\subsection{One point of contact}

It can be easily shown that the optimum circle must contact at least one input point. For this, consider the small inscribing circle $C_{I}$ in Fig. 5 that keeps all the input points outside of it. Such a small circle of arbitrarily small radius can always be found, and let $r_{c}$ be its variable radius. Grow $C_{I}$ concentrically (keeping its center at $c$ ) by increasing its radius $r_{c}$ gradually while maintaining its inscribing constraints given by Eq. (2). Then every term in the sum of Eq. (1) is reduced, leading to a reduction in the objective function till a first input point is contacted. Stop at that instant because proceeding any further will violate the constraints in Eq. (2). Since this is true for any center $c$, including the one for the optimal solution, it leads to the following result.

Lemma 1a: $\mathrm{CL}_{2} \mathrm{IC}$ must contact at least one input point.

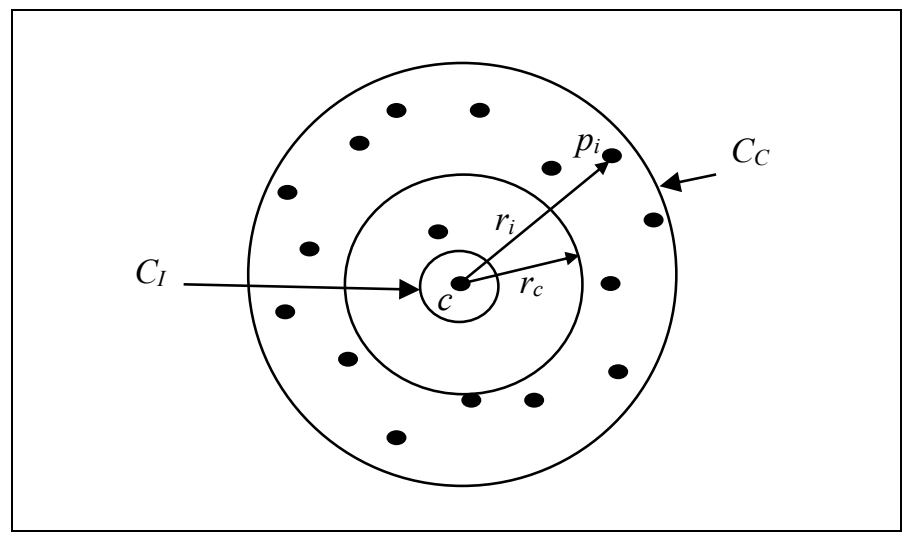

Figure 5. A set of points and concentric circles in a plane.

Next consider the large circumscribing circle $C_{C}$ in Fig. 5 that keeps all the input points inside of it. Such a large circle with arbitrarily large radius can always be found, and let $r_{c}$ be its 
variable radius. Shrink $C_{C}$ concentrically (keeping its center at $c$ ) by decreasing its radius $r_{c}$ gradually while maintaining its circumscribing constraints given by Eq. (3). Then every term in the sum of Eq. (1) is reduced, leading to a reduction in the objective function till a first input point is contacted. Stop at that instant because proceeding any further will violate the constraints in Eq. (3). Since this is true for any center $c$, including the one for the optimal solution, it leads to the following result.

Lemma 1b: $\mathrm{CL}_{2} \mathrm{CC}$ must contact at least one input point.

These results can be generalized to spheres. For this, just replace the word 'circle' with 'sphere' in every exposition so far in this section and consider the input points to be located in space instead of a plane. The following results then emerge.

Lemma 2a: $\mathrm{CL}_{2} \mathrm{IS}_{\mathrm{p}}$ must contact at least one input point.

Lemma 2b: $\mathrm{CL}_{2} \mathrm{CS}_{\mathrm{p}}$ must contact at least one input point.

It can be seen that these results apply to cylinders as well. Again, replace the word 'circle' with 'cylinder' and consider the input points located in space. Then 'concentric' circles become 'coaxial' cylinders. Also, set the viewpoint along the axis of the cylinder so that all the objects are projected onto a plane perpendicular to the axis. Then the axis itself is projected onto the center $c$ as in Fig. 5, and all the arguments will hold for the cylinders as well leading to the following results.

Lemma 3a: $\mathrm{CL}_{2} \mathrm{IC}_{\mathrm{y}}$ must contact at least one input point.

Lemma 3b: $\mathrm{CL}_{2} \mathrm{CC}_{\mathrm{y}}$ must contact at least one input point.

\subsection{Two points of contact}

Can the optimal circles, cylinders, and spheres contact more than one input point? To answer this question, consider the circles again to establish the following result.

Theorem 1a: $\mathrm{CL}_{2} \mathrm{IC}$ must contact at least two input points.

Proof: By Lemma 1a, there should be at least one contact point for the inscribing circle. Without any loss of generality, let $p_{1}$ be that first contact point from the input set. The proof relies on growing the inscribing circle co-tangentially at $p_{1}$ while maintaining the inscribing constraints of Eq. (2). Figure 6 shows the construction to aid the proof.

In Fig. $6, C_{l}$ is the inscribing circle with center $c_{l}$ that makes the first contact with the input point $p_{l} . C_{2}$ is another inscribing circle with center $c_{2}$ that is co-tangential to $C_{1}$ at $p_{1}$ having $T_{1}$ as the co-tangent. $C_{2}$ is obtained by growing $C_{1}$ gradually and cotangentially while maintaining the inscribing constraints of Eq. (2). By this assumption, all the other input points are outside of both $C_{1}$ and $C_{2}$. Let $p_{i}$ be one such input point.

In Fig. $6, C_{3}$ is a circle centered $p_{i}$ and tangential to $C_{2}$ at the point $q_{2}$ with common tangent $T_{2}$. The radial line joining centers $c_{2}$ and $p_{i}$ will obviously intersect circle $C_{2}$ at $q_{2}$. Let $q_{1}$ be the intersection point of the radial line between centers $c_{l}$ and $p_{i}$. It is clear from this construction that point $q_{l}$ lies strictly outside of the circle $C_{3}$ because circle $C_{1}$ is completely contained within circle $C_{2}$. Therefore, $p_{i} q_{2}<p_{i} q_{1}$. (A special case arises when $p_{i}$ lies directly below $p_{1}$ on the vertical line through $c_{2}, c_{1}$, and $p_{1}$ in Fig. 6. Then $q_{1}, q_{2}$, and $p_{1}$ coincide, resulting in $p_{i} q_{2}=p_{i} q_{1}$.)

Note that $p_{i} q_{2}$ is the distance of the point $p_{i}$ to the circle $C_{2}$ and $p_{i} q_{1}$ is the distance of the $p_{i}$ to the circle $C_{l}$. Therefore, each term in the sum of the objective function in Eq. (1) is strictly reduced as $C_{l}$ is grown co-tangentially to $C_{2}$. (In the special case of a point $p_{i}$ discussed above, where $p_{i} q_{2}=p_{i} q_{1}$, that term remains unchanged.) Such reduction in the objective function continues till $C_{2}$ contacts a second input point. Stop at that instant, because proceeding any further will violate the inscribing constraints in Eq. (2). This proves the assertion that $\mathrm{CL}_{2} \mathrm{IC}$ must contact at least two input points.

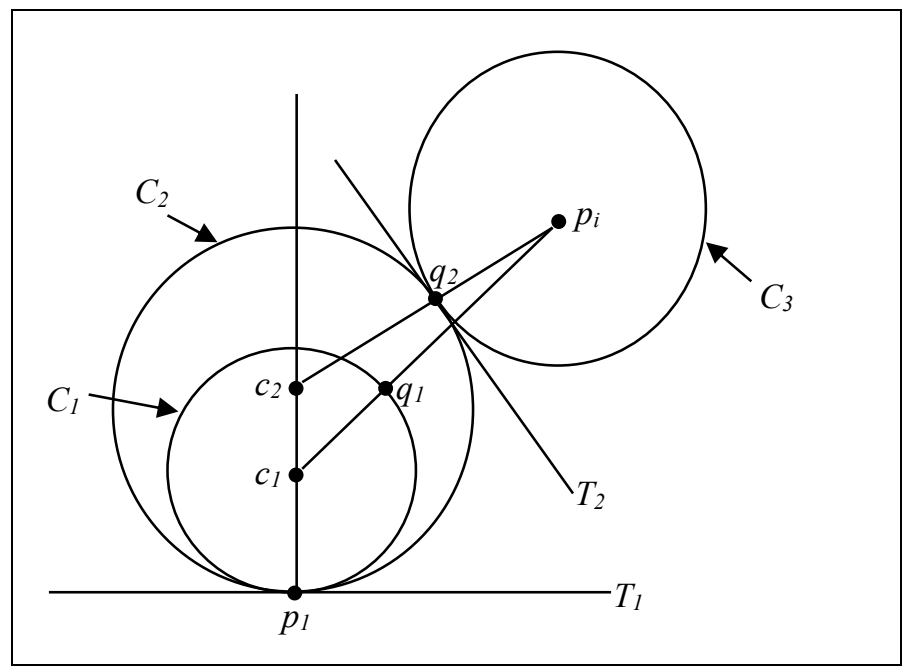

Figure 6. Construction for the proof of Theorem 1a.

Pursuing the same strategy, the following assertion can be proved.

Theorem 1b: $\mathrm{CL}_{2} \mathrm{CC}$ must contact at least two input points.

Proof: By Lemma 1b, there should be at least one contact point for the circumscribing circle. Without any loss of generality, let $p_{I}$ be that first contact point from the input set. The proof relies on shrinking the circumscribing circle co-tangentially at $p_{1}$ while maintaining the circumscribing constraints of Eq. (3). Figure 7 shows the construction to aid the proof.

In Fig. $7, C_{1}$ is the circumscribing circle with center $c_{1}$ that makes the first contact with the input point $p_{1} . C_{2}$ is another circumscribing circle with center $c_{2}$ that is co-tangential to $C_{1}$ at $p_{1}$ having $T_{1}$ as the co-tangent. $C_{2}$ is obtained by shrinking $C_{1}$ gradually and co-tangentially while maintaining the circumscribing constraints of Eq. (3). By this assumption, all the 
other input points are inside of both $C_{l}$ and $C_{2}$. Let $p_{i}$ be one such input point.

In Fig. 7, $C_{3}$ is a circle centered $p_{i}$ and tangential to $C_{2}$ at the point $q_{2}$ with common tangent $T_{2}$. The radial line joining centers $c_{2}$ and $p_{i}$ will obviously intersect circle $C_{2}$ at $q_{2}$. Let $q_{1}$ be the intersection point on $C_{l}$ of the radial line joining centers $c_{l}$ and $p_{i}$. It is clear from this construction that point $q_{1}$ lies outside of the circle $C_{3}$ because circle $C_{1}$ lies completely outside of circle $C_{2}$, which lies outside of circle $C_{3}$. Therefore, $p_{i} q_{2}<p_{i} q_{1}$. (A special case arises when $p_{i}$ coincides with $c_{2}$ in Fig. 7. Then $q_{1}$, $q_{2}$, and $p_{1}$ coincide, resulting in $p_{i} q_{2}=p_{i} q_{1}$.)

Note that $p_{i} q_{2}$ is the distance of the point $p_{i}$ to the circle $C_{2}$ and $p_{i} q_{1}$ is the distance of the $p_{i}$ to the circle $C_{l}$. Therefore, each term in the sum of the objective function in Eq. (1) is strictly reduced as $C_{1}$ is shrunk co-tangentially to $C_{2}$. (In the special case discussed above, where $p_{i} q_{2}=p_{i} q_{1}$, that term remains unchanged.) Such reduction in the objective function continues till $C_{2}$ contacts a second input point. Stop at that instant, because proceeding any further will violate the circumscribing constraints in Eq. (3). This proves the assertion that $\mathrm{CL}_{2} \mathrm{CC}$ must contact at least two input points.

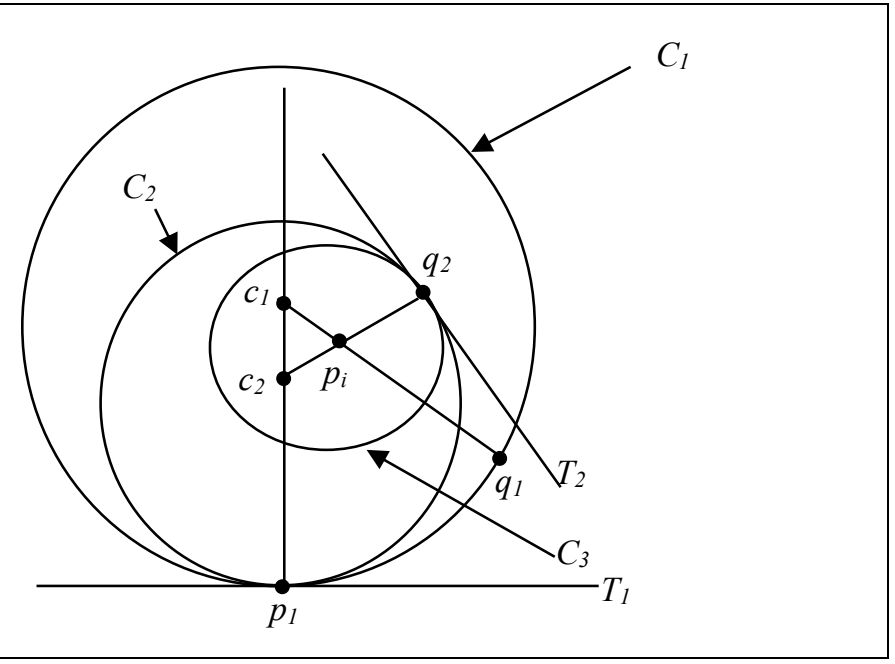

Figure 7. Construction for the proof of Theorem $1 \mathrm{~b}$.

By replacing the word 'circle' with 'sphere' in Eqs. (1), (2) and (3), and in the proofs and statements of Theorems $1 \mathrm{a}$ and $1 \mathrm{~b}$, the following results are obtained.

Theorem 2a: $\mathrm{CL}_{2} \mathrm{IS}_{\mathrm{p}}$ must contact at least two input points.

Theorem 2b: $\mathrm{CL}_{2} \mathrm{CS}_{\mathrm{p}}$ must contact at least two input points.

Obtaining similar results for cylinders requires only slightly more work. The words 'circle' and 'center' should be replaced by 'cylinder' and 'axis,' respectively, in Eqs. (1), (2), and (3). Also, the same replacement should be applied in the proofs and statements of Theorems $1 \mathrm{a}$ and $1 \mathrm{~b}$, while the constructions in the Figs. 6 and 7 should be viewed as projections on a plane perpendicular to the axes of the cylinders. (Note that the axes of these cylinders are parallel, and the term 'coaxial' replaces 'concentric'; the term 'co-tangential' remains the same.) After making these replacements, it is possible to grow and shrink cotangential cylinders in the proofs of Theorems $1 \mathrm{a}$ and $1 \mathrm{~b}$ even if the first contact, shown as $p_{l}$ in Figs. 6 and 7, is made by cylinder $C_{l}$ at two or more points that lie on the same generator (also known as generatrix) line of $C_{l}$. Then, the following results are obtained.

Theorem 3a: $\mathrm{CL}_{2} \mathrm{IC}_{\mathrm{y}}$ must contact at least two input points that do not lie on the same generator line.

Theorem 3b: $\mathrm{CL}_{2} \mathrm{CC}_{\mathrm{y}}$ must contact at least two input points that do not lie on the same generator line.

\subsection{Cases with only two points of contact}

Can the minimum number of contact points be more than two for any of the geometries considered? No, as this subsection will show that there are cases that have only two contact points at the optimal solution. The first case involves circles in a plane, and this case is then expanded to space to produce a case for spheres. Finally, a case is constructed for cylinders in space.

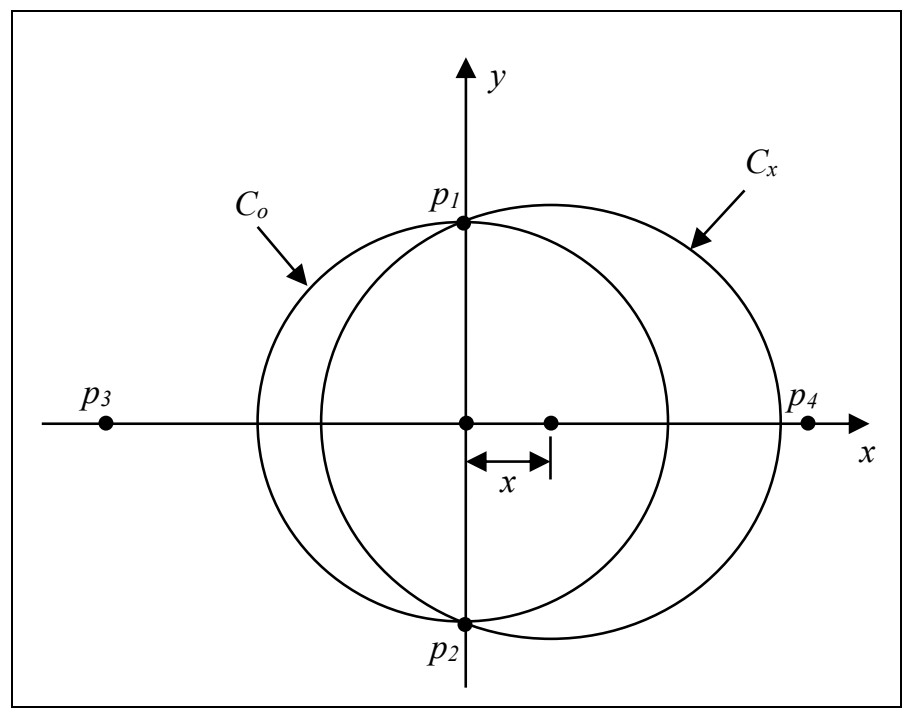

Figure 8. Construction of an example case with only two contact points for an optimal circle.

To start with circles, consider four input points in a plane as shown in Fig. 8, with the following coordinates:

$$
p_{1}:(0,1), p_{2}:(0,-1), p_{3}:(-1-\Delta, 0) \text {, and } p_{4}:(1+\Delta, 0) \text {. }
$$

Here $\Delta$ can be any arbitrarily small positive or negative number. Initially, $\Delta$ will be assigned an arbitrarily small positive value for producing the case of an inscribing circle. In this case, one can view the four input points as representing a 'pinched' circle. Let $C_{o}$ be the unit circle centered at the origin with unit radius, 
contacting points $p_{1}$ and $p_{2}$. With a small positive value for $\Delta, C_{o}$ is an inscribing circle for the four input points. It can be proved that $C_{o}$ is also the inscribing circle that minimizes the sum of the squares of its distances from the input points; that is, $C_{o}$ is the $\mathrm{CL}_{2} \mathrm{IC}$ for these four input points. To prove this assertion, consider another inscribing circle $C_{x}$ centered at $(x, 0)$ for a very small value of $x$, and contacting points $p_{1}$ and $p_{2}$ as shown in Fig. 8.

From Theorem 1a, $C_{x}$ can aspire to be an optimal solution because it is an inscribing circle maintaining contact with at least the two input points $p_{1}$ and $p_{2}$. The objective function (the sum of the squared distances of the four input points to a circle) for $C_{x}$ in terms of $x$, and the first and second derivatives of the objective function can be easily seen to be the following:

$$
\begin{gathered}
f(x)=\left\{(1+\Delta)-x-\sqrt{x^{2}+1}\right\}^{2} \\
+\left\{(1+\Delta)+x-\sqrt{x^{2}+1}\right\}^{2}, \\
f^{\prime}(x)=8 x-4(1+\Delta) x\left(x^{2}+1\right)^{-1 / 2}, \text { and } \\
f^{\prime \prime}(x)=8-4(1+\Delta)\left\{\left(x^{2}+1\right)^{-1 / 2}-x^{2}\left(x^{2}+1\right)^{-3 / 2}\right\} .
\end{gathered}
$$

Therefore at $x=0$,

$$
f(0)=2 \Delta^{2}, f^{\prime}(0)=0 \text {, and } f^{\prime \prime}(0)=4(1-\Delta) .
$$

It means that $f^{\prime \prime}(0)>0, \forall \Delta<1$ at $x=0$. This proves that $C_{0}$ achieves a local minimum for the objective function [12]. Since there are only four input points, $C_{0}$ also achieves the global minimum and is the $\mathrm{CL}_{2} \mathrm{IC}$ that contacts only two input points. (Note that the two circles that contact three points without contacting both $p_{1}$ and $p_{2}$ (i.e., are not centered at $\left.(x, 0)\right)$ are not inscribing circles.)

To present a circumscribing case with only two contact points, consider Fig. 8 again and the related discussions with Theorem $2 \mathrm{~b}$ in mind, but now with an arbitrarily small negative value for $\Delta$. The expressions for the objective function and its first and second derivatives will still remain the same as above, leading to the result that $f^{\prime}(0)=0$ and $f^{\prime \prime}(0)>0$ at $x=0$. So $C_{0}$ achieves a local minimum. Since there are only four input points, $C_{0}$ also achieves the global minimum, and is the $\mathrm{CL}_{2} \mathrm{CC}$ that contacts only two input points.

Proving the existence of optimal spheres with only two contact points requires a different construction. For this, consider six input points in space as shown in Fig. 9, with the following coordinates:

$$
\begin{gathered}
p_{1}:(0,0,1), p_{2}:(0,0,-1), p_{3}:(1+\Delta, 0,0), p_{4}:(-1-\Delta, 0,0), \\
p_{5}:(0,1+\Delta, 0), \text { and } p_{6}:(0,-1-\Delta, 0)
\end{gathered}
$$

For a small positive value of $\Delta$, these six points may be viewed as representing a sphere 'pinched' at the poles. Let $S_{0}$ be a unit sphere centered at the origin with unit radius that contacts input points $p_{1}$ and $p_{2}$. The goal is to show that $S_{0}$ is the $\mathrm{CL}_{2} \mathrm{IS}_{\mathrm{p}}$ for a small positive value of $\Delta$, and $S_{0}$ is the $\mathrm{CL}_{2} \mathrm{CS}_{\mathrm{p}}$ for a small negative value of $\Delta$.

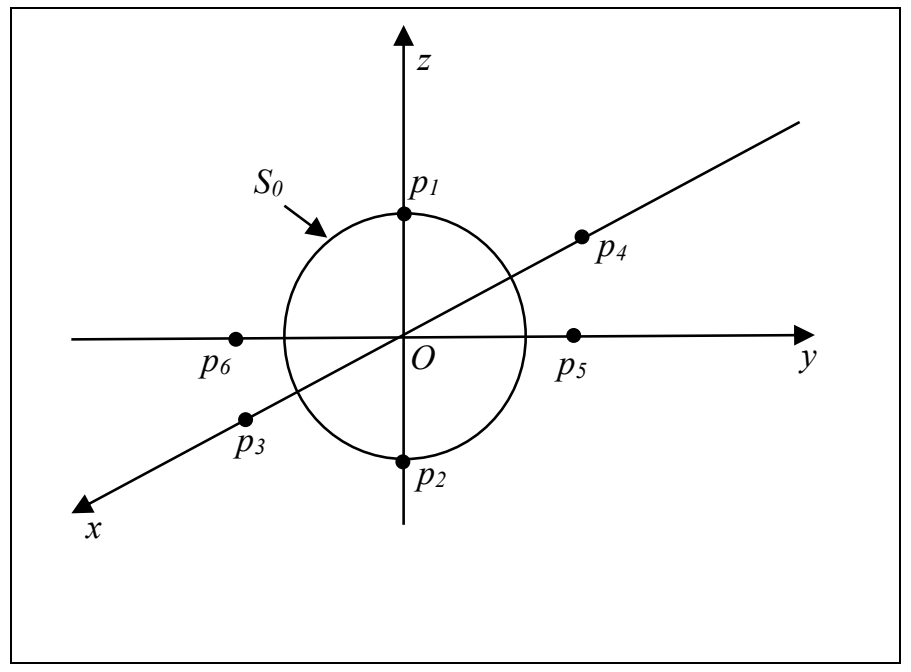

Figure 9. Construction of an example case with only two contact points for an optimal sphere.

For this, consider another sphere $S_{x}$ centered at $(x, y, 0)$ for small values of $x$ and $y$. Also assume that $S_{x}$ contacts only $p_{l}$ and $p_{2}$ from the input set of points. An objective function $F(x, y)$ for the sum of the squared distances of the six input points to $S_{x}$, and the first and second partial derivatives of $F(x, y)$ can be derived in closed form. At $x=y=0$ these closed form expressions yield the following values for the objective function, its gradient vector, and Hessian matrix:

$$
\begin{gathered}
F(0,0)=4 \Delta^{2}, \\
\nabla F(0,0)=\left\{\begin{array}{l}
0 \\
0
\end{array}\right\}, \text { and } \\
H(0,0)=\left[\begin{array}{cc}
8(1-\Delta)-\frac{4}{(1+\Delta)} & 0 \\
0 & 8(1-\Delta)-\frac{4}{(1+\Delta)}
\end{array}\right] .
\end{gathered}
$$

So the gradient vanishes at the origin. The eigenvalues of the Hessian matrix $H$ are both close to 4 at the origin for small positive or negative values of $\Delta$. Therefore, $H$ is positive definite for any small positive or negative value of $\Delta$. These conditions are sufficient to show that $S_{0}$ achieves a local minimum for the objective function [12]. Since there are only six input points, $S_{0}$ also achieves the global minimum contacting only two input points. Thus the following fact is established: when $\Delta$ is a small positive number, $S_{0}$ is the $\mathrm{CL}_{2} \mathrm{IS}_{\mathrm{p}}$; similarly, when $\Delta$ is a small negative number, $S_{0}$ is the $\mathrm{CL}_{2} \mathrm{CS}_{\mathrm{p}}$.

The examples for the case of circles also motivate similar examples for the case of cylinders. In addition to the four input 
points (for circles) in the $z=0$ plane as shown in Fig. 8, consider additional eight points with coordinates given by

$$
\begin{gathered}
p_{5}:(0,1+\Delta,-1), p_{6}:(0,-1-\Delta,-1), \\
p_{7}:(-1-\Delta, 0,-1), p_{8}:(1+\Delta, 0,-1), \\
p_{9}:(0,1+\Delta, 1), p_{10}:(0,-1-\Delta, 1), \\
p_{11}:(-1-\Delta, 0,1), \text { and } p_{12}:(1+\Delta, 0,1)
\end{gathered}
$$

One can view these twelve points representing a 'pinched' cylinder for a small positive value of $\Delta$. Reasoning with these twelve input points in space in a manner very similar to the cases of the circles discussed above, it can be shown that there are the cases where $\mathrm{CL}_{2} \mathrm{IC}_{\mathrm{y}}$ and $\mathrm{CL}_{2} \mathrm{CC}_{\mathrm{y}}$ have only two contact points $p_{1}$ and $p_{2}$. For this, let a cylinder be represented by four parameters $(x, y, a, b)$ using the point $(x, y, 0)$ where the axis of the cylinder pierces the $x y$-plane, and the direction cosines of the axis as $\left(a, b, \sqrt{1-a^{2}-b^{2}}\right)$. (The radius is taken to be the largest/smallest possible while still inscribing/circumscribing the points.) Then the values for the objective function $F(x, y, a, b)$, its gradient, and Hessian matrix entries can be obtained using a symbolic derivative calculations software as

$$
\begin{gathered}
F(0,0,0,0)=10 \Delta^{2}, \\
\nabla F(0,0,0,0)=\left\{\begin{array}{l}
0 \\
0 \\
0 \\
0
\end{array}\right\} \text {, and } \\
H(0,0,0,0)=\left[\begin{array}{cccc}
12+O(\Delta) & 0 & 0 & 0 \\
0 & \text { large positive } & 0 & 0 \\
0 & 0 & 8+O(\Delta) & 0 \\
0 & 0 & 0 & 8+O(\Delta)
\end{array}\right] .
\end{gathered}
$$

The phrase 'large positive' in the Hessian matrix indicates that the function has a convex corner (much like $y=|x|$ has at $x=$ $0)$. These results of a zero gradient and a positive definite Hessian (for a small positive or negative value of $\Delta$ ) indicate that the solution is a local minimum, and the simplicity of the construction of the example is sufficient to show that this local minimum is also the global minimum.

\subsection{Possibility of more than two points of contact}

The existence of cases with only two input points of contact does not preclude the possibility that there can be more points of contact when the input points are still in general position. For example, the construction of Fig. 8 suggests a procedure for a $\mathrm{co}$ chordal traversal to seek a better solution.

Consider the case shown in Fig. 10, where $p_{1}$ and $p_{2}$ are two contact points from the input set for an inscribing circle $C_{l}$. A one-parameter family of co-chordal circles, all contacting $p_{l}$ and $p_{2}$ (hence having $\overline{p_{1} p_{2}}$ as the common chord) and yet inscribing all the input points, can be considered for possible reduction in the objective function. Let $C_{2}$ be one member in such a family of co-chordal circles.

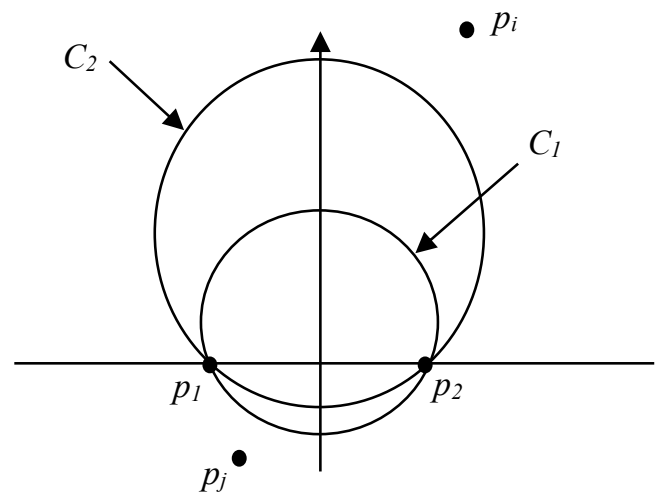

Figure 10. A one-parameter family of inscribing circles having a common chord.

It can be easily seen that for points such as $p_{i}$ in Fig. 10, $C_{2}$ will be closer than $C_{l}$ thus contributing a reducing term to the objective function in Eq. (1). But for points such as $p_{j}$ in Fig. 10, $C_{2}$ will be farther away than $C_{1}$ leading to an increase in the objective function. So it is possible that when all the terms are summed up in Eq. (1), there might be a net reduction in the objective function for $C_{2}$. This reduction might continue as $C_{2}$ is grown in this co-chordal traversal process till $C_{2}$ contacts a third input point.

Such optimal conditions involving three contact points for $\mathrm{CL}_{2} \mathrm{IC}$ have been observed in computational investigations [11], leading to the pleasing result that $\mathrm{MIC}$ and $\mathrm{CL}_{2} \mathrm{IC}$ may turn out to be the same in these cases. Similar results have been observed for cylinders and spheres. These empirical observations in numerical trials seem to suggest that in many cases, where the input points are in general position, the constrained least-squares solutions may coincide with the maximum inscribing and minimum circumscribing solutions. And where small perturbations in the input points can cause instabilities in the maximum inscribing and minimum circumscribing solutions, an example of which is shown in Fig. 4, the constrained leastsquares approach can lead to a stable solution.

\section{Summary and Concluding Remarks}

This paper addressed the problem of establishing datums using constrained least-squares fitting to input points sampled on non-linear elements such as circles, cylinders, and spheres. Combinatorial characterizations of the optimality conditions for these constrained non-linear optimization problems were provided with rigorous proofs. These results have been verified by computational investigations using research software [11].

These necessary conditions, in the form of the minimum number of points of contact, not only enable a check of optimal solution candidates but also inspire design of new algorithms to find global solutions. For circles and spheres, the fact that the optimal solution must contact at least two input points implies that the centers of such circles and spheres must lie on the edges 
or surfaces of the Voronoi diagram of the input points. This leads to a dramatic reduction the search space for globally optimal solution. Algorithms that exploit this fact may also employ GPUs to speed up the search.

Further research is necessary to investigate such algorithmic issues. Another important open research problem is the extension of the constrained least-squares fitting to a continuous set of points, such as the one shown in Fig. 3(a). Robust implementations of constrained least-squares fitting algorithms in commercial software will be important for their acceptance by industry. Preliminary response from leading CMS vendors indicates that they are indeed beginning to implement and test their software using the results of this paper.

\section{Acknowledgment and Disclaimer}

The authors thank ISO and ASME standards experts whose advice and suggestions were invaluable in initiating and sustaining this research investigation. Any mention of commercial products or systems in this article is for information only; it does not imply recommendation or endorsement by NIST.

\section{References}

[1] ASME Y14.5-2009, Dimensioning and Tolerancing, The American Society of Mechanical Engineers, New York, 2009.

[2] ASME Y14.5.1-1994, Mathematical Definition of Dimensioning and Tolerancing Principles, The American Society of Mechanical Engineers, New York, 1994.

[3] ISO 1101:2012, Geometrical product specifications (GPS) Geometrical tolerancing - Tolerances of form, orientation, location and run-out, International Organization for Standardization, Geneva, 2012.

[4] ISO 5459:2011, Geometrical product specifications (GPS) Geometrical tolerancing - Datums and datum systems, International Organization for Standardization, Geneva, 2011.

[5] Nielsen, H.S., The ISO Geometrical Product Specifications Handbook - Find your way in GPS, International Organization for Standardization, Geneva, 2012.

[6] Krulikowski, A., Fundamentals of Geometric Dimensioning and Tolerancing, $3^{\text {rd }}$ Edition, Effective Training Inc., Westland, MI, 2012.

[7] Srinivasan, V., Geometrical Product Specification, in CIRP Encyclopedia of Production Engineering, Laperrière, L. and Reinhart, G. (Eds), Springer, 2014.

[8] Wick, C. and Veilleux, R.F., Tool and Manufacturing Engineers Handbook, Vol. IV Quality control and assembly, $4^{\text {th }}$ Edition, Society of Manufacturing Engineers, Dearborn, MI, 1987.

[9] Shakarji, C.M. and Srinivasan, V., A constrained $\mathrm{L}_{2}$ based algorithm for standardized planar datum establishment, ASME IMECE2015-50654, Proceedings of the ASME 2015 International Mechanical Engineering Congress and Exposition, Houston, TX, Nov. 13-19, 2015.
[10] Shakarji, C.M. and Srinivasan, V., Theory and algorithm for planar datum establishment using constrained total leastsquares, $14^{\text {th }}$ CIRP Conference on Computer Aided Tolerancing, Gothenburg, Sweden, 2016.

[11] Shakarji, C.M. and Srinivasan, V., Computational investigations for a new, constrained least-squares datum definition for circles, cylinders, and spheres, ASME IMECE2016-66753, Proceedings of the ASME 2016 International Mechanical Engineering Congress and Exposition, Phoenix, AZ, Nov. 11-17, 2016.

[12] Gill, P.E., Murray, W., and Wright, M.H., Practical Optimization, Emerald Group Publishing Ltd., 1982.

[13] Hopp, T.H., The mathematics of datums, ASPE Newsletter, The American Society of Precision Engineers, Raleigh, NC, 1990.

[14]Zhang, H. and Rou, U., Criteria for establishing datums in manufactured parts, Journal of Manufacturing Systems, 12(1), pp. 36-50, 1993.

[15] Anthony, G.T. et al., Chebyshev Best-Fit Geometric Elements, NPL Report DITC 221/93, National Physical Laboratory, U.K., 1993.

[16] Butler, B.P., Forbes, A.B., and Harris, P.M., Algorithms for geometric tolerance assessment, NPL Report DITC 228/94, National Physical Laboratory, U.K., 1994.

[17] Srinivasan, V., Reflections on the role of science in the evolution of dimensioning and tolerancing standards, Proceedings of the Institution of Mechanical Engineers, Part B: Journal of Engineering Manufacture, 227(1), pp. 3-11, 2013.

[18] Srinivasan, V., Shakarji, C.M. and Morse, E.P., On the enduring appeal of least-squares fitting in computational coordinate metrology, ASME Journal of Computing and Information Science in Engineering, Vol. 12, March 2012.

[19] Shakarji, C.M. and Srinivasan, V., Theory and algorithms for weighted total least-squares fitting of lines, planes, and parallel planes to support tolerancing standards, ASME Journal of Computing and Information Science in Engineering, 13(3), 2013.

[20] Shakarji, C.M. and Srinivasan, V., Theory and algorithms for $\mathrm{L}_{1}$ fitting used for planar datum establishment in support of tolerancing standards, ASME DETC2013-12372, Proceedings of the ASME 2013 IDETC/CIE Conferences, Portland, OR, 2013.

[21] Shakarji, C.M. and Srinivasan, V., An improved $\mathrm{L}_{1}$ based algorithm for standardized planar datum establishment, ASME DETC2014-35461, Proceedings of the ASME 2014 IDETC/CIE Conferences, Buffalo, NY, 2014.

[22] Shakarji, C.M. and Srinivasan, V., Datum planes based on a constrained $\mathrm{L}_{1}$ norm, ASME Journal of Computing and Information Science in Engineering, Vol. 15, December 2015.

[23] O'Rourke, J., Computational Geometry in C, $2^{\text {nd }}$ Edition, Cambridge University Press, 1998. 